WSRC-TR-92-532

\title{
FLOWTRAN-TF Software Design (U)
}

S. E. Aleman, G. P. Flach, L. L. Hamm, S. Y. Lee \&
F. G. Smith III

\section{Publication Date: February, 1993}


TASK NUMBER: 89-021-1

DOCUMENT TTTLE: FLOWTRAN-TF Software Design (U)

DOCUMENT NUMBER: WSRC-TR-92-532

REVISION NUMBER: 0

APPROVALS
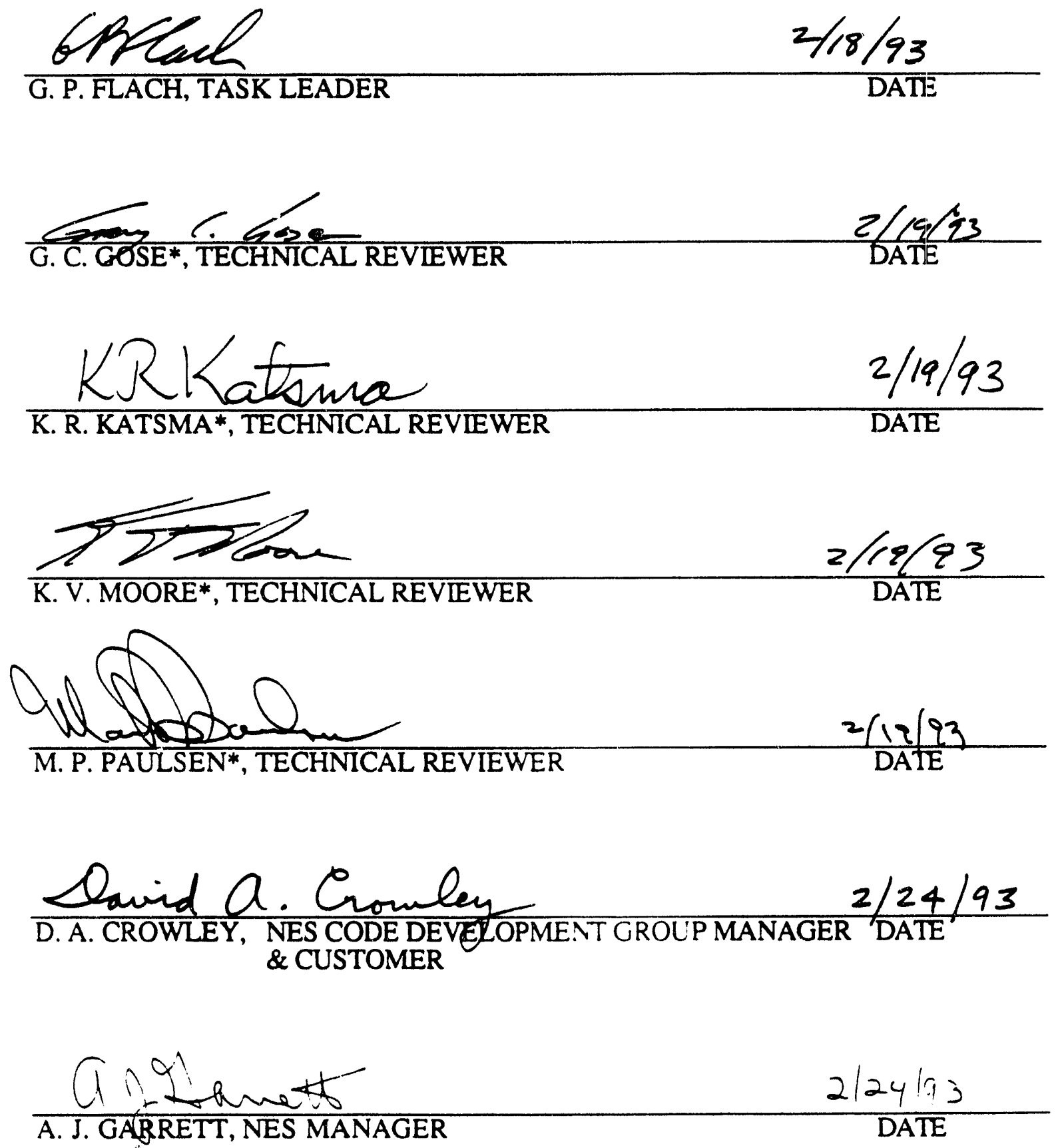

* Computer Simulation \& Analysis, Inc. 


\section{Acknowledgements}

The FLOWTRAN-TF code development effort was initiated in early 1989. Several members of the Code Development Group have contributed to FLOWTRAN-TF development as summarized alphabetically below:

\section{Contributor Principal Contributions}

Sebastian Aleman Axial wall drag, neutronic power distribution, splines, code architecture, documentation, coding, graphics, testing

Roger Cooper Coding, graphics

Greg Flach

Program leadership, interfacial drag, axial wall drag, phase transition logic, fluid finite-differencing and solution methods, equations of state, documentation, coding, testing, benchmarking

Larry Hamm Program concept, initiation and leadership, all aspects of physical model development and computational implementation, interfacial heat and mass transfer, code architecture, coding, testing, benchmarking

Andrea Kielpinski Interfacial heat and mass transfer coefficients, flow regime map, documentation

Larry Koffman Documentation

Si Young Lee

Channel inlet void distribution, CCFL correlation, inter-subchannel mixing, interfacial heat and mass transfer coefficients, rib void model, equations of state, documentation, coding, testing, benchmarking

Andy Shadday

Azimuthal wall drag, documentation, testing

Frank Smith

Solid heat conduction, neutronic power distribution, wall heat transfer, interfacial heat and mass transfer, fluid finite differencing and solution methods, code architecture and $\mathrm{V} O \mathrm{O}$, documentation, coding, testing, benchmarking

The developers are grateful to the many technical reviewers of FLOWTRAN-TF. These include Ken Moore, Garry Gose, Ken Katsma and M. Paulsen of Computer Simulation \& Analysis, Inc. and Steve Hensel and Jeff Jerrell of WSRC. The helpful suggestions of the DOE Independent Review Panel (DIRP) are also appreciated.

The developers also appreciate the support of the DOE, former WSRC Nuclear Engineering Section manager Ken Huffman and present manager Al Garrett, and former Code Development Group managers Larry Hamm and Bill Horton and current manager Dave Crowley. 
This page intentionally left blank 


\section{Table of Contents}

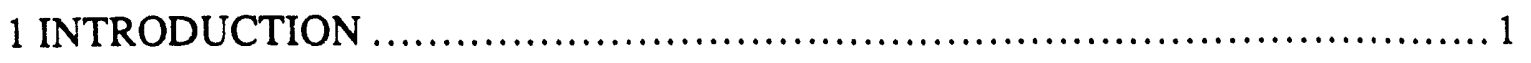

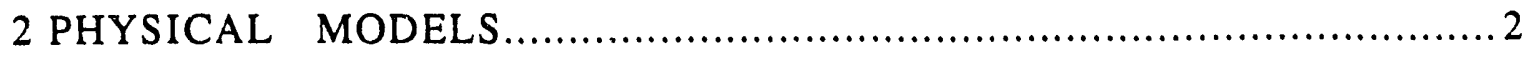

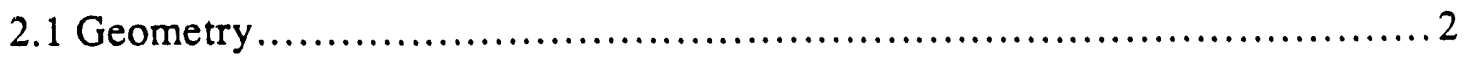

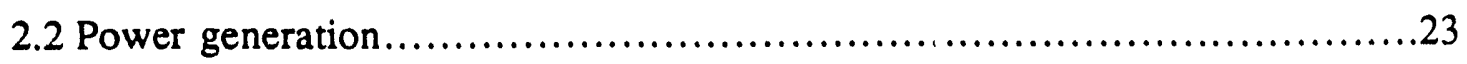

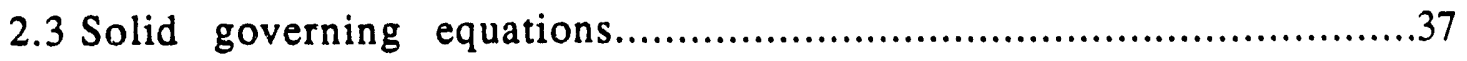

2.4 Fluid governing equations........................................................56

2.4.1 Space/time averaged two-phase two-component fluid field equations ........56

2.4.2 Equations of state.............................................................74

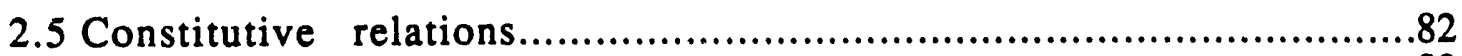

2.5.1 Wall heat transfer.......................................................

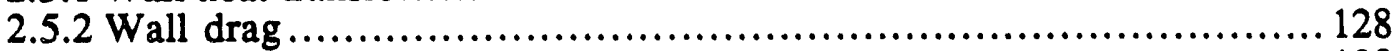

2.5.2.1 Axial wall drag model ...................................... 128

2.5.2.2 Azimuthal wall drag model........................................ 134

2.5.3 Flow regime map and interfacial area .................................. 146

2.5.3.1 Flow regime map....................................................... 146

2.5.3.2 Interfacial area concentration........................................... 160

2.5.4 Interfacial heat and mass transfer.................................... 172

2.5.4.1 Rate coefficients........................................ 172

2.5.4.2 Interfacial heat and mass transfer in bulk flow .................... 183

2.5.4.3 Interfacial heat and mass transfer at walls..........................207

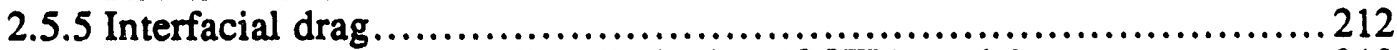

2.5.5.1 Counter-current flow limitation (CCFL) models .................212

2.5.5.2 Interfacial drag model................................................220

2.5.6 Channel inlet void distribution model for Mk22 assembly .................259

2.5.7 Rib void model ..................................................... 276

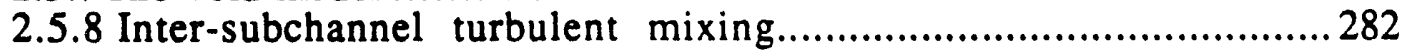

2.6 Boundary conditions................................................. 294

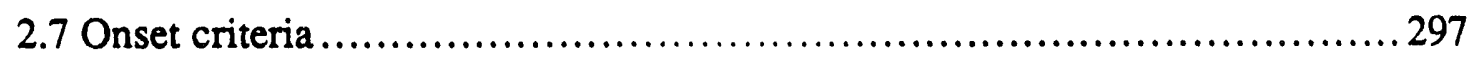

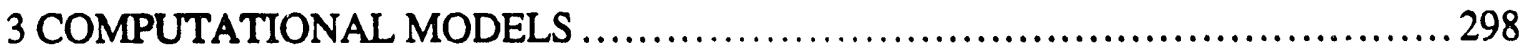

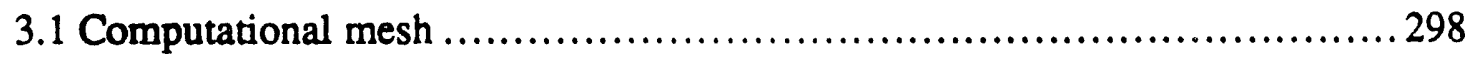

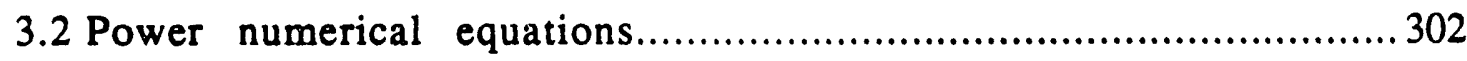

3.3 Solid finite difference equations .............................................. 317

3.4 Fluid finite difference equations ............................................. 331

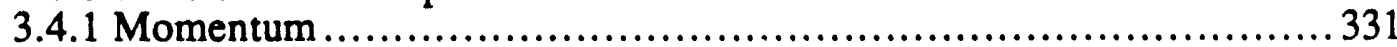

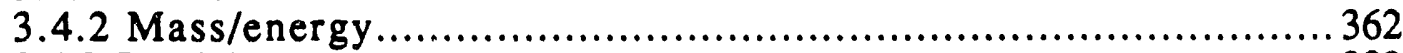

3.4.3 Special cases ...................................................... 382 


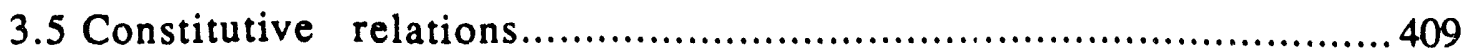

3.6 Boundary conditions................................................... 410

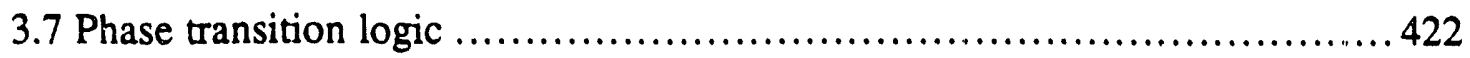

3.8 Solution methods...................................................... 446

3.8.1 Solid/power ....................................................... 448

3.8.2 Solid-fluid ....................................................... 450

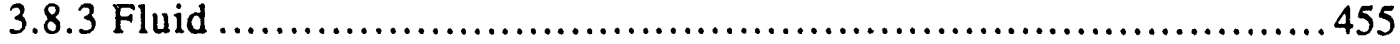

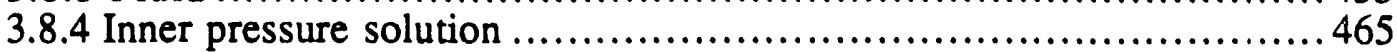

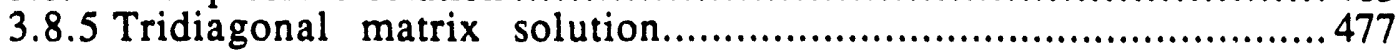

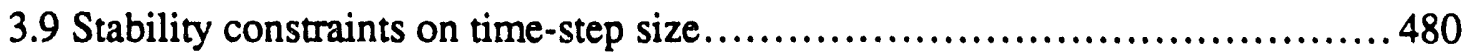

3.10 Onset criteria checking \& power iteration method ............................ 487

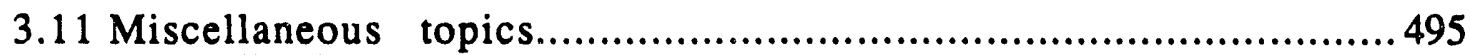

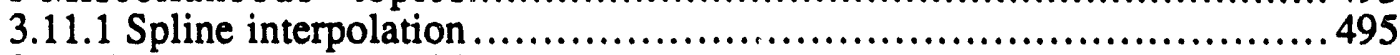

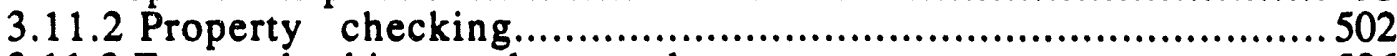

3.11.3 Error checking and control...............................................506

3.11.4 Time-step control ........................................................ 509

3.11.5 Steady state checking ................................................ 511

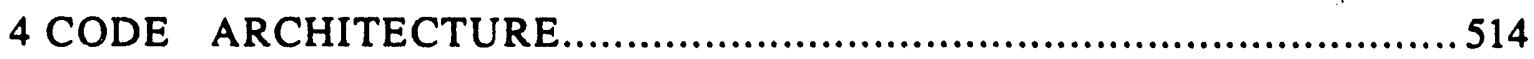

APPENDIX - Fluid thermodynamic and transport property data correlations .......... 526 


\begin{tabular}{|l|ll|l|}
\hline $2 / 15 / 93$ & WSRC-TR-92-532 Rev. 0 & FLOWTRAN-TF Software Design & Page 1 \\
\hline
\end{tabular}

\section{INTRODUCTION}

FLOWTRAN-TF was created to analyze an individual Mk22 fuel assembly during a large break Loss Of Coolant Accident (LOCA) scenario involving the Savannah River Site $\mathrm{K}$-reactor after the initial few seconds of the transient. During the initial few seconds reactor cooling is limited by the static or Ledinegg flow instability phenomenon. The predecessor FLOWTRAN code was developed to analyze this portion of a LOCA. In the several seconds following the break, a significant fraction of the reactor coolant inventory leaks out the break, Emergency Cooling System (ECS) flow is initiated, and air enters the primary coolant circulation loops. Reactor fuel assemblies are cooled by a low flowrate airwater downflow. Existing commercial nuclear industry thermal-hydraulic codes were judged inadequate for detailed modeling of a Mk22 fuel assembly because the application involves a ribbed annular geometry, low pressure, downflow and an air-water mixture.

FLOWTRAN-TF is a two-phase thermal-hydraulics code of similar technology to existing commercial codes such as RELAP and TRAC but customized for Savannah River Site applications. The main features and capabilities of FLOWTRAN-TF are

- Detailed Mk22 fuel assembly ribbed annular geometry

- Conjugate heat transfer

- Detailed neutronic power distribution

- Three-dimensional heat conduction in Mk22 fuel and target tubes

- Two-dimensional coolant flow in channels (axial, azimuthal)

- Single-phase and/or two-phase fluid (gas, liquid and/or gas-liquid)

- Two-component (air, water)

- Constitutive models applicable to low pressure air-water downflow in ribbed annular channels

The design of FLOWTRAN-TF is described in detail in this report which serves as the Software Design Report in accordance with Quality Assurance Procedure IV-4, Rev. 0 "Software Design and Implementation" in the 1Q34 manual. 


\section{PHYSICAL MODELS}

This section focuses primarily on the physical models which comprise the FLOWTRAN-TF code. In some cases computational implementation is also discussed here instead of section 3 "Computational Models" to keep the subject material together for convenience.

\subsection{Geometry}

The basic geometrical capabilities of the FLOWTRAN-TF code are similar to those of the predecessor FLOWTRAN code (Aleman et al., 1989). As with the FLOWTRAN code, FLOWTRAN-TF is restricted to modeling a cvlindrical geometry which is general enough to include all current SRS reactor assemblies and most experimental rigs. The model divides the fluid flow path into three coupled sections termed the top, middle and bottom sections. Within the middle section heared metal structures can be modeled. Geometric information must be specified for each of these sections. The basic characteristics of these flow channels are summarized below.

\section{One-Dimensional Top and Bottom Sections}

The top and bottom sections of the fluid mesh are one-dimensional axial flow channels which can be annular, tubular, or tapered in geometry. The flow channel can be subdivided into an arbitrary number of axial cells. This is illustrated in Fig. 2.1-1 which shows a generalized one-dimensional mesh containing four fluid cells. 


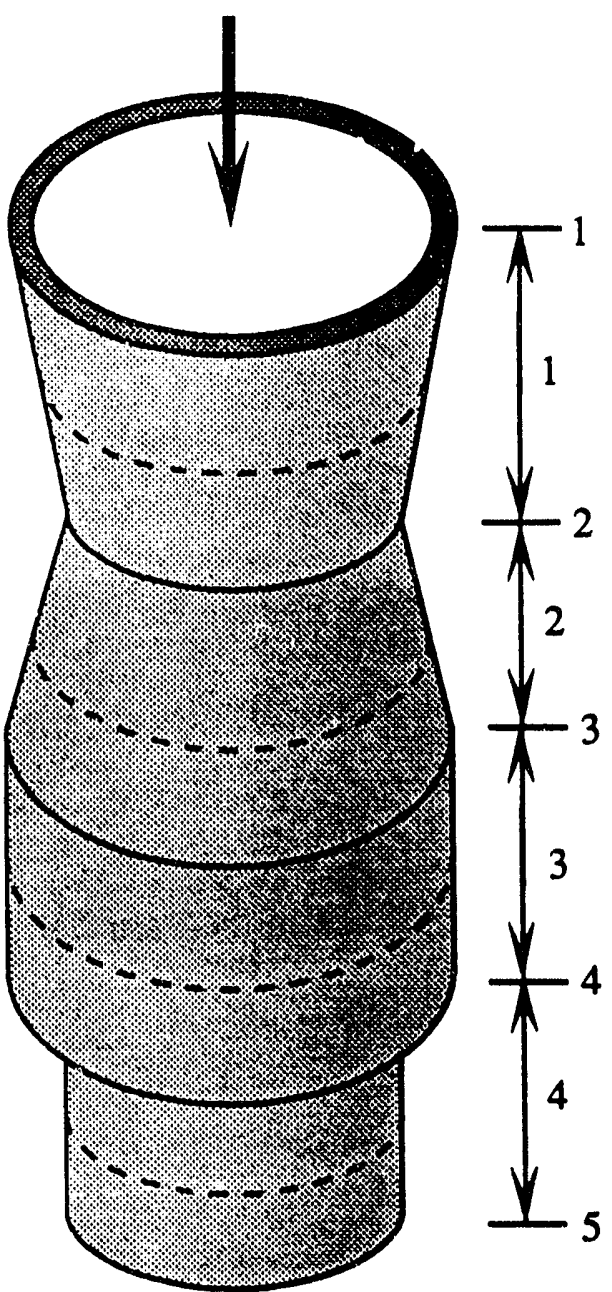

Figure 2.1-1 General fluid mesh for top and bottom section.

Cross-sectional areas are specified by the code user at the faces (solid boundaries in Fig. 2.1-1) and centers (dashed curves in Fig. 2.1-1) of the fluid computational cells and hydraulic diameters are specified for each cell. Form losses at the fluid cell faces and frictional losses within the momentum cell volume can be specified. As illustrated in Fig. 2.1-1, we would locate fluid cell boundaries at points of abrupt area changes and specify form losses at these locations to correctly match actual pressure drops through the flow channel. There is no heat transfer between the solid and fluid in the top and bottom sections.

\section{Two-Dimensional Middle Section}

The middle section of the fluid mesh is comprised of one or more flow channels separated by solid conductors. The flow channels are annular in geometry with the exception of the inner most channel which can be tubular or annular. The general geometries available to model the middle section of an assembly or test rig are illustrated in Fig. 2.1-2. Each annular flow channel can be divided into one or more subchannels (azimuthal fluid cells) 
and an arbitrary number of axial cells. Two-dimensional fluid flow in both the axial and azimuthal directions is modelled in the middle section.

In the middle section of the mesh, FLOWTRAN-TF models three-dimensional power distributions within the solid materials and computes heat transfer between the solid and fluid. The code performs three-dimensional $(r, \theta, z)$ heat conduction calculations to determine temperature distributions within the solids. The solid mesh is assumed to be in a cylindrical geometry. The innermost solid structure can be a rod or hollow cylinder and surrounding solid structures are hollow cylinders (see Fig. 2.1-2). The radial mesh within a solid structure is controlled by the code user through the input. Different materials can be specified at various radial mesh locations so that composite heaters can be modeled. This capability is required to model the clad SRS reactor assemblies and to model some of the heaters used in experimental rigs. In the azimuthal direction, the annular flow channels in SRS assemblies are each divided into four subchannels by the spacer ribs (shown in Fig. 2.1-4 below). Since the ribs in successive channels are rotated through $45^{\circ}$, the overall azimuthal mesh naturally divides into eight segments. Therefore the azimuthal grid in the solid structures is restricted to be a multiple of eight uniformly spaced segments. Figure 2.1-2 illustrates eight azimuthal cells uniformly spaced around the example model geometry. More information on the solid computational mesh is given in section 3.3. To construct the fluid computational mesh, we take each flow subchannel to bound a mesh cell. The code user could subdivide the fluid cells into a finer azimuthal mesh; however, our current two-phase modeling capabilities do not generally warrant a more detailed grid. The code will automatically align the surfaces of the fluid and solid cells to compute the heat exchange.

A minimum of three axial cells must be specified in the middle section. In SRS assemblies, the top and bottom axial cells of each subchannel interface to the top and bottom sections of the mesh, respectively, and are adiabatic along the solid-fluid interface. Given that there are $n z$ axial cells per subchannel, the remaining $n z-2$ axial cells compose the heated section of the fluid mesh. However, the code user is not restricted to this convention and can specify through input the exact group of cells in the middle section that form the active core. The code user inputs areas at the cell faces and cell center locations in both the axial and azimuthal directions and hydraulic diameters for the axial and azimuthal cells. Form losses can be specified at all fluid cell faces within the middle section and frictional losses can be applied within each momentum cell of the fluid mesh. At a given axial layer, there is mass and heat transfer between the subchannels in an annular flow channel through exchange of fluid across the rib gaps (azimuthal crossflow). Heat transfer coupling between the flow channels is also achieved by conduction through the solid cylinders. 


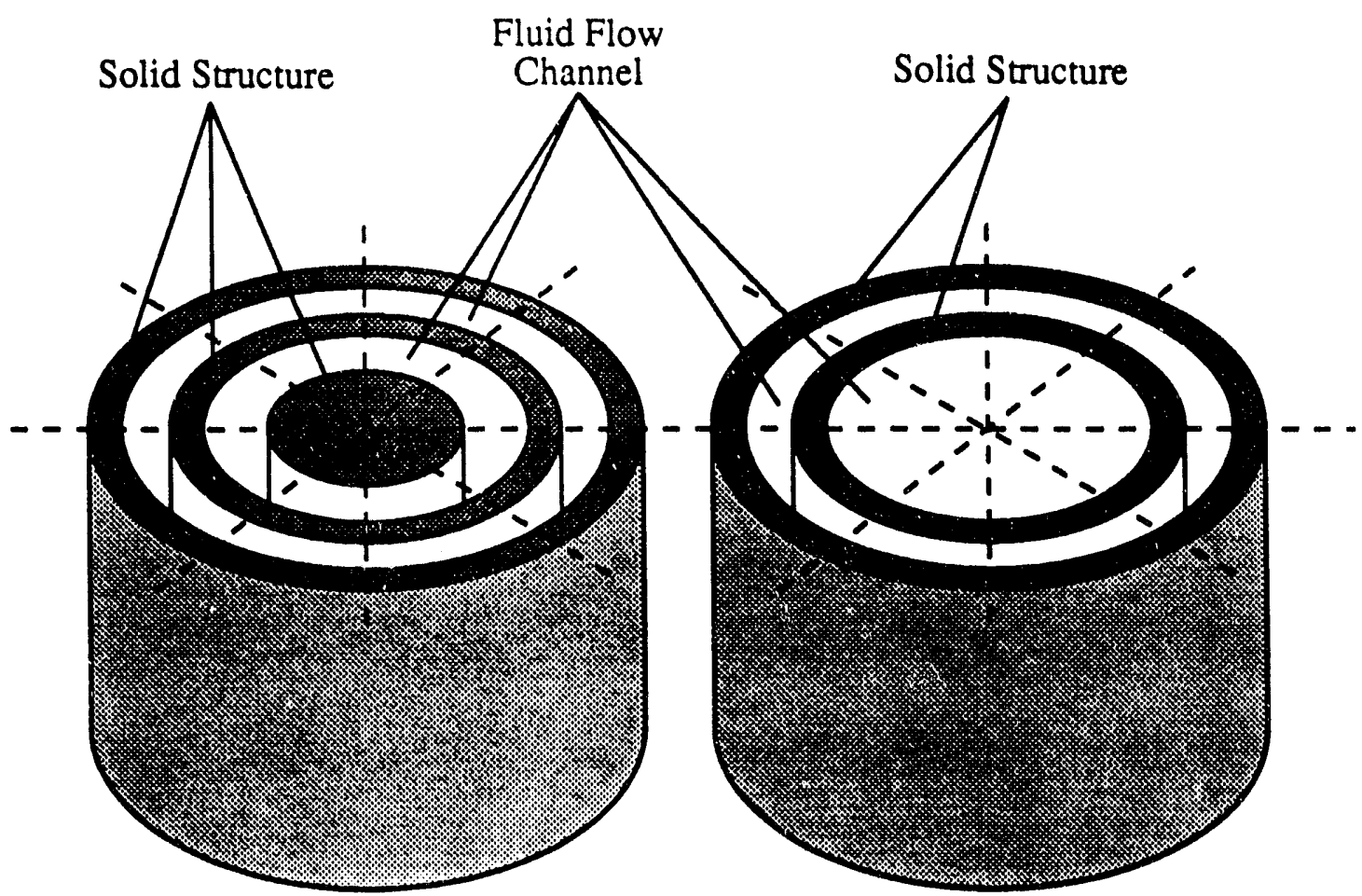

Figure 2.1-2 Two configurations of FLOWTRAN-TF middle section geometry.

\section{SRS Assembly Model}

In this subsection of the report we will use the SRS Mark 22 fuel assembly to illustrate how a reactor assembly can be modeled using the FLOWTRAN-TF code. This example will serve to further illustrate and explain the geometric modeling capabilities of the code. We note that all of the geometric information is specified through the input to retain code generality and flexibility.

The active core region of a Mark 22 assembly is a nested series of two concentric fuel tubes contained within a Universal Sleeve Housing (USH). Within the innermost fuel tube is the inner target and between the USH and the outer fuel tube is the outer target. All of these tubes are aluminum allo's extruded with aluminum cladding and ribs for centering. A representation of the overall structure of an assembly is shown in Fig. 2.1-3 and a horizontal slice through the active fuel region of this assembly is shown in Fig. 2.1-4. In these figures the cladding, fuel regions, and details of the rib structure are drawn approximately to scale. The outer diameter of the USH is 4.11 inches. 


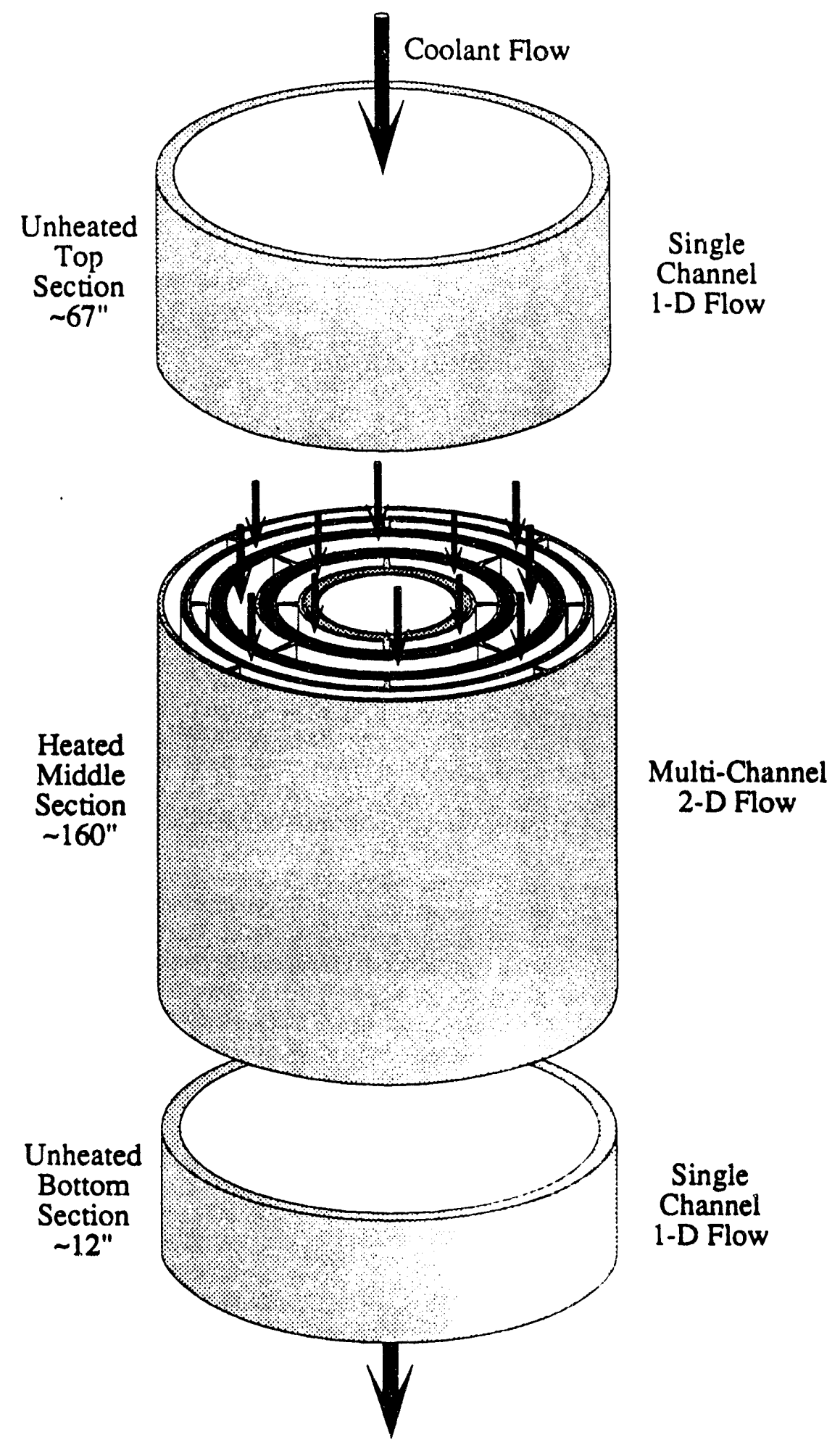

Figure 2.1-3 Representation of SRS assembly model. 


\begin{tabular}{|l|ll|r|}
\hline $2 / 15 / 93$ & WSRC-TR-92.532 Rev. 0 & FLOWTRAN-TF Software Design & Page 7 \\
\hline
\end{tabular}



Figure 2.1-4

Cross-sectional $(r-\theta)$ view of entire Mark 22 assembly shuwing all metal cylinders and coolant flow channels. 


\begin{tabular}{|l|ll|l|}
\hline $2 / 15 / 93$ & WSRC-TR-92-532 Rev. 0 & FLOWTRAN-TF Software Design & Page 8 \\
\hline
\end{tabular}

The Mark 22 fuel assembly depicted in Fig. 2.1-4 contains five nested cylinders, three annular flow regions, and two low flow purge channels within the inner target cylinder and between the outer target and the USH. Each of the three annular flow channels is in turn divided by the ribs into four "subchannels", producing a total of twelve subchannels not counting those in the purge channels. Gaps exist bet'ween the ribs and their abutting surfaces to allow assemblage of the components so that each subchannel is not hydraulically independent of all the others. FLOWTRAN-TF has two dimensional (axial and azimuthal) fluid modeling capabilities that consider the mass and energy exchange across these rib gaps. The dashed lines in Fig. 2.1-4 indicate the nodalization used to create azimuthal mesh cells in the solid cylinders. The presence of the spacing ribs requires at least eight azimuthal divisions in the solid to correctly align solid cell surfaces with their corresponding fluid cells.

Figure 2.1-5 illustrates a cross-sectional $(r-\theta)$ view of the computational mesh used to model the active core region (Middle Section) of a Mark 22 assembly. As shown in Fig. 2.1-4, the actual Mark 22 assembly consists of five metal cylinders and five flow channels. However, since most of the heat generation occurs in the fuel tubes, the innermost and outermost flow channels are purge channels that take a negligible fraction of the total assembly flow. The Universal Sleeve Housing (the outermost solid cylinder) generates no power. Since the numerical solution method employed in FLOWTRAN-TF requires using time steps at or below the Courant limit, computational time is of significant concern. Including the purge channels would increase the number of fluid computational cells in the middle section by over $40 \%$ and would significantly increase the computational time without affecting the final result. Therefore, we choose to model the reduced geometry illustrated in Fig. 2.1-5 where only the three primary flow channels and the fuel and target tubes are considered. That is, our assembly model neglects the two assembly purge channels and FLOWTRAN-TF calculations are performed for four solid cylinders and three flow channels. Each flow channel contains four subchannels (numbered 1-4 as illustrated) each of which is modeled with a single azimuthal fluid cell. 


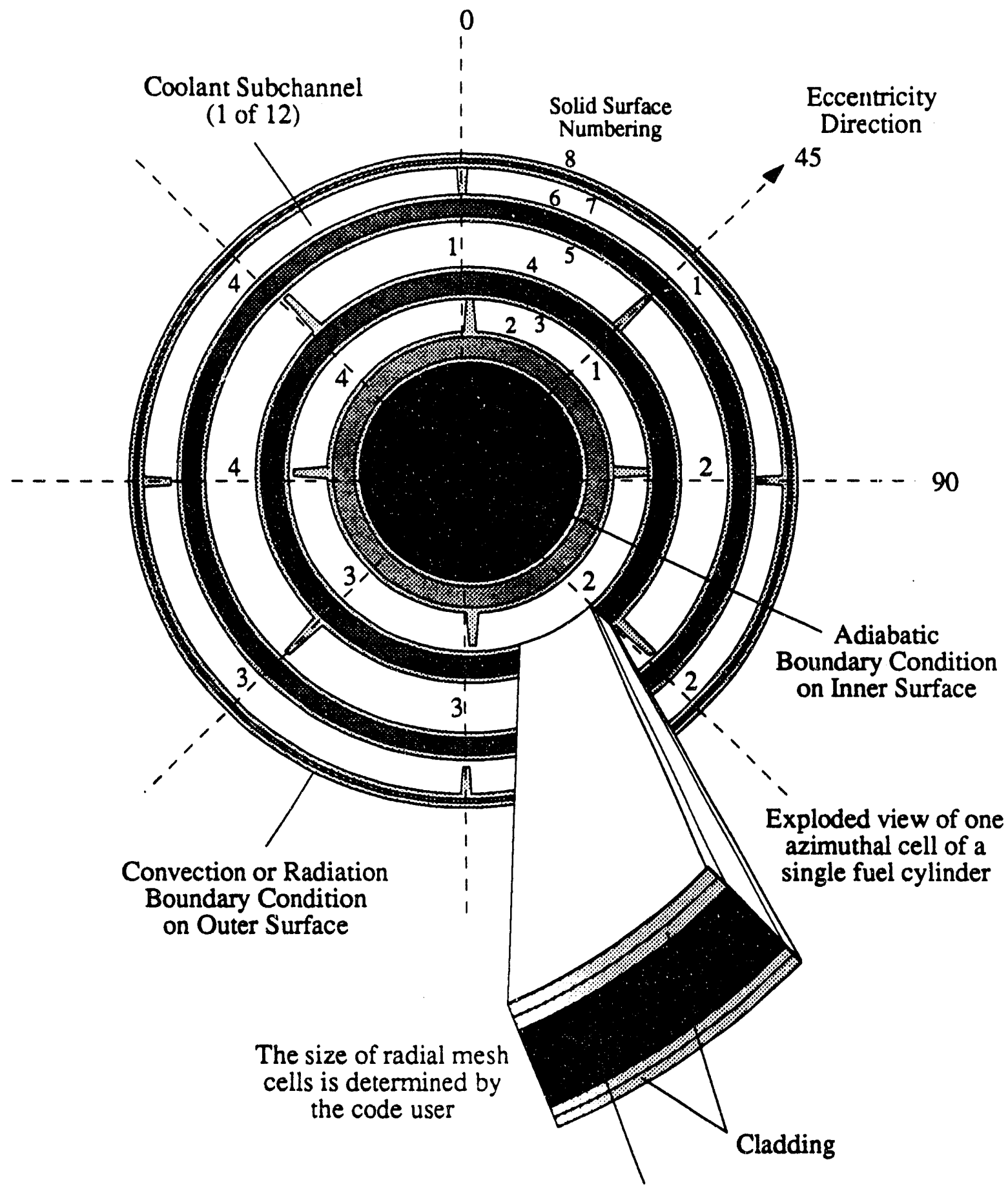

Fuel

Figure 2.1-5 Cross-sectional view of FLOWTRAN-TF model of eccentric Mark 22 assembly showing the metal cylinders and coolant flow channels. The numbering conventions for solid surfaces and coolant subchannels used by the code are shown. 
A full three-dimensional $(r, \theta, z)$ cylindrical heat conduction calculation is performed in each of the four metal cylinders, with boundary conditions furnished by the fluid calculations in each of the twelve subchannels. We apply an adiabatic boundary condition at the inner surface and have the option of using a fixed heat transfer coefficient and reference bulk temperature to compute convective heat transfer on the outer surface or using a simple radiation model to approximate radiative heat transfer to this surface. The reference temperature and boundary heat transfer coefficient are input by the code user. If the input heat transfer coefficient is set equal to zero, an adiabatic boundary condition can be specified at the outer surface as well.

In Fig. 2.1-5, we show a typical radial mesh in an exploded view of a part of a single azimuthal cell at a particular axial layer to indicate a possible solid radial mesh. Typically, we use a somewhat coarser nodalization and represented the cladding layer by a single radial node while the metal core contains three nodes. The quantity and spacing of the radial mesh cells is completely arbitrary; the axial mesh spacing is also arbitrary while the azimuthal mesh must be uniform. Figure 2.1-5 also illustrates modeling a composite heater since the cladding material (aluminum) is different from the fuel or target material. The total number of azimuthal cells must be a multiple of eight due to the staggered arrangement of the ribs. Cell sizes are specified by the user through the code input.

Figure 2.1-5 also illustrates how the solid and fluid cells are coupled. For example, in each azimuthal sector, the heat fluxes computed over surfaces 4 and 5 are multiplied by their respective surface areas and summed to calculate the total heat deposited to the fluid in the subchannels of the middle flow channel (subchannels 5-8). As shown in Figs. 2.1-4 and 2.1-5, eight azimuthal solid cells are routinely used in the calculations so that two azimuthal cells fall into each fluid subchannel. Since the rib gaps allow some movement of the tubes once they are assembled, we usually assume that the tubes are positioned eccentrically in the model. This eccentric positioning is also illustrated in Fig. 2.1-5 where we assume that all of the tubes are moved as far as possible in the $45^{\circ}$ direction.

Let us now focus on the assembly as a whole, rather than just a horizontal slice through the active fuel region. The FLOWTRAN-TF view of the entire assembly involves a connected series of flow paths. These flow paths, and a typical subdivision of them into fluid mesh cells, are shown in Fig. 2.1-6. Although this figure is strictly diagrammatic and not to scale, it does represent the actual number and approximate location of the mesh cells that have been used in some of the deposited power limits calculations for the Mark 22 assemblies. We begin in the plenum, which is divided from the assembly itself by a set of slots in the permanent housing and holes in the USH itself. We place a single mesh cell in the plenum, whose primary function is the specification of the boundary conditions for each of the primary state variables $\left(P, \alpha, T_{g}, T_{f}\right.$, and $\left.X_{a}\right)$ at the inlet to the assembly. FLOWTRAN-TF also allows the specification of flow boundary conditions at the top of the assembly. In this case, the flow through the slots and USH holes is specified directly. We also place a single mesh cell in the bottom of the tank, which serves to set boundary conditions for the primary state variables at the assembly exit. 


\begin{tabular}{|l|ll|l|}
\hline $2 / 15 / 93$ & WSRC-TR-92-532 Rev. 0 & FLOWTRAN-TF Software Design & Page 11 \\
\hline
\end{tabular}

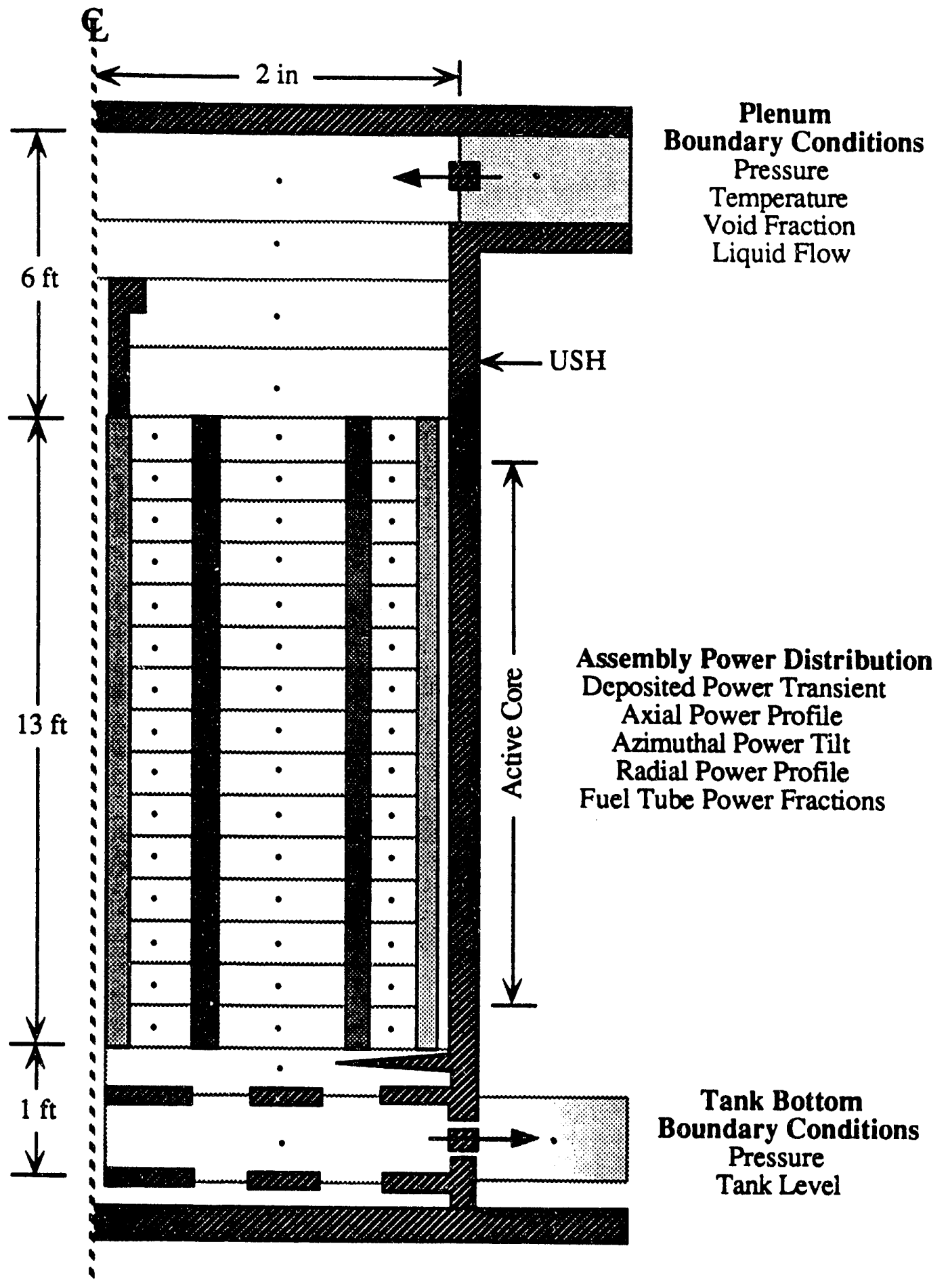

Figure 2.1-6 Schematic representation of fluid computational mesh used for Mark 22 assembly calculations. 
As discussed above, the assembly itself is divided by the FLOWTRAN-TF model into three regions: the top, middle, and bottom sections. The top section consists of a single flow path divided into a arbitrary number of mesh cells of user specified volumes and flow areas. Flow restrictions, such as orifice plates and the USH holes, can be modeled by reducing the flow area and by specifying additional form losses at these locations. The middle section comprises the entire fuel region, and it is only in this section that solid heat conduction calculations are performed. We assume that no energy is transferred through the USH to the fluid in the top and bottom sections. In general, the middle section consists of one or more flow paths. As we indicated above, for the Mark 22 assembly, the FLOWTRAN-TF model utilizes three primary flow paths each of which is subdivided into four hydraulically coupled subchannels (subchannels are not shown in Fig. 2.1-6). Frictional losses and form losses can be specified at any location in any section. Form losses in the middle section have been included to allow modeling of experiments using spacer pegs in place of continuous ribs. The bottom section is used to model the bottom end fitting and the shell holes in the USH at the assembly exit. Like the top section, the bottom section consists of a single flow path with an arbitrary mesh.

Within each secion the fluid mass and energy cells coincide while the momentum cells are staggered one-half cell length. The arrangement of the axial mass cells within the fluid mesh is illustrated in Fig. 2.1-6. The middle section of the fluid mesh can be discretized into concentric annuli or channels. Each channel can further be subdivided into subchhannels. This discretization is illustrated in Fig. 2.1-5 for a Mark 22 assembly where the spacer ribs naturally subdivide the three flow channels into a total of twelve subchannels. Figure 2.1-6 illustrates the typical fluid nodalization used for Mark 22 assembly calculations. The assembly model consists of a top section having four fluid cells, a middle section with 15 axial cells in both the fluid and solid mesh, and a bottom section with two fluid cells. The middle section contains the three annular flow paths and only the 13 central axial cells in the middle section are heated (active core region). Loss coefficients are set in the bottom section to model flow through a single pressure plate as illustrated in the figure. Figure 2.1-6 also indicates some of the boundary condition information required by the FLOWTRAN-TF code.

\section{Assembly Bowing}

The FLOWTRAN-TF code is programmed to account for bowing in the assembly. Assemblies within the reactor experience an asymmetric azimuthal power distribution. Some of the experimental rigs have also been constructed to simulate this azimuthal power distribution. The effect of an azimuthal non-uniformity in power is to preferentially heat one side of the assembly which may cause the assembly to bow outward. This bowing is illustrated schematically in Fig. 2.1-7 where we assume that the center of the assembly bows outward some distance $\delta$. Since the ends of the assembly are held fixed, we assume that the bowed assembly takes the shape of a sine curve which can be expressed as

$$
\mathrm{d}_{\mathrm{z}}=\delta \sin \left(\frac{\mathrm{z} \pi}{\mathrm{L}}\right)
$$

where $d_{z}$ is the deflection of the rig from vertical at distance $z$ along the length.

The effect of the bowing is to alter the orientation of the fluid cells with respect to the vertical direction. This shifts the alignment of the cells with respect to the gravity vector and changes the gravitational body forces acting on the fluid volumes. This is illustrated in the exploded view of a single axial cell shown in Fig. 2.1-7 and in the lower part of Fig. 
2.1-7 where we show the gravitational components acting on the azimuthal face of one fluid cell in terms of the rib orientation direction $\alpha$ and the local angle of inclination of the fluid cell $\phi$. The slops of the bowed shape is the derivative of equation $(2.1-1)$ with respect to $\mathrm{z}$ which gives the angle of inclination at axial location $\mathrm{z}$ as

$$
\phi=\tan ^{-1}\left[\frac{\delta \pi}{L} \cos \left(\frac{z \pi}{L}\right)\right]
$$

The magnitude of the assembly bow $(\delta)$ and the direction of bowing $(\omega)$ are input by the code user. The angle of inclination $(\phi)$ and adjusted directional cosines are calculated at the faces of each fluid computational cell automatically within the code. Figure 2.1-7 illustrates bowing in an initially vertical rig; however, the vertical orientations of the axial cells can in general be specified through the input. Then the gravitational component in the axial direction for fluid momentum cells in the bowed rig is giv's by

$$
g_{z}=g \cos \left(\theta_{z}+\phi\right)
$$

For the example shown in Fig. 2.1-7, inclination angles in the upper half of the assembly will be positive while angles on the lower half of the assembly will be negative. Equation (2.1-3) is evaluated at the upper and lower faces of each fluid mass cell. To estimate the gravitational forces for the azimuthal momentum cells we first reevaluate equation $(2.1-2)$ to find $\phi$ at the axial midpoint of the fluid cell $\left(z_{\text {mid }}\right)$. The midpoints of the azimuthal momentum cells will be located at the rib gaps. We assume that the rib is oriented at some angle $\alpha$ with respect to our coordinate system as shown in Fig. 2.1-7. In general, directional cosines for the azimuthal faces of each fluid cell can also be specified through the input. We assume that the cell face is initially oriented at some angle $\theta_{x}$ with respect to the vertical. Incorporating the input cell orientation by using the angle convention explained in the next section, the gravitational component in the azimuthal direction is given by

$$
g_{x}=g \cos \left(\theta_{x}-\phi\right) \sin (\alpha)
$$

Finally, the angle $\alpha$ will depend upon the direction of bowing and the location of the ribs within a subchannel. As shown in Fig. 2.1-4, we take the ribs in odd number flow channels to be oriented in the directions $n \pi / 2$ with $n=0,1,2,3$. In even numbered flow channels the ribs are rotated $-\pi / 4$ from these directions. This configuration is illustrated in Fig. 2.1-7 where the centers of the azimuthal momentum cells (rib locations) and the flow subchannels are numbered in order. The angle $\alpha$ is calculated as

$$
\alpha=\left(n_{\text {sub }}-1-\beta\right) \frac{\pi}{2}-\omega
$$

where

$$
\beta=\left\{\begin{array}{cc}
0 & \text { for odd numbered flow channels } \\
1 / 2 & \text { for even numbered flow channels }
\end{array}\right.
$$

and $n_{\text {sub }}$ is the subchannel (or rib) number $(1,2,3,4)$. The bowing angle $(\omega)$ is subtracted in equation (2.1-5) to correctly orient the assembly in the direction of the bow. 

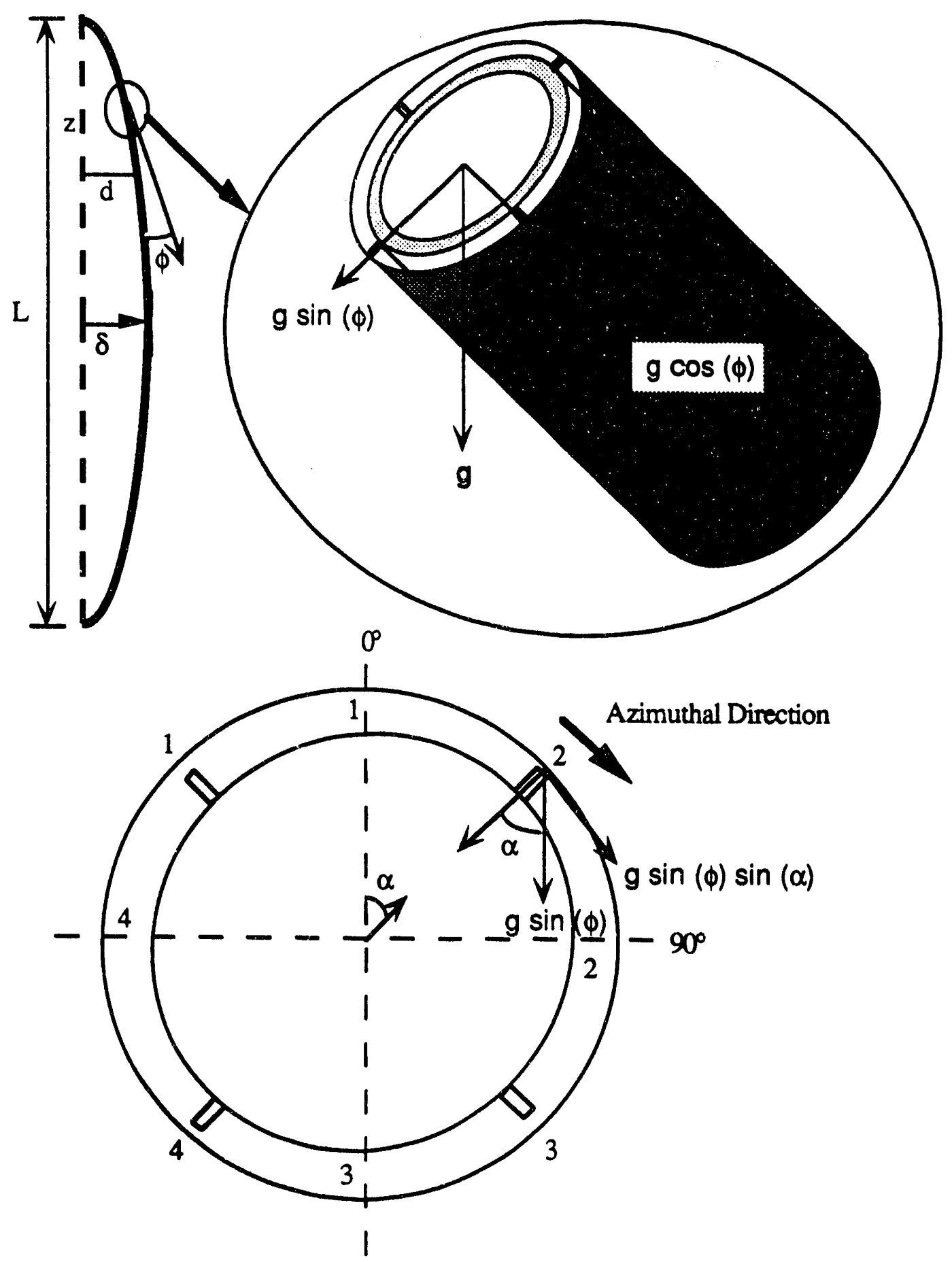

Figure 2.1-7 Schematic representation of bowed assembly geometry. 


\section{Fluid Cell Angle Conventions}

We assume that fluid cells are initially oriented at some angle $\theta_{z}$ with respect to a downward pointing vertical and that azimuthal faces are oriented at some angle $\theta_{x}$. For a vertical assembly with fluid downflow we take $\theta_{z}=0$ with $\cos \left(\theta_{z}\right)=1$ and $\theta_{x}=\pi / 2$ with $\cos \left(\theta_{\mathrm{x}}\right)=0$.

To obtain equations (2.1-3) and (2.1-4), we assume that the fluid cell acts as a rigid body so that, as it rotates through an angle $\phi$ in the vertical direction, the axial and azimuthal faces both rotate by $\phi$. This is illustrated in the following diagram where we show rotation of a fluid cell from an initially vertical position through an angle of $90^{\circ}$ to a horizontal position
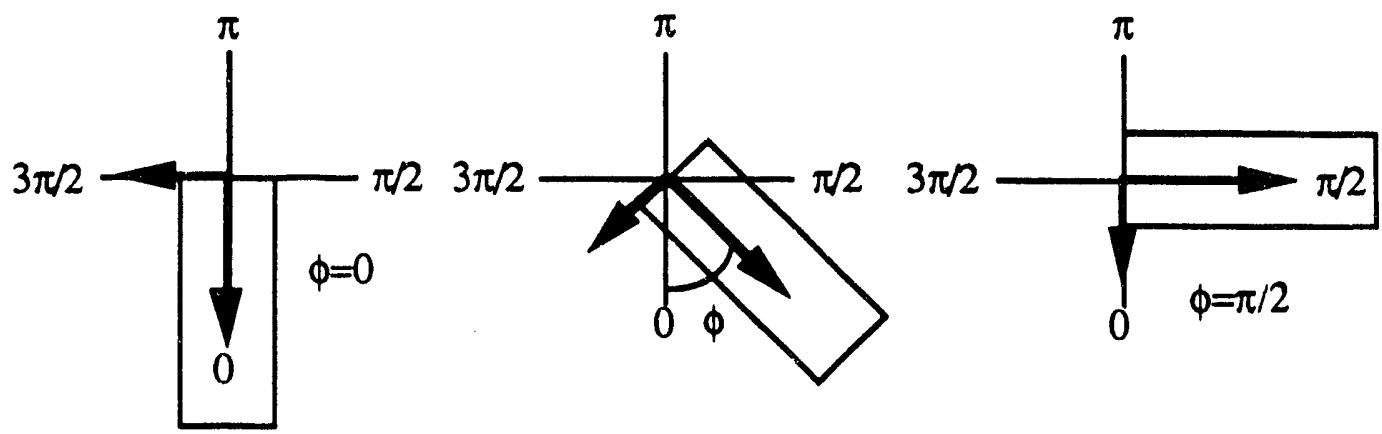

The axial orientation angle $\theta_{2}$ starts at an angle of 0 and increases by $\phi$ as the rotation occurs. The orientation angle for the azimuthal cell face, $\theta_{x}$, starts at a true angle of $3 \pi / 2$ and also increases by $\phi$ as the rotation occurs. Using simple trigonometric identities

$$
\begin{aligned}
\cos (3 \pi / 2+\phi) & =\cos (2 \pi-\pi / 2+\phi) \\
& =\cos (-\pi / 2+\phi) \\
& =\cos (\pi / 2-\phi)
\end{aligned}
$$

Therefore we can specify the azimuthal face angle as ranging from $\pi / 2$ for a horizontal orientation to 0 for a vertical orientation and subtract the bowing angle as shown in equation (2.1-4). Similarly, the axial angle will vary from 0 for a vertical cell up to $\pi / 2$ for horizontal orientation with the bowing angle added as shown in equation $(2.1-3)$. This is the convention adopted for FLOWTRAN-TF code input.

We note that equation (2.1-4) reduces to the correct form for the case of an initially vertical cell. Using the trigonometric identity for the cosine of the difference between two angles

$$
g_{x}=g\left\{\cos \left(\theta_{x}\right) \cos (\phi) \sin (\alpha)+\sin \left(\theta_{x}\right) \sin (\phi) \sin (\alpha)\right\}
$$

For $\theta_{x}=\pi / 2$, this correctly reduces to the relationship shown in Fig. 2.1-7

$$
g_{x}=g \sin (\phi) \sin (\alpha)
$$




\section{Subchannel Eccentricity Formulas}

Formulas for computing eccentric subchannel flow areas and wetted perimeters are derived in this section. These formulas are not part of the FLOWTRAN-TF code itself, but, are provided as a reference to aid the user in computing fluid cell areas and hydraulic diameters for code input.

Fundamental area and perimeter formulas for an eccentric quadrant: The eccentric subchannel area and perimeter formulas are constructed from the areas and perimeters of various eccentric quadrants of a circle. Figure 2.1-8 illustrates an eccentric quadrant of circle of radius $r$. The region is created by perturbing the quadrant corner point from $(0,0)$ to $(\delta x, \delta y)$. The area of the eccentric quadrant shown in Figure 2.1-8 is

$$
A_{x y}=\frac{r^{2}}{2}\left[\frac{\pi}{2}-\sin ^{-1} \rho_{x}-\sin ^{-1} \rho_{y}-\rho_{x}\left(1-\rho_{x}^{2}\right)^{1 / 2}+2 \rho_{x} \rho_{y}-\rho_{y}\left(1-\rho_{y}^{2}\right)^{1 / 2}\right]
$$

where

$$
\begin{aligned}
& \rho_{x}=\frac{\delta x}{r} \\
& \rho_{y}=\frac{\delta y}{r}
\end{aligned}
$$

Useful special cases of equation (2.1-6) are the perturbations $(0, \delta y)$ and $(\delta x, 0)$ for which

$$
A_{0 y}=\frac{r^{2}}{2}\left[\frac{\pi}{2}-\sin ^{-1} \rho_{y}-\rho_{y}\left(1-\rho_{y}^{2}\right)^{1 / 2}\right]
$$

and

$$
A_{x 0}=\frac{r^{2}}{2}\left[\frac{\pi}{2}-\sin ^{-1} \rho_{x}-\rho_{x}\left(1-\rho_{x}^{2}\right)^{1 / 2}\right]
$$

respectively. Similarly, for the eccentric quadrant perimeter the following formulas can be derived as

$$
\begin{gathered}
P_{x y}=r\left(\frac{\pi}{2}-\sin ^{-1} \rho_{x}-\sin ^{-1} \rho_{y}\right) \\
P_{0 y}=r\left(\frac{\pi}{2}-\sin ^{-1} \rho_{y}\right) \\
P_{x 0}=r\left(\frac{\pi}{2}-\sin ^{-1} \rho_{x}\right)
\end{gathered}
$$

Here $\mathrm{P}_{0 \mathrm{y}}$ and $\mathrm{P}_{\mathrm{x} 0}$ are the perimeters for the perturbations $(0, \delta \mathrm{y})$ and $(\delta \mathrm{x}, 0)$, respectively. 


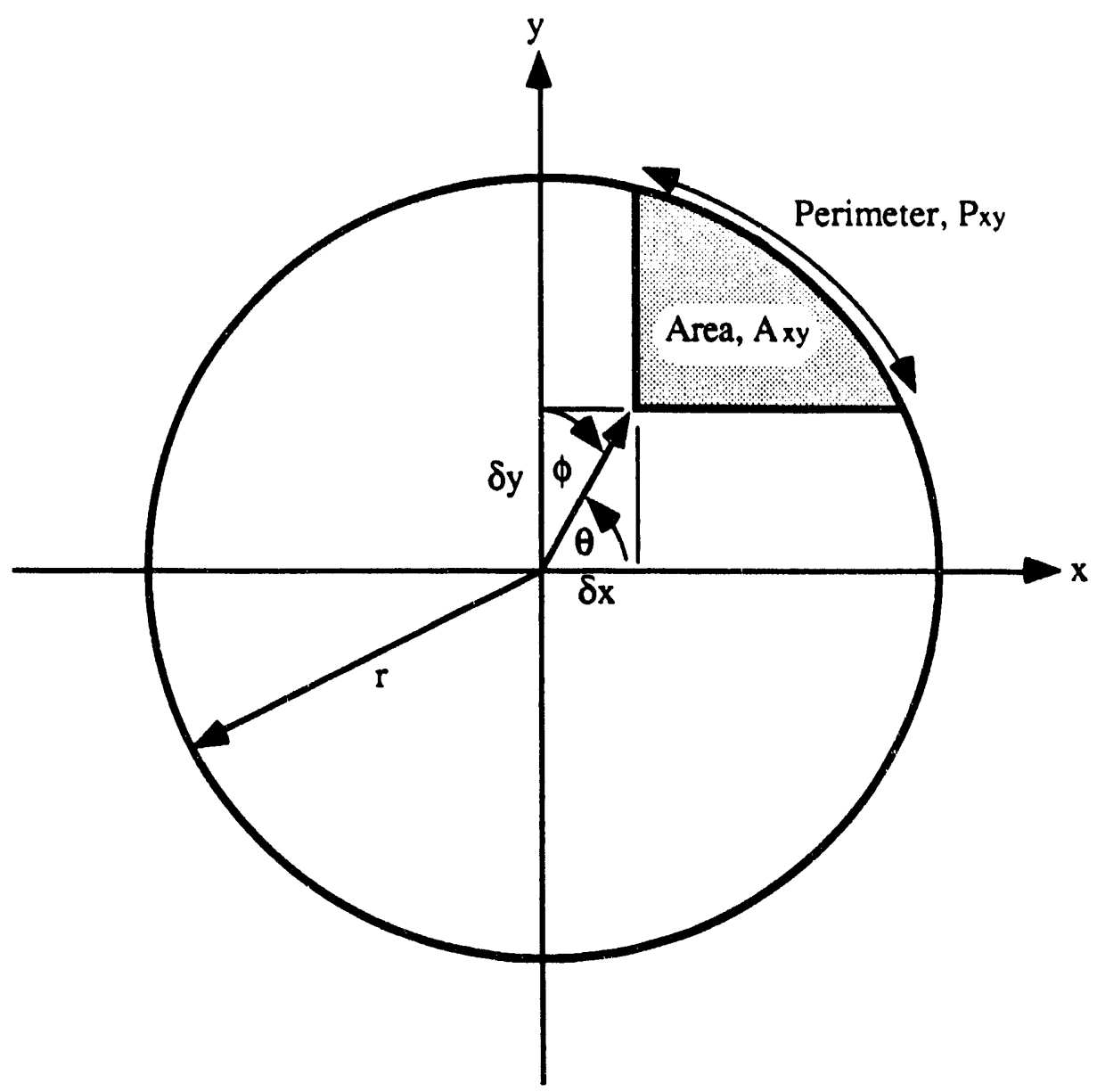

Figure 2.1-8 Eccentric quadrant geometry.

Quadrant areas and perimeters for ribs on the outer radius (case I): Figure 2.1-9 illustrates an eccentric ribbed annulus with the longitudinal ribs on the outer cylinder. For this case the subchannel areas are

$$
\begin{gathered}
A_{1}^{\text {outer }}=\frac{\pi r_{0}^{2}}{4}-\pi r_{i}^{2}+2 A_{x 0}+2 A_{0 y}-A_{x y}-A_{\text {rib }} \\
A_{2}^{\text {outer }}=\frac{\pi r_{0}^{2}}{4}-2 A_{0 y}+A_{x y}-A_{\text {rib }} \\
A_{3}^{\text {outer }}=\frac{\pi r_{0}^{2}}{4}-A_{x y}-A_{\text {rib }}
\end{gathered}
$$

and 


$$
A_{4}^{\text {outer }}=\frac{\pi r_{0}^{2}}{4}-2 A_{x 0}+A_{x y}-A_{\text {rib }}
$$

Note that

$$
\sum_{i=1}^{4} A_{i}^{\text {outer }}=\pi r_{0}^{2}-\pi r_{i}^{2}-4 A_{\text {rib }}
$$

as expected. The perimeters are

$$
\begin{gathered}
\mathrm{P}_{1}^{\text {outer }}=\frac{\pi r_{0}}{2}+2 \pi r_{i}-2 P_{x 0}-2 P_{0 y}+P_{x y}+P_{\text {rib }} \\
P_{2}^{\text {outer }}=\frac{\pi r_{0}}{2}+2 P_{0 y}-P_{x y}+P_{\text {rib }} \\
P_{3}^{\text {outer }}=\frac{\pi r_{0}}{2}+P_{x y}+P_{\text {rib }}
\end{gathered}
$$

and

$$
P_{4}^{\text {outer }}=\frac{\pi r_{0}}{2}+2 P_{x 0}-P_{x y}+P_{\text {rib }}
$$

Note that

$$
\sum_{i=1}^{4} P_{i}^{\text {outer }}=2 \pi r_{0}+2 \pi r_{i}+4 P_{\text {rib }}
$$

as expected. The rib area and perimeter are defined in terms of the rib length $R_{L}$ and rib half-width $\mathbf{R}_{\mathrm{T}}$ to be

$$
A_{\text {rib }}=2 R_{T} R_{L}
$$

and

$$
P_{\text {rib }}=2 R_{L}-4 R_{T}
$$

Note that the area of the rib tip and opposing cylinder area are not included in the perimeter calculation. The perimeter values are ultimately used to compute a subchannel hydraulic diameter. The hydraulic diameter is thought to be more appropriate if the wetted perimeter associated with the rib gap is not included. The hydraulic diameter is readily computed from its definition once the subchannel area and perimeter are known. 


\begin{tabular}{|l|ll|l|}
\hline $2 / 15 / 93$ & WSRC-TR-92-532 Rev. 0 & FLOWTRAN-TF Software Design & Page 19 \\
\hline
\end{tabular}

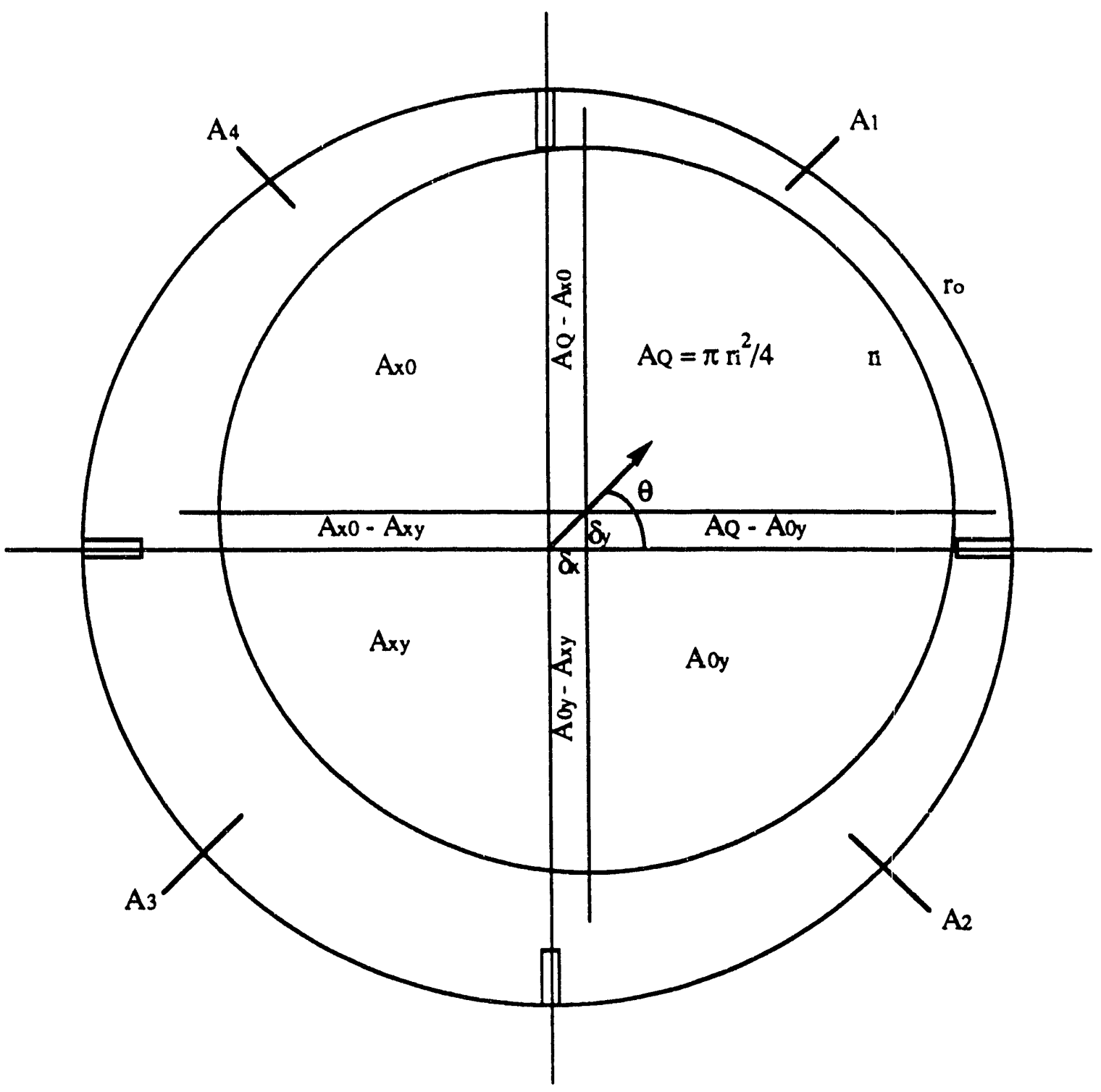

Figure 2.1.9 Eccentricity geometry for case I - ribs on outer radius.

Quadrant areas and perimeters for ribs on the inner radius (case $I$ ): Similar formulas result for the case of ribs on the inner cylinder shown in Figure 2.1-10. The results are summarized below:

$$
\begin{gathered}
A_{1}^{i n n e r}=A_{x y}-\frac{\pi r_{i}^{2}}{4}-A_{\text {rib }} \\
A_{2}^{i n n e r}=2 A_{x 0}-A_{x y}-\frac{\pi r_{i}^{2}}{4}-A_{r i b}
\end{gathered}
$$




$$
\begin{gathered}
A_{3}^{i n n e r}=\pi r_{0}^{2}-2 A_{x 0}-2 A_{0 y}+A_{x y}-\frac{\pi r_{i}^{2}}{4}-A_{r i b} \\
A_{4}^{i n n e r}=2 A_{0 y}-A_{x y}-\frac{\pi r_{i}^{2}}{4}-A_{r i b} \\
P_{1}^{\text {inner }}=\frac{\pi r_{i}}{2}+P_{x y}+P_{r i b} \\
P_{2}^{\text {inner }}=\frac{\pi r_{i}}{2}+2 P_{x 0}-P_{x y}+P_{r i b} \\
P_{3}^{\text {inner }}=\frac{\pi r_{i}}{2}+2 \pi r_{0}-2 P_{x 0}-2 P_{0 y}+P_{x y}+P_{\text {rib }} \\
P_{4}^{\text {inner }}=\frac{\pi r_{i}}{2}+2 P_{0 y}-P_{x y}+P_{r i b}
\end{gathered}
$$

Note that

$$
\sum_{i=1}^{4} A_{i}^{i n n e r}=\pi r_{0}^{2}-\pi r_{i}^{2}-4 A_{\text {rib }}
$$

and

$$
\sum_{i=1}^{4} P_{i}^{i n n e r}=2 \pi r_{i}+2 \pi r_{0}+4 P_{r b}
$$




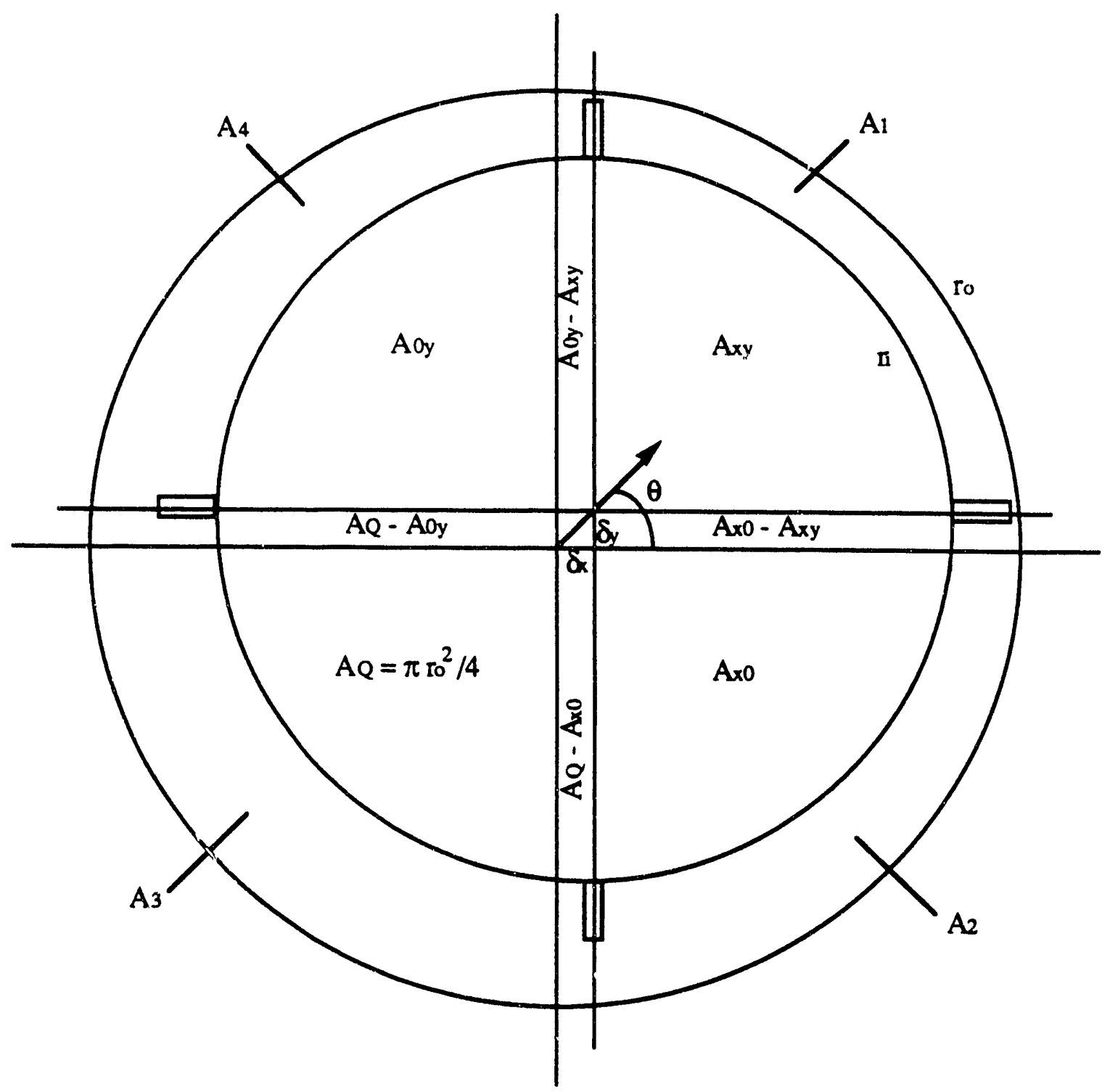

Figure 2.1-10 Eccentricity geometry for case II - ribs on inner radius.

Maximum eccentric deflections for ribs on the outer radius: $100 \%$ eccentricity is defined to be the maximum deflection possible for a given orientation, $\phi$. For case I these are

$$
\begin{gathered}
\delta y^{\text {outer }}=g \\
\delta x^{\text {outer }}=g / \tan \theta
\end{gathered}
$$

where $\mathrm{g}$ is the nominal rib gap (1/2 diametrical gap). 
Maximum eccentric deflections for ribs on the outer radius: For case $I I$ the results are

$$
\begin{gathered}
\delta x^{\text {inner }}=\frac{1}{m^{2}+1}\left[\sqrt{r_{0}^{2}\left(m^{2}+1\right)-\left(y_{0}-m x_{0}\right)^{2}}-m y_{0}-x_{0}\right] \\
\delta y^{\text {inner }}=\delta x^{\text {inner } \tan \theta}
\end{gathered}
$$

where

$$
\begin{aligned}
& \mathrm{x}_{0}=\left\{\begin{array}{cc}
\mathrm{r}_{\mathrm{i}}+\mathrm{R}_{\mathrm{L}} & \theta \leq \pi / 4 \\
\mathrm{R}_{\mathrm{T}} & \theta>\pi / 4
\end{array}\right. \\
& \mathrm{y}_{0}=\left\{\begin{array}{cc}
\mathrm{R}_{\mathrm{T}} & \theta \leq \pi / 4 \\
\mathrm{r}_{\mathrm{i}}+\mathrm{R}_{\mathrm{L}} & \theta>\pi / 4
\end{array}\right.
\end{aligned}
$$

and

$$
\mathrm{m}=\tan \theta
$$

\section{References}

Aleman, S. E., M. V. Gregory, L. L. Hamm, L. D. Koffman, R. E. Pevey, W. H. Reed and F. G. Smith, III, 1989, "FLOWTRAN: An Algorithm for Describing the Thermal-Hydraulic Behavior of SRP Assemblies (U)", DPSTM-140, September 15. 


\subsection{Power Generation}

In FLOWTRAN-TF, reactor fuel cylinders are represented as collections of individual volume elements called nodes. The radial, azimuthal and axial boundaries of the nodes are determined by the chosen number of subdivisions in each dimension of the cylindrical mesh. The heat conduction calculation in FLOWTRAN-TF requires a deposited power source for each computational node in this finite difference representation. The representation of the deposited power distributions depends upon whether the code is being used to model a reactor assembly or an electrically heated experimental rig. The first section of this report describes the formulation of the assembly deposited power distribution. The second section addresses the simplification of this formulation for the case of electrically heated experimental rigs.

\section{Assembly Deposited Power}

For reactor assemblies, the deposited power is derived from several sources:

- charged particle emissions due to the fission fragment interactions with surrounding matter

- distributed $\gamma$-ray absorptions from prompt fission

- distributed $\gamma$-ray absorptions from fission products

- fission product charged particle emissions (principally $\beta$ )

- distributed $\gamma$-ray absorptions from neutron capture

- neutron capture charged particle emissions ( $\alpha$ and $\beta$ )

These six sources are grouped into two major categories: neutronic and fission product. The fission product component accounts for the contribution from the decay of fission products: energy carried by primary particles (primarily $\beta$ 's) and the redistributed (fission product) gamma energy. The neutronic component includes all other sources. Consequently, the neutronic component includes the contribution from fission, including the absorbed fission gamma energy, and the contribution from the absorbed neutron capture gamma energy and neutron capture primary particle energy.

We should emphasize that no fundamental power calculation is performed in the FLOWTRAN-TF code. There is no neutronics solution algorithm in the code. Instead, the code takes input power data and unfolds it into a detailed nodal power distribution. That input data is generated by four other computer codes (Fig. 2.2-1). The response of total reactor power versus time is generated by the reactor system code AA3 (Smith and Church, 1990), which contains a point kinetics model. Detailed assembly power shapes are provided by the GLASS system of codes (Graves, 1989), which uses two-dimensional integral transport theory for unit supercell calculations. The response of fission product decay power versus time is generated by the HMTABLE code (Baxter and Apperson, 1982). Finally, the time-dependent axial shape is calculated by the one-dimensional spacetime kinetics code AXLIB (Pevey, 1987). 
The fundamental basis for the FLOWTRAN-TF power algorithm is the formulation for the total assembly deposited power developed by Parks and Graves (1987) and modified by Gregory (1992):

$$
\begin{aligned}
\Pi(t) & =\Pi(0)\left\{\left(1-x(t) \delta_{\text {mod }}\right)\left[\frac{H(t)}{H}\right] F^{n p}(t)+x(t) \delta_{\bmod }\left[\frac{H(t)}{H}\right] F_{\bmod }^{d p}(t)\right. \\
& \left.+x(t) \delta_{d r y}\left[1-\frac{H(t)}{H}\right] F_{d r y}^{d p}(t)\right\}
\end{aligned}
$$

where

$$
\begin{aligned}
& \Pi \equiv \text { Total assembly deposited power } \\
& \delta_{k} \equiv \text { Fraction of deposited power from decay heat ("k=dry" for regions where } \\
& H(t) \equiv \text { Height of water in the reactor tank relative to the bottom of active core } \\
& H \equiv \text { Length of active core region } \\
& F^{n p} \equiv \text { Fission power plus neutron capture power amplitude, normalized to } 1.0 \text { at } \\
& \begin{aligned}
F_{k}^{d p} \equiv & \text { Decay heat power amplitude ("dry" and "mod"), normalized to } 1.0 \text { at time } \\
0.0 &
\end{aligned} \\
& x(t) \equiv \text { Conservative decay heat multiplier. }
\end{aligned}
$$

The three terms in Eq. (2.2-1) represent the three contributions to the time-dependent total assembly power: from the neutronic component, from the fission product component in the moderated part of the tank, and from the fission product component in the dry part of the tank. Figure 2.2-2 is a plot of these three terms and the total, $\Pi(t)$. The time-dependent factors are the tank level $H(t)$, the conservative decay heat multiplier $x(t)$, and the amplitude of each power component. The decay heat factors $\delta_{\kappa}$ 's are assumed to be constant.

Gregory (1992) has revised the Parks and Graves (1987) decay heat model to implement best estimate or conservative estimate values calculated by HMTABLE. The procedure involves augmenting the $\delta_{K}$ 's by the function $x(t)$ which is identically 1.0 for the bestestimate case or the multiplier greater than 1.0 for the conservative case, while $F$ mp and $F_{\text {dry }}^{d p}$ take on either the best estimate or conservative values calculated by HMTABLE.

\section{Nodal Deposited Power}

The expression for the nodal deposited power is obtained by spatially distributing the threeterm form of Eq. $(2.2-1)$ over the nodes in the active core region in the middle section of the FLOWTRAN-TF assembly model. Five assumptions are made:

1. The power shape is the product of three separable shapes: radial, azimuthal and axial. 
2. Three separate radial power shapes are required, one for the neutronic component, one for the wet tank fission product component, and one for the dry tank fission product component. Figure $2.2-3$ is an example of the neutronic component of the radial power shape for a Mark 22 inner fuel.

3. The radial and azimuthal power shapes are assumed to be independent of time, but the radial shape is dependent on reactor exposure (Fig. 2.2-3). Figure 2.2-4 represents a bounded Mark 22 azimuthal flux shape for various orientations from the inner target rib. The markers on each curve are the values that FLOWTRAN-TF would use at each of 8 azimuthal cells within the tube.

4. The neutronic axial shape depends on time, so that we can model the effects of safety rod insertion. Figure 2.2-5 is a Mark 22 axial flux shape that has been bounded for LOCA-ECS analysis. The axial decay heat shaje is independent of time.

5. Three separate power fractions are required for the inner clad, fuel, and outer clad regions of each tube (neutronic, moderated decay and dry decay components). The power fractions are independent of time and are functions of reactor exposure. Figure 2.2-6 is a plot of the Mark 22 inner fuel power fraction as a function of $U^{235}$ burn-up for each of the three deposited power components. Typically, the inner and outer clad power fractions are set to zero.

We now derive the nodal deposited power. Let the deposited neutronic or decay heat power density at a given point be expressed as:

$$
q^{\prime \prime \prime}(r, \theta, z, t)=C R(r) \Theta(\theta) Z(z, t)
$$

where

$$
\begin{aligned}
& C \equiv \text { Power normalization parameter } \\
& R \equiv \text { Non-normalized radial shape magnitude } \\
& \Theta \equiv \text { Non-normalized azimuthal shape magnitude } \\
& Z \equiv \text { Non-normalized axial shape magnitude. }
\end{aligned}
$$

For a given tube region (i.e. clad or fuel region) we integrate Eq. (2.2-2) over the volume contained within the region as:

$$
\text { Region deposited power }=\iiint_{V_{r}} q^{\prime \prime \prime} r d r d \theta d z=C \iiint_{V_{r}} R \Theta Z r d r d \theta d z
$$

This triple-integral represents the total deposited power in a tube region and is computed for the neutronic, moderated decay power, and dry decay power components as: 


$$
\Pi_{n r e g}(t)=\iiint_{V_{n r e g}} q^{\prime \prime \prime} r d r d \theta d z=\left\{\begin{array}{l}
p^{n p}(n r e g) \Pi(0)\left(1-x(t) \delta_{\text {mod }}\right)\left[\frac{H(t)}{H}\right] F^{n p}(t) \\
p f_{\bmod }^{d p}(n r e g) \Pi(0) x(t) \delta_{\bmod }\left[\frac{H(t)}{H}\right] F_{\text {mod }}^{d p}(t) \\
p f_{d r y}^{d p}(n r e g) \Pi(0) x(t) \delta_{d r y}\left[1-\frac{H(t)}{H}\right] F_{d r y}^{d p}(t)
\end{array}\right.
$$

where

$$
\begin{aligned}
\mathrm{pf}^{\mathrm{np}} & \equiv \text { Tube region neutronic power fraction, } \\
\mathrm{pf}_{\text {mod }}^{\mathrm{dp}} & \equiv \text { Tube region moderated decay heat power fraction, } \\
\mathrm{pf}_{\mathrm{dry}}^{\mathrm{dp}} & \equiv \text { Tube region dry decay heat power fraction, } \\
\text { nreg } & \equiv \text { Tube region index. }
\end{aligned}
$$

The sum of all the tube region deposited powers must equal the total assembly deposited power defined in Eq. (2.2-1):

$$
\Pi(t)=\sum_{\text {nreg }} \Pi_{\text {nreg }}(t)
$$

For each deposited power component, we compute the corresponding power normalization parameter for each region by combining Eqs. (2.2-3) and $(2.2-4)$ with the appropriate limits of integration. The neutronic power normalization parameter is:

$$
\begin{aligned}
C^{n p} & =\frac{p^{n p}(n r e g) \Pi(0)\left(1-x(t) \delta_{m o d}\right)\left[\frac{H(t)}{H}\right] F^{n p}(t)}{\int_{0}^{H(t)} Z^{n p}(z, t) d z \int_{0}^{2 \pi} \int_{r_{1}}^{r_{2}} R^{n p} \Theta r d r d \theta} \\
& =\frac{p^{n p}(n r e g) \Pi(0)\left(1-x(t) \delta_{m o d}\right)\left[\frac{H(t)}{H}\right] F^{n p}(t)}{\sum_{i z=1}^{n z} z^{n p}(i z, t) \delta z(i z) \sum_{i a=1}^{n a} \sum_{i r=1 r_{1}}^{I_{2}} R^{n p}(i r) \Theta(i a) A(i r)} \\
\delta z(i z) & =\left\{\begin{array}{cl}
H(t)-b o t n o d(i z) & \text { partially moderated node } \\
\Delta z(i z) & \text { wet node }
\end{array}\right.
\end{aligned}
$$

where

$$
\mathrm{R}^{\mathrm{np}} \equiv \text { Neutronic radial shape magnitude }
$$




$$
\begin{aligned}
\mathrm{Z}^{\mathrm{np}} & \equiv \text { Neutronic axial shape magnitude } \\
\mathrm{r}_{1}, \mathrm{r}_{2} & \equiv \text { Inner and outer radii, respectively, for a given region } \\
\mathrm{A} & \equiv \text { Node cross-sectional area } \\
\mathrm{ir}, \mathrm{ia}, \mathrm{iz} & \equiv \text { Spatial nodal index } \\
\mathrm{na} & \equiv \text { Number of azimuthal divisions } \\
\mathrm{nz} & \equiv \text { Number of axial divisions } \\
\text { botnod } & \equiv \text { Elevation of lower face of axial node iz relative to bottom of active core } \\
\Delta z & \equiv \text { Axial cell length in active core region. }
\end{aligned}
$$

Similarly, the moderated decay heat power normalization parameter is computed as:

$$
\begin{aligned}
C_{m o d}^{d p} & =\frac{p_{m o d}^{d p}(n r e g) \Pi(0) x(t) \delta_{m o d}\left[\frac{H(t)}{H}\right] F_{m o d}^{d p}(t)}{\int_{0}^{H(t)} Z^{n p}(z, 0) d z \int_{0}^{2 \pi} \int_{r_{1}}^{r_{2}} R_{m o d}^{d p} \Theta r d r d \theta} \\
= & \frac{p f_{m o d}^{d p}(n r e g) \Pi(0) x(t) \delta_{m o d}\left[\frac{H(t)}{H}\right] F_{m o d}^{d p}(t)}{\sum_{i z=1}^{n z} Z^{n p}(i z, 0) \delta z(i z) \sum_{i a=1}^{n a} \sum_{i r=i r_{1}}^{i_{2}} R_{m o d}^{d p}(i r) \Theta(i a) A(i r)}
\end{aligned}
$$

where

$$
\mathrm{R}_{\text {mod }}^{\mathrm{dp}} \equiv \text { Moderated decay heat radial shape magnitude. }
$$

The decay heat azimuthal power shape is assumed to be identical to the neutronic shape. In addition, the axial shape function is assumed to be identical to the neutronic axial shape function at steady-state $(t=0)$. This identity arises because the effect of safety rod insertion on the distribution of fission products (and hence the $\beta$ absorption distribution) is negligible. The safety rod insertion will also have a negligible effect on the fission product the pre-incident stable redistributed, only the pre-incident stable neutron distribution and fission product power distribution. 
The dry decay heat power normalization parameter is:

$$
\begin{aligned}
& C_{d r y}^{d p}=\frac{p f_{d r y}^{d p}(n r e g) \Pi(0) x(t) \delta_{d r y}\left[1-\frac{H(t)}{H}\right] F_{d r y}^{d p}(t)}{\int_{H(t)}^{H} Z^{n p}(z, 0) d z \int_{0}^{2 \pi} \int_{r_{1}}^{r_{2}} R_{d r y}^{d p} \Theta r d r d \theta} \\
&=\frac{p_{d r y}^{d p}(n r e g) \Pi(0) x(t) \delta_{d r y}\left[1-\frac{H(t)}{H}\right] F_{d r y}^{d p}(t)}{\sum_{i z=1}^{n z} Z^{n p}(i z, 0) \delta z(i z) \sum_{i a=1}^{n a} \sum_{\text {ir }=i r_{1}}^{i r_{2}} R_{d r y}^{d p}(i r) \Theta(i a) A(i r)} \\
& \delta z(i z)=\left\{\begin{array}{cl}
\Delta z(i z) & \text { dry node } \\
\operatorname{topnod}(i z)-H(t) & \text { partially moderated node } \\
0 & \text { wet node }
\end{array}\right.
\end{aligned}
$$

where

$$
R_{d r y}^{d p} \equiv \text { Dry decay heat radial shape magnitude, }
$$

topnod $\equiv$ Elevation of upper face of axial node iz relative to bottom of active core

The nodal deposited power is computed in FLOWTRAN-TF by summing over the contributions to the deposited power density weighted by the volume of the node that is dry or wet. The result is:

$$
\begin{aligned}
P(i r, i a, i z, i c y l n, t) & =C^{n p} R^{n p}(i r, i c y l n) \Theta(i a, i c y l n) Z^{n p}(i z, t) A(i r, i c y l n) \delta z(i z) \\
& +C_{m o d}^{d p} R_{m o d}^{d p}(i r, i c y l n) \Theta(i a, i c y l n) Z^{n p}(i z, 0) A(i r, i c y l n) \delta z(i z) \\
& +C_{d r y}^{d p} R_{d r y}^{d p}(i r, i c y l n) \Theta(i a, i c y l n) Z^{n p}(i z, 0) A(i r, i c y l n) \delta z(i z)
\end{aligned}
$$

where

$$
\text { icyln } \equiv \text { Cylinder index. }
$$

\section{Power Shape Approximations}

The selection of appropriate power shapes to use in the FLOWTRAN-TF power model is not entirely straight-forward. FLOWTRAN-TF models a single assembly which represents a single flowzone. The same flowzone occurs at multiple positions throughout the reactor core. Thus variations in local power shapes (due to differing neighboring assemblies and due to changes in local control rod positioning) must be accounted for indirectly in the model. Generally, a pad is added to the calculated limiting power to account for interassembly power shape variations in the horizontal plane, while variations in the axial shape 
are handled by doing multiple calculations with bounding axial shapes and simply selecting the worst (i.e., most conservative) result.

In addition, the complete power shape database is not yet in hand. In particular, the current model assumes that, for a given assembly type, the radial shape is solely a function of operating power history. The effect of other variables is currently under study (see Webb and Parks, 1988 and Webb, 1988). In one final simplification, the azimuthal shape is assumed to be the same for the neutronic and decay heat shapes (i.e., $\Theta^{\mathrm{np}}=\Theta^{\mathrm{dp}}$ ).

\section{Experimental Rig Heat Generation}

Experimental rigs and mock-ups are primarily heated through resistance heating or magnetic induction. Tube wall thicknesses and/or material compositions are varied axially and azimuthally to generate heat generation profiles that simulate those of neutron flux peaking. FLOWTRAN-TF utilizes the dry tank power distribution described above to calculate heat generation in experimental rigs. Experimental rig tube power fractions and radial shape factors are specified using the at-power (neutronic) input section. These values are then assigned internally in FLOWTRAN-TF to their corresponding dry decay values.

For experimental rigs, Eq. $(2.2-11)$ can be simplified and written as:

$$
P(\text { ir, ia, iz, icyln }, t)=C R(\text { ir }, \text { icyln }) \Theta(\text { ia }, \text { icyln }) Z(i z) A(i r, \text { icyln }) \Delta z(i z)
$$

where

$$
\begin{aligned}
C & \equiv \text { Heat generation normalization parameter, } \\
R & \equiv \text { Radial shape factor, nominally set to } 1.0, \text { for rigs, } \\
\Theta & \equiv \text { Azimuthal shape factor, } \\
Z & \equiv \text { Axial shape factor, } \\
A & \equiv \text { Cross-sectional area of node, } \\
\Delta \mathbf{z} & \equiv \text { Axial cell length. }
\end{aligned}
$$

The axial flux shape is not allowed to vary whth ume since experimental simulation of that effect is difficult to do.

Similarly, Eq. (2.2-4) simplifies to

$$
\Pi_{\text {nreg }}(t)=p f(\text { nreg }) \Pi(0) F(t)
$$

where

$$
\begin{aligned}
\mathrm{pf} & \equiv \text { Tube region power fraction, } \\
F(t) & \equiv \text { Heat generation transient response. }
\end{aligned}
$$


Since the radial, azimuthal and axial shape factors are static, the normalization parameter, $\mathrm{C}$, is computed as

$$
C=\frac{p f(n r e g) \Pi(0) F(t)}{\int_{0}^{H} Z d z \int_{0}^{2 \pi} \int_{r_{1}}^{r_{2}} R \Theta r d r d \theta}=\frac{p f(n r e g) \Pi(0) F(t)}{\sum_{i z=1}^{n z} Z(i z) \Delta z(i z) \sum_{i a=1}^{n a} \sum_{i r=i r_{1}}^{i r_{2}} R(i r) \Theta(i a) A(i r)}
$$

\section{References}

Baxter, C. A. and C. E. Apperson, Jr., 1982, "Revision of the Hydraulic Manual Decay Heat Tables," DPST-82-646, August 1982. (also DPST-81-785, DPST-82-432).

Graves, W. E., 1989, "Glass User's Manual," DPSTM-89-100-1, July 1989.

Gregory, M. V., 1992, "Revised Conservative Decay Heat Model," VIM-MVG-90005, February 6, 1990.

Parks, P. B. and W. E. Graves, 1987, "Derivation of the Assembly Deposited Power Ratio for Use in ECS Analyses - Revision 2,"DPST-85-239, October 28, 1987.

Pevey, R. E., 1987, "Axial Power Profiles in SRP Reactors During LOCA,."DPST-87511, June 26, 1987.

Smith, J. A. and J. P. Church, 1990, "Accident Analysis Computer Program," DPSTM120, February 171990.

Webb, R. L. and P. B. Parks, 1988, "Program to Provide Power Shape Data for FLOWTRAN," NEDP-88-1, January 15, 1988.

Webb, R. L., 1988, "Radial Power Profiles in Mark 16-31A/B Assemblies Resulting from the Deposition of Fission Product Decay Energy, "NEDP-88-8, May 6, 1988. 


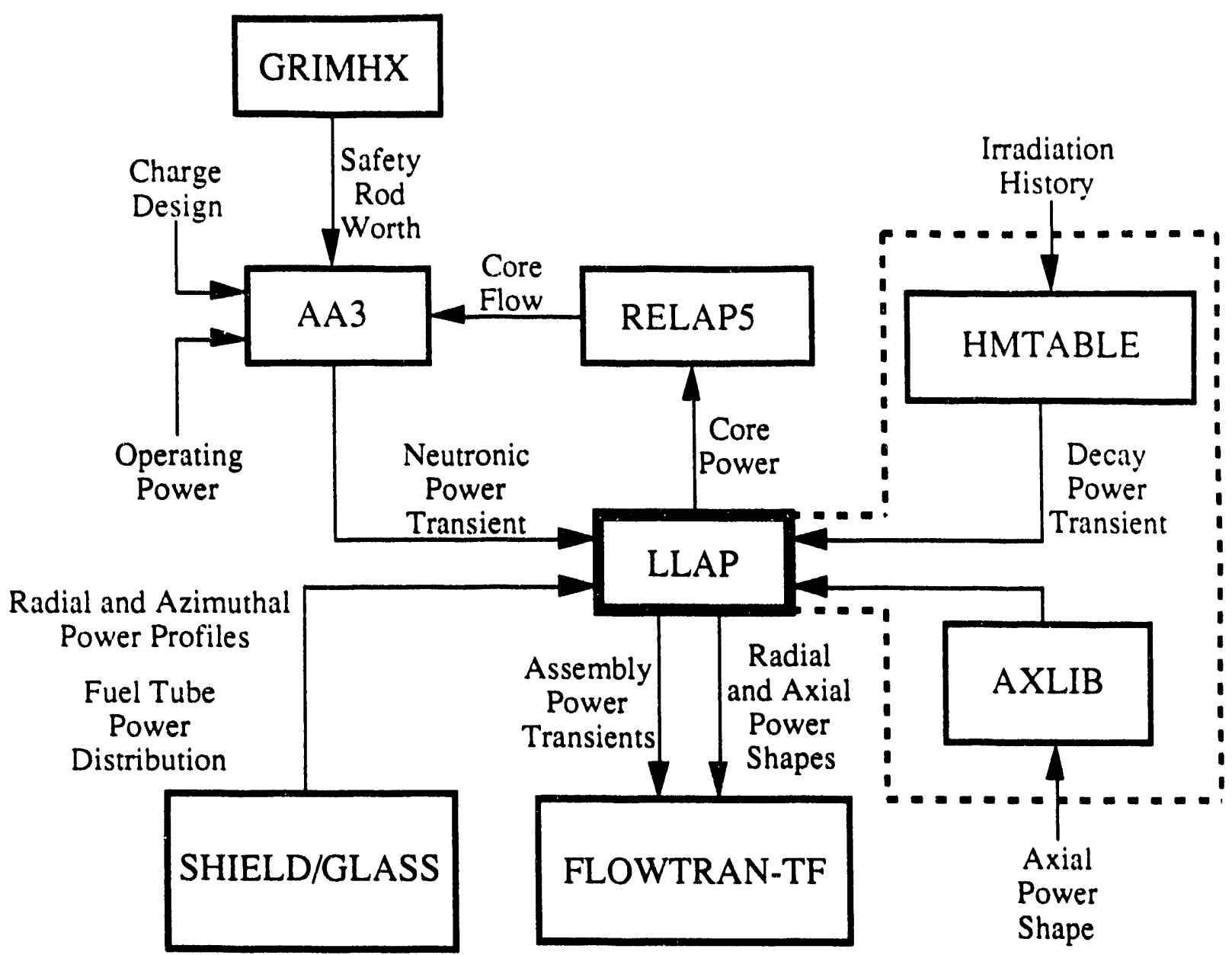

Figure 2.2.1 Assembly Deposited Power Computational Diagram 


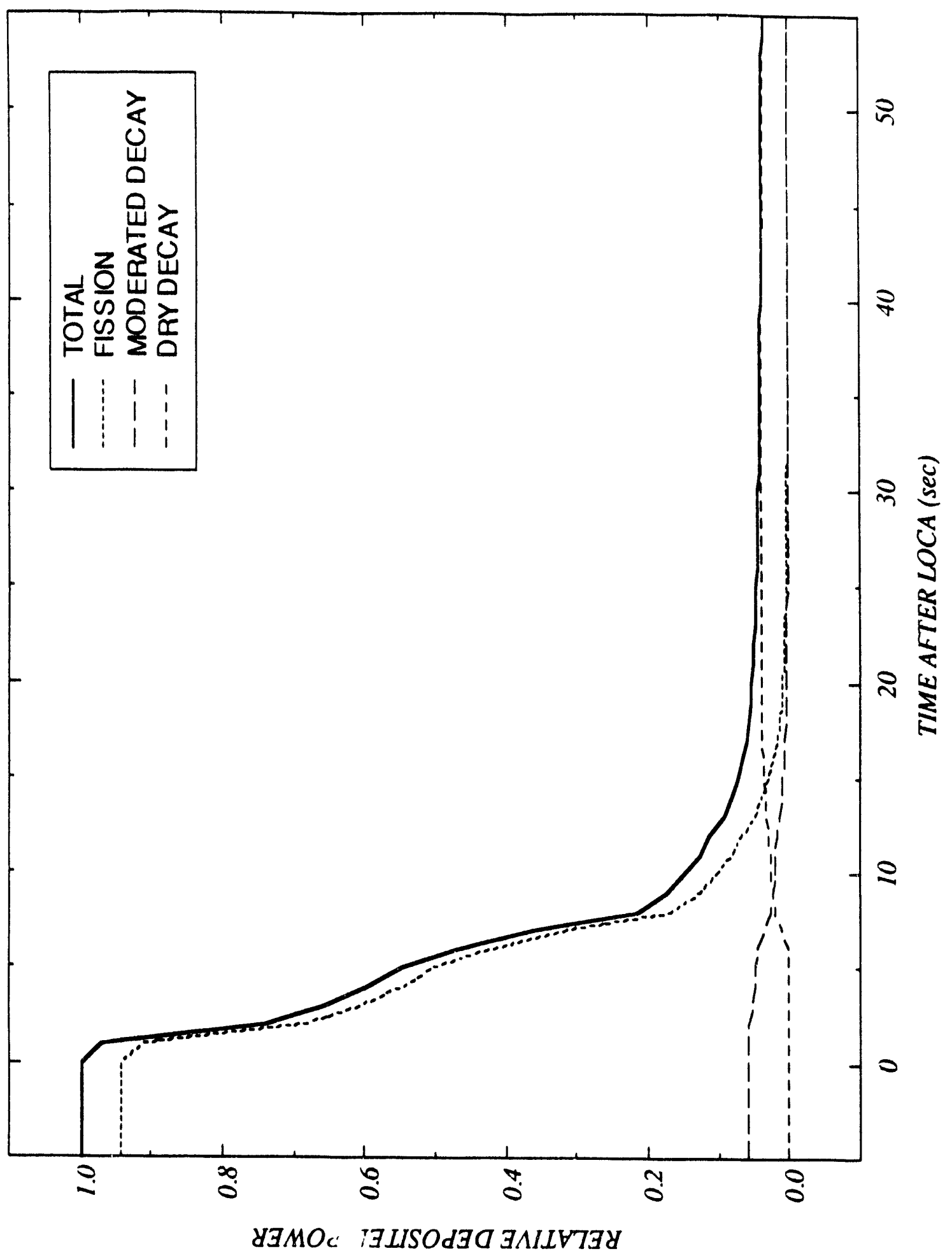

Figure 2.2.2 Constituents of Deposited Assembly Power 


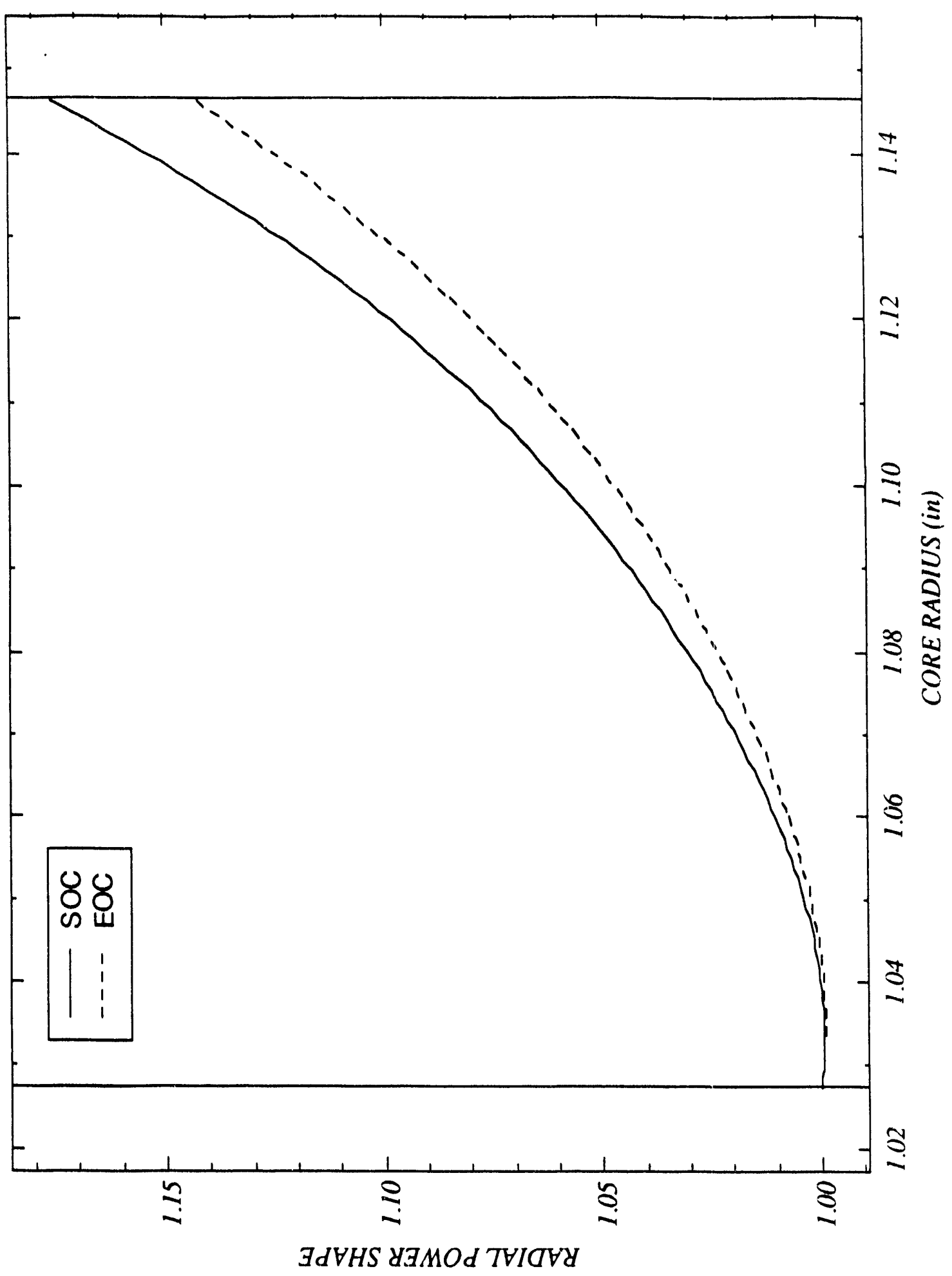

Figure 2.2.3 Mark 22 Inner Fuel Neutronic Radial Power Shape 


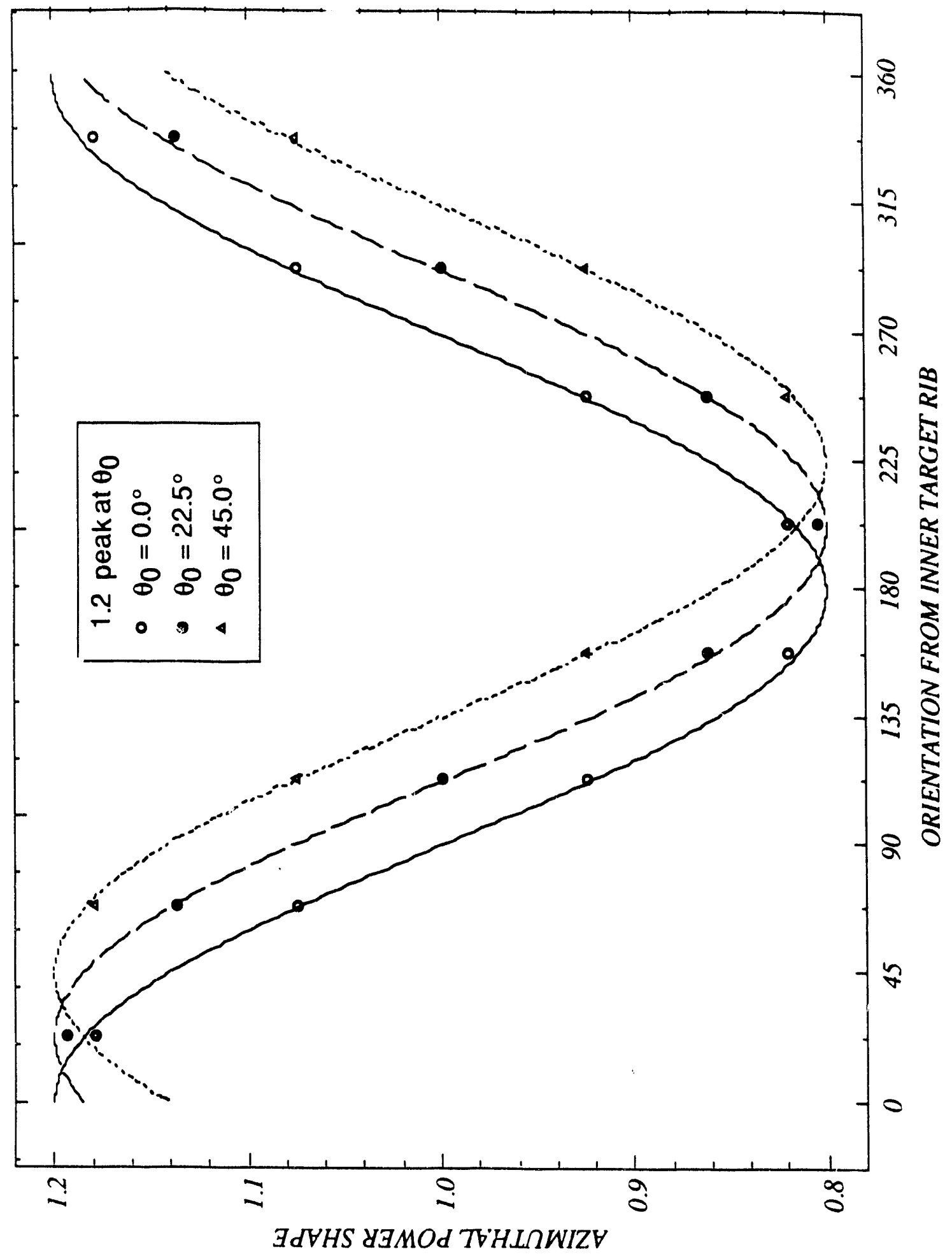

Figure 2.2.4 Mark 22 Azimuthal Flux Shapes (Bounded) 


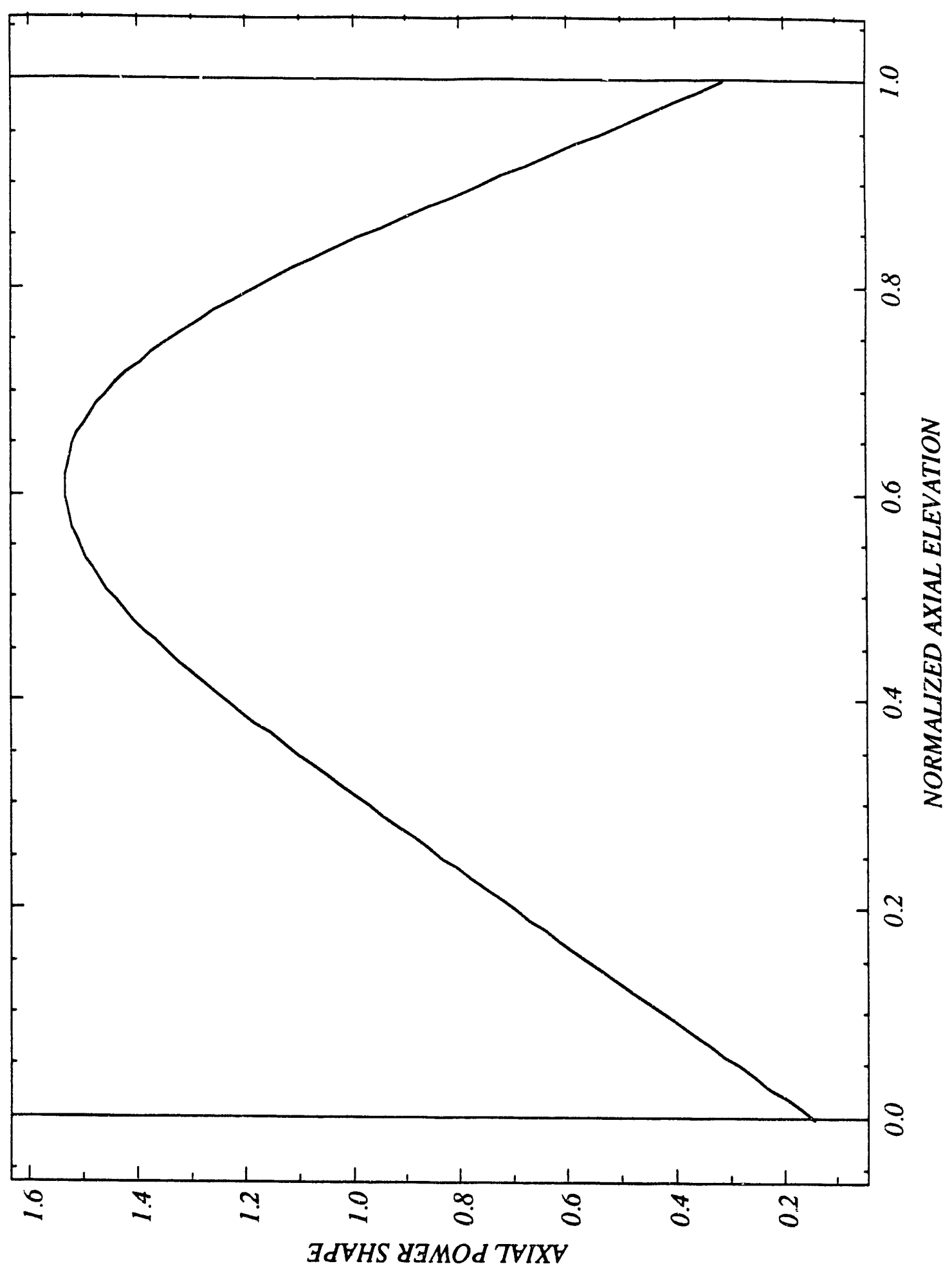

Figure 2.2-5 Axial Flux Shape (Bounded) 


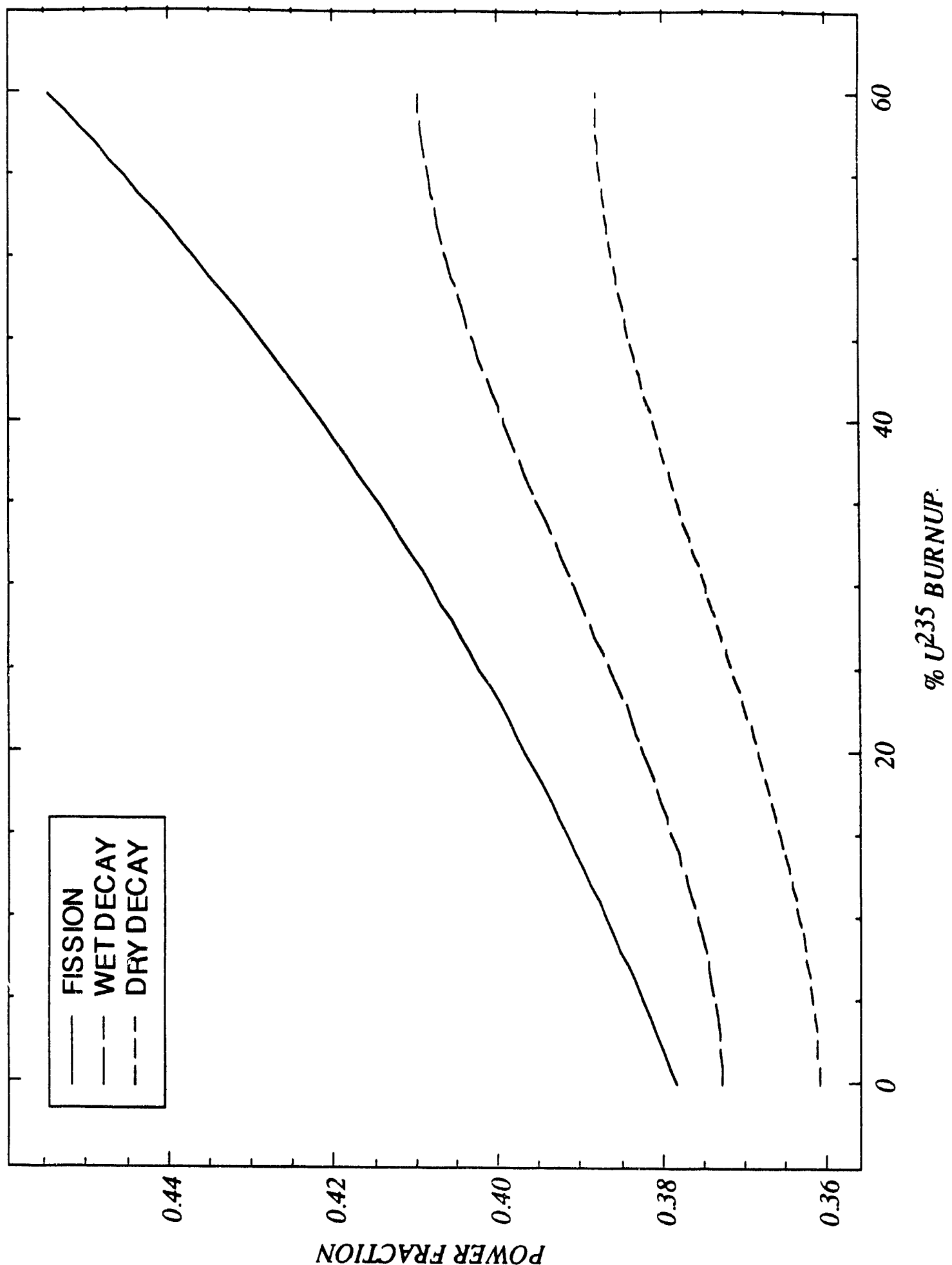

Figure 2.2-6 Mark 22 Inner Fuel Power Fraction 


\subsection{Solid Governing Equations}

The FLOWTRAN-TF code solves the full unsteady three-dimensional heat conduction equations in a cylindrical geometry to determine the temperature distribution within solid reactor assembly components and the assembly surface temperatures.

\section{Solid Geometry}

A schematic representation of a single metal cylinder within a reactor assembly illustrating the differencing mesh used in FLOWTRAN-TF is presented in Fig. 2.3-1. Several such cylinders concentrically positioned around each other exist within a reactor assembly. Heat transfer coupling between the cylinders occurs through the intermediate fluid in the surrounding flow annuli. The heat conduction equations are solved for each metal cylinder independently and the overall solution iterated to convergence using the numerical scheme presented later. 


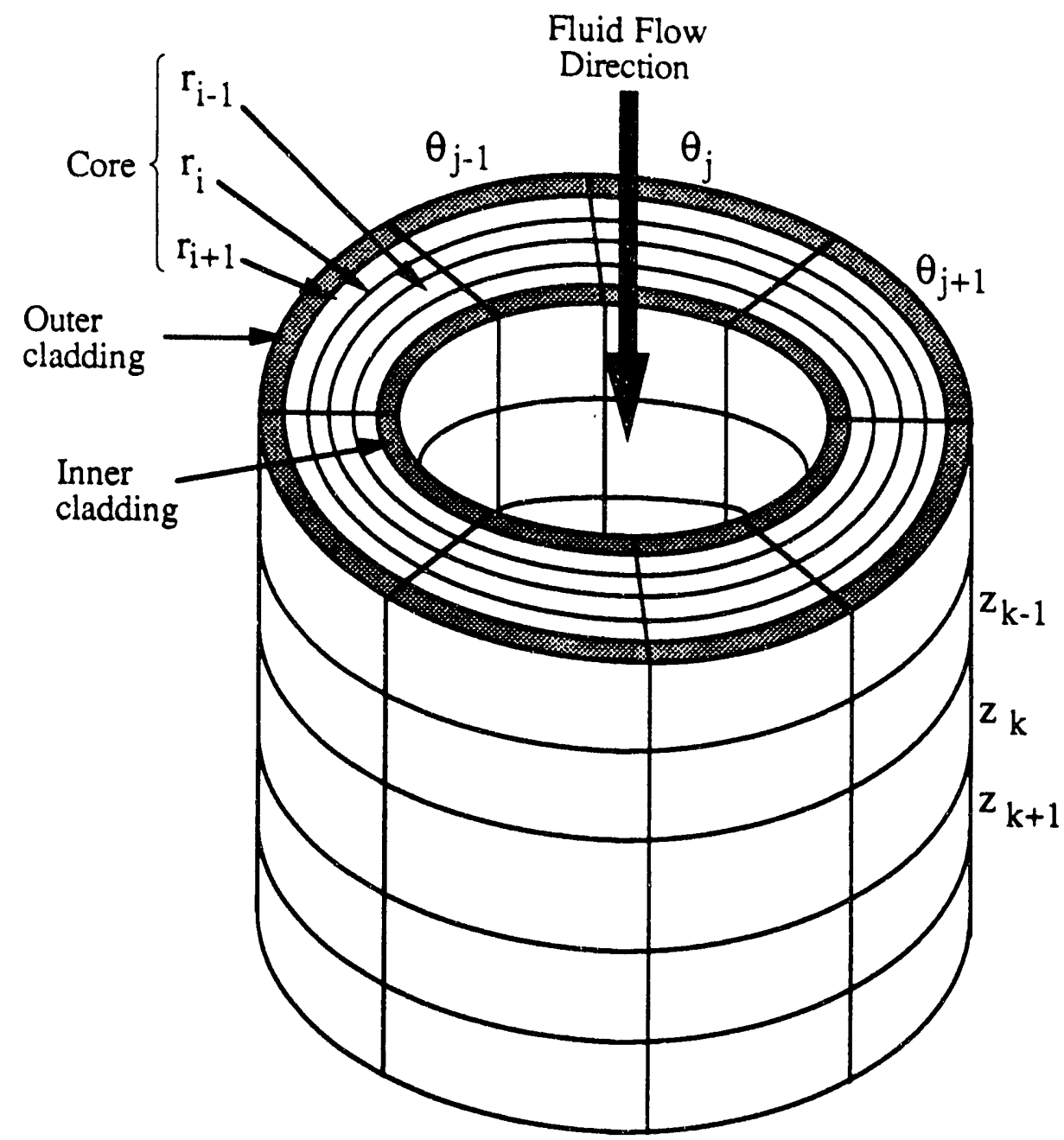

Figure 2.3-1 Schematic representation of mesh used for FLOWTRAN-TF solid heat conduction calculations in a single cylinder.

\section{Governing Equations}

The fundamental equation describing unsteady heat conduction in a solid material in terms of the solid internal energy per unit volume $\left(\mathrm{E}, \mathrm{J} / \mathrm{m}^{3}\right)$ is given by

$$
\frac{\partial E}{\partial t}=\nabla \cdot(k(T) \nabla T)+q^{\prime \prime \prime}
$$

where

$$
\begin{aligned}
k(T) & \equiv \text { Solid thermal conductivity in W/m C } \\
q^{\prime \prime \prime}(r, \theta, z ; t) & \equiv \text { Rate of internal energy generation in } W / \mathrm{m}^{3}
\end{aligned}
$$


In writing Eq. (2.3-1) we use Fourier's law of heat conduction to determine the heat flux vector in the solid and assume that the velocity in the solid is identically zero. We are then neglecting the increase in internal energy arising from expansion or compression of the solid. Since the solution strategy is designed to conserve energy, the basic equations are cast in terms of the solid energy and Eq. (2.3-1) may be viewed as an equation for solid energy conservation. The solid internal energy may be expressed as a function of temperature $(\mathrm{T}, \mathrm{C})$ by

$$
E(T)=E_{0}+\int_{T_{0}}^{T} \rho_{0} c_{p}(T) d T
$$

where

$$
\begin{aligned}
\rho_{0} & \equiv \text { Solid density at reference temperature in } \mathrm{kg} / \mathrm{m}^{3} \\
c_{\mathrm{p}}(\mathrm{T}) & \equiv \text { Solid heat capacity at constant pressure in } \mathrm{J} /(\mathrm{kg} \mathrm{C}) \\
\mathrm{T}_{0} & \equiv \text { Reference temperature in } \mathrm{C} \\
\mathrm{E}_{0} & \equiv \text { Solid internal energy at the reference temperature. }
\end{aligned}
$$

As indicated by the above notation, in general the solid heat capacity will be a function of temperature and the solution strategy developed here will account for this temperature dependence. However, the solid density is fixed at a reference value since we use a fixed solid grid mesh and do not alter the cell volume with changes in temperature. Therefore, a constant solid density is required in the calculations to conserve solid mass.

A general three-dimensional control volume for the solid heat conduction calculation is illustrated in Fig. 2.3-2. In cylindrical coordinates, we use the indexing $\mathrm{i}, \mathrm{j}, \mathrm{k}$ for the radial, azimuthal, and axial directions, respectively. Integrating Eq. (2.3-1) over the general control volume $\left(\mathrm{V}_{\mathrm{ijk}}\right)$ yields

$$
\frac{\partial}{\partial t}\left\{\int_{V_{i j k}} E d V\right\}=\int_{S_{i j k}}(k \nabla T) \cdot d \vec{S}+\int_{V_{i j k}} q^{\prime \prime \prime} d V
$$

where the divergence theorem has been applied to convert the volume integral of the divergence of the heat fluxes to the corresponding surface integral. 


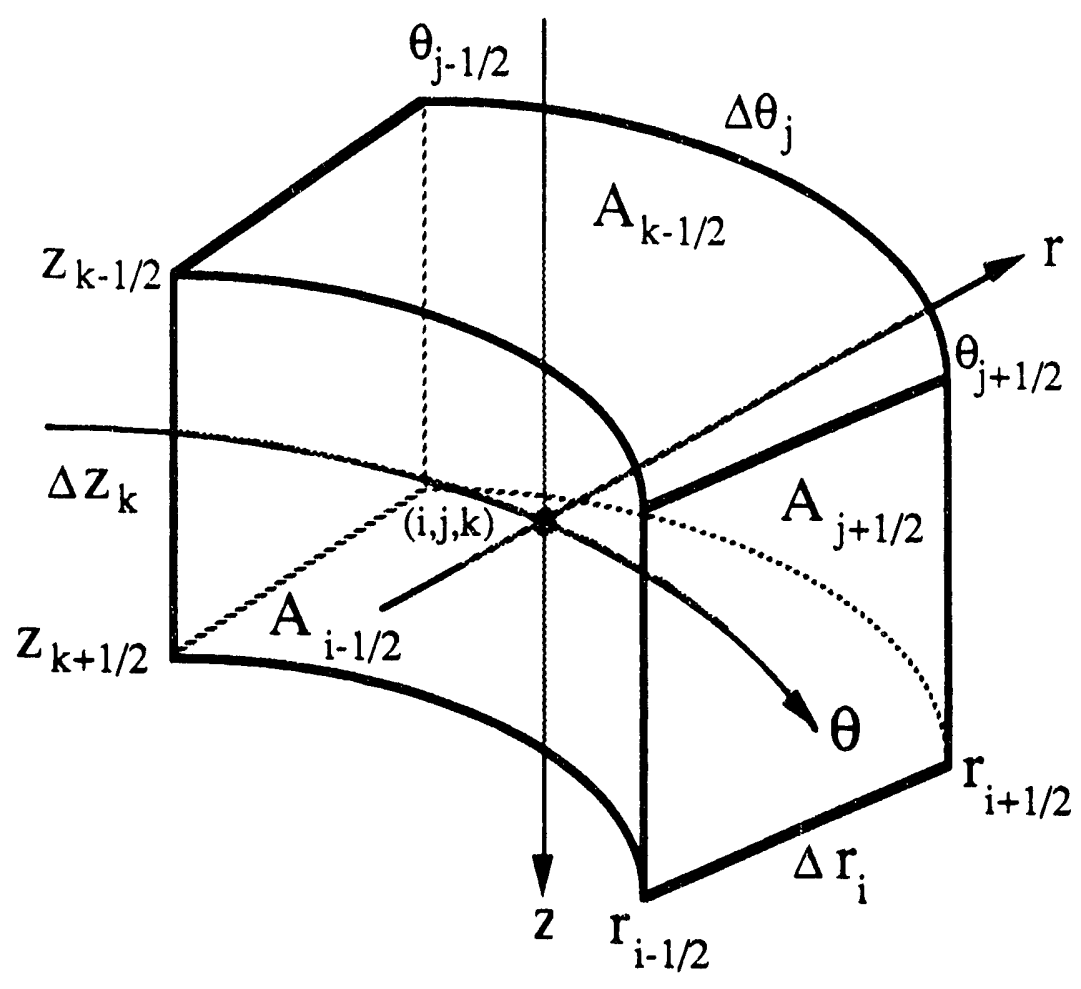

Figure 2.3-2 Schematic representation of a single solid mesh cell.

Replacing the variables in Eq. (2.3-3) with volume averaged values gives a final form of the basic governing equation as

$$
V_{i j k} \frac{\partial E}{\partial t}=\sum_{s=1}^{6}\left\{\left(k_{s} \nabla T_{s} \cdot N_{s}\right) A_{s}\right\}+V_{i j k} q^{\prime \prime \prime}
$$

where the summation is over the six faces of the volume element, $\mathbf{N}_{\mathbf{s}}$ is the unit normal to surface $s$, and $A_{s}$ is the area of the $s^{\text {th }}$ face. Figure 2.3-2 indicates that variable mesh spacings are allowed in all three directions. In practice, the radial and axial mesh spacings may vary with nodal position however the azimuthal mesh spacing is fixed at a constant value. Inserting expressions for the cell face areas into Eq. (2.3-4) and collecting like terms gives the heat conduction equation in the form used to develop the FLOWTRAN-TF finite differencing scheme as 


$$
\begin{aligned}
\mathrm{V}_{\mathrm{ijk}} \frac{\partial \mathrm{E}}{\partial \mathrm{t}} & =\left\{\mathrm{k}_{\mathrm{i}+1 / 2}\left[\frac{\partial \mathrm{T}}{\partial \mathrm{r}}\right]_{\mathrm{i}+1 / 2} \mathrm{r}_{\mathrm{i}+1 / 2}-\mathrm{k}_{\mathrm{i}-1 / 2}\left[\frac{\partial \mathrm{T}}{\partial \mathrm{r}}\right]_{\mathrm{i}-1 / 2} \mathrm{r}_{\mathrm{i}-1 / 2}\right\} \Delta \theta_{\mathrm{j}} \Delta \mathrm{z}_{\mathrm{k}} \\
& +\left\{\mathrm{k}_{\mathrm{j}+1 / 2} \frac{1}{\mathrm{r}_{\mathrm{i}}}\left[\frac{\partial \mathrm{T}}{\partial \theta}\right]_{\mathrm{j}+1 / 2}-\mathrm{k}_{\mathrm{j}-1 / 2} \frac{1}{\mathrm{r}_{\mathrm{i}}}\left[\frac{\partial \mathrm{T}}{\partial \theta}\right]_{\mathrm{j}-1 / 2}\right\} \Delta \mathrm{r}_{\mathrm{i}} \Delta \mathrm{z}_{\mathrm{k}} \\
& +\left\{\mathrm{k}_{\mathrm{k}+1 / 2}\left[\frac{\partial \mathrm{T}}{\partial \mathrm{z}}\right]_{\mathrm{k}+1 / 2}-\mathrm{k}_{\mathrm{k}-1 / 2}\left[\frac{\partial \mathrm{T}}{\partial \mathrm{z}}\right]_{\mathrm{k}-1 / 2}\right\} \mathrm{r}_{\mathrm{i}} \Delta \mathrm{r}_{\mathrm{i}} \Delta \theta_{\mathrm{j}} \\
& +\mathrm{V}_{\mathrm{ijk}} \mathrm{q}^{\prime \prime \prime}
\end{aligned}
$$

The rate of energy generation ( $\left.\mathrm{q}^{\prime \prime \prime}\right)$ in Eq. (2.3-5) will in general depend on the mesh cell location and on time.

\section{Rib Heat Transfer}

Some of the metal cylinders within reactor assemblies at the Savannah River Site have aluminum spacing ribs on their surfaces. These ribs serve to keep the fuel and target tubes separated and are formed from the cladding material during the extrusion process when the tubes are manufactured. The FLOWTRAN-TF code uses a simple model to account for heat transfer effects at the ribbed surfaces.

A schematic representation of a rib is shown in Fig. 2.3-3 where we assume that a rib of length $R_{L}$ and half thickness $R_{T}$ is present on the inner surface (labeled Solid Surface 1 ) of a coolant flow channel. Figure 2.3-3 illustrates parts of two fluid computational cells with one cell on either side of the rib. Along Surface 1, the fluid cell has a nominal surface area $A_{T 1}$ and actual wetted surface area $A_{W_{1}}$. The wetted surface is smaller than the nominal cell surface area by $R_{T} \Delta z$ where $\Delta z$ is the axial cell length and $R_{T}$ is one half of the thickness of the rib. Similarly, along Surface 2 , the fluid cell has total surface area $A_{T 2}$ and actual wetted surface area Aw2 where we assume that the surface within the rib gap is not wetted. Since we are dealing with a cylindrical geometry, the areas along Surface 2 will be slightly greater than the corresponding areas along Surface 1. For simplicity, we assume that the rib has a uniform rectangular cross-section. The fluid and solid heat transfer calculations in FLOWTRAN-TF assume that all of the heat transfer takes place across the nominal total surface areas $A_{T 1}$ and $A_{T 2}$. We wish to develop a simple scheme that will account for the modified heat transfer that takes place at these surfaces from the presence of the rib without explicitly performing an exact heat transfer calculation along the rib. 


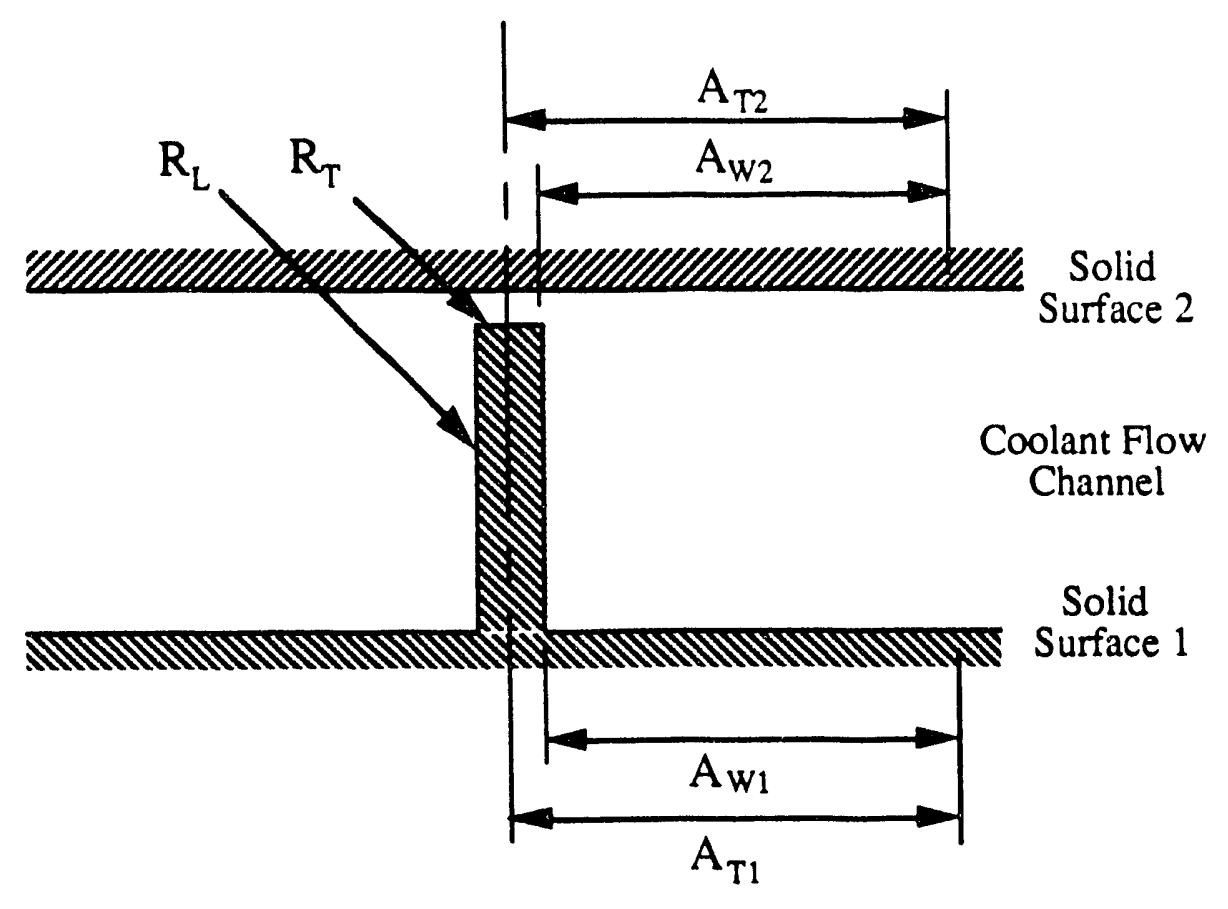

Figure 2.3-3 Schematic representation of rib geometry within a fluid computational cell.

We first consider Surface 1 which has the rib attached to it. The total heat deposited in the flow channel is equal to the sum of the heat transferred from the wetted cylinder surface and the heat transferred from the rib surface. Writing this relationship in terms of surface heat fluxes and surface areas gives

$$
q^{\prime \prime} A_{T}=q_{W}^{\prime \prime} A_{W}+q_{R}^{\prime \prime} N_{R i b} R_{L} \Delta z
$$

where

$$
\begin{aligned}
A_{T} & \equiv \text { Total cell surface area } \\
A_{W} & \equiv \text { Wetted metal surface area } \\
N_{R i b} & \equiv \text { Number of half ribs in the computational cell } \\
q^{\prime \prime} & \equiv \text { Effective or apparent surface heat flux } \\
q_{W}^{\prime \prime} & \equiv \text { True surface heat flux at wetted metal surface } \\
q_{R}^{\prime \prime} & \equiv \text { True surface heat flux at rib surface } \\
R_{L} & \equiv \text { Rib length } \\
\Delta z & \equiv \text { Cell axial length }
\end{aligned}
$$

The product $\left(\mathrm{N}_{\mathrm{Rib}} \mathrm{R}_{\mathrm{L}} \Delta \mathrm{z}\right.$ ) in Eq. (2.3-6) is the total rib surface area within the cell where we include the number of rib sides for generality. For three-dimensional solid heat 
conduction calculations, where a fluid computational cell may include all or a portion of a single flow subchannel, the cell would have either two, one, or zero rib surfaces present. For two-dimensional calculations, where a fluid computational cell corresponds to an entire annulus, each cell would have either eight or zero rib surfaces. That is, we assume that an assembly will have either four ribs or no ribs in each flow channel. The number of ribs, the rib dimensions, and their orientation are set by the code user through input. The apparent heat flux is the heat flux used as a boundary condition to calculate solid and fluid heat transfer within the FLOWTRAN-TF code. That is, FLOWTRAN-TF uses a heat flux that corresponds to total surface area $A_{T}$ in heat transfer calculations. Since this treatment does not explicitly account for the heat capacity of the rib, transient effects from the presence of the ribs on the surface nodes are not modeled.

We assume that the surface heat fluxes can be written as the product of heat transfer coefficients and the temperature difference between the surfaces and the bulk fluid (see Eqs. (2.3-11) to (2.3-13) below). Further assuming that all of the surfaces see the same temperature difference and that the rib heat transfer coefficient is equal to the heat transfer coefficient for the wetted surface multiplied by a rib (fin) efficiency factor, substituting for the heat fluxes in Eq. (2.3-6) gives

$$
\tilde{h}_{\mathrm{FC}} A_{T}=h_{\mathrm{FC}} A_{\mathrm{W}}+\eta_{\mathrm{f}} h_{\mathrm{FC}} \mathrm{N}_{\mathrm{Rib}} R_{\mathrm{L}} \Delta z
$$

where

$$
\begin{aligned}
\tilde{\mathrm{h}}_{\mathrm{FC}} & \equiv \text { Effective forced convection heat transfer coefficient for the cell surface } \\
\mathrm{h}_{\mathrm{FC}} & \equiv \text { Forced convection heat transfer coefficient for the wetted surface } \\
\eta_{\mathrm{f}} & \equiv \text { Rib or fin efficiency factor }
\end{aligned}
$$

Equation (2.3-7) has been simplified by assuming that all of the terms contain the same temperature difference. This implies that the metal surface temperature is uniform across a cell and over the attached ribs. Since SRS assemblies are constructed of aluminum we expect that local temperature gradients over the metal surface are small and that this is a reasonable approximation.

In writing Eq. (2.3-7) we have neglected heat transfer from the small surface at the tip of the rib. The ribs on Savannah River assembly tubes are closely approximated by rectangular fins. Therefore, we can simplify our analysis by restricting FLOWTRAN-TF to the calculation of an efficiency factor for a rib having a rectangular cross-section that is insulated on the tip. The efficiency factor for such a rectangular fin, based on a steady state heat transfer analysis, is given by Holman (1976) as (following Holman's notation)

$$
\eta_{\mathrm{f}}=\frac{\tanh \left(m R_{L}\right)}{m R_{L}}
$$

where the function $\left(m R_{L}\right)$ is defined to be

$$
m R_{L}=\sqrt{\frac{h_{F C}}{k_{w} R_{T}}} R_{L}
$$

where $k_{w}$ is the metal thermal conductivity and $R_{T}$ is the half thickness of the rib. 
Equation (2.3-7) can be used to determine a rib effectiveness parameter $(\psi)$ which we define to be the ratio of the effective surface heat transfer coefficient to the true wetted surface heat transfer coefficient

$$
\begin{aligned}
\psi \equiv \frac{\tilde{h}_{F C}}{h_{F C}} & =\frac{A_{W}}{A_{T}}+\eta_{f} P_{R i b} R_{L} \\
& =1.0+P_{R i b}\left(\eta_{f} R_{L}-R_{T}\right)
\end{aligned}
$$

where

$$
P_{R \text { ib }} \equiv \begin{aligned}
& \text { Number of ribs divided by the wetted perimeter of the cell wall, } N_{\text {Rib }} \\
& \Delta z / A_{T}
\end{aligned}
$$

The second equality in Eq. $(2.3-10)$ is derived using the relationship $A_{W}=A_{T}-N_{R i b} R_{T} \Delta z$ and, as shown below, is particularly useful in simplifying the treatment of the rib heat transfer for both solid surfaces.

Rewriting the various surface heat fluxes in terms of the rib effectiveness parameter and the rib efficiency then gives us the following expressions

$$
\begin{gathered}
\mathrm{q}^{\prime \prime}=\tilde{h}_{\mathrm{FC}} \Delta \mathrm{T}=\psi \mathrm{h}_{\mathrm{FC}} \Delta \mathrm{T} \\
\mathrm{q}_{\mathrm{W}}^{\prime \prime}=\mathrm{h}_{\mathrm{FC}} \Delta \mathrm{T}=\frac{\mathrm{q}^{\prime \prime}}{\psi} \\
\mathrm{q}_{\mathrm{R}}^{\prime \prime}=\eta_{\mathrm{f}} \mathrm{h}_{\mathrm{FC}} \Delta \mathrm{T}=\eta_{\mathrm{f}} \frac{\mathrm{q}^{\prime \prime}}{\psi}
\end{gathered}
$$

FLOWTRAN-TF calculates $h_{F C}$ from the heat transfer correlations (see section 2.5.1) and determines $\eta_{\mathrm{f}}$ and $\psi$ from Eqs. (2.3-8) through (2.3-10). The metal thermal conductivity is calculated using the current temperature (i.e. old time step value). Equation (2.3-11) then allows the calculation of an apparent heat flux based on the total wall surface area. When evaluating criteria that depend on surface heat flux, Eqs. (2.3-12) and (2.3-13) should be used to estimate actual heat fluxes over the wetted surface and the attached rib. Since the fin efficiency factor $\eta_{\mathrm{f}}$ is less than one, the largest heat flux will be obtained on the non-rib wetted surface of the metal cylinder. As a conservative approach, this heat flux should then be used to evaluate power limit criteria expressions that depend on the surface heat flux. Currently, the FLOWTRAN-TF code does not employ any heat flux criteria to set power limits.

Since the gaps between ribs on SRS assemblies and the opposite metal surfaces are quite small, it is possible that water flow in this region is restricted and does not contribute to heat removal. Therefore, as a conservative approximation, the small surface area immediately across from a rib is considered to be adiabatic and heat transfer from this surface area (and from the rib tip) is not included in our treatment. We account for the reduction in heat transfer at the opposite surface by reducing the surface area available for heat transfer by a factor of $A_{W} / A_{T}$. That is, the effective heat transfer area on a surface opposite a rib (Surface 2 in Fig. 2.3-3) is determined by subtracting off the rib area from the true surface area. Equations (2.3-10) and (2.3-11) can be used directly as developed 
above to calculate an effective heat transfer coefficient for this surface by simply setting the rib efficiency factor for the surface to zero $\left(\eta_{\mathrm{f}}=0\right)$.

\section{Solid Material Properties}

Transient heat conduction calculations in FLOWTRAN-TF require the density, heat capacity, and thermal conductivity of the solid materials. Correlations of these properties for uranium, aluminum, 304L stainless steel, lithium-aluminum alloy, and uraniumaluminum alloy were obtained from the literature survey of Nash (1987) and from Touloukian (1970). These correlations were programmed into the code so that the user can select appropriate properties for these materials by simply specifying the material identification number through the code input. The correlations used in FLOWTRAN-TF are reproduced here for completeness and to provide the user with a guide to the range of applicability of the material property functions.

Density: For all of the single component materials, the temperature dependence of the density $\left(\rho, \mathrm{kg} / \mathrm{m}^{3}\right)$ is accurately approximated by the simple linen- relationship in temperature $(\mathrm{T}, \mathrm{C})$

$$
\rho=\alpha-\beta T
$$

where $\alpha$ and $\beta$ are constants. As noted above, we must use a constant solid density to conserve mass in the heat conduction solution. We have chosen to evaluate the metal density at a standard temperature of $\mathrm{T}_{0}=20^{\circ} \mathrm{C}$ since this should be approximately the temperature at which the solid dimensions were measured and therefore should give a accurate estimation of the true solid mass. Table 2.3-1 lists the density coefficients $\alpha$ and $\beta$ and gives the value of $\rho_{0}=\rho\left(T_{0}\right)$ for the three single component solids specified internally to the FLOWTRAN-TF code.

Heat Capacity and Thermal Conductivity: The heat capacity at constant pressure $\left(c_{p}, \mathrm{~kJ} / \mathrm{kg}-\mathrm{K}\right)$ and thermal conductivity $(\mathrm{k}, \mathrm{W} / \mathrm{m}-\mathrm{K})$ of the single component materials are well correlated as quadratic or cubic functions of temperature $\left(\mathrm{T}\right.$ in ${ }^{\circ} \mathrm{C}$ ) using

$$
c_{p}=c_{0}+c_{1} T+c_{2} T^{2}
$$

and

$$
k=k_{0}+k_{1} T+k_{2} T^{2}+k_{3} T^{3}
$$

respectively. The fitting coefficients used in FLOWTRAN-TF for Eqs. (2.3-15) and (2.3-16) are listed in Tables $2.3-2$ and $2.3-3$ below along with the fit correlation coefficients. The heat capacity of lithium-6 is taken from Touloukian (1970) and is also included in the Table 2.3-2 for use in the alloy property determinations. The heat capacity of 316 stainless steel as reported in Touloukian (1970) was used in the physical property determinations since these data were more consistent than the values reported for $304 \mathrm{~L}$ stainless steel. The heat capacity correlations are plotted in Figs. 2.3-4 and 2.3-5 while the thermal conductivity correlations are plotted in Figs. 2.3-6 and 2.3-7 below.

Alloy Properties: Alloy physical properties are correlated as functions of temperature and the weight percent of lithium or uranium in aluminum. Alloy heat capacities are assumed to obey the simple ideal-mixture mixing rules given by 


$$
c_{p}(L i-A l)=c_{p}(L i) W_{L i}+c_{p}(A l)\left[1.0-W_{L i}\right]
$$

and

$$
c_{p}(U-A l)=c_{p}(U) W_{U}+c_{p}(A l)\left[1.0-W_{U}\right]
$$

where $W_{L i}$ and $W_{U}$ are the weight fractions of lithium and uranium in the alloys, respectively. The thermal conductivities and densities of the two alloys were fit to correlating equations over the range of available data. Specifically, these properties were correlated as:

\section{Lithium-Aluminum Alloy}

$$
\mathrm{k}_{\mathrm{Li}-\mathrm{Al}}=\frac{242.168+\left(6.0405 \times 10^{3}\right) \mathrm{W}_{\mathrm{Li}}}{1.0+128.184 \mathrm{~W}_{\mathrm{Li}}}
$$

Equation (2.3-19) was fit to thermal conductivity data for lithium weight fractions from 0 to 0.104 with a correlation coefficient of 0.977 . Equation $(2.3-19)$ represents a simplified version of the expression reported by Nash (1987). Note that the temperature dependence of the alloy thermal conductivity was reported to be negligible over the temperature range of $20^{\circ} \mathrm{C}$ to $260^{\circ} \mathrm{C}$.

$$
\rho_{0}(\mathrm{Li}-\mathrm{Al})=\left(2.733-8.799 \mathrm{~W}_{\mathrm{Li}}+22.671 \mathrm{~W}_{\mathrm{Li}}^{2}\right) \times 10^{3}
$$

Equation (2.3-20) was evaluated at $20^{\circ} \mathrm{C}$ from a fit to data for lithium weight fractions ranging from 0 to 0.10 with a correlation coefficient of 0.995 .

Uranium-Aluminum Alloy

$$
\begin{aligned}
& \mathrm{k}_{\mathrm{U}-\mathrm{Al}}=222.43-354.52 \mathrm{~W}_{\mathrm{U}}+3.30 \times 10^{-2} \mathrm{~T} \\
&+280.38 \mathrm{~W}_{\mathrm{U}}^{2}+11.84 \times 10^{-2} \mathrm{~W}_{\mathrm{U}} \mathrm{T}-137.07 \times 10^{-6} \mathrm{~T}^{2} \\
& \rho_{0}(\mathrm{U}-\mathrm{Al})=\left(2.749+0.966 \mathrm{~W}_{\mathrm{U}}+5.397 \mathrm{~W}_{\mathrm{U}}^{2}\right) \times 10^{3}
\end{aligned}
$$

Equation (2.3-21) was fit to thermal conductivity data for uranium weight fractions from 0 to 0.305 with a correlation coefficient of 0.975 . As with the lithium-aluminum alloy, Eq. (2.3-22) represents the material density evaluated at $20^{\circ} \mathrm{C}$ and is a simplification of the density correlation presented by Nash (1987) that includes a temperature dependence. The correlation coefficient for the fit given by Eq. (2.3-22) is 0.998 . The thermal conductivity and density correlations for the alloy materials are shown below in Figs. 2.3-8 and 2.3-9, respectively.

Complete literature references, the data used to obtain the correlations, and graphs and tables showing the accuracy of the fits are given by Nash (1987). We have converted the correlations to SI units and, in some cases, we have simplified the correlations to improve computational efficiency without loss of accuracy. Graphs of the correlation fits to the experimental data for metal heat capacities and thermal conductivities as used in 
FLOWTRAN-TF are shown in Figs. 2.3-4 to 2.3-9 below to give the reader an indication of the accuracy and the range of applicability of the correlations. Correlation ranges for the material properties are listed in Table 2.3-4. The heat capacity data for lithium and stainless steel were taken directly from Touloukian (1970) where improved data were found allowing better correlations to be developed.

Table 2.3.1 Density coefficients $\left(\rho, \mathrm{kg} / \mathrm{m}^{3}\right)$ used in FLOWTRAN-TF code.

\begin{tabular}{|c|c|c|c|}
\hline Material & $\boldsymbol{\alpha} \times \mathbf{1 0}^{-3}$ & $\beta$ & $\rho_{0} \times \mathbf{1 0}^{-3}$ \\
\hline Uranium & 19.042 & 1.560 & 19.011 \\
Aluminum & 2.709 & 0.208 & 2.705 \\
$\begin{array}{c}\text { Stainless } \\
\text { Steel }\end{array}$ & 8.025 & 0.447 & 8.016 \\
\hline
\end{tabular}

Table 2.3-2 Heat capacity coefficients $\left(c_{p}, \mathrm{~kJ} / \mathrm{kg}-\mathrm{K}\right)$ used in FLOWTRAN-TF code and correlation coefficients.

\begin{tabular}{|c|c|c|c|c|}
\hline Material & $c_{0}$ & $c_{1} \times 10^{4}$ & $c_{2} \times 10^{7}$ & $r^{2}$ \\
\hline Uranium & 0.11624 & 0.43641 & 1.2957 & 0.998 \\
Aluminum & 0.88016 & 2.9376 & 3.4389 & 0.868 \\
$\begin{array}{c}\text { Stainless } \\
\text { Steel }\end{array}$ & 0.46004 & 2.8259 & -1.1067 & 0.980 \\
Lithium & 3.4591 & 32.869 & 58.337 & 0.999 \\
\hline
\end{tabular}


Table 2.3-3 Thermal conductivity coefficients $(\mathrm{k}, \mathrm{W} / \mathrm{m} \cdot \mathrm{K})$ used in FLOWTRAN-TF code and correlation coefficients.

\begin{tabular}{|c|c|c|c|c|c|}
\hline Material & $k_{0}$ & $k_{1} \times 10^{2}$ & $k_{2} \times 10^{6}$ & $k_{3} \times 10^{7}$ & $r^{2}$ \\
\hline Uranium & 27.009 & 1.9424 & 5.5425 & - & 1.000 \\
Aluminum & 235.94 & 6.5734 & -301.22 & 2.2005 & 0.996 \\
$\begin{array}{c}\text { Stainless } \\
\text { Steel }\end{array}$ & 14.780 & 1.5552 & -3.7095 & - & 0.999 \\
\hline
\end{tabular}

Table 2.3.4 Temperature range in ${ }^{\circ} \mathrm{C}$ and weight percent range of applicability for FLOWTRAN-TF physical property correlations.

\begin{tabular}{|c|c|c|c|c|}
\hline Material & Density & $\begin{array}{c}\text { Specific } \\
\text { Heat }\end{array}$ & $\begin{array}{c}\text { Thermal } \\
\text { Conductivity }\end{array}$ & $\begin{array}{c}\text { Weight } \\
\text { Fraction }\end{array}$ \\
\hline Uranium & $0-650$ & $0-650$ & $0-650$ & - \\
$\begin{array}{c}\text { Aluminum } \\
\text { Stainless } \\
\text { Steel } \\
\text { Lithium- }\end{array}$ & $0-650$ & $0-650$ & $0-650$ & - \\
$\begin{array}{c}\text { Aluminum } \\
\text { Uranium- }\end{array}$ & $0-500$ & $0-500$ & $0-500$ & - \\
Aluminum & $0-400$ & $0-400$ & $0-400$ & $0-0.305$ \\
\hline
\end{tabular}



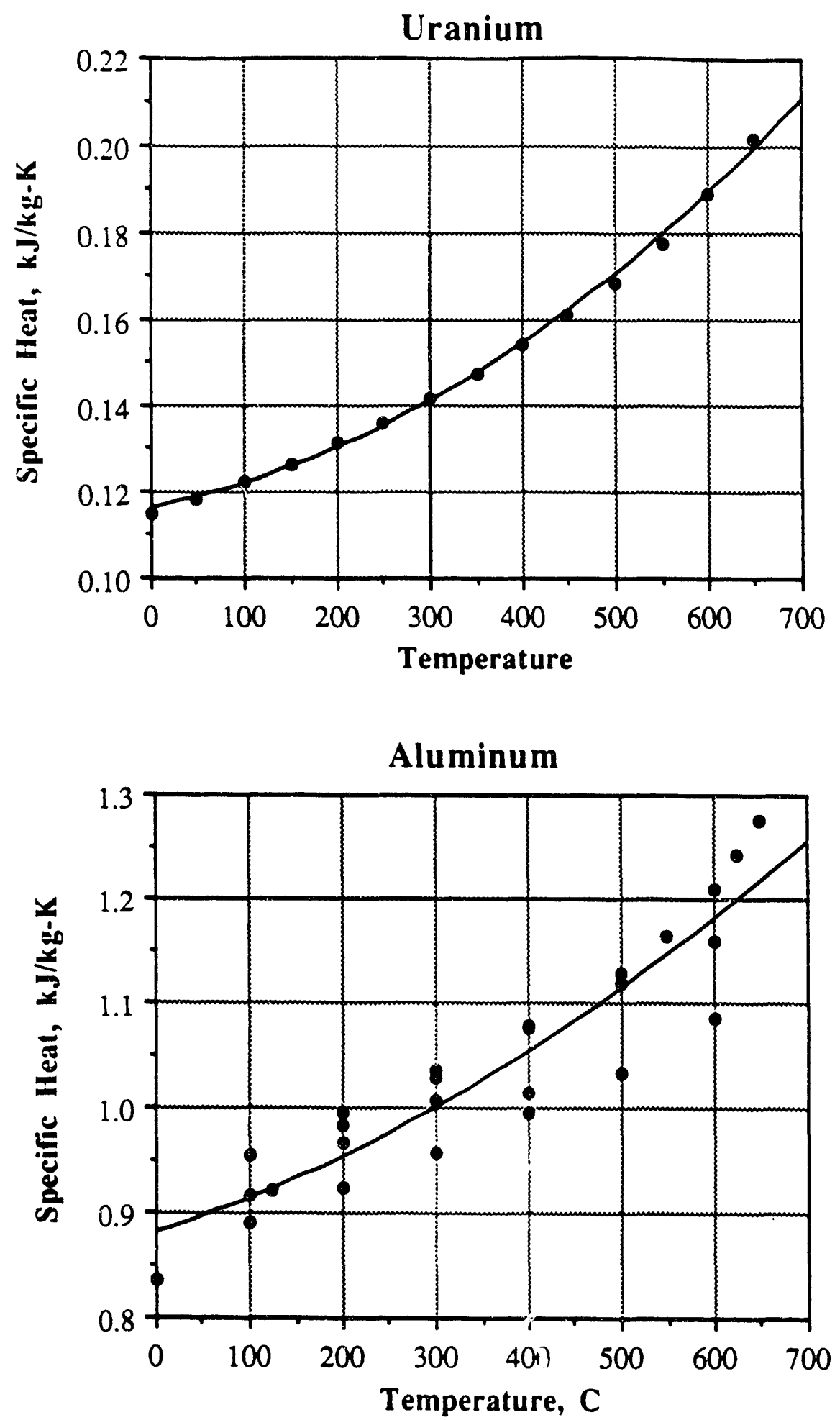

Figure 2.3-4 Heat capacity correlations for uranium and aluminum. 


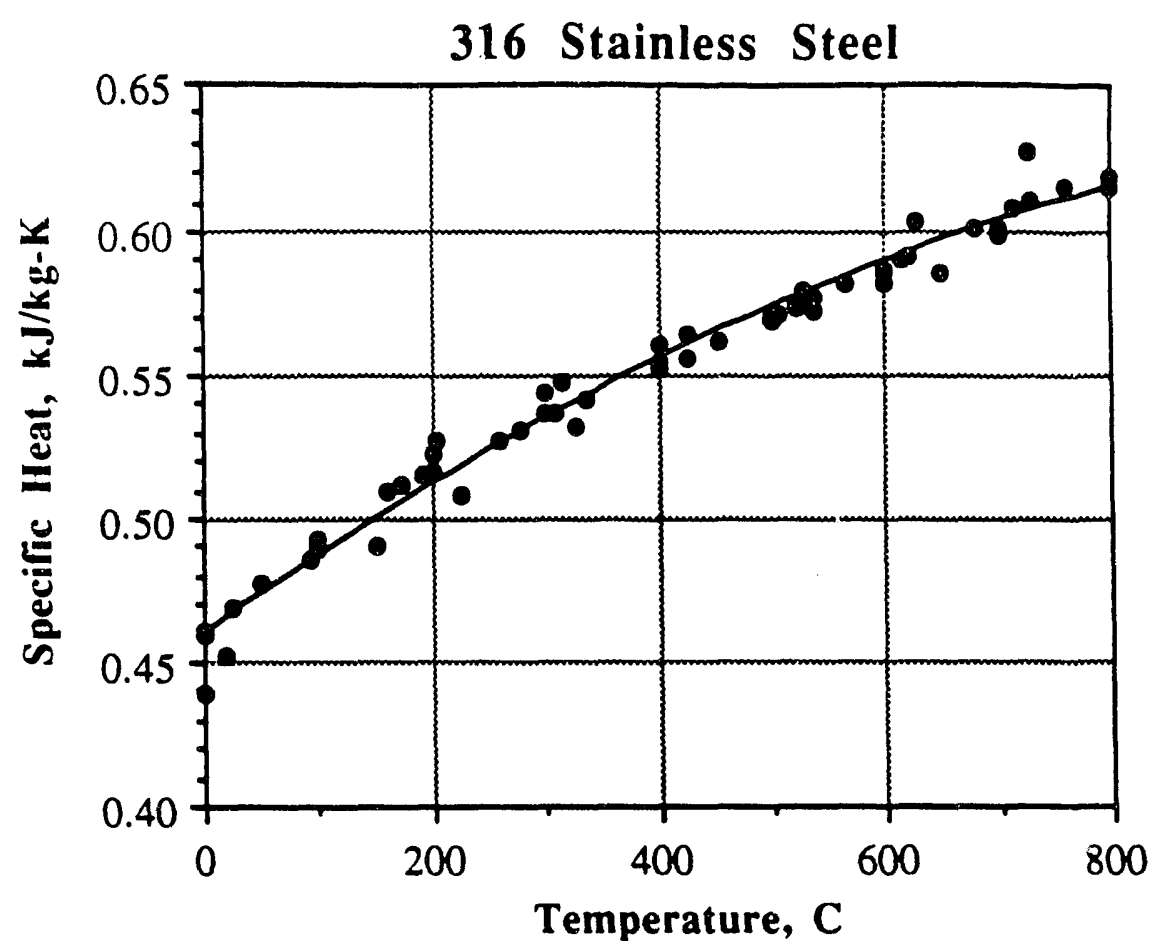

Lithium

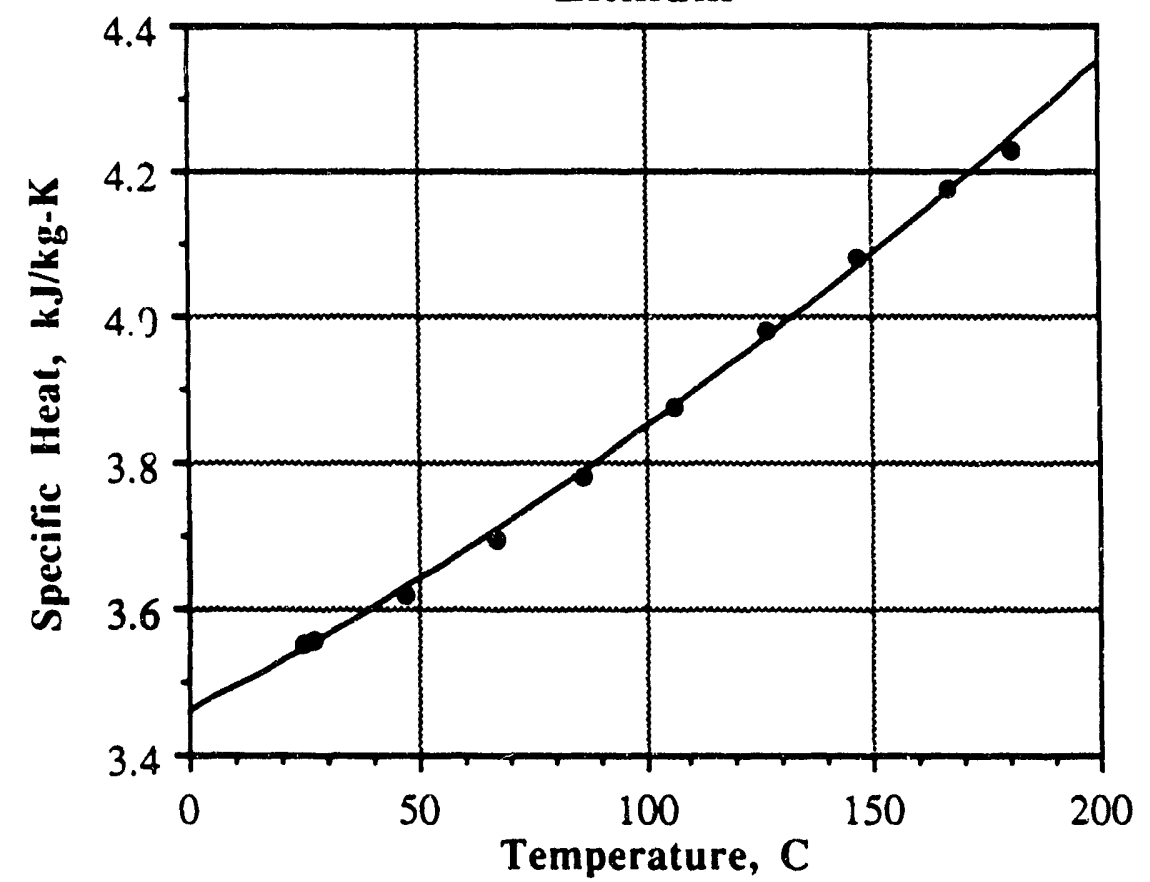

Figure 2.3-5 Heat capacity correlations for 316 stainless steel and lithium. 


\begin{tabular}{|l|lll|l|}
\hline $2 / 15 / 93$ & WSRC-TR-92-532 Rev. 0 & FLOWTRAN-TF Software Design & Page 51 \\
\hline
\end{tabular}
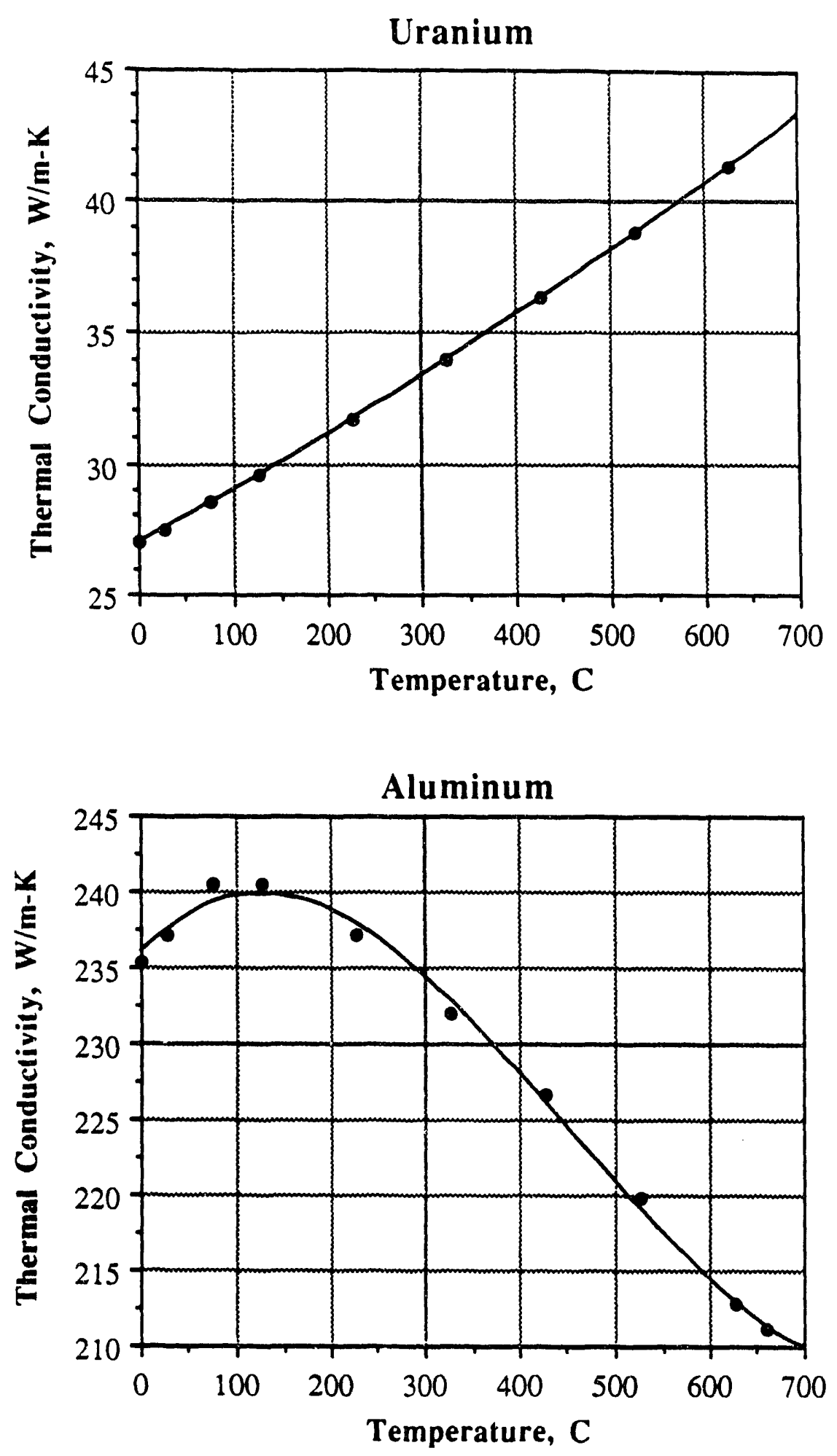

Figure 2.3.6 Thermal conductivity correlations for uranium and aluminum. 


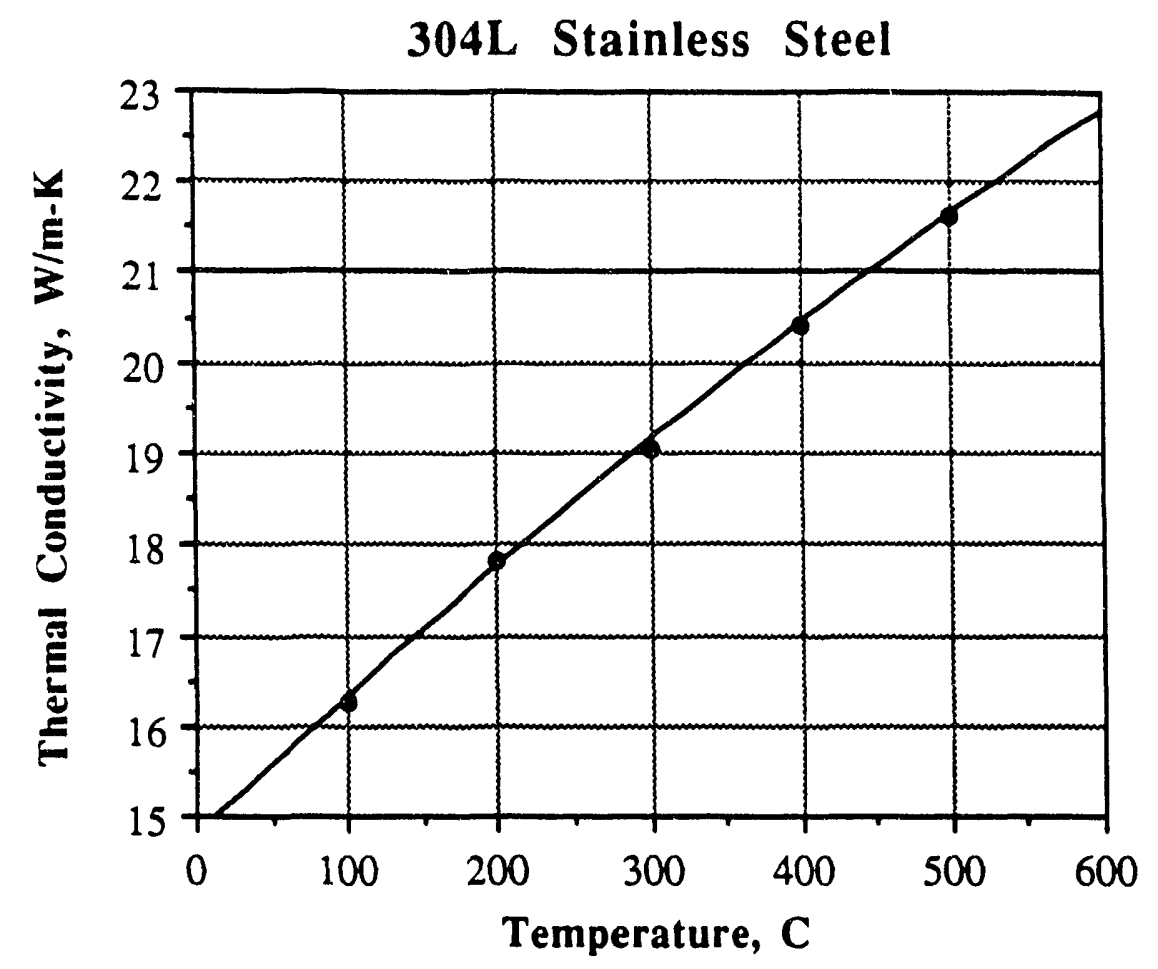

Figure 2.3-7 Thermal conductivity correlation for 304L stainless steel. 


\begin{tabular}{|l|lrr|r|r|}
\hline $2 / 15 / 93$ & WSRC-TR-92-532 Rev. 0 & FLOWTRAN-TF Software Design & Page 53 \\
\hline
\end{tabular}
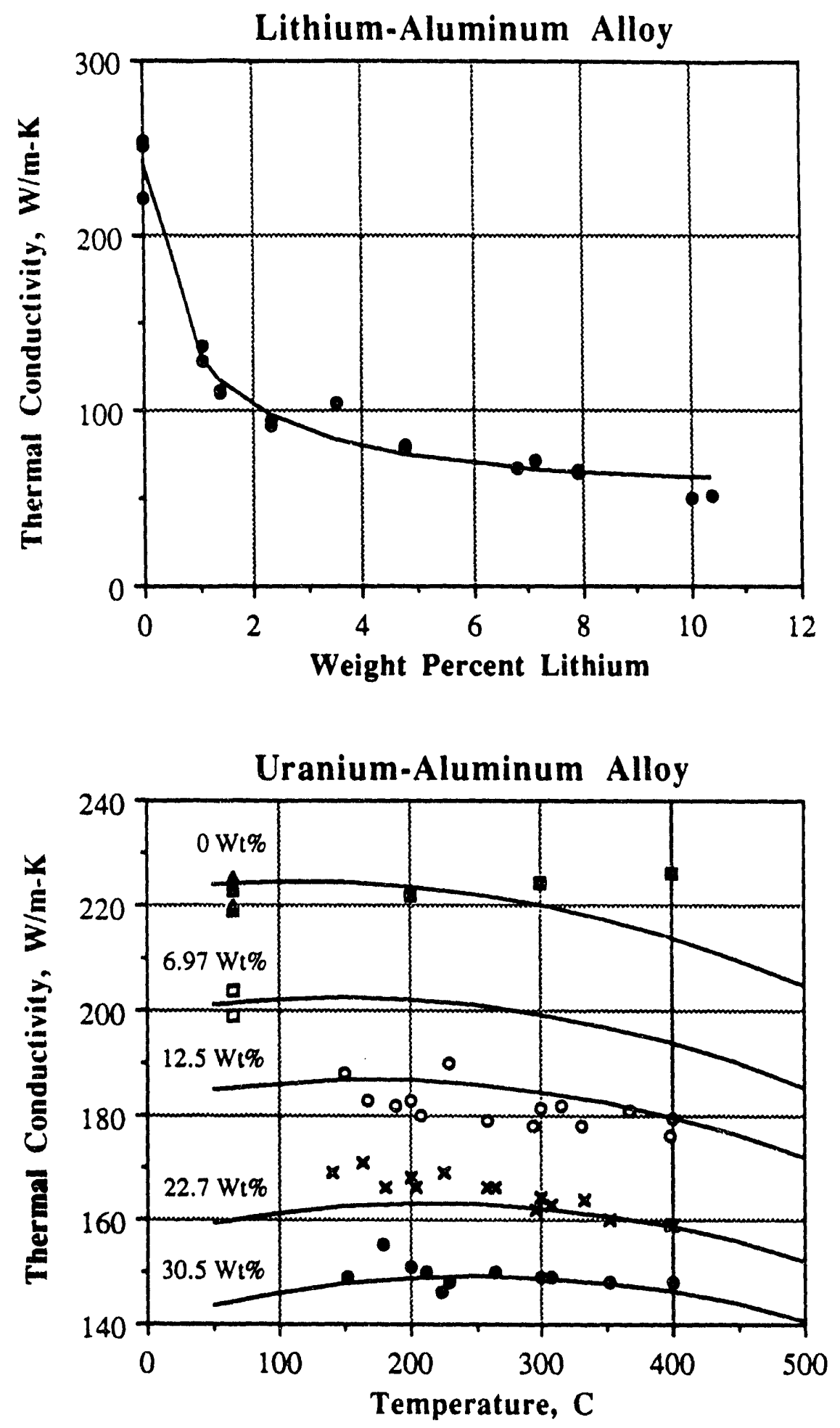

Figure 2.3-8 Thermal conductivity correlations for lithium-aluminum and uranium-aluminum alloys. 

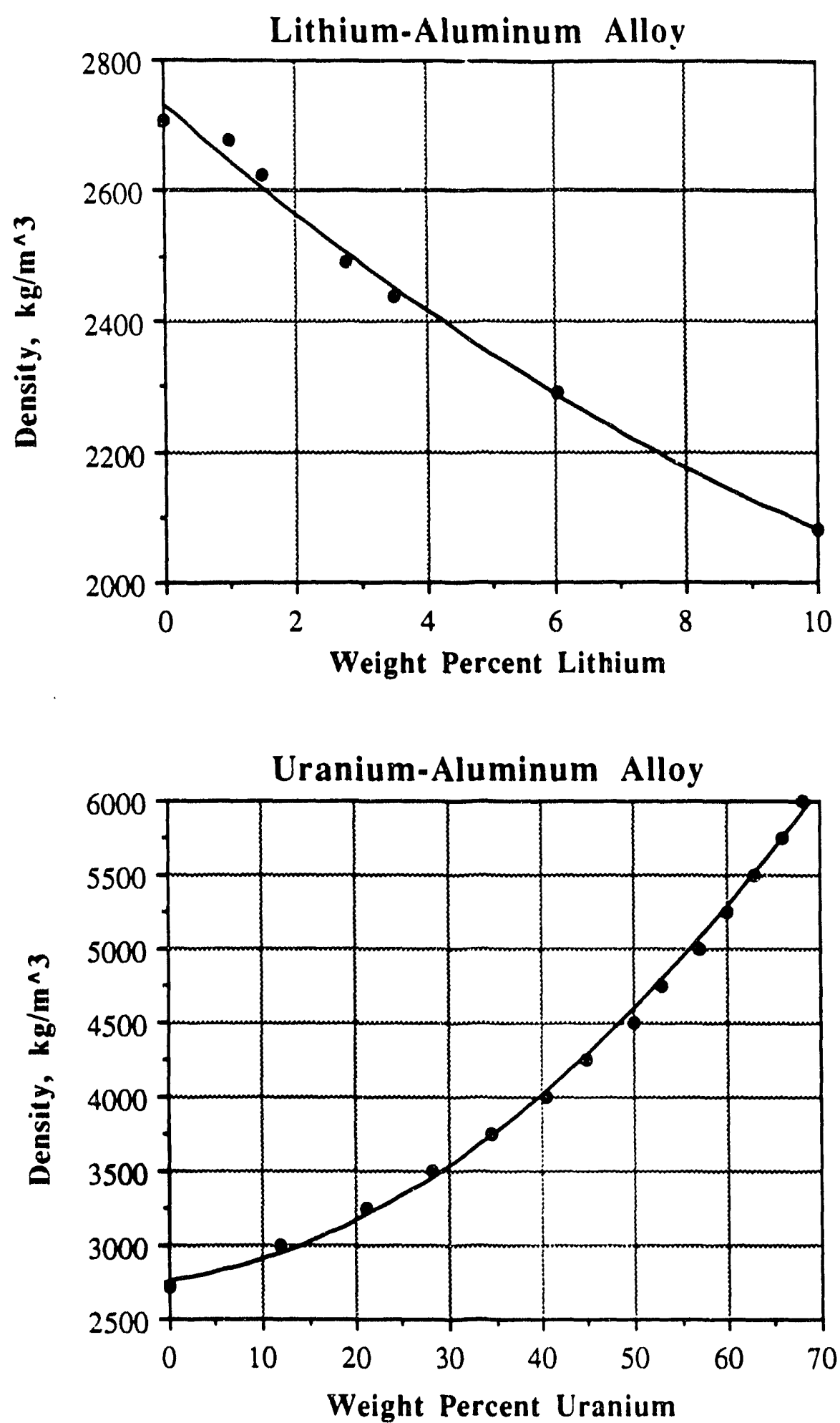

Figure 2.3.9 Density correlations for lithium-aluminum and uranium-aluminum alloys. 


\section{References}

Holman, J. P., 1976, Heat Transfer, McGraw-Hill Book Company, Inc., New York, NY.

Nash, C. A., 1987, "Reactor Material Property Correlations for Flowtran", DPST-87-692, September 29, 1987.

Touloukian, Y. S., 1970, Thermophysical Properties of Matter, Vol. 4, IFI/Plenum, New York, NY. 


\subsection{Fluid Governing Equations}

The space and time averaged mass, momentum and energy conservation equations for an air-water mixture are presented first followed by the gas and liquid equations of state.

\subsubsection{Space/Time Averaged Two-Phase Two-Component Fluid Field Equations}

Within each phase of a two-phase flow, the familiar single-phase mass, momentum, and energy conservation equations apply. At the interfaces between the phases, mass, momentum. and energy balances across the interfaces yield interface or jump conditions. In principl a two-phase flow could be modeled by tracking the phase inter:aces and solving the local ins. ntaneous conservation equations within each phase subject to appropriate boundary and interface conditions. In practice however, the computational effort and complexity are far too great to perform such a computation.

Rather, the standard approach is to space and/or time average the local instantaneous phasic conservation equations and solve for averaged flow quantities. Fortunately, information about the average behavior of a two-phase flow is sufficient for most applications. The process is similar to that employed in the solution of turbulent single-phase flow problems although much more complex and difficult. The averaged equations contain terms which must be related to the averaged quantities in order to close the problem. That is, the information lost in the averaging process must be recovered through largely empirical modeis typically termed 'closure' or 'constitutive' relations. These are roughly analogous to the Reynolds stress turbulence model required to solve the time averaged single-phase momentum balance for an incompressible fluid.

The derivation of averaged two-phase flow equations is well established. Excellent references are the works of Ishii (1975), Boure and Delhaye (1982) and Lahey and Drew (1988) among others. Space or time averaging alone yields continuous but not necessarily smooth averaged variables. Space and time averaging yields averaged quantities which vary smoothly and therefore the averaged equations are more suited to numerical modeling. The derivation of the general three-dimensional space-time averaged two-phase conservation equations as performed by Lahey and Drew, with minor modifications, are summarized in this note. Then the assumptions invoked to yield the set of simpler conservation equations solved by FLOWTRAN-TF are given. Details of the derivation are given by Lahey and Drew (1988) and in FLOWTRAN-TF development report TF-8 (Flach and Lee, 1992). The reader is also generally referred to Lahey and Drew (1988) for nomenclature definitions although nomenclature is defined here as well.

\section{Local, instantaneous conservation equations}

The local, instantaneous mass, momentum and energy conservation equations for the $k$ phase are ( $k=g$ for gas, $k=f$ for liquid)

$$
\frac{\partial \rho_{k}}{\partial t}+\nabla \cdot\left(\rho_{k} \mathbf{v}_{k}\right)=0
$$




\begin{tabular}{|l|lll|r|}
\hline $2 / 15 / 93$ & WSRC-TR-92-532 Rev.0 & FLOWTRAN-TF Software Design & Page 57 \\
\hline
\end{tabular}

$$
\begin{gathered}
\frac{\partial \rho_{k} v_{k}}{\partial t}+\nabla \cdot\left(\rho_{k} v_{k} v_{k}\right)=-\nabla P_{k}+\nabla \cdot \underline{\tau_{k}}+\rho_{k} g_{k} \\
\frac{\partial \rho_{k} e_{k}}{\partial t}+\nabla \cdot\left(\rho_{k} e_{k} v_{k}\right)=\frac{\partial P_{k}}{\partial t}-\nabla \cdot q_{k}^{\prime \prime}+\nabla \cdot\left[\underline{\tau_{k}} \cdot v_{k}\right]+\rho_{k} g_{k} \cdot v_{k}+q_{k}^{\prime \prime \prime}
\end{gathered}
$$

where

$$
\begin{aligned}
& \rho_{k} \equiv \text { density } \\
& v_{k} \equiv \text { velocity vector } \\
& P_{k} \equiv \text { pressure } \\
& \frac{\tau_{k}}{\mathbf{g}_{k}} \equiv \text { deviatoric stress tensor } \\
& h_{k} \equiv \text { enthalpy } \\
& e_{k} \equiv h_{k}+\left(v_{k} \cdot v_{k}\right) / 2 \\
& q_{k}^{\prime \prime} \equiv \text { heat flux vector } \\
& q_{k}^{\prime \prime \prime} \equiv \text { volumetric heat generation rate }
\end{aligned}
$$

as shown in FLOWTRAN-TF development report TF-8 (Flach and Lee, 1992). Lahey and Drew (1988) give the corresponding interface or jump conditions which couple the phases at their interfaces.

\section{Space averaging}

The space and time averaging operators are known to be commutative so the order of the operations does not affect the final result (Lahey and Drew, 1988). However, the derivation is easier to follow if space averaging is performed first.

First a consistent manner of choosing a total volume V, occupied completely by fluid (gas and/or liquid), is defined. With pipe flow for example, the control volume might be defined as the entire cross-section from $z-\Delta z / 2$ to $z+\Delta z / 2$ where $z$ is measured along the pipe axis. Area averaging could be obtained in the limit as $\Delta z \rightarrow 0$. Figure 2.4.1-1 shows an arbitrary control volume $V$ at location $(x, t)$ which is occupied completely by gas, liquid, or more typically, a gas-liquid mixture. The surface of $V$ may be in contact with gas, liquid, and/or a solid wall. The volume within $V$ occupied by phase $k$ is defined to be $V_{k}$. The total surface area of phase $k$ within $V$ is defined to be $A V_{k}$. The surface area of $V$ occupied by phase $k$ and in contact with the wall is $A_{k w}$. The surface of area of $V$ occupied by phase $k$ and in contact with phase $k$ outside of $V$ is $A_{k V}$. The internal surface area of $V_{k}$ in contact with the other phase is $A_{i}$. Therefore,

$$
A_{V k}=A_{k w}+A_{k V}+A_{i}
$$


The k-phase space averaging operator is defined by

$$
\left\langle f_{k}\right\rangle \equiv \frac{1}{V_{k}(x, t)} \int_{V_{k}(x, t)} f_{k}\left(x^{\prime}, t\right) d V^{\prime}
$$

where $f_{k}$ is an arbitrary property of the $k$-phase. The spatial averaging process requires the use of Leibnitz's Rule, Gauss' Theorem and the Reynolds Rules which are now introduced in preparation for space averaging.

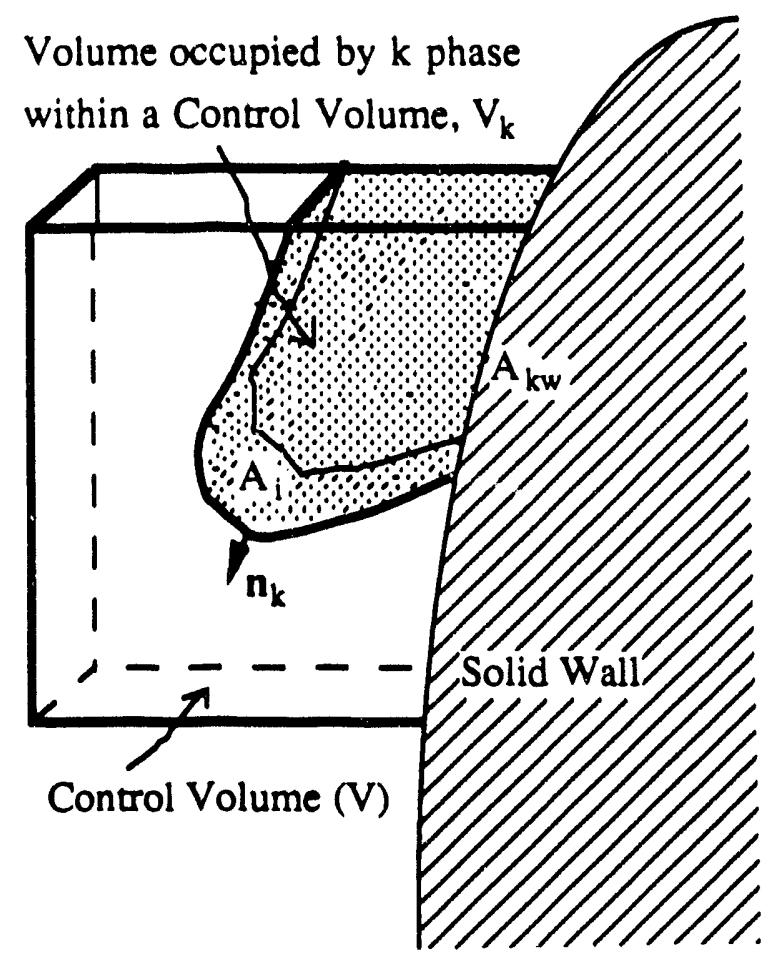

Figure 2.4.1-1 Control volume for spatial averaging

Leibnitz's Rule applied to volume $V_{k}$ is

$$
\int_{V_{k}(x, t)} \frac{\partial f_{k}}{\partial t} d V=\frac{\partial}{\partial t} \int_{V_{k}(x, t)} f_{k} d V-\int_{A_{i}(x, t)} f_{k}\left(v_{i} \cdot n_{k}\right) d S
$$

where $f_{k}$ is an arbitrary vector property of phase $k$. Leibnitz's Rule is used to express the space average of the time derivative of a quantity in terms of the time derivative of the space averaged quantity plus an additional surface integral term. Here $\mathbf{n}_{\mathbf{k}}$ is the outward-directed normal vector of the interfacial surface of $V_{k}$ and $v_{i}$ is the interface velocity. The interface velocity is generally not equal to either of the phasic velocities at the interface.

Gauss' Theorem is 


$$
\int_{V_{k}(x, l)} \nabla \cdot B d V=\int_{A_{V k}(x, l)} B \cdot n_{k} d S=\int_{A_{k V}(x, t)} B \cdot n_{k} d S+\int_{A_{i}(x, t)} B \cdot n_{k} d S+\int_{A_{k w}(x, l)} B \cdot n_{k} d S
$$

where B may be a vector or tensor quantity. The first integral on the r.h.s of the second equality is usually expressed in an alternate form. For the control volume described above and defined as the entire pipe cross-section from $z-\Delta z / 2$ to $z+\Delta z / 2$

$$
\int_{A_{k V}(x, l)} B \cdot n_{k} d S=\frac{\partial}{\partial z} \int_{V_{k}(z, l)} n_{z} \cdot B d V
$$

as shown in FLOWTRAN-TF development report TF-8 (Flach and Lee, 1992). Lahey and Drew (1988) show in general that

$$
\int_{A_{k V}(x, t)} B \cdot n_{k} d S=\nabla \cdot \int_{V_{k}(x, t)} B d V
$$

and equation (2.4.1-5) becomes

$$
\int_{V_{k}(x, t)} \nabla \cdot B d V=\nabla \cdot \int_{V_{k}(x, t)} B d V+\int_{A_{i}(x, t)} B \cdot n_{k} d S+\int_{A_{k, ~}(x, t)} B \cdot \mathbf{n}_{k} d S
$$

which is the preferred form of Gauss' Theorem. A special case of equation $(2.4 .1-8)$ is the gradient theorem

$$
\int_{V_{k}(x, t)} \nabla B d V=\nabla \int_{V_{k}(x, t)} B d V+\int_{A_{1}(x, t)} B n_{k} d S+\int_{A_{k w}(x, t)} B n_{k} d S
$$

where B is an arbitrary scalar. Equation $(2.4 .1-9)$ is obtained by replacing B with BI in equation $(2.4 .1-5)$.

Finally, the following Reynold's Rules (approximate) are used to simplify the space averaged equations:

$$
\begin{gathered}
\left\langle f_{k}\left\langle g_{k}\right\rangle\right\rangle \equiv\left\langle f_{k}\right\rangle\left\langle g_{k}\right\rangle \\
\left\langle\left\langle f_{k}\right\rangle\right\rangle \equiv\left\langle f_{k}\right\rangle
\end{gathered}
$$

With the above mathematical tools in hand, the space averaged conservation equations are derived by integrating the local instantaneous equations over the volume $V_{k}$ and dividing by $\mathrm{V}$. The resulting space averaged mass conservation equation for phase $\mathrm{k}$ is

$$
\frac{\partial}{\partial t}\left[\beta_{k}\left\langle\rho_{k}\right\rangle\right]+\frac{1}{V} \nabla \cdot\left[V \beta_{k}\left\langle\rho_{k} v_{k}\right\rangle\right]=\Gamma_{k}
$$

where 


$$
\begin{gathered}
\beta_{k} \equiv \frac{V_{k}(x, t)}{V} \\
\Gamma_{k} \equiv-\frac{1}{V} \int_{A_{i}(x, t)} m_{k}^{\prime \prime} d S
\end{gathered}
$$

and

$$
m_{k}^{\prime \prime} \equiv \rho_{k} n_{k} \cdot\left(v_{k}-v_{i}\right)
$$

The quantity $\beta_{k}$ is the instantaneous volume fraction at location $(x, t)$. The quantity $\Gamma_{k}$ is the instantaneous rate of phase $k$ mass creation at the interface. The space averaged momentum balance is

$$
\begin{aligned}
& \frac{\partial}{\partial t}\left[\beta_{k}\left\langle\rho_{k} v_{k}\right\rangle\right]+\frac{1}{V} \nabla \cdot\left[V \beta_{k}\left\langle\rho_{k} v_{k} v_{k}\right\rangle\right]=-\frac{1}{V} \nabla \cdot\left[V \beta_{k}\left\langle P_{k}\right)\right]=\frac{1}{V} \nabla \cdot\left[V \beta_{k}\left\langle\tau_{k}\right\rangle\right] \\
& +\beta_{k}\left\langle\rho_{k} g_{k}\right\rangle-\frac{1}{V} \int_{A_{i}(x, t)} m_{k}^{\prime \prime} v_{k} d S-\frac{1}{V} \int_{A_{i}(x, t)} P_{k_{i}} n_{k} d S+\frac{1}{V} \int_{A_{i}(x, t)} \tau_{k_{i}} \cdot n_{k} d S \\
& -\frac{1}{V} \int_{A_{k w}(x, t)} P_{k_{w}} n_{k_{w}} d S+\frac{1}{V} \int_{A_{k w}(x, t)} \tau_{k_{w}} \cdot n_{k_{w}} d S
\end{aligned}
$$

Lahey and Drew (1988) choose to rearrange equation $(2.4 .1-15)$ in the following form

$$
\begin{aligned}
& \frac{\partial}{\partial t}\left[\beta_{k}\left\langle\rho_{k} \mathbf{v}_{k}\right\rangle\right]+\frac{1}{V} \nabla \cdot\left[V \beta_{k}\left\langle\rho_{k} v_{k} v_{k}\right\rangle\right]=-\beta_{k} \nabla \cdot\left[\left\langle P_{k}\right\rangle\right]-\left\langle\Delta P_{k_{i}}\right\rangle S_{k_{i}}^{\prime \prime \prime}-\left\langle\Delta P_{k_{w}}\right\rangle S_{k_{w}}^{\prime \prime \prime} \\
& +\frac{1}{V} \nabla \cdot\left[V \beta_{k}\left\langle\underline{\tau_{k}}\right\rangle\right]+\beta_{k}\left\langle\rho_{k} g_{k}\right\rangle+\Gamma_{k} v_{k_{i}}^{\Gamma}+\mathbf{M}_{k_{i}}^{n d}+\mathbf{M}_{k_{i}}^{d}+\mathbf{M}_{k_{w}}^{n d}+\mathbf{M}_{k_{w}}^{d}+\left\langle\underline{\tau_{k}}\right\rangle_{i} \cdot S_{k_{i}}^{\prime \prime \prime}
\end{aligned}
$$

where

$$
\begin{aligned}
& v_{k_{i}}^{\Gamma} \equiv \frac{\int_{A_{i}(x, t)} m_{k_{i}}^{\prime \prime} v_{k} d S}{\int_{A_{i}(x, t)}^{\prime \prime} m_{k_{i}} d S}=-\frac{1}{V} \frac{\int A_{i}(x, t)}{m_{k_{i}}^{\prime \prime} v_{k} d S} \\
& \mathbf{M}_{\mathrm{k}_{\mathrm{i}}}^{\mathrm{nd}} \equiv-\frac{1}{\mathrm{~V}} \int_{\mathrm{A}_{\mathrm{i}}(\mathbf{x}, \mathrm{t})} \Delta \tilde{\mathrm{P}}_{\mathrm{k}_{\mathrm{i}}}^{\mathrm{nd}} \mathbf{n}_{\mathrm{k}} \mathrm{dS}
\end{aligned}
$$




$$
\begin{aligned}
& \mathbf{M}_{\mathrm{k}_{i}}^{\mathrm{d}} \equiv-\frac{1}{\mathrm{~V}} \int_{\mathbf{A}_{1}(\mathbf{x}, \mathrm{t})}\left[\Delta \tilde{\mathrm{P}}_{\mathrm{k}_{1}}^{\mathrm{d}} \underline{\mathbf{I}}-\Delta \tilde{\boldsymbol{\tau}}_{\mathrm{k}_{\mathrm{i}}}\right] \cdot \mathbf{n}_{\mathrm{k}} \mathrm{d} S \\
& \mathbf{M}_{\mathrm{k}_{w}}^{\mathrm{nd}} \equiv-\frac{1}{\mathrm{~V}} \int_{\mathbf{A}_{\mathrm{k} w}(\mathbf{x}, t)} \Delta \tilde{\mathrm{P}}_{\mathrm{k}_{w}} \mathbf{n}_{\mathrm{k}_{\mathrm{w}}} \mathrm{dS} \\
& \mathbf{M}_{k_{w}}^{d} \equiv \frac{1}{V} \int_{A_{k w}(x, l)} \tau_{k_{w}} \cdot n_{k_{w}} \mathrm{~d} S \\
& \left\langle P_{k}(x, t)\right\rangle_{i} \equiv \frac{1}{A_{i}(x, t)} \int_{A_{i}(x, t)} P_{k_{i}} d S \\
& \Delta \tilde{P}_{k_{i}} \equiv P_{k_{i}}-\left\langle P_{k}\right\rangle_{i}
\end{aligned}
$$

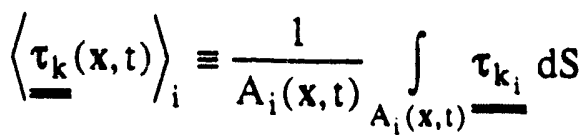

$$
\begin{aligned}
& \Delta \tilde{\tau}_{k_{i}} \equiv \tau_{k_{i}}-\left\langle\underline{\tau_{k}}\right\rangle_{i} \\
& S_{k_{i}}^{\prime \prime \prime} \equiv \frac{1}{V} \int_{A_{i}(x, t)} n_{k} d S \\
& \left\langle P_{k}(x, t)\right\rangle_{w} \equiv \frac{1}{A_{k w}(x, t)} \int_{A_{k w}(x, t)} P_{k_{w}} d S \\
& \Delta \tilde{\mathrm{P}}_{\mathrm{k}_{\mathrm{w}}} \equiv \mathrm{P}_{\mathrm{k}_{\mathrm{w}}}-\left\langle\mathrm{P}_{\mathrm{k}}\right\rangle_{\mathrm{w}} \\
& S_{k_{w}}^{\prime \prime \prime} \equiv \frac{1}{V} \int_{A_{k w}(x, t)} n_{k_{w}} d S \\
& \left\langle\Delta \mathrm{P}_{\mathrm{k}_{\mathrm{i}}}\right\rangle \equiv\left\langle\mathrm{P}_{\mathrm{k}}\right\rangle_{\mathrm{i}}-\left\langle\mathrm{P}_{\mathrm{k}}\right\rangle
\end{aligned}
$$

and

$$
\left\langle\Delta \mathrm{P}_{\mathrm{k}_{w}}\right\rangle \equiv\left\langle\mathrm{P}_{\mathrm{k}}\right\rangle_{w}-\left\langle\mathrm{P}_{\mathrm{k}}\right\rangle
$$


The reader is referred to Lahey and Drew (1988) more information on the derivation of equations $(2.4 .1-15)$ and $(2.4 .1-16)$ and for interpretations of the symbols defined above. The space averaged energy conservation equation is

$$
\begin{aligned}
& \frac{\partial}{\partial t}\left[\beta_{k}\left\langle\rho_{k} e_{k}\right\rangle\right]+\frac{1}{V} \nabla \cdot\left[V \beta_{k}\left\langle\rho_{k} e_{k} v_{k}\right\rangle\right]=-\left\langle P_{k}\right\rangle_{i} \frac{\partial \beta_{k}}{\partial t}+\frac{\partial\left[\beta_{k}\left\langle P_{k}\right\rangle\right]}{\partial t}-\frac{1}{V} \nabla \cdot\left[v \beta_{k}\left\langle q_{k}^{\prime \prime}\right\rangle\right] \\
& +\frac{1}{V} \nabla \cdot\left[v \beta_{k}\left\langle\underline{\tau_{k}} \cdot v_{k}\right\rangle\right]+\beta_{k}\left\langle\rho_{k} g_{k} \cdot v_{k}\right\rangle+\beta_{k}\left\langle q_{k}^{\prime \prime \prime}\right\rangle+\Gamma_{k} e_{k_{i}}+M_{k_{i}}^{n d} \cdot v_{k_{i}}^{n d}+M_{k_{i}}^{d} \cdot v_{k_{i}}^{d}+q_{k_{i}}^{\prime \prime} A_{i}^{\prime \prime \prime} \\
& +q_{k_{w}}^{\prime \prime} A_{k_{w}}^{\prime \prime \prime}+v_{k_{i}}^{\tau} \cdot\left\langle\underline{\tau_{k}}\right\rangle_{i} \cdot S_{k_{i}}^{\prime \prime \prime}-\Gamma_{k}\left\langle\frac{\Delta \tilde{P}_{k}^{d}}{\rho_{k}}\right\rangle_{i}
\end{aligned}
$$

where

$$
\begin{aligned}
& e_{k_{i}} \equiv \frac{\int_{A_{i}(x, t)} m_{k}^{\prime \prime} e_{k} d S}{\int_{A_{i}(x, t)} m_{k}^{\prime \prime} d S}=-\frac{1}{V} \frac{\int A_{i}(x, t)}{\Gamma_{k}^{\prime \prime} e_{k} d S} \\
& M_{k_{i}}^{n d} \cdot v_{k_{i}}^{n d} \equiv-\frac{1}{V} \int_{A_{i}(x, t)} \Delta \tilde{P}_{k_{1}}^{\text {nd }} n_{k} \cdot v_{i} d S \\
& \mathbf{M}_{k_{i}}^{d} \cdot v_{k_{i}}^{d} \equiv-\frac{1}{V} \int_{A_{i}(x, t)} n_{k} \cdot\left[\Delta \tilde{P}_{k_{i}}^{d} I-\Delta \tilde{\tau}_{k_{i}}\right] \cdot v_{k} d S \\
& q_{k_{i}}^{\prime \prime} A_{i}^{\prime \prime \prime} \equiv-\frac{1}{V} \int_{A_{i}(x, t)} n_{k} \cdot q_{k}^{\prime \prime} d S \\
& q_{k_{w}}^{\prime \prime} A_{k w}^{\prime \prime \prime} \equiv-\frac{1}{V} \int_{A_{k w}(x, t)} n_{k_{w}} \cdot q_{k}^{\prime \prime} d S \\
& v_{k_{i}}^{\tau} \cdot\left\langle\underline{\underline{\tau_{k}}}\right\rangle_{i} \cdot S_{k_{i}}^{\prime \prime \prime} \equiv \frac{1}{V} \int_{A_{i}(x, t)} n_{k} \cdot\left\langle\underline{\underline{\tau_{k}}}\right\rangle_{i} \cdot v_{k} d S
\end{aligned}
$$

and

$$
\Gamma_{k}\left\langle\frac{\Delta \tilde{P}_{k}^{d}}{\rho_{k}}\right\rangle_{i} \equiv-\frac{1}{V} \int_{A_{i}(x, l)} \Delta \tilde{P}_{k_{i}}^{d}\left(\frac{m_{k}^{\prime \prime}}{\rho_{k}}\right) d S
$$


Again the reader is referred to Lahey and Drew (1988) for more information on the derivation of equation (2.4.1-32) and the interpretation of definitions $(2.4 .1-33)$ through (2.4.1-39).

\section{Time averaging}

The time averaging operator is defined by

$$
\overline{f_{k}} \equiv \frac{1}{T} \int_{t-T}^{t} f_{k} d t^{\prime}
$$

Lahey and Drew (1988) show that

$$
\frac{\overline{\partial f_{k}}}{\partial t}=\frac{\partial \overline{f_{k}}}{\partial t}
$$

and

$$
\overline{\nabla \cdot \mathbf{B}}=\nabla \cdot \overline{\mathbf{B}}
$$

Integrating equations $(2.4 .1-11),(2.4 .1-16)$ and $(2.4 .1-32)$ over the time interval $t-T$ to $t$, dividing by $\mathrm{T}$, and using equations $(2.4 .1-41)$ and $(2.4 .1-42)$ yields the following space and time averaged conser ration equations:

$$
\begin{aligned}
& \frac{\partial}{\partial t} \overline{\left[\beta_{k}\left\langle\rho_{k}\right\rangle\right]}+\frac{1}{V} \nabla \cdot\left[v \overline{\beta_{k}\left\langle\rho_{k} v_{k}\right\rangle}\right]=\overline{\Gamma_{k}} \\
& \left.\frac{\partial}{\partial t} \overline{\left[\beta_{k}\left\langle\rho_{k} v_{k}\right\rangle\right]}+\frac{1}{v} \nabla \cdot\left[v \overline{\beta_{k}\left\langle\rho_{k} v_{k} v_{k}\right\rangle}\right]=-\overline{\beta_{k} \nabla \cdot\left[\left\langle P_{k}\right\rangle I\right.}\right]-\overline{\left\langle\Delta P_{k_{i}}\right\rangle \mathbf{S}_{k_{i}}^{\prime \prime \prime}}-\overline{\left\langle\Delta P_{k_{w}}\right\rangle S_{k_{w}}^{\prime \prime \prime}} \\
& +\frac{1}{V} \nabla \cdot\left[V \overline{\beta_{k}\left\langle\underline{\tau_{k}}\right\rangle}\right]+\overline{\beta_{k}\left\langle\rho_{k} g_{k}\right\rangle}+\overline{\Gamma_{k} v_{k_{i}}^{\Gamma}}+\overline{M_{k_{i}}^{n d}}+\overline{M_{k_{i}}^{d}}+\overline{M_{k_{w}}^{n d}}+\overline{M_{k_{w}}^{d}}+\overline{\left\langle\tau_{k}\right\rangle_{i} \cdot S_{k_{i}}^{\prime \prime \prime}}
\end{aligned}
$$

$$
\begin{aligned}
& \frac{\partial}{\partial t} \overline{\left[\beta_{k}\left\langle\rho_{k} e_{k}\right\rangle\right]}+\frac{1}{V} \nabla \cdot\left[v \overline{\beta_{k}\left\langle\rho_{k} e_{k} v_{k}\right\rangle}\right]=-\overline{\left\langle p_{k}\right\rangle_{i} \frac{\partial \beta_{k}}{\partial t}}+\frac{\partial \overline{\left[\beta_{k}\left\langle P_{k}\right\rangle\right]}}{\partial t}-\frac{1}{V} \nabla \cdot\left[v \overline{\beta_{k}\left\langle q_{k}^{\prime \prime}\right\rangle}\right] \\
& +\frac{1}{V} \nabla \cdot\left[V \overline{\beta_{k}\left\langle\tau_{k} \cdot v_{k}\right\rangle}\right]+\overline{\beta_{k}\left\langle\rho_{k} g_{k} \cdot v_{k}\right\rangle}+\overline{\beta_{k}\left\langle q_{k}^{\prime \prime \prime}\right\rangle}+\overline{\Gamma_{k} e_{k_{i}}}+\overline{M_{k_{i}}^{n d} \cdot v_{k_{i}}^{n d}}+\overline{M_{k_{i}}^{d} \cdot v_{k_{i}}^{d}}+\overline{q_{k_{i}}^{\prime \prime} A_{i}^{\prime \prime \prime}} \\
& +\overline{q_{k_{w}}^{\prime \prime} A_{k_{w}}^{\prime \prime \prime}}+\overline{v_{k_{i}}^{\tau} \cdot\left\langle\tau_{k}\right\rangle_{i} \cdot S_{k_{i}}^{\prime \prime \prime}}-\overline{\Gamma_{k}\left\langle\frac{\Delta \tilde{P}_{k}^{d}}{\rho_{k}}\right\rangle_{i}}
\end{aligned}
$$




\section{Transformed conservation equations}

The quantities in equations (2.4.1-43) through (2.4.1-45) have been space averaged first and then time averaged. For constitutive relation development, quantities which have been time averaged first are typically more convenient. As stated earlier the averaging operations are commutative so the transformation is relatively easy. Let the phase presence function be defined by

$$
X_{k}(x, t) \equiv\left\{\begin{array}{lc}
1 & \text { when phase } k \text { is at position } \mathbf{x} \text { at time } t \\
0 & \text { otherwise }
\end{array}\right.
$$

Then the local time fraction of phase $\mathrm{k}$ presence is

$$
\alpha_{k} \equiv \frac{T_{k}}{T}=\overline{X_{k}}
$$

If the $\mathrm{X}_{\mathrm{k}}$-weighted time average of an arbitrary function $\mathrm{f}_{\mathrm{k}}$ is defined as

$$
\overline{f_{k}}=\frac{\overline{X_{k} f_{k}}}{\overline{X_{k}}}
$$

and the total volume average of an arbitrary function $\mathrm{g}$ is defined as

$$
\varangle g \triangleright \equiv \frac{1}{V} \int_{V} g d V
$$

then the commutativity property of the space and time averaging processes can be written

$$
\begin{gathered}
\overline{\beta_{k}\left\langle f_{k}\right\rangle}=\nless \alpha_{k} \bar{f}_{k}^{X} \triangleright \\
\overline{\int_{A_{i}} B_{k} \cdot \mathbf{n}_{k} \mathrm{dS}}=\int_{V} \sum_{j} \ell_{j}^{-1}\left(\underline{B}_{k} \cdot n_{k}\right) d V \\
\ell_{j}=T\left|v_{i} \cdot n_{k}\right|
\end{gathered}
$$

The latter two expressions are from Boure and Delhaye (1982). Expression (2.4.1-50a) will be used to recast many terms in the space-time averaged equations given by $(2.4 .1-43)$ to $(2.4 .1-45)$. However, the interfacial terms will be left as is; that is, equation (2.4.1-50 b) will not be used. Before doing so, the following definitions are introduced so that the various averages of variable products can be formally written as the product of averages:

$$
\overline{\varangle f_{k} \triangleright_{k}} \equiv \frac{\varangle \alpha_{k} \bar{f}_{k}^{X} \triangleright}{\varangle \alpha_{k} \triangleright}
$$




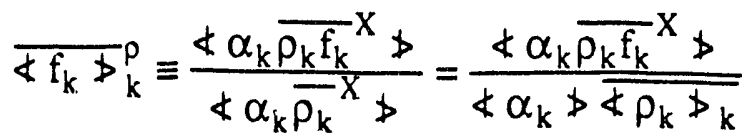

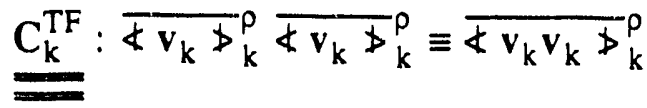

$$
\begin{aligned}
& C_{k}^{M} \nless \alpha_{k} \triangleright \nabla \overline{\left\langle P_{k}\right\rangle}=C_{k}^{M} \overline{\beta_{k}} \nabla \overline{\left\langle P_{k}\right\rangle} \equiv \overline{\beta_{k} \nabla\left\langle P_{k}\right\rangle}=\overline{\beta_{k} \nabla \cdot\left\langle P_{k}\right\rangle I}
\end{aligned}
$$

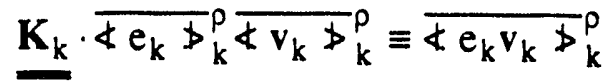

$$
\begin{aligned}
& C_{k}^{E} 1 \overline{\left\langle P_{k}\right\rangle_{i}} \frac{\partial \nless \alpha_{k} \triangleright}{\partial t}=C_{k}^{E 1} \overline{\left\langle P_{k}\right\rangle_{i}} \frac{\partial \overline{\beta_{k}}}{\partial t} \equiv \overline{\left\langle P_{k}\right\rangle_{i} \frac{\partial \beta_{k}}{\partial t}} \\
& C_{k}^{E 2} \Varangle \alpha_{k} \triangleright \overline{\left\langle P_{k}\right\rangle}=C_{k}^{E 2} \overline{\beta_{k}} \overline{\left\langle P_{k}\right\rangle} \equiv \overline{\beta_{k}\left\langle P_{k}\right\rangle}
\end{aligned}
$$

Equations (2.4.1-51) and (2.4.1-52) define volume-weighted and mass-weighted averages, respectively. Equations (2.4.1-53) through (2.4.1-57) introduce various scalar, secondorder tensor and fourth-order tensor distribution coefficients. Note that the distribution coefficient defined by equation (2.4.1-53) differs from the corresponding term in Lahey and Drew (1988). Also, definitions (2.4.1-54), (2.4.1-56) and (2.4.1-57) are an extension of Lahey and Drew (1988).

Using the above relations and definitions, the space-time averaged conservation equations can be transformed to

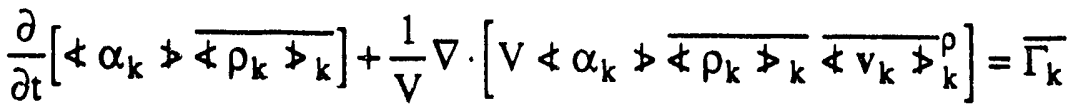

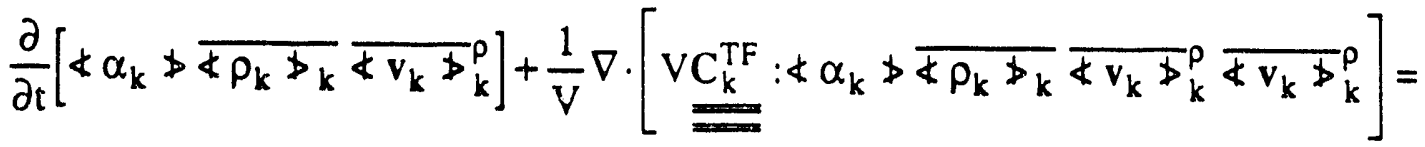

$$
\begin{aligned}
& -C_{k}^{M} \nless \alpha_{k} \triangleright \nabla \overline{\left\langle P_{k}\right\rangle}-\overline{\left\langle\Delta P_{k_{i}}\right\rangle S_{k_{i}}^{\prime \prime \prime}}-\overline{\left\langle\Delta P_{k_{w}}\right\rangle S_{k_{w}}^{\prime \prime \prime}}+\frac{1}{V} \nabla \cdot\left[V \nless \alpha_{k} \ngtr \overline{\& \tau_{k} \triangleright_{k}}\right] \\
& +\Varangle \alpha_{k} \gg \overline{\left\langle\rho_{k} g_{k} \nabla_{k}\right.}+\overline{\Gamma_{k} v_{k_{i}}^{\Gamma}}+\overline{M_{k_{i}}^{n d}}+\overline{M_{k_{1}}^{d}}+\overline{M_{k_{w}}^{n d}}+\overline{M_{k_{w}}^{d}}+\overline{\left\langle\tau_{k}{ }_{i} \cdot S_{k_{i}}^{\prime \prime \prime}\right.}
\end{aligned}
$$




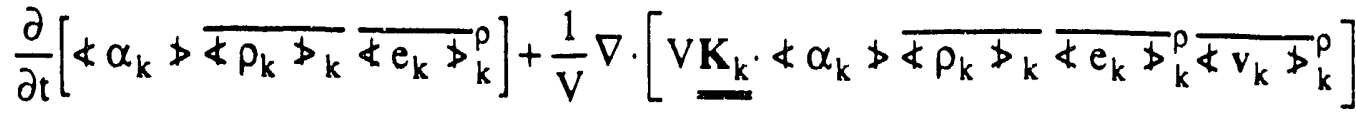

$$
\begin{aligned}
& =-C_{k}^{E} 1 \overline{\left\langle P_{k}\right\rangle_{i}} \frac{\partial \nless \alpha_{k} \downarrow}{\partial t}+\frac{\partial C_{k}^{E 2} \nless \alpha_{k} \triangleright \overline{\left\langle P_{k}\right\rangle}}{\partial t}-\frac{1}{V} \nabla \cdot\left[V \nless \alpha_{k} \triangleright \overline{\psi q_{k}^{\prime \prime} \triangleright_{k}}\right] \\
& +\frac{1}{V} \nabla \cdot\left[V \nless \alpha_{k} \triangleright \overline{\phi \tau_{k} \cdot v_{k} \triangleright_{k}}\right]+\varangle \alpha_{k} \triangleright \overline{\varangle \rho_{k} \triangleright_{k}} \overline{\varangle g_{k} \cdot v_{k} b_{k}^{\rho}} \\
& +\nless \alpha_{k} \ngtr \overline{\nless q_{k}^{\prime \prime \prime} \triangleright_{k}}+\overline{\Gamma_{k} e_{k_{i}}}+\overline{M_{k_{i}}^{n d} \cdot v_{k_{i}}^{n d}}+\overline{M_{k_{i}}^{d} \cdot v_{k_{i}}^{d}}+\overline{q_{k_{i}}^{\prime \prime} A_{i}^{\prime \prime \prime}}+\overline{q_{k_{w}}^{\prime \prime} A_{k_{w}}^{\prime \prime \prime}} \\
& +\overline{v_{k_{i}}^{\tau} \cdot\left\langle\tau_{k}\right\rangle_{i} \cdot S_{k_{i}}^{\prime \prime \prime}}-\overline{\Gamma_{k}\left\langle\frac{\Delta \tilde{P}_{k}^{d}}{\rho_{k}}\right\rangle_{i}}
\end{aligned}
$$

as shown in FLOWTRAN-TF development report TF-8 (Flach and Lee, 1992). None of the newly defined terms have reduced the complexity of the conservation equations or changed them in substance.

The mass-weighted average velocity, $\overline{\varangle v_{k} b_{k}^{p}}$, was introduced for convenience above because it allows the product of an average to be expressed as the product of averages. Fortunately, the mass-weighted velocity has a straightforward interpretation and is actually preferred over other average velocity definitions. Consider one-dimensional two-phase flow through a pipe. If area averaging is chosen then the phasic density is constant within the averaging area because the pressure is constant for practical purposes. Then

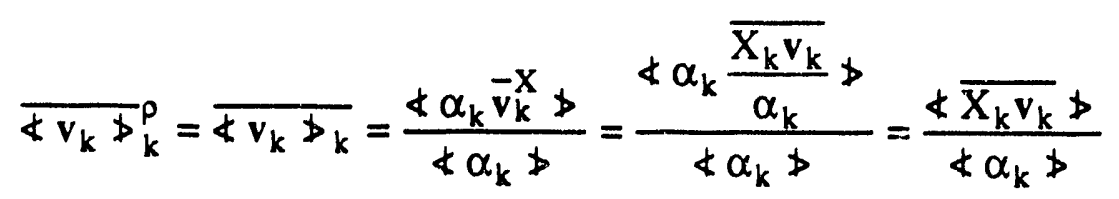

The r.h.s. of the last equality is recognized as the phasic flowrate divided by area (channel averaged superficial velocity $\varangle j_{k} \$$ ) divided by channel average volume fraction, both of which can be determined experimentally. Therefore, $\varangle v_{k} b_{k}$ can be readily determined experimentally.

\section{Approximate conservation equations}

The transformed equations given by equations $(2.4 .1-58)$ to $(2.4 .1-60)$ are still exact with the exception of the use of Reynolds Rules early on. Several approximations are now invoked to simplify the transport equations while still capturing the dominant effects with regard to FLOWTRAN-TF applications. The resulting equations are typical of those solved by other two-phase thermal-hydraulic codes.

The explicit averaging notation will be dropped to abbreviate the resulting conservation equations. In particular, the following changes in notation are introduced among others:

$$
\begin{aligned}
& \nless \alpha_{k} \triangleright \rightarrow \alpha_{k} \\
& \overline{\& \rho_{k}{ }_{k}} \rightarrow \rho_{k}
\end{aligned}
$$




$$
\begin{gathered}
\Varangle \bar{v}_{k} P_{k}^{p} \rightarrow v_{k} \\
\Varangle \overline{e_{k} P_{k}^{p}} \rightarrow e_{k} \\
\overline{\left\langle P_{k}\right\rangle} \rightarrow P_{k}
\end{gathered}
$$

No approximations are made to the mass conservation equation given by $(2.4 .1-58)$ and it remains unchanged. The average pressure in each phase is assumed to be equal

$$
P_{g}=P_{f} \equiv P
$$

in general and each phase experiences the same constant gravitational field so

$$
\mathbf{g}_{\mathbf{k}}=\mathbf{g}
$$

An exception is made to assumption (2.4.1-62) for the case of horizontal, stratified twophase flow and will be discussed in sections 2.6 and 3.6. In the momentum balance, the following quantities/terms are neglected

$$
\begin{aligned}
& \mathrm{C}_{\mathrm{k}}^{\mathrm{TF}}, \mathrm{C}_{\mathrm{k}}^{\mathrm{M}} \\
& \left\langle\Delta \mathrm{P}_{\mathrm{k}_{\mathrm{i}}}\right\rangle \\
& \mathbf{M}_{\mathbf{k}_{\mathrm{i}}}^{\text {nd }}, \mathbf{M}_{\mathbf{k}_{\mathbf{w}}}^{\text {nd }} \\
& \frac{1}{V} \nabla \cdot\left[V \nless \alpha_{k} \ngtr \overline{\underline{\tau_{k}} \nabla_{k}}\right] \\
& \overline{\Gamma_{k} v_{k_{i}}^{\Gamma}}
\end{aligned}
$$

Models have been developed for distribution coefficients for special cases. For example, Ishii and Mishima (1984) present a model for the equivalent of

$$
\underline{\underline{C_{k}^{T F}}}
$$

for the special case of one-dimensional, fully-developed, two-phase flow in a constant area circular pipe. The distribution coefficient is a scalar for this case. However, models applicable to the geometries encountered in FLOWTRAN-TF applications are not available so the distribution coefficients given by (2.4.1-64) are neglected for lack of a better alternative. Neglecting the term given by $(2.4 .1-65)$ is equivalent to assuming the average phasic and average interfacial pressures are equal. For FLOWTRAN-TF applications involving predominantly vertical two-phase flow, the assumption is quite reasonable. An exception would be horizontal, stratified flow. As mentioned above, this situation will be treated in sections 2.6 and 3.6. Nondrag interfacial forces are considered small compared 


\begin{tabular}{|l|lll|l|}
\hline $2 / 15 / 93$ & WSRC-TR-92-532 & Rev. 0 & FLOWTRAN-TF Software Design & Page 68 \\
\hline
\end{tabular}

to drag forces and and can be saiely neglected. Recalling relation (2.4.1-7), neglecting the term given by (2.4.1-67) is interpreted as neglecting fluid-fluid shear stresses. For onedimensional pipe flow for example, the term becomes

$$
\frac{1}{V} \nabla \cdot\left[V \nless \alpha_{k} \ngtr \overline{\tau_{k}}{ }_{k}\right] \rightarrow \frac{1}{V} \frac{\partial V \alpha_{k} \tau_{z z_{k}}}{\partial z}
$$

Neglecting this term is the same as neglecting the normal deviatoric shear stress at the ends $z-\Delta z / 2$ and $z+\Delta z / 2$ of the control volume. For two-dimensional flow $\tau_{z x}$ stresses also appear in (2.4.1-67), that is, lateral fluid-fluid coupling forces are present. Both of these fluid-fluid shear stresses are small compared to fluid-wall stresses and pressure forces in FLOWTRAN-TF applications because a fuel assembly subchannel forms a typical fluid computational cell. In the axial (z) direction, the only significant fluid-fluid contact occurs at the $z$-directed faces. The normal deviatoric stresses which occur here are small compared to the pressure force. In the azimuthal ( $x$ ) direction, the rib gap clearance (y direction) is much smaller than the $x$-direction (azimuthal) dimension. Therefore the dominant shear occurs due to wall contact, not fluid-fluid contact. Finally, the term given by $(2.4 .1-68)$ represents the momentum created with the creation of phase $k$ mass at the interface. For FLOWTRAN-TF power limit calculations using the $T_{\text {wall }}=T_{\text {sat }}$ criterion, $\Gamma_{k}$ is small and $(2.4 .1-68)$ can be safely neglected.

In the energy balance, the following quantities/terms are neglected

$$
\begin{aligned}
& \mathbf{K}_{\mathbf{k}}, C_{k}^{E 1}, C_{k}^{E 2} \\
& \frac{v_{k} \cdot v_{k}}{2} \text { (i.e., } e_{k} \equiv h_{k} \text { ) } \\
& \left\langle\Delta \mathrm{P}_{\mathrm{k}_{\mathrm{i}}}\right\rangle \text { (i.e. } \overline{\left\langle\mathrm{P}_{\mathrm{k}}\right\rangle_{\mathrm{i}}} \equiv \overline{\left\langle\mathrm{P}_{\mathrm{k}}\right\rangle} \text { ) } \\
& \frac{1}{V} \nabla \cdot\left[V \nless \alpha_{k} \triangleright \overline{\varangle q_{k}^{\prime \prime} \triangleright_{k}}\right] \\
& {\overline{\Varangle g_{k} \cdot v_{k}}}_{k}^{\rho} \\
& \overline{\mathbf{M}_{k_{i}}^{\text {nd }} \cdot v_{k_{i}}^{\text {nd }}} \\
& \overline{\mathbf{M}_{k_{i}}^{d} \cdot v_{k_{i}}^{d}} \\
& \overline{v_{k_{i}}^{\tau} \cdot\left\langle\tau_{k}\right\rangle_{i} \cdot S_{k_{i}}^{\prime \prime \prime}} \\
& \overline{\Gamma_{k}\left\langle\frac{\Delta \tilde{\mathrm{P}}_{k}^{d}}{\rho_{k}}\right\rangle_{i}}
\end{aligned}
$$




$$
\begin{gathered}
\nless \alpha_{k} \triangleright \overline{\nless q_{k}^{\prime \prime \prime} \triangleright_{k}} \\
\frac{1}{V} \nabla \cdot\left[V \nless \alpha_{k} \triangleright \overline{\overline{\tau_{k} \cdot v_{k} \triangleright_{k}}}\right]
\end{gathered}
$$

The distribution coefficients given by $(2.4 .1-69)$ are neglected for the same reason given above for the momentum equation. Thermal effects are assumed to be dominate in the energy balance compared to kinetic energy, viscous dissipation, etc. which results in the neglecting (2.4.1-70), (2.4.1-71), (2.4.1-73) through (2.4.1-77), and (2.4.1-79). Term $(2.4 .1-78)$ can be neglected because there is no internal heat generation present in FLOWTRAN-TF applications. Finally, term (2.4.1-72) is analogous to term (2.4.1-67) above. For one-dimensional flow in the $z$-direction the term becomes

$$
\frac{1}{V} \nabla \cdot\left[V \nless \alpha_{k} \triangleright \overline{\nless q_{k}^{\prime \prime} \downarrow_{k}}\right] \rightarrow \frac{1}{V} \frac{\partial V \alpha_{k} q_{z_{k}}}{\partial z}
$$

which is interpreted as axial conduction. The term is neglected for the same reasons as given above for the momentum conservation equation.

To further abbreviate the notation the following groupings are defined

$$
\begin{gathered}
-F_{i k} \equiv \overline{\underline{\left.\underline{\tau_{k}}\right\rangle_{i} \cdot S_{k_{i}}^{\prime \prime \prime}}}+\overline{M_{k_{i}}^{d}}=\underline{\tau_{k_{i}}} \cdot S_{k_{i}}^{\prime \prime \prime}+M_{k_{i}}^{d} \\
-F_{w k} \equiv-\overline{\left.\Delta P_{k_{w}}\right\rangle S_{k_{w}}^{\prime \prime \prime}}+\overline{M_{k_{w}}^{d}}=-\Delta P_{k_{w}} S_{k_{w}}^{\prime \prime \prime}+M_{k_{w}}^{d} \\
Q_{i k} \equiv \overline{q_{k_{i}}^{\prime \prime} A_{i}^{\prime \prime \prime}} \\
Q_{w k} \equiv \overline{q_{k_{w}}^{\prime \prime} A_{k_{w}}^{\prime \prime \prime}} \\
h_{k i} \equiv \frac{\overline{\Gamma_{k} h_{k_{i}}}}{\overline{\Gamma_{k}}} \\
E_{i k} \equiv Q_{i k}+\Gamma_{k} h_{k i}
\end{gathered}
$$

As with single-phase flow, flow fluctuations of a scale smaller than the computational discretization cannot be modeled directly (Anderson et al., 1984, Chap. 5). This leads to the introduction of the Reynolds stress term in the single-phase time-averaged momentum balance for incompressible flow. For compressible single-phase flow, additional terms accounting for small scale fluctuations in both velocity and density occur. Velocity, density, and volume fraction perturbations must be considered in two-phase, compressible flow. To account for these small-scale fluctuations, the terms $-W_{\mathfrak{i k}},-\mathbf{M}_{\mathfrak{i k}}$, and $-Q_{\mathrm{tk}}$ are added to the mass, momentum and energy balances, respectively. These terms are typically called 'turbulent mixing' terms, however, their presence is due to fluctuations in all of the two-phase flow variables (including volume fraction), not just turbulence within each phase. Two-phase computational models are coarse by comparison with single-phase 
numerical models. Therefore, the turbulent mixing models must account for fluctuations up to a relatively large scale.

Finally, we generate the non-conservative form of the momentum balance by making use of the mass conservation equation and neglecting $\bar{\Gamma}_{\mathrm{k}} \mathbf{v}_{\mathrm{k}}$. Actually, term (2.4.1-68) could be retained until now and then

$$
\overline{\Gamma_{k} v_{k_{i}}^{\Gamma}}-\overline{\Gamma_{k} v_{k}}
$$

is being neglected.

The resulting approximate conservation equations become

$$
\begin{gathered}
\frac{\partial \alpha_{k} \rho_{k}}{\partial t}+\frac{1}{V} \nabla \cdot\left[V \alpha_{k} \rho_{k} v_{k}\right]=\Gamma_{k}-W_{i k} \\
\alpha_{k} \rho_{k} \frac{\partial v_{k}}{\partial t}+\alpha_{k} \rho_{k} v_{k} \cdot \nabla v_{k}=-\alpha_{k} \nabla P+\alpha_{k} \rho_{k} g-F_{w k}-F_{i k}-M_{t k} \\
\frac{\partial \alpha_{k} \rho_{k} h_{k}}{\partial t}+\frac{1}{V} \nabla \cdot\left[V \alpha_{k} \rho_{k} h_{k} v_{k}\right]=\alpha_{k} \frac{\partial P}{\partial t}+E_{i k}+Q_{w k}-Q_{t k}
\end{gathered}
$$

The interface conditions alluded to earlier must also be space and time averaged to obtain the interfacial coupling conditions associated with the above conservation equations. The reader is referred to Lahey and Drew (1988) for more information. Like the conservation equations, approximations must made to the averaged interface conditions, however, care must be taken to ensure the approximations made to the conservation and interface equations are compatible. The appropriate approximate interface conditions for equations (2.4.1-86) through (2.4.1-87) are

$$
\begin{gathered}
\Gamma_{\mathrm{g}}+\Gamma_{\mathrm{f}}=0 \\
\mathbf{F}_{\mathrm{ig}}+\mathbf{F}_{\mathrm{if}}=0
\end{gathered}
$$

and

$$
E_{i g}+E_{i f}=0
$$

respectively. Observe that when the phasic space-time averaged equations are added together to produce the mixture conservation equations, the interfacial terms cancel. The interface conditions can be incorporated into the conservation equations by defining

$$
\begin{aligned}
\Gamma & \equiv \Gamma_{g} \\
F_{i} & \equiv F_{i g} \\
E_{i} & \equiv E_{i g}
\end{aligned}
$$


and writing out explicitly the gas and liquid phase conservation equations as

$$
\begin{aligned}
& \frac{\partial \alpha \rho_{g}}{\partial t}+\frac{1}{V} \nabla \cdot\left[V \alpha \rho_{g} v_{g}\right]=\Gamma-W_{t g} \\
& \frac{\partial(1-\alpha) \rho_{\mathrm{f}}}{\partial \mathrm{t}}+\frac{1}{\mathrm{~V}} \nabla \cdot\left[\mathrm{V}(1-\alpha) \rho_{\mathrm{f}} \mathbf{v}_{\mathrm{f}}\right]=-\Gamma-\mathrm{W}_{\mathrm{tf}} \\
& \alpha \rho_{g} \frac{\partial v_{g}}{\partial t}+\alpha \rho_{g} v_{g} \cdot \nabla v_{g}=-\alpha \nabla P+\alpha \rho_{g} g-F_{w_{g}}-F_{i}-M_{t g} \\
& (1-\alpha) \rho_{\mathrm{f}} \frac{\partial \mathbf{v}_{\mathrm{f}}}{\partial \mathrm{t}}+(1-\alpha) \rho_{\mathrm{f}} \mathbf{v}_{\mathrm{f}} \cdot \nabla \mathbf{v}_{\mathrm{f}}=-(1-\alpha) \nabla \mathrm{P}+(1-\alpha) \rho_{\mathrm{f}} \mathbf{g}-\mathbf{F}_{\mathrm{wf}}+\mathbf{F}_{\mathrm{i}}-\mathbf{M}_{\mathrm{tf}} \\
& \frac{\partial \alpha \rho_{g} h_{g}}{\partial t}+\frac{1}{V} \nabla \cdot\left[V \alpha \rho_{g} h_{g} v_{g}\right]=\alpha \frac{\partial P}{\partial t}+E_{i}+Q_{w g}-Q_{t g} \\
& \frac{\partial(1-\alpha) \rho_{f} h_{f}}{\partial t}+\frac{1}{V} \nabla \cdot\left[V(1-\alpha) \rho_{f} h_{f} v_{f}\right]=(1-\alpha) \frac{\partial P}{\partial t}-E_{i}+Q_{w f}-Q_{t f}
\end{aligned}
$$

\section{Two-component, two-phase conservation equations}

If the gas phase is comprised of a homogeneous air-water vapor mixture, an additional mass balance is required. Let $\mathrm{X}_{\mathrm{a}}$ be the mass fraction of air in the gas phase. Then the air mass balance is

$$
\frac{\partial \alpha \rho_{g} X_{a}}{\partial t}+\frac{1}{V} \nabla \cdot\left[V \alpha \rho_{g} X_{a} v_{g}\right]=-X_{a} W_{t g}
$$

Equation (2.4.1-95) is the gas mixture mass balance and the water vapor balance is obtained (if desired) by subtracting (2.4.1-101) from (2.4.1-95). No additional momentum or energy balances are needed because the air and water vapor velocities and temperatures are implicitly assumed to be equal.

Now that the final forms of the two-component, two-phase averaged mass, momentum, and energy balances have been derived, the various constitutive terms are now interpreted

$$
\begin{aligned}
& \Gamma \equiv \begin{array}{l}
\text { rate of gas phase mass creation at two-phase interfaces per unit volume } \\
(\text { steam })
\end{array} \\
& F_{i} \equiv \text { interfacial drag force on the gas phase per unit volume } \\
& E_{i} \equiv \begin{array}{l}
\text { energy transfer rate to the gas phase through heat and mass transfer at } \\
\text { two-phase interfaces per unit volume }
\end{array}
\end{aligned}
$$

$F_{w k} \equiv$ wall drag force on the $k$ phase per unit volume 
$\mathrm{Q}_{\mathrm{wk}} \equiv$ wall heat rate into the $\mathrm{k}$ phase per unit total volume

$\mathrm{W}_{\mathrm{tk}} \equiv$ rate of $\mathrm{k}$ phase mass transfer out of a control volume due to turbulent mixing per unit volume

$F_{1 k} \equiv$ rate of $k$ phase momentum transfer out of a control volume due to turbulent mixing per unit volume

$Q_{\mathrm{tk}} \equiv$ rate of $k$ phase heat transfer out of a control volume due to turbulent mixing per unit volume

\section{Integration of the averaged conservation equations}

In developing finite difference counterparts to the continuum two-component, two-phase conservation equations given by $(2.4 .1-95)$ through $(2.4 .1-101)$, the various terms in the continuum equations are integrated over a computational control volume. Most of the integrations can be handled straightforwardly. The proper treatment of the divergence terms is not as obvious and is discussed here. As an example, consider integration of the divergence term in the gas mass balance

$$
\int_{V_{c}} \frac{1}{V} \nabla \cdot\left[V \alpha \rho_{g} v_{g}\right] d V_{c}
$$

where $V_{c}$ denotes the computational cell control volume. Although not immediately obvious, Gauss' Theorem can be used in general to transform (2.4.1-102) into an area integral as shown below. For one-dimensional flow direct integration is used.

In FLOWTRAN-TF, the averaging volume $\mathrm{V}$ for a one-dimensional (z-direction) twophase flow is defined to be $A_{x y}(z) d z$. That is, area averaging is chosen. The computational control volume is defined to comprise the entire cross-section in the $x y$-plane between $z-\Delta z / 2$ and $z+\Delta z / 2$. The integration is performed as

$$
\begin{aligned}
\int_{V_{c}(z)} \frac{1}{A_{x y}} \frac{\partial}{\partial z}\left[A_{x y} \alpha \rho_{g} u_{g}\right] d V_{c} & =\int_{z-\Delta z / 2}^{z+\Delta z / 2} \frac{1}{A_{x y}} \frac{\partial}{\partial z}\left[A_{x y} \alpha \rho_{g} u_{g}\right] A_{x y} d z \\
& =\int_{z-\Delta z / 2}^{z+\Delta z / 2} \frac{\partial}{\partial z}\left[A_{x y} \alpha \rho_{g} u_{g}\right] d z \\
& =\left.A_{x y} \alpha \rho_{g} u_{g}\right|_{z-\Delta z / 2} ^{z+\Delta z / 2}
\end{aligned}
$$

For a two-dimensional two-phase flow in the $x-z$ plane, the averaging 'volume' is defined to be the line segment along the $y$-axis spanning the flow passage, $L_{y}(x, z)$ (i.e. $V=$ $\left.L_{y} d x d z\right)$. Then 


$$
\begin{aligned}
\int_{V_{c}(x, z)} \frac{1}{L_{y}} \nabla \cdot\left[L_{y} \alpha \rho_{g} v_{g}\right] d V_{c} & =\int_{z-\Delta z / 2}^{z+\Delta z / 2} \int_{x-\Delta x / 2}^{x+\Delta x / 2} \frac{1}{L_{y}} \nabla \cdot\left[L_{y} \alpha \rho_{g} v_{g}\right] L_{y} d x d z \\
& =\int_{z-\Delta z / 2}^{z+\Delta z / 2} \int_{x-\Delta x / 2}^{x+\Delta x / 2} \nabla \cdot\left[L_{y} \alpha \rho_{g} v_{g}\right] d x d z \\
& =\int_{S} L_{y} \alpha \rho_{g} v_{g} \cdot n d S \\
& =\left.A_{x y} \alpha \rho_{g} u_{g}\right|_{z-\Delta z / 2} ^{z+\Delta z / 2}+\left.A_{y z} \alpha \rho_{g} w_{g}\right|_{x-\Delta x / 2} ^{x+\Delta x / 2}
\end{aligned}
$$

Observe that the final result in each case is the net mass flowrate out of the computational cell as one intuitively expects. The divergence terms in the other conservation equations are handled in a similar manner.

\section{References}

Anderson, D. A., Tannehill, J. C. and Pletcher, R. H., 1984, Computational Fluid Mechanics and Heat Transfer, Hemisphere and McGraw-Hill.

Boure, J. A. and J. M. Delhaye, 1982, "General Equations and Two-Phase Flow Modeling" in Handbook of Multiphase Systems, ed. G. Hetsroni, Hemisphere and McGraw-Hill.

Flach, G. P., and S. Y. Lee, 1992, "Space/Time Averaged Two-Component Two-Phase Fluid Field Equations", QA Task 89-021-1 document TF-8 Rev. 2.

Ishii, M., 1975, Thermo-Fluid Dynamic Theory of Two-Phase Flow, Eryolles.

Ishii, M. and K. Mishima, 1984, "Two-Fluid Model and Hydrodynamic Constitutive Relations", Nucl. Engr. and Design, v 82, pp. 107-126.

Lahey, R. T. Jr. and D. A. Drew, "The Three-Dimensional Time and Volume Averaged Conservation Equations of Two-Phase Flows" in Advances in Nuclear Science and Technology, v 20, eds. J. Lewis and M. Becker, Plenum Press, New York. 


\subsubsection{Equations of State}

Classical thermodynamics imposes certain restrictions of the equations of state of a simple, multi-component system, as described by Callen (1960) and Modell and Reid (1974) for example. These consistency constraints are summarized in the first section below. In FLOWTRAN-TF, strict thermodynamic consistency is sacrificed in favor of computational efficiency as described in the following section. Finally, the FLOWTRAN-TF equations of state are given in detail.

\section{Thermodynamic equations of state}

The fundamental equation of a system contains all thermodynamic information about the system. For a two-component simple system, the Gibbs free energy representation of the fundamental equation is

$$
G=F_{G}\left(T, P, N_{1}, N_{2}\right)
$$

where

$$
\begin{aligned}
G & \equiv \text { extensive Gibbs free energy of the two-component mixture } \\
T & \equiv \text { mixture temperature } \\
P & \equiv \text { pressure } \\
N_{1} & \equiv \text { number of moles of component } 1 \\
N_{2} & \equiv \text { number of moles of component } 2
\end{aligned}
$$

All thermodynamic properties, extensive as well as intensive, can be obtained from equation (2.4.2-1). The intensive Gibbs free energy can be derived from equation $(2.4 .2-1)$ and takes the form

$$
g=F_{g}\left(T, P, y_{1}\right)
$$

where

$$
\begin{aligned}
& g \equiv \text { intensive Gibbs free energy, } G / N \text {, where } N \equiv N_{1}+N_{2} \\
& y_{1} \equiv \text { mole fraction of component } 1, N_{1} / N
\end{aligned}
$$

Equation (2.4.2-2) is sufficient to define all intensive properties of the system. For example,

$$
s=-\left(\frac{\partial g}{\partial T}\right)_{P, y_{1}}
$$




$$
\begin{gathered}
v=\left(\frac{\partial g}{\partial P}\right)_{T, y_{1}} \\
h=g+T s=g-T\left(\frac{\partial g}{\partial T}\right)_{P, y_{1}}
\end{gathered}
$$

where $s, v$ and $h$ are the molar intensive entropy, volume and enthalpy of the twocomponent mixture. Equation (2.4.2-2) contains less information than equation (2.4.2-1) because information about the extent of the system is not present ( $\mathrm{N}$ not specified). The following equation set contains all the information of equation (2.4.2-2), to within a constant of integration:

Mixture P-v-T equation:

$$
v=F_{v}\left(T, P, y_{1}\right)
$$

Pure component 1 P-v-T equation:

$$
v_{1}=F_{v_{1}}(T, P)
$$

Pure component 1 specific heat at low $P$ :

$$
\mathrm{C}_{1}^{0}=\mathrm{F}_{\mathrm{C}_{1}^{0}}(\mathrm{~T})
$$

Pure component 2 P-v-T equation:

$$
v_{2}=F_{v_{2}}(T, P)
$$

Pure component 2 specific heat at low P:

$$
C_{2}^{0}=F_{C_{2}^{0}}(T)
$$

The specific heats may be $C_{v}$ or $C_{p}$. Equations (2.4.2-6) through $(2.4 .2-10)$ can be integrated and manipulated to determine the intensive Gibbs free energy to within a constant of integration and therefore contain all the information needed to define intensive properties. It is sufficient to determine properties to within an arbitrary datum because changes in properties are what are of interest.

While expressions for intensive properties in addition to equation (2.4.2-2), or alternatively equations (2.4.2-6) through (2.4.2-10), can be written down, these additional relations provide no new fundamental information. Auxiliary expressions must be derived from equation $(2.4 .2-2)$ or equations $(2.4 .2-6)$ through $(2.4 .2-10)$ to be thermodynamically consistent. 


\begin{tabular}{|l|lll|r|}
\hline $2 / 15 / 93$ & WSRC.TR-92.532 Rev. 0 & FLOWTRAN.TF Software Design & Page 76 \\
\hline
\end{tabular}

\section{FLOWTRAN-TF approach}

In FLOWTRAN-TF, the air-water mixture is assumed to be an ideal solution and the mixture P-v-T equation (2.4.2-6) becomes

$$
v=\Sigma y_{i} v_{i}(T, P)
$$

A P-v-T relation (ideal gas) and specific heat expression $\left(\mathrm{C}_{\mathrm{pa}}\left(\mathrm{T}_{\mathrm{g}}\right)\right)$ are given for pure air corresponding to equations (2.4.2-7) and (2.4.2-8). All additional air properties are derived from these.

A complicated P-v-T expression would be required to accurately model the physical behavior of water from compressed liquid to superheated gas. When highly accurate values are desired, such as the ASME Steam Tables, the expressions must be extremely complicated. FLOWTRAN-TF requires low accuracy by comparison, so a less complicated P-v-T expression is needed, but the expression would surely still be relatively complicated. Phase equilibrium points (saturation pressure-temperature information) are determined by finding those points at the same $T$ and $P$ which have the same Gibbs free energy through an iterative process. In general, much computational effort would be required to generate the thermodynamic properties of water needed by FLOWTRAN-TF.

Unfortunately two-phase thermal-hydraulic codes spent a significant fraction of time in computing properties. To save significant computational effort, each thermodynamic property of water, including saturation properties, is modeled by a simple, usually empirical, expression. The formulation is not strictly thermodynamically consistent because the chosen equations of state do not satisfy a single underlying fundamental equation of the form given by equation $(2.4 .2-2)$. Strict thermodynamic consistency does not appear to be necessary however, as no problems associated with consistency have been observed during code development.

Even with extremely simple equations of state chosen below, the computational effort is a major contributor to the overall run-time of FLOWTRAN-TF. For example, a typical simulation involving two-phase fluid calculations and no solid calculations spent about $1 / 8$ of the execution time in equation of state subroutines. If the computational effort of the equations of state were increased by a factor of 5 for example, the overall run time would go up by $50 \%$.

\section{Summary of FLOWTRAN-TF equations of state}

The thermodynamic and transport properties which are not considered constitutive relations in solving the fluid transport equations are evaluated by the equation of state. The main variables in FLOWTRAN-TF code are taken as system pressure $P$, gas void fraction $\alpha$, gas temperature $T_{g}$, liquid temperature $T_{f}$, air mass fraction $X_{a}$, gas phasic velocity $v_{g}$, and liquid phasic velocity $\mathbf{v}_{\mathrm{f}}$. State variables are computed as functions of the 5 primary state variables in the code $\left(P, \alpha, T_{g}, T_{f}\right.$, and $\left.X_{a}\right)$. External to the equation of state subroutine in the code, the gas properties are viewed as functions of $\mathrm{P}, \mathrm{T}_{\mathrm{g}}$, and $\mathrm{X}_{\mathrm{a}}$. The liquid $\left(\mathrm{H}_{2} \mathrm{O}\right.$ or $D_{2} O$ ) properties are functions of $P$ and $T_{f}$. Due to the relatively low pressures and temperatures encountered in SRS reactors, simple mixture rules are sufficient. Internal to the equation of state subroutine, the gas density is computed assuming Dalton's law and ideal gas behavior and the gas enthalpy is computed assuming an ideal gas solution. The resulting internal equations of state, in functional form, are 


$$
\begin{aligned}
& \rho_{f}=\rho_{f}\left(P, T_{f}\right) \\
& h_{f}=h_{f}\left(P, T_{f}\right) \\
& \rho_{a}=\rho_{a}\left(P_{a}, T_{g}\right) \\
& h_{a}=h_{a}\left(P, T_{g}\right) \\
& \rho_{s}=\rho_{s}\left(P_{s}, T_{g}\right) \\
& h_{s}=h_{s}\left(P, T_{g}\right)
\end{aligned}
$$

and the gas mixture equations are

$$
\begin{gathered}
P=P_{a}+P_{s} \\
\rho_{a}=X_{a} \rho_{g} \\
\rho_{g}=\rho_{a}+\rho_{s} \\
h_{g}=X_{a} h_{a}+\left(1-X_{a}\right) h_{s}
\end{gathered}
$$

where

$$
\begin{aligned}
& P_{a} \equiv \text { partial pressure of air } \\
& P_{s} \equiv \text { partial pressure of steam } \\
& \rho_{a} \equiv \text { mass of air per unit total gas volume } \\
& \rho_{s} \equiv \text { mass of steam per unit total gas volume }
\end{aligned}
$$

Note from equation $(2.4 .2-21)$ that the gas specific heat is obtained as

$$
C_{p g}=X_{a} C_{p a}+\left(1-X_{a}\right) C_{p s}
$$

where

$$
\begin{aligned}
& C_{p a} \equiv \text { constant pressure specific heat of air } \\
& C_{p s} \equiv \text { constant pressure specific heat of steam }
\end{aligned}
$$

Using the ideal gas law and equations $(2.4 .2-18)$ to $(2.4 .2-21)$ the air and steam partial pressures are computed from

$$
P_{a}=P_{a}\left(P, X_{a}\right)=P \frac{X_{a} M_{s}}{X_{a} M_{s}+\left(1-X_{a}\right) M_{a}}
$$




\begin{tabular}{|l|lll|l|}
\hline $2 / 15 / 93$ & WSRC-TR-92-532 Rev. 0 & FLOWTRAN-TF Software Design & Page 78 \\
\hline
\end{tabular}

and

$$
P_{s}=P-P_{a}
$$

where

$$
\begin{aligned}
& \mathrm{M}_{\mathrm{a}} \equiv \text { molecular weight of air } \\
& \mathrm{M}_{\mathrm{s}} \equiv \text { molecular weight of steam }
\end{aligned}
$$

Simple equation of state relations, denoted functionally by equations $(2.4 .2-12)$ to $(2.4 .2-17)$, were developed taking advantage of the relatively low pressures and temperatures encountered in SRS applications. The liquid thermodynamic property models are valid for pressures up to $1 \mathrm{MPa}$ and temperatures from $275 \mathrm{~K}$ to $450 \mathrm{~K}$. For gas thermodynamic properties, temperatures up to $700 \mathrm{~K}$ can be handled. Within these ranges of temperature and pressure, the ideal gas assumption is generally quite good. The largest density error occurs for saturated steam at $1 \mathrm{MPa}$ where the code underpredicts by $7 \%$.

Transport properties of gas mixture (air and steam) are computed in terms of air mass fraction $\left(X_{\mathrm{a}}\right)$ based on the homogeneous mixture.

$$
\begin{aligned}
& \mu_{\mathrm{g}}=\mathrm{X}_{\mathrm{a}} \mu_{\mathrm{a}}+\left(1-\mathrm{X}_{\mathrm{a}}\right) \mu_{\mathrm{s}} \\
& \mathrm{k}_{\mathrm{g}}=\mathrm{X}_{\mathrm{a}} \mathrm{k}_{\mathrm{a}}+\left(1-\mathrm{X}_{\mathrm{a}}\right) \mathrm{k}_{\mathrm{s}}
\end{aligned}
$$

where

$$
\begin{aligned}
& \mu_{\mathrm{a}} \equiv \text { viscosity of air } \\
& \mu_{\mathrm{s}} \equiv \text { viscosity of steam } \\
& \mathrm{k}_{\mathrm{a}} \equiv \text { thermal conductivity of air } \\
& \mathrm{k}_{\mathrm{s}} \equiv \text { thermal conductivity of steam }
\end{aligned}
$$

The FLOWTRAN-TF code computes the thermodynamic and transport properties of liquid and steam phases for light water and heavy water as well as those of air directly from curve fit functions. The functions re listed in the Appendix along with their associated coefficients. In addition, all the fluid property correlations which were used by the FLOWTKAN-TF code are reproduced there in order to provide the user with a guide to the range of appli ability and the uncertainty of the fluid property functions.

\section{Gas Property Derivatives}

The liquid properties, their dervatives, and the gas properties were specified completely in terms of the primary variables $\left(P, \alpha, T_{f}, T_{g}, X_{a}\right)$ in the Appendix. For closure of the equation of state, the derivatives of gas density and enthalpy with respect to the primary variables should be specified. That is, 


$$
\frac{\partial \rho_{g}}{\partial P}, \frac{\partial \rho_{g}}{\partial T_{g}}, \frac{\partial \rho_{g}}{\partial X_{a}}, \frac{\partial h_{g}}{\partial P}, \frac{\partial h_{g}}{\partial T_{g}}, \frac{\partial h_{g}}{\partial X_{a}}
$$

are needed. From the equation of state,

$$
\begin{gathered}
\rho_{g}=\rho_{a}+\rho_{s} \\
P=P_{a}+P_{s} \\
h_{g}=X_{a} h_{a}\left(P, T_{g}\right)+\left(1-X_{a}\right) h_{s}\left(P, T_{g}\right) \\
X_{a}=\frac{\rho_{a}}{\rho_{a}+\rho_{s}}
\end{gathered}
$$

From equation (2.4.2-31) and the ideal gas relation, the air mass fraction $\left(\mathrm{X}_{\mathrm{a}}\right)$ becomes

$$
X_{a}=\frac{M_{a} P_{a}}{\left(P_{a} M_{a}+P_{s} M_{s}\right)}
$$

The partial derivatives of gas density with respect to gas temperature, system pressure, and air mass fraction are obtained by differentiating equation $(2.4 .2-28)$.

$$
\begin{aligned}
& \frac{\partial \rho_{g}}{\partial T_{g}}=\frac{\partial \rho_{a}}{\partial T_{g}}+\frac{\partial \rho_{s}}{\partial T_{g}} \\
& \frac{\partial \rho_{g}}{\partial P}=\frac{\partial \rho_{a}}{\partial P}+\frac{\partial \rho_{s}}{\partial P} \\
& \frac{\partial \rho_{g}}{\partial X_{a}}=\frac{\partial \rho_{a}}{\partial X_{d}}+\frac{\partial \rho_{s}}{\partial X_{a}}
\end{aligned}
$$

From equation (2.4.2-30), the partial derivaluves of gas mixture enthalpy with respect to the primary variables $\left(P, T_{g}, X_{a}\right)$ are evaluated.

$$
\begin{gathered}
\frac{\partial h_{g}}{\partial P}=X_{a} \frac{\partial h_{s}}{\partial P}+\left(1-X_{a}\right) \frac{\partial h_{s}}{\partial P} \\
\frac{\partial h_{g}}{\partial^{\prime} T_{g}}=X_{a} \frac{\partial h_{a}}{\partial T_{g}}+\left(1-X_{a}\right) \frac{\partial h_{s}}{\partial T_{g}} \\
\frac{\partial h_{g}}{\partial X_{a}}=h_{a}-h_{s}
\end{gathered}
$$


Now, the right hand side terms of equations $(2.4 .2-34)$ and $(2.4 .2-35)$ are to be evaluated. When $\chi$ is denoted as $\rho_{a}$ or $\rho_{s}, \chi$ is a fuction of air mass fraction $\left(X_{a}\right)$ and local system pressure $(\mathrm{P})$, that is,

$$
\begin{aligned}
& \chi=f \operatorname{cn}\left(X_{a}, P\right) \\
& X_{a}=f \operatorname{cn}\left(P_{a}, P_{s}\right) \\
& P=f \operatorname{cn}\left(P_{a}, P_{s}\right) .
\end{aligned}
$$

The partial derivatives of air and steam densities with respect to the air mass fraction and system pressure, $\left(\partial \chi / \partial \mathrm{X}_{\mathrm{a}}\right)$ and $(\partial \chi / \partial \mathrm{P})$, are obtained by using the chain rule and the Cramer's rule. Using the chain rule,

$$
\left(\begin{array}{l}
\frac{\partial \chi}{\partial \mathrm{P}_{\mathrm{a}}} \\
\frac{\partial \chi}{\partial \mathrm{P}_{\mathrm{s}}}
\end{array}\right)=\left(\begin{array}{ll}
\frac{\partial \mathrm{X}_{\mathrm{a}}}{\partial \mathrm{P}_{\mathrm{a}}} & \frac{\partial \mathrm{P}}{\partial \mathrm{P}_{\mathrm{a}}} \\
\frac{\partial \mathrm{X}_{\mathrm{a}}}{\partial \mathrm{P}_{\mathrm{s}}} & \frac{\partial \mathrm{P}}{\partial \mathrm{P}_{\mathrm{s}}}
\end{array}\right)\left(\begin{array}{l}
\frac{\partial \chi}{\partial \mathrm{X}_{\mathrm{a}}} \\
\frac{\partial \chi}{\partial \mathrm{P}}
\end{array}\right)
$$

In equation (2.4.2-42) the following derivatives are available from the differentiation of equations (2.4.2-29) and (2.4.2-32) with repect to the gas partial pressure, $\mathrm{P}_{\mathrm{a}}$ or $\mathrm{P}_{\mathrm{s}}$,:

$$
\begin{gathered}
\frac{\partial \mathrm{P}}{\partial \mathrm{P}_{\mathrm{a}}}=1 \\
\frac{\partial \mathrm{P}}{\partial \mathrm{P}_{\mathrm{s}}}=1 \\
\frac{\partial \mathrm{X}_{\mathrm{a}}}{\partial \mathrm{P}_{\mathrm{a}}}=\frac{\mathrm{M}_{\mathrm{a}} \mathrm{M}_{\mathrm{s}} \mathrm{P}_{\mathrm{s}}}{\left(\mathrm{P}_{\mathrm{a}} \mathrm{M}_{\mathrm{a}}+\mathrm{P}_{\mathrm{s}} \mathrm{M}_{\mathrm{s}}\right)^{2}} \\
\frac{\partial \mathrm{X}_{\mathrm{a}}}{\partial \mathrm{P}_{\mathrm{s}}}=-\frac{\mathrm{M}_{\mathrm{a}} \mathrm{M}_{\mathrm{s}} \mathrm{P}_{\mathrm{a}}}{\left(\mathrm{P}_{\mathrm{a}} \mathrm{M}_{\mathrm{a}}+\mathrm{P}_{\mathrm{s}} \mathrm{M}_{\mathrm{s}}\right)^{2}}
\end{gathered}
$$

After applying the Cramer's rule to equation (2.4.2-42), the partial derivatives of gas densities are obtained using equations $(2.4 .2-43)$ to $(2.4 .2-46)$.

$$
\begin{aligned}
& \frac{\partial \chi}{\partial \mathrm{X}_{\mathrm{a}}}=\frac{\left(\mathrm{M}_{\mathrm{a}} \mathrm{P}_{\mathrm{a}}+\mathrm{M}_{\mathrm{s}} \mathrm{P}_{\mathrm{s}}\right)^{2}}{\mathrm{M}_{\mathrm{a}} \mathrm{M}_{\mathrm{s}}\left(\mathrm{P}_{\mathrm{a}}+\mathrm{P}_{\mathrm{s}}\right)}\left(\frac{\partial \chi}{\partial \mathrm{P}_{\mathrm{a}}}-\frac{\partial \chi}{\partial \mathrm{P}_{\mathrm{s}}}\right) \\
& \frac{\partial \chi}{\partial \mathrm{P}}=\frac{\mathrm{P}_{\mathrm{a}}}{\left(\mathrm{P}_{\mathrm{a}}+\mathrm{P}_{\mathrm{s}}\right)}\left(\frac{\partial \chi}{\partial \mathrm{P}_{\mathrm{a}}}\right)+\frac{\mathrm{P}_{\mathrm{s}}}{\left(\mathrm{P}_{\mathrm{a}}+\mathrm{P}_{\mathrm{s}}\right)}\left(\frac{\partial \chi}{\partial \mathrm{P}_{\mathrm{s}}}\right)
\end{aligned}
$$

where $\chi$ is $\rho_{\mathrm{a}}$ oi $\rho_{\mathrm{s}}$. 


\begin{tabular}{|l|lll|}
\hline $2 / 15 / 93$ & WSRC.TR-92-532 Rev. 0 & FLOWTRAN-TF Software Design & Page 81 \\
\hline
\end{tabular}

\section{References}

Callen, H. B., 1960, Thermodynamics, Wiley, New York.

Modell, M. and R. C. Reid, 1974, Thermodynamics and its Applications, Prentice-Hall, Englewood Cliffs, N. J. 


\subsection{Constitutive Relations}

The two-component, two-phase fluid conservation equations for an air-water mixture have been presented in the preceding section. These governing equations contain constitutive terms for wall heat transfer, wall drag, interfacial drag, interfacial heat and mass transfer, etc. Models for the constitutive terms are presented in this section to complete the formulation of the fluid governing equations.

\subsubsection{Wall Heat Transfer}

FLOWTRAN-TF contains a complete boiling curve for air-water, two-phase flow. Except for the non-boiling portion of the curve, the wall heat transfer models are largely based on untested adaptations of single-component correlations from the literature; it is an open question whether the models predict correct behavior for two-component (air-water) flows. An extensive literature survey was not made nor have novel models been incorporated into the heat transfer scheme. Rather, we have looked to existing codes to provide guidance in the development of a heat transfer module that uses tested correlations (for singlecomponent flows) capable of modeling a complete boiling curve. In particular, model components have been abstracted from the existing FLOWTRAN-FI (Aleman et al., 1989) code and from the RELAP5/MOD2 (Ransom et al., 1985; Dimenna, et al., 1988), COBRA/TRAC (Thurgood et al., 1983), TRAC (Liles et al., 1988), and THERMIT (Kelly, 1980) codes. We have selected heat transfer correlations appropriate for the low pressure conditions that prevail in SRS reactors. We note that the fluid flow channels in SRS assemblies are narrow annuli heated from both sides. The heat transfer correlations presented below are primarily derived from data in circular tubes. We have not attempted to modify the correlations to account for flow in annuli or for two-sided heating effects (Kays, 1966).

One of the nonboiling heat transfer models is based on SRS data taken for air-water twophase downflows in a ribbed annulus. Fortunately, boiling at metal surfaces does not occur using the current ECS power limit criterion of $T_{\text {wall }}=T_{\text {sat }}$ (Smith et al., 1990a; Smith et al., 1990b). For this criterion the maximum surface temperature in the heated assembly core is restricted to remain below the local fluid saturation temperature. Therefore, a non-boiling heat transfer correlation appropriate for two-phase air-water downflows is sufficient to calculate the wall heat flux throughout the LOCA-ECS transient for ECS power limit computations. However, an assessment of the safety margin between the physical damage limit and the conservative $T_{w a l l}=T_{\text {sat }}$ limit requires calculations in the boiling heat transfer regime. For these FLOWTRAN-TF calculations, the entire boiling curve logic may be exercised.

We first discuss the overall wall heat transfer computational strategy and then describe the individual heat transfer correlations selected and indicate their source. In the subsection, we show a few representative boiling curves calculated using the correlations. 


\section{Overall Heat Transfer Strategy}

The basic FLOWTRAN-TF heat transfer computational strategy is illustrated in Fig. 2.5.1-1. Subroutine QWALL, which is called at the start of each time step from subroutine TRANS, performs all of the heat transfer calculations required to advance the computation another time step. That is, QWALL provides explicit approximations to the calculated quantities. QWALL calculates the heat flux to the liquid phase $\left(q_{f}^{\prime \prime}\right)$, heat flux to the gas phase $\left(\mathrm{q}_{g}^{\prime \prime}\right)$, the derivatives of heat fluxes with respect to wall temperature $\left(\mathrm{T}_{w}\right)$, and the wall vapor generation rate $\left(\Gamma_{w}\right)$ at all computational cell solid surfaces. Subroutine SOLID then uses the total heat flux at each surface to calculate new time values for the solid temperature distribution $\left(T_{s}\right)$ and wall temperature. With new wall temperatures, the derivative with respect to wall temperature is used to obtain an improved estimate for the surface heat fluxes. The new estimates for the surface heat fluxes are then used to formulate the fluid energy equations. Finally, subroutine NEWTON solves for fluid temperatures at the new time. 


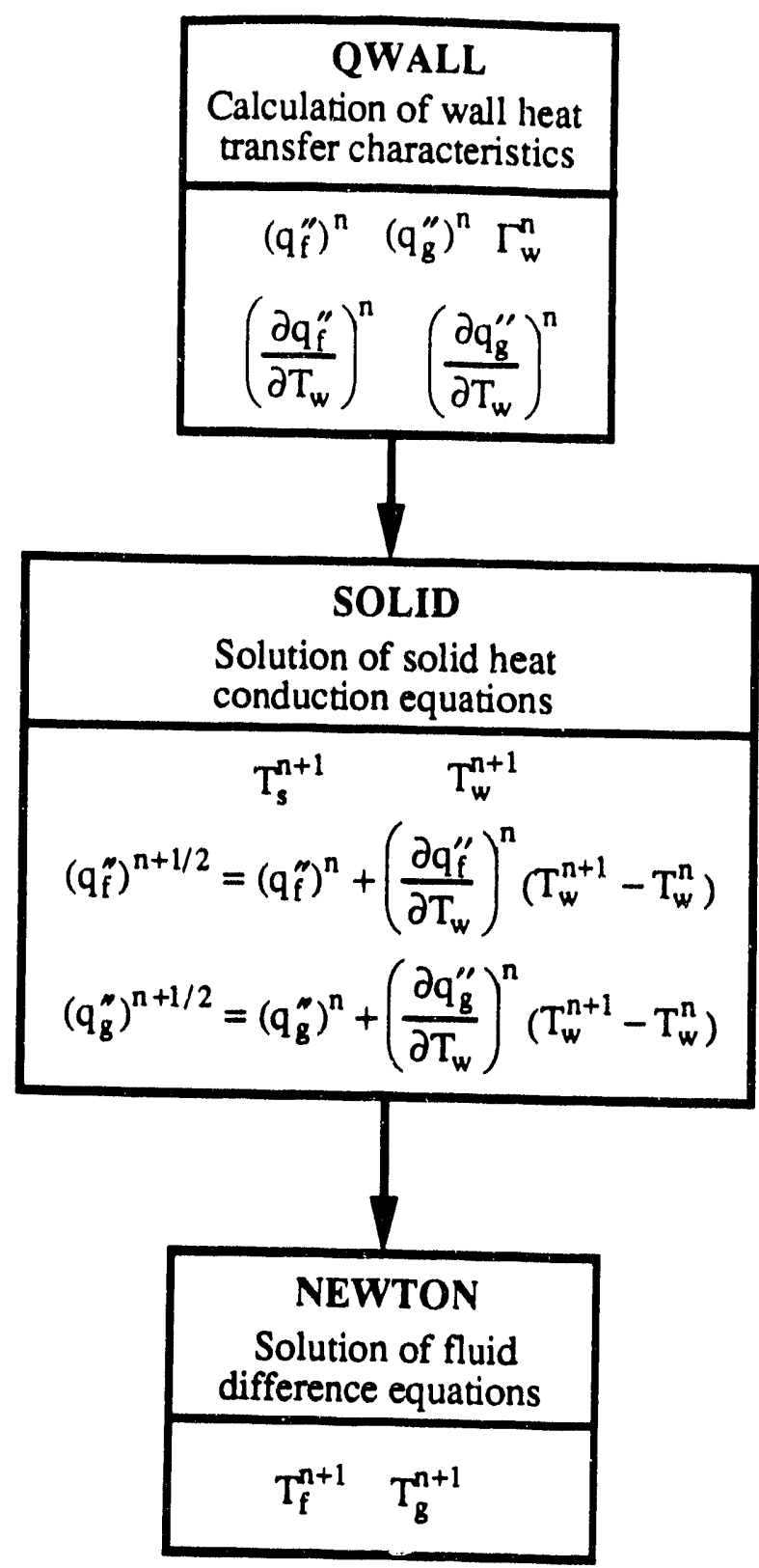
$\begin{array}{ll}\text { Figure 2.5.1-1 } & \text { FLOWTRAN-TF wall heat flux and temperature computational } \\ \text { strategy. }\end{array}$ 
The heat transfer correlations are coded in a set of seven subroutines that are called by QWALL. These subroutines (HCONV, SRLHTC, CDENS, MIKIC, CHEN, CHF, and FILMB) are used to solve for heat transfer coefficients and the surface heat fluxes in the various heat transfer regimes considered. In general, the package models a boiling curve with four heat transfer regimes:

1. Single phase heat transfer to liquid when the surface temperature is below the local fluid saturation temperature $\left(\mathrm{T}_{\mathrm{SAT}}\right)$.

2. Subcooled and saturated nucleate boiling heat transfer when the surface temperature is between saturation and the critical heat flux temperature $\left(\mathrm{T}_{\mathrm{CHF}}\right)$.

3. Transition heat transfer for wall temperatures between the critical heat flux temperature and the minimum stable film boiling temperature $\left(\mathrm{T}_{\mathrm{MSFB}}\right)$.

4. Film heat transfer when surface temperatures are greater than $T_{M S F B}$.

The basic structure of subroutine QWALL is illustrated in Fig. 2.5.1-2 which shows the logic used to select a particular heat transfer regime and the subroutines used to perform heat transfer calculations within each regime. An input parameter (IBOIL) is specified by the user to select the particular heat transfer options for the calculation. Each of the subroutines and their associated heat transfer correlations is discussed below. For reference, a schematic representation of the general boiling curve is presented in Fig. 2.5.1-3.

Subroutine QWALL also contains logic to adjust heat transfer to the moderator space as the tank drains. That is, if the tank level covers the outer surface of the assembly for a given axial cell, heat transfer at that surface is calculated using an input heat transfer coefficient (HMOD) and input moderator temperature (TMOD). As the tank drains and outer surfaces become uncovered, the heat transfer coefficient is adjusted so that for bare surfaces an adiabatic condition applies. For partial coverage, the external heat transfer coefficient is interpo'ated between the input value and zero to approximately account for surface dryout. 


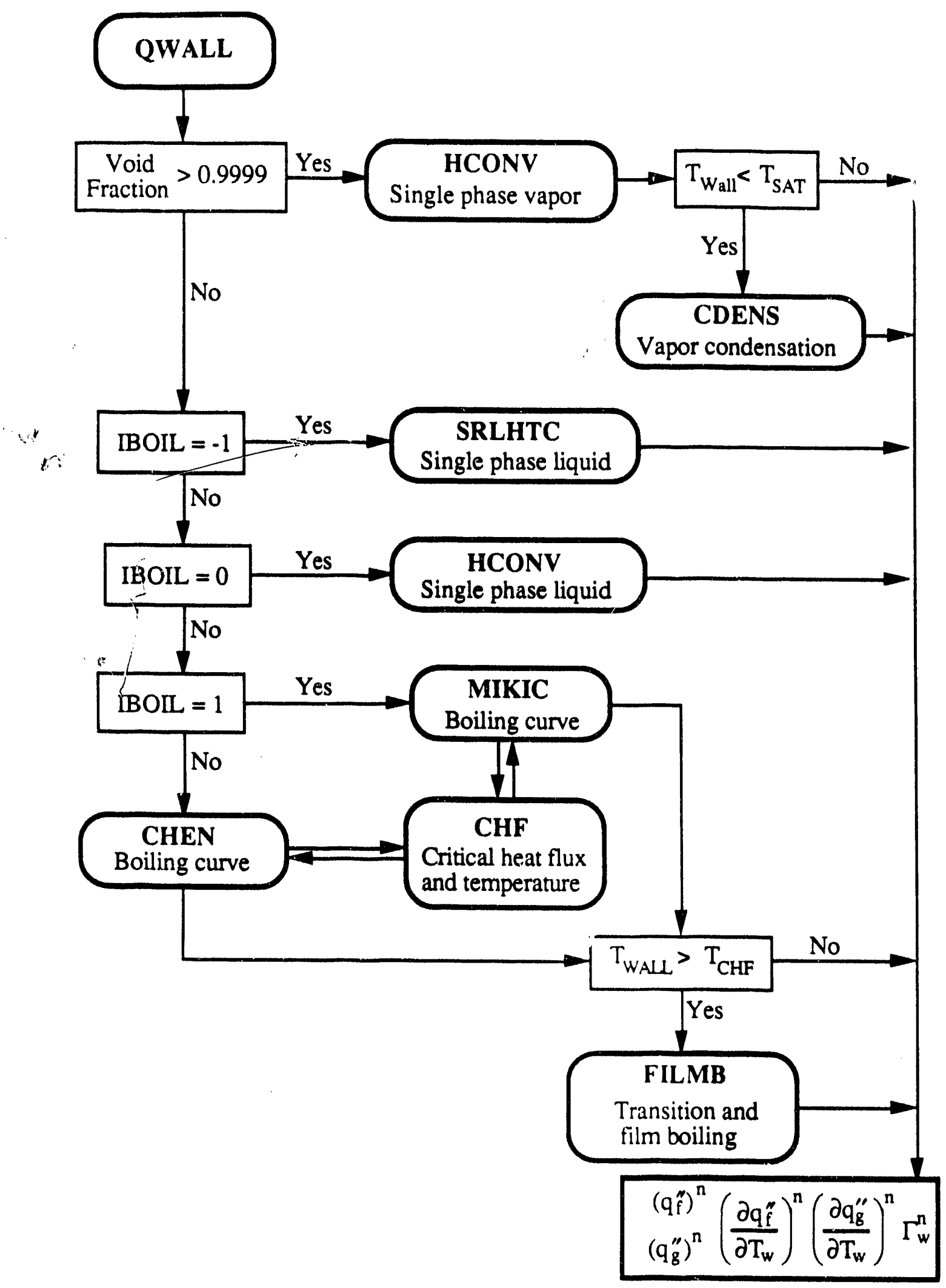

Figure 2.5.1-2 Heat transfer regime selection logic in FLOWTRAN-TF. 


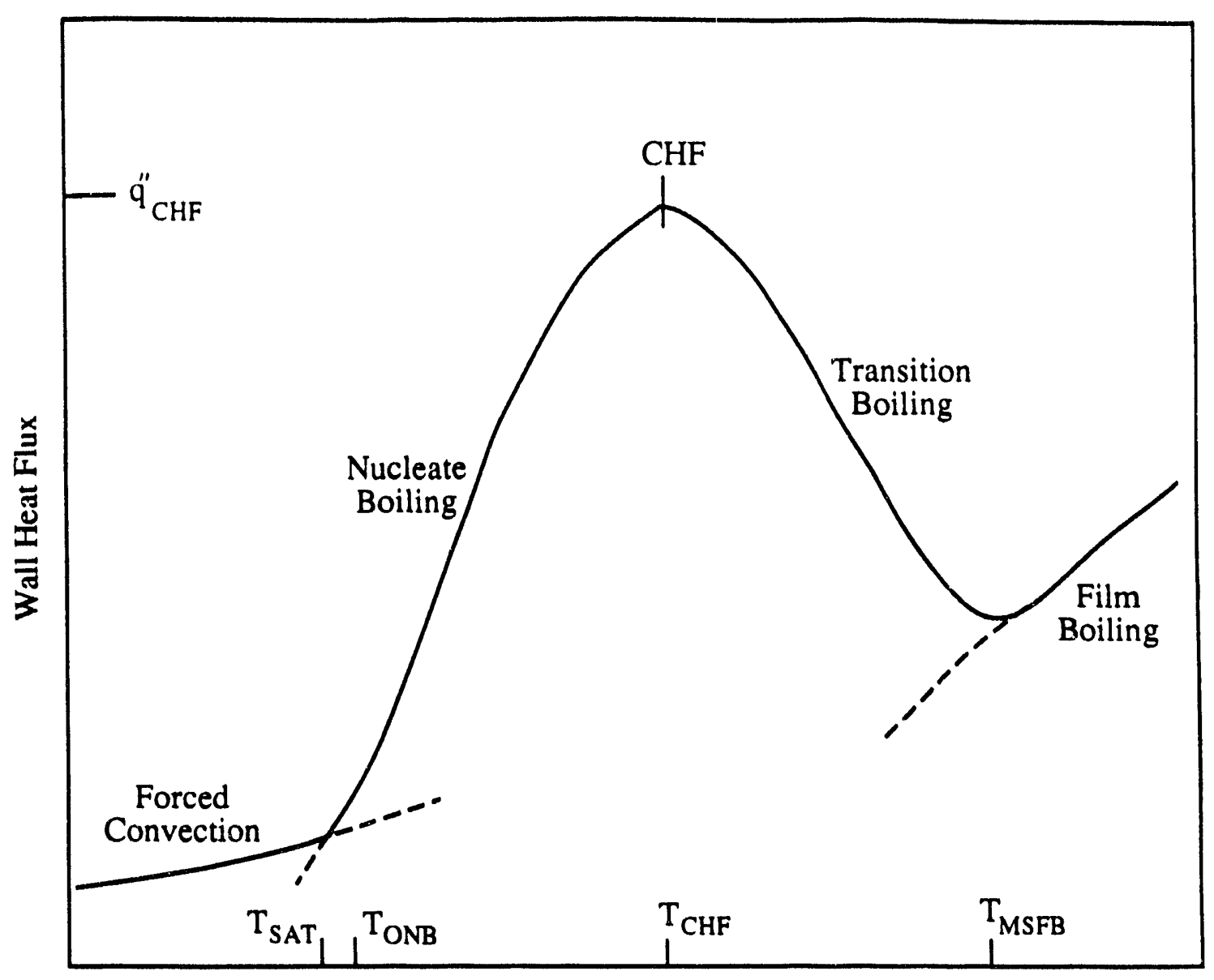

Wall Temperature

Figure 2.5.1-3 Schematic representation of boiling curve.

\section{Heat Transfer Correlations}

The individual models that form the overall boiling curve are discussed below. The models are for wall heat fluxes $\left(q_{f}^{\prime \prime}, q_{g}^{\prime \prime}\right)$ and mass flux $\left(\Gamma_{w}\right)$ quantities. The rates of wall heat exchange $\left(Q_{w f}, Q_{w g}\right)$ and phase change $\left(\Gamma_{w}\right)$ per unit volume are obtained by multiplying the flux quantities by the appropriate cell surface area and dividing by fluid cell volume.

Single-Phase Fluids (HCONV): Heat transfer to a single-phase vapor is assumed to occur when the cell void fraction is greater than 0.9999 . The single-phase heat transfer correlations in subroutine HCONV are also used for the liquid phase when the input parameter IBOIL is set equal to 0 . That is, when the IBOIL parameter is specified as 0 , the code does not test for CHF and will use the single-phase heat transfer correlation in subroutine HCONV to calculate the surface heat flux for any wall temperature.

For heat transfer to either single-phase gas or liquid in turbulent flow, subroutine HCONV uses the Sieder-Tate (1936) correlation which includes a viscosity correction to account for heated wall effects. The correlation is 


$$
h_{F C}=0.027\left(\frac{k}{D_{H}}\right) \operatorname{Re}^{0.8} \operatorname{Pr}^{1 / 3}\left(\frac{\mu}{\mu_{w}}\right)^{0.14}
$$

where

$$
\begin{aligned}
h_{\mathrm{FC}} & \equiv \text { forced convection heat transfer coefficient, } \mathrm{W} / \mathrm{m}^{2}{ }^{\circ} \mathrm{C} \\
\mathrm{k} & \equiv \text { fluid thermal conductivity, } \mathrm{W} / \mathrm{m}^{\circ} \mathrm{C} \\
\mathrm{D}_{\mathrm{H}} & \equiv \text { hydraulic diameter of flow channel, } \mathrm{m} \\
\mathrm{Re} & \equiv \text { superficial Reynolds number }=\mathrm{G} \mathrm{D}_{\mathrm{H}} / \mu \\
\mathrm{G} & \equiv \text { fluid (liquid or gas) mass flux, } \mathrm{kg} / \mathrm{m}^{2} \mathrm{~s} \\
\mathrm{Pr} & \equiv \text { Prandtl number }=\mathrm{c}_{\mathrm{p}} \mu / \mathrm{k} \\
\mathrm{c}_{\mathrm{p}} & \equiv \text { fluid specific heat, } \mathrm{J} / \mathrm{kg}{ }^{\circ} \mathrm{C} \\
\mu & \equiv \text { fluid viscosity at bulk temperature, } \mathrm{kg} / \mathrm{m} \mathrm{s} \\
\mu_{\mathrm{w}} & \equiv \text { fluid viscosity at wall temperature, } \mathrm{kg} / \mathrm{m} \mathrm{s}
\end{aligned}
$$

For single-phase laminar flow, the heat transfer coefficient is evaluated using (Rohsenow and Choi, 1961)

$$
h_{L}=4.0\left(\frac{k}{D_{H}}\right)
$$

The Nusselt number of 4.0 used in equation (2.5.1-2) is the mean of the values for constant wall heat flux $(\mathrm{Nu}=4.364)$ and constant wall temperature $(\mathrm{Nu}=3.658)$ that apply for fully-developed laminar flow through a circular tube. Nusselt numbers for fullydeveloped laminar flow through annuli are approximately $20 \%$ higher than for tubes with a circular cross-section. We have not included this effect in our laminar heat transfer correlation. In practice, the subroutine evaluates both equation $(2.5 .1-1)$ and equation $(2.5 .1-2)$ and takes the maximum value for the single-phase heat transfer coefficient. This procedure gives a continuous transition between the flow regimes.

Having obtained the heat transfer coefficient, the forced convection heat flux to either liquid or gas is then calculated from

$$
\mathrm{q}_{\mathrm{FC}}^{\prime \prime}=\mathrm{h}\left(\mathrm{T}_{\mathrm{w}}-\mathrm{T}\right)
$$

where

$$
\begin{aligned}
h & \equiv \max \left(, h_{F C}\right) \\
T_{w} & \equiv \text { wall temperature, }{ }^{\circ} \mathrm{C} \\
T & \equiv \text { fluid (gas or liquid) temperature, }{ }^{\circ} \mathrm{C}
\end{aligned}
$$


In the single-phase liquid regime, the surface is assumed to be fully wetted and no vapor generation at the wall takes place.

Single-Phase Liquid or Two-Phase Fluid (SRLHTC): Correlations suitable for air-water downflow in ribbed annuli were developed using experimental data obtained from Rig B at SRS (Guerrero, 1990). Rig B consisted of a single annular channel (divided into four subchannels by non-conducting ribs) that was heated over the outer radial surface. The rig was constructed of stainless steel and had uniform axial and azimuthal power profiles. Experiments were conducted over a wide range of water flowrates and pressure boundary conditions. The data clearly divided into two groups according to void fraction; this was attributed to a change in flow regime. The void fraction in Rig B for a particular set of operating conditions was estimated from FLOWTRAN-TF calculations. At calculated void fractions less than 0.75 a dispersed (bubbly, slug or churn-turbulent) flow regime was inferred while at higher void fractions the flow regime was assumed to be annular (separated). When the IBOIL parameter is set to -1 , the following correlations derived from a statistical analysis of the Rig B data are used.

In the dispersed flow regime, $\alpha<0.75$, FLOWTRAN-TF uses the following slightly modified form of the Dittus-Boelter (1930) correlation for turbulent liquid heat transfer

$$
h_{T}=0.0217\left(\frac{k}{D_{H}}\right) \operatorname{Re}^{0.8} \operatorname{Pr}^{0.4}
$$

with

$$
\operatorname{Re}=\frac{G_{f} D_{H}}{\mu_{f}(1-\alpha)}
$$

As indicated above, phasic liquid velocity is used to calculate the Reynolds number and bulk liquid properties are used to evaluate physical properties. Correlation (2.5.1-4a) and the experimental data are shown in Fig. 2.5.1-4. The figure legend gives the range of data that the correlation is based on where the indicated pressure is the difference between inlet and outlet pressures. Figure 2.5.1-4 also shows $95 \%$ confidence bounds on the correlation, the correlation coefficient for the fit $\left(\mathrm{r}^{2}=0.47\right)$, and the standard estimate of the error $\left(s_{e}=18.88\right)$. The standard estimate of the error is defined by

$$
s_{e}=\sqrt{\frac{\sum_{i=1}^{n}\left(N u_{i}^{E x p}-N u_{i}^{C a l c}\right)^{2}}{n-2}}
$$




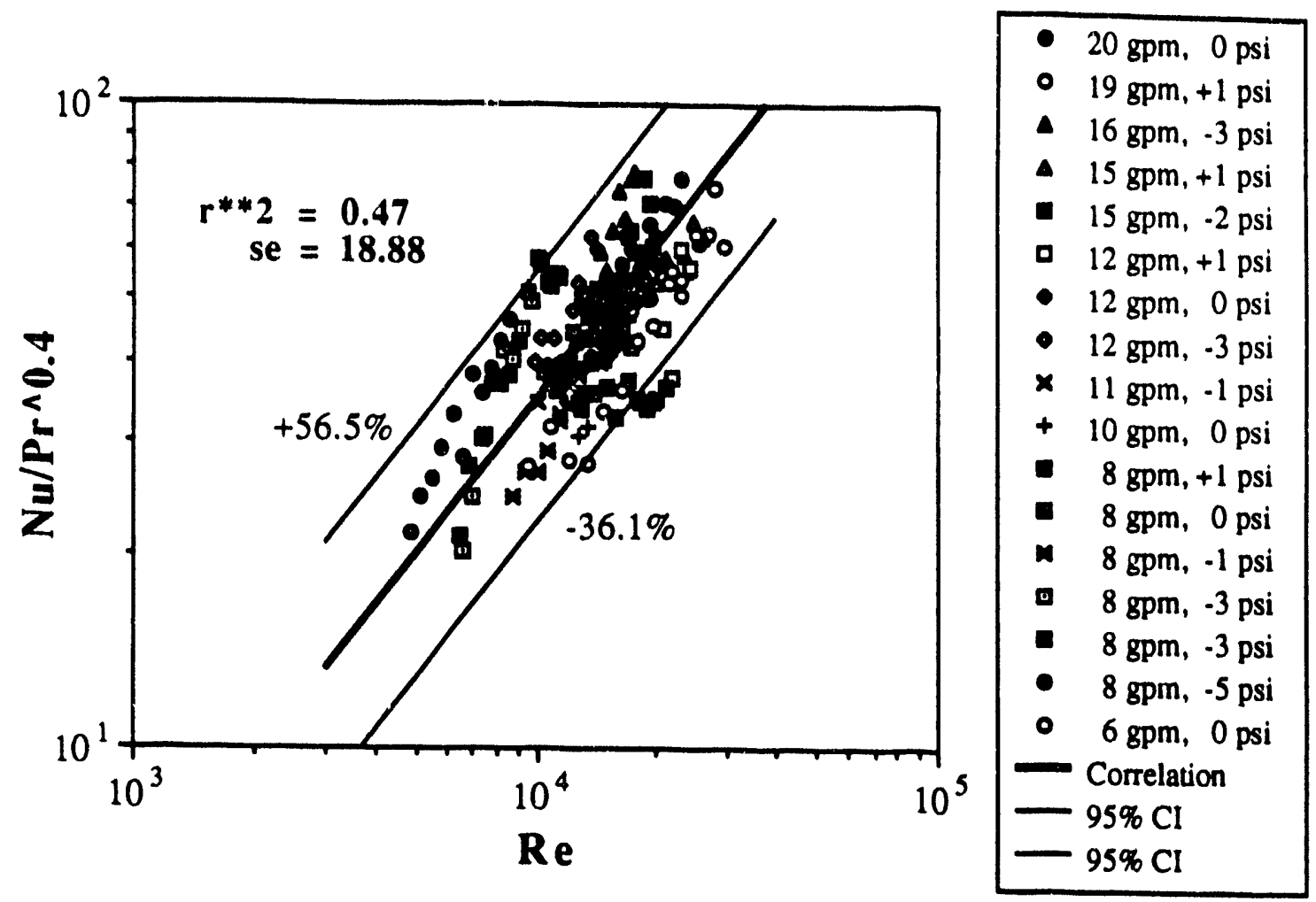

Figure 2.5.1.4 Correlation of Rig B dispersed regime heat transfer data $(\alpha<0.75)$.

Figure 2.5.1-5 shows the dispersed regime data with experimental error bars added along with correlation (2.5.1-4a). The error bars on the data were calculated assuming a $1.2 \%$ error in the power measurement and a $2^{\circ} \mathrm{C}$ error in thermocouple readings. Analysis of the SRS data indicated that the viscosity correction used in the Sieder-Tate model did not improve the correlation. 

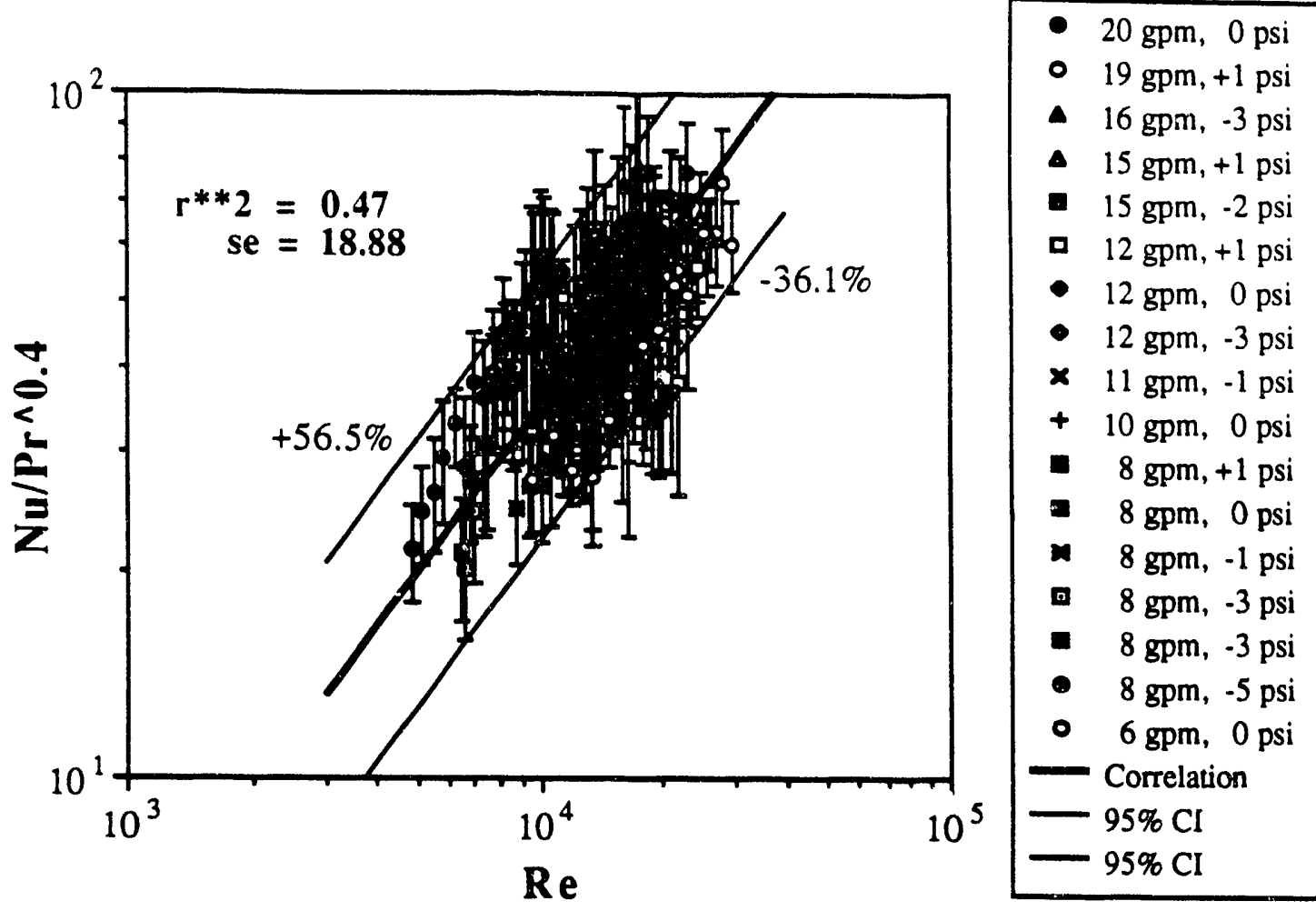

Figure 2.5.1-5 Rig B data with experimental error bars shown.

In the annular flow regime, $\alpha>0.75$, the data were correlated with the equation

$$
\mathrm{h}_{\mathrm{A}}=6.77 \times 10^{-5}\left(\frac{\mathrm{k}}{\mathrm{D}_{\mathrm{H}}}\right) \operatorname{Re}^{1.5} \mathrm{Pr}^{0.5}
$$

with

$$
\operatorname{Re}=\frac{G_{f} D_{H}}{\mu_{f}}
$$

Equation (2.5.1-5a), which is based on an analogous expression for annular regime mass transfer (Treybal, 1980), uses the superficial liquid velocity to evaluate the Reynolds number since this is typically used for annular flow correlations. The correlation to the experimental data with corresponding 95\% confidence bounds is shown in Fig. 2.5.1-6. The SRS data indicated that the viscosity correction used in the Sieder-Tate model did not improve correlation (2.5.1-5a). 


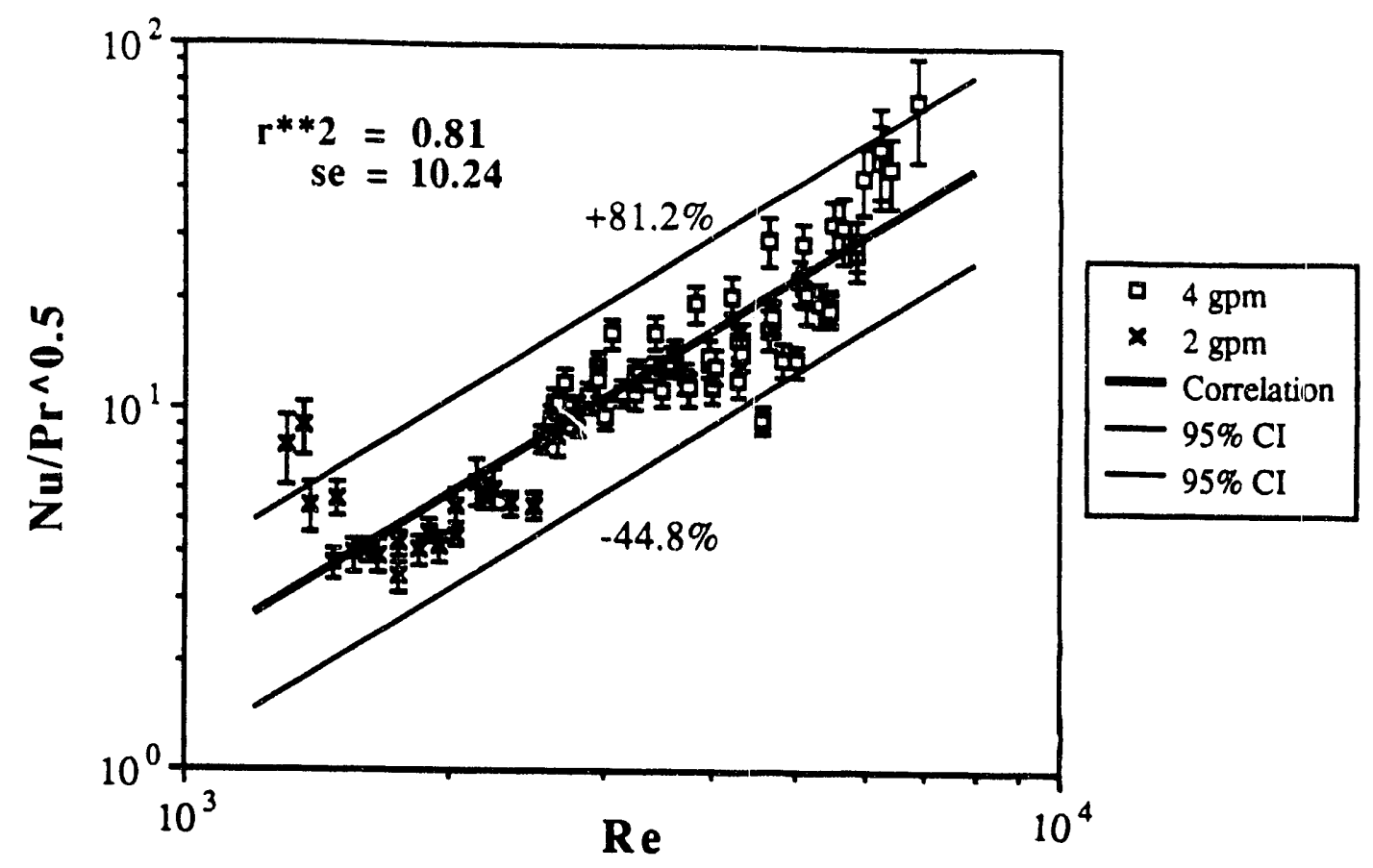

Figure 2.5.1-6 Correlation of Rig B annular regime heat transfer data $(\alpha>0.75)$.

Two methods for transitioning between the turbulent and annular correlations are considered. The first method uses the turbulent correlation at low void fractions, the annular correlation at high void fractions, and a weighted average of the correlations within a user controlled transition regime. A parameter $\Delta \alpha$ is used to define the range of void fraction in the transition regime. This scheme can be represented as

$$
\mathrm{h}_{\mathrm{FC}}=\omega \mathrm{h}_{\mathrm{A}}+(1-\omega) \mathrm{h}_{\mathrm{T}}
$$

where the linear interpolation parameter is defined to be

$$
\omega=\frac{\alpha-\alpha_{\min }}{\alpha_{\max }-\alpha_{\min }}
$$

with the additional constraints that $\omega=\max (\omega, 0)$ and $\omega=\min (\omega, 1)$. A schematic representation of the interpolation scheme is shown below. The width of the transition region is controlled through the input parameter $\Delta \alpha$. 


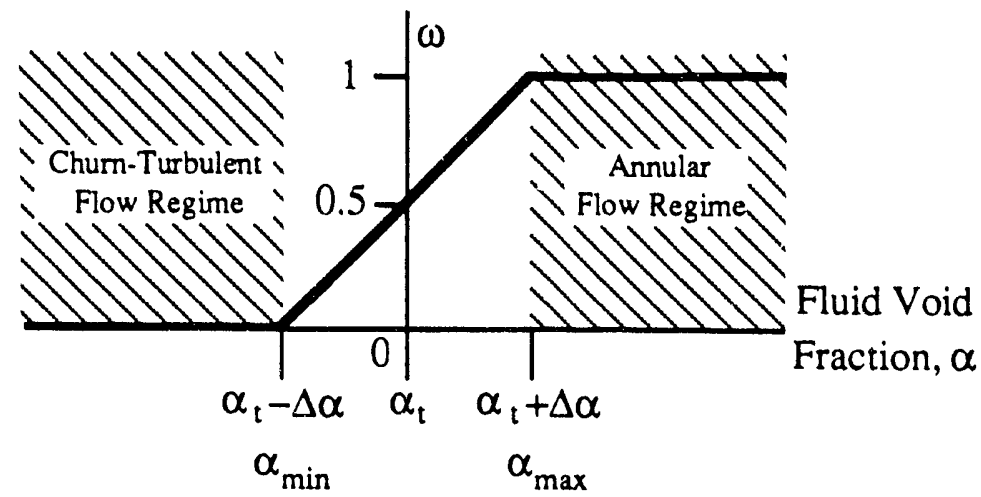

The minimum and maximum void fractions are computed from the void fraction at the point of transition to annular flow $\left(\alpha_{t}=0.75\right)$ and the input parameter $\Delta \alpha$ as

$$
\begin{aligned}
& \alpha_{\min }=\alpha_{t}-\Delta \alpha \\
& \alpha_{\max }=\alpha_{t}+\Delta \alpha
\end{aligned}
$$

To keep the void fractions within their physical limits we apply the constraints

$$
\begin{aligned}
& \alpha_{\min }=\max \left(\alpha_{\min }, 0\right) \\
& \alpha_{\max }=\min \left(\alpha_{\max }, 1\right)
\end{aligned}
$$

A second method of smoothly combining the heat transfer correlations is to set the convective heat transfer coefficient equal to the minimum of the values calculated equations $(2.5 .1-4)$ and $(2.5 .1-5)$

$$
\mathrm{h}_{\mathrm{FC}}=\operatorname{minimum}\left(\mathrm{h}_{\mathrm{T}}, \mathrm{h}_{\mathrm{A}}\right)
$$

The first method is preferable since it associates the heat transfer correlations with their respective flow regimes. Using the second scheme is clearly conservative and avoids introducing the arbitrary parameter $\Delta \alpha$. However, this method does not guarantee that the heat transfer correlation being used corresponds to the existing flow regime. In FLOWTRAN-TF, we choose the first method given by equation (6a) and set $\Delta \alpha$ to 0.01 .

Also, as in subroutine HCONV, the laminar correlation given by equation (2.5.1-2) is used as a lower bound on the heat transfer coefficient

$$
\mathrm{h}_{\mathrm{FC}}=\operatorname{maximum}\left(\mathrm{h}_{\mathrm{FC}}, \mathrm{h}_{\mathrm{L}}\right)
$$

Since we assume that the surface is wetted, all of the heat is transferred between the surface and the liquid phase.

We account for the presence of ribs on the metal surfaces by using a rib efficiency factor to augment the heat transfer coefficient on the surface where the rib is attached. The gaps between ribs on SRS assemblies and the opposite metal surfaces are quite small (approximately 30 mil diametrical gap). Therefore, water flow in the rib gap is restricted and we assume that the metal surfaces within the gap do not contribute to heat removal. That is, we assume that rib tip and the small surface area immediately across from a rib are adiabatic and heat transfer from these surface areas is not included in our calculation. 

Using the solution for a rcctangular fin insulated on its tip (Holman, 1976), we define the
function $\phi$ as

$$
\phi=r_{L} \sqrt{\frac{h_{F C}}{k_{w} r_{T}}}
$$

where

$$
\begin{aligned}
\mathrm{k}_{\mathrm{w}} & \equiv \text { thermal conductivity of wall, } \mathrm{W} / \mathrm{m}{ }^{\circ} \mathrm{C} \\
\mathrm{r}_{\mathrm{L}} & \equiv \text { rib length, } \mathrm{m} \\
\mathrm{r}_{\mathrm{T}} & \equiv \text { rib half thickness, } \mathrm{m}
\end{aligned}
$$

The fin efficiency is then given by

$$
\psi=\frac{\tanh (\phi)}{\phi}
$$

and an adjusted heat transfer coefficient is calculated from

$$
\tilde{h}_{\mathrm{FC}}=\mathrm{h}_{\mathrm{FC}}\left[1.0+\mathrm{P}_{\mathrm{rib}}\left(\psi \mathrm{r}_{\mathrm{L}}-\mathrm{r}_{\mathrm{T}}\right)\right]
$$

where

$$
\begin{aligned}
& \mathrm{P}_{\mathrm{rib}} \equiv \text { rib perimeter factor }=\mathrm{n}_{\mathrm{rib}} / \mathrm{P}_{\mathrm{S}} \\
& \mathrm{n}_{\mathrm{rib}} \equiv \begin{array}{l}
\text { number of rib side surfaces (or the number of half ribs) on solid cell } \\
\text { surface corresponding to the fluid cell }
\end{array} \\
& \mathrm{P}_{\mathrm{S}} \equiv \text { wetted perimeter of solid cell without ribs, } m
\end{aligned}
$$

Equation (2.5.1-9) accounts for rib efficiency and also decreases the effective heat transfer coefficient on surfaces opposite to the rib when $\psi$ equals zero. We account for the decreased heat transfer at the opposite surface by reducing the surface area available for heat transfer. That is, the effective heat transfer area on a surface opposite to a rib is determined by subtracting off the rib area from the true surface area.

Vapor Condensation (CDENS): When two-phase mixtures are present, vapor condensation is accounted for through the interfacial heat and mass transfer calculations. However, when the fluid phase is entirely vapor $(\alpha>0.9999)$ and the wall temperature is below saturation, the vapor will condense on the wall. To calculate wall condensation heat transfer we adopt a slight modification to the method used by RELAP5/MOD2.

A local heat transfer coefficient for condensation on a vertical plane surface with a frationary (Collier, 1972; Nusselt 1916) 


$$
h_{\text {con } 1}=1.1025 \mathrm{k}_{\mathrm{f}} \operatorname{Re}_{\mathrm{f}}^{-1 / 3}\left[\frac{\rho_{\mathrm{f}}\left(\rho_{\mathrm{f}}-\rho_{\mathrm{g}}\right) \mathrm{g}}{\mu_{\mathrm{f}}^{2}}\right]^{1 / 3}
$$

A local heat transfer correlation for condensation on a vertical tube with significant interfacial shear between the condensate and vapor is (Collier, 1972; Carpenter and Colburn, 1951)

$$
h_{c o n}=0.043 \frac{k_{f}}{\mu_{f}} \sqrt{\rho_{f} P_{f} \tau_{i}}
$$

where the term $\tau_{\mathrm{i}}$ in equation $(2.5 .1-10)$ is defined by

$$
\tau_{\mathrm{i}}=0.0792 \operatorname{Re}_{\mathrm{g}}^{-1 / 4}\left(\frac{\rho_{\mathrm{g}} \mathrm{V}_{\mathrm{g}}^{2}}{2}\right)
$$

In the above equations

$$
\begin{aligned}
\mathrm{k}_{\mathrm{f}} & \equiv \text { liquid thermal conductivity, } \mathrm{W} / \mathrm{m}^{\circ} \mathrm{C} \\
\rho_{\mathrm{f}} & \equiv \text { liquid density, } \mathrm{kg} / \mathrm{m}^{3} \\
\rho_{\mathrm{g}} & \equiv \text { gas density, } \mathrm{kg} / \mathrm{m}^{3} \\
\mu_{\mathrm{f}} & \equiv \text { liquid viscosity, } \mathrm{kg} / \mathrm{m} \mathrm{s} \\
\mathrm{g} & \equiv \text { gravitational acceleration, } 9.8066 \mathrm{~m} / \mathrm{s}^{2} \\
\mathrm{~V}_{\mathrm{g}} & \equiv \text { superficial gas velocity, } \mathrm{m} / \mathrm{s} \\
\operatorname{Pr}_{\mathrm{f}} & \equiv \text { Prandtl number for liquid phase }=\mathrm{c}_{\mathrm{pf}} \mu_{\mathrm{f}} / \mathrm{k}_{\mathrm{f}} \\
\operatorname{Re}_{\mathrm{f}} & \equiv \text { superficial liquid Reynolds number }=\mathrm{G}_{\mathrm{f}} \mathrm{D}_{\mathrm{H}} / \mu_{\mathrm{f}} \\
\operatorname{Re}_{\mathrm{g}} & \equiv \text { superficial gas Reynolds number }=\mathrm{G}_{\mathrm{g}} D_{\mathrm{H}} / \mu_{\mathrm{g}}
\end{aligned}
$$

In writing equation (2.5.1-11a), we have replaced the coefficient of 0.065 given in the references, which applies for an average heat transfer coefficient over the entire surface, with the value 0.043 to convert it into a local heat transfer coefficient. The logic for this conversion is that the local heat transfer coefficient is proportional to one over the cube root of the axial distance. Therefore, averaging the coefficient over the surface introduces a factor of $3 / 2$ into the pre-multiplying constant. The maximum of these two estimates of the heat transfer coefficient is used to calculate the condensation wall heat flux in order to cover the range of vapor flow situations:

$$
\begin{gathered}
h_{\text {con }}=\max \left(h_{\text {con } 1}, h_{\text {con } 2}\right) \\
q_{c o n}^{\prime \prime}=h_{\text {con }}\left(T_{w}-T_{\text {sat }}\right)
\end{gathered}
$$


We assume that the total heat flux to the gas phase is equal to the sum of the condensation heat flux and the forced convection heat flux calculated previously.

$$
q_{g}^{\prime \prime}=q_{\text {con }}^{\prime \prime}+q_{F C}^{\prime \prime}
$$

The mass flux of vapor condensing at the wall is calculated as

$$
\hat{\Gamma}_{w}=\frac{q_{g}^{\prime \prime}-q_{F C}^{\prime \prime}}{h_{f g}+c_{p g}\left(T_{g}-T_{\text {sat }}\right)} \equiv \frac{q_{c o n}^{\prime \prime}}{h_{f g}}
$$

where

$$
\begin{aligned}
\hat{\Gamma}_{\mathrm{w}} & \equiv \text { rate of vapor condensation per unit surface area, } \mathrm{kg} / \mathrm{s}-\mathrm{m}^{2} \\
\mathrm{q}_{\mathrm{FC}}^{\prime \prime} & \equiv \text { single phase heat flux to gas phase given by equation }(2.5 .1-3), \mathrm{W} / \mathrm{m}^{2} \\
\mathrm{~h}_{\mathrm{fg}} & \equiv \text { latent heat of vaporization, } \mathrm{J} / \mathrm{kg} \\
\mathrm{c}_{\mathrm{pg}} & \equiv \text { gas specific heat, } \mathrm{J} / \mathrm{kg}{ }^{\circ} \mathrm{C} \\
\mathrm{T}_{\mathrm{g}} & \equiv \text { gas temperature, }{ }^{\circ} \mathrm{C} \\
\mathrm{T}_{\mathrm{sat}} & \equiv \text { saturation temperature, }{ }^{\circ} \mathrm{C}
\end{aligned}
$$

Note that, since the wall temperature must be colder than the gas temperature for condensation to occur, $\mathrm{q}_{\mathrm{g}}^{\prime \prime}, \mathrm{q}_{\mathrm{FC}}^{\prime \prime}$, and the rate of vapor condensation will be negative numbers. The correction term to the latent heat in the denominator of equation $(2.5 .1-14)$ accounts for the energy required to cool the condensing vapor from the bulk gas temperature $T_{g}$ to saturation temperature. This is a small correction term (Rohsenow et al., 1973) and is neglected in our application. Rohsenow has also derived a correction to the latent heat during condensation to account for liquid subcooling (Whalley, 1987). However, we assume that the gas is condensed at the saturation temperature and neglect this small correction as well.

Boiling Heat Transfer - Mikic/Rohsenow Correlation (MIKIC): When the wall temperature exceeds the local saturation temperature but is less than the temperature for critical heat flux and liquid is present, the fluid is in the boiling heat transfer regime. We have implemented two procedures to estimate heat transfer to the liquid phase under boiling conditions in FLOWTRAN-TF. When the input parameter IBOIL equals one, the code branches to subroutine MIKIC while any other value of IBOIL causes the code to use subroutine CHEN. We have used the SRS correlation for two-phase non-boiling heat transfer to compute the forced convection heat transfer coefficient in the Mikic-Rohsenow boiling correlation. Therefore, we recommend using this correlation for air-water boiling calculations until a better correlation can be developed from experimental data. Nevertheless; existing thermal-hydraulics codes such as RELAP and TRAC use the Chen correlation to compute boiling heat transfer. Therefore, we have included this option in FLOWTRAN-TF as well so that direct comparisons to results computed with other codes can be more easily made. In this part of the code development report we describe the MIKIC boiling curve while in the following section the CHEN boiling curve is explained. 

(1964) is used to construct a smooth curve for forced convection boiling. The boiling
curve is approximated by the relationship

$$
q_{f}^{\prime \prime}=\sqrt{\left(q_{F C}^{\prime \prime}\right)^{2}+\left(q_{B}^{\prime \prime}-q_{B i}^{\prime \prime}\right)^{2}}
$$

where

$$
\begin{aligned}
\mathrm{q}_{\mathrm{FC}}^{\prime \prime} & =\text { forced convection heat flux } \\
\mathrm{q}_{\mathrm{B}}^{\prime \prime} & =\text { fully developed boiling heat flux } \\
\mathrm{q}_{B i}^{\prime \prime} & =\text { fully developed boiling heat flux at point of incipient boiling (ONB) }
\end{aligned}
$$

For $\mathrm{q}_{\mathrm{FC}}^{\prime \prime}\left(\mathrm{h}_{\mathrm{FC}}\right)$ we use the SRS correlations shown in equation (2.5.1-4a) and equation (2.5.1-5a) above. The Mikic and Rohsenow (1969) correlation given by

$$
q_{B}^{\prime \prime}=B\left[\frac{g^{1 / 2} h_{f g}^{m-15 / 8} k_{f}^{1 / 2} \rho_{f}^{17 / 8} c_{p f}^{19 / 8} \rho_{g}^{m-15 / 8}}{\left(\rho_{f}-\rho_{g}\right)^{5 / 8} \sigma^{m-7 / 8} T_{s a t}^{m-15 / 8}}\right]\left(T_{w}-T_{s a t}\right)^{m+1}={ }_{h}^{o}\left(T_{w}-T_{s a t}\right)^{m+1}
$$

is used to calculate the fully developed boiling heat flux where

$$
\begin{aligned}
\mathrm{k}_{\mathrm{f}} & \equiv \text { liquid thermal conductivity, } \mathrm{W} / \mathrm{m}^{\circ} \mathrm{C} \\
\mathrm{h}_{\mathrm{fg}} & \equiv \text { latent heat of vaporization, } \mathrm{J} / \mathrm{kg} \\
\rho_{\mathrm{f}} & \equiv \text { liquid density, } \mathrm{kg} / \mathrm{m}^{3} \\
\rho_{\mathrm{g}} & \equiv \text { gas density, } \mathrm{kg} / \mathrm{m}^{3} \\
\mathrm{c}_{\mathrm{pf}} & \equiv \text { liquid specific heat, } \mathrm{J} / \mathrm{kg}^{\circ} \mathrm{C} \\
\mathrm{g} & \equiv \text { gravitational acceleration, } 9.8066 \mathrm{~m} / \mathrm{s}^{2} \\
\sigma & \equiv \text { surface tension, } \mathrm{N} / \mathrm{m}
\end{aligned}
$$

All of the fluid properties used in equation (2.5.1-16a) are evaluated at the local saturation functions of the cavity size distribution for surface in equation $(2.5 .1-16 \mathrm{a})$ are solely distribution is generally not known, these factors must be determined from cavity size Using coefficient values $\mathrm{m}=2$ and $\mathrm{B}=1.89 \times 10^{-14}$ (SI units), the Mikic-Rohsenow equation has been found to agree well with fully developed boiling data for water (Rohsenow et al., 1973). In the absence of better information, we have used these coefficient values in FLOWTRAN-TF. Substituting these values for B and $m$ into equation (2.5.1-16a) gives 


$$
q_{B}^{\prime \prime}=1.89 \times 10^{-14}\left[\frac{g^{1 / 2} h_{f g}^{1 / 8} k_{f}^{1 / 2} \rho_{f}^{17 / 8} c_{p f}^{19 / 8} \rho_{g}^{1 / 8}}{\left(\rho_{f}-\rho_{g}\right)^{5 / 8} \sigma^{9 / 8} T_{s a t}^{1 / 8}}\right]\left(T_{w}-T_{s a t}\right)^{3}=h_{B}\left(T_{w}-T_{\text {sat }}\right)^{3}
$$

The heat flux at the point of incipient boiling is calculated from equation (2.5.1-16b) using the temperature difference at the point of incipient boiling $\left(T_{w}-T_{s a t}\right)_{i}$ :

$$
\mathrm{q}_{\mathrm{Bi}}^{\prime \prime}=\mathrm{o}_{\mathrm{B}}\left(\Delta \mathrm{T}_{\mathrm{ONB}}\right)^{3}
$$

The temperature difference at ONB (onset of nucleate boiling) is estimated using the procedure developed by Bjorge and Rohsenow (1982) modified to account for the presence of noncondensable gases. A detailed description of this procedure is provided by Aleman et al. (1989). Slightly different notation is used here; $\Sigma$ in this report is $2 \Gamma$ in Aleman et al. (1989). Also, the first sign under the radical of equation (2.4-24) in Aleman et al. should be positive, not negative. In summary, $\Delta \mathrm{T}_{\mathrm{ONB}}$ is calculated from the expression

$$
\Delta T_{\mathrm{ONB}}= \begin{cases}\frac{\Delta \mathrm{T}_{\mathrm{sub}} N \mathrm{u}_{\mathrm{cav}}+\frac{1}{2 N u_{\mathrm{cav}} \Sigma}-\gamma}{1-N u_{\mathrm{cav}}}, & \mathrm{h}_{\mathrm{FC}}<\mathrm{h}_{\mathrm{tan}} \\ \frac{1}{N u_{\mathrm{tan}} \Sigma}-\gamma, & \mathrm{h}_{\mathrm{FC}} \geq h_{\mathrm{tan}}\end{cases}
$$

In equation $(2.5 .1-18), \mathrm{Nu}_{c a v}$ is the nucleation site cavity Nusselt number defined to be

$$
\mathrm{Nu}_{\mathrm{cav}}=\frac{\mathrm{h}_{\mathrm{FC}} \mathrm{r}_{\max }}{\mathrm{k}_{\mathrm{w}}}
$$

and $\mathrm{Nu}_{\tan }$ is the Nusselt number at the point of bubble tangency defined by the expression

$$
\mathrm{Nu}_{\mathrm{tan}}=\frac{\mathrm{h}_{\mathrm{tan}} \mathrm{r}_{\max }}{\mathrm{k}_{\mathrm{w}}}=\frac{1}{1+\sqrt{\tau}}
$$

where the parameter $\tau$ is given by

$$
\tau=\max \left\{0,1+2\left(\Delta \mathrm{T}_{\text {sub }}-\gamma\right) \Sigma\right\}
$$

Equation (2.5.1-20) is used to calculate the heat transfer coefficient at the point of bubble tangency $\left(h_{\text {tan }}\right)$. In defining the Nusselt numbers, we have used the parameters:

$$
\begin{aligned}
\mathrm{k}_{\mathrm{w}} & \equiv \text { thermal conductivity of fluid at wall temperature, } \mathrm{W} / \mathrm{m}{ }^{\circ} \mathrm{C} \\
\mathrm{r}_{\max } & \equiv \operatorname{maximum}_{\left(\mathrm{r}_{\max } \text { is taken to be } 1 \mu \mathrm{m}\right)}
\end{aligned}
$$




$$
\Delta \mathrm{T}_{\text {sub }} \equiv \mathrm{T}_{\text {sat }}-\mathrm{T}_{\mathrm{f}}
$$

The other terms in equations $(2.5 .1-18)$ and $(2.5 .1-21)$ are defined by the relationships

$$
\Sigma=\left(\frac{k_{w}}{4 T_{\text {sat }} h_{\mathrm{FC}}}\right)\left(\frac{h_{\mathrm{fg}}}{\sigma v_{\mathrm{fg}}}\right)_{\text {sat }}
$$

and

$$
\gamma=p_{g} T_{s a t}\left(\frac{v_{f g}}{h_{f g}}\right)_{s a t}
$$

where

$$
\begin{aligned}
\mathrm{v}_{\mathrm{fg}} & \equiv \text { difference in specific volumes of saturated liquid and saturated gas, } \mathrm{m}^{3} / \mathrm{kg} \\
\mathrm{p}_{\mathrm{g}} & \equiv \text { partial pressure of non-condensible gas, } \mathrm{Pa}
\end{aligned}
$$

The $\gamma$ term in the above equations accounts for the presence of non-condensible gas. The partial pressure of the non-condensible component in the gas phase is calculated from an application of Henry's law

$$
\mathrm{p}_{\mathrm{g}}=\mathrm{x}_{\mathrm{c}} \mathrm{H}_{\mathrm{sat}}
$$

where $\mathrm{H}_{\text {sat }}$ is the Henry's law constant at the local saturation temperature. The liquid concentration of the dissolved gas $\left(\mathbf{x}_{\mathbf{c}}\right)$ is calculated from

$$
x_{c}=y_{c}\left(\frac{p_{s a t}-P}{p_{s a t}-H_{T}}\right)
$$

where

$$
\begin{aligned}
\mathrm{y}_{\mathrm{c}} & \equiv \text { gas phase molar concentration of non-condensible component } \\
\mathrm{p}_{\mathrm{sat}} & \equiv \text { vapor pressure of pure liquid at } \mathrm{T}_{\mathrm{f}}, \mathrm{Pa} \\
\mathrm{P} & \equiv \text { total local pressure, } \mathrm{Pa} \\
\mathrm{H}_{\mathrm{T}} & \equiv \text { Henry's law constant at local temperature }
\end{aligned}
$$

To calculate the vapor generation rate, we assume that the nucleate boiling heat flux, given by $\left(\mathrm{q}_{\mathrm{f}}^{\prime \prime}-\mathrm{q}_{\mathrm{FC}}^{\prime \prime}\right)$, is available for vapor generation. The amount of vapor generated at the wall is then calculated as

$$
\hat{\Gamma}_{w}=\frac{\left(q_{f}^{\prime \prime}-q_{F C}^{\prime \prime}\right)}{h_{f g}}
$$

Boiling Heat Transfer - Chen Correlation (CHEN): In subroutine CHEN, we use the correlation developed by Chen (Chen, 1963; Collier,1972) for both subcooled and 
saturated nucleate boiling to calculate a heat flux to the liquid phase. The Chen correlation is used by all of the standard codes mentioned previously in a similar fashion. This correlation assumes a superposition of a forced-convection heat transfer correlation and a pool boiling correlation so that the total heat transfer coefficient and corresponding heat flux are given by

$$
h_{C H E N}=h_{F C}+h_{N B}
$$

and

$$
\mathrm{q}_{f}^{\prime \prime}=\mathrm{h}_{\mathrm{FC}}\left(\mathrm{T}_{\mathrm{w}}-\mathrm{T}_{\mathrm{f}}\right)+\mathrm{h}_{\mathrm{NB}}\left(\mathrm{T}_{\mathrm{w}}-\mathrm{T}_{\mathrm{sat}}\right)
$$

The forced-convection heat transfer coefficient is calculated from a modified Dittus-Boelter correlation

$$
h_{F C}=0.023 F\left(\frac{k_{f}}{D_{H}}\right) \operatorname{Re}_{f}^{0.8} \operatorname{Pr}^{0.4}
$$

where $\operatorname{Re}_{\mathrm{f}}$ is the superficial liquid phase Reynolds number evaluated using saturation properties. The nucleate boiling heat transfer coefficient is obtained using the correlation

$$
h_{N B}=\frac{0.00122 S}{\operatorname{Pr}^{0.05}}\left[\frac{k_{f} \rho_{f}}{h_{f g} \mu_{f} \rho_{g}}\right]^{0.24}\left[\frac{k_{f} c_{p f} \sqrt{g_{c} \rho_{f}}}{\sigma}\right]^{0.5}\left(T_{w}-T_{s a t}\right)^{0.24}\left(P_{w}-P\right)^{0.75}
$$

which can be rearranged into the equivalent form

$$
h_{N B}=0.00122 \mathrm{~S}\left(\frac{k_{f}}{D_{H}}\right) W e^{0.5} \operatorname{Re}_{f}^{0.5} \operatorname{Pr}^{0.21}\left[\frac{\rho_{f} c_{p f}\left(T_{w}-T_{s a t}\right)}{\rho_{g} h_{f g}}\right]^{0.24}\left(\frac{P_{w}-P}{\rho_{f} V_{f}^{2}}\right)^{0.75}
$$

In the above equations

$$
\begin{aligned}
\mathrm{F} & \equiv \text { Reynolds number factor } \\
\mathrm{S} & \equiv \text { suppression factor } \\
\mathrm{k}_{\mathrm{f}} & \equiv \text { liquid thermal conductivity, } \mathrm{W} / \mathrm{m}^{\circ} \mathrm{C} \\
\mathrm{h}_{\mathrm{fg}} & \equiv \text { latent heat of vaporization, } \mathrm{J} / \mathrm{kg} \\
\rho_{\mathrm{f}} & \equiv \text { liquid density, } \mathrm{kg} / \mathrm{m}^{3} \\
\rho_{\mathrm{g}} & \equiv \text { gas density, } \mathrm{kg} / \mathrm{m}^{3} \\
\mu_{\mathrm{f}} & \equiv \text { liquid viscosity, } \mathrm{kg} / \mathrm{m} \mathrm{s} \\
\mathrm{c}_{\mathrm{pf}} & \equiv \text { liquid specific heat, } \mathrm{J} / \mathrm{kg}{ }^{\circ} \mathrm{C}
\end{aligned}
$$




$$
\begin{aligned}
\mathrm{g}_{\mathrm{c}} & \equiv \text { gravitational conversion constant } \\
\sigma & \equiv \text { surface tension, } \mathrm{N} / \mathrm{m} \\
\mathrm{P}_{\mathrm{w}} & \equiv \text { saturation pressure corresponding to } \mathrm{T}_{\mathrm{w}}, \mathrm{Pa} \\
\mathrm{P} & \equiv \text { system pressure, } \mathrm{Pa} \\
\mathrm{We} & \equiv \text { Weber number, }\left(\mathrm{V}_{\mathrm{f}}^{2} \rho_{\mathrm{f}} \mathrm{D}_{\mathrm{H}}\right) / \sigma \\
\mathrm{V}_{\mathrm{f}} & \equiv \text { superficial liquid velocity, } \mathrm{m} / \mathrm{s}
\end{aligned}
$$

All liquid properties are evaluated at the saturation temperature. Equation $(2.5 .1-29)$ is identical to the usual Dittus-Boelter (1930) correlation except that a Reynolds number factor $F$ is included to account for two-phase flow effects. The parameters $F$ and $S$ are in turn obtained using the relationships shown below due to Collier (1981). We first calculate an inverse Martinelli factor $X_{t t}^{-1}$ (Collier, 1972; Lockhart and Martinelli,
1949 ) as

$$
X_{t t}^{-1}=\left(\frac{x}{1-x}\right)^{0.9}\left(\frac{\rho_{f}}{\rho_{g}}\right)^{0.5}\left(\frac{\mu_{g}}{\mu_{f}}\right)^{0.1}
$$

where

$$
\begin{aligned}
x & \equiv \text { fluid quality } \\
\mu_{\mathrm{g}} & \equiv \text { gas viscosity, } \mathrm{kg} / \mathrm{m} \mathrm{s}
\end{aligned}
$$
The Reynolds number factor used in equation $(2.5 .1-29)$ is given by the relationship
(Collier, 1981)

$$
F= \begin{cases}1.0 & \text { for } X_{\mathfrak{t t}}^{-1} \leq 0.1 \\ 2.35\left(X_{\mathfrak{t t}}^{-1}+0.213\right)^{0.736} & \text { for } X_{\mathfrak{t t}}^{-1}>0.1\end{cases}
$$

We next calculate an effective two-phase Reynolds number as

$$
\operatorname{Re}_{\mathrm{TP}}=1.0 \times 10^{-4} \operatorname{Re}_{\mathrm{f}} \mathrm{F}^{1.25}
$$

The two-phase Reynolds number is used to calculate the suppression factor $\mathrm{S}$ from

$$
S= \begin{cases}1.0 /\left[1.0+0.12\left(\operatorname{Re}_{\mathrm{TP}}\right)^{1.14}\right] & , \quad \operatorname{Re}_{\mathrm{TP}}<32.5 \\ 1.0 /\left[1.0+0.42\left(\operatorname{Re}_{\mathrm{TP}}\right)^{0.78}\right] & , 32.5 \leq \operatorname{Re}_{\mathrm{TP}}<50.9 \\ 0.1 & , \operatorname{Re}_{\mathrm{TP}} \geq 50.9\end{cases}
$$


Following COBRA/TRAC (Thurgood, 1983), we have used an upper limit of 50.9 for $\mathrm{Re}_{\mathrm{TP}}$ rather than the original value of 70.0 used in most other codes. As noted in the COBRA/TRAC manual, the modified Reynolds number limit is used to make the correlation continuous.

The Chen correlation was developed to describe saturated boiling but may also be employed for subcooled nucleate boiling with minor modification (Thurgood, 1983). In the subcooled regime, the suppression factor is computed from equation $(2.5 .1-34)$ using the single-phase liquid Reynolds number to evaluate $\operatorname{ReTP}_{\mathrm{TP}}$ from equation (2.5.1-33) with the Reynolds number factor $F$ set equal to unity. Fluid properties in these correlations and in equation (2.5.1-29) are evaluated at the subcooled liquid temperature. Equations $(2.5 .1-29)$ and $(2.5 .1-30)$ are then used as before.

To calculate the vapor generation rate, we assume that the nucleate boiling heat flux, given by the term

$$
\mathrm{q}_{\mathrm{NB}}^{\prime \prime}=\mathrm{h}_{\mathrm{NB}}\left(\mathrm{T}_{\mathrm{w}}-\mathrm{T}_{\text {sat }}\right)
$$

is available for vapor generation. Rouhani and Axelsson (1970) suggest that a fraction of this energy is used to heat liquid entering the saturated thermal boundary layer. The remaining fraction available for vapor generation is given by

$$
\varepsilon_{\Gamma}=\frac{h_{f g}}{h_{f g}+\left(\rho_{f} / \rho_{g}\right)\left(h_{f}-h_{1}\right)}
$$

where $h_{f}$ is the saturated liquid enthalpy and $h_{l}$ is the liquid enthalpy at bulk fluid conditions. The amount of vapor generated at the wall is then calculated as

$$
\hat{\Gamma}_{w}=\frac{\varepsilon_{\Gamma} h_{N B}\left(T_{w}-T_{s a t}\right)}{h_{f g}}=\frac{\varepsilon_{\Gamma} q_{N B}^{\prime \prime}}{h_{f g}}
$$

Film Boiling (FILMB): When the wall temperature exceeds the minimum stable film boiling temperature, the fluid enters the film boiling regime. In this regime, we follow the TRAC heat transfer formulation and calculate separate heat fluxes to the liquid and to the gas phases. When film boiling takes place with relatively low void fractions, we assume that the liquid is in an inverted annular flow regime and that heat transfer to the liquid phase occurs by convection through a thin vapor film and by radiation. For convective heat transfer to the liquid in inverted annular flow, we use the Bromley-Pomeranz (Pomeranz, 1964) correlation given by

$$
h_{B P}=0.620\left(\frac{D_{H}}{\lambda_{c}}\right)^{0.172}\left[\frac{k_{g}^{3} \rho_{g}\left(\rho_{f}-\rho_{g}\right) \hat{h}_{f g} g}{D_{H} \mu_{g}\left(T_{w}-T_{s a t}\right)}\right]^{0.25}
$$

where

$$
\lambda_{\mathrm{c}}=2, \pi \sqrt{\frac{\sigma}{\mathrm{g}\left(\rho_{\mathrm{f}}-\rho_{\mathrm{g}}\right)}}
$$


and a modified heat of vaporization is defined as

$$
\hat{h}_{\mathrm{fg}}=\mathrm{h}_{\mathrm{fg}}+0.5 \mathrm{c}_{\mathrm{pg}}\left(\mathrm{T}_{\mathrm{w}}-\mathrm{T}_{\mathrm{sat}}\right)
$$

At higher void fractions, the liquid will be dispersed as droplets. The model assumes that some liquid will still contact the surface through drop impingement resulting in heat transfer directly to the liquid phase. In this flow regime, subroutine FILMB uses the ForslundRohsenow (1968) correlation for liquid heat transfer. This correlation is

$$
h_{F R}=0.2552(1-\alpha)^{2 / 3}\left[\frac{k_{g}^{3} \rho_{g} \rho_{f} h_{f g}^{*} g}{D_{D} \mu_{g}\left(T_{w}-T_{\text {sat }}\right)}\right]^{0.25}
$$

where

$$
h_{f g}^{*}=h_{f g}\left[1+\frac{0.35 c_{p g}\left(T_{w}-T_{\text {sat }}\right)}{h_{f g}}\right]^{-3}
$$

In equation (2.5.1-41) $D_{D}$ is the droplet diameter which is estimated from

$$
D_{D}=\frac{4 \sigma}{\rho_{g}\left(u_{g}-u_{f}\right)^{2}}
$$

with the limits that $1.0 \times 10^{-4}<D_{D}<3.0 \times 10^{-3} \mathrm{~m}$. To interpolate between the inverted annular flow regime and the dispersed liquid droplet flow regime, we use the parameter $\zeta$ defined by (Liles et al., 1988)

$$
\zeta=\left\{\begin{array}{lr}
0 & \alpha<\alpha_{\text {IA }} \\
\frac{\alpha-\alpha_{I A}}{\alpha_{\text {DIS }}-\alpha_{I A}} & \alpha_{I A} \leq \alpha \leq \alpha_{\text {DIS }} \\
1 & \alpha>\alpha_{\text {DIS }}
\end{array}\right.
$$

where

$$
\begin{aligned}
& \alpha_{\mathrm{IA}} \equiv \text { Void fraction limit for inverted annular flow }=0.5 \\
& \alpha_{\text {DIS }} \equiv \text { Void fraction for fully dispersed flow }=0.75
\end{aligned}
$$

For void fractions less than 0.5 we assume that the flow is entirely inverted annular and for void fractions greater than 0.75 we assume that the liquid flow is entirely in the form of dispersed droplets.

For heat transfer to the gas phase, we use the Dougall-Rohsenow (1963) correlation which is simply an application of the Dittus-Boelter correlation using gas properties and a mixture velocity in the Reynolds number. The correlation is 


$$
\begin{gathered}
h_{D R}=0.023\left(\frac{k_{g}}{D_{H}}\right) \operatorname{Re}_{T P}^{0.8} \operatorname{Pr}_{g}^{0.4} \\
\operatorname{Re}_{T P}=\frac{D_{H} u_{m i x} \rho_{g}}{\mu_{g}}
\end{gathered}
$$

with

$$
u_{\operatorname{mix}}=\alpha\left|u_{g}\right|+(1-\alpha)\left|u_{f}\right|
$$

where $\alpha$ is the void fraction and $u_{g}$ and $u_{f}$ are the phasic gas and liquid velocities, respectively. In this high temperature boiling regime, we also include contributions to the gas and liquid phase heat fluxes from radiation heat transfer. Assuming a surface view factor of unity and a surface emissivity of unity, the radiative heat fluxes to each phase are given by

$$
\left(\mathrm{q}_{\mathrm{f}}^{\prime \prime}\right)_{\mathrm{rad}}=\varepsilon\left(\mathrm{T}_{\mathrm{w}}^{4}-\mathrm{T}_{\mathrm{sat}}^{4}\right)
$$

and

$$
\left(\mathrm{q}_{\mathrm{g}}^{\prime \prime}\right)_{\mathrm{rad}}=\varepsilon\left(\mathrm{T}_{\mathrm{w}}^{4}-\mathrm{T}_{\mathrm{g}}^{4}\right)
$$

where $\varepsilon$ is Boltzman's constant.

The contributions to the heat fluxes to the gas and liquid are then summed and weighted by their respective volume fractions to obtain total film boiling heat fluxes as

$$
\begin{gathered}
\mathrm{q}_{\mathrm{f}}^{\prime \prime}=(1-\alpha)\left\{(1-\zeta) \mathrm{h}_{\mathrm{BP}}\left(\mathrm{T}_{\mathrm{w}}-\mathrm{T}_{\mathrm{sat}}\right)+\zeta \mathrm{h}_{\mathrm{FR}}\left(\mathrm{T}_{\mathrm{w}}-\mathrm{T}_{\mathrm{sat}}\right)+\left(\mathrm{q}_{\mathrm{f}}^{\prime \prime}\right)_{\mathrm{rad}}\right\} \\
\mathrm{q}_{\mathrm{g}}^{\prime \prime}=\alpha\left\{\mathrm{h}_{\mathrm{DR}}\left(\mathrm{T}_{\mathrm{w}}-\mathrm{T}_{\mathrm{g}}\right)+\left(\mathrm{q}_{\mathrm{g}}^{\prime \prime}\right)_{\mathrm{rad}}\right\}
\end{gathered}
$$

The vapor generation term is computed as

$$
\hat{\Gamma}_{w}=\frac{q_{f}^{\prime \prime}}{h_{f g}+0.5 c_{p g}\left(T_{w}-T_{s a t}\right)}
$$

Transition Boiling (FILMB): Transition boiling takes place when the surface temperature is greater than the critical heat flux temperature but below the minimum stable film temperature. In this boiling regime, the FLOWTRAN-TF formulation is similar to the TRAC scheme (Liles, 1988) and interpolates a surface heat flux between the critical heat flux and the value at the minimum stable film temperature. That is, in the transition regime we calculate heat fluxes using

$$
\mathrm{q}_{\mathrm{f}}^{\prime \prime}=(1-\delta)\left(\mathrm{q}_{\mathrm{f}}^{\prime \prime}\right)_{\mathrm{MSFB}}+\delta \mathrm{q}_{\mathrm{CHF}}^{\prime \prime}
$$


and

$$
\mathrm{q}_{\mathrm{g}}^{\prime \prime}=(1-\delta)\left(\mathrm{q}_{\mathrm{g}}^{\prime \prime}\right)_{\mathrm{MSFB}}
$$

The interpolation parameter $\delta$, which represents the fraction of the surface that is wetted, is
given by the relationship

$$
\delta=1-\xi^{2}(3-2 \xi)
$$

where

$$
\xi=\frac{\mathrm{T}_{w}-\mathrm{T}_{\mathrm{CHF}}}{\mathrm{T}_{\mathrm{MSFB}}-\mathrm{T}_{\mathrm{CHF}}}
$$

The functional form assumed in equation (2.5.1-53) defines a smooth cubic polynomial the heat fluxes at the minimum heat fluxes ( $q_{C H F}^{\prime \prime}$ for the liquid and zero for the gas) and slope at both end points.

Heat fluxes to the liquid and gas at the minimum stable film boiling temperature are calculated from the film boiling correlations given in equations $(2.5 .1-38)$ through determine $T_{M S F B}$, FLOWTRAN-TF usle film temperature ( $T_{M S F B}$ ) in place of $T_{w}$. To Groeneveld and Stewart (1983)

$$
\mathrm{T}_{\mathrm{MSFB}}=284.7+\left(4.41 \times 10^{-5}-3.72 \times 10^{-12} \mathrm{P}\right) \mathrm{P}-\frac{1.0 \times 10^{4} \mathrm{X}}{\left(2.82+1.22 \times 10^{-6} \mathrm{P}\right)}
$$

where

$$
\begin{aligned}
\mathrm{T}_{\mathrm{MSFB}} & \equiv \text { minimum stable film boiling temperature, }{ }^{\circ} \mathrm{C} \\
\mathrm{P} & \equiv \text { pressure, } \mathrm{Pa} \\
\mathrm{X} & \equiv \text { quality }
\end{aligned}
$$

This expression is applicable at the lower pressures of interest with SRS assemblies. The quality $X$ is replaced with $\min (X, 0)$ to account for positive values of $X$ in code small and to a good approximation, we are us the leading term in equation $(2.5 .1-55)$ are

Critical Heat Flux and Critical Heat Flux Temperature (CHF): If the wall the critire is greater than the saturation temperature, subroutine $\mathrm{CHF}$ is called to calculate some form of the Biasi corrature and heat flux. All of the standard codes listed above use was developed for commercial upflow and downflow conditions reactor applications. The correlation includes both sections and high pressures. THERMIT database is restricted to uniformly heated test increase the range of applicability. FL supplements this correlation with two others to 
from SRS data that may be more directly applicable to aluminum clad surfaces near atmospheric pressure. We have provided the user option of selecting the Biasi correlation or the SRS correlation. Neither correlation is verified for two-component air-water systems.

The Biasi correlation uses three separate calculations of the critical heat flux and selects the final value depending on the mass flux. For high mass fluxes $\left(G>300 \mathrm{~kg} / \mathrm{m}^{2}-\mathrm{s}\right)$, a low quality heat flux is calculated from

$$
\mathrm{q}_{\text {crit }}^{\prime \prime}=\frac{1.883 \times 10^{7}}{D_{\mathrm{H}}^{\mathrm{n}}} \mathrm{G}^{-1 / 6}\left[\mathrm{~F}(\mathrm{P}) \mathrm{G}^{-1 / 6}-\mathrm{Xe}\right]
$$

and a high quality heat flux is calculated from

$$
\mathrm{q}_{\text {crit }}^{\prime \prime}=\frac{3.78 \times 10^{7}}{D_{H}^{n}} G^{-0.6} H(P)[1.0-X e]
$$

The maximum value of the two determinations of $\mathrm{q}_{\text {crit }}^{\prime \prime}$ is then used for $\mathrm{q}_{\mathrm{CHF}}^{\prime \prime}$. In applying equation (2.5.1-56), the pressure function $F(P)$ is given by

$$
F(P)=0.7249+0.099 P e^{-0.032 P}
$$

Similarly, in applying equation (2.5.1-57), the corresponding pressure function $H(P)$ is given by

$$
H(P)=-1.159+0.149 P e^{-0.019 P}+\frac{8.99 P}{\left(10+P^{2}\right)}
$$

In both equations (2.5.1-56) and (2.5.1-57), $\mathrm{Xe}$ is the equilibrium quality and the exponent on the hydraulic diameter $(n)$ is chosen according to

$$
\mathfrak{n}= \begin{cases}0.4 & , D_{H} \geq 0.01 \mathrm{~m} \\ 0.6 & , D_{H}<0.01 \mathrm{~m}\end{cases}
$$

In evaluating equations (2.5.1-56) through (2.5.1-59), the mass flux $G$ is in units of $\mathrm{g} / \mathrm{cm}^{2}-\mathrm{s}$, the pressure $\mathrm{P}$ is in bars, and the hydraulic diameter is in centimeters. We note that at pressures near one atmosphere (one bar), the function $H(P)$ becomes negative which indicates that it is not applicable at low pressure. Therefore, when the Biasi CHF correlation is selected for SRS assemblies, equation (2.5.1-56) is actually being used to calculate the critical heat flux.

As a user option, when the mass flux through the flow channel is greater than $300 \mathrm{~kg} / \mathrm{m}^{2}-\mathrm{s}$, the critical heat flux can alternatively be calculated using the correlation developed at SRL (Hodges and Knoebel, 1973). This correlation is based on data taken with light water in a narrow rectangular channel having an aluminum surface. The correlation is presented in two parts: 


$$
\mathrm{q}_{\mathrm{CHF}}^{\prime \prime}=5.264 \times 10^{5}(1.0+0.476 \mathrm{~V})\left[1.0+0.031\left(\mathrm{~T}_{\text {sat }}-\mathrm{T}_{\mathrm{f}}\right)\right]
$$

and

$$
\mathrm{q}_{\mathrm{CHF}}^{\prime \prime}=2.021 \times 10^{6}(1.0+0.169 \mathrm{~V})
$$

where

$$
\begin{aligned}
\mathrm{q}_{\mathrm{CHF}}^{\prime \prime} & \equiv \text { critical heat flux, } \mathrm{W} / \mathrm{m}^{2}{ }^{\circ} \mathrm{C} \\
\mathrm{T}_{\text {sat }} & \equiv \text { saturation temperature, }{ }^{\circ} \mathrm{C} \\
\mathrm{T}_{\mathrm{f}} & \equiv \text { fluid temperature, }{ }^{\circ} \mathrm{C} \\
\mathrm{V} & \equiv \text { superficial liquid velocity, } \mathrm{m} / \mathrm{s}
\end{aligned}
$$

The SRL correlations are based on experimental data obtained over the following range of
conditions:

1) Liquid velocity: 1.68 to $12.95 \mathrm{~m} / \mathrm{s}$

2) Liquid subcooling: 4 to $107^{\circ} \mathrm{C}$

3) Pressure: 40 to 80 psig

4) Channel equivalent diameter: 1.12 to $1.27 \mathrm{~cm}$

5) Channel width: 2.54 to $5.21 \mathrm{~cm}$

6) Channel width/Channel thickness: 4 to 9.3

7) Nonconducting fiberglass ribs having a rectangular geometry attached to the
heater surface ( 0 to $0.635 \mathrm{~cm}$ thick rib)

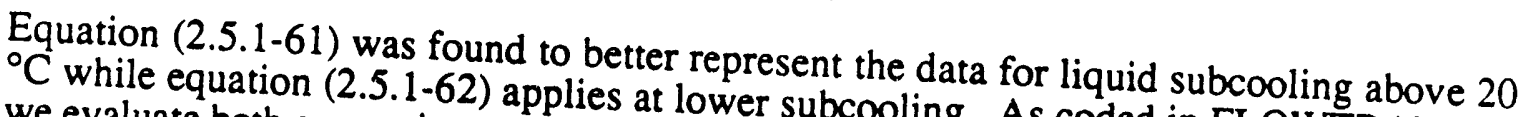
${ }^{\circ} \mathrm{C}$ while equation (2.5.1-62) applies at lower subcooling. As coded in FLOWTRAN-TF, flux. This procedure correctly transitions maximum value as an estimate of the critical heat subcooling decreases.

At liquid mass fluxes less than $200 \mathrm{~kg} / \mathrm{m}^{2}-\mathrm{s}$, Griffith's modification (Bjornard and Griffith critical heat flux:

$$
q_{\text {pool }}^{\prime \prime}=\frac{0.9 \pi}{24}(1.0-\alpha) h_{f_{g}} \rho_{g}^{0.5}\left[g_{c} g \sigma\left(\rho_{f}-\rho_{g}\right)\right]^{0.25}
$$

We note that this correlation (also employed by COBRA/TRAC) predicts a critical heat flux increases. At mass fluxes between 200 and $300 \mathrm{~kg} / \mathrm{m}^{2}-\mathrm{s}$, decreases as the void fraction 
\begin{tabular}{|l|lll|r|}
\hline $2 / 15 / 93$ & WSRC-TR-92-532 Rev. 0 & FLOWTRAN-TF Software Design & Page 108 \\
\hline
\end{tabular}

Biasi or SRL correlation and the Zuber pool boiling correlation is used to estimate the critical heat flux.

Finally, knowing $\mathrm{q}_{\mathrm{CHF}}^{\prime \prime}$, we need to determine the wall temperature when the critical heat flux is reached $\left(\mathrm{T}_{\mathrm{CHF}}\right)$. This is found by solving for the temperature at which the Chen or Mikic-Rohsenow correlations yield a heat flux equal to q⿳⺈冂大 The Chen boiling correlations are based on assuming a nucleate boiling and a forced convection contribution to the overall heat flux. Using equations $(2.5 .1-29)$ and $(2.5 .1-30)$ to calculate heat transfer coefficients and replacing $T_{w}$ with $T_{C H F}$ yields the expression

$$
\mathrm{q}_{\mathrm{CHF}}^{\prime \prime}=\mathrm{h}_{\mathrm{LNB}}\left(\mathrm{T}_{\mathrm{CHF}}-\mathrm{T}_{\mathrm{f}}\right)^{1.24}\left(\mathrm{P}_{\mathrm{w}}-\mathrm{P}\right)^{0.75}+\mathrm{h}_{\mathrm{LFC}}\left(\mathrm{T}_{\mathrm{CHF}}-\mathrm{T}_{\mathrm{f}}\right)
$$

for the total heat flux. In equation (2.5.1-64), $h_{L N B}$ and $h_{L F C}$ are the constant portions of the heat transfer coefficients. Since $P_{w}$ will depend upon $T_{C H F}$, the pressure dependence is left explicit in equation (2.5.1-64). Equation (2.5.1-64) is solved using a Newton iteration scheme until the critical heat flux temperature is determined within $0.1^{\circ} \mathrm{C}$. The critical heat flux temperature is restricted to be greater than the local saturation temperature and less than the critical temperature for water $\left(647.14^{\circ} \mathrm{K}\right)$. A similar procedure again involving a Newton iteration is used with the Mikic-Rohsenow correlation to determine $\mathrm{T}_{\mathrm{CHF}}$. This completes determination of all points along the boiling curve.

\section{Heat Fluxes at Boundary Surfaces}

The heat transfer boundary condition at the outermost surface of the assembly is specified separately through the input. The code user has two options:

1. Use of a fixed heat transfer coefficient coupling the outer surface to a heat sink that follows an input temperature transient. That is the code computes the heat flux at the outer surface $\left(\mathrm{q}_{0}^{\prime \prime}\right)$ as

$$
q_{0}^{\prime \prime}=f h_{0}\left(T_{w}-T_{0}\right)
$$

where

$\mathrm{f} \equiv$ fraction of the surface that is wetted

$h_{o} \equiv$ constant heat transfer coefficient at outer surface

$\mathrm{T}_{0} \equiv$ time varying fluid temperature

The code user inputs $h_{0}$ and the fluid temperature $T_{0}$ which may vary with time. The tank level transient is used to compute the fraction of the outer surface that is wet (f). If the outer surface is dry, the code applies an adiabatic boundary condition.

2. Use of a simple radiation heat transfer boundary condition. We assume that radiative heat transfer between concentric cylinders applies at the outer surface. The heat flux at the outer surface is then computed as 


$$
\mathrm{q}_{o}^{\prime \prime}=\frac{\sigma\left(\mathrm{T}_{\mathrm{w}}^{4}-\mathrm{T}_{\mathrm{o}}^{4}\right)}{1 / \varepsilon_{\mathrm{w}}+\left(\mathrm{D}_{\mathrm{w}} / \mathrm{D}_{\mathrm{o}}\right)\left(1 / \varepsilon_{\mathrm{o}}-1\right)}
$$

where

$\varepsilon_{\mathrm{w}} \equiv$ emissivity of outermost surface

$\varepsilon_{0} \equiv$ emissivity of radiating surface

$D_{w} \equiv$ diameter of outermost surface

$D_{0} \equiv$ diameter of radiating surface

$T_{0} \equiv$ time varying surrounding surface temperature

\section{Sample Boiling Curve Calculations}

In this section of the report, we present a few representative sample calculations to illustrate the application of the FLOWTRAN-TF heat transfer correlations. All of the calculations were performed assuming the following flow conditions:

Liquid and gas velocities $=2.0 \mathrm{~m} / \mathrm{s}$

Liquid and gas bulk temperatures $=50^{\circ} \mathrm{C}$

Channel hydraulic diameter $=1.2065 \times 10^{-2} \mathrm{~m}$

Mole fraction water vapor in air $=0.985$

Channel area $=1.3935 \times 10^{-3} \mathrm{~m}^{2}$

Pressure $=0.101325 \mathrm{MPa}$

The channel dimensions correspond to those for a single subchannel within the middle flow channel of an SRS Mark 22 assembly and the selected flow conditions are representative of those that pertain during the ECS phase of the DEGB-LOCA.

In Fig. 2.5.1-7, we have plotted boiling curves computed using the various code options at $0 \%$ and $50 \%$ void fraction in the flow channel. Both curves use the SRL critical heat flux correlation to set the upper limit to the boiling heat flux. We find that the Mikic-Rohsenow correlation produces a steeper boiling curve than the Chen correlation.

Figure 2.5.1-8 presents a comparison of boiling curves computed using the SRL and the Biasi critical heat flux correlations along with the Mikic-Rohsenow boiling correlation. The SRL CHF correlation produces a lower estimate of the critical heat flux for this particular
case.

Finally, Fig. 2.5.1-9 shows the behavior of the FLOWTRAN-TF boiling curves as a function of void fraction. At low void fraction the boiling curve is relatively insensitive to the void. As the void fraction approaches one, the liquid mass flux decreases and the boiling curve transitions to the Zuber pool boiling correlation. The FLOWTRAN-TF scheme provides for a smooth transition of the surface heat transfer characteristics as the void fraction within the flow channels varies. 

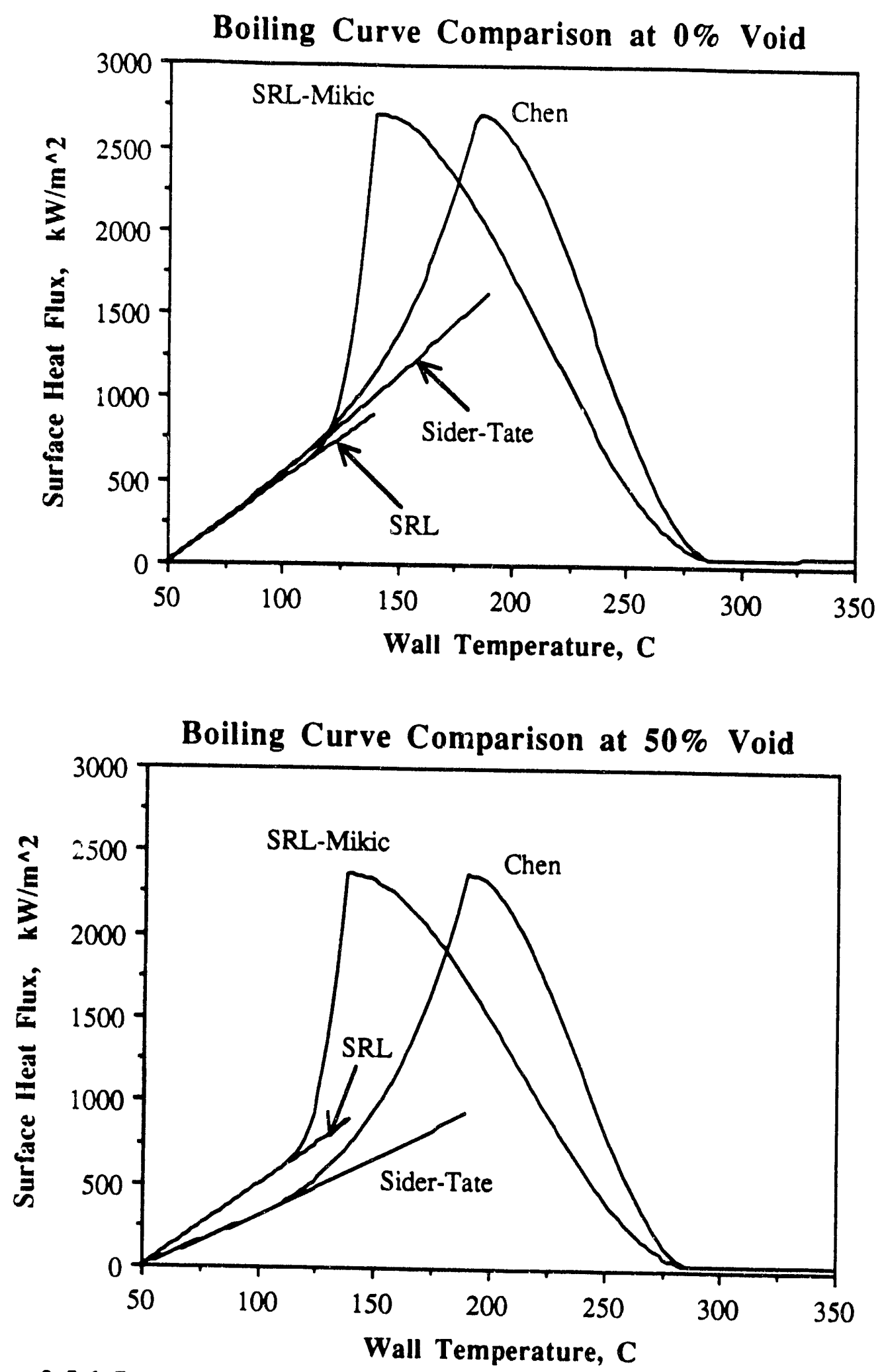

Figure 2.5.1.7 FLOWTRAN-TF boiling curves at $0 \%$ and $50 \%$ void. 


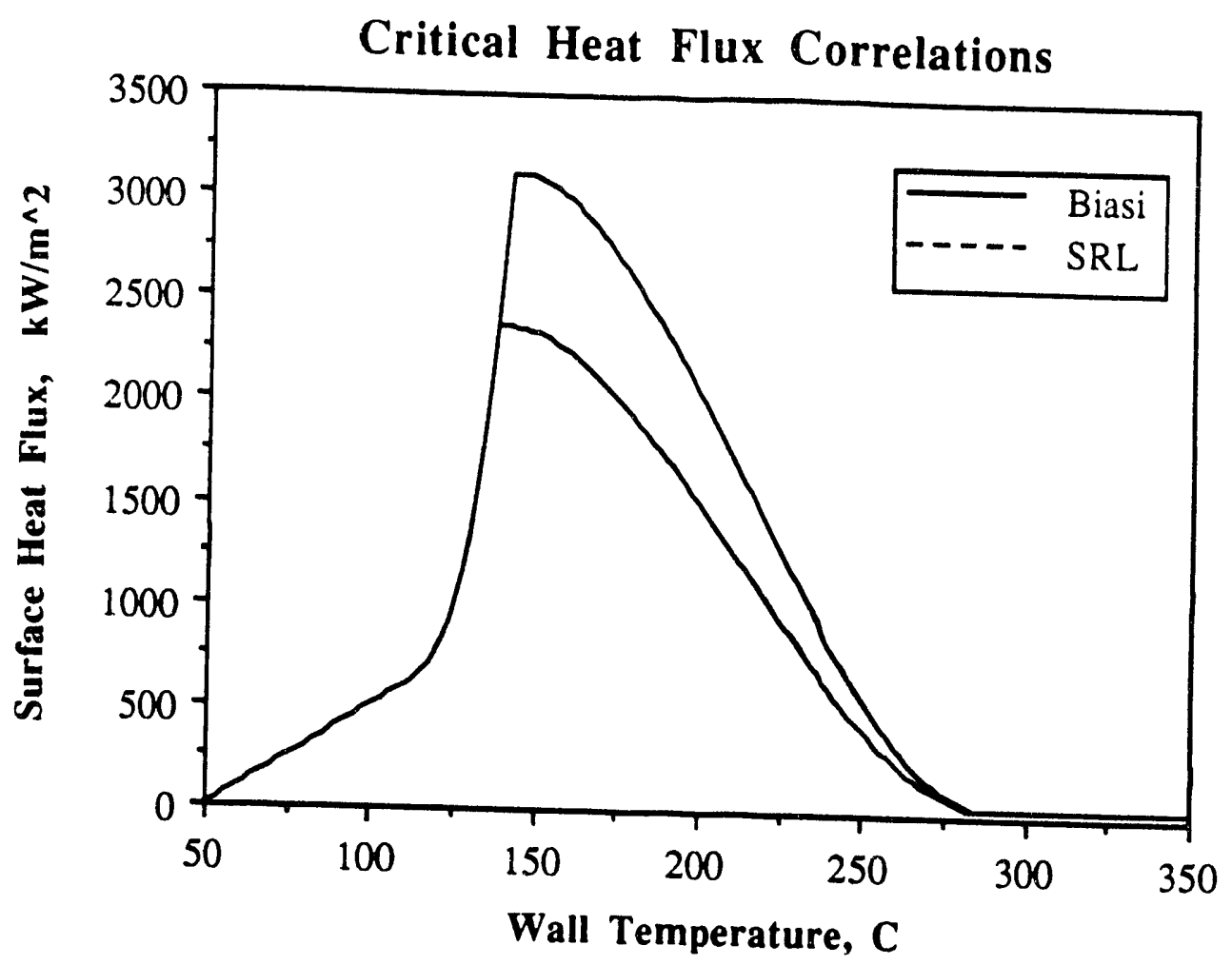

Figure 2.5.1-8 Comparison of FLOWTRAN-TF CHF correlations. 


\begin{tabular}{|l|lll|l|}
\hline $2 / 15 / 93$ & WSRC-TR-92-532 Rev. 0 & FLOWTRAN-TF Software Design & Page 112 \\
\hline
\end{tabular}



Figure 2.5.1-9 Dependence of FLOWTRAN-TF boiling curve on void fraction. 


\begin{tabular}{|l|lll|l|}
\hline $2 / 15 / 93$ & WSRC-TR-92-532 Rev. 0 & FLOWTRAN-TF Software Design & Page 113 \\
\hline
\end{tabular}

Non-condensible gas solubility in water and Mikic-Rohsenow's fullydeveloped boiling curve coefficient

In this section, auxiliary constitutive equations required to evaluate the Mikic-Rohsenow correlation are presented. First correlations for Henry's law constant are given. Then a more computationally efficient version of the fully-developed boiling heat transfer coefficient is given.

Henry's law constant $(\eta)$ : The partial pressure of the non-condensible component such as air or helium gas in the gas mixture is computed by Henry's law. The concentration of non-condensible gas dissolved in liquid water is also considered in terms of Henry's law constant, which is related to the calculation of the minimum wall superheat at the onset of nucleate boiling on the heateci wall surface.

Henry's law constant for helium gas dissolved in liquid water is given by

$$
\eta=6894.8\left\{10^{\left(A+B \chi+C \chi^{2}+D \chi^{3}\right)}\right\}, \quad \chi=\frac{1000}{T}
$$

where $\eta$ is in $\mathrm{Pa}$ and $\mathrm{T}$ is in $\mathrm{K}$. For light water

$$
\begin{aligned}
& A \equiv 3.4527291 \\
& B \equiv 8.7992069 \times 10^{-1} \\
& C \equiv 2.7718148 \times 10^{-1} \\
& D \equiv-8.4275100 \times 10^{-2}
\end{aligned}
$$

and for heavy water

$$
\begin{aligned}
& A \equiv-2.6034660 \\
& B \equiv 8.3240746 \\
& C \equiv-2.6612343 \\
& D \equiv 2.8897693 \times 10^{-1}
\end{aligned}
$$

The applicable range of the correlation is $283 \mathrm{~K}<\mathrm{T}<573 \mathrm{~K}$ for light water and $323 \mathrm{~K}<\mathrm{T}$ $<553 \mathrm{~K}$ for heavy water. The constants for light water and heavy water were determined by curve fitting data from Stephan et al. (1962).

Henry's law constant for air dissolved in liquid water is given by

$$
\eta=1.01325 \times 10^{5} \mathrm{e}^{\left(104.208-\frac{137.296}{\tau}-58.7394 \ln \tau+5.7669 \tau\right)}, \tau=\frac{T}{100}
$$


where $\eta$ is in $\mathrm{Pa}$ and $\mathrm{T}$ is in $\mathrm{K}$. The correlation is given by Battino et al. (1984) as shown above. This correlation is used for the computation of Henry's law constant for light water $\left(\mathrm{H}_{2} \mathrm{O}\right)$ or heavy water $\left(\mathrm{D}_{2} \mathrm{O}\right)$.

Mikic-Rohsenow's fully-developed boiling curve coefficient: The fullydeveloped boiling heat transfer coefficient $\left(h_{b}^{0} ; \mathrm{W} /\left(\mathrm{m}^{2} \mathrm{~K}^{3}\right)\right)$ is given by equations (2.5.1-16a). Computationally, FLOWTRAN-TF uses the following more efficient approximation

$$
h_{b}^{0}=e^{\left(A+B \tau_{r}+C \tau_{r}^{2}+D \tau_{r}^{3}+E \tau_{r}^{4}+F \tau_{r}^{5}\right)}, \tau_{r}=\frac{T}{T_{c r}}
$$

where for light water

$$
\begin{aligned}
T_{c t} & \equiv 647.14 \mathrm{~K} \\
A & \equiv-1.0201219 \times 10^{1} \\
B & \equiv 9.0042537 \times 10^{1} \\
C & \equiv-2.7087881 \times 10^{2} \\
D & \equiv 4.4721796 \times 10^{2} \\
E & \equiv-3.8294426 \times 10^{2} \\
F & \equiv 1.3526261 \times 10^{2}
\end{aligned}
$$

and for heavy water

$$
\begin{aligned}
T_{C r} & \equiv 643.89 \mathrm{~K} \\
A & \equiv-1.8932962 \times 10^{1} \\
B & \equiv 1.501543 \times 10^{2} \\
C & \equiv-4.2238743 \times 10^{2} \\
D & \equiv 6.2298006 \times 10^{2} \\
E & \equiv-4.7386928 \times 10^{2} \\
F & \equiv 1.5028174 \times 10^{2}
\end{aligned}
$$

The correlational results are compared with those of Mikic-Rohsenow's equation as shown in the following tabular and graphical results. 
Table 2.5.1-1 Henry's saw constant of helium gas dissolved in light water

\begin{tabular}{|l|c|c|c|}
\hline $\mathrm{T}(\mathrm{K})$ & $\eta \times 10^{-5}(\mathrm{~Pa})-\mathrm{Data}$ & $\eta \times 10^{-5}(\mathrm{~Pa})-$ Corr. & \% error \\
\hline 283.15 & 141395 & 139239 & 1.525 \\
293.15 & 148201 & 148938 & -0.497 \\
303.15 & 151334 & 153094 & -1.163 \\
313.15 & 150903 & 152530 & -1.078 \\
323.15 & 147323 & 148300 & -0.663 \\
333.15 & 141216 & 141468 & -0.178 \\
343.15 & 133291 & 132973 & 0.239 \\
353.15 & 124234 & 123576 & 0.529 \\
363.15 & 114642 & 113859 & 0.683 \\
373.15 & 104987 & 104234 & 0.718 \\
383.15 & 95608 & 94978 & 0.659 \\
393.15 & 86729 & 86263 & 0.537 \\
403.15 & 78479 & 78183 & 0.378 \\
413.15 & 70919 & 70774 & 0.204 \\
423.15 & 64059 & 64038 & 0.033 \\
433.15 & 57879 & 57949 & -0.121 \\
443.15 & 52338 & 52470 & -0.252 \\
453.15 & 47388 & 47555 & -0.352 \\
463.15 & 42973 & 43153 & -0.420 \\
473.15 & 39040 & 39217 & -0.455 \\
483.15 & 35536 & 35698 & -0.456 \\
493.15 & 32415 & 32553 & -0.427 \\
503.15 & 29631 & 29740 & -0.369 \\
513.15 & 27146 & 27223 & -0.285 \\
523.15 & 24924 & 24968 & -0.177 \\
533.15 & 22935 & 22946 & -0.049 \\
543.15 & 21150 & 21130 & 0.096 \\
553.15 & 19547 & 19497 & 0.256 \\
563.15 & 18103 & 18025 & 0.428 \\
573.15 & 16800 & 16698 & \\
\hline
\end{tabular}

* Relative \% error 
Table 2.5.1-2 Henry's law constant of helium gas dissolved in heavy water

\begin{tabular}{|c|c|c|c|}
\hline $\mathrm{T}(\mathrm{K})$ & $\eta \times 10^{-5}(\mathrm{~Pa})-$ Data & $\eta \times 10^{-5}(\mathrm{~Pa})-$ Corr. & $\%$ error \\
\hline $\begin{array}{l}283.15 \\
293.15 \\
303.15 \\
313.15 \\
323.15 \\
333.15 \\
343.15 \\
353.15 \\
363.15 \\
373.15 \\
383.15 \\
393.15 \\
403.15 \\
413.15 \\
423.15 \\
433.15 \\
443.15 \\
453.15 \\
463.15 \\
473.15 \\
483.15 \\
493.15 \\
503.15 \\
513.15 \\
523.15 \\
533.15 \\
543.15 \\
553.15 \\
563.15 \\
573.15\end{array}$ & $\begin{array}{l}118039 \\
99906 \\
69500 \\
48195 \\
30613\end{array}$ & $\begin{array}{l}147728 \\
136090 \\
128340 \\
122781 \\
118383 \\
114501 \\
110731 \\
106827 \\
102656 \\
98166 \\
93361 \\
88284 \\
83001 \\
77591 \\
72136 \\
66715 \\
61400 \\
56251 \\
51317 \\
46636 \\
42234 \\
38127 \\
34322 \\
30820 \\
27613 \\
24693 \\
22044 \\
19651 \\
17497 \\
15564\end{array}$ & $\begin{array}{l}1.741 \\
-3.793 \\
3.234 \\
-0.676 \\
-0.3 .57\end{array}$ \\
\hline
\end{tabular}


Table 2.5.1-3 Henry's law constant of air dissolved in liquid water

\begin{tabular}{|c|c|c|c|}
\hline $\mathrm{T}(\mathrm{K})$ & $\eta \times 10^{-5}(\mathrm{~Pa})-$ Data & $\eta \times 10^{-5}(\mathrm{~Pa})-$ Corr & $\%$ error \\
\hline $\begin{array}{l}273.15 \\
278.15 \\
283.15 \\
288.15 \\
293.15 \\
298.15 \\
303.15 \\
308.15 \\
313.15 \\
318.15 \\
323.15 \\
328.15 \\
333.15 \\
338.15 \\
343.15 \\
348.15 \\
353.15 \\
358.15 \\
363.15 \\
368.15 \\
373.15 \\
378.15 \\
383.15 \\
388.15 \\
398.15 \\
408.15 \\
418.15 \\
428.15 \\
448.15 \\
448.15 \\
468.15 \\
488.15 \\
528.15 \\
543.15\end{array}$ & $\begin{array}{l}43750 \\
49620 \\
55551 \\
61521 \\
67370 \\
73001 \\
78364 \\
83395 \\
88032 \\
92197 \\
96043 \\
99338 \\
102142 \\
104523 \\
106423 \\
107815 \\
108788 \\
109328 \\
109552 \\
109446 \\
108893\end{array}$ & $\begin{array}{l}43702 \\
49590 \\
55548 \\
61484 \\
67309 \\
72940 \\
78304 \\
83337 \\
87987 \\
92213 \\
95986 \\
99288 \\
102109 \\
104452 \\
106325 \\
107743 \\
108728 \\
109304 \\
109499 \\
109345 \\
108871 \\
108111 \\
107096 \\
105857 \\
102826 \\
99237 \\
95283 \\
91123 \\
82680 \\
82680 \\
74632 \\
67371 \\
55753 \\
52399\end{array}$ & $\begin{array}{l}0.109 \\
0.062 \\
0.006 \\
0.061 \\
0.092 \\
0.083 \\
0.077 \\
0.069 \\
0.051 \\
-0.017 \\
0.059 \\
0.051 \\
0.032 \\
0.068 \\
0.092 \\
0.067 \\
0.055 \\
0.022 \\
0.048 \\
0.093 \\
0.020\end{array}$ \\
\hline
\end{tabular}



Table 2.5.1-4 Light water Mikic-Rohsenow fully-developed boiling heat transfer

\begin{tabular}{|c|c|c|l|}
\hline $\mathrm{T}(\mathrm{K})$ & $\mathrm{h}_{\mathrm{b}}\left(\mathrm{W} / \mathrm{m}^{2} \mathrm{~K}^{3}\right)-$ Data & $\mathrm{h}_{\mathrm{b}}\left(\mathrm{W} / \mathrm{m}^{2} \mathrm{~K}^{3}\right)-$ Corr. & \% error \\
\hline 283.15 & 18.8 & 18.79 & 0.061 \\
293.15 & 20.7 & 20.67 & 0.143 \\
303.15 & 22.6 & 22.64 & -0.196 \\
313.15 & 24.7 & 24.72 & -0.071 \\
323.15 & 26.9 & 26.90 & 0.008 \\
333.15 & 29.2 & 29.19 & 0.019 \\
343.15 & 31.6 & 31.62 & -0.056 \\
353.15 & 34.2 & 34.18 & 0.059 \\
363.15 & 36.9 & 36.89 & 0.017 \\
373.15 & 39.8 & 39.78 & 0.062 \\
383.15 & 42.9 & 42.84 & 0.132 \\
393.15 & 46.1 & 46.12 & -0.046 \\
403.15 & 49.6 & 49.64 & -0.076 \\
413.15 & 53.4 & 53.43 & -0.054 \\
423.15 & 57.5 & 57.54 & -0.071 \\
433.15 & 62.0 & 62.03 & -0.055 \\
443.15 & 66.9 & 66.99 & -0.130 \\
453.15 & 72.4 & 72.50 & -0.139 \\
463.15 & 78.7 & 78.71 & -0.011 \\
473.15 & 85.8 & 85.79 & 0.013 \\
483.15 & 94.0 & 93.98 & 0.026 \\
493.15 & 103.7 & 103.58 & 0.111 \\
503.15 & 115.2 & 115.04 & 0.136 \\
513.15 & 129.1 & 128.94 & 0.125 \\
523.15 & 146.3 & 146.09 & 0.144 \\
533.15 & 167.7 & 167.65 & 0.028 \\
543.15 & 195.0 & $195.31)$ & -0.154 \\
553.15 & 230.9 & 231.50 & -0.258 \\
563.15 & 279.4 & 279.95 & -0.198 \\
573.15 & 347.5 & 346.42 & 0.310 \\
\hline
\end{tabular}


Table 2.5.1-5 Heavy water Mikic-Rohsenow fully-developed boiling heat transfer coefficient

\begin{tabular}{|c|c|c|c|}
\hline $\mathrm{T}(\mathrm{K})$ & $h_{b}\left(W / m^{2} K^{3}\right)-$ Data & $h_{b}\left(W / m^{2} K^{3}\right)$-Corr. & $\%$ error \\
\hline 283.15 & 21.9 & 21.83 & 0.333 \\
\hline 293.15 & 24.1 & 24.15 & -0.221 \\
\hline 303.15 & 26.5 & 26.48 & 0.065 \\
\hline 313.15 & 28.8 & 28.82 & -0.053 \\
\hline 323.15 & 31.1 & 31.16 & -0.179 \\
\hline 333.15 & 33.5 & 33.51 & -0.042 \\
\hline 343.15 & 35.9 & 35.91 & -0.015 \\
\hline 353.15 & 38.4 & 38.35 & 0.135 \\
\hline 363.15 & 40.9 & 40.86 & 0.087 \\
\hline 373.15 & 43.5 & 43.48 & 0.046 \\
\hline 383.15 & 46.3 & 46.23 & 0.161 \\
\hline 393.15 & 49.2 & 49.14 & 0.131 \\
\hline 403.15 & 52.2 & 52.25 & -0.096 \\
\hline 413.15 & 55.6 & 55.62 & -0.032 \\
\hline 423.15 & 59.2 & 59.29 & -0.159 \\
\hline 433.15 & 63.3 & 63.35 & -0.078 \\
\hline 443.15 & 67.8 & 67.87 & -0.102 \\
\hline 453.15 & 72.9 & 72.96 & -0.081 \\
\hline 463.15 & 78.7 & 78.75 & -0.070 \\
\hline 473.15 & 85.4 & 85.43 & -0.035 \\
\hline 483.15 & 93.3 & 93.21 & 0.097 \\
\hline 493.15 & 102.5 & 102.39 & 0.106 \\
\hline 503.15 & 113.6 & 113.37 & 0.202 \\
\hline 513.15 & 126.9 & 126.68 & 0.170 \\
\hline 523.15 & 143.2 & 143.07 & 0.091 \\
\hline 533.15 & 163.5 & 163.55 & -0.034 \\
\hline 543.15 & 189.3 & 189.60 & -0.161 \\
\hline 553.15 & 222.7 & 223.34 & -0.288 \\
\hline 563.15 & 267.3 & 267.91 & -0.227 \\
\hline 573.15 & 329.2 & 328.06 & 0.345 \\
\hline
\end{tabular}




\begin{tabular}{|l|lll|r|}
\hline $2 / 15 / 93$ & WSRC-TR-92-532 Rev. 0 & FLOWTRAN-TF Software Design & Page 120 \\
\hline
\end{tabular}

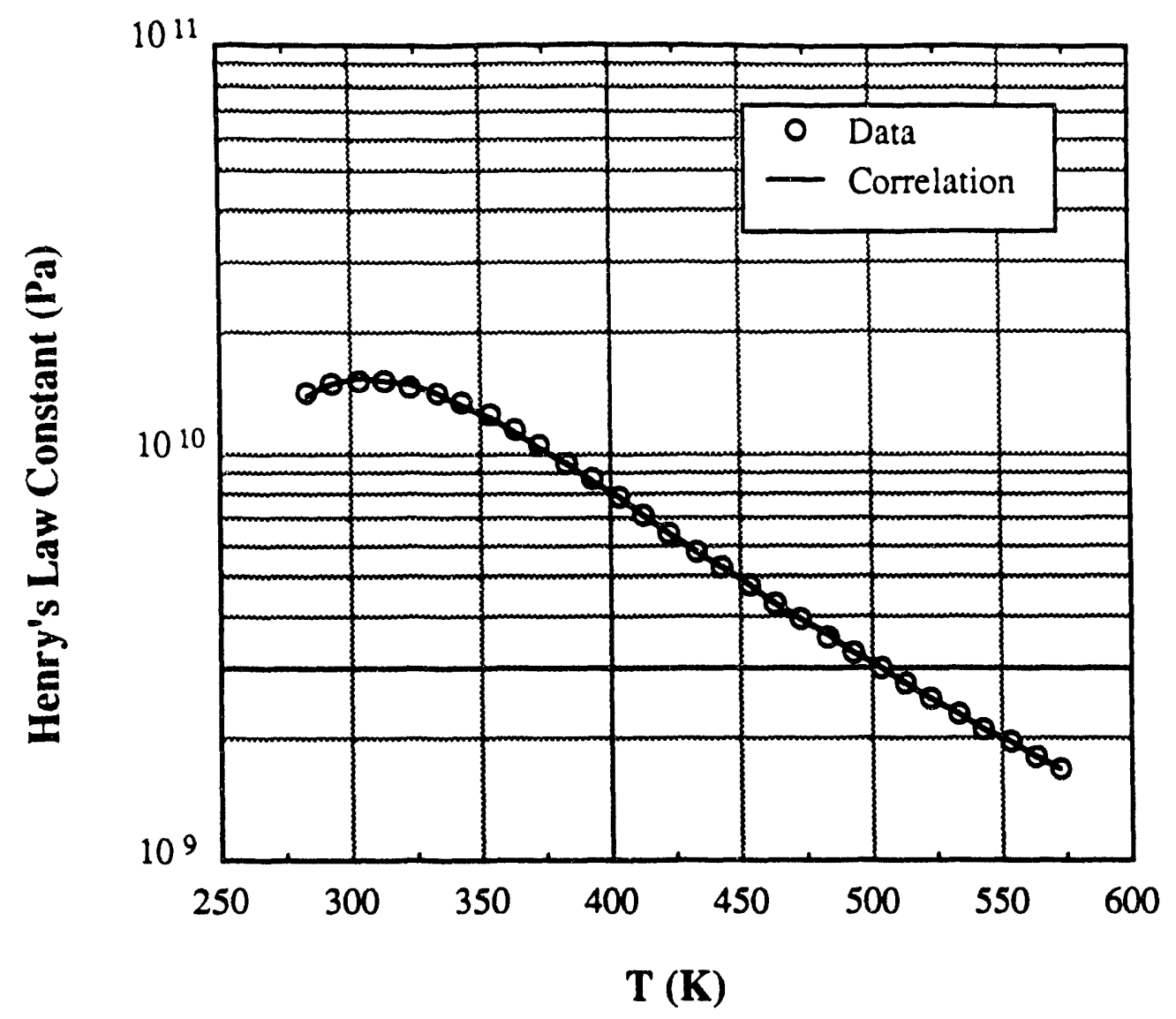

Figure 2.5.1-10 Henry's law constant of helium gas dissolved in light water 


\begin{tabular}{|l|lll|l|}
\hline $2 / 15 / 93$ & WSRC-TR-92-532 Rev. 0 & FLOWTRAN-TF Software Design & Page 121 \\
\hline
\end{tabular}

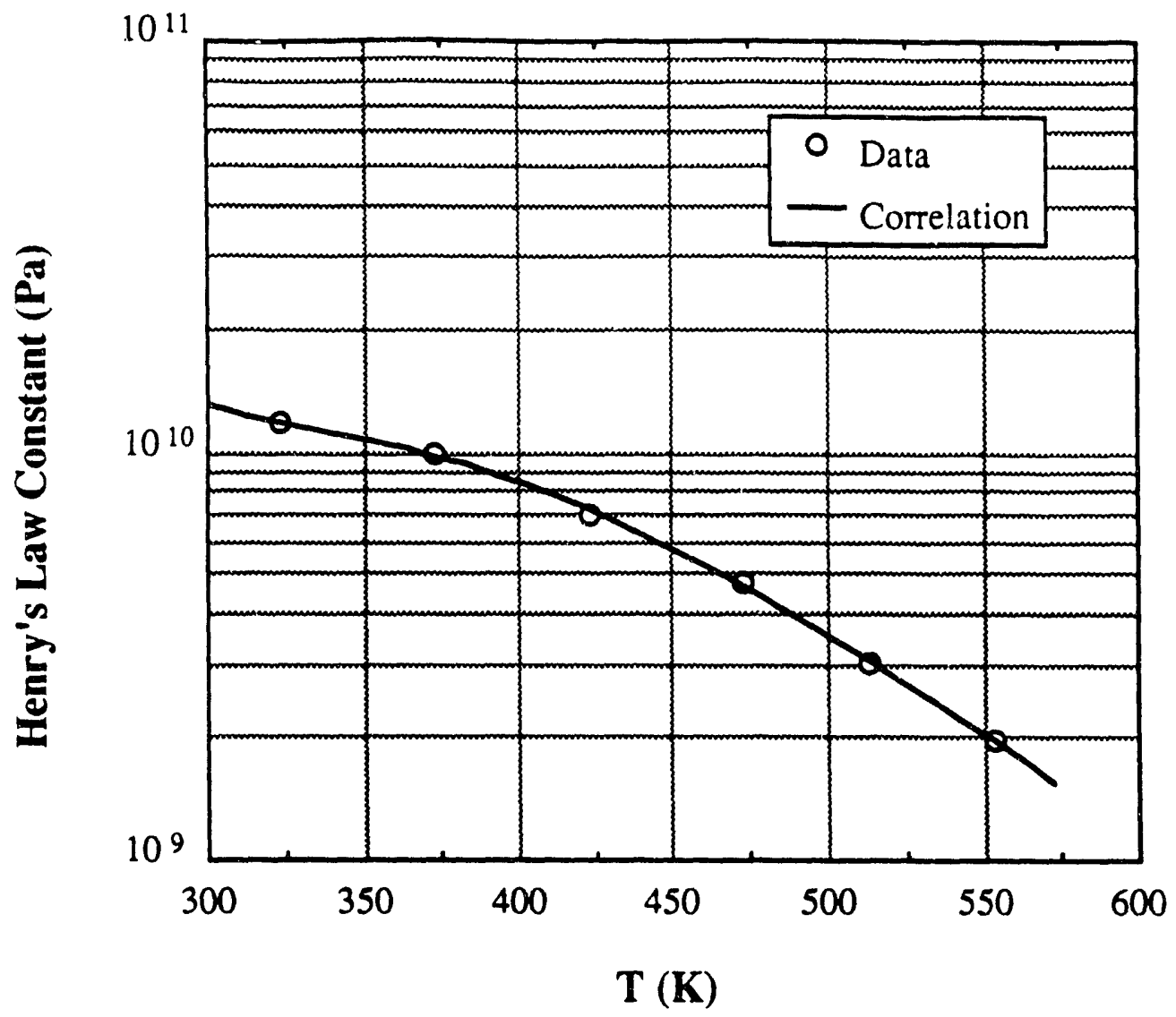

Figure 2.5.1-11 Henry's law constant of helium gas dissolved in heavy water 


\begin{tabular}{|l|lll|r|}
\hline $2 / 15 / 93$ & WSRC-TR-92-532 Rev. 0 & FLOWTRAN-TF Software Design & Page 122 \\
\hline
\end{tabular}

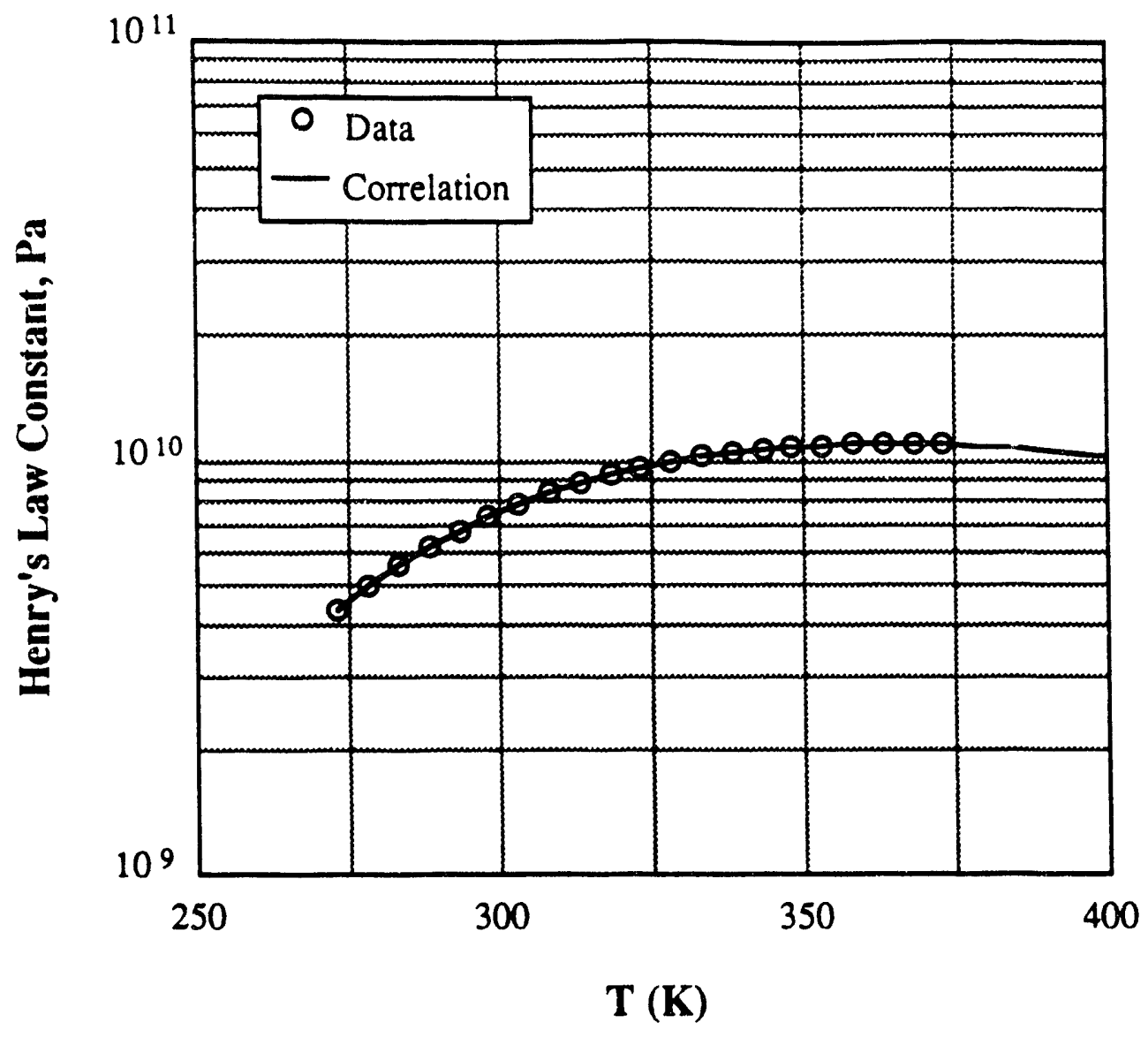

Figure 2.5.1-12 Henry's law constant of air dissolved in liquid water 


\begin{tabular}{|l|lll|l|}
\hline $2 / 15 / 93$ & WSRC-TR-92-532 Rev. 0 & FLOWTRAN-TF Software Design & Page 123 \\
\hline
\end{tabular}

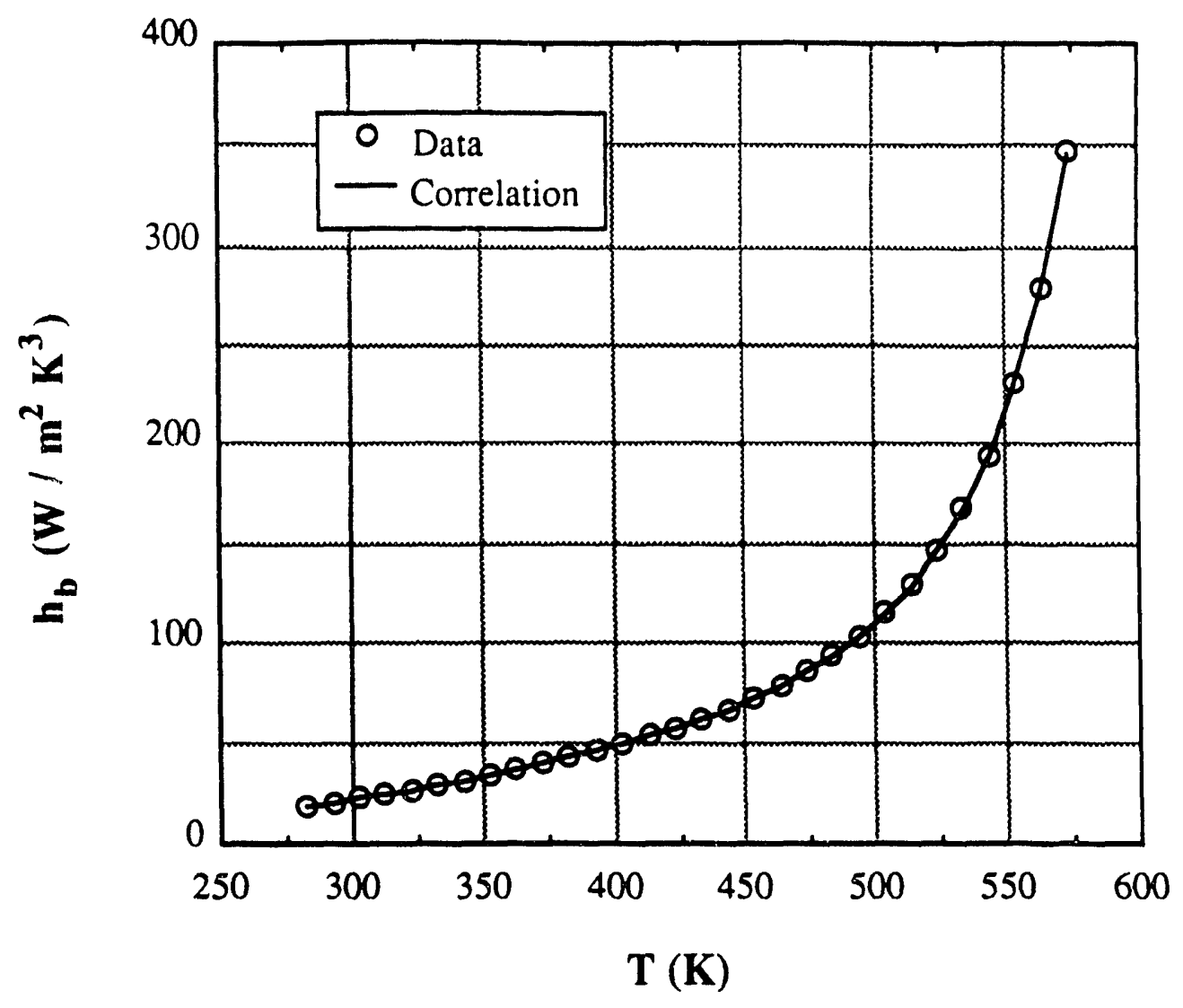

Figure 2.5.1-13 Mikic-Rohsenow fully-developed boiling heat transfer coefficient of light water 


\begin{tabular}{|l|ll|r|r|}
\hline $2 / 15 / 93$ & WSRC.TR-92-532 Rev. 0 & FLOWTRAN-TF Software Design & Page 124 \\
\hline
\end{tabular}

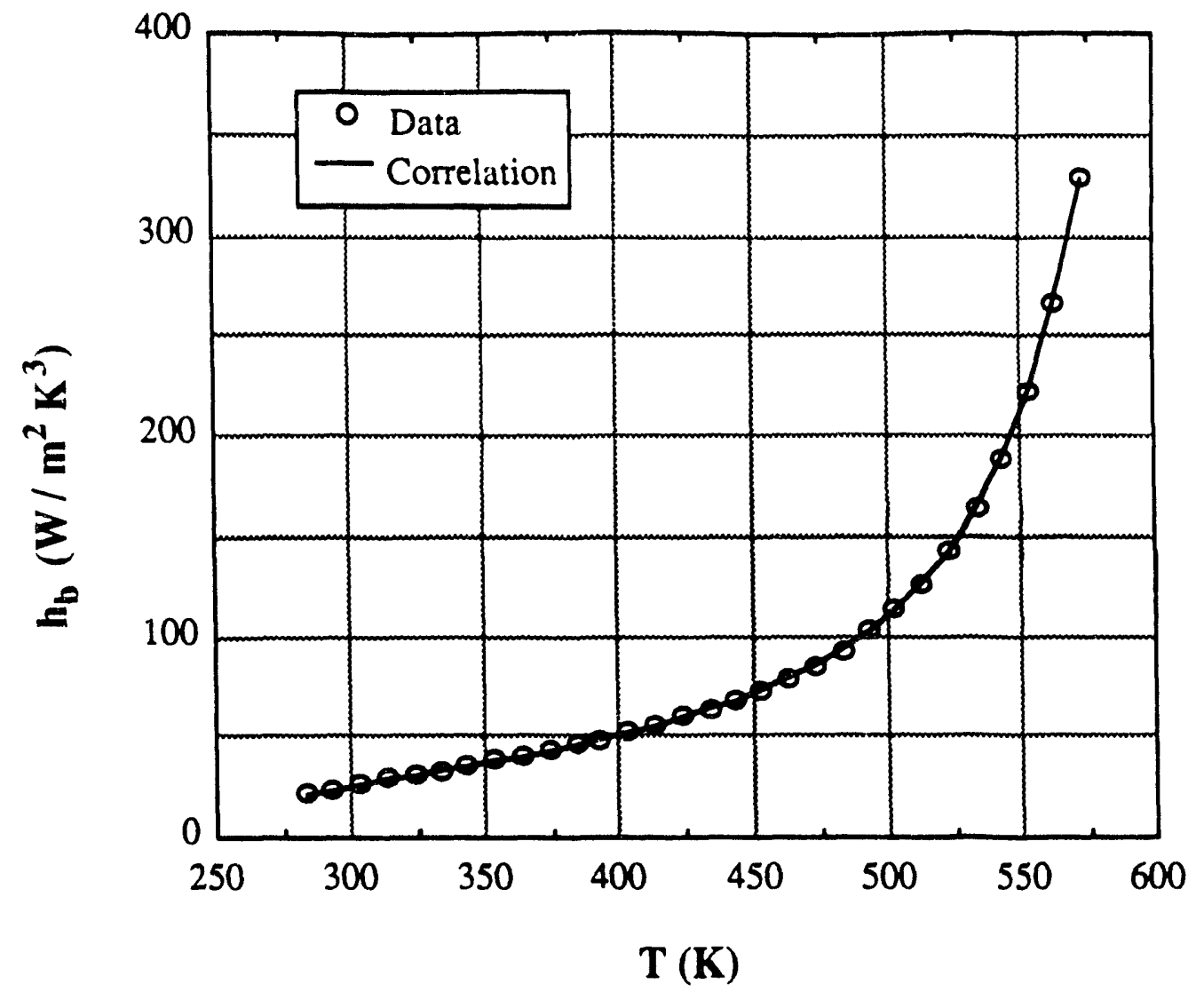

Figure 2.5.1-14 Mikic-Rohsenow fully-developed boiling heat transfer coefficient of heavy water 


\section{References}

Aleman, S. E., Gregory, M. V., Hamm, L. L., Koffman, L. D., Pevey, R. E, Reed, W. H., and Smith, III, F. G., 1989, "FLOWTRAN: An Algorithm for Describing the Thermal-Hydraulic Behavior of SRP Assemblies", DPSTM-140.

Battino, R., Rettich, T. R., and Tominaga, T., 1984, "The Solubility of Nitrogen and Air in Liquids", Journal of Physical and Chemical Reference Data, Vol. 13, No. 2, pp. 563-600.

Bergles, A. E., and Rohsenow, W. M., 1964, "The Determination of Forced-Convection Surface Boiling Heat Transfer", J. Heat Transfer 86, p. 365.

Biasi, L., Clerici, G. C., Garribba, S., Sala, R., and Tozzi, A., 1967, "Studies on Burnout, Part 3: A New Correlation for Round Ducts and Uniform Heating and Its Comparison with World Data", Energia Nucleare 14, p. 530.

Bjorge, R. W., and Rohsenow, W. M., 1982, "Correlation of Forced Convection Boiling Heat Transfer Data", Int. J. Heat and Mass Transfer 25 (6), p. 753.

Bjornard, T. A., and Griffith, P., 1977, "PWR Blowdown Heat Transfer", in Thermal and Hydraulic Aspects of Nuclear Reactor Safety 1, American Society of Mechanical Engineers, New York, p. 17.

Carpenter, E. F., and Colburn, A. P., 1951, "The Effect of Vapour Velocity on Condensation Inside Tubes", Proc. of General Discussion on Heat Transfer, 2026, IMechE/ASME.

Chen, J. C., 1963, "A Correlation for Boiling Heat Transfer to Saturated Fluids in Convective Flow", ASME 63-HT-34, American Society of Mechanical Engineers.

Collier, J. G., 1972, Convective Boiling and Condensation, 2nd edition, McGraw-Hill Book Company, Inc., p. 330.

Collier, J. G., 1981, "Forced Convection Boiling", in Two Phase Flow and Heat Transfer, ed. A. E. Bergles, J. G. Collier, J. M. Delhaye, G. F. Hewitt, and F. Mayinger, Chap. 8, Hemisphere, Washington.

Dimenna, R. A., Larson, J. R., Johnson, R. W., Larson, T. K., Miller, C. S., Streit, J. E., Hanson, R. G., and Kiser, D. M., 1988, "RELAP5/MOD2 Models and Correlations", NUREG/CR-5194, Idaho National Engineering Laboratory, Idaho Falls, Idaho.

Dittus, F. W., and Boelter, L. M. K., 1930, "Heat Transfer in Automobile Radiators of the Tubular Type", University of California (Berkley), Publications in Engineering 2, p. 443.

Dougall, R. S., and Rohsenow, W. M., 1963, "Film Boiling on the Inside of Vertical Tubes with Upward Flow of the Fluid at Low Qualities", Massachusetts Institute of Technology Report 9079-76. 
Forslund, R. P., and Rohsenow, W. M., 1968, "Dispersed Flow Film Boiling", J. Heat
Transfer 90 (6), p. 399.

Groeneveld, D. C., and Stewart, J. C., 1983, "The Minimum Film Boiling Temperature for Water During Film Boiling Collapse", in Proc. 2nd Int. Topical Meeting on Nuclear Reactor Thermal-Hydraulics, Santa Barbara, Calif., p. 393.

Guerrero, H. N., 1990, "Heated Annulus ECS Tests in Rig B", WSRC-TR-90-489.

Hodges, M. W., and Knoebel, D. H., 1973, "Subcooled Burnout Phenomena Adjacent to a Spacer Rib", ASME 73-HT-40, American Society of Mechanical Engineers.

Holman, J. P., 1976, Heat Transfer, 4th edition, McGraw-Hill Book Company, Inc., p. Kays, W. M., 1966, Convective Heat and Mass Transfer, McGraw-Hill Book Company,
Inc.

Kelly, J. E., 1980, "Development of a Two-Fluid, Two-Phase Model for Light Water Reactor Subchannel Analysis," PhD. Thesis, Massachusetts Institute of
Technology.

Liles, D. R., Spore, J. W., Knight, T. D., Nelson, R. A., Cappiello, M. W., Pasamehmetoglu, K. O., Mahaffy, J. H., Guffee, L. A., Stumpf, H. J., Dotson, P. J., Steinke, R. G., Shire, P. R., Greiner, S. E., and Sherwood, K. B., 1988, "TRAN-PF1/MOD1 Correlations and Models", NUREG/CR-5069.

Lockhart, R. W., and Martinelli, R. C., 1949, "Proposed Correlation of Data for Isothermal Two-Phase Two-Component Flow in Pipes", Chem. Eng. Prog. 45, p.
39.

Mikic, B. B., and Rohsenow, W. M., 1969, "A New Correlation of Pool Boiling Data Including the Effect of Heating Surface Characteristics", Trans. Am. Soc. Mech. Engrs., Series C, J. Heat Transfer 91, p. 245.

Nusselt, W. 1916, "Die Oberflachenkondensation des Wasserdampfes", Zeitsch. Ver. deutsch. Ing., 60, 541 and 569.

Pomeranz, M. L., 1964, "Film Boiling on a Horizontal Tube in Increased Gravity Fields",
J. Heat Transfer, 86, p. 213.

Ransom, V. H., Wagner, R. J., Trapp, J. A., Feinauer, L. R., Johnsen, G. W., Kiser, D. M., and Riemke, R. A., 1985, "RELAP5/MOD2 Code Manual Volume 1: Code Structure, System Models, and Solution Methods", NUREG/CR-4312, EG\&G
Idaho, Inc. Rohsenow, W. M. and Choi, H., 1961, Heat, Mass, and Momentum Transfer, Prentice-
Hall, Inc.

Rohsenow, W. M., Hartnett, J. P., and Ganic, E. N., 1973, Handbook of Heat Transfer Fundamentals, 2nd edition, McGraw-Hill Book Company, Inc. 
Rouhani, S. Z., and Axelsson, E., 1970, "Calculation of Void Volume Fraction in Subcooled and Quality Boiling Regions", Int. J. Heat and Mass Transfer 13, p. 383.

Sieder, E. N., and Tate, C. E., 1936, "Heat Transfer and Pressure Drop of Liquids in Tubes", Ind. Eng. Chem. 28, p. 1429.

Stephan, E. F., Berry, W. E., and Fink, F. W., 1962, Helium Solubility in D2O at Elevated Temperatures and Pressures, Report No. BMI-1587, Battelle Memorial Institute.

Thurgood, M. J., Kelly, J. M., Guidotti, T. E., Kohrt, R. J., and Crowell, K. R., 1983, "COBRA/TRAC - A Thermal Hydraulics Code for Transient Analysis of Nuclear Reactor Vessels and Primary Coolant Systems", NUREG/CR-3046, Pacific Northwest Laboratory.

Treybal, R. E., 1980, Mass-Transfer Operations, 3rd edition, McGraw-Hill Book Company, Inc.

Whalley, P. B., 1987, Boiling, Condensation and Gas-Liquid Flow, Oxford University Press.

Zuber, N., Tribus, M., and Westwater, J. W., 1961, "The Hydrodynamic Crisis in Pool Boiling of Saturated and Subcooled Liquids", International Developments in Heat Transfer, Part II, p. 230. 


\begin{tabular}{|l|lll|l|}
\hline $2 / 15 / 93$ & WSRC.TR-92-532 Rev.0 & FLOWTRAN-TF Software Design & Page 128 \\
\hline
\end{tabular}

\subsubsection{Wall Drag}

Due to the particular geometry of the Mk22 assembly, separate although similar wall drag models are used in the axial and azimuthal momentum balances. The models are developed individually in the following two sub-sections.

\subsubsection{Axial Wall Drag Model}

The mixture or total wall drag force in the axial ( $z$ ) direction is constructed by summing phasic contributions. For single-phase liquid and two-phase flows, the wall is assumed to be completely wetted and, except for single-phase gas flow, all frictional drag is attributed to the liquid phase. Both phases may experience a form loss however. One-dimensional axial flow is assumed in developing the wall drag model. Application of the model to twodimensional flows is considered subsequently.

Model development: The wall frictional drag model is an extension of familiar singlephase flow concepts. The wall frictional drag for a single-phase liquid flow through a circular channel is given by

$$
F_{w f}=\frac{f_{f}}{D} \frac{1}{2} \rho_{f}\left|u_{f}\right| u_{f}
$$

where

$$
\begin{aligned}
& F_{w f} \equiv \text { wall frictional drag force per unit total volume; frictional pressure drop } \\
&\left(\mathrm{N} / \mathrm{m}^{3}=\mathrm{Pa} / \mathrm{m}\right) \\
& \mathrm{f}_{\mathrm{f}} \equiv \text { D'Arcy friction factor }^{\prime} \\
& D \equiv \text { Diameter } \\
& \rho_{\mathrm{f}} \equiv \text { liquid density } \\
& u_{\mathrm{f}} \equiv \text { liquid average velocity }
\end{aligned}
$$

The basic concept behind the general FLOWTRAN-TF frictional drag model is illustrated in Figure 2.5.2.1-1. Any two flows having the same average liquid phasic velocity are assumed to have a similar liquid velocity gradient, and therefore shear stress, at the completely wetted wall. In this case, equation (2.5.2.1-1) is valid for two-phase as well single-phase flows. Flach (1991) has shown this assumption to be quite good. The COBRA/TRAC thermal-hydraulic code also models two-phase frictional drag in terms of phasic velocity squared (Thurgood et al., 1983). Note that model correctly predicts a twophase flow of a given liquid flowrate will have a higher drag than a single-phase liquid flow at the same flowrate.

Equation (2.5.2.1-1) applies to circular cross-sections so extensions are needed to handle the non-circular geometries encountered in FLOWTRAN-TF applications. Based on the works of Jones (1976) and Jones and Leung (1981), Blevins (1984, Chap. 6) proposes the characteristic dimension in the Reynolds number used to determine the friction factor be a modified hydraulic diameter, $\mathrm{D}_{\mathrm{e}}$, defined by 


$$
D_{e}=K_{h} D_{h}
$$

where

$$
\begin{aligned}
& D_{e} \equiv \text { corrected hydraulic diameter } \\
& D_{h} \equiv \text { hydraulic diameter, } 4 A_{c} / P \\
& K_{h} \equiv \text { hydraulic diameter correction factor }
\end{aligned}
$$

The hydraulic diameter in equation $(2.5 .2 .1-1)$ is left as is however. Blevins (1984) has tabulated the information needed to compute $K_{h}$ for a wide variety of geometries. Table 2.5.2.1-II gives the hydraulic diameter correction factors for the geometries encountered in FLOWTRAN-TF applications. For convenient evaluation of the turbulent regime friction factor, an explicit approximation to the Colebrook-White equation due to Jain (1976) is chosen (Blevins, 1984, Chap. 6).

The complete FLOWTRAN-TF axial frictional drag model for both phases is given by

$$
\begin{gathered}
F_{w f}^{z}=\frac{\theta \Phi^{2} f_{f}^{2}}{D_{h}^{2}} \frac{1}{2} \rho_{f}\left|u_{f}\right| u_{f} \\
F_{w g}^{z}=\frac{(1-\theta) \Phi^{2} f_{g}^{z}}{D_{h}^{2}} \frac{1}{2} \rho_{g}\left|u_{g}\right| u_{g} \\
\operatorname{Re}_{k}^{z}=\frac{\rho_{k}\left|u_{k}\right| K_{h} D_{h}}{\mu_{k}}, \quad k=f, g
\end{gathered}
$$

and the friction factor expressions are given in Table 2.5.2.1-I. The 'wetted wall parameter' $\theta$ is defined to be the fraction of the wall in contact with the liquid phase. The wall heat transfer logic defines this parameter. For pre-CHF conditions $\theta=1$ and in the film boiling regime or single-phase gas region $\theta=0$. In the transition boiling regime, $\theta$ is defined by equation (2.5.1-53) $(\theta=\delta)$. The 'L/D correction factor' $\Phi^{2}$ is included to allow the user to compensate for minor differences in chaninel length between the computational mesh and the actual geometry. For example, all channels in the Middle Section of the computational mesh must have the same axial length (\$2.1). The actual channels vary slightly in length. The correct frictional pressure loss is obtained by setting $\Phi^{2}$ equal to the aciual channel length divided by the computational channel length. The $\Phi^{2}$ parameter can also be used as a switch to turn off frictional pressure losses if only form losses are desired by choosing $\Phi^{2}=0$.

The wall form drag model is an extension of homogeneous two-phase flow concepts. For a homogeneous flow $\left(u_{g}=u_{f} \equiv u\right)$ form loss can be modeled as

$$
\begin{aligned}
F_{w} & =\frac{K}{\Delta z} \frac{1}{2} \rho|u| u=\frac{K}{\Delta z} \frac{1}{2}\left[\alpha \rho_{g}+(1-\alpha) \rho_{f}\right]|u| u \\
& =\frac{(1-\alpha) K}{\Delta z} \frac{1}{2} \rho_{f}|u| u+\frac{\alpha K}{\Delta z} \frac{1}{2} \rho_{g}|u| u
\end{aligned}
$$


where

$$
\begin{aligned}
F_{w} & \equiv \text { mixture (total) form pressure loss } \\
K & \equiv \text { form loss coefficient } \\
\Delta z & \equiv \text { computational cell length } \\
\rho & \equiv \text { mixture density } \\
u & \equiv \text { velocity (both phases) } \\
\alpha & \equiv \text { void fraction } \\
\rho_{k} & \equiv \text { density of } k \text { phase }
\end{aligned}
$$

The FLOWTRAN-TF form loss model extends equation (2.5.2.1-6) to non-homogeneous flows by replacing $\mathrm{u}$ with phasic velocities. The result is

$$
\begin{gathered}
F_{w f}^{z}=\frac{(1-\alpha) K^{z}}{\Delta z} \frac{1}{2} \rho_{f}\left|u_{f}\right| u_{f} \\
F_{w g}^{z}=\frac{\alpha K^{2}}{\Delta z} \frac{1}{2} \rho_{g}\left|u_{g}\right| u_{g}
\end{gathered}
$$

The form loss coefficient $\mathrm{K}^{2}$ may be determined from calibration to experimental data or literature recommendations (also based on experimental data). Form loss coefficients are typically quite geometry dependent. Therefore it is important that the experimental data used to define $\mathrm{K}^{2}$ be for the exact geometry under consideration.

The combined wall drag expressions for both frictional and form losses become

$$
\begin{aligned}
& F_{w f}^{z}=\left[\frac{\theta \Phi^{z} f_{f}^{z}}{D_{h}^{z}}+\frac{(1-\alpha) K^{z}}{\Delta z}\right] \frac{1}{2} \rho_{f}\left|u_{f}\right| u_{f} \\
& F_{w g}^{z}=\left[\frac{(1-\theta) \Phi^{z} f_{g}^{z}}{D_{h}^{z}}+\frac{\alpha K^{z}}{\Delta z}\right] \frac{1}{2} \rho_{g}\left|u_{g}\right| u_{g}
\end{aligned}
$$




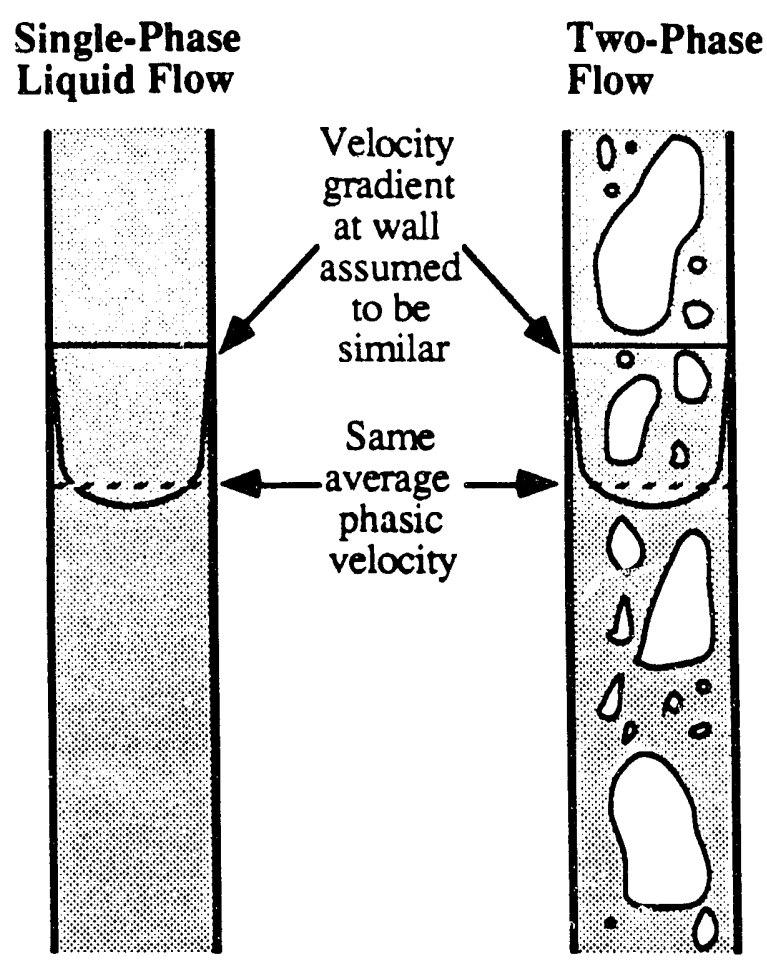

Figure 2.5.2.1-1 Comparison of velocity profiles for single-phase liquid and twophase flows having the same phasic liquid velocity.

Table 2.5.2.1-I Friction factor expressions in FLOWTRAN-TF.

\begin{tabular}{|c|c|c|}
\hline Regime & Friction Factor & Reference \\
\hline $\begin{array}{c}\text { Laninar: } \\
\operatorname{Re} \leq 2000\end{array}$ & $\begin{array}{c}\text { Exact solution: } \\
\mathrm{f}_{\mathrm{L}}=\frac{64}{\mathrm{Re}}\end{array}$ & \\
\hline $\begin{array}{c}\text { Transition: } \\
2000<\operatorname{Re}<4000\end{array}$ & $\begin{array}{c}\text { Interpolation: } \\
\mathrm{f}_{\mathrm{L}-\mathrm{T}}=\left(2-\frac{4000}{\mathrm{Re}}\right)\left(\mathrm{f}_{\mathrm{T}, 4000}-\mathrm{f}_{\mathrm{L}, 2000}\right)+\mathrm{f}_{\mathrm{L}, 2000}\end{array}$ \\
\hline $\begin{array}{c}\text { Turbulent: } \\
\operatorname{Re} \geq 4000\end{array}$ & $\begin{array}{c}\text { Explicit approximation to Colebrook-White: } \\
\mathrm{f}_{\mathrm{T}}=\left[1.14-2 \log _{10}\left(\frac{\varepsilon}{\mathrm{D}_{\mathrm{h}} \mathrm{K}_{\mathrm{h}}}+\frac{21.25}{\operatorname{Re}^{0.9}}\right)\right]^{-2}\end{array}$ & $\begin{array}{c}\text { Jain (1976), } \\
\text { Blevins } \\
(1984)\end{array}$ \\
\hline
\end{tabular}


Table 2.5.2.1-II Hydraulic diameter correction factors for selected geometries.

\begin{tabular}{|c|c|c|}
\hline Geometry & Hydraulic Diameter Correction Factor & Reference \\
\hline $\begin{array}{c}\text { Rectangular } \\
\widetilde{a}_{\mathrm{b}<\mathrm{a}}^{\mathrm{b}}\end{array}$ & $\begin{array}{cc}\mathrm{K}_{\mathrm{h}}=\frac{2}{3}+\frac{11}{24}\left(\frac{\mathrm{b}}{\mathrm{a}}\right)\left(2-\frac{\mathrm{b}}{\mathrm{a}}\right) \\
\mathrm{b} / \mathrm{a} & \mathrm{K}_{\mathrm{h}} \overline{1.55} \\
1.00 & 1.010 \\
0.50 & 1.010 \\
0.10 & 0.867 \\
0.05 & 0.754 \\
0.00 & 0.711 \\
& 0.667\end{array}$ & $\begin{array}{c}\text { Row 12 } \\
\text { Blevins } \\
(1984) \\
\text { Table 6-2 }\end{array}$ \\
\hline Ribbed Annular & \begin{tabular}{cc}
\multicolumn{2}{c}{ Interpolate using: } \\
$\mathrm{r}_{\mathrm{i}} / \mathrm{r}_{\mathrm{O}}$ & $\mathrm{K}_{\mathrm{h}}$ \\
0.1 & $1.08 \overline{1}$ \\
0.3 & 1.080 \\
0.5 & 0.998 \\
0.7 & 0.859 \\
0.9 & 0.726
\end{tabular} & $\begin{array}{c}\text { Row } 7 \\
\text { Blevins } \\
\text { (1984) } \\
\text { Table 6-2 }\end{array}$ \\
\hline Annular & 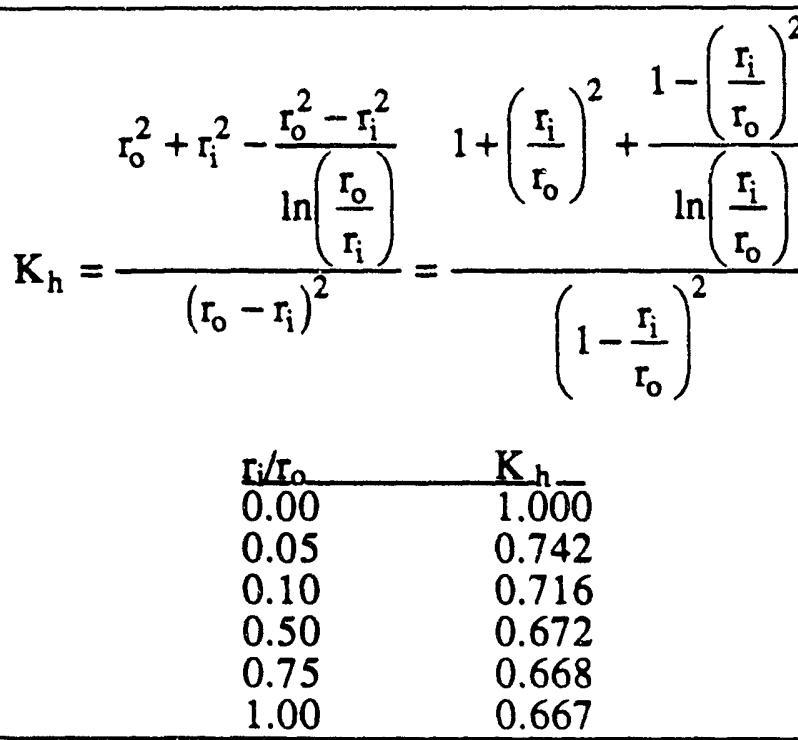 & $\begin{array}{c}\text { Row } 5 \\
\text { Blevins } \\
\text { (1984) } \\
\text { Table 6-2 }\end{array}$ \\
\hline Circular & $\mathrm{K}_{\mathrm{h}}=1.0$ & $\begin{array}{l}\text { Row 1 } \\
\text { Blevins } \\
\text { (1984) } \\
\text { Table 6-2 }\end{array}$ \\
\hline
\end{tabular}


Two-dimensional flow considerations: The above wall drag model was developed implicitly assuming a one-dimensional axial flow. The validity of the model for predicting the axial wall drag in the presence of a two-dimensional flow field is a concern. Indeed, a more natural derivation of the scalar components of a total wall frictional drag $\mathbf{F}_{\mathrm{w}}$ is

$$
F_{w}=\frac{f}{D_{h}} \frac{1}{2} \rho|v| v=\frac{f}{D_{h}} \frac{1}{2} \rho|v|(u i+v j)=\left(\frac{f}{D_{h}} \frac{1}{2} \rho|v| u\right) i+\left(\frac{f}{D_{h}} \frac{1}{2} \rho|v| w\right) j
$$

as opposed to

$$
F_{w}=\left(\frac{f}{D_{h}} \frac{1}{2} \rho|u| u\right) i+\left(\frac{f}{D_{h}} \frac{1}{2} p|w| w\right) \mathbf{j}
$$

which is essentially the approach chosen for FLOWTRAN-TF. Ebeling-Koning et al. (1986) have addressed this issue for single-phase flow. The approaches indicated by (2.5.2.1-11) and (2.5.2.1-12) were considered as well as two additional models. Fortunately, the model given by $(2.5 .2 .1-12)$ is demonstrated by Ebeling-Koning et al. (1986) to be the overall best model among the 4 alternatives considered for modeling multidimensional flows. Their evaluation criteria included predictive capability and ease of implementation. The same conclusion is assumed to hold for two-phase flow too. Therefore models (2.5.2.1-9) and (2.5.2.1-10) are affirmed for two-dimensional flows as well as one-dimensional flows.

Computational implementation: In order to avoid a divide by zero and illconditioning, the laminar friction factor is computed as

$$
f_{L}=\frac{64}{\max (\operatorname{Re}, 50)}
$$

instead as indicated in Table 2.5.2.1-I. Computational versions of physical models (2.5.2.1-9) and (2.5.2.1-10) are also needed. All quantities are evaluated at the previous time-step (n) except velocities which are evaluated at the current time level $(n+1)$. Following \$3.4.1 equations (2.5.2.1-9) and (2.5.2.1-10) are then spatially averaged over a momentum computational cell. The resulting computational versions of (2.5.2.1-9) and $(2.5 .2 .1-10)$ at arbitrary location $j+1 / 2$ are

$$
\begin{aligned}
& F_{w f, j+1 / 2}^{z}=\left[\frac{\theta \Phi^{z} f_{f}^{z}\left(\langle\cdot\rangle_{j+1 / 2}^{n}\right)}{D_{h}^{z}}+\frac{\langle(1-\alpha) A\rangle_{j+1 / 2}^{n} K^{z}}{V_{z, j+1 / 2}}\right] \frac{1}{2}\left\langle\rho_{f}\right\rangle_{j+1 / 2}^{n}\left|u_{f, j+1 / 2}\right| u_{f, j+1 / 2} \\
& F_{w g, j+1 / 2}^{z}=\left[\frac{(1-\theta) \Phi^{z} f_{g}^{z}\left(\langle\cdot\rangle_{j+1 / 2}^{n}\right)}{D_{h}^{z}}+\frac{\langle\alpha A\rangle_{j+1 / 2}^{n} K^{z}}{V_{z, j+1 / 2}}\right] \frac{1}{2}\left\langle\rho_{g}\right\rangle_{j+1 / 2}^{n}\left|u_{g, j+1 / 2}\right| u_{g, j+1 / 2}
\end{aligned}
$$


Consult $\$ 3.4 .1$ for precise definitions of the various numerical quantities in the above expressions.

\section{References:}

Blevins, R. D., 1984, Applied Fluid Dynamics Handbook, Van Nostrand Reinhold, New York.

Ebeling-Koning, D. B., Robinson, J. T., and Todreas, N. E., 1986, "Models for the Fluid-Solid Interaction Force for Multidimensional Single Phase Flow Within Tube Bundles", Nucl. Engr. \& Design 91, pp. 29-40.

Flach, G. P., 1991, "Void Fraction Estimation Program Available", NES-CDG-910062.

Jain, A. K., 1976, "Accurate Explicit Equation for Friction Factor," ASCE J. Hydraulics Div. 102, pp. 674-677.

Jones, O. C., Jr., 1976, "An Improvement in the Calculation of Turbulent Friction in Rectangular Ducts", J. Fluids Engr. 98, pp. 173-180.

Jones, O. C., Jr., and Leung, J. C. M., 1981, "An Improvement in the Calculation of Turbulent Friction in Smooth Concentric Annuli", J. Fluids Engr. 103, pp. 615623.

Thurgood, M. J., Kelly, J. M., Guidotti, T. E., Kohrt, R. J., and Crowell, K. R., 1983, "COBRA/TRAC - A Thermal-Hydraulics Code for Transient Analysis of Nuclear Reactor Vessels and Primary Coolant Systems", NUREG/CR-3046, Volume 1, p. 3.29 .

\subsubsection{Azimuthal Wall Drag Model}

The experimental basis for modelling azimuthal cross-flow between sub-channels through the rib gaps of a Mk22 assembly is the study conducted by Tapucu (1977). He measured the pressure induced cross-flow between two parallel channels through long narrow slots, and used the data to calculate loss coefficients for a variety of flow rates and gap geometries. The result was a bewildering collection of curves. The Tapucu data has been analyzed by considering both axial and transverse momentum balances for the slot, and a coherent model has emerged. The development of this model is described in this section. Then the model is adapted for use in FLOWTRAN-TF.

Description of the Tapucu Experiment: Mass and momentum exchanges between parallel channels that are connected by a narrow slot occur due to turbulent mixing and cross-flow due to a lateral pressure gradient. The latter phenomena is herein analyzed. Tapucu (1977) measured the cross-flow between parallel channels. Figure 2.5.2.2-1 is a schematic of his experiment. The two channels have 0.5 in. square cross-sections, and they are connected by a slot narrow slot that is approximately three feet long. Slots with several widths and transverse lengths were tested. The controlled quantities in these tests were the inlet flow rates to the two channels. The axial variations in channel flow rates, cross-flow velocity and channel pressures were determined.

Most of the results are presented by Tapucu (1977) in the form of plots of loss coefficients versus non-dimensional parameters, so all of the measured quantities are not available. However, the presented data is complete for the test with the slot geometric parameters and 
channel inlet flow rates listed in Table 2.5.2.2-I. This data was used to develop the crossflow model.

Table 2.5.2.2-I Geometric and flow parameters of the Tapucu test used to develop the cross-flow model.

Slot gap width (W)

Transverse slot length

Axial slot length

Donor channel inlet flow rate

Recipient channel inlet flow rate
$1.016 \mathrm{~mm}$

$3.17 \mathrm{~mm}$

$846 \mathrm{~mm}$

$0.905 \mathrm{~m}^{3} / \mathrm{hr}$

$0.0 \mathrm{~m}^{3} / \mathrm{hr}$

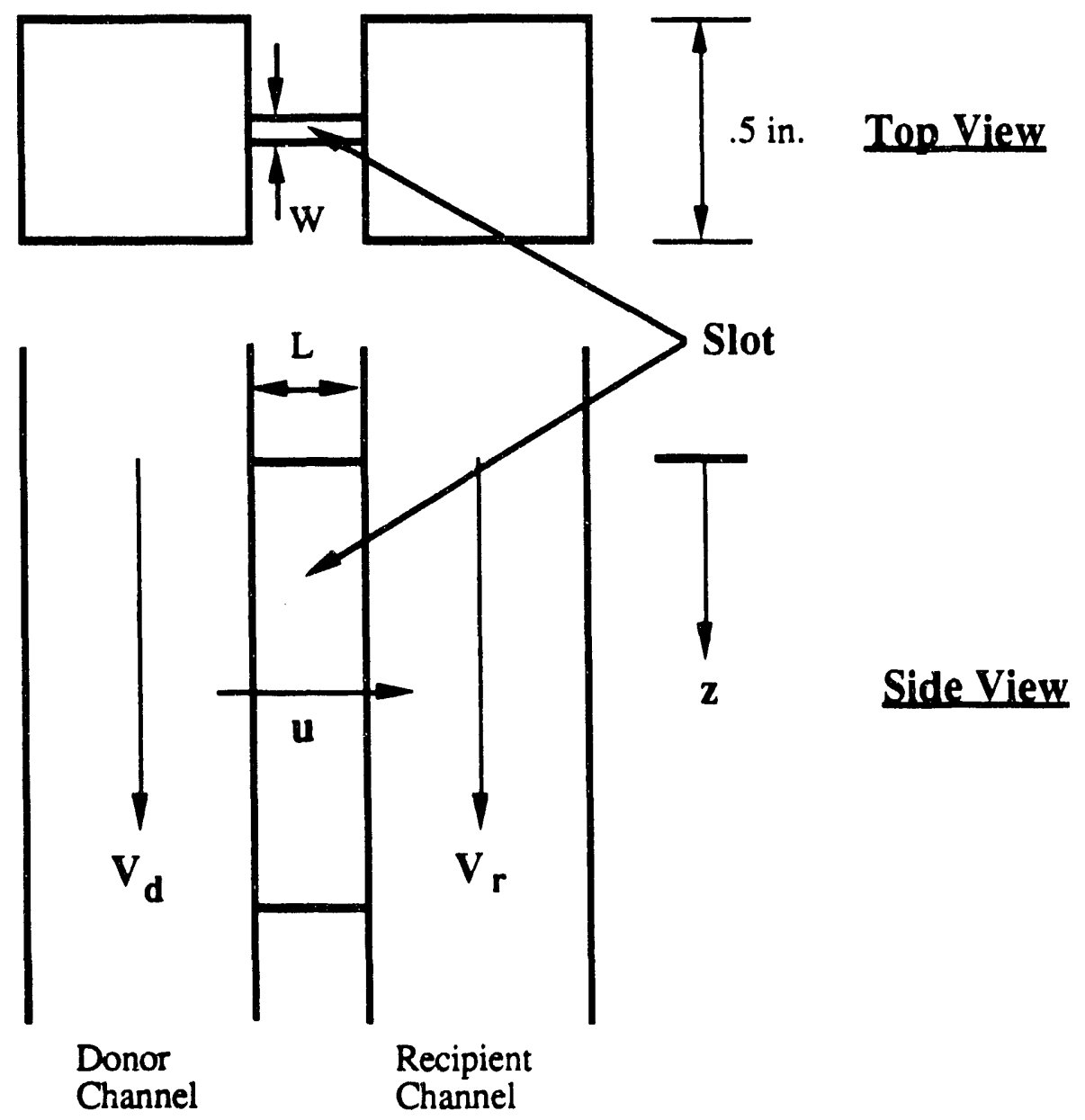

Figure 2.5.2.2-1 Schematic of the Tapucu cross-flow experiment .

Axial Component of the Slot Velocity: The flow in the slot has both axial and transverse velocity components, and both must be correctly modelled in order to calculate the correct axial momentum exchange between the two channels. The axial momentum of the slot flow is convected into the recipient channel. Equation (2.5.2.2-1) is an expression 
for the axial component of the slot velocity, and it is derived from the integral form of the momentum equation, applied to the differential section of the slot shown in Figure 2.5.2.2-2.

$$
V_{s}=\frac{\rho k V_{d}\left(\frac{u}{L}+\frac{1}{2} \frac{\partial V_{s}}{\partial z}\right)-\frac{\partial P}{\partial z}}{\rho\left(\frac{u}{L}+\frac{3}{2} \frac{\partial V_{s}}{\partial z}+\frac{f V}{2 D_{h}}\right)}
$$

where

$$
V=\sqrt{u^{2}+V_{s}^{2}}
$$

The friction force is evaluated with the slot velocity, $\mathrm{V}$, and axial and transverse components are used in the appropriate momentum equations. The term representing the convection of axial momentum from the donor channel into the slot has a form loss coefficient, $k$. This coefficient is the ratio of the actual to ideal momentum convected into the slot. The calculated axial components of the slot velocities, with $k=1$, are shown in Figure 2.5.2.2-3. These are the ideal values. Also shown are the values of $\mathrm{V}_{s}$ required by the axial pressure gradients in the recipient channel. The two curves are qualitatively similar, and the differences are due to form losses. The good qualitative agreement between the required and calculated ideal slot velocities supports the assumption that the slot axial pressure gradient is the mean of the two channel pressure gradients. The calculated values of $\mathrm{V}_{\mathrm{s}}$ with the friction factor set to zero are also shown. Friction losses in the slot are important, and they are of increasing relative importance as the transverse pressure drop and the cross-flow velocity decreases. The calculated slot velocity is insensitive to the axial gradient of the slot velocity.

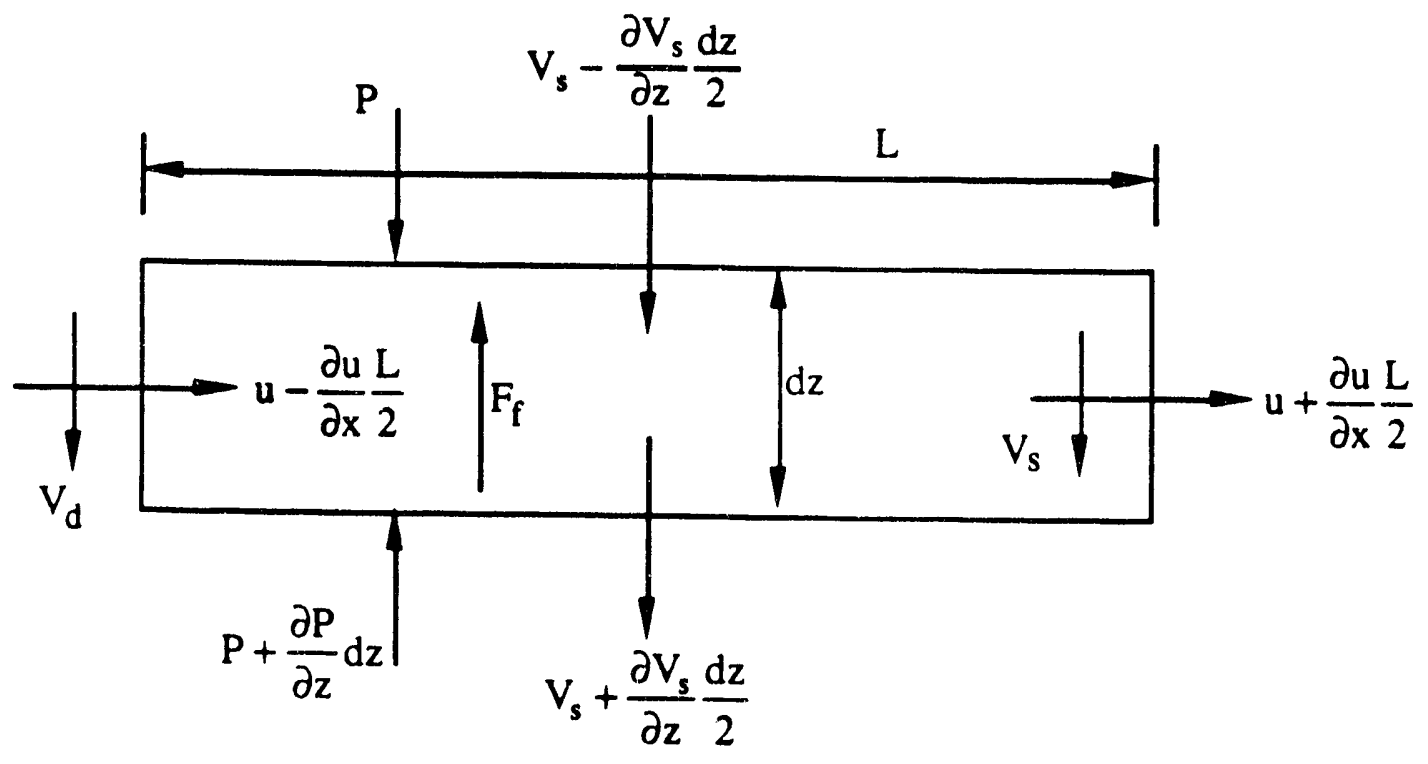

Figure 2.5.2.2-2 Differential control volume for the axial component of the slot momentum equation. 
The slot axial velocity form loss coefficients were calculated by manipulating equation (2.5.2.2-1) and using required values of Vs for the recipient channel pressure gradient. The results are shown in Figure 2.5.2.2-4. There is a sharp drop in the loss coefficient at low values of the cross-flow velocity. This corresponds to a relative increase in the importance of form losses.

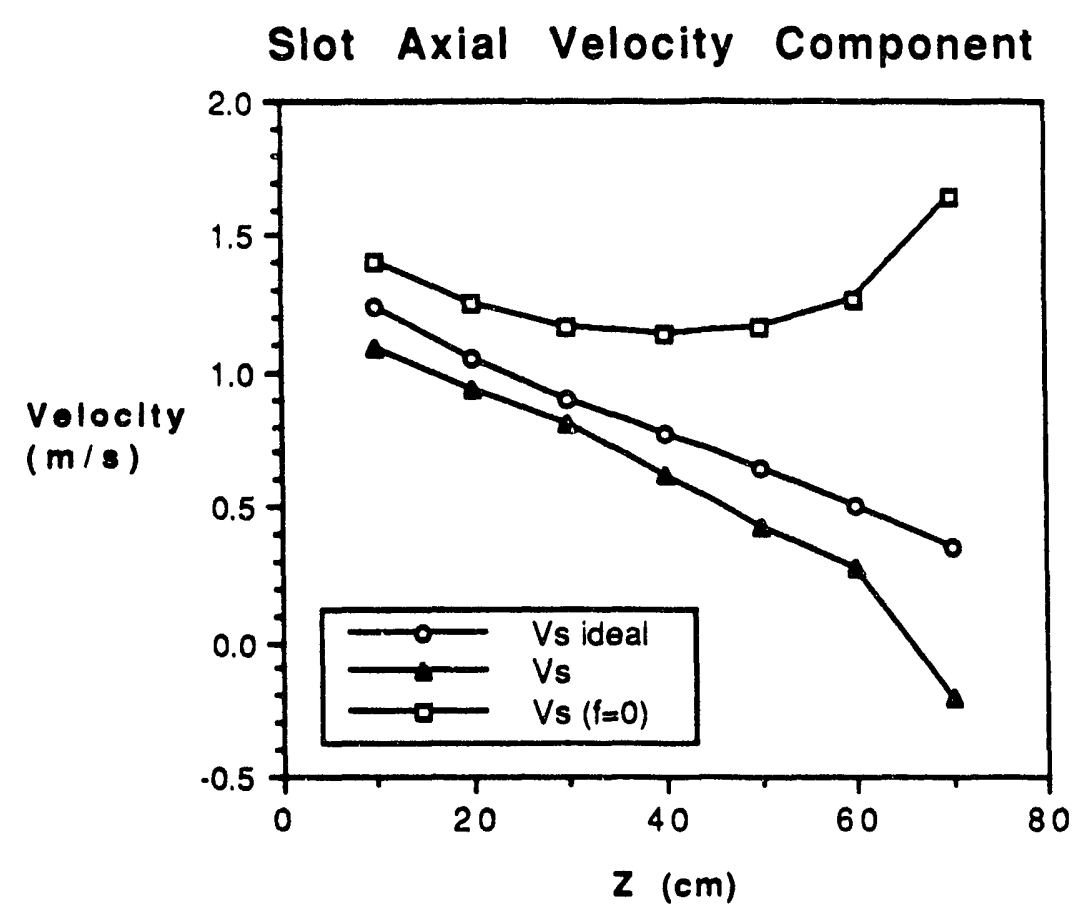

Figure 2.5.2.2-3 Slot axial velocity. 


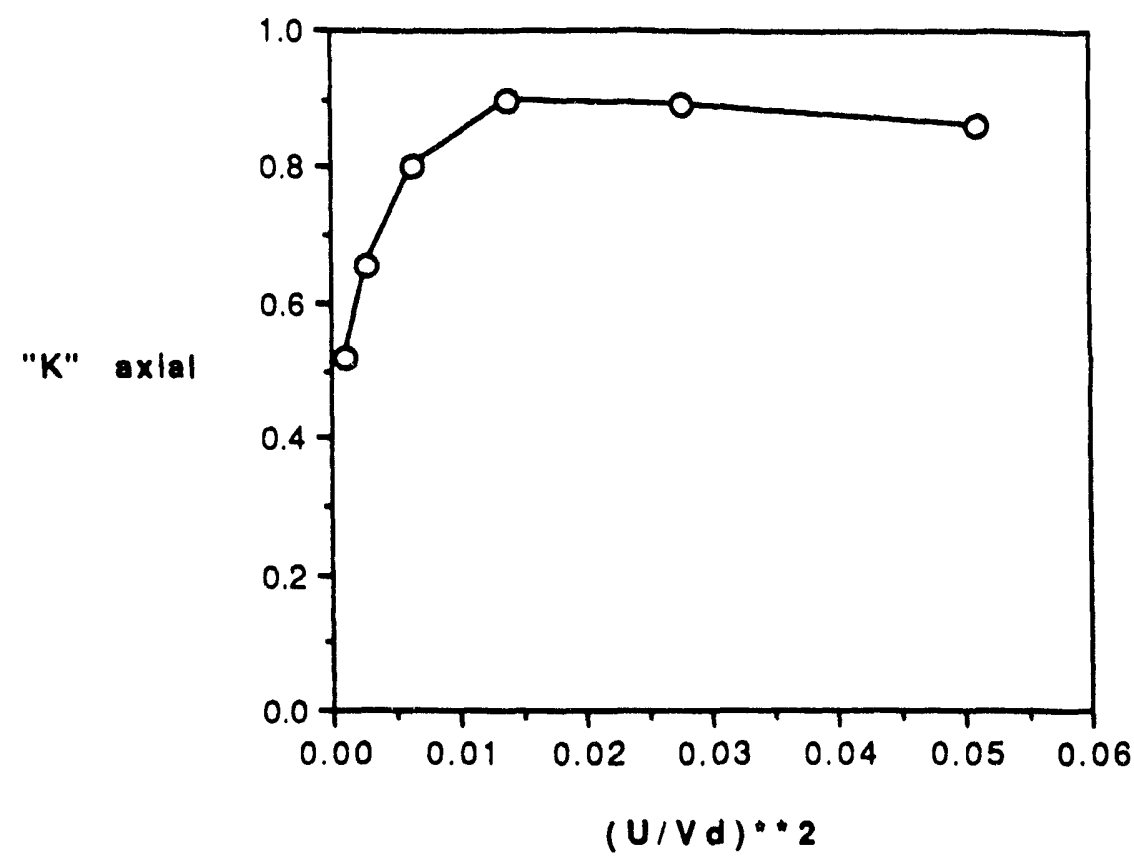

Figure 2.5.2.2.4 Slot axial velocity form loss coefficient.

Transverse Component of the Slot Velocity: The expression for the transverse component of the slot velocity is derived in a similar manner to that for the axial component. Figure 2.5.2.2-5 is a schematic of a differential section of the slot that is used to derive the transverse component of the momentum equation. The two lateral sides of this control volume are assumed to be stream lines. This simplifies the treatment of the convective terms. The friction force is handled in exactly the same manner as in the derivation of the expression for the axial slot velocity. Equation (2.5.2.2-2) is the expression for the pressure drop across the slot.

$$
\Delta P=P_{d}-P_{r}=\rho L\left(V_{s} \frac{\partial u}{\partial z}+\frac{f u V}{2 D_{h}}\right)
$$

The calculated and experimental transverse slot pressure drops are shown in Figure 2.5.2.2-6. The difference between the two curves is due to form losses, and it is evident for this slot that the dominant irreversible losses are form losses. The transverse form losses, equation (2.5.2.2-3), are defined in terms of the cross-flow velocity.

$$
\Delta \mathrm{P}_{\text {form }}=\frac{1}{2} \rho \mathrm{ku}^{2}
$$

Required values of the loss coefficient were calculated from the data, and the results are shown in Figure 2.5.2.2-7. Also shown is a fit of the data, equation (2.5.2.2-4). 


$$
k=1.55+.01068\left(\frac{u}{V_{d}}\right)^{-2}
$$

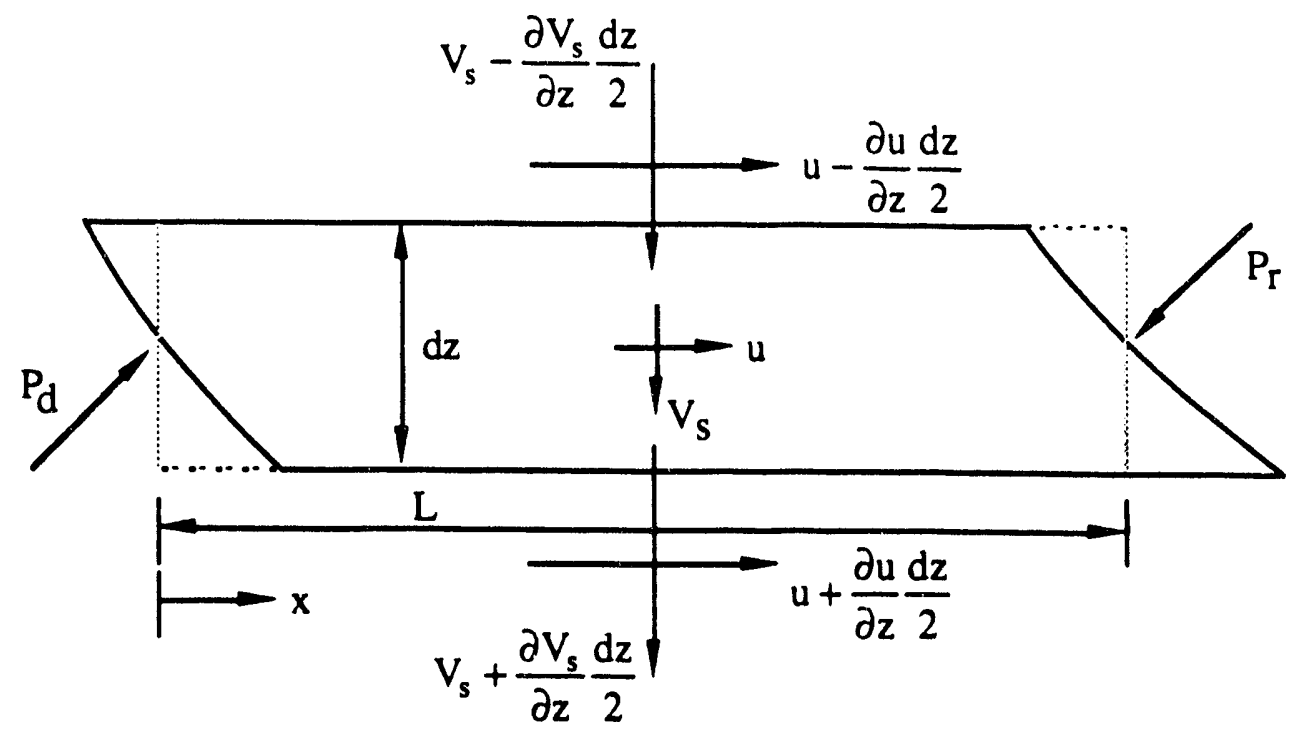

Figure 2.5.2.2-5 Differential control volume for the transverse component of the slot momentum equation.

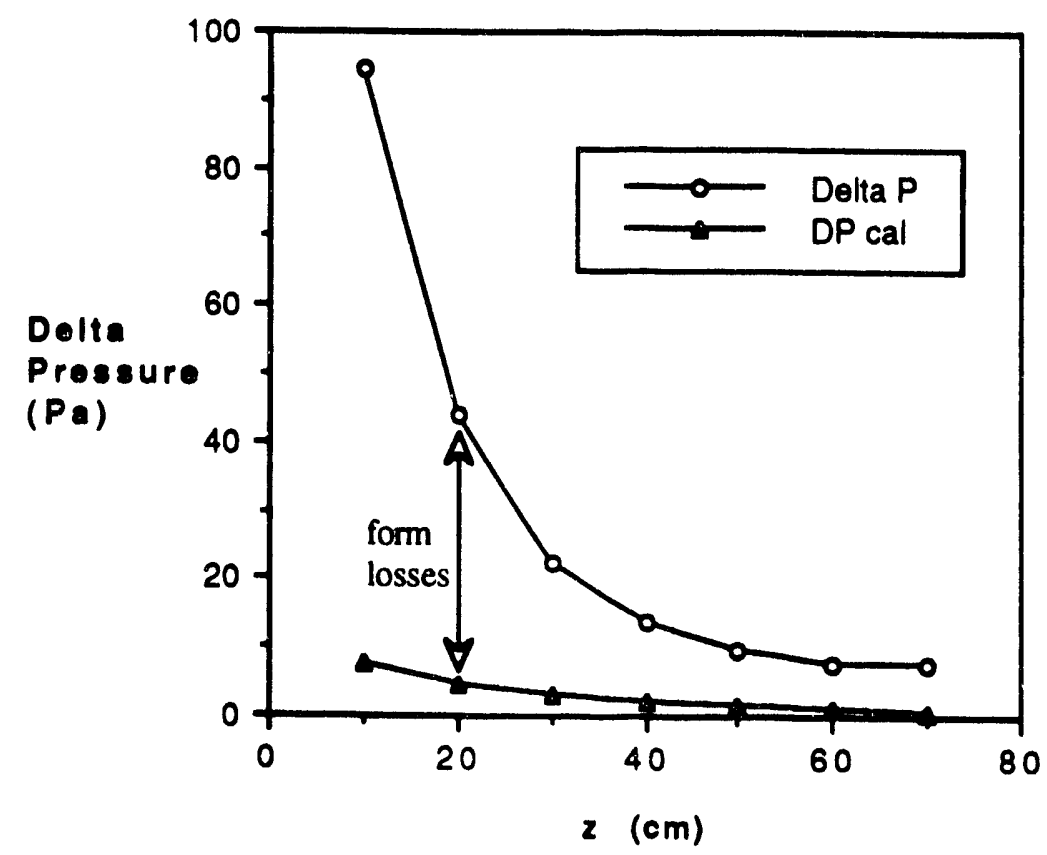

Figure 2.5.2.2-6 Slot transverse pressure drop. 


\begin{tabular}{|l|lll|l|}
\hline $2 / 15 / 93$ & WSRC-TR-92-532 Rev. 0 & FLOWTRAN-TF Software Design & Page 140 \\
\hline
\end{tabular}

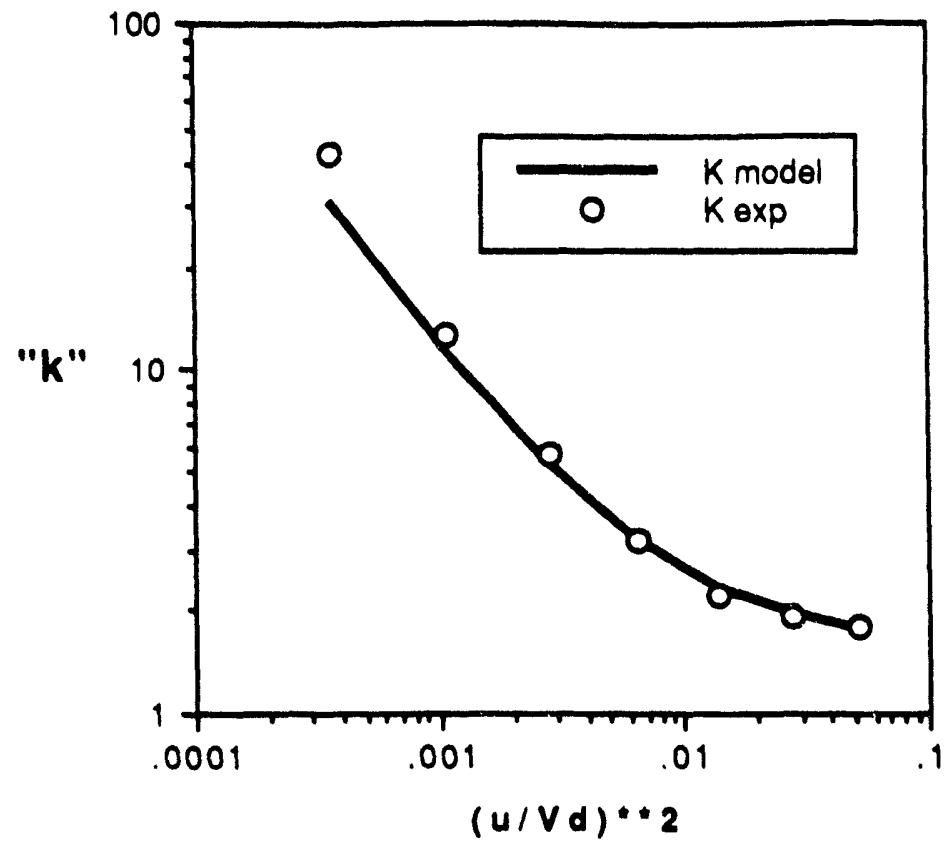

Figure 2.5.2.2-7 Cross-flow velocity loss coefficient.

Adding the form loss pressure drop to equation (2.5.2.2-2) yields the following quadratic equation for the cross-flow velocity.

$$
\left(\frac{1}{2} \rho k\right) u^{2}+\left(\rho \frac{f L V}{2 D_{h}}\right) u+\left(\rho L V_{s} \frac{\partial u}{\partial z}-\Delta P_{s l o t}\right)=0
$$

This equation and equation (2.5.2.2-1) are sufficient to solve for the cross-flow velocity. The axial momentum convected out of the donor channel is calculated with the channel axial velocity, and the axial momentum convected into the recipient channel is calculated with the slot axial velocity. Figure 2.5.2.2-3 shows that friction losses are important in the calculation of the slot axial velocity, and the friction losses are strongly geometry dependent.

Model Verification: A reasonable success criterion for the cross-flow model is the ability to simultaneously predict the axial velocity and pressure distributions in a laboratory test. The axial distributions of channel velocities, pressures, and the cross-flow velocity are strongly coupled. Figure 2 of Tapucu et. al. (1977b) shows the axial variations of pressure in both channels for the test described in Table 2.5.2.2-I. The ability to model the results of this test demonstrates that the parts of the cross-flow model are consistent. A numerical model of this test was developed. The model used a marching technique, simultaneously calculating the downstream values of the channel velocities and pressures and the axial and transverse components of the slot velocity. Test pressures and velocities at $\mathrm{z}=10 \mathrm{~cm}$. were used as the initial conditions for the model. This avoided the necessity of modelling entrance effects at the start of the channel. Results are shown in Figures 2.5.2.2-8 and 2.5.2.2-9, along with Tapucu's data. Figure 2.5.2.2-8 shows the channel 


\begin{tabular}{|l|lll|l|}
\hline $2 / 15 / 93$ & WSRC.TR-92.532 Rev. 0 & FLOWTRAN-TF Software Design & Page 141 \\
\hline
\end{tabular}

axial pressure distributions. The pressures are reduced pressures, with the local hydrostatic pressure subtracted out. The agreement is pretty good up to approximately $z=35 \mathrm{~cm}$. At $z=39 \mathrm{~cm}$, the predicted pressure profiles cross and the recipient channel pressure becomes larger than the donor channel pressure. Since the direction of cross-flow is assumed in the code, this is the end of the computation. Tapucu noted that the slot flow oscillated in regions where the channel pressure differences were small, so the pressure cross-over is not alarming.

Figure 2.5.2.2-9 shows the channel and cross-flow velocities, and again there is good agreement up to approximately $z=35 \mathrm{~cm}$. Thereafter the cross-flow velocity components drop sharply. This is understandable since pressure driven flow is assumed and the pressure difference drops to zero. Tapucu measured the cross-flow by injecting dye into one of the channels and determining the concentration changes in the channels optically. He assumed that turbulent mixing was negligible. At low channel pressure differences this assumption was probably poor and consequently the cross-flow flow rates were overpredicted.

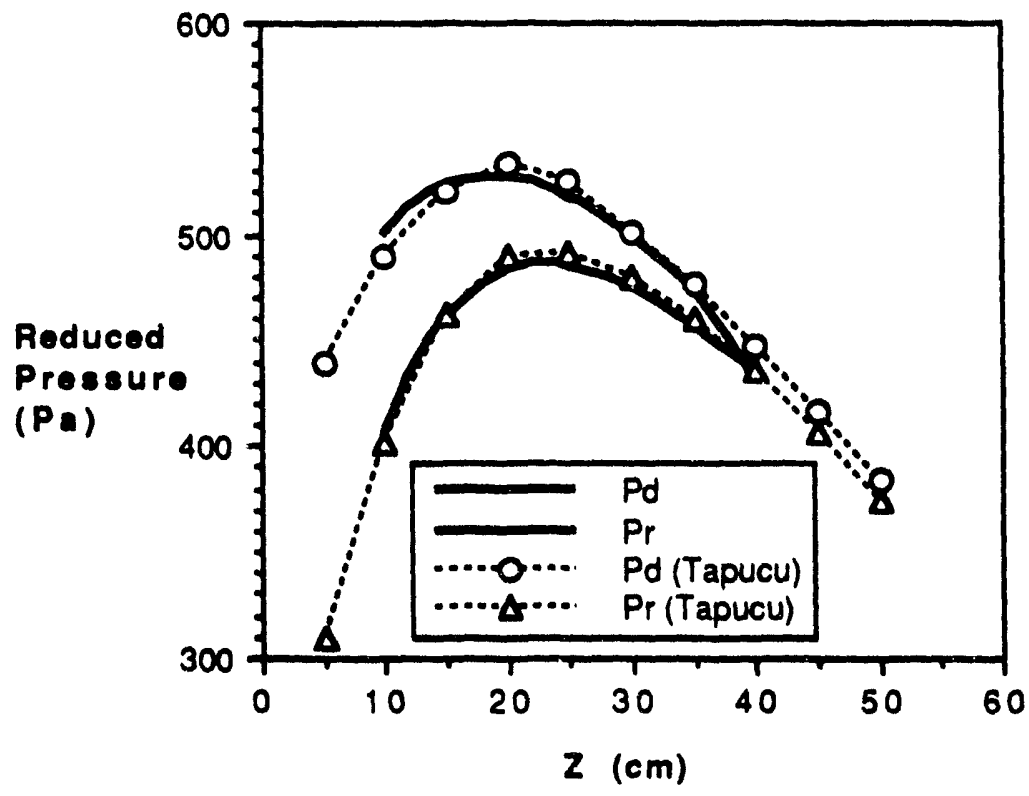

Figure 2.5.2.2-8 Theoretical and experimental channel axial pressure profiles. 


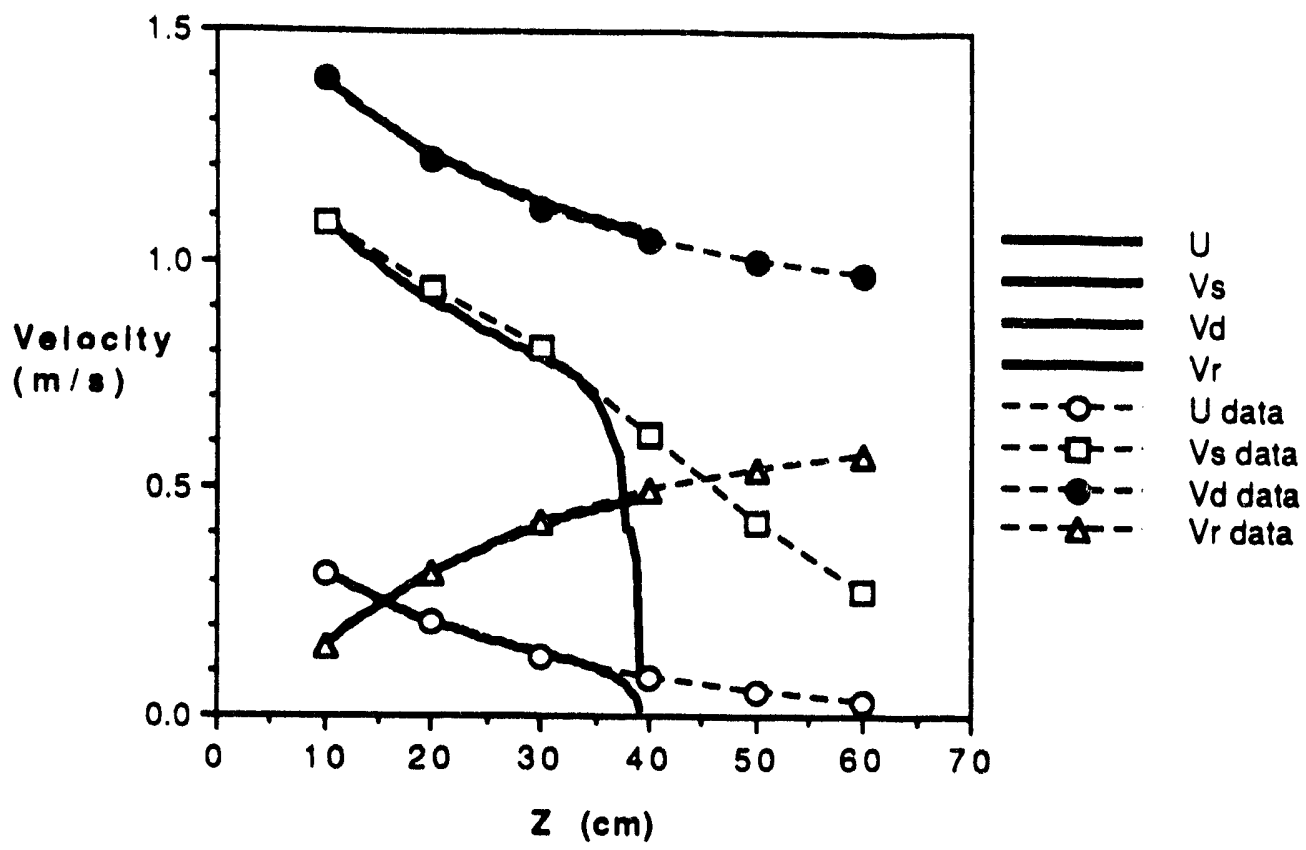

Figure 2.5.2.2.9 Theoretical and experimental channel and slot velocities.

Application to FLOWTRAN-TF: The azimuthal wall drag in FLOWTRAN-TF is the sum of friction and form losses. The model of the Mk-22 assembly used for LOCA/ECS power limits calculations has four azimuthal cells in each channel. Each subchannel is a cell, and the ribs form the lateral cell boundaries. All of the azimuthal pressure drop is assumed to occur across the ribs, and this is modelled as a form loss. Schedule constraints prohibit large scale modifications to the code architecture at this time, so the results of the cross-flow analysis were used to determine the correct form for the azimuthal form losses. The goal is to have the correct flow rate/ pressure drop relationship for the ribs. It is realized that the calculation of axial momentum exchange between the subchannels will be incorrect, since axial momentum is not dissipated under the ribs, but the mass exchange will be appropriately calculated.

To determine the appropriate functional relationship for the rib form loss coefficient, the model of the Tapucu experiment was modified to that of two parallel channels with the cross-section areas and hydraulic diameters of subchannels in the center flow channel of a Mk-22 assembly, connected by a slot with the width and transverse length of the nominal rib gap. This model was run with three different donor channel inlet velocities and slot pressure drops, shown in Table 2.5.2.2-II.

Table 2.5.2.2-II Subchannel cross-flow model inlet parameters

\begin{tabular}{llll} 
Case \# & $\mathrm{V}_{\mathrm{d}}(\mathrm{m} / \mathrm{s})$ & $\mathrm{V}_{\mathrm{r}}(\mathrm{m} / \mathrm{s})$ & $\Delta \mathrm{P}(\mathrm{Pa})$ \\
\hline 1 & 2.78 & 0.6 & 600 \\
2 & 1.39 & 0.15 & 300 \\
3 & 0.695 & 0.15 & 150
\end{tabular}


Figure 2.5.2.2-10 is a plot of the computed cross-flow velocities versus the rib pressure drops. It is evident that the cross-flow velocity is a single-valued function of the pressure drop, and since the three cases have significantly different donor channel axial velocities, the functional relationship is essentially independent of the donor channel axial velocity. Equation $(2.5 .2 .2-6)$ is the expression for the rib form loss.

$$
\Delta P_{\text {rib }}=\frac{1}{2} \rho K u^{2}
$$

Form loss coefficients for the ribs were calculated from the parallel channel results. These are plotted in Figure 2.5.2.2-11 along with a curve fit. The fit expression is equation $(2.5 .2 .2-7)$. The loss coefficient is a function of the rib gap Reynolds number.

$$
\mathrm{K}=1.56522+\frac{200}{\operatorname{Re}}
$$

where

$$
\operatorname{Re}=\frac{\rho u D_{h_{\mathrm{ab}}}}{\mu}
$$

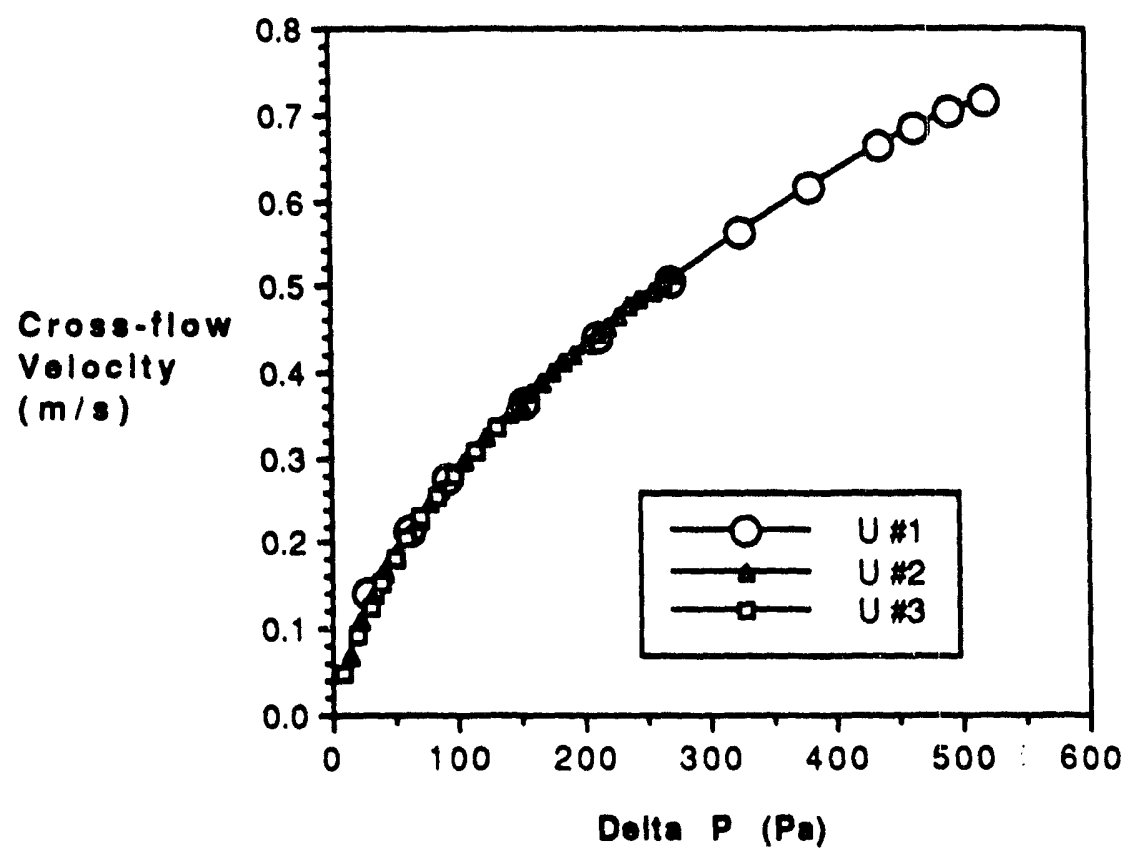

Figure $2.5 .2 .2-10$ Rib cross-flow for a Mk-22 assembly. 


\begin{tabular}{|l|l|l|l|}
\hline $2 / 15 / 93$ & WSRC-TR-92-532 Rev. 0 & FLOWTRAN-TF Software Design & Page 144 \\
\hline
\end{tabular}

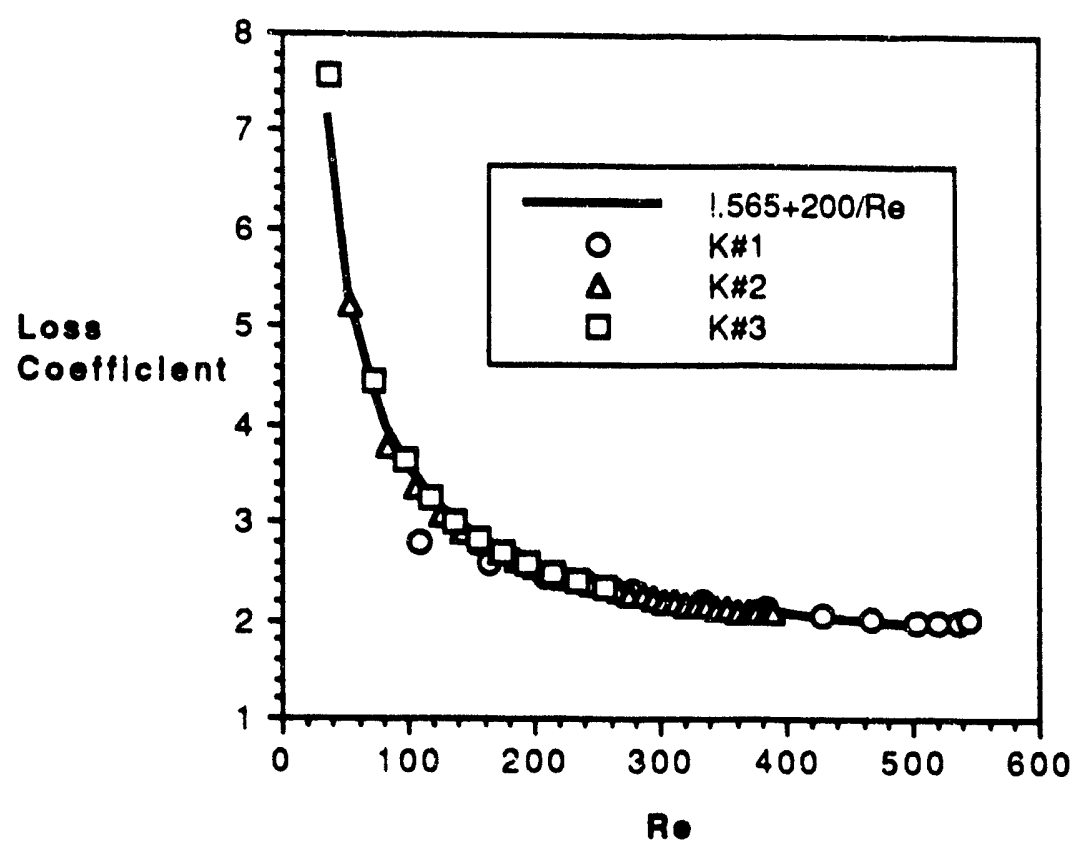

Figure 2.5.2.2-11 Rib form loss coefficient.

Complete azimuthal wall drag model: The complete wall drag expressions for both frictional and form losses in the azimuthal direction are

$$
\begin{aligned}
& F_{w f}^{x}=\left[\frac{\theta \Phi_{f_{f}^{x}}^{x}}{D_{h}^{x}}+K^{x} \frac{(1-\alpha) K}{\Delta x}\right] \frac{1}{2} \rho_{f}\left|w_{f}\right| w_{f} \\
& F_{w g}^{x}=\left[\frac{(1-\theta) \Phi^{x} f_{g}^{x}}{D_{h}^{x}}+K^{x} \frac{\alpha K}{\Delta x}\right] \frac{1}{2} \rho_{g}\left|w_{g}\right| w_{g}
\end{aligned}
$$

where the symbols are analogous to those in equations (2.5.2.1-9) and (2.5.2.1-9) with one exception. In equations (2.5.2.2-8) and $(2.5 .2 .2-8), \mathrm{K}$ is given by equation $(2.5 \cdot 2.2-7 \mathrm{a})$ and $\mathrm{K}^{\mathrm{x}}$ is a form loss multiplier. The symbol $w_{k}$ denotes the phasic azimuthal velocity of phase $k$. 


\section{References:}

Tapucu A., 1977a, "Studies on Diversion Cross-Flow Between Two Parallel Channels Communicating by a Lateral Slot. I: Transverse Flow Resistance Coefficient", Nuclear Engineering and Design, 42, pp 297-306.

Tapucu A. and M. Merilo, 1977b, "Studies on Diversion Cross-Flow Between Two Parallel Channels Communicating by a Lateral Slot. II: Axial Pressure Variations", Nisclear Engineering and Design, 42, pp 307-318. 
\begin{tabular}{|l|lll|r|}
\hline $2 / 15 / 93$ & WSRC-TR-92-532 Rev. 0 & FLOWTRAN-TT. Soft vare Design & Page 146 \\
\hline
\end{tabular}

\subsubsection{Flow Regime Map and Interfacial Area}

The topology of a two-phase flow typically has a strong influence on the various constitutive terms appearing in the two-phase conservation equations, such as interfacial drag, wall heat transfer and interfacial heat and mass transfer among others. Universal models for the constitutive terms, applicable to all flow regimes, are generally not available. Rather the flow regime is first deduced from global flow information such as void fraction and superficial velocities. Then a flow regime specific model is selected to model each constitutive term. This approach is taken in FLOWTRAN-TF. Clearly, accurate inference of flow regime is an important prerequisite for modeling each constitutive term. The flow regime map employed by FLOWTRAN-TF is described in section 2.5.3.1 below.

Associated with each flow regime is the area per unit volume at the interface between the gas and liquid phases termed 'interfacial area'. Knowledge of the interfacial area is necessary in defining the rates of interfacial heat and mass transfer. The FLOWTRAN-TF interfacial area model is described in section 2.5.3.2.

\subsubsection{Flow Regime Map}

Many flow regime works in the open literature are compiled in the References subsection, especially recent papers and those particularly pertinent to FLOWTRAN-TF applications. In addition Kielpinski (1992) has reported unqualifies flow regime data for a ribbed annular flow channel. These data must unfortunately be ignored henceforth pending data qualification for critical applications. Historically, the FLOWTRAN-TF flow regime map was based on the logic used in RELAP (Ransom et al., 1985) for vertically upward twophase flow, which in turn was based mainly on Taitel et al. (1980). The FLOWTRAN-TF map now relies directly on Taitel et al. (1980) and in addition on the works of Barnea (1987), Barnea (1986) and Barnea et al. (1982b) which address downward flow. The regime transition logic is still based on RELAP. The flow regime transition models developed by Barnea (1987), Barnea (1986), Barnea et al. (1982b) and Taitel et al. (1980) were chosen due to their relatively strong mechanistic basis. These four references represent an evolution of flow regime logic applicable to the whole range of pipe inclinations with the later references building upon earlier ones. For brevity, the collection of Barnea (1987), Barnea (1986), Barnea et al. (1982b) and Taitel et al. (1980) will frequently be cited as "Barnea et ai." (no date specified) in the following discussion.

The discussion below begins with a summary of the flow regime transition logic developed by Barnea et al. for the special case on vertically downward two-phase flow encountered in FLOWTRAN-TF applications. The resulting flow regime map for the dimensions of the VOID Rig (Whatley, 1990) is presented to illustrate the logic for a typical FLOWTRAN-TF application. Computational implementation is presented next with the bulk of the discussion covering interpolation between flow regimes. Finally, the FLOWTRAN-TF flow regime map exactly as coded is illustrated.

Flow regime transition models of Barnea et al.: Barnea et al. define four flow regimes for the purpose of deriving regime transition models: bubbly, dispersed bubble, intermittent (slug or churn) and annular. The topology of the bubbly and dispersed bubble regimes is nominally the same. The difference is the mechanism for their creation. Bubbly refers to sufficiently low void fraction bubble flows thai bubble coalescence is negligible. Dispersed bubble refers to flows having a wider range of void fractions which have 
sufficient turbulence to break up large bubbles as they form by coalescence. Taitel et al. (1980) define the various flow regimes for upward flow as

" 1. Bubble Flow: The gas phase is approximately uniformly distributed in the form of discrete bubbles in a continuous liquid phase.

2. Slug Flow: Most of the gas is located in large bullet shaped bubbles which have a diameter almost equal to the pipe diameter. They move uniformly upward and are sometimes designated as "Taylor bubbles." Taylor bubbles are separated by slugs of continuous liquid which bridge the pipe and contain small gas bubbles. Between the Taylor bubbles and the pipe wall, liquid flows downward in the form of a thin falling film. (This pattern has been designated by others as plug, or piston, flow at low rates where the gas liquid boundaries are well defined, and as slug flow at higher rates where the boundaries are less clear.)

3. Churn Flow: Churn flow is somewhat similar to slug flow. It is, however, much more chaotic, frothy and disordered. The bullet-shaped Taylor bubble becomes narrow, and its shape distorted. The continuity of the liquid in the slug between successive Taylor bubbles is repeatedly destroyed by a high local gas concentration in the slug. As this happens, and liquid slug falls [sic]. This liquid accumulates, forms a bridge and is again lifted by the gas. Typical of churn flow is this oscillatory or alternating direction of motion of the liquid. (Some observers refer to a froth flow pattern for higher liquid and gas rates where the system appears more finely dispersed.)

4. Annular Flow: Annular flow is characterized by the continuity of the gas phase along the pipe in the core. The liquid phase moves upwards partly as wavy liquid film and partially in the form of drops entrained in the gas core. (Annular flow has been described as a whispy-annular pattern when the entrained phase is in the form of large lumps or "wisps." Froth, mist or semiannular flow patterns have also been used to describe the churn and annular patterns.) "

Figure 2.5.3.1-1, reproduced from Taitel et al. (1980), illustrates the above flow regimes. The bubble flow definition above and in Figure 2.5.3.1-1 applies to both the bubbly and dispersed bubble regimes. Barnea et al. consider slug and churn flow to have nearly the same configuration and be subdivisions of an 'intermittent' flow regime. Barnea (1987) defined churn flow as "highly aerated slugs with repeated destruction of liquid continuity in the slug" and noted that this definition is "somewhat different from the usual association of chum flow with oscillatory motion of the liquid slug". Barnea (1987) also observed that "there is, however, no contradiction between the two criteria since the oscillatory motion is caused by the high void fraction in the slug". Therefore oscillatory behavior of the chum regime, while typical, is not required. The above descriptions and Figure 2.5.3.1-1 largely carry over as is for downflow.

With respect to constitutive term models, no distinction is made in FLOWTRAN-TF between the bubbly and dispersed bubble regimes and the slug and churn regimes. Therefore, the bubbly and dispersed bubble regimes and the slug and churn regimes may be combined leaving three fundamental flow regimes which will be termed 'bubbly', 'intermittent or slug', and 'annular'. Barnea et al. use 'intermittent' to describe the slug and churn regimes and this terminology will be used initially in discussing the works of Barnea et al. for easy reference to Barnea et al. Later in discussing the FLOWTRAN-TF implementation, the slug and churn regimes are termed 'slug' rather than 'intermittent' 
because, historically, the 'slug' terminology has been used in FLOWTRAN-TF development following RELAP, and the terminology has already been used extensively
elsewhere such as in interfacial drag documentation.

In the following discussion, attention is confined to downward flows. According to Barnea et al., the dispersed bubble regime occurs for void fractions between 0 and 0.52 when the turbulence level is sufficiently high. The criterion for sufficient turbulence is when the liquid superficial velocity exceeds a threshold. The bubbly regime occurs for liquid flowrates below this threshold when the void fraction is less than 0.25 and if the Taylor bubble rise velocity is greater than the velocity of small bubbles. This is the case except for very small pipes. For void fractions less than 0.25 and the Taylor bubble rise velocity less than the bubble rise velocity, the flow is intermittent. The flow regime is intermittent regardless of pipe size for void fractions between 0.25 and 0.52 if the liquid superficial velocity is less than the threshold mentioned above. The regime is also intermittent, without qualification, if the void fraction is between 0.52 and 0.76 . The flow regime is annular if the void fraction is greater than 0.76. Figure 2.5.3.1-2 illustrates the form of the Barnea et al. flow regime map in superficial velocity coordinates for the case of downflow. The mechanistic regime transition models which yield this map are discussed
in more detail below.
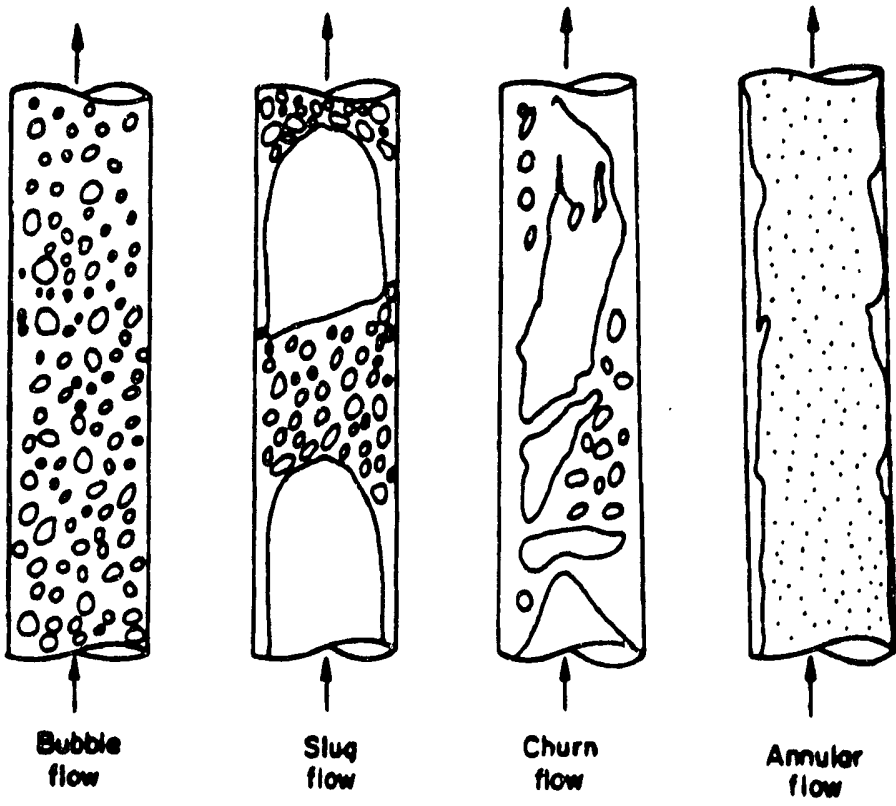
Figure 2.5.3.1-1 $\begin{aligned} & \text { Schematic illustrations of bubble, slug, churn and annular two- } \\ & \text { phase flows. }\end{aligned}$ 


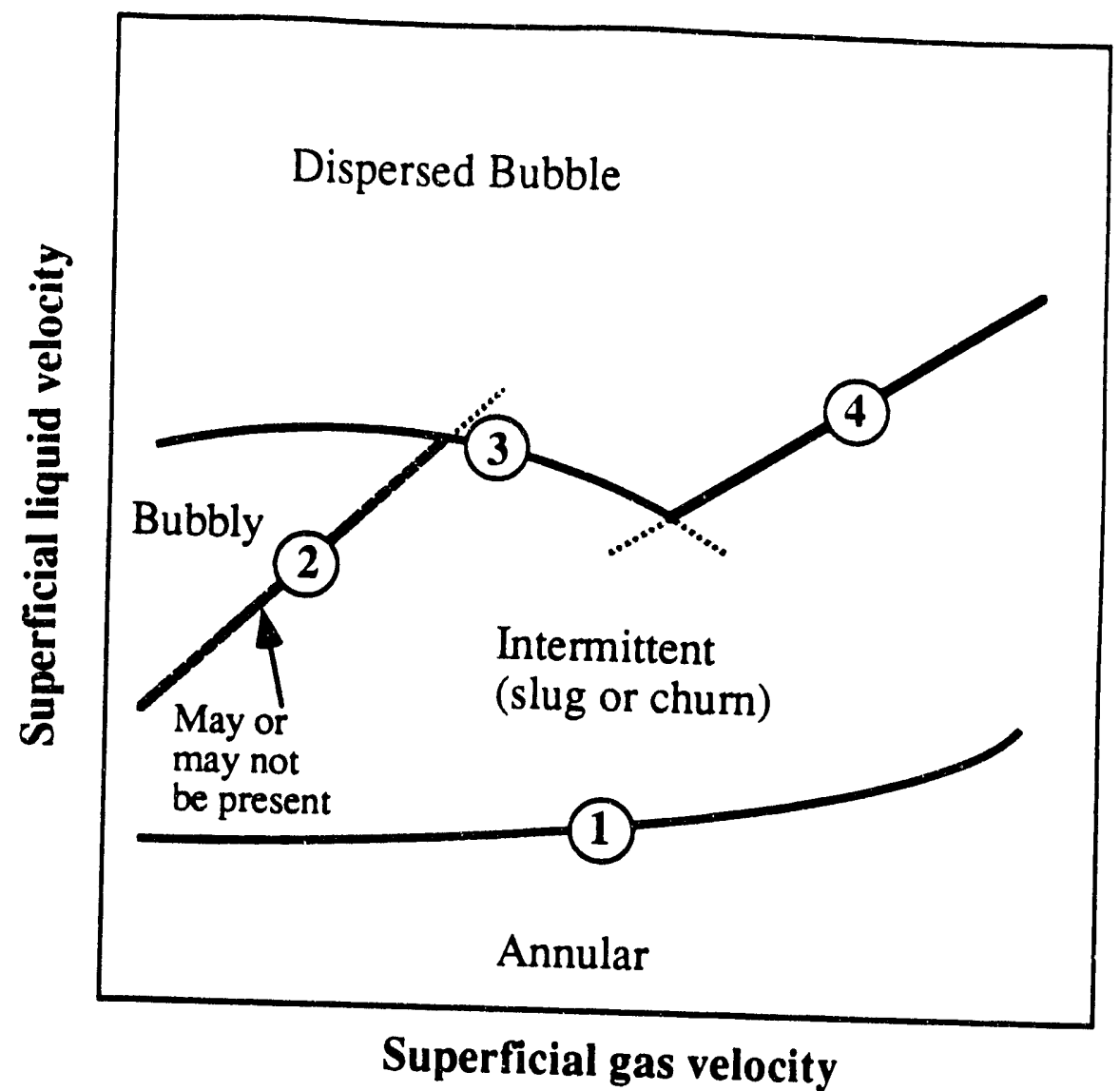

Figure 2.5.3.1-2 Schematic illustration of the flow regime transition criteria of Barnea et al. for downflow. Transition line 1: Barnea et al. (1982b) proposed that a "stable slug will be formed when
the supply of liquid in the [annular] film is large enough to provide the liquid needed to
maintain such a slug". In other words, when the space and maintain the blockage, the flowen there is sufficient liquid to bridge the gas churn) flow. Barnea et al. (1982b) proposed a void from annular to intermittent (slug or based on this liquid bridging (1982b) proposed a void fraction of 0.65 as the transition point recommendation to $\alpha=0.76$ despite. However, Barnea (1986) apparently changed the transition line agrees well with their for the annular transition but showed it is not Barnea (1986) presented a second mechanism transition is given by

$$
\alpha=0.76
$$
The transition line can be cast in terms of superficial velocities, as is commonly done in the
literature, using a drift-flux model. The drift-flux model may be written

$$
\mathrm{j}_{\mathrm{f}}=\mathrm{j}_{\mathrm{g}}\left(\frac{1}{\mathrm{C}_{0} \alpha}-1\right)+\frac{\left|\mathrm{u}_{\mathrm{gj}}\right|}{\mathrm{C}_{0}}
$$

where 


$$
\begin{aligned}
\mathrm{j}_{\mathrm{g}} & \equiv \text { superficial gas velocity; positive for downflow } \\
\mathrm{j}_{\mathrm{f}} & \equiv \text { superficial liquid velocity; positive for downflow } \\
\alpha & \equiv \text { void fraction } \\
\mathrm{C}_{0} & \equiv \text { distribution coefficient } \\
\left|\mathrm{u}_{\mathrm{gj}}\right| & \equiv \text { drift velocity magnitude }
\end{aligned}
$$

Transition line 2: Transition line 2 may or may not exist depending on pipe size. Taitel et al. (1980) proposed that the bubbly regime will exist only if the Taylor bubble rise velocity exceeds that of small bubbles. If the Taylor bubble velocity is larger then the bubbles are swept past the nose of the Taylor bubble without effective coalescence. Otherwise, the small bubbles coalesce at the trailing end of the Taylor bubbles and augment them. The speed of small bubbles is modeled as

$$
\left|u_{g j}\right|=K_{b}\left[\frac{\Delta \rho g \sigma}{\rho_{f}^{2}}\right]^{1 / 4}
$$

where the leading coefficient varies somewhat in the literature. Ishii (1977) proposed $K_{b}=\sqrt{2}$ and Harmathy (1960) proposed $K_{b}=1.53$. The Taylor bubble velocity is
modeled as

$$
\left|u_{g j}\right|=K_{s}\left[\frac{\Delta \rho g D_{c}}{\rho_{f}}\right]^{1 / 2} \cong K_{s}\left[g D_{c}\right]^{1 / 2}
$$

where $D_{c}$ is a characteristic dimension for the channel geometry. For a circular channel $D_{c}$ is the pipe diameter $D$ and $K_{s}=0.35$ (Ishii, 1977). For a circular pipe the criterion for existence of the bubbly regime is

$$
0.35\left[\frac{\Delta \rho g D}{\rho_{\mathrm{f}}}\right]^{1 / 2}>1.53\left[\frac{\Delta \rho g \sigma}{\rho_{\mathrm{f}}^{2}}\right]^{1 / 4}
$$

or

$$
\mathrm{D}>19.1\left[\frac{\sigma}{\Delta \rho \mathrm{g}}\right]^{1 / 2}
$$

Equation (2.5.3.1-6) differs slightly from that given by Taitel et al. (1980). Taitel et al. (1980) used the approximate expression given by the second term of equation (2.5.3.1-4) and therefore equation (2.5.3.1-6) is preferred. For a ribbed annular channel typical of FLOWTRAN-TF applications, the Taylor bubble rise velocity can be inferred from the drift-flux results presented in $\$ 2.5 .5 .2$ to be approximately 


$$
\left|\mathrm{u}_{\mathrm{gj}}\right|=0.5\left[\frac{\Delta \rho \mathrm{gD}_{\mathrm{c}}}{\rho_{\mathrm{f}}}\right]^{1 / 2}
$$

where the characteristic dimension is given in terms of the inner and outer annulus
diameters by

$$
D_{c}=\frac{\pi}{2} \bar{D}=\frac{\pi}{4}\left(D_{i}+D_{o}\right)
$$

For a ribbed annular channel, the bubbly regime existence criterion becomes

$$
D_{c}>9.4\left[\frac{\sigma}{\Delta \rho g}\right]^{1 / 2}
$$

At $20^{\circ} \mathrm{C}$ equation (2.5.3.1-9) yields

$$
D_{c}>0.026 m
$$

For comparison, the characteristic dimensions of the inner, middle and outer channels of the Mk22 assembly are given $\$ 2.5 .5 .2$ as $0.0715,0.105$ and $0.135 \mathrm{~m}$ respectively. All (2.5.3.1-10) so the bubbly regime is predicted to exceed the criterion given by equation

Transition line 2, provided it exists, is based on the following bubble coalescence
arguments given by Taitel et al. (1980). Experimental flow bubblyts given by Taitel et al. (1980). Experimental flow regime data suggests the to Taitel et al. (1980), transition occurs at a void fraction around 0.25 to 0.30 . According show that the frequency of bubble collision for fluc2) used a semi-theoretical approach to increases at a void fraction of about 0.30 . for fluctuating bubbles in a cubic lattice sharply high coalescence. Data indicates 0.30 . High collision rates presumably correspond to fractions below 0.20 . These observation bubble coalescence is rarely observed for void

$$
\alpha=0.25
$$

as the transition line between bubbly and slug flow, provided the bubbly regime exists. As with criterion (1), the drift-flux model can be used to recast equation $(2.5 .3 .1-11)$ in terms
of superficial velocities.

Transition line 3: The dispersed bubble regime occurs when the liquid turbulence level is coalescence. Low void and disperse large bubbles that would otherwise form due to in the first place $(\alpha<0.25)$ are turbulence is high to break-up large bubbles if ed as dispersed bubble when the liquid by Taitel et al. (1980) was superseded by an they existed. The transition criterion given (1982b). Both developments are lengthy and improved version given by Barnea et al. (1982b) give the more recent criterion as 


\begin{tabular}{|l|lll|r|}
\hline $2 / 15 / 93$ & WSRC-TR-92-532 Rev. 0 & FLOWTRAN-TF Software Design & Page 152 \\
\hline
\end{tabular}

$$
2\left(\frac{0.4 \sigma}{\Delta \rho g}\right)^{0.5}\left(\frac{\rho_{f}}{\sigma}\right)^{0.6}\left[\frac{0.092}{D_{h}}\left(\frac{D_{h}}{v_{f}}\right)^{-0.2}\right]^{0.4}\left(j_{g}+j_{f}\right)^{1.12}=0.725+4.15\left(\frac{j_{g}}{j_{g}+j_{f}}\right)^{0.5}
$$

where circular pipe diameter $D$ in Barnea et al. (1982b) has been replaced with hydraulic diameter $D_{h}$ in equation (2.5.3.1-12). Incidentally, Barnea et al. (1982b, equation (18)) contains a typographical error which has been corrected by equation $(2.5 .3 .1-12)$.

Transition line 4: Taitel et al. (1980) observed that spherical bubbles arranged in a cubic lattice have a maximum packing density corresponding to a void fraction of $52 \%$. Therefore, large bubbles must form for void fractions greater than $\alpha=0.52$ regardless of the turbulence level. This observation introduces an upper limit to the dispersec bubble regime and transition line 4 is defined by

$$
\alpha=0.52
$$

As with criterion (1), the drift-flux model can be used to recast equation (2.5.3.1-13) in terms of superficial velocities.

VOID Rig: Figure 2.5.3.1-2 shows the form of the flow regime map resulting from the transition criteria discussed above in superficial velocity coordinates. The above transition criteria are now applied to a particular channel geometry of interest and plotted quantitatively to better illustrate the flow regime model of Barnea et al. The VOID Rig channel geometry is chosen for this purpose (Whatley, 1990). The criteria given by equations (2.5.3.1-1), (2.5.3.1-11) and (2.5.3.1-13) are expressed in terms of void fraction. To plot the equivalent criteria in terms of superficial velocities, the drift-flux model will be used. VOID Rig data are given in two sets by Whatley (1990) and Flach (1991) and by Steimke and Fowley (1992). Drift-flux fits to the data surrounding void fractions of $0.25,0.52$ and 0.76 are respectively:

$$
\begin{aligned}
& \mathrm{j}_{\mathrm{f}}=1.9630 \mathrm{j}_{\mathrm{g}}+0.4741 \mathrm{~m} / \mathrm{s} \\
& \mathrm{j}_{\mathrm{f}}=0.3543 \mathrm{j}_{\mathrm{g}}+0.4296 \mathrm{~m} / \mathrm{s} \\
& \mathrm{j}_{\mathrm{f}}=0.0785 \mathrm{j}_{\mathrm{g}}+0.2213 \mathrm{~m} / \mathrm{s}
\end{aligned}
$$

The criterion given by equation (2.5.3.1-12) when applied to the VOID Rig and $20^{\circ} \mathrm{C}$ conditions is

$$
1.4046\left(j_{g}+j_{f}\right)^{1.12}-0.725-4.15\left(\frac{j_{g}}{j_{g}+j_{f}}\right)^{1 / 2}=0
$$

The above flow regime transition criteria are plotted in Figure 2.5.3.1-3 along with the VOID Rig data. Experimental observations of flow regime were not acquired as part of the test so the predictions shown by Figure 2.5.3.1-3 cannot be verified. More detailed information concerning development of equations $(2.5 .3 .1-14)$ to $(2.5 .3 .1-17)$ is given in the FLOWTRAN-TF development report TF-5. 
Flow Regime Map

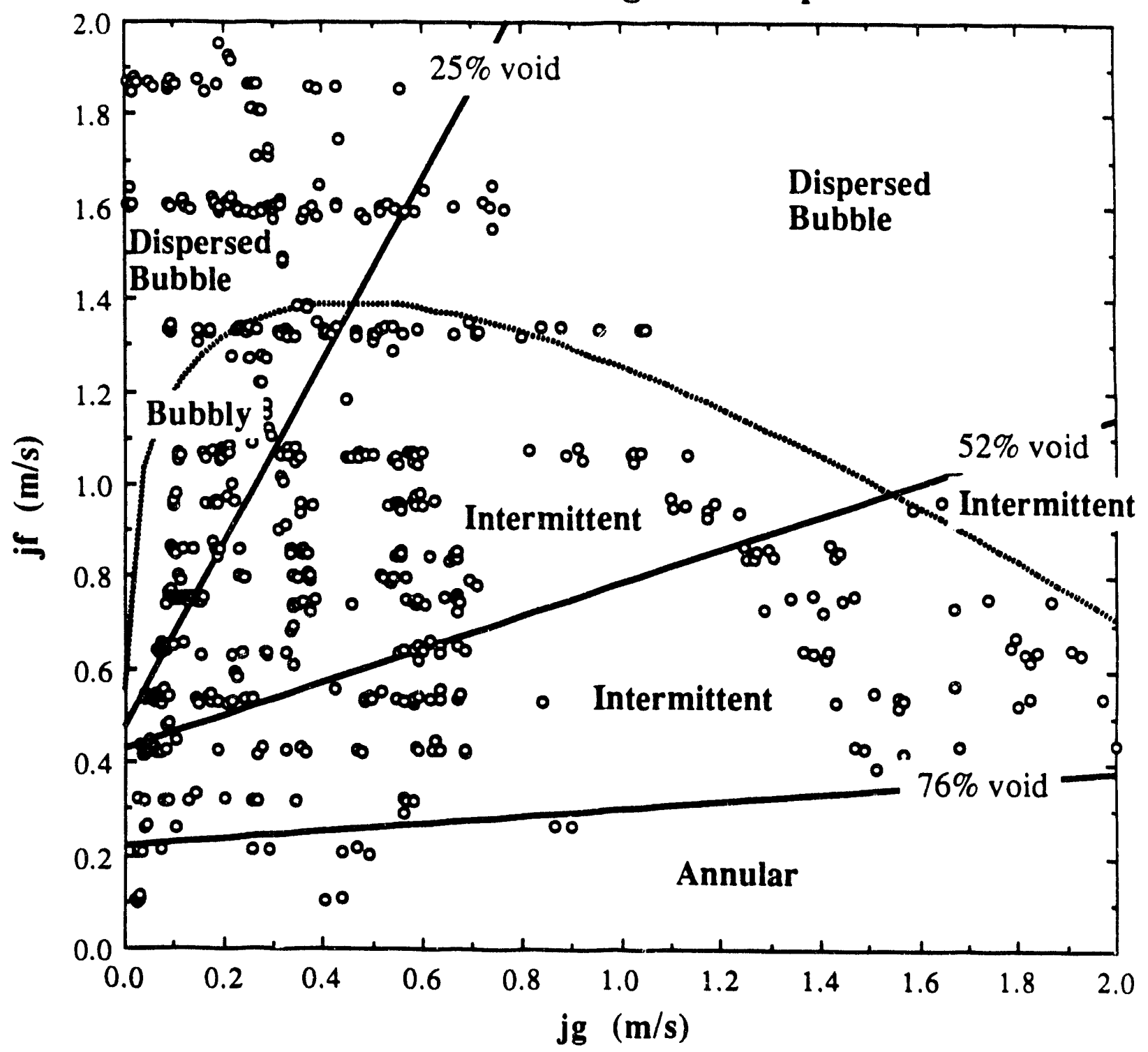

Figure 2.5.3.1-3 Flow regime logic of Barnea et al. applied to VOID Rig channel dimensions and $20^{\circ} \mathrm{C}$ conditions. 
FLOWTRAN-TF implementation: For all FLOWTRAN-TF applications identified to-date, the channel sizes are sufficiently large that the bubble regime exists. Recognizing this situation, the bubbly-slug transition defined by equation $(2.5 .3 .1-11)$ is always assumed to apply in FLOWTRAN-TF; that is, an explicit check for bubble existence using a criterion such as equation $(2.5 .3 .1-6)$ or $(2.5 .3 .1-10)$ is not performed by FLOWTRAN-TF. In addition, all FLOWTRAN-TF applications identified to-date involve relatively low velocities such that dispersed bubble flows with void fractions between $25 \%$ and 52\% are rarely encountered; see Figure 2.5.3.1-3 for example. As a simplification, FLOWTRAN-TF assumes that the criterion given by equation $(2.5 .3 .1-12)$ is never exceeded. This approximation is made recognizing that at the high liquid flowrates required to exceed criterion (2.5.3.1-12), the error in computed interfacial drag due to incorrectly identifying the flow regime is small. This is because the error in the calculated relative velocity between the phases is relatively small compared to the (high) liquid velocity. The interfacial heat and mass transfer model is also affected by incorrectly identifying the flow regime. However, interfacial heat and mass transfer phenomena are generally not important in FLOWTRAN-TF applications so the error introduced is not significant. An exception could be thermal excursion simulations. In FLOWTRAN-TF, the annular transition is defined to occur at $75 \%$ void instead of $76 \%$ void following RELAP. Also, the intermittent regime comprised of slug and churn subregimes is termed 'slug' in FLOWTRAN-TF again following RELAP. With these modifications the FLOWTRAN-TF flow regime map becomes simply

$$
\begin{array}{ll}
\text { Bubbly (bubbly or dispersed bubble) } & 0 \leq \alpha \leq 0.25 \\
\text { Slug (slug and churn) } & 0.25<\alpha<0.75 \\
\text { Annular } & 0.75 \leq \alpha \leq 1
\end{array}
$$

Note that FLOWTRAN-TF uses the terminology 'slug' instead of 'intermittent' used by Barnea et al. as discussed previously. Therefore the 'slug' regime in FLOWTRAN-TF includes both the slug and chum flow regimes described by Barnea et al. Similarly, the 'bubbly' regime includes the bubbly and dispersed bubble regimes. Further note that void fraction al ne defines the flow regime in FLOWTRAN-TF.

In order to transition continuously between the three fundamental flow regimes of bubbly, slug and annular, FLOWTRAN-TF uses an interpolation scheme following RELAP. For void fractions up to and including 0.25 , the regime is considered purely bubbly. Likewise, for void fractions down to and including 0.75 , the regime is considered purely annular. At $\alpha=0.52$ the regime is considered to be purely slug. For the interval $0.25<\alpha<0.52$ the regime is considered to be a mixture of bubbly and slug. Similarly the interval $0.52<\alpha<0.75$ comprises the slug-annular regime. With this approach 5 two-phase flow regimes (bubbly, bubbly-slug, slug, slug-annular, annular) are created from three components defined by

$$
\begin{aligned}
& \alpha_{b} \equiv \text { bubbly regime void fraction, } V_{b} / V \\
& \alpha_{s} \equiv \text { slug regime void fraction, } V_{s} / V \\
& \alpha_{a} \equiv \text { annular regime void fraction, } V_{a} / V
\end{aligned}
$$

so that 


$$
\alpha=\alpha_{b}+\alpha_{s}+\alpha_{a}
$$

Note that no more than two flow regime void fractions are non-zero for a given total void.

The parameters $\alpha_{b}$ and $\alpha_{s}$ are not convenient interpolation parameters in the bubbly-slug region. Rather, the bybbly-slug interpolation scheme is formulated in terms of a local bubble void fraction $\alpha_{b}$ defined to be the volume of small bubbles divided by the liquid volume; that is, $\alpha_{b}$ is the local void fraction in the predominantly liquid slugs separating Taylor bubbles. This local bubble void fraction may be written as

$$
\alpha_{b}^{*}=\frac{\alpha-\alpha_{s}}{1-\alpha_{s}}
$$

and rearangement gives

$$
\alpha_{s}=\frac{\alpha-\alpha_{b}^{*}}{1-\alpha_{b}^{*}}
$$

Because $\alpha_{b}^{*}$ varies from 0.25 to 0 between the endpoints $\alpha=0.25$ and 0.52 , respectively, the desired interpolation is easily accomplished by choosing the power law function

$$
\alpha_{b}^{*}=\alpha_{b}^{* *}\left[\frac{\alpha_{s}^{* *}-\alpha}{\alpha_{s}^{* *}-\alpha_{b}^{* *}}\right]^{n_{b s}}
$$

where

$$
\begin{aligned}
& \alpha_{b}^{* *} \equiv 0.25 \\
& \alpha_{s}^{* *} \equiv 0.52 \\
& n_{b s} \equiv 4
\end{aligned}
$$

The selection $n_{b s}=4$ maintains a relatively sharp transition consistent with the physical models presented above without making it so sharp that numerical difficulties might be experienced. The chosen interpolation scheme is not unique; other schemes could be devised which serve the same purpose. Following calculation of the local void fraction using equation (2.5.3.1-20) and the slug void fraction using equation (2.5.3.1-19), the bubbly void fraction is computed from

$$
\alpha_{b}=\alpha-\alpha_{s}
$$

using equation (2.5.3.1-18). In an analogous manner, the slug-annular transition is defined by the equations

$$
\alpha_{s}=\frac{\alpha-\alpha_{a}^{*}}{1-\alpha_{a}^{*}}
$$




$$
\begin{gathered}
\alpha_{\mathrm{a}}^{*}=\alpha_{\mathrm{a}}^{* *}\left[\frac{\alpha-\alpha_{\mathrm{s}}^{* *}}{\alpha_{\mathrm{a}}^{* *}-\alpha_{\mathrm{s}}^{* *}}\right]^{\mathrm{n}_{\mathrm{sa}}} \\
\alpha_{\mathrm{a}}=\alpha-\alpha_{\mathrm{s}}
\end{gathered}
$$

where

$$
\begin{aligned}
\alpha_{\mathrm{s}}^{* *} & \equiv 0.52 \\
\alpha_{\mathrm{a}}^{* *} & \equiv 0.75 \\
\mathrm{n}_{\mathrm{sa}} & \equiv 4
\end{aligned}
$$

The two interpolation schemes yield continuous, but relatively sharp, regime transitions. Figure 2.5.3.1-4 illustrates the final flow regime map implemented in the
FLOWTRAN-TF.

For some constitutive term models, the only flow regime distinction required is that of drag models. For these cases, the bubbly. Examples are the wall heat transfer and while annular is synonymous with separated flow. The flow regime map shown in Figure exception. For horizontal stratified flow at he and constitutive terms with one notable first cell of the Top Section, the flow regime the cell face between the Plenum cell and the $\$ 3.6$ for more information. 


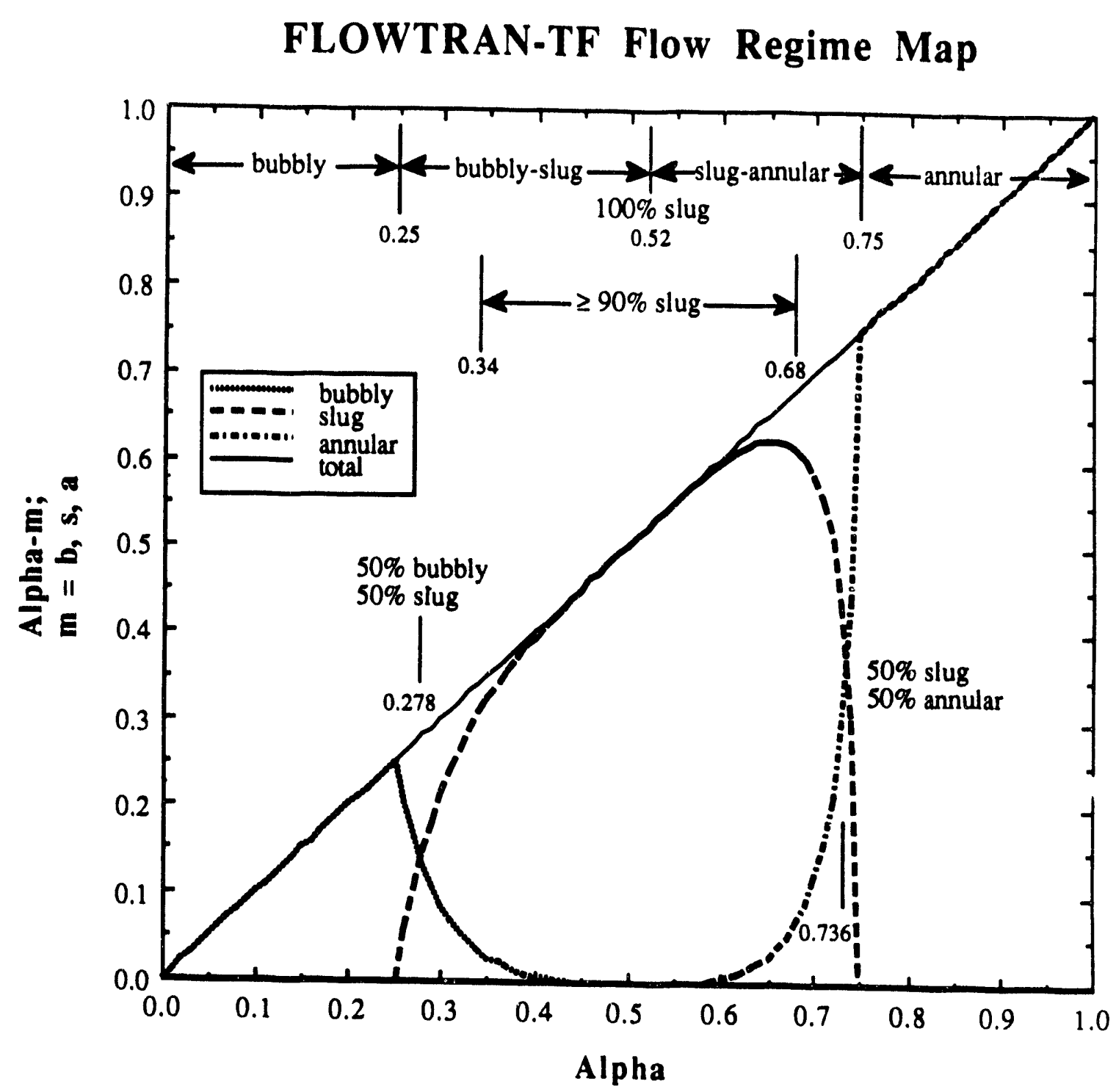

Figure 2.5.3.1-4 FLOWTRAN-TF flow regime map. 


\begin{tabular}{|l|lll|l|}
\hline $2 / 15 / 93$ & WSRC-TR-92-532 Rev.0 & FLOWTRAN-TF Software Design & Page 158 \\
\hline
\end{tabular}

\section{References:}

Barnea, D., 1986, "Transition from Annular Flow and from Dispersed Flow-Unified Models for the Whole Range of Pipe Inclinations", Int. J. Multiphase Flow, v12 n5, pp. 733-744.

Barnea, D., 1987, "A Unified Model for Predicting Flow-Pattern Transitions for the Whole Range of Pipe Inclinations", Int. J. Multiphase Flow, v13 n1, pp. 1-12.

Barnea, D., O. Shoham, and Y. Taitel, 1982a, "Flow Pattern Transition for Downward Inclined Two-Phase Flow; Horizontal to Vertical", Chem. Engr. Science, v37 n5, pp. 735-740.

Barnea, D., O. Shoham, and Y. Taitel, 1982b, "Flow Pattern Transition for Vertical Downward Two Phase Flow", Chem. Engr. Science, v37 n5, pp. 741-744.

Barnea, D., and Taitel, Y., 1986, "Flow Pattern Transition in Two-Phase Gas-Liquid Flows", Chapter 16 in Encyclopedia of Eluid Mechanics. Volume 3 edited by N. P. Cheremisinoff, Gulf Publishing Company, Houston.

Bilicki, Z., and J. Kestin, 1987, "Transition Criteria for Two-Phase Flow Patterns in Vertical Upward Flow", Int. J. Multiphase Flow, v13 n3, pp. 283-294.

Crawford, T. J., and C. B. Weinberger, 1985, "Two-Phase Flow Patterns and Void Fractions in Downward Flow. Part I: Steady-State Flow Patterns", Int. J. Multiphase Flow, v11 n6, pp. 761-782.

Crawford, T. J., and C. B. Weinberger, 1986, "Two-Phase Flow Fatterns and Void Fractions in Downward Flow. Part II: Void Fractions and Transient Flow Patterns", Int. J. Multiphase Flow, v12 n2, pp. 219-236.

Flach, G. P., 1991, "WSRC-TR-90-328 Errata and Corrections (U)", WSRC-TR-91-466.

Harmathy, T. Z., 1960, "Velocity of Large Drops and Bubbles in Media of Restricted or Infinite Extent", AIChE J. v6 pp. 281-??.

Ishii, M., 1977, "One-Dimensional Drift-Flux Model and Constitutive Equations for Relative Motion Between Phases in Various Two-Phase Flow Regimes", ANL-7747.

Kelessidis, V. C., and A. E. Dukler, 1989, "Modeling Flow Pattern Transitions for Upward Gas-Liquid Flow in Vertical Concentric and Eccentric Annuli", Int. J. Multiphase Flow, v15 n2, pp. 173-191.

Kielpinski, A. L., 1992, "Flow Regime Mapping for Vertical Two-Phase Downflow in a Ribbed Annulus", WSRC-TR-92-30 draft.

McQuillan, K. W., and P. B. Whalley, 1985, "Flow Patterns in Vertical Two-Phase Flow", Int. J. Multiphase Flow, v11 n2, pp. 161-175.

Mishima, K., and M. Ishii, 1984, "Flow Regime Transition Criteria for Upward TwoPhase Flow in Vertical Tubes", Int. J. Multiphase Flow, v27 n 5, pp. 723-737. 
Mukherjee, H., and J. P. Brill, 1985, "Empirical Equations to Predict Flow Patterns in Two-Phase Inclined Flow", Int. J. Multiphase Flow, v11 n3, pp. 299-315.

Radovicich, N. A., and R. Moissis, 1962, "The Transition from Two-Phase Bubbly Flow to Slug Flow", MIT Report 7-7673-22.

Ransom, V. H., R. J. Wagner, J. A. Trapp, L. R. Feinauer, G. W. Johnsen, D. M. Kiser, and R. A. Riemke, 1985, "RELAP5/MOD2 Code Manual; Volume 1: Code Structure, Systems Models, and Solution Methods", NUREG/CR-4312, EGG2396.

Spedding, P. L., and V. T. Nguyen, 1980, "Regime Maps for Air Water Two Phase Flow", Chem. Engr. Science, v35, pp. 779-793.

Steimke, J. L., and M. D. Fowley, 1992, "Azimuthal Variation of Void Fraction in a Ribbed Annulus (U)", WSRC-TR-91-661, February.

Taitel, Y., D. Barnea, and A. E. Dukler, 1980, "Modelling Flow Pattern Transitions for Steady Upward Gas-Liquid Flow in Vertical Tubes", AIChE J., v26 n3, pp. 345354.

Taitel, Y., and A. E. Dukler, 1976, "A Model for Predicting Flow Regime Transitions in Horizontal Gas-Liquid Flow", AIChE J., v22 n1, pp. 47-55.

Whatley, V., 1990, "Measurements of Void Fractions and Pressure Profiles for Downward Flowing Air-Water Mixtures in Single Test Annulus (U)", WSRC-TR-90-328.

Yamaguchi, K., and Y. Yamazaki, 1984, "Combinated Flow Pattern Map for Cocurrent and Countercurrent Air-Water Flows in Vertical Tube", J. Nucl. Sci. and Technology, v21 n5, pp. 321-327.

Ying, A., and J. Weisman, 1989, "The Relationship Between Interfacial Shear and Flow Patterns in Vertical Flow", Int. J. Multiphase Flow, v! 5 n1, pp. 23-34. 


\begin{tabular}{|l|lll|l|}
\hline $2 / 15 / 93$ & WSRC-TR-92-532 Rev.0 & FLOWTRAN-TF Software Design & Page 160 \\
\hline
\end{tabular}

\subsubsection{Interfacial Area Concentration}

The code basically considers the flow regime of two-phase flow to be a superposition of the contributions from the three flow regimes of bubbly, slug and annular. The local interfacial area of the two-phase mixture is computed by summing the interfacial areas contributed by each of three flow' regimes.

$$
a_{i}=\sum_{m} a_{i, m}=a_{i, b}+a_{i, s}+a_{i, a}
$$

In equation (2.5.3.2-1) $\mathrm{m}$ stands for three flow regimes (bubbly, slug, annular flow) and $\mathrm{a}_{\mathrm{i}}$ is the interfacial area per unit two-phase mixed volume. In addition, the subscripts $(b, s$, and a) in equation (2.5.3.2-1) represent the three basic flow regimes.

The present section establishes the basic formulation of the interfacial area concentration in pure bubbly, annular, and slug flows, as well as in the bubbly-slug and slug-annular transition regimes. The FLOWTRAN-TF code implementation of the limiting cases of pure liquid and pure gas is also considered.

Bubbly Flow: At void fractions below $\alpha_{g}=0.25$, purely bubbly flow is assumed to exist. For the interfacial area concentration calculations, bubbles are assumed to be spherical since bubble size in this flow regime is relatively small. A minimum bubble diameter is chosen, somewhat arbitrarily, as $\left(d_{b}\right)_{\min }=1 \times 10^{-5} \mathrm{~m}$, which is sufficient to prevent numerical problems. The maximum allowable bubble diameter is chosen as the minimum of three different criteria:

1) An Eotvos number (Eo), which is defined as $\Delta \rho \mathrm{gd}_{b}{ }^{2} / \sigma$, is equal to 6.0. This value comes directly from equating the surface tension force to the buoyancy force in a static equilibrium. In this situation, all the bubbles within stagnant liquid are assumed to be spherical.

2) The hydraulic diameter of a thin annular channel with ribs is approximately equal to $\left(D_{0}-D_{i}\right)$. Since maximum bubble size can not exceed the equivalent channel diameter on a physical basis, the criterion is imposed on the bubble size for the assumedly spherical bubbles. That is, the criterion appropriate for spherical bubbles is the channel hydraulic diameter $\left(D_{h}\right)$.

3) The bubble diameter is constrained by the entire volume of gas phase in the computational mesh cell forming a single bubble. This is a mesh-dependent quantity, as well as being dependent on void fraction. Given the assumed upper bound on pure bubbly flow of $\alpha_{g}=0.25$ for the minimum fluid cell volume, the maximum bubble diameter associated with this criterion is about 2 cm.

For bubble properties at room temperature, criterion 1), i.e., Eo $=6$, yields a bubble diameter of about $0.7 \mathrm{~cm}$. This is less than any of the three hydraulic diameters (criterion 2 ): $\left(D_{h}\right)_{\min } \approx 0.76 \mathrm{~cm}$ ). The single-bubble criterion (criterion 3$)$ ) will yield the minimum value of the three criteria only at very low void fractions $\left(\alpha_{8}<0.01\right)$. Thus, the Eo criterion $(E 0=6)$ mainly limits the maximum bubble size for bubbly flow regime. 
Following RELAP5, the average bubble diameter $\left(\mathrm{d}_{\mathrm{o}}\right)$ is taken, a priori, as one half the maximum diameter $\left(d_{b, \max }\right)$. RELAP5 uses a Weber number criterion to determine the maximum bubble size (Ransom, et al., 1985). The Weber number is related to the Eotvos number when drag force for a single bubble is balanced by its buoyancy force. That is,

$$
\frac{1}{2}\left(\frac{\pi d_{b}{ }^{2}}{4}\right) C_{d} \rho_{f} u_{r}{ }^{2}=\Delta \rho g\left(\frac{\pi d_{b}{ }^{3}}{6}\right)
$$

where $C_{d}$ is drag coefficient on the bubble surface and $d_{b}$ and $u_{r}$ are the bubble diameter and liquid velocity relative to the bubble, respectively. Then, equation $(2.5 .3 .2-2)$
becomes

$$
W e=\frac{4 \text { Eo }}{3 C_{d}}
$$

where Weber number (We) is defined as the ratio of inertia to surface tension forces, that is,

$$
W e \equiv \frac{\rho_{f} d_{b} u_{r}^{2}}{\sigma}
$$

In equation (2.5.3.2-3) drag coefficient $\left(C_{d}\right)$ for the flow over a spherical bubble is dependent on liquid Reynolds number (Bird et al., 1960). When $C_{d}$ is assumed to be unity over a wide range of Reynolds numbers, FLOWTRAN-TF's Eotvos criterion corresponds to a Weber number of 8 . RELAP5/MOD2 used $\mathrm{We}=10$ to compute maximum bubble
diameter.

The determination of interfacial area concentration follows Wallis (1969) and a mean bubble diameter is determined as was done in the RELAP5 code (Dimenna et al., 1988). The Sauter mean diameter is defined as

$$
d_{s m}=\frac{\int_{0}^{\infty} d_{b}^{3} P\left(d_{b}\right) d d_{b}}{\int_{0}^{\infty} d_{b}^{2} P\left(d_{b}\right) d d_{b}}
$$

where $P\left(d_{b}\right)$ is the probability density function of a bubble having diameter between $d_{b}$ and $d_{b}+o d_{i}$ within control volume. Physically, the Sauter mean diameter represents a bubble whose surface area to volume ratio is the same as that of the whole mixture (Wallis, 1969). For drop sprays, Wallis recommends the Nukiyama-Tarasawa probability function for the distribution of drop diameters. In dimensionless form with the coefficients recommended by Wallis (1969), which is corresponding to quantization on a basis of bubble volume and a specified value of bubble mean diameter

$$
P\left(d_{b}^{*}\right)=4\left(d_{b}^{*}\right)^{2} e^{-2 d_{b}^{*}}
$$

where $d_{b}{ }^{*}=d_{b} / d_{b}{ }^{\prime}$. Figure 2.5.3.2-1 shows that $d_{b}$ ' is the most probable bubble diameter in the bubble size distribution function. Following RELAP5, this probability function is applied to the bubble size distribution of interest in these thermal-hydraulics codes. 
Evaluation of the integral in equation (2.5.3.2-5) yields the following relationship between Sauter mean diameter and the most probable diameter using the distribution function and the Euler's integral:

$$
\mathrm{d}_{\mathrm{sm}}^{*} \equiv \frac{\mathrm{d}_{\mathrm{sm}}}{\mathrm{d}_{\mathrm{b}}^{\prime}}=\frac{5}{2}
$$

In this evaluation, Euler's integral, defined by equation (2.5.3.2-8), was used (Abramowitz and Stegun, 1972).

$$
\begin{aligned}
\Gamma(x) & =\int_{0}^{\infty} u^{(x-1)} e^{-x} d x \\
& =\lim _{n \rightarrow \infty} \frac{n ! n^{x}}{x(x+1)(x+2) \ldots \ldots . .(x+n)}
\end{aligned}
$$

Likewise, the dimensionless average droplet diameter is defined as

$$
\mathrm{d}_{\mathrm{o}}^{*} \equiv \frac{\mathrm{d}_{\mathrm{o}}}{\mathrm{d}_{\mathrm{b}}{ }^{\prime}}=\int_{0}^{\infty} \mathrm{d}_{\mathrm{o}}^{*} \mathrm{P}\left(\mathrm{d}_{\mathrm{o}}^{*}\right) \mathrm{dd}_{\mathrm{o}}^{*}
$$

$\mathrm{d}_{0}$ * is equal to 1.5 using the distribution function, equation (2.5.3.2-6).

The interfacial area per unit two-phase mixed volume can be expressed by using the definition of the Sauter mean diameter as

$$
a_{i, b}=\frac{6 \alpha_{g, b}}{d_{s m}}
$$

where $\alpha_{\mathrm{g}, b}$ is the void fraction due to the contribution of bubbly flow in total mixed void fraction. This is also given by Ishii and Mishima (1980). Using the results of equations $(2.5 .3 .2-7)$ and $(2.5 .3 .2-9)$, equation $(2.5 .3 .2-10)$ can be expressed in terms of the mean bubble diameter $\left(\mathrm{d}_{0}\right)$ as

$$
a_{i, b}=\frac{3.6 \alpha_{g, b}}{d_{o}}
$$

It is noted that these results are dependent on the chosen probability distribution function, that is, $\mathrm{d}_{\mathrm{sm}}=5 \mathrm{~d}_{\mathrm{o}} / 3$ for the present distribution function, equation $(2.5 .3 .2-6)$.

In a two-phase flow system, the size distribution of bubbles is commonly determined by the dynamics of bubble breakup and coalescence due to a number of the processes such as strong velocity gradient, turbulent flow field, and flow oscillation. In the FLOWTRAN-TF code, the maximum bubble size in the bubbly flow regime is sasically determined by the critical Eotvos number ( $\mathrm{Eo}_{\text {crit }}$ ). The value of Eo $\mathrm{O}_{\text {crit }}$ is chosen as 6 based on the static force balance between the surface tension force and the buoyancy force of a single bubble in stagnant liquid since there is no definite value in the two-phase literature. With the 
spherical shape and size distribution of the bubbles within a continuous liquid phase given by the above model, the interfacial area per unit volume can be determined by equation $(2.5 .3 .2-11)$ for a given void fraction in the bubbly flow regime.

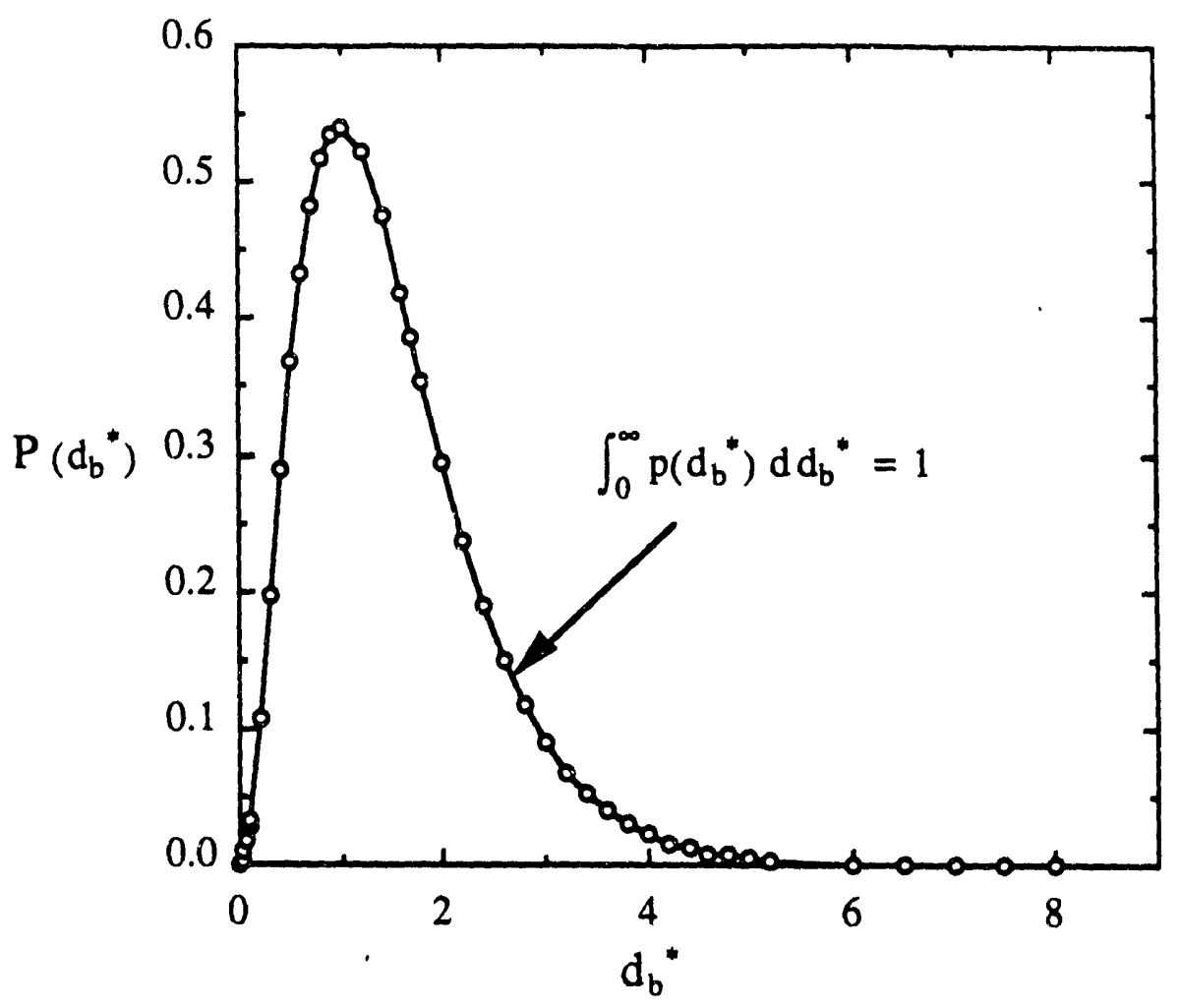

Figure 2.5.3.2-1 Bubble size distribution function given by equation (2.5.3.2-6).

Annular Regime: Above a void fraction of 0.75 , annular flow is assumed to occur. For the ribbed annular geometry shown in Figure 2.5.3.2-1, a constant value of film thickness $\delta$ on the surfaces is assumed. The basic definition of interfacial area concentration is

$$
a_{i, a} \equiv-\frac{\text { Interfacial area }}{\text { Computational cell volume }}
$$

The interfacial area per unit channel height is just the perimeter between the two phases, while the volume per unit channel height is the cross-sectional area of the flow channel. Then, the interfacial area per unit volume becomes

$$
a_{i, a}=\frac{P_{i}}{A_{c}}
$$

where $P_{i}$ is the interfacial perimeter and $A_{c}$ is the cross-sectional flow area of the computational cell which is illustrated in Figure 2.5.3.2-2. $P_{i}$ is the interfacial perimeter of two phases along the computational fluid cell boundary. 
In the FLOWTRAN-TF code, the interfacial perimeter is computed from the geometrical input information and the computed cell averaged void fraction. That is,

$$
P_{i}=P_{w}-8 \delta
$$

where $P_{w}$ is the wetted wall perimeter and $\delta$ is the mean film thickness along the computational cell boundary.

$$
P_{w}=\frac{4 A_{c}}{D_{h}}
$$

where $D_{h}$ is the hydraulic diameter of the computational cell.

$$
\delta=\frac{\left(1-\alpha_{g, a}\right) A_{c}}{P_{w}}
$$

where $\alpha_{\mathrm{g}, \mathrm{a}}$ is the cell averaged void fraction due to the contribution of pure annular flow regime as shown in Figure 2.5.3.2-2. The resulting equation for the interfacial area concentration is obtained from equation $(2.5 .3 .2-13)$ by using equations $(2.5 .3 .2-14)$ to (2.5.3.2-16).

$$
a_{i, a}=\frac{4}{D_{h}}-\frac{2 D_{h}}{A_{c}}\left(1-\alpha_{g, a}\right)
$$

It is noted that equation (2.5.3.2-17) is derived from the condition that mean film thickness is uniform along the channel wall surface which is contacted by water as illustrated in Figure 2.5.3.2-2. 


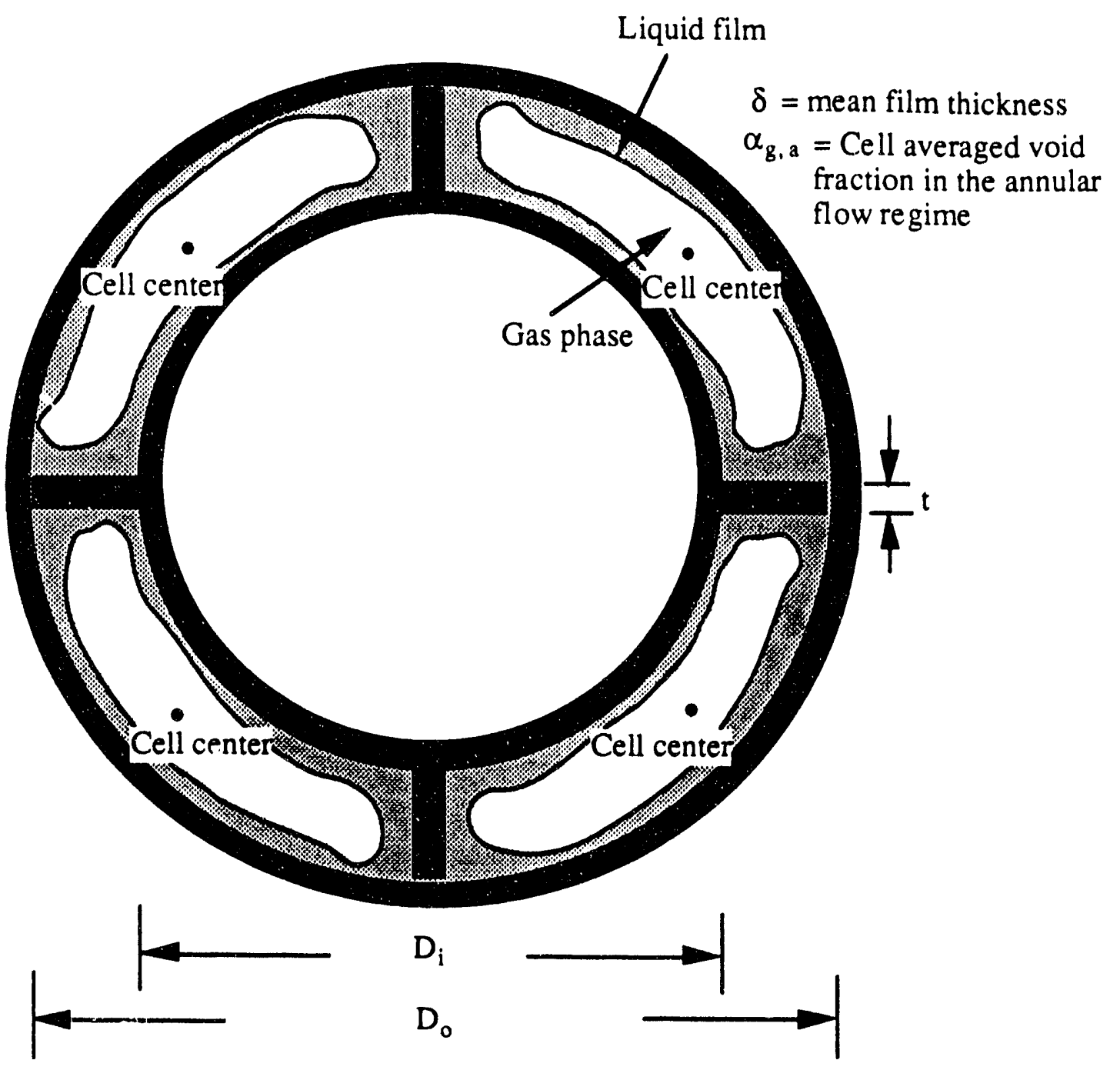

Figure 2.5.3.2-2 The computational model for the interfacial area concentration in the annular flow regime.

Slug Regime: We derive here the expression appropriate for interfacial area for a pure slug flow. Pure slug flow is assumed to exist only at a single value of void fraction, $\alpha=$ 0.52 . In practice, the derivation for slug flow is of interest mainly in computing the slugflow contribution to interfacial area for the bubbly-slug and slug-annular flow regimes.

The interfacial area for a pure slug flow is computed using the results of Ishii and Mishima (1980) for a slug (Taylor) bubble in a tube. The ratio of diameter to length for large slug bubbles is usually much less than 4 and the bubble diameter is constrained by the flow geometry, that is, approximately,

$$
\mathrm{d}_{\mathrm{b}} \approx 0.88 \mathrm{D}
$$

where $d_{b}$ is the bubble diameter and $D$ is the channel diameter. In addition, they considered the roughness of gas-liquid interface. Then, for the pure slug flow portion of two-phase flow, the interfacial area is given by 


$$
a_{i, s}=\frac{4.5 C}{D} \alpha_{g, s}
$$

where $\alpha_{g, s}$ is the void fraction for the slug flow portion. $C$ in equation (2.5.3.2-19) is the roughness parameter. The parameter accounts for the irregular interfacial surface which occurs as Taylor bubbles are replaced with those of churn-turbulent flow. Ishii and Mishima (1980) state that the roughness coefficient should be greater than unity for such churn-turbulent bubbles.

An equivalent derivation for one quadrant of an annulus can be written by approximating the quadrant as a rectangular channel of wide span a and width $b$. The long slug bubble is then approximated as a right rectangular parallelpiped with wide span of slug bubble $\left(d_{b}\right)$ $\gg$ narrow span $\left(b_{b}\right)$ and bubble length $\left(\ell_{b}\right)$. The distance between noses of successive bubbles is denoted as $\mathrm{L}$ in Figure 2.5.3.2-3. When $\mathrm{V}_{c}$ is the volume of control volume for the computation of interfacial area concentration in slug flow, the resulting equation becomes

$$
\begin{aligned}
a_{i, s} & =\frac{A_{i, s}}{V_{c}} \approx \frac{2\left(d_{b} \ell_{b}+b_{b} \ell_{b}+d_{b} b_{b}\right)}{a b L} \\
& =\frac{2 d_{b} \ell_{b}\left(1+\frac{b_{b}}{d_{b}}+\frac{b_{b}}{\ell_{b}}\right)}{a b L} \\
& =\frac{2 d_{b} \ell_{b} C_{l}}{a b L}
\end{aligned}
$$

where $\mathrm{C}_{1}$ is the correction factor associated with the geometrical shape of the slug bubble as illustrated in Figure 2.5.3.2-3 and $C_{1}$ is always greater than unity. In a thin rectangular channel, the hydraulic diameter is given by

$$
D_{h}=\frac{4 A_{c}}{P_{w}} \approx 2 b
$$

where $A_{c}$ and $P_{w}$ are the channel cross-sectional area and wetted perimeter. From equation (2.5.3.2-18), the ratio of the parameters denoted in Figure 2.5.3.2-3 can be approximated as

$$
\frac{\mathrm{d}_{\mathrm{b}}}{\mathrm{a}} \approx 0.88
$$

Void fraction for the slug flow $\left(\alpha_{\mathrm{g}, \mathrm{s}}\right)$ in the control volume of Figure 2.5.3.2-3 can be estimated as

$$
\alpha_{g, s}=\frac{V_{b, s}}{V_{c}}=\frac{C_{2} d_{b} b_{b} \ell_{b}}{a b L}=\frac{(0.88)^{2} C_{2} \ell_{b}}{L}
$$


where $V_{b, s}$ is the volume of a single slug bubble within the control volume and $C_{2}$ is the geometrical correction factor generated from the computation of slug bubble volume. $C_{2}$ is expected to be closely equal to unity from the model assumption.

The interfacial area per unit two-phase mixed volume in equation (2.5.3.2-20) can be obtained in terms of $\alpha_{g, s}$ and hydraulic channel diameter by using equations (2.5.3.2-21) to $(2.5 .3 .2-23)$.

$$
a_{i, s}=\frac{2 d_{b} \ell_{b} C_{1}}{a b L}=4.545\left(\frac{C_{1}}{C_{2}}\right) \frac{\alpha_{g, s}}{D_{h}}
$$

where $\left(C_{1} / C_{2}\right)$ is greater than unity. When $\left(C_{1} / C_{2}\right)$ in equation (2.5.3.2-24) is equal to $C$ (roughness coefficient), equation (2.5.3.2-24) becomes identical to equation (2.5.3.2-19). In the FLOWTRAN-TF code, $\mathrm{C}$ is set to be 1.5 for the computation of interfacial area concentration, while a value of 2 is used for the parameter in the RELAP5/MOD2 code.

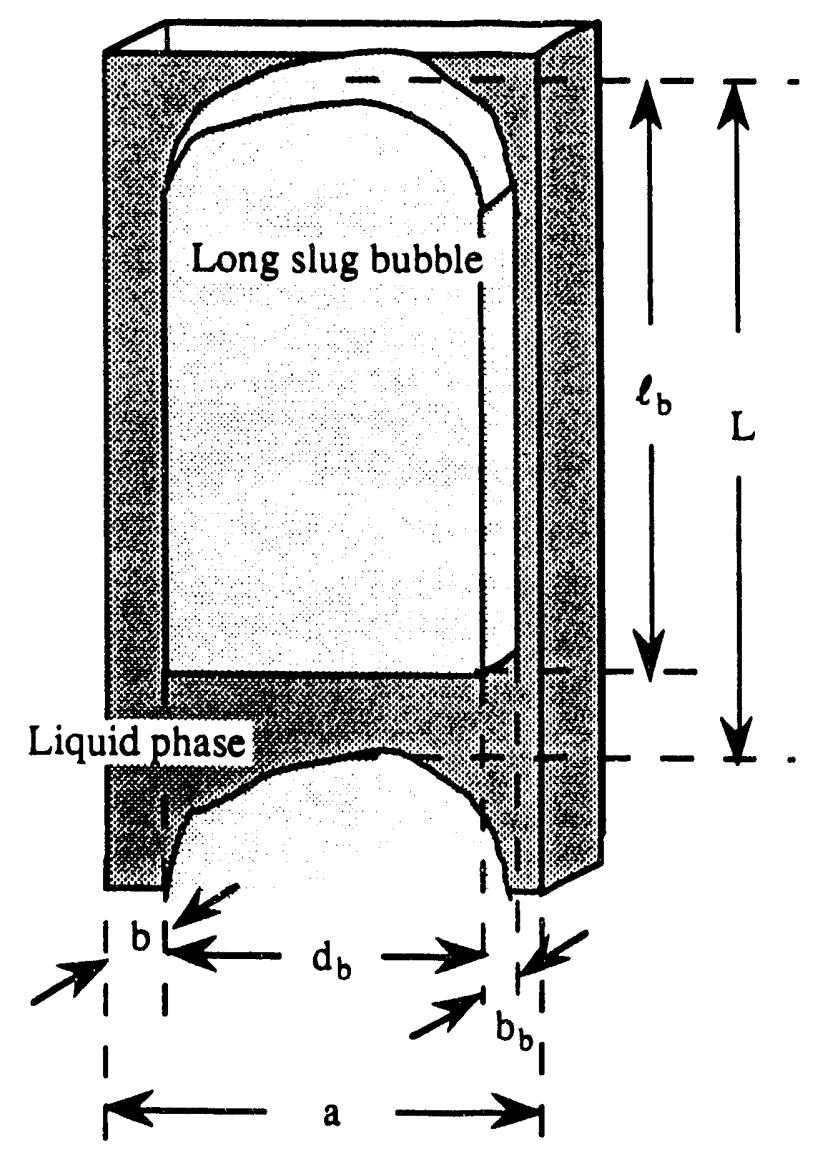

Flgure 2.5.3.2-3 Long slug bubble in a thin slit channel.

Bubbly-Slug Regime: The bubbly-slug mixed region in the two-phase flow regime map of the FLOWTRAN-TF code is considered to occur at void fractions between 0.25 and 0.52 . As discussed previously, the interfacial area concentration for the mixed flow regime is the sum of the interfacial area concentration for each of the two flow regimes, 
bubbly and slug flows, considered independently. Based on the computed void fraction for each flow regime $\left(\alpha_{g, b}\right.$ and $\left.\alpha_{g, s}\right)$, the total interfacial area concentration for the bubbly-slug regime is evaluated as

$$
\begin{gathered}
a_{i}=a_{i, b}+a_{i, s} \\
=\frac{3.6 \alpha_{g, b}}{d_{0}}+\frac{4.5 C \alpha_{g, s}}{D_{h}}
\end{gathered}
$$

Slug-Annular Regime: For a void fraction of 0.52 to 0.75 , two-phase flow regime is assumed to be in a transition between the slug and annular regimes. The partitioning of the void fraction between these two regimes is computed in a manner analogous to that described above for the bubbly-slug regime. The expression for the total interfacial area concentration for slug-annular flow is given by using equations $(2.5 .3 .2-17)$ and
$(2.5 .3 .2-19)$. That is,

$$
\begin{aligned}
a_{i} & =a_{i, s}+a_{i, a}\left(\frac{\alpha_{g, a}}{\alpha_{g}}\right) \\
& =\left(\frac{\alpha_{g, a}}{\alpha_{g}}\right)\left[\frac{4}{D_{h}}-\frac{2 D_{h}}{A_{c}}\left(1-\alpha_{g, a}\right)\right]+\frac{4.5 C \alpha_{g, s}}{D_{h}}
\end{aligned}
$$

In equation (2.5.3.2-26), interfacial area concentration due to annular flow contribution in this transition region is interpolated in terms of the ratio of annular flow void to total void fraction to satisfy a physical constraint. That is, annular void fraction $\left(\alpha_{g}, a\right)$ should approach zero as slug flow contribution to total void fraction becomes dominant.

Single Phase Limit near Zero Void Flow: For a physical system at zero void fraction, the interfacial area should become zero since there is no bubble present within a system. For the purpose of the code implementation, the formulation of the interfacial area concentration for the bubbly flow regime should satisfy the limiting condition. That is, from equation $(2.5 .3 .2-11)$,

$$
a_{i}=a_{i, b}=\frac{3.6 \alpha_{g}}{d_{0}}
$$

where $\mathrm{a}_{\mathrm{i}}$ goes to zero as $\alpha_{\mathrm{g}}$ approaches zero.

Single Phase Limit near Pure Void Flow: For no liquid flow within a gas-liquid system, the interfacial area concentration should become zero as a physical constraint. Under the present flow regime map, the formulation of the annular interfacial area concentration, equation (2.5.3.2-19), should be satisfied with this physical requirement. However, it is noted that $a_{i}$ goes to a finite non-zero value $\left(4 / \mathrm{D}_{h}\right)$ as void fraction approaches unity. In order to remedy this situation, the following forcing function is used to make the interfacial area concentration near unity void fraction become zero and well-
posed numerically: 
For $\alpha_{g}<\alpha_{0}$,

$$
\varphi\left(\alpha_{8}\right)=1
$$

For $\alpha_{0} \leq \alpha_{g} \leq 1.0$

$$
\varphi\left(\alpha_{g}\right)=\frac{\left(1-\alpha_{g}\right)}{\left(1-\alpha_{0}\right)}
$$

In the FLOWTRAN-TF code, $\alpha_{0}$ is taken as 0.999 . The smooth behavior of the forcing function is illustrated in Figure 2.5.3.2-4 when $\alpha_{0}=0.999$. For the code implementation near pure void region, the interfacial area concentration for the annular flow regime, $a_{i, a}$, is modified as

$$
a_{i}=a_{i, a}=\varphi\left(\alpha_{g}\right)\left(a_{i, a}\right)_{0} \quad \alpha_{g}>0.999
$$

where $\left(a_{i}, a\right)$ is given by equation $(2 \cdot 5 \cdot 3 \cdot 2-17)$.

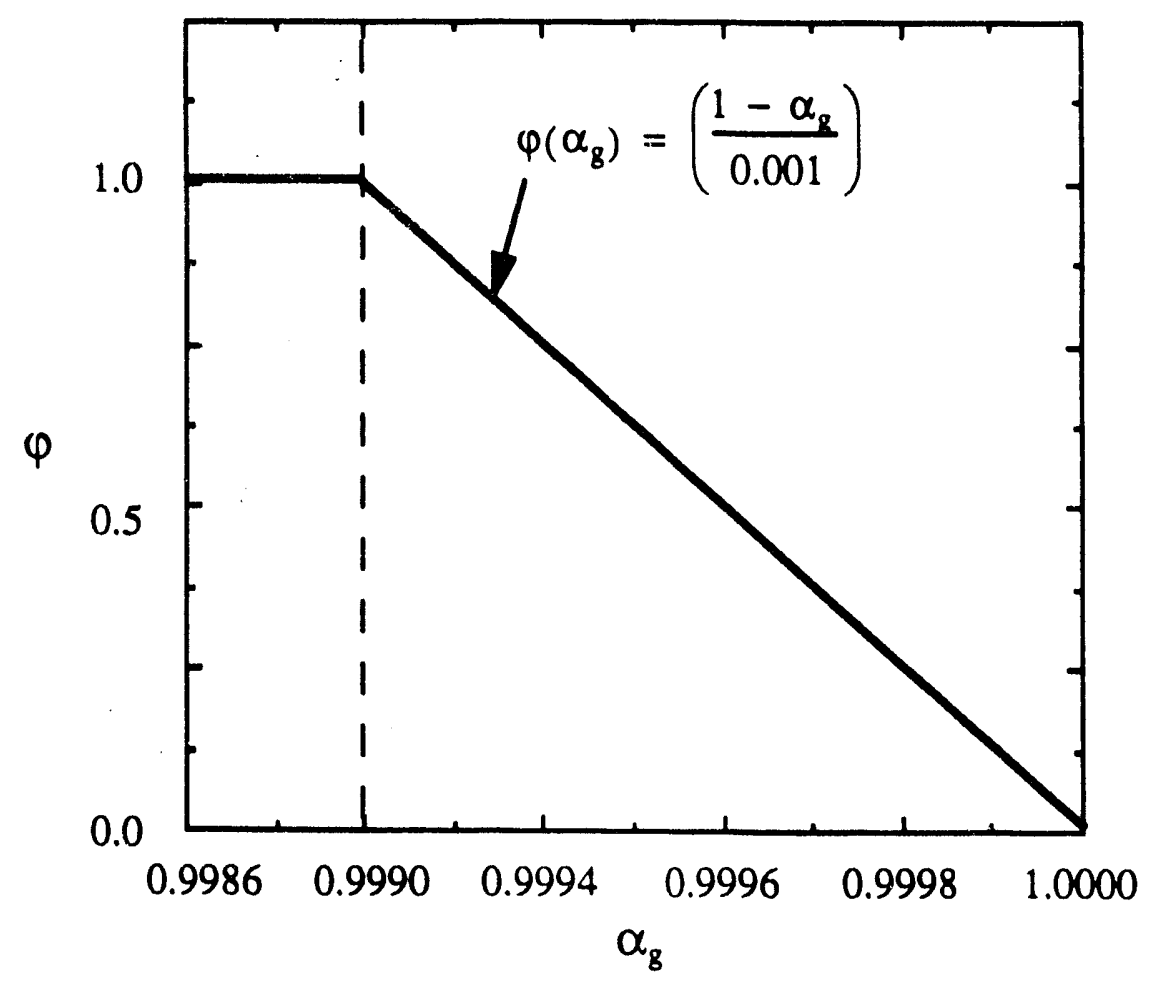

Figure 2.5.3.2-4 Forcing function for single phase limit near pure void region under annular flow regime. 
Table 2.5.3.2-1 summarizes the interfacial area models associated with each flow regime in FLOWTRAN-TF.

Table 2.5.3.2.1 Formulation of interfacial area concentration for each flow regime used in the FLOWTRAN-TF code.

\begin{tabular}{|c|c|}
\hline Flow Regime & Formulation of Interfacial Area Concentration \\
\hline $\begin{array}{l}\text { - Basic flow regime: } \\
\text { Bubbly flow } \\
\left(\alpha_{\mathrm{g}}<0.25\right) \\
\text { Annular flow } \\
\left(\alpha_{\mathrm{g}}>0.75\right) \\
\text { Slug flow } \\
\left.\text { (pure slug: } \alpha_{\mathrm{g}}=0.52\right)\end{array}$ & $\begin{aligned} a_{i, b} & =\frac{3.6 \alpha_{g, b}}{d_{o}} \\
a_{i, a} & =\frac{4}{D_{h}}-\frac{2 D_{b}}{A_{c}}\left(1-\alpha_{g, a}\right) \\
a_{i, s} & =\frac{4.5 C}{D} \alpha_{g, s}\end{aligned}$ \\
\hline $\begin{array}{r}- \text { Transition flow regime: } \\
\text { Bubbly - slug flow } \\
\left(0.25<\alpha_{\mathrm{g}}<0.52\right) \\
\text { Slug - annular flow } \\
\left(0.52<\alpha_{\mathrm{g}}<0.75\right)\end{array}$ & $\begin{aligned} a_{i} & =a_{i, b}+a_{i, s} \\
& =\frac{3.6 \alpha_{g, b}}{d_{0}}+\frac{4.5 C \alpha_{g, s}}{D_{h}} \\
a_{i} & =a_{i, s}+a_{i, a}\left(\frac{\alpha_{g, a}}{\alpha_{g}}\right) \\
& =\left(\frac{\alpha_{g, a}}{\alpha_{g}}\right)\left[\frac{4}{D_{h}}-\frac{2 D_{h}}{A_{c}}\left(1-\dot{\alpha}_{g, a}\right)\right]+\frac{4.5 C \alpha_{g, s}}{D_{h}}\end{aligned}$ \\
\hline $\begin{array}{l}\text { - Single phase limit: } \\
\text { Near zero void } \\
\text { Near pure void }\end{array}$ & $\begin{aligned} a_{i}= & a_{i, b}= \\
a_{i}= & a_{i, a}= \\
\text { where } & \left.\alpha_{g}>0.6 \alpha_{g}\right)\left(a_{i, a}\right)_{0} \\
& \varphi\left(\alpha_{g}\right) \text { is given by equation }(2.5 .3 .2-29)\end{aligned}$ \\
\hline
\end{tabular}




\begin{tabular}{|l|lll|l|}
\hline $2 / 15 / 93$ & WSRC.TR.92.532 Rev. 0 & FLOWTRAN-TF Software Design & Page 171 \\
\hline
\end{tabular}

\section{References:}

Abramowitz, M. and Stegun, I. A., 1972, Handbook of Mathematical Functions, Dover Publications, Inc., New York.

Bird, R. B., Stewart, W. E., and Lightfoot, E. N., 1960, Transport Phenomena, John Wiley \& Sons, New York.

Clift, R., Grace, J. R., and Weber, M. E., 1978, Bubbles, Drops, and Particles, Academic Press, New York.

Dimenna, R. A., Larson, J. R., Johnson, R. W., Larson, T. K., Miller, C. S., Streit, J. E., Hanson, R. G., and Kiser, D. M., 1988, "RELAP5/MOD2 Models and Correlations," NUREG/CR-5194, EGG-2531.

Ishii, M. and Mishima, K., 1980, "Study of Two-Fluid Model and Interfacial Area," NUREG/CR-1873.

Ransom, V. H., Wagner, R. J., Trapp, J. A., Feinauer, L. R., Johnson, G. W., Kiser, D. M., and Riemke, R. A., 1985, "RELAP5/MOD2 Code Manual Volume 1: Code Structure, System Models, and Solution Methods," NUREG/CR-4312, EGG2396.

Wallis, G., 1969, One-Dimensional Two Phase Flow, McGraw-Hill, New York. 


\subsubsection{Interfacial Heat and Mass Transfer}

Interfacial heat and mass transfer at the gas-liquid interface is modeled by FLOWTRAN-TF as described in section 2.5.4.2. First the rate coefficients for heat and mass transfer needed by the model are defined in section 2.5.4.1 however. In section 2.5.4.3, the models for subcooled boiling and superheated condensation at the solid wall are presented.

\subsubsection{Rate Coefficients}

This section presents the heat and mass transfer rate coefficients used in the bulk interfacial heat and mass transfer model described in the next section. Interfacial heat and mass exchange occurs due to temperature and concentration gradients between gas/liquid interface and bulk fluid. Correlations are employed for each phase and in each flow regime. Correlations are applicable to low mass transfer rates. A correction, which is referred to as the Ackermann correction, is applied to the resulting coefficient to consider the impact of high mass transfer rates on the simultaneous heat and mass transfer rates at the interface. The Ackermann correction is discussed in the section 2.5.4.2. Table 2.5.4.1-2 summarizes all the interfacial heat and mass transfer correlations used in the FLOWTRAN-TF code.

Bubbly flow regime: In the FLOWTRAN-TF code, the applicable range of the correlation is chosen from the smooth transition of the curve for each correlation due to the lack of definite boundary for the present problem, i.e., heat transfer from bubble in liquid fluid, as illustrated numerically in Figures 2.5.4.1-1 and 2.5.4.1-2. 


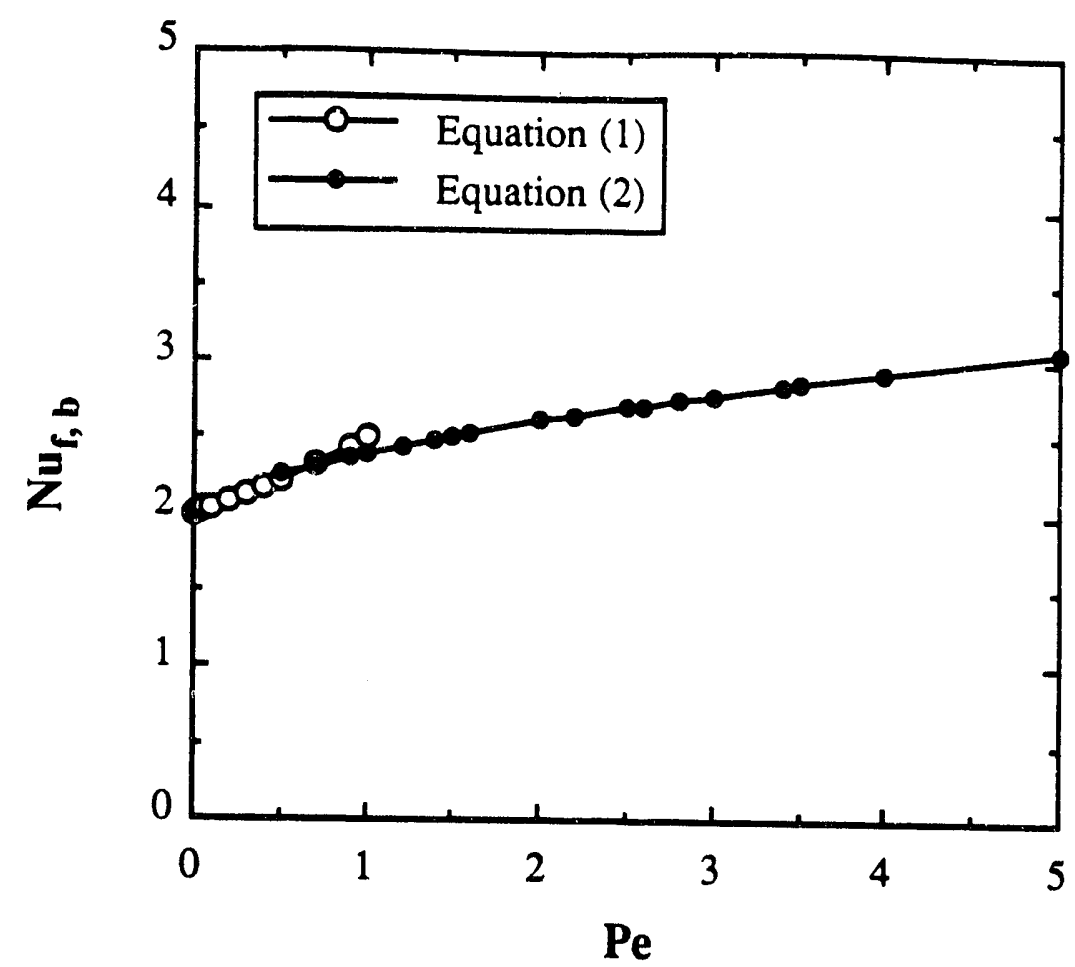

Figure 2.5.4.1-1 Average interfacial heat transfer coefficient external to a bubble in Stokes flow $(\operatorname{Re}<1)$

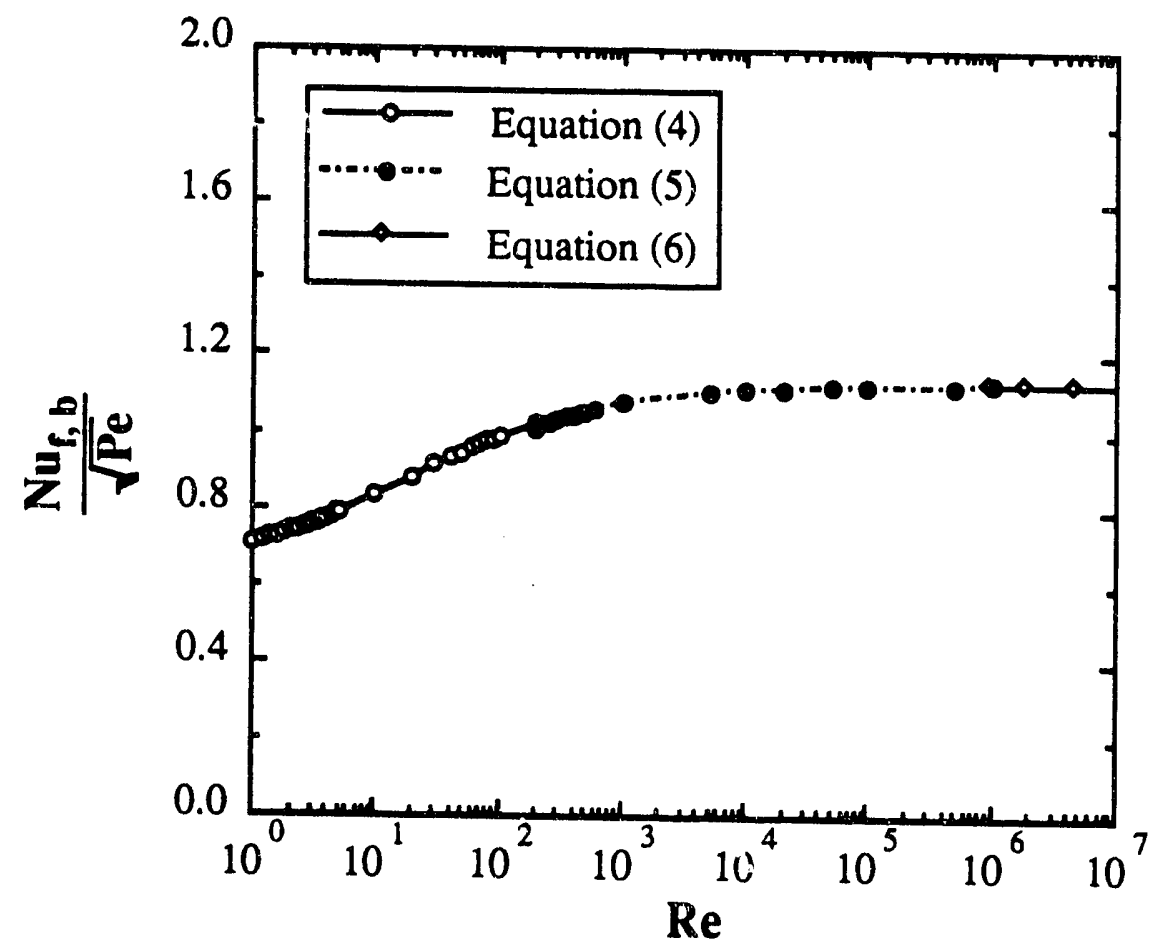

Figure 2.5.4.1-2 Average interfacial heat transfer coefficient external to a bubble at higher Reynolds number $(\operatorname{Re}>1$ ), 
The code computes the interfacial transfer coefficient on the liquid fluid side according to the following correlations given under steady state condition. For the low Reynolds number (Stokes) flow relative to a fluid bubble of spherical shape, equations $(2.5 .4 .1-1)$ and (2.5.4.1-2) are used for forced convective heat transfer coefficient around a bubble. Equation (2.5.4.1-1) is an approximate result for average Nusselt number under steady state forced convection heat transfer from an isothermal fluid particle to an external fluid flowing in small Peclet number. This equation was derived by Brenner (1963) for a fluid sphere when Peclet number goes near zero. Equation (2.5.4.1-2), which is an approximation to Oellrich et al.'s (1973) numerical results for all non-zero Peclet numbers, is given by Clift et al. (1978).

For $\operatorname{Re}<1$

$$
N u_{f, b}=2+0.5 \mathrm{Pe}+\frac{\left(2 \mu_{f}+3 \mu_{g}\right)}{12\left(\mu_{f}+\mu_{g}\right)} \mathrm{Pe}^{2} \ln (\mathrm{Pe})
$$

where $\mathrm{Pe}<0.5$ and

$$
\mathrm{Nu}_{\mathrm{f}, \mathrm{b}}=1+\left(1+0.564 \mathrm{Pe}^{2 / 3}\right)^{0.75}
$$

where $\mathrm{Pe}>0.5$. $\mathrm{Nu}_{\mathrm{f}, \mathrm{b}}$ is the Nusselt number of fluid in the bubbly flow regime and

$$
\mathrm{Nu}_{\mathrm{f}, \mathrm{b}}=\frac{\mathrm{h}_{\mathrm{f}, \mathrm{b}} \mathrm{d}}{\mathrm{k}_{\mathrm{f}}}
$$

with $\mathrm{d}$ equal to bubble diameter, $\mathrm{k}_{\mathrm{f}}$ the thermal conductivity of liquid fluid, and $\mathrm{h}_{\mathrm{f}, \mathrm{b}}$ the convective interfacial heat transfer coefficient on the liquid phase side in the bubbly flow regime. Reynolds number $(\mathrm{Re})$ is also definc 1 as

$$
\operatorname{Re}=\frac{d u_{\mathrm{r}} \rho_{\mathrm{f}}}{\mu_{\mathrm{f}}}
$$

where $u_{\mathrm{r}}$ is the relative velocity between gas and liquid phases and $\rho_{\mathrm{f}}$ and $\mu_{\mathrm{f}}$ are liquid density and viscosity respectively.

For the intermediate ileynolds number, the code uses equation (2.5.4.1-5) recommended by Clift et al. (1978). The equation was obtained by fitting the calculated values, which were based on the asymf . otic solutions using the surface velocities of Abdel-Alim and Hamielec (1975). That is, for $1<\operatorname{Re}<200$

$$
N u_{f, b}=\frac{2}{\sqrt{\pi}}\left(1-\frac{2}{3\left(1+0.1415(\operatorname{Re})^{2 / 3}\right)^{0.75}}\right)^{0.5}(\mathrm{Pe})^{0.5}
$$

For the heat transfer from the spherical bubble at high Reynolds and Peclet numbers, the interfacial heat transfer coefficient from the gas bubble surface within liquid fluid was 
obtained by Weber (1975) using the thin boundary layer assumption and the interfacial velocity of Harper and Moor (1968). The equation is given by

$$
\mathrm{Nu}_{\mathrm{f}, \mathrm{b}}=\frac{2}{\sqrt{\pi}}\left(1-\frac{2.89}{\mathrm{Re}^{0.5}}\right)^{0.5} \mathrm{Pe}^{0.5}
$$

where the recommended applicable range of Reynolds number is from 200 to sufficiently high number, i.e., 1000000 .

When Reynolds number of fluid flow external to a spherical bubble approaches infinity as an asymptotic situation, the external heat transfer coefficient is derived using thin boundary layer approximation and potential flow velocity distribution external to the bubble surface, which is known as Boussinesq equation, namely,

$$
\mathrm{Nu}_{\mathrm{f}, \mathrm{b}}=\frac{2}{\sqrt{\pi}}(\mathrm{Pe})^{0.5}
$$

In the code, equation (2.5.4.1-7) is used at greater than 1000000 of Reynolds number.

The heat transfer coefficient for the gas phase side is given by (Dimenna et al., 1988)

$$
\mathrm{Nu}_{\mathrm{g}, \mathrm{b}}=200
$$

where

$$
\mathrm{Nu}_{g, b}=\frac{h_{g, b} \mathrm{~d}}{\mathrm{k}_{\mathrm{g}}}
$$

The intent is to drive the gas bubble temperature to the interfacial temperature and to ensure that gas phase side is not controlling the interfacial heat transfer resistance [Treybal, 1980] as with RELAP5 [Dimenna et al., 1988]. During FLOWTRAN-TF development $\mathrm{Nu}_{\mathrm{g}, \mathrm{b}}=$ $10^{4}$ was used following RELAP5. However, convergence problems were sometimes encountered in the iteration for interfacial steam mole fraction $\left(y_{s i}\right)$, temperature $\left(T_{i}\right)$ and mass transfer rate $(\Gamma)$, apparently due to numerically large values of gas-side heat transfer coefficient $\left(\mathrm{H}_{\mathrm{gi}}\right)$. The present selection of $\mathrm{Nu}_{\mathrm{g}, \mathrm{b}}=200$ yields a heat transfer coefficient on the order of $10^{3} \mathrm{~W} / \mathrm{m}^{2} \mathrm{~K}$ which is sufficiently large to force the gas temperature to effectively equal the interfacial temperature. This is reasonable for most processes since gas temperature within bubble is increased rapidly toward the saturation temperature corresponding to local system pressure.

The mass transfer coefficient on the gas phase side is computed using the Chilton-Colburn heat and mass transfer analogy. The relation is given by Sherwood et al. (1975).

$$
\mathrm{Sh}_{\mathrm{g}, \mathrm{b}}=\mathrm{Nu}_{\mathrm{g}, \mathrm{b}} \mathrm{Le} \mathrm{e}^{1 / 3}
$$

where Le is the Lewis number and is defined as the ratio of Sc to Pr. The non-dimensional numbers used in equation (2.5.4.1-10) are described as 


$$
\begin{aligned}
S h_{g, b} & =\frac{k_{g, b} d}{C \delta_{A B}} \\
S c & =\frac{\mu}{\rho \delta_{A B}} \\
\operatorname{Pr} & =\frac{C_{p} \mu}{k_{g}}
\end{aligned}
$$

where

$$
\begin{aligned}
\boldsymbol{K}_{\mathrm{g}, \mathrm{b}} & \equiv \text { mass transfer coefficient }\left[\mathrm{kmol} / \mathrm{m}^{2} \mathrm{sec}\right] \\
\mathrm{d} & \equiv \text { bubble diameter }[\mathrm{m}] \\
\mathrm{C} & \equiv \text { gas phase concentration }\left[\mathrm{kmol} / \mathrm{m}^{3}\right] \\
\delta_{\mathrm{AB}} & \equiv \text { diffusivity }\left[\mathrm{m}^{2} / \mathrm{sec}\right] \\
\mathbf{k}_{\mathrm{g}} & \equiv \text { gas thermal conductivity }[\mathrm{W} / \mathrm{m} \mathrm{K}] \\
\mathrm{C}_{\mathrm{p}} & \equiv \text { specific heat capacity }[\mathrm{J} / \mathrm{kg} \mathrm{K}]
\end{aligned}
$$

In general, the approach of using the analogous relationship beiween heat and mass transfers is chosen as illustrated in Table 2.5.4.1-1.

Slug flow regime: This flow regime is never strictly stable and fully-developed since the following bubble is continuously tending to catch up with the preceding bubble and coalesce with it. Therefore, the purely slug flow is virtually never postulated by the code as specified by the flow regime map section. For the liquid side external to the slug bubble, the heat transfer coefficient is computed from the mass transfer experiments of Van Heuven and Beek (1963). Their experimental data are correlated by Clift et al. (1978) as

$$
\kappa_{\mathrm{f}, \mathrm{s}}{ }^{\prime}=\mathrm{C}_{\mathrm{s}} \delta_{\mathrm{AB}} 0.5\left(\frac{\mathrm{g}}{\mathrm{L}_{\mathrm{s}}}\right)^{0.25}
$$

where $\kappa_{\mathrm{f},}{ }^{\prime}$ is the bubble surface-averaged mass transfer coefficient on the liquid side based on the concentration difference and $L_{s}$ is the length of slug bubble. Equation (2.5.4.1-14) can be rewritten in terms of Sherwood number as follows:

$$
\mathrm{Sh}_{\mathrm{f}, \mathrm{s}}=\mathrm{C}_{\mathrm{s}} \mathrm{d}_{\mathrm{s}} \delta_{\mathrm{AB}}-0.5\left(\frac{\mathrm{g}}{\mathrm{L}_{\mathrm{s}}}\right)^{0.25}
$$

where $d_{s}$ is the equivalent diameter of top portion of cylindrical slug bubble.

From the analogy of heat and mass transfers given in Table 2.5.4.1-1, the corresponding heat transfer correlation is obtained by replacing the mass diffusivity by the thermal diffusivity. 


$$
\mathrm{Nu}_{\mathrm{f}, \mathrm{s}}=\mathrm{C}_{\mathrm{s}} \mathrm{d}_{\mathrm{s}}\left(\frac{\mathrm{k}_{\mathrm{f}}}{\rho_{\mathrm{f}} \mathrm{C}_{\mathrm{p}, \mathrm{f}}}\right)^{-0.5}\left(\frac{\mathrm{g}}{\mathrm{L}_{\mathrm{s}}}\right)^{0.25}
$$

When the values of $d_{s} C_{s}$, and $L_{s}$ in the equation are given, convective heat transfer coefficient on the liquid phase side can be computed. In the FLOWTRAN-TF code $d_{s}$ is set equal to 0.88 times channel hydraulic diameter $\left(\mathrm{d}_{h}\right)$ from the work of Ishii and Mishima (1980) and $\mathrm{L}_{\mathrm{s}}$ is give in terms of non-dimensional ratio $\left(\mathrm{L}_{\mathrm{s}} / \mathrm{d}_{\mathrm{h}}=4.5\right)$ from the laboratory observation of rising bubbles within stagnant liquid in single annular channel with ribs [Kielpinski, 1991]. The values of the constant $C_{s}$ in equation (2.5.4.1-16) are given as near unity for $L_{s} / d_{h}>1$. For the present channel geometry, $C_{s}$ can be taken as unity.

For the gas phase side in the slug flow, Filla et al. (1976) recommended the correlation for gas phase mass transfer coefficient for $1.0<\mathrm{L}_{\mathrm{s}} / \mathrm{d}_{\mathrm{h}}<2.0$, but their correlation can be reasonably extended to higher $L_{s} / d_{h}(=4.5)$ based on their experimental data.

$$
\begin{gathered}
\mathrm{Sh}_{\mathrm{g}, \mathrm{s}}=9.3 \quad \text { for } \mathrm{Pe}_{\mathrm{m}}<25 \\
\mathrm{Sh}_{\mathrm{g}, \mathrm{s}}=2.05 \sqrt{\mathrm{Pe}_{\mathrm{m}}} \quad \text { for } \mathrm{Pe}_{\mathrm{m}}>25
\end{gathered}
$$

In the code implementation, 10.25 was used instead of 9.3 in equation (2.5.4.1-17) for smooth transition between equations, $(2.5 .4 .1-17)$ and $(2.5 .4 .1-18)$ at $\mathrm{Pe}_{\mathrm{m}}=25$. In equations (2.5.4.1-17) and (2.5.4.1-18), $\mathrm{Pe}_{\mathrm{m}}$ is the Peclet number in the mass transfer defined as

$$
P e_{m}=\frac{u_{s} d_{h}}{\delta_{A B}}
$$

where $u_{s}$ is the velocity of slug bubble within channel.

Th heat transfer correlation in the slug flow regime corresponding to equations $(2.5 .4 .1-17)$ and $(2.5 .4 .1-18)$ can be found from the heat and mass transfer analogy given in Table 2.5.4.1-1.

Annular flow regime: Emmert and Pigford (1954) developed correlation of the mass transfer from the gas phase to the liquid film on the channel wall assuming equilibrium at the interface, a parabolic velocity distribution in the falling liquid film, uniform initial gas phase concentration, and no rippling waves at the gas-liquid interface. They also indicated that the transition from laminar to turbulent flow occurs at a Reynold number of about 1200 , which was in an agreement with the results of previous investigators. The correlation for the laminar film flow $\left(\operatorname{Re}_{\mathrm{f}}<1200\right)$ is given by

$$
\mathrm{Sh}_{\mathrm{f}, \mathrm{a}}=\left(\frac{3 \delta_{\mathrm{f}}}{2 \pi \mathrm{L}} \operatorname{Re}_{\mathrm{f}} \mathrm{Sc}\right)^{0.5}
$$

where

$$
\delta_{\mathrm{f}} \equiv \text { liquid film thickness }
$$




$$
\begin{aligned}
\operatorname{Re}_{\mathrm{f}} & \equiv 4 \Gamma / \mu \\
\Gamma & \equiv \text { mass flow rate per unit time per unit length of wetted periphery } \\
L & \equiv \text { channel height with liquid film }
\end{aligned}
$$

The mass transfer coefficient on the turbulent liquid phase side was correlated by Lamourelle and Sandall (1972) from the experimental work over a Reynolds number range of 1300 to 8300 . The correlation for $1300<\operatorname{Re}_{\mathrm{f}}<8300$ is

$$
\mathrm{Sh}_{\mathrm{f}, \mathrm{a}}=1.76 \times 10^{-5} \mathrm{Re}^{1.506} \mathrm{Sc}^{0.5}
$$

For the transition region $\left(1200<\operatorname{Re}_{\mathrm{f}}<1300\right)$, the mass transfer coefficient is computed by the linear interpolation, that is,

$$
S h_{f, a}=\left.S h_{f, a}\right|_{\operatorname{Re}_{\mathrm{f}}=1200}+0.01\left(\left.S h_{\mathrm{f}, \mathrm{a}}\right|_{\operatorname{Re}_{\mathrm{f}}=1300}-\left.S h_{\mathrm{f}, \mathrm{a}}\right|_{\operatorname{Re}_{\mathrm{f}}=1200}\right)\left(\operatorname{Re}_{\mathrm{f}}-1200\right)
$$

The heat transfer correlations corresponding to the equations, (2.5.4.1-20) to (2.5.4.1-22), are obtained by the heat-mass transfer analogy given in Table 2.5.4.1-1.

The mass transfer coefficient on the gas phase side is given by Sherwood et al. (1975) based on the experimental work of Gilliland and Sherwood (1934) on the diffusion of water vapor into ir stream.

$$
\left(\frac{K_{g_{1} a} d_{h}}{\delta_{A B}}\right)\left(\frac{P_{B M}}{P}\right)=0.023 \operatorname{Re}^{0.83} S^{0.44}
$$

where

$$
\begin{aligned}
\mathrm{P}_{\mathrm{BM}} & \equiv \text { logarithmic mean partial pressure of non-diffusion gas phase medium } \\
\mathrm{P} & \equiv \text { total system pressure }
\end{aligned}
$$

For the code implementation, equation (2.5.4.1-23) is converted into the following in terms of the Sherwood number using logarithmic mean difference of non-diffusing gas (air) concentration:

$$
\mathrm{Sh}_{\mathrm{g}, \mathrm{a}} \mathrm{C}_{\mathrm{BM}}=0.023 \mathrm{Re}^{0.83} \mathrm{Sc}^{0.44}
$$

where $\mathrm{C}_{\mathrm{BM}}$ is the logarithmic mean difference of air concentration between the interface and bulk region.

The corresponding correlation of heat transfer coefficient is given by

$$
\mathrm{Nu}_{\mathrm{g}_{\mathrm{a}} \mathrm{a}}=0.023 \mathrm{Re}^{0.83} \operatorname{Pr}^{0.44}
$$


Table 2.5.4.1-1 Analogies of heat and mass transfers

\begin{tabular}{|c|c|}
\hline Heat Transfer & Mass Transfer \\
\hline Temperature $[\mathrm{K}]$ & Concentration $\left[\mathrm{kmol} / \mathrm{m}^{3}\right]$ \\
\hline $\begin{array}{l}\text { Thermal diffusivity } \\
\qquad \alpha=\frac{k}{\rho C_{p}}\end{array}$ & $\begin{array}{l}\text { Mass diffusivity } \\
\qquad \delta_{\mathrm{AB}}\end{array}$ \\
\hline $\begin{array}{l}\text { Prandtl number } \\
\text { (Pr) }\end{array}$ & $\begin{array}{l}\text { Schmidt number } \\
(\mathrm{Sc})\end{array}$ \\
\hline $\begin{array}{l}\text { Péclet number }\left(\mathrm{Pe}_{\mathrm{h}}\right) \\
\mathrm{Pe}_{\mathrm{h}}=\frac{\rho \mathrm{C}_{\mathrm{p}} \mathrm{ud}_{\mathrm{h}}}{\mathrm{k}}\end{array}$ & $\begin{array}{l}\text { Péclet number }\left(\mathrm{Pe}_{\mathrm{m}}\right) \\
\mathrm{Pe}_{\mathrm{m}}=\frac{\mathrm{u} \mathrm{d}_{\mathrm{h}}}{\delta_{\mathrm{AB}}}\end{array}$ \\
\hline $\begin{array}{l}\text { Nusselt number }(\mathrm{Nu}) \text { : } \\
\begin{array}{l}\mathrm{Nu}=\frac{\mathrm{hd}}{\mathrm{k}} \\
\text { where } \\
\mathrm{h}=\text { heat transfer coefficient }\end{array}\end{array}$ & $\begin{array}{l}\text { Sherwood number }(\mathrm{Sh}): \\
\text { Sh }=\frac{\kappa \mathrm{d}}{\mathrm{C} \delta_{\mathrm{AB}}} \\
\text { where } \\
\boldsymbol{K}=\text { mass transfer coefficient }\end{array}$ \\
\hline
\end{tabular}


Table 2.5.4.1-2 Interfacial heat and mass transfer coefficient correlations in FLOWTRAN-TF code (The same correlations are used for mass transfer by heat-mass transfer analogy in Table 2.5.4.1-1)

\begin{tabular}{|c|c|c|}
\hline Flow Regime & $\begin{array}{l}\text { Liquid or } \\
\text { Gas Phase }\end{array}$ & Correlation \\
\hline \multirow[t]{2}{*}{ Bubbly Flow } & Liquid Phase & 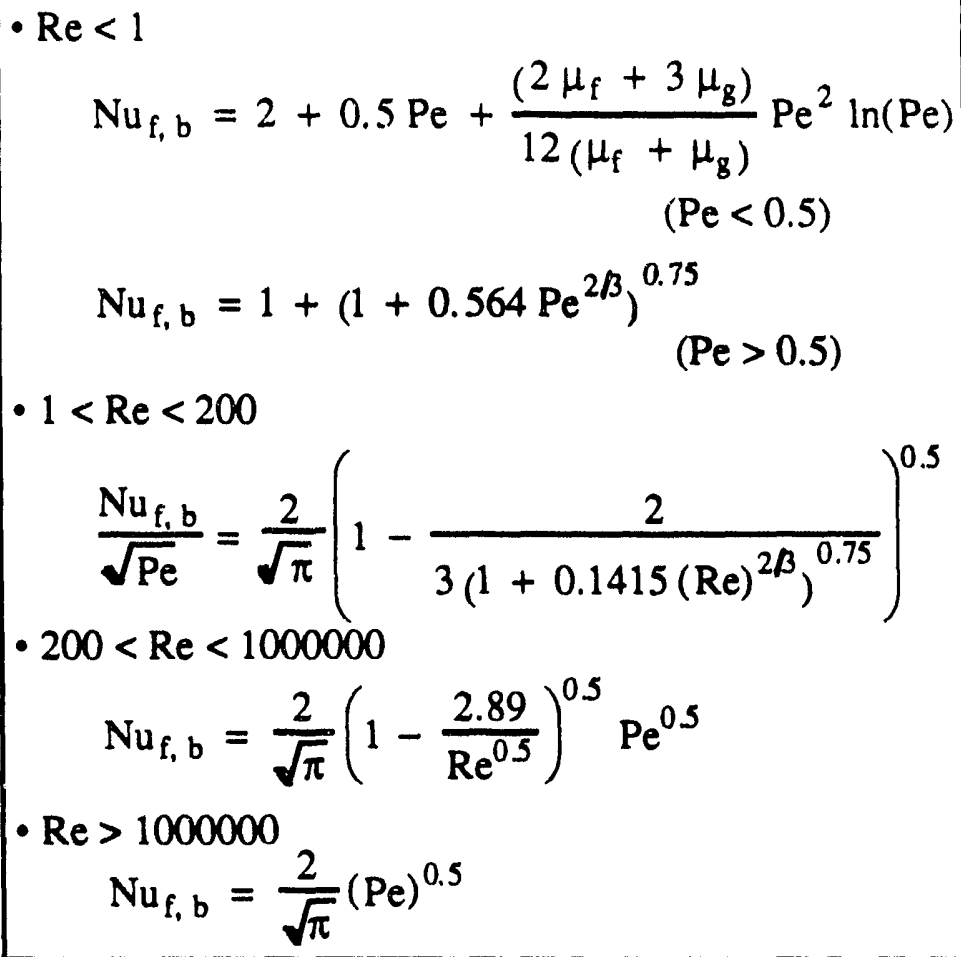 \\
\hline & Gas Phase & $\mathrm{Nu}_{\mathrm{g}, \mathrm{b}}=200$ \\
\hline \multirow{2}{*}{ Slug Flow } & Liquid Phase & $\mathrm{Nu}_{\mathrm{f}, \mathrm{s}}=\mathrm{C}_{\mathrm{s}} \mathrm{d}_{\mathrm{s}}\left(\frac{\mathrm{k}_{\mathrm{f}}}{\rho_{\mathrm{f}} \mathrm{C}_{\mathrm{p}, \mathrm{f}}}\right)^{-0.5}\left(\frac{\mathrm{g}}{\mathrm{L}_{\mathrm{s}}}\right)^{0.25}$ \\
\hline & Gas Phase & $\begin{array}{ll}\mathrm{Nu}_{\mathrm{g}, \mathrm{s}}=10.25 & \left(\mathrm{Pe}_{\mathrm{h}}<25\right) \\
\mathrm{Nu}_{\mathrm{g}, \mathrm{s}}=2.05 \sqrt{\mathrm{Pe}_{\mathrm{h}}} & \left(\mathrm{Pe}_{\mathrm{h}}>25\right)\end{array}$ \\
\hline \multirow[t]{2}{*}{ Annular Flow } & Liquid Phase & $\begin{array}{l}\text { - Laminar flow }\left(\operatorname{Re}_{\mathrm{f}}<1200\right) \\
\mathrm{Nu}_{\mathrm{f}, \mathrm{a}}=\left(\frac{3 \delta_{\mathrm{f}}}{2 \pi \mathrm{L}} \operatorname{Re}_{\mathrm{f}} \operatorname{Pr}\right)^{0.5} \\
\text { - Turbulent flow }\left(\operatorname{Re}_{\mathrm{f}}>1300\right) \\
\mathrm{Nu}_{\mathrm{f}, \mathrm{a}}=1.76 \times 10^{-5} \operatorname{Re}^{1.506} \operatorname{Pr}^{0.5} \\
\text { - Transition region } \\
\text { Linear interpolation between laminar and } \\
\text { turbulent values }\end{array}$ \\
\hline & Gas Phase & $\mathrm{Nu}_{\mathrm{g}_{\mathrm{a}}}=0.023 \mathrm{Re}^{0.83} \operatorname{Pr}^{0.44}$ \\
\hline
\end{tabular}




\section{References:}

Clift, R. Grace, J. R., and Weber, M. E., 1978, Bubbles, Drops, and Particles, Academic Press, New York.

Gilliland, E. R. and Sherwood, T. K., 1934, "Diffusion of Vapors into Air Streams", Industrial Engineering Chemistry, Vol. 26, pp. 516.

Van Heuven, J. W. and Beek, J. W., 1963, "Gas Absorption in Narrow Gas Lifts", Chemical Engineering Science, Vol. 18, pp. 377-390.

Sherwood, T. K., Pigford, R. L., and Wilke, C. R., 1975, Mass Transfer, McGraw-Hill Book Company, New York.

Treybal, R. E., 1980, Mass Transfer Operations, McGraw-Hill Book Company, New York.

Acrivos, A. and Taylor, T. D., 1962, "Heat and Mass Transfer from Single Spheres in Stokes Flow", The Physics of Fluids, Vol. 5, No. 4, pp. 387-394.

Filla, M. Davidson, J. F.. Bates, J. F., and Eccles, M. A., 1976, "Gas Phase Controlled Mass Transfer from a Bubble", Chemical Engineering Science, Vol. 31, pp. 359 367.

Weber, M. E., 1975, "Mass Transfer from Spherical Drops at High Reynolds Numbers", Industrial and Engineering Chemistry, Fundamentals, Vol. 14, No. 4, pp. 365366.

Dimenna, R. A., Larson, J. R., Johnson, R. W., Larson, T. K., Miller, C. S., Streit, J. E., Hans'sn, R. G., and Kiser, D. M., 1988, "RELAP5/MOD 2 Models and Correlations", NUREG/CR-5194, EGG-2531.

Oellrich, L., Schmidt-Traub, H., and Brauer, H., 1973, "Theoretische Berechnung des Stofftransports in der Umgebung einer Einzelblase", Chemical Engineering Science, Vol. 28, pp. 711-721.

Gilliland, E. R., and Sherwood, T. K., 1934, "Diffusion of Vapors into Air Streams", Industrial and Engineering Chemistry, Vol. 26, No. 5, pp. 516-523.

Harper, J. E. and Moore, D. W., 1968, "The Motion of a Spherical Liquid Drop at High Reynolds Number", Journal of Fluid Mechanics, Vol. 32, Part 2, pp. 367-391.

Abdel-Alim, A. H. and Hamielec, A. E., 1975, "A Theoretical and Experimental Investigation of the Effect of Internal Circulation on the Drag of Spherical Droplets Falling at Terminal Velocity in Liquid Media", Industrial and Engineering Chemistry, Fundamentals, Vol. 14, No. 4, pp. 308-312.

Hieber C. A. and Gebhart, B., 1968, "Low Reynolds Number Heat Transfer from a Circular C'ylinder", Journal of Fluid Mechanics, Vol. 32, Part 1, pp. 21-28.

Brenner, H., 1963, "Forced Convection Heat and Mass Transfer at Small Peclet Numbers from a Particle of Arbitrary Shape", Chemical Engineering Science, Vol. 18, pp. 109-122. 
Lamourelle, A. P. and Sandall, O. C., 1972, "Gas Absorption "nto a Turbulent Liquid", Chemical Engineering Science, Vol. 27, pp. 1035-1043.

Emmert, R. E. and Pigford, R. L., 1954, "A Study of Gas Absorption in Falling Liquid Films", Chemical Engineering Progress, Vol. 50, No. 2, pp. 87-93.

Ishii, M. and Mishima, K., 1980, "Study of Two-Fluid Model and Interfacial Area", NUREG/CR-1873. 


\begin{tabular}{|l|lll|l|}
\hline $2 / 15 / 93$ & WSRC-TR-92.532 Rev.0 & FLOWTRAN-TF Software Design & Page 183 \\
\hline
\end{tabular}

\subsubsection{Interfacial Heat and Mass Transfer in Bulk Flow}

We consider the simultaneous transport of heat and mass across a gas-liquid interface as illustrated in Fig. 2.5.4.2-1. For simplicity we will restrict the discussion to the air-water interface of interest in FLOWTRAN-TF. We treat the liquid phase as a single component (neglecting dissolved air) and the gas phase as a two component mixture of air and water vapor. Then, adopting a film theory model as shown in Fig. 2.5.4.2-1, we postulate the existence of a temperature profile across a thin stagnant film on the liquid side of the boundary and both temperature and concentration profiles across similar films on the vapor side. In general, as indicated in Fig. 2.5.4.2-1, the concentration film thickness can be different from the temperature film thickness on the gas side. We assume no accumulation of mass or energy at the interface and adopt the convention that positive fluxes are directed from the interface into the bulk gas phase. The actual interfacial heat and mass transfer processes are extremely complicated since the real interfaces are irregular and moving. We obviously cannot hope to model this complexity in detail. To make the modeling tractable, 've consider a one-dimensional stationary interface as illustrated in Fig. 2.5.4.2-1.

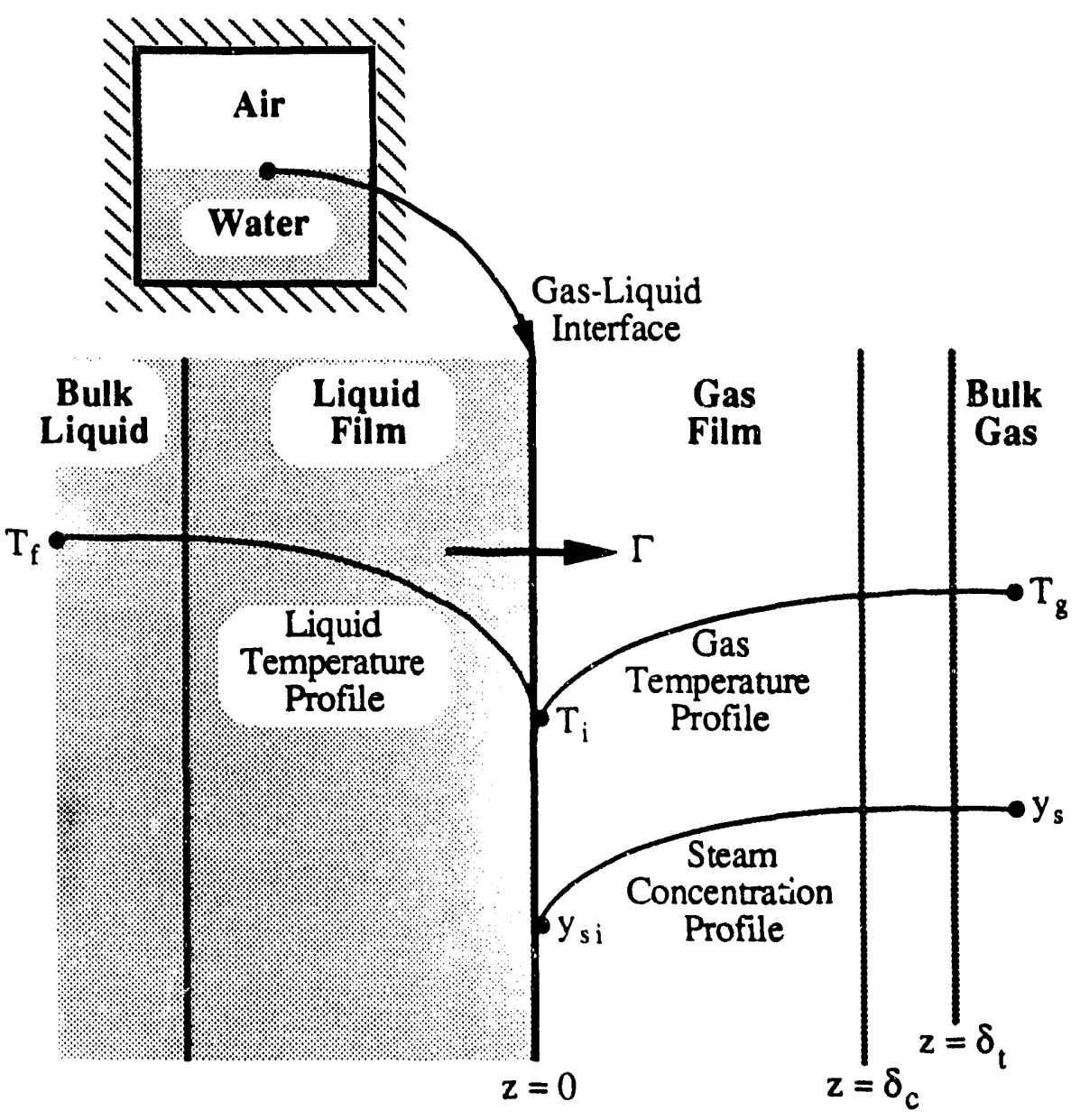

Figure 2.5.4.2-1 Diagram of simultaneous heat and mass transfer at a gas-liquid interface. 
Mass Transfer: We wish to estimate the mass transfer across the phase boundary in such a way that it will be applicable at large mass fluxes (Bird et al., 1960, Sherwood et al., 1975). Since there is no accumulation at the interface, the mass flux is entirely characterized by the gas side mass transfer. Under steady state conditions at any location within the gas film, the mass flux of water vapor (steam) can be expressed as the sur. of a diffusive flux component and a convective flux component

$$
\mathrm{N}_{\mathrm{s}}=-\mathrm{c} D_{s a} \frac{\mathrm{dy}}{\mathrm{dz}}+\mathrm{y}_{\mathrm{s}}\left(\mathrm{N}_{\mathrm{s}}+\mathrm{N}_{\mathrm{a}}\right)
$$

where

$$
\begin{aligned}
\mathrm{N}_{\mathrm{s}} & \equiv \text { Molar flux of water vapor (steam), } \mathrm{kg}-\mathrm{mole} / \mathrm{m}^{2}-\mathrm{s} \\
\mathrm{N}_{\mathrm{a}} & \equiv \text { Molar flux of air, } \mathrm{kg}-\mathrm{mole} / \mathrm{m}^{2}-\mathrm{s} \\
\mathrm{y}_{\mathrm{s}} & \equiv \text { Mole fraction of water vapor (steam) } \\
\mathrm{c} & \equiv \text { Molar concentration of gas phase, } \mathrm{kg}-\mathrm{mole} / \mathrm{m}^{3} \\
D_{s a} & \equiv \text { Binary diffusion coefficient for water vapor in air, } \mathrm{m}^{2} / \mathrm{s} \\
\mathrm{z} & \equiv \text { Direction coordinate normal to interface, } \mathrm{m}
\end{aligned}
$$

The molar fluxes $\mathrm{N}_{\mathrm{s}}$ and $\mathrm{N}_{\mathrm{a}}$ are taken with respect to a fixed coordinate system. Since we assume a fixed interface, these fluxes are also with respect to the interface. Because the air component is assumed to be noncondensible in the liquid phase, the flux of air perpendicular to the interface in the gas film is zero $\left(\mathrm{N}_{\mathrm{a}}=0\right)$ and we can then rearrange Eq. $(2.5 .4 .2-1)$ to separate variables as

$$
\frac{\mathrm{dz}}{\mathrm{c} D_{s a}}=\frac{-\mathrm{dy}_{\mathrm{s}}}{\mathrm{N}_{\mathrm{s}}\left(1-\mathrm{y}_{\mathrm{s}}\right)}
$$

Integrating both sides of Eq. (2.5.4.2-2) across the concentration film assuming a constant mass flux of water vapor yields

$$
\begin{gathered}
\int_{0}^{\delta_{c}} \frac{d z}{c D_{s a}}=\frac{\delta_{c}}{c D_{s a}} \\
-\frac{1}{N} \int_{y_{i}}^{y_{s}} \frac{d y_{s}}{\left(1-y_{s}\right)}=\frac{1}{N_{s}} \ln \left[\frac{1-y_{s}}{1-y_{s i}}\right]
\end{gathered}
$$

where

$\delta_{c} \equiv$ Concentration film thickness, $m$

Dsa $\equiv$ Average binary diffusion coefficient for water vapor in air, $\mathrm{m}^{2} / \mathrm{s}$

$y_{s i} \equiv$ Mole fraction of water vapor at the interface 


$$
y_{s} \equiv \text { Mole fraction of water vapor in the bulk gas }
$$

Note that the integrated diffusion coefficient on the right hand side of Eq. (2.5.4.2-3) is taken to be an average value representative of conditions across the film.

Equating the two resultant expressions given in Eq. (2.5.4.2-3) we obtain

$$
\left[\frac{1-y_{s}}{1-y_{s i}}\right]=\exp \left(\frac{N_{s} \delta_{c}}{c D_{s a}}\right)
$$

Equation (2.5.4.2-4) can then be rearranged by observing that

$$
\frac{1-y_{s}}{1-y_{s i}}=\frac{1-y_{s i}+y_{s i}-y_{s}}{1-y_{s i}}=1+\frac{y_{s i}-y_{s}}{1-y_{s i}}
$$

Substituting Eq. (2.5.4.2-5) into Eq. (2.5.4.2-4) yields the equivalent result

$$
\frac{y_{s i}-y_{s}}{1-y_{s i}}=\exp \left(\frac{N_{s} \delta_{c}}{c D_{s a}}\right)-1
$$

Evaluating Eq. (2.5.4.2-1) at the gas-liquid interface yields

$$
\mathrm{N}_{\mathrm{s}}=\left.\multimap D_{s a} \frac{\mathrm{dy}}{\mathrm{dz}}\right|_{\mathrm{z}=0}+\mathrm{y}_{\mathrm{si}} \mathrm{N}_{\mathrm{s}}
$$

Using Eqs. (2.5.4.2-7) and (2.5.4.2-6), the diffusive component of the mass flux $\left(\mathrm{J}_{\mathrm{s}}\right)$ can be written as

$$
\mathrm{J}_{\mathrm{s}}=\left.\multimap D_{s a} \frac{\mathrm{dy}}{\mathrm{dz}}\right|_{\mathrm{z}=0}=\mathrm{N}_{\mathrm{s}}\left(1-\mathrm{y}_{\mathrm{si}}\right)=\frac{\mathrm{N}_{\mathrm{s}}\left(\mathrm{y}_{\mathrm{si}}-\mathrm{y}_{\mathrm{s}}\right)}{\exp \left(\frac{\mathrm{N}_{\mathrm{s}} \delta_{\mathrm{c}}}{\mathrm{c} \mathrm{D_{sa }}}\right)-1}
$$

In the limit of low mass flux, as $\mathrm{N}_{\mathrm{s}} \rightarrow 0$, by expanding the exponential term in Eq. $(2.5 .4 .2-8)$ we find

$$
J_{s} \rightarrow \frac{c D_{s a}}{\delta_{c}}\left(y_{s i}-y_{s}\right) \equiv k_{y}\left(y_{s i}-y_{s}\right)
$$

which serves to define the miss transfer coefficient at low levels of mass flux $(\mathrm{ky}, \mathrm{kg}$ mole/m²-s) as

$$
k_{y} \equiv \frac{c D_{s a}}{\delta_{c}}
$$

To correct the mass transfer for conditions at high mass flux, we define a correction factor $\theta$ through the relationship 


$$
J_{s} \equiv \theta k_{y}\left(y_{s i}-y_{s}\right)
$$

We then use Eq. $(2 \cdot 5 \cdot 4 \cdot 2-11)$ and Eq. $(2 \cdot 5 \cdot 4.2-8)$ to solve for the correction factor as

$$
\theta=\frac{N_{s}}{k_{y}} \frac{\left(1-y_{s i}\right)}{\left(y_{s i}-y_{s}\right)}=\frac{\left(N_{s} / k_{y}\right)}{\exp \left(N_{s} / k_{y}\right)-1}
$$

where the second equality in Eq. $(2.5 .4 .2-12)$ is obtained by using the definition of $k_{y}$ in Eq. $(2 \cdot 5 \cdot 4 \cdot 2-8)$.

To complete the analysis we define a mass transfer correction parameter or rate factor $\beta$ as

$$
\beta=-\frac{N_{s}}{k_{y}}
$$

so that Eq. (2.5.4.2-12) can be written more simply as the equivalent expression

$$
\theta=\frac{\beta}{1-\exp (-\beta)}
$$

Heat Transfer: We now wish to develop interfacial heat transfer relationships that will account for the simultaneous presence of mass transfer (Bird et al., 1960, Sherwood et al., 1975). Under steady state conditions, at any location within the thermal films in either phase, the enthalpy transport can be expressed as the sum of a conduction heat flux component and a flux of enthalpy due to convection. In general, the convective enthalpy transport is the sum of the contributions from both the water and air components.

Focusing on the gas phase, the steady-state enthalpy transport can be expressed as

$$
e_{g}=-k_{g} \frac{d T}{d z}+n_{s} h_{s}+n_{a} h_{a}
$$

where

$$
\begin{aligned}
e_{\mathrm{g}} & \equiv \text { Enthalpy flux across gas film, W/m } \\
k_{\mathrm{g}} & \equiv \text { Thermal conductivity, W/m-K } \\
\mathrm{T} & \equiv \text { Temperature, } \mathrm{K} \\
\mathrm{n}_{\mathrm{s}} & \equiv \mathrm{N}_{\mathrm{s}} \mathrm{M}_{\mathrm{s}}, \text { mass flux of water vapor, } \mathrm{kg} / \mathrm{m}^{2}-\mathrm{s} \\
\mathrm{n}_{\mathrm{a}} & \equiv \mathrm{N}_{\mathrm{a}} \mathrm{M}_{\mathrm{a}} \text {, mass flux of air, } \mathrm{kg} / \mathrm{m}^{2}-\mathrm{s} \\
\mathrm{h}_{\mathrm{s}} & \equiv \text { Specific enthalpy of water vapor, } \mathrm{J} / \mathrm{kg} \\
\mathrm{h}_{\mathrm{a}} & \equiv \text { Specific enthalpy of air, J/kg }
\end{aligned}
$$

As before, we assume that the air transport across the interfice is identically zero $\left(n_{a}=0\right)$ and integrate Eq. (2.5.4.2-15) across the gas thermal film 


$$
\int_{0}^{\delta_{\mathrm{t}}} \frac{\mathrm{dz}}{k_{\mathrm{g}}}=\frac{\delta_{\mathrm{t}}}{\mathrm{k}_{\mathrm{g}}}=-\int_{\mathrm{T}_{\mathrm{i}}}^{\mathrm{T}_{\mathrm{g}}} \frac{\mathrm{dT}}{\mathrm{e}_{\mathrm{g}}-\mathrm{n}_{\mathrm{s}} \mathrm{h}_{\mathrm{s}}}
$$

where $\delta_{t}$ is the thermal film thickness and we replace the local thermal conductivity with a value averaged over the film thickness $\left(\mathrm{k}_{\mathrm{g}}\right)$.

The enthalpy of the water vapor in the gas phase can be represented in terms of a standard state value adjusted to the prevailing conditions by pressure and temperature corrections as

$$
h_{s}=h_{s}^{0}\left(T_{0}, P_{0}\right)+\Delta h_{T}\left(T, T_{0}\right)+\Delta h_{P}\left(P, P_{0}\right)
$$

where

$$
\begin{aligned}
& h_{s}^{0}\left(T_{0}, P_{0}\right) \equiv \text { Enthalpy of steam at standard state conditions, } J / k g \\
& \Delta h_{T}\left(T, T_{0}\right) \equiv \text { Enthalpy correction for temperature difference, } J / k g \\
& \Delta h_{P}\left(P, P_{0}\right) \equiv \text { Enthalpy correction for pressure difference, } J / k g
\end{aligned}
$$

At the prevailing conditions in the SRS reactors of low pressure and moderate temperatures, steam may be considered an ideal gas and the pressure correction can be neglected. Assuming that the heat capacity for steam can be represented by an appropriate average value $\left(c_{p s}\right)$ which can be removed from the integral, the enthalpy is evaluated as

$$
h_{s}=h_{s}^{0}\left(T_{0}, P_{0}\right)+\int_{T_{0}}^{T} c_{p s} d T=h_{s}^{0}+c_{p s}\left(T-T_{0}\right)
$$

For convenience, we choose the enthalpy at standard state conditions to be zero $\left(h_{s}^{0}=0\right)$. Then, substituting Eq. (2.5.4.2-18) into Eq. (2.5.4.2-16) and performing the integration across the film thickness yields

$$
\frac{\delta_{t}}{k_{g}}=-\int_{T_{i}}^{T_{g}} \frac{d T}{e_{g}-n_{s} c_{p s}\left(T-T_{0}\right)}=\frac{1}{n_{s} c_{p s}} \ln \left[\frac{e_{g}-n_{s} c_{p s}\left(T_{g}-T_{0}\right)}{e_{g}-n_{s} c_{p s}\left(T_{i}-T_{0}\right)}\right]
$$

Solving Eq. (2.5.4.2-19) for the total enthalpy flux across the gas film $\left(e_{g}\right)$ we obtain

$$
e_{g}=n_{s} c_{p s}\left(T_{i}-T_{0}\right)+\frac{n_{s} c_{p s}\left(T_{g}-T_{i}\right)}{1-\exp \left(\frac{n_{s} c_{p s} \delta_{i}}{k_{g}}\right)}
$$

Evaluating Eq. (2.5.4.2-15) at the gas-liquid interface and using Eq. (2.5.4.2-18) to determine $h_{s}$ at the interface yields the relationship

$$
e_{g}=-\left.k_{g} \frac{d T}{d z}\right|_{z=0}+n_{s} c_{p s}\left(T_{i}-T_{0}\right)
$$


Then the flux of heat at the interface $\left(q_{c}\right)$ due to conduction is given by

$$
\mathrm{q}_{\mathrm{c}}=-\left.k_{\mathrm{g}} \frac{\mathrm{dT}}{\mathrm{dz}}\right|_{\mathrm{z}=0}=\mathrm{e}_{\mathrm{g}}-\mathrm{n}_{\mathrm{s}} \mathrm{c}_{\mathrm{ps}}\left(\mathrm{T}_{\mathrm{i}}-\mathrm{T}_{0}\right)=\frac{\mathrm{n}_{\mathrm{s}} \mathrm{c}_{\mathrm{ps}}\left(\mathrm{T}_{\mathrm{g}}-\mathrm{T}_{\mathrm{i}}\right)}{1-\exp \left(\frac{\mathrm{n}_{\mathrm{s}} \mathrm{c}_{\mathrm{ps}} \delta_{\mathrm{q}}}{\mathrm{k}_{\mathrm{g}}}\right)}
$$

In the limit of low mass transfer, as $n_{s} \rightarrow 0$, we can expand the exponential term to find

$$
\mathrm{q}_{\mathrm{c}} \rightarrow \frac{\mathrm{k}_{\mathrm{g}}}{\delta_{\mathrm{t}}}\left(\mathrm{T}_{\mathrm{i}}-\mathrm{T}_{\mathrm{g}}\right) \equiv \mathrm{h}_{\mathrm{g}}\left(\mathrm{T}_{\mathrm{i}}-\mathrm{T}_{\mathrm{g}}\right)
$$

which serves to define the gas phase heat transfer coefficient in the absence of mass flux $\left(\mathrm{h}_{\mathrm{g}}\right)$ as

$$
h_{g}=\frac{k_{g}}{\delta_{t}}
$$

To correct the heat transfer in the gas phase for the effects of simultaneous mass transfer, we define a correction factor $\theta_{\mathrm{g}}$ through the relationship

$$
q_{c} \equiv \theta_{g} h_{g}\left(T_{i}-T_{g}\right)
$$

Setting Eq. (2.5.4.2-25) equal to Eq. (2.5.4.2-22), and solving for the correction factor we obtain

$$
\theta_{g}=\frac{-\left(\frac{n_{s} c_{p s}}{h_{g}}\right)}{1-\exp \left(\frac{n_{s} c_{p s}}{h_{g}}\right)}=\frac{\beta_{g}}{1-\exp \left(-\beta_{g}\right)}
$$

where we have used the definition of the heat transfer coefficient in the limit of no mass transfer given by Eq. (2.5.4.2-24) to simplify the exponential term. The correction factor is the ratio of the conduction heat flux including mass transfer effects to the limiting value in the absence of mass flux. To complete the analysis, we have also defined a heat transfer correction parameter $\beta_{8}$ as

$$
\beta_{g}=-\frac{n_{s} c_{p s}}{h_{g}}
$$

so that Eq. (2.5.4.2-26) can be written more simply as the equivalent expression shown in the second equality. We note that Eq. $(2.5 .4 .2-26)$ is of exactly the same form as the correction factor for high mass transfer shown in Eq. (2.5.4.2-14). Both terms are the so called Ackermann correction factor for finite mass flux (Ackermann, 1937).

Combining Eq. (2.5.4.2-26) with Eq. (2.5.4.2-20) shows that the constant enthalpy flux in the gas phase can be evaluated as 


$$
e_{g}=n_{s} c_{p s}\left(T_{i}-T_{0}\right)+h_{g} \theta_{g}\left(T_{i}-T_{g}\right)
$$

Equation (2.5.4.2-28) shows that the enthalpy flux is composed of two terms. The first term, which is just $n_{s} h_{s}$, is the energy added to the gas phase due to gas mass creation at the interface and the second term represents the convective heat transfer from the bulk phase to the interface corrected for net mass flux.

We note that an exactly analogous development can be applied to describe heat transfer across the film within the liquid phase as well. The resulting expression for the liquid phase enthalpy transport $\left(e_{f}\right)$ is

$$
e_{f}=-n_{s} c_{p w}\left(T_{i}-T_{0}\right)+h_{f} \theta_{f}\left(T_{i}-T_{f}\right)
$$

In writing Eq. (2.5.4.2-29) we recognize that the mass flux in the liquid phase must be equal and opposite to the mass flux in the gas phase $\left(n_{s}\right)$ and we use the average specific heat of liquid water $\left(c_{\mathrm{pw}}\right)$. The liquid phase heat transfer coefficient in the absence of mass transfer $\left(h_{f}\right)$ and the corresponding correction factor $\left(\theta_{f}\right)$ are defined as

$$
h_{f}=\frac{k_{f}}{\delta_{t}}
$$

where

$$
\begin{aligned}
& \delta_{\mathrm{t}} \equiv \text { Liquid phase heat transfer film thickness, } \mathrm{m} \\
& \mathrm{k}_{\mathrm{f}} \equiv \text { Average liquid thermal conductivity, W/m-K }
\end{aligned}
$$

and

$$
\theta_{\mathrm{f}}=\frac{\beta_{\mathrm{f}}}{1-\exp \left(-\beta_{\mathrm{f}}\right)}
$$

with the parameter $\beta_{\mathrm{f}}$ defined to be

$$
\beta_{\mathrm{f}}=\frac{\mathrm{n}_{\mathrm{s}} \mathrm{c}_{\mathrm{pw}}}{\mathrm{h}_{\mathrm{f}}}
$$

The correction factors $\theta_{\mathrm{g}}$ and $\theta_{\mathrm{f}}$ are the ratios of the convective heat transfer coefficients with high mass flux to the convective heat transfer coefficients without mass transfer.

Linear Approximation to Correction Factors: The correction factors for the transport coefficients can be approximated by using a series expansion of the exponential terms. We recognize that the $\beta$ parameters are less than one so that the approximation

$$
\exp (-\beta) \cong 1-\beta+\frac{\beta^{2}}{2}+O\left(\beta^{3}\right)
$$

is valid. Then 


$$
\theta \equiv \frac{\beta}{\beta-\frac{\beta^{2}}{2}+O\left(\beta^{3}\right)}=\left[1-\frac{\beta}{2}+O\left(\beta^{2}\right)\right]^{-1} \cong 1+\frac{\beta}{2}+O\left(\beta^{2}\right)
$$

The above analysis is based on a film theory model of the interphase transport. Using penetration theory or boundary layer theory will give correction terms of the same form (see Eq. (2.5.4.2-14)) but with somewhat different numerical values. The results from different transport theories are plotted in Fig. 2.5.4.2-2 taken from Bird et al. (1960). We assume that a good approximation to the correction factor may be obtained by using the simple linear relationship

$$
\theta \cong 1+\phi \beta
$$

For computational purposes, we have chosen a value of $\phi=0.625$ which is representative of the average conditions predicted by the various models for use in FLOWTRAN-TF calculations. This linear approximation is also shown in Fig. 2.5.4.2-2 where it is compared to predictions from several theoretical treatments. We find that this simple linear approximation falls between the various theoretical values and gives a good representation of the theories over a wide range of conditions.

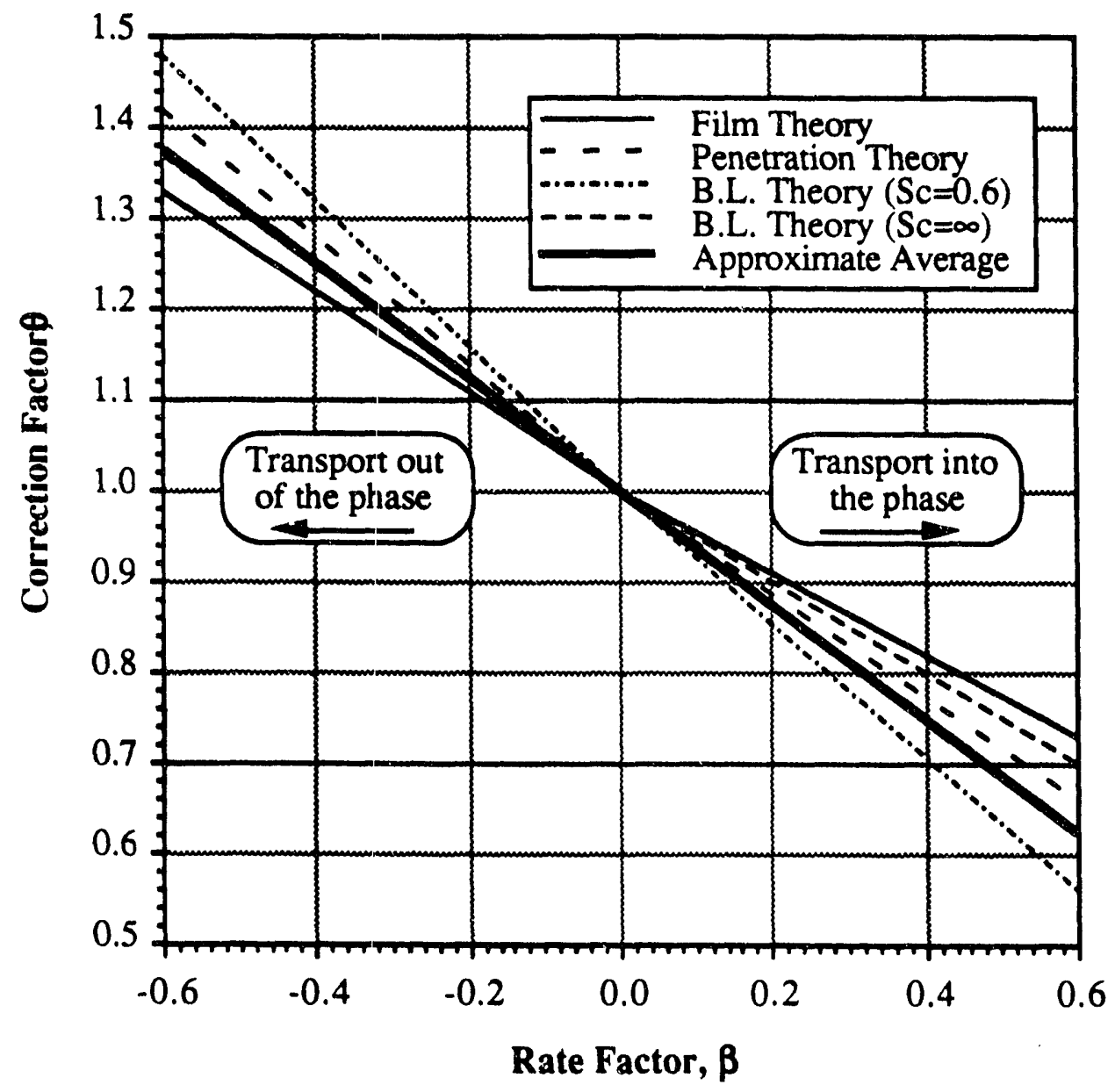

Figure 2.5.4.2-2 Ackermann correction factors from different transport theories. 
Equation (2.5.4.2-33) is intended to apply for all three correction factors derived above. That is, we also use

$$
\theta_{\mathrm{g}} \equiv 1+\phi \beta_{\mathrm{g}}
$$

and

$$
\theta_{\mathrm{f}} \equiv 1+\phi \beta_{\mathrm{f}}
$$

with $\phi=0.625$ to approximate the heat transfer correction factors.

Low mass transfer rate coefficients are defined as the diffusivity or conductivity divided by film thickness by Eqs. (2.5.4.2-10), (2.5.4.2-24) and (2.5.4.2-30) in the film theory derivations. Analogous to abandoning $\phi=0.5$ in favor of $\phi=0.625$, we also replace equations (2.5.4.2-10), (2.5.4.2-24) and (2.5.4.2-30) with more accurate expressions based on data correlations. The correlations chosen for the FLOWTRAN-TF air-water application are fully discussed in $\$ 2.5 .4 .1$. The rate coefficients are functions of fluid properties and the flow regime.

Development of Modified Interfacial Heat and Mass Transfer Relations: Substituting Eq. (2.5.4.2-33) into Eq. (2.5.4.2-11), using the definition of $\beta$ from Eq. (2.5.4.2-13) and rearranging the resulting expression gives

$$
N_{s}\left[1-y_{s i}+\phi\left(y_{s i}-y_{s}\right)\right]-k_{y}\left(y_{s i}-y_{s}\right)=0
$$

We now multiply through Eq. $(2 \cdot 5 \cdot 4.2-34)$ by the bulk interfacial area $A_{i}\left(m^{2}\right)$ and the molecular weight of steam $M_{s}$, and divide by the fluid cell volume $V_{c}\left(\mathrm{~m}^{3}\right)$ to obtain

$$
\Gamma\left(1-\mathrm{y}_{\mathrm{si}}+\phi \Delta \mathrm{y}_{\mathrm{s}}\right)-\mathrm{K}_{\mathrm{g}} \Delta \mathrm{y}_{\mathrm{s}}=0
$$

where

$$
\begin{aligned}
& \Gamma \equiv A_{i} N_{s} M_{s} / N_{c}=\text { Mass transfer rate per unit volume, } \mathrm{kg} / \mathrm{m}^{3}-\mathrm{s} \\
& K_{\mathrm{g}} \equiv A_{\mathrm{i}} \mathrm{k}_{\mathrm{y}} \mathrm{M}_{\mathrm{s}} / \mathrm{V}_{\mathrm{c}}=\text { Gas phase mass transfer coefficient, } \mathrm{kg} / \mathrm{m}^{3}-\mathrm{s} \\
& \Delta y_{s} \equiv \underset{\text { phase }}{\left(y_{s i}-y_{s}\right)=\text { Molar concentration difference for mass transfer in the gas }}
\end{aligned}
$$

Substituting the version of Eq. (2.5.4.2-33) for $\theta_{\mathrm{g}}$ into Eq. (2.5.4.2-28), using the definition of $\beta_{\mathrm{g}}$ from Eq. (2.5.4.2-27), multiplying through by the interfacial area, and dividing by the cell volume gives

$$
E_{g i}=\Gamma c_{p s}\left(T_{i}-T_{0}\right)+H_{g i}\left(T_{i}-T_{g}\right)-\phi \Gamma c_{p s}\left(T_{i}-T_{g}\right)
$$

where

$$
\begin{aligned}
& E_{g i} \equiv A_{i} e_{g} / V_{c}=\text { Gas phase energy transfer rate per unit volume, } W / m^{3} \\
& \mathrm{H}_{\mathrm{gi}} \equiv \mathrm{A}_{\mathrm{i}} \mathrm{h}_{\mathrm{g}} / \mathrm{V}_{\mathrm{c}}=\text { Gas phase heat transfer coefficient multiplied by the interfacial }
\end{aligned}
$$


Equation (2.5.4.2-36) can be written in the slightly simpler form

$$
\mathrm{E}_{\mathrm{gi}}=\Gamma \mathrm{h}_{\mathrm{gi}}+\mathrm{H}_{\mathrm{gi}} \Delta \mathrm{T}_{\mathrm{g}}-\phi \Gamma \mathrm{c}_{\mathrm{ps}} \Delta \mathrm{T}_{\mathrm{g}}=\Gamma\left(\mathrm{h}_{\mathrm{gi}}-\phi \mathrm{c}_{\mathrm{ps}} \Delta \mathrm{T}_{\mathrm{g}}\right)+\mathrm{H}_{\mathrm{gi}} \Delta \mathrm{T}_{\mathrm{g}}
$$

by defining the terms

$$
\begin{aligned}
h_{g i} \equiv & c_{p s}\left(T_{i}-T_{0}\right)=\text { Gas enthalpy at the interface, } J / k g \\
\Delta T_{g} \equiv & \left(T_{i}-T_{g}\right)=\text { Temperature difference between interface and bulk fluid in the } \\
& \text { gas phase, } K
\end{aligned}
$$

Performing similar transformations on Eq. (2.5.4.2-29) yields

$$
E_{f i}=-\Gamma\left(h_{f i}-\phi c_{p w} \Delta T_{f}\right)+H_{f i} \Delta T_{f}
$$

where

$$
\begin{aligned}
& E_{f i} \equiv A_{i} e_{f} / V_{c}=\text { Liquid phase energy transfer rate per unit volume, } W / m^{3} \\
& \mathrm{H}_{\mathrm{fi}} \equiv \mathrm{A}_{\mathrm{i}} \mathrm{h}_{\mathrm{f}} / \mathrm{V}_{\mathrm{c}}=\text { Liquid phase heat transfer coefficient multiplied by the } \\
& \text { interfacial area concentration, } \mathrm{W} / \mathrm{m}^{3}-\mathrm{K} \\
& h_{f_{1}} \equiv c_{p w}\left(T_{i}-T_{0}\right)=\text { Liquid enthalpy at the interface, } J / k g \\
& \Delta T_{f} \equiv\left(T_{i}-T_{f}\right)=\text { Temperature difference between interface and bulk fluid in the }
\end{aligned}
$$

We then recognize that the summation of the liquid and gas energy transfer rates must be zero to conserve total energy in the system since we do not allow energy accumulation at the interface. That is, the energy transport to the interface is equal to $E_{g i}=-E_{f i}$. Performing the addition of Eq. (2.5.4.2-37) and Eq. (2.5.4.2-38) and setting the result to zero gives

$$
\Gamma\left[\lambda_{i}+\phi\left(c_{p w} \Delta T_{f}-c_{p s} \Delta T_{g}\right)\right]+\left(H_{f i} \Delta T_{f}+H_{g i} \Delta T_{g}\right)=0
$$

where we have introduced the latent heat of vaporization at the interface defined to be

$$
\lambda_{\mathrm{i}} \equiv \mathrm{h}_{\mathrm{gi}}-\mathrm{h}_{\mathrm{fi}}
$$

Finally, we assume that the interface is in a state of vapor-liquid equilibrium such that the partial pressure of steam at the interface $\left(\mathrm{p}_{\mathrm{si}}\right)$ is equal to the saturation vapor pressure at the interfacial temperature $T_{i}$. Therefore, $y_{s i}$ and $T_{i}$ are related through the equation

$$
T_{i}=T_{s a t}\left(p_{s i}\right)=T_{s a t}\left(y_{s i} P\right)
$$

In the FLOWTRAN-TF code, enthalpies $h_{g i}$ and $h_{f i}$ are evaluated using the equations of state described in $\$ 2.4 .2$.

Solution of Interfacial Equations: Equations (2.5.4.2-35) and (2.5.4.2-39) and the constitutive relation in Eq. (2.5.4.2-40) form a system of three equations in the three unknowns $y_{s i}, T_{i}$, and $\Gamma$. We use these equations to define the three functions 


$$
\begin{gathered}
f_{1}\left(\mathrm{y}_{\mathrm{si}}, \Gamma\right)=0=\Gamma\left(1-\mathrm{y}_{\mathrm{si}}+\phi \Delta \mathrm{y}_{\mathrm{s}}\right)-\mathrm{K}_{\mathrm{g}} \Delta \mathrm{y}_{\mathrm{s}} \\
f_{2}\left(\mathrm{~T}_{\mathrm{i}}, \Gamma\right)=0=\Gamma\left[\lambda_{\mathrm{i}}+\phi\left(\mathrm{c}_{\mathrm{pw}} \Delta \mathrm{T}_{\mathrm{f}}-\mathrm{c}_{\mathrm{ps}} \Delta \mathrm{T}_{\mathrm{g}}\right)\right]+\left(\mathrm{H}_{\mathrm{fi}} \Delta \mathrm{T}_{\mathrm{f}}+\mathrm{H}_{\mathrm{gi}} \Delta \mathrm{T}_{\mathrm{g}}\right) \\
f_{3}\left(\mathrm{y}_{\mathrm{si}}, \mathrm{T}_{\mathrm{i}}\right)=0=\mathrm{T}_{\mathrm{i}}-\mathrm{T}_{\mathrm{sat}}\left(\mathrm{y}_{\mathrm{si}} \mathrm{P}\right)
\end{gathered}
$$

This equation set is solved simultaneously in the FLOWTRAN-TF subroutine GAMMAI. Taking the mass transfer rate to be a function of $y_{s i}, \Gamma\left(y_{s i}\right)$, we view Eq. (2.5.4.2-35) as a function of the interfacial concentration of water vapor $y_{s i}$

$$
f_{1}\left(\mathrm{y}_{\mathrm{si}}, \Gamma\left(\mathrm{y}_{\mathrm{si}}\right)\right)=0
$$

where $\Gamma\left(y_{s i}\right)$ is defined implicitly by equations (2.5.4.2-39) and (2.5.4.2-40). We use a Newton iteration strategy to find the root of this equation

$$
y_{s i}^{k+1}=y_{s i}^{k}-\frac{f_{1}\left(y_{s i}^{k}\right)}{f_{1}{ }^{\prime}\left(y_{s i}^{k}\right)}
$$

where

$$
f_{1}\left(y_{s i}^{k}\right)=\Gamma\left(y_{s i}^{k}\right)\left(1-y_{s i}^{k}+\phi \Delta y_{s}^{k}\right)-K_{g} \Delta y_{s}^{k}
$$

and

$$
f_{1}^{\prime}\left(y_{s i}^{k}\right)=\left(1-y_{s i}^{k}+\phi \Delta y_{s}^{k}\right) \Gamma\left(y_{s i}^{k}\right)-(1-\phi) \Gamma\left(y_{s i}^{k}\right)-K_{g}
$$

The derivative of $\Gamma$ with respect to $y_{\text {si }}$ in Eq. (2.5.4.2-43) is evaluated using the chain rule and the derivative of Eq. (2.5.4.2-40) to obtain

$$
\begin{aligned}
\Gamma\left(y_{s i}^{k}\right) & =\left[\frac{d \Gamma}{d T_{i}}\right]\left[\frac{d T_{i}}{d y_{s i}}\right] \\
& =\left[\frac{d \Gamma}{d T_{i}}\right]\left[\frac{d T_{s a t}}{d p_{s i}}\right] P
\end{aligned}
$$

The derivative of $\Gamma$ with respect to $T_{i}$ is obtained from Eq. (2.5.4.2-39) as

$$
\frac{d \Gamma}{d T_{i}}=-\frac{\Gamma\left[\left(\frac{\partial h_{g i}}{\partial T_{i}}\right)_{\bar{x}}-\left(\frac{\partial h_{f i}}{\partial T_{i}}\right)_{\bar{x}}\right]+\left(H_{f i}+\phi \Gamma c_{p w}\right)+\left(H_{g i}-\phi \Gamma c_{p s}\right)}{\lambda_{i}+\phi\left(c_{p w} \Delta T_{f}-c_{p s} \Delta T_{g}\right)}
$$

We replace the partial derivatives of enthalpy with respect to temperature with the corresponding specific heats and rearrange to obtain the last equality in Eq. (2.5.4.2-45). 
The solution procedure is then as follows:

0 . Make the initial guess $y_{s i}^{1}=y_{s}$.

1. Evaluate the interfacial temperature $T_{i}$ corresponding to $y_{s i}^{k}$ from Eq. (2.5.4.2-40).

2. Given $T_{i}$, evaluate $\Gamma\left(y_{s i}^{k}\right)$ from Eq. $(2 \cdot 5 \cdot 4.2-39)$ and $\Gamma^{2}\left(y_{s i}^{k}\right)$ from Eqs. (2.5.4.2-44-45).

3. Evaluate $f_{1}\left(\mathrm{y}_{\mathrm{si}}^{\mathrm{k}}\right)$ from Eq. $(2 \cdot 5 \cdot 4 \cdot 2-42)$ and $f_{1}^{\prime}\left(\mathrm{y}_{\mathrm{si}}^{\mathrm{k}}\right)$ from Eq. (2.5.4.2-43).

4. Apply Newton's method as shown in Eq. (2.5.4.2-41) to obtain $y_{s i}^{k+1}$.

5. If $\left|y_{s i}^{k+1}-y_{s i}^{k}\right|<10^{-4}$ and $\left|f_{1}\left(y_{s i}^{k}\right)\right|<1$ we assume that the solution has converged to the correct interfacial conditions; otherwise, the index $k i s$ incremented by one and the procedure in steps $1-5$ is repeated until convergence is achieved.

Once the interfacial conditions have been established, the rate of energy transfer per unit volume to the gas phase at the interface is evaluated as $\mathrm{E}_{\mathrm{gi}}$ using Eq. (2.5.4.2-37).

Composite Interfacial Heat and Mass Transfer Coefficients: As noted above, the heat and mass transfer rate coefficients used in the FLOWTRAN-TF code depend upon fluid properties and flow regime. The code employs a simple flow regime map that identifies three basic flow regimes (bubble, slug, and annular) and two transition regimes (bubble-slug and slug-annular). We need to generalize the above equations somewhat to account for the transition flow regimes where the interfacial heat and mass transfer coefficients must be composite functions accounting for the presence of two separate mechanisms. For example, the heat transfer coefficient in the gas phase is in general given by

$$
H_{g i}=\sum_{j}\left(H_{g i}\right)_{j}=\frac{1}{V_{c}} \sum_{j}\left(h_{g j} A_{i, j}\right)
$$

where $\mathrm{j}$ indicates the flow regime and we sum the contributions from individual regimes to calculate a composite coefficient. Exactly analogous expressions apply for the liquid phase heat transfer coefficient $\mathrm{H}_{\mathrm{f}}$ and the mass transfer coefficient $\mathrm{K}_{\mathrm{g}}$.

Equation (2.5.4.2-46) does not directly indicate a dependence of the interfacial transport coefficients on the void fraction. For computational purposes, it is desirable to express the void fraction functionality by rewriting Eq. (2.5.4.2-46) as

$$
H_{g i}^{m}=\alpha^{m}(1-\alpha)^{m}\left\{\frac{1}{V_{c}} \sum_{j}\left(\frac{h_{g j} A_{i, j}}{\alpha(1-\alpha)}\right)^{n}\right\}
$$

In writing Eq. (2.5.4.2-47), we have noted that the quantity in brackets is evaluated explicitly at the previous time step $n$ while the pre-multiplying function of void fraction is allowed to vary implicitly within the overall Newton iteration scheme used to solve the fluid governing equations at each time step. Using the formulation in Eq. (2.5.4.2-47) 
introduces an implicit dependence on the void fraction into the interfacial heat and mass transport equations. Equation $(2.5 .4 .2-47)$ has the correct functional form in the limits of $\alpha^{\mathrm{m}} \rightarrow 0$ and $\alpha^{\mathrm{m}} \rightarrow 1$ since interfacial heat and mass transfer must vanish when only a single phase is present. As noted above, exactly analogous expressions apply for the liquid phase heat transfer coefficient $\mathrm{H}_{\mathrm{fi}}$ and the mass transfer coefficient $\mathrm{K}_{\mathrm{g}}$ and we write

$$
\begin{aligned}
& \mathrm{H}_{\mathrm{gi}}=\alpha(1-\alpha) \hat{\mathrm{H}}_{\mathrm{gi}} \\
& \mathrm{H}_{\mathrm{fi}}=\alpha(1-\alpha) \hat{\mathrm{H}}_{\mathrm{fi}} \\
& \mathrm{K}_{\mathrm{g}}=\alpha(1-\alpha) \hat{\mathrm{K}}_{\mathrm{g}}
\end{aligned}
$$

where $\hat{\mathrm{H}}_{\mathrm{gi}}, \hat{\mathrm{H}}_{\mathrm{fi}}$, and $\hat{\mathrm{K}}_{\mathrm{g}}$ are the respective summation terms analogous to the bracketed quantity in Eq. (2.5.4.2-47). In the FLOWTRAN-TF code, these explicit terms are computed in subroutine INTARA for subsequent use in subroutine GAMMAI. A schematic diagram illustrating the overall strategy in FLOWTRAN-TF for solving the heat and mass transfer equations is shown in Fig. 2.5.4.2-3. 


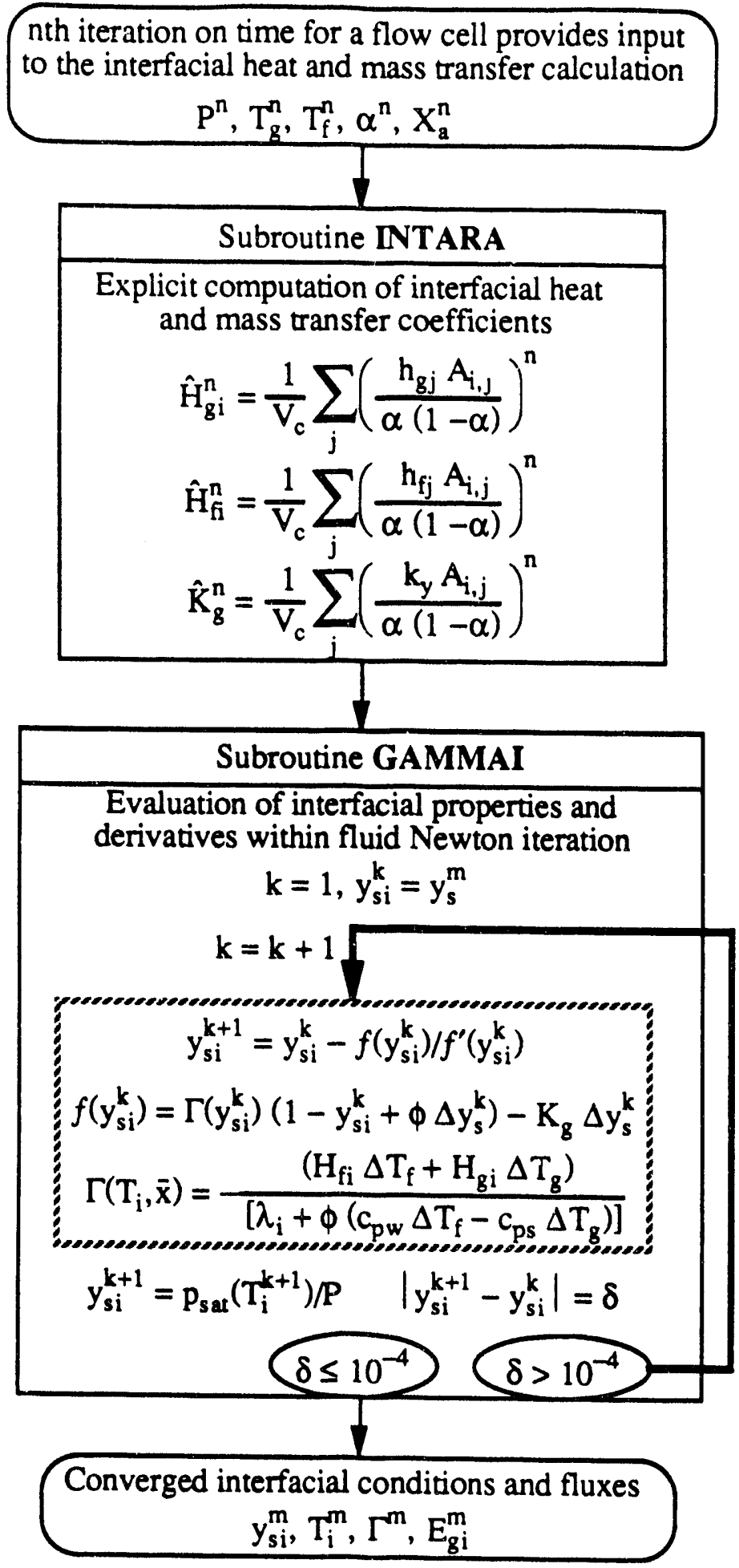

Figure 2.5.4.2-3 Schematic diagram for interfacial heat and mass transfer solution.

Interfacial Transport at Limiting Conditions: Figure 2.5.4.2-4 illustrates all the combinations of single/two-phase and single/two component situations that can occur in a general flow system. Cases A and B indicate limits where the interface disappears and only 
a single phase exists while Cases $C$ and $D$ indicate limiting conditions of pure air or pure steam in the gas phase. The interfacial heat and mass transfer model was developed for the two-phase two-component situation that applies away from these boundaries but it must also be valid for these other situations. Therefore, we must check the equations to be sure of their general applicability.

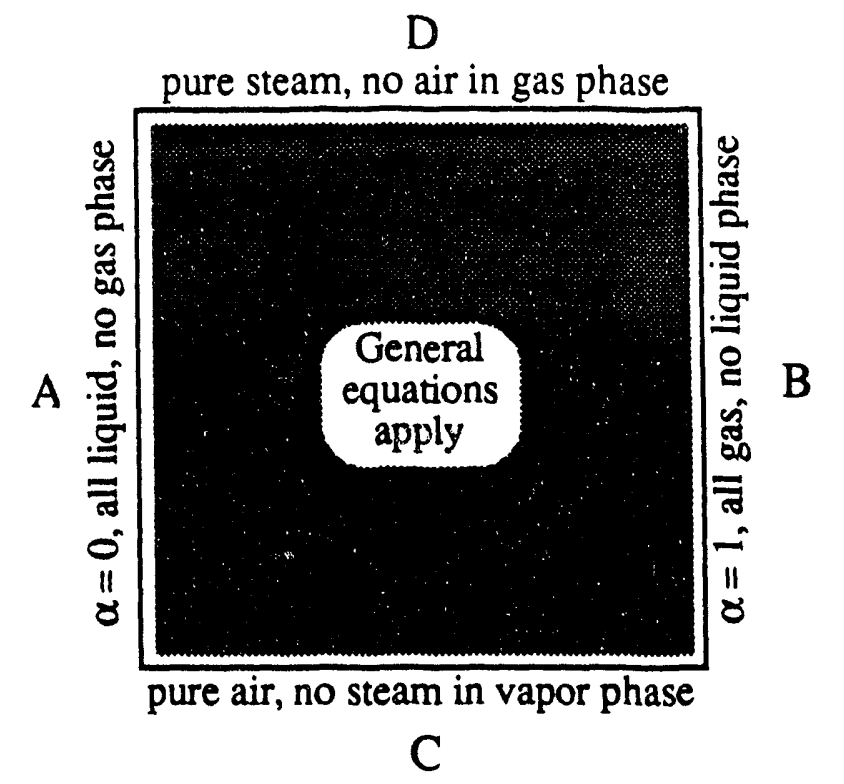

Figure 2.5.4.2-4 Schematic gas phase map showing limiting conditions.

Equation (2.5.4.2-35) shows that we have a function of $y_{\text {si }}$ given by

$$
\Gamma\left(1-y_{s i}\right)-\left(K_{g}-\phi \Gamma\right)\left(y_{s i}-y_{s}\right)=0
$$

where $\mathrm{K}_{\mathrm{g}}$ is the uncorrected mass transfer coefficient and the term $\phi \Gamma$ is a correction for finite mass flux. In the limit of pure steam in the bulk gas phase (Case $D, y_{s}=1$ ), we find

$$
\begin{aligned}
& {\left[\mathrm{K}_{\mathrm{g}}+\Gamma(1-\phi)\right]\left(\mathrm{y}_{\mathrm{si}}-1\right)=0} \\
& \therefore \mathrm{y}_{s i} \rightarrow 1 \text { as } \mathrm{y}_{\mathrm{s}} \rightarrow 1
\end{aligned}
$$

Similarly, in the limit of pure air in the bulk gas phase (Case $C, y_{s}=0$ ), the equation is well behaved giving

$$
y_{s i}=\frac{\Gamma}{K_{g}+\Gamma(1-\phi)}
$$

Thie interfacial heat and mass transfer must go to zero in the single phase limits. The convective coefficients contain the interfacial area concentration $\left(A_{i} / V_{c}\right)$ which goes to zero in the limits of a single phase (see \$2.5.3.2). However, the interfacial area is evaluated based on the void fraction at the old time step $\alpha^{n}$ and we also need for the convective coefficients to recognize the occurrence of single phase conditions within the Newton iteration. The form of Eqs. (2.5.4.2-48) forces the coefficients to approach zero as $\alpha^{\mathrm{m}} \rightarrow$ 0,1 satisfying this constraint. Therefore, the convective terms in the transport equations 
will go to zero when either the gas or liquid phase is not present. The net effect is to have the interfacial mass and energy fluxes go to zero for Cases A and B.

$$
\begin{aligned}
& \lim _{\alpha \rightarrow 0} \mathrm{H}_{\mathrm{gi}} \rightarrow 0 \Rightarrow \lim _{\alpha \rightarrow 0} \mathrm{~T}_{\mathrm{g}} \rightarrow \mathrm{T}_{\mathrm{i}}=\mathrm{T}_{\mathrm{sat}}(\mathrm{P}) \\
& \lim _{\alpha \rightarrow 1} \mathrm{H}_{\mathrm{fi}} \rightarrow 0 \Rightarrow \lim _{\alpha \rightarrow 1} \mathrm{~T}_{\mathrm{f}} \rightarrow \mathrm{T}_{\mathrm{i}}=\mathrm{T}_{\text {sat }}\left(\mathrm{y}_{\mathrm{si}} \mathrm{P}\right)
\end{aligned}
$$

Equations (2.5.4.2-37) and (2.5.4.2-39) can then be used to show that

$$
\lim _{\substack{\alpha \rightarrow 0 \\ \alpha \rightarrow 1}} \Gamma \rightarrow 0 \text { and } \lim _{\substack{\alpha \rightarrow 0 \\ \alpha \rightarrow 1}} E_{g i} \rightarrow 0
$$

The gradients of the interfacial mass and energy fluxes also approach zero in the limit as a single phase condition is approached. This can be seen by examining the derivative expressions developed in the following section.

Derivatives of Interfacial Transport Functions: To solve the fluid governing equations as developed in Section 2.5.1, we must evaluate the derivatives of the interfacial mass transfer and heat transfer rates $\left(\Gamma\right.$ and $\left.E_{g i}\right)$ with respect to the set of primary variables $\left(P, T_{f}, T_{g}, \alpha\right.$, and $\left.X_{a}\right)$. Including the first order derivative terms in our numerical scheme is used to improve the analytical approximation. Evaluating the required derivatives is a relatively complicated process that will be explained in detail in this section of the report. Using the equations developed here, the reader should be able to follow the FORTRAN programming in the code without difficulty. The calculation of the derivative values is performed in subroutine GAMMAI within the code.

We begin by defining

$$
\mathrm{D} \equiv \lambda_{\mathrm{i}}+\phi\left(\mathrm{c}_{\mathrm{pw}} \Delta \mathrm{T}_{\mathrm{f}}-\mathrm{c}_{\mathrm{ps}} \Delta \mathrm{T}_{\mathrm{g}}\right)
$$

and

$$
\mathrm{N} \equiv \mathrm{H}_{\mathrm{fi}} \Delta \mathrm{T}_{\mathrm{f}}+\mathrm{H}_{\mathrm{gi}} \Delta \mathrm{T}_{\mathrm{g}}
$$

so that Eq. (2.5.4.2-39), derived from the interfacial energy balance, can be written in the equivalent form

$$
\Gamma\left(T_{i}, \bar{x}\right)=-\frac{\left(H_{f i} \Delta T_{f}+H_{g i} \Delta T_{g}\right)}{\left[\lambda_{i}+\phi\left(c_{p w} \Delta T_{f}-c_{p s} \Delta T_{g}\right)\right]}=-\frac{N}{D}
$$

In general, the derivative of $\Gamma$ with respect to any primary variable (denoted by $x$ ) can be written as

$$
\left(\frac{\partial \Gamma}{\partial x}\right)_{x_{j}}=\left(\frac{\partial \Gamma}{\partial T_{i}}\right)_{\bar{x}}\left(\frac{\partial T_{i}}{\partial x}\right)_{x_{j}}+\left(\frac{\partial \Gamma}{\partial x}\right)_{T_{i}, x_{j}}
$$

where the subscript $x_{j}$ is used to indicate that all primary variables other than $x$ are held constant and the subscript $\bar{x}$ is used to indicate that the entire vector of primary variables is held constant. 
Taking the derivative of Eq. (2.5.4.2-52) with respect to $T_{i}$ we find

$$
\left(\frac{\partial \Gamma}{\partial \mathrm{T}_{\mathrm{i}}}\right)_{\overline{\mathrm{x}}}=-\frac{1}{\mathrm{D}}\left\{\Gamma\left(\frac{\partial \mathrm{D}}{\partial \mathrm{T}_{\mathrm{i}}}\right)_{\overline{\mathrm{x}}}+\left(\frac{\partial \mathrm{N}}{\partial \mathrm{T}_{\mathrm{i}}}\right)_{\overline{\mathrm{x}}}\right\}
$$

Differentiating Eqs. (2.5.4.2-50) and (2.5.4.2-51) yields the two required relationships

$$
\left(\frac{\partial \mathrm{D}}{\partial \mathrm{T}_{\mathrm{i}}}\right)_{\overline{\mathrm{x}}}=\left(\frac{\partial \mathrm{h}_{\mathrm{gi}}}{\partial \mathrm{T}_{\mathrm{i}}}\right)_{\overline{\mathrm{x}}}-\left(\frac{\partial \mathrm{h}_{\mathrm{fi}}}{\partial \mathrm{T}_{\mathrm{i}}}\right)_{\overline{\mathbf{x}}}+\phi\left(\mathrm{c}_{\mathrm{pw}}-\mathrm{c}_{\mathrm{ps}}\right)
$$

and

$$
\left(\frac{\partial \mathrm{N}}{\partial \mathrm{T}_{\mathrm{i}}}\right)_{\overline{\mathbf{x}}}=\mathrm{H}_{\mathrm{fi}}+\mathrm{H}_{\mathrm{gi}}
$$

To write the final result in E.q. (2.5.4.2-55), we recognize that, of the five primary variables, the interfacial fluid enthalpies depend only on pressure (i.e. $\bar{x} \rightarrow P$ ) and that the heat capacity at constant pressure is equal to the derivative of the enthalpy with respect to temperature at constant pressure. Therefore, the enthalpy derivatives can be replaced with the fluid heat capacities (see definitions of $h_{g i}$ and $h_{f i}$ above). Combining Eqs. $(2.5 .4 .2-55)$ and $(2.5 .4 .2-56)$ with $(2.5 .4 .2-54)$ then gives the complete derivative

$$
\left(\frac{\partial \Gamma}{\partial T_{i}}\right)_{\bar{x}}=-\frac{1}{D}\left\{\Gamma\left[\left(\frac{\partial h_{g i}}{\partial T_{i}}\right)_{\bar{x}}-\left(\frac{\partial h_{f i}}{\partial T_{i}}\right)_{\bar{x}}\right]+\left(H_{f i}+\phi \Gamma c_{p w}\right)+\left(H_{g i}-\phi \Gamma c_{p s}\right)\right\}
$$

The derivatives of the gas and liquid enthalpies with respect to temperature (interfacial heat capacities) are supplied by the property evaluation subroutines. The right hand side of Eq. (2.5.4.2-57) can then be immediately evaluated to obtain the indicated derivative.

In a similar fashion, the last derivative in Eq. (2.5.4.2-53) can be evaluated from

$$
\left(\frac{\partial \Gamma}{\partial x}\right)_{T_{i}, x_{j}}=-\frac{1}{D}\left\{\Gamma\left(\frac{\partial D}{\partial x}\right)_{T_{i}, x_{j}}+\left(\frac{\partial N}{\partial x}\right)_{T_{i}, x_{j}}\right\}
$$

Differentiating Eqs. (2.5.4.2-50) and (2.5.4.2-51) yields the two relationships

$$
\left(\frac{\partial D}{\partial x}\right)_{T_{i}, x_{j}}=\left(\frac{\partial h_{g i}}{\partial x}\right)_{T_{i}, x_{j}}-\left(\frac{\partial h_{f i}}{\partial x}\right)_{T_{i}, x_{j}}-\phi\left[c_{p w}\left(\frac{\partial T_{f}}{\partial x}\right)_{x_{j}}-c_{p s}\left(\frac{\partial T_{g}}{\partial x}\right)_{x_{j}}\right]
$$

and 


$$
\begin{aligned}
\left(\frac{\partial N}{\partial x}\right)_{T_{i}, x_{j}} & =\Delta T_{f}\left(\frac{\partial H_{f i}}{\partial x}\right)_{T_{i}, x_{j}}-H_{f i}\left(\frac{\partial T_{f}}{\partial x}\right)_{x_{j}} \\
& +\Delta T_{g}\left(\frac{\partial H_{g i}}{\partial x}\right)_{T_{i}, x_{j}}-H_{g i}\left(\frac{\partial T_{g}}{\partial x}\right)_{x_{j}}
\end{aligned}
$$

Derivatives with respect to specific heats have been neglected in equation (2.5.4.2-59). The primary variables are not functions of the interfacial temperature. Therefore, we drop the $T_{i}$ dependence from those derivatives in writing Eqs. (2.5.4.2-59) and (2.5.4.2-60). To evaluate the required derivatives of the interfacial enthalpies in Eq. (2.5.4.2-59), we use the relationships

$$
h_{g i}=h_{g i}\left(T_{i}, P\right) \therefore\left(\frac{\partial h_{g i}}{\partial x}\right)_{T_{i}, x_{j}}=\left(\frac{\partial h_{g i}}{\partial P}\right)_{T_{i}}\left(\frac{\partial P}{\partial x}\right)_{x_{j}}
$$

and

$$
h_{\mathrm{fi}}=\mathrm{h}_{\mathrm{fi}}\left(\mathrm{T}_{\mathrm{i}}\right) \therefore\left(\frac{\partial \mathrm{h}_{\mathrm{fi}}}{\partial \mathrm{x}}\right)_{\mathrm{T}_{\mathrm{i}}, \mathrm{x}_{\mathrm{j}}}=0
$$

The liquid enthalpy is only a function of temperature and therefore the interfacial enthalpy does not exhibit any dependence on the primary variables. The gas enthalpy is a function of temperature and pressure. The derivative of the gas enthalpy with respect to pressure is supplied by the physical property evaluation subroutine.

We use the definitions given in Eqs. (2.5.4.2-48) to evaluate the derivatives of the heat transfer coefficients that are required in Eq. (2.5.4.2-60) as

$$
\begin{aligned}
& \left(\frac{\partial H_{g i}}{\partial x}\right)_{T_{i}, x_{j}}=(1-2 \alpha) \hat{H}_{g i}\left(\frac{\partial \alpha}{\partial x}\right)_{x_{j}} \\
& \left(\frac{\partial H_{f i}}{\partial x}\right)_{T_{i}, x_{j}}=(1-2 \alpha) \hat{H}_{f i}\left(\frac{\partial \alpha}{\partial x}\right)_{x_{j}}
\end{aligned}
$$

Combining Eqs. (2.5.4.2-58) through (2.5.4.2-63) yields the final result

$$
\begin{array}{r}
\left(\frac{\partial \Gamma}{\partial x}\right)_{T_{i}, x_{j}}=\frac{1}{D}\left\{\left(H_{g i}-\phi \Gamma c_{p s}\right)\left(\frac{\partial T_{g}}{\partial x}\right)_{x_{j}}+\left(H_{f i}+\phi \Gamma c_{p w}\right)\left(\frac{\partial T_{f}}{\partial x}\right)_{x_{j}}\right. \\
-(1-2 \alpha)\left(\hat{H}_{f i} \Delta T_{f}+\hat{H}_{g i} \Delta T_{g}\right)\left(\frac{\partial \alpha}{\partial x}\right)_{x_{j}} \\
\left.-\Gamma\left(\frac{\partial h_{g i}}{\partial P}\right)_{T_{i}}\left(\frac{\partial P}{\partial x}\right)_{x_{j}}\right\}
\end{array}
$$


When Eq. (2.5.4.2-64) is used to compute derivatives, terms of the general form $\left(\partial \mathrm{x}_{\mathrm{i}} / \partial \mathrm{x}\right)_{\mathrm{x}_{j}}$ where $x_{i}=\left\{T_{g}, T_{f}, \alpha, P\right\}$ will be one when $x_{i}=x$ and zero otherwise.

Eqs. (2.5.4.2-57) and (2.5.4.2-64) represent two of the three functions required to completely evaluate the derivatives of $\Gamma$ as given by Eq. (2.5.4.2-53). Momentarily postponing the development of the interfacial temperature derivative, we turn to the interfacial energy term. As with the mass transfer rate, a general expression for the derivative of the interfacial energy transfer rate with respect to an arbitrary primary variable is given by

$$
\left(\frac{\partial E_{g i}}{\partial x}\right)_{x_{j}}=\left(\frac{\partial E_{g i}}{\partial T_{i}}\right)_{\bar{x}}\left(\frac{\partial T_{i}}{\partial x}\right)_{x_{j}}+\left(\frac{\partial E_{g i}}{\partial x}\right)_{T_{i}, x_{j}}
$$

Taking the indicated derivatives of Eq. (2.5.4.2-37), we obtain the relationships

$$
\left(\frac{\partial \mathrm{E}_{\mathrm{gi}}}{\partial \mathrm{T}_{\mathrm{i}}}\right)_{\overline{\mathrm{x}}}=\left(\mathrm{h}_{\mathrm{gi}}-\phi c_{\mathrm{ps}} \Delta \mathrm{T}_{\mathrm{g}}\right)\left(\frac{\partial \Gamma}{\partial \mathrm{T}_{\mathrm{i}}}\right)_{\overline{\mathrm{x}}}+\mathrm{H}_{\mathrm{gi}}+\Gamma\left[\left(\frac{\partial \mathrm{h}_{\mathrm{gi}}}{\partial \mathrm{T}_{\mathrm{i}}}\right)_{\overline{\mathrm{x}}}-\phi \mathrm{c}_{\mathrm{ps}}\right]
$$

and

$$
\begin{aligned}
\left(\frac{\partial E_{g i}}{\partial x}\right)_{T_{i}, x_{j}} & =\left(h_{g i}-\phi c_{p s} \Delta T_{g}\right)\left(\frac{\partial \Gamma}{\partial x}\right)_{T_{i}, x_{j}}+\Gamma\left(\frac{\partial h_{g i}}{\partial P}\right)_{T_{i}}\left(\frac{\partial P}{\partial x}\right)_{x_{j}} \\
& -\left(H_{g i}-\phi \Gamma c_{p s}\right)\left(\frac{\partial T_{g}}{\partial x}\right)_{x_{j}}+(1-2 \alpha) \hat{H}_{g i} \Delta T_{g}\left(\frac{\partial \alpha}{\partial x}\right)_{x_{j}}
\end{aligned}
$$

In writing Eq. (2.5.4.2-67), we have used Eq. (2.5.4.2-61) and Eq. (2.5.4.2-63) to express the result in terms of the primary variables. Eqs. (2.5.4.2-57) and (2.5.4.2-64) can be used to complete the evaluation of the energy derivatives given in Eqs. (2.5.4.2-66) and (2.5.4.2-67). Alternatively, Eqs. (2.5.4.2-65) through (2.5.4.2-67) can be combined and simplified using Eq. (2.5.4.2-53) to obtain

$$
\begin{array}{r}
\left(\frac{\partial \mathrm{E}_{\mathrm{gi}}}{\partial \mathrm{x}}\right)_{\mathrm{x}_{\mathrm{j}}}=\left(\mathrm{h}_{\mathrm{gi}}-\phi \mathrm{c}_{\mathrm{ps}} \Delta \mathrm{T}_{\mathrm{g}}\right)\left(\frac{\partial \Gamma}{\partial \mathrm{x}}\right)_{\mathrm{x}_{\mathrm{j}}}+\left[\mathrm{H}_{\mathrm{gi}}+\Gamma\left(\frac{\partial \mathrm{h}_{\mathrm{gi}}}{\partial \mathrm{T}_{\mathrm{i}}}\right)_{\overline{\mathrm{x}}}-\phi \Gamma \mathrm{c}_{\mathrm{ps}}\right]\left(\frac{\partial \mathrm{T}_{\mathrm{i}}}{\partial \mathrm{x}}\right)_{\mathrm{x}_{\mathrm{j}}} \\
+\Gamma\left(\frac{\partial \mathrm{h}_{\mathrm{gi}}}{\partial \mathrm{P}}\right)_{\mathrm{T}_{\mathrm{i}}}\left(\frac{\partial \mathrm{P}}{\partial \mathrm{x}}\right)_{\mathrm{x}_{\mathrm{j}}}-\left(\mathrm{H}_{\mathrm{gi}}-\phi \Gamma \mathrm{c}_{\mathrm{ps}}\right)\left(\frac{\partial \mathrm{T}_{\mathrm{g}}}{\partial \mathrm{x}}\right)_{\mathrm{x}_{\mathrm{j}}} \\
+(1-2 \alpha) \hat{\mathrm{H}}_{\mathrm{gi}} \Delta \mathrm{T}_{\mathrm{g}}\left(\frac{\partial \alpha}{\partial \mathrm{x}}\right)_{\mathrm{x}_{\mathrm{j}}}
\end{array}
$$

The code also requires the partial derivatives of the interfacial energy transfer rate into the fluid phase $\left(2.5 .4 .2-E_{f i}\right)$ with respect to $T_{g}$ and $T_{f}$. Since $E_{f i}=-E_{g i}$, these derivatives are immediately evaluated as 


$$
\left(\frac{\partial \mathrm{E}_{\mathrm{fi}}}{\partial \mathrm{T}_{\mathrm{g}}}\right)=-\left(\frac{\partial \mathrm{E}_{\mathrm{gi}}}{\partial \mathrm{T}_{\mathrm{g}}}\right) \text { and }\left(\frac{\partial \mathrm{E}_{\mathrm{fi}}}{\partial \mathrm{T}_{\mathrm{f}}}\right)=-\left(\frac{\partial \mathrm{E}_{\mathrm{gi}}}{\partial \mathrm{T}_{\mathrm{f}}}\right)
$$

Finally, to complete the computation of the derivative expressions, we must determine the derivative of the interfacial temperature used in Eqs. (2.5.4.2-53) and (2.5.4.2-65). This derivative can be computed from the remaining independent equation for the interfacial mass transfer as given by Eq. (2.5.4.2-35). We take this equation to represent a function of the interfacial temperature and the set of primary variables as

$$
\begin{aligned}
F\left(T_{i}, \bar{x}\right) & =\Gamma\left(1-y_{s i}+\phi \Delta y_{s}\right)-K_{g} \Delta y_{s} \\
& =\Gamma\left(1-y_{s i}\right)-\left(K_{g}-\phi \Gamma\right)\left(y_{s i}-y_{s}\right)=0
\end{aligned}
$$

As before, we write a general expression for the derivative of the function $F$ with respect to an arbitrary primary variable $x$ as

$$
\left(\frac{\partial F}{\partial x}\right)_{x_{j}}=\left(\frac{\partial F}{\partial T_{i}}\right)_{\bar{x}}\left(\frac{\partial T_{i}}{\partial x}\right)_{x_{j}}+\left(\frac{\partial F}{\partial x}\right)_{T_{i}, x_{j}}=0
$$

This shows that the derivative of the interfacial temperature $T_{i}$ with respect to the primary variable can be calculated from

$$
\left(\frac{\partial T_{i}}{\partial x}\right)_{x_{j}}=-\frac{\left(\frac{\partial F}{\partial x}\right)_{T_{i}, x_{j}}}{\left(\frac{\partial F}{\partial T_{i}}\right)_{\bar{x}}}
$$

Taking the derivative of Eq. (2.5.4.2-69) with respect to $T_{i}$ gives the result

$$
-\left(\frac{\partial \mathrm{F}}{\partial \mathrm{T}_{\mathrm{i}}}\right)_{\overline{\mathrm{x}}}=\left[\mathrm{K}_{\mathrm{g}}+\Gamma(1-\phi)\right]\left(\frac{\partial \mathrm{y}_{\mathrm{si}}}{\partial \mathrm{T}_{\mathrm{i}}}\right)_{\overline{\mathrm{x}}}-\left(1-\mathrm{y}_{\mathrm{si}}+\phi \Delta \mathrm{y}_{\mathrm{s}}\right)\left(\frac{\partial \Gamma}{\partial \mathrm{T}_{\mathrm{i}}}\right)_{\overline{\mathrm{x}}}
$$

From the relationship for $T_{i}$ as a function of $y_{s i}$ given in Eq. (2.5.4.2-40) we define a function of $T_{i}, y_{s i}$, and $P$ as

$$
\begin{aligned}
f\left(T_{i}, y_{s i}, P\right) & \equiv T_{i}-T_{s a t}\left(p_{s i}\right) \\
& =T_{i}-T_{s a t}\left(y_{s i} P\right)
\end{aligned}
$$

Applying the chain rule for differentiation we obtain the required derivative as 


$$
\begin{aligned}
\left(\frac{\partial y_{s i}}{\partial T_{i}}\right)_{\bar{x}} & =-\frac{\left(\partial f / \partial T_{i}\right)_{\bar{x}}}{\left(\partial f / \partial y_{s i}\right) \bar{x}} \\
& =-\frac{1}{-\frac{d T_{s a t}}{d p_{s i}}\left(\frac{\partial p_{s i}}{\partial y_{s i}}\right)_{P}} \\
& =-\frac{1}{-P \frac{d T_{s a t}}{d p_{s i}}}=\frac{1}{P}\left(\frac{d T_{s a t}}{d_{s i}}\right)^{-1}
\end{aligned}
$$

The physical property relationship giving saturation temperature as a function of pressure is used to evaluate the last derivative in Eq. (2.5.4.2-73). Substituting the derivative relationship from Eq. $(2.5 .4 .2-73)$ into Eq. (2.5.4.2-72) yields our final expression for the derivative of function $F$ as

$$
-\left(\frac{\partial \mathrm{F}}{\partial \mathrm{T}_{\mathrm{i}}}\right)_{\overline{\mathrm{x}}}=\left[\mathrm{K}_{\mathrm{g}}+\Gamma(1-\phi)\right]\left[\frac{1}{\mathrm{P}}\left(\frac{\mathrm{dT}_{\mathrm{sat}}}{\mathrm{dp}_{\mathrm{si}}}\right)^{-1}\right]-\left(1-\mathrm{y}_{\mathrm{si}}+\phi \Delta \mathrm{y}_{\mathrm{s}}\right)\left(\frac{\partial \Gamma}{\partial \mathrm{T}_{\mathrm{i}}}\right)_{\overline{\mathrm{x}}}
$$

Next, taking the derivative of Eq. (2.5.4.2-69) with respect to $\mathrm{x}$ we find

$$
\begin{array}{r}
\left(\frac{\partial F}{\partial x}\right)_{T_{i}, x_{j}}=\left(1-y_{s i}+\phi \Delta y_{s}\right)\left(\frac{\partial \Gamma}{\partial x}\right)_{T_{i}, x_{j}}-\left[K_{g}+\Gamma(1-\phi)\right]\left(\frac{\partial y_{s i}}{\partial x}\right)_{T_{i}, x_{j}} \\
+\left(K_{g}-\phi \Gamma\right)\left(\frac{\partial y_{s}}{\partial x}\right)_{T_{i}, x_{j}}-\Delta y_{s}\left(\frac{\partial K_{g}}{\partial x}\right)_{T_{i}, x_{j}}
\end{array}
$$

Note that two additional terms appear in Eq. (2.5.4.2-75) over Eq. (2.5.4.2-72) since we include the possibility that $\mathrm{y}_{s}$ and $\mathrm{K}_{\mathrm{g}}$ may depend upon the primary variables. In fact, $\mathrm{y}_{\mathrm{si}}$ is a function of pressure $P_{,} y_{s}$ is a function of air mass fraction $X_{a}$, and $K_{g}$ is a function of void fraction $\alpha$. Since $y_{s i}$ is only a function of pressure, we can write

$$
\left(\frac{\partial y_{s i}}{\partial x}\right)_{T_{i}, x_{j}}=\left(\frac{\partial y_{s i}}{\partial P}\right)_{T_{i}}\left(\frac{\partial P}{\partial x}\right)_{x_{j}}
$$

Using the function $f\left(T_{i}, y_{s i}, P\right)$ defined above, we can calculate the derivative of $y_{s i}$ with respect to pressure as 


$$
\begin{aligned}
\left(\frac{\partial y_{s i}}{\partial \mathrm{P}}\right)_{T_{i}} & =-\frac{(\partial f / \partial \mathrm{P})_{T_{i}}}{\left(\partial f / \partial y_{s i} T_{i}\right.} \\
& =-\frac{d T_{s a t}\left(\frac{\partial p_{s i}}{\partial p_{s i}}\right)_{T_{i}}}{-\frac{d T_{s a t}}{d p_{s i}}\left(\frac{\partial p_{s i}}{\partial y_{s i}}\right)_{T_{i}}}=-\frac{y_{s i}}{P}
\end{aligned}
$$

Combining the two relationships immediately above gives the final result

$$
\left(\frac{\partial y_{s i}}{\partial x}\right)_{T_{i}, x_{j}}=-\frac{y_{s i}}{P}\left(\frac{\partial P}{\partial x}\right)_{x_{j}}
$$

The definition of the mole fraction of water vapor is

$$
y_{s}=\frac{\frac{X_{s}}{M_{s}}}{\frac{X_{s}}{M_{s}}+\frac{X_{a}}{M_{a}}}=\frac{1}{1+\left(\frac{M_{s}}{M_{a}}\right)\left(\frac{X_{a}}{1-X_{a}}\right)}
$$

where:

$$
\begin{aligned}
& X_{s} \equiv \text { Mass fraction of water vapor in gas phase }=\left(1-X_{a}\right) \\
& X_{a} \equiv \text { Mass Fraction of air in gas phase } \\
& M_{s} \equiv \text { Molecular weight of water vapor } \\
& M_{a} \equiv \text { Molecular weight of air }
\end{aligned}
$$

Differentiating Eq. (2.5.4.2-77) and rearranging the results gives

$$
\left(\frac{\partial y_{s}}{\partial x}\right)_{T_{j}, x_{j}}=-\left(\frac{M_{s}}{M_{a}}\right)\left(\frac{y_{s}}{1-X_{a}}\right)^{2}\left(\frac{\partial X_{a}}{\partial x}\right)_{x_{j}}
$$

As was done in writing Eq. (2.5.4.2-63), we use Eq. (2.5.4.2-48) to evaluate the derivative of the mass transfer coefficient as

$$
\left(\frac{\partial K_{g}}{\partial x}\right)_{T_{i}, x_{j}}=(1-2 \alpha) \hat{K}_{g}\left(\frac{\partial \alpha}{\partial x}\right)_{x_{j}}
$$

Equation (2.5.4.2-79) is used to evaluate the last term in Eq. (2.5.4.2-75) which can then be rearranged to show that 


$$
\begin{aligned}
-\Delta \mathrm{y}_{\mathrm{s}}\left(\frac{\partial \mathrm{K}_{\mathrm{g}}}{\partial \mathrm{x}}\right)_{\mathrm{T}_{\mathrm{i}}, \mathrm{x}_{\mathrm{j}}} & =-(1-2 \alpha) \frac{\mathrm{K}_{\mathrm{g}} \Delta \mathrm{y}_{\mathrm{s}}}{\alpha(1-\alpha)}\left(\frac{\partial \alpha}{\partial \mathrm{x}}\right)_{\mathrm{x}_{\mathrm{j}}} \\
& =-(1-2 \alpha) \frac{\Gamma\left(1-\mathrm{y}_{\mathrm{si}}+\phi \Delta \mathrm{y}_{\mathrm{s}}\right)}{\alpha(1-\alpha)}\left(\frac{\partial \alpha}{\partial \mathrm{x}}\right)_{\mathrm{x}_{\mathrm{j}}} \\
& =(1-2 \alpha) \frac{\left(1-\mathrm{y}_{\mathrm{si}}+\phi \Delta \mathrm{y}_{\mathrm{s}}\right)}{\mathrm{D}} \frac{\left(\mathrm{H}_{\mathrm{fi}} \Delta \mathrm{T}_{\mathrm{f}}+\mathrm{H}_{\mathrm{gi}} \Delta \mathrm{T}_{\mathrm{g}}\right)}{\alpha(1-\alpha)}\left(\frac{\partial \alpha}{\partial \mathrm{x}}\right)_{\mathrm{x}_{\mathrm{j}}} \\
& =\frac{\left(1-\mathrm{y}_{\mathrm{si}}+\phi \Delta \mathrm{y}_{\mathrm{s}}\right)}{\mathrm{D}}\left\{(1-2 \alpha)\left(\hat{\mathrm{H}}_{\mathrm{fi}} \Delta \mathrm{T}_{\mathrm{f}}+\hat{\mathrm{H}}_{\mathrm{gi}} \Delta \mathrm{T}_{\mathrm{g}}\right)\left(\frac{\partial \alpha}{\partial \mathrm{x}}\right)_{\mathrm{x}_{\mathrm{j}}}\right\}
\end{aligned}
$$

To obtain the resultant in Eq. $(2.5 .4 .2-80)$ we first replace $\hat{K}_{\mathrm{g}}$ with the equivalent expression in terms of $\mathrm{K}_{\mathrm{g}}$ from Eq. (2.5.4.2-48). We then use Eq. (2.5.4.2-69) to replace $\mathrm{K}_{\mathrm{g}} \Delta \mathrm{y}_{\mathrm{s}}$ and next replace $\mathrm{F}$ with its equivalent expression from Eq. (2.5.4.2-52). Finally $\mathrm{H}_{\mathrm{fi}}$ and $\mathrm{H}_{\mathrm{gi}}$ are replaced with their equivalent terms in $\hat{\mathrm{H}}_{\mathrm{fi}}$ and $\mathrm{H}_{\mathrm{gi}}$. The point of this exercise is that when all of the above expressions are combined to obtain a final result for the derivative of the interfacial temperature, we find that Eq. $(2.5 .4 .2-80)$ shows that this term exactly cancels with a term in Eq. (2.5.4.2-64). Therefore, combining Eqs. (2.5.4.2-74) through (2.5.4.2-80) along with Eq. (2.5.4.2-64) into Eq. (2.5.4.2-71), we obtain the final simplified result that

$$
\begin{array}{r}
\left(\frac{\partial T_{i}}{\partial x}\right)_{x_{j}}\left\{\left[K_{g}+\Gamma(1-\phi)\right]\left[\frac{1}{P}\left(\frac{d T_{s a t}}{d p_{s i}}\right)^{-1}\right]-\left(1-y_{s i}+\phi \Delta y_{s}\right)\left(\frac{\partial \Gamma}{\partial T_{i}}\right)_{\bar{x}}\right\}= \\
\left\{\left[K_{g}+\Gamma(1-\phi)\right]\left(\frac{y_{s i}}{P}\right)-\frac{K_{g} \Delta y_{s}}{D}\left(\frac{\partial h_{g i}}{\partial P}\right)_{T_{i}}\right\}\left(\frac{\partial P}{\partial x}\right)_{x_{j}} \\
+\frac{\left(1-y_{s i}+\phi \Delta y_{s}\right)}{D}\left\{\left(H_{g i}-\phi \Gamma c_{p s}\right)\left(\frac{\partial T_{g}}{\partial x}\right)_{x_{j}}\right. \\
\left.+\left(H_{f i}+\phi \Gamma c_{p w}\right)\left(\frac{\partial T_{f}}{\partial x_{s}}\right)_{x_{j}}\right\}_{x_{j}}^{2}\left(\frac{\partial X_{a}}{\partial-X_{a}}\right)
\end{array}
$$

The derivatives of the interfacial heat and mass transfer rates are evaluated directly from the expressions derived above. 
The computational procedure is as follows:

- Make a single evaluation of $\left(\partial \Gamma / \partial \mathrm{T}_{\mathrm{i}}\right)_{\overline{\mathrm{x}}}$ from Eq. (2.5.4.2-57)

- For $\mathrm{x}=\mathrm{P}, \mathrm{T}_{\mathrm{g}}, \mathrm{T}_{\mathrm{f}}, \alpha$, and $\mathrm{X}_{\mathrm{a}}$

- Evaluate $\left(\partial \mathrm{T}_{\mathrm{i}} / \partial \mathrm{x}\right)_{\mathrm{x}_{\mathrm{j}}}$ from Eq. (2.5.4.2-81)

- Evaluate $(\partial \Gamma / \partial x)_{T_{i}, x_{j}}$ from Eq. $(2.5 .4 .2-64)$

- Evaluate $(\partial \Gamma / \partial)_{x_{j}}$ using Eq. (2.5.4.2-53)

- Evaluate $\left(\partial \mathrm{E}_{\mathrm{gi}} \mathrm{I} / \partial \mathrm{x}\right)_{\mathrm{x}_{\mathrm{j}}}$ using Eq. (2.5.4.2-68).

Note that, in all of the above derivative relations

$$
\left(\frac{\partial x_{i}}{\partial x}\right)_{x_{j}}= \begin{cases}1, & x=x_{i} \\ 0, & x \neq x_{i}\end{cases}
$$

where $x_{i}=\left(P, T_{f}, T_{g}, \alpha\right.$, or $\left.X_{\mathrm{a}}\right)$.

Interfacial Transport Model as Coded: As developed above, the diffusion coefficient and heat capacities in the model equations are averaged values representative of conditions across the film. In the code we have chosen to simply evaluate these properties using the bulk gas and liquid temperatures. A better choice would perhaps be to use the average of the bulk and interfacial temperatures. However, since we are solving for the interfacial temperature this would require repeated property evaluations with little increase in the expected accuracy of the model. Otherwise, the interfacial transport models are coded exactly as explained above with the exception of the single phase limits which is covered in \$3.7.

\section{References:}

Ackermann, G., 1937, "Waermeuebergang und Molekulare Stoffuebertragung in Gleichen Feld", Ver. Disch. Ing. Forschungsh. 382, pp. 1-16.

Bird, R. B., Stewart, W. E., and Lightfoot, E. N., 1960, Transport Phenomena, Wiley, New York, pp. 656-676.

Sherwood, T. K., Pigford, R. L., and Wilke, C. R., 1975, Mass Transfer, McGraw-Hill, New York, Pp. 172-188 and pp. 257-260. 


\subsubsection{Interfacial Heat and Mass Transfer at Walls}

Phase change $\alpha$ curs at two distinct locations. At the interfaces between coexisting gas and liquid phases, interfacial heat and mass exchange occurs due to temperature and concentration gradients between the interface and the bulk flow. This situation is treated in $\$ 2.5 .4$.2. At a heated wall in contact with the liquid phase, boiling can occur due to local superheating of the liquid which flashes into steam. Similarly, condensation can occur due to local subcooling of the gas phase at a cooled wall. The latter cases we term interfacial heat and mass transfer at walls. Explic it zxpressions for boiling and condensation rates at solid surfaces have already been developed in the Wall Heat Transfer section $(\$ 2.5 .1)$. In this section, we complete the discussion of boiling/condensation at solid walls.

General Considerations: In general, several surface cells will generate heat and create or condense vapor that is deposited into a single fluid cell. This is expressed in Eq. $(2.5 .4 .3-1)$ where the total vapor generation or condensation rate within a single fluid computational cell $\left(\Gamma_{w}\right)$ is the summation of vapor generation or condensation rates over each of the $\mathrm{j}$ surface cells. As shown in the fluid equation development, we compute the vapor generation rate on a volumetric basis. This volumetric vapor generation rate is obtained by multiplying the rate of vapor generation per unit surface area by the cell surface areas $\left(A_{w j}\right)$ and dividing by the fluid cell volume $\left(V_{c}\right)$. This concept is illustrated in Fig. 2.5.4.3-1 where it is seen that the summation over $\mathrm{j}$ includes summation over solid azimuthal cells and over the inner and outer surfaces of the fluid channel.

$$
\Gamma_{w}=\sum_{j} \Gamma_{w j}=\sum_{j}\left(\frac{A_{w j} \hat{\Gamma}_{w j}}{V_{c}}\right)
$$

where

$$
\begin{aligned}
& \Gamma_{w} \equiv \text { total rate of vapor transport at the wall, } \mathrm{kg} / \mathrm{s}-\mathrm{m}^{3} \\
& \Gamma_{w j} \equiv \text { vapor transport rate for solid cell } \mathrm{j}, \mathrm{kg} / \mathrm{s}-\mathrm{m}^{3} \\
& \hat{\Gamma}_{w j} \equiv \text { vapor transport rate per unit surface area for solid cell } \mathrm{j}, \mathrm{kg} / \mathrm{s}-\mathrm{m}^{2} \\
& A_{w j} \equiv \text { surface area of } \mathrm{jth} \text { solid cell, } \mathrm{m}^{2} \\
& V_{c} \equiv \text { fluid cell volume, } \mathrm{m}^{3}
\end{aligned}
$$

Vaporization and condensation rates at solid surfaces have already been defined in $\$ 2.5 .1$ describing the wall heat transfer calculations. These rates of vaporization (or condensation) can be used directly in the fluid mass and energy equations. Since wall heat fluxes are calculated at the start of a time step using temperatures from the previous iteration, this gives a fully explicit representation for the vapor generation or condensation at the wall as far as the fluid calculations are concerned. 


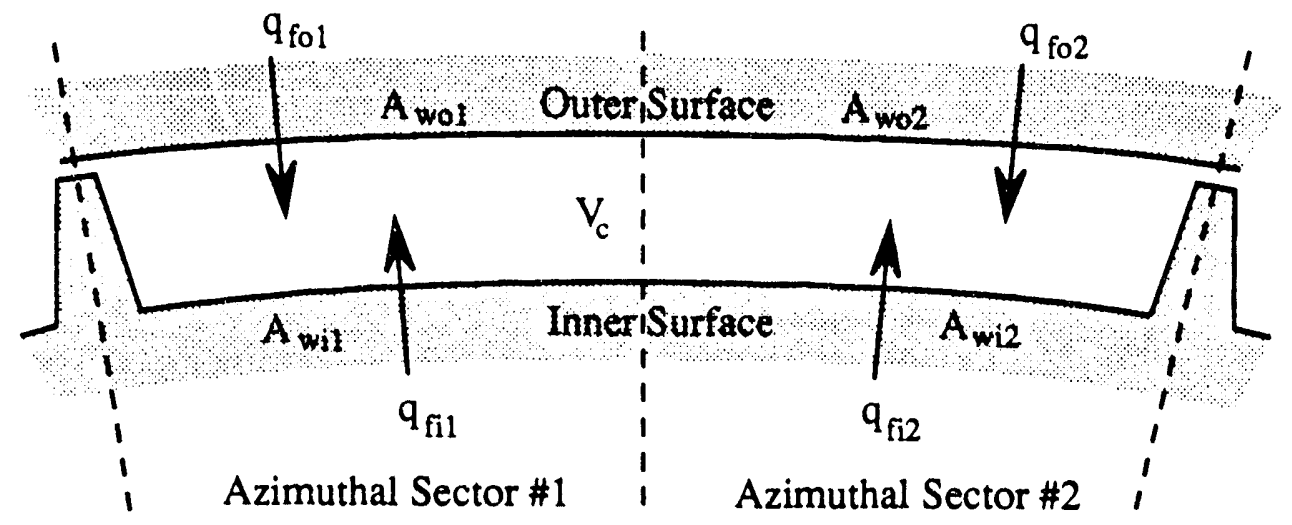

Figure 2.5.4.3-1 Illustration of heat transfer into a fluid cell from several solid surfaces.

Boiling at Solid Surfaces: For boiling heat transfer, the vapor generation rate is calculated by assuming that the heat flux for nucleate boiling, given by $\Delta q_{B}^{\prime \prime}=\left(q_{f}^{\prime \prime}-q_{F}^{\prime \prime}\right)$, is available for vapor generation. The amount of vapor generated at the wall is then calculated as

$$
\hat{\Gamma}_{w}=\frac{q_{f}^{\prime \prime}-q_{F C}^{\prime \prime}}{\lambda_{w}}=\frac{\Delta q_{B}^{\prime \prime}}{\lambda_{w}}
$$

where

$$
\begin{aligned}
\hat{\Gamma}_{\mathrm{w}} & \equiv \text { rate of vapor generation at the wall, } \mathrm{kg} / \mathrm{s}-\mathrm{m}^{2} \\
\mathrm{q}_{\mathrm{f}}^{\prime \prime} & \equiv \text { total heat flux into the fluid phase, } \mathrm{W} / \mathrm{m}^{2} \\
\mathrm{q}_{\mathrm{FC}}^{\prime \prime} & \equiv \text { forced convection (non-boiling) heat flux, W/m } / \mathrm{m}^{2} \\
\lambda_{\mathrm{w}} & \equiv \text { latent heat of vaporization at the wall, } \mathrm{J} / \mathrm{kg} \\
\Delta \mathrm{q}_{\mathrm{B}}^{\prime \prime} & \equiv \text { boiling heat flux, } \mathrm{W} / \mathrm{m}^{2}
\end{aligned}
$$

The wall heat transfer correlations supply values for the heat flux between the wall and the liquid phase $\left(\mathrm{q}_{\mathrm{f}}^{\prime \prime}\right)$ and for the forced convection heat flux. A schematic representation of a general boiling curve is shown in Fig. 2.5.4.3-2. As illustrated in the figure, we assume that the boiling heat flux available for vapor generation is the difference between the total heat flux to the fluid and the heat flux for forced convection.

We express the forced convection heat transfer at solid surface $j$ as

$$
q_{F C_{j}}^{\prime \prime}=h_{F C_{j}}^{n}\left(T_{w j}^{n+1}-T_{f}^{n}\right)
$$

where

$\mathrm{h}_{\mathrm{FC}}^{\mathrm{n}} \mathrm{j}$. forced convection heat transfer coefficient evaluated at time $\mathrm{n}$ 


$$
\begin{aligned}
T_{w j}^{n+1} & \equiv \begin{array}{l}
\text { surface temperature at wall sector } j \text { estimated at the new } n+1 \text { time (see Eq. } \\
(3.3-16))
\end{array} \\
T_{f}^{n} & \equiv \text { fluid temperature at the previous time } n
\end{aligned}
$$

Equation (2.5.4.3-2) becomes

$$
\hat{\Gamma}_{w j}^{n+1}=\frac{\left(q_{f}^{\prime \prime}\right)^{n+1}-h_{F C_{j}}^{n}\left(T_{w j}^{n+1}-T_{f}^{n}\right)}{\lambda_{w}^{n}}
$$

When the total interfacial mass transfer has been computed, the interfacial energy exchange to the gas phase at the wall for a boiling process is calculated as

$$
E_{g w}^{n+1}=\Gamma_{w}^{n+1} h_{s}^{\text {sat }}(P)
$$

Equation (2.5.4.3-5) assumes that all of the energy transferred to the gas phase goes into creating saturated vapor and that none of the energy is used to superheat the gas. Assuming that no energy can accumulate at the interface, an interfacial energy balance gives the energy transferred to the liquid phase at the wall as

$$
E_{f w}^{n+1}=-E_{g w}^{n+1}
$$

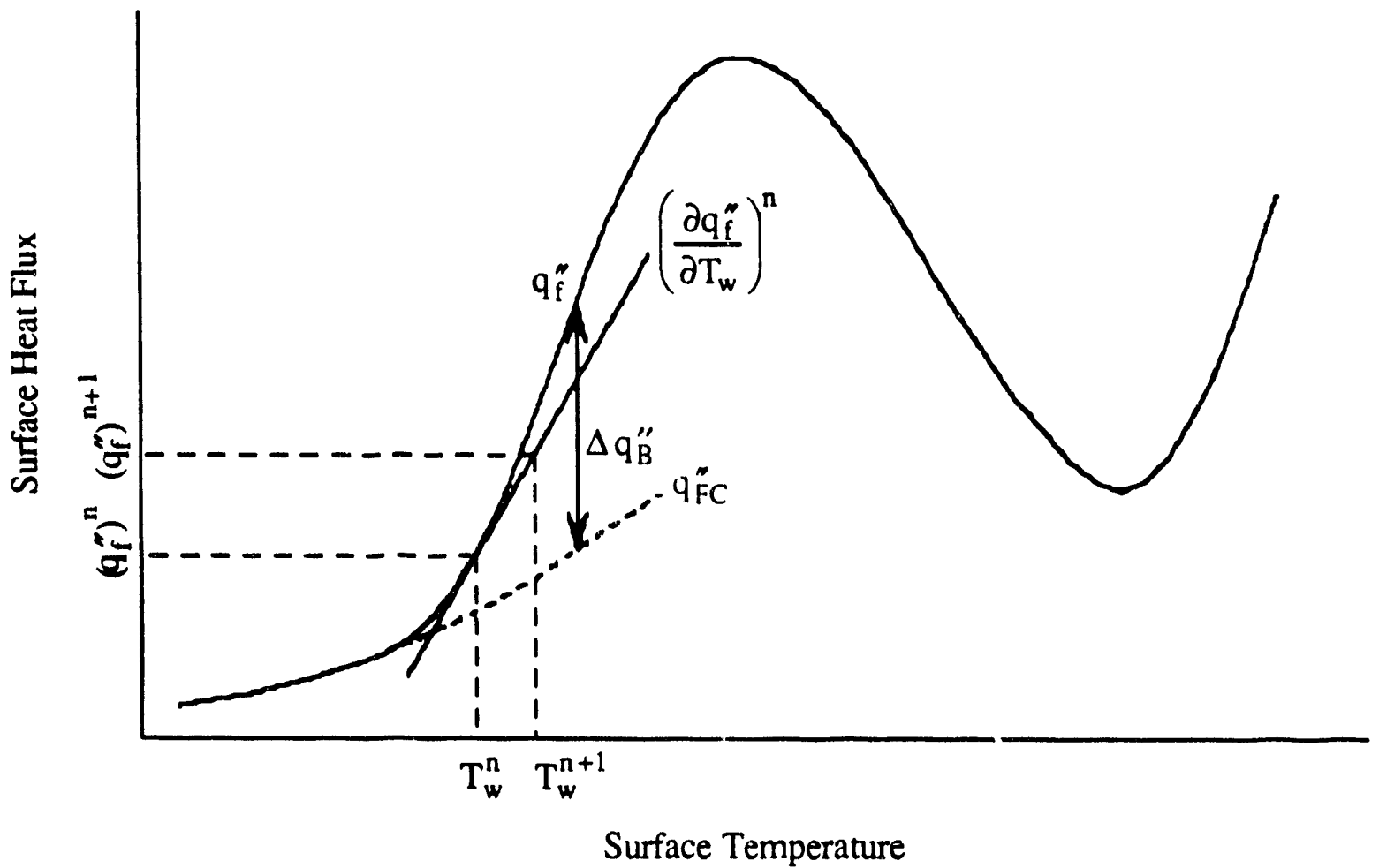

Figure 2.5.4.3-2 Schematic representation of boiling curve. 
Condensation at Solid Surfaces: Arguments exactly analogous to those used to describe the boiling process can be applied to describe condensation phenomena. For condensation heat transfer, the vapor deposition rate is calculated by assuming that the heat flux difference given by $\Delta q_{C}^{\prime \prime}=\left(q_{B}^{\prime \prime}-q_{F C}^{\prime \prime}\right)$, is available for vapor condensation. The amount of vapor generated at the wall is then calculated as

$$
\hat{\Gamma}_{w}=\frac{q_{g}^{\prime \prime}-q_{F C}^{\prime \prime}}{\lambda_{w}}=\frac{\Delta q_{C}^{\prime \prime}}{\lambda_{w}}
$$

where

$$
\begin{aligned}
\hat{\Gamma}_{w} & \equiv \text { rate of vapor condensation at the wall, } \mathrm{kg} / \mathrm{s}-\mathrm{m}^{2} \\
\mathrm{q}_{\mathrm{g}}^{\prime \prime} & \equiv \text { total heat flux into the gas phase, } \mathrm{W} / \mathrm{m}^{2} \\
\mathrm{q}_{\mathrm{FC}}^{\prime \prime} & \equiv \text { forced convection heat flux, W/m } / \mathrm{m}^{2} \\
\lambda_{\mathrm{w}} & \equiv \text { latent heat of condensation (vaporization) at the wall, } \mathrm{J} / \mathrm{kg} \\
\Delta \mathrm{q}_{\mathrm{C}}^{\prime \prime} & \equiv \text { condensing heat flux, } \mathrm{W} / \mathrm{m}^{2}
\end{aligned}
$$

A schematic representation of a portion of a generalized condensation curve is shown in Fig. 2.5.4.3-3. Note that in Fig. 2.5.4.3-3 we use the convention that $T_{w}^{n+1}<T_{w}^{n}$ and $\left(\mathrm{q}_{\mathrm{g}}^{\prime \prime}\right)^{\mathrm{n}+\mathrm{T}}<\left(\mathrm{q}_{\mathrm{g}}^{\prime \prime}\right)^{\mathrm{n}}$. As illustrated in the figure, we assume that the heat flux available for vapor condensation is the difference between the total heat flux to the gas and the heat flux for forced convection. The condensing heat flux is a negative quantity and the rate of vapor condensation will also be negative indicating mass transfer from the gas phase to the liquid. Condensation will only occur if heat is being removed from the gas phase.

We compute the forced convection heat transfer at solid surface $j$ as

$$
q_{F C_{j}}^{\prime \prime}=h_{F C_{j}}^{n}\left(T_{w j}^{n+1}-T_{8}^{n}\right)
$$

where

$$
\begin{aligned}
& \mathrm{h}_{\mathrm{FC}}^{\mathrm{n}} \equiv \text { forced convection heat transfer coefficient evaluated at time } \mathrm{n} \\
& \mathrm{T}_{\mathrm{wj}}^{\mathrm{n}+1} \equiv \begin{array}{l}
\text { surface temperature at wall sector } \mathrm{j} \text { estimated at the new } \mathrm{n}+1 \text { time (see Eq. } \\
(3.3-16))
\end{array} \\
& \mathrm{T}_{\mathrm{g}}^{\mathrm{n}} \equiv \text { gas temperature at the previous time } \mathrm{n}
\end{aligned}
$$

Equation (2.5.4.3-7) becomes

$$
\hat{\Gamma}_{w j}^{n+1}=\frac{\left(q_{g}^{\prime \prime}\right)^{n+1}-h_{F C_{j}}^{n}\left(T_{w j}^{n+1}-T_{g}^{n}\right)}{\lambda_{w}^{n}}
$$

When the total interfacial mass transfer has been computed, the interfacial energy exchange to the liquid phase at the wall for a condensing process is calculated as 


$$
E_{f w}^{n+1}=-\Gamma_{w}^{n+1} h_{f}^{\text {sat }}\left(T_{f}\right)
$$

Equation (2.5.4.3-10) assumes that all of the energy transferred to the liquid phase goes into creating saturated liquid and that none of the energy is used to subcool the liquid. Assuming that no energy can accumulate at the interface, an interfacial energy balance gives the energy transferred to the gas phase at the wall as

$$
E_{g w}^{m}=-E_{f w}^{m}
$$

Since the rate of mass exchange is negative, the heat exchange to the gas phase is also negative which is physically correct for condensation.

\section{Surface Temperature}

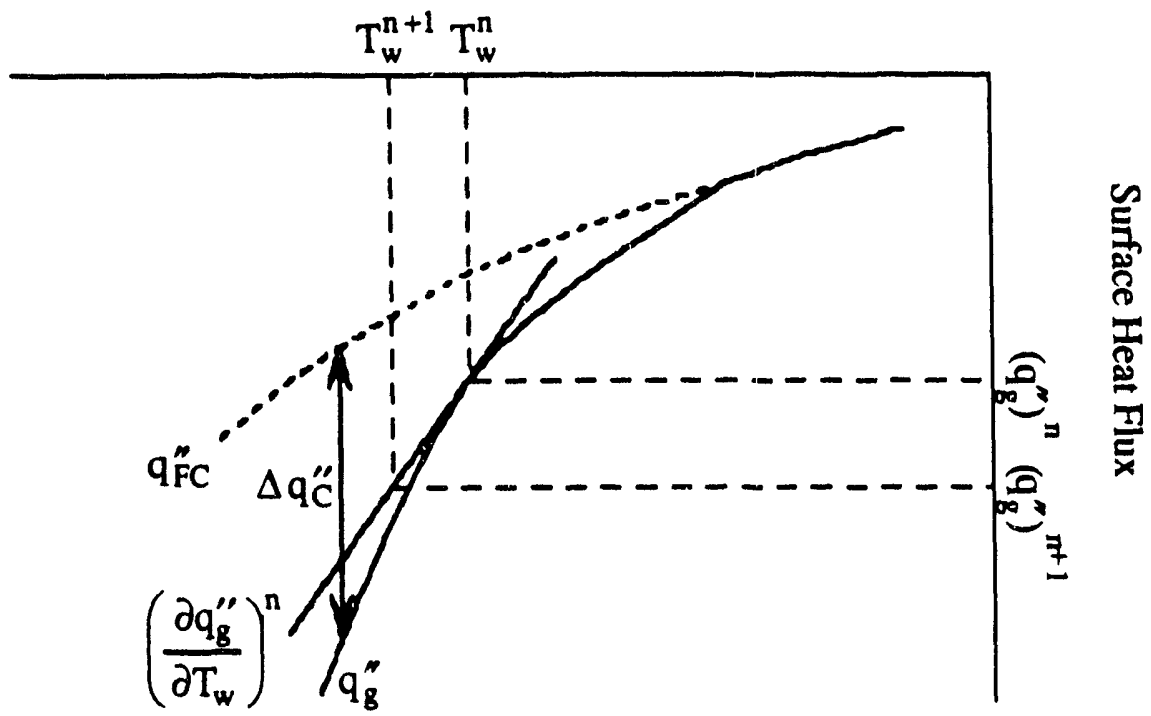

Figure 2.5.4.3-3 Schematic representation of condensation curve. 


\subsubsection{Interfacial Drag}

The FLOWTRAN-TF interfacial drag model is presented in section 2.5.5.2. The model makes use of the counter-current flow limitation models presented in section 2.5.5.1.

\subsubsection{Counter-Current Flow Limitation (CCFL) Models}

For a given liquid flow rate in a channel geometry, there is a certain gas upflow at which very large waves appear on the air-water interface in a countercurrent two-phase flow regime and the whole flow becomes chaotic. Then the gas pressure drop increases suddenly and liquid is expelled from the inlet of the channel. This condition is known as flooding phenomenon, that is, countercurrent flow limitation (CCFL). Unlike the flow phenomenon which occurs in dispersions of bubbles, the flooding inception point is the result of a sudden instability. Under some abnormal operational transients and accidents, the flooding condition can cause local dryout spot on the heated channel wall surface, which may lead to the onset of thermal excursion (OTE).

The configuration of such a subchannel that is composed of annular channels with ribs in Mark 22 multi-channel assembly is assumed to be rectangular as illustrated in Figure 2.5.5.1-1. From the countercurrent flow visualization studies in thin rectangular channel done by Sudo and Kaminaga (1989), it was indicated that mosi of the liquid film downflow occurred along the narrow side walls of thin slit channel as illustrated in Figure 2.5.5.1-2. Using the shannel hydraulic diameter as the characteristic dimension failed to collapse the flooding data for various aspect ratios. Several authors used different characteristic lengths for thin rectangular channels in order to analyze their experimental data as shown in Table 2.5.5.1-1. For the present work, non-dimensional superficial velocities are defined using two times the wide span of rectangular channel on the following basis:

For a cylindrical channel in Figure 2.5.5.1-2, the liquid flow fraction is

$$
(1-\alpha) \equiv \frac{4 \delta}{D}
$$

where $\delta$ is the film thickness of annular liquid downflow and $D$ is the channel hydraulic diameter.

For a thin rectangular channel of Figure 2.5.5.1-2, the liquid flow fraction is based on the flow visualization studies:

$$
(1-\alpha) \cong \frac{2 \delta}{a}
$$

where $a$ is the wide span of rectangular channel. From equations, (2.5.5.1-1) and (2.5.5.1-2), hydraulic characteristic diameter for thin rectangular channel is equal to $2 \mathrm{a}$. Mishima and Nishihara (1985) and Cheng (1990) also used twice the wide span (2a) as a characteristic dimension to non-dimensionalize superficial velocity for each phase. 
Two length scales have been available in the flooding literature, corresponding to two dimensionless superficial velocities. One is the characteristic diameter of flow passage, leading to the widely-used scaling parameter introduced by Wallis

$$
J_{k}^{*}=j_{k} \sqrt{\frac{\rho_{k}}{g d\left(\rho_{1}-\rho_{g}\right)}}
$$

where $j_{k}$ is the superficial velocity of the gas $(k=g)$ or liquid $(k=1)$ and $d$ is the characteristic diameter of flow passage. Another characteristic length has been constructed from the properties of the gas-liquid interface incorporated by Kutateladze in the dimensionless number

$$
K_{k}^{*}=j_{k}\left[\frac{\rho_{k}^{2}}{g \sigma\left(\rho_{1}-\rho_{g}\right)}\right]^{0.25}
$$

where $\sigma$ is the liquid surface tension.

In cases where both the interfacial properties and the characteristic diameter are important, no satisfactory means exists at present for interpolating between these two scales.

Hence, Wallis-type non-dimensional correlation was used for the formulation of flooding criterion based on the literature data with the characteristic diameter of $2 \mathrm{a}$ since geometrical scaling iras more important impact on hydraulic performance of thin slit channel than airwater interfacial property does. This correlation applies to air-water countercurrent flow near the atmospheric pressure in a thin rectangular passage. The results for various aspect ratios irom the literature are shown in Figure 2.5.5.1-3. FLOWTRAN-TF development report TF-3 (Lee, 1991) also shows single-slit channel data of several authors employed in the flooding model development including the multiple channel data of ORNL (1990). The detailed informations about experimental conditions and test section geometry are given in Table 2.5.5.1-1. This functional form ${ }_{i}$ can also be derived from the basic momentum balance because flooding corresponds to the state of countercurrent flow limitation, which is represented mathematically by the locus of this family of curves, $f\left(j_{g}, j_{l}, \alpha\right)=0$ (Lee, 1990).

$$
\sqrt{\mathrm{J}_{\mathrm{g}}^{*}}+m \sqrt{\mathrm{j}_{1}^{*}}=\mathrm{C}
$$

Based on the experimental data available in the literature, $\mathrm{m}$ and $\mathrm{C}$ in equation (2.5.5.1-5) were correlated in terms of aspect ratio (b/a), where $b$ is the channel gap size on the narrow side of the rectangular channel. As shown in Figure 2.5.5.1-4, the curve was fitted by the least square method, that is,

$$
\begin{gathered}
m=0.5194+3.4019\left(\frac{b}{a}\right) \\
C=0.6959+0.1618 \log _{10}\left(\frac{b}{a}\right)
\end{gathered}
$$


As illustrated in Figure 2.5.5.1-3, the present flooding correlation is in good agreement with ORNL data, i.e., multi-channel (parallel slit channels) data although the correlation was developed by single slit channel data. This result indicates that the countercurrent flow performance of an individual channel in the parallel multichannel system is consistent with data taken by experiments of a single channel. Ruggles (1990) also drew the same conclusion from his multi-channel data analysis. As demonstrated in Figure 2.5.5.1-1, Mark 22 assembly is assumed to be a multichannel system which is composed of single equivalent rectangular channels. From this viewpoint, it is expected that the present flooding correlation developed for a single thin rectangular channel is applicable to a Mark 22 assembly. Table 2.5.5.1-2 lists the flooding correlation parameters used in FLOWTRAN-TF simulations for various geometries of interest. 


\begin{tabular}{|l|lll|l|}
\hline $2 / 15 / 93$ & WSRC-TR-92-532 Rev. 0 & FLOWTRAN-TF Software Design & Page 215 \\
\hline
\end{tabular}

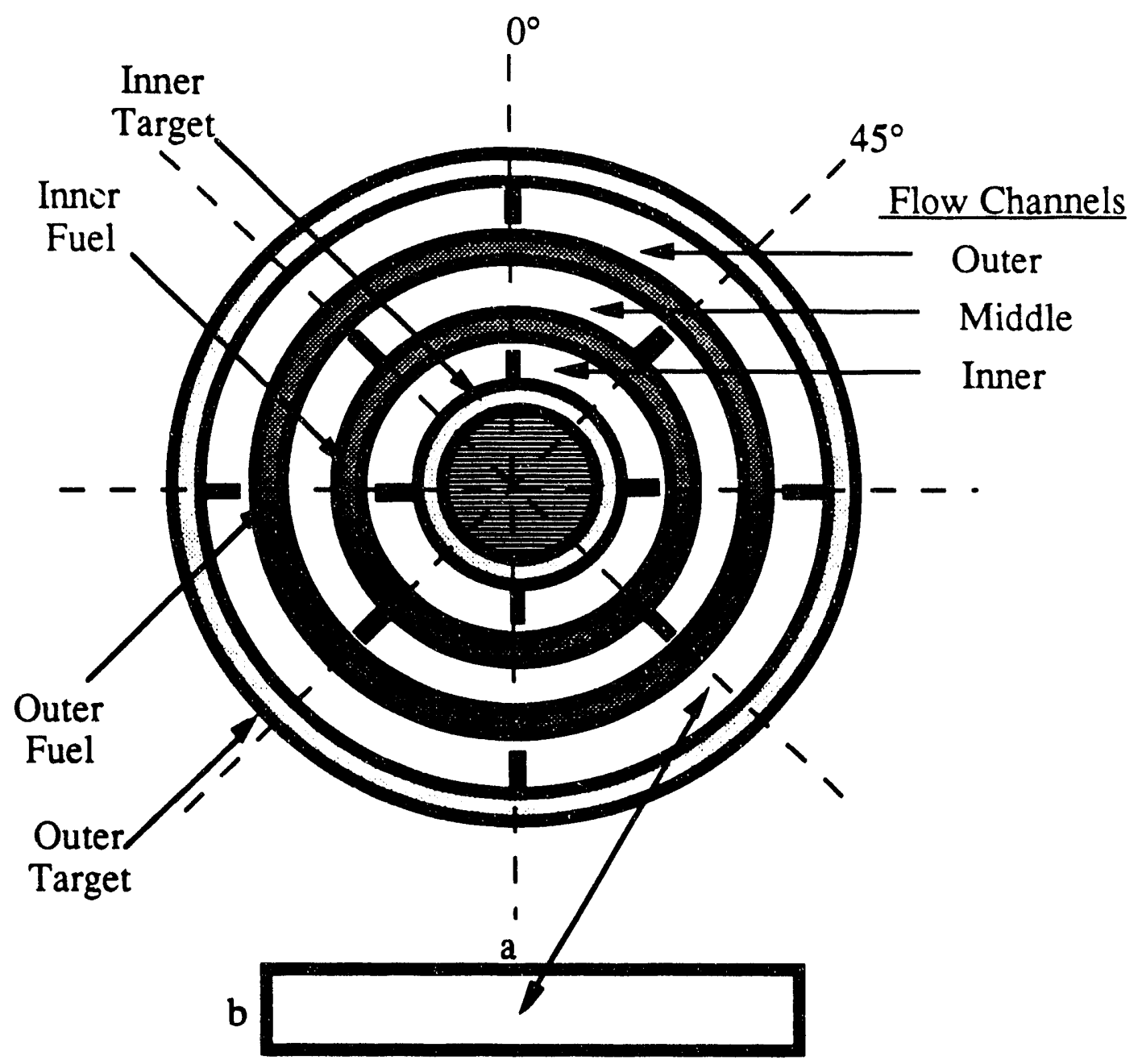

Single rectangular slit equivalent to a Mark 22 subchannel

\begin{tabular}{|l|c|c|c|c|}
\hline Mark 22 Channel & $\mathrm{a}(\mathrm{mm})$ & $\mathrm{b}(\mathrm{mm})$ & $\mathrm{b} / \mathrm{a}$ & $\begin{array}{c}\text { Channel Height } \\
(\mathrm{L}: \mathrm{mm})\end{array}$ \\
\hline Inner Channel & 36 & 5.1 & 0.14 & 4115 \\
Middle Channel & 52 & 6.8 & 0.13 & 4115 \\
Outer Channel & 67 & 4.3 & 0.064 & 4115 \\
\hline
\end{tabular}

Figure 2.5.5.1-1 Multi-channel goemetry composed of equivalent thin rectangular channels in Mark 22 assembly. 


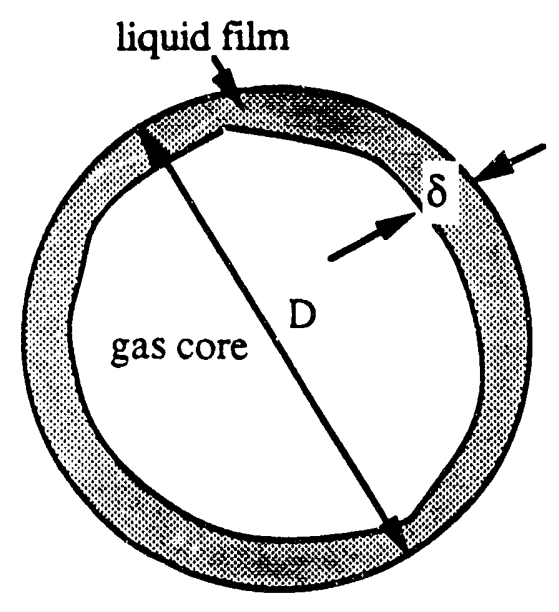

Cylindrical Channel

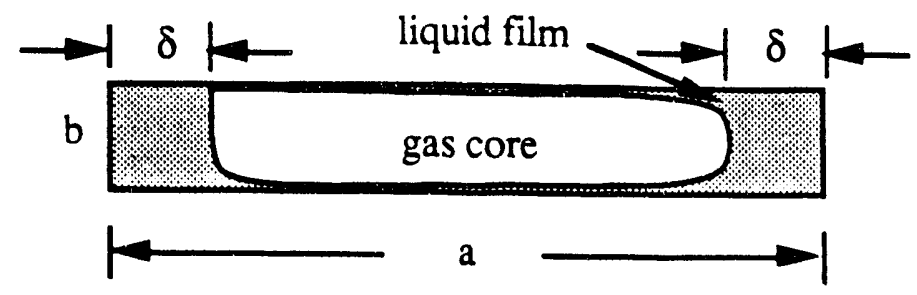

Thin Rectangular Channel

Figure 2.5.5.1-2 Experimental Observations of CCFL Liquid Film for Different Channel Geometries 
Table 2.5.5.1-1 Experimental studies on flooding for slit geometry in the literature

\begin{tabular}{|c|c|c|c|c|}
\hline Author (Pub. Year) & Mishima (1984) & $\begin{array}{l}\text { Osakabe and } \\
\text { Kawasaki (1989) }\end{array}$ & $\begin{array}{l}\text { Sudo and } \\
\text { Kaminaga (1989) }\end{array}$ & $\begin{array}{l}\text { ORNL } \\
(1990)\end{array}$ \\
\hline $\begin{array}{l}\text { Test Section } \\
\text { (b } \times \text { a: } \mathrm{mm} \times \mathrm{mm})\end{array}$ & $\begin{array}{l}1.5 \times 40 \\
2.4 \times 40 \\
5.0 \times 40\end{array}$ & $\begin{array}{l}2 \times 100 \\
5 \times 100 \\
10 \times 100\end{array}$ & $\begin{array}{l}8.3 \times 66 \\
12.3 \times 66\end{array}$ & $1.27 \times 84$ \\
\hline$\underset{(\mathrm{mm})}{\text { Plenum Height }}$ & $300,400,600$ & $19,60,61$ & 550 & - \\
\hline $\begin{array}{l}\text { Characteristic } \\
\text { Length used for } \\
\text { Data Analysis }\end{array}$ & $2 a$ & a & b & $\mathbf{a}$ \\
\hline $\begin{array}{l}\text { Channel Height } \\
\text { (L: mm) }\end{array}$ & 470 & 1235 & 362,782 & 610 \\
\hline $\begin{array}{l}\text { Two-phase } \\
\text { Fluid }\end{array}$ & Air-Water & Air-Water & Air-Water & Air-Water \\
\hline $\begin{array}{l}\text { System } \\
\text { Pressure }\end{array}$ & about $1 \mathrm{~atm}$ & about $1 \mathrm{~atm}$ & about $1 \mathrm{~atm}$ & about $1 \mathrm{~atm}$ \\
\hline $\begin{array}{l}\text { Test Section } \\
\text { Geometry }\end{array}$ & & & & $\begin{array}{l}\text { Multichannel } \\
\text { (Parallel thin } \\
\text { rectangular } \\
\text { slits) }\end{array}$ \\
\hline
\end{tabular}




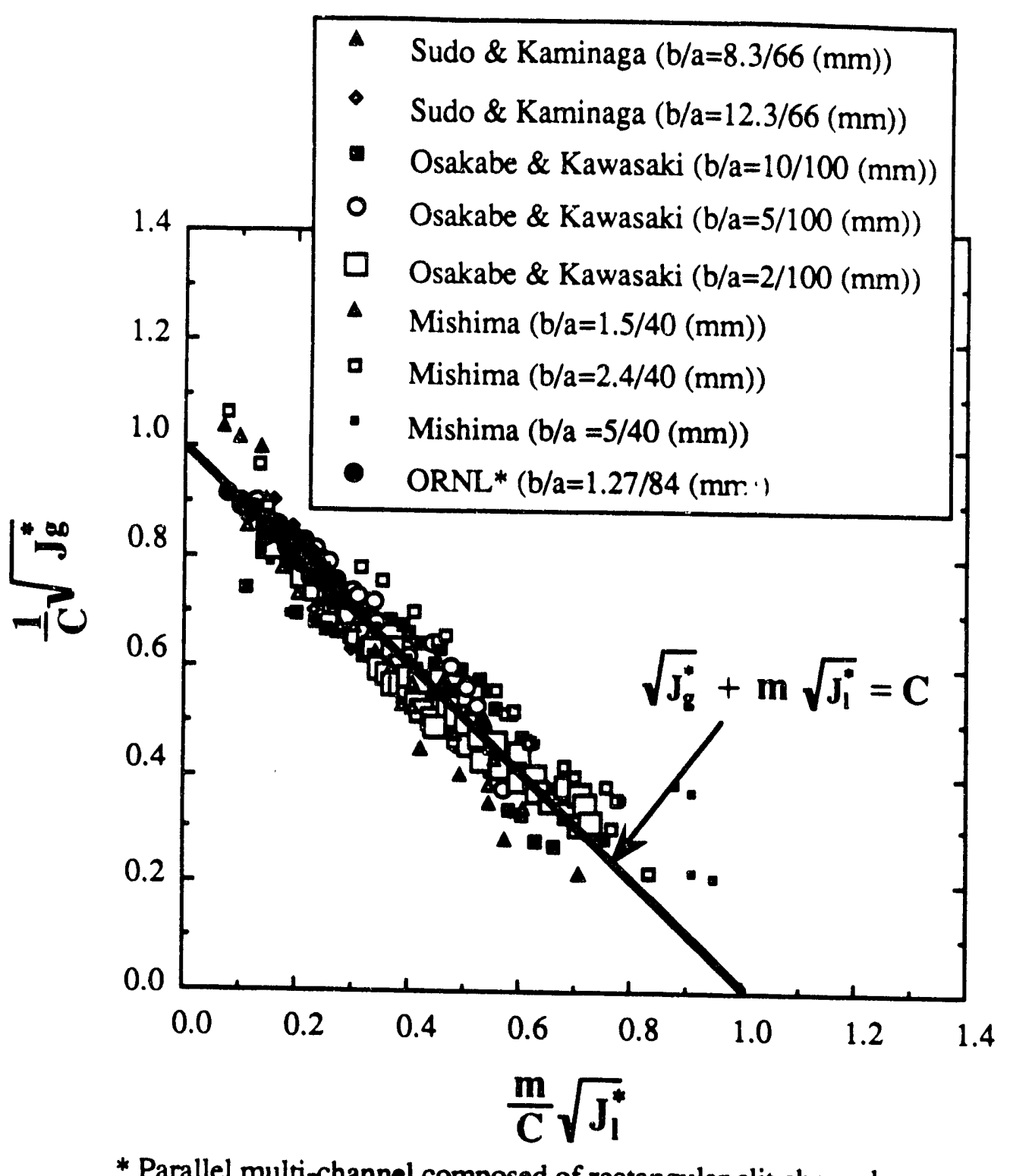

* Parallel multi-channel composed of rectangular slit channels

Figure 2.5.5.1-3 Flooding correlation for thin rectangular geometry 


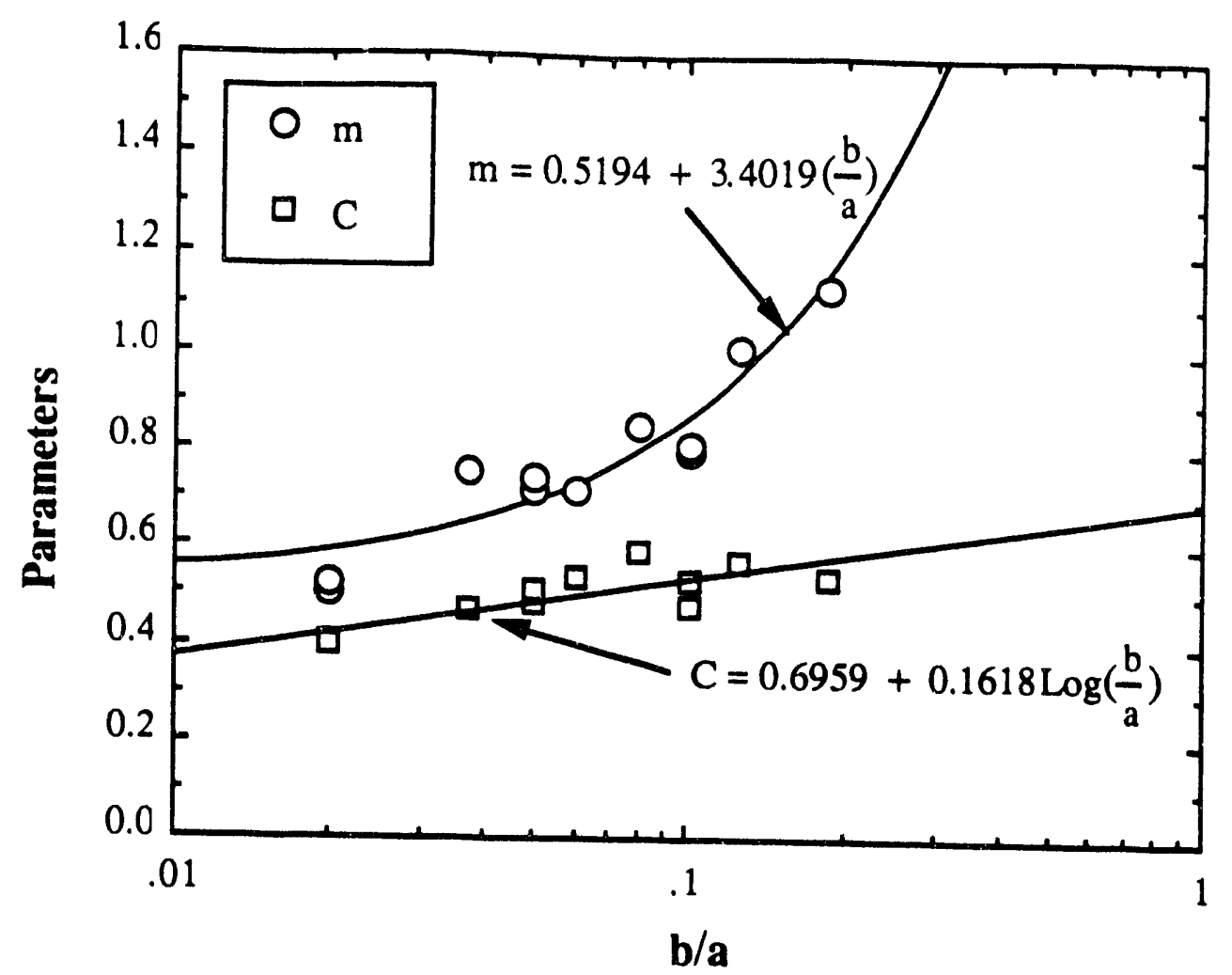

Figure 2.5.5.1.4 Correlations of the parameters employed in flooding formulation

Table 2.5.5.1-2 Flooding parameters associated with CCFL constraint in interfacial drag for SRS geometries of interest

\begin{tabular}{|c|c|c|c|c|}
\hline Geometry & $\mathrm{D}_{\mathrm{C}}$ & $\mathbf{m}$ & $\mathrm{C}$ & References \\
\hline $\begin{array}{l}\text { Thin } \\
\text { Rectangular } \\
\frac{a}{b} \text { b }\end{array}$ & $2 a$ & $\begin{array}{c}\min [0.5194+ \\
3.4019(\mathrm{~b} / \mathrm{a}), \\
1.0]\end{array}$ & $\begin{array}{c}0.6959+ \\
0.1618 \log _{10}(b / a)\end{array}$ & $\begin{array}{c}\text { Sudo \& } \\
\text { Kaminaga, 1989; } \\
\text { Osakabe \& } \\
\text { Kawasaki, 1989; } \\
\text { Mishima, 1984; } \\
\text { Ruggles, 1990 }\end{array}$ \\
\hline $\begin{array}{c}\text { Ribbed } \\
\text { Annulus } \\
\mathrm{a} \equiv \frac{\pi}{4}\left(r_{i}+r_{0}\right) \\
b \equiv r_{0}-r_{i}\end{array}$ & $\frac{\pi}{2}\left(r_{i}+r_{0}\right) \equiv 2 a$ & same as above & same as above & \\
\hline Annulus & $\frac{\pi}{2}\left(r_{i}+r_{0}\right)$ & 0.8 & 0.45 & $\begin{array}{c}\text { Osakabe \& } \\
\text { Kawasaki, } 1989\end{array}$ \\
\hline Circular & $\mathrm{D}$ & 1.0 & 0.94 & Wallis, 1969 \\
\hline
\end{tabular}




\subsubsection{Interfacial Drag Model}

The FLOWTRAN-TF interfacial drag model is based heavily on the approaches taken with other reactor thermal-hydraulic codes such as RELAP5 (Ransom et al., 1985), TRAC (Taylor et al., 1984; Weaver et al., 1986), THERMIT (Reed and Stewart), COBRA/TRAC (Thurgood et al., 1983) and VIPRE (Stewart et al., 1989), and on the works of Ishii (1977, 1979), Ishii and Mishima (1984), Anderson and Chu (1982), and Putney (1988, 1991). Development of the FLOWTRAN-TF interfacial drag model is organized according to the following outline:

1) Approximate space/time averaged two-phase momentum conservation equations

2) Transformation of $\tau_{i} \cdot S_{i}$

3) Interfacial drag moacling approach

4) Axial, dispersed two-phase flow (bubbly \& slug flow regimes)

5) Axial, separated two-phase flow (annular flow regime)

6) Flow regime transitions

7) Dispersed regime interfacial drag data correlations for a ribbed annular channel

8) General recommendations for axial interfacial drag parameters

9) Azimuthal two-phase flow

10) Computational implementation

References

Subsection 1 reviews the approximate space/time averaged momentum conservation equations presented in section 2.4. An alternative, preferred form of one of of the two interfacial drag terms is derived in subsection 2 . The overall approach to interfacial drag modeling is discussed on subsection 3. Different interfacial drag models are used for the axial and azimuthal directions. Subsections 4 and 5 address the dispersed and separated flow regimes of an axial flow and subsection 6 covers regime transitions between bubbly, slug and annular flow. Experimental data for ribbed thin annular channels taken in the SRS Heat Transfer Laboratory are analyzed in subsection 7; these include Rig C, FA/FB, and VOID data. Subsection 8 establishes recommendations for parameter values as a function of channel geometry and flow regime for the axial direction. The azimuthal interfacial drag model is developed in subsection 9. Finally, modifications needed to numerically implement the models are described in subsection 10 .

1) Approximate space/time averaged two-phase momentum conservation equations: The gas and liquid momentum balances are given in section 2.4 as

$$
\begin{gathered}
\alpha \rho_{\mathrm{g}} \frac{\partial v_{\mathrm{g}}}{\partial \mathrm{t}}+\alpha \rho_{\mathrm{g}} \mathrm{v}_{\mathrm{g}} \cdot \nabla \mathrm{v}_{\mathrm{g}}=-\alpha \nabla \mathrm{P}+\alpha \rho_{\mathrm{g}} \mathrm{g}-\mathrm{F}_{\mathrm{wg}}-\mathrm{F}_{\mathrm{i}}-\mathbf{M}_{\mathrm{tg}} \\
(1-\alpha) \rho_{\mathrm{f}} \frac{\partial \mathrm{v}_{\mathrm{f}}}{\partial \mathrm{t}}+(1-\alpha) \rho_{\mathrm{f}} \mathrm{v}_{\mathrm{f}} \cdot \nabla \mathrm{v}_{\mathrm{f}}=-(1-\alpha) \nabla \mathrm{P}+(1-\alpha) \rho_{\mathrm{f}} \mathrm{g}-\mathrm{F}_{\mathrm{wf}}+\mathrm{F}_{\mathrm{i}}-\mathbf{M}_{\mathrm{tf}}
\end{gathered}
$$

where the interfacial drag term is defined by

$$
F_{i k} \equiv-\tau_{k_{i}} \cdot S_{k_{i}}^{\prime \prime \prime}-M_{k_{i}}^{d}
$$




$$
\begin{gathered}
S_{k_{i}}^{\prime \prime \prime} \equiv \frac{1}{V} \int_{A_{i}} n_{k} d S \\
\mathbf{M}_{k_{i}}^{d} \equiv-\frac{1}{V} \int_{A_{i}}\left[\Delta \tilde{P}_{k_{i}}^{d} I-\Delta \tilde{\tau}_{k_{i}}\right] \cdot n_{k} d S
\end{gathered}
$$

and $\mathbf{F}_{\mathrm{i}} \equiv \mathbf{F}_{\mathrm{ig}}$. Explicit averaging notation has been omitted. The notation can be simplified by examining the local instantaneous momentum jump condition given by Lahey and Drew (1988) as

$$
\sum_{k=1}^{2}\left[\rho_{k} v_{k}\left(v_{k}-v_{i}\right)+\left(P_{k_{i}} I-\tau_{k_{i}}\right)\right] \cdot n_{k}=\nabla_{s} \cdot \sigma \underline{a}+2 H \sigma n_{k}
$$

where a and $\mathrm{H}$ quantify surface curvature (see Flach (1992)). In deriving the momentum balancess above, mass transfer and surface tension effects were neglected. Doing the same with the momentum jump condition yields

$$
\sum_{k=1}^{2}\left(P_{k_{i}=} I-\tau_{k_{i}}\right) \cdot n_{k}=0
$$

That is, in the absence of mass transfer and surface tension effects the local instantaneous shear stress tensor and pressure are continuous across the phase interface. Therefore define

$$
\begin{aligned}
& \underline{\underline{\tau_{i}}} \equiv \underline{\underline{\tau_{i}}}=\underline{\underline{\tau_{f_{i}}}} \\
& \Delta \tilde{\tau}_{i} \equiv \Delta \tilde{\tau}_{g_{i}}=\Delta \tilde{\tau}_{f_{i}} \\
& \Delta \tilde{\mathrm{P}}_{\mathrm{i}}^{\mathrm{d}} \equiv \Delta \tilde{\mathrm{P}}_{\mathrm{g}_{\mathrm{i}}}^{\mathrm{d}}=\Delta \tilde{\mathrm{P}}_{\mathrm{f}_{\mathrm{i}}}^{\mathrm{d}}
\end{aligned}
$$

Note also that because $\mathbf{n}_{\mathbf{g}}=-\mathbf{n}_{\mathrm{f}}$

$$
S_{B_{i}}^{\prime \prime \prime}=-S_{f_{i}}^{\prime \prime \prime} \equiv S_{i}^{\prime \prime \prime}
$$

and

$$
\mathbf{M}_{\mathbf{g}_{\mathrm{i}}}^{\mathrm{d}}=-\mathbf{M}_{\mathrm{f}_{\mathrm{i}}}^{\mathrm{d}} \equiv \mathbf{M}_{\mathrm{i}}^{\mathrm{d}}
$$

With these results, definition (2.5.5.2-3) can be rewritten as

$$
F_{i} \equiv-\tau_{i} \cdot S_{i}^{\prime \prime \prime}-M_{i}^{d}
$$


Recall that the two-phase conservation equations were derived in section 2.4 by space averaging followed by time averaging. For constitutive (closure) relation development, quantities which have been time averaged first are frequently preferred over the space/time averaged counterpart (as will be evident later). Many of the terms in equations (2.5.5.2-1) and $(2.5 .5 .2-2)$ have already been transformed to the equivalent time/space averaged form. However, the first term in definition (2.5.5.2-13) is still in space/time averaged form, although not explicitly shown. In the next subsection the commutativity of the space and time averaging operators is used to transform the term to the time/space averaged version.

2) Transformation of $\tau_{i} \cdot S_{i}^{\prime \prime \prime}:$ First the origin of

$$
\left\langle\underline{\tau_{k}}\right\rangle_{i} \cdot S_{k_{i}}^{\prime \prime \prime}
$$

is reviewed. Recall that

$$
\begin{aligned}
\Delta \underline{\tilde{\tau}_{k_{i}}} \equiv & \underline{\tau_{k_{i}}}-\left\langle\underline{\tau_{k}}\right\rangle_{i} \\
\left\langle\underline{\tau_{k}}\right\rangle_{i} & \equiv \frac{1}{A_{i}} \int_{A_{i}}{\underline{\tau_{k_{i}}}} d S \\
S_{k_{i}}^{\prime \prime \prime} & \equiv \frac{1}{V} \int_{A_{i}} n_{k} d S
\end{aligned}
$$

A Reynolds averaging rule was used to obtain

$$
\begin{aligned}
\frac{1}{V} \int_{A_{i}} \tau_{k_{i}} \cdot n_{k} d S & \equiv\left\langle\underline{\tau_{k}}\right\rangle_{i} \cdot \frac{1}{V} \int_{A_{i}} n_{k} d S+\frac{1}{V} \int_{A_{i}} \Delta \tilde{\tau}_{k_{i}} \cdot n_{k} d S \\
& =\left\langle\underline{\tau_{k}}\right\rangle_{i} \cdot S_{k_{i}}^{\prime \prime \prime}+\frac{1}{V} \int_{A_{i}} \Delta \tilde{\tau}_{k_{i}} \cdot n_{k} d S
\end{aligned}
$$

Time averaging yields

$$
\frac{1}{V} \int_{A_{i}} \tau_{k_{i}} \cdot n_{k} d S \equiv \overline{\left\langle\tau_{k}\right\rangle_{i} \cdot S_{k_{i}}^{\prime \prime \prime}}+\frac{1}{V} \overline{\int_{A_{i}} \Delta \tilde{\tau}_{k_{i}} \cdot n_{k} d S}
$$

The first term on the r.h.s. of equation (2.5.5.2-19) is the term under consideration and the second is part of $\mathbf{M}_{k_{k}}^{d}$. The transformation is performed by using the following identity given by Boure and Delhaye (1982)

$$
\overline{\int_{A_{i}} B_{k} \cdot n_{k} d S}=\int_{V} \sum_{j} \ell_{j}^{-1}\left(B_{k} \cdot n_{k}\right)_{j} d V
$$




$$
\ell_{\mathrm{j}} \equiv \mathrm{T}\left|\mathbf{v}_{\mathrm{i}} \cdot \mathbf{n}_{\mathrm{k}}\right|
$$

which expresses the commutativity property of the space and time averaging operators with respect to interfacial terms. In Lahey and Drew's notation (generally used here) the generic tensor

$$
\underline{\mathbf{B}_{\mathrm{k}}}
$$

would have the additional subscript $i$. This notation is actually redundant and will not be used in order to be consistent with Boure and Delhaye (1982) for the present development. Replacing $\mathbf{B}_{k}$ with $\tau_{k}$, dividing by $V$, and recalling the definition of the space averaging operator $\Varangle \rightarrow$ gives -

$$
\frac{1}{V} \int_{A_{i}} \tau_{k} \cdot n_{k} \mathrm{~d} S=\frac{1}{V} \int_{V} \sum_{j} \ell_{j}^{-1}\left(\underline{\tau_{k}} \cdot n_{k}\right)_{j} d V=\Varangle \sum_{j} \ell_{j}^{-1}\left(\tau_{k} \cdot n_{k}\right)_{j} \downarrow
$$

Define a time average interfacial stress tensor by

$$
{\overline{\tau_{k}}}^{i} \equiv \frac{\sum_{j} \ell_{j}^{-1} \tau_{k}}{\sum_{j} \ell_{j}^{-1}}
$$

and split the stress tensor into average and fluctuating components:

$$
\underline{\tau_{k}} \equiv \overline{\tau_{k}} i+\Delta \hat{\tau}_{k}
$$

Then using a Reynolds averaging rule

$$
\begin{aligned}
& \Varangle \sum_{j} \ell_{j}^{-1}\left(\underline{\tau_{k}} \cdot n_{k}\right)_{j} \triangleright=\Varangle \sum_{j} \ell_{j}^{-1}\left[\left(\bar{\tau}_{k}^{i}+\Delta \hat{\tau}_{k}\right) \cdot n_{k}\right]_{j} \downarrow \\
& \equiv \Varangle \bar{\tau}_{k}^{i} \cdot \sum_{j} \ell_{j}^{-1}\left[n_{k}\right]_{j} \downarrow+\nless \sum_{j} \ell_{j}^{-1}\left[\Delta \hat{\tau}_{k} \cdot n_{k}\right]_{j} \downarrow
\end{aligned}
$$

Boure and Delhaye (1982) give the following form of Gauss' Theorem

$$
\int_{T_{k}} \nabla \cdot B_{k} d t=\nabla \cdot \int_{T_{k}} B_{k} d t+T \sum_{j} \ell_{j}^{-1}\left(\underline{B_{k}} \cdot n_{k}\right)_{j}
$$

Replacing $\mathbf{B}_{k}$ with the identity matrix $I$ and dividing by $T$ gives

$$
-\frac{1}{\mathrm{~T}} \nabla \mathrm{T} \alpha_{\mathrm{k}}=\sum_{\mathrm{j}} \ell_{\mathrm{j}}^{-1}\left[\mathrm{n}_{\mathrm{k}}\right]_{\mathrm{j}}
$$


Lahey and Drew (1988) show using Gauss' Theorem that

$$
S_{k_{i}}^{\prime \prime \prime}+S_{k_{w}}^{\prime \prime \prime}=-\frac{1}{V} \nabla V \beta_{k}
$$

Frequently $\mathbf{S}_{\mathrm{k}_{\mathrm{w}}}^{\prime \prime \prime}=0$, such as if $\mathrm{V}$ does not contact a wall or if the flow is one-dimensional and the walls are straight. Equations (2.5.5.2-19) and (2.5.5.2-25) are equally valid and either may be used depending on which is more convenient. In both cases the second term on the r.h.s. is contained in $\mathbf{M}_{\mathbf{k}_{i}}^{\mathrm{d}}$.

3) Interfacial drag modeling approach: Section 2.1 discusses the physical geometries encountered in FLOWTRAN-TF applications and the computational geometry capabilities of FLOWTRAN-TF. Two basic geometries typically arise in FLOWTRAN-TF applications corresponding to the Top/Bottom and Middle sections of the computational mesh. The Top and Bottom sections simulate a one-dimensional and usually vertical downflow through a complex channel. The Middle section typically models ribbed annular channels formed by nested tubes with longitudinal ribs (uniform flow passage). The dominant flow direction is parallel to the ribs. Because the ribs do not form a perfect seal between the 4 subchannels of a channel, crossflow through the rib gaps may occur. The main flow direction in all sections is assigned the $\mathrm{z}$ coordinate and 'axial' flow label. Flow perpendicular to the longitudinal ribs in the Middle section is assigned the $\mathrm{x}$ coordinate and 'azimuthal' identifier. The positive z-direction is chosen to be downward $\left(\mathbf{g} \cdot \mathbf{n}_{\mathbf{z}}=\mathbf{g}\right)$. This convention is convenient for downflow but opposite that typically chosen; therefore, many expressions in this document differ in sign from their counterparts in the literature.

Section 2.5.3 discusses the flow regime map in FLOWTRAN-TF. Five two-phase flow regimes are possible with the map, these being bubbly, bubbly-slug, slug, slug-annular, and annular. The bubbly-slug regime is modeled as a blend of the pure bubbly and pure slug regimes. The slug-annular regime is treated analogously. The interfacial drag model for the azimuthal direction is the same for all flow regimes. In the axial direction, functional forms for dispersed and separated axial flow are developed initially. Separated flow is synonymous with the annular regime and dispersed refers to the bubbly and slug regimes. The dispersed flow interfacial drag model is subsequently subdivided into bubbly and slug regime versions.

The functional forms of the interfacial drag models are developed by considering simplified one-dimensional two-phase flows. For these special cases, various terms in the momentum conservation equations are negligible permitting the interfacial drag closure term to be somewhat decoupled. Simplifying approximations are made to make the development tractable. The resulting functional form is assumed to apply under more general circumstances. Values for parameters in the model are derived from experimental data taken under less restrictive conditions.

4) Axial, dispersed two-phase flow (bubbly \& slug regimes): The interfacial drag force per unit volume given by equation (2.5.5.2-13) contains two elements, both of which are important in a dispersed two-phase flow. The time/space averaged version of the first term in definition $(2.5 .5 .2-13)$ is preferred for the dispersed regime interfacial drag model (see equation (2.5.5.2-25)): 


$$
-\nless \underline{\bar{\tau}_{g}} \cdot \sum_{j} \ell_{j}^{-1}\left[n_{g}\right]_{j} \ngtr=\nless \bar{\tau}_{g}^{i} \cdot \frac{1}{T} \nabla T \alpha_{g} \downarrow=\Varangle \underline{\underline{\tau}} \cdot \nabla \alpha \ngtr
$$

The latter equality was obtained by abbreviating the notation and assuming the time averaging interval is constant. A functional form for the above term has been derived by Ishii and Mishima (1984). Their analysis is summarized below and repeated in detail in Flach (1992). To deduce the proper form they consider an axially uniform dispersed twophase flow in a circular pipe. The axial ( $z$ ) component of the vector given by (2.5.5.2-29) is

$$
\varangle \bar{\tau}_{r 2}^{i} \frac{\partial \alpha}{\partial r} \triangleright
$$

Power law variations in the void fraction and shear stress profiles are postulated as

$$
\begin{aligned}
& \frac{\alpha}{\alpha_{0}}=1-\left(\frac{r}{r_{w}}\right)^{n} \\
& \bar{\tau}_{r z}^{i}=\tau_{w}\left(\frac{r}{r_{w}}\right)^{m}
\end{aligned}
$$

where $r_{w}$ is the pipe radius, $\tau_{w}$ is the wall shear stress and $n$ and $m$ are unspecified for the moment. As shown in Flach (1992), the integration indicated by the spatial averaging operator in term $(2.5 .5 .2-30)$ can readily be performed yielding

$$
\nless \bar{\tau}_{\mathrm{rz}} \mathrm{i} \frac{\partial \alpha}{\partial \mathrm{r}} \downarrow=\frac{\mathrm{n}+2}{\mathrm{n}+\mathrm{m}+1} \nless \alpha \triangleright \mathrm{F}_{\mathrm{w}}^{\mathrm{z}} \equiv \mathrm{C}_{\tau} \alpha \mathrm{F}_{\mathrm{w}}^{\mathrm{z}} \equiv \mathrm{F}_{\mathrm{i}}^{\mathrm{wz}}
$$

Ishii and Mishima state that $\mathrm{C}_{\tau}$ is expected to close to 1 . The statement is reasonable for reasonable choices of $\mathbf{n}$ and $\mathrm{m}$, although most choices yield a value consistently less than 1 (see Flach (1992)). Term (2.5.5.2-33) is the wall drag induced component of the axial interfacial drag denoted by $F_{i}{ }^{w 2}$ for brevity.

Using expression (2.5.5.2-33) the axial gas and liquid momentum balances for onedimensional, steady, axially uniform flow and fully wetted wall become

$$
\begin{gathered}
0=-\alpha \frac{d P}{d z}+\alpha \rho_{g} g^{z}-C_{\tau} \alpha F_{w}^{z}+M_{i}^{d z} \\
0=-(1-\alpha) \frac{d P}{d z}+(1-\alpha) \rho_{f} g^{z}-\left(1-C_{\tau} \alpha\right) F_{w}^{z}-M_{i}^{d z}
\end{gathered}
$$

The mixture momentum balance is the sum of the phasic conservation equations:

$$
0=-\frac{d P}{d z}+\left[\alpha p_{g}+(1-\alpha) \rho_{f}\right] g^{z}-F_{w}^{z}
$$


Another particularly useful auxiliary equation is obtained by eliminating pressure gradient between the gas and mixture momentum balances. The result is

$$
M_{i}^{\mathrm{dz}}=\alpha(1-\alpha) \Delta \rho g^{2}-\left(1-\mathrm{C}_{\tau}\right) \alpha \mathrm{F}_{\mathrm{w}}^{2}
$$

Recalling definition (2.5.5.2-5), the term $\mathrm{M}_{\mathrm{i}}^{\mathrm{dz}}$ accounts for localized perturbations from the average interfacial shear stress. For no liquid flow $\left(F_{w}^{z}=0\right)$ or $C_{\tau}=1$ the secend term on the r.h.s. of (2.5.5.2-37) disappears and the 'local' interfacial drag $\mathrm{M}_{\mathrm{i}}^{\mathrm{dz}}$ is balanced by a buoyancy force.

The local interfacial drag $\mathrm{M}_{\mathrm{i}}^{\mathrm{dz}}$ is postulated to be proportional to an average relative velocity between the phases. An interfacial drag coefficient can then be defined by

$$
M_{i}^{d z} \equiv-C_{i}^{d z}\left|u_{r}\right| u_{r}
$$

As shown in Flach (1992), if the drift velocity $u_{g j}$ varies weakly with position (a good assumption for a dispersed regime) the average relative velocity $\downarrow u_{r} \downarrow$ is well approximated by

$$
u_{r}=\frac{\varangle u_{g j} b_{g}}{\downarrow 1-\alpha \downarrow}
$$

Combining equations $(2.5 .5 .2-37),(2.5 .5 .2-38)$ and $(2.5 .5 .2-39)$ yields

$$
C_{i}^{d z}=-\frac{(1-\alpha)^{2}}{\left|u_{g j}\right| u_{g j}}\left[\alpha(1-\alpha) \Delta \rho g^{z}-\left(1-C_{\tau}\right) \alpha F_{w}^{z}\right]
$$

For no liquid flow or $\mathrm{C}_{\tau}=1$

$$
C_{i}^{d z}=-\frac{\alpha(1-\alpha)^{3} \Delta \rho g^{z}}{\left|u_{g j}\right| u_{g j}}
$$

The drift velocity is negative valued so $C_{i}^{d z}$ is a positive coefficient. Data correlations of the drift velocity are available for dispersed flow regimes. Equation (2.5.5.2-41) relates the local interfacial drag coefficient to these correlations.

The relative velocity must be expressed in terms of the mass-weighted phasic velocities $\Varangle u_{b} p$ and $\$ u_{f} p_{f}$ which are two of the primary variables computed by FLOWTRAN-TF. Because pressure and temperature are constant throughout a crosssection of the flow, the phasic densities are as well and the mass-weighted and volume fraction-weighted velocities are essentially identical. As Flach (1992) shows drift-flux model concepts can be used to relate the average relative velocity to the volume fractionweighted velocities as

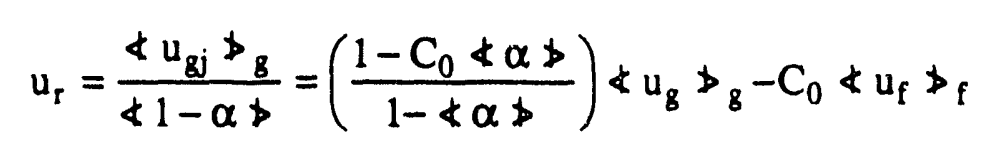


An important observation from Flach (1992) is that if $C_{\tau}=1$ the overall momentum balance satisfies the drift-flux equation

$$
\nless u_{g} \triangleright_{g}=C_{0} \nless j \ngtr+\nless u_{g j} \triangleright_{g}
$$

Because the drift-flux model has proved very successful in modeling dispersed two-phase flows, a clear motivation for choosing $C_{\tau}=1$ has emerged in addition to the Ishii and Mishima's approximate power law profile analysis. So that the interfacial drag model for dispersed two-phase flow will reduce to the drift-flux model for one-dimensiona!, steady, axially uniform flow (negligible inertial terms) and completely wetted wall, this choice is now made. The choice has a second benefit in that local interfacial drag coefficient is now decoupled from the wall drag model; this simplifies the model development process. Although Ishii and Mishima's work indicates $C_{\tau}$ should be somewhat iess than one, the analysis is not sufficiently rigorous to require $C_{\tau}<1$ over $C_{\tau}=1$.

The momentum balances and interfacial drag model become

$$
\begin{gathered}
0=-\alpha \frac{d P}{d z}+\alpha \rho_{g} g^{z}-\alpha F_{w}^{z}-F_{i}^{d z} \\
0=-(1-\alpha) \frac{d P}{d z}+(1-\alpha) \rho_{f} g^{z}-(1-\alpha) F_{w}^{z}+F_{i}^{d z}
\end{gathered}
$$

where

$$
\begin{gathered}
\mathrm{F}_{\mathrm{i}}^{\mathrm{dz}}=-\frac{\left.\alpha_{1} 1-\alpha\right)^{3} \Delta \rho g^{z}}{\left|\mathrm{u}_{\mathrm{gj}}\right| \mathrm{u}_{\mathrm{gj}}}\left|\left(\frac{1-\mathrm{C}_{0} \alpha}{1-\alpha}\right) \mathrm{u}_{\mathrm{g}}-\mathrm{C}_{0} \mathrm{u}_{\mathrm{f}}\right|\left[\left(\frac{1-\mathrm{C}_{0} \alpha}{1-\alpha}\right) \mathrm{u}_{\mathrm{g}}-\mathrm{C}_{0} \mathrm{u}_{\mathrm{f}}\right] \\
\mathrm{F}_{\mathrm{i}}^{\mathrm{dz}} \equiv-\mathrm{M}_{\mathrm{i}}^{\mathrm{dz}}
\end{gathered}
$$

The local drag nomenclature has been changed above so that a positive value of $\mathrm{F}_{\mathrm{i}}^{\mathrm{dz}}$ corresponds to a drag on the gas phase (momentum sink). Note that the total interfacial drag $F_{i}^{z}$ is the sum of $F_{i}^{w z}$ and $F_{i}^{d z}$ defined by equations (2.5.5.2-33) and (2.5.5.2-46), respectively.

As Putney (1988) points out, the total interfacial drag is comprised of three effects. Even though only the liquid phase is assumed to contact the wall, the dispersed gas phase experiences the wall friction indirectly through an interfacial drag equal to $\alpha \mathrm{F}_{w}^{2}$ because both phases experience the same average stress field at their interface. In effect the wall drag has been repartitioned between the phases according to void fraction. The two remaining effects involve the local drag term given by (2.5.5.2-46). Locally there is slip, and therefore interfacial drag between the phases, due to the buoyancy of the gas phase. This effect is captured by the leading coefficient of (2.5.5.2-46) involving essentially the local drift velocity of the gas phase. An additional effect arises because the gas phase is generaliy not uniformly dispersed within the liquid phase $\left(C_{0} \neq 1\right)$. The average relative velocity therefore depends on the phase distribution.

Drift velocity correlations are needed for the bubbly and slug regimes so that the interfacial drag coefficient given by $(25.5 .2-41)$ may be evaluated. For now only the functional 
forms of the drift velocity correlations are established. Recommended parameter values are given subsequently in subsection 8 after SRS data for ribbed thin annular geometries have been presented. Four geometries are encountered in present and anticipated FLOWTRAN-TF applications: circular, thin annular, ribbed thin annular, and thin rectangular. Drift velocity models for the bubbly and slug regimes are needed for each of these geometries.

The bubbly flow regime in FLOWTRAN-TF is assumed to be best modeled as a churnturbulent regime in the sense used by Ishii (1977). This regime is commonly observed in bubbly flows and the drift velocity is modeled with expressions of the form (Ishii, 1977)

$$
u_{g j}=-K_{b}\left[\frac{\Delta \rho g \sigma}{\rho_{\mathrm{f}}^{2}}\right]^{1 / 4}
$$

Equation (2.5.5.2-48) is geometry iridependent and is assumed to be valid for each of the four geometries of interest. The leading coefficient varies somewhat in the literature with $\mathrm{K}_{\mathrm{b}}=\sqrt{2}$ being a typical value (Ishii, 1977).

For slug flow in a circular pipe the drift velocity is correlated as

$$
u_{g j}=-K_{s}\left[\frac{\Delta \rho g D_{c}}{\rho_{f}}\right]^{1 / 2} \equiv-K_{s}\left[g D_{c}\right]^{1 / 2}
$$

where the characteristic dimension $D_{c}$ is the pipe diameter $D$ and $K_{s} \approx 0.35$ (Ishii, 1977). For other geometries the characteristic dimension differs but the leading coefficient typically remains near 0.35 . Experimental data are available for thin annular and thin rectangular channels but not for a ribbed annular cross-section. The correct characteristic dimension for a ribbed annular channel is assumed to be related to those for an unribbed thin annulus and a thin $90^{\circ}$ annular sector (perfectly sealing ribs) which may be approximated by a thin rectangular channel.

For a thin annulus with $D_{i} / D_{0} \rightarrow 1$, Griffith $(1964$, Fig. 2) recommends

$$
u_{\mathrm{gj}} \cong-0.43\left[\mathrm{gD}_{\mathrm{o}}\right]^{1 / 2}
$$

and Sadatomi et al. (1982, Table 3) correlated data for a $15 \mathrm{~mm} \times 30 \mathrm{~mm}$ annular channel as

$$
u_{g j}=-0.32 \sqrt{g\left(D_{i}+D_{o}\right)}
$$

Barnea and Shemer (1986) considered the data of Griffith (1964) and Sadatomi (1982) and suggest using (2.5.5.2-49) with

$$
\begin{gathered}
D_{c}=\frac{D_{0}}{4}\left[\pi+1+\frac{D_{i}}{D_{0}}(\pi-1)\right] \equiv \frac{\pi}{2} D_{0} \\
K_{s}=0.35
\end{gathered}
$$


A recent analytical and experimental work of Kelessidis and Dukler (1990) for a 2" $\times 3$ " $(50.8 \mathrm{~mm} \times 76.2 \mathrm{~mm}$ ) annulus leads to (see Flach (1992))

$$
u_{g j}=-0.37\left[g \frac{\pi \bar{D}}{2}\right]^{1 / 2}
$$

where $\bar{D}$ is the average of the inner and outer diameters. For thin channels $D_{0} \approx \bar{D}$ so all four correlations show essentially a square root of average diameter dependence. Griffith's result can be recast as

$$
u_{g j} \equiv-0.34\left[g \frac{\pi \bar{D}}{2}\right]^{1 / 2}
$$

and Sadatomi et al.'s correlation is equivalent to

$$
u_{g j} \cong-0.36\left[g \frac{\pi \bar{D}}{2}\right]^{1 / 2}
$$

Hence, all three correlations are similar and suggest

$$
D_{c}=\frac{\pi \bar{D}}{2}
$$

for a characteristic dimension with $0.34 \leq \mathrm{K}_{\mathrm{s}} \leq 0.37$.

For a thin rectangular channel Griffith (1964, Fig. 2) proposes

$$
\mathrm{u}_{\mathrm{gj}} \cong-0.24[\mathrm{ga}]^{1 / 2}
$$

Sadatomi et al. (1982, Table 3) present bubble rise velocity data for a number of rectangular channels and correlate in terms of the equi-periphery diameter, perimeter divided by $\pi$. Table 2.5.5.2-I summarizes their and Griffith's data for thin rectangular channels ('thin' defined to be b/a $\leq 1 / 2$ ). Barnea and Shemer (1986), again considering the data of Griffith and Sadatomi et al., propose

$$
\begin{gathered}
D_{c}=\frac{a+b}{2} \equiv \frac{a}{2} \\
K_{s}=0.35
\end{gathered}
$$

Counter-Current Flow Limitation (CCFL) experiments and analyses involving thin rectangular channels can also be used to ascertain the proper characteristic dimension. CCFL data correlations for this geometry are typically of the Wallis form (\$2.5.5.1)

$$
\left|j_{\mathrm{B}}^{*}\right|^{1 / 2}+m\left|j_{\mathrm{f}}^{*}\right|^{1 / 2}=\mathrm{C}
$$


where

$$
j_{k}^{*} \equiv j_{k}\left[\frac{\rho_{k}}{\Delta \rho g D_{c}}\right]^{1 / 2}
$$

The characteristic dimension in (2.5.5.2-58) is assumed to the same as that in the slug regime drift velocity correlation. Osakabe and Kawasaki (1989) and Ruggles (1990) chose

$$
\mathrm{D}_{\mathrm{c}}=\mathrm{a}
$$

to successfully correlate experimental CCFL data using the above Wallis form. Mishima and Nishihara (1985) and Cheng (1990) developed analytical models of the CCFL line and deduced that the correct characteristic dimension is

$$
D_{c}=2 a
$$

Observe that all of the drift velocity and CCFL correlations indicate the characteristic dimension should be proportional to the channel width a for thin channels $(b / a \rightarrow 0)$. For FLOWTRAN-TF modeling, the slug regime characteristic dimension is chosen as $2 \mathrm{a}$ on the strength of the analyses of Mishima and Nishihara (1985) and Cheng (1990) and because when converted to the $90^{\circ}$ sector geometry equation $(2.5 .5 .2-60)$ is identical to (2.5.5.2-54) for an annular passage. When $2 \mathrm{a}$ is chosen as the characteristic dimension, the coefficient values for the Griffith and Sadatomi et al. data range from 0.18 to 0.23 as shown in Table 2.5.5.2-I.

Both a thin annular channel and thin $90^{\circ}$ annular sector (assumed equivalent to a thin rectangular channel) can be associated with the same characteristic dimension. Assuming a thin ribbed annular channel has the same characteristic dimension yields

$$
\mathrm{D}_{\mathrm{c}}=2 \mathrm{a}=\frac{\pi \overline{\mathrm{D}}}{2}
$$

for a ribbed annular channel. Table 2.5.5.2-II summarizes the drift-velocity functional forms recommended for the slug regime. 
Table 2.5.5.2-I Data of Sadatomi et al. (1982) and Griffith (1964) for thin rectangular channels (b/a $\leq 1 / 2)$ (Sadatomi et al., 1982, Table 3).

\begin{tabular}{|c|c|c|c|c|}
\hline $\begin{array}{l}\text { Dimensions } \\
\begin{array}{c}(\mathrm{mm}) \\
\mathrm{a} b \\
a=\text { width } \\
b=\text { gap }\end{array}\end{array}$ & Data Source & $\begin{array}{c}\text { Rise Velocity } \\
u(\mathrm{~m} / \mathrm{s})\end{array}$ & $\begin{array}{c}\text { Based on } \\
\text { Equi- } \\
\text { Periphery } \\
\text { Diameter } \\
\mathrm{K}_{\mathrm{s}} \equiv \frac{\mathrm{u}}{\sqrt{\mathrm{g} \frac{\mathrm{P}}{\pi}}}\end{array}$ & $\begin{array}{l}\text { Based on } \\
\text { Twice Width } \\
\mathrm{K}_{\mathrm{s}} \equiv \frac{\mathrm{u}}{\sqrt{\mathrm{g} 2 \mathrm{a}}}\end{array}$ \\
\hline $7 \times 20.6$ & Sadatomi et al. & 0.142 & 0.34 & 0.22 \\
\hline $7 \times 50$ & Sadatomi et al. & 0.228 & 0.38 & 0.23 \\
\hline $10 \times 50$ & Sadatomi et al. & 0.204 & 0.33 & 0.21 \\
\hline $17 \times 50$ & Sadatomi et al. & 0.216 & 0.33 & 0.22 \\
\hline $30 \times 120$ & Sadatomi et al. & 0.336 & 0.35 & 0.22 \\
\hline $8.9 \times 37.8$ & Griffith & 0.167 & 0.31 & 0.19 \\
\hline $11 \times 133$ & Griffith & 0.292 & 0.31 & 0.18 \\
\hline
\end{tabular}

Table 2.5.5.2-II Drift-velocity functional forms for selected geometries.

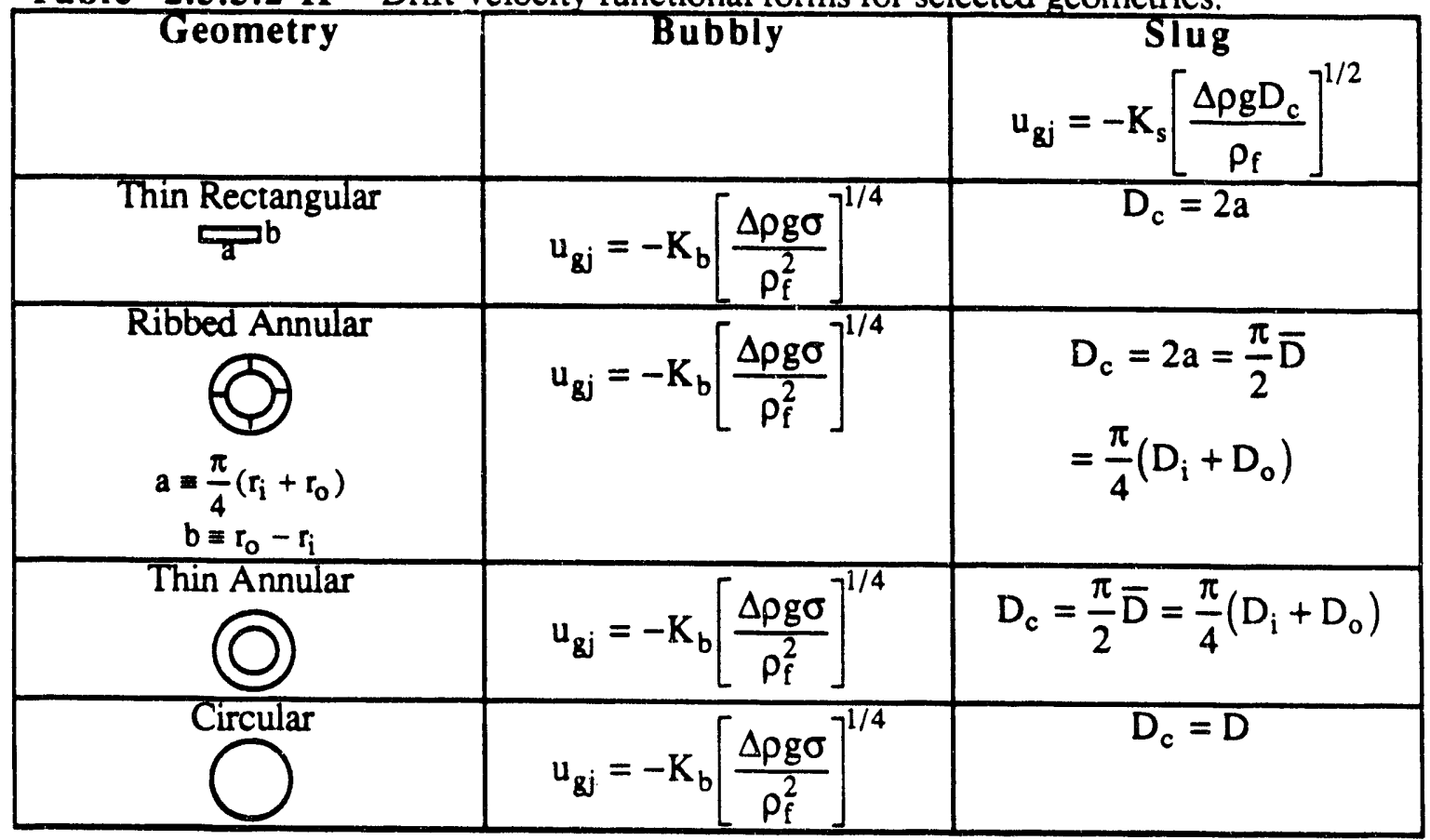

5) Axial, separated two-phase flow (annular regime): The general interfacial drag term is given by equation (2.5.5.2-13). Both terms on the r.h.s. are important to dispersed flow regime modeling as discussed above. The proper functional forms of interfacial drag per unit volume for an annular two-phase flow are deduced by again considering a one-dimensional, axially uniform flow through a circular pipe. The liquid phase is assumed to form an annular film which completely wets the wall. The gas phase occupies the circular core. Because of axial and azimuthal uniformity in the interfacial pressure and shear stress 


$$
\begin{aligned}
& \Delta \tilde{\mathrm{P}}_{\mathrm{i}}^{\mathrm{d}}=0 \\
& \Delta \tilde{\boldsymbol{\tau}}_{\mathrm{i}}=0
\end{aligned}
$$

and therefore

$$
\mathbf{M}_{\mathrm{i}}^{\mathbf{d}}=0
$$

That is, the total interfacial shear stress is comprised only of an average shear contribution given by the first term of equation (2.5.5.2-13), which for separated flow is written most conveniently in the space and then time averaged nomenclature:

$$
F_{i} \equiv-\tau_{i} \cdot S_{i}^{\prime \prime \prime}=-\overline{\left\langle\tau_{g}\right\rangle_{i} \cdot S_{k_{i}}^{\prime \prime \prime}}=-\overline{\frac{1}{A_{i}} \int_{A_{i}} \tau_{g_{i}} d S \cdot \frac{1}{V} \int_{A_{i}} n_{k} d S}
$$

For the assumed geometry and an $(r, z)$ coordinate system

$$
S_{i}^{\prime \prime \prime}=\frac{1}{V} \int_{A_{i}} n_{k} d S=\frac{1}{V} \int_{A_{i}}\left[\begin{array}{l}
1 \\
0
\end{array}\right] d S=\left[\begin{array}{l}
A_{i} \\
V \\
0
\end{array}\right]
$$

The shear stress tensor was assumed to be spatially constant above so

$$
\left\langle\underline{\tau_{g}}\right\rangle_{i}=\frac{1}{A_{i}} \int_{A_{i}} \tau_{g_{i}} d S=\frac{1}{A_{i}}\left[\begin{array}{ll}
\tau_{r z} & \tau_{r z} \\
\tau_{z x} & \tau_{z z}
\end{array}\right] A_{i}=\left[\begin{array}{cc}
0 & \tau_{r z} \\
\tau_{r z} & 0
\end{array}\right]
$$

If the interfacial area and stress are constant with time, the interfacial drag force per unit volume in the axial direction becomes

$$
F_{i}^{2}=-\frac{\overline{\tau_{r z} A_{i}}}{V}=-\frac{\tau_{r z} A_{i}}{V}
$$

In analogy with single-phase gas flow in a pipe, the interfacial shear stress is modeled as

$$
\begin{aligned}
& -\tau_{r z}=f_{i} \frac{1}{2} \rho_{g}\left|u_{r}\right| u_{r}
\end{aligned}
$$

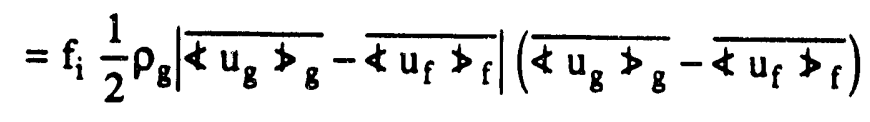

$$
\begin{aligned}
& =f_{i} \frac{1}{2} \rho_{g}\left|u_{g}-u_{f}\right|\left(u_{g}-u_{f}\right)
\end{aligned}
$$
where $f_{i}$ is an interfacial friction factor. The interfacial drag force per unit volume for
separated flow becomes 


\begin{tabular}{|l|ll|l|}
\hline $2 / 15 / 93$ & WSRC-TR-92-532 Rev. 0 & FLOWTRAN-TF Software Design & Page 233 \\
\hline
\end{tabular}

$$
F_{i}^{z}=\frac{A_{i}}{V} f_{i} \frac{1}{2} \rho_{g}\left|u_{g}-u_{f}\right|\left(u_{g}-u_{f}\right)
$$

Models for the annular regime interfacial friction factor are now developed.

The annular flow regime is generally characterized by relatively low interfacial momentum coupling $\left(\mathrm{f}_{\mathrm{i}}\right)$ compared to dispersed flow regimes. An exception is counter-current annular flow at Counter-Current Flow Limitation (CCFL) conditions for which the interfacial momentum coupling is orders of magnitude greater. The large difference in non-CCFL and CCFL interfacial coupling motivates the two-part approach taken here. First a separated flow interfacial friction factor model applicable to non-CCFL conditions is presented. Then modifications are introduced to handle CCFL conditions as well.

The work of Asali, Hanratty and Andreussi (1985) is the basis for the annular flow regime interfacial drag model for non-CCFL flow. Based on annular air-water dowr.flow data involving no liquid entrainment and circular pipe sizes from 2.4 to $6.35 \mathrm{~cm}$ diameter, Asali et al. (1985) recommend the following interfacial shear model

$$
-\tau_{i}=f_{i} \frac{1}{2} \rho_{g}\left|u_{g}\right| u_{g}
$$

where

$$
\begin{aligned}
& \frac{\mathrm{f}_{\mathrm{i}}}{\mathrm{f}_{\mathrm{s}}}-1=\left\{\begin{array}{cc}
0.045\left(\mathrm{~m}_{\mathrm{g}}^{+}-5.9\right) & \mathrm{m}_{\mathrm{g}}^{+} \geq 5.9 \\
0 & \mathrm{~m}_{\mathrm{g}}^{+}<5.9
\end{array}\right. \\
& \mathrm{f}_{\mathrm{s}}=0.046 \operatorname{Re}_{\mathrm{g}}^{-0.20}, \text { gas-phase friction factor for a smooth pipe } \\
& \mathrm{m}_{\mathrm{g}}^{+} \equiv \frac{\mathrm{m}}{\mathrm{v}_{\mathrm{g}}}\left(\frac{-\tau_{\mathrm{i}}}{\rho_{\mathrm{g}}}\right)^{1 / 2} \text {, non-dimensional film thickness } \\
& \operatorname{Re}_{\mathrm{g}} \equiv \frac{4 \mathrm{~W}_{\mathrm{g}}}{\mu_{\mathrm{g}} \mathrm{P}}, \text { Reynolds number assuming gas-phase occupies whole channel } \\
& \mathrm{m} \equiv \text { film thickness } \\
& \mathrm{W}_{\mathrm{g}} \equiv \text { gas-phase mass flowrate }
\end{aligned}
$$

Asali et al. (1985) state that the interfacial shear is approximately independent of the pipe size and correlate the data in terms of film thickness non-dimensionalized with respect to the friction length instead of pipe diameter (see definition (2.5.5.2-74)). In applying these above results to FLOWTRAN-TF applications, any effects due to channel shape are neglected in addition to channel size effects.

The data correlated by Asali et al. (1985) presumably involved co-current flow with the gas being driven by a favorable pressure gradient. The gas phase velocities are assumed to be 
relatively large by comparison to FLOWTRAN-TF applications which typically involve a falling film annular flow with the gas phase being entrained by the liquid (adverse pressure gradient). For the Asali et al. (1985) data the gas phasic velocity probably does not differ greatly from the relative phasic velocity, so either velocity could have been chosen; certainly $u_{g}$ is more convenient. With typical FLOWTRAN-TF applications the phasic velocities are relatively low so $u_{g}$ and $u_{r}$ differ significantly. Also note that interfacial shear model given by $(2.5 .5 .2-71)$ does not give the correct sign for a cocurrent downflow with an adverse pressure gradient. Therefore, equation $(2.5 .5 .2-69)$ is chosen over equation (2.5.5.2-71) in FLOWTRAN-TF. Also, the gas phasic velocity is replaced by the absolute value of the relative velocity in the definition of $\mathrm{Re}_{\mathrm{g}}$. To adapt the model to non-circular cross-sections the pipe diameter is replaced with the equivalent circular pipe diameter defined by

$$
D_{e} \equiv K_{h} D_{h}
$$

where $K_{h}$ is a hydraulic diameter correction factor.

As shown in Flach (1992), the resulting model and computational procedure become:

- Compute

$$
\operatorname{Re}_{g}=\frac{\rho_{g}\left|u_{r}\right| D_{e}}{\mu_{g}}
$$

- Compute

$$
R e_{m}=\frac{\rho_{g}\left|u_{r}\right| m}{\mu_{g}}
$$

- Compute

$$
f_{s}=0.046 R e_{g}^{-0.20}
$$

- Compute $\left(\mathfrak{f}_{i}^{1 / 2}\right)$ from the quadratic

$$
\left(f_{i}^{1 / 2}\right)^{2}-0.03182 \operatorname{Re}_{m} f_{s}\left(f_{i}^{1 / 2}\right)-0.7345 f_{s}=0
$$

- Compute

$$
\mathrm{m}_{\mathrm{g}}^{+}=\frac{\operatorname{Re}_{\mathrm{m}}}{\sqrt{2}} \mathrm{f}_{\mathrm{i}}^{1 / 2}
$$

- Compute

$$
f_{i}=f_{s} \cdot \max \left[1, \quad 1+0.045\left(m_{s}^{+}-5.9\right)\right]
$$

In summary, the momentum balances and interfacial drag model for separated flow under non-CCFL conditions become

$$
\begin{gathered}
0=-\alpha \frac{d P}{d z}+\alpha \rho_{g} g^{z}-F_{i}^{z} \\
0=-(1-\alpha) \frac{d P}{d z}+(1-\alpha) \rho_{f} g^{z}-F_{w}^{z}+F_{i}^{z}
\end{gathered}
$$




\begin{tabular}{|l|lll|l|}
\hline $2 / 15 / 93$ & WSRC.TR-92-532 Rev.0 & FLOWTRAN-TF Software Design & Page 235 \\
\hline
\end{tabular}

where

$$
\begin{gathered}
F_{i}^{2}=C_{i}^{2}\left|u_{g}-u_{f}\right|\left(u_{g}-u_{f}\right) \\
C_{i}^{2}=\frac{A_{i}}{V} f_{i} \frac{1}{2} \rho_{g}
\end{gathered}
$$

and the interfacial friction factor $f_{i}$ is defined by equations $(2.5 .5 .2-77-82)$. The wall is assumed to be completely wetted in the above expressions.

Figure 2.5.5.2-1 qualitatively illustrates the two-phase flow conditions possible under steady-state conditions. The CCFL line is characterized by a sudden increase in the interfacial drag coefficient not accounted for in the preceding drag model. Section 2.5.5.1 presents a detailed discussion of the CCFL data correlations applicable to FLOWTRAN-TF applications. The appropriate form is shown to be the Wallis type

$$
\left|\mathrm{j}_{\mathrm{B}}^{*}\right|^{1 / 2}+\mathrm{m}\left|\mathrm{j}_{\mathrm{f}}^{*}\right|^{1 / 2}=\mathrm{C}
$$

where

$$
j_{k} \equiv j_{k}\left(\frac{\rho_{k}}{\Delta \rho g D_{c}}\right)^{1 / 2}
$$

Ohkawa and Lahey (1980) show how to make the drift-flux model

$$
u_{g}=C_{0}(\alpha) j+u_{g j}(\alpha)
$$

consistent with a CCFL correlation of the Kutateladze form. Note that the drift-flux parameters may be at most functions of void fraction. For the Wallis form and a downward directed z-coordinate, Ohkawa and Lahey's results become

$$
-u_{g j}=C^{2} \sqrt{\frac{\Delta \rho g D_{c}}{\rho_{f}}} \cdot \frac{C_{0}}{m^{2}+\frac{C_{0} \alpha}{1-C_{0} \alpha}\left(\frac{\rho_{g}}{\rho_{f}}\right)^{1 / 2}}
$$

That is, if equation (2.5.5.2-90) is imposed as a constraint on the drift-flux parameters $\mathrm{C}_{0}$ and $u_{\mathrm{gij}}$, then the locus of all the constant void fraction operating lines will trace out the CCFL line as shown in Figure 2.5.5.2-1.

Recall from the axial, dispersed interfacial drag modeling development in subsection 4 that the two-fluid model exactly consistent with the drift-flux model given by $(2.5 .5 .2-89)$ is

$$
0=-\alpha \frac{d P}{d z}+\alpha \rho_{g} g^{z}-\alpha F_{w}^{z}-F_{i}^{d z}
$$




$$
0=-(1-\alpha) \frac{d P}{d z}+(1-\alpha) \rho_{f} g^{z}-(1-\alpha) F_{w}^{z}+F_{i}^{d z}
$$

where

$$
\begin{gathered}
F_{i}^{d z}=C_{i}^{d z}\left|\left(\frac{1-C_{0} \alpha}{1-\alpha}\right) u_{g}-C_{0} u_{f}\right|\left[\left(\frac{1-C_{0} \alpha}{1-\alpha}\right) u_{g}-C_{0} u_{f}\right] \\
C_{i}^{d z}=-\frac{\alpha(1-\alpha)^{3} \Delta \rho g^{2}}{\left|u_{g j}\right| u_{g j}}
\end{gathered}
$$

Equations (2.5.5.2-91-94) can be made more similar to equations $(2.5 .5 .2-83-86)$ by setting $\mathrm{C}_{0}=1$. If equations (2.5.5.2-91) and (2.5.5.2-92) did not contain the wall drag partitioning terms $\alpha \mathrm{F}_{w}$ and $(1-\alpha) \mathrm{F}_{w}$ then the correct interfacial drag coefficient under CCFL conditions would be given by equation (2.5.5.2-94) using the drift velocity computed from equation (2.5.5.2-90) with $\mathrm{C}_{0}=1$ instead of equation (2.5.5.2-86). Of course, the wall drag partitioning terms are present so equations (2.5.5.2-90) and (2.5.5.2-94) cannot be used directly.

Fortunately, the interfacial drag coefficient defined by equations (2.5.5.2-90) and (2.5.5.2-94) with $\mathrm{C}_{0}=1$, when used in conjunction with equations $(2.5 .5 .2-83-85)$ instead of equations (2.5.5.2-91-93), overpredicts the interfacial coupling at CCFL as will now be shown. Let the coefficient derived from equations (2.5.5.2-94) and (2.5.5.2-90) with $\mathrm{C}_{0}=1$ be denoted $\mathrm{C}_{\mathrm{i}} \mathrm{CCFL}$. The gas momentum balance which $\mathrm{C}_{\mathrm{i}}^{\mathrm{CCF}}$ is based upon is

$$
0=-\alpha \frac{d P}{d z}+\alpha p_{g} g^{z}-\alpha F_{w}^{z}-F_{i}^{d z}
$$

but suppose it is used instead with the momentum balance

$$
\begin{array}{r}
0=-\alpha \frac{d P}{d z}+\alpha \rho_{g} g^{2}-F_{i}^{2} \\
-\quad++
\end{array}
$$

The sign of each term is given for a counter-current flow and a downward directed $z$ coordinate. If $\mathrm{C}_{\mathrm{i}}$ CCFL is used with equation (2.5.5.2-95) then the CCFL data correlation is satisfied. Equation (2.5.5.2-96) differs from equation (2.5.5.2-95) by a negative valued term. Because a negative valued term represents an upward directed force, the absence of such a term in equation (2.5.5.2-96) means the upward gas flow will be lower with equation (2.5.5.2-96) compared with equation (2.5.5.2-95), all other quantities being equal. The gravitational term in equations (2.5.5.2-95) and (2.5.5.2-96) is negligible. Interfacial shear is the only significant force retarding the upward flow of gas. Because equation (2.5.5.2-96) yields a lower upward gas flow than equation (2.5.5.2-95) which is consistent with the CCFL correlation, $\mathrm{C}_{i}{ }^{C C L}$ overpredicts the interfacial coupling at CCFL when used with equation (2.5.5.2-96).

The interfacial drag coefficient given by equation (2.5.5.2-86) and accompanying expressions underpredicts the interfacial coupling at CCFL. Therefore the correct 
interfacial drag coefficient at CCFL can be obtained by the following blending between the non-CCFL coefficient given by equation $(2.5 .5 .2-86)$ and $C_{i}{ }^{C C L}$ :

$$
C_{i}=(1-\omega) C_{i}^{\text {nonCCFL }}+\omega C_{i}^{C C F L}
$$

where $\omega$ is a weighting factor. If the flow is co-current up or down then $\omega=0$. For counter-current flow, the following quantity is computed at each current time step $n$ (see
equation (2.5.5.2-87))

$$
C^{n}=\left|j_{g}^{* n}\right|^{1 / 2}+m\left|j_{f}^{* n}\right|^{1 / 2}
$$

If $C^{n} \leq C$ then $\omega$ remains zero. However, if $C^{n}>C$ then the computed conditions at time step $n$ are beyond the counter-current flow limitation and $\omega$ is increased for the next time step to force the predicted solution below the CCFL line as shown in Figure 2.5.5.2-2. Numerical experimentation during the development of this logic indicated that the scheme produces a solution which oscillates with time about the CCFL line when boundary conditions producing CCFL conditions are imposed. While the $\omega$ logic does permit the solution at particular time steps to exceed the CCFL line, the perturbations are reasonably small compared to the uncertainty in the CCFL data correlation and the time-averaged response agrees even better with the chosen CCFL correlation. 


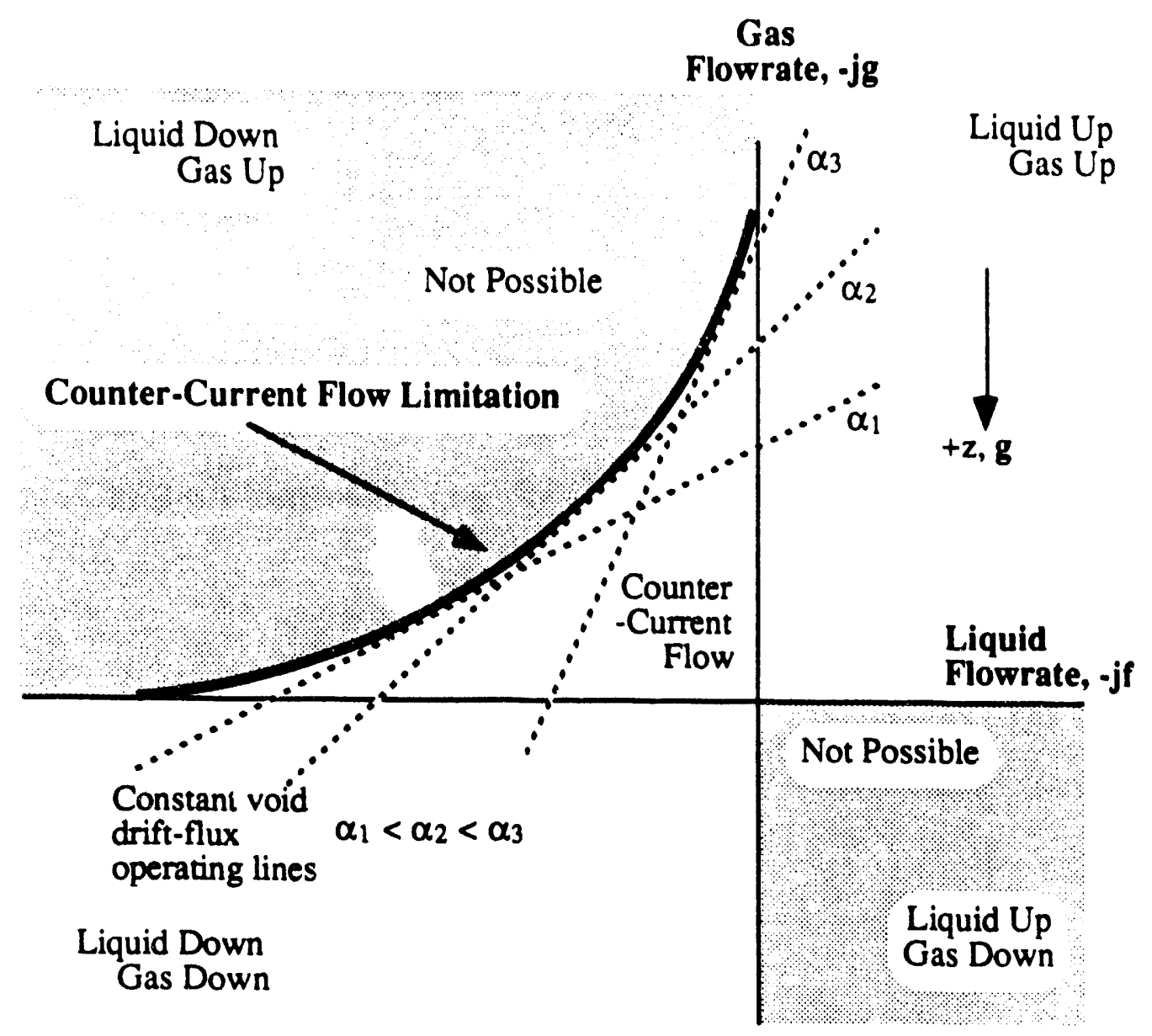

Figure 2.5.5.2-1 Possible steady-state two-phase flow operating points. 


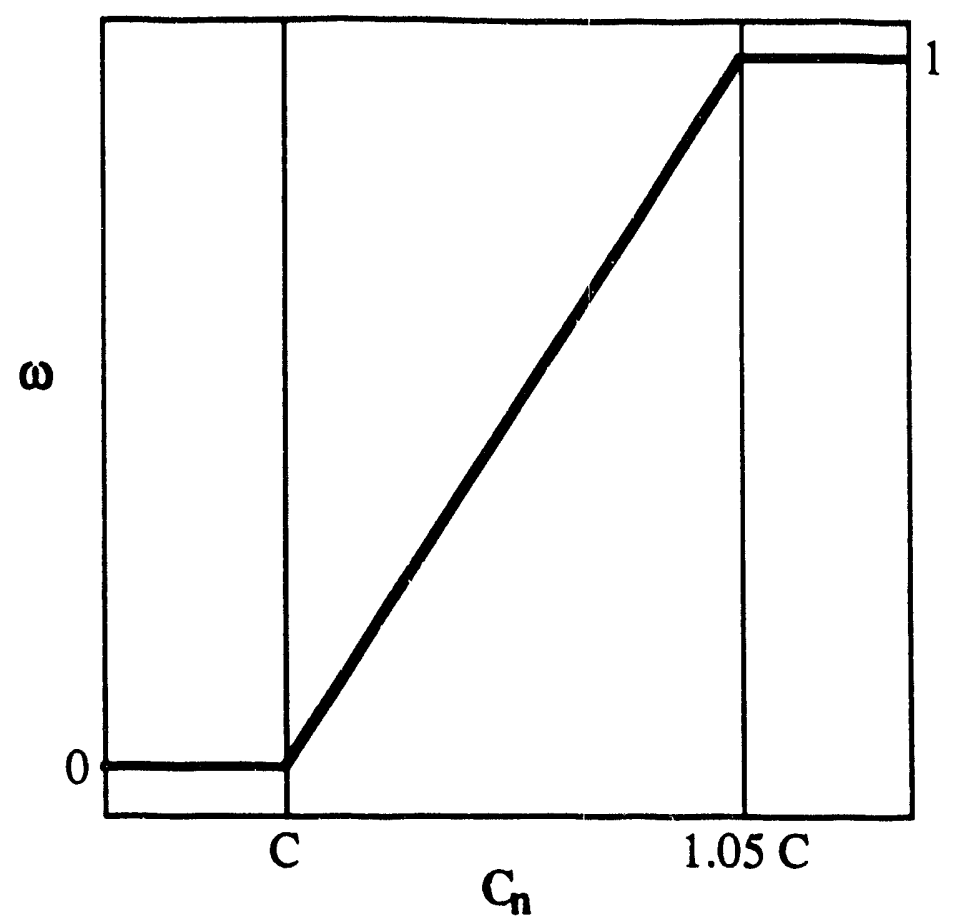

Figure 2.5.5.2-2 Logic for selecting the weighting factor between the non-CCFL and CCFL interfacial drag coefficients in the counter-current annular flow regime.

6) Flow regime transitions: The FLOWTRAN-TF flow regime map is based upon 3 fundamental flow regimes; bubbly, slug, and annular. From the fundamental regimes 5 two-phase flow regimes are constructed; bubbly, bubbly-slug, slug, slug-annular, and annular. Figure 2.5.5.2-3 illustrates the flow regime map logic. Interfacial drag per unit volume models have been established for the three fundamental flow regimes above. Now the above results are extended to the bubbly-slug and slug-annular transition regimes. All three elements of interfacial drag modeling (discussed above) require modifications as discussed in Flach (1992) and summarized below.

The modifications required to the local interfacial drag are deduced by performing separate force balances on the pair of fundamental regimes in question and then adding the results. The local drag force on the $m$ dispersed flow regime $(\mathrm{m}=$ bubbly or slug) is balanced by the buoyancy force:

$$
C_{D} A_{p} \frac{1}{2} \rho_{f} u_{r}^{2}=\left(\rho_{m i x}-\rho_{g}\right) v_{m g}
$$

where

$$
\begin{aligned}
& C_{D} \equiv \text { drag coefficient } \\
& A_{p} \equiv \text { projected frontal area } \\
& u_{r} \equiv \text { local relative velocity, } u_{g, m}-u_{f}
\end{aligned}
$$




$$
\mathrm{V}_{\mathrm{m}} \equiv \text { volume of } \mathrm{m} \text { dispersed phase }
$$

The local relative velocity is related to the drift velocity of the $\mathrm{m}$ flow regime by

$$
u_{r}=\frac{u_{g j \cdot m}}{(1-\alpha)}
$$

Eliminating $u_{r}$ between (2.5.5.2-99) and (2.5.5.2-100) and solving for $C_{D} A_{p}$ yields

$$
C_{D} A_{p}=\frac{2 \alpha_{m}(1-\alpha)^{3} \Delta \rho g}{\rho_{f} u_{g, m}^{2}} \cdot v
$$

and the drag force on the $\mathrm{m}$ dispersed regime per unit volume becomes

$$
\mathrm{F}_{\mathrm{im}}^{\mathrm{dz}}=\frac{\alpha_{\mathrm{m}}(1-\alpha)^{3} \Delta \rho g}{u_{\mathrm{g}, \mathrm{i} m}^{2}} \cdot\left|\mathrm{u}_{\mathrm{r}}\right| \mathrm{u}_{\mathrm{r}} \equiv C_{\mathrm{im}}^{\mathrm{dz}} \cdot\left|\mathrm{u}_{\mathrm{r}}\right| \mathrm{u}_{\mathrm{r}}
$$

From equations (2.5.5.2-85) and (2.5.5.2-86), the drag force per unit volume for the separated flow regime is

$$
F_{i a}^{d z}=\frac{A_{i a}}{V} f_{i} \frac{1}{2} \rho_{g} \cdot\left|u_{r}\right| u_{r} \equiv C_{i a}^{d z} \cdot\left|u_{r}\right| u_{r}
$$

where $A_{i a}$ is the fraction of the total area attributed to the annular regime. From equations $(2.5 .5 .2-102)$ and $(2.5 .5 .2-103)$ the bubbly-slug and slug-annular interfacial drag models are derived as

$$
F_{i}^{d z}=\left(C_{i b}^{d z}+C_{i s}^{d z}\right) \cdot\left|u_{r}\right| u_{r}=\left[\frac{\alpha_{b}(1-\alpha)^{3} \Delta \rho g}{u_{g j, b}^{2}}+\frac{\alpha_{s}(1-\alpha)^{3} \Delta \rho g}{u_{g j, s}^{2}}\right] \cdot\left|u_{r}\right| u_{r}
$$

and

$$
F_{i}^{d z}=\left(C_{i s}^{d z}+C_{i a}^{d z}\right) \cdot\left|u_{r}\right| u_{r}=\left[\frac{\alpha_{s}(1-\alpha)^{3} \Delta \rho g}{u_{g j, s}^{2}}+\frac{A_{i a}}{V} f_{i} \frac{1}{2} \rho_{g}\right] \cdot\left|u_{r}\right| u_{r}
$$

respectively, where

$\alpha_{m} \equiv$ volume of $m$ regime per total volume

$A_{\text {ia }} \equiv$ annular interfacial area

To evaluate the relative velocity in equations (2.5.5.2-104) and (2.5.5.2-105) the distribution coefficient in the bubbly-slug and slug-annular transition regimes is defined by 


$$
C_{0}=\left(\frac{\alpha_{b}}{\alpha}\right) C_{0, b}+\left(\frac{\alpha_{s}}{\alpha}\right) C_{0, s}
$$

and

$$
\mathrm{C}_{0}=\left(\frac{\alpha_{\mathrm{s}}}{\alpha}\right) \mathrm{C}_{0, \mathrm{~s}}+\left(\frac{\alpha_{\mathrm{a}}}{\alpha}\right) \mathrm{C}_{0, \mathrm{a}}
$$

respectively.

The dispersed fundamental flow regimes introduce a wall drag induced interfacial drag (recall definition (2.5.5.2-33))

$$
-F_{i}^{w z}=-\alpha F_{w}^{z}
$$

The separated (annular) flow regime does not introduce such a term, i.e., $F_{j}^{\text {wz }}=0$. In order to smoothly transition between the dispersed and separated interfacial drag models, the wall shear induced drag term is defined by

$$
F_{i}^{w z}=\left(1-\frac{\alpha_{a}}{\alpha}\right) \cdot \alpha F_{w}^{z}
$$

where $\alpha_{\downarrow} / \alpha$ is the fraction of the gas phase attributed to the annular regime. 


\section{FLOWTRAN-TF Flow Regime Map}

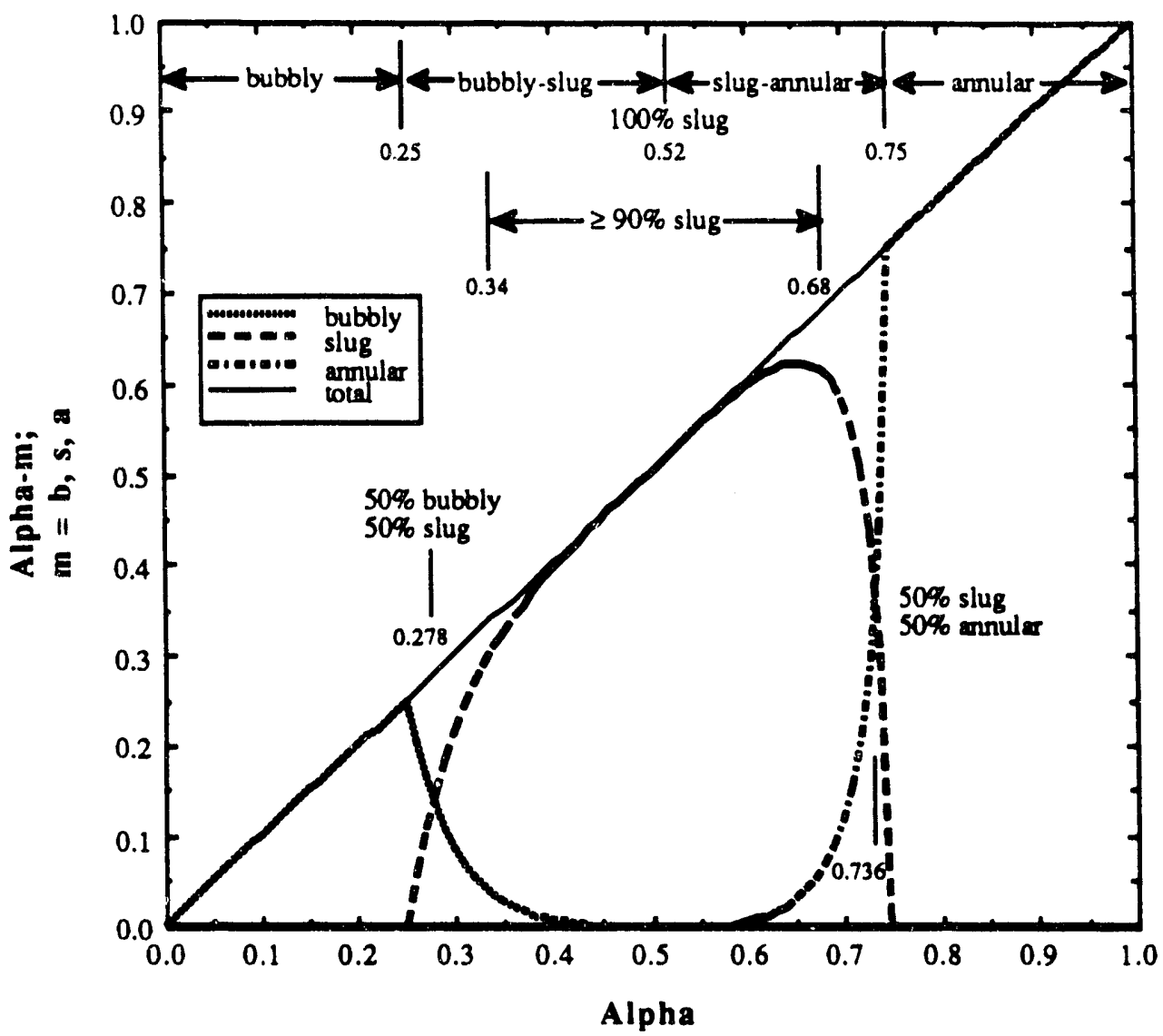

Figure 2.5.5.2-3 FLOWTRAN-TF flow regime map.

7) Dispersed regime interfacial drag data correlations for a ribbed annular channel: A large amount of air-water downflow data from thin ribbed annular channels are available from SRS Heat Transfer Laboratory experiments. Table 2.5.5.2-III lists the four SRS experiments which will be used to generate dispersed regime interfacial drag correlations for use with FLOWTRAN-TF (Flach, 1992). Table 2.5.5.2-IV gives the hydraulic parameters of the three main flow channels of a Mk22 fuel assembly for comparison (Flach, 1992). Observe that the experimental rigs cover the range of hydraulic parameters associated with the Mk22 fuel assembly. The geometries of Rigs FA and FB are nominally identical so the data from these experiments are lumped together.

As discussed in subsection 4), the dispersed flow regime interfacial drag model reduces to the drift-flux model for one-dimensional, steady, axially uniform two-phase flow (1.h.s. of momentum balances equal to zero). Furthermore, the interfacial drag model is expressed in terms of the drift-flux model parameters. Therefore if a drift-flux model is fit to the experimental data, the results can be easily incorporated into the FLOWTRAN-TF model. 
This approach is taken below for each of the three SRS databases. All of the SRS experimental rigs have a constant area ribbed annular section and the tests were conducted under steady-state conditions. For the two-phase flow to be axially uniform, the void fraction must be constant in addition to flow area. Local void fraction measurements are not available for any of the SRS experiments. However, the pressure profile is linear if the void fraction is axially constant, so, pressure readings can readily be used to detect axially uniform conditions.

With all three databases listed in Table 2.5.5.2-III the subset of data involving axially uniform conditions is identified. Figure 2.5.5.2-3 illustrates the FLOWTRAN-TF definition of 'bubbly' and 'slug' flow. Because no experimental flow regime observations are available, this map must be used to assign a flow regime to the data. Much of the map involves transitions among the three fundamental regimes of bubbly, slug, and annular. For simplicity in fitting the experimental data, all experimental data which is $50 \%$ or more bubbly is grouped together to develop the bubbly regime drift-flux parameters. Likewise for the slug regime. That is, experimental data for which $0<\alpha<0.278$ are defined to be bubbly regime data while $0.278<\alpha<0.736$ data is assigned to the slug regime. Then the drift-flux model given by equation $(2.5 .5 .2-43)$ is least-squares fit to the $\left(j, u_{g}\right)$ data to determine optimal values of the $C_{0}$ and $u_{g j}$ parameters for bubbly and slug regimes.

Table 2.5.5.2-III Hydraulic parameters of selected SRS ribbed annular experiments.

\begin{tabular}{|c|c|c|c|}
\hline $\begin{array}{l}\text { Hydraulic } \\
\text { Parameter }\end{array}$ & $\begin{array}{l}\text { Rig C } \\
\text { Kielpinski (1992) } \\
\text { WSRC-TR-92-30 }\end{array}$ & $\begin{array}{l}\text { Rigs FA \& FB } \\
\text { Steimke (1990) and } \\
\text { Johnston (1990) } \\
\text { WSRC-TR-90-44 \& } \\
\text { WSRC-TR-90-45 } \\
\text { Rig FA values given, Rig } \\
\text { FB nominally identical. } \\
\text { Hydraulic parameters in } \\
\text { WSRC-TR-90-44 } \\
\text { apparently do not } \\
\text { consider the rib gap and } \\
\text { differ slightly from the } \\
\text { values below }\end{array}$ & $\begin{array}{l}\text { VOID Rig } \\
\text { Whatley (1990) and } \\
\text { Flach (1991a) } \\
\text { WSRC-TR-90-328 \& } \\
\text { WSRC-TR-91-466 }\end{array}$ \\
\hline Inner diameter, $D_{i}$ & $0.0404 \mathrm{~m}$ & $0.0683 \mathrm{~m}$ & $0.0940 \mathrm{~m}$ \\
\hline Outer diameter, $\mathrm{D}_{\mathrm{o}}$ & $0.0508 \mathrm{~m}$ & $0.0761 \mathrm{~m}$ & 4.010 in \\
\hline Area, $A_{c}$ & $1.11 \mathrm{in}^{2} \quad 7.14 \times 10^{-4} \mathrm{~m}^{2}$ & 131 in $8.42 \times 10^{-4} \mathrm{~m}^{2}$ & 1.85 in $^{2} \quad 11.9 \times 10^{-4} \mathrm{~m}^{2}$ \\
\hline $\begin{array}{l}\text { Hydraulic diameter, } \\
D_{h}\end{array}$ & 0.343 in $8.71 \times 10^{-3} \mathrm{~m}$ & 0.275 in $6.99 \times 10^{-3} \mathrm{~m}$ & 0.292 in $7.41 \times 10^{-3} \mathrm{~m}$ \\
\hline $\begin{array}{l}\text { Hydraulic diameter } \\
\text { correction factor, } K_{h}\end{array}$ & 0.796 & 0.728 & 0.712 \\
\hline $\begin{array}{l}\text { Characteristic } \\
\text { dimension for slug } \\
\text { regime drift-velocity } \\
\text { correiation and } \\
\text { CCFL correlation, } \\
D_{c}\end{array}$ & $0.0716 \mathrm{~m}$ & $0.113 \mathrm{~m}$ & $0.154 \mathrm{~m}$ \\
\hline
\end{tabular}


Table 2.5.5.2-IV Hydraulic parameters of Mk22 fuel assembly channels.

\begin{tabular}{|c|c|c|c|c|c|c|}
\hline \multirow{2}{*}{$\begin{array}{l}\text { Hydraulic } \\
\text { Parameter } \\
\text { Inner diameter, } \mathrm{D}_{\mathrm{i}} \\
\end{array}$} & \multicolumn{2}{|c|}{$\begin{array}{l}\text { Inner Channel } \\
\text { (between inner target and } \\
\text { inner fuel) }\end{array}$} & \multicolumn{2}{|c|}{$\begin{array}{l}\text { Middle Channel } \\
\text { (between inner and outer } \\
\text { fuel) }\end{array}$} & \multicolumn{2}{|c|}{$\begin{array}{l}\text { Outer Channel } \\
\text { (between outer fuel and } \\
\text { Outer target) }\end{array}$} \\
\hline & 1.590 in & $0.0404 \mathrm{~m}$ & 2.353 in & $0.0598 \mathrm{~m}$ & 3.200 in & $0.0813 \mathrm{~m}$ \\
\hline Outer diameter, $\mathrm{D}_{0}$ & 1.995 in & $0.0507 \mathrm{~m}$ & $2.892 \mathrm{in}$ & $0.0735 \mathrm{~m}$ & 3.540 in & $0.0899 \mathrm{~m}$ \\
\hline Area, $A_{c}$ & \multicolumn{2}{|c|}{$1.08 \mathrm{in}^{2} \quad 6.95 \times 10^{-4} \mathrm{~m}^{2}$} & \multicolumn{2}{|c|}{2.15 in $13.8 \times 10^{-4} \mathrm{~m}^{2}$} & \multicolumn{2}{|c|}{$1.75 \mathrm{in}^{2} \quad 11.3 \times 10^{-4} \mathrm{~m}^{2}$} \\
\hline $\begin{array}{l}\text { Hydraulic diameter, } \\
D_{h}\end{array}$ & \multicolumn{2}{|c|}{0.338 in $8.57 \times 10^{-3} \mathrm{~m}$} & \multicolumn{2}{|c|}{0.464 in $11.8 \times 10^{-3} \mathrm{~m}$} & \multicolumn{2}{|c|}{0.313 in $7.94 \times 10^{-3} \mathrm{~m}$} \\
\hline $\begin{array}{l}\text { Hydraulic diameter } \\
\text { correction: factor, } K_{h}\end{array}$ & \multicolumn{2}{|c|}{0.794} & \multicolumn{2}{|c|}{0.783} & \multicolumn{2}{|c|}{0.723} \\
\hline $\begin{array}{l}\text { Charactenstic } \\
\text { dimension for slug } \\
\text { regime drift-velocity } \\
\text { correlation and } \\
\text { CCFL correlation, } \\
D_{c} \\
\end{array}$ & 2.82 in & $0.0715 \mathrm{~m}$ & 4.12 in & $0.105 \mathrm{~m}$ & 5.29 in & $0.135 \mathrm{~m}$ \\
\hline
\end{tabular}

VOID Rig: The VOID Rig experiment and data are discussed by Whatley (1990) and Flach (1991a). Hydraulically important dimensions are given in Table 2.5.5.2-III. Inlet liquid flowrate, inlet pressure and outlet standpipe level (pressure) were all controlled and measured. Quick-closing valves were positioned at both ends of the constant area ribbed annular section. The void fraction between the valves, air mass flowrate, and pressures between the valves were measured. Pressure and flowrates were measured every 5 seconds and time-averaged over a 5 minute interval. The void fraction measurements are instantaneous axially-averaged gas volume fractions. The experiment was run under steady-state boundary conditions. From the raw data the $\left(j, u_{g}\right)$ data illustrated in Figures 2.5.5.2-4 and 2.5.5.2-5 can be generated as shown in Flach (1992). The optimal drift-flux parameters for the bubbly and slug regimes using a weighted least-squares technique are shown in Figures 2.5.5.2-4 and 2.5.5.2-5 too. The data in Figure 2.5.5.2-4 exhibits significant scatter. An uncertainty analysis demonstrates the scatter is due to experimental uncertainty rather than model deficiency. 
VOID Rig - Bubbly Regime

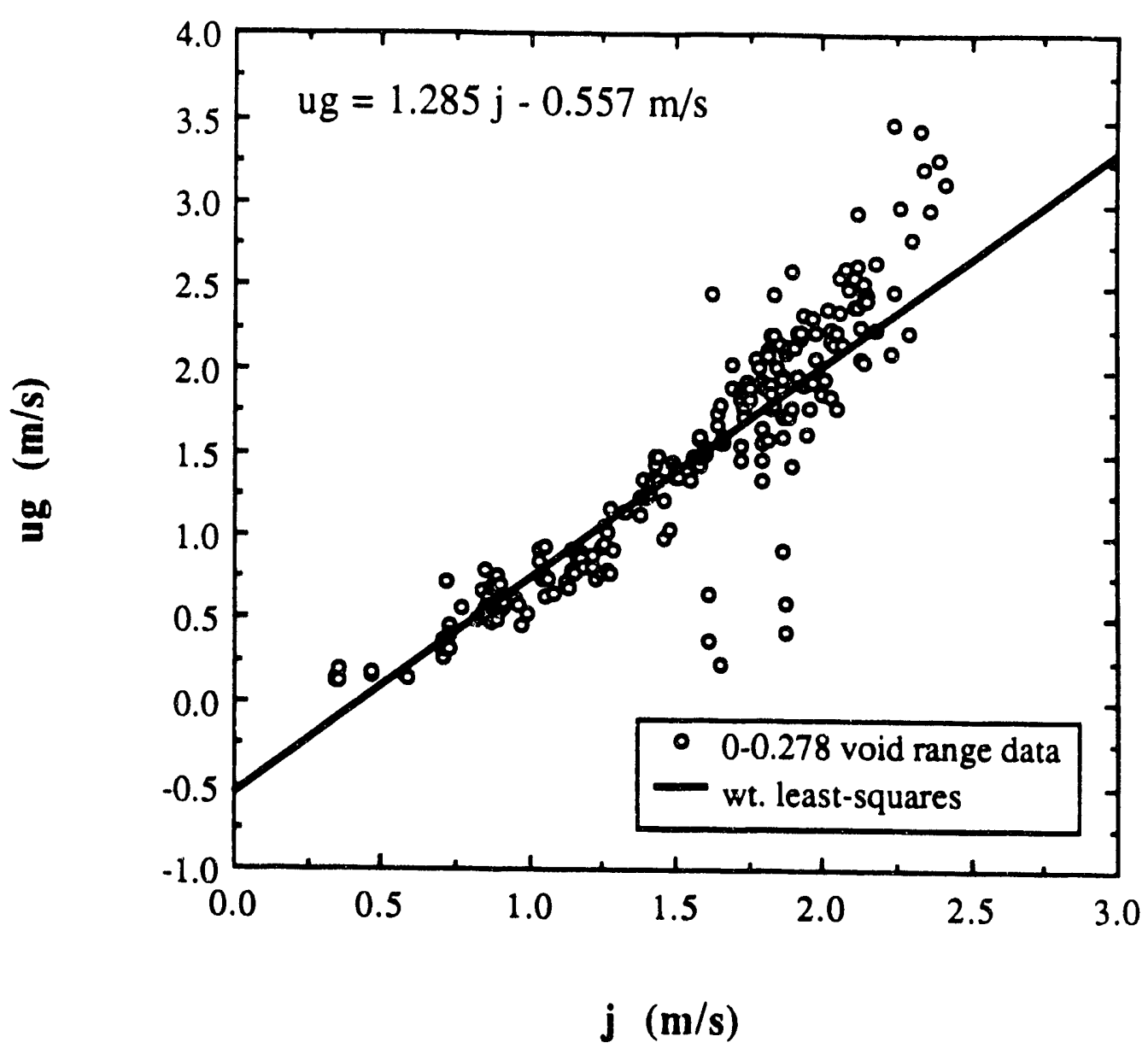

Figure 2.5.5.2-4 VOID Rig bubbly regime data and best-fit drift-flux model. 


\section{VOID Rig - Slug Regime}

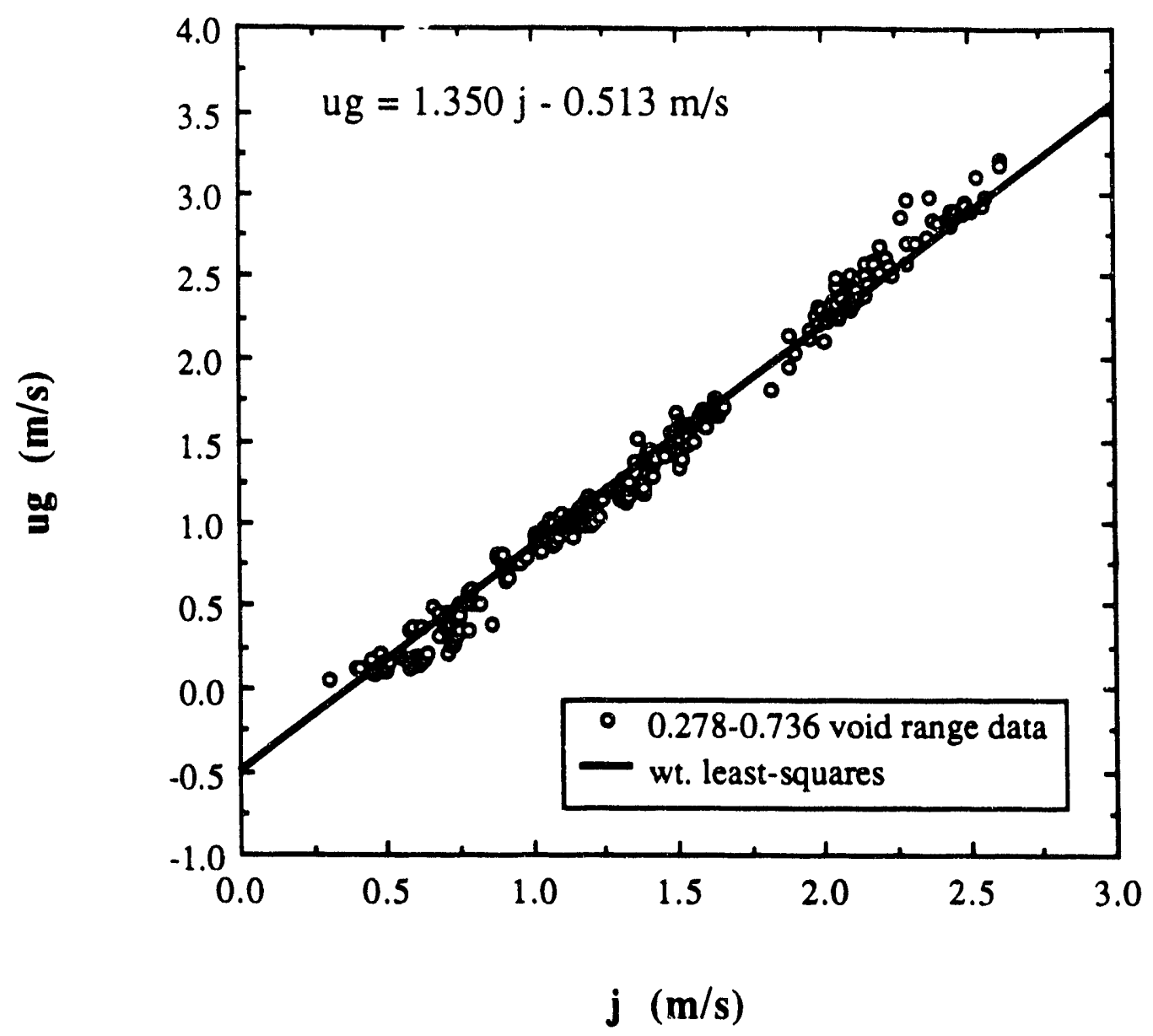

Figure 2.5.5.2-5 VOID Rig slug regime data and best-fit drift-flux model.

Rig Ci The Rig C experiment and data are discussed by Kielpinski (1992). Hydraulically important dimensions are given in Table 2.5.5.2-III. The experiment is similar to the VOID Rig except that void fraction was not measured. Also gas superficial velocity replaces inlet pressure as a controlled parameter. All the data involve a positive, non-zero gas superficial velocity so axially uniform conditions were observed for nearly all the data. (Nonuniform conditions as detected by a nonlinear pressure profile are typically observed when the gas throughput is zero.) Void fraction is needed to compute the gas phasic velocity from superficial velocity to develop a drift-flux correlation. Lacking direct void fraction measurements, the void fraction can be accurately estimated from liquid flowrate and pressure gradient measurements as described by Flach (1991b) and shown in Flach (1992). Estimated void fractions were used to generate the $u_{g}$ data. There are too few bubbly regime data points to develop a meaningful correlation. Therefore the bubbly and slug data were combined to develop a single correlation for both regimes. The data and best-fit correlation are shown in Figure 2.5.5.2-6. 


\section{Rig C - Bubbly \& Slug Regimes}

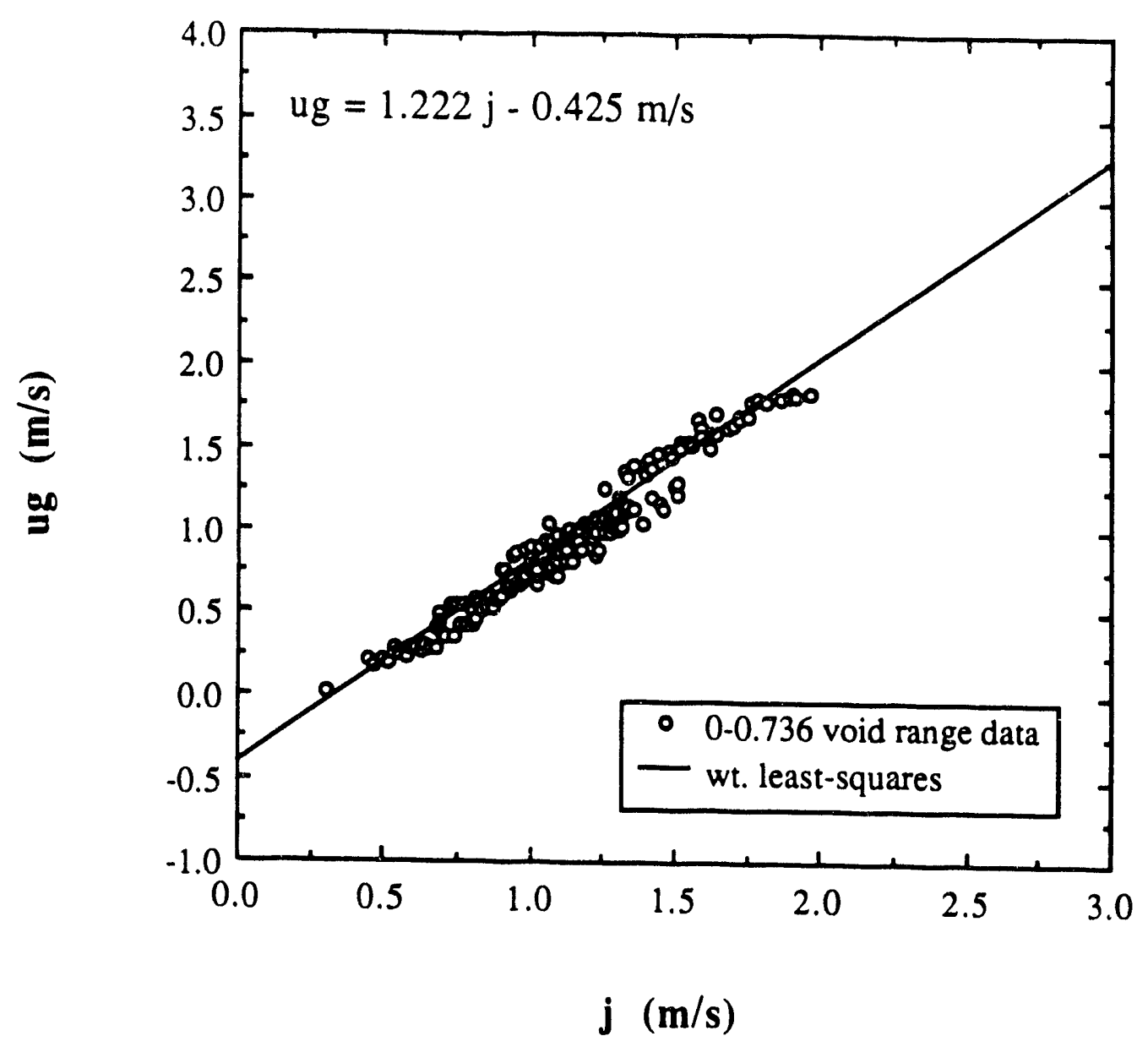

Figure 2.5.5.2-6 Rig $\mathrm{C}$ bubbly/slug regime data and best-fit drift-flux model.

Rig_FA/FB: Rigs FA and FB are described by Steimke (1990) and Johnston (1990), respectively. Rig FA has a nominally uniform axial and azimuthal power shape while Rig FB has an approximately $\pm 10 \%$ azimuthal power tilt. Hydraulically the apparatuses are nominally identical so the isothermal data from these experiments can be lumped together. Like Rig C void fraction was not measured so the void fraction must be estimated from liquid flowrate and pressure gradient data in order to generate a drift-flux correlation. The details of data manipulation are given in Flach (1992). Figures 2.5.5.2-7 and 2.5.5.2-8 illustrate the results for the bubbly and slug regimes, respectively. 


\section{Rigs FA/FB - Bubbly Regime}

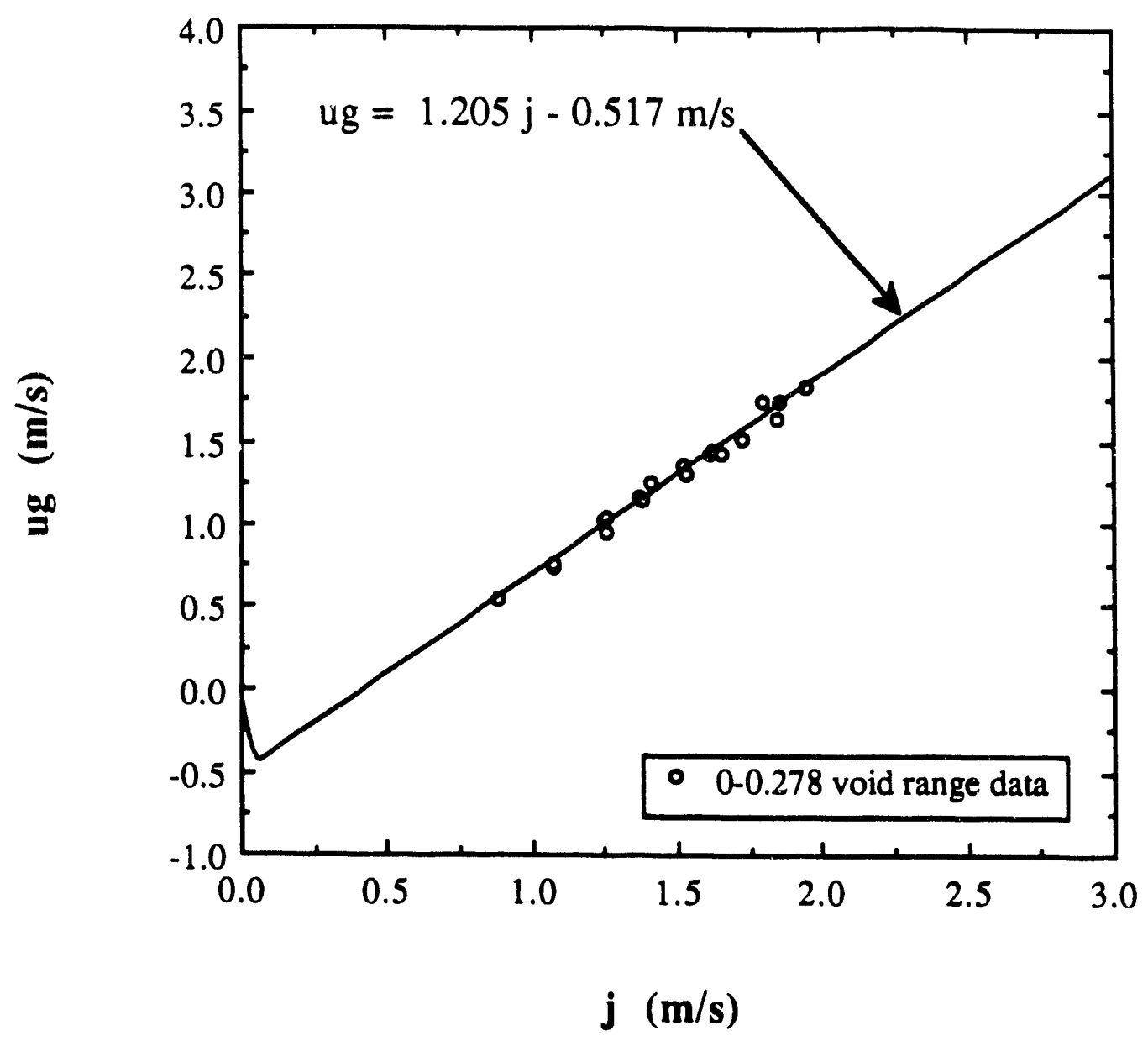

Figure 2.5.5.2-7 Rigs FA and FB bubbly regime data and best-fit drift-flux model. 


\section{Rigs FA/FB - Slug Regime}

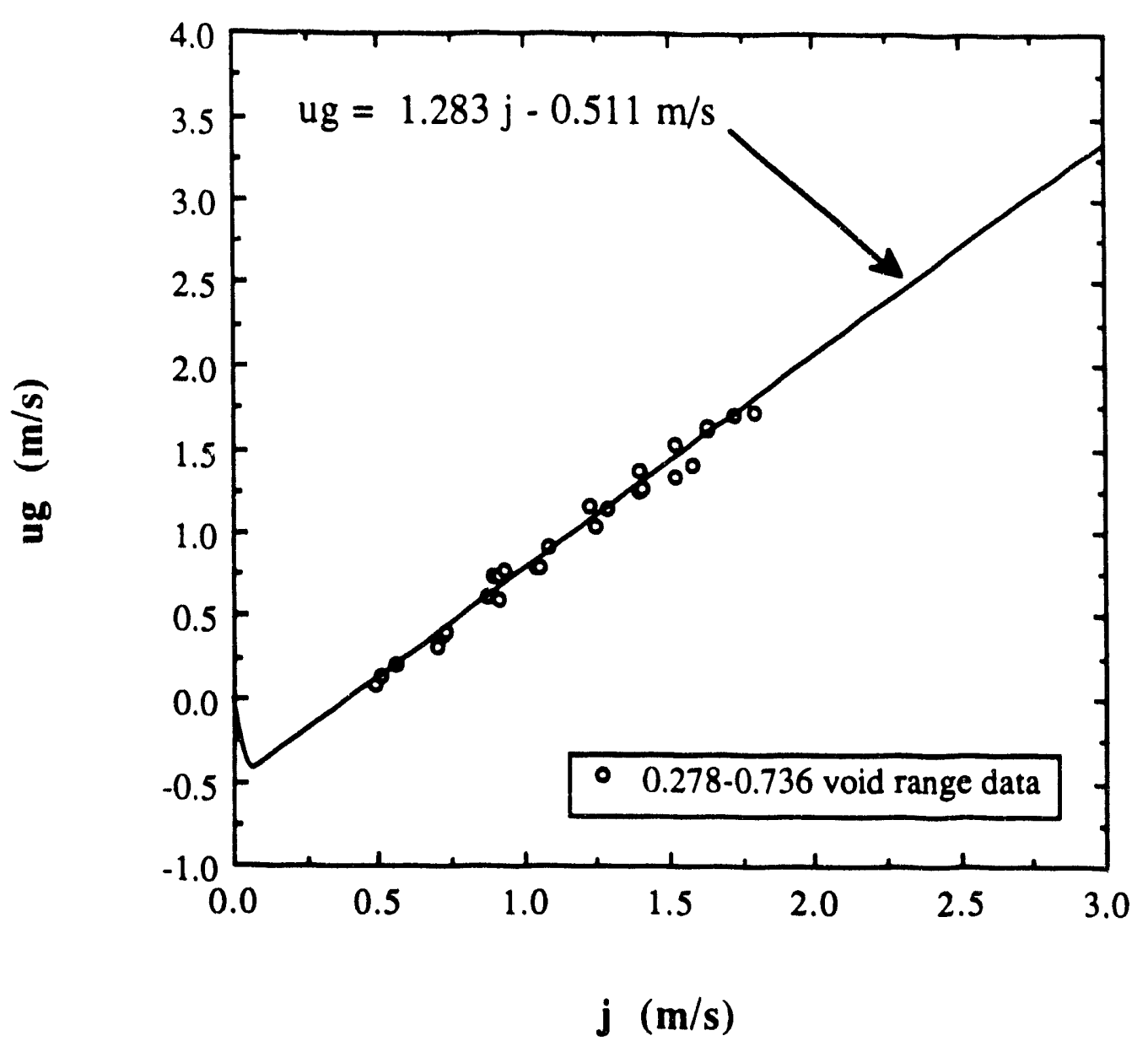

Figure 2.5.5.2 -8 Rigs FA and FB slug regime data and best-fit drift-flux model.

Comments: Table 2.5.5.2-V summarizes the best-fit drift-flux models for Rigs C, FA, FB and VOID. When interpreting the results given in Table 2.5.5.2-V, the reader should keep in mind the uncertainty in the correlations. Figures 2.5.5.2-4 through 2.5.5.2-8 show the data scatter about the correlations. The slope $\left(C_{0}\right)$ and intercept $\left(u_{g j}\right)$ of the best-fit line exhibit a strong correlation. That is, there exists a significant range of parameter selections which give nearly as good of a fit as the best-fit. A more positive $\mathrm{C}_{0}$ coupled with a corresponding decrease in $u_{g j}$ will fit the data well too. Therefore, while values are given with three or four figures in Table $2.5 .5 .2-\mathrm{V}$, the results are probably only good to about 2 significant figures.

The bubbly regime drift velocity correlation coefficient, $K_{b}$, is significantly larger than typical values in the literature such as $K_{b}=\sqrt{2}$ (Ishii, 1977). Furthermore, the driftvelocity increases with the slug regime characteristic dimension, $D_{c}$ and furthermore varies roughly as $\sqrt{ } \mathrm{D}_{\mathrm{c}}$. This suggests that data assigned to the bubbly regime was actually in the slug regime. Also note that the drift velocities for Rigs FA/FB and VOID are nearly the 
same (Rig $\mathrm{C}$ dispersed data was combined into a single correlation so the drift velocities are defined to be identical). This suggests the drift velocity is independent of $D_{c}$ for large channels. More interpretation of the data correlations is given in the next subsection.

Table 2.5.5.2.V Summary of best-fit drift-flux models for selected SRS experiments.

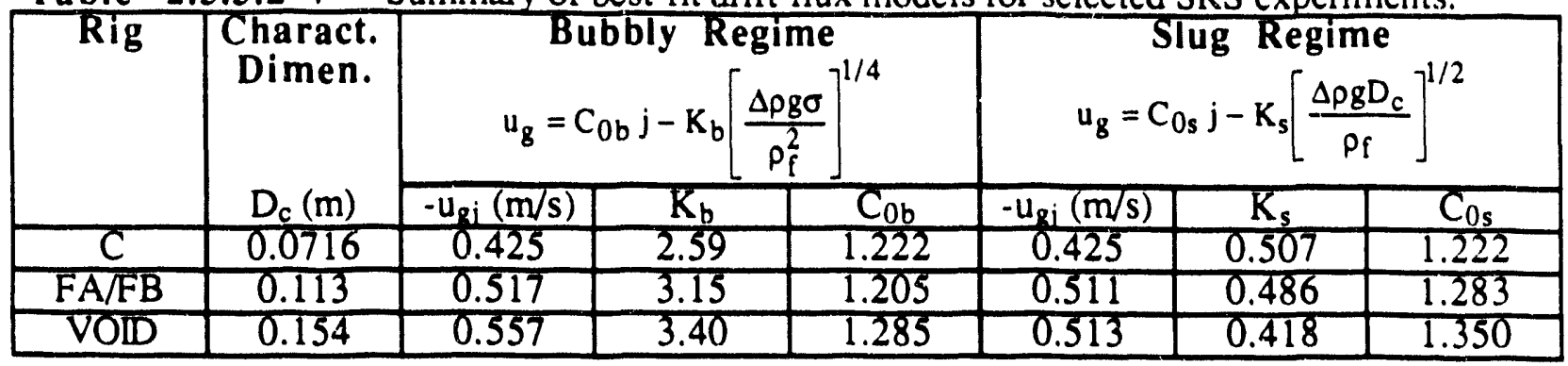

8) General recommendations for axial interfacial drag parameters: The interfacial drag functional forms for bubbly, slug and annular flow are given in subsections 4 and 5 . In this subsection, parameter value recommendations for typical FLOWTRAN-TF applications are established. FLOWTRAN-TF applications typically involve non-circular geometries, co-current down or counter-current flow, low velocities, low pressure, and relatively small channels. Unfortunately, little data addressing these aspects, especially simultaneously, is generally available. The data just presented in subsection 7 for ribbed annular channels are an important exception. Table 2.5.5.2-VI summarizes the parameter recommendations for typical FLOWTRAN-TF applications.

For the annular regime, the friction factor under non-CCFL conditions is assumed to be the same for each geometry. The CCFL correlation depends on geometry however; see Lee (1991) for more information on the recommendations for $m$ and $C$ given in Table 2.5.5.2-VI.

The bubbly and slug regime recommendations for a circular duct are based on the works of Ishii (1977), Martin (1976) and Kawanishi et al. (1990). Ishii (1977) recommends the following $\mathrm{C}_{0}$ for both upflow and downflow

$$
C_{0}=1.2-0.2 \sqrt{\frac{\rho_{g}}{\rho_{f}}}
$$

and the drift velocity recommendations for bubbly and slug flows are

$$
\begin{aligned}
& u_{g j}=-\sqrt{2}\left[\frac{\Delta \rho g \sigma}{\rho_{f}^{2}}\right]^{1 / 4} \\
& u_{g j}=-0.35\left[\frac{\Delta \rho g D_{c}}{\rho_{f}}\right]^{1 / 2}
\end{aligned}
$$


respectively. Ishii (1977) based the recommendations on data taken over a wide range of up and down flows. However, Martin (1976) presented slug regime downflow data at low velocities which indicates

$$
\mathrm{C}_{0} \cong 0.9
$$

For a $2.6 \mathrm{~cm}$ diameter tube, Martin (1976) found equation (2.5.5.2-112) adequate, but, for 10.16 and $14.0 \mathrm{~cm}$ pipes the observed drift velocities were much larger than predicted by (2.5.5.2-112). The experimentally derived coefficients for the 10.16 and $14.0 \mathrm{~cm}$ tubes are 0.66 and 0.58 . The measured drift velocities are nearly the same for the larger pair of pipes which lead Martin (1976) to suggest that the drift velocity is independent of pipe size above a certain diameter (10 cm or less). Martin (1976) did not recommend a drift velocity model for large pipes apparently due to insufficient data. More recently Kawanishi et al. (1990, Table 1) recommended that $\mathrm{C}_{0}$ be given by $(2.5 .5 .2-110)$ for upflows of any velocity and downflows with a mixture superficial velocity greater than $3.5 \mathrm{~m} / \mathrm{s}$. For downflow and $\mathrm{j}<2.5 \mathrm{~m} / \mathrm{s}$ Kawanishi et al. (1990, Table 1) recommend

$$
C_{0}=0.9+0.1 \sqrt{\frac{\rho_{g}}{\rho_{f}}}
$$

The downflow region $2.5<\mathrm{j}<3.5 \mathrm{~m} / \mathrm{s}$ is handled through interpolation between (2.5.5.2-110) and (2.5.5.2-114). For the slug regime Kawanishi et al. (1990, Fig. 11) show that the drift velocity is a function of pipe diameter with $(2.5 .5 .2-112)$ being applicable for $D<0.05 \mathrm{~m}$. For larger pipes the data yields a coefficient of 0.52 which corroborates Martin's observations. However, Kawanishi et al. (1990) suggest that only the bubbly regime drift velocity correlation (2.5.5.2-111) should be used for downflows.

The recommendation for $\mathrm{C}_{0}$ of Kawanishi et al. (1990) is substantially consistent with Ishii (1977) and Martin (1976). However, a flow dependence is not permitted with FLOWTRAN-TF so either (2.5.5.2-110) or (2.5.5.2-114) must be chosen. The scheme given in Table 2.5.5.2-VI is reasonable method for choosing between $(2.5 .5 .2-110)$ and (2.5.5.2-114). For most TF applications equation (2.5.5.2-114) is applicable. Although Kawanishi et al. (1990) do not apply their slug regime correlation to downflow, the model agrees with the observations of Martin (1976) for downflow and the FLOWTRAN-TF flow regime map does contains a slug regime for downflow. Therefore, the Kawanishi et al. (1990) model is chosen for the slug regime drift velocity. For the bubbly regime, equation (2.5.5.2-111) is selected.

For a thin rectangular channel, the appropriate drift velocity correlations have already been discussed in subsection 4. The slug regime recommendation of $K_{s}=0.21$ is the average of the Griffith (1964) and Sadatomi et al. (1982) data given in Table 2.5.5.2-I. The proper distribution coefficient $C_{0}$ is less certain. Ishii (1977) recommends

$$
1.35-0.35 \sqrt{\frac{\rho_{\mathrm{g}}}{\rho_{\mathrm{f}}}}
$$

but Sadatomi et al. (1982, Table 2) report data in the range $1.16 \leq \mathrm{C}_{0} \leq 1.24$ for slug flow. Equation (2.5.5.2-115) is chosen for both dispersed regimes in Table 2.5.5.2-VI. 


\begin{tabular}{|l|lll|l|}
\hline $2 / 15 / 93$ & WSRC-TR-92.532 Rev.0 & FLOWTRAN-TF Software Design & Page 252 \\
\hline
\end{tabular}

For a thin annular channel, the drift velocity correlations have been discussed in subsection 4. The recommendation in Table 2.5.5.2-VI for the slug regime is $K_{s}=0.35$ which is an average of the available data. The proper distribution coefficient is even less certain than that for a rectangular channel. Sadatomi et al. (1982, Table 2) report $\mathrm{C}_{0}=1.30$ for slug flow in a $15 \mathrm{~mm} \times 30 \mathrm{~mm}$ annulus. However, Kelessidis and Dukler (1990) measured $\mathrm{C}_{0}$ $=1.55$ for a $2 " \times 3 "(50.8 \mathrm{~mm} \times 76.2 \mathrm{~mm})$ annulus (slug flow). Because the data are very sparse and inconsistent, Table 2.5.5.2-VI lists equation (2.5.5.2-115) on the assumption that thin annular and rectangular channels ought to have similar phase/velocity distributions.

Finally, Table 2.5.5.2-VI summarizes the drift flux parameter recommendations for ribbed annular channels established in the preceding subsection. The slug regime coefficients, $K_{s}$, for the ribbed annular geometry appear to be consistent with their circular geometry counterparts. Note that $D_{c}$ is greater than $0.05 \mathrm{~m}$ and $\mathrm{K}_{\mathrm{s}}$ is significantly larger than 0.35 for each ribbed annular channel. Also note from Table 2.5.5.2-V that the drift velocities for Rigs FA/FB and VOID are essentially the same. This suggests that the drift velocity has become independent of characteristic dimension for $D_{c}>0.1 \mathrm{~m}$. These observations are similar to those of Martin (1976) and Kawanishi et al. (1990). However, the thin annular data does not exhibit any dependence of $K_{s}$ on $D_{c}$. For example, $D_{c}=0.1 \mathrm{~m}(>0.05 \mathrm{~m})$ for the data of Kelessidis and Dukler (1990) yet $K_{\mathbf{s}}=0.37$. Likewise, the rectangular channel data given in Table 2.5.5.2-I does not exhibit any $K_{s}$ dependence on $D_{c}$. The ribbed annular bubbly regime coefficient $\mathrm{K}_{\mathrm{b}}$ is much larger than 1.41. As mentioned earlier, the data were segregated according to the FLOWTRAN-TF flow regime partly because flow regime observations were not available. The large values of $\mathrm{K}_{\mathrm{b}}$ and their roughly $V D_{c}$ dependence suggest that data for flows actually in a slug regime were assigned to the bubbly regime according to the TF flow regime map. These observations suggest improvements can be made to the TF flow regime map. However, the ribbed annular correlations are based on the present TF flow regime map and are therefore expected to yield good results when used with the map. 


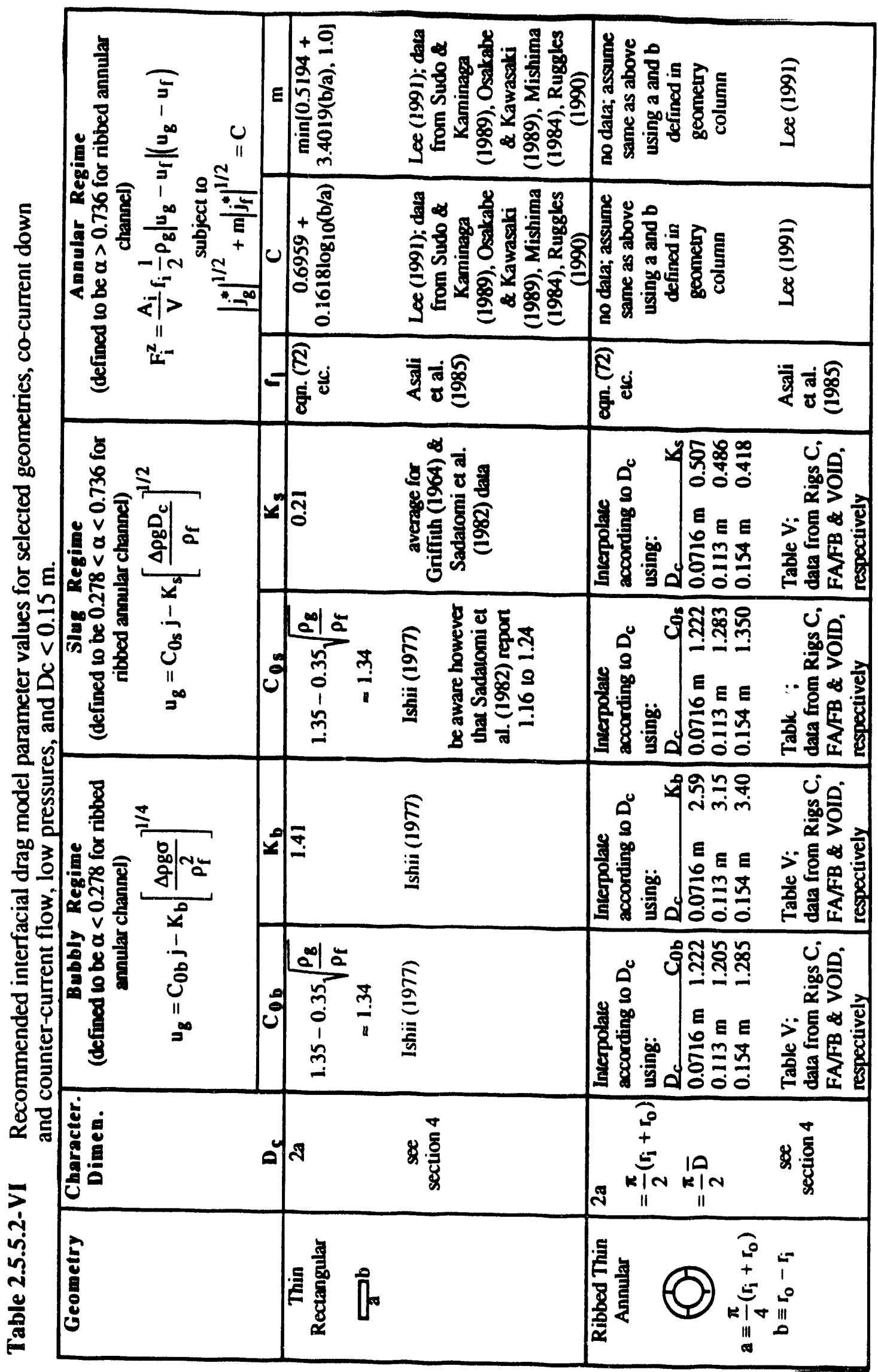




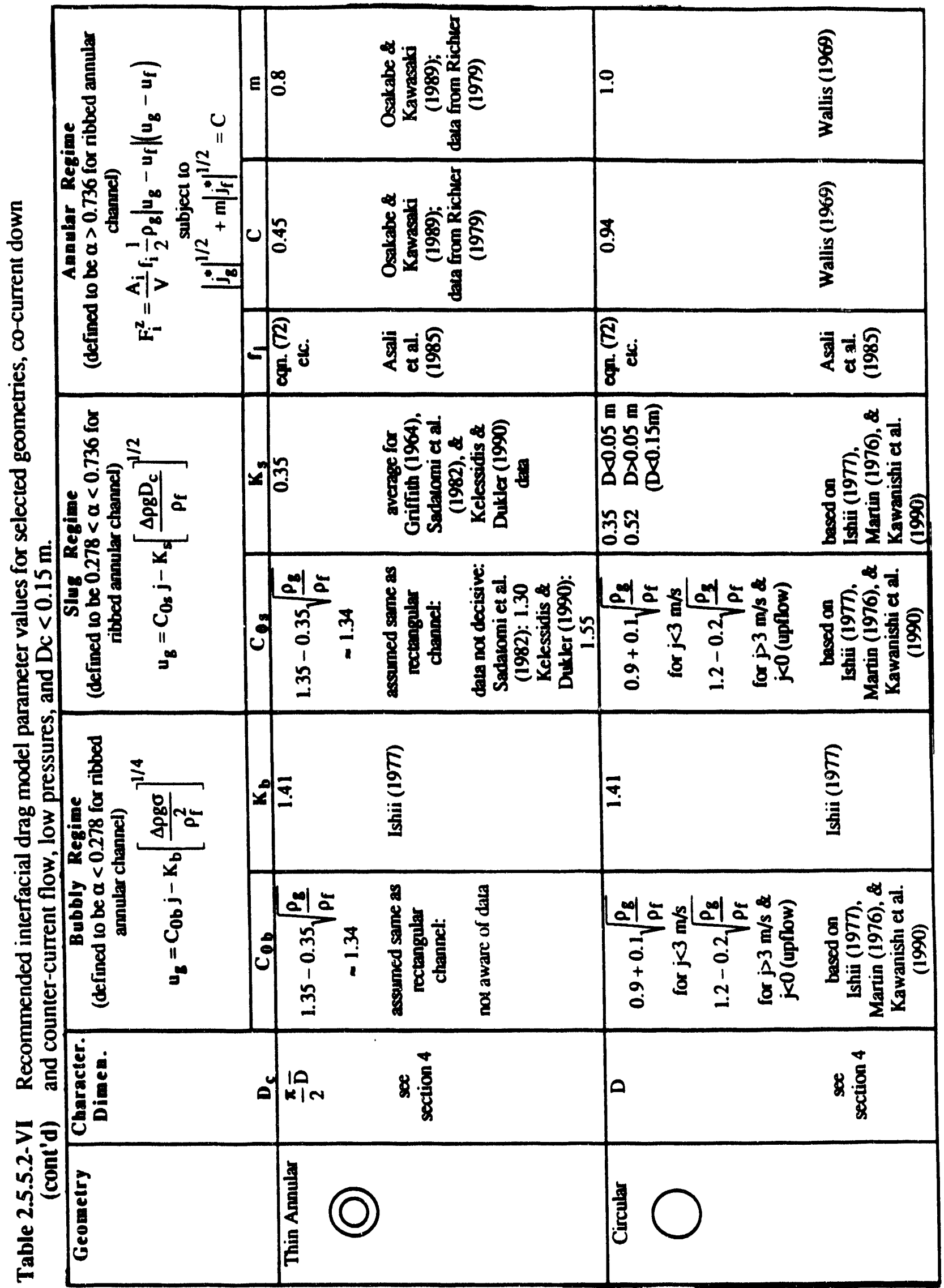


9) Azimuthal two-phase flow: All FLOWTRAN-TF applications to-date having an azimuthal flow component involve flow past the rib gap of a longitudinally ribbed annular channel. The typical gap size is $0-30$ mils with 15 mils being nominal. Interfacial drag data for this geometry are not available so physical reasoning is used to establish the TF azimuthal interfacial drag model. Because the rib gaps are small the phases are assumed to be highly coupled as they flow through the gap. Specifically, the two-phase flow is assumed to be homogeneous in the azimuthal direction for all two-phase flows. The resulting azimuthal momentum model is

$$
\begin{gathered}
0=-\alpha \frac{d P}{d x}+\alpha \rho_{g} g^{x}-F_{i}^{d x} \\
0=-(1-\alpha) \frac{d P}{d x}+(1-\alpha) \rho_{f} g^{x}-F_{w}^{x}+F_{i}^{d x}
\end{gathered}
$$

where

$$
\begin{gathered}
F_{i}^{d x}=C_{i}^{d x}\left|w_{g}-w_{f}\right|\left[w_{g}-w_{f}\right] \\
C_{i}^{d x} \rightarrow \infty
\end{gathered}
$$

The interfacial drag per unit total volume, $F_{i}{ }^{d x}$, is a finite quantity. By setting the interfacial drag coefficient ve $y$ large the relative velocity between the phases must become very small. Assessment of the homogeneous flow assumption through benchmarking to experimental data is clearly needed.

10) Computational implementation: To implement the physical interfacial drag model presented above, three additional issues must be addressed. The first is the timestep at which the various variables in tho interfacial drag models are evaluated. All quantities except velocities are evaluated at the previous time-step (n). Velocities are evaluated at the current time-step $(n+1)$. For example the equations $(2.5 .5 .2-102)$ and (2.5.5.2-103) take the form

$$
\left(F_{i}^{d z}\right)^{n+1}=\left(C_{i}^{d z}\right)^{n} \cdot\left|\left(\frac{1-C_{0} \alpha}{1-\alpha}\right)^{n} u_{g}^{n+1}-C_{0}^{n} u_{f}^{n+1}\right|\left[\left(\frac{1-C_{0} \alpha}{1-\alpha}\right)^{n} u_{g}^{n+1}-C_{0}^{n} u_{f}^{n+1}\right]
$$

Another computational issue involves restrictions on the newly computed interfacial drag coefficient intended to yield more stable, well-behaved numerical solutions. Either of two schernes may be chosen. Both make use of a 'decay factor' defined by

$$
\gamma^{n}=2^{-\frac{\Delta t^{n}}{\tau}}
$$

where $\Delta \mathrm{t}^{\mathrm{n}}$ is the time-step and $\tau$ is a time constant, both in seconds. The time constant is user specified. The first scheme is 


$$
C_{i}^{n} \leftarrow \max \left[\min \left(C_{i}^{n}, \frac{C_{i}^{n-1}}{\gamma^{n}}\right), C_{i}^{n-1} \gamma^{n}\right]
$$

and the second option is

$$
C_{i}^{n} \leftarrow \gamma^{n} C_{i}^{n-1}+\left(1-\gamma^{n}\right) C_{i}^{n}
$$

Both schemes are effective in limiting the change in the interfacial drag coefficient between time-steps and yield a smoother two-phase solution in time.

\section{$\$ 2.5 .5$ References}

Anderson, J. G. M., and K. H. Chu, 1982, "BWR Refill-Reflood Program, Task 4.7: Constitutive Correlations For Shear and Heat Transfer For the BWR Version of TRAC", NUREG/CR-2134, EPRI NP-1582, GEAP-24940.

Asali, J. C., T. J. Hanratty, and P. Andreussi, 1985, "Interfacial Drag and Film Height for Vertical Annular Flow", AIChE Journal v31 n6, pp. 895-902.

Barnea, D., and L. Shemer, 1986, "Rise Velocity of Large Bubbles in Stagnant Liquid in Non-Circular Ducts", Int. J. Multiphase Flow v12 n6, pp. 1025-1027.

Blevins, R. D., 1984, Applied Fluid Dynamics Handbook, Van Nostrand Reinhold, New York.

Boure, J. A. and J. M. Delhaye, 1982, "General Equations and Two-Phase Flow Modeling" in Handbook of Multiphase Systems, ed. G. Hetsroni, Hemisphere and McGraw-Hill.

Cheng, L. Y., 1990, "Counter-Current Flow Limitation in Thin Rectangular Channels", The Safety, Status and Future of Non-Commercial Reactors and Irradiation Facilities, ANS International Topical Meeting, Proceedings v2, Sept. 31 to Oct. 4.

Flach, G. P., 1991a, "WSRC-TR-90-328 Errata and Corrections (U)", WSRC-TR-91466.

Flach, G. P., 1991b, "Void Fraction Estimation Program Available", NES-CDG-910062.

Flach, G. P., 1992, "Interfacial Drag Model", QA Task 89-021-1 document TF-23 Rev. 1.

Griffith, P., 1964, "The Prediction of Low-Quality Boiling Voids", J. Heat Transfer, August, pp. 327-333.

Ishii, M., 1977, "One-Dimensional Drift-Flux Model and Constitutive Equations for Relative Motion Between Phases in Various Two-Phase Flow Regimes", ANL-7747.

Ishii, M., 1979, "Local Drag Laws in Dispersed Two-Phase Flow", NUREG/CR-1230, ANL-79-105. 
Ishii, M., and K. Mishima, 1984, "Two-Fluid Model and Hydrodynamic Constitutive Relations", Nucl. Engr. \& Design 82, pp. 107-126.

Johnston, B. S., 1990, "Thermal Excursions Under ECS Conditions in a Single Annulus With Azimuthal Tilt (U)", WSRC-TR-90-45.

Kawanishi, K., Y. Hirao, and A. Tsuge, 1990, "An Experimental Study on Drift Flux Parameters for Two-Phase Flow in Vertical Round Tubes", Nucl. Engr. \& Design v120, pp. 447-458.

Kelessidis, V. C., and A. E. Dukler, 1990, "Motion of Large Gas Bubbles Through Liquids in Vertical Concentric and Eccentric Annuli", Int. J. Multiphase Flow v16 n3, pp. 375-390.

Kielpinski, A. L., 1992, "Flow Regime Mapping for Vertical Two-Phase Downflow in a Ribbed Annulus", WSRC-TR-92-30 draft.

Lahey, R. T. Jr. and D. A. Drew, "The Three-Dimensional Time and Volume Averaged Conservation Equations of Two-Phase Flows" in Advances in Nuclear Science and Technology, v 20, eds. J. Lewis and M. Becker, Plenum Press, New York.

Lee, S. Y., 1990, Lab. notebook: Theoretical approaches on onset of flooding phenomenon, WSRC-NB-89-211, P. 97 - 105.

Lee, S. Y., 1991, "Flooding Model", QA Task 89-021-1 document TF-3 Rev. 1.

Martin, C. S., 1976, "Vertically Downward Two-Phase Slug Flow", J. Fluids Engr., December, pp. 715-722.

Mishima, K., 1984, "Boiling Burnout at Low Flow Rate and Low Pressure Conditions", PhD Dissertation, Research Reactor Institute, Kyoto University, Japan.

Mishima, K., and H. Nishihara, 1985, "The Effect of Flow Direction and Magnitude on CHF for Low Pressure Water in Thin Rectangular Channels", Nucl. Engr. \& Design v86, pp. 165-181.

Ohkawa, K., and R. T. Lahey, Jr., 1980, "The Analysis of CCFL Using Drift-Flux Models", Nucl. Engr. \& Design v61, pp. 245-255.

Osakabe, M., and M. Kaminaga, 1989, "Top Flooding in Thin Rectangular and Annular Passages", Int. J. Multiphase Flow v15, pp. 747-754.

Pushkina, O. L. and Sorokin, Y. L., 1969, "Breakdown of Liquid Film Motion in Vertical Tubes", Heat Transfer Research, Vol. 1, pp. 5.

Putney, J. M., 1988, "Equations for Calculating Interfacial Drag and Shear From Void Fraction Correlations", PWR/HTWG/P(88)630, Central Electricity Research Laboratories.

Putney, J. M., 1991, "Development of a New Bubbly-Slug Interfacial Friction Model for RELAP5", Nucl. Engr. \& Design 131, pp. 223-240. 
Ransom, V. H., R. J. Wagner, J. A. Trapp, L. R. Feinauer, G. W. Johnsen, D. M. Kiser, and R. A. Riemke, 1985, "RELAP5/MOD2 Code Manual; Volume 1: Code Structure, Systems Models, and Solution Methods", NUREG/CR-4312, EGG2396.

Reed, W. H., and H. B. Stewart, "THERMIT: A Computer Program for ThreeDimensional Thermal-Hydraulic Analysis of Light Water Reactor Cores".

Richter, H. J., 1981, "Flooding in Tubes and Annuli", Int. J. Multiphase Flow v7, pp. 647-658.

Ruggles, A. E., 1990, "Countercurrent Flow Limited (CCFL) Heat Flux in the High Flux Isotope Reactor (HFIR) Fuel Element", ORNL/TM-11638, Oak Ridge National Laboratory.

Sadatomi, M., Y. Sato, and S. Saruwatari, 1982, "Two-Phase Flow in Vertical Noncircular Channels", Int. J. Multiphase Flow v8 n6, pp. 641-655.

Steimke, J. L., 1990, "Status of Heat Transfer Rig FA (U)", WSRC-TR-90-44.

Stewart, C. W., J. M. Cuta, S. D. Montgomery, J. M. Kelly, K. L. Basehore, T. I. George, and D. S. Rowe, "VIPRE-01: A Thermal-Hydraulic Code for Reactor Cores; Volume 1: Mathematical Modeling (Revision 3)", EPRI NP-2511-CCM-A.

Sudo, Y., and M. Kaminaga, 1989, "A CHF Characteristic for Downward Flow in a Narrow Vertical Rectangular Channel Heated From Both Sides", Int. J. Multiphase Flow v15 n5, pp.755-766.

Taylor, D. D. et al., 1984, "TRAC-BD1/MOD1: An Advanced Best Estimate Computer Program for Boiling Water Reactor Transient Analysis; Volume 1: Model Description", NUREG/CR-3633, EGG-2294.

Thurgood, M. J., J. M. Kelly, T. E. Guidotti, R. J. Kohrt, and K. R. Crowell, 1983, "COBRA/TRAC - A Thermal-Hydraulics Code for Transient Analysis of Nuclear Reactor Vessels and Primary Coolant Systems", NUREG/CR-3046, PNL-4385.

Wallis, G. B., 1969, One-Dimensional Two-Phase Flow, McGraw-Hill, New York.

Weaver, W. L., R. W. Shumway, G. L. Singer, and S. Z. Rouhani, 1986, "TRAC-BF1 Manual: Extensions to TRAC-BD1/MOD1 (Draft)", NUREG/CR-4391, EGG2417.

Whatley, V., 1990, "Measurements of Void Fractions and Pressure Profiles for Downward Flowing Air-Water Mixtures in Single Test Annulus (U)", WSRC-TR-90-328. 


\begin{tabular}{|l|lll|l|}
\hline $2 / 15 / 93$ & WSRC-TR-92.532 Rev. 0 & FLOWTRAN-TF Software Design & Page 259 \\
\hline
\end{tabular}

\subsubsection{Channel Inlet Void Distribution Model for Mk22 Assembly}

The main application of FLOWTRAN-TF is the Mk22 fuel assembly shown schematically in Figure 2.5.6-1. In two-component two-phase thermal-hydraulic codes such as FLOWTRAN-TF, the spatial nodalization is relatively coarse with mesh cells typically spanning the entire cross-sectional area of the dominant flow direction as shown in Figure 2.5.6-2. Code calculations yield only cross-sectional average values; the details of the flow behavior within a mesh cell are inherently imbedded in the constitutive relations. Explicit tracking of the detailed flow within a cell is generally unnecessary to perform the transport calculations. One exception occurs at the junction between the top and middle sections of the Mark 22 assembly mesh, illustrated in Figure 2.5.6-3, where the main channel is divided into multiple flow paths. Local void fraction advected across each channel entrance of the middle section is not equal to the upstream cell-average value available from the normal code computations because the phase distribution is not generally uniform within the cell above the multiple channel inlet. In this section, we describe an empirical model for the middle section channel inlet void distribution for the Mark 22 assembly which we use to specify void fraction at the middle section entrance. Liquid flow splits, gas flow splits, and total assembly air flow are all strongly dependent on the channel inlet void distribution for a given assembly liquid flowrate and pressure boundary condition.

Before presenting the detailed channel inlet void model for the Mark 22 assembly, we first qualitatively describe the expected phase distribution in the upstream cell based on physical reasoning and experimental observation. Because of symmetry in the azimuthal direction, we assume local void fraction does not vary in this direction. In the radial direction, local void fraction is presumed to be generally nonuniform. Phase distribution phenomena are known to depend on channel geometry, flow direction and flow conditions (i.e. flowrates, pressure gradient, etc.). For downward flows, void peaking has generally been observed toward the center of the conduit with the lowest void near the wall (Oshinowa and Charles, 1974; Wang et al., 1987). Accordingly, we expect the local void advected across the middle channel inlet to tend to be higher than that advected across the inner and outer channel entrances. Also we expect this effect to be more pronounced in the slug and annular flow regimes. A complicating factor is the 'overhang' immediately above the inlet to the outer channel as illustrated in the schematic diagram of Figure 2.5.6-3. Under an adverse pressure gradient situation (i.e., the case in which top plenum pressure is lower than tank bottom pressure) for which the liquid flow is gravity-dominated and the gas flow is due to the entrainment of the liquid flow, we expect the overhanging lip to tend to divert liquid away from the outer channel. That is, we anticipate a tendency toward higher void at the outer channel inlet due to the Mark 22 geometry for an adverse pressure gradient. For a favorable pressure gradient, on the other hand, air flows are relatively high and water is swept along with the air flow. Because of the low inertia of the gas phase, the overhanging lip is not a significant obstacle and we anticipate less impact on the channel inlet void distribution compared to the adverse pressure gradient case.

An examination of the Annular Flow Distribution (AFD) liquid flow fraction data taken at Babcock \& Wilcox (B\&W) (Kielpinski et al., 1990) supports the anticipated phase distribution behavior discussed above. Table I shows AFD liquid flow split data obtained under the prototypic experiments of Mark 22 assembly, which was used to develop the channel inlet void model. Furthermore, channel inlet void values inferred from the liquid flow fraction data can be successfully correlated as a function of computed upstream average void fraction and local average pressure gradient at the Top/Middle Section 
junction. The Mark 22 channel inlet void model in FLOWTRAN-TF code was constructed by assuming the inner and middle channel inlet void could be empirically modeled as

$$
\begin{aligned}
& \alpha_{\mathrm{i}}=\mathrm{fcn}(\alpha, \nabla \mathrm{P}) \\
& \alpha_{\mathrm{m}}=\mathrm{fcn}(\alpha, \nabla \mathrm{P})
\end{aligned}
$$

and the outer channel inlet void fraction could be determined from the mass balance

$$
\alpha=\sum_{k=i, m, 0} \alpha_{k} \frac{V_{k}}{V}=\sum_{k=i, m, 0} \alpha_{k} \frac{A_{k}}{A}
$$

where the upstream cell volume is assumed to be partitioned by the channel inlet flow area. Symbols in the above equations are defined by

$$
\begin{aligned}
i & \equiv \text { inner channel } \\
\mathrm{m} & \equiv \text { middle channel } \\
0 & \equiv \text { outer channel } \\
\alpha & \equiv \text { average upstream void fraction denoted in Figure } 2.5 .6-3 \\
\alpha_{k} & \equiv \mathrm{k}^{\text {th }} \text { channel inlet void; } \mathrm{k}=\mathrm{i}, \mathrm{m}, \mathrm{o} \\
\nabla \mathrm{P} & \equiv \text { local average pressure gradient } \\
\mathrm{V} & \equiv \text { upstream cell volume } \\
\mathrm{V}_{\mathrm{k}} & \equiv \text { volume partitioned to the } \mathrm{k}^{\text {th }} \text { channel } \\
\mathrm{A} & \equiv \text { total channel inlet area } \\
\mathrm{A}_{\mathrm{k}} & \equiv \mathrm{k}^{\text {th }} \text { channel inlet area }
\end{aligned}
$$

The procedure for inferring 'experimental' channel inlet void fraction values from the AFD data is based on matching the experimental and predicted liquid flow splits as shown in Figure 2.5.6-4. For a given local pressure gradient, equations $(2.5 .6-1)$ and $(2.5 .6-2)$ become

$$
\alpha_{k}^{\ell}=\mathrm{fen}(\alpha)
$$

where $\ell=\mathrm{adv}$ or fav depending on the local pressure gradient at the inlet of multiple channel section, i.e., $\ell=$ adv for $\Delta \mathrm{P}<\Delta \mathrm{P}_{1}$ and $\ell=$ fav for $\Delta \mathrm{P}>\Delta \mathrm{P}_{2}$. Additional symbols related to the above equation are defined by

$\mathrm{adv} \equiv$ adverse pressure gradient

fav $\equiv$ favorable pressure gradient 


\begin{tabular}{|l|lll|l|}
\hline $2 / 15 / 93$ & WSRC-TR-92-532 & Rev. 0 & FLOWTRAN-TF Software Design & Page 261 \\
\hline
\end{tabular}

Pressure difference $\Delta \mathrm{P}$ in equation $(2.5 .6-4)$ is defined to be the upstream cell pressure minus the arithmetic average of the cell pressures at the top axial layer of the middle section. That is, using the symbols denoted in Figure 2.5.6-3,

$$
\Delta \mathrm{P}=\mathrm{P}-\frac{1}{3}\left(\mathrm{P}_{\mathrm{i} 1}+\mathrm{P}_{\mathrm{m} 1}+\mathrm{P}_{\mathrm{ol}}\right)
$$

In addition, the parameters, $\Delta \mathrm{P}_{1}$ and $\Delta \mathrm{P}_{2}$, were established by the empiricism obtained from the inferred AFD data trend for both cases, i.e., adverse and favorable pressure gradients. For the present work, 0.0 and $200 \mathrm{~Pa}$ were used for $\Delta \mathrm{P}_{1}$ and $\Delta \mathrm{P}_{2}$ respectively. For a smooth change of channel inlet void fraction in the transition region, $\Delta \mathrm{P}_{1}<\Delta \mathrm{P}<$ $\Delta \mathrm{P}_{2}$, the linear weighting between the adverse and favorable correlations was employed, that is,

$$
\alpha_{k}=(1-\varphi) \alpha_{k}^{\mathrm{adv}}+\varphi \alpha_{k}^{\mathrm{fav}}
$$

where

$$
\varphi=\frac{\left(\Delta \mathrm{P}-\Delta \mathrm{P}_{1}\right)}{\left(\Delta \mathrm{P}_{2}-\Delta \mathrm{P}_{1}\right)}
$$

The results of this process for the subset of the AFD data given in Table I are shown in Figures 2.5.6-5 through 2.5.6-8. The inferred data was subsequently correlated using polynomial functions of upstream average void. In developing the correlations, the following constraints were imposed on the polynomial forms:

$$
\begin{gathered}
\alpha_{k}^{\ell}(0)=0 \\
\alpha_{k}^{\ell}(1)=1
\end{gathered}
$$

Channel inlet void distribution near zero void of inner and middle channels and near pure void of inner channel was assumed to be homogeneous $\left(\alpha_{k}=\alpha\right)$ for adverse and favorable pressure gradient conditions from the data trend in these regions. That is, equations, (2.5.6-12) through (15), satisfy the following additional constraints:

$$
\begin{aligned}
& \frac{\partial \alpha_{k}^{\ell}(0)}{\partial \alpha}=1 \\
& \frac{\partial \alpha_{i}^{\ell}(1)}{\partial \alpha}=1
\end{aligned}
$$

The detailed FLOWTRAN-TF channel inlet void distribution is given below. 
For $\Delta \mathrm{P}<0 \mathrm{~Pa}$ (adverse pressure gradient),

$$
\begin{aligned}
\alpha_{i}^{a d v}=\alpha & +\left(a_{i 1}+2 a_{i 2}+3 a_{i 3}+4 a_{i 4}\right) \alpha^{2}-\left(2 a_{i 1}+3 a_{i 2}+4 a_{i 3}+5 a_{i 4}\right) \alpha^{3} \\
+ & a_{i 1} \alpha^{4}+a_{i 2} \alpha^{5}+a_{i 3} \alpha^{6}+a_{i 4} \alpha^{7} \\
\alpha_{m}^{a d v}= & \alpha-\left(a_{m 1}+a_{m 2}+a_{m 3}+a_{m 4}+a_{m 5}\right) \alpha^{2} \\
& +a_{m 1} \alpha^{3}+a_{m 2} \alpha^{4}+a_{m 3} \alpha^{5}+a_{m 4} \alpha^{6}+a_{m 5} \alpha^{7}
\end{aligned}
$$

where

$$
\begin{aligned}
& \mathrm{a}_{\mathrm{i} 1}=-27.0749 \\
& \mathrm{a}_{\mathrm{i} 2}=+19.1607 \\
& \mathrm{a}_{\mathrm{i} 3}=0.0000 \\
& \mathrm{a}_{\mathrm{i} 4}=-3.96141 \\
& \mathrm{a}_{\mathrm{m} 1}=-25.8320 \\
& \mathrm{a}_{\mathrm{m} 2}=+89.9370 \\
& \mathrm{a}_{\mathrm{m} 3}=-141.192 \\
& \mathrm{a}_{\mathrm{m} 4}=+101.411 \\
& \mathrm{a}_{\mathrm{m} 5}=-27.3417
\end{aligned}
$$

For $\Delta \mathrm{P}>\Delta \mathrm{P}_{0}=200 \mathrm{~Pa}$ (favorable pressure gradient),

$$
\begin{aligned}
\alpha_{\mathrm{i}}^{\mathrm{fav}}=\alpha+\left(\mathrm{f}_{\mathrm{i} 1}\right. & \left.+2 \mathrm{f}_{\mathrm{i} 2}+3 \mathrm{f}_{\mathrm{i} 3}+4 \mathrm{f}_{\mathrm{i} 4}\right) \alpha^{2}-\left(2 \mathrm{f}_{\mathrm{i} 1}+3 \mathrm{f}_{\mathrm{i} 2}+4 \mathrm{f}_{\mathrm{i} 3}+5 \mathrm{f}_{\mathrm{i} 4}\right) \alpha^{3} \\
+ & \mathrm{f}_{\mathrm{i} 1} \alpha^{4}+\mathrm{f}_{\mathrm{i} 2} \alpha^{5}+\mathrm{f}_{\mathrm{i} 3} \alpha^{6}+\mathrm{f}_{\mathrm{i} 4} \alpha^{7} \\
\alpha_{\mathrm{m}}^{\mathrm{fav}}= & \alpha-\left(\mathrm{f}_{\mathrm{m} 1}+\mathrm{f}_{\mathrm{m} 2}+\mathrm{f}_{\mathrm{m} 3}+\hat{f}_{44}+\mathrm{f}_{\mathrm{m} 5}\right) \alpha^{2} \\
& +\mathrm{f}_{\mathrm{m} 1} \alpha^{3}+\mathrm{f}_{\mathrm{m} 2} \alpha^{4}+\mathrm{f}_{\mathrm{m} 3} \alpha^{5}+\mathrm{f}_{\mathrm{m} 4} \alpha^{6}+\mathrm{f}_{\mathrm{m} 5} \alpha^{7}
\end{aligned}
$$

where

$$
\begin{aligned}
f_{i} 1 & =-6.28314 \\
f_{i} 2 & =+2.57^{\prime} .41 \\
f_{i} 3 & =0.0000 \\
f_{i} 4 & =-0.636891 \\
f_{m} 1 & =+29.6763 \\
f_{m} 2 & =-53.3518 \\
f_{m} 3 & =+46.4944 \\
f_{m} 4 & =-22.6892 \\
f_{m} 5 & =+5.59218
\end{aligned}
$$

These correlations were developed under developmental version 1.1 of the FLOWTRAN-TF code with a current Mark 22 input deck. The impact of code updates in going from v1.1 to $v 1.2$ on the channel inlet void correlations is expected to be essentially zero. However, version 1.2 of the code will be benchmarked against the AFD liquid split 

data to confirm the validity of the model. See the FLOWTRAN-TF Software Testing
report for more information.

Figures 2.5.6-9 compare the correlations derived for adverse and favorable pressure gradients and show the outer channel void fraction resulting from equation (2.5.6-3).

Top of Assembly
Hardware

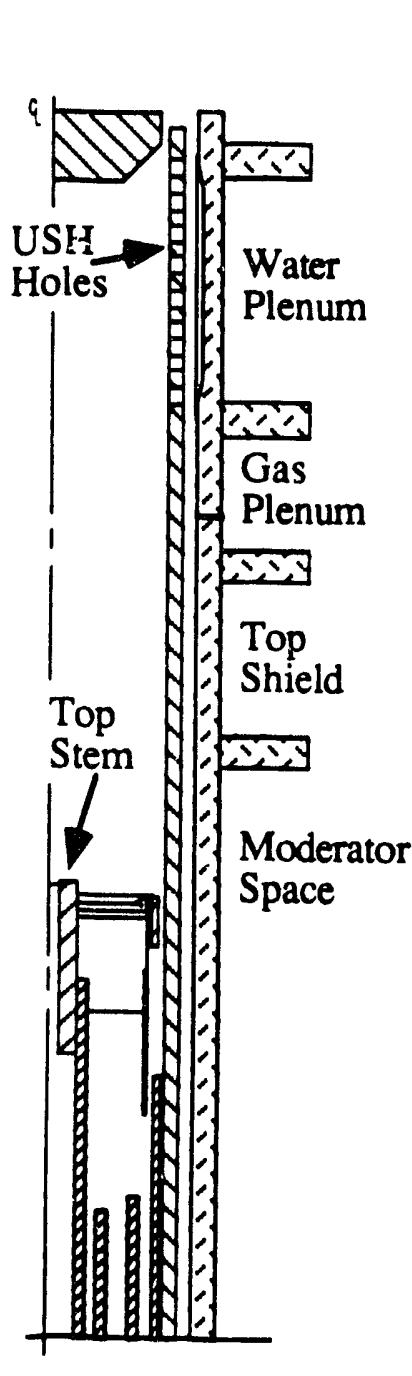

Core
Hardware

Bottom End Fitting Hardware
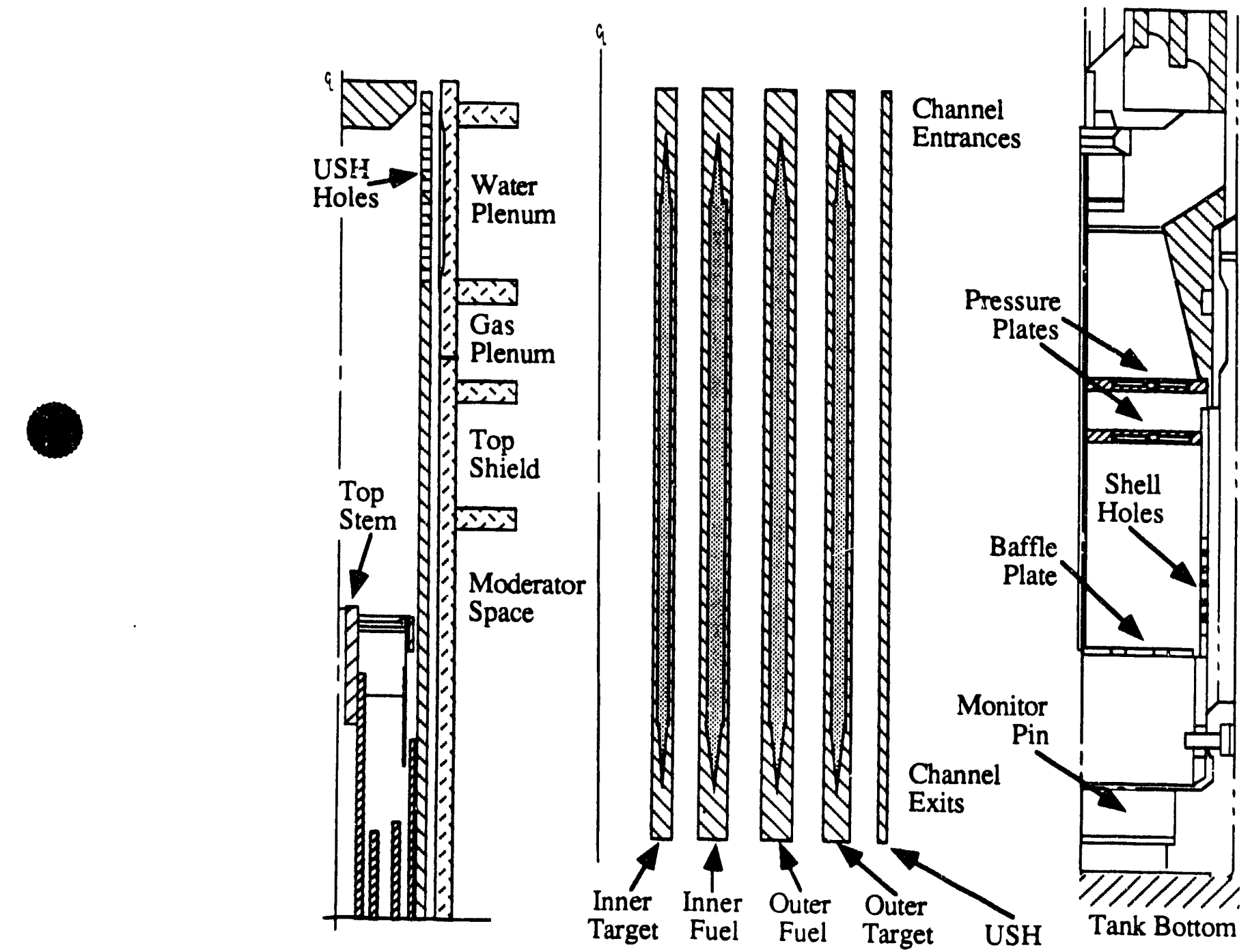

Figure 2.5.6-1 Schematic illustration of Mark 22 fuel assembly 


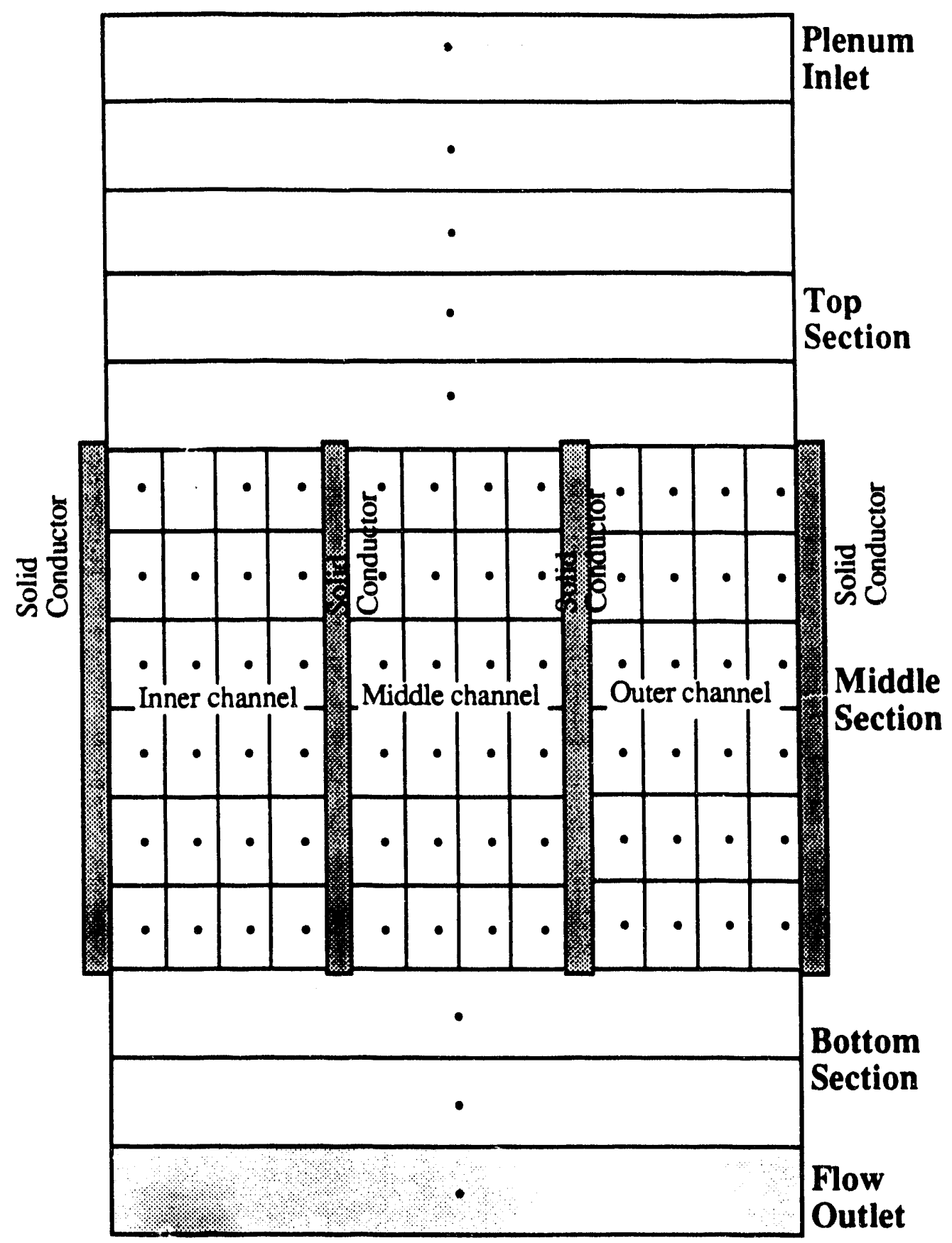

Figure 2.5.6-2 FLOWTRAN-TF fluid mesh for a typical Mark 22 assembly calculation 


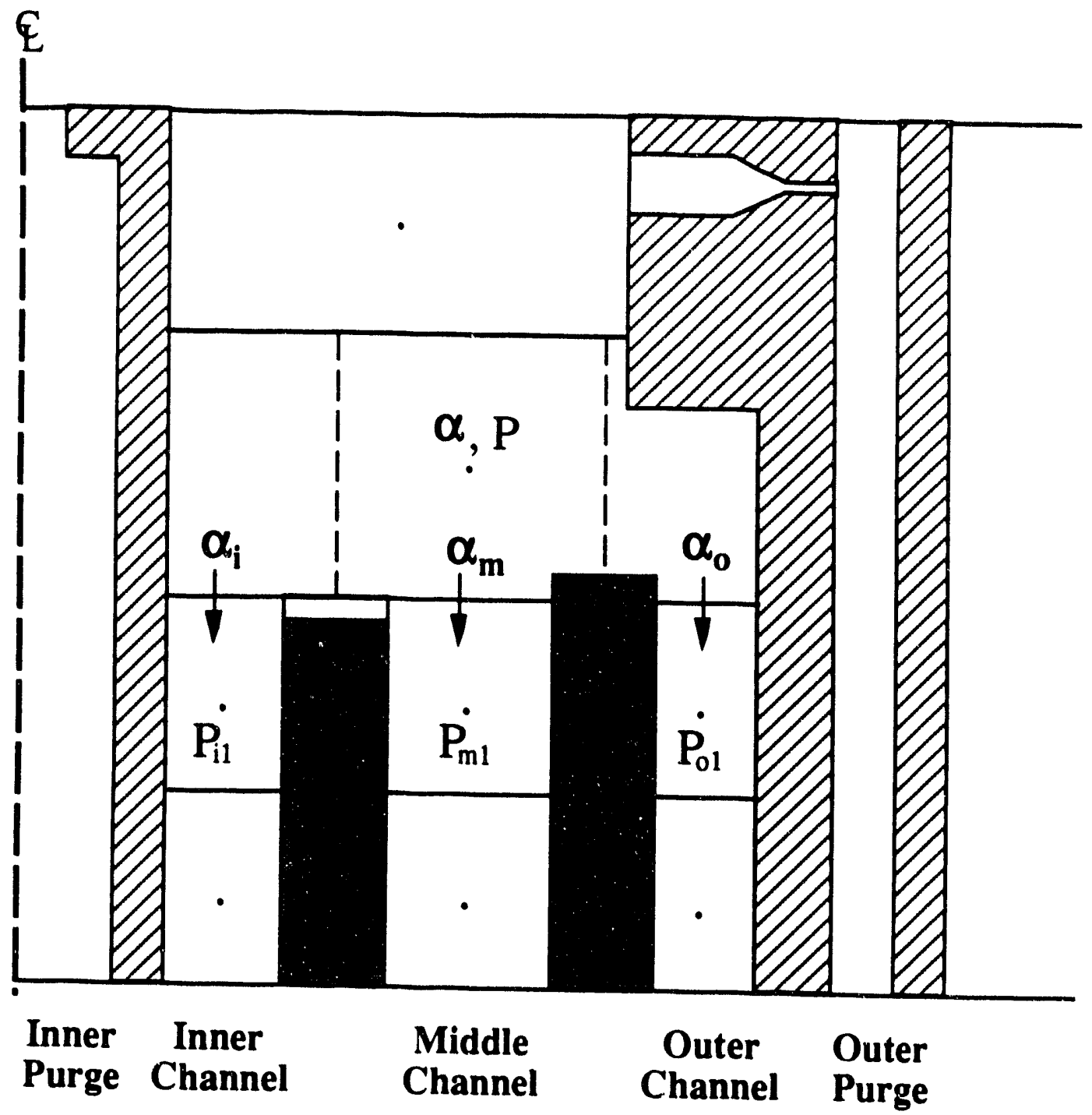

Figure 2.5.6-3 Schematic diagram of Mark 22 assembly geometry and FLOWTRAN-TF nodalization in the vicinity of the middle section channel entrance (axi-symmetric slice) 


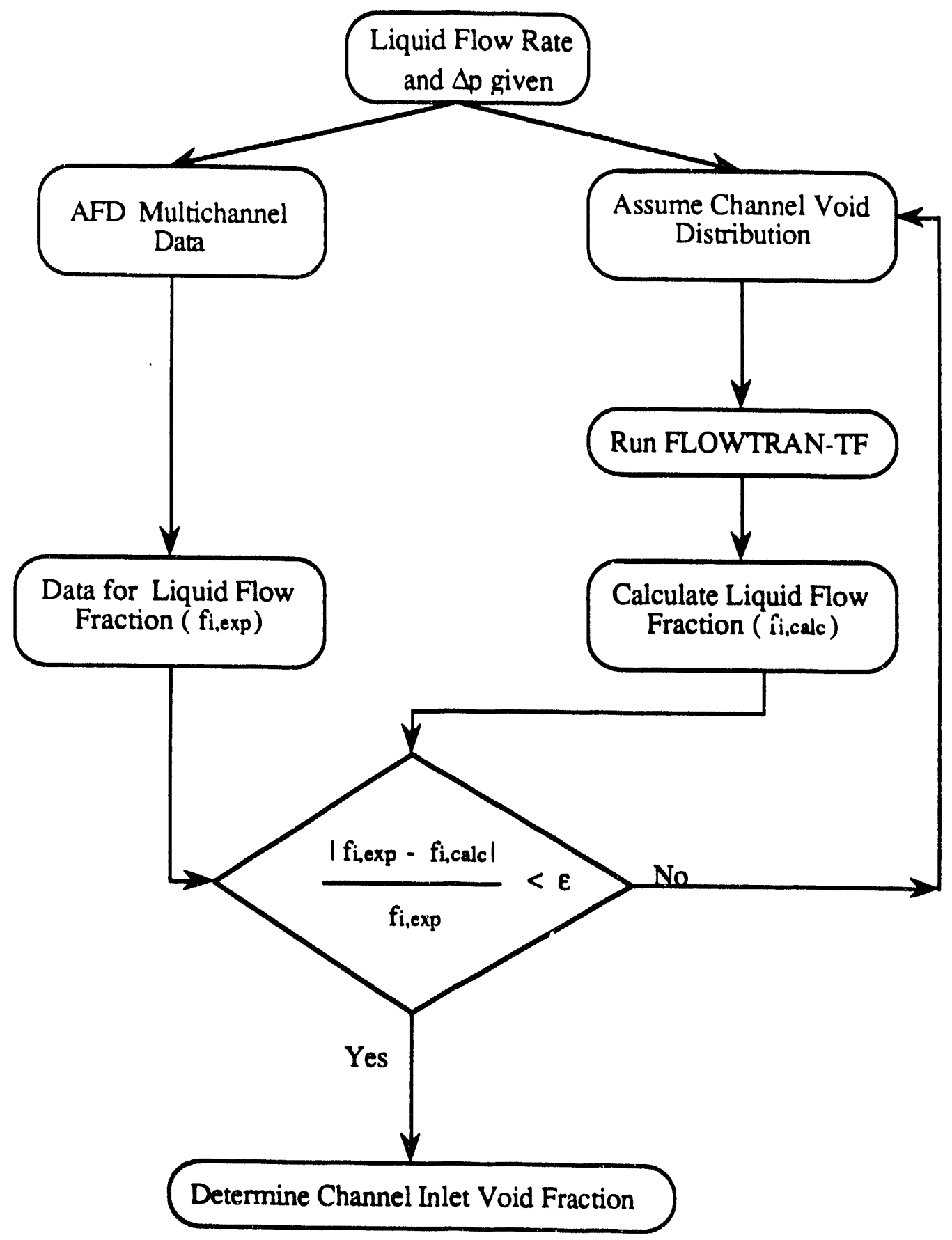

Figure 2.5.6-4 Strategy for determining experimental channel inlet void distribution values from AFD data 
Table 2.5.6-I B\&W annular flow distribution (AFD) data for the prototypic rig of Mark 22 assembly

\begin{tabular}{|c|c|c|c|c|c|c|c|}
\hline \multirow[t]{2}{*}{ Test No } & \multirow{2}{*}{$\begin{array}{l}\text { Liquid flow } \\
\text { (gpm) }\end{array}$} & \multirow{2}{*}{$\begin{array}{l}\text { Plenum } \\
\text { Inlet } \\
\text { (psia) } \\
\end{array}$} & \multirow{2}{*}{$\begin{array}{l}\text { Tank } \\
\text { bottom } \\
\text { (psia) }\end{array}$} & \multirow{2}{*}{$\begin{array}{l}\Delta p * \\
\text { (psid) }\end{array}$} & \multicolumn{3}{|c|}{ Channel liquid flow fraction } \\
\hline & & & & & Outer & Middle & Inner \\
\hline 964001 & 23.921 & 14.26 & 16.43 & -2.17 & 0.328 & 0.259 & 0.413 \\
\hline 964003 & 26.173 & 13.801 & 15.61 & -1.809 & 0.302 & 0.288 & 0.410 \\
\hline 964004 & 25.949 & 14.394 & 15.359 & -0.965 & 0.328 & 0.257 & 0.415 \\
\hline 964005 & 37.242 & 12.733 & 16.205 & -3.472 & 0.262 & 0.401 & 0.337 \\
\hline 964006 & 47.593 & 13.221 & 15.878 & -2.657 & 0.233 & 0.442 & 0.325 \\
\hline 964007 & 59.508 & 13.24 & 15.875 & -2.635 & 0.18 & 0.524 & 0.295 \\
\hline 964008 & 70.365 & 12.78 & 16.177 & -3.397 & 0.253 & 0.496 & 0.251 \\
\hline 964009 & 26.544 & 13.164 & 15.765 & -2.601 & 0.296 & 0.297 & 0.408 \\
\hline 964010 & 24.292 & 14.16 & 16.331 & -2.171 & 0.329 & 0.250 & 0.422 \\
\hline 964011 & 37.3 & 12.282 & 16.393 & -4.111 & 0.277 & 0.405 & 0.318 \\
\hline 964012 & 48.433 & 13.728 & 15.514 & -1.786 & 0.203 & 0.454 & 0.343 \\
\hline 964013 & 69.884 & 12.259 & 16.465 & -4.206 & 0.29 & 0.469 & 0.241 \\
\hline 964017 & 23.768 & 14.124 & 16.306 & -2.182 & 0.31 & 0.281 & 0.409 \\
\hline 964018 & 37.223 & 13.161 & 15.742 & -2.581 & 0.248 & 0.386 & 0.366 \\
\hline 964019 & 37.3 & 13.566 & 15.4 & -1.834 & 0.218 & 0.388 & 0.394 \\
\hline 964020 & 37.112 & 14.063 & 15.137 & -1.074 & 0.218 & 0.377 & 0.406 \\
\hline 964021 & 48.114 & 12.147 & 16.281 & 4.134 & 0.286 & 0.440 & 0.274 \\
\hline 964024 & 47.923 & 12.651 & 16.116 & -3.465 & 0.254 & 0.441 & 0.305 \\
\hline 964025 & 14.742 & 13.295 & 15.814 & -2.519 & 0.375 & 0.168 & 0.457 \\
\hline 964026 & 24.13 & 14.212 & 16.388 & -2.176 & 0.333 & 0.246 & 0.421 \\
\hline 964027 & 15.49 & 13.657 & 15.507 & -1.85 & 0.374 & 0.165 & 0.462 \\
\hline 964028 & 14.861 & 14.19 & 15.242 & -1.052 & 0.377 & 0.154 & 0.470 \\
\hline 964029 & 70.121 & 13.183 & 15.823 & -2.64 & 0.192 & 0.535 & 0.273 \\
\hline 964031 & 25.968 & 14.685 & 14.911 & -0.226 & 0.394 & 0.194 & 0.412 \\
\hline 964032 & 26.138 & 15.561 & 14.959 & 0.602 & 0.45 & 0.176 & 0.374 \\
\hline 964033 & 25.81 & 16.242 & 14.857 & 1.385 & 0.501 & 0.157 & 0.342 \\
\hline 964034 & 36.894 & 14.653 & 14.857 & -0.204 & 0.316 & 0.283 & 0.401 \\
\hline 964035 & 36.838 & 15.545 & 14.948 & 0.597 & 0.376 & 0.243 & 0.382 \\
\hline 964036 & 36.557 & 16.24 & 14.865 & 1.375 & 0.417 & 0.226 & 0.358 \\
\hline 964037 & 48.314 & 14.147 & 15.249 & -1.102 & 0.196 & 0.463 & 0.341 \\
\hline 964038 & 47.687 & 14.643 & 14.852 & -0.209 & 0.204 & 0.431 & 0.365 \\
\hline 964039 & 47.609 & 15.492 & 14.918 & 0.574 & 0.3 & 0.325 & 0.375 \\
\hline 964040 & 47.845 & 16.253 & 14.858 & 1.395 & 0.344 & 0.294 & 0.363 \\
\hline 964041 & 58.948 & 13.654 & 15.498 & -1.844 & 0.191 & 0.504 & 0.305 \\
\hline 964042 & 58.431 & 14.127 & 15.251 & -1.124 & 0.189 & 0.501 & 0.310 \\
\hline 964045 & 59.294 & 15.556 & 14.952 & 0.604 & 0.25 & 0.418 & 0.332 \\
\hline 964046 & 59.009 & 16.177 & 14.799 & 1.378 & 0.283 & 0.377 & 0.341 \\
\hline 964047 & 23.553 & 14.138 & 16.311 & -2.173 & 0.303 & 0.291 & 0.406 \\
\hline
\end{tabular}

* (Plenum inlet pressure - tank bottom pressure) 
Table 2.5.6-I B\&W annular flow distribution (AFD) data for the prototypic rig of Mark 22 assembly (Continued)

\begin{tabular}{|c|c|c|c|c|c|c|c|}
\hline \multirow[t]{2}{*}{ Test No } & \multirow{2}{*}{$\begin{array}{l}\text { Liquid flow } \\
\text { (gpm) }\end{array}$} & \multirow{2}{*}{$\begin{array}{l}\text { Plenum } \\
\text { Inlet } \\
\text { (psia) }\end{array}$} & \multirow{2}{*}{$\begin{array}{l}\text { Tank } \\
\text { bottom } \\
\text { (psia) }\end{array}$} & \multirow{2}{*}{$\begin{array}{l}\Delta p^{*} \\
\text { (psid) }\end{array}$} & \multicolumn{3}{|c|}{ Channel liquid flow fraction } \\
\hline & & & & & Outer & Middle & Inner \\
\hline 964050 & 69.545 & 15.56 & 14.953 & 0.607 & 0.201 & 0.498 & 0.301 \\
\hline 964051 & 69.945 & 16.276 & 14.853 & 1.423 & 0.243 & 0.446 & 0.311 \\
\hline 964052 & 15.164 & 14.678 & 14.9 & -0.222 & 0.429 & 0.124 & 0.447 \\
\hline 964053 & 14.695 & 15.6 & 14.973 & 0.627 & 0.542 & 0.108 & 0.350 \\
\hline 964054 & 14.693 & 16.287 & 14.903 & 1.384 & 0.544 & 0.135 & 0.321 \\
\hline 964055 & 37.025 & 13.725 & 15.534 & -1.809 & 0.21 & 0.400 & 0.389 \\
\hline 964057 & 59.04 & 13.204 & 15.83 & -2.626 & 0.154 & 0.544 & 0.303 \\
\hline 964058 & 70.17 & 12.219 & 16.393 & -4.174 & 0.289 & 0.471 & 0.240 \\
\hline 964060 & 14.963 & 16.272 & 14.889 & 1.383 & 0.543 & 0.137 & 0.321 \\
\hline 964061 & 69.878 & 16.235 & 14.885 & 1.35 & 0.237 & 0.454 & 0.309 \\
\hline 964062 & 26.157 & 14.664 & 14.886 & -0.222 & 0.391 & 0.207 & 0.402 \\
\hline 964063 & 26.124 & 17.912 & 14.897 & 3.015 & 0.524 & 0.156 & 0.320 \\
\hline 964064 & 70.349 & 17.859 & 14.889 & 2.97 & 0.288 & 0.385 & 0.327 \\
\hline 964066 & 9.12 & 13.721 & 15.517 & -1.796 & 0.496 & 0.090 & 0.414 \\
\hline 964067 & 9.087 & 14.653 & 14.867 & -0.214 & 0.55 & 0.070 & 0.379 \\
\hline 964068 & 8.904 & 16.21 & 14.872 & 1.338 & 0.53 & 0.150 & 0.320 \\
\hline 964069 & 23.871 & 14.247 & 16.419 & -2.172 & 0.302 & 0.286 & 0.412 \\
\hline 964070 & 108.99 & 12.893 & 14.838 & -1.945 & 0.293 & 0.491 & 0.216 \\
\hline 964071 & 106.64 & 17.855 & 14.819 & 3.036 & 0.234 & 0.520 & 0.246 \\
\hline 964072 & 95.618 & 12.988 & 16.387 & -3.399 & 0.293 & 0.492 & 0.216 \\
\hline 964073 & 95.246 & 13.684 & 15.515 & -1.831 & 0.281 & 0.488 & 0.231 \\
\hline 964075 & 94.066 & 14.653 & 14.832 & -0.179 & 0.248 & 0.512 & 0.240 \\
\hline 964076 & 95.393 & 16.256 & 14.848 & 1.408 & 0.221 & 0.525 & 0.254 \\
\hline 964077 & 2.918 & 14.606 & 14.8 & -0.194 & 0.581 & 0.108 & 0.312 \\
\hline 964079 & 39.78 & 16.18 & 16.34 & -0.16 & 0.282 & 0.336 & 0.382 \\
\hline 964080 & 10.28 & 14.16 & 15.04 & -0.88 & 0.477 & 0.141 & 0.382 \\
\hline 964081 & 23.79 & 15.25 & 15.61 & -0.36 & 0.349 & 0.236 & 0.415 \\
\hline 964082 & 37.301 & 14.54 & 15.154 & -0.614 & 0.243 & 0.358 & 0.398 \\
\hline 964083 & 36.874 & 15.254 & 15.006 & 0.248 & 0.353 & 0.264 & 0.383 \\
\hline 964084 & 42.99 & 14.63 & 14.87 & -0.24 & 0.23 & 0.394 & 0.377 \\
\hline 964086 & 23.86 & 14.19 & 16.38 & -2.19 & 0.302 & 0.288 & 0.411 \\
\hline 964087 & 48 & 14.64 & 15.23 & -0.59 & 0.191 & 0.467 & 0.342 \\
\hline 964088 & 47.51 & 15.26 & 15.06 & 0.2 & 0.264 & 0.374 & 0.362 \\
\hline 964089 & 25.9 & 14.68 & 15.31 & -0.63 & 0.324 & 0.267 & 0.409 \\
\hline 964090 & 15.23 & 14.69 & 15.28 & -0.59 & 0.364 & 0.166 & 0.469 \\
\hline 964091 & 24.08 & 14.22 & 16.39 & -2.17 & 0.297 & 0.291 & 0.413 \\
\hline 964092 & 14.68 & 15.25 & 15.05 & 0.2 & 0.511 & 0.090 & 0.399 \\
\hline 964093 & 25.45 & 15.29 & 15.05 & 0.24 & 0.413 & 0.197 & 0.390 \\
\hline
\end{tabular}

* (Plenum inlet pressure - tank bottom pressure) 


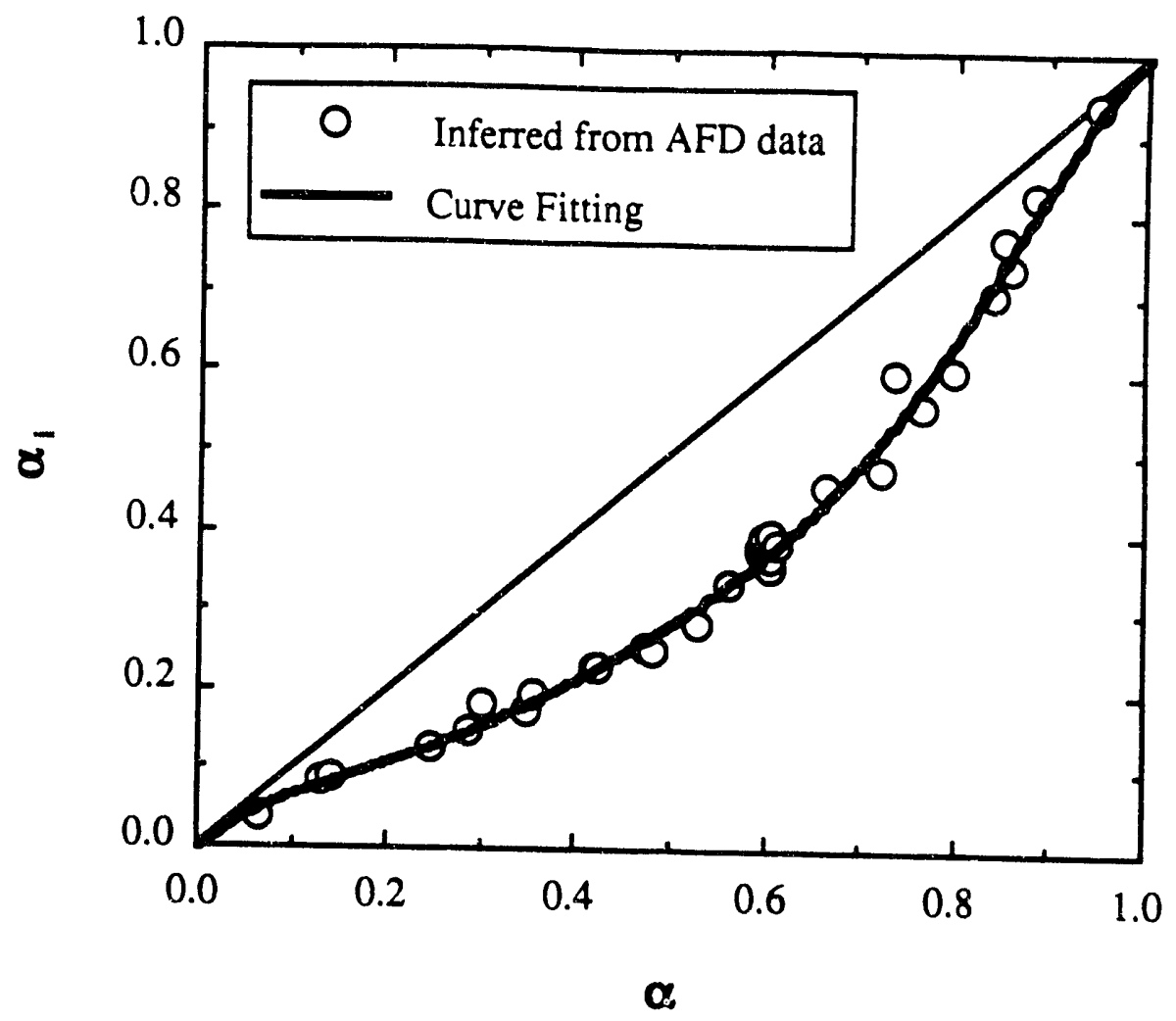

Figure 2.5.6-5 Inner channel inlet void fraction versus average upstream void fraction for adverse pressure gradient ( - ; homogeneous void
distribution) 


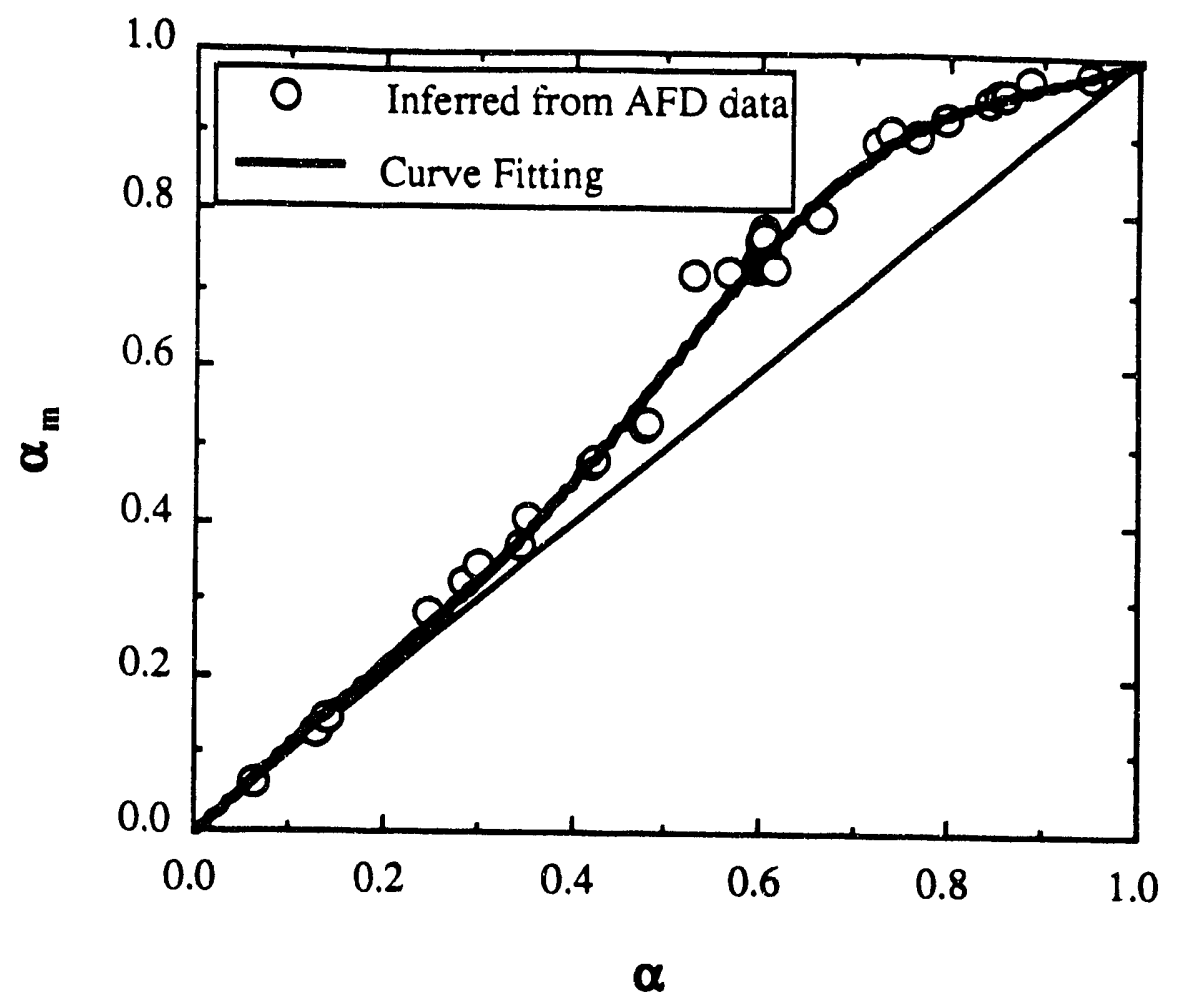

Figure 2.5.6-6 Middle channel inlet void fraction versus average upstream void fraction for adverse pressure gradient ( - ; homogeneous void distribution) 


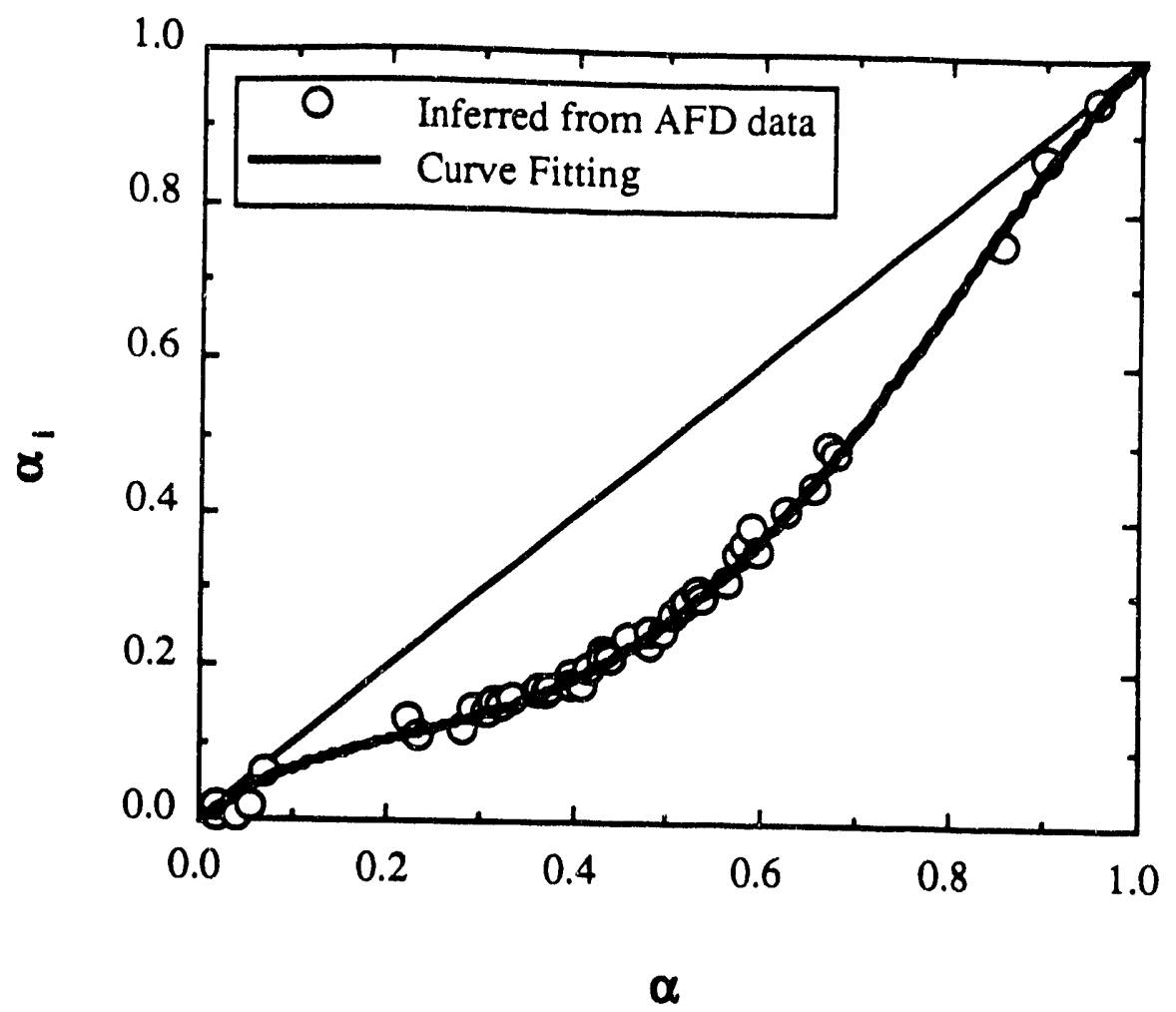

Figure 2.5.6-7 Inner channel inlet void fraction versus average upstream void fraction for favorable pressure gradient ( - ; homogeneous void distribution) 


\begin{tabular}{|l|lll|r|}
\hline $2 / 15 / 93$ & WSRC-TR-92-532 Rev.0 & FLOWTRAN-TF Software Design & Page 272 \\
\hline
\end{tabular}

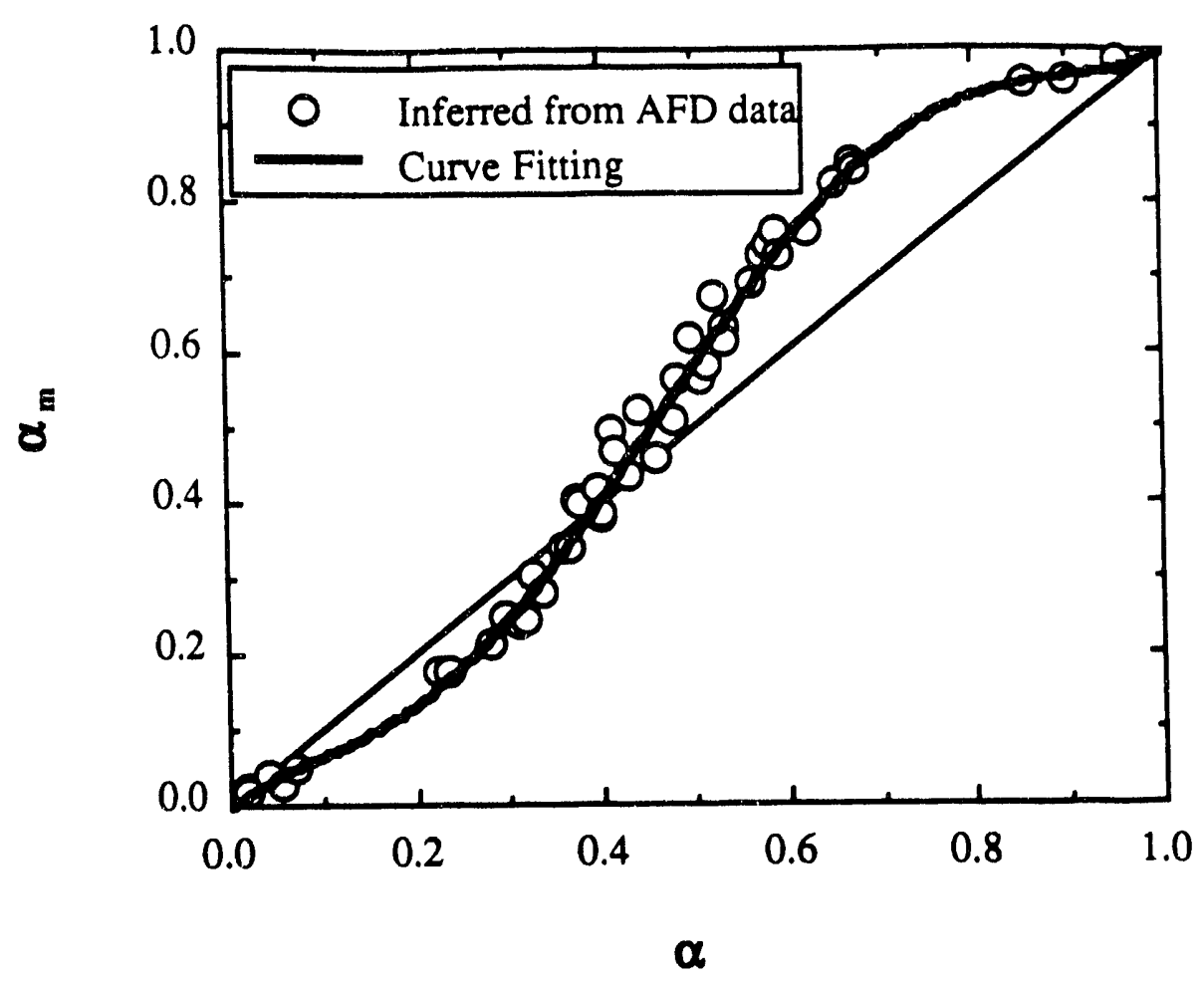

Figure 2.5.6-8 Middle channel inlet void fraction versus average upstream void fraction for favorable pressure gradient ( - ; homogeneous void distribution) 


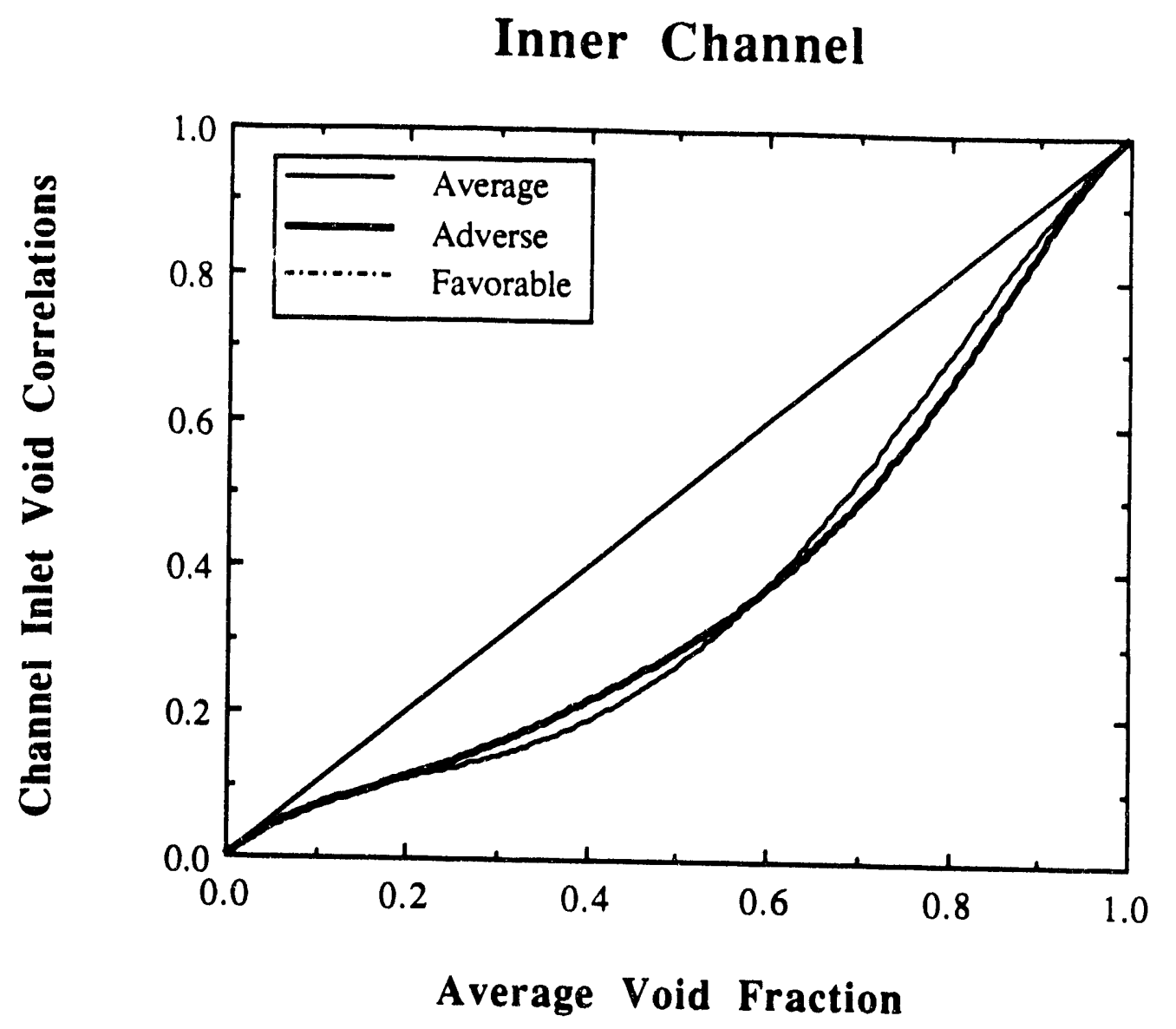

Figure 2.5.6.9a Comparison of channel inlet void correlations for the inner channel of the Mk22 fuel assembly 


\section{Middle Channel}

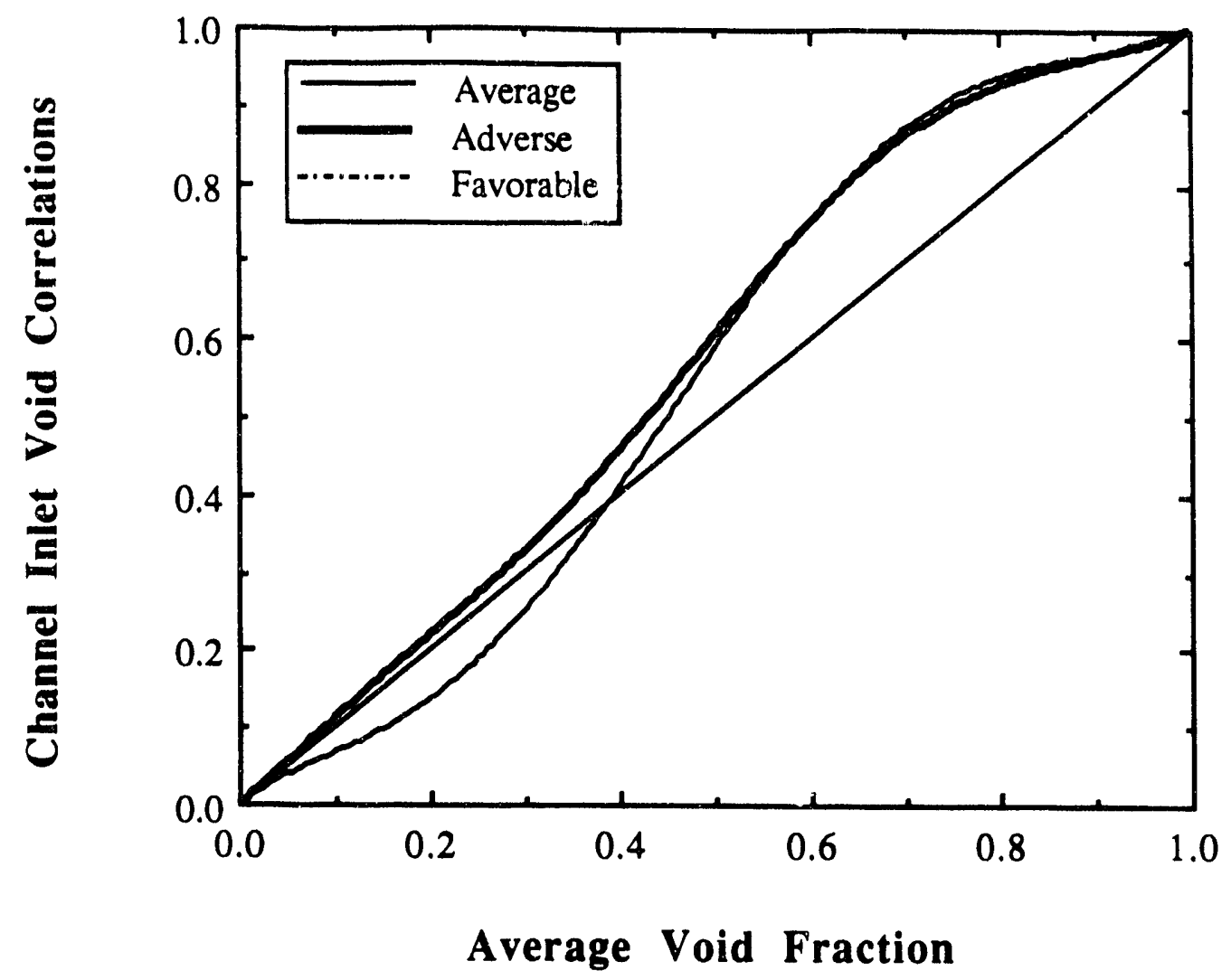

Figure 2.5.6-9b Comparison of channel inlet void correlations for the middle channel of the Mk22 fuel assembly 


\section{Outer Channel}

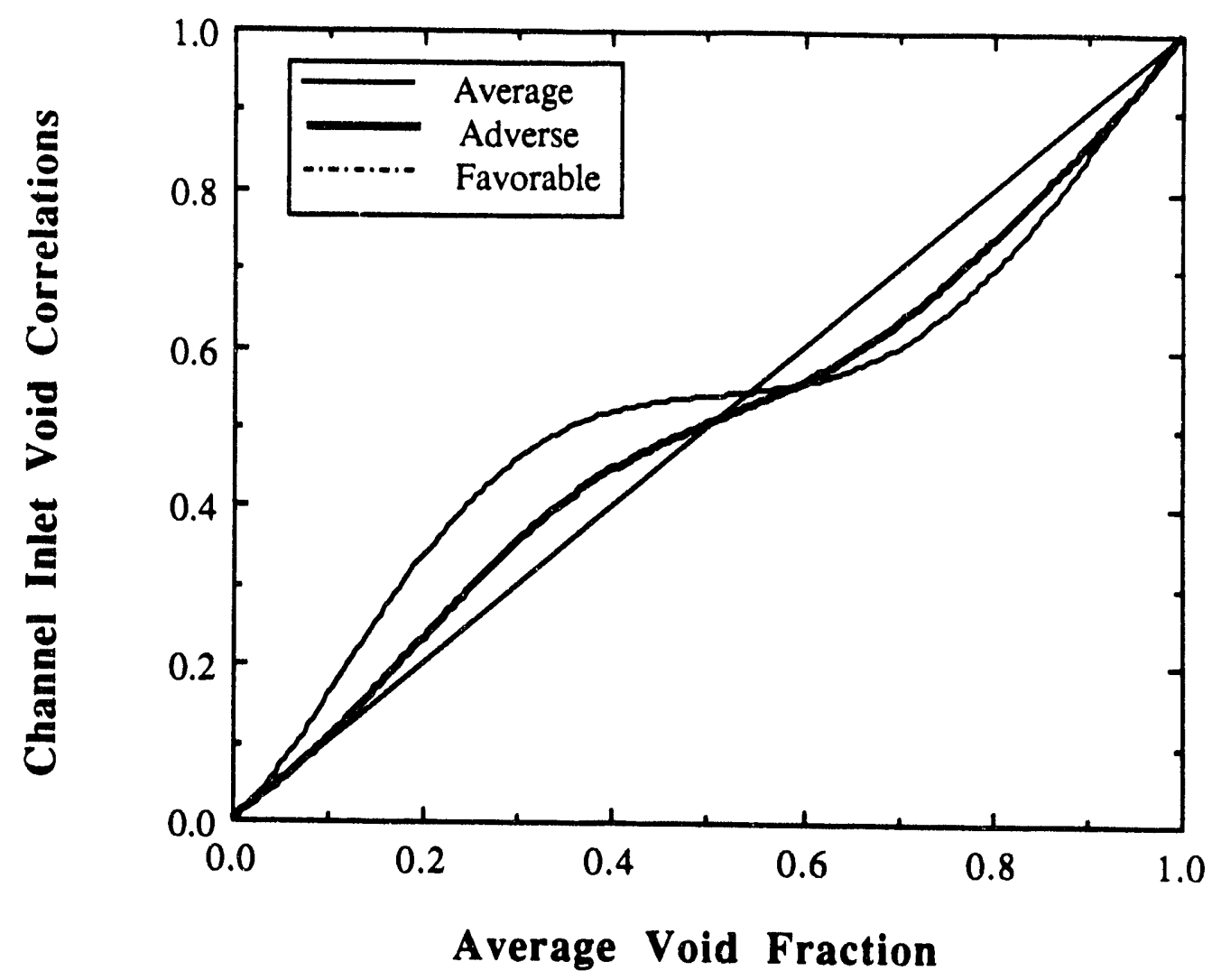

Figure 2.5.6-9c Comparison of channel inlet void correlations for the outer channel of the Mk22 fuel assembly

\section{References}

Kielpinski, A. L., Childerson, M. T., Knoll, K. E., Manolescu, M. I., and Reed, M. J., "Annular Flow Distribution Test: Final Report (U)", WSRC-TR-90-551, December, 1990.

Oshinowa, T., and Charles, M. E., 1974, "Vertical Two-Phase Flow, Part 1, Flow Pattern Correlations", Can. J. Chem. Engr. 52, pp. 25-35.

Wang, S. K., Lee, S. J., Jones, O. C., Jr., and Lahey, R. T., Jr., 1987, "3-D Turbulence Structure and Phase Distribution Measurements in Bubbly Two-Phase Flows", Int. J. Multiphase Flow 13, pp. 327-343. 


\subsubsection{Rib void model}

The detailed azimuthal phase distribution, especially, near the rib region within a fluid computational cell is important to capture the physical phenomenon of flow redistribution within each subchannel of a Mark 22 assembly as illustrated schematically in Figure 2.5.7-1. Within a ribbed annular channel, a high void occurs near the center of each quadrant subchannel and a high fraction of the liquid phase contained within subchannel occupies the region near each rib gap since there is a tendency for the lighter phase, i.e., gas phase, of the two phases to migrate towards the central region of high velocity within a quadrant of each subchannel of Mark 22 assembly as illustrated in Figure 2.5.7-1. Referring to Figure 2.5.7-2, properties at a cell face are usually taken from one of the adjoining cell centers depending on the velocity direction at the cell interface. Specifically, cell face properties are taken to be upwind cell center values and are called donored properties. For the situation illustrated in Figure 2.5.7-1, the donored local void fraction at

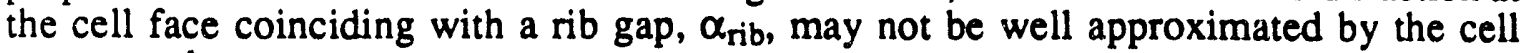
average value, $\alpha$.

However, no direct experimental measurements have been available of local void fraction, in particular, near rib gap region within an annular channel with ribs. Recently, SRL Heat Transfer Laboratory (Steimke and Fowley, 1992) conducted two-phase, air-water, experiments in a vertical ribbed annular geometry 13 feet long under hydraulic steady state condition to measure annular channel average void fraction and azimuthal void distribution. Channel average void fraction was measured using a quick closing valve technique, and local azimuthal void fractions were measured by a gamma densitometer containing a cesium-137 gamma source. Figure 2.5.7-3 shows the experimental results in which local void fraction near rib region is about half of channel averaged void fraction except for near zero and very high void regions. They also found that the variation of void distribution along the axial downflow direction can be neglected for linear axial pressure profile and local void distribution in the azimuthal direction is nearly flat except near the rib gap. Local void fraction near the rib gap region was found to be significantly less than that of the other region. However, as shown in their data, there is usually a difference between channel averaged void fraction and the azimuthal averaged void fraction because of nonlinear pressure profile along the axial direction. For all cases except for two, the azimuthal averaged void fraction at the location of rib void measurement was obtained by applying an additive correction factor to the channei averaged void fraction. The correction factor was based on a quadratic fit of five axial pressure data to determine local void fraction corresponding to the pressure gradient at the elevation of the gamma densitometer. Local void fraction was evaluated by using the simplified steady-state mixed momentum balance of the FLOWTRAN-TF code (Flach, 1991). These two data sets correspond to low liquid flowrates ( 3 and $6 \mathrm{gpm}$ ) for high tank level (56 inches). For $3 \mathrm{gpm}$ liquid flowrate, a straight line was fit through the first three pressures rather than a quadratic fit through all five pressures. The pressure gradient was used in the estimation process of azimuthal averaged void fraction. The other case corresponds to annular flow regime at the upper portion of channel where the gamma densitometer is located, i.e., zero pressure gradient, and bubbly flow region at the lower part of channel. In this case, the azimuthal average void fractions at the elevation of rib void measurement were estimated by using the analytical solution for laminar film thickness from the literature (Flach, 1992).

Now, Rib void fraction $\left(\alpha_{\text {rib }}\right)$ is correlated as a function of local cell average void fraction $(\alpha)$ (Lee, 1992) using the best estimate of azimuthal averaged void fraction data at the elevation of gamma densitometer in Table 2.5.7-1, i.e., 


$$
\alpha_{\text {rib }}=\mathrm{fcn}(\alpha)
$$

In developing the correlation, the following constraints were imposed on the polynomial equation, equation (2.5.7-1):

$$
\begin{array}{ll}
\alpha_{\text {rib }}=0 & \text { at } \alpha=0 \\
\alpha_{\text {rib }}=1 & \text { at } \alpha=1
\end{array}
$$

Azimuthal phase distribution within a ribbed annular channel is nearly uniform near zero channel average void since bubbly dispersed flow regime is established on a physical basis for low void fraction region, that is, the following additional constraint is imposed:

$$
\left.\frac{\partial \alpha_{\text {rib }}}{\partial \alpha}\right|_{\alpha=0}=1
$$

Correlation for the rib void, $\alpha_{\text {rib, }}$ was obtained by the least square fitting of SRL/HTL data considering the above constraints, equations $(2.5 .7-1)$ to $(2.5 .7-3)$ for the fifth order polynomial equation.

$$
\alpha_{\text {rib }}=\alpha-\left(a_{1}+a_{2}+a_{3}\right) \alpha^{2}+a_{1} \alpha^{3}+a_{2} \alpha^{4}+a_{3} \alpha^{5}
$$

where

$$
\begin{aligned}
& a_{1}=18.7005 \\
& a_{2}=-25.8207 \\
& a_{3}=12.7963
\end{aligned}
$$

The curve fit function, equation (2.5.7-5), is shown in Figure 2.5.7-3. The straight line in this graph corresponds to homogeneous phase distribution along the azimuthal directio * at a given elevation of ribbed annular channel. Table 2.5.7-1 also shows local void fracion data used for rib void model development.

The FLOWTRAN-TF code also employs a flexible donoring scheme to allow the users to choose the option of the default mode, that is, the normal donoring scheme of $\alpha_{\text {rib }}=\alpha$. Experience with FLOWTRAN-TF indicates that azimuthal crossflow can be sensitive to the rib void fraction model depending on the selections for other parameters (i.e, azimuthal form loss coefficient, interfacial drag coefficient, and rib gap change due to channel eccentricity, etc.). 


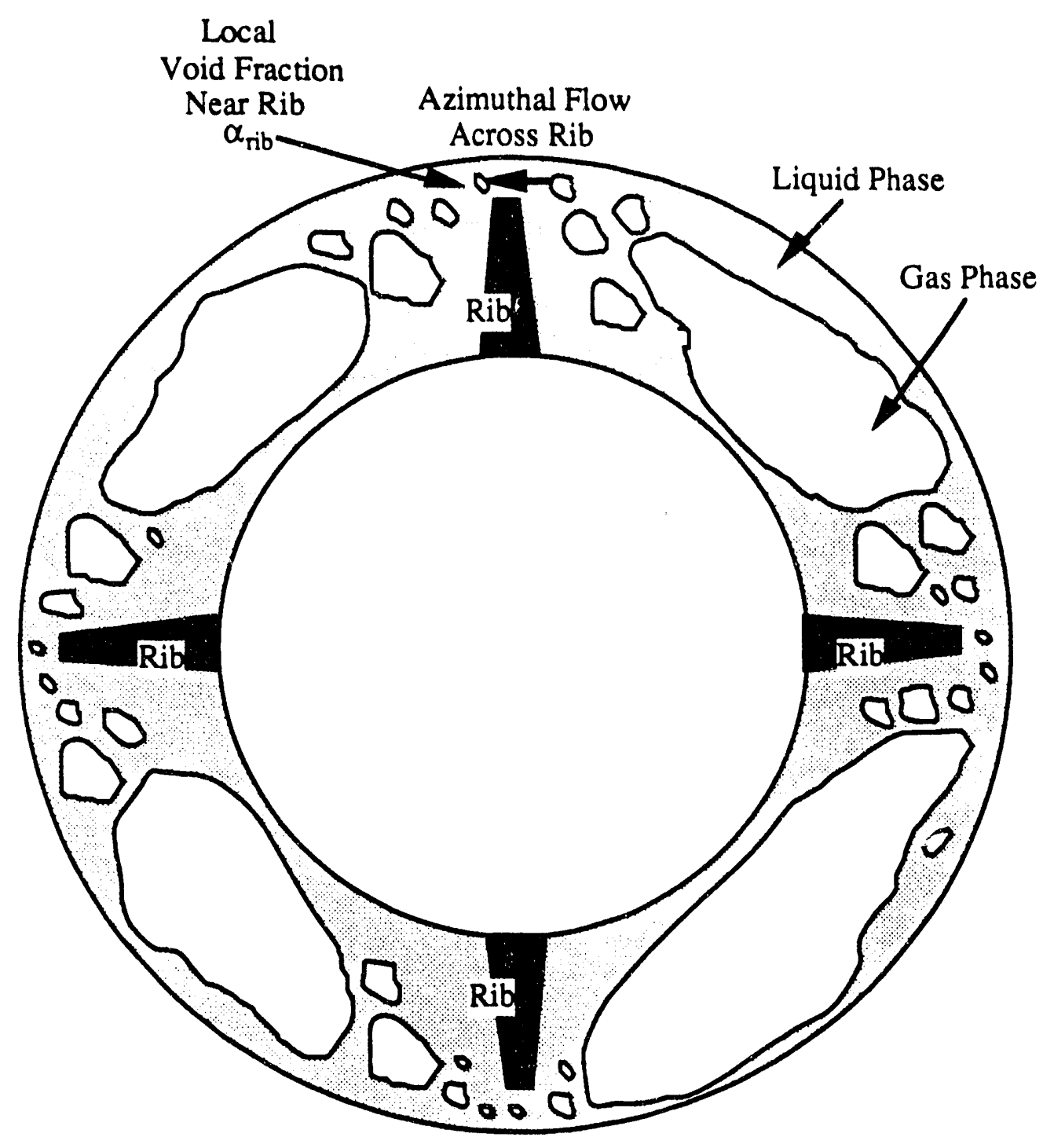

Figure 2.5.7-1 Schematic azimuthal pnase distribution at a given elevation of a ribbed annular channel. 


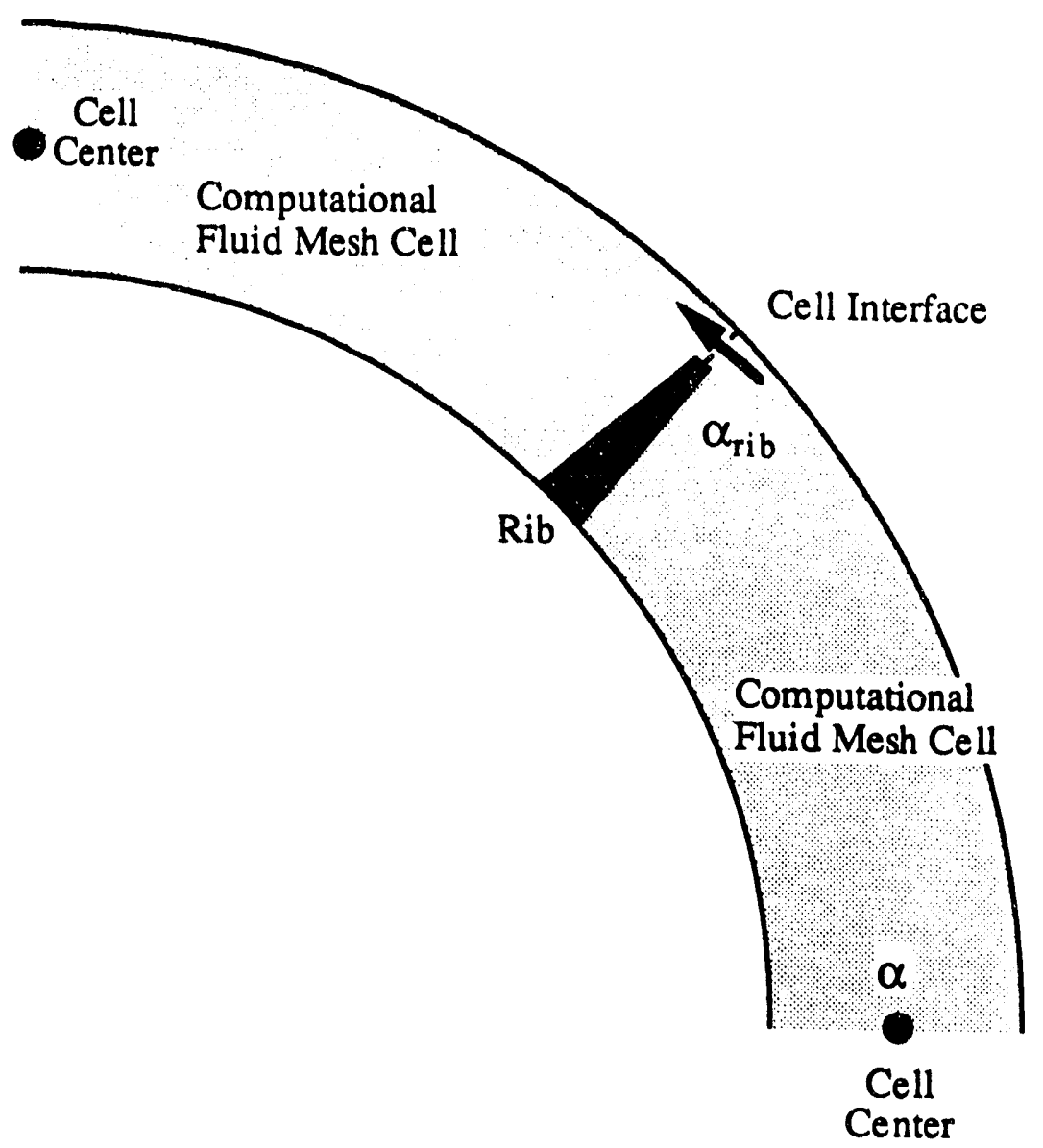

Figure 2.5.7-2 Typical fluid computational meshes of the FLOWTRAN-TF code for a quadrant of ribbed annular channel. 


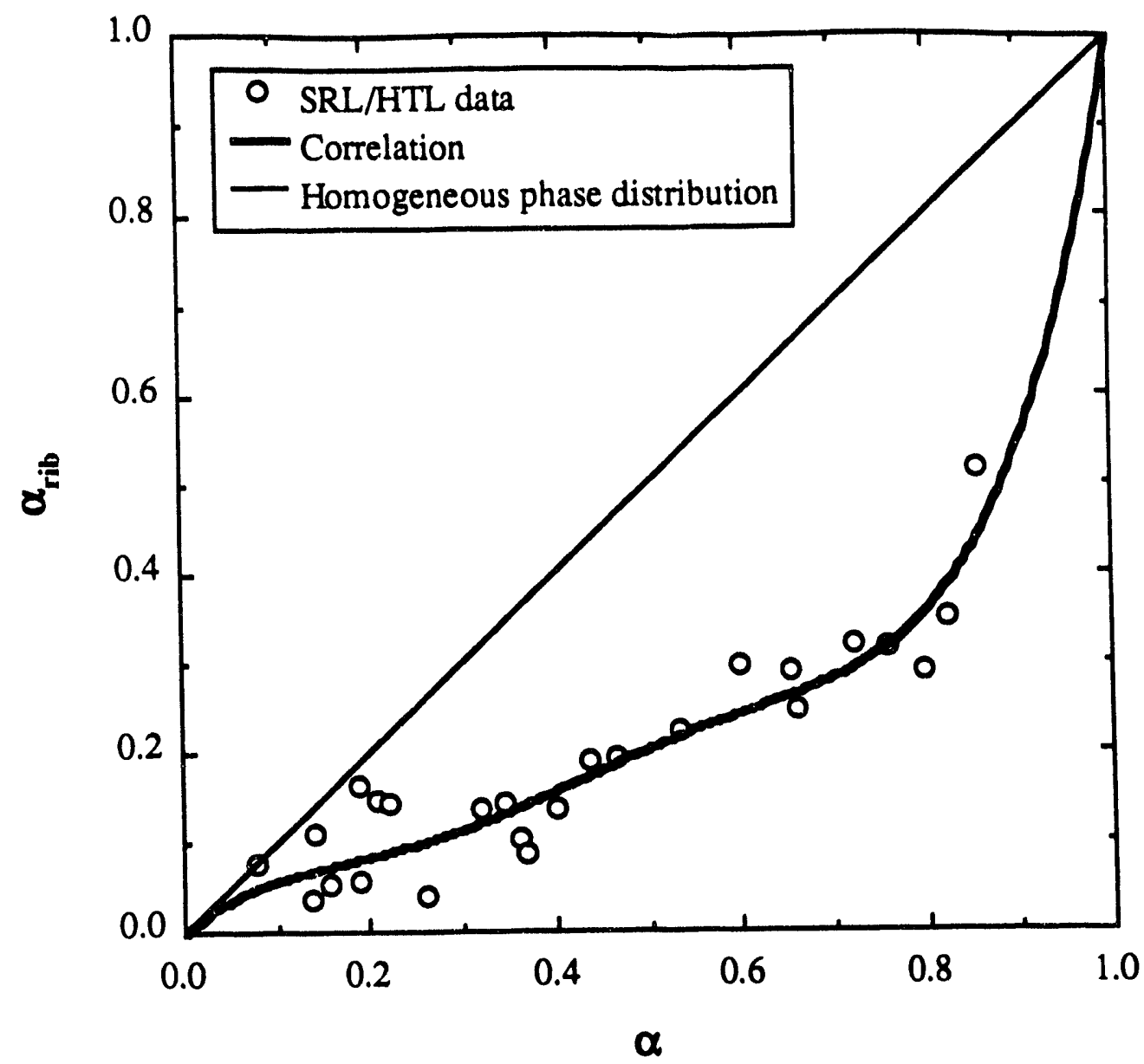

Figure 2.5.7-3 Experimental data of rib void as function of cell averaged void fraction. 
Table 2.5.7-1 SRL data of local void fraction near rib gap region used for rib void correlation (plenum inlet pressure: atmospheric pressure, fluid temperature: about $20^{\circ} \mathrm{C}$ )

\begin{tabular}{|ccccc|}
$\begin{array}{c}\text { Tank Level } \\
\text { (inch) }\end{array}$ & $\begin{array}{c}\text { Water Flowrate } \\
(\mathrm{gpm})\end{array}$ & $\begin{array}{c}\text { Avg. Void } \\
(\alpha)\end{array}$ & $\begin{array}{c}\text { Rib Void - Data } \\
\left(\alpha_{\text {ib }}\right)\end{array}$ & $\begin{array}{c}\alpha_{\text {ib }}-\text { Corr. } \\
\text { (from eq. }(5) \text { ) }\end{array}$ \\
\hline 4.5 & 2.0 & 0.858 & 0.517 & 0.4481 \\
4.5 & 4.0 & 0.799 & 0.290 & 0.3577 \\
4.5 & 8.0 & 0.656 & 0.290 & 0.2654 \\
4.5 & 14.0 & 0.465 & 0.192 & 0.1889 \\
4.5 & 26.0 & 0.207 & 0.145 & 0.0871 \\
4.5 & 32.0 & 0.139 & 0.111 & 0.0706 \\
56 & 12.0 & 0.361 & 0.102 & 0.1410 \\
56 & 24.0 & 0.190 & 0.162 & 0.0829 \\
56 & 30.0 & 0.077 & 0.078 & 0.0510 \\
4.5 & 20.0 & 0.319 & 0.136 & 0.1233 \\
4.5 & 3.0 & 0.825 & 0.351 & 0.3914 \\
4.5 & 6.0 & 0.723 & 0.319 & 0.2961 \\
4.5 & 10.0 & 0.599 & 0.298 & 0.2442 \\
4.5 & 12.0 & 0.534 & 0.222 & 0.2191 \\
4.5 & 16.0 & 0.400 & 0.136 & 0.1587 \\
4.5 & 18.0 & 0.346 & 0.142 & 0.1345 \\
4.5 & 30.0 & 0.136 & 0.036 & 0.0698 \\
56 & 3.0 & 0.760 & 0.317 & 0.3207 \\
56 & 6.0 & 0.662 & 0.245 & 0.2677 \\
56 & 10.0 & 0.435 & 0.190 & 0.1750 \\
56 & 12.0 & 0.368 & 0.085 & 0.1441 \\
56 & 16.0 & 0.262 & 0.040 & 0.1028 \\
56 & 18.0 & 0.223 & 0.143 & 0.0913 \\
56 & 22.0 & 0.190 & 0.056 & 0.0829 \\
56 & 24.0 & 0.157 & 0.053 & 0.0750 \\
& & & & \\
\hline
\end{tabular}

\section{References}

Steimke, J. L. and Fowley M. D., Azimuthal Variation of Void Fraction in Ribbed Annulus (U), WSRC-TR-91-661, February, 1992. Flach, G. P., "Void Fraction Estimation Program Available", NES-CDG-910062, April,
199i.

Flach, G. P., "Esstimated Area Averaged Void Fractions for WRSC-TR-91-661", NESCDG-920014, January, 1992.

Lee, S. Y. , "Rib Void Model Development", WSRC-NB-89-211, p. 113-116, February, 1992. 


\subsubsection{Inter-Subchannel Turbulent Mixing}

The local thermal-hydraulic performance within a fuel assembly is related to the air-water flow structure through the process of crossflow mixing between subchannels since this mixing provides a mechanism to equalize subchannel coolant temperatures. Using an averaged parameter approach, the azimuthal momentum control volume, like the axial momentum control volume, is also staggered with respect to the mass and energy control volume and solved numerically taking into account inter-subchannel interactions such as mixing and diversion crossflow. A subchannel analysis of fluid flow in a fuel assembly includes the effects of heat, mass and momentum transfer in the direction perpendicular to the bulk downward flow. The end product is a map of mass flow rates, enthalpies and void fractions throughout each coolant channel for a given boundary condition. In this case a continuously intercornected set of parallel subchannel flow is coupled to each other by the crossflow. The ccmmunications of fluid-fluid interactions across the rib gap in the azimuthal direction may be driven by the laterally nonuniform phase distribution of twophase flow. The azimuthal two-phase crossflow may be classified into three categories depending on the driving mechanism as follows:

- Diversion crossflow due to azimuthal pressure gradients

- Mixing due to flow fluctuations under zero azimuthal pressure gradient

- Void migration (or void drift) due to the geometrical effect of fuel subchannel on assembly power such as fuel geometry bowing in Mark 22 assembly

Diversion crossflow due to azimuthal pressure gradients is discussed in the section on azimuthal wall drag. Two-phase mixing is defined as the flow interaction between adjacent subchannels that takes place in the absence of any mean pressure difference in the azimuthal direction. As a result of that, there is a net effect on the energy transport across the rib gap between subchannels. For the present analysis, the mixing parameters are described empirically because a definite correlating equation for subchannel mixing of two-phase flow under a given channel geometry has not been developed. This section deals with mixing model including the effect of void migration on phase relaxation within subchannel. The literature data are subsequently compared with the model predictions to ascertain how well they can be predicted.

The mixing due to turbulent flow fluctuation is modeled phenomenologically. When the void fractions in the adjacent subchannels are different, the mixing process transfers momentum as well as mass and energy. This concept is employed in the present modeling. The main assumptions for the model are listed as follows:

- The exchange of equal fluid volume but of different void fraction between adjacent subchannels is assumed as illustrated in Figure 2.5.8-1. This means that the transverse fluctuating velocity for each phase is assumed to be equal.

- The net energy transfer due to two-phase turbulent mixing between two neighboring subchannels is caused by net mass exchange only for the differences in the void fractions of adjacent subchannels.

From the assumption of equal fluid volume exchange between adjacent subchannels across their separating gap, 


$$
Q_{i j}^{\prime}=Q_{j i}^{\prime}
$$

where the superscript ' means the fluctuating quantity.

For the appropriate cross sectional area,

$$
u_{i j}^{\prime}=u_{j i}^{\prime}
$$

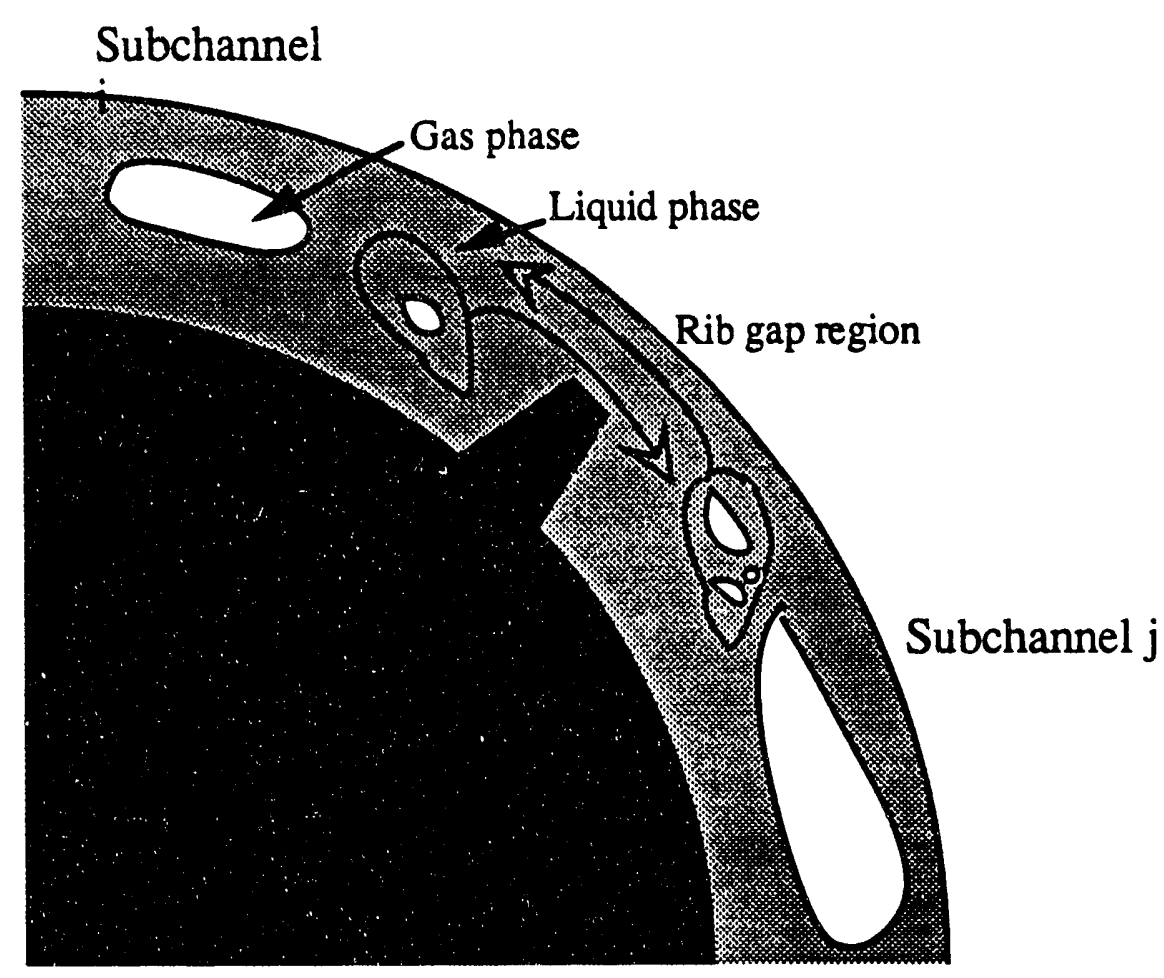

Figure 2.5.8-1 Equal fluid volume mixing between adjacent two subchannels.

The net fluctuating transverse mass flowrate per unit length across the gap between subchannels $i$ and $j\left(W_{\text {net }}\right.$ ij) is written in terms of Prandtl mixing length theory and equation (2.5.8-2) as follows?

$$
\begin{aligned}
W_{\text {net, }, \mathrm{ij}}^{\prime} & =\rho_{\mathrm{i}} \mathrm{u}_{\mathrm{ij}}^{\prime} s_{\mathrm{ij}}-\rho_{\mathrm{j}} \mathrm{u}_{\mathrm{ji}}^{\prime} s_{\mathrm{ij}} \\
& =u_{\mathrm{ij}}^{\prime} s_{\mathrm{ij}}\left(\rho_{\mathrm{i}}-\rho_{\mathrm{j}}\right) \\
& =\left(\frac{\varepsilon}{\ell}\right)_{t \mathrm{p}} s_{\mathrm{ij}}\left(\rho_{\mathrm{i}}-\rho_{\mathrm{j}}\right)
\end{aligned}
$$

where $s_{i j}$ is the clearance of rib gap and $\rho_{i}$ and $\rho_{j}$ mixture density for subchannels $i$ and $j$ respectively. 
In equation (2.5.8-3), $\varepsilon$ and $\ell$ are the eddy diffusivity and mixing length respectively, and $(\varepsilon / \ell)_{\mathrm{tp}}$ is two-phase mixing velocity. The mixing length represents the lateral distance travelled by the fluid packet associated with the fluctuating velocity $\mathrm{u}_{\mathrm{ij}}^{\prime}$. In single phase case where the subchannel densities are equal, equation (2.5.8-3) becomes zero, i.e., the net mass transfer between $i$ and $j$ subchannels is zero. In two-phase flow, there is nonzero net mass transfer rate due to flow fluctuation since the mixture density within each subchannel may not be equal due to nonuniform phase distribution. Equation (2.5.8-3) can be written in terms of average subchannel void fraction difference between neighboring subchannels.

$$
W_{\text {net. }, \mathrm{ij}}^{\prime}=\left(\frac{\varepsilon}{\ell}\right)_{\mathrm{qp}} s_{\mathrm{ij}}\left(\rho_{\mathrm{f}}-\rho_{\mathrm{g}}\right)\left(\alpha_{\mathrm{j}}-\alpha_{\mathrm{i}}\right)
$$

where $\rho_{f}$ and $\rho_{g}$ are the average liquid and gas densities respectively.

When the net mass transfer rates per unit length for gas and liquid phase are defined as equations (2.5.8-5) and (2.5.8-6), i.e.,

$$
\mathrm{W}_{\mathrm{gij}}^{\prime} \equiv\left(\frac{\varepsilon}{\ell}\right)_{\varphi} s_{\mathrm{ij}} \rho_{\mathrm{g}}\left(\alpha_{\mathrm{i}}-\alpha_{\mathrm{j}}\right)
$$

and

$$
W_{\mathrm{fij}}^{\prime} \equiv\left(\frac{\varepsilon}{\ell}\right)_{\mathrm{tp}} s_{i j} \rho_{\mathrm{f}}\left(\alpha_{\mathrm{j}}-\alpha_{\mathrm{i}}\right)
$$

equation $(2.5 .8-4)$ becomes

$$
W_{\text {net, }, \mathrm{ij}}^{\prime}=W_{\text {fij }}^{\prime}+W_{g i j}^{\prime}
$$

Thus, the net mass transfer rate consists of a net gas mass flowrate from $j$ to $i$ subchannels and a net liquid mass flowrate from $i$ to $j$ subchannels when void fraction of $j$ subchannel $\left(\alpha_{j}\right)$ is greater than that of $i$ subchannel $\left(\alpha_{i}\right)$.

The net energy transfer rate per unit length due to flow fluctuation between $i$ and $j$ subchannels is written as follows:

$$
W_{\text {net, }, \mathrm{ij}}^{\mathrm{H}}=\left(\frac{\varepsilon}{\ell}\right)_{\mathrm{tp}} s_{\mathrm{ij}}\left(\rho_{\mathrm{i}} h_{\mathrm{i}}-\rho_{\mathrm{j}} \mathrm{h}_{\mathrm{j}}\right)
$$

where $h_{i}$ and $h_{j}$ are mixture enthalpies of subchannel $i$ and $j$ respectively.

When the net energy transfer rate for each phase is defined as

$$
W_{g i j}^{\prime H} \equiv\left(\frac{\varepsilon}{\ell}\right)_{t p} s_{i j j} \rho_{g} h_{g}\left(\alpha_{i}-\alpha_{j}\right)
$$

and 


$$
W_{\mathrm{fij}}^{\mathrm{H}} \equiv\left(\frac{\varepsilon}{\ell}\right)_{\mathrm{qp}} s_{\mathrm{ij}} \rho_{\mathrm{f}} h_{\mathrm{f}}\left(\alpha_{\mathrm{j}}-\alpha_{\mathrm{i}}\right)
$$

equation (2.5.8-8) can be written as follows:

$$
W_{\text {net, }, \mathrm{ij}}^{\mathrm{H}}=\mathrm{W}_{\mathrm{fij}}^{\mathrm{H}}+\mathrm{W}_{\mathrm{gij}}^{\mathrm{H}}
$$

From the above equations, the mixing rate is finite whenever there is a difference in subchannel void fraction between the adjacent subchannels $\mathrm{i}$ and $\mathrm{j}$. Figure 2.5.8-2 illustrates the net exchange of mass and energy for each phase between subchannels $i$ and j. This implies that subchannel mixing drives adjacent subchannel void fractions in each coolant channel (inner, middle and outer channels) toward equality. In this case, void drift effect, which makes the void migrated to the subchannel with the larger cross sectional area and higher velocity, may be negligible because all four subchannels in each annular channel are almost identical in terms of geometrical shape and dimension even if annular fuel rod eccentricity occurs. The net momentum mixing rate per unit length between $i$ and $j$ subchannels is determined based on equal fluid volume exchange.

$$
W_{\text {net, }, \mathrm{ij}}^{\prime M}=\left(\frac{\varepsilon}{\ell}\right)_{\mathrm{tp}} s_{\mathrm{ij}}\left(\rho_{\mathrm{i}} u_{\mathrm{i}}-\rho_{\mathrm{j}} u_{\mathrm{j}}\right)
$$

In equation (2.5.8-12) $u_{i}$ and $u_{j}$ are the average velocities of subchannels $i$ and $j$ respectively. When net mixing momentum transfer rate per unit length for each phase is defined as

$$
W_{g i j}^{M} \equiv\left(\frac{\varepsilon}{\ell}\right)_{t p} s_{i j}\left(G_{g i}-G_{g j}\right)
$$

and

$$
W_{f i j}^{\prime M} \equiv\left(\frac{\varepsilon}{\ell}\right)_{t p} s_{i j}\left(G_{f i}-G_{f j}\right)
$$

equation (2.5.8-12) becomes

$$
W_{n e t, i j}^{\prime M}=W_{f i j}^{\prime M}+W_{g i j}^{\prime M}
$$




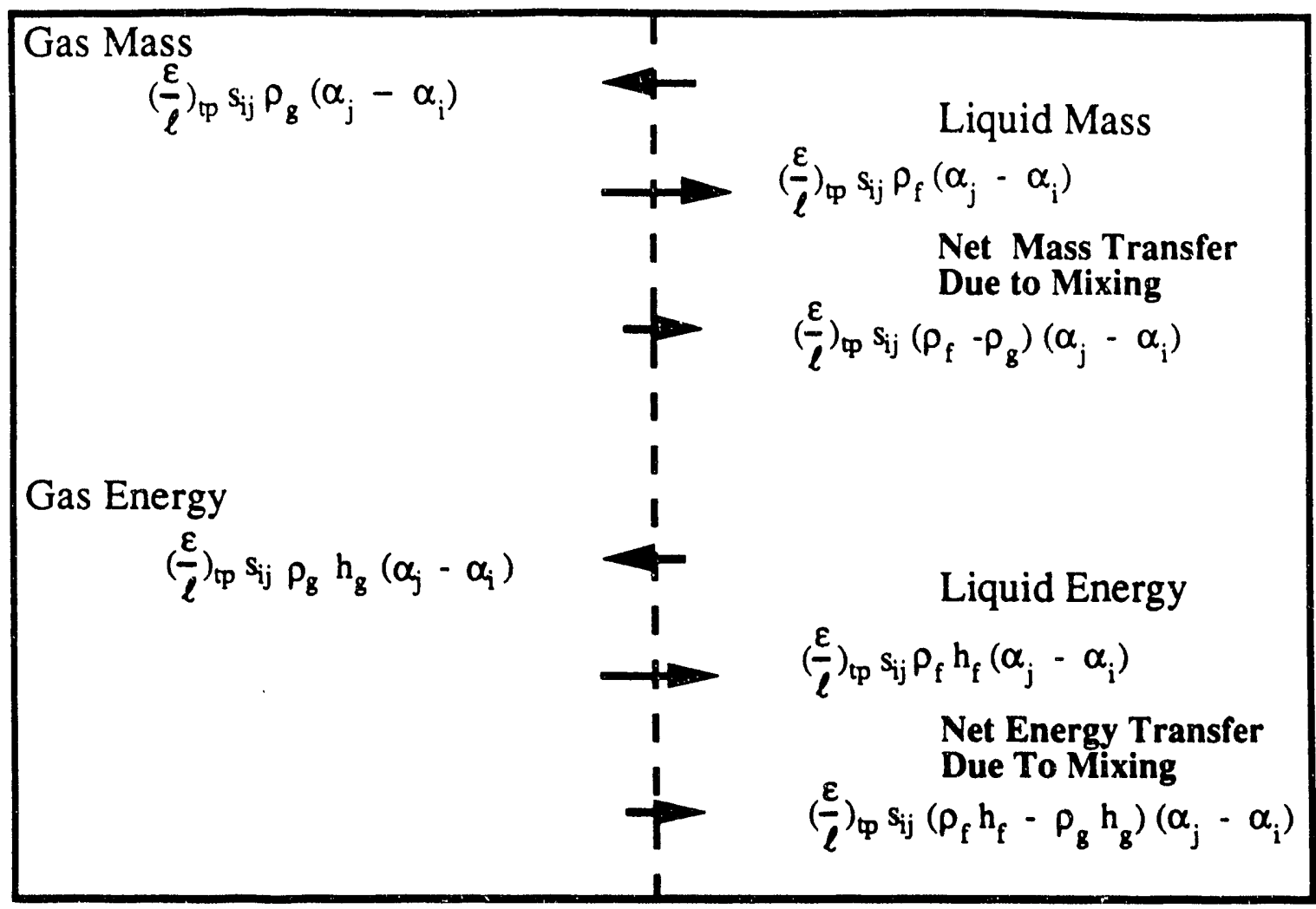

(i subchannel)

( $\mathrm{j}$ subchannel)

Figure 2.5.8-2 Mass and energy exchange rate per unit height between adjacent subchannels based on equal fluid volume mixing for $\alpha_{i}<\alpha_{j}$.

The terms, which represent the subchannel mixing effects in mass, momentum and energy equations, have been identified. The resulting conservation equations are listed in Table $2.5 .8-1$. In mixing terms, $(\varepsilon / \ell)_{\text {tp }}$, which has the dimension of a velocity and is referred to as two-phase mixing velocity, can be found from the two-phase mixing measurements by the following equation:

$$
\left(\frac{\varepsilon}{\ell}\right)_{\varphi}=\frac{W^{\prime}}{\rho_{m} s_{i j}}
$$

where $\rho_{m}$ is the averaged density based on the two-phase mixture, i.e., $0.5\left(\rho_{i}+\rho_{j}\right)$ and $W^{\prime}$ two-phase mixing rate per unit height.

Once the mixing velocity is found from the experimental data, mixing terms in each conservation equation can be determined. From the experimental observations of several authors in the literature, mixing rate must be dependent on the flow regime and subchannel geometry (Lahey and Shraub, 1969: Gonzalez-Santalo and Griffith, 1972).

The flow regime effect is considered by utilizing the ratio of air-water turbulent mixing velocity, $(\varepsilon / \ell)_{\mathrm{tp}}$, to single phase value, $(\varepsilon / \ell)_{\mathrm{sp}}$, and the geometrical impact on subchannel 
mixing is included through the single phase correlation for turbulent velocity. Following the Beus' work (1970), two-phase turbulent velocity is written as

$$
\left(\frac{\varepsilon}{\ell}\right)_{\mathrm{tp}}=\theta\left(\frac{\varepsilon}{\ell}\right)_{\mathrm{sp}}
$$

where $\theta$ is a two-phase multiplication factor and a function of flow regime.

In equation (2.5.8-17), Rogers and Tahir's (1975) correlation for single phase turbulent velocity in the square-square subchannel can be used for the present work. That is,

$$
\left(\frac{\varepsilon}{\ell}\right)_{s p}=0.0025 \operatorname{Re}_{i}^{-0.1}\left\{1+\left(\frac{D_{h j}}{D_{h i}}\right)^{1.5}\right\}\left(\frac{W-s_{i j}}{s_{i j}}\right)^{0.894}\left(\frac{D_{h i}}{W-s_{i j}}\right)\left(\frac{G_{m i}}{\rho_{m}}\right)
$$

where

$$
\operatorname{Re}_{i}=\frac{G_{m i} D_{h i}}{\alpha_{i} \mu_{g}+\left(1-\alpha_{i}\right) \mu_{f}}
$$

In equation (2.5.8-18), mass velocity $\left(G_{m i}\right)$ and density $\left(\rho_{m}\right)$ are based on the two-phase mixture for $i$ subchannel and $D_{h i}$ and $D_{h j}$ are the hydraulic diameters for $i$ and $j$ subchannels respectively. Parameters of $\mathrm{W}$ and $s_{\mathrm{ij}}$ are denoted in Table 2.5.8-2. With equation (2.5.8-17) $\theta$ is determined by the air-water literature data through equation (2.5.8-16). In the literature, two-phase mixing rate was measured in terms of flow quality under air-water cocurrent upward flow experiment related to the subchannel analysis for the application of commercial nuclear power plant. 
Table 2.5.8-1 Governing equation set including subchannel mixing terms.

\begin{tabular}{|c|c|}
\hline & Conservation Equations \\
\hline $\begin{array}{l}\text { Liquid } \\
\text { Phase }\end{array}$ & $\begin{array}{l}\text { Mass: } \\
\qquad \begin{array}{l}\frac{\partial \alpha \rho_{g}}{\partial t}+\frac{\partial \alpha \rho_{g} w_{g}}{\partial x}+\frac{\partial \alpha \rho_{g} u_{g}}{\partial z}=\Gamma_{I}+\Gamma_{w}-W_{t g} \\
\frac{\partial \alpha \rho_{g} X_{a}}{\partial t}+\frac{\partial \alpha \rho_{g} w_{g} X_{a}}{\partial x}+\frac{\partial \alpha \rho_{g} u_{g} X_{a}}{\partial z}=-X_{a} W_{t g} \\
\frac{\partial(1-\alpha) \rho_{f}}{\partial t}+\frac{\partial(1-\alpha) \rho_{f} w_{f}}{\partial x}+\frac{\partial(1-\alpha) \rho_{f} u_{f}}{\partial z}=-\Gamma_{I}-\Gamma_{w}-W_{t f}\end{array}\end{array}$ \\
\hline $\begin{array}{l}\text { Gas } \\
\text { Phase } \\
\text { Liquid } \\
\text { Phase }\end{array}$ & 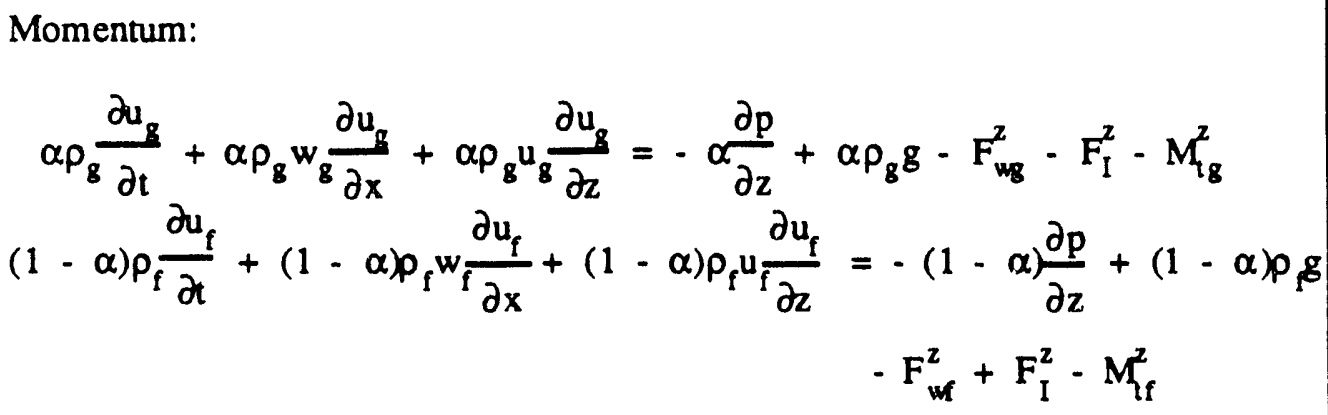 \\
\hline $\begin{array}{l}\text { Gas } \\
\text { Phase }\end{array}$ & $\begin{array}{l}\text { Energy: } \\
\frac{\partial \alpha \rho_{g} h_{g}}{\partial t}+\frac{\partial \alpha \rho_{g} h_{g} w_{g}}{\partial x}+\frac{\partial \alpha \rho_{g} h_{g} u_{g}}{\partial z}=\alpha \frac{\partial p}{\partial t}+Q_{w g}+Q_{f}-Q_{g} \\
\frac{\partial(1-\alpha) \rho_{f} h_{f}}{\partial t}+\frac{\partial\left(1-\alpha p_{f} h_{f} w_{f}\right.}{\partial x}+\frac{\partial(1-\alpha) p_{f} h_{f} u_{f}}{\partial z}=(1-\alpha) \frac{\partial p}{\partial t}+Q_{w f}-Q_{q}-Q_{f f}\end{array}$ \\
\hline Mixir & $\begin{aligned} W_{t k}= & \sum_{j} \frac{1}{A_{i}} W_{k j}^{\prime} \quad M_{t k}^{2}=\sum_{j} \frac{1}{A_{i}} W_{k, j}^{M} Q_{k}=\sum_{j} \frac{1}{A_{i}} W_{k j}^{H} \\
& \text { where } k=g \text { or } f \text { and } A_{i}=\text { flow area of } i \text { subchannel. }\end{aligned}$ \\
\hline
\end{tabular}

Most of two-phase mixing data have been collected from the simulated rod bundle geometry as seen in Table 2.5.8-2. For the present subchannel mixing analysis, the turbulent velocity fitted by these data is applicable to the present cocurrent downward twophase flow when the flow fluctuation is assumed not to be sensitive to the flow orientation. A rigorous basis for this assumption is not referenced, but from the viewpoint of the mixing phenomenon defined under zero pressure gradient it is considered as a reasonable approach. As a major simplification in the subchannel analysis, it is also assumed that any 


\begin{tabular}{|l|lll|l|}
\hline $2 / 15 / 93$ & WSRC-TR-92-532 & Rev. 0 & FLOWTRAN-TF Software Design & Page 289 \\
\hline
\end{tabular}

crossflow through the gap region between subchannels loses its sense of flow direction after leaving the gap region.

However, flow quality information is not always available for the present cocurrent downward air-water flow situation since it can not be defined if flow regime is changed to countercurrent two-phase flow due to the dominant buoyancy of gas phase. In order to avoid this situation, the flow quality information was converted to the void fraction data base for given hydraulic conditions (total mass flux, flow quality and axial pressure gradient) and test section geometry using the axial two-phase mixture momentum balance employed in the wall drag benchmarking of the FLOWTRAN-TF code (Flach et al., 1990 and Flach, 1991) since void fraction was not measured in the literature experiment.

Beus developed the turbulent mixing rate for rod bundle geometry in terms of flow quality. For the present analysis, a functional form for two-phase multiplier $(\theta)$ is written in terms of void fraction. By substituting appropriate constants into his correlational form based on Singh's (1972) data, the two-phase multiplier $(\theta)$ is given depending on the flow regime map.

For the bubbly slug flow regime $\left(\alpha<\alpha_{m l}\right)$,

$$
\theta=1+\left(\theta_{\mathrm{m}}-1\right) \frac{\alpha}{\alpha_{\mathrm{ml}}}
$$

For the slug-annular transition regime $\left(\alpha_{m l}<\alpha<\alpha_{m u}\right)$,

$$
\theta=\theta_{\mathrm{m}}
$$

For the annular flow regime $\left(\alpha>\alpha_{\mathrm{mu}}\right)$,

$$
\theta=1+\left(\theta_{m}-1\right) \frac{\alpha_{m u}-c}{\alpha-c}
$$

where

$$
c=0.52 \alpha_{\mathrm{mu}} \operatorname{Re}^{0.045} .
$$

$\theta_{\mathrm{m}}$ was found to be 11.0 based on the literature data (Singh, 1972) since the gap clearance and subchannel hydraulic diameters of his test section are similar to those of equivalent Mark 22 assembly as illustrated in Table 2.5.8-2. The maximum two-phase multiplier $\left(\theta_{m}\right)$ was shown to occur near the slug-annular flow regime from the experimental observations (Petrunik and Pierre (1970); Singh and Pierre (1972)). 
Table 2.5.8-2 Comparison of equivalent Mark 22 subchannel with the test sections of air-water mixing experiments in the literature.

\section{Mark 22 Subchannel Geometry}

\begin{tabular}{|c|cc|c|}
\hline Equivalent Subchannel Geometry & $\begin{array}{c}\text { Nominal Gap Size }\left(\mathrm{s}_{\mathrm{ij}}\right) \\
\text { (mils) }\end{array}$ & $\begin{array}{c}\text { Hydraulic Diameter } \\
(\mathrm{ft})\end{array}$ \\
\hline & Inner Channel & 15.0 & 0.0282 \\
\hline & Middle Channel & 15.0 & 0.0387 \\
\hline & Outer Channel & 15.0 & 0.0261 \\
\hline
\end{tabular}

\section{Literature Geometry for Air-Water Experiment}

\begin{tabular}{|c|c|c|c|c|}
\hline $\begin{array}{l}\text { Author } \\
\text { (Pub. yr.) }\end{array}$ & Test Section Geometry & $\begin{array}{l}\operatorname{Gap} \operatorname{Size}\left(\mathbf{s}_{\mathrm{ij}}\right) \\
\text { (mils) }\end{array}$ & $\begin{array}{l}\text { System } \\
\text { Pressure } \\
\text { (psia) }\end{array}$ & $\begin{array}{l}\text { Hydraulic Diameter } \\
\text { (ft) }\end{array}$ \\
\hline $\begin{array}{l}\text { Petrunik } \\
\text { (1968) }\end{array}$ & & 100 & 50 & 0.0661 \\
\hline $\begin{array}{l}\text { Singh } \\
(1972)\end{array}$ & & $15,35,80$ & 50 & $\begin{array}{l}0.0272,0.0287 \\
0.0315\end{array}$ \\
\hline
\end{tabular}

If the range of void fraction for the slug-annular regime, i.e., $\alpha_{\mathrm{ml}}$ and $\alpha_{\mathrm{mu}}$, is prescribed by the flow regime map, the function describing the mixing rate is complete. In the FLOWTRAN-TF code, the slug-annular transition occurs at the void fraction between $\alpha_{m}$ $=0.52$ and $\alpha_{\mathrm{mu}}=0.75$ (Flach et al., 1991). The resulting behavior for $\theta$ is shown in Figure 2.5.8-3.

In order to examine the predictive capability of mixing rate against the data, the model predictions were compared with the data available in the literature. Equations (2.5.8-16) through (2.5.8-22) were employed to compute two-phase mixing rate (W') under the square-square subchannel geometry (e.g., Petrunik's test section), which is found in the literature as illustrated in Table 2.5.8-2. The predicted results were compared to the data in 


\begin{tabular}{|l|lll|l|}
\hline $2 / 15 / 93$ & WSRC-TR-92-532 Rev. 0 & FLOWTRAN-TF Software Design & Page 291 \\
\hline
\end{tabular}

Figure 2.5.8-4. The experimental data and predictions are also tabulated in the Lee (1992). Although there is considerable scatter for the very high void region as illustrated in Figure 2.5.8-4, benchmarking results show reasonable predictions considering experimental uncertainty and the fact that the single-phase mixing correlation was developed from a limited number of experimental data.

As mentioned earlier, it is apparent that the mixing effect under two-phase condition is strongly enhanced relative to the single-phase flow situation. This enhancement increases with void fraction until the slug-annular transition. It then decreases rapidly through the annular flow regime with increased void fraction. This tendency has been confirmed by several investigators (Petrunik and Pierre (1970); Gonzalez-Santalo and Griffith (1972); Singh and Pierre (1973)) in the low-pressure air-water cocurrent upward experiment. When the mixing data for Mark 22 subchannel geometry under cocurrent downward twophase flow are available, the present mixing correlation will be benchmarked against the experimental data.

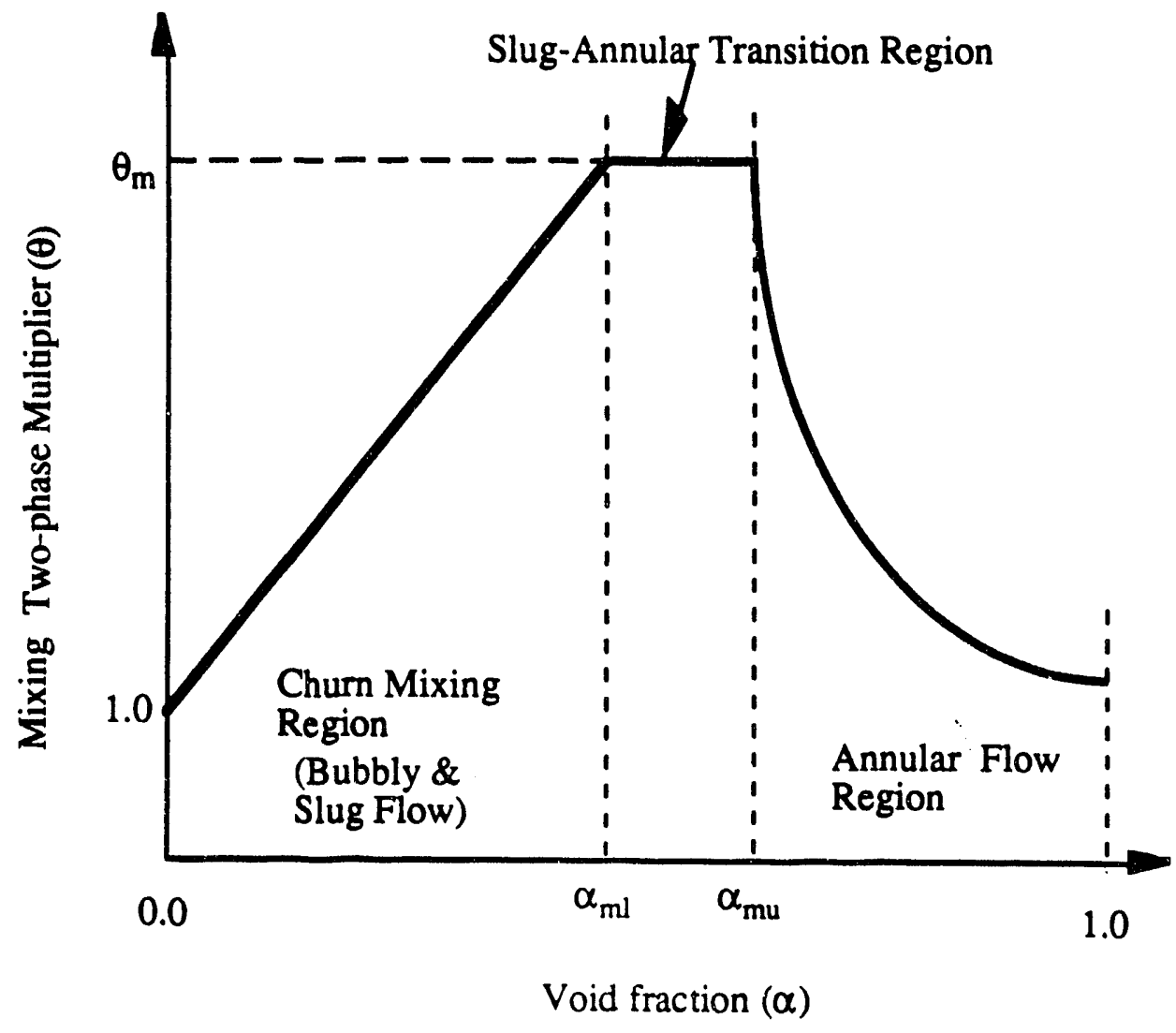

Figure 2.5.8-3 Air-water turbulent mixing rate as a function of flow regime. 


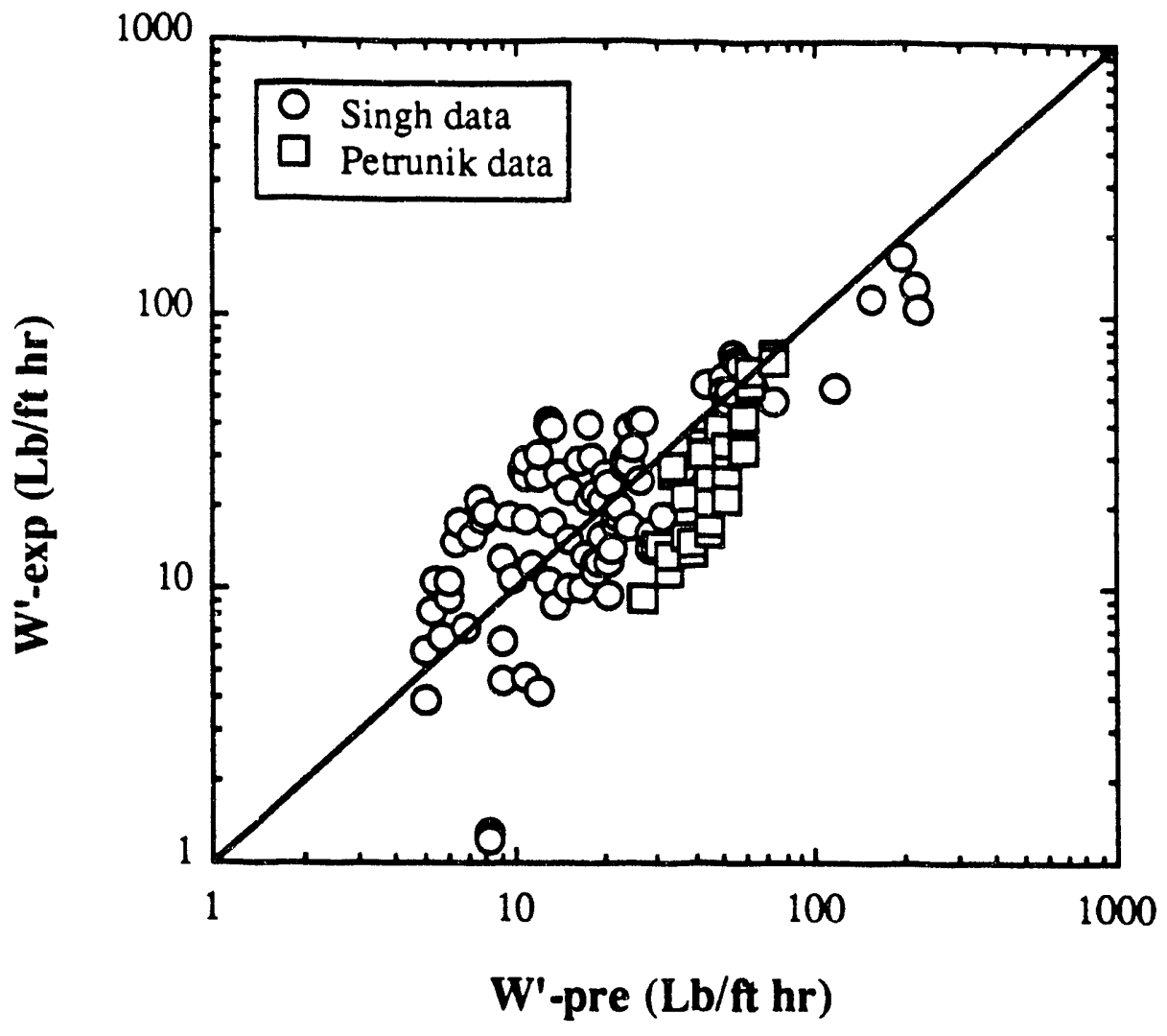

Figure 2.5.8-4 Comparison of subchannel mixing predictions with experimental data.

\section{References}

R. T. Lahey and F. A. Shraub, "Mixing, Flow Regimes and Void Fraction for Two-phase Flow in Rod Bundles", Two-phase Flow and Heat Transfer in Rod Bundles, Winter Annual Meeting, ASME, 1969.

R. T. Lahey and F. J. Moody, "The Thermal-hydraulics of a Boiling Water Nuclear Reactor", ANS Monograph, 1979

J. M. Gonzalez-Santalo and P. Griffith, "Two-phase Flow Mixing in Rod Bundle Subchannels", ASME Winter Annual Meeting, New York, November 1972.

S. Sugawara and Y. Miyamoto, "FIDAS: Detailed Subchannel Analysis Code Based On the Three-Fluid and Three-Field Model", Nuclear Engineering and Design, Vol. 120 , pp. 147-161, 1991.

G. P. Flach, S. E. Aleman, L. L. Hamm, S. Y. Lee, F. G. Smith, and V. Van Brunt, "FLOWTRAN-TF Code Description (U)", WSRC-TR-90-413 Rev. 1, September 1991.

G. P. Flach, S. E. Aleman, S. Y. Lee, and F. G. Smith, "FLOWTRAN-TF Code Benchmarking (U)", WSRC-TR-90-414, December, 1990. 
J. T. Rogers and R. G. Rosehart, "Mixing by Turbulent Interchange in Fuel Bundles: Correlations and Influences", ASME 72-HT-53, 1972. G. P. Flach, "Void Fraction Estimation Program Available", NES-CDG-910062, April 22,
1991.

J. T. Rogers and A. E. E. Tahir, "Turbulent Interchange Mixing in Fuel Bundles and the Role of Secondary Flows", ASME 75-HT-31, 1975.

G. B. Wallis, "One-dimensional Two-phase Flow", McGraw-Hill, 1969.

S. G. Beus, "Two-phase Turbulent Mixing Model for Flow in Rod Bundles", WAPD-T2438, 1970.

K. Singh, "Air-Water Turbulent Mixing in Simulated Rod Bundle Geometries", Ph. D. Dissertation, Chemical Engineering, University of Windsor, Windsor, Ontario, 1972.

K. Singh and C. C. St. Pierre, "Two-phase Mixing for Annular Flow in Simulated Rod Bundle Geometries", Nuclear Science and Engineering, Vol. 50, pp. 382-401, 1973.

K. J. Petrunik, "Turbulent Mixing Measurements for Single Phase Air, Single Phase Water, and Two-phase Air-Water Flows in Adjacent Rectangular Subchannels", MASc Thesis, Chemical Engineering, University of Windsor, Windsor, Ontario, 1968.

K. J. Petrunik and C. C. St. Pierre, "Turbulent Mixing Rate for Air-Water Two-phase Flows in Adjacent Rectangular Channels,", The Canadian J. of Chemical Engineering, Vol. 48, pp. 123-125, 1970.

S. Y. Lee, "Subchannel Mixing", QA Task 89-021-1 document number TF-4 Rev. 3, 1992. 


\subsection{Boundary Conditions}

Four inlet boundary conditions options are provided to the user in FLOWTRAN-TF. The basic inlet boundary condition is prescribed pressure $P$, void fraction $\alpha$, gas temperature $T_{g}$, liquid temperature $T_{f}$, and air mass fraction $X_{a}$. Inlet densities and enthalpies are computed from $P, T_{g}, T_{f}$ and $X_{a}$. Pressure and void fraction, in conjunction with gas and liquid finite differenced momentum balances at the inlet cell face, provide the driving force for gas and liquid flow. The inlet gas and liquid flowrates are unspecified and part of the FLOWTRAN-TF solution. The basic boundary condition is user option \#1 and termed "Prescribed pressure and void".

Option \#2 is termed "Prescribed liquid volumetric flowrate". This boundary condition is the same as option \#1 except that the liquid momentum balance at the inlet cell face is replaced with a prescribed velocity expression which yields the desired liquid flowrate. The gas momentum balance is retained as is. Inlet pressure and void fraction no longer provide the driving force for liquid flow because the liquid momentum balance has effectively been discarded. Option \#3, "Prescribed gas volumetric flowrate", is analogous to option \#2 with the gas and liquid phases reversing roles. With Option \#4, "Prescribed liquid and gas volumetric flowrate", both momentum balances at the inlet face between the Plenum and Top Section are replaced with prescribed velocity statements. Option \#1 is generally preferred over options \#2-4 because both momentum balances are prescribed at the inlet cell face. Options \#2-4 are provided to allow the user to easily prescribe flowrate(s) but are less mechanistic. Options for correcting the Plenum pressure for options \#2 and 4 such that the liquid momentum balance is approximately satisfied are presented in section 3.7. A recommended technique for effectively prescribing liquid flowrate using option $\# 1$ is discussed in section 3.7 too.

Only a prescribed pressure and void fraction boundary condition is rermitted at the Tank Bottom boundary cell.

\section{Horizontal, stratified flow}

For a horizontal, stratified flow an additional term appears in each of the momentum balances. As discussed in section 2.4 .1, both phases are generally assumed to have the same pressure (see eqn. (2.4.1-62)). The assumption is accurate except for horizontal, stratified two-phase flows as shown in Figure 2.6-1. Note that the average pressures of the phases differ significantly. Modifications to the gas and liquid momentum balances needed to accurately model horizontal, stratified flow are derived in section 3.7. In both reactor assembly and experimental rig FLOWTRAN-TF applications, horizontal, stratified flow frequently exists at the cell face between the Plenum boundary cell and the first Top Section cell in the fluid computational mesh as shown schematically in Figure 2.6-2. All other cell faces are typically either vertical or if horizontal do not involve stratified flow. Therefore attention can be confined to the cell face at $j=1 / 2$ shown in Figure $2.6-3$ in the treatment of horizontal, stratified flow. The required modifications to the one-dimensional phasic momentum balances at $j=1 / 2$ could be derived by reeviluating certain terms derived in section 2.4.1. However, a simpler approach is presented in section 3.7 which better illustrates the physics involved. 


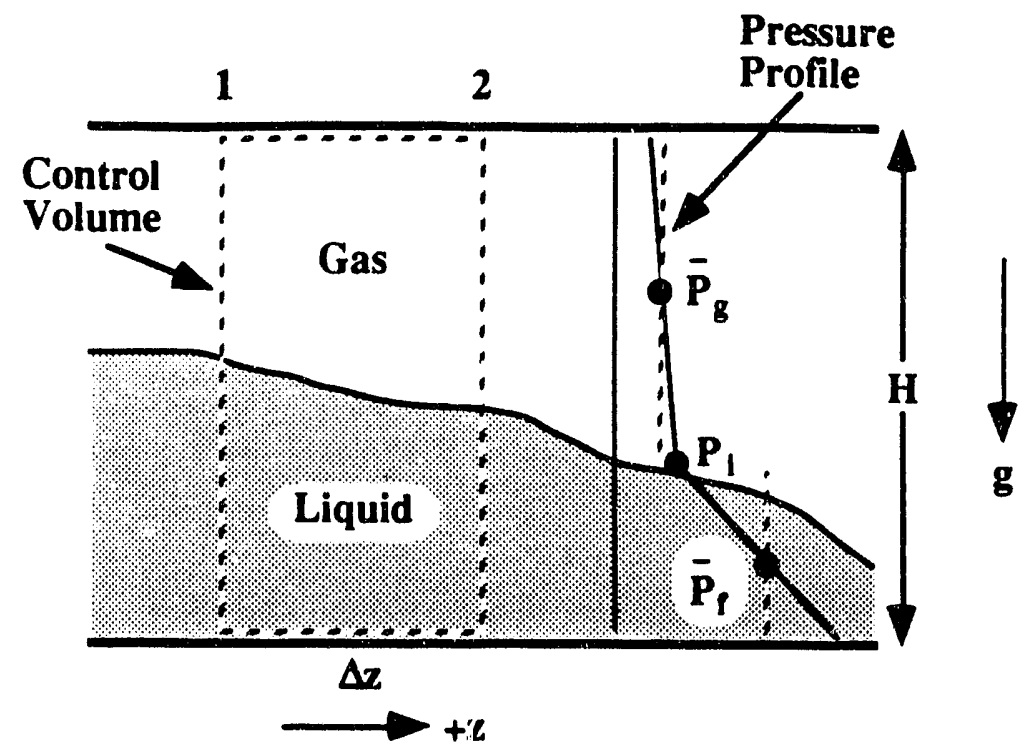

Figure 2.6-1 Control volume and nomenclature for horizontal, stratified flow.

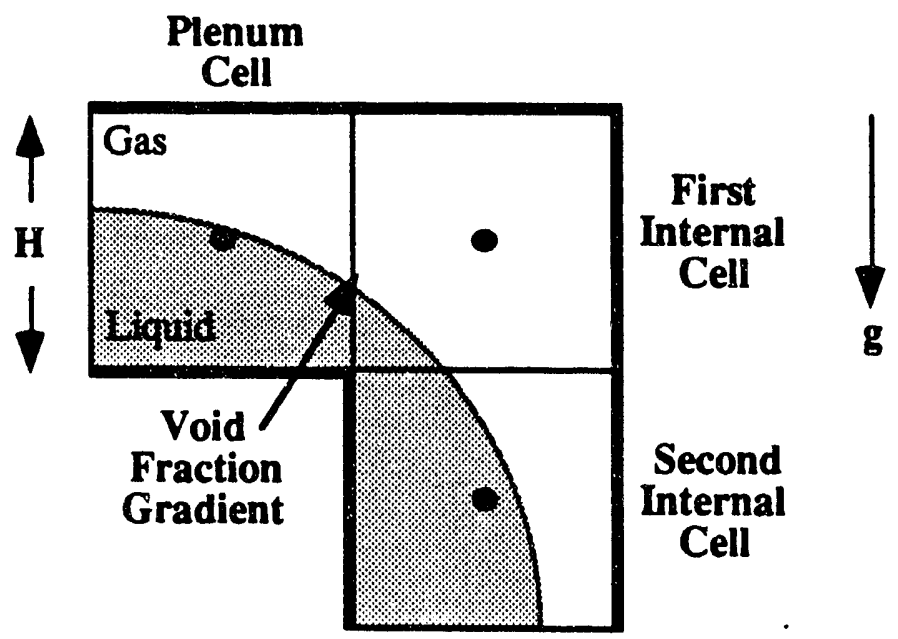

Figure 2.6-2 Horizontal, stratified flow at the cell face between the Plenum and Top Section. 


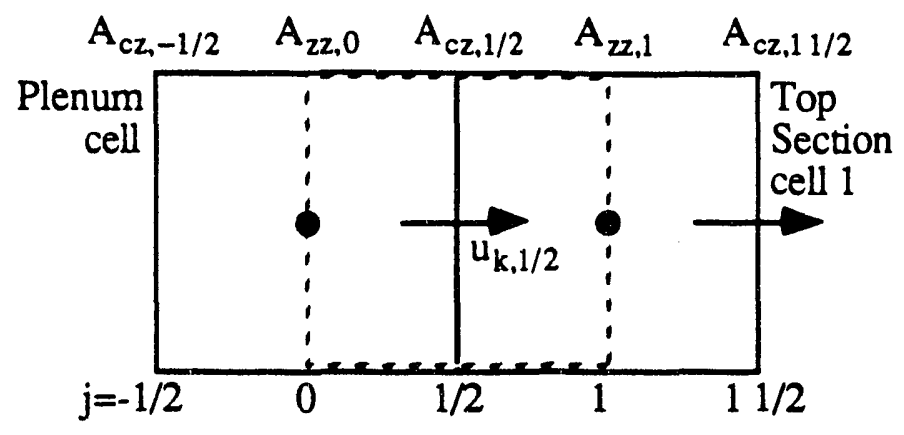

Figure 2.6-1 Momentum computational cell at inlet plenum. 


\begin{tabular}{|l|lll|l|}
\hline $2 / 15 / 93$ & WSRC-TR-92.532 Rev. 0 & FLOWTRAN-TF Software Design & Page 297 \\
\hline
\end{tabular}

\subsection{Onset Criteria}

The FLOWTRAN-TF code is capable of automatically iterating to find the pre-incident assembly power (i.e. the assembly power at time zero) such that the most limiting operational criterion is just satisfied during the flow transient. The criteria that have been selected to establish power limits under air-water flow conditions are presented in this section.

The primary application of FLOWTRAN-TF is computing the reactor power limit for the Emergency Cooling System (ECS) phase of a Loss Of Coolant Accident (LOCA). The thermal-hydraulic criteria chosen for this accident are based on prevention of significant net steam generation and wall temperatures in excess of the critical heat flux temperature. Depending on the two-phase flow conditions, significant steam generation can initiate a static flow instability or restrict coolant flow because of a counter-current flow limitation (CCFL). Significant net steam generation is avoided if wall temperatures are not permitted to be superheated and fluid temperatures are below the saturation temperalure. The specific thermal-hydraulic acceptance criteria chosen for LOCA-ECS are

- Fluid temperature less than or equal to the local saturation temperature ( $\mathrm{T}_{\text {fluid }} \leq \mathrm{T}_{\text {sat }}$ )

- Surface temperature less than or equal to the local fluid saturation temperature $\left(T_{\text {wall }} \leq \mathrm{T}_{\text {sat }}\right)$

The $T_{\text {wall }} \leq T_{\text {sat }}$ criterion is invariably limiting because flashing in the Bottom Fitting Insert (BFI) does not occur under ECS conditions. The term 'flashing' is being used loosely here because in a two-component (air-water) system steam generation occurs for any decrease in pressure; however, steam generation only becomes significant near the saturation point of water and resembles true flashing in a single-component system in this sense. The $T_{\text {wall }} \leq T_{\text {sat }}$ condition does not correspond to a physical thermal excursion initiator. Rather, the criterion is a conservative precursor chosen to make the analytical modeling tractable and reliable. The criterion typically yields reactor operating powers 20 $40 \%$ lower than those at the thermal excursion threshold based on experimental data.

The four criteria options which may be used to determine the maximum operating power are:

1. The bulk fluid temperature must remain below saturation.

2. The maximum instantaneous metal surface temperature must remain below the local fluid saturation temperature.

3. The maximum running time averaged metal surface temperature must remain below the local fluid saturation temperature.

4. A criterion that the Counter-current Flow Limitation (CCFL) cannot be exceeded locally at any instant.

The second and third selections permit the user to apply the $\mathrm{T}_{\text {wall }} \leq \mathrm{T}_{\text {sat }}$ criterion on either an instantaneous or time-averaged basis. Typically the first criterion with either the second or third option would be selected. Either pair of criteria will prevent significant net steam generation so CCFL conditions would not be expected. The fourth choice is provided mainly to allow the user to confirm that that a CCFL condition has not occurred. 


\section{COMPUTATIONAL MODELS}

Numerical implementation of the physical models described in section 2 is discussed in this section.

\subsection{Computational Mesh}

Many aspects of the computational mesh have already been discussed in section 2.1. In this section additional information is presented concerning the fluid computational mesh. The solid computational mesh is discussed further in section 3.3 in connection with the solid finite difference equations.

The fluid computations employ a staggered mesh that defines fluid properties at the centers of the mesh cells and fluid velocities at the centers of the cell faces. A representative fluid mesh is shown in Fig. 3.1-1 which illustrates mesh cells within a single flow channel unfolded from its cylindrical configuration. In the top and bottom sections of the mesh, the fluid cells form a one-dimensional axial grid. Within the middle section, the fluid computations are two-dimensional (axial and azimuthal). The mesh cells shown in Fig. 3.1-1. comprise the control volumes for the derivation of the fluid mass and energy finite difference equations. The arrows in Fig. 3.1-1 show the direction of positive fluid (gas or liquid) velocities at each of the cell faces.

Figure 3.1-1 illustrates the numbering conventions adopted in the FLOWTRAN-TF code for fluid cells and faces. Axial cells are labeled in plain text, axial faces are labeled in bold text, azimuthal cells are labeled in italics, and azimuthal faces are labeled in bold italic text. In reading Fig. 3.1-1, nzt is the number of axial cells in the top section, nzb the number of axial cells in the bottom section, $\mathrm{nz}$ is the number of axial cells in the middle, and $\mathrm{nx}$ is the number of azimuthal cells in the middle section. In the top section, cell face numbers start with one at the interface between the plenum and the top. In the bottom section, cell face numbers end with nzb at the interface between the tank bottom and the bottom section. In the middle section, for coding purposes, it was advantageous to place fictitious cells above and below the section to more conveniently handle the coupling between the multiple flow paths through the middle and the single flow paths on either end. The fictitious cell above the middle section mimics the last cell in the top section and the fictitious cell below the middle section has the properties of the first cell in the bottom section. Within the code, these cells are labeled as cell numbers 1 and $n z+2$ respectively. However, in this code documentation, we will adopt the convention of numbering the actual physical cells in the middle section from 1 to $\mathrm{nz}$ and numbering the corresponding cell faces from 1 to $\mathrm{nz}+1$ as shown in Fig. 3.1-1.

Control volumes for the momentum equations are staggered on half step from the mass and energy mesh cells. The axial staggered mesh at an arbitrary grid point $(i, j)$ is illustrated in Fig. 3.1-2 which shows one representative mass cell and a single momentum cell. This axial mass and momentum cell arrangement also applies in the top and bottom sections of the mesh (without the azimuthal velocities). The azimuthal staggered mesh at grid point $(i, j)$ is illustrated in Fig. 3.1-3 which shows a mass cell and one azimuthal momentum cell. The azimuthal momentum equations are only solved in the middle section of the assembly. At the mass/energy cell center $(i, j)$, we compute values for fluid temperature, gas temperature, pressure, void fraction, and air mass fraction $\left(T_{f i, j}, T_{g i, j}, P_{i, j}, \alpha_{i, j}\right.$, and 
$\mathrm{Xa}_{\mathrm{i}, \mathrm{j}}$. The fluid velocities are defined at the cell face locations shown in the figures. In Figs. 3.1-2 and 3.1-3, $u$ and $w$ are used to represent components of either gas or liquid velocity.

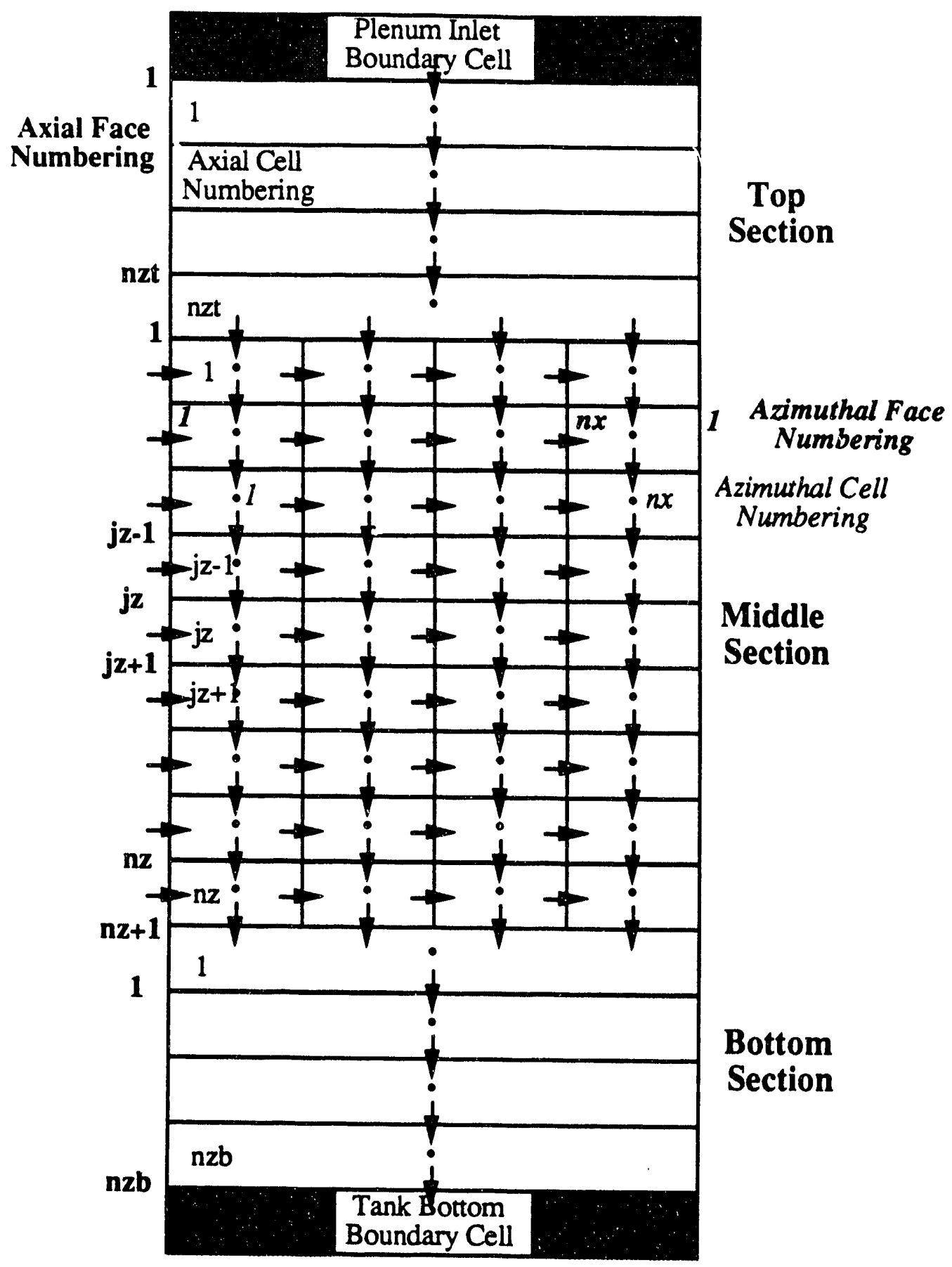

Figure 3.1-1 Schematic representation of FLOWTRAN-TF fluid mesh for a single channel. 


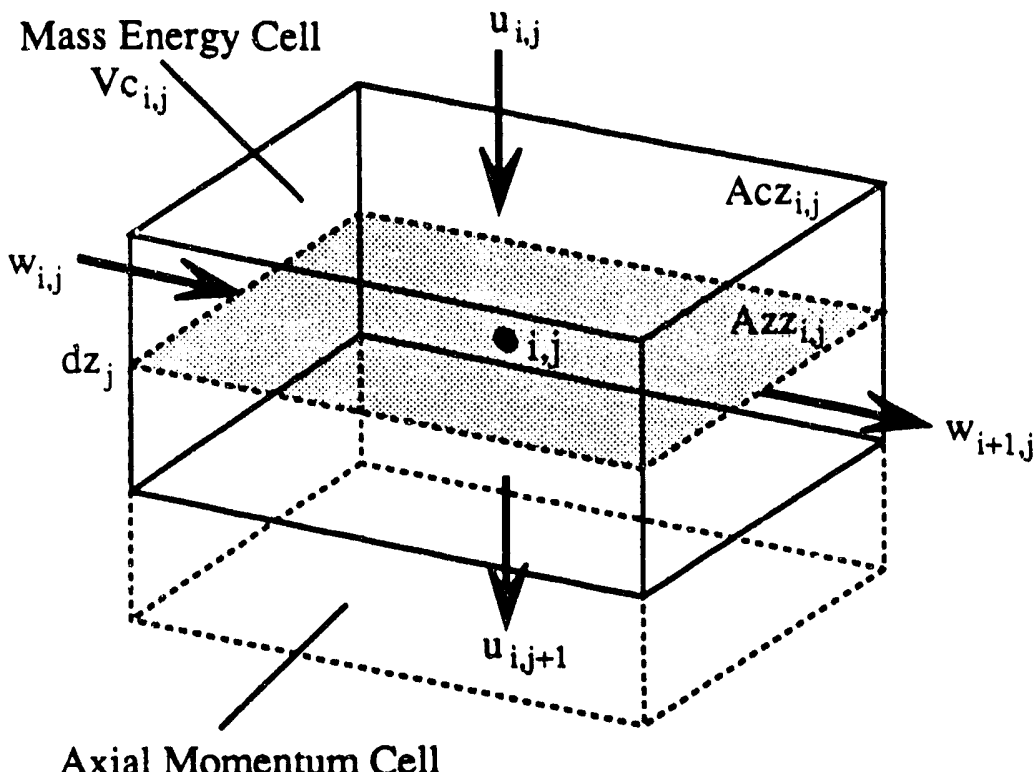

Figure 3.1-2 Axial staggered mesh used for fluid computations.

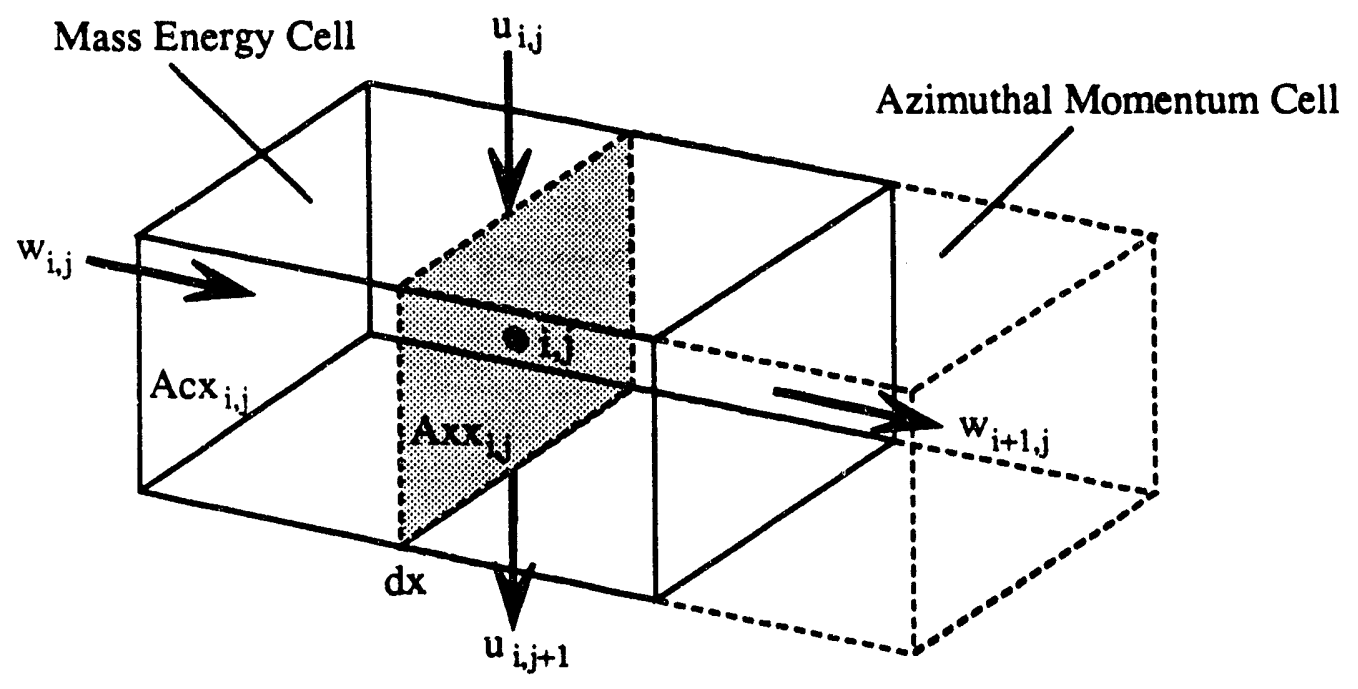

Figure 3.1-3 Azimuthal staggered mesh used for fluid computations.

Figures 3.1-2 and 3.1-3 indicate some of the geometric information that is input to the FLOWTRAN-TF code to define the fluid computational mesh. The code user inputs axial cell face areas $\left(A c z_{i, j}\right)$ and cell center areas $\left(\mathrm{Azz}_{\mathrm{i}, \mathrm{j}}\right)$. Similarly, in the azimuthal direction the user inputs cell face areas $\left(\mathbf{A c x}_{\mathrm{i}, \mathrm{j}}\right)$ and cell center areas $\left(\mathbf{A x x}_{\mathrm{i}, \mathrm{j}}\right)$. Since both cell face and cell center areas are input, variation in the cross sectional area within the flow channels can be accounted for. Axial cell lengths $\left(\mathrm{dz}_{\mathrm{j}}\right)$ are input to the computations. In the middle section, the azimuthal cell length is computed as 


$$
d x=\frac{\pi\left(D_{i}+D_{0}\right)}{2 N_{\text {sub }}}
$$

where

$$
\begin{aligned}
D_{i} & \equiv \text { inner diameter of the flow channel } \\
D_{0} & \equiv \text { outer diameter of the flow channel } \\
N_{\text {sub }} & \equiv \text { number of subchannels within the flow channel }
\end{aligned}
$$

This calculation assumes that the azimuthal subchannels are of equal size and does not correct for the slight distortion that arises from eccentric positioning of the metal tubes.

In the top and bottom sections, the code user must input mass cell volumes $\left(\mathrm{Vc}_{\mathrm{j}}\right)$. This allows the user to include volume reductions from irregular cross sectional areas within the cells. In the middle section of the assembly, we assume that the cell cross sectional areas are either uniform or tapered. The volumes of these mass cells are then computed from the input cell face areas and axial cell lengths as

$$
V c_{i, j}=\frac{\left(A c z_{i, j}+A c z_{i, j+1}\right)}{2} d z_{j}
$$




\subsection{Power Numerical Equations}

This paper describes the finite difference implementation in FLOWTRAN-TF of the power generation methodology derived in Aleman (1992). The power generation methodology is implemented in three steps:

I. Input Processing - Subroutine INPUT (Fig. 3.2-1)

II. Initialization of Variables - Subroutine INITL (Fig. 3.2-2)

III. Transient Calculations - Subroutine TRANS (Fig. 3.2-3)

\section{Input Processing}

\section{Subroutine INPUT}

\section{Call INPOWR.}

\section{Subroutine DNPOWR}

A. Initialize assembly exposure, $\delta_{\text {mod }}, \delta_{\text {dry }}$ to zero. If reactor assembly, iexp $=0$, then read values for these variables.

B. Call INDPWR to process fission power tube region power fractions and radial shape functions (iwtdry $=1$ ).

\section{Subroutine INDPWR}

a. Read exposure coefficients to fission power tube region power fraction polynomial.

b. Adjust the tube region fission power fractions for reactor exposure as:

$$
\mathrm{pf}^{\mathrm{np}}(\text { ireg })=\sum_{n c=1}^{\text {ncoeff }} \mathrm{pf}_{\mathrm{region}}^{\mathrm{np}}(\text { icyln, nc }) \exp ^{\mathrm{nc}-1}
$$

where

$\mathrm{pf} \equiv$ Coefficients to tube region power fraction polynomial,

icyln $\equiv$ Cylinder index,

ireg $\equiv$ Tube region index,

ncoeff $\equiv$ Number of coefficients in polynomial,

$\exp \equiv$ Reactor exposure (MWD or \%burn-up). 
c. Read coefficients to polynomial fit in $r$ or exposure for fission power radial shape function.

d. If the number of radial points specified is nrpa $\leq 0$, rphi array contains coefficients to polynomial fit in $r$ as:

$$
\operatorname{rphi}(\mathrm{nc}, \text { icyln })=\operatorname{coeff}(\mathrm{nc}, 1, \text { iset })
$$

where

coeff $\equiv$ Coefficients to radial shape function polynomial,

nc $\equiv$ Polynomial coefficient index,

icyln $\equiv$ Cylinder index,

iset $\equiv$ Cylinder set index.

or else the rphi array is adjusted for exposure as:

$$
\operatorname{mph}(\mathrm{np}, \text { icyln })=\sum_{n c=1}^{\text {ncoeff }} \operatorname{coeff}(\mathrm{nc}, \mathrm{np}, \text { iset }) \exp ^{\mathrm{nc}-1}
$$

where

$\mathrm{np} \equiv$ Radial interval index.

e. Set iwtdry $=0$ and return to INPOWR.

C. Call INDPWR to process moderated decay power tube region power fractions and radial shape functions (iwtdry $=2$ ).

a. Read skip control variable, iskip. If iskip=0, set iwtdry=100 and return to INPOWR.

b. Read exposure coefficients to moderated decay power tube region power fraction polynomial.

c. Adjust the tube region fission power fractions for reactor exposure as:

$$
\mathrm{pf}_{\text {mod }}^{\mathrm{dp}}(\mathrm{ireg})=\sum_{n c=1}^{\text {ncoeff }} \mathrm{pf}_{\text {mod,region }}^{\mathrm{dp}}(\mathrm{icyln}, \mathrm{nc}) \exp ^{\mathrm{nc}-1}
$$

d. Read coefficients to polynomial fit in $r$ or exposure for moderated decay power radial shape polynomial.

e. If the number of radial points specified is nrpa $\leq 0$, rphdw array contains coefficients to polynomial fit in $r$ as: 


$$
\operatorname{rphdw}(\mathrm{nc}, \mathrm{icyln})=\operatorname{coeff}(\mathrm{nc}, 1, \text { iset })
$$

or else the rphdw array is adjusted for exposure as:

$$
\operatorname{rphdw}(n p, i c y l n)=\sum_{n c=1}^{n c o e f f} \operatorname{coeff}(n c, n p, \text { iset }) \exp ^{n c-1}
$$

f. Set iwtdry=0 and return to INPOWR.

D. If iwtdry $\neq 0$ then set moderated decay power data to fission power data as:

$$
\begin{aligned}
\mathrm{pf}_{\text {mod }}^{\mathrm{dp}}(\text { ireg }) & =\mathrm{pf}^{\mathrm{np}}(\text { ireg }) \\
\operatorname{rphdw}(\mathrm{np}, \mathrm{icyln}) & =\operatorname{rphi}(\mathrm{np}, \text { icyln })
\end{aligned}
$$

E. Call INDPWR to process dry decay power tube region power fractions and radial shape functions (iwtdry=3).

a. Read skip control variable, iskip. If iskip=0, set iwtdry=100 and return to INPOWR. If iskip=2, set iwtdry $=200$ and return to INPOWR.

b. Read exposure coefficients to dry decay power tube region power fraction polynomial.

c. Adjust the tube region fission power fractions for reactor exposure as:

$$
\mathrm{pf}_{\mathrm{dry}}^{\mathrm{dp}}(\mathrm{ireg})=\sum_{n c=1}^{\text {ncoeff }} \mathrm{pf}_{\mathrm{dry}, \text { region }}^{\mathrm{dp}}(\mathrm{icyln}, \mathrm{nc}) \exp ^{\mathrm{nc}-1}
$$

d. Read coefficients to polynomial fit in $r$ or exposure for dry decay power radial shape polynomial.

e. If the number of radial points specified is nrpa $\leq 0$, rphdd array contains coefficients to polynomial fit in $r$ as:

$$
\operatorname{rphdd}(\mathrm{nc}, \mathrm{icyln})=\operatorname{coeff}(\mathrm{nc}, 1, \text { iset })
$$

or else the rphdd array is adjusted for exposure as:

$$
\operatorname{rphdd}(n p, i c y l n)=\sum_{n c=1}^{\text {ncoeff }} \operatorname{coeff}(n c, n p, \text { iset }) \exp ^{n c-1}
$$

f. Set iwtdry=0 and return to INPOWR.

F. If iwtdry $=200$ then set dry decay power data to moderated decay power data as: 


$$
\begin{aligned}
\operatorname{pf}_{\text {dry }}^{\mathrm{dp}}(\text { ireg }) & =\mathrm{pf}_{\bmod }^{\mathrm{dp}}(\text { ireg }) \\
\operatorname{rphdd}(\mathrm{np}, \text { icyln }) & =\operatorname{rphdw}(\mathrm{np}, \text { icyln })
\end{aligned}
$$

G. Else if iwtdry $\neq 0$ then set dry decay power data to fission power data as:

$$
\begin{aligned}
\operatorname{pf}_{\mathrm{dry}}^{\mathrm{dp}}(\text { ireg }) & =\mathrm{pf}^{\mathrm{np}}(\text { ireg }) \\
\operatorname{rphdd}(\mathrm{np}, \mathrm{icyln}) & =\operatorname{rphi}(\mathrm{np}, \mathrm{icyln})
\end{aligned}
$$

H. Initialize azimuthal shape function array, $\Theta($ ia, icyln) to 1 . Read in array.

I. Read in spline pointers to time-dependent terms in Eq. (2.2-1).

J. Read in axial power spline pointers and corresponding times.

2. Call INSPLN.

Subroutine INSPLN

A. Read the axial flux shape and time-dependent power data.

B. Call SPLINE for each set of data.

\section{Initialization of Variables}

Subroutine NITL ,

1. Clear the deposited power array, pows(ir,ia,iz,icyln).

2. Call PNORM to compute the $\mathbf{R}-\boldsymbol{\theta}$ component of the fission power normalization parameter.

\section{Subroutine PNORM}

A. Initialize the fission power radial power density, $\mathrm{R}^{\mathrm{np}}(\mathrm{ir}, \mathrm{icyln})_{\mathrm{a}}$ to 1 .

B. Call RADSHP to compute the area-averaged fission power radial power density, $\mathbf{R}^{\mathrm{mP}}$ (ir,icyln), for the core region of each tube.

\section{Subroutine RADSHP}

a. If $\mathrm{nrp}(\mathrm{icyln})<0$, then coefficients to radial power density polynomial have been read. Proceed to step e and compute area-averagell radial power density.

b. The rphi array represents tabulated values of the fission power radial power density at equally spaced boundaries within the core region of each tube. This shape is given as a polynomial in $r$ of degree $n r-1$ as: 


$$
\operatorname{mphi}(n p, i c y l n)=\sum_{k=1}^{n r} a_{k} r(n p, i c y l n)^{k-1}
$$

where

$a_{k} \equiv$ Coefficients to rphi polynomial in $r$,

$\mathrm{nr} \equiv$ Number of radial intervals for polynomial,

$r \equiv$ Radii of intervals within core region for which rphi values are given.

c. The system of equations, $\mathrm{np} \times \mathrm{np}$, generated from Eqn. (3.2-13) is known as a Vandermode system. This system can be quite ill-conditioned. To alleviate this problem we recast Eqn. $(3.2-13)$ in non-dimensional form as:

$$
\begin{aligned}
& \operatorname{rphi}(n p, i c y l n)=\sum_{k=1}^{m} b_{k} \tilde{r}(n p, i c y l n)^{k-1} \\
& \bar{r}(n p, i c y l n)=\frac{R_{0}(\text { icyln })-r(n p, i c y l n)}{R_{0}(i c y l n)-R_{i}(i c y l n)}
\end{aligned}
$$

where

$b_{k} \equiv$ Coefficients to rphi polynomial in $\bar{r}$,

$\mathbf{R}_{\mathrm{i}} \equiv$ Inner radius of core region,

$R_{0} \equiv$ Outer radius of core region.

d. Solve Eq. (3.2-14) for the $b_{k}$ coefficients with the CHLSKI routine.

e. Compute the area-averaged fission power radial power density as

$$
\begin{gathered}
R^{\mathrm{np}}(\mathrm{ir}, \mathrm{icyln})=\frac{\sum_{k=1}^{\mathrm{nr}} \frac{b_{k} \Delta r}{k(k+1)}\left\{\left(R_{0}+k r_{i}\right)\left(\frac{R_{0}-r_{i}}{R_{0}-R_{i}}\right)^{k}-\left(R_{0}+k r_{0}\right)\left(\frac{R_{0}-r_{0}}{R_{0}-R_{i}}\right)^{k}\right\}}{\frac{1}{2}\left(r_{0}^{2}-r_{i}^{2}\right)} \\
R_{i}=\frac{1}{2} \operatorname{diam}(n f, i c y l n) \\
R_{0}=\frac{1}{2} \operatorname{diam}(n l+1, i c y l n) \\
r_{i}=\frac{1}{2} \operatorname{diam}(i r, i c y l n)
\end{gathered}
$$




$$
\begin{gathered}
r_{0}=\frac{1}{2} \operatorname{diam}(i r+1, i c y l n) \\
\Delta r=R_{0}-R_{i}
\end{gathered}
$$

where

ir $\equiv$ Index of solid node in core region,

$\mathrm{nf}, \mathrm{nl} \equiv$ Index of first and last nodes in core region.

A detailed derivation of Eqn. (3.2-15) is given below.

C. Compute the R- $\Theta$ component of the fission power normalization parameter as:

$$
\begin{gathered}
\operatorname{bigm}(\mathrm{ir}, \mathrm{ia}, \mathrm{icyln})=\frac{\mathrm{pf}^{\mathrm{np}}(\mathrm{ireg}) \mathrm{R}^{\mathrm{np}}(\mathrm{ir}, \mathrm{icyln}) \Theta(\mathrm{ia}, \mathrm{icyln}) \mathrm{A}(\mathrm{ir}, \mathrm{icyln})}{\sum_{\mathrm{ir}=\mathrm{ir}_{1}}^{\mathrm{ir}_{2}} \sum_{\mathrm{i}=1}^{\mathrm{na}} \mathrm{R}^{\mathrm{np}}(\mathrm{ir}, \mathrm{icyln}) \Theta(\mathrm{ia}, \mathrm{icyln}) \mathrm{A}(\mathrm{ir}, \mathrm{icyln})} \\
\mathrm{A}(\mathrm{ir}, \mathrm{icyln})= \\
\frac{\pi\left(\operatorname{diam}(\mathrm{ir}+1, \mathrm{icyln})^{2}-\operatorname{diam}(\mathrm{ir}, \mathrm{icyln})^{2}\right)}{4 \mathrm{na}}
\end{gathered}
$$

where

$A \equiv$ Node cross-sectional area,

diam $\equiv$ Inner or outer diameter of node,

ir, ia, iz $\equiv$ Radial, azimuthal and axial node index,respectively,

$\mathrm{ir}_{1}, \mathrm{i} \mathrm{r}_{2} \equiv$ Inner and outer radii, respectively, for a given region,

na $\equiv$ Number of azimuthal divisions.

3. Call PNORM to compute the R- $\Theta$ component of the moderated decay power normalization parameter. Repeat the procedure in step 2 . The results are

$$
R_{\bmod }^{d p}(i r, i c y l n)=\frac{\sum_{k=1}^{n r} \frac{c_{k} \Delta r}{k(k+1)}\left\{\left(R_{o}+k r_{i}\right)\left(\frac{R_{o}-r_{i}}{R_{o}-R_{i}}\right)^{k}-\left(R_{o}+k r_{0}\right)\left(\frac{R_{0}-r_{0}}{R_{o}-R_{i}}\right)^{k}\right\}}{\frac{1}{2}\left(r_{0}^{2}-r_{i}^{2}\right)}
$$

where

$c_{k} \equiv$ Coefficients to rphdw polynomial in $\overline{\mathrm{r}}$. 
and

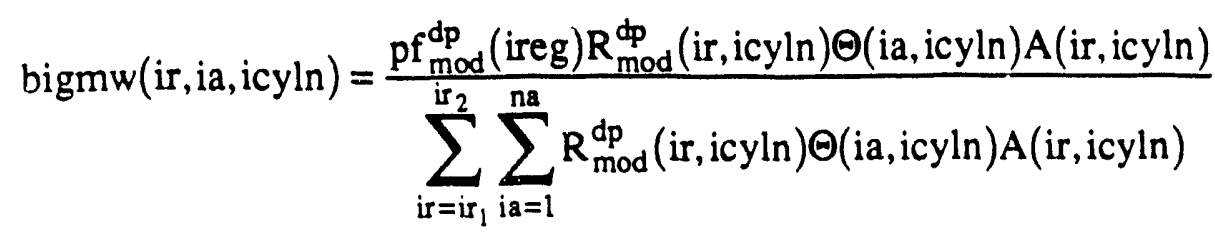

4. Call PNORM to compute the R- $\Theta$ component of the dry decay power normalization parameter. Repeat the procedure in step 2 . The results are

$$
R_{\text {mod }}^{d p}(i r, i c y l n)=\frac{\sum_{k=1}^{n r} \frac{d_{k} \Delta r}{k(k+1)}\left\{\left(R_{0}+k r_{i}\right)\left(\frac{R_{0}-r_{i}}{R_{0}-R_{i}}\right)^{k}-\left(R_{o}+k r_{0}\right)\left(\frac{R_{0}-r_{0}}{R_{0}-R_{i}}\right)^{k}\right\}}{\frac{1}{2}\left(r_{0}^{2}-r_{i}^{2}\right)}
$$

where

$$
\mathrm{d}_{\mathrm{k}} \equiv \text { Coefficients to rphdd polynomial in } \overrightarrow{\mathrm{r}} \text {. }
$$

and

$$
\operatorname{bigmd}(\mathrm{ir}, \mathrm{ia}, \mathrm{icyln})=\frac{\mathrm{pf}_{\mathrm{dry}}^{\mathrm{dp}}(\mathrm{ireg}) \mathrm{K}_{\mathrm{dy}}^{\mathrm{dp}}(\mathrm{ir}, \mathrm{icyln}) \Theta(\mathrm{ia}, \mathrm{icyln}) \mathrm{A}(\mathrm{ir}, \mathrm{icyln})}{\sum_{\mathrm{ir}=\mathrm{ir}_{1}}^{\mathrm{ir}_{2}} \sum_{\mathrm{ia}=1}^{\mathrm{na}} \mathrm{R}_{\mathrm{dry}}^{\mathrm{dp}}(\mathrm{ir}, \mathrm{icyln}) \Theta(\mathrm{ia}, \mathrm{icyln}) \mathrm{A}(\mathrm{ir}, \mathrm{icyln})}
$$

5. Call AXIALP at tsec $=0$ and compute normalized axial flux shapes.

\section{Subroutine AXIALR}

A. If tsec=0, evaluate $Z^{\text {np }}(i z, 0)$ from spline interpolation routine YVALU and normalize. Evaluate $Z^{n p}(i z, t)$ from spline interpolation routine and normalize.

6. Call PDIST at time $=0$ and init $=1$ to compute the deposited power at each solid node, pows(ir,ia,iz,icyln).

\section{Subroutine PDIST}

A. If experimental rig, iexp=1, then initialize $\delta_{\text {mod }}, F^{n p}, F_{\bmod }^{d p}, H(t)$ to 0.0 and $\delta_{d r y}$, $x(t)$, to 1.0. If reactor assembly, iexp=0, then evaluate time-dependent terms using YVALU at tsec $=0$.

B. Compute term1, term2, and term3 of total deposited power in Eq. 1 of Aleman (1992) as: 


$$
\begin{aligned}
& \text { term } 1=\Pi(0)\left(1-x(t) \delta_{\text {mod }}\right)\left[\frac{H(t)}{H}\right] F^{n p}(t) \\
& \text { term } 2=\Pi(0) x(t) \delta_{\bmod }\left[\frac{H(t)}{H}\right] F_{\text {mod }}^{d p}(t) \\
& \text { term } 3=\Pi(0) x(t) \delta_{d r y}\left[1-\frac{H(t)}{H}\right] F_{d r y}^{d p}(t)
\end{aligned}
$$

where

$\Pi \equiv$ Total assembly deposited power,

$\delta_{x} \equiv$ Fraction of deposited power from decay heat ("k=dry" for regions where tank is empty, " $k=$ mod" where assembly is moderated),

$H(t) \equiv$ Height of water in the reactor tank relative to the bottom of active core,

$\mathrm{H} \equiv$ Length of active core region,

$F^{n p} \equiv$ Fission power plus neutron capture power amplitude, normalized to 1.0 at time 0.0 ,

$\mathrm{F}_{\mathbf{k}}^{\mathrm{dp}} \equiv$ Decay heat power amplitude ("dry" and "mod"), normalized to 1.0 at time $x(t) \equiv$ Conservative decay heat multiplier.

C. Compute summations over product of axial flux shape and cell length for fission power and moderated decay components as:

$$
\begin{aligned}
& \text { sumfis }=\sum_{\mathrm{iz}=\mathrm{izht}}^{\mathrm{izhb}} \mathrm{Z}^{\mathrm{np}}(\mathrm{iz}, \mathrm{t}) \delta \mathrm{z}(\mathrm{iz}) \\
& \text { summod }=\sum_{i z=i z h t}^{i z h b} z^{n p}(i z, 0) \delta z(i z) \\
& \delta z(i z)=\left\{\begin{array}{cl}
0 & \text { dry node } \\
H(t)-\text { botnod(iz) } & \text { partially moderated node } \\
\Delta z(i z) & \text { wet node }
\end{array}\right.
\end{aligned}
$$

where

izht $\equiv$ Index of top axial cell that is heated,

izhb $\equiv$ Index of bnttom axial cell that is heared,

botnod $\equiv$ Elevation sf lower face of axial node relative to bottom of active core, 
$\Delta z \equiv$ Axial cell length in active core region.

D. Compute summations over product of axial flux shape and cell length for dry decay component as:

$$
\begin{gathered}
\text { sumdry }=\sum_{\mathrm{iz}=\mathrm{izht}}^{\mathrm{izhb}} \mathrm{Z}^{\mathrm{np}}(\mathrm{iz}, 0) \delta \mathrm{z}(\mathrm{iz}) \\
\delta \mathrm{z}(\mathrm{iz})=\left\{\begin{array}{cl}
\Delta \mathrm{z}(\mathrm{iz}) & \text { dry node } \\
\operatorname{topnod}(\mathrm{iz})-\mathrm{H}(\mathrm{t}) & \text { partially moderated node } \\
0 & \text { wet node }
\end{array}\right.
\end{gathered}
$$

where

topnod $\equiv$ Elevation of upper face of axial node relative to bottom of active core.

E. Compute the product of the time-dependent power term and the contribution by the axial flux shape term for each deposited power component as:

$$
\begin{gathered}
\text { comb }=\operatorname{term} 1 \frac{Z^{\mathrm{np}}(\mathrm{iz}, \mathrm{t}) \delta \mathrm{z}(\mathrm{iz})}{\text { sumfis }} \\
\text { combw }=\operatorname{term} 2 \frac{\mathrm{Z}^{\mathrm{np}}(\mathrm{iz}, 0) \delta z(i z)}{\text { summod }} \\
\text { combd }=\operatorname{term} 3 \frac{\mathrm{Z}^{\mathrm{nq}}(\mathrm{iz}, 0) \delta z(i z)}{\text { sumdry }}
\end{gathered}
$$

F. The nodal deposited pownr is computed in FLOWTRAN-TF by combining Eqs. $(3.2-16),(3.2-18),(3.2-23)$ and $(3.2-24)$ as:

$$
\begin{aligned}
\operatorname{pows}(\mathrm{ir}, \mathrm{ia}, \mathrm{iz}, \mathrm{icyln}) & =\operatorname{bigm}(\mathrm{ir}, \mathrm{ia}, \mathrm{icyln}) \operatorname{comb}+\operatorname{bigmw}(\mathrm{ir}, \mathrm{ia}, \mathrm{icyln}) \operatorname{combw} \\
& +\operatorname{bigmd}(\mathrm{ir}, \mathrm{ia}, \mathrm{icyln}) \operatorname{combd}
\end{aligned}
$$

7. If tmid $>0$ then repeat steps 5 and 6 with $t=t m i d$ and init $=0$. tmid represents the time between the old and new time, $n+1 / 2$.

8. Call PRTPOW to print deposited power data at time tsec. 


\begin{tabular}{|l|lll|l|}
\hline $2 / 15 / 93$ & WSRC-TR-92-532 Rev. 0 & FLOWTRAN-TF Software Design & Page 311 \\
\hline
\end{tabular}

\section{Transient Calculations}

\section{Subroutine TRANS}

1. If solid calculations requested, isolid $=1$, then repeat steps 5 and 6 with $t=t$ mid.

2. If solid calculations requested and steady-state conditions achieved, call PRTPOW.

3. If solid calculations requested and print frequency for power prints exceeded, call PRTPOW.

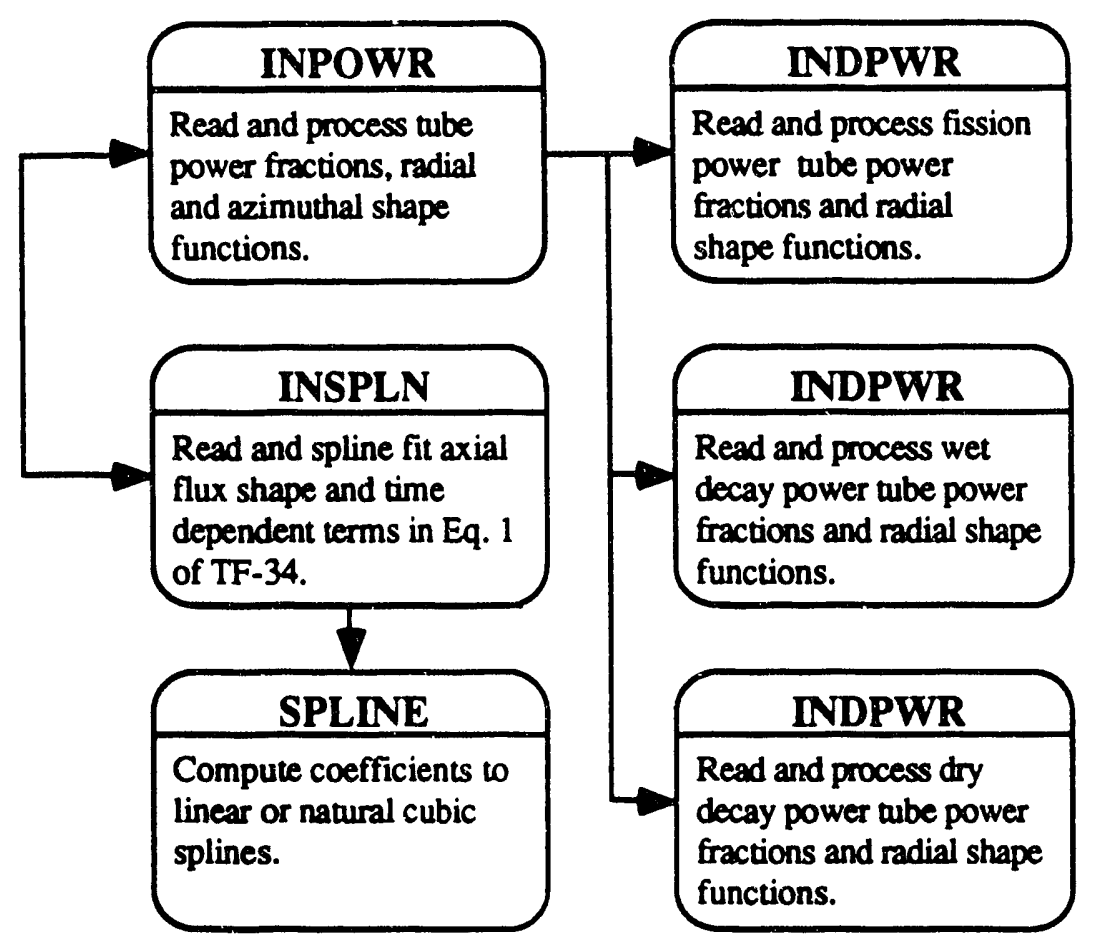

Figure 3.2.1 Flow Diagram of power portion of INPUT 


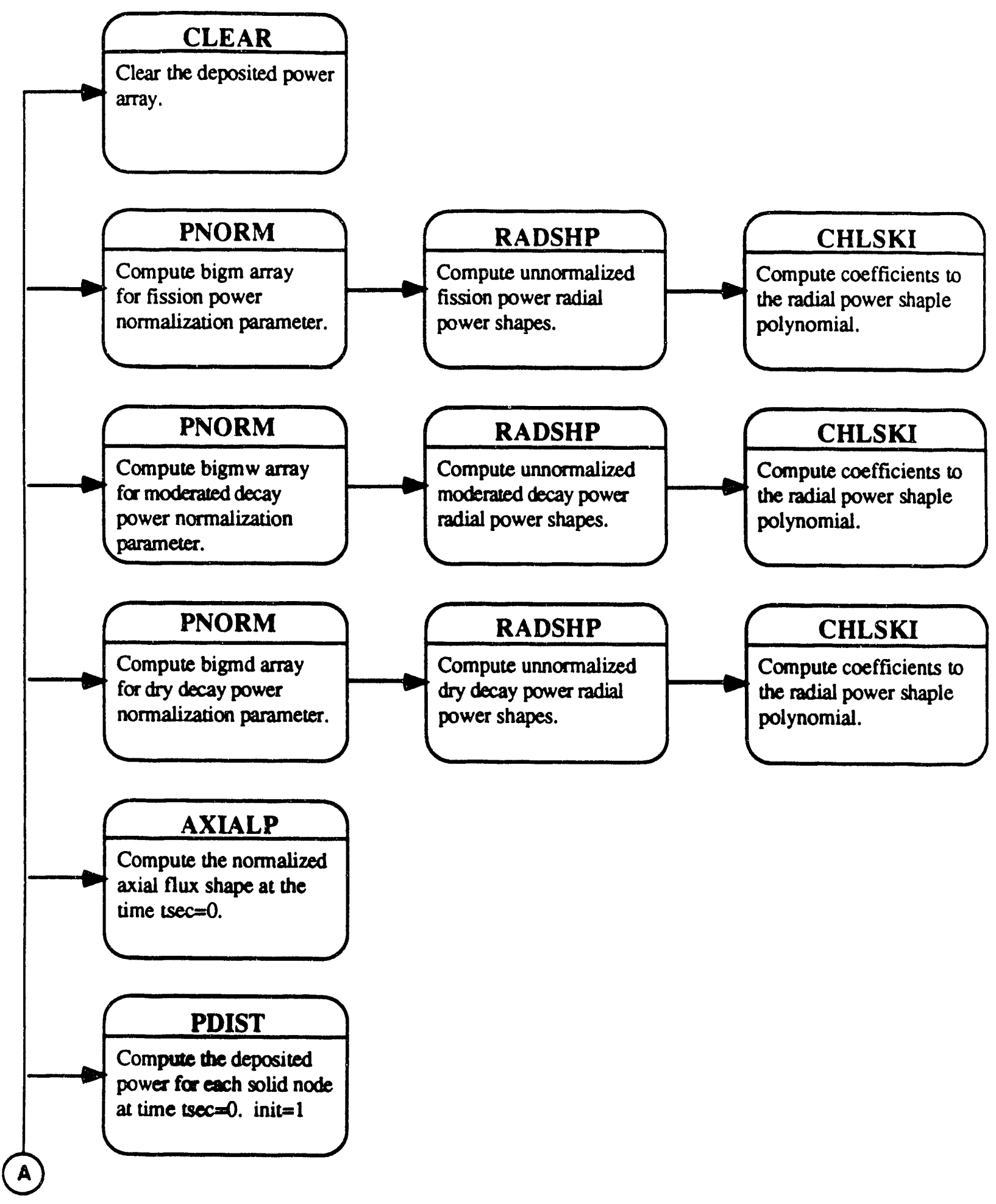

Figure 3.2-2 Flow Diagram of power portion of INITL 


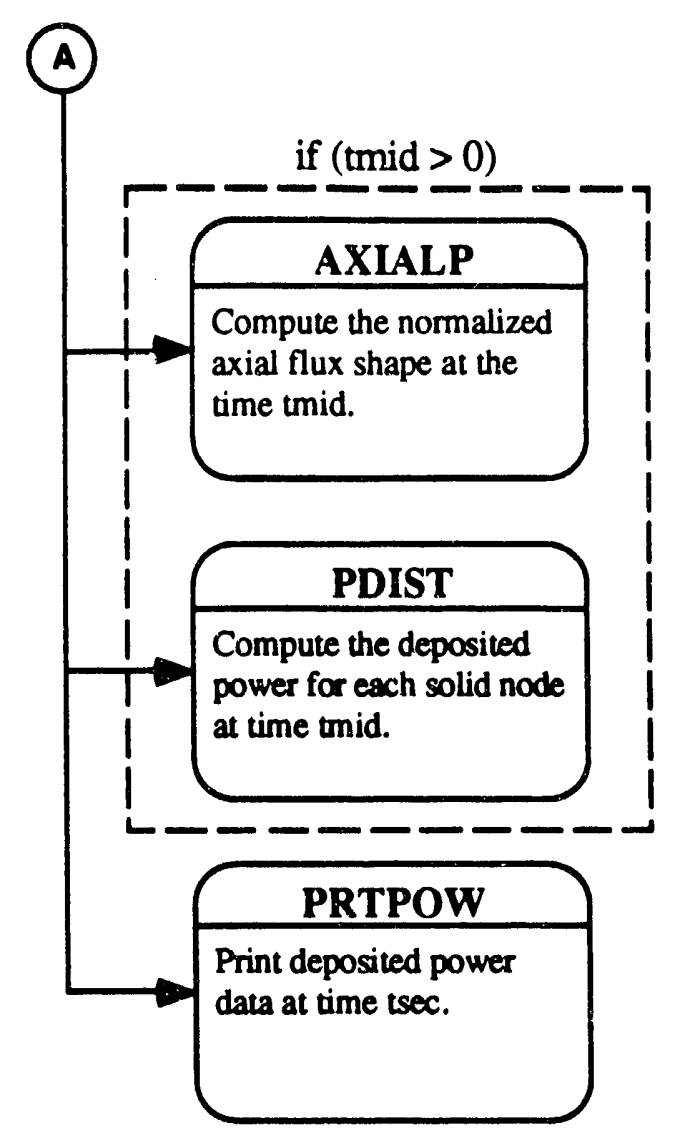

Figure 3.2-2 Flow Diagram of power portion of INITL (Continued) 


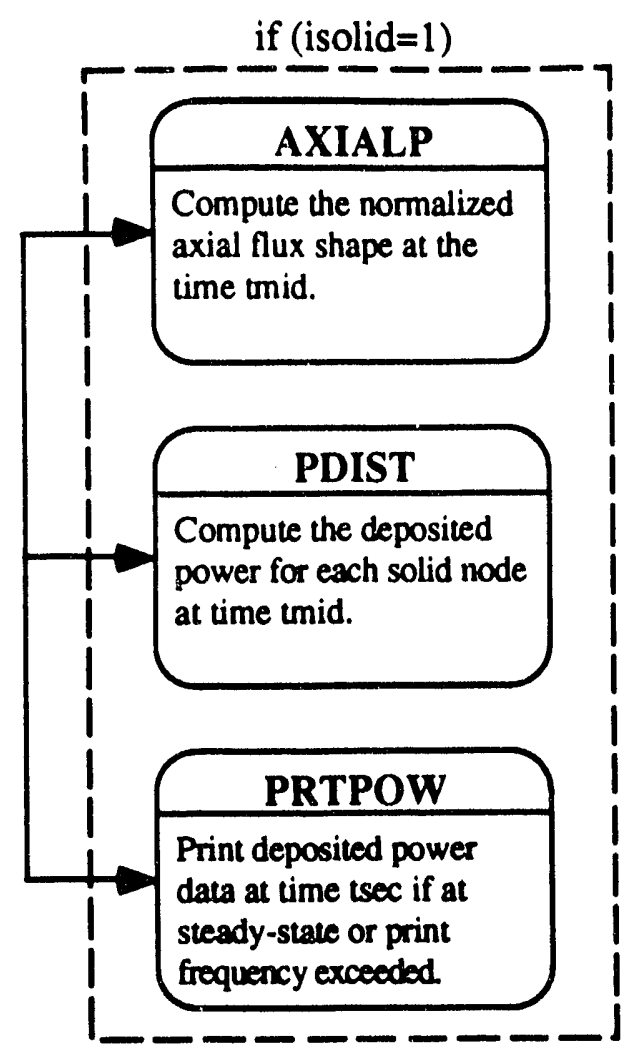

Figure 3.2.3 Flow Diagram of power portion of TRANS 
Detailed Derivation of the Area-Averaged Radial Power Density

The rphi array can be expressed as

$$
\begin{gathered}
\text { rphi }=\sum_{k=1}^{n r} a_{k} r^{k-1}=\sum_{k=1}^{n r} b_{k} \bar{r}^{k-1} \\
\bar{r}=\frac{R_{0}-r}{R_{0}-R_{i}}
\end{gathered}
$$

The area-average radial power density, $R$, is computed from Eqn. (3.2-26) as

$$
R=\langle\mathrm{rphi}\rangle=\frac{\int_{r_{j}}^{r_{j}} \sum_{k=1}^{n r} b_{k} \bar{r}^{k-1} r d r}{\int_{r_{i}}^{r_{0}} r d r}
$$

Substituting for rdr in the numerator of Eqn. (3.2-28) using Eqn. (3.2-27) gives

$$
\begin{gathered}
R=\frac{\int_{\bar{t}_{j}}^{\bar{r}_{0}} \sum_{k=1}^{n \pi} b_{k} \bar{r}^{k-1}\left(R_{0}-\Delta r \bar{r}\right)(-\Delta r) d r}{\int_{r_{i}} r d r} \\
\Delta r=R_{0}-R_{i}
\end{gathered}
$$

Interchanging integration and summation gives

$$
R=\frac{\sum_{k=1}^{n r} b_{k} \Delta r\left(\frac{\Delta r^{-k+1}}{k+1}-\frac{R_{0} \bar{r}^{k}}{k}\right)_{\bar{r}_{i}}^{\bar{s}_{0}}}{\frac{1}{2}\left(r_{0}^{2}-r_{i}^{2}\right)}
$$

Rearranging the numerator of Eqn. (3.2-31) yields 


$$
\begin{aligned}
R & =\frac{\sum_{k=1}^{n r} b_{k} \Delta r\left(\frac{k \Delta r^{k+1}-(k+1) R_{0} \bar{r}^{k}}{k(k+1)}\right)_{\bar{r}_{i}}^{\tilde{r}_{0}}}{\frac{1}{2}\left(r_{0}^{2}-r_{i}^{2}\right)} \\
& =\frac{\sum_{k=1}^{n r} b_{k} \Delta r \bar{r}^{k}\left(\frac{k \Delta r \bar{r}-(k+1) R_{0}}{k(k+1)}\right)_{\bar{r}_{i}}^{\bar{r}_{0}}}{\frac{1}{2}\left(r_{0}^{2}-r_{i}^{2}\right)} \\
& =\frac{\sum_{k=1}^{n r} b_{k} \Delta \bar{r}^{k}\left(\frac{k\left(R_{0}-r\right)-(k+1) R_{0}}{k(k+1)}\right)_{\tilde{r}_{i}}^{\bar{r}_{0}}}{\frac{1}{2}\left(r_{0}^{2}-r_{i}^{2}\right)} \\
& =\frac{\sum_{k=1}^{n r} b_{k} \Delta \bar{r}^{k}\left(\frac{-\left(R_{0}+k r\right)}{k(k+1)}\right)_{\bar{r}_{i}}^{\bar{t}_{0}}}{\frac{1}{2}\left(r_{0}^{2}-r_{i}^{2}\right)}
\end{aligned}
$$

Finally, applying the limits of integration to the numerator yields

$$
\begin{aligned}
R & =\frac{\sum_{k=1}^{n r} \frac{b_{k} \Delta r}{k(k+1)}\left(\left(R_{0}+k r_{i}\right) \bar{r}_{i}^{k}-\left(R_{0}+k r_{0}\right) \bar{r}_{0}^{k}\right)}{\frac{1}{2}\left(r_{0}^{2}-r_{i}^{2}\right)} \\
& =\frac{\sum_{k=1}^{n r} \frac{b_{k} \Delta r}{k(k+1)}\left\{\left(R_{0}+k r_{i}\right)\left(\frac{R_{0}-r_{i}}{R_{0}-R_{i}}\right)^{k}-\left(R_{0}+k r_{0}\right)\left(\frac{R_{0}-r_{0}}{R_{0}-R_{i}}\right)^{k}\right\}}{\frac{1}{2}\left(r_{0}^{2}-r_{i}^{2}\right)}
\end{aligned}
$$




\begin{tabular}{|l|lll|r|}
\hline $2 / 15 / 93$ & WSRC-TR-92-532 Rev. 0 & FLOWTRAN-TF Software Design & Page 317 \\
\hline
\end{tabular}

\subsection{Solid Finite Difference Equations}

The solid heat conduction equation is solved in conjunction with the equations for fluid flow and heat transfer. At each time step in the iteration, surface heat flux boundary conditions are calculated and solid properties are evaluated using current estimates of the temperatures. The heat conduction equations are then solved, using the spatial and temporal differencing techniques described in this section, to produce an updated estimate of the solid and surface temperatures and the surface heat fluxes. The differencing scheme is designed to conserve energy in the solid material.

To simplify the notation in the differencing equations presented below, the convention is adopted that cell centered temperatures and temperatures at off-center locations are indicated by using only the subscript index that changes. That is, the solid temperature at the center of a mesh cell $\left(T_{i, j, k}\right)$ will be represented by $T_{i}, T_{j}$, or $T_{k}$. For example, when discussing the radial difference equations we simplify the notation $T_{i+1, j, k}$ to $T_{i+1}$. This notation is used for thermal conductivities, temperatures, heat fluxes, solid energies, and geometric parameters in the equations that follow.

Spatial Differencing: The heat flux terms in Eq. (2.3-5) contain thermal conductivities and temperature gradients evaluated at the cell faces. To convert these quantities into terms depending on the cell center temperatures, the heat fluxes to each cell surface from adjacent cells are equated and thermal conductivities are approximated with cell centered values. Requiring that the heat flux between adjacent mesh cells exactly balance in the differencing scheme serves to conserve energy for the solid calculations. That is, the increase in total solid energy will be equal to the internal generation plus the boundary energy flux at each time step.

To illustrate the spatial differencing procedure, several mesh cells in the radial direction are shown in Fig. 3.3-1. Cell centered mesh points are indicated by whole integers and cell face points by half integer indices. We take the cell center locations to be exactly midway between the cell faces. 


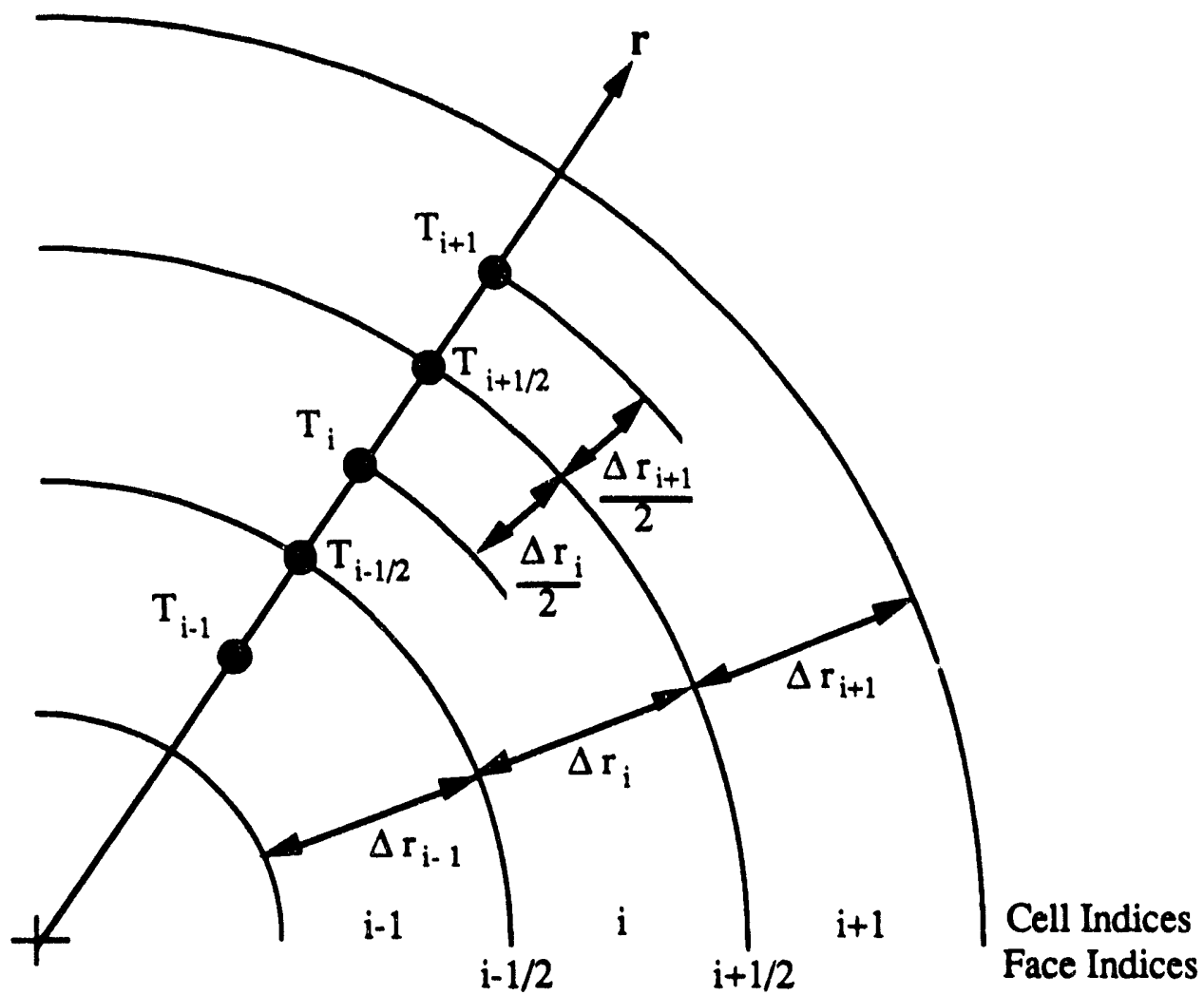

Figure 3.3-1 One dimensional illustration of spatial finite differencing of solid temperatures in the radial direction.

The heat flux at the $i+1 / 2$ interior cell surface is given by

$$
\begin{aligned}
{\left[\mathrm{q}^{\prime \prime}\right]_{i+1 / 2} } & =-k_{i+1 / 2}\left(\frac{\partial T}{\partial T}\right)_{i+1 / 2} \\
& =-k_{i}\left(\frac{T_{i+1 / 2}-T_{i}}{\Delta r_{i} / 2}\right) \\
& =-k_{i+1}\left(\frac{T_{i+1}-T_{i+1 / 2}}{\Delta r_{i+1} / 2}\right)
\end{aligned}
$$

where the subscript convention introduced above has been used in writing the thermal conductivities and temperatures. Equation (3.3-1) sets the heat fluxes evaluated on either side of the cell face equal to each other. Setting the last two expressions in Eq. (3.3-1) equal to each other and solving for the cell face temperature $T_{i+1 / 2}$ gives

$$
T_{i+1 / 2}=\left[\frac{\frac{k_{i}}{\Delta r_{i}} T_{i}+\frac{k_{i+1}}{\Delta r_{i+1}} T_{i+1}}{\frac{k_{i}}{\Delta r_{i}}+\frac{k_{i+1}}{\Delta r_{i+1}}}\right]
$$


Substituting Eq. (3.3-2) into either of the expressions for the surface heat flux in Eq. (3.3-1) and multiplying by the surface area to obtain the total rate of heat transfer across the surface we find

$$
-q_{i+1 / 2}=2 A_{i+1 / 2}\left[\frac{\frac{k_{i}}{\Delta r_{i}} \frac{k_{i+1}}{\Delta r_{i+1}}}{\frac{k}{\Delta r_{i}}+\frac{k_{i+1}}{\Delta r_{i+1}}}\right]\left(T_{i+1}-T_{i}\right) \equiv \Phi_{i+1}\left(T_{i+1}-T_{i}\right)
$$

as the final form for the radial surface heat transfer. Similarly, in the azimuthal and axial directions we obtain

$$
-q_{j+1 / 2}=2 A_{j+1 / 2}\left[\frac{\frac{k_{j}}{r_{i} \Delta \theta} \frac{k_{j+1}}{r_{i} \Delta \theta}}{\frac{k_{j}}{r_{i} \Delta \theta}+\frac{k_{j+1}}{r_{i} \Delta \theta}}\right]\left(T_{j+1}-T_{j}\right) \equiv \Psi_{j+1}\left(T_{j+1}-T_{j}\right)
$$

and

$$
-\cdot q_{k+1 / 2}=2 A_{k+1 / 2}\left[\frac{\frac{k_{k}}{\Delta z_{k}} \frac{k_{k+1}}{\Delta z_{k+1}}}{\frac{k_{k}}{\Delta z_{k}}+\frac{k_{k+1}}{\Delta z_{k+1}}}\right]\left(T_{k+1}-T_{k}\right) \equiv \Lambda_{k+1}\left(T_{k+1}-T_{k}\right)
$$

For the cylindrical geometry of interest, the area terms in these equations are given by

$$
\begin{gathered}
A_{i+1 / 2}=r_{i+1 / 2} \Delta \theta \Delta z_{k} \\
A_{j+1 / 2}=\Delta r_{i} \Delta z_{k} \\
A_{k+1 / 2}=\left(\pi r_{i+1 / 2}^{2}-\pi r_{i-1 / 2}^{2}\right) \frac{\Delta \theta}{2 \pi} \\
=\left(r_{i+1 / 2}+r_{i-1 / 2}\right)\left(r_{i+1 / 2}-r_{i-1 / 2}\right) \frac{\Delta \theta}{2} \\
=r_{i} \Delta r_{i} \Delta \theta
\end{gathered}
$$

The above equations and the area expressions assume that the radial and axial mesh spacing may vary with nodal position but that the azimuthal mesh spacing is constant. This practical constraint on the azimuthal mesh simplifies the numerical implementation.

The coefficients $\Phi_{i+1}, \Psi_{j+1}, \Lambda_{k+1}$ which are functions of the cell surface areas, thermal conductivities, and mesh spacings are introduced in Eqs. (3.3-3) through (3.3-5) with the obvious definitions to simplify the notation. Substituting Eqs. (3.3-3) through (3.3-5) and the equivalent expressions at the $-1 / 2$ step cell faces into Eq. (2.3-5) yields the spatially differenced form of the heat conduction equation used in the FLOWTRAN-TF code as 


$$
\begin{aligned}
V \frac{\partial E}{\partial t}=V q^{\prime \prime \prime} & +\Phi_{i+1}\left(T_{i+1}-T_{i}\right)-\Phi_{i}\left(T_{i}-T_{i-1}\right) \\
& +\Psi_{j+1}\left(T_{j+1}-T_{j}\right)-\Psi_{j}\left(T_{j}-T_{j-1}\right) \\
& +\Lambda_{k+1}\left(T_{k+1}-T_{k}\right)-\Lambda_{k}\left(T_{k}-T_{k-1}\right)
\end{aligned}
$$

In writing Eq. (3.3-9), we have dropped the subscripts on the volume element $V_{i, j, k}$ and on the heat generation term $\mathrm{q}_{i, j, k}^{\prime \prime \prime}$ since it is understood that cell center indices apply.

Time Differencing: We obtain an approximation to the left hand side of Eq. (3.3-9) by using a Taylor series expansion. From the definition of solid internal energy, the product $\left(\rho c_{p}\right)$ is equal to the rate of change in the solid internal energy (E) with respect to temperature. Expanding the solid energy for a differential volume element in a Taylor series about the current temperature gives an approximation for the energy at time step $n+1$ in terms of the energy at time step $n$ as

$$
\begin{aligned}
E^{n+1} & =E^{n}+\left[\frac{\partial E}{\partial T}\right]_{i, j, k}^{n}\left(T^{n+1}-T^{n}\right) \\
& =E^{n}+p_{0}\left(c_{p}\right)_{i, j, k}^{n}\left(T^{n+1}-T^{n}\right)
\end{aligned}
$$

Note that a constant solid density is used to conserve solid mass but the heat capacity can vary as a function of temperature. The left hand side of Eq. (3.3-9) may then be approximated by the relationship

$$
\begin{aligned}
V \frac{\partial E}{\partial t} & =V \rho_{0}\left(c_{p}\right)_{i, j, k}^{n} \frac{\left(T^{n+1}-T^{n}\right)}{\Delta t} \\
& =V \xi^{n}\left(T^{n+1}-T^{n}\right)
\end{aligned}
$$

where the local cell variable

$$
\xi^{n}=\frac{\rho_{0}\left(c_{p}\right)_{i, j, k}^{n}}{\Delta t}
$$

has been introduced to simplify the notation. Using the approximation in Eq. (3.3-11) avoids the necessity of evaluating solid physical properties at the new time step temperatures. Since the FLOWTRAN-TF time step increments are small this approximation is adequate.

The time differencing is made fully implicit in the radial and azimuthal directions by evaluating the associated terms on the right hand side of Eq. (3.3-9) at the new time level. In the axial direction, the time differencing can be varied by the code user. The axial differencing is made semi-implicit by setting the input parameter WSS equal to one while the differencing is made fully explicit in the axial direction when the parameter WSS is equal to zero. By semi-implicit we mean that terms involving $T_{i, j, k}$ are evaluated at the new time step ( $n+1$ values) while all other temperatures are evaluated at the old $(n)$ time step. The semi-implicit axial scheme is sufficiently stable to allow a solution to be achieved by 
taking large time steps and is particularly useful in obtaining rapid convergence to steady state solutions. For transient calculations we usually employ the explicit axial representation. The contribution to the solid energy balance from axial conduction is small for reactor assemblies and prototypic experimental rigs since the solid structures are long thin cylinders and therefore have a very large axial aspect ratio.

The full differencing scheme of Eq. (3.3-9) for a semi-implicit calculation is shown in Eq. (3.3-13). For explicit calculations, the axial temperatures evaluated at $n+1$ are simply replaced with values from the $\mathrm{n}^{\text {th }}$ time step. The heat generation term is evaluated at the midpoint of the time step. This is indicated in Eq. $(3.3-13)$ by using the $n+1 / 2$ superscript.

$$
\begin{aligned}
V \xi^{n}\left(T^{n+1}-T^{n}\right) & =V\left(q^{\prime \prime \prime}\right)^{n+1 / 2} \\
& +\Phi_{i+1}^{n}\left(T_{i+1}^{n+1}-T_{i}^{n+1}\right)-\Phi_{i}^{n}\left(T_{i}^{n+1}-T_{i-1}^{n+1}\right) \\
& +\Psi_{j+1}^{n}\left(T_{j+1}^{n+1}-T_{j}^{n+1}\right)-\Psi_{j}^{n}\left(T_{j}^{n+1}-T_{j-1}^{n+1}\right) \\
& +\Lambda_{k+1}^{n}\left(T_{k+1}^{n}-T_{k}^{n+1}\right)-\Lambda_{k}^{n}\left(T_{k}^{n+1}-T_{k-1}^{n}\right)
\end{aligned}
$$

Equation (3.3-13) can be rearranged to collect known quantities evaluated explicitly with temperatures and property values from the old time step on the right hand side and the unknown temperatures at the new time and their associated coefficients on the left hand side as shown in Eq. (3.3-14). In writing Eq. $(3.3-14)$, we have reintroduced complete subscripts on the temperatures for clarity. The coefficient values, cell volume, heat capacity, and deposited power are understood to vary with spatial location as well. We have also introduced the parameter $\delta$ into Eq. (3.3-14) which serves the function of the WSS input flag described above. That is, $\delta=1$ shows the semi-implicit axial differencing scheme while $\delta=0$ shows the fully explicit axial formulation.

$$
\begin{aligned}
{\left[V \xi^{n}+\Phi_{i}^{n}+\right.} & \left.\Phi_{i+1}^{n}+\Psi_{j}^{n}+\Psi_{j+1}^{n}+\delta\left(\Lambda_{k}^{n}+\Lambda_{k+1}^{n}\right)\right] T_{i, j, k}^{n+1} \\
-\Phi_{i}^{n} T_{i-1, j, k}^{n+1} & -\Phi_{i+1}^{n} T_{i+1, j, k}^{n+1}-\Psi_{j}^{n} T_{i, j-1, k}^{n+1}-\Psi_{j+1}^{n} T_{i, j+1, k}^{n+1} \\
= & V \xi^{n} T_{i, j, k}^{n}+\Lambda_{k}^{n} T_{i, j, k-1}^{n}+\Lambda_{k+1}^{n} T_{i, j, k+1}^{n} \\
& -(1-\delta)\left(\Lambda_{k}^{n}+\Lambda_{k+1}^{n}\right) T_{i, j, k}^{n}+V\left(q^{\prime \prime \prime}\right)^{n+1 / 2}
\end{aligned}
$$

To complete the solution of Eq. (3.3-14), boundary conditions must be applied in the spatial directions. In effect, special forms of the coefficients $\Phi_{i}, \Psi_{j}, \Lambda_{k}$ must be specified at the boundary cells.

Axial Boundary Conditions: To solve Eq. (3.3-14) at each axial level in a cylinder we increment the index $\mathrm{k}$ from 1 up to $\mathrm{nz}$ the number of axial levels. The top and bottom levels where the axial boundary conditions are applied must be treated specially. In particular we specify

$$
\Lambda_{1}^{\mathrm{n}} \equiv 0
$$

and 


$$
\Lambda_{\mathrm{nz}+1}^{\mathrm{n}} \equiv 0
$$

which in effect applies adiabatic boundary conditions in the axial direction. Other boundary conditions could be specified by (for example) defining values for solid temperatures $T_{i, j, 0}$ and $T_{i, j, n z+1}$ and evaluating the required coefficients. However, the adiabatic assumption is sufficient for the assembly analysis of interest to us since the geometry characteristically has a large axial aspect ratio and the interior solid temperatures are little influenced by conditions at the axial boundaries.

Azimuthal Boundary Conditions: In the azimuthal direction, we must apply puriodic boundary conditions as the index $\mathrm{j}$ is looped from 1 to $\mathrm{nx}$ where $\mathrm{nx}$ is the number of azimuthal cells. This implies that we set $T_{i, 0, k}=T_{i, n x, k}$ and $T_{i, n x+1, k}=T_{i, 1, k}$ and that, when applying Eq. (3.3-4) to determine $\Psi_{n x+1}^{n}$, we substitute 1 where the subscript $n x+1$ occurs in the metal conductivities. Similarly, to obtain $\Psi_{1}^{n}$ we apply Eq. (3.3-4) with $j=0$ and substitute $\mathrm{nx}$ where the subscript 0 occurs. Note that this also implies that $\Psi_{1}^{n}=\Psi_{n x+1}^{n}$.

Radial Boundary Conditions: The FLOWTRAN-TF code determines heat fluxes at exterior surfaces of the solid assembly cylinders as a function of the current surface temperatures, flow conditions, and boiling regime. These surface heat fluxes provide boundary conditions in the radial direction that are applied in the heat conduction calculations. For example, assuming that the $n \mathrm{r}+1 / 2$ surface represents a solid boundary (the cylinder exterior surface at node $\mathrm{nr}+1 / 2$ where $\mathrm{nr}$ represents the number of radial mesh cells), we write a heat balance at that surface similar to Eq. (3.3-2) as

$$
\mathrm{q}_{n \mathrm{r}+1 / 2}^{\prime \prime}=-\mathrm{k}_{\mathrm{nr}+1 / 2}\left(\frac{\partial \mathrm{T}}{\partial \mathrm{r}}\right)_{\mathrm{nr}+1 / 2}=-\mathrm{k}_{\mathrm{nr}}\left(\frac{\mathrm{T}_{\mathrm{nr}+1 / 2}-\mathrm{T}_{\mathrm{nr}}}{\Delta \mathrm{r}_{\mathrm{nr}} / 2}\right)=\mathrm{q}_{\mathrm{w}}^{\prime \prime}
$$

where $q_{w}^{\prime \prime}$ is the heat flux at the outer surface (wall). A similar expression would apply at the interior surface (subscript $1 / 2$ ). The heat flux at the surface is actually the sum of two terms representing wall heat transfer to the liquid phase and heat transfer to the gas phase. Calculation of the surface heat flux is discussed in section 2.5.1.

A Taylor series expansion of the surface heat flux about the current surface (or wall) temperature $\left(\mathrm{T}_{\mathrm{w}}\right)$ is used to calculate an improved estimate of the radial heat flux boundary condition to more completely capture the temperature dependence and to improve convergence properties. A first order Taylor series expansion is written for the heat flux at the outer radial surface to obtain an approximation for the heat flux at the next time step as

$$
\left[\mathrm{q}_{\mathrm{w}}^{\prime \prime}\right]_{\mathrm{nr}+1 / 2}^{\mathrm{n}+1}=\left[\mathrm{q}_{\mathrm{w}}^{\prime \prime}\right]_{\mathrm{nr}+1 / 2}^{\mathrm{n}}+\left[\frac{\partial \mathrm{q}_{\mathrm{w}}^{\prime \prime}}{\partial \mathrm{T}_{\mathrm{w}}}\right]_{\mathrm{nr}+1 / 2}^{\mathrm{n}}\left(\mathrm{T}_{\mathrm{n}+1 / 2}^{\mathrm{n}+1}-\mathrm{T}_{\mathrm{nr}+1 / 2}^{\mathrm{n}}\right)
$$

In writing Eq. (3.3-16) the subscript $\mathrm{nr}$ indicates the last cell in the radial direction and $n r+1 / 2$ indicates the outer metal surface. In general, the terms in Eq. (3.3-16) will be functions of the azimuthal and axial position within the cylinder. As : vith the surface heat flux, the derivative of the heat flux in Eq. (3.3-16) is the sum of two terms from the wall heat transfer to the liquid phase and heat transfer to the gas phase. 
Substituting Eq. (3.3-16) into the expression for the radial boundary condition given by Eq. (3.3-15) evaluated at the $n+1$ time step and solving for the surface temperature at the $\mathrm{n}+1$ time step then yields

$$
T_{n r+1 / 2}^{n+1}=\frac{\frac{k_{n r}}{\Delta r_{n r}} T_{n r}^{n+1}-\frac{\left[q_{w}^{\prime \prime}\right]_{n r+1 / 2}^{n}}{2}+\frac{1}{2}\left[\frac{\partial q_{w}^{\prime \prime}}{\partial T_{w}}\right]_{n r+1 / 2}^{n} T_{n r+1 / 2}^{n}}{\frac{k_{n r}}{\Delta r_{n r}}+\frac{1}{2}\left[\frac{\partial q_{w}^{\prime \prime}}{\partial T_{w}}\right]_{n r+1 / 2}^{n}}
$$

Using Eq. (3.3-17) to eliminate $T_{n+1 / 2}^{n+1}$ from Eq. (3.3-15), we derive an expression for the heat flux at the radial boundary (analogous to rq. (3.3-3) which applies at interior radial nodes) as

$$
-q_{n r+1 / 2}^{\prime \prime}=2\left(\frac{\frac{k_{n r}}{\Delta r_{n r}} \frac{1}{2}\left[\frac{\partial q_{w}^{\prime \prime}}{\partial T_{w}}\right]_{n r+1 / 2}^{n}}{\frac{k_{n r}}{\Delta r_{n r}}+\frac{1}{2}\left[\frac{\partial q_{w}^{\prime \prime}}{\partial T_{w}}\right]_{n r+1 / 2}^{n}}\right)\left\{\left(T_{n r+1 / 2}^{n}-T_{n r}^{n+1}\right)-\frac{\left[q_{w}^{\prime \prime}\right]_{n r+1 / 2}^{n}}{\left[\frac{\partial q_{w}^{\prime \prime}}{\partial T_{w}}\right]_{n r+1 / 2}^{n}}\right\}
$$

Equation (3.3-18) and the equivalent expression for the heat flux on the inner surface are applied at the boundary cells in the radial direction in solving Eq. (3.3-14). We define

$$
\Phi_{1}^{n} \equiv 2 A_{1 / 2}\left(\frac{\frac{k_{1}}{\Delta r_{1}} \frac{1}{2}\left[\frac{\partial q_{w}^{\prime \prime}}{\partial T_{w}}\right]_{1 / 2}^{n}}{\frac{k_{1}}{\Delta r_{1}}+\frac{1}{2}\left[\frac{\partial q_{w}^{\prime \prime}}{\partial T_{w}}\right]_{1 / 2}^{n}}\right)
$$

and

$$
\Phi_{n r+1}^{n} \equiv 2 A_{n r+1 / 2}\left(\frac{\frac{k_{n r}}{\Delta r_{n r}} \frac{1}{2}\left[\frac{\partial q_{w}^{\prime \prime}}{\partial T_{w}}\right]_{n+1 / 2}^{n}}{\frac{k_{n r}}{\Delta r_{n r}}+\frac{1}{2} \cdot\left[\frac{\partial q_{w}^{\prime \prime}}{\partial T_{w}}\right]_{n r+1 / 2}^{n}}\right)
$$

as radial coefficient values on the inner and outer radial surfaces respectively. Then Eq. (3.3-14) can be applied for $i=1$ and $i=n r$ by eliminating the terms where a temperature $T_{0, j, k}^{n+1}$ or $\mathrm{T}_{\mathrm{nr}+1, j, \mathrm{k}}^{\mathrm{n}+1}$ ocur and by including the additional terms appearing in Eq. (3.3-18) on the right hand side. These additional terms account for heat addition or removal through the exterior metal surfaces at the radial boundaries. For example, at the outer radial surface Eq. (3.3-14) becomes 


$$
\begin{aligned}
{\left[V \xi^{n}+\Phi_{n r}^{n}+\right.} & \left.\Phi_{n r+1}^{n}+\Psi_{j}^{n}+\Psi_{j+1}^{n}+\delta\left(\Lambda_{k}^{n}+\Lambda_{k+1}^{n}\right)\right] T_{n r, j, k}^{n+1} \\
-\Phi_{n r}^{n} T_{n r-1, j, k}^{n+1}- & \Psi_{j}^{n} T_{n r, j-1, k}^{n+1}-\Psi_{j+1}^{n} T_{n r, j+1, k}^{n+1} \\
= & V \xi^{n} T_{n r, j, k}^{n}+\Lambda_{k}^{n} T_{n r, j, k-1}^{n}+\Lambda_{k+1}^{n} T_{n r, j, k+1}^{n} \\
& -(1-\delta)\left(\Lambda_{k}^{n}+\Lambda_{k+1}^{n}\right) T_{n r, j, k}^{n}+V\left(q^{\prime \prime \prime}\right)^{n+1 / 2} \\
& +\Phi_{n r+1}^{n}\left\{T_{n r+1 / 2, j, k}^{n}-\frac{\left[q_{w}^{\prime \prime}\right]_{n r+1 / 2}^{n}}{\left[\frac{\partial q_{w}^{\prime \prime}}{\partial T_{w}}\right]_{n r+1 / 2}^{n}}\right\}
\end{aligned}
$$

Equatio (3.3-, , is also used to compute new values of the surface temperature in FLOWTRAN-T. This expression for the surface temperature is then used to obtain an improved estimate of the surface heat flux from Eq. (3.3-16). This updated estimate of the surface heat flux is used in the fluid energy calculations. We note that the thermal conductivity in Eq. (3.3-17) should be evaluated using the solid temperature at time $n+1$. In practice, we have used the thermal conductivity based on the time $n$ teinperatures in this calculation within the code. Since small time steps are taken and the thermal conductivity will only change slightly this approximation is adequate and avoids reevaluating the property function.

Solution Coding: For each cylinder of a solid assembly, Eq. (3.3-14) is solved at each axial position and each azimuthal position for an updated radial temperature distribution. Flow diagrams of the solution strategy for the solid heat conduction calculations as implemented in the FLOWTRAN-TF code are shown in Figs. 3.3-2, 3.3-3, and 3.3-4. Subroutine SOLID controls the heat conduction solution, evaluates solid physical properties, and loops the calculation over the individual solid cylinders. Subroutine CYLNDR controls the computation for each solid cylinder by calculating the axial terms in the difference equations (which form parts of the diagonal and right hand side terms of the solution matrix) and looping over the axial positions.

Subroutine RING then solves for the temperature distribution at a particular axial layer of the solid cylinder. Holding the temperatures at adjacent axial and azimuthal positions at their current values, a tridiagonal system of equations is solved for the new radial temperatures at each azimuthal position. That is, the azimuthal terms in Eq. (3.3-14) are actually transferred to the right hand side to give the modified equation

$$
\begin{aligned}
{\left[V \xi^{n}+\Phi_{i}^{n}+\right.} & \left.\Phi_{i+1}^{n}+\Psi_{j}^{n}+\Psi_{j+1}^{n}+\delta\left(\Lambda_{k}^{n}+\Lambda_{k+1}^{n}\right)\right] T_{i, j, k}^{n+1} \\
& -\Phi_{i}^{n} T_{i-1, j, k}^{n+1}-\Phi_{i+1}^{n} T_{i+1, j, k}^{n+1} \\
& =V \xi^{n} T_{i, j, k}^{n}+\Lambda_{k}^{n} T_{i, j, k-1}^{n}+\Lambda_{k+1}^{n} T_{i, j, k+1}^{n} \\
& -(1-\delta)\left(\Lambda_{k}^{n}+\Lambda_{k+1}^{n}\right) T_{i, j, k}^{n}+V\left(q^{\prime \prime \prime}\right)^{n+1 / 2} \\
& +\Psi_{j}^{n} T_{i, j-1, k}^{n+1}+\Psi_{j+1}^{n} T_{i, j+1, k}^{n}
\end{aligned}
$$


Equation (3.3-20) is now tridiagonal in the radial temperatures and can be solved efficiently. This solution is swept around over each azimuthal mesh position for the single axial layer using the newly calculated radial temperatures for $n+1$ time step values on the right hand side (the $T_{i, j-1, k}^{n+1}$ temperatures in Eq. (3.3-20)). When solving the first azimuthal stripe $(j=1)$ this temperature is the old value at the $n x$ azimuthal location $\left(T_{i, n x, k}^{n}\right)$. For subsequent azimuthal stripes (see Fig. 3.3-2), the temperatures are evaluated as shown in Eq. (3.3-20). After a complete azimuthal sweep, a coarse mesh rebalance calculation is performed to give improved temperature estimates. The azimuthal sweep and rebalance calculations are performed several times every time step. The number of sweeps is controlled by the code user through input. When the problem does not have any azimuthal dependence (two dimensional case), this procedure is bypassed to simplify the numerics. For two-dimensional problems, FLOWTRAN-TF is coded to solve a reduced form of Eq. (3.3-14) having only radial and axial dependence. For these cases, the azimuthal coefficients in Eq. (3.3-14) $\left(\Psi_{\mathrm{j}}\right.$ and $\left.\Psi_{\mathrm{j}+1}\right)$ vanish identically and the solution is somewhat simplified.

The above calculation procedure is efficient in that only a tridiagonal matrix needs to be solved at each azimuthal and axial position within the cylinders for new radial temperature values. Since the solution proceeds around an axial ring azimuthally, the updated temperatures appear only in the j-1 positions in Eq. (3.3-20). The coarse mesh rebalance is used to bring all of the temperatures into alignment at each step in the iteration. The coarse mesh equations are periodic tridiagonal and can also be solved efficiently (see section 3.8). After a complete azimuthal calculation has been made, the solution is advanced to the next axial layer and the procedure repeated. This solution strategy is then repeated for each cylinder in the assembly. A partial listing of the variables used in the heat conduction equations and the corresponding variables internal to the FLOWTRAN-TF code is presented in Table 3.3-1. 
Table 3.3-1

Heat conduction variables.

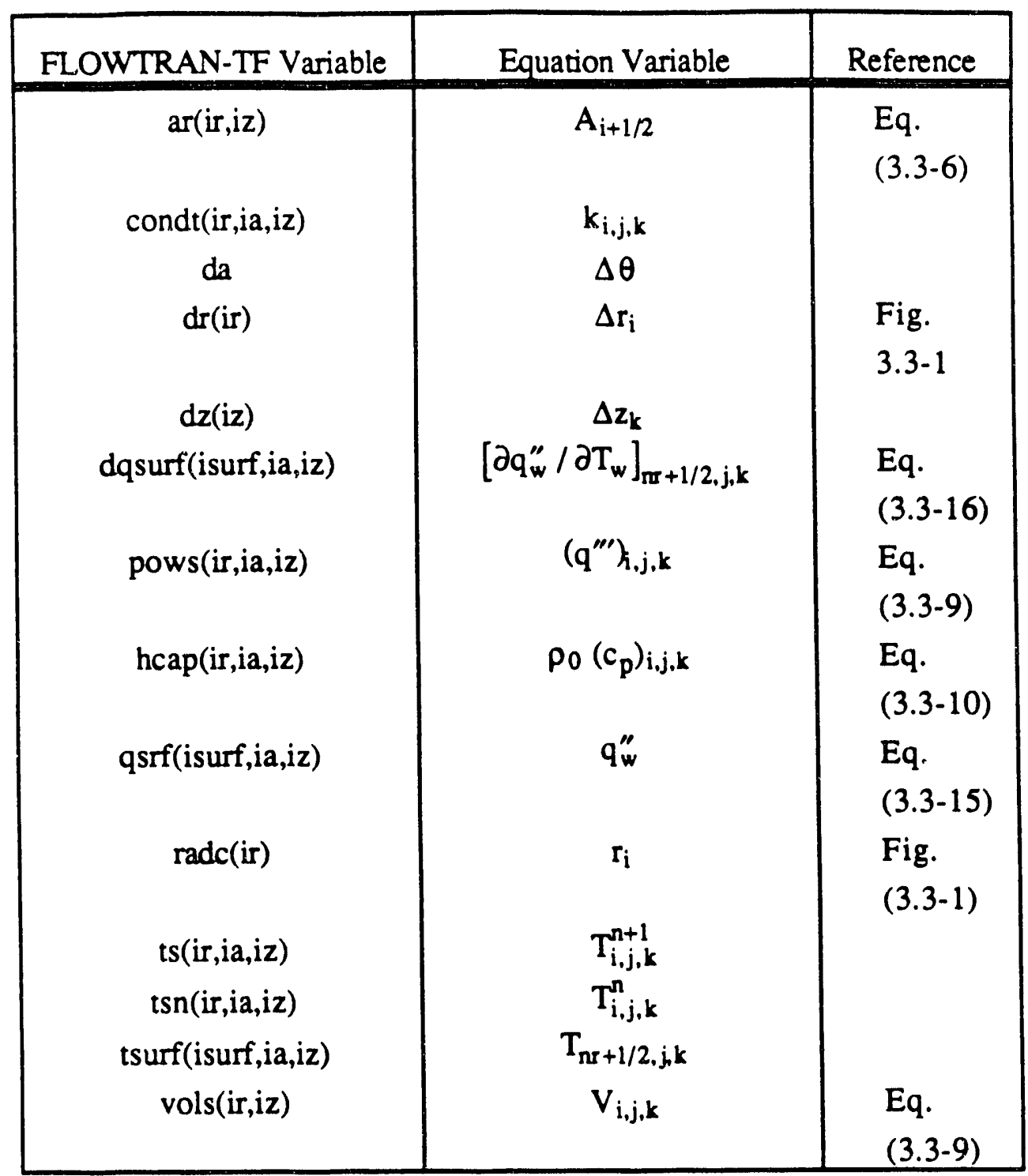

Coarse Mesh Rebalance: As explained above, the solid solution scheme calculates updated estimates of the radial temperatures at a particular azimuthal location using current values of temperatures in adjacent azimuthal and axial cells. We assume that improved estimates for the temperatures at each time step and axial level may be obtained by lumping the radial cells at each azimuthal position into single nodes and solving Eq. (3.3-14) for residual values that will balance the temperatures over the azimuthal direction. That is, we take an improved temperature estimate at the new time step $(n+1)$ as

$$
T_{i, j, k}^{n+1, p+1}=T_{i, j, k}^{n+1, p}+R_{j}
$$

where $R_{j}$ is the residual correction factor which is a function of azimuthal position and the superscript $p$ is used to indicate the step in the coarse mesh rebalance iteration. 
Substituting Eq. (3.3-21) into Eq. (3.3-14), summing over all nr cells in the radial direction and rearranging the result to collect like terms multiplying correction factors on the left hand side yields

$$
\begin{aligned}
& \left\{\sum_{i=1}^{n r}\left[V \xi^{n}+\Psi_{j}^{n}+\Psi_{j+1}^{n}+\delta\left(\Lambda_{k}^{n}+\Lambda_{k+1}^{n}\right)\right]+\Phi_{1}^{n}+\Phi_{n r+1}^{n}\right\} R_{j} \\
& -\left\{\sum_{i=1}^{n r} \Psi_{j}^{n}\right\} R_{j-1}-\left\{\sum_{i=1}^{n r} \Psi_{j+1}^{n}\right\} R_{j+1} \\
& =\sum_{i=1}^{n x}\left\{V \xi^{n}\left(T_{i, j, k}^{n}-T_{i, j, k}^{n+1}\right)+\Psi_{j}^{n}\left(T_{i, j-1, k}^{n+1}-T_{i, j, k}^{n+1}\right)+\Psi_{j+1}^{n}\left(T_{i, j+1, k}^{n+1}-T_{i, j, k}^{n+1}\right)\right. \\
& +\Lambda_{k}^{n}\left(T_{i, j, k-1}^{n}-T_{i, j, k}^{n}\right)+\Lambda_{k+1}^{n}\left(T_{i, j, k+1}^{n}-T_{i, j, k}^{n}\right) \\
& \left.+\delta\left(\Lambda_{k}^{n}+\Lambda_{k+1}^{n}\right)\left(T_{i, j, k}^{n}-T_{i, j, k}^{n+1}\right)+V\left(q^{\prime \prime \prime}\right)^{n+1 / 2}\right\} \\
& +\Phi_{1}^{n}\left\{T_{1 / 2, j, k}^{n}-T_{1, j, k}^{n+1}-\frac{\left[q_{w}^{\prime \prime}\right]_{1 / 2}^{n}}{\left[\frac{\partial q_{w}^{\prime \prime}}{\partial T_{w}}\right]_{1 / 2}^{n}}\right\}+\Phi_{n r+1}^{n}\left\{T_{n r+1 / 2, j, k}^{n}-T_{n r, j, k}^{n+1}-\frac{\left[q_{w}^{\prime \prime}\right]_{n+1 / 2}^{n}}{\left[\frac{\partial q_{w}^{\prime \prime}}{\partial T_{w}}\right]_{n r+1 / 2}^{n}}\right\}
\end{aligned}
$$

where the superscript $p$ has been dropped from the $n+1$ temperatures for simplification. In effect, the $n+1$ values in Eq. (3.3-22) are understood to be current estimates from previous radial solution and azimuthal rebalance iterations. In the radial direction, the contribution to the summations from adjacent cells identically cancel leaving only the boundary terms. Equation (3.3-22) is solved for the temperature residuals and improved estimates of the solid temperatures are computed by adding the residual at every azimuthal position to all radial solid temperatures as indicated by Eq. (3.3-21). Since the first and last cells in the azimuthal direction are coupled, the solution matrix is periodic tridiagonal containing additional terms in the comer positions of a simple tridiagonal matrix. However, with a slight modification of the usual tridiagonal solution technique, Eq. (3.3-22) can be solved efficiently (see section 3.8.5). 


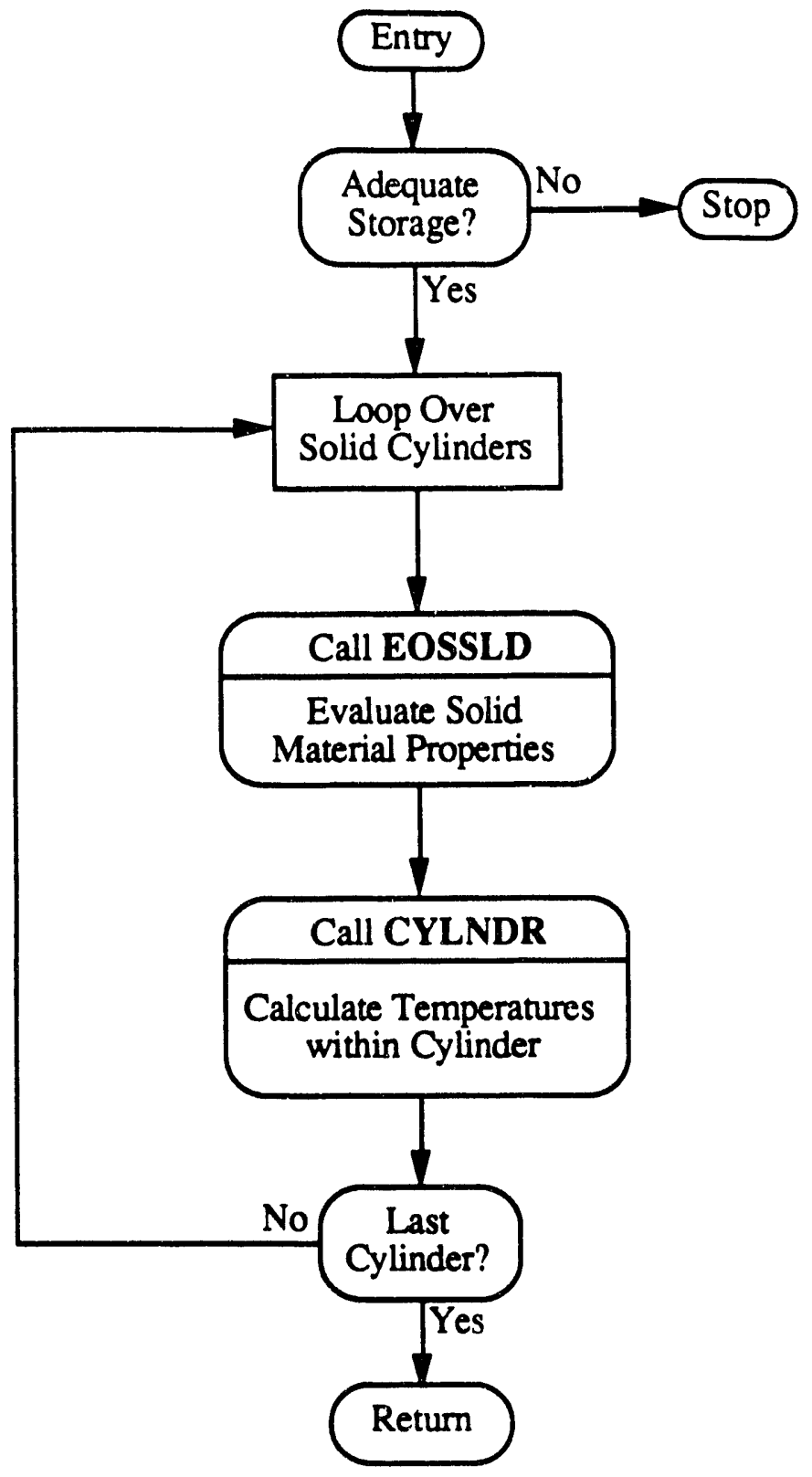

Figure $\quad 3.3-2 \quad$ Flowchart for subroutine SOLID. 


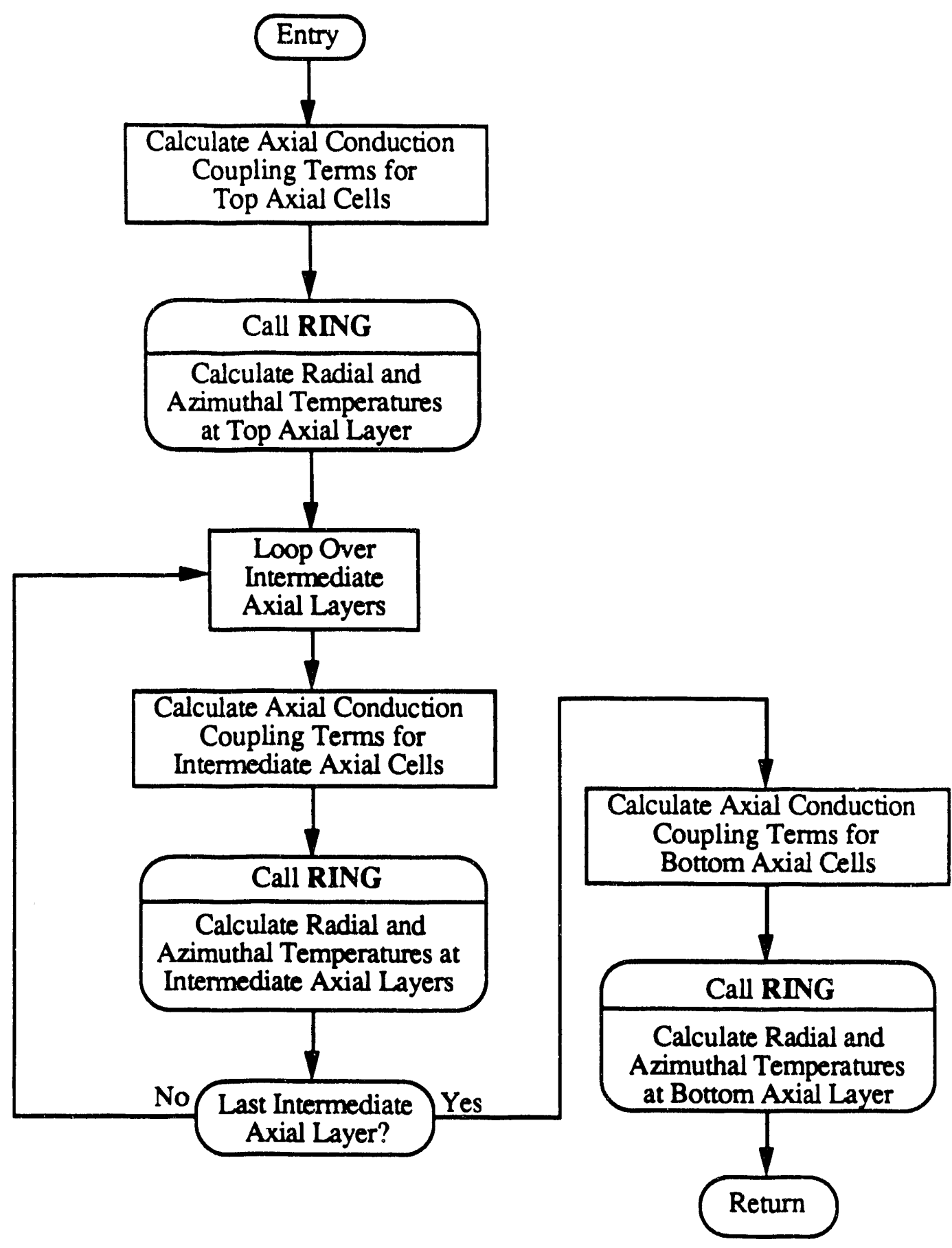

Figure 3.3-3 Flowchart for subroutine CYLNDR. 


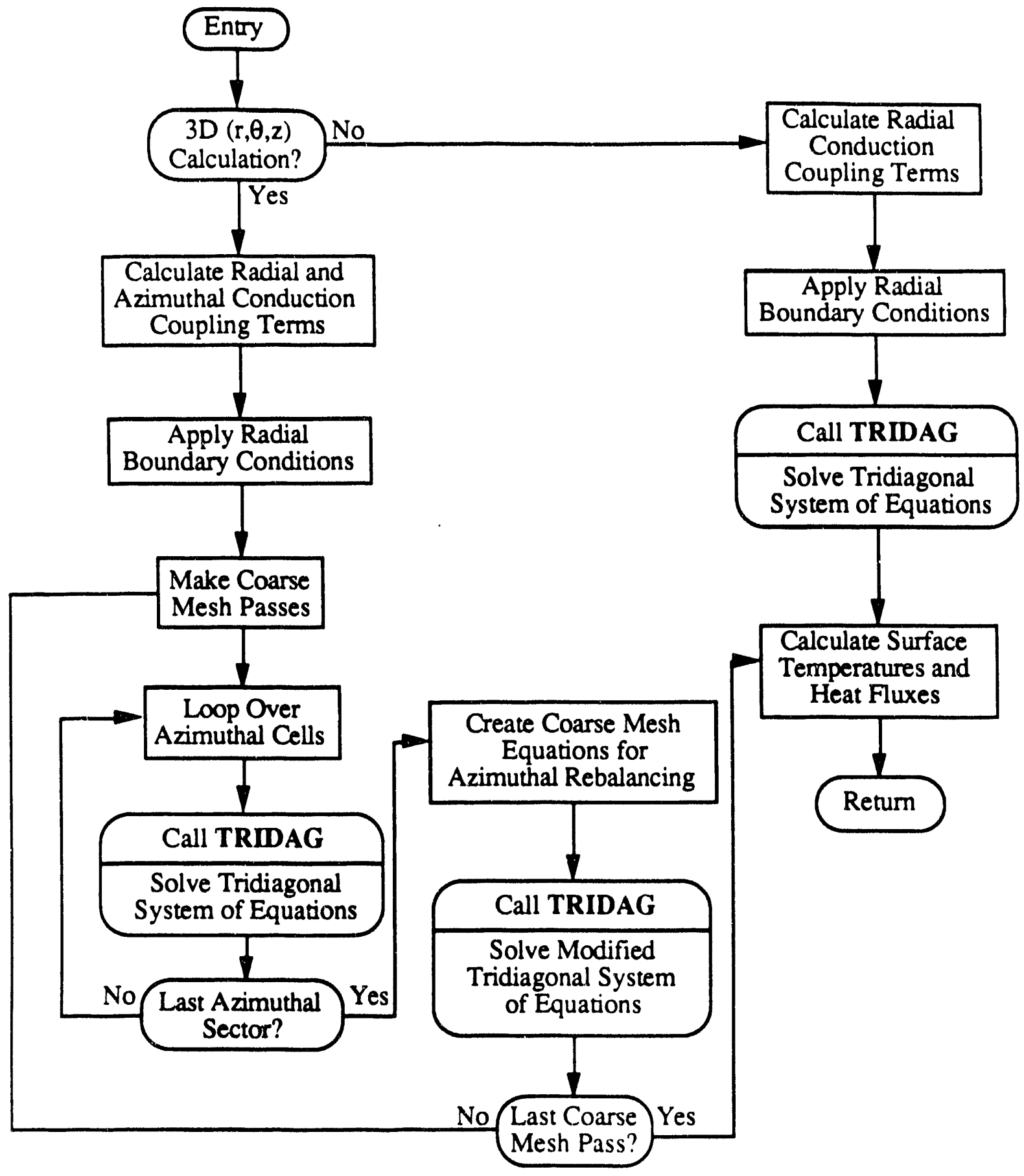

Figure 3.3.4 Flowchart for subroutine RING. 


\subsection{Fluid Finite Difference Equations}

The discussion is broken into three parts. The first is the basic finite difference scheme for the axial and azimuthal momentum balances associated with the two-dimensional part of the FLOWTRAN-TF mesh. The corresponding mass and energy finite differenced equations are derived in the second subsection. The third subsection presents the variants of the general two-dimensional mass, momentum and energy finite differenced equations needed for one-dimensional regions and junctions between one- and two-dimensional mesh sections.

\subsubsection{Momentum}

In this section of the manual, we derive the finite difference approximations used to solve the fluid governing equations. In general the fluid equations are two-dimensional (axial and azimuthal) so we will use the two-dimensional forms of the equations to develop our difference equations. The two-dimensional fluid cells are located in the middle section of the assembly as shown by the cross-hatched iegion in Fig. 3.4.1-1. In the top and bottom sections of the assembly, the fluid equations reduce to one-dimensional (axial) forms and the azimuthal momentum equations do not apply.

Some of the nomenclature used to describe the fluid variables is illustrated in Figs. 3.4.1-2 through 3.4.1-4. Figure 3.4.1-2 shows a general mass and energy fluid control volume, Fig. 3.4.1-3 shows an axial momentum control volume, and Fig. 3.4.1-4 shows an azimuthal momentum control volume. In drawing these diagrams and in the equation development that follows, we have chosen to use whole integers for the grid locations at the centers of the mass/energy control volumes and half integer indices for the face center locations. Index $\mathrm{i}$ is used for the azimuthal direction and index $\mathrm{j}$ for the axial direction.

Figure 3.4.1-2 illustrates the staggered mesh used for the fluid computations where the primary variables are defined at the cell center and fluid velocities are defined at the centers of the cell faces. The axial and azimuthal momentum cells are composed of half volumes of adjacent mass cells as shown in Figs. 3.4.1-3 and 3.4.1-4. The subscript nomenclature on the cell face areas (e.g. $A_{c z}$ ) follows the notation convention:

First subscript denotes the associated control volume;

$$
\begin{aligned}
& c \Rightarrow \text { mass/energy cell } \\
& x \Rightarrow x \text {-momentum face } \\
& z \Rightarrow z \text {-momentum face }
\end{aligned}
$$

Second subscript identifies the direction of the normal vector to the area;

$$
\begin{aligned}
& z \Rightarrow z \text {-direction } \\
& x \Rightarrow x \text {-direction }
\end{aligned}
$$




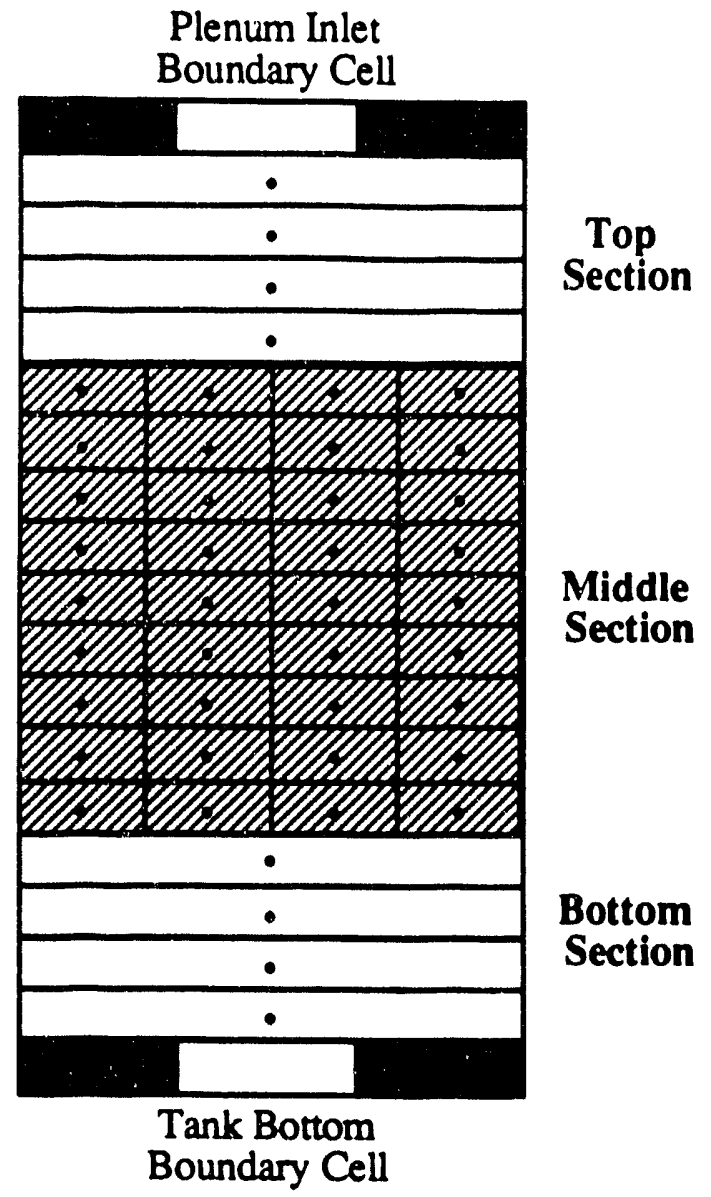

Figure 3.4.1-1 Two-dimensional fluid cells.

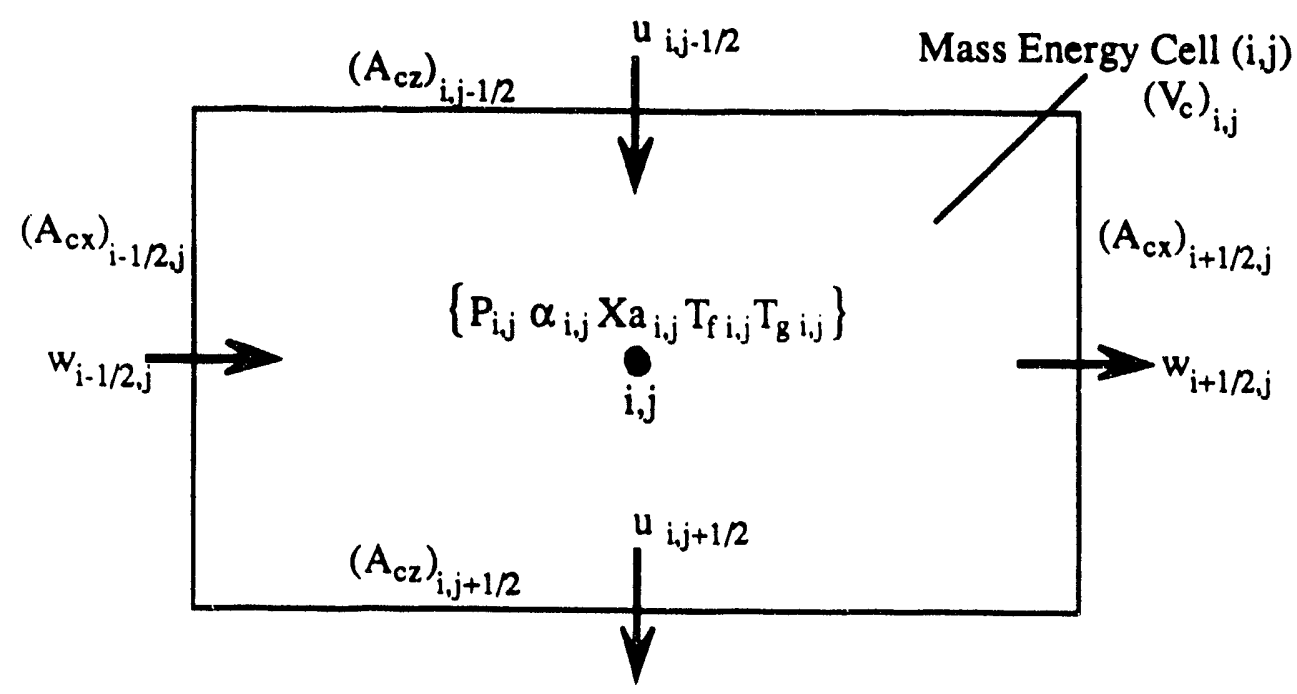

Figure 3.4.1-2 Mass/energy control volume. 


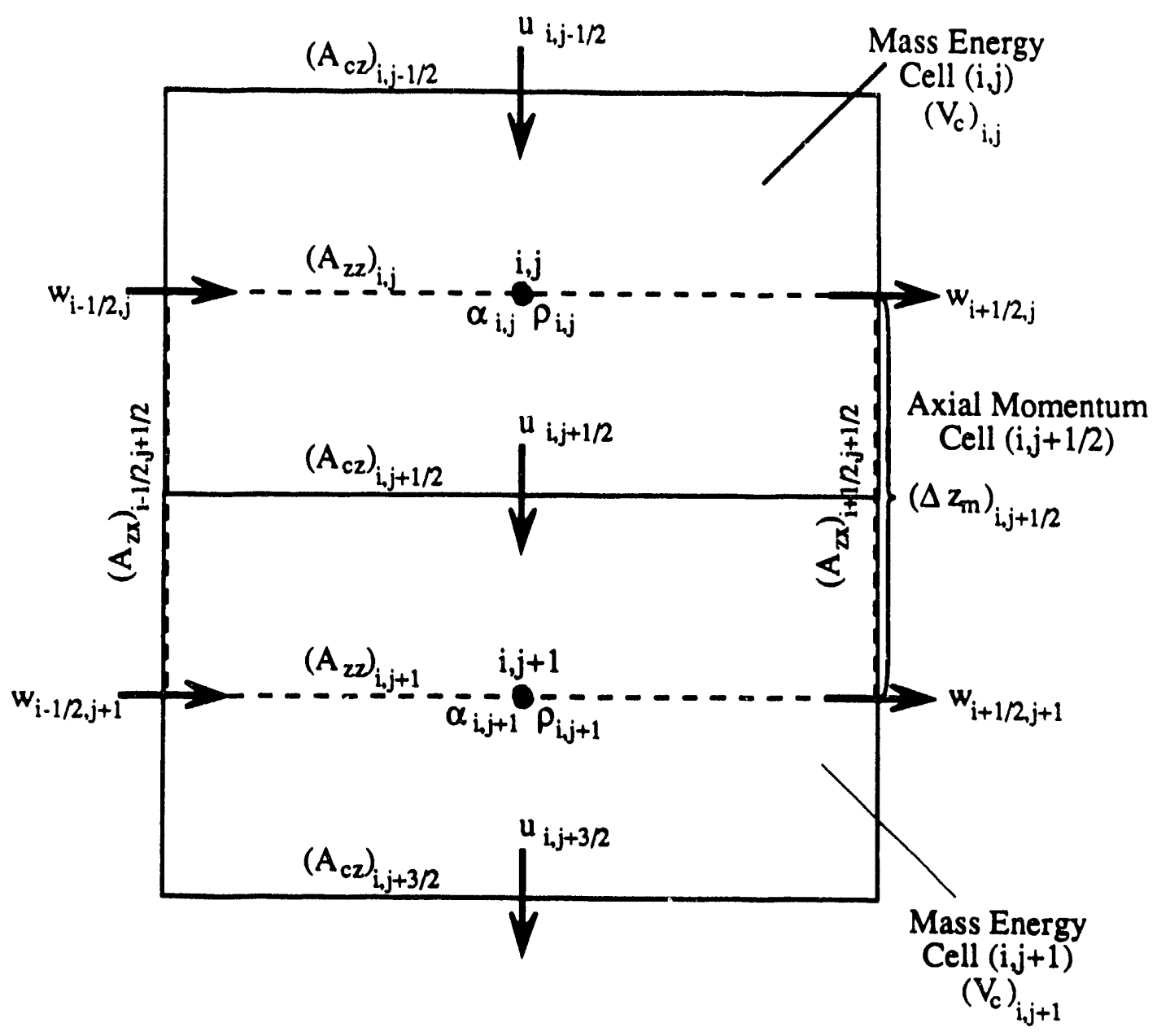

Figure 3.4.1-3 Axial momentum control volume. 


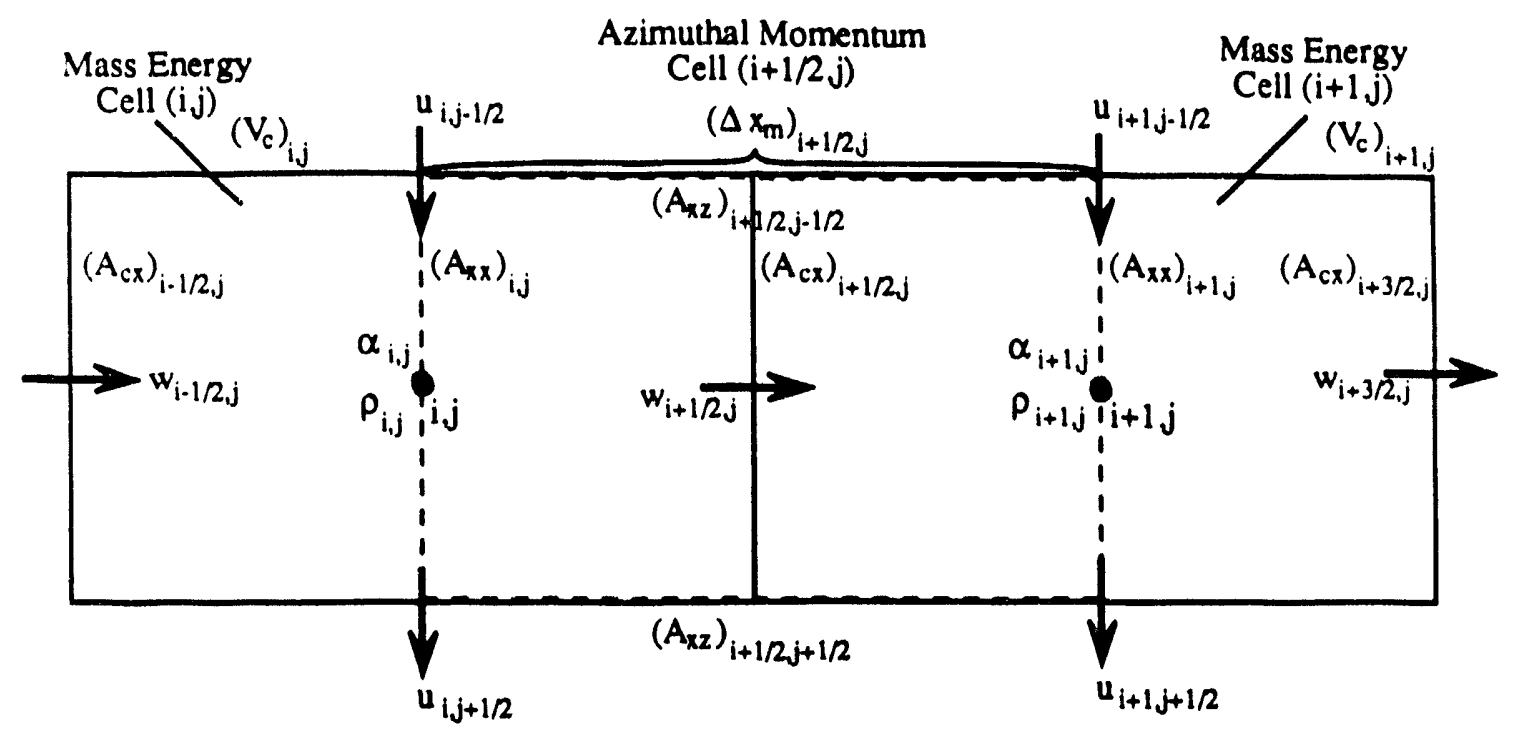

Figure 3.4.1-4 Azimuthal momentum control volume.

Differencing Strategy: In developing the fluid difference equations, we have followed the same basic logic used with the THERMIT (Reed and Stewart, 1978) and RELAP5 (Dimenna et al., 1988) codes. That is, the differencing is neither fully explicit nor fully implicit. Local phenomena such as interfacial mass and energy exchange that occur on a short time scale are treated implicitly. Likewise, velocities in the mass and energy transport equations and the pressure gradient term in the momentum equation which represent sonic propagation effects are also treated implicitly. Other terms in the mass and energy conservation equations and in the momentum transport equations are treated explicitly to increase computational efficiency. These terms represent physical phenomena that occur on a longer time scale. In particular, advection terms in the momentum equations are evaluated explicitly.

Since some of the differencing is explicit, Courant limits must be placed on the time step to ensure numerical stability. Donor-cell differencing is also used to improve the stability characteristics of the fluid difference equations. In addition, time step constraints are imposed such that nonlinear implicit functions can be adequately approximated by Taylor series expansions about the old time values. This produces function approximations that are linear in the implicit variables and avoids the need to iteratively solve systems of nonlinear equations.

Since momentum advection terms are evaluated explicitly and Taylor series expansions are used to linearize implicit terms, the momentum equations can be directly solved for the fluid velocity as a function of the local pressure differences. The coefficients in these velocity functions are all evaluated explicitly. These functions will be used to eliminate the explicit velocities from the finite difference equations for mass and energy conservation developed in the following sections. This leaves a set of five equations in the five unknown state variables $\left(P, \alpha, T_{g}, T_{f}\right.$, and $\left.X_{a}\right)$. These equations are then combined to reduce the problem of solving the fluid equation set to that of solving for the pressure field at the new time.

Momentum Equations: We begin our development of the fluid difference equations by treating the axial and azimuthal momentum equations. Essentially, the methodology used to approximate the fluid momentum equations is to evaluate advection terms explicitly and 
pressure gradients implicitly. Further implicit dependence on the fluid velocities is introduced through Taylor series expansions of the wall and interfacial drag forces. The resulting equations are then solved simultaneously for expressions relating the local velocity components at the new time step to the local pressure difference.

Before beginning the analysis, we wish to clarify our use of the sharp bracket $(<>)$ nomenclature. Frequently, the brackets are used to denote volume averaged quantities but their application is more general. Basically, we use the brackets to denote auxiliary quantities which are ultimately defined in terms of the fundamental quantities. By fundamental quantities we mean the state variables at $(i, j)$, the axial velocities at $(i, j+1 / 2)$, and the azimuthal velocities at $(i+1 / 2, j)$ that are calculated from the governing equations. The bracket notation is useful for explicitly flagging those quantities for which auxiliary expressions must be provided because one or more of the fundamental variables is not present at the location in question.

Starting with Eqs. (2.4.1-97) and (2.4.1-98), the full gas and liquid momentum equations can be written in a slightly modified form as

$$
\begin{aligned}
\alpha \rho_{g} \frac{\partial \bar{v}_{g}}{\partial t}+\frac{1}{V} \nabla \cdot\left[V \alpha \rho_{g} \bar{v}_{g} \bar{v}_{g}\right] & -\frac{\bar{v}_{g}}{V} \nabla \cdot\left[V \alpha \rho_{g} \bar{v}_{g}\right]= \\
& -\alpha \nabla P+\alpha \rho_{g} \bar{g}-\bar{F}_{w g}-\bar{F}_{I}-\bar{M}_{t g}
\end{aligned}
$$

and

$$
\begin{aligned}
(1-\alpha) \rho_{f} \frac{\partial \bar{v}_{f}}{\partial t}+\frac{1}{V} \nabla \cdot\left[V(1-\alpha) \rho_{f} \bar{v}_{f} \bar{v}_{f}\right]-\frac{\bar{v}_{f}}{V} \nabla \cdot\left[V(1-\alpha) \rho_{f} \bar{v}_{f}\right]= \\
-(1-\alpha) \nabla P+(1-\alpha) \rho_{f} \bar{g}-\bar{F}_{w f}+\bar{F}_{I}-\bar{M}_{t f}
\end{aligned}
$$

The advection terms in the above equations have been written in an expanded form to make the finite differencing process more straightforward. The development of the gas and liquid equations is almost identical so we will treat the gas equation in detail and then simply give the modifications required to form the equivalent liquid equation. We will first consider the axial component of the momentum equations.

Axial Momentum Equations: The axial component of Eq. (3.4.1-1) is

$$
\alpha \rho_{g} \frac{\partial u_{g}}{\partial t}+\frac{1}{V} \nabla \cdot\left[V \alpha \rho_{g} u_{g} \bar{v}_{g}\right]-\frac{u_{g}}{V} \nabla \cdot\left[V \alpha \rho_{g} \bar{v}_{g}\right]=-\alpha \frac{\partial P}{\partial z}+\alpha \rho_{g} g^{2}-F_{w_{g}}^{z}-F_{I}^{z}-M_{t g}^{z}
$$

We integrate each term in Eq. (3.4.1-3) over the axial momentum control volume $\left(\mathrm{V}_{\mathrm{m}}\right.$, shown in Fig. 3.4.1-3) and divide by the momentum cell volume. Integrating the first term yields 


$$
\begin{aligned}
\frac{1}{V_{m}} \int_{V_{m}} \alpha \rho_{g} \frac{\partial u_{g}}{\partial t} d V_{m} & \rightarrow \frac{1}{V_{m}} \int_{V_{m}} \alpha \rho_{g} d V_{m} \cdot \frac{\partial}{\partial t}\left\{\frac{1}{V_{m}} \int_{V_{m}} u_{g} d V_{m}\right\} \\
& =\left\langle\alpha \rho_{g}\right\rangle_{i, j+1 / 2}^{n}\left[\frac{\left\langle u_{g}\right\rangle_{i, j+1 / 2}^{n+1}-\left\langle u_{g}\right\rangle_{i, j+1 / 2}^{n}}{\Delta t}\right]
\end{aligned}
$$

To obtain the first equality in Eq. (3.4.1-4) (indicated by the arrow) we assume that the volume average of the product of the terms inside the integral is equal to the product of the volume averages and we interchange the operations of taking the partial derivative with respect to time and volume averaging the velocity. To obtain the final equality, we approximate the cell center velocity using an appropriate volume averaged velocity and replace the partial derivative with a forward difference approximation.

At the $(i, j+1 / 2)$ mesh point an axial velocity is available and we could simply use this as an approximation to the momentum cell average value. However, we have chosen to define a volume averaged momentum cell velocity through the relationship

$$
\begin{aligned}
\left\langle u_{g}\right\rangle_{i, j+1 / 2}^{n} & =\frac{\left(A_{c z}\right)_{i, j+1 / 2}}{\left\langle A_{c z}\right\rangle_{i, j+1 / 2}}\left(u_{g}\right)_{i, j+1 / 2}^{n} \\
& =R_{i, j+1 / 2}^{z}\left(u_{g}\right)_{i, j+1 / 2}^{n}
\end{aligned}
$$

where we have introduced the average area

$$
\left\langle A_{c z}\right\rangle_{i, j+1 / 2} \equiv \frac{\left(V_{z}\right)_{i, j+1 / 2}}{\left(\Delta z_{m}\right)_{i, j+1 / 2}}
$$

which represents the average cross sectional area of the momentum cell. Dividing the momentum cell volume by the momentum cell length $\left(\Delta z_{m}\right)_{i, j+1 / 2}$ gives an average cell cross sectional area which may be different from $\left(A_{c z}\right)_{i, j+1 / 2}$ the input cross sectional area at grid location $(i, j+1 / 2)$. We take the computational mesh points to be located at the centers of the mass/energy control volumes. The momentum cell volume is then equal to one half of the upper mass cell plus one half of the lower mass cell. That is, we compute the momentum cell volume introduced in Eq. $(3.4 .1-6)$ as

$$
\left(V_{z}\right)_{i, j+1 / 2}=\frac{\left(V_{c}\right)_{i, j}+\left(V_{c}\right)_{i, j+1}}{2}
$$

Similarly, the momentum cell length is equal to one half of the sum of lengths of the two mass/energy cells that make up the momentum cell (see \$2.1 and \$3.1)

$$
\left(\Delta z_{m}\right)_{i, j+1 / 2}=\frac{\Delta z_{i, j}+\Delta z_{i, j+1}}{2}
$$


The mass/energy cell volumes and axial lengths are input values. Equation (3.4.1-5) then represents a true momentum cell averaged velocity. We have introduced the axial area ratio $R_{i, j+1 / 2}^{R}$ in Eq. (3.4.1-5) to simplify the notation.

Returning to Eq. (3.4.1-4), we indicate the volume average of the product of void fraction and gas density using the angle bracket notation and evaluate the average at the old time $(n)$ and at the center of the momentum cell. In general, the volume average of any physical property is computed by weighting the property values at the centers of the mass cells by the respective cell volumes as

$$
\langle\phi\rangle_{i, j+1 / 2}^{n} \equiv \frac{(\phi)_{i, j}^{n}\left(V_{c}\right)_{i, j}+(\phi)_{i, j+1}^{n}\left(V_{c}\right)_{i, j+1}}{\left(V_{c}\right)_{i, j}+\left(V_{c}\right)_{i, j+1}}
$$

We apply Eq. (3.4.1-9) to the product of void fraction and gas density by setting $\phi=\alpha \rho_{\mathrm{g}}$. Integratirig the second term in Eq. (3.4.1-3) gives

$$
\begin{aligned}
\frac{1}{V_{m}} \int_{V_{m}} \frac{1}{V} \nabla \cdot\left[V \alpha \rho_{g} u_{g} \bar{v}_{g}\right] d V_{m} & \rightarrow \frac{1}{V_{m}} \int_{A_{g}}\left(\alpha \rho_{g} u_{g} \bar{v}_{g}\right) \cdot \bar{n} d A_{s} \\
\cong \frac{1}{\left(V_{z}\right)_{i, j+1 / 2}}\left[-\left\langle A_{z z} \alpha \rho_{g} u_{g} u_{g}\right\rangle_{i, j}^{n}+\left\langle A_{z z} \alpha \rho_{g} u_{g} u_{g}\right\rangle_{i, j+1}^{n}\right. & \quad-\left\langle A_{z x} \alpha \rho_{g} w_{g}\right\rangle_{i-1 / 2, j+1 / 2}^{n}\left\langle u_{g}\right\rangle_{i-1 / 2, j+1 / 2}^{n} \\
& \left.+\left\langle A_{z x} \alpha \rho_{g} w_{g}\right\rangle_{i+1 / 2, j+1 / 2}^{n}\left\langle u_{g}\right\rangle_{i+1 / 2, j+1 / 2}^{n}\right]
\end{aligned}
$$

Equation (3.4.1-10) is obtained by applying the divergence theorem to convert the volume integral of the divergence of the momentum flux into an integral over the surface of the momentum control volume. The surface integral is evaluated by taking appropriate averages of the indicated momentum flux terms over the four sides of the momentum control volume. The first two terms represent axial m/smentum advected into the control volume by the axial velocity while the last two terms refresent axial momentum advected into the control volume by the azimuthal velocity. Wirt the staggered mesh used for the fluid differencing, axial and azimuthal velocities are defined at mesh points that do not easily correspond to each other. Therefore, it is con venient to introduce the approximation shown in the last two terms of Eq. (3.4.1-10) where we separate the axial velocity from the azimuthal component to the momentum and average it separately. All of the momentum advection terms are evaluated explicitly using velocities and property values from the previous time step. Approximations for these average quantities will be derived below.

Integrating the third term in Eq. (3.4.1-3) gives 


$$
\begin{aligned}
\frac{1}{V_{m}} \int_{V_{m}} \frac{u_{g}}{V} \nabla \cdot\left[V \alpha \rho_{g} \bar{v}_{g}\right] d V_{m} & \rightarrow \frac{1}{V_{m}} \int_{V_{m}} u_{g} d V_{m} \cdot \frac{1}{V_{m}} \int_{A_{g}}\left(\alpha \rho_{g} \bar{v}_{g}\right) \cdot \bar{n} d A_{s} \\
= & \frac{\left\langle u_{g}\right\rangle_{i, j+1 / 2}^{n}}{\left(V_{z}\right)_{i, j+1 / 2}}\left[-\left\langle A_{z z} \alpha \rho_{g} u_{g}\right\rangle_{i, j}^{n}+\left\langle A_{z z} \alpha \rho_{g} u_{g}\right\rangle_{i, j+1}^{n}\right. \\
& \left.-\left\langle A_{z x} \alpha \rho_{g} w_{g}\right\rangle_{i-1 / 2, j+1 / 2}^{n}+\left\langle A_{z x} \alpha \rho_{g} w_{g}\right\rangle_{i+1 / 2, j+1 / 2}^{n}\right]
\end{aligned}
$$

Equation (3.4.1-11) is derived by first assuming that the volume average of the product of the velocity and the divergence of the mass flux can be separated into the product of volume averages of the individual terms. We then apply the divergence theorem to convert the volume integral of the mass flux into an integral over the surface of the momentum control volume. The second equality is obtained by evaluating the surface integral by taking appropriate averages of the mass flows over the four sides of the momentum control volume. Approximations for the spatially averaged mass flowrates will be derived below. The gas velocity and other spatial averages are evaluated explicitly using values from the previous time step. Note that the azimuthal mass flowrates in Eq. (3.4.1-11) also appear in Eq. (3.4.1-10).

Integrating the first term on the right hand side of Eq. (3.4.1-3) gives

$$
\begin{aligned}
\frac{1}{V_{m}} \int_{V_{m}} \alpha \frac{\partial P}{\partial z} d V_{m} & =\frac{1}{V_{m}} \int_{z_{m}} \alpha A(z) \frac{\partial P}{\partial z} d z_{m} \\
& =\frac{\langle\alpha A\rangle_{i, j+1 / 2}^{n}}{\left(V_{z}\right)_{i, j+1 / 2}}\left(P_{i, j+1}^{n+1}-P_{i, j}^{n+1}\right)
\end{aligned}
$$

To derive Eq. (3.4.1-12), we first use the relationship

$$
d V_{m}=A(z) d z_{m}
$$

to replace the integral over the momentum control volume with the equivalent integral over the cell length. Applying the mean value theorem, we evaluate the integral of the axial pressure gradient as the pressure difference across the momentum cell in the axial direction multiplied by an average product of the void fraction and cell area. The term $\langle\alpha A\rangle_{j, j+1 / 2}^{h}$ is some as yet undefiped average phasic area to be applied to the local pressure difference. The value of $\langle\alpha A\rangle_{j, j+1 / 2}$ is chosen such that the Bernoulli equation is satisfied in certain limiting cases. A derivation of the appropriate form of the average phasic area is given in the "Bernoulli Differencing Scheme" section below. The pressures in Eq. (3.4.1-12) are evaluated implicitly at the new time $(n+1)$.

Differencing the gravitational head term [second term on the right hand side of Eq. $(3.4 .1-3)$ ] must be done carefully so that the Bernoulli equation will be recovered correctly. From Eq. (3.4.1-12), we see that the pressure difference is multiplied by the factor 


$$
\frac{\langle\alpha A\rangle_{i, j+1 / 2}^{n}}{\left(V_{z}\right)_{i, j+1 / 2}}
$$

To recover pressure changes predicted by Bernoulli's equation, we must have the gravity head term multiplied by the momentum cell length $\left(\Delta z_{m}\right)_{i, j+1 / 2}$. Therefore, we use the approximation

$$
\begin{aligned}
\frac{1}{V_{m}} \int_{V_{m}} \alpha \rho_{g} g^{2} d V_{m} & =\frac{1}{V_{m}} \int_{z_{m}} \alpha \rho_{g} g^{z} A(z) d z_{m} \\
& \cong\left\langle\rho_{g}\right\rangle_{i, j+1 / 2}^{n} g_{i, j+1 / 2}^{z} \frac{\langle\alpha A\rangle_{i, j+1 / 2}^{n}\left(\Delta z_{m}\right)_{i, j+1 / 2}}{\left(V_{z}\right)_{i, j+1 / 2}}
\end{aligned}
$$

The volume averaged gas density is evaluated using Eq. (3.4.1-9) with $\phi=\rho_{\mathrm{g}}$.

The wall frictional drag and interfacial drag terms are both evaluated in a similar manner. These drag forces are nonlinear functions of the liquid and gas velocities. We linearize these expressions by expanding in Taylor series about the old time values. The motivation for performing this linearization is to eventually allow us to solve the momentum equations for velocity as an explicit function of pressure and ultimately reduce the entire problem to that of solving for the pressure only. For the wall frictional drag we obtain

$$
\begin{aligned}
\frac{1}{V_{m}} \int_{V_{m}} F_{w_{g}}^{z} d V_{m} \rightarrow\left(F_{w_{g}}^{z}\right)_{i, j+1 / 2}^{n+1} \equiv\left(F_{w_{g}}^{z}\right)_{i, j+1 / 2}^{n} & +\left(\frac{\partial F_{w_{g}}^{z}}{\partial u_{g}}\right)_{i, j+1 / 2}^{n}\left[u_{g}^{n+1}-u_{g}^{n}\right]_{i, j+1 / 2} \\
& +\left(\frac{\partial F_{w_{g}}^{z}}{\partial u_{f}}\right)_{i, j+1 / 2}^{n}\left[u_{f}^{n+1}-u_{f}^{n}\right]_{i, j+1 / 2}
\end{aligned}
$$

Rearranging to collect terms evaluated at the same time step and simplifying the notation by writing the common spatial subscripts on the outer brackets gives

$$
\begin{aligned}
\left(F_{w g}^{z}\right)_{i, j+1 / 2}^{n+1} & \equiv\left[\left(\frac{\partial F_{w_{g}}^{z}}{\partial u_{g}}\right)^{n} u_{g}^{n+1}+\left(\frac{\partial F_{w_{g}}^{z}}{\partial u_{f}}\right)^{n} u_{f}^{n+1}\right]_{i, j+1 / 2} \\
& +\left[\left(F_{w_{g}}^{z}\right)^{n}-\left(\frac{\partial F_{w_{g}}^{z}}{\partial u_{g}}\right)^{n} u_{g}^{n}-\left(\frac{\partial F_{w_{g}}^{z}}{\partial u_{f}}\right)^{n} u_{f}^{n}\right]_{i, j+1 / 2}
\end{aligned}
$$

Using a similar expansion for the interfacial drag we obtain 


$$
\begin{aligned}
\left(F_{I}^{z}\right)_{i, j+1 / 2}^{n+1} & \equiv\left[\left(\frac{\partial F_{I}^{z}}{\partial u_{g}}\right)^{n} u_{g}^{n+1}+\left(\frac{\partial F_{I}^{z}}{\partial u_{f}}\right)^{n} u_{f}^{n+1}\right]_{i, j+1 / 2} \\
& +\left[\left(F_{I}^{z}\right)^{n}-\left(\frac{\partial F_{I}^{z}}{\partial u_{g}}\right)^{n} u_{g}^{n}-\left(\frac{\partial F_{I}^{z}}{\partial u_{f}}\right)^{n} u_{f}^{n}\right]_{i, j+1 / 2}
\end{aligned}
$$

Introducing a dependence on both the liquid and gas velocities into the two drag forces couples the liquid and gas momentum equations.

The final term in the momentum equation representing momentum exchange due to turbulent fluctuations is evaluated explicitly as

$$
\frac{1}{V_{m}} \int_{V_{m}} M_{t g}^{z} d V_{m} \rightarrow\left(M_{t g}^{z}\right)_{i, j+1 / 2}^{n}
$$

Substituting the volume averaged expressions derived above into Eq. (3.4.1-3) yields the basic difference equation for axial momentum in the gas phase as 


$$
\begin{aligned}
& \left\langle\alpha \rho_{g}\right\rangle_{i, j+1 / 2}^{n}\left[\frac{\left\langle u_{g}\right\rangle_{i, j+1 / 2}^{n+1}-\left\langle u_{g}\right\rangle_{i, j+1 / 2}^{n}}{\Delta t}\right] \\
& +\frac{1}{\left(V_{z}\right)_{i, j+1 / 2}}\left[-\left\langle A_{z z} \alpha \rho_{g} u_{g} u_{g}\right\rangle_{i, j}^{n}+\left\langle A_{z z} \alpha \rho_{g} u_{g} u_{g}\right\rangle_{i, j+1}^{n}\right. \\
& -\left\langle A_{2 x} \alpha \rho_{g} w_{g}\right\rangle_{i-1 / 2, j+1 / 2}^{n}\left\langle u_{g}\right\rangle_{i-1 / 2, j+1 / 2}^{n} \\
& \left.+\left\langle A_{z x} \alpha \rho_{g} w_{g}\right\rangle_{i+1 / 2, j+1 / 2}^{n}\left\langle u_{g}\right\rangle_{i+1 / 2, j+1 / 2}^{n}\right] \\
& -\frac{\left\langle u_{g}\right\rangle_{i, j+1 / 2}^{n}}{\left(V_{z}\right)_{i, j+1 / 2}}\left[-\left\langle A_{z z} \alpha \rho_{g} u_{g}\right\rangle_{i, j}^{n}+\left\langle A_{z z} \alpha \rho_{g} u_{g}\right\rangle_{i, j+1}^{n}\right. \\
& \left.-\left\langle A_{z x} \alpha \rho_{g} w_{g}\right\rangle_{i-1 / 2, j+1 / 2}^{n}+\left\langle A_{z x} \alpha \rho_{g} w_{g}\right\rangle_{i+1 / 2, j+1 / 2}^{n}\right] \\
& =-\frac{\langle\alpha A\rangle_{i, j+1 / 2}^{n}}{\left(V_{z}\right)_{i, j+1 / 2}}\left(P_{i, j+1}^{n+1}-P_{i, j}^{n+1}\right)+\left\langle\rho_{g}\right\rangle_{i, j+1 / 2}^{n} g_{i, j+1 / 2}^{z} \frac{\langle\alpha A\rangle_{i, j+1 / 2}^{n}\left(\Delta z_{m}\right)_{i, j+1 / 2}}{\left(V_{z}\right)_{i, j+1 / 2}} \\
& -\left[\left(\frac{\partial F_{w_{g}}^{z}}{\partial u_{g}}\right)^{n} u_{g}^{n+1}+\left(\frac{\partial F_{w_{g}}^{z}}{\partial u_{f}}\right)^{n} u_{f}^{n+1}\right]_{i, j+1 / 2} \\
& -\left[\left(F_{w_{g}}^{z}\right)^{n}-\left(\frac{\partial F_{w_{g}}^{z}}{\partial u_{g}}\right)^{n} u_{g}^{n}-\left(\frac{\partial F_{w_{g}}^{z}}{\partial u_{f}}\right)^{n} u_{f}^{n}\right]_{i, j+1 / 2} \\
& -\left[\left(\frac{\partial F_{I}^{z}}{\partial u_{g}}\right)^{n} u_{g}^{n+1}+\left(\frac{\partial F_{l}^{z}}{\partial u_{f}}\right)^{n} u_{f}^{n+1}\right]_{i, j+1 / 2} \\
& -\left[\left(F_{l}^{2}\right)^{n}-\left(\frac{\partial F_{l}^{2}}{\partial u_{g}}\right)^{n} u_{g}^{n}-\left(\frac{\partial F_{l}^{z}}{\partial u_{f}}\right)^{n} u_{f}^{n}\right]_{i, j+1 / 2}-\left(M_{t g}^{z}\right)_{i, j+1 / 2}^{n}
\end{aligned}
$$

Collecting terms in Eq. (3.4.1-19) and rearranging gives the equivalent expression which is the final form of the momentum equation implemented in FLOWTRAN-TF 


$$
\begin{aligned}
& {\left[\frac{R^{z}\left\langle\alpha \rho_{g}\right\rangle^{n}}{\Delta t}+\left(\frac{\partial F_{w_{g}}^{z}}{\partial u_{g}}\right)^{n}+\left(\frac{\partial F_{l}^{z}}{\partial u_{g}}\right)^{n}\right]_{i, j+1 / 2}\left(u_{g}\right)_{i, j+1 / 2}^{n+1}} \\
& +\left[\left(\frac{\partial F_{w g}^{z}}{\partial u_{f}}\right)^{n}+\left(\frac{\partial F_{l}^{z}}{\partial u_{f}}\right)^{n}\right]_{i, j+1 / 2}\left(u_{f}\right)_{i, j+1 / 2}^{n+1} \\
& =\frac{\langle\alpha A\rangle_{i, j+1 / 2}^{n}}{\left(V_{2}\right)_{i, j+1 / 2}}\left(P_{i, j}^{n+1}-P_{i, j+1}^{n+1}\right) \\
& +\frac{1}{\left(V_{z}\right)_{i, j+1 / 2}}\left\{\left\langle A_{z z} \alpha \rho_{g} u_{g} u_{g}\right\rangle_{i, j}^{n}-\left\langle A_{z z} \alpha \rho_{g} u_{g}\right\rangle_{i, j}^{n}\left\langle u_{g}\right\rangle_{i, j+1 / 2}^{n}\right. \\
& -\left\langle A_{z z} \alpha \rho_{g} u_{g} u_{g}\right\rangle_{i, j+1}^{n}+\left\langle A_{z z} \alpha \rho_{g} u_{g}\right\rangle_{i, j+1}^{n}\left\langle u_{g}\right\rangle_{i, j+1 / 2}^{n} \\
& +\left\langle A_{2 x} \alpha \rho_{g} w_{g}\right\rangle_{i-1 / 2, j+1 / 2}^{n}\left[\left\langle u_{g}\right\rangle_{i-1 / 2, j+1 / 2}^{n}-\left\langle u_{g}\right\rangle_{i, j+1 / 2}^{n}\right] \\
& \left.-\left\langle A_{2 x} \alpha \rho_{g} w_{g}\right\rangle_{i+1 / 2, j+1 / 2}^{n}\left[\left\langle u_{g}\right\rangle_{i+1 / 2, j+1 / 2}^{n}-\left\langle u_{g}\right\rangle_{i, j+1 / 2}^{n}\right]\right\} \\
& +R^{2}\left\langle\alpha \rho_{g}\right\rangle_{i, j+1 / 2}^{n} \frac{\left\langle u_{g}\right\rangle_{i, j+1 / 2}^{n}}{\Delta t}+\left\langle\rho_{g}\right\rangle_{i, j+1 / 2}^{n} g_{i, j+1 / 2}^{z} \frac{\langle\alpha A\rangle_{i, j+1 / 2}^{n}\left(\Delta z_{m}\right)_{i, j+1 / 2}}{\left(V_{z}\right)_{i, j+1 / 2}} \\
& -\left[\left(F_{w_{g}}^{z}\right)^{n}-\left(\frac{\partial F_{w_{g}}^{z}}{\partial u_{g}}\right)^{n} u_{g}^{n}-\left(\frac{\partial F_{w g}^{z}}{\partial u_{f}}\right)^{n} u_{f}^{n}\right]_{i, j+1 / 2} \\
& -\left[\left(F_{T}^{z}\right)^{n}-\left(\frac{\partial F_{I}^{z}}{\partial u_{g}}\right)^{n} u_{g}^{n}-\left(\frac{\partial F_{I}^{z}}{\partial u_{f}}\right)^{n} u_{f}^{n}\right]_{i, j+1 / 2}-\left(M_{t g}^{z}\right)_{i, j+1 / 2}^{n}
\end{aligned}
$$

Equation (3.4.1-20) has the general functional form

$$
a_{11}^{z}\left(u_{g}\right)_{i, j+1 / 2}^{n+1}+a_{12}^{z}\left(u_{f}\right)_{i, j+1 / 2}^{n+1}=b_{1}+c_{1}\left(P_{i, j}^{n+1}-P_{i, j+1}^{n+1}\right)
$$

where $a_{11}^{2}, a_{12}^{z}, b_{1}$, and $c_{1}$ are constant numbers evaluated explicitly from parameter values at the old time step. These coefficients vary with the grid location; however, we have omitted the $i, j+1 / 2$ subscripts for brevity.

To complete our treatment of the gas axial momentum equation we must derive appropriate definitions of the spatially averaged advection terms and velocities that appear in Eq. $(3.4 .1-20)$. We first consider momentum advection in the axial direction from the axial velocity component. We use an upwind differencing or donoring scheme to compute axial 
mass and momentum flows at the momentum cell faces. This scheme has desirable numerical properties as will be demonstrated in the code development report on our verification calculations. Axial mass flowrates at the faces of the two mass cells that form the momentum control volume (see Fig. 3.4.1-3) are computed directly as

$$
\left(A_{c z} \hat{\alpha} \hat{\rho}_{g} u_{g}\right)_{i, j-1 / 2}^{n},\left(A_{c z} \hat{\alpha} \hat{\rho}_{g} u_{g}\right)_{i, j+1 / 2}^{n} \text {, and }\left(A_{c z} \hat{\alpha} \hat{\rho}_{g} u_{g}\right)_{i, j+3 / 2}^{n}
$$

The void fractions and gas densities in Eq. (3.4.1-22) are values donored to the cell faces. For example, in the axial direction, the void fraction donored to mesh location $(i, j-1 / 2)$ is defined to be

$$
(\hat{\alpha})_{i, j-1 / 2}^{n}=\omega \alpha_{i, j-1}^{n}+(1-\omega) \alpha_{i, j}^{n}
$$

and the donored gas density is given by

$$
\left(\hat{\rho}_{g}\right)_{i, j-1 / 2}^{n}=\omega\left(\rho_{g}\right)_{i, j-1}^{n}+(1-\omega)\left(\rho_{g}\right)_{i, j}^{n}
$$

The interpolation parameter $\omega$ used in the above relations is defined to be

$$
\omega \equiv\left\{\begin{array}{cr}
1 & v \geq v_{\text {min }} \\
\frac{1}{2}\left(1+\frac{v}{v_{\min }}\right) & v_{\min }>v>-v_{\min } \\
0 & v \leq-v_{\min }
\end{array}\right.
$$

where the velocity $(v)$ is the value at the $(i, j-1 / 2)$ face, $v=\left(u_{g}\right)_{i, j-1 / 2}^{n}$. The interpolation procedure shown by Eqs. (3.4.1-23) and (3.4.1-24) is used with all of our property donoring to provide a smooth transition between property values as the flow changes direction. The interpolation scheme is illustrated in Fig. 3.4.1-5 below. Note that we take fluid velocities in the downward direction to be positive. Typically, we choose $v_{\min }=0.05$ $\mathrm{m} / \mathrm{s}$ in the axial direction and $0 \mathrm{~m} / \mathrm{s}$ in the azimuthal direction although the exact value is controlled through user input.

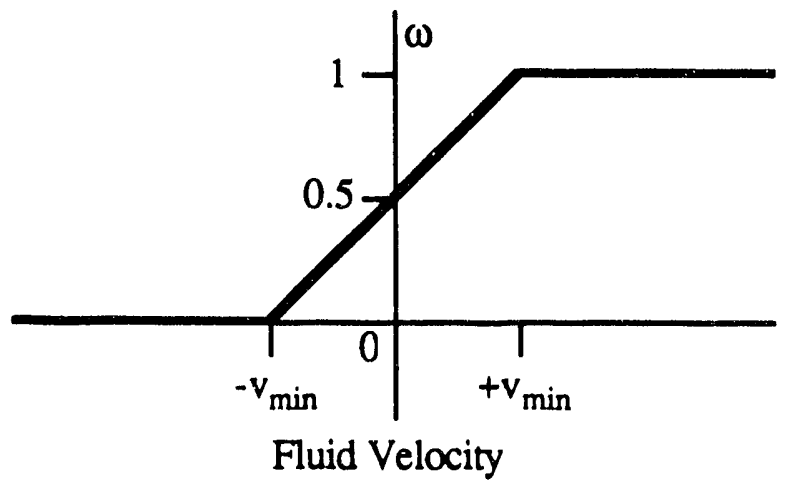

Figure 3.4.1-5 Illustration of interpolation parameter calculation. 
Similar definitions of the donored physical properties apply at the $j+1 / 2$ and $j+3 / 2$ mesh locations. These definitions for the mass flowrates are consistent with the mass flowrates computed for the mass/energy balances (see \$3.4.2).

We must then decide which mass flows apply at the faces of the bounded momentum cell. To accomplish this, we estimate axial velocities at the faces (centers of the mass cells) and again use a donoring scheme. We use an area weighted average of the mass flows in the axial direction to estimate the axial velocity at the center of the mass control volume. For example, for the upper mass cell shown in Fig. 3.4.1-3, we compute an axial velocity at the cell center as

$$
\left(\tilde{u}_{g}\right)_{i, j} \cong \frac{\left(A_{c z} \hat{\alpha} \hat{\rho}_{g} u_{g}\right)_{i, j-1 / 2}^{n}\left(A_{c z}\right)_{i, j-1 / 2}+\left(A_{c z} \hat{\alpha} \hat{\rho}_{g} u_{g}\right)_{i, j+1 / 2}^{n}\left(A_{c z}\right)_{i, j+1 / 2}}{\left[\left(A_{c z} \hat{\alpha} \hat{\rho}_{g}\right)_{i, j-1 / 2}^{n}+\left(A_{c z} \hat{\alpha} \hat{\rho}_{g}\right)_{i, j+1 / 2}^{n}\right]\left(A_{z z}\right)_{i, j}}
$$

Equation (3.4.1-25) is identical to the relationship used by the RELAP5 code (Ransom et al., 1985, Dimenna et al., 1988) to compute velocities at the centers of the mass cells and was also used in the FLOWTRAN-FI code (Aleman et al., 1989). The cell center velocity is only used to determine the donoring direction for momentum and mass flux evaluations and therefore the exact value computed is relatively unimportant. In the single phase limit as $\hat{\alpha} \rightarrow 0$ Eq. (3.4.1-25) becomes indeterminate. The code tests for this condition and bypasses the computation of momentum transport for a phase that is not present.

Applying our donoring scheme to determine the axial mass flow at the upper face of the momentum cell gives

$$
\left\langle A_{z z} \alpha \rho_{g} u_{g}\right\rangle_{i, j}^{n}=\omega\left(A_{c z} \hat{\alpha} \hat{\rho}_{g} u_{g}\right)_{i, j-1 / 2}^{n}+(1-\omega)\left(A_{c z} \hat{\alpha} \hat{\rho}_{g} u_{g}\right)_{i, j+1 / 2}^{n}
$$

where the interpolation parameter $\omega$ in Eq. (3.4.1-24) is determined using the velocity $v=\left(\tilde{u}_{g}\right)_{i, j}$. We use the same donoring scheme to compute the momentum terms at the cell faces

$$
\left\langle A_{z z} \alpha \rho_{g} u_{g} u_{g}\right\rangle_{i, j}^{n}=\omega\left(A_{c z} \hat{\alpha} \hat{\rho}_{g} u_{g} u_{g}\right)_{i, j-1 / 2}^{n}+(1-\omega)\left(A_{c z} \hat{\alpha} \hat{\rho}_{g} u_{g} u_{g}\right)_{i, j+1 / 2}^{n}
$$

Relationships similar to those shown in Eqs. (3.4.1-25) - (3.4.1-27) are applied to compute the axial mass and momentum flow at the $j+1$ momentum cell axial face.

We next evaluate momentum advection in the axial direction from the azimuthal velocity components. Azimuthal velocities are available at the comers of the momentum control volume. First, considering the upper mass cell forming the momentum control volume (see Figs. 3.4.1-2 and 3.4.1-3), we compute mass flowrates at the left and right hand faces of the mass cell as

$$
\left(A_{c x} \hat{\alpha} \hat{\rho}_{g} w_{g}\right)_{i-1 / 2, j}^{n} \text { and }\left(A_{c x} \hat{\alpha} \hat{\rho}_{g} w_{g}\right)_{i+1 / 2, j}^{n}
$$


We again use donored physical quantities such that the void fraction donored to the $(i-1 / 2, j)$
mesh location is given by

$$
(\hat{\alpha})_{i-1 / 2, j}^{n}=\omega \alpha_{i-1, j}^{n}+(1-\omega) \alpha_{i, j}^{n}
$$

and the donored gas density is

$$
\left(\hat{\rho}_{g}\right)_{i-1 / 2, j}^{n}=\omega\left(\rho_{g}\right)_{i-1, j}^{n}+(1-\omega)\left(\rho_{g}\right)_{i, j}^{n}
$$

where $v=\left(w_{g}\right)_{i-1 / 2, j}^{n}$ is used to determine the interpolation parameter in Eq. $(3.4 .1-24)$. Similar definitions of the donored physical properties apply at the $i+1 / 2$ mesh location.

Applying Eqs. (3.4.1-28) - (3.4.1-30) we evaluate the contribution to azimuthal mass flow into the left hand face of the momentum control volume from the upper mass cell as one
half of the azimuthal mass flow into the mass cell

$$
\left\{\left\langle A_{z x} \alpha \rho_{g} w_{g}\right\rangle_{i-1 / 2, j+1 / 2}^{n}\right\}_{U p p e r}=\frac{1}{2}\left(A_{c x} \hat{\alpha} \hat{\rho}_{g} w_{g}\right)_{i-1 / 2, j}^{n}
$$

An equivalent expression that applies at the right hand face of the momentum cell is obtained from Eq. (3.4.1-31) by replacing $i-1 / 2$ with $i+1 / 2$.

We use cell average velocities and our donoring scheme to define the axial velocities at the right and left hand faces of the momentum cell. At the left hand face of the upper mass cell
[mesh point $(i-1 / 2, j+1 / 2)]$ we use

$$
\left\{\left\langle u_{g}\right\rangle_{i-1 / 2, j+1 / 2}^{n}\right\}_{U_{\text {pper }}}=\omega\left\langle u_{g}\right\rangle_{i-1, j+1 / 2}^{n}+(1-\omega)\left\langle u_{g}\right\rangle_{i, j+1 / 2}^{n}, \quad v=\left(w_{g}\right)_{i-1 / 2, j}^{n}
$$

and at the right hand face [mesh point $(i+1 / 2 j+1 / 2)]$ we use

$$
\left\{\left\langle u_{g}\right\rangle_{i+1 / 2, j+1 / 2}^{n}\right\}_{U_{p p e r}}=\omega\left\langle u_{g}\right\rangle_{i, j+1 / 2}^{n}+(1-\omega)\left\langle u_{g}\right\rangle_{i+1, j+1 / 2}^{n}, \quad v=\left(w_{g}\right)_{i+1 / 2, j}^{n}
$$

When the lower mass cell is considered, similar equations apply. The contribution to azimuthal mass flows into the momentum control volume from the lower mass cell is computed as one half of the azimuthal mass flows into the mass cell faces. For example, at
the left hand face we use

$$
\left\{\left\langle A_{z x} \alpha \rho_{g} w_{g}\right\rangle_{i-1 / 2, j+1 / 2}^{n}\right\}_{\text {Lower }}=\frac{1}{2}\left(A_{c x} \hat{\alpha} \hat{\rho}_{g} w_{g}\right)_{i-1 / 2, j+1}^{n}
$$

The donoring schemes applied to the lower part of the momentum cell are: at the left hand face [mesh point $(i-1 / 2, j+1 / 2)]$ 


$$
\left\{\left\langle u_{g}\right\rangle_{i-1 / 2, j+1 / 2}^{n}\right\}_{\text {Lower }}=\omega\left\langle u_{g}\right\rangle_{i-1, j+1 / 2}^{n}+(1-\omega)\left\langle u_{g}\right\rangle_{i, j+1 / 2}^{n}, \quad v=\left(w_{g}\right)_{i-1 / 2, j+1}^{n}
$$

at the right hand face [mesh point $(i+1 / 2, j+1 / 2)]$

$$
\left\{\left\langle u_{g}\right\rangle_{i+1 / 2, j+1 / 2}^{n}\right\}_{\text {Lower }}=\omega\left\langle u_{g}\right\rangle_{i, j+1 / 2}^{n}+(1-\omega)\left\langle u_{g}\right\rangle_{i+1, j+1 / 2}^{n}, \quad v=\left(w_{g}\right)_{i+1 / 2, j+1}^{n}
$$

We note that, since a different azimuthal velocity is used to test the donoring direction in the lower mass cell, the axial velocities at the faces of the lower half of the momentum cell may be different from those used with the upper half. The complete momentum advection term for the gas phase at the left hand face of the momentum control volume is computed as

$$
\begin{aligned}
\left\langle A_{2 x}\right. & \left.\alpha \rho_{g} w_{g}\right\rangle_{i-1 / 2, j+1 / 2}^{n}\left[\left\langle u_{g}\right\rangle_{i-1 / 2, j+1 / 2}^{n}-\left\langle u_{g}\right\rangle_{i, j+1 / 2}^{n}\right]= \\
& \frac{1}{2}\left(A_{c x} \hat{\alpha} \hat{\rho}_{g} w_{g}\right)_{i-1 / 2, j}^{n}\left[\left\{\left\langle u_{g}\right\rangle_{i-1 / 2, j+1 / 2}^{n}\right\}_{\text {Upper }}-\left\langle u_{g}\right\rangle_{i, j+1 / 2}^{n}\right] \\
+ & \frac{1}{2}\left(A_{c x} \hat{\alpha} \hat{\rho}_{g} w_{g}\right)_{i-1 / 2, j+1}^{n}\left[\left\{\left\langle u_{g}\right\rangle_{i-1 / 2, j+1 / 2}^{n}\right\}_{\text {lower }}-\left\langle u_{g}\right\rangle_{i, j+1 / 2}^{n}\right]
\end{aligned}
$$

A similar expression applies for the right hand face $(i+1 / 2)$. This completes evaluation of all of the momentum advection terms and velocities in Eq. (3.4.1-20).

Turning to the liquid momentum equation, the axial component of Eq. (3.4.1-2) is

$$
\begin{aligned}
(1-\alpha) \rho_{f} \frac{\partial u_{f}}{\partial t}+\nabla \cdot\left[(1-\alpha) \rho_{f} u_{f} \bar{v}_{f}\right]-u_{f} \nabla \cdot\left[(1-\alpha) \rho_{f} \bar{v}_{f}\right]= \\
-(1-\alpha) \frac{\partial P}{\partial z}+(1-\alpha) \rho_{f} g^{z}-F_{w f}^{z}+F_{I}^{2}-M_{t f}^{2}
\end{aligned}
$$

Comparison of Eq. (3.4.1-38) with the gas momentum equation given in Eq. (3.4.1-3) shows that the gas and liquid momentum equations are nearly identical. To convert the above analysis for the axial liquid momentum equation we need only reverse the sign of the interfacial drag terms, replace the gas void fraction $\alpha$ with the equivalent liquid void fraction $(l-\alpha)$, and substitute $\rho_{f}$ for $\rho_{g}, u_{f}$ for $u_{g}$, and $w_{f}$ for $w_{g}$. The difference equation for the liquid axial momentum is 


$$
\begin{aligned}
& {\left[\left(\frac{\partial F_{w f}^{z}}{\partial u_{g}}\right)^{n}-\left(\frac{\partial F_{l}^{2}}{\partial u_{g}}\right)^{n}\right]_{i, j+1 / 2}\left(u_{g}\right)_{i, j+1 / 2}^{n+1}} \\
& +\left[\frac{R^{z}\left\langle(1-\alpha) \rho_{f}\right\rangle^{n}}{\Delta t}+\left(\frac{\partial F_{w f}^{z}}{\partial u_{f}}\right)^{n}-\left(\frac{\partial F_{f}^{2}}{\partial u_{f}}\right)^{n}\right]_{i, j+1 / 2}\left(u_{f}\right)_{i, j+1 / 2}^{n+1} \\
& =\frac{\langle(1-\alpha) A\rangle_{i, j+1 / 2}^{n}}{\left(V_{z}\right)_{i, j+1 / 2}}\left(P_{i, j}^{n+1}-P_{i, j+1}^{n+1}\right) \\
& +\frac{1}{\left(V_{z}\right)_{i, j+1 / 2}}\left\{\left\langle A_{z z}(1-\alpha) \rho_{f} u_{f} u_{f}\right\rangle_{i, j}^{n}-\left\langle A_{z z}(1-\alpha) \rho_{f} u_{f}\right\rangle_{i, j}^{n}\left\langle u_{f}\right\rangle_{i, j+1 / 2}^{n}\right. \\
& -\left\langle A_{2 z}(1-\alpha) \rho_{f} u_{f} u_{f}\right\rangle_{i, j+1}^{n}+\left\langle A_{z z}(1-\alpha) \rho_{f} u_{f}\right\rangle_{i, j+1}^{n}\left\langle u_{f}\right\rangle_{i, j+1 / 2}^{n} \\
& +\left\langle A_{z x}(1-\alpha) \rho_{f} w_{f}\right\rangle_{i-1 / 2, j+1 / 2}^{n}\left[\left\langle u_{f}\right\rangle_{i-1 / 2, j+1 / 2}^{n}-\left\langle u_{f}\right\rangle_{i, j+1 / 2}^{n}\right] \\
& \left.-\left\langle A_{2 x}(1-\alpha) \rho_{f} w_{f}\right\rangle_{i+1 / 2, j+1 / 2}^{n}\left[\left\langle u_{f}\right\rangle_{i+1 / 2, j+1 / 2}^{n}-\left\langle u_{f}\right\rangle_{i, j+1 / 2}^{n}\right]\right\} \\
& +R^{2}\left\langle(1-\alpha) \rho_{f}\right\rangle_{i, j+1 / 2}^{n} \frac{\left\langle u_{f}\right\rangle_{i, j+1 / 2}^{n}}{\Delta t} \\
& +\left\langle\rho_{f}\right\rangle_{i, j+1 / 2}^{n} g_{i, j+1 / 2}^{z} \frac{\langle(1-\alpha) A\rangle_{i, j+1 / 2}^{n}\left(\Delta z_{m}\right)_{i, j+1 / 2}}{\left(V_{z}\right)_{i, j+1 / 2}} \\
& -\left[\left(F_{w f}^{z}\right)^{n}-\left(\frac{\partial F_{w f}^{z}}{\partial u_{g}}\right)^{n} u_{g}^{n}-\left(\frac{\partial F_{w f}^{z}}{\partial u_{f}}\right)^{n} u_{f}^{n}\right]_{i, j+1 / 2} \\
& +\left[\left(F_{I}^{2}\right)^{n}-\left(\frac{\partial F_{I}^{2}}{\partial u_{g}}\right)^{n} u_{g}^{n}-\left(\frac{\partial F_{l}^{2}}{\partial u_{f}}\right)^{n} u_{f}^{n}\right]_{i, j+1 / 2}-\left(M_{t f}^{z}\right)_{i, j+1 / 2}^{n}
\end{aligned}
$$

As with Eq. (3.4.1-20), Eq. (3.4.1-39) has the general functional form

$$
a_{21}^{z}\left(u_{g}\right)_{i, j+1 / 2}^{n+1}+a_{22}^{z}\left(u_{f}\right)_{i, j+1 / 2}^{n+1}=b_{2}+c_{2}\left(P_{i, j}^{n+1}-P_{i, j+1}^{n+1}\right)
$$

where $a_{21}^{2}, a_{22}^{z}, b_{2}$, and $c_{2}$ are constant numbers evaluated explicitly from old time values at each $i, j+1 / 2$ grid point. Equations (3.4.1-22) through (3.4.1-37) are applied to define the terms appearing in Eq. (3.4.1-39) with the obvious modifications.

Equations (3.4.1-21) and (3.4.1-40) form a $2 \times 2$ system of equations which can be inverted to give a set of equations relating the gas and fluid axial velocities to the axial pressure 
difference at each mesh location. Combining these two equations yields the equivalent matrix equation

$$
\left[\begin{array}{ll}
a_{11}^{z} & a_{12}^{z} \\
a_{21}^{z} & a_{22}^{z}
\end{array}\right]\left[\begin{array}{l}
\left(u_{g}\right)_{i, j+1 / 2}^{n+1} \\
\left(u_{f}\right)_{i, j+1 / 2}^{n+1}
\end{array}\right]=\left[\begin{array}{l}
b_{1} \\
b_{2}
\end{array}\right]+\left[\begin{array}{l}
c_{1} \\
c_{2}
\end{array}\right]\left(P_{i, j}^{n+1}-P_{i, j+1}^{n+1}\right)
$$

Equation (3.4.1-41) is easily inverted to give the velocity relationships

$$
\begin{aligned}
& \left(u_{g}\right)_{i, j+1 / 2}^{n+1}=B_{g}+C_{g}\left(P_{i, j}^{n+1}-P_{i, j+1}^{n+1}\right) \\
& \left(u_{f}\right)_{i, j+1 / 2}^{n+1}=B_{f}+C_{f}\left(P_{i, j}^{n+1}-P_{i, j+1}^{n+1}\right)
\end{aligned}
$$

where the coefficients are calculated as

$$
\begin{aligned}
& B_{g}=\left(a_{22}^{2} b_{1}-a_{12}^{z} b_{2}\right)\left|\underline{A}^{2}\right|^{-1} \\
& C_{8}=\left(a_{22}^{2} c_{1}-a_{12}^{2} c_{2}\right)\left|A^{z}\right|^{-1} \\
& B_{f}=\left(a_{11}^{z} b_{2}-a_{21}^{z} b_{1}\right)\left|A^{2}\right|^{-1} \\
& C_{f}=\left(a_{11}^{2} c_{2}-a_{21}^{z} c_{1}\right)\left|A^{z}\right|^{-1}
\end{aligned}
$$

and the determinant of the matrix $\underline{\underline{\underline{A}}}^{2}$ is

$$
\left|\mathbf{A}^{z}\right|=\left(a_{11}^{2} a_{22}^{2}-a_{12}^{2} a_{21}^{z}\right)
$$

Azimuthal Momentum Equations: The azimuthal momentum equations for the gas and liquid phases can be developed in an manner analogous to that presented above for the axial momentum equations. The azimuthal component of the gas momentum equation (see Eq. $(3.4 .1-1))$ is

$$
\begin{aligned}
& \alpha \rho_{g} \frac{\partial w_{g}}{\partial t}+\nabla \cdot\left[\alpha \rho_{g} w_{g} \bar{v}_{g}\right]-w_{g} \nabla \cdot\left[\alpha \rho_{g} \bar{v}_{g}\right]= \\
&-\alpha \frac{\partial P}{\partial x}+\alpha \rho_{g} g^{x}-F_{w g}^{x}-F_{I}^{x}-M_{t g}^{x}
\end{aligned}
$$

Using the averaging techniques developed above the finite difference analog of Eq. (3.4.1-45) becomes 


$$
\begin{aligned}
& {\left[\frac{R^{x}\left\langle\alpha \rho_{g}\right\rangle^{n}}{\Delta t}+\left(\frac{\partial F_{w_{g}}^{x}}{\partial w_{g}}\right)^{n}+\left(\frac{\partial F_{l}^{x}}{\partial w_{g}}\right)^{n}\right]_{i+1 / 2, j}\left(w_{g}\right)_{i+1 / 2, j}^{n+1}} \\
& +\left[\left(\frac{\partial F_{w g}^{x}}{\partial w_{f}}\right)^{n}+\left(\frac{\partial F_{l}^{x}}{\partial w_{f}}\right)^{n}\right]_{i+1 / 2, j}\left(w_{f}\right)_{i+1 / 2, j}^{n+1} \\
& =\frac{\langle\alpha A\rangle_{i+1 / 2, j}^{n}}{\left(V_{x}\right)_{i+1 / 2, j}}\left(P_{i, j}^{n+1}-P_{i+1, j}^{n+1}\right) \\
& +\frac{1}{\left(V_{x}\right)_{i+1 / 2, j}}\left\{\left\langle A_{x x} \alpha \rho_{g} w_{g} w_{g}\right\rangle_{i, j}^{n}-\left\langle A_{x x} \alpha \rho_{g} w_{g}\right\rangle_{i, j}^{n}\left\langle w_{g}\right\rangle_{i+1 / 2, j}^{n}\right. \\
& -\left\langle A_{x x} \alpha \rho_{g} w_{g} w_{g}\right\rangle_{i+1, j}^{n}+\left\langle A_{x x} \alpha \rho_{g} w_{g}\right\rangle_{i+1, j}^{n}\left\langle w_{g}\right\rangle_{i+1 / 2, j}^{n} \\
& +\left\langle A_{x z} \alpha \rho_{g} u_{g}\right\rangle_{i+1 / 2, j-1 / 2}^{n}\left[\left\langle w_{g}\right\rangle_{i+1 / 2, j-1 / 2}^{n}-\left\langle w_{g}\right\rangle_{i+1 / 2, j}^{n}\right] \\
& \left.-\left\langle A_{x z} \alpha \rho_{g} u_{g}\right\rangle_{i+1 / 2, j+1 / 2}^{n}\left[\left\langle w_{g}\right\rangle_{i+1 / 2, j+1 / 2}^{n}-\left\langle w_{g}\right\rangle_{i+1 / 2, j}^{n}\right]\right\} \\
& +R^{x}\left\langle\alpha \rho_{g}\right\rangle_{i+1 / 2, j}^{n} \frac{\left\langle w_{g}\right\rangle_{i+1 / 2, j}^{n}}{\Delta t}+\left\langle\rho_{g}\right\rangle_{i+1 / 2, j}^{n} g_{i+1 / 2, j}^{x} \frac{\langle\alpha A\rangle_{i+1 / 2, j}^{n}\left(\Delta x_{m}\right)_{i+1 / 2, j}}{\left(V_{x}\right)_{i+. ~}, j} \\
& -\left[\left(F_{w_{g}}^{x}\right)^{n}-\left(\frac{\partial F_{w_{g}}^{x}}{\partial w_{g}}\right)^{n} w_{g}^{n}-\left(\frac{\partial F_{w_{g}}^{x}}{\partial w_{f}}\right)^{n} w_{f}^{n}\right]_{i+1 / 2, j} \\
& -\left[\left(F_{I}^{x}\right)^{n}-\left(\frac{\partial F_{I}^{x}}{\partial w_{g}}\right)^{n} w_{g}^{n}-\left(\frac{\partial F_{I}^{x}}{\partial w_{f}}\right)^{n} w_{f}^{n}\right]_{i+1 / 2, j}-\left(M_{t g}^{x}\right)_{i+1 / 2, j}^{n}
\end{aligned}
$$

Equation (3.4.1-46) has the general functional form

$$
a_{11}^{x}\left(w_{g}\right)_{i+1 / 2, j}^{n+1}+a_{12}^{x}\left(w_{f}\right)_{i+1 / 2, j}^{n+1}=d_{1}+e_{1}\left(P_{i, j}^{n+1}-P_{i+1, j}^{n+1}\right)
$$

where $a_{11}^{x}, a_{12}^{x}, d_{1}$, and $e_{1}$ are constant numbers evaluated explicitly using parameter values from the previous time step. In deriving Eq. (3.4.1-46), we define a volume averaged velocity for the azimuthal
momentum cell as 


$$
\begin{aligned}
\left\langle w_{g}\right\rangle_{i+1 / 2, j}^{n} & =\frac{\left(A_{c x}\right)_{i+1 / 2, j}}{\left\langle A_{c x}\right\rangle_{i+1 / 2, j}}\left(w_{g}\right)_{i+1 / 2, j}^{n} \\
& =R_{i+1 / 2, j}^{x}\left(w_{g}\right)_{i+1 / 2, j}^{n}
\end{aligned}
$$

where the average momentum cell area is defined to be

$$
\left\langle A_{c x}\right\rangle_{i+1 / 2, j} \equiv \frac{\left(V_{x}\right)_{i+1 / 2, j}}{\left(\Delta x_{m}\right)_{i+1 / 2, j}}
$$

Assuming that fluid mesh points are located at the centers of the mass cells, the azimuthal momentum cell volume is given by

$$
\left(V_{x}\right)_{i+1 / 2, j}=\frac{\left(V_{c}\right)_{i, j}+\left(V_{c}\right)_{i+1, j}}{2}
$$

and the momentum cell length by

$$
\left(\Delta x_{m}\right)_{i+1 / 2, j}=\frac{\Delta x_{i, j}+\Delta x_{i+1, j}}{2}
$$

As with the axial momentum equations, we must define the appropriate spatially averaged advection and velocity terms that appear in Eq. (3.4.1-46). We again use an upwind donoring scheme to determine the azimuthal mass and momentum flows at the cell faces. At the left face of the azimuthal momentum cell (see Fig. 3.4.1-4), the mass flow in the azimuthal direction is given by

$$
\left\langle A_{x x} \alpha \rho_{g} w_{g}\right\rangle_{i, j}^{n}=\omega\left(A_{c x} \hat{\alpha} \hat{\rho}_{g} w_{g}\right)_{i-1 / 2, j}^{n}+(1-\omega)\left(A_{c x} \hat{\alpha} \hat{\rho}_{g} w_{g}\right)_{i+1 / 2, j}^{n}
$$

and the flow of azimuthal momentum into this face of the control volume is

$$
\left\langle A_{x x} \alpha \rho_{g} w_{g} w_{g}\right\rangle_{i, j}^{n}=\omega\left(A_{c x} \hat{\alpha} \hat{\rho}_{g} w_{g} w_{g}\right)_{i-1 / 2, j}^{n}+(1-\omega)\left(A_{c x} \hat{\alpha} \hat{\rho}_{g} w_{g} w_{g}\right)_{i+1 / 2, j}^{n}
$$

In applying Eqs. (3.4.1-52) and (3.4.1-53), we use $v=\left(\tilde{w}_{g}\right)_{i, j}$ to compute the weighting parameter $\omega$ from Eq. (3.4.1-24).

We use an area weighted average of the mass flows in the azimuthal direction to estimate the azimuthal velocity at the center of the mass control volume which is then used to decide the donoring direction. For the left mass cell shown in Fig. 3.4.1-4, we compute an azimuthal velocity at the cell center as 


$$
\left(\tilde{w}_{g}\right)_{i, j} \equiv \frac{\left(A_{c x} \hat{\alpha} \hat{\rho}_{g} w_{g}\right)_{i-1 / 2, j}^{n}\left(A_{c x}\right)_{i-1 / 2, j}+\left(A_{c x} \hat{\alpha} \hat{\rho}_{g} w_{g}\right)_{i+1 / 2, j}^{n}\left(A_{c x}\right)_{i+1 / 2, j}}{\left[\left(A_{c x} \hat{\alpha} \hat{\rho}_{g}\right)_{i-1 / 2, j}^{n}+\left(A_{c x} \hat{\alpha} \hat{\rho}_{g}\right)_{i+1 / 2, j}^{n}\right]\left(A_{x x}\right)_{i, j}}
$$

The void fractions and gas densities in the above equations are values donored to the cell faces. For example, in the azimuthal direction, the void fraction donored to mesh location $(i-1 / 2, j)$ is defined to be

$$
(\hat{\alpha})_{i-1 / 2, j}^{n}=\omega \alpha_{i-1, j}^{n}+(1-\omega) \alpha_{i, j}^{n}, \quad v=\left(w_{g}\right)_{i-1 / 2, j}^{n}
$$

and the donored gas density is given by

$$
\left(\hat{\rho}_{g}\right)_{i-1 / 2, j}^{n}=\omega\left(\rho_{g}\right)_{i-1, j}^{n}+(1-\omega)\left(\rho_{g}\right)_{i, j}^{n}, \quad v=\left(w_{g}\right)_{i-1 / 2, j}^{n}
$$

Relationships similar to those in Eqs. (3.4.1-52) through (3.4.1-56) are applied to compute physical properties and the azimuthal mass and momentum flows at the $i+1$ momentum cell face.

We next evaluate momentum advection in the azimuthal direction from the axial velocity components. First, consider the mass cell that forms the left half of the azimuthal momentum control volume (see Figs. 3.4.1-2 and 3.4.1-4). We use donored physical quantities such that the void fraction donored to the $(i, j-1 / 2)$ mesh location is given by

$$
(\hat{\alpha})_{i, j-1 / 2}^{n}=\omega \alpha_{i, j-1}^{n}+(1-\omega) \alpha_{i, j}^{n}, \quad v=\left(u_{g}\right)_{i, j-1 / 2}^{n}
$$

and the donored gas density is

$$
\left(\hat{\rho}_{g}\right)_{i, j-1 / 2}^{n}=\omega\left(\rho_{g}\right)_{i, j-1}^{n}+(1-\omega)\left(\rho_{g}\right)_{i, j}^{n}, \quad v=\left(u_{g}\right)_{i, j-1 / 2}^{n}
$$

Similar definitions of the donored physical properties apply at the $j+1 / 2$ mesh location.

We evaluate the axial mass flow into the upper face of the momentum control volume that comes from the left hand mass cell as one half of the total axial mass flow at that face

$$
\left\{\left\langle A_{x z} \alpha \rho_{g} u_{g}\right\rangle_{i+1 / 2, j-1 / 2}^{n}\right\}_{\text {Left }}=\frac{1}{2}\left(A_{c z} \hat{\alpha} \hat{\rho}_{g} u_{g}\right)_{i, j-1 / 2}^{n}
$$

An equivalent expression for the lower face of the momentum cell is obtained by replacing $\mathrm{j}-1 / 2$ with $\mathrm{j}+1 / 2$.

We then use cell average velocities and our donoring procedure to define azimuthal velocities at the upper and lower faces of the left half of the azimuthal momentum cell. For the left hand mass cell at the upper face [mesh point $(i+1 / 2, j-1 / 2)]$ ive use 


$$
\left\{\left\langle w_{g}\right\rangle_{i+1 / 2, j-1 / 2}^{n}\right\}_{\text {Left }}=\omega\left\langle w_{g}\right\rangle_{i+1 / 2, j-1}^{n}+(1-\omega)\left\langle w_{g}\right\rangle_{1+1 / 2, j}^{n}, \quad v=\left(u_{g}\right)_{i, j-1 / 2}^{n}
$$

and on the lower face [mesh point $(i+1 / 2, j+1 / 2)]$ we use

$$
\left\{\left\langle w_{g}\right\rangle_{i+1 / 2, j+1 / 2}^{n}\right\}_{\text {Left }}=\omega\left\langle w_{g}\right\rangle_{i+1 / 2, j}^{n}+(1-\omega)\left\langle w_{g}\right\rangle_{i+1 / 2, j+1}^{n}, \quad v=\left(u_{g}\right)_{i, j+1 / 2}^{n}
$$

When the mass cell on the right hand side is considered, similar equations apply. The axial mass flow into the upper face of the momentum control volume from the mass cell forming the right hand side of the momentum cell is

$$
\left\{\left\langle A_{x z} \alpha \rho_{g} u_{g}\right\rangle_{i+1 / 2, j-1 / 2}^{n}\right\}_{\text {Right }}=\frac{1}{2}\left(A_{c z} \hat{\alpha} \hat{\rho}_{g} u_{g}\right)_{i+1, j-1 / 2}^{n}
$$

The donoring schemes applied to the right half of the momentum cell are:

At the upper face [mesh point $(i+1 / 2, j-1 / 2)]$ -

$$
\left\{\left\langle w_{g}\right\rangle_{i+1 / 2, j-1 / 2}^{n}\right\}_{\text {Right }}=\omega\left\langle w_{g}\right\rangle_{i+1 / 2, j-1}^{n}+(1-\omega)\left\langle w_{g}\right\rangle_{i+1 / 2, j}^{n}, \quad v=\left(u_{g}\right)_{i+1, j-1 / 2}^{n}
$$

At the lower face [mesh point $(i+1 / 2, j+1 / 2)]$ -

$$
\left\{\left\langle w_{g}\right\rangle_{i+1 / 2, j+1 / 2}^{n}\right\}_{\text {Right }}=w\left\langle w_{g}\right\rangle_{i+1 / 2, j}^{n}+(1-\omega)\left\langle w_{g}\right\rangle_{i+1 / 2, j+1}^{n}, \quad v=\left(u_{g}\right)_{i+1, j+1 / 2}^{n}
$$

As with the axial momentum computations, since different velocities are used to test the donoring directions in the right hand mass cell, axial velocities used at the right half of the momentum cell faces may be different from those used at the left half. The complete momentum advection term for the upper face of the azimuthal momentum control volume is computed as

$$
\begin{aligned}
\left\langle A_{x z}\right. & \left.\propto \rho_{g} u_{g}\right\rangle_{i+1 / 2, j-1 / 2}^{n}\left[\left\langle w_{g}\right\rangle_{i+1 / 2, j-1 / 2}^{n}-\left\langle w_{g}\right\rangle_{i+1 / 2, j}^{n}\right]= \\
& \frac{1}{2}\left(A_{c z} \hat{\alpha} \hat{\rho}_{g} u_{g}\right)_{i, j-1 / 2}^{n}\left[\left\{\left\langle w_{g}\right\rangle_{i+1 / 2, j-1 / 2}^{n}\right\}_{\text {Left }}-\left\langle w_{g}\right\rangle_{i+1 / 2, j}^{n}\right] \\
+ & \frac{1}{2}\left(A_{c z} \hat{\alpha} \hat{\rho}_{g} u_{g}\right)_{i+1, j-1 / 2}^{n}\left[\left\{\left\langle w_{g}\right\rangle_{i+1 / 2, j-1 / 2}^{n}\right\}_{\text {Right }}-\left\langle w_{g}\right\rangle_{i+1 / 2, j}^{n}\right]
\end{aligned}
$$

A similar expression applies for the lower face $(j+1 / 2)$. 
We complete our treatment of the azimuthal momentum equation for the gas phase by defining volume averaged physical properties through the relationship

$$
\langle\phi\rangle_{i+1 / 2, j}^{n} \equiv \frac{(\phi)_{i, j}^{n}\left(V_{c}\right)_{i, j}+(\phi)_{i+1, j}^{n}\left(V_{c}\right)_{i+1, j}}{\left(V_{c}\right)_{i, j}+\left(V_{c}\right)_{i+1, j}}
$$

Equation (3.4.1-66) is applied to the product of void fraction and gas density by setting $\phi$ $=\alpha \rho_{g}$ and to the gas density with $\phi=\rho_{g}$ to compute terms appearing in Eq. (3.4.1-46).

Similarly, the azimuthal component of the liquid momentum equation is

$$
\begin{aligned}
& (1-\alpha) \rho_{\mathrm{f}} \frac{\partial w_{f}}{\partial t}+\nabla \cdot\left[(1-\alpha) \rho_{\mathrm{f}} w_{f} \bar{v}_{\mathrm{f}}\right]-w_{\mathrm{f}} \nabla \cdot\left[(1-\alpha) \rho_{\mathrm{f}} \bar{v}_{\mathrm{f}}\right]= \\
& -(1-\alpha) \frac{\partial \mathrm{P}}{\partial \mathrm{x}}+(1-\alpha) \rho_{\mathrm{f}} \mathrm{g}^{\mathrm{x}}-\mathrm{F}_{\mathrm{wf}}^{\mathrm{x}}+\mathrm{F}_{\mathrm{I}}^{\mathrm{x}}-\mathrm{M}_{\mathrm{tf}}^{\mathrm{x}}
\end{aligned}
$$

The finite difference analog of Eq. $(3.4 \cdot 1-67)$ is 


$$
\begin{aligned}
& {\left[\left(\frac{\partial F_{w f}^{x}}{\partial w_{g}}\right)^{n}-\left(\frac{\partial F_{I}^{x}}{\partial w_{g}}\right)^{n}\right]_{i+1 / 2, j}\left(w_{g}\right)_{i+1 / 2, j}^{n+1}} \\
& +\left[\frac{R^{x}\left\langle(1-\alpha) \rho_{f}\right\rangle^{n}}{\Delta t}+\left(\frac{\partial F_{w f}^{x}}{\partial w_{f}}\right)^{n}-\left(\frac{\partial F_{l}^{x}}{\partial w_{f}}\right)^{n}\right]_{i+1 / 2, j}\left(w_{f}\right)_{i+1 / 2, j}^{n+1} \\
& =\frac{\langle(1-\alpha) A\rangle_{i+1 / 2, j}^{n}}{\left(V_{x}\right)_{i+1 / 2, j}}\left(P_{i, j}^{n+1}-P_{i+1, j}^{n+1}\right) \\
& +\frac{1}{\left(V_{x}\right)_{i+1 / 2, j}}\left\{\left\langle A_{x x}(1-\alpha) \rho_{f} w_{f} w_{f}\right\rangle_{i, j}^{n}-\left\langle A_{x x}(1-\alpha) \rho_{f} w_{f}\right\rangle_{i, j}^{n}\left\langle w_{f}\right\rangle_{i+1 / 2, j}^{n}\right. \\
& -\left\langle A_{x x}(1-\alpha) \rho_{f} w_{f} w_{f}\right\rangle_{i+1, j}^{n}+\left\langle A_{x x}(1-\alpha) \rho_{f} w_{f}\right\rangle_{i+1, j}^{n}\left\langle w_{f}\right\rangle_{i+1 / 2, j}^{n} \\
& +\left\langle A_{x z}(1-\alpha) \rho_{f} u_{f}\right\rangle_{i+1 / 2, j-1 / 2}^{n}\left[\left\langle w_{f}\right\rangle_{i+1 / 2, j-1 / 2}^{n}-\left\langle w_{f}\right\rangle_{i+1 / 2, j}^{n}\right] \\
& \left.-\left\langle A_{x z}(1-\alpha) \rho_{f} u_{f}\right\rangle_{i+1 / 2, j+1 / 2}^{n}\left[\left\langle w_{f}\right\rangle_{i+1 / 2, j+1 / 2}^{n}-\left\langle w_{f}\right\rangle_{i+1 / 2, j}^{n}\right]\right\} \\
& +R^{x}\left\langle(1-\alpha) \rho_{f}\right\rangle_{i+1 / 2, j}^{n} \frac{\left\langle w_{f}\right\rangle_{i+1 / 2, j}^{n}}{\Delta i} \\
& +\left\langle\rho_{f}\right\rangle_{i+1 / 2, j}^{n} g_{i+1 / 2, j}^{x} \frac{\langle(1-\alpha) A\rangle_{i+1 / 2, j}^{n}\left(\Delta x_{m}\right)_{i+1 / 2, j}}{\left(V_{x}\right)_{i+1 / 2, j}} \\
& -\left[\left(F_{w f}^{x}\right)^{n}-\left(\frac{\partial F_{w f}^{x}}{\partial w_{g}}\right)^{n} w_{g}^{n}-\left(\frac{\partial F_{w f}^{x}}{\partial w_{f}}\right)^{n} w_{f}^{n}\right]_{i+1 / 2, j} \\
& +\left[\left(F_{1}^{x}\right)^{n}-\left(\frac{\partial F_{l}^{x}}{\partial w_{g}}\right)^{n} w_{g}^{n}-\left(\frac{\partial F_{I}^{x}}{\partial w_{f}}\right)^{n} w_{f}^{n}\right]_{i+1 / 2, j}-\left(M_{f f}^{x}\right)_{i+1 / 2, j}^{n}
\end{aligned}
$$

Equation (3.4.1-68) has the general functional form

$$
a_{21}^{x}\left(w_{g}\right)_{i+1 / 2, j}^{n+1}+a_{22}^{x}\left(w_{f}\right)_{i+1 / 2, j}^{n+1}=d_{2}+e_{2}\left(P_{i, j}^{n+1}-P_{i+1, j}^{n+1}\right)
$$

where $a_{21}^{x}, a_{22}^{x}, d_{2}$, and $e_{2}$ are constant numbers evaluated explicitly from old time values at each $i+1 / 2, j$ mesh point.

Equations (3.4.1-47) and (3.4.1-69) form a $2 \times 2$ matrix equation which can be inverted to give a set of equations relating the azimuthal fluid and gas velocities to the azimuthal pressure difference across a mesh point. Combining the two equations yields the matrix equation 


$$
\left[\begin{array}{ll}
a_{11}^{x} & a_{12}^{x} \\
a_{21}^{x} & a_{22}^{x}
\end{array}\right]\left[\begin{array}{l}
\left(w_{g}\right)_{i+1 / 2, j}^{n+1} \\
\left(w_{f}\right)_{i+1 / 2, j}^{n+1}
\end{array}\right]=\left[\begin{array}{l}
d_{1} \\
d_{2}
\end{array}\right]+\left[\begin{array}{l}
e_{1} \\
e_{2}
\end{array}\right]\left(P_{i, j}^{n+1}-P_{i+1, j}^{n+1}\right)
$$

Inverting Eq. (3.4.1-70) gives the velocity relationships

$$
\begin{aligned}
& \left(w_{g}\right)_{i+1 / 2, j}^{n+1}=D_{g}+E_{g}\left(P_{i, j}^{n+1}-P_{i+1, j}^{n+1}\right) \\
& \left(w_{f}\right)_{i+1 / 2, j}^{n+1}=D_{f}+E_{f}\left(P_{i, j}^{n+1}-P_{i+1, j}^{n+1}\right)
\end{aligned}
$$

where the coefficients are computed as

$$
\begin{aligned}
& D_{g}=\left(a_{22}^{x} d_{1}-a_{12}^{x} d_{2}\right)\left|\underline{A}^{x}\right|^{-1} \\
& E_{g}=\left(a_{22}^{x} e_{1}-a_{12}^{x} e_{2}\right)\left|\underline{A}^{x}\right|^{-1} \\
& D_{f}=\left(a_{11}^{x} d_{2}-a_{21}^{x} d_{1}\right)\left|\underline{A}^{x}\right|^{-1} \\
& E_{f}=\left(a_{11}^{x} e_{2}-a_{21}^{x} e_{1}\right)\left|\underline{A}^{x}\right|^{-1}
\end{aligned}
$$

and the determinant of the matrix $\underline{\underline{A}}^{\mathbf{x}}$ is given by

$$
\left|\underline{A}^{x}\right|=\left(a_{11}^{x} a_{22}^{x}-a_{12}^{x} a_{21}^{x}\right)
$$

This completes our analysis of the fluid momentum conservation equations. In summary, momentum advection is evaluated explicitly and the momentum equations are used to derive equations relating the velocity components to the differences in the pressure at each mesh point. These relationships are given symbolically by Eqs. (3.4.1-42) and (3.4.1-71) shown above.

Bernoulli Differencing Scheme: In this section, we present a differencing scheme for the FLOWTRAN-TF momentum equations that will preserve Bernoulli equation pressure differences under certain restricted conditions. The analysis is restricted to a steady-state, one-dimensional, frictionless momentum equation. Evaluating Eq. (3.4.1-19) under these restrictions yields a simplified form of the gas momentum equation 


$$
\begin{aligned}
& \frac{1}{\left(V_{z}\right)_{i, j+1 / 2}}\left[-\left\langle A_{z z} \alpha \rho_{g} u_{g} u_{g}\right\rangle_{i, j}^{n}+\left\langle A_{z z} \alpha \rho_{g} u_{g} u_{g}\right\rangle_{i, j+1}^{n}\right] \\
& -\frac{\left\langle u_{g}\right\rangle_{i, j+1 / 2}^{n}}{\left(V_{z}\right)_{i, j+1 / 2}}\left[-\left\langle A_{z z} \alpha \rho_{g} u_{g}\right\rangle_{i, j}^{n}+\left\langle A_{z z} \alpha \rho_{g} u_{g}\right\rangle_{i, j+1}^{n}\right]= \\
& -\frac{\langle\alpha A\rangle_{i, j+1 / 2}^{n}}{\left(V_{z}\right)_{i, j+1 / 2}}\left(P_{i, j+1}^{n+1}-P_{i, j}^{n+1}\right)+\left\langle\rho_{g}\right\rangle_{i, j+1 / 2}^{n} g_{i, j+1 / 2}^{z} \frac{\langle\alpha A\rangle_{i, j+1 / 2}^{n}\left(\Delta z_{m}\right)_{i, j+1 / 2}}{\left(V_{z}\right)_{i, j+1 / 2}}
\end{aligned}
$$

For steady flow, the last term on the left hand side of Eq. (3.4.1-74) is identically zero from conservation of mass. Solving for the local pressure difference, Eq. $(3.4 .1-74)$ then reduces to

$$
\begin{aligned}
\left(P_{i, j+1}-P_{i, j}\right) & =\frac{\left\langle A_{z z} \alpha \rho u u\right\rangle_{i, j}-\left\langle A_{z z} \alpha \rho u u\right\rangle_{i, j+1}}{\langle\alpha A\rangle_{i, j+1 / 2}} \\
& +\langle\rho\rangle_{i, j+1 / 2} g_{i, j+1 / 2}^{z}\left(\Delta z_{m}\right)_{i, j+1 / 2}
\end{aligned}
$$

where we have dropped the $g$ subscript since the equation applies equally well to either fluid phase (replacing $\alpha$ with $1-\alpha$ for the liquid phase) and we have dropped the $n$ and $n+1$ superscripts indicating the time steps since they are not important to the analysis. Corresponding to the usual assumption applied in Bernoulli's equation, we restrict the analysis to an isothermal, incompressible (constant density) fluid which gives the simplified equation

$$
\begin{aligned}
\left(P_{i, j+1}-P_{i, j}\right) & =\frac{\rho}{\langle\alpha A\rangle_{i, j+1 / 2}}\left[\left\langle A_{z z} \alpha u u\right\rangle_{i, j}-\left\langle A_{z z} \alpha u u\right\rangle_{i, j+1}\right] \\
& +\rho g_{i, j+1 / 2}^{z}\left(\Delta z_{m}\right)_{i, j+1 / 2}
\end{aligned}
$$

We apply the donoring scheme described above to evaluate the momentum transport terms as

$$
\begin{aligned}
& \left\langle A_{z z} \alpha u u\right\rangle_{i, j}=\omega\left(A_{c z} \hat{\alpha} u u\right)_{i, j-1 / 2}+(1-\omega)\left(A_{c z} \hat{\alpha} u u\right)_{i, j+1 / 2} \\
& \left\langle A_{z z} \alpha u u\right\rangle_{i, j+1}=\omega\left(A_{c z} \hat{\alpha} u u\right)_{i, j+1 / 2}+(1-\omega)\left(A_{c z} \hat{\alpha} u u\right)_{i, j+3 / 2}
\end{aligned}
$$

Neglecting low velocity cases, for downflow $\omega=1$ and for upflow $\omega=0$. We then have the two forms of Eq. (3.4.1-76) 
Downflow:

$$
\begin{aligned}
\left(P_{i, j+1}-P_{i, j}\right) & =\rho g_{i, j+1 / 2}^{z}\left(\Delta z_{m}\right)_{i, j+1 / 2} \\
& +\frac{\rho}{\langle\alpha A\rangle_{i, j+1 / 2}}\left[\left(\hat{\alpha} A_{c z}\right)_{i, j-1 / 2} u_{i, j-1 / 2}^{2}-\left(\hat{\alpha} A_{c z}\right)_{i, j+1 / 2} u_{i, j+1 / 2}^{2}\right]
\end{aligned}
$$

Upflow:

$$
\begin{aligned}
\left(P_{i, j+1}-P_{i, j}\right) & =\rho g_{i, j+1 / 2}^{2}\left(\Delta z_{m}\right)_{i, j+1 / 2} \\
& +\frac{\rho}{\langle\alpha A\rangle_{i, j+1 / 2}}\left[\left(\hat{\alpha} A_{c z}\right)_{i, j+1 / 2} u_{i, j+1 / 2}^{2}-\left(\hat{\alpha} A_{c z}\right)_{i, j+3 / 2} u_{i, j+3 / 2}^{2}\right]
\end{aligned}
$$

Since we are using a staggered mesh and the pressure and fluid velocity do not occur at the same grid locations, it is difficult to compare the FLOWTRAN-TF momentum equations directly to the usual Bernoulli equation. If we try to apply Bernoulli's equation to compute $\left(P_{i, j+1}-P_{i, j}\right)$ for each fluid phase as

$$
\left(P_{i, j+1}-P_{i, j}\right)=\rho g_{i, j+1 / 2}^{z}\left(\Delta z_{m}\right)_{i, j+1 / 2}+\frac{\rho}{2}\left[u_{i, j}^{2}-u_{i, j+1}^{2}\right]
$$

we need the fluid velocities at the $i, j$ and $i, j+1$ mesh locations. In fact, the FLOWTRAN-FI code takes this approach and modifies the momentum difference equations to use cell centered velocities so that they will correspond to the Bernoulli equation formulation. Alternatively, we could equate Eq. (3.4.1-81) and Eqs. (3.4.1-79) and (3.4.1-80) to derive expressions for the required average phasic area $\langle\alpha A\rangle_{i, j+1 / 2}$; however, in practice this leads to physically unreasonable results for certain special cases. For example, for single phase flow with no gravity term, if $u_{i, j}=u_{i, j+1}$ but $u_{i, j-1 / 2} \neq u_{i, j+1 / 2}$ FLOWTRAN-TF predicts a pressure difference at this mesh location but the Bernoulli equation does not and the average area must be infinite to make the two results equal. Therefore, the best approach is to compute the pressure difference from Bernoulli's equation using velocity components displaced one half cell. This locates pressure changes at the points where velocity changes occur in our fluid mesh. Then we use modified Bernoulli equations for each phase of the form

Downflow:

$$
\left(P_{i, j+1}-P_{i, j}\right)=\rho g_{i, j+1 / 2}^{z}\left(\Delta z_{m}\right)_{i, j+1 / 2}+\frac{\rho}{2}\left[u_{i, j-1 / 2}^{2}-u_{i, j+1 / 2}^{2}\right]
$$

Upflow:

$$
\left(P_{i, j+1}-P_{i, j}\right)=\rho g_{i, j+1 / 2}^{z}\left(\Delta z_{m}\right)_{i, j+1 / 2}+\frac{\rho}{2}\left[u_{i, j+1 / 2}^{2}-u_{i, j+3 / 2}^{2}\right]
$$


Setting the pressure differences computed by Eq. (3.4.1-79) and (3.4.1-82) equal to each other,

$$
\begin{aligned}
& \frac{1}{2}\left[u_{i, j-1 / 2}^{2}-u_{i, j+1 / 2}^{2}\right]= \\
& \frac{1}{\langle\alpha A\rangle_{i, j+1 / 2}}\left[\left(\hat{\alpha} A_{c z}\right)_{i, j-1 / 2} u_{i, j-1 / 2}^{2}-\left(\hat{\alpha} A_{c z}\right)_{i, j+1 / 2} u_{i, j+1 / 2}^{2}\right]
\end{aligned}
$$

From the finite differenced mass balance (see §3.4.2), we have

$$
\left(\hat{\alpha} A_{c z} u\right)_{i, j-1 / 2}=\left(\hat{\alpha} A_{c z} u\right)_{i, j+1 / 2}
$$

or

$$
\frac{(u)_{i, j-1 / 2}}{(u)_{i, j+1 / 2}}=\frac{\left(\hat{\alpha} A_{c z}\right)_{i, j+1 / 2}}{\left(\hat{\alpha} A_{c z}\right)_{i, j-1 / 2}}
$$

Substituting Eqs. (3.4.1-85) or (3.4.1-86) into (3.4.1-84) and rearranging we obtain the area relationship for downflow as

$$
\frac{1}{\langle\alpha A\rangle_{i, j+1 / 2}}=\frac{1}{2}\left[\frac{1}{\left(\hat{\alpha} A_{c z}\right)_{i, j-1 / 2}}+\frac{1}{\left(\hat{\alpha} A_{c z}\right)_{i, j+1 / 2}}\right]
$$

or equivalently

$$
\langle\alpha A\rangle_{i, j+1 / 2}=\frac{2\left(\hat{\alpha} A_{c z}\right)_{i, j-1 / 2}\left(\hat{\alpha} A_{c z}\right)_{i, j+1 / 2}}{\left.\left(\hat{\alpha} A_{c z}\right)_{i, j-1 / 2}+\left(\hat{\alpha} A_{c z}\right)_{i, j+1 / 2}\right]}
$$

Setting the pressure differences computed by Eq. (3.4.1-80) and (3.4.1-83) equal to each other, applying the same analysis for upflow yields the area relationship

$$
\frac{1}{\langle\alpha A\rangle_{i, j+1 / 2}}=\frac{1}{2}\left[\frac{1}{\left(\hat{\alpha} A_{c z}\right)_{i, j+1 / 2}}+\frac{1}{\left(\hat{\alpha} A_{c z}\right)_{i, j+3 / 2}}\right]
$$

or equivalently

$$
\langle\alpha A\rangle_{i, j+1 / 2}=\frac{2\left(\hat{\alpha} A_{c z}\right)_{i, j+1 / 2}\left(\hat{\alpha} A_{c z}\right)_{i, j+3 / 2}}{\left[\left(\hat{\alpha} A_{c z}\right)_{i, j+1 / 2}+\left(\hat{\alpha} A_{c z}\right)_{i, j+3 / 2}\right]}
$$

Equations (3.4.1-88) and (3.4.1-90) then give expressions for the average phasic areas that, when applied to our differenced momentum equations, will produce pressure 
differences consistent with the Bernoulli equation. The above logic is implemented in general by defining

$$
\langle\alpha A\rangle_{i, j+1 / 2}^{n}=\omega\langle\alpha A\rangle_{i, j+1 / 2}^{\text {Downflow }}+(1-\omega)\langle\alpha A\rangle_{i, j+1 / 2}^{\text {Upflow }} \quad v=\left(u_{g}\right)_{i, j+1 / 2}^{n}
$$

where the downflow and upflow versions of $\langle\alpha A\rangle_{i, j+1 / 2}$ are given by equations (3.4.1-88) and $(3.4 .1-90)$, respectively.

The pressure differences will in some cases be displaced one cell length because of the staggered mesh. However, this offset can be eliminated (as long as the flow direction does not change) by the correct choice of the cell areas. Since it is clear from the above development that the gravity head loss is treated correctly, we will consider horizontal flow to simplify the presentation. Then Eq. $(3.4 .1-82)$ reduces to

$$
\left(P_{i, j+1}-P_{i, j}\right)=\frac{P}{2}\left[u_{i, j-1 / 2}^{2}-u_{i, j+1 / 2}^{2}\right]
$$

From Eq. (3.4.1-92), we see that the change in pressure takes place where the change in fluid velocity occurs. For steady-state flows, conservation of mass implies that the velocity change corresponds to a change in the flow area. Therefore, to match the pressure changes to the correct physical location in the flow channel, we must specify flow areas so that velocity changes are correctly located. This procedure is illustrated by the following two examples.

First consider the sudden change in area illustrated in Fig. 3.4.1-6. Physically, the pressure change should take place between $P_{i, j+1}$ and $P_{i, j}$ where the change in area occurs. From Eq. (3.4.1-92) this implies that the velocity must change between mesh locations $(i, j-$ $1 / 2)$ and $(i, j+1 / 2)$. Therefore, the flow area that should be specified at mass cell face $(i, j+1 / 2)$ is the area at the cell center location $(i, j+1)$. 


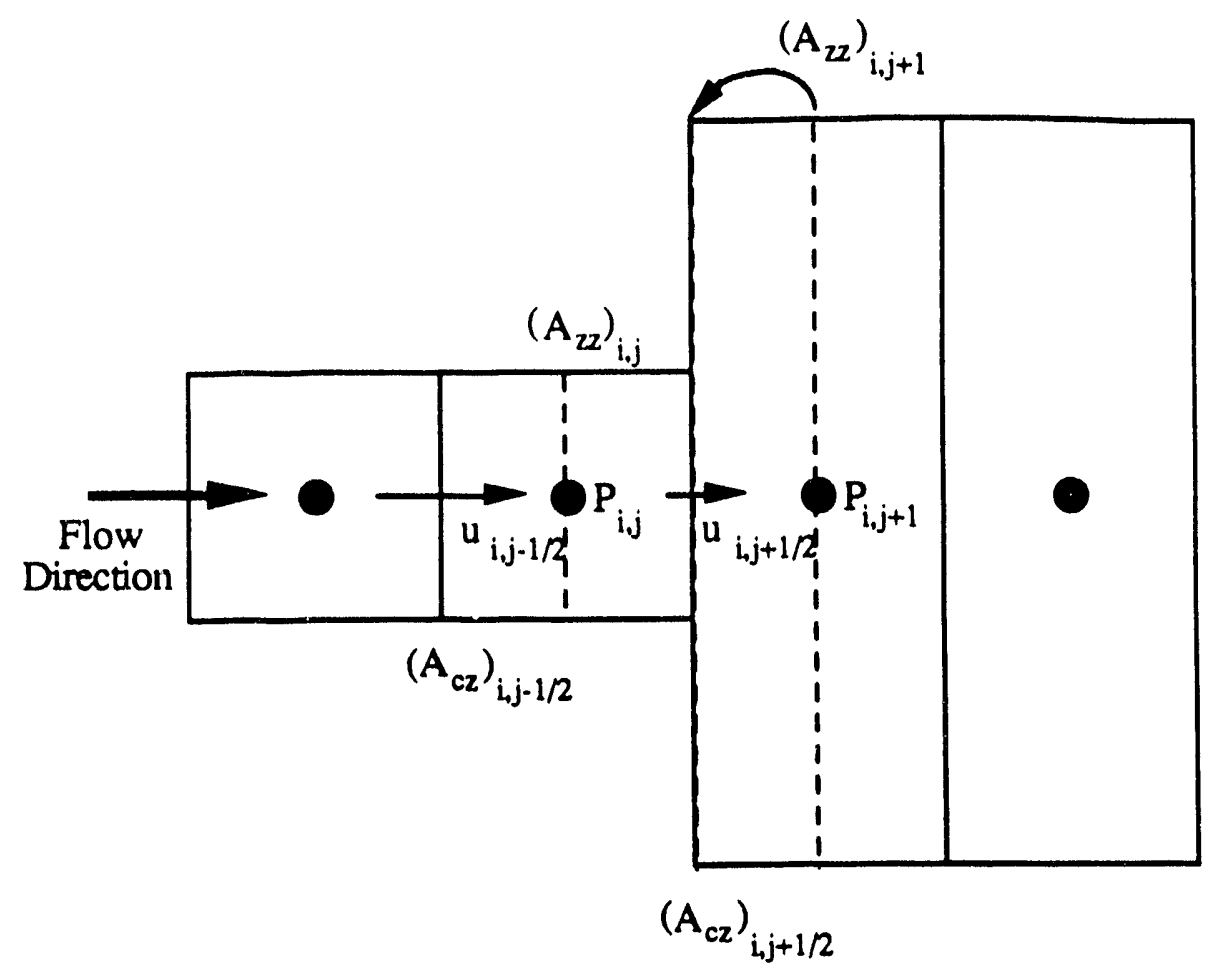

Figure 3.4.1-6 Dlustration of area adjustment technique at an abrupt change in area.

As a second example, consider the gradual change in area illustrated in Fig. 3.4.1-7 where we assume that several fluid cells are placed along the section where the area changes. To correctly compute the pressure change between $P_{i, j+1}$ and $P_{i, j}$ the fluid velocities must correspond to the flow areas at the pressure locations. This implies that we should specify flow areas as

$$
\begin{aligned}
& \left(A_{c z}\right)_{i, j-1 / 2}=\left(A_{z z}\right)_{i, j} \\
& \left(A_{c z}\right)_{i, j+1 / 2}=\left(A_{z z}\right)_{i, j+1}
\end{aligned}
$$

In effect, when we anticipate changes in the flow area and approximate the shape of the gradual area change by the dashed step changes shown in the figure we are able to exactly reproduce Bernoulli pressure changes across the flow channel. More complicated geometries can be treated in a similar fashion. As both examples illustrate, to match Bernoulli pressure changes, we must specify flow areas at the velocity mesh locations that actually correspond to the downstream pressure mesh points. This procedure is useful as long as a predominant flow direction exists. If the flow is expected to reverse direction, the code user may decide to enter physical areas at the velocity and pressure mesh points and accept the resulting offset in the pressure profile. 


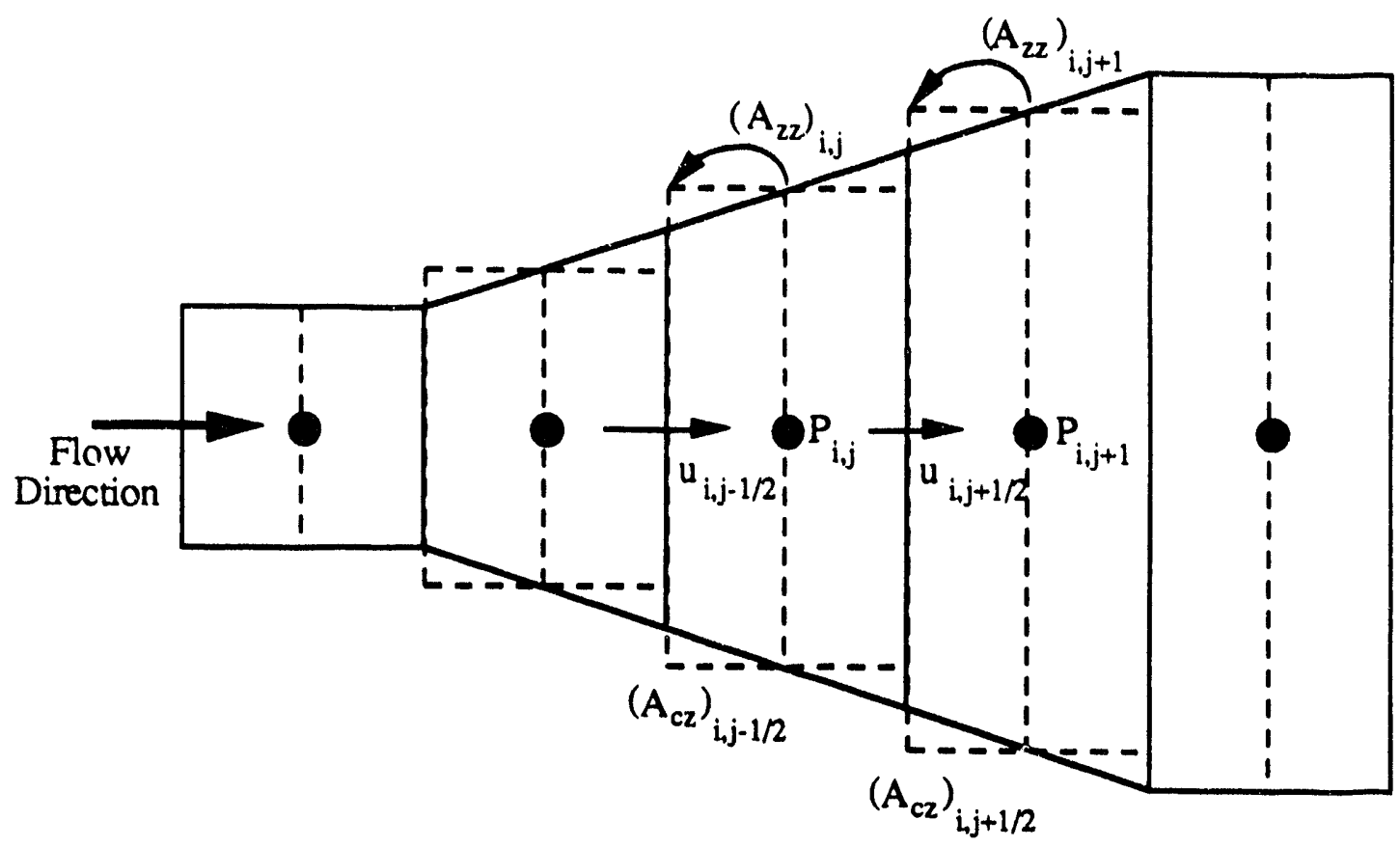

Figure 3.4.1-7 Illustration of area adjustment technique for a gradual change in area.

\section{References:}

Aleman, S. E., Gregory, M. V., Hamm, L. L., Koffman, L. D., Pevey, R. E, Reed, W. H., and Smith, III, F. G., 1989, "FLOWTRAN: An Algorithm for Describing the Thermal-Hydraulic Behavior of SRP Assemblies", DPSTM-140.

Dimenna, R. A., Larson, J. R., Johnson, R. W., Larson, T. K., Miller, C. S., Streit, J. E., Hanson, R. G., and Kiser, D. M., 1988, "RELAP5/MOD2 Models and Correlations", NUREG/CR-5194, Idaho National Engineering Laboratory, Idaho Falls, Idaho.

Ransom, V. H., Wagner, R. J., Trapp, J. A., Feinauer, L. R., Johnsen, G. W., Kiser, D. M., and Riemke, R. A., 1985, "RELAP5/MOD2 Code Manual Volume 1: Code Structure, System Models, and Solution Methods", NUREG/CR-4312, EG\&G Idaho, Inc.

Reed, W. H. and Stewart, H. B., 1978, "THERMIT: A Computer Program for ThreeDimensional Thermal-Hydraulic Analysis of Light Water Reactor Cores", M.I.T. Report prepared for EPRI. 


\subsubsection{Mass/Energy}

In this section of the code documentation, we derive finite difference equations to represent the mass and energy conservation equations for the gas and liquid phases. The five remaining conservation equations are solved simultaneously for values of the five primary state variables at the new time step. The differencing scheme uses explicit representations for the velocities and the equations relating local velocity components to the local pressure differences (derived from the solution of the momentum equations presented above) are then used to eliminate the velocity from the mass and energy finite difference equations. The final set of difference equations at each mesh point are combined and all unknown variables except for the pressure are eliminated. A solution for the change in pressure is obtained and the difference equations developed here are then used to determine the changes in the other primary state variables.

We will use the two dimensional forms of the equations which apply in the middle section of the assembly to derive the difference equations. In the top and bottom sections, the equations are reduced to one dimensional axial forms. Also the top and bottom sections of the assembly are unheated so that wall heat transfer and phase changes do not apply in these sections.

Differential Equations: Differential forms of the conservation equations for gas and liquid mass and energy are presented in Eqs. (2.4.1-95), (2.4.1-96) and (2.4.1-99) (2.4.1-101). We reproduce the differential equations here for completeness and for immediate reference.

Gas Mixture Mass -

$$
\frac{\partial \alpha \rho_{g}}{\partial t}+\frac{1}{V} \nabla \cdot\left[V \alpha \rho_{g} \bar{v}_{g}\right]=\Gamma_{I}+\Gamma_{w}-W_{t g}
$$

Liquid Mass -

$$
\frac{\partial(1-\alpha) \rho_{f}}{\partial t}+\frac{1}{V} \nabla \cdot\left[V(1-\alpha) \rho_{f} \bar{v}_{f}\right]=-\Gamma_{I}-\Gamma_{w}-W_{d f}
$$

Air Mass -

$$
\frac{\partial \alpha \rho_{g} X_{a}}{\partial t}+\frac{1}{V} \nabla \cdot\left[V \alpha \rho_{g} X_{a} \bar{v}_{g}\right]=-X_{a} W_{t g}
$$

Gas Energy -

$$
\frac{\partial \alpha \rho_{g} h_{g}}{\partial t}+\frac{1}{V} \nabla \cdot\left[V \alpha \rho_{g} h_{g} \bar{v}_{g}\right]=\alpha \frac{\partial P}{\partial t}+E_{I}+E_{w}+Q_{w_{g}}-Q_{t g}
$$


Liquid Energy -

$$
\frac{\partial(1-\alpha) \rho_{f} h_{f}}{\partial t}+\frac{1}{V} \cdot \nabla \cdot\left[V(1-\alpha) \rho_{f} h_{f} \bar{v}_{f}\right]=(1-\alpha) \frac{\partial P}{\partial t}-E_{I}-E_{w}+Q_{w f}-Q_{t f}
$$

In Eqs. (3.4.2-4) and (3.4.2-5), we have used the definition

$$
E_{I} \equiv E_{g i}=-E_{f i}
$$

to represent interfacial energy exchange between the bulk gas and liquid phases (see code development report TF-13). Similarly,

$$
E_{w} \equiv E_{g w}=-E_{f w}
$$

(see \$2.5.4.3). The terms $\Gamma_{I}$ and $\Gamma_{w}$ comprise $\Gamma$ in $\$ 2.4 .1$ and the terms $E_{I}$ and $E_{w}$ comprise $\mathrm{E}_{\mathrm{i}}$.

General Form of the Conservation Equations: We note that Eqs. (3.4.2-1) through (3.4.2-5) are all of the general form

$$
\frac{\partial\left(\alpha_{k} \rho_{k} \phi_{k}\right)}{\partial t}+\frac{1}{V} \nabla \cdot\left[V \alpha_{k} \rho_{k} \phi_{k} \bar{v}_{k}\right]=\sum_{i} S_{i}
$$

where the subscript $k$ and the term $\alpha_{k}$ are used as follows:

$$
\begin{aligned}
& \mathrm{k} \rightarrow \mathrm{g} \text { designates the gas phase and } \alpha_{\mathrm{g}}=\alpha \\
& \mathrm{k} \rightarrow \mathrm{f} \text { designates the liquid phase and } \alpha_{\mathrm{f}}=1-\alpha
\end{aligned}
$$

Equation (3.4.2-6) represents a generalized conservation equation that sets the time rate of change in a quantity plus the rate of input by advective transport equal to the sum of all source terms for the quantity. The following table summarizes the definitions required to cast Eqs. (3.4.2-1) through (3.4.2-5) into the general form of Eq. (3.4.2-6). 


\begin{tabular}{|c|c|c|c|}
\hline $\begin{array}{c}\text { Conservation } \\
\text { Equation }\end{array}$ & $k$ & $\phi_{\mathrm{k}}$ & $\sum_{\mathrm{i}} \mathrm{S}_{\mathrm{i}}$ \\
\hline Gas Mixture Mass & $\mathrm{g}$ & 1 & $\Gamma_{\mathrm{I}}+\Gamma_{\mathrm{w}}-\mathrm{W}_{\mathrm{tg}}$ \\
Liquid Mass & $\mathrm{f}$ & 1 & $-\Gamma_{\mathrm{I}}-\Gamma_{\mathrm{w}}-\mathrm{W}_{\mathrm{tf}}$ \\
Air Mass & $\mathrm{g}$ & $\mathrm{X}_{\mathrm{g}}$ & $-\mathrm{X}_{\mathrm{a}} \mathrm{W}_{\mathrm{tg}}$ \\
Gas Energy & $\mathrm{g}$ & $\mathrm{h}_{\mathrm{g}}$ & $\alpha_{\mathrm{g}} \frac{\partial \mathrm{P}}{\partial \mathrm{t}}+\mathrm{E}_{\mathrm{I}}+\mathrm{E}_{\mathrm{w}}+\mathrm{Q}_{\mathrm{wg}}-\mathrm{Q}_{\mathrm{tg}}$ \\
& $\mathrm{f}$ & $\mathrm{h}_{\mathrm{f}}$ & $\alpha_{\mathrm{f}} \frac{\partial \mathrm{P}}{\partial \mathrm{t}}-\mathrm{E}_{\mathrm{I}}-\mathrm{E}_{\mathrm{w}}+\mathrm{Q}_{\mathrm{wf}}-\mathrm{Q}_{\mathrm{tf}}$ \\
\hline
\end{tabular}

Since Eq. (3.4.2-6) can be considered a general representation of the basic conservation equations, we will use it to develop a generalized finite difference equation. In particular, the left hand side of the generalized equation can be treated one time and the results immediately applied to all of the conservation equations. To develop a difference equation, we integrate each term in Eq. (3.4.2-6) over the mass/energy control volume $\left(\mathrm{V}_{\mathrm{m} / \mathrm{e}}\right.$, shown in Fig. 3.4.1-2) and divide by the volume. Integrating the first term yields

$$
\begin{aligned}
\frac{1}{V_{m / e}} \int_{V_{m / e}} \frac{\partial\left(\alpha_{k} \rho_{k} \phi_{k}\right)}{\partial t} d V_{m / e} & \rightarrow \frac{\partial}{\partial t}\left\{\frac{1}{V_{m / e}} \int_{V_{m / e}}\left(\alpha_{k} \rho_{k} \phi_{k}\right) d V_{m / e}\right\} \\
& =\frac{\left(\alpha_{k} \rho_{k} \phi_{k}\right)_{i, j}^{n+1}-\left(\alpha_{k} \rho_{k} \phi_{k}\right)_{i, j}^{n}}{\Delta t}
\end{aligned}
$$

To derive Eq. (3.4.2-7), we first interchange the operations of taking the partial derivative with respect to time and volume averaging the product of void fraction, density, and the associated function. We then assume that cell center properties represent volume average quantities. Finally, we use a forward in time finite difference approximation to the partial derivative.

Integrating the second term in Eq. (3.4.2-6), which represents the advection of mass or energy, gives 


$$
\begin{aligned}
\frac{1}{V_{m / e}} \int_{V_{m / e}} \frac{1}{V} \nabla \cdot\left[V \alpha_{k} \rho_{k} \phi_{k} \bar{v}_{k}\right] d V_{m / e} \rightarrow \frac{1}{V_{m / e}} \int_{A_{s}}\left(\alpha_{k} \rho_{k} \phi_{k} \bar{v}_{k}\right) \cdot \bar{n} d A_{s} \\
=\frac{1}{\left(V_{c}\right)_{i, j}}\left[-\left\langle A_{c z} \alpha_{k} \rho_{k} \phi_{k} u_{k}\right\rangle_{i, j-1 / 2}^{n+1}+\left\langle A_{c z} \alpha_{k} \rho_{k} \phi_{k} u_{k}\right\rangle_{i, j+1 / 2}^{n+1}\right. \\
\left.\quad-\left\langle A_{c x} \alpha_{k} \rho_{k} \phi_{k} w_{k}\right\rangle_{i-1 / 2, j}^{n+1}+\left\langle A_{c x} \alpha_{k} \rho_{k} \phi_{k} w_{k}\right\rangle_{i+1 / 2, j}^{n+1}\right]
\end{aligned}
$$

The first equality in Eq. (3.4.2-8) is obtained by applying the divergence theorem to convert the volume integral of the divergence of the flux term into an integral over the surface of the control volume. A detailed justification for this procedure that directly treats the presence of the volume terms inside the integral is given in \$2.4.1. Evaluating the surface integral by taking appropriate averages of the indicated flows over the four sides of the mass control volume we obtain the second equality. The first two terms represent advection into the control volume by the axial component of the velocity while the last two terms represent advection into the control volume by the azimuthal velocity. The advection terms are evaluated semi-explictly using property values calculated from conditions at the previous time step and velocities at the new time step. Property values are donored to the control volume faces. For example, at the top and bottom faces of the mass/energy control volume, we define the axial flow terms to be

$$
\begin{aligned}
& \left\langle A_{c z} \alpha_{k} \rho_{k} \phi_{k} u_{k}\right\rangle_{i, j-1 / 2}^{n+1}=\left(A_{c z} \hat{\alpha}_{k} \hat{\rho}_{k} \hat{\phi}_{k}\right)_{i, j-1 / 2}^{n}\left(u_{k}\right)_{i, j-1 / 2}^{n+1} \\
& \left\langle A_{c z} \alpha_{k} \rho_{k} \phi_{k} u_{k}\right\rangle_{i, j+1 / 2}^{n+1}=\left(A_{c z} \hat{\alpha}_{k} \hat{\rho}_{k} \hat{\phi}_{k}\right)_{i, j+1 / 2}^{n}\left(u_{k}\right)_{i, j+1 / 2}^{n+1}
\end{aligned}
$$

and at the right and left hand faces of the control volume flows in the azimuthal direction are evaluated as

$$
\begin{aligned}
& \left\langle A_{c x} \alpha_{k} \rho_{k} \phi_{k} w_{k}\right\rangle_{i-1 / 2, j}^{n+1}=\left(A_{c x} \hat{\alpha}_{k} \hat{\rho}_{k} \hat{\phi}_{k}\right)_{i-1 / 2, j}^{n}\left(w_{k}\right)_{i-1 / 2, j}^{n+1} \\
& \left\langle A_{c x} \alpha_{k} \rho_{k} \dot{\phi}_{k} w_{k}\right\rangle_{i+1 / 2, j}^{n+1}=\left(A_{c x} \hat{\alpha}_{k} \hat{\rho}_{k} \hat{\phi}_{k}\right)_{i+1 / 2, j}^{n}\left(w_{k}\right)_{i+1 / 2, j}^{n+1}
\end{aligned}
$$

Donored physical properties have already been defined in our description of the momentum difference equations (see \$3.4.1). In general. the following relations apply:

At the top face of the mass/energy control volume, $v=\left(u_{g}\right)_{i, j-1 / 2}^{n}$

$$
\left(\hat{\phi}_{k}\right)_{i, j-1 / 2}^{n}=\omega\left(\phi_{k}\right)_{i, j-1}^{n}+(1-\omega)\left(\phi_{k}\right)_{i, j}^{n}
$$

At the bottom face of the mass/energy control volume, $v=\left(u_{g}\right)_{i, j+1 / 2}^{n}$

$$
\left(\hat{\phi}_{k}\right)_{i, j+1 / 2}^{n}=\omega\left(\phi_{k}\right)_{i, j}^{n}+(1-\omega)\left(\phi_{k}\right)_{i, j+1}^{n}
$$


At the left face of the mass/energy control volume, $v=\left(w_{g}\right)_{i-1 / 2, j}^{n}$

$$
\left(\hat{\phi}_{k}\right)_{i-1 / 2, j}^{n}=\omega\left(\phi_{k}\right)_{i-1, j}^{n}+(1-\omega)\left(\phi_{k}\right)_{i, j}^{n}
$$

At the right face of the mass/energy control volume, $v=\left(w_{g}\right)_{i+1 / 2, j}^{n}$

$$
\left(\hat{\phi}_{k}\right)_{i+1 / 2, j}^{n}=\omega\left(\phi_{k}\right)_{i, j}^{n}+(1-\omega)\left(\phi_{k}\right)_{i+1, j}^{n}
$$

Using the velocities indicated with Eqs. $(3.4 .2-11)$ - (3.4.2-14), the interpolation parameter $\omega$ for each of the above relations is defined as

$$
\omega \equiv\left\{\begin{array}{cc}
1 & v \geq v_{\min } \\
\frac{1}{2}\left(1+\frac{v}{v_{\min }}\right) & v_{\min }>v>-v_{\min } \\
0 & v \leq-v_{\min }
\end{array}\right.
$$

The velocity-pressure relations derived from the momentum equations (e.g. see Eqs. (3.4.1-42) and (3.4.1-71)) are used to eliminate the velocities from Eqs. (3.4.2-9) and $(3.4 .2-10)$ and obtain alternative expressions for the advection terms. For the axial advection terms we find

$$
\begin{aligned}
& \left\langle A_{c z} \alpha_{k} \rho_{k} \phi_{k} u_{k}\right\rangle_{i, j-1 / 2}^{n+1}= \\
& \left(A_{c z} \hat{\alpha}_{k} \hat{\rho}_{k} \hat{\phi}_{k}\right)_{i, j-1 / 2}^{n}\left[\left(B_{k}\right)_{i, j-1 / 2}^{n}+\left(C_{k}\right)_{i, j-1 / 2}^{n}\left(P_{i, j-1}^{n+1}-r_{i, j}^{n+1}\right)\right] \\
& \left\langle A_{c z} \alpha_{k} \rho_{k} \phi_{k} u_{k}\right\rangle_{i, j+1 / 2}^{n+1}= \\
& \left(A_{c z} \hat{\alpha}_{k} \hat{\rho}_{k} \hat{\phi}_{k}\right)_{i, j+1 / 2}^{n}\left[\left(B_{k}\right)_{i, j+1 / 2}^{n}+\left(C_{k}\right)_{i, j+1 / 2}^{n}\left(P_{i, j}^{n+1}-P_{i, j+1}^{n+1}\right)\right]
\end{aligned}
$$

Similarly, for the azimuthal a vection terms we obtain

$$
\begin{aligned}
& \left\langle A_{r x} \alpha_{k} \rho_{k} \phi_{k} w_{k}\right\rangle_{i-1 / 2, j}^{n+1}= \\
& \left(A_{c x} \hat{\alpha}_{k} \hat{\rho}_{k} \hat{\phi}_{k}\right)_{i-1 / 2, j}^{n}\left[\left(D_{k}\right)_{i-1 / 2, j}^{n}+\left(E_{k}\right)_{i-1 / 2, j}^{n}\left(P_{i-1, j}^{n+1}-P_{i, j}^{n+1}\right)\right] \\
& \left\langle A_{c x} \alpha_{k} \rho_{k} \phi_{k} w_{k}\right\rangle_{i+1 / 2, j}^{n+1}= \\
& \left(A_{c x} \hat{\alpha}_{k} \hat{\rho}_{k} \hat{\phi}_{k}\right)_{i+1 / 2, j}^{n}\left[\left(D_{k}\right)_{i+1 / 2, j}^{n}+\left(E_{k}\right)_{i+1 / 2, j}^{n}\left(P_{i, j}^{n+1}-P_{i+1, j}^{n+1}\right)\right]
\end{aligned}
$$


Coefficients $\left(B_{g}\right)_{i, j \pm 1 / 2}^{n},\left(C_{g}\right)_{i, j \pm 1 / 2}^{n},\left(D_{g}\right)_{i \pm 1 / 2, j}^{n}$, and $\left(E_{g}\right)_{i \pm 1 / 2, j}^{n}$ are all known quantities that depend solely on parameter values at the previous time step.

In general, the source terms on the right hand side of Eq. (3.4.2-6) are also integrated over the control volume and divided by the volume to replace them with appropriate volume averaged quantities. We will represent this process through the relationship

$$
\frac{1}{V_{m / e}} \int_{V_{m / e}} S_{i} d V_{m / e} \rightarrow\left(S_{i}\right)_{i, j}^{n+1}
$$

Specific representations for each of the source terms will be developed below where the individual equations are considered.

Combining Eqs. (3.4.2-7) and (3.4.2-8) with Eq. (3.4.2-6) and applying the relationships given in Eqs. (3.4.2-15) through (3.4.2-17) yields a general difference equation of the form

$$
\begin{aligned}
\frac{\left(\alpha_{k} \rho_{k} \phi_{k}\right)_{i, j}^{n+1}-}{\Delta t} & \left(\alpha_{k} \rho_{k} \phi_{k}\right)_{i, j}^{n}+ \\
\frac{1}{\left(V_{c}\right)_{i, j}} & \left\{-\left(A_{c z} \hat{\alpha}_{k} \hat{\rho}_{k} \hat{\phi}_{k}\right)_{i, j-1 / 2}^{n}\left[\left(B_{k}\right)_{i, j-1 / 2}^{n}+\left(C_{k}\right)_{i, j-1 / 2}^{n}\left(P_{i, j-1}^{n+1}-P_{i, j}^{n+1}\right)\right]\right. \\
& +\left(A_{c z} \hat{\alpha}_{k} \hat{\rho}_{k} \hat{\phi}_{k}\right)_{i, j+1 / 2}^{n}\left[\left(B_{k}\right)_{i, j+1 / 2}^{n}+\left(C_{k}\right)_{i, j+1 / 2}^{n}\left(P_{i, j}^{n+1}-P_{i, j+1}^{n+1}\right)\right] \\
& -\left(A_{c x} \hat{\alpha}_{k} \hat{\rho}_{k} \hat{\phi}_{k}\right)_{i-1 / 2, j}^{n}\left[\left(D_{k}\right)_{i-1 / 2, j}^{n}+\left(E_{k}\right)_{i-1 / 2, j}^{n}\left(P_{i-1, j}^{n+1}-P_{i, j}^{n+1}\right)\right] \\
& \left.+\left(A_{c x} \hat{\alpha}_{k} \hat{\rho}_{k} \hat{\phi}_{k}\right)_{i+1 / 2, j}^{n}\left[\left(D_{k}\right)_{i+1 / 2, j}^{n}+\left(E_{k}\right)_{i+1 / 2, j}^{n}\left(P_{i, j}^{n+1}-P_{i+1, j}^{n+1}\right)\right]\right\} \\
= & \sum_{i}\left(S_{i}\right)_{i, j}^{n+1}
\end{aligned}
$$

Equation (3.4.2-18) can not be solved directly because it contains nonlinear functions of the state variables evaluated at the $n+1$ time step. The material properties (e.g. density and $\phi_{\mathrm{k}}$ function) and the source terms for interfacial mass and energy exchange are all functions of the primary state variables such as pressure and temperature. Therefore, Eq. (3.4.2-18) contains imbedded functional dependencies which preclude a direct solution to the final set of equations. To solve the difference equations we use an iteration scheme that:

1. Estimates values for the five primary state variables.

2. Evaluates the implicit functions.

3. Solves the difference equations for the changes in values of the state variables.

This procedure is iterated until changes in the state variables are sufficiently small. To improve convergence properties, the functional dependences on the primary state variables 
are accounted for in the difference equations by expanding the dependent terms in Taylor series about the most recent estimate of their value.

It is advantageous to cast the difference equations in terms of the changes in the primary state variables. We then solve for these changes directly and use them to update the values of the variables. For example, denoting the iteration step by the superscript $m$, updated pressures at each step in the iteration are computed as

$$
P_{i, j}^{n+1} \equiv P_{i, j}^{m+1} \equiv P_{i, j}^{m}+(\delta P)_{i, j}^{m+1}
$$

In the limit, as the iteration converges, the pressure at the new time $\mathrm{P}_{i, j}^{n+1}$ is equal to the pressure at the $m+1$ iteration $P_{i, j}^{m+1}$. Alternatively, the $m+1$ pressure iterate can be thought of as an approximation to the pressure at the $n+1$ time step as indicated in Eq. (3.4.2-19). In a similar fashion, for the other state variables, we use the expressions

$$
\begin{gathered}
\alpha_{i, j}^{m+1} \equiv \alpha_{i, j}^{m}+(\delta \alpha)_{i, j}^{m+1} \\
\left(T_{g}\right)_{i, j}^{m+1} \equiv\left(T_{g}\right)_{i, j}^{m}+\left(\delta T_{g}\right)_{i, j}^{m+1} \\
\left(T_{f}\right)_{i, j}^{m+1} \equiv\left(T_{f}\right)_{i, j}^{m}+\left(\delta T_{f}\right)_{i, j}^{m+1} \\
\left(X_{a}\right)_{i, j}^{m+1} \equiv\left(X_{a}\right)_{i, j}^{m}+\left(\delta X_{a}\right)_{i, j}^{m+1}
\end{gathered}
$$

Substituting Eq. (3.4.2-19) into Eq. (3.4.2-18) and rearranging the result to put known values on the right hand side and collect like terms multiplying the unknown pressure differentials yields 


$$
\begin{aligned}
& \frac{\left(\alpha_{k} \rho_{k} \phi_{k}\right)_{i, j}^{n+1}}{\Delta t} \\
& +\frac{1}{\left(V_{c}\right)_{i, j}}\left\{\left(A_{c z} \hat{\alpha}_{k} \hat{\rho}_{k} \hat{\phi}_{k} C_{k}\right)_{i, j-1 / 2}^{n}+\left(A_{c z} \hat{\alpha}_{k} \hat{\rho}_{k} \hat{\phi}_{k} C_{k}\right)_{i, j+1 / 2}^{n}\right. \\
& \left.+\left(A_{c x} \hat{\alpha}_{k} \hat{\rho}_{k} \hat{\phi}_{k} E_{k}\right)_{i-1 / 2, j}^{n}+\left(A_{c x} \hat{\alpha}_{k} \hat{\rho}_{k} \hat{\phi}_{k} E_{k}\right)_{i+1 / 2, j}^{n}\right\}(\delta P)_{i, j}^{m+1} \\
& -\frac{1}{\left(V_{c}\right)_{i, j}}\left\{\left(A_{c z} \hat{\alpha}_{k} \hat{\rho}_{k} \hat{\phi}_{k} C_{k}\right)_{i, j-1 / 2}^{n}\right\}(\delta P)_{i, j-1}^{m+1} \\
& -\frac{1}{\left(V_{c}\right)_{i, j}}\left\{\left(A_{c z} \hat{\alpha}_{k} \hat{\rho}_{k} \hat{\phi}_{k} C_{k}\right)_{i, j+1 / 2}^{n}\right\}(\delta P)_{i, j+1}^{m+1} \\
& -\frac{1}{\left(V_{c}\right)_{i, j}}\left\{\left(A_{c x} \hat{\alpha}_{k} \hat{\rho}_{k} \hat{\phi}_{k} E_{k}\right)_{i-1 / 2, j}^{n}\right\}(\delta P)_{i-1, j}^{m+1} \\
& -\frac{1}{\left(V_{c}\right)_{i, j}}\left\{\left(A_{c x} \hat{\alpha}_{k} \hat{\rho}_{k} \hat{\phi}_{k} E_{k}\right)_{i+1 / 2, j}^{n}\right\}(\delta P)_{i+1, j}^{m+1} \\
& =\frac{1}{\left(V_{c}\right)_{i, j}}\left\{\left(A_{c z} \hat{\alpha}_{k} \hat{\rho}_{k} \hat{\phi}_{k}\right)_{i, j-1 / 2}^{n}\left[\left(B_{k}\right)_{i, j-1 / 2}^{n}+\left(C_{k}\right)_{i, j-1 / 2}^{n}\left(P_{i, j-1}^{m}-P_{i, j}^{m}\right)\right]\right. \\
& -\left(A_{c z} \hat{\alpha}_{k} \hat{\rho}_{k} \hat{\phi}_{k}\right)_{i, j+1 / 2}^{n}\left[\left(B_{k}\right)_{i, j+1 / 2}^{n}+\left(C_{k}\right)_{i, j+1 / 2}^{n}\left(P_{i, j}^{m}-P_{i, j+1}^{m}\right)\right] \\
& +\left(A_{c x} \hat{\alpha}_{k} \hat{\rho}_{k} \hat{\phi}_{k}\right)_{i-1 / 2, j}^{n}\left[\left(D_{k}\right)_{i-1 / 2, j}^{n}+\left(E_{k}\right)_{i-1 / 2, j}^{n}\left(P_{i-1, j}^{m}-P_{i, j}^{m}\right)\right] \\
& \left.-\left(A_{c x} \hat{\alpha}_{k} \hat{\rho}_{k} \hat{\phi}_{k}\right)_{i+1 / 2, j}^{n}\left[\left(D_{k}\right)_{i+1 / 2, j}^{n}+\left(E_{k}\right)_{i+1 / 2, j}^{n}\left(P_{i, j}^{m}-P_{i+1, j}^{m}\right)\right]\right\} \\
& +\frac{\left(\alpha_{k} \rho_{k} \phi_{k}\right)_{i, j}^{n}}{\Delta t}+\sum_{i}\left(s_{i}\right)_{i, j}^{n+1}
\end{aligned}
$$

Equation (3.4.2-20) will serve as our general difference equation which will be used to develop individual relationships for each of the five conservation equations. To accomplish this, we must expand each of the functions evaluated at the new time step (denoted by the $\mathrm{n}+1$ superscript) in terms of the values computed within the iteration scheme. These derivations will be presented below for each conservation equation.

Gas Mixture Mass $\left(k=g, \phi_{k}=1\right)$ : As noted above, Taylor series expansions of terms evaluated at the new time about the most recent estimate of their value are used to linearize the difference equations. Gas density is a function of pressure, gas temperature, and air mass fraction. Denoting the iteration step by the superscript $m$, we write the following expansion for the product of void fraction and gas density at the new time step 


$$
\begin{aligned}
\left(\alpha \rho_{g}\right)_{i, j}^{n+1} \cong\left(\alpha \rho_{g}\right)_{i, j}^{m} & +\left[\alpha\left(\frac{\partial \rho_{g}}{\partial P}\right)\right]_{i, j}^{m}(\delta P)_{i, j}^{m+1}+\left(\rho_{g}\right)_{i, j}^{m}(\delta \alpha)_{i, j}^{m+1} \\
& +\left[\alpha\left(\frac{\partial \rho_{g}}{\partial T_{g}}\right)\right]_{i, j}^{m}\left(\delta T_{g}\right)_{i, j}^{m+1}+\left[\alpha\left(\frac{\partial \rho_{g}}{\partial X_{a}}\right)\right]_{i, j}^{m}\left(\delta X_{a}\right)_{i, j}^{m+1}
\end{aligned}
$$

We treat the source terms in a similar fashion. Mass exchange at the gas-liquid interface $\left(\Gamma_{\mathrm{I}}\right)$ is evaluated as an implicit function and assuming that cell center values can be used to represent the volume averages. The required expansions are

$$
\begin{aligned}
\left(\Gamma_{I}\right)_{i, j}^{n+1} \cong\left(\Gamma_{I}\right)_{i, j}^{m} & +\left(\frac{\partial \Gamma_{I}}{\partial P}\right)_{i, j}^{m}(\delta P)_{i, j}^{m+1}+\left(\frac{\partial \Gamma_{I}}{\partial \alpha}\right)_{i, j}^{m}(\delta \alpha)_{i, j}^{m+1} \\
& +\left(\frac{\partial \Gamma_{I}}{\partial T_{g}}\right)_{i, j}^{m}\left(\delta T_{g}\right)_{i, j}^{m+1}+\left(\frac{\partial \Gamma_{I}}{\partial T_{f}}\right)_{i, j}^{m}\left(\delta T_{f}\right)_{i, j}^{m+1}+\left(\frac{\partial \Gamma_{I}}{\partial X_{a}}\right)_{i, j}^{m}\left(\delta X_{a}\right)_{i, j}^{m+1}
\end{aligned}
$$

where $\delta x$ for $x=\left\{P, \alpha, T_{g}, T_{f}, X_{a}\right\}$ is the change in the indicated primary state variable. As explained in TF-14, the mass exchange at the wall $\left(\Gamma_{w}\right)$ is evaluated explicitly.

$$
\left(\Gamma_{w}\right)_{i, j}^{n+1} \cong\left(\Gamma_{w}\right)_{i, j}^{n}
$$

The final term on the right hand side of the gas mass equation representing mass exchange due to turbulent fluctuations is evaluated explicitly as

$$
\left(w_{t g}\right)_{i, j}^{n+1} \cong\left(w_{t g}\right)_{i, j}^{n}
$$

We substitute Eqs. (3.4.2-21) - (3.4.2-24) into Eq. (3.4.2-20), grouping terms that multiply changes in the primary state variables and placing known quantities on the right hand side, to obtain a complete difference equation for gas mass conservation as 
\begin{tabular}{|l|l|}
\hline $2 / 15 / 93$ & WSRC-TR-92-532 Rev. 0 \\
\hline
\end{tabular}

FLOWTRAN-TF Software Design

Page 371

$$
\begin{aligned}
& \left\{\frac{\alpha_{i, j}^{m}}{\Delta t}\left(\frac{\partial \rho_{g}}{\partial P}\right)_{i, j}^{m}-\left(\frac{\partial \Gamma_{I}}{\partial P}\right)_{i, j}^{m}\right. \\
& +\frac{1}{\left(V_{c}\right)_{i, j}}\left[\left(A_{c z} \hat{\alpha} \hat{\rho}_{g} C_{g}\right)_{i, j-1 / 2}^{n}+\left(A_{c z} \hat{\alpha} \hat{\rho}_{g} C_{g}\right)_{i, j+1 / 2}^{n}\right. \\
& \left.\left.+\left(A_{c x} \hat{\alpha} \hat{\rho}_{g} E_{g}\right)_{i-1 / 2, j}^{n}+\left(A_{c x} \hat{\alpha} \hat{\rho}_{g} E_{g}\right)_{i+1 / 2, j}^{n}\right]\right\}(\delta P)_{i, j}^{m+1} \\
& +\left\{\frac{\left(\rho_{g}\right)_{i, j}^{m}}{\Delta t}-\left(\frac{\partial \Gamma_{I}}{\partial \alpha}\right)_{i, j}^{m}\right\}(\delta \alpha)_{i, j}^{m+1} \\
& +\left\{\frac{\alpha_{i, j}^{m}}{\Delta t}\left(\frac{\partial \rho_{g}}{\partial T_{g}}\right)_{i, j}^{m}-\left(\frac{\partial \Gamma_{I}}{\partial T_{g}}\right)_{i, j}^{m}\right\}\left(\delta T_{g}\right)_{i, j}^{m+1} \\
& +\left\{-\left(\frac{\partial \Gamma_{I}}{\partial T_{f}}\right)_{i, j}^{m}\right\}\left(\delta T_{f}\right)_{i, j}^{m+1} \\
& +\left\{\frac{\alpha_{i, j}^{m}}{\Delta t}\left(\frac{\partial \rho_{g}}{\partial X_{a}}\right)_{i, j}^{m}-\left(\frac{\partial \Gamma_{I}}{\partial X_{a}}\right)_{i, j}^{m}\right\}\left(\delta X_{a}\right)_{i, j}^{m+1} \\
& -\frac{1}{\left(V_{c}\right)_{i, j}}\left\{\left(A_{c z} \hat{\alpha} \hat{\rho}_{g} C_{g}\right)_{i, j-1 / 2}^{n}(\delta P)_{i, j-1}^{m+1}+\left(A_{c z} \hat{\alpha} \hat{\rho}_{g} C_{g}\right)_{i, j+1 / 2}^{n}(\delta P)_{i, j+1}^{m+1}\right. \\
& \left.+\left(A_{c x} \hat{\alpha} \hat{\rho}_{g} E_{g}\right)_{i-1 / 2, j}^{n}(\delta P)_{i-1, j}^{m+1}+\left(A_{c x} \hat{\alpha} \hat{\rho}_{g} E_{g}\right)_{i+1 / 2, j}^{n}(\delta P)_{i+1, j}^{m+1}\right\} \\
& =\frac{1}{\Delta t}\left[\left(\alpha \rho_{g}\right)_{i, j}^{n}-\left(\alpha \rho_{g}\right)_{i, j}^{m}\right]+\left(\Gamma_{I}\right)_{i, j}^{m}+\left(\Gamma_{w}\right)_{i, j}^{n}-\left(W_{t g}\right)_{i, j}^{n} \\
& +\frac{1}{\left(V_{c}\right)_{i, j}}\left\{\left(A_{c z} \hat{\alpha} \hat{\rho}_{g}\right)_{i, j-1 / 2}^{n}\left[\left(B_{g}\right)_{i, j-1 / 2}^{n}+\left(C_{g}\right)_{i, j-1 / 2}^{n}\left(P_{i, j-1}^{m}-P_{i, j}^{m}\right)\right]\right. \\
& -\left(A_{c z} \hat{\alpha} \hat{\rho}_{g}\right)_{i, j+1 / 2}^{n}\left[\left(B_{g}\right)_{i, j+1 / 2}^{n}+\left(C_{g}\right)_{i, j+1 / 2}^{n}\left(P_{i, j}^{m}-P_{i, j+1}^{m}\right)\right] \\
& +\left(A_{c x} \hat{\alpha} \hat{\rho}_{g}\right)_{i-1 / 2, j}^{n}\left[\left(D_{g}\right)_{i-1 / 2, j}^{n}+\left(E_{g}\right)_{i-1 / 2, j}^{n}\left(P_{i-1, j}^{m}-P_{i, j}^{m}\right)\right] \\
& \left.-\left(A_{c x} \hat{\alpha} \hat{\rho}_{g}\right)_{i+1 / 2, j}^{n}\left[\left(D_{g}\right)_{i+1 / 2, j}^{n}+\left(E_{g}\right)_{i+1 / 2, j}^{n}\left(P_{i, j}^{m}-P_{i+1, j}^{m}\right)\right]\right\}
\end{aligned}
$$


Liquid Mass ( $\left.k=f, \phi_{k}=1\right)$ : Equation (3.4.2-2) is basically identical to Eq. (3.4.2-1) with the gas void fraction replaced with the equivalent liquid void fraction $(1-\alpha)$, gas density and velocity replaced with liquid density and velocity, and the signs of the mass exchange terms reversed. The derivation of the difference equation for liquid mass conservation is therefore very similar to that for the gas mixture mass.

Liquid density is a function of pressure and liquid temperature. Therefore, the Taylor series expansion of the product of liquid void fraction and density is

$$
\begin{aligned}
\left((1-\alpha) \rho_{f}\right)_{i, j}^{n+1} & \equiv\left((1-\alpha) \rho_{f}\right)_{i, j}^{m}+\left[(1-\alpha)\left(\frac{\partial \rho_{f}}{\partial P}\right)\right]_{i, j}^{m}(\delta P)_{i, j}^{m+1} \\
& -\left(\rho_{f}\right)_{i, j}^{m}(\delta \alpha)_{i, j}^{m}+\left[(1-\alpha)\left(\frac{\partial \rho_{f}}{\partial T_{f}}\right)\right]_{i, j}^{m}\left(\delta T_{f}\right)_{i, j}^{m+1}
\end{aligned}
$$

Equations (3.4.2-22) and (3.4.2-23) remain unchanged.

Including these series expansions in Eq. (3.4.2-20), we derive a difference equation for liquid mass conservation in terms of the changes in the primary state variables as 


$$
\begin{aligned}
& \left\{\frac{1-\alpha_{i, j}^{m}}{\Delta t}\left(\frac{\partial \rho_{f}}{\partial P}\right)_{i, j}^{m}+\left(\frac{\partial \Gamma_{I}}{\partial P}\right)_{i, j}^{m}\right. \\
& +\frac{1}{\left(V_{c}\right)_{i, j}}\left[\left(A_{c z}(1-\hat{\alpha}) \hat{\rho}_{f} C_{f}\right)_{i, j-1 / 2}^{n}+\left(A_{c z}(1-\hat{\alpha}) \hat{\rho}_{f} C_{f}\right)_{i, j+1 / 2}^{n}\right. \\
& \left.\left.+\left(A_{c x}(1-\hat{\alpha}) \hat{\rho}_{f} E_{f}\right)_{i-1 / 2, j}^{n}+\left(A_{c x}(1-\hat{\alpha}) \hat{\rho}_{f} E_{f}\right)_{i+1 / 2, j}^{n}\right]\right\}(\delta P)_{i, j}^{m+1} \\
& +\left\{-\frac{\left(\rho_{\mathrm{f}}\right)_{i, j}^{m}}{\Delta t}+\left(\frac{\partial \Gamma_{I}}{\partial \alpha}\right)_{i, j}^{m}\right\}(\delta \alpha)_{i, j}^{m+1} \\
& +\left\{\left(\frac{\partial \Gamma_{I}}{\partial T_{g}}\right)_{i, j}^{m}\right\}\left(\delta T_{g}\right)_{i, j}^{m+1} \\
& +\left\{\frac{1-\alpha_{i, j}^{m}}{\Delta t}\left(\frac{\partial \rho_{f}}{\partial T_{f}}\right)_{i, j}^{m}+\left(\frac{\partial \Gamma_{I}}{\partial T_{f}}\right)_{i, j}^{m}\right\}\left(\delta T_{f}\right)_{i, j}^{m+1} \\
& +\left\{\left(\frac{\partial \Gamma_{I}}{\partial X_{a}}\right)_{i, j}^{m}\right\}\left(\delta X_{a}\right)_{i, j}^{m+1}
\end{aligned}
$$

$-\frac{1}{\left(V_{c}\right)_{i, j}}\left\{\left(A_{c z}(1-\hat{\alpha}) \hat{\rho}_{f} C_{f}\right)_{i, j-1 / 2}^{n}(\delta P)_{i, j-1}^{m+1}+\left(A_{c z}(1-\hat{\alpha}) \hat{\rho}_{f} C_{f}\right)_{i, j+1 / 2}^{n}(\delta P)_{i, j+1}^{m+1}\right.$$$
\left.+\left(A_{c x}(1-\hat{\alpha}) \hat{\rho}_{f} E_{f}\right)_{i-1 / 2, j}^{n}(\delta P)_{i-1, j}^{m+1}+\left(A_{c x}(1-\hat{\alpha}) \hat{\rho}_{f} E_{f}\right)_{i+1 / 2, j}^{n}(\delta P)_{i+1, j}^{m+1}\right\}
$$$$
=\frac{1}{\Delta t}\left[\left((1-\alpha) \rho_{f}\right)_{i, j}^{n}-\left((1-\alpha) \rho_{f}\right)_{i, j}^{m}\right]-\left(\Gamma_{I}\right)_{i, j}^{m}-\left(\Gamma_{w}\right)_{i, j}^{n}-\left(W_{t f}\right)_{i, j}^{n}
$$$$
+\frac{1}{\left(V_{c}\right)_{i, j}}\left\{\left(A_{c z}(1-\hat{\alpha})_{\rho_{f}}\right)_{i, j-1 / 2}^{n}\left[\left(B_{f}\right)_{i, j-1 / 2}^{n}+\left(C_{f}\right)_{i, j-1 / 2}^{n}\left(P_{i, j-1}^{m}-P_{i, j}^{m}\right)\right]\right.
$$$$
-\left(A_{c z}(1-\hat{\alpha}) \hat{\rho}_{f}\right)_{i, j+1 / 2}^{n}\left[\left(B_{f}\right)_{i, j+1 / 2}^{n}+\left(C_{f}\right)_{i, j+1 / 2}^{n}\left(P_{i, j}^{m}-P_{i, j+1}^{m}\right)\right]
$$$$
+\left(A_{c x}(1-\hat{\alpha}) \hat{\rho}_{f}\right)_{i-1 / 2, j}^{n}\left[\left(D_{f}\right)_{i-1 / 2, j}^{n}+\left(E_{f}\right)_{i-1 / 2, j}^{n}\left(P_{i-1, j}^{m}-P_{i, j}^{m}\right)\right]
$$$$
\left.-\left(A_{c x}(1-\hat{\alpha}) \hat{\rho}_{f}\right)_{i+1 / 2, j}^{n}\left[\left(D_{f}\right)_{i+1 / 2, j}^{n}+\left(E_{f}\right)_{i+1 / 2, j}^{n}\left(P_{i, j}^{m}-P_{i+1, j}^{m}\right)\right]\right\}
$$ 
Air Mass $\left(k=g, \phi_{k}=X_{a}\right)$ : Derivation of the basic difference equation is essentially identical to that used for the gas mixture mass. As note $J$ above, gas density is a function of pressure, gas temperature, and air mass fraction. A Taylor series expansion of the product term $\left(\alpha \rho_{\mathrm{g}} \mathrm{X}_{\mathrm{a}}\right)$ about the $\mathrm{m}^{\text {th }}$ estimate values then gives the expression

$$
\begin{aligned}
\left(\alpha \rho_{g} X_{a}\right)_{i, j}^{n+1} & \equiv\left(\alpha \rho_{g} X_{a}\right)_{i, j}^{m}+\left[\alpha X_{a}\left(\frac{\partial \rho_{g}}{\partial P}\right)\right]_{i, j}^{m}(\delta P)_{i, j}^{m+1}+\left(\rho_{g} X_{a}\right)_{i, j}^{m}(\delta \alpha)_{i, j}^{m+1} \\
& +\left[\alpha X_{a}\left(\frac{\partial \rho_{g}}{\partial T_{g}}\right)\right]_{i, j}^{m}\left(\delta T_{g}\right)_{i, j}^{m+1}+\left[\alpha X_{a}\left(\frac{\partial \rho_{g}}{\partial X_{a}}\right)+\alpha \rho_{g}\right]_{i, j}^{m}\left(\delta X_{a}\right)_{i, j}^{m+1}
\end{aligned}
$$

Combining the above relationships with Eq. $(3.4 .2-20)$ gives the final difference equation as 


$$
\begin{aligned}
& \left\{\frac{\left(\alpha X_{a}\right)_{i, j}^{m}}{\Delta t}\left(\frac{\partial \rho_{g}}{\partial P}\right)_{i, j}^{m}\right. \\
& +\frac{1}{\left(V_{c}\right)_{i, j}}\left[\left(A_{c z} \hat{\alpha} \hat{\rho}_{g} \hat{x}_{a} C_{g}\right)_{i, j-1 / 2}^{n}+\left(A_{c z} \hat{\alpha} \hat{\rho}_{g} \hat{X}_{a} C_{g}\right)_{i, j+1 / 2}^{n}\right. \\
& \left.\left.+\left(A_{c x} \hat{\alpha} \hat{\rho}_{g} \hat{X}_{a} E_{g}\right)_{i-1 / 2, j}^{n}+\left(A_{c x} \hat{\alpha} \hat{\rho}_{g} \hat{x}_{a} E_{g}\right)_{i+1 / 2, j}^{n}\right]\right\}(\delta P)_{i, j}^{m+1} \\
& +\left\{\frac{\left(\rho_{g} X_{a}\right)_{i, j}^{m}}{\Delta t}\right\}(\delta \alpha)_{i, j}^{m+1} \\
& +\left\{\frac{\left(\alpha X_{a}\right)_{i, j}^{m}}{\Delta t}\left(\frac{\partial \rho_{g}}{\partial T_{g}}\right)_{i, j}^{m}\right\}\left(\delta T_{g}\right)_{i, j}^{m+1} \\
& +\left\{\frac{\alpha_{i, j}^{m}}{\Delta t}\left(X_{a} \frac{\partial \rho_{g}}{\partial X_{a}}+\rho_{g}\right)_{i, j}^{m}\right\}\left(\delta X_{a}\right)_{i, j}^{m+1} \\
& -\frac{1}{\left(V_{c}\right)_{i, j}}\left\{\left(A_{c z} \hat{\alpha} \hat{\rho}_{g} \hat{x}_{a} C_{g}\right)_{i, j-1 / 2}^{n}(\delta P)_{i, j-1}^{m+1}+\left(A_{c z} \hat{\alpha} \hat{\rho}_{g} \hat{x}_{a} C_{g}\right)_{i, j+1 / 2}^{n}(\delta P)_{i, j+1}^{m+1}\right. \\
& \left.+\left(A_{c x} \hat{\alpha} \hat{\rho}_{g} \hat{X}_{a} E_{g}\right)_{i-1 / 2, j}^{n}(\delta P)_{i-1, j}^{m+1}+\left(A_{c x} \hat{\alpha} \hat{\rho}_{g} \hat{X}_{a} E_{g}\right)_{i+1 / 2, j}^{n}(\delta P)_{i+1, j}^{m+1}\right\} \\
& =\frac{1}{\Delta t}\left[\left(\alpha \rho_{g} X_{a}\right)_{i, j}^{n}-\left(\alpha \rho_{g} X_{a}\right)_{i, j}^{m}\right]-\left(X_{a} W_{t g}\right)_{i, j}^{n} \\
& +\frac{1}{\left(V_{c}\right)_{i, j}}\left\{\left(A_{c z} \hat{\alpha} \hat{\rho}_{g} \hat{X}_{a}\right)_{i, j-1 / 2}^{n}\left[\left(B_{g}\right)_{i, j-1 / 2}^{n}+\left(C_{g}\right)_{i, j-1 / 2}^{n}\left(P_{i, j-1}^{m}-P_{i, j}^{m}\right)\right]\right. \\
& -\left(A_{c z} \hat{\alpha} \hat{\rho}_{g} \hat{X}_{a}\right)_{i, j+1 / 2}^{n}\left[\left(B_{g}\right)_{i, j+1 / 2}^{n}+\left(C_{g}\right)_{i, j+1 / 2}^{n}\left(P_{i, j}^{m}-P_{i, j+1}^{m}\right)\right] \\
& +\left(A_{c x} \hat{\alpha}_{\rho_{g}} \hat{X}_{a}\right)_{i-1 / 2, j}^{n}\left[\left(D_{g}\right)_{i-1 / 2, j}^{n}+\left(E_{g}\right)_{i-1 / 2, j}^{n}\left(P_{i-1, j}^{m}-P_{i, j}^{m}\right)\right] \\
& \left.-\left(A_{c x} \hat{\alpha} \hat{\rho}_{g} \hat{x}_{a}\right)_{i+1 / 2, j}^{n}\left[\left(D_{g}\right)_{1+1 / 2, j}^{n}+\left(E_{g}\right)_{i+1 / 2, j}^{n}\left(P_{i, j}^{m}-P_{i+1, j}^{m}\right)\right]\right\}
\end{aligned}
$$


Gas Energy $\left(k=g, \phi_{k}=h_{g}\right)$ : The first term on the right hand side of Eq. $(3.4 .2-4)$ is treated in a semi-implicit manner. Averaging over the mass control volume we find

$$
\begin{aligned}
\frac{1}{V_{m / e}} \int_{V_{m / e}}\left(\alpha \frac{\partial P}{\partial t}\right) d V_{m / e} & \rightarrow \frac{1}{V_{m / e}} \int_{V_{m / e}} \alpha d V_{m / e} \cdot \frac{\partial}{\partial t}\left\{\frac{1}{V_{m / e}} \int_{V_{m / e}} P d V_{m / e}\right\} \\
& =\alpha_{i, j}^{n}\left(\frac{P_{i, j}^{n+1}-P_{i, j}^{n}}{\Delta t}\right)
\end{aligned}
$$

To derive Eq. (3.4.2-30), we first approximate the volume average of the product as the product of the volume averaged quantities. We then interchange the order of averaging and differentiation for the last term. To obtain the second equality, we take cell center void fractions and pressures to be volume average values and approximate the derivative using a forward in time finite difference.

The gas energy sources at the wall $\left(Q_{w g}\right)$ and at the gas-liquid interface $\left(E_{I}\right)$ contain implicit dependences on the primary state variables. These terms are approximated using the following Taylor series expansions

$$
\begin{aligned}
\left(Q_{w_{g}}\right)_{i, j}^{n+1} & \equiv\left(Q_{w_{g}}\right)_{i, j}^{m}+\left(\frac{\partial Q_{w_{g}}}{\partial P}\right)_{i, j}^{m}(\delta P)_{i, j}^{m+1}+\left(\frac{\partial Q_{w_{g}}}{\partial \alpha}\right)_{i, j}^{m}(\delta \alpha)_{i, j}^{m+1} \\
& +\left(\frac{\partial Q_{w_{g}}}{\partial T_{g}}\right)_{i, j}^{m}\left(\delta T_{g}\right)_{i, j}^{m+1}+\left(\frac{\partial Q_{w_{g}}}{\partial T_{f}}\right)_{i, j}^{m}\left(\delta T_{f}\right)_{i, j}^{m+1}+\left(\frac{\partial Q_{w g}}{\partial X_{a}}\right)_{i, j}^{m}\left(\delta X_{a}\right)_{i, j}^{m+1}
\end{aligned}
$$

and

$$
\begin{aligned}
\left(E_{I}\right)_{i, j}^{n+1} & \equiv\left(E_{I}\right)_{i, j}^{m}+\left(\frac{\partial E_{I}}{\partial P}\right)_{i, j}^{m}(\delta P)_{i, j}^{m+1}+\left(\frac{\partial E_{I}}{\partial \alpha}\right)_{i, j}^{m}(\delta \alpha)_{i, j}^{m+1} \\
& +\left(\frac{\partial E_{I}}{\partial T_{g}}\right)_{i, j}^{m}\left(\delta T_{g}\right)_{i, j}^{m+1}+\left(\frac{\partial E_{I}}{\partial T_{f}}\right)_{i, j}^{m}\left(\delta T_{f}\right)_{i, j}^{m+1}+\left(\frac{\partial E_{I}}{\partial X_{a}}\right)_{i, j}^{m}\left(\delta X_{a}\right)_{i, j}^{m+1}
\end{aligned}
$$

The gas enthalpy is a function of pressure, gas temperature, and air mass fraction. We can then develop a Taylor series expansion for the product of 'oid fraction, gas density, and gas enthalpy as 


$$
\begin{aligned}
\left(\alpha \rho_{g} h_{g}\right)_{i, j}^{n+1} \cong\left(\alpha \rho_{g} h_{g}\right)_{i, j}^{m}+ & {\left[\alpha h_{g}\left(\frac{\partial \rho_{g}}{\partial P}\right)+\alpha \rho_{g}\left(\frac{\partial h_{g}}{\partial P}\right)\right]_{i, j}^{m}(\delta P)_{i, j}^{m+1} } \\
+\left(\rho_{g} h_{g}\right)_{i, j}^{m}(\delta \alpha)_{i, j}^{m+1}+ & {\left[\alpha h_{g}\left(\frac{\partial \rho_{g}}{\partial T_{g}}\right)+\alpha \rho_{g}\left(\frac{\partial h_{g}}{\partial T_{g}}\right)\right]_{i, j}^{m}\left(\delta T_{g}\right)_{i, j}^{m+1} } \\
+ & {\left[\alpha h_{g}\left(\frac{\partial \rho_{g}}{\partial X_{g}}\right)+\alpha \rho_{g}\left(\frac{\partial h_{g}}{\partial X_{a}}\right)\right]_{i, j}^{m}\left(\delta X_{a}\right)_{i, j}^{m+1} }
\end{aligned}
$$

Combining all of the above relationships into Eq. (3.4.2-20) gives our complete difference equation for the gas energy as 


$$
\begin{aligned}
& \left\{\frac{\left(\alpha h_{g}\right)_{i, j}^{m}}{\Delta t}\left(\frac{\partial \rho_{g}}{\partial P}\right)_{i, j}^{m}+\frac{\left(\alpha \rho_{g}\right)_{i, j}^{m}}{\Delta t}\left(\frac{\partial h_{g}}{\partial P}\right)_{i, j}^{m}-\frac{\alpha_{i, j}^{n}}{\Delta t}-\left(\frac{\partial E_{I}}{\partial P}\right)_{i, j}^{m}-\left(\frac{\partial Q_{w g}}{\partial P}\right)_{i, j}^{m}\right. \\
& +\frac{1}{\left(V_{c}\right)_{i, j}}\left[\left(A_{c z} \hat{\alpha} \hat{\rho}_{g} \hat{h}_{g} C_{g}\right)_{i, j-1 / 2}^{n}+\left(A_{c z} \hat{\alpha} \hat{\rho}_{g} \hat{h}_{g} C_{g}\right)_{i, j+1 / 2}^{n}\right. \\
& \left.\left.+\left(A_{c x} \hat{\alpha} \hat{\rho}_{g} \hat{h}_{g} E_{g}\right)_{i-1 / 2, j}^{n}+\left(A_{c x} \hat{\alpha} \hat{\rho}_{g} \hat{h}_{g} E_{g}\right)_{i+1 / 2, j}^{n}\right]\right\}(\delta P)_{i, j}^{m+1} \\
& +\left\{\frac{\left(\rho_{g} h_{g}\right)_{i, j}^{m}}{\Delta t}-\left(\frac{\partial E_{I}}{\partial \alpha}\right)_{i, j}^{m}-\left(\frac{\partial Q_{w g}}{\partial \alpha}\right)_{i, j}^{m}\right\}(\delta \alpha)_{i, j}^{m+1} \\
& +\left\{\frac{\left(\alpha h_{g}\right)_{i, j}^{m}}{\Delta t}\left(\frac{\partial \rho_{g}}{\partial T_{g}}\right)_{i, j}^{m}+\frac{\left(\alpha \rho_{g}\right)_{i, j}^{m}}{\Delta t}\left(\frac{\partial h_{g}}{\partial T_{g}}\right)_{i, j}^{m}-\left(\frac{\partial E_{I}}{\partial T_{g}}\right)_{i, j}^{m}-\left(\frac{\partial Q_{w g}}{\partial T_{g}}\right)_{i, j}^{m}\right\}\left(\delta T_{g}\right)_{i, j}^{m+1} \\
& +\left\{-\left(\frac{\partial \mathrm{E}_{\mathrm{I}}}{\partial \mathrm{T}_{\mathrm{f}}}\right)_{i, j}^{\mathrm{m}}-\left(\frac{\partial \mathrm{Q}_{w_{\mathrm{g}}}}{\partial \mathrm{T}_{\mathrm{f}}}\right)_{i, j}^{\mathrm{m}}\right\}\left(\delta \mathrm{T}_{\mathrm{f}}\right)_{i, j}^{\mathrm{m}+1} \\
& +\left\{\frac{\left(\alpha h_{g}\right)_{i, j}^{m}}{\Delta t}\left(\frac{\partial \rho_{g}}{\partial X_{a}}\right)_{i, j}^{m}+\frac{\left(\alpha \rho_{g}\right)_{i, j}^{m}}{\Delta t}\left(\frac{\partial h_{g}}{\partial X_{a}}\right)_{i, j}^{m}-\left(\frac{\partial E_{I}}{\partial X_{a}}\right)_{i, j}^{m}-\left(\frac{\partial Q_{w_{g}}}{\partial X_{a}}\right)_{i, j}^{m}\right\}\left(\delta X_{a}\right)_{i, j}^{m+1} \\
& -\frac{1}{\left(V_{c}\right)_{i, j}}\left\{\left(A_{c z} \hat{\alpha} \hat{\rho}_{g} \hat{h}_{g} C_{g}\right)_{i, j-1 / 2}^{n}(\delta P)_{i, j-1}^{m+1}+\left(A_{c z} \hat{\alpha} \hat{\rho}_{g} \hat{h}_{g} C_{g}\right)_{i, j+1 / 2}^{n}(\delta P)_{i, j+1}^{m+1}\right. \\
& \left.+\left(A_{c x} \hat{\alpha} \hat{\rho}_{g} \hat{h}_{g} E_{g}\right)_{i-1 / 2, j}^{n}(\delta P)_{i-1, j}^{m+1}+\left(A_{c x} \hat{\alpha} \hat{\rho}_{g} \hat{h}_{g} E_{g}\right)_{i+1 / 2, j}^{n}(\delta P)_{i+1, j}^{m+1}\right\} \\
& =\frac{1}{\Delta t}\left[\left(\alpha \rho_{g} h_{g}\right)_{i, j}^{n}-\left(\alpha \rho_{g} h_{g}\right)_{i, j}^{m}\right]+\frac{\alpha_{i, j}^{n}}{\Delta t}\left[P_{i, j}^{m}-P_{i, j}^{n}\right]+\left(E_{I}\right)_{i, j}^{m}+\left(E_{w}\right)_{i, j}^{n}+\left(Q_{w g}\right)_{i, j}^{m}-\left(Q_{t g}\right)_{i, j}^{n} \\
& +\frac{1}{\left(V_{c}\right)_{i, j}}\left\{\left(A_{c z} \hat{\alpha} \hat{p}_{g} \hat{h}_{g}\right)_{i, j-1 / 2}^{n}\left[\left(B_{g}\right)_{i, j-1 / 2}^{n}+\left(C_{g}\right)_{i, j-1 / 2}^{n}\left(P_{i, j-1}^{m}-P_{i, j}^{m}\right)\right]\right. \\
& -\left(A_{c z} \hat{\alpha} \hat{\rho}_{g} \hat{h}_{g}\right)_{i, j+1 / 2}^{n}\left[\left(B_{g}\right)_{i, j+1 / 2}^{n}+\left(C_{g}\right)_{i, j+1 / 2}^{n}\left(P_{i, j}^{m}-P_{i, j+1}^{m}\right)\right] \\
& +\left(A_{c x} \hat{\alpha} \hat{\rho}_{g} \hat{h}_{g}\right)_{i-1 / 2, j}^{n}\left[\left(D_{g}\right)_{i-1 / 2, j}^{n}+\left(E_{g}\right)_{i-1 / 2, j}^{n}\left(P_{i-1, j}^{m}-P_{i, j}^{m}\right)\right] \\
& \left.-\left(A_{c x} \hat{\alpha} \hat{\rho}_{g} \hat{h}_{g}\right)_{i+1 / 2, j}^{n}\left[\left(D_{g}\right)_{i+1 / 2, j}^{n}+\left(E_{g}\right)_{i+1 / 2, j}^{n}\left(P_{i, j}^{m}-P_{i+1, j}^{m}\right)\right]\right\}
\end{aligned}
$$


Liquid Energy $\left(k=f, \phi_{k}=h_{f}\right)$ : The liquid energy equation is almost identical in form to the gas energy equation. We simply replace $\alpha$ with $(1-\alpha)$, change the subscripts from $g$ to $f$, and reverse the sign of the interfacial heat exchange. The Taylor series expansions shown in Eqs. (3.4.2-31) and (3.4.2-32) apply here without change. Taking liquid enthalpy to be a function of pressure and liquid temperature, the expansion for the product of liquid void fraction, density, and enthalpy is given by

$$
\begin{aligned}
&\left((1-\alpha) \rho_{\mathrm{f}} \mathrm{h}_{\mathrm{f}}\right)_{\mathrm{i}, \mathrm{j}}^{\mathrm{n}+1} \equiv\left((1-\alpha) \rho_{\mathrm{f}} \mathrm{h}_{\mathrm{f}}\right)_{\mathrm{i}, \mathrm{j}}^{\mathrm{m}}+\left[(1-\alpha) \mathrm{h}_{\mathrm{f}}\left(\frac{\partial \rho_{\mathrm{f}}}{\partial \mathrm{P}}\right)+(1-\alpha) \rho_{\mathrm{f}}\left(\frac{\partial \mathrm{h}_{\mathrm{f}}}{\partial \mathrm{P}}\right)\right]_{\mathrm{i}, \mathrm{j}}^{\mathrm{m}}(\delta \mathrm{P})_{\mathrm{i}, \mathrm{j}}^{\mathrm{m}+1} \\
&-\left(\rho_{\mathrm{f}} \mathrm{h}_{\mathrm{f}}\right)_{\mathrm{i}, \mathrm{j}}^{\mathrm{m}}(\delta \alpha)_{\mathrm{i}, \mathrm{j}}^{\mathrm{m}+1}+\left[(1-\alpha) \mathrm{h}_{\mathrm{f}}\left(\frac{\partial \rho_{\mathrm{f}}}{\partial \mathrm{T}_{\mathrm{f}}}\right)+(1-\alpha) \rho_{\mathrm{f}}\left(\frac{\partial \mathrm{h}_{\mathrm{f}}}{\partial \mathrm{T}_{\mathrm{f}}}\right)\right]_{\mathrm{i}, \mathrm{j}}^{\mathrm{m}}\left(\delta \mathrm{T}_{\mathrm{f}}\right)_{\mathrm{i}, \mathrm{j}}^{\mathrm{m}+1}
\end{aligned}
$$

Combining all of the appropriate relationships into Eq. (3.4.2-20) gives the complete difference equation for liquid energy as 


$$
\begin{aligned}
& \left\{\frac{\left[(1-\alpha) h_{f}\right]_{i, j}^{m}}{\Delta t}\left(\frac{\partial p_{f}}{\partial P}\right)_{i, j}^{m}+\frac{\left[(1-\alpha) \rho_{f}\right]_{i, j}^{m}}{\Delta t}\left(\frac{\partial h_{f}}{\partial P}\right)_{i, j}^{m}-\frac{1-\alpha_{i, j}^{n}}{\Delta t}+\left(\frac{\partial E_{I}}{\partial P}\right)_{i, j}^{m}-\left(\frac{\partial Q_{w f}}{\partial P}\right)_{i, j}^{m}\right. \\
& +\frac{1}{\left(V_{c}\right)_{i, j}}\left[\left(A_{c z}(1-\hat{\alpha}) \hat{\rho}_{f} \hat{h}_{f} C_{f}\right)_{i, j-1 / 2}^{n}+\left(A_{c z}(1-\hat{\alpha}) \hat{\rho}_{f} \hat{h}_{f} C_{f}\right)_{i, j+1 / 2}^{n}\right. \\
& \left.\left.+\left(A_{c x}(1-\hat{\alpha}) \hat{\rho}_{f} \hat{h}_{f} E_{f}\right)_{i-1 / 2, j}^{n}+\left(A_{c x}(1-\hat{\alpha}) \hat{\rho}_{f} \hat{h}_{f} E_{f}\right)_{i+1 / 2, j}^{n}\right]\right\}(\delta P)_{i, j}^{m+1} \\
& +\left\{-\frac{\left(\rho_{f} h_{f}\right)_{i, j}^{m}}{\Delta t}+\left(\frac{\partial E_{I}}{\partial \alpha}\right)_{i, j}^{m}-\left(\frac{\partial Q_{w f}}{\partial \alpha}\right)_{i, j}^{m}\right\}(\delta \alpha)_{i, j}^{m+1} \\
& +\left\{\left(\frac{\partial E_{I}}{\partial T_{g}}\right)_{i, j}^{m}-\left(\frac{\partial Q_{w f}}{\partial T_{g}}\right)_{i, j}^{m}\right\}\left(\delta T_{g}\right)_{i, j}^{m+1} \\
& +\left\{\frac{\left[(1-\alpha) h_{f}\right]_{i, j}^{m}}{\Delta t}\left(\frac{\partial \rho_{f}}{\partial T_{f}}\right)_{i, j}^{m}+\frac{\left[(1-\alpha) \rho_{f}\right]_{i, j}^{m}}{\Delta t}\left(\frac{\partial h_{f}}{\partial T_{f}}\right)_{i, j}^{m}+\left(\frac{\partial E_{I}}{\partial T_{f}}\right)_{i, j}^{m}-\left(\frac{\partial Q_{w f}}{\partial T_{f}}\right)_{i, j}^{m}\right\}\left(\delta T_{f}\right)_{i, j}^{m+1} \\
& +\left\{\left(\frac{\partial E_{I}}{\partial X_{a}}\right)_{i, j}^{m}-\left(\frac{\partial Q_{w f}}{\partial X_{a}}\right)_{i, j}^{m}\right\}\left(\delta X_{a}\right)_{i, j}^{m+1} \\
& -\frac{1}{\left(V_{c}\right)_{i, j}}\left\{\left(A_{c z}(1-\hat{\alpha}) \hat{\rho}_{f} \hat{h}_{f} C_{f}\right)_{i, j-1 / 2}^{n}(S P)_{i, j-1}^{m+1}+\left(A_{c z}(1-\hat{\alpha}) \hat{\rho}_{f} \hat{h}_{f} C_{f}\right)_{i, j+1 / 2}^{n}(\delta P)_{i, j+1}^{m+1}\right. \\
& \left.+\left(A_{c x}(1-\hat{\alpha}) \hat{\rho}_{f} \hat{h}_{f} E_{f}\right)_{i-1 / 2, j}^{n}(\delta P)_{i-1, j}^{m+1}+\left(A_{c x}(1-\hat{\alpha}) \hat{\rho}_{f} \hat{h}_{f} E_{f}\right)_{i+1 / 2, j}^{n}(\delta P)_{i+1, j}^{m+1}\right\} \\
& =\frac{1}{\Delta t}\left[\left((1-\alpha) \rho_{f} h_{f}\right)_{i, j}^{n}-\left((1-\alpha) \rho_{f} h_{f}\right)_{i, j}^{m}\right]+\frac{1-\alpha_{i, j}^{n}}{\Delta t}\left[P_{i, j}^{m}-P_{i, j}^{n}\right] \\
& -\left(E_{I}\right)_{i, j}^{m}-\left(E_{w}\right)_{i, j}^{n}+\left(Q_{w f}\right)_{i, j}^{m}-\left(Q_{t f}\right)_{i, j}^{n} \\
& +\frac{1}{\left(V_{c}\right)_{i, j}}\left\{\left(A_{c z}(1-\hat{\alpha}) \hat{p}_{f} \hat{h}_{f}\right)_{i, j-1 / 2}^{n}\left[\left(B_{f}\right)_{i, j-1 / 2}^{n}+\left(C_{f}\right)_{i, j-1 / 2}^{n}\left(P_{i, j-1}^{m}-P_{i, j}^{m}\right)\right]\right. \\
& -\left(A_{c z}(1-\hat{\alpha}) \hat{\rho}_{f} \hat{h}_{f}\right)_{i, j+1 / 2}^{n}\left[\left(B_{f}\right)_{i, j+1 / 2}^{n}+\left(C_{f}\right)_{i, j+1 / 2}^{n}\left(P_{i, j}^{m}-P_{i, j+1}^{m}\right)\right] \\
& +\left(A_{c x}(1-\hat{\alpha}) \hat{\rho}_{f} \hat{h}_{f}\right)_{i-1 / 2, j}^{n}\left[\left(D_{f}\right)_{i-1 / 2, j}^{n}+\left(E_{f}\right)_{i-1 / 2, j}^{n}\left(P_{i-1, j}^{m}-P_{i, j}^{m}\right)\right] \\
& \left.-\left(A_{c x}(1-\hat{\alpha}) \hat{\rho}_{f} \hat{h}_{f}\right)_{i+1 / 2, j}^{n}\left[\left(D_{f}\right)_{i+1 / 2, j}^{n}+\left(E_{f}\right)_{i+1 / 2, j}^{n}\left(P_{i, j}^{m}-P_{i+1, j}^{m}\right)\right]\right\}
\end{aligned}
$$


The set of five difference equations derived above are all of the same general form and can be combined into the equivalent matrix equation

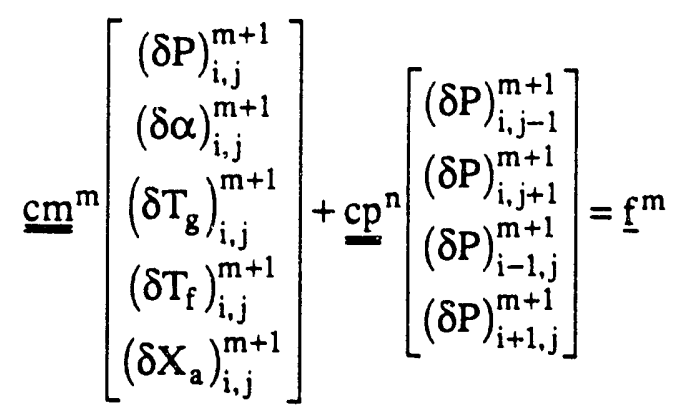

where $\mathrm{cm}^{\mathrm{m}}$ is a $5 \times 5$ matrix of coefficient values, $\mathrm{cp}^{\mathrm{n}}$ is a $5 \times 4$ matrix of pressure coupling coefficients, and $\underline{\mathrm{f}}^{\mathrm{m}}$ is the 5 element vector of right hand side terms. The solution of Eq. $(3.4 .2-37)$ is discussed in the solution methods section of the manual. 


\begin{tabular}{|lll|r|r}
\hline $2 / 15 / 93$ & WSRC-TR-92-532 Rev.0 & FLOWTRAN-TF Software Design & Page 382 \\
\hline
\end{tabular}

\subsubsection{Special Cases}

In this section, we consider special forms of the finite difference approximations used to solve the fluid goveming equations in one-dimensional sections of the assembly and at locations that interface between the sections. These equations will apply to the onedimensional mass cells indicated by the cross-hatching in Fig. 3.4.3-1 and to the momentum cells across their interior faces. One-dimensional flow channels occur in the top and bottom sections of the assembly and are indicated by the lighter cross-hatching in Fig. 3.4.3-1. The location of fluid cells at section interfaces also occur within the top and bottom sections and are indicated by the denser cross-hatching in Fig. 3.4.3-1.

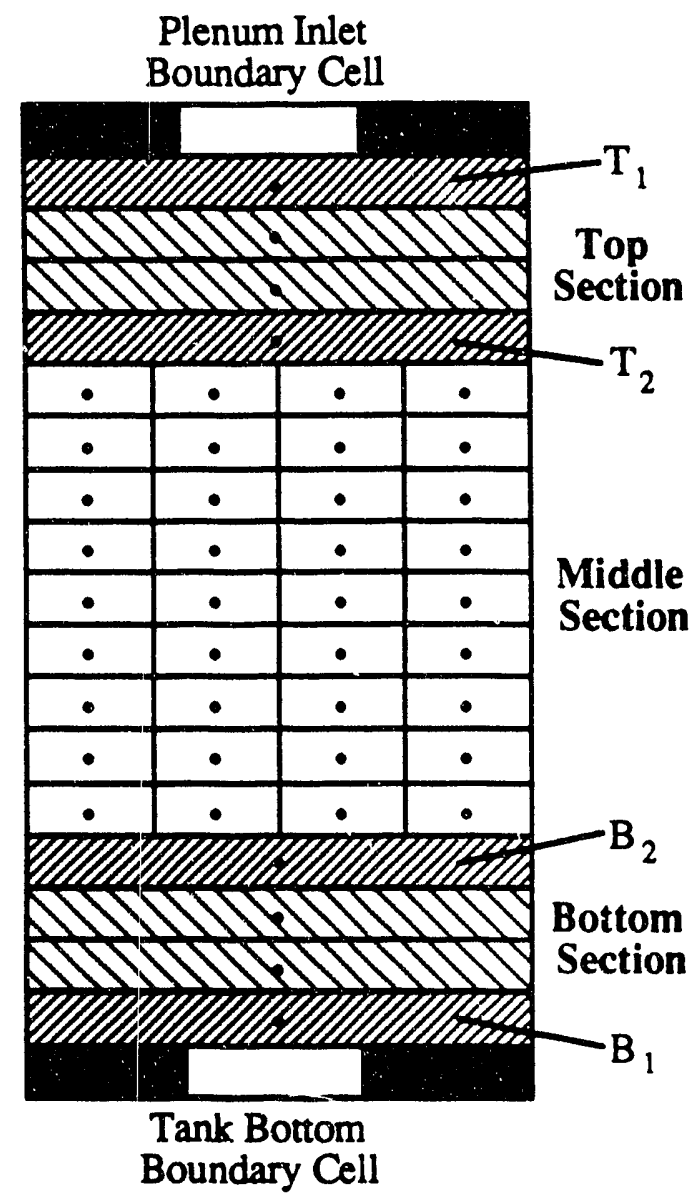

Figure 3.4.3-1 Location of special one-dimensional flow cells.

Some of the nomenclature used to describe the fluid variables for one-dimensional cells is illustrated in Figs. 3.4.3-2 and 3.4.3-3 below. Figure 3.4.3-2 shows a general onedimensional mass and energy fluid control volume and illustrates the staggered mesh used for the fluid computations where primary state variables are defined at the cell center and fluid velocities are defined at the centers of the cell faces. Figure 3.4.3-3 shows a onedimensional momentum control volume composed of half volumes from adjacent mass cells. 


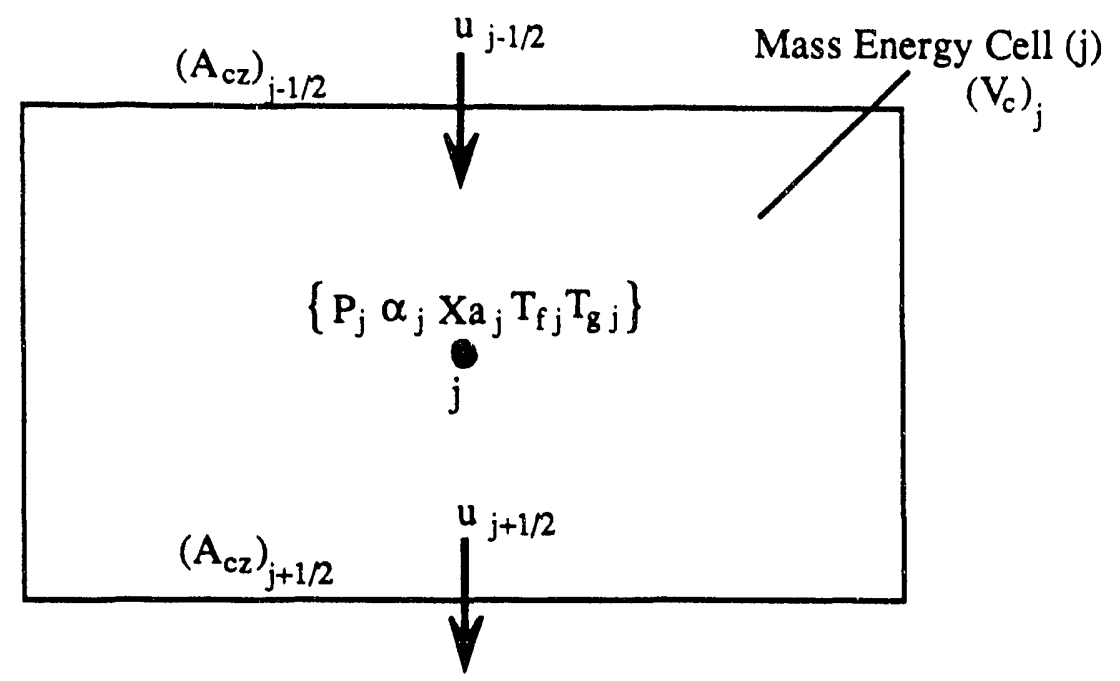

Figure 3.4.3-2 One-dimensional mass/energy control volume.

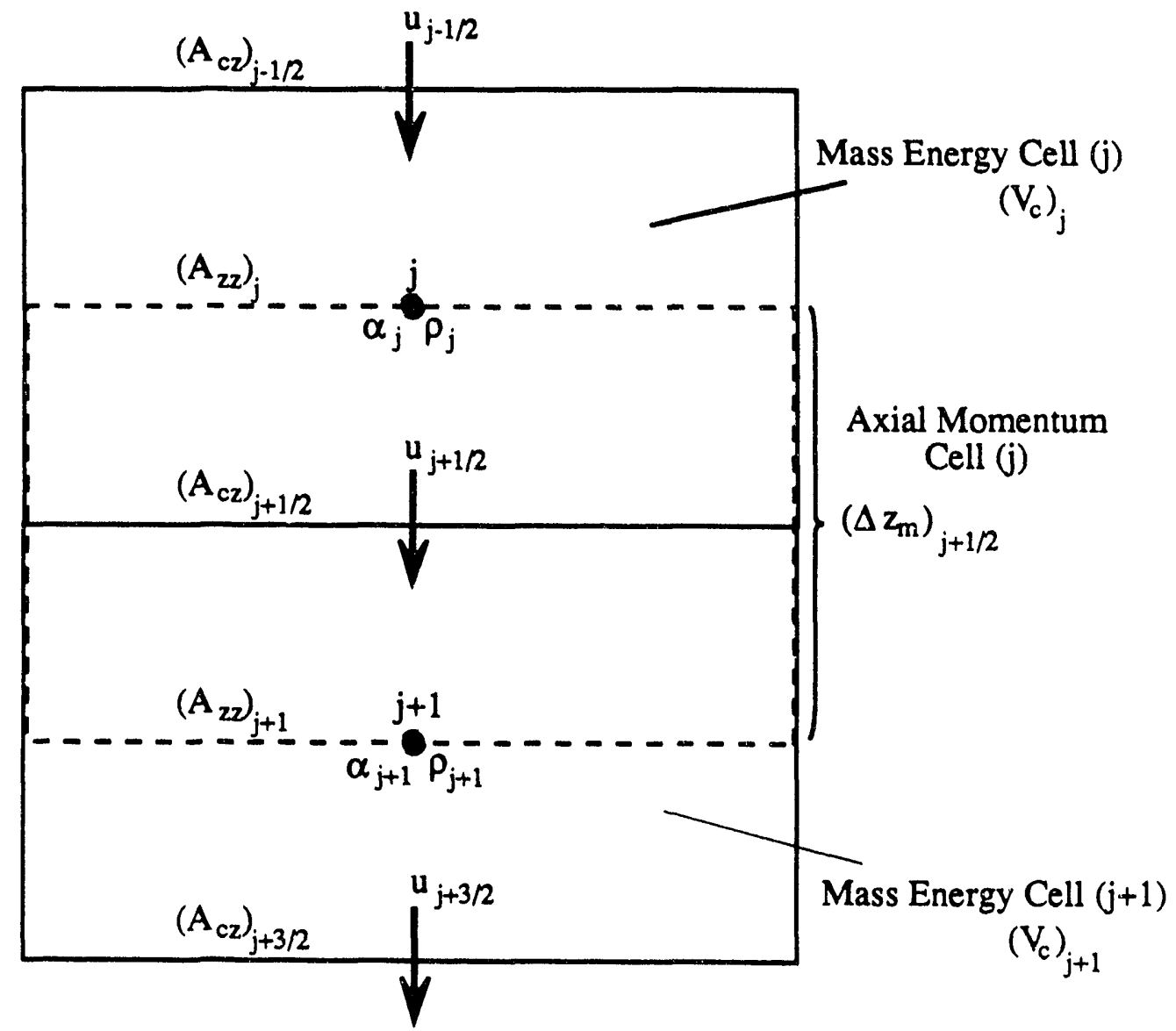

Figure 3.4.3-3 One-dimensional axial momentum control volume. 
As in the two-dimensional case, we use whole integers for the grid points at the centers of the mass/energy control volumes and half integer indices for the face center locations. For consistency, the index $\mathrm{j}$ is again used to indicate the axial direction.

One-Dimensional Momentum Equations: Development of the one-dimensional gas and liquid momentum equations is almost identical to the development of the twodimensional equations presented in $\$ 3.4 .1$. Therefore, we will not present detailed derivations but will briefly describe the significant differences.

The one-dimensional gas momentum equation (see Eq. (1) of TF-17) is given by

$$
\alpha \rho_{g} \frac{\partial u_{g}}{\partial t}+\nabla \cdot\left[\alpha \rho_{g} u_{g} u_{g} \bar{j}\right]-u_{g} \nabla \cdot\left[\alpha \rho_{g} u_{g} \bar{j}\right]=-\alpha \frac{\partial P}{\partial z}+\alpha \rho_{g} g^{z}-F_{w g}^{z}-F_{Y}^{z}
$$

where $\bar{j}$ is a unit vector in the positive axial direction. Equation (3.4.3-1) does not contain the source term for turbulent exchange which was included in the two-dimensional equation to model momentum transfer between adjacent flow channels arising from turbulent exchange across the rib gaps. As before, we integrate each term in Eq. (3.4.3-1) over the axial momentum control volume $\left(\mathrm{V}_{\mathrm{m}}\right)$ shown in Fig. 3.4.3-3. The wall frictional drag and interfacial drag terms are both evaluated as one-dimensional Taylor series expansions about the old time values (see TF-17). Substituting the volume averaged finite difference expressions into Eq. (3.4.3-1), collecting terms, and rearranging gives the one-dimensional version of the gas momentum equation implemented in FLOWTRAN-TF 


$$
\begin{aligned}
& {\left[\frac{R^{2}\left\langle\alpha \rho_{g}\right\rangle^{n}}{\Delta t}+\left(\frac{\partial F_{w g}^{z}}{\partial u_{g}}\right)^{n}+\left(\frac{\partial F_{I}^{2}}{\partial u_{g}}\right)^{n}\right]_{j+1 / 2}\left(u_{g}\right)_{j+1 / 2}^{n+1} } \\
&+\left[\left(\frac{\partial F_{w g}^{z}}{\partial u_{f}}\right)^{n}+\left(\frac{\partial F_{I}^{z}}{\partial u_{f}}\right)^{n}\right]_{j+1 / 2}\left(u_{f}\right)_{j+1 / 2}^{n+1} \\
&=\frac{\langle\alpha A\rangle_{j+1 / 2}^{n}}{\left(V_{z}\right)_{j+1 / 2}}\left(P_{j}^{n+1}-P_{j+1}^{n+1}\right) \\
&+\frac{1}{\left(V_{z}\right)_{j+1 / 2}}\left\{\left\langle A_{z z} \alpha \rho_{g} u_{g} u_{g}\right\rangle_{j}^{n}-\left\langle A_{z z} \alpha \rho_{g} u_{g}\right\rangle_{j}^{n}\left\langle u_{g}\right\rangle_{j+1 / 2}^{n}\right. \\
&+-\left\langle A_{z z}^{z} \alpha \rho_{g} u_{g} u_{g}\right\rangle_{j+1}^{n}+\left\langle A_{z z}\right\rangle_{j+1 / 2}^{n} \frac{\left.\left.\left\langle u_{g}\right\rangle_{j+1 / 2}^{n} u_{g}\right\rangle_{j+1}^{n}\left\langle u_{g}\right\rangle_{j+1 / 2}^{n}\right\}}{\Delta t}+\left\langle\rho_{g}\right\rangle_{j+1 / 2}^{n} g_{j+1 / 2}^{z} \frac{\langle\alpha A\rangle_{j+1 / 2}^{n}\left(\Delta z_{m}\right)_{j+1 / 2}}{\left(V_{z}\right)_{j+1 / 2}} \\
&-\left[\left(F_{w g}^{z}\right)^{n}-\left(\frac{\partial F_{w g}^{z}}{\partial u_{g}}\right)^{n} u_{g}^{n}-\left(\frac{\partial F_{w g}^{z}}{\partial u_{f}}\right)^{n} u_{f}^{n}\right]_{j+1 / 2} \\
&-\left[\left(F_{I}^{z}\right)^{n}-\left(\frac{\partial F_{I}^{z}}{\partial u_{g}}\right)^{n} u_{g}^{n}-\left(\frac{\partial F_{I}^{z}}{\partial u_{f}}\right)^{n} u_{f}^{n}\right]_{j+1 / 2}
\end{aligned}
$$

As in the two-dimensional case, Eq. (3.4.3-2) has the general functional form

$$
a_{11}^{z}\left(u_{g}\right)_{j+1 / 2}^{n+1}+a_{12}^{z}\left(u_{f}\right)_{j+1 / 2}^{n+1}=b_{1}+c_{1}\left(P_{j}^{n+1}-P_{j+1}^{n+1}\right)
$$

where $a_{11}^{z}, a_{12}^{z}, b_{1}$, and $c_{1}$ are constant numbers evaluated directly from old time values.

We apply the donoring scheme previously described to compute axial mass and momentum flows at the momentum cell faces. Definitions of the donored physical properties and mass flows are exactly analogous to the two-dimensional forms presented in \$3.4.1.

The one-dimensional liquid momentum equation is

$$
\begin{aligned}
(1-\alpha) \rho_{\mathrm{f}} \frac{\partial u_{f}}{\partial t}+\nabla \cdot\left[(1-\alpha) \rho_{f} u_{f} u_{f} \bar{j}\right]-u_{f} \nabla \cdot\left[(1-\alpha) \rho_{f} u_{f} \bar{j}\right]= \\
-(1-\alpha) \frac{\partial P}{\partial z}+(1-\alpha) \rho_{f} g^{z}-F_{w f}^{z}+F_{I}^{z}
\end{aligned}
$$


Comparison to the gas momentum equation in Eq. (3.4.3-1) shows that to convert the above analysis for the liquid momentum equation we need only reverse the sign of the interfacial drag terms, replace the gas void fraction $\alpha$ with the equivalent liquid void fraction $(1-\alpha)$, and substitute $\rho_{f}$ for $\rho_{g}$, and $u_{f}$ for $u_{g}$. The difference equation for liquid momentum is then immediately written as

$$
\begin{aligned}
& {\left[\left(\frac{\partial F_{w f}^{z}}{\partial u_{g}}\right)^{n}-\left(\frac{\partial F_{l}^{z}}{\partial u_{g}}\right)^{n}\right]_{j+1 / 2}\left(u_{g}\right)_{j+1 / 2}^{n+1}} \\
& +\left[\frac{R^{2}\left\langle(1-\alpha) \rho_{f}\right\rangle^{n}}{\Delta t}+\left(\frac{\partial F_{w f}^{z}}{\partial u_{f}}\right)^{n}-\left(\frac{\partial F_{l}^{z}}{\partial u_{f}}\right)^{n}\right]_{j+1 / 2}\left(u_{f}\right)_{j+1 / 2}^{n+1} \\
& =\frac{\langle(1-\alpha) A\rangle_{j+1 / 2}^{n}}{\left(V_{z}\right)_{j+1 / 2}}\left(P_{j}^{n+1}-P_{j+1}^{n+1}\right) \\
& +\frac{1}{\left(V_{z}\right)_{j+1 / 2}}\left\{\left\langle A_{z z}(1-\alpha) \rho_{f} u_{f} u_{f}\right\rangle_{j}^{n}-\left\langle A_{z z}(1-\alpha) \rho_{f} u_{f}\right\rangle_{j}^{n}\left\langle u_{f}\right\rangle_{j+1 / 2}^{n}\right. \\
& \left.-\left\langle A_{z z}(1-\alpha) \rho_{f} u_{f} u_{f}\right\rangle_{j+1}^{n}+\left\langle A_{z z}(1-\alpha) \rho_{f} u_{f}\right\rangle_{j+1}^{n}\left\langle u_{f}\right\rangle_{j+1 / 2}^{n}\right\} \\
& +R^{2}\left\langle(1-\alpha) \rho_{f}\right\rangle_{j+1 / 2}^{n} \frac{\left\langle u_{f}\right\rangle_{j+1 / 2}^{n}}{\Delta t} \\
& +\left\langle\rho_{f}\right\rangle_{j+1 / 2}^{n} g_{j+1 / 2}^{z} \frac{\langle(1-\alpha) A\rangle_{j+1 / 2}^{n}\left(\Delta z_{m}\right)_{j+1 / 2}}{\left(V_{z}\right)_{j+1 / 2}} \\
& -\left[\left(F_{w f}^{z}\right)^{n}-\left(\frac{\partial F_{w f}^{z}}{\partial u_{g}}\right)^{n} u_{g}^{n}-\left(\frac{\partial F_{w f}^{z}}{\partial u_{f}}\right)^{n} u_{f}^{n}\right]_{j+1 / 2} \\
& +\left[\left(F_{I}^{z}\right)^{n}-\left(\frac{\partial F_{I}^{z}}{\partial u_{g}}\right)^{n} u_{g}^{n}-\left(\frac{\partial F_{I}^{z}}{\partial u_{f}}\right)^{n} u_{f}^{n}\right]_{j+1 / 2}
\end{aligned}
$$

Equation (3.4.3-5) has the general functional form

$$
a_{21}^{z}\left(u_{g}\right)_{j+1 / 2}^{n+1}+a_{22}^{z}\left(u_{f}\right)_{j+1 / 2}^{n+1}=b_{2}+c_{2}\left(P_{j}^{n+1}-P_{j+1}^{n+1}\right)
$$

where $a_{21}^{z}, a_{22}^{z}, b_{2}$, and $c_{2}$ are constant numbers that are evaluated explicitly from old time values.

Equations (3.4.3-3) and (3.4.3-6) form a $2 \times 2$ system of equations which can be inverted to give a set of equations relating the gas and fluid axial velocities to the axial pressure 
difference at each mesh location. Combining these two equations yields the equivalent matrix equation

$$
\left[\begin{array}{ll}
a_{11}^{z} & a_{12}^{z} \\
a_{21}^{z} & a_{22}^{z}
\end{array}\right]\left[\begin{array}{l}
\left(u_{g}\right)_{j+1 / 2}^{n+1} \\
\left(u_{f}\right)_{j+1 / 2}^{n+1}
\end{array}\right]=\left[\begin{array}{l}
b_{1} \\
b_{2}
\end{array}\right]+\left[\begin{array}{l}
c_{1} \\
c_{2}
\end{array}\right]\left(P_{j}^{n+1}-P_{j+1}^{n+1}\right)
$$

One-Dimensional Mass/Energy Equations: In the top and bottom sections of an assembly, the conservation equations for mass and energy in the gas and liquid phases reduce to one-dimensional axial forms. The top and bottom sections of the assembly are also unheated so that heat transfer and phase changes at the solid surfaces do not apply in these sections. Otherwise, the differencing scheme is the same as was used for the twodimensional equations in the middle section. Explicit representations for the velocities are used and the momentum equations relating local velocity components to the local pressure differences are used to eliminate the velocity from the mass and energy finite difference equations. The final set of difference equations at each mesh point are combined and all unknown variables except for the pressure are eliminated. The difference equations from the top and bottom sections are solved simultaneously with the equations from the middle section to obtain the change in pressure throughout the entire assembly.

The one-dimensional differential conservation equations are all of the general form

$$
\frac{\partial\left(\alpha_{k} \rho_{k} \phi_{k}\right)}{\partial t}+\frac{1}{V} \nabla \cdot\left[V \alpha_{k} \rho_{k} \phi_{k} u_{k} j\right]=\sum_{i} S_{i}
$$

where the subscript $\mathrm{k}$ and the term $\alpha_{\mathrm{k}}$ are used as follows:

$$
\begin{aligned}
& \mathrm{k} \rightarrow \mathrm{g} \text { designates the gas phase and } \alpha_{\mathrm{g}}=\alpha \\
& \mathrm{k} \rightarrow \mathrm{f} \text { designates the liquid phase and } \alpha_{\mathrm{f}}=1-\alpha
\end{aligned}
$$

The velocity vector in Eq. (3.4.3-8) has been replaced with the axial velocity where $\bar{j}$ is a vector in the axial direction. The following table summarizes the definitions required to cast the one-dimensional conservation equations into the general form of Eq. (3.4.3-8). Note that turbulent exchange is not modelled in the one-dimensional sections of the assembly. 


\begin{tabular}{|c|c|c|c|}
\hline $\begin{array}{c}\text { Conservation } \\
\text { Equation }\end{array}$ & $\mathrm{k}$ & $\phi_{\mathrm{k}}$ & $\sum_{\mathrm{i}} \mathrm{S}_{\mathrm{i}}$ \\
\hline Gas Mixture Mass & $\mathrm{g}$ & 1 & $\Gamma_{\mathrm{I}}+\Gamma_{\mathrm{w}}$ \\
Liquid Mass & $\mathrm{f}$ & 1 & $-\Gamma_{\mathrm{I}}-\Gamma_{\mathrm{w}}$ \\
Air Mass & $\mathrm{g}$ & $\mathrm{X}_{\mathrm{a}}$ & 0 \\
Gas Energy & $\mathrm{g}$ & $\mathrm{h}_{\mathrm{g}}$ & $\alpha_{\mathrm{g}} \frac{\partial \mathrm{P}}{\partial \mathrm{t}}+\mathrm{E}_{\mathrm{I}}$ \\
& & $\mathrm{f}$ & $\alpha_{\mathrm{f}} \frac{\partial \mathrm{P}}{\partial \mathrm{t}}-\mathrm{E}_{\mathrm{I}}$ \\
\hline
\end{tabular}

Equation (3.4.3-8) is a general representation of the basic conservation equations that is used to develop a general difference equation by integrating each term over the mass/energy control volume $\left(\mathrm{V}_{\mathrm{m} / \mathrm{e}}\right)$ shown in Fig. 3.4.3-2. The integrations are nearly identical to those used to derive the two-dimensional equations and details of the methods will not be repeated. From the solution of the momentum equations, velocities are eliminated from the advection terms in the mass and energy conservation equations. Performing these steps yields

$$
\begin{aligned}
& \frac{\left(\alpha_{k} \rho_{k} \phi_{k}\right)_{j}^{n+1}}{\Delta t} \\
& +\frac{1}{\left(V_{c}\right)_{j}}\left\{\left(A_{c z} \hat{\alpha}_{k} \hat{\rho}_{k} \hat{\phi}_{k} C_{k}\right)_{j-1 / 2}^{n}+\left(A_{c z} \hat{\alpha}_{k} \hat{\rho}_{k} \hat{\phi}_{k} C_{k}\right)_{j+1 / 2}^{n}\right\}(\delta P)_{j}^{m+1} \\
& -\frac{1}{\left(V_{c}\right)_{j}}\left\{\left(A_{c z} \hat{\alpha}_{k} \hat{\rho}_{k} \hat{\phi}_{k} C_{k}\right)_{j-1 / 2}^{n}\right\}(\delta P)_{j-1}^{m+1} \\
& =\frac{1}{\left(V_{c}\right)_{j}}\left\{\left(A_{c z} \hat{\alpha}_{k} \hat{\rho}_{k} \hat{\phi}_{k}\right)_{j-1 / 2}^{n}\left[\left(B_{k}\right)_{j-1 / 2}^{n}+\left(C_{k}\right)_{j-1 / 2}^{n}\left(P_{j-1}^{m}-P_{j}^{m}\right)\right]\right. \\
& \left.\quad-\left(A_{c z} \hat{\alpha}_{k} \hat{\rho}_{k} \hat{\phi}_{k}\right)_{j+1 / 2}^{n}\left[\left(B_{k}\right)_{j+1 / 2}^{n}+\left(C_{k}\right)_{j+1 / 2}^{n}\left(P_{j}^{m}-P_{j+1}^{m}\right)\right]\right\} \\
& +\frac{\left.\left.\left(\alpha_{k} \rho_{k} \phi_{k}\right)_{j}^{n} \hat{\rho}_{k} \hat{\phi}_{k} C_{k}\right)_{j+1 / 2}^{n}\right\}(\delta P)_{j+1}^{m+1}}{\Delta t}+\sum_{i}\left\langle S_{i}\right\rangle_{j}^{n+1}
\end{aligned}
$$


To obtain Eq. (3.4.3-9), source terms on the right hand side of Eq. (3.4.3-8) are replaced with appropriate volume averaged quantities. Equation (3.4.3-9) serves as a general difference equation which is used to develop individual difference equations for each of the five conservation equations. To accomplish this, we expand each of the functions evaluated at the new time step (denoted by the $n+1$ superscript) in terms of the values computed within the iteration scheme. These derivations are essentially identical to those given for the two-dimensional case and will not be repeated. Instead we simply present the one-dimensional forms for the five mass and energy conservation equations.

Gas Mixture Mass $\left(k=g, \phi_{k}=1\right)$;

$$
\begin{aligned}
& \left\{\frac{\alpha_{j}^{m}}{\Delta t}\left(\frac{\partial \rho_{g}}{\partial P}\right)_{j}^{m}-\left(\frac{\partial \Gamma_{I}}{\partial P}\right)_{j}^{m}\right. \\
& \left.+\frac{1}{\left(V_{c}\right)_{j}}\left[\left(A_{c z} \hat{\alpha} \hat{\rho}_{g} C_{g}\right)_{j-1 / 2}^{n}+\left(A_{c z} \hat{\alpha} \hat{\rho}_{g} C_{g}\right)_{j+1 / 2}^{n}\right]\right\}(\delta P)_{j}^{m+1} \\
& +\left\{\frac{\left(\rho_{g}\right)_{j}^{m}}{\Delta t}-\left(\frac{\partial \Gamma_{I}}{\partial \alpha}\right)_{j}^{m}\right\}(\delta \alpha)_{j}^{m+1} \\
& +\left\{\frac{\alpha_{j}^{m}}{\Delta t}\left(\frac{\partial \rho_{g}}{\partial T_{g}}\right)_{j}^{m}-\left(\frac{\partial \Gamma_{I}}{\partial T_{g}}\right)_{j}^{m}\right\}\left(\delta T_{g}\right)_{j}^{m+1} \\
& +\left\{-\left(\frac{\partial \Gamma_{\mathrm{I}}}{\partial \mathrm{T}_{\mathrm{f}}}\right)_{\mathrm{j}}^{\mathrm{m}}\right\}\left(\delta \mathrm{T}_{\mathrm{f}}\right)_{\mathrm{j}}^{\mathrm{m}+1} \\
& +\left\{\frac{\alpha_{j}^{m}}{\Delta t}\left(\frac{\partial \rho_{g}}{\partial X_{a}}\right)_{j}^{m}-\left(\frac{\partial \Gamma_{I}}{\partial X_{a}}\right)_{j}^{m}\right\}\left(\delta X_{a}\right)_{j}^{m+1} \\
& -\frac{1}{\left(V_{c}\right)_{j}}\left\{\left(A_{c z} \hat{\alpha} \hat{\rho}_{g} C_{g}\right)_{j-1 / 2}^{n}(\delta P)_{j-1}^{m+1}+\left(A_{c z} \hat{\alpha} \hat{\rho}_{g} C_{g}\right)_{j+1 / 2}^{n}(\delta P)_{j+1}^{m+1}\right\} \\
& =\frac{1}{\Delta t}\left[\left(\alpha \rho_{g}\right)_{j}^{n}-\left(\alpha \rho_{g}\right)_{j}^{m}\right]+\left(\Gamma_{I}\right)_{j}^{m} \\
& +\frac{1}{\left(V_{c}\right)_{j}}\left\{\left(A_{c z} \hat{\alpha} \hat{\rho}_{g}\right)_{j-1 / 2}^{n}\left[\left(B_{g}\right)_{j-1 / 2}^{n}+\left(C_{g}\right)_{j-1 / 2}^{n}\left(P_{j-1}^{m}-P_{j}^{m}\right)\right]\right. \\
& \left.-\left(A_{c z} \hat{\alpha} \hat{\rho}_{g}\right)_{j+1 / 2}^{n}\left[\left(B_{g}\right)_{j+1 / 2}^{n}+\left(C_{g}\right)_{j+1 / 2}^{n}\left(P_{j}^{m}-P_{j+1}^{m}\right)\right]\right\}
\end{aligned}
$$


Liquid Mass $\left(k=f, \phi_{k}=1\right)$ :

$$
\begin{aligned}
& \left\{\frac{1-\alpha_{j}^{m}}{\Delta t}\left(\frac{\partial \rho_{f}}{\partial P}\right)_{j}^{m}+\left(\frac{\partial \Gamma_{I}}{\partial P}\right)_{j}^{m}\right. \\
& \left.+\frac{1}{\left(V_{c}\right)_{j}}\left[\left(A_{c z}(1-\hat{\alpha}) \hat{\rho}_{f} C_{f}\right)_{j-1 / 2}^{n}+\left(A_{c z}(1-\hat{\alpha})_{\rho_{f}} C_{f}\right)_{j+1 / 2}^{n}\right]\right\}(\delta P)_{j}^{m+1} \\
& +\left\{-\frac{\left(\rho_{f}\right)_{i, j}^{m}}{\Delta t}+\left(\frac{\partial \Gamma_{I}}{\partial \alpha}\right)_{j}^{m}\right\}(\delta \alpha)_{j}^{m+1} \\
& +\left\{\left(\frac{\partial \Gamma_{I}}{\partial T_{g}}\right)_{j}^{m}\right\}\left(\delta T_{g}\right)_{j}^{m+1} \\
& +\left\{\frac{1-\alpha_{j}^{m}}{\Delta t}\left(\frac{\partial \rho_{f}}{\partial T_{f}}\right)_{j}^{m}+\left(\frac{\partial \Gamma_{I}}{\partial T_{f}}\right)_{j}^{m}\right\}\left(\delta T_{f}\right)_{j}^{m+1} \\
& +\frac{1}{\left(V_{c}\right)_{j}}\left\{\left(A_{c z}(1-\hat{\alpha}) \hat{\rho}_{f}\right)_{j-1 / 2}^{n}\left[\left(B_{f}\right)_{j-1 / 2}^{n}+\left(C_{f}\right)_{j-1 / 2}^{n}\left(P_{j-1}^{m}-P_{j}^{m}\right)\right]\right. \\
& -\frac{1}{\left(V_{c}\right)_{j}}\left\{\left(A_{c z}(1-\hat{\alpha}) \hat{\rho}_{f} C_{f}\right)_{j-1 / 2}^{n}(\delta P)_{j-1}^{m+1}+\left(A_{c z}(1-\hat{\alpha}) \hat{\rho}_{f} C_{f}\right)_{j+1 / 2}^{n}(\delta P)_{j+1}^{m+1}\right\} \\
& \left.=\frac{1}{\Delta t}\left[\left((1-\alpha) \rho_{f}\right)_{j}^{n}-\left((1-\alpha)_{f}\right)_{j}^{m}\right)_{j+1 / 2}^{n}\left[\left(B_{f}\right)_{j+1 / 2}^{n}+\left(C_{f}\right)_{j+1 / 2}^{n}\left(P_{j}^{m}-P_{j+1}^{m}\right)\right]\right\}
\end{aligned}
$$


$\operatorname{Air}$ Mass $\left(k=g, \phi_{k}=X_{2}\right)$;

$$
\begin{aligned}
& \left\{\frac{\left(\alpha X_{a}\right)_{j}^{m}}{\Delta t}\left(\frac{\partial p_{g}}{\partial P}\right)_{j}^{m}\right. \\
& \left.+\frac{1}{\left(V_{c}\right)_{j}}\left[\left(A_{c z} \hat{\alpha} \hat{\rho}_{g} \hat{X}_{a} C_{g}\right)_{j-1 / 2}^{n}+\left(A_{c z} \hat{\alpha} \hat{\rho}_{g} \hat{X}_{a} C_{B}\right)_{j+1 / 2}^{n}\right]\right\}(\delta P)_{j}^{m+1} \\
& +\left\{\frac{\left(\rho_{g} X_{a}\right)_{j}^{m}}{\Delta t}\right\}(\delta \alpha)_{j}^{m+1} \\
& +\left\{\frac{\left(\alpha X_{a}\right)_{j}^{m}}{\Delta t}\left(\frac{\partial \rho_{g}}{\partial T_{g}}\right)_{j}^{m}\right\}\left(\delta T_{g}\right)_{j}^{m+1} \\
& +\left\{\frac{\alpha_{j}^{m}}{\Delta t}\left(X_{a} \frac{\partial \rho_{g}}{\partial X_{a}}+\rho_{g}\right)_{j}^{m}\right\}\left(\delta X_{a}\right)_{j}^{m+1} \\
& -\frac{1}{\left(V_{c}\right)_{j}}\left\{\left(A_{c z} \hat{\alpha} \hat{\rho}_{g} \hat{X}_{\mathbf{z}} C_{g}\right)_{j-1 / 2}^{n}(\delta P)_{j-1}^{m+1}+\left(A_{c z} \hat{\alpha} \hat{\rho}_{g} \hat{X}_{z} C_{g}\right)_{j+1 / 2}^{n}(\delta P)_{j+1}^{m+1}\right\} \\
& =\frac{1}{\Delta t}\left[\left(\alpha \rho_{\mathrm{g}} \mathbf{X}_{\mathrm{a}}\right)_{\mathrm{j}}^{\mathrm{n}}-\left(\alpha \rho_{\mathrm{g}} \mathrm{X}_{\mathrm{a}}\right)_{\mathrm{j}}^{\mathrm{m}}\right] \\
& +\frac{1}{\left(V_{c}\right)_{j}}\left\{\left(A_{c z} \hat{\alpha} \hat{\rho}_{g} \hat{X}_{a}\right)_{j-1 / 2}^{n}\left[\left(B_{g}\right)_{j-1 / 2}^{n}+\left(C_{g}\right)_{j-1 / 2}^{n}\left(P_{j-1}^{m}-P_{j}^{m}\right)\right]\right. \\
& \left.-\left(A_{c z} \hat{\alpha} \hat{\rho}_{g} \hat{\mathbf{X}}_{\mathbf{a}}\right)_{j+1 / 2}^{\mathrm{n}}\left[\left(B_{g}\right)_{j+1 / 2}^{n}+\left(C_{g}\right)_{j+1 / 2}^{n}\left(P_{j}^{m}-P_{j+1}^{m}\right)\right]\right\}
\end{aligned}
$$




\section{Gas Energy $\left(k=g, \quad \phi_{k}=h_{g}\right.$ :}

$$
\begin{aligned}
& \left\{\frac{\left(\alpha h_{g}\right)_{j}^{m}}{\Delta t}\left(\frac{\partial \rho_{g}}{\partial P}\right)_{j}^{m}+\frac{\left(\alpha \rho_{g}\right)_{j}^{m}}{\Delta t}\left(\frac{\partial h_{g}}{\partial P}\right)_{j}^{m}-\frac{\alpha_{j}^{n}}{\Delta t}-\left(\frac{\partial E_{I}}{\partial P}\right)_{j}^{m}\right. \\
& \left.+\frac{1}{\left(V_{c}\right)_{j}}\left[\left(A_{c z} \hat{\alpha} \hat{\rho}_{g} \hat{h}_{g} C_{g}\right)_{j-1 / 2}^{n}+\left(A_{c z} \hat{\alpha} \hat{\rho}_{g} \hat{h}_{g} C_{g}\right)_{j+1 / 2}^{n}\right]\right\}(\delta P)_{j}^{m+1} \\
& +\left\{\frac{\left(\rho_{g} h_{g}\right)_{j}^{m}}{\Delta t}-\left(\frac{\partial E_{I}}{\partial \alpha}\right)_{j}^{m}\right\}(\delta \alpha)_{j}^{m+1} \\
& +\left\{\frac{(\alpha h \cdot)_{j}^{m}}{\Delta t}\left(\frac{\partial \rho_{g}}{\partial T_{g}}\right)_{j}^{m}+\frac{\left(\alpha \rho_{g}\right)_{j}^{m}}{\Delta t}\left(\frac{\partial h_{g}}{\partial T_{g}}\right)_{j}^{m}-\left(\frac{\partial E_{I}}{\partial T_{g}}\right)_{j}^{m}\right\}\left(\delta T_{g}\right)_{j}^{m+1} \\
& +\left\{-\left(\frac{\partial \mathrm{E}_{\mathrm{I}}}{\partial \mathrm{T}_{\mathrm{f}}}\right)_{\mathrm{j}}^{\mathrm{m}}\right\}\left(\delta \mathrm{T}_{\mathrm{f}}\right)_{\mathrm{j}}^{\mathrm{m}+1} \\
& +\left\{\frac{\left(\alpha h_{g}\right)_{j}^{m}}{\Delta t}\left(\frac{\partial \rho_{g}}{\partial X_{a}}\right)_{j}^{m}+\frac{\left(\alpha \rho_{g}\right)_{j}^{m}}{\Delta t}\left(\frac{\partial h_{g}}{\partial X_{a}}\right)_{j}^{m}-\left(\frac{\partial E_{I}}{\partial X_{a}}\right)_{j}^{m}\right\}\left(\delta X_{a}\right)_{j}^{m+1} \\
& -\frac{1}{\left(V_{c}\right)_{j}}\left\{\left(A_{c z} \hat{\alpha} \hat{\rho}_{g} \hat{h}_{g} C_{g}\right)_{j-1 / 2}^{n}(\delta P)_{j-1}^{m+1}+\left(A_{c z} \hat{\alpha}_{\rho_{g}} \hat{h}_{g} C_{g}\right)_{j+1 / 2}^{n}(\delta P)_{j+1}^{m+1}\right\} \\
& =\frac{1}{\Delta t}\left[\left(\alpha \rho_{g} h_{g}\right)_{j}^{n}-\left(\alpha \rho_{g} h_{g}\right)_{j}^{m}\right]+\frac{\alpha_{j}^{n}}{\Delta t}\left[P_{j}^{m}-P_{j}^{n}\right]+\left(E_{I}\right)_{j}^{m} \\
& +\frac{1}{\left(V_{c}\right)_{j}}\left\{\left(A_{c z} \hat{\alpha} \hat{\rho}_{g} \hat{h}_{g}\right)_{j-1 / 2}^{n}\left[\left(B_{g}\right)_{j-1 / 2}^{n}+\left(C_{g}\right)_{j-1 / 2}^{n}\left(P_{j-1}^{m}-P_{j}^{m}\right)\right]\right. \\
& \left.-\left(A_{c z} \hat{\alpha} \hat{\rho}_{g} \hat{h}_{g}\right)_{j+1 / 2}^{n}\left[\left(B_{g}\right)_{j+1 / 2}^{n}+\left(C_{g}\right)_{j+1 / 2}^{n}\left(P_{j}^{m}-P_{j+1}^{m}\right)\right]\right\}
\end{aligned}
$$


Liquid Energy $\left(k=f . \phi_{k}=L_{f}\right)$ :

$$
\begin{aligned}
& \left\{\frac{\left[(1-\alpha) h_{f}\right]_{j}^{m}}{\Delta t}\left(\frac{\partial \rho_{f}}{\partial P}\right)_{j}^{m}+\frac{\left[(1-\alpha) \rho_{f}\right]_{j}^{m}}{\Delta t}\left(\frac{\partial h_{f}}{\partial P}\right)_{j}^{m}-\frac{1-\alpha_{j}^{n}}{\Delta t}+\left(\frac{\partial E_{I}}{\partial P}\right)_{j}^{m}\right. \\
& \left.+\frac{1}{\left(V_{c}\right)_{j}}\left[\left(A_{c z}(1-\hat{\alpha}) \hat{\rho}_{f} \hat{h}_{f} C_{f}\right)_{j-1 / 2}^{n}+\left(A_{c z}(1-\hat{\alpha}) \hat{\rho}_{f} \hat{h}_{f} C_{f}\right)_{j+1 / 2}^{n}\right]\right\}(\delta P)_{j}^{m+1} \\
& +\left\{-\frac{\left(\rho_{\mathrm{f}} \mathrm{h}_{\mathrm{f}}\right)_{j}^{\mathrm{m}}}{\Delta \mathrm{t}}+\left(\frac{\partial \mathrm{E}_{\mathrm{I}}}{\partial \alpha}\right)_{\mathrm{j}}^{\mathrm{m}}\right\}(\delta \alpha)_{j}^{\mathrm{m}+1} \\
& +\left\{\left(\frac{\partial \mathrm{E}_{\mathrm{I}}}{\partial \mathrm{T}_{8}}\right)_{j}^{\mathrm{m}}\right\}\left(\delta \mathrm{T}_{8}\right)_{\mathrm{j}}^{\mathrm{m}+1} \\
& +\left\{\frac{\left[(1-\alpha) h_{f}\right]_{j}^{m}}{\Delta t}\left(\frac{\partial \rho_{f}}{\partial T_{f}}\right)_{j}^{m}+\frac{\left[(1-\alpha) \rho_{f}\right]_{j}^{m}}{\Delta t}\left(\frac{\partial h_{f}}{\partial T_{f}}\right)_{j}^{m}+\left(\frac{\partial E_{I}}{\partial T_{f}}\right)_{j}^{m}\right\}\left(\delta T_{f}\right)_{j}^{m+1} \\
& +\left\{\left(\frac{\partial E_{I}}{\partial X_{a}}\right)_{j}^{m}\right\}\left(\delta X_{a}\right)_{j}^{m+1} \\
& -\frac{1}{\left(V_{c}\right)_{j}}\left\{\left(A_{c z}(1-\hat{\alpha}) \hat{\rho}_{f} \hat{h}_{f} C_{f}\right)_{j-1 / 2}^{n}(\delta P)_{j-1}^{m+1}+\left(A_{c z}(1-\hat{\alpha}) \hat{\rho}_{f} \hat{h}_{f} \mathcal{L}_{f}\right)_{j+1 / 2}^{n}\right\}(\delta P)_{j+1}^{m+1} \\
& =\frac{1}{\Delta t}\left[\left((1-\alpha) \rho_{f} h_{f}\right)_{j}^{n}-\left((1-\alpha) \rho_{f} h_{f}\right)_{j}^{m}\right]+\frac{1-\alpha_{j}^{n}}{\Delta t}\left[P_{j}^{m}-P_{j}^{n}\right]-\left(E_{I}\right)_{j}^{m} \\
& +\frac{1}{\left(V_{c}\right)_{j}}\left\{\left(A_{c z}(1-\hat{\alpha}) \hat{p}_{f} \hat{h}_{f}\right)_{j-1 / 2}^{n}\left[\left(B_{f}\right)_{j-1 / 2}^{n}+\left(C_{f}\right)_{j-1 / 2}^{n}\left(P_{j-1}^{m}-P_{j}^{m}\right)\right]\right. \\
& \left.-\left(A_{c z}(1-\hat{\alpha}) \hat{p}_{f} \hat{h}_{f}\right)_{j+1 / 2}^{n}\left[\left(B_{f}\right)_{j+1 / 2}^{n}+\left(C_{f}\right)_{j+1 / 2}^{n}\left(P_{j}^{m}-P_{j+1}^{m}\right)\right]\right\}
\end{aligned}
$$

The set of five difference equations derived above are all of the same general form and can be combined into the equivalent matrix equation 


$$
\underline{c m}^{m}\left[\begin{array}{c}
(\delta P)_{j}^{m+1} \\
(\delta \alpha)_{j}^{m+1} \\
\left(\delta T_{g}\right)_{j}^{m+1} \\
\left(\delta T_{f}\right)_{j}^{m+1} \\
\left(\delta X_{a}\right)_{j}^{m+1}
\end{array}\right]+\underline{p p}^{n}\left[\begin{array}{c}
(\delta P)_{j-1}^{m+1} \\
(\delta P)_{j+1}^{m+1}
\end{array}\right]=\underline{f}^{m}
$$

where $\mathrm{cm}^{\mathrm{m}}$ is a $5 \times 5$ matrix of coefficient values, $\mathrm{cp}^{\mathrm{n}}$ is a $5 \times 2$ matrix of pressure coupling coefficients, and $\underline{\mathrm{f}}^{\mathrm{m}}$ is the 5 element vector of right hand side terms. The solution of Eq. (3.4.3-15) is discussed in the solution methods section of the manual. The formulation of the mass and energy finite difference equations and the inversion of the $\mathrm{cm}^{\mathrm{m}}$ matrix is programmed in subroutine JACOBT for the top section of the assembly and in subroutine JACOBB for the bottom section.

Mesh Cells at Interfaces Between Sections: We will next treat the special cases of fluid mesh cells at the interfaces between the one-dimensional flow channels in the top and bottom sections of the assembly and the multi-channel middle section. We find that no special logic is required for the momentum or the mass/energy cells in the top and bottom rows of the middle section. Each of these cells is coupled to a single fluid cell at each face and this coupling can be treated exactly as shown by the general two-dimensional formulation in $\$ 3.4 .1$ and $\$ 3.4 .2$. The computations for the fluid cells in the middle section at these boundaries need only be able to correctly select parameter values from the adjacent fluid cells in either the top or bottom section. In practice it is convenient to set up two rows of fictitious fluid cells at either end of the middle section. Fictitious cells adjacent to the top of the middle section are assigned the properties of the last cell in the top section and the fictitious cells adjacent to the bottom of the middle section are assigned the properties of the first cell in the bottom section. This artifice facilitates coding since computations for fluid cells in the middle section can simply treat these fictitious cells as extensions ot the middle section and no special logic is then required to assign parameters from the top and bottom sections of the assembly. With the addition of the two fictitious rows of cells, each real fluid computational cell in the middle section (momentum cells and mass/energy cells) can be treated identically using the equations already presented.

A schematic representation of these fictitious cells is given in Fig. 3.4.3-4 which shows the fluid cells at the interfaces between the top and middle sections and between the middle and bottom sections. We assume that the flow in the middle section splits between $\mathrm{nx}$ azimuthal fluid cells. We note that the fictitious cells physically overlap the last fluid cell in the top section and the first fluid cell in the bottom section. Velocities at the cell faces and the values of state variables at the cell centers are taken from the corresponding real fluid cell as shown in the figure. We then need only specify appropriate physical dimensions for these cells to implement their usage. 


\begin{tabular}{|l|lll|l|}
\hline $2 / 15 / 93$ & WSRC-TR-92-532 Rev.0 & FLOWTRAN-TF Software Design & Page 395 \\
\hline
\end{tabular}
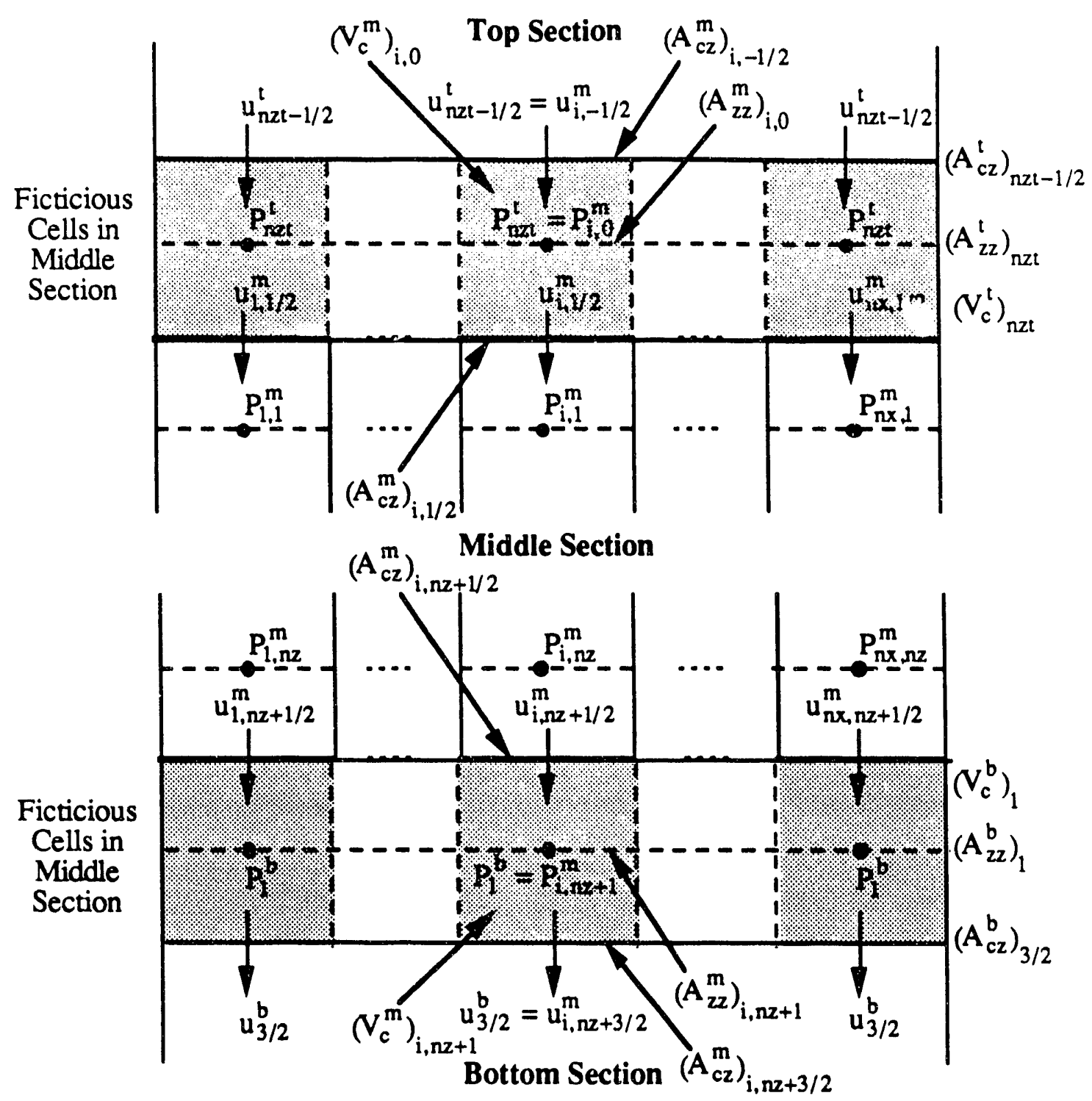

Figure 3.4.3-4 Schematic representation of fictitious fluid cells.

The cell volumes and cross-sectional areas at the tops and centers of the fictitious middle section fluid cells corresponding to the last cell in the top section are computed as

$$
\begin{gathered}
\left(V_{c}^{m}\right)_{i, 0}=f_{i, 1 / 2}\left(V_{c}^{t}\right)_{n z t} \\
\left(A_{z z}^{m}\right)_{i, 0}=f_{i, 1 / 2}\left(A_{z z}^{t}\right)_{n z t} \\
\left(A_{c z}^{m}\right)_{i,-1 / 2}=f_{i, 1 / 2}\left(A_{c z}^{t}\right)_{n z t-1 / 2}
\end{gathered}
$$

The parameter $f_{i, 1 / 2}$ which represents the fraction of the physical cell volume and crosssectional areas assigned to fictitious cell $i, 1 / 2$ is given by 


$$
f_{i, 1 / 2}=\frac{\left(A_{c z}^{m}\right)_{i, 1 / 2}}{\sum_{i=1}^{n z}\left(A_{c z}^{m}\right)_{i, 1 / 2}}
$$

Similarly, for the fictitious cells corresponding to the first cell in the bottom section we have

$$
\begin{gathered}
\left(V_{c}^{m}\right)_{i, n z+1}=f_{i, n z+1 / 2}\left(V_{c}^{b}\right)_{1} \\
\left(A_{z z}^{m}\right)_{i, n z+1}=f_{i, n z+1 / 2}\left(A_{z z}^{b}\right)_{1} \\
\left(A_{c z}^{m}\right)_{i, n z+3 / 2}=f_{i, n z+1 / 2}\left(A_{c z}^{b}\right)_{3 / 2}
\end{gathered}
$$

The parameter $f_{i, n z+1 / 2}$ which represents the fraction of the physical cell volume and crosssectional areas assigned to fictitious cell $i, n z+1 / 2$ is given by

$$
f_{i, n z+1 / 2}=\frac{\left(A_{c z}^{m}\right)_{i, n z+1 / 2}}{\sum_{i=1}^{n z}\left(A_{c z}^{m}\right)_{i, n z+1 / 2}}
$$

Summing Eqs. (3.4.3-16) and (3.4.3-18) over all cells shows that the real cell volumes and flow areas of the last fluid cell in the top section and first fluid cell in the bottom section are conserved within the fictitious cells since

$$
\sum_{i=1}^{n z} f_{i, 1 / 2}=\sum_{i=1}^{n z} f_{i, n z+1 / 2}=1
$$

A deficiency of the above scheme is that the finite-differenced momentum balances at the junctions between the Middle section and either Top or Bottom sections do not satisfy the modified Bernoulli equation (see equation (3.4.1-82) or (3.4.1-83) depending on flow direction) for flow into the Middle section. The scheme defined by equations (3.4.3-16) through (3.4.3-20) introduces undesirable numerical source/sink terms to the momentum balances. There is no problem for flow exiting the Middle section whether at the Top or Bottom section junctions. To see this fact consider the case of flow entering the Middle section from the Top section. The reason behind the deficiency is that the areas

$$
\left(A_{c 2}^{m}\right)_{i,-1 / 2}
$$

do not align with the dividing streamlines of the entering flow field. That is, the mass flow rate into a fictitious mass/energy cell $i$ does not agree with that leaving the cell and entering the Middle section: 


$$
\left(A_{c z}^{m} \hat{\alpha}_{k} \hat{\rho}_{k} u_{k}\right)_{i,-1 / 2} \neq\left(A_{c z}^{m} \hat{\alpha}_{k} \hat{\rho}_{k} u_{k}\right)_{i, 1 / 2}
$$

To remedy this deficiency, the scheme

$$
\left(A_{c z}^{m}\right)_{i,-1 / 2}=f_{i, 1 / 2}\left(A_{c z}^{t}\right)_{n z t-1 / 2}
$$

is replaced by

$$
\left(A_{c z, k}^{m}\right)_{i,-1 / 2}=\frac{\left(A_{c z}^{m} \hat{\alpha}_{k} \hat{\rho}_{k} u_{k}\right)_{i, 1 / 2}}{\left(\hat{\alpha}_{k} \hat{\rho}_{k} u_{k}\right)_{i,-1 / 2}}
$$

for $k=g$ and/or $f$ provided each of the following three conditions is satisfied:

- phase k exists, $\left(\hat{\alpha}_{k}\right)_{i,-1 / 2}>0$

- $\left(u_{k}\right)_{i,-1 / 2}$ and $\left(u_{k}\right)_{i, 1 / 2}$ have the same sign

- the Middle section is comprised of 2 or more axial flow paths.

Otherwise the original scheme is retained. The analogous logic is applied to

$$
\left(A_{c z}^{m}\right)_{i, n z+3 / 2}
$$

With this change, the modified Bernoulli balance is satisfied. We will now examine the momentum cells at the interfaces between the top and bottom sections and the middle section.

Last Momentum Cell in Top Section: Figure 3.4.3-5 shows a schematic representation of the !ast two fluid cells in the top section and the first axial layer of fluid cells in the middle section. In Fig. 3.4.3-5, we have highlighted the last momentum cell in the top section and one of the first momentum cells in the middle section. 


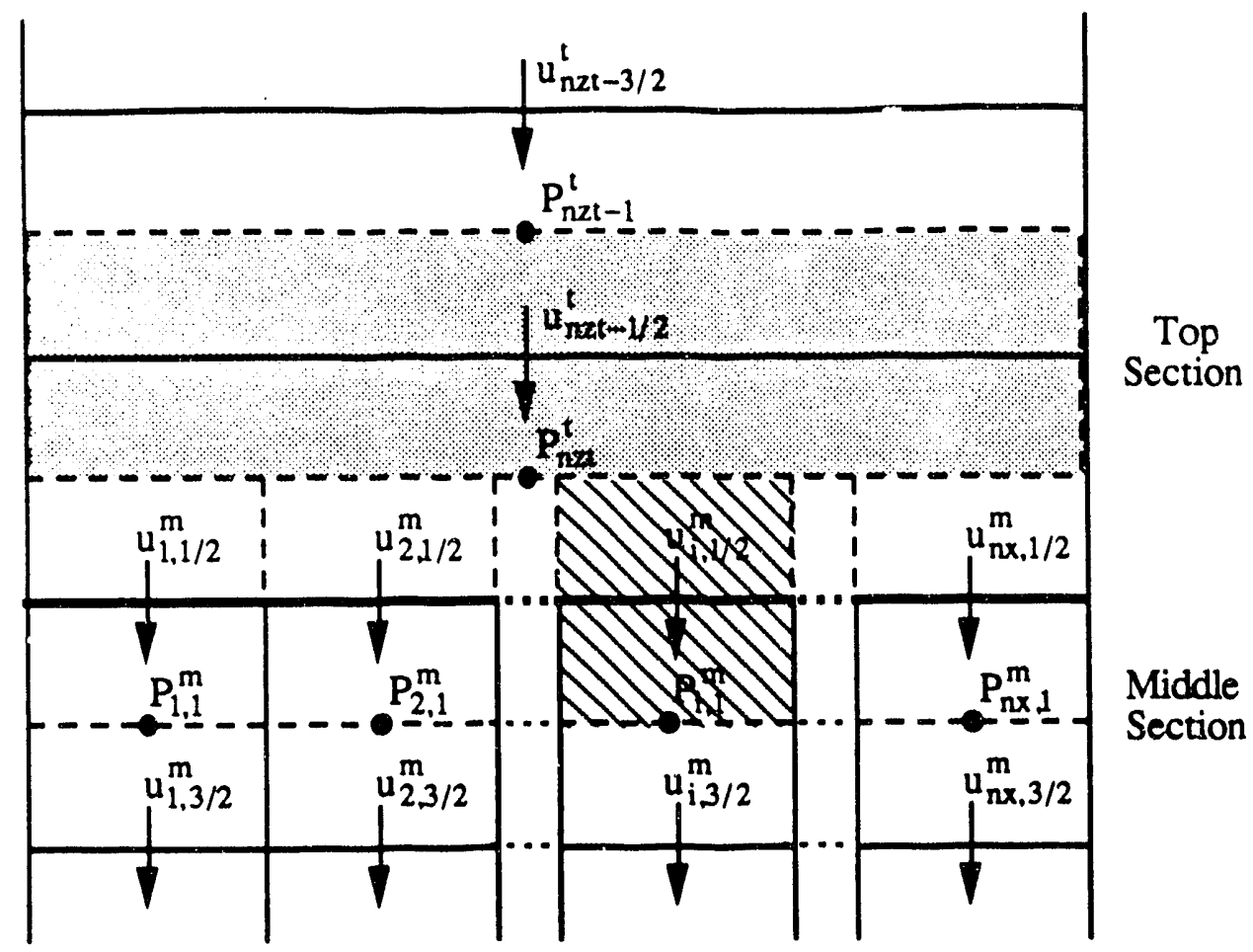

Figure 3.4.3-5 Momentum cells at interface between top and middle sections.

Considering the last momentum cell in the top section, Eqs. (3.4.3-2) and (3.4.3-5) apply as written above for the gas and liquid phases, respectively using

$$
\mathrm{j} \Rightarrow \mathrm{nzt}-1, \mathrm{j}+1 / 2 \Rightarrow \mathrm{nzt}-1 / 2, \mathrm{j}+1 \Rightarrow \mathrm{nzt}
$$

However, when evaluating the cell center velocity in the last mass cell and the donoring of mass and momentum flow rates to the bottom face of the momentum cell, we must account for the multi-channel flows. The modified equations (see TF-17) are

$$
\begin{aligned}
& \left(\tilde{u}_{g}\right)_{n z t}^{t} \equiv \frac{\left(A_{c z}^{t} \hat{\alpha} \hat{\rho}_{g} u_{g}^{t}\right)_{n z t-1 / 2}^{n}\left(A_{c z}^{t}\right)_{n z t-1 / 2}+\sum_{i=1}^{n x}\left(A_{c z}^{m} \hat{\alpha}_{\rho_{g}} u_{g}^{m}\right)_{i, 1 / 2}^{n} \sum_{i=1}^{n x}\left(A_{c z}^{m}\right)_{i, 1 / 2}}{\left[\left(A_{c z}^{t} \hat{\alpha} \hat{\rho}_{g}\right)_{n z t-1 / 2}^{n}+\sum_{i=1}^{n x}\left(A_{c z}^{m} \hat{\alpha}_{\rho_{g}}\right)_{i, 1 / 2}^{n}\right]\left(A_{z z}\right)_{n z t}} \\
& \left\langle A_{z z} \alpha \rho_{g} u_{g}\right\rangle_{n z t}^{n}=\omega\left(A_{c z}^{t} \hat{\alpha} \hat{\rho}_{g} u_{g}^{t}\right)_{n z t-1 / 2}^{n}+(1-\omega) \sum_{i=1}^{n x}\left(A_{c z}^{m} \hat{\alpha} \hat{\rho}_{g} u_{g}^{m}\right)_{i, 1 / 2}^{n}
\end{aligned}
$$




$$
\left\langle A_{z z} \alpha \rho_{g} u_{g} u_{g}\right\rangle_{n z t}^{n}=\omega\left(A_{c z}^{t} \hat{\alpha} \hat{\rho}_{g} u_{g}^{t} u_{g}^{t}\right)_{n z t-1 / 2}^{n}+(1-\omega) \sum_{i=1}^{n x}\left(A_{c z}^{m} \hat{\alpha} \hat{\rho}_{g} u_{g}^{m} u_{g}^{m}\right)_{i, 1 / 2}^{n}
$$

First Momentum Cell in Bottom Section: Exactly analogous arguments apply to the momentum cells at the interface between the middle section and the bottom section. Figure 3.4.3-6 shows a schematic representation of the first two fluid cells in the bottom section and the last axial layer of fluid cells in the middle section. In Fig. 3.4.3-6, we have highlighted the first momentum cell in the bottom section and one of the last momentum cells in the middle section.

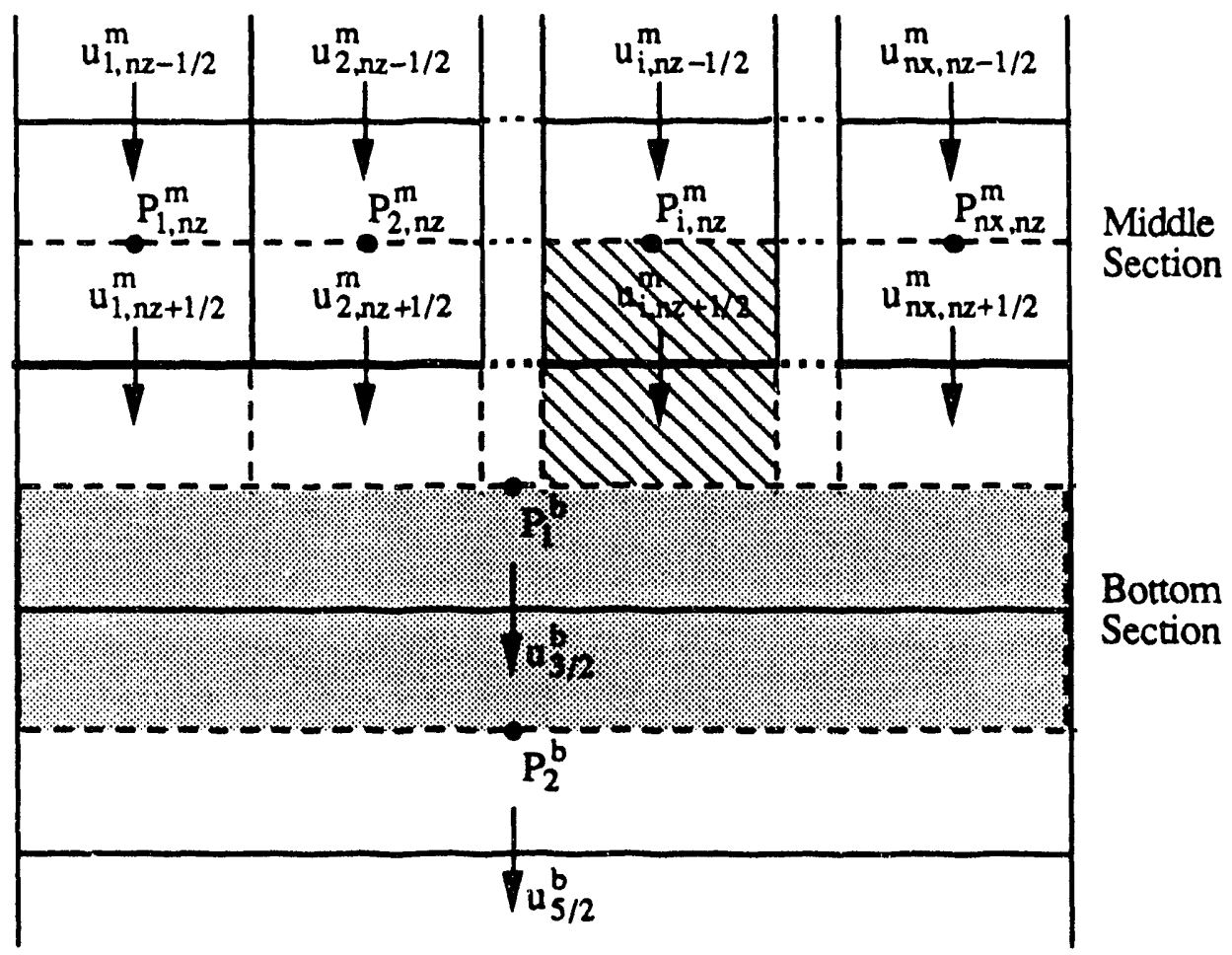

Figure 3.4.3-6 Momentum cells at interface between middle and bottom sections.

Considering the first momentum cell in the bottom section, Eqs. (3.4.3-2) and (3.4.3-5) again apply as written above for the gas and liquid phases, respectively with

$$
\mathrm{j} \Rightarrow 1, \mathrm{j}+1 / 2 \Rightarrow 3 / 2, \mathrm{j}+1 \Rightarrow 2
$$

When evaluating the cell center velocity in the first mass cell and the donoring of mass and momentum flow rates to the top face of this momentum cell, we must account for the multichannel flows. The required equations are 


$$
\begin{aligned}
& \left(\tilde{u}_{g}\right)_{1}^{b} \equiv \frac{\sum_{i=1}^{n x}\left(A_{c z}^{m} \hat{\alpha} \hat{\rho}_{g} u_{g}^{m}\right)_{i, n z+1 / 2}^{n} \sum_{i=1}^{n x}\left(A_{c z}^{m}\right)_{i, n z+1 / 2}+\left(A_{c z}^{b} \hat{\alpha} \hat{\rho}_{g} u_{g}^{b}\right)_{3 / 2}^{n}\left(A_{c z}^{b}\right)_{3 / 2}}{\left[\sum_{i=1}^{n x}\left(A_{c z}^{m} \hat{\alpha} \hat{\rho}_{g}\right)_{i, n z+1 / 2}^{n}+\left(A_{c z}^{b} \hat{\alpha} \hat{\rho}_{g}\right)_{3 / 2}^{n}\right]\left(A_{z z}\right)_{1}} \\
& \left\langle A_{z z} \alpha \rho_{g} u_{g}\right\rangle_{1}^{n}=\omega \sum_{i=1}^{n x}\left(A_{c z}^{m} \hat{\alpha}_{\rho_{g}} u_{g}^{m}\right)_{i, n z+1 / 2}^{n}+(1-\omega)\left(A_{c z}^{b} \hat{\alpha} \hat{\rho}_{g} u_{g}^{b}\right)_{3 / 2}^{n}(3.4 .4 \\
& \left\langle A_{z z} \alpha \rho_{g} u_{g} u_{g}\right\rangle_{1}^{n}=\omega \sum_{i=1}^{n x}\left(A_{c z}^{m} \hat{\alpha} \hat{\rho}_{g} u_{g}^{m} u_{g}^{m}\right)_{i, n z+1 / 2}^{n}+(1-\omega)\left(A_{c z}^{b} \hat{\alpha} \hat{\rho}_{g} u_{g}^{b} u_{g}^{b}\right)_{3 / 2}^{n}
\end{aligned}
$$

Equations (3.4.3-3), (3.4.3-6), and (3.4.3-7) apply directly to the momentum equations without modification. We next examine mass/energy cells at the interfaces between the top and bottom sections and the middle section.

Last Mass/Energy Cell in Top Section: Figure 3.4.3-7 shows a schematic representation of the interface between the top and middle sections with the last mass/energy cell in the top section (cell $\mathrm{T}_{2}$ in Fig. 3.4.3-1) and a typical mass/energy cell in the first axial layer of the middle section highlighted.

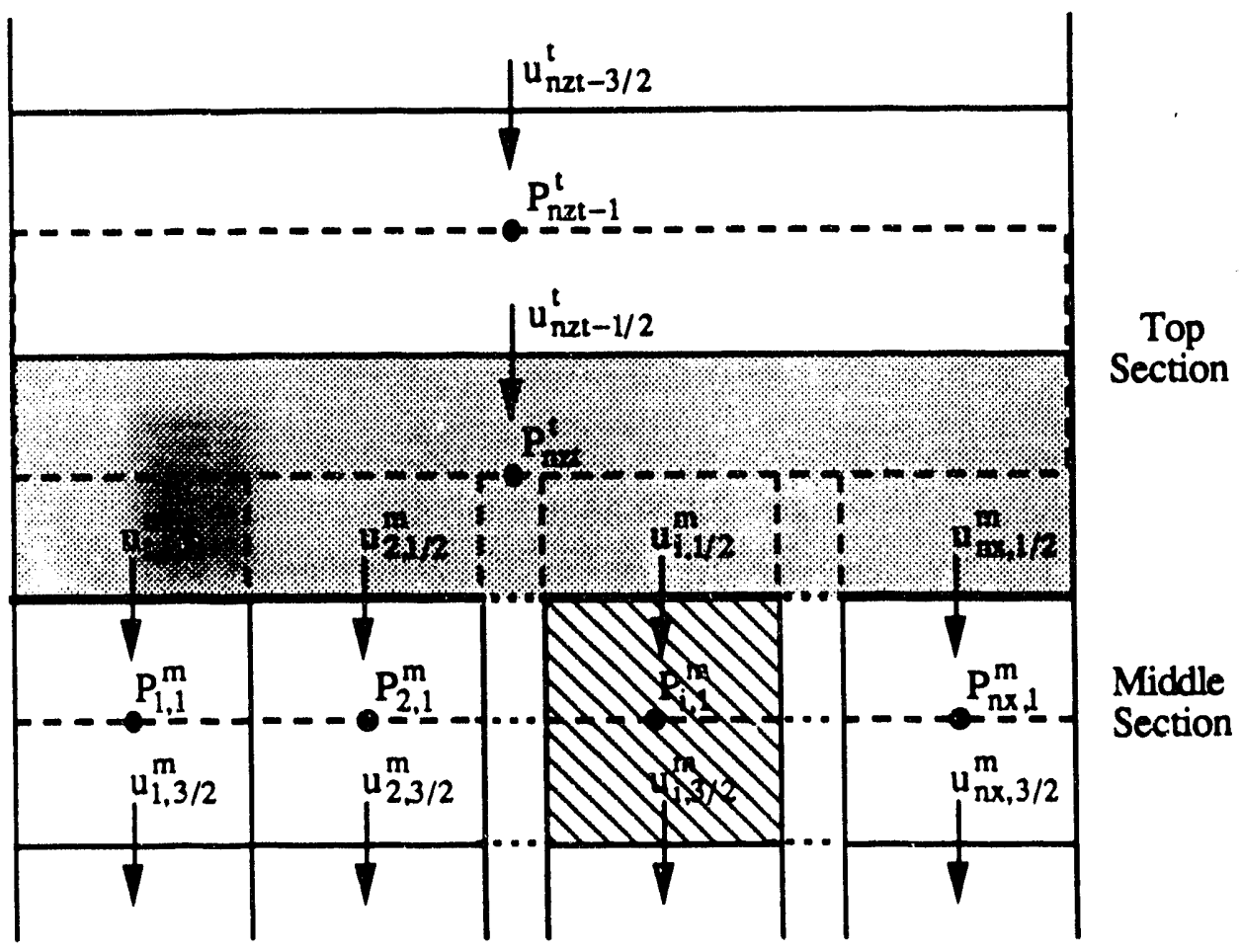

Figure 3.4.3-7 Mass/energy cells at interface between top and middle sections. 
Considering the last fluid cell in the top section, the general differenced form of the mass/energy conservation equation shown in Eq. (3.4.3-9) can be applied using

$$
j \Rightarrow n z t, j-1 / 2 \Rightarrow n z t-1 / 2, j-1 \Rightarrow n z t-1, j+1 / 2 \Rightarrow i, 1 / 2, j+1 \Rightarrow i, 1
$$

Nodes $j+1 / 2$ and $j+1$ point into the middle section and the contribution from these terms must be summed over all of the mass/energy cells in the first axial layer of the middle section. The general mass/energy finite difference equation for this cell is given by

$$
\begin{aligned}
& \frac{\left(\alpha_{k} \rho_{k} \phi_{k}\right)_{n z t}^{n+1}}{\Delta t} \\
& +\frac{1}{\left(V_{c}^{t}\right)_{n z t}}\left\{\left(A_{c z}^{t} \hat{\alpha}_{k} \hat{\rho}_{k} \hat{\phi}_{k} C_{k}\right)_{n z t-1 / 2}^{n}+\sum_{i=1}^{n x}\left(A_{c z}^{m} \hat{\alpha}_{k} \hat{\rho}_{k} \hat{\phi}_{k} C_{k}\right)_{i, 1 / 2}^{n}\right\}\left(\delta P^{t}\right)_{n z t}^{m+1} \\
& -\frac{1}{\left(V_{c}^{t}\right)_{n z t}}\left\{\left(A_{c z}^{t} \hat{\alpha}_{k} \hat{\rho}_{k} \hat{\phi}_{k} C_{k}\right)_{n z t-1 / 2}^{n}\right\}\left(\delta P^{t}\right)_{n z t-1}^{m+1} \\
& -\frac{1}{\left(V_{c}^{i}\right)_{n z t}} \sum_{i=1}^{n x}\left\{\left(A_{c z}^{m} \hat{\alpha}_{k} \hat{\rho}_{k} \hat{\phi}_{k} C_{k}\right)_{i, 1 / 2}^{n}\right\}\left(\delta P^{m}\right)_{i, 1}^{m+1} \\
& =\frac{1}{\left(V_{c}^{t}\right)_{n z t}}\left\{\left(A_{c z}^{t} \hat{\alpha}_{k} \hat{\rho}_{k} \hat{\phi}_{k}\right)_{n z t-1 / 2}^{n}\left[\left(B_{k}\right)_{n z t-1 / 2}^{n}+\left(C_{k}\right)_{n z t-1 / 2}^{n}\left(P_{n z t-1}^{t}-P_{n z t}^{t}\right)^{m}\right]\right. \\
& \left.-\sum_{i=1}^{n x}\left(A_{c z}^{m} \hat{\alpha}_{k} \hat{\rho}_{k} \hat{\phi}_{k}\right)_{i, 1 / 2}^{n}\left[\left(B_{k}\right)_{i, 1 / 2}^{n}+\left(C_{k}\right)_{i, 1 / 2}^{n}\left(P_{n z t}^{t}-P_{i, 1}^{m}\right)^{m}\right]\right\} \\
& +\frac{\left(\alpha_{k} \rho_{k} \phi_{k}\right)_{n z t}^{n}}{\Delta t}+\sum_{i}\left\langle S_{i}\right\rangle_{n z t}^{n+1}
\end{aligned}
$$

In writing Eq. (3.4.3-28), we have placed $t$ and $m$ superscripts on pressures, areas, and cell volumes to more clearly show the derivation of these terms. This creates some conflict since the superscript $\mathrm{m}$ has also been used to indicate the $\mathrm{m}^{\text {th }}$ iteration during the calculations for time step $n+1$. We have consistently moved the $m$ iteration superscript outside of the brackets and placed the positional superscript:; ( $t$ and $m$ ) directly on the indicated parameters. With this convention the meaning of Eq. (3.4.3-28) is clear and we have kept the double definition for the $m$ superscript. Specific conservation equations for this mass/energy cell analogous to those shown in Eqs. (3.4.3-10) through (3.4.3-14) above can readily be derived from Eq. (3.4.3-28) and will not be presented here. The only modification required is to insert the appropriate summations over the middle section cells as shown in Eq. (3.4.3-28). 
The set of five difference equations for the last mass/energy cell in the top section can be combined into the equivalent matrix equation

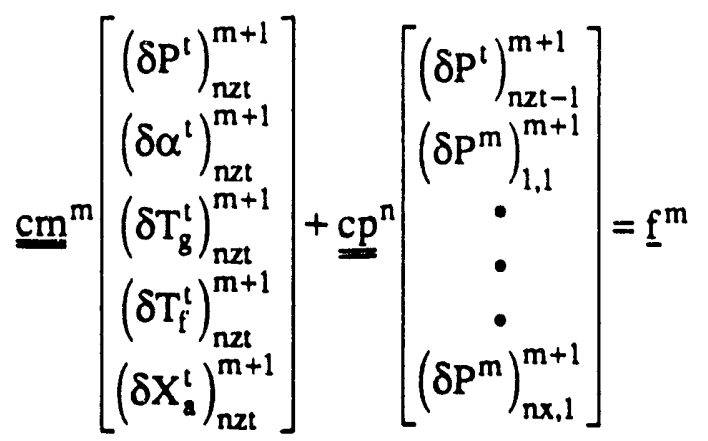

where $\mathrm{cm}^{\mathrm{m}}$ is a $5 \times 5$ matrix of coefficient values, $\mathrm{cp}^{\mathrm{n}}$ is now a $5 \times(\mathrm{nx}+1)$ matrix of pressure coupling coefficients, and $\mathrm{f}^{\mathrm{m}}$ is the 5 element vector of right hand side terms. Formulation of the mass and energy finite difference equations described above and the inversion of the $\mathrm{cm}^{\mathrm{m}}$ matrix are programmed in subroutine JACOBT for the top section of the assembly.

First Mass/Energy Cell in Bottom Section: Figure 3.4.3-8 shows a schematic representation of the interface between the middle section and the bottom section of the assembly.

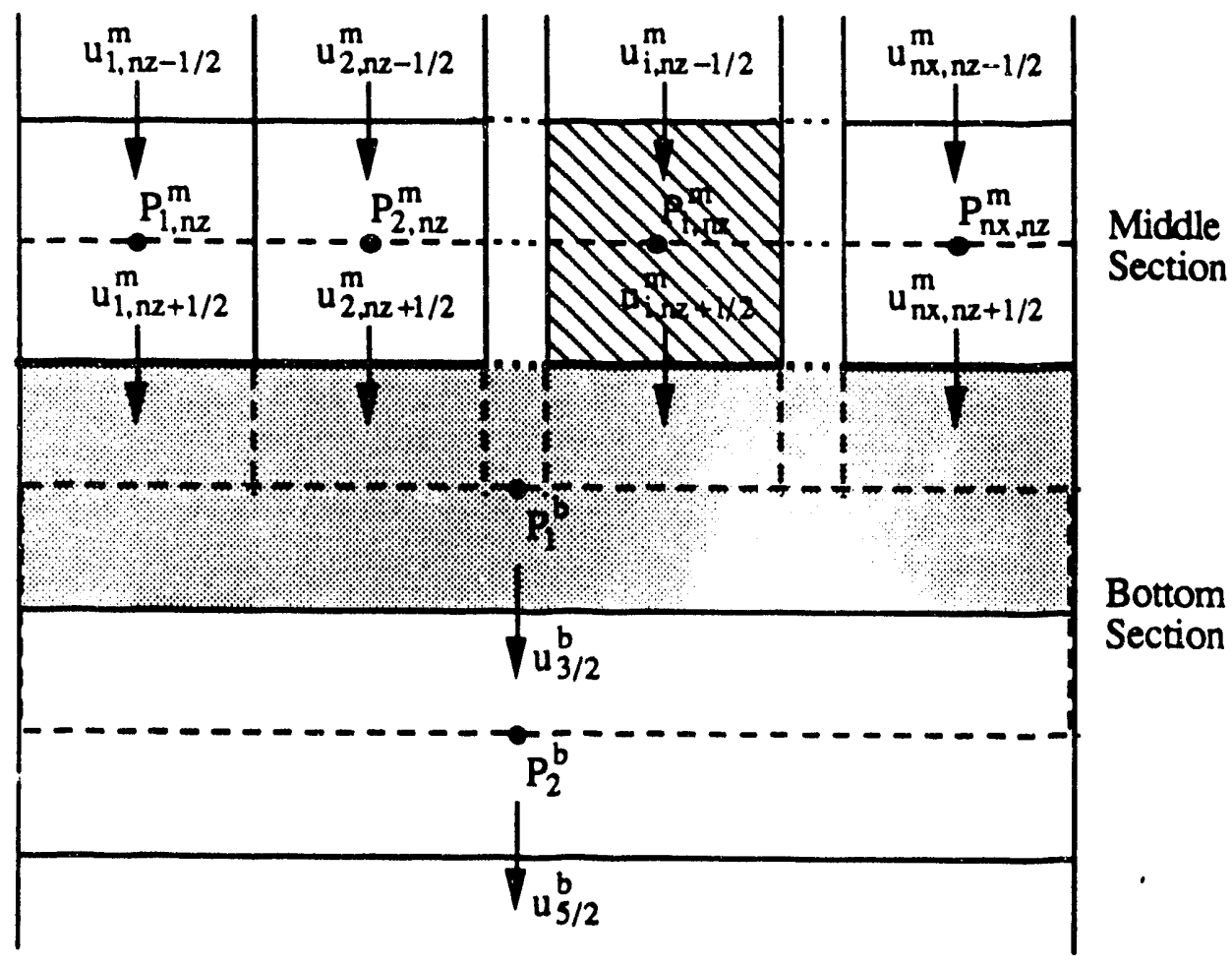

Figure 3.4.3-8 Mass/energy cells at interface between middle and bottom sections.

In Fig. 3.4.3-8, the first mass/energy cell in the bottom section (cell $\mathrm{B}_{2}$ in Fig. 3.4.3-1) and one of the last mass/energy cells in the middle section are highlighted. Considering the 

first fluid cell in the bottom section, the general differenced form of the mass/energy
conservation equation shown in Eq. (3.4.3-9) can be applied using

$$
\mathrm{i} \Rightarrow 1, \mathrm{j}-1 / 2 \Rightarrow \mathrm{i}, \mathrm{nz}+1 / 2, \mathrm{j}+1 / 2 \Rightarrow 3 / 2, \mathrm{j}-1 \Rightarrow \mathrm{i}, \mathrm{nz}, \mathrm{j}+1 \Rightarrow 2
$$

Nodes $j-1 / 2$ and $j-1$ point into the middle section and the contribution from these terms must be summed over all of the mass/energy cells in the last axial layer of the middle section. The general mass/energy finite difference equation for this cell is given by

$$
\begin{aligned}
& \frac{\left(\alpha_{k} \rho_{k} \phi_{k}\right)_{1}^{n+1}}{\Delta t} \\
& +\frac{1}{\left(V_{c}^{b}\right)_{1}}\left\{\sum_{i=1}^{n x}\left(A_{c z}^{m} \hat{\alpha}_{k} \hat{\rho}_{k} \hat{\phi}_{k} C_{k}\right)_{i, n z+1 / 2}^{n}+\left(A_{c z}^{b} \hat{\alpha}_{k} \hat{\rho}_{k} \hat{\phi}_{k} C_{k}\right)_{3 / 2}^{n}\right\}\left(\delta P^{b}\right)_{1}^{m+1} \\
& -\frac{1}{\left(V_{c}^{b}\right)_{1}} \sum_{i=1}^{n x}\left\{\left(A_{c z}^{m} \hat{\alpha}_{k} \hat{\rho}_{k} \hat{\phi}_{k} C_{k}\right)_{i, n z+1 / 2}^{n}\right\}\left(\delta P^{m}\right)_{i, n z}^{m+1} \\
& =\frac{1}{\left(V_{c}^{b}\right)_{1}}\left\{\sum_{i=1}^{n x}\left(A_{c z}^{m} \hat{\alpha}_{k} \hat{\rho}_{k} \hat{\phi}_{k}\right)_{i, n z+1 / 2}^{n}\left[\left(B_{k}\right)_{i, n z+1 / 2}^{n}+\left(C_{k}\right)_{i, n z+1 / 2}^{n}\left(P_{i, n z}^{m}-P_{1}^{b}\right)^{m}\right]\right. \\
& +\frac{\left(\alpha_{k} \rho_{k} \phi_{k}\right)_{1}^{n}}{\Delta t}+\sum_{i}\left\langle\left(A_{c z}^{b} \hat{\alpha}_{k} \hat{\rho}_{k} \hat{\phi}_{k} C_{k}\right)_{3 / 2}^{n}\right\}\left(\delta P^{b}\right)_{2}^{m+1}
\end{aligned}
$$

In writing Eq. (3.4.3-30), we have placed $b$ and $m$ superscripts on pressures, areas, and cell volumes to more clearly define these terms. Specific conservation equations for this can again be readily derived from Eq $(3.43-30)$ ans. $(3.4 .3-10)$ through $(3.4 .3-14)$ above modification required in each of these equations is will not be presented here. The only over the middle section cells exactly as shown in to insert the appropriate summations

The set of five difference equations for the first mass/energy cell in the bottom section can
be combined into the equivalent matrix equation 


$$
\mathrm{cm}^{\mathrm{m}}\left[\begin{array}{l}
\left(\delta \mathrm{P}^{\mathrm{b}}\right)_{1}^{\mathrm{m}+1} \\
\left(\delta \alpha^{\mathrm{b}}\right)_{1}^{\mathrm{m}+1} \\
\left(\delta \mathrm{T}_{\mathrm{g}}^{\mathrm{b}}\right)_{1}^{\mathrm{m}+1} \\
\left(\delta \mathrm{T}_{\mathrm{f}}^{\mathrm{b}}\right)_{1}^{\mathrm{m}+1} \\
\left(\delta \mathrm{X}_{\mathrm{a}}^{\mathrm{b}}\right)_{1}^{\mathrm{m}+1}
\end{array}\right]+\underline{\mathrm{cp}}^{\mathrm{n}}\left[\begin{array}{c}
\left(\delta \mathrm{P}^{\mathrm{b}}\right)_{2}^{\mathrm{m}+1} \\
\left(\delta \mathrm{P}^{\mathrm{m}}\right)_{1, \mathrm{nz}}^{\mathrm{m}+1} \\
\dot{\cdot} \\
\cdot \\
\left(\delta \mathrm{P}^{\mathrm{m}}\right)_{n x, n z}^{\mathrm{m}+1}
\end{array}\right]=\underline{\mathrm{f}}^{\mathrm{m}}
$$

where $\mathrm{cm}^{\mathrm{m}}$ is a $5 \times 5$ matrix of coefficient values, $\mathrm{cp}^{\mathrm{n}}$ is a $5 \times(\mathrm{nx}+1)$ matrix of pressure coupling coefficients, and $\mathrm{f}^{\mathrm{m}}$ is the 5 element vector of right hand side terms. Formulation of the mass and energy finite difference equations and inversion of the $\mathrm{cm}^{\mathrm{m}}$ matrix are programmed in subroutine JACOBB for the bottom section of the assembly. The solution of Eqs. (3.4.3-29) and (3.4.3-31) is discussed in the solution methods section of the code manual.

In addition to the cases examined above, special forms of the finite difference equations are required for the fluid cells adjacent to the plenum and tank bottom boundary cells (labeled $\mathrm{T}_{1}$ and $\mathrm{B}_{1}$ in Fig. 3.4.3-1). The exact form of the momentum difference equations applied at these locations depends upon the boundary conditions specified for the problem. These cases will not be discussed here but will be treated in the code development report describing the specification of boundary conditions. However, the development of mass/energy difference equations is essentially unchanged from our previous results and will be treated here.

First Mass/Energy Cell in Top Section: Figure 3.4.3-9 shows a schematic representation of the plenum boundary cell and the first mass/energy cell in the top section (cell $T_{1}$ in Fig. 3.4.3-1). 


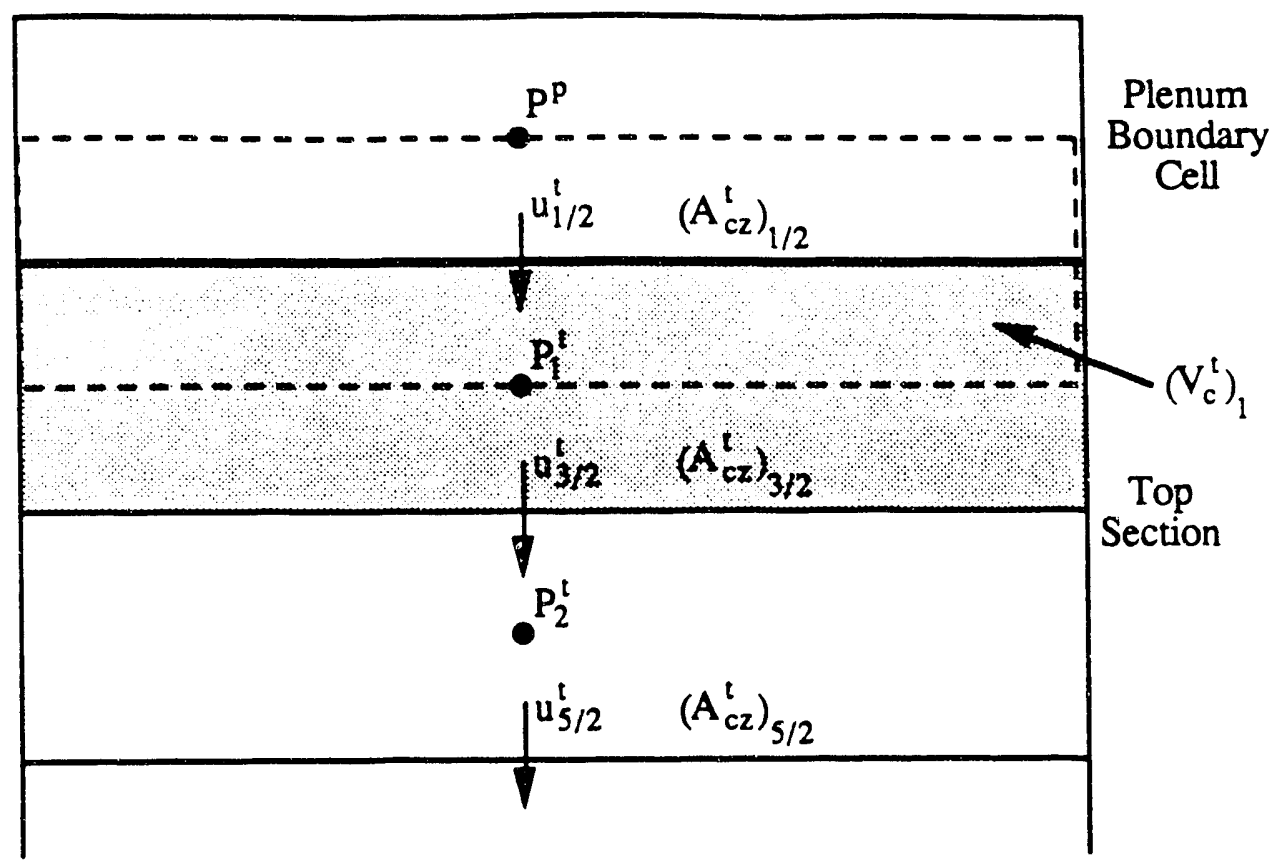

Figure 3.4.3-9 First mass/energy cell in top section.

The general differenced form of the mass/energy conservation equation shown in Eq. (3.4.3-9) can be applied using

$$
\mathrm{j} \Rightarrow 1, \mathrm{j}-1 / 2 \Rightarrow 1 / 2, \mathrm{j}+1 / 2 \Rightarrow 3 / 2, \mathrm{j}-1 \Rightarrow \mathrm{p}, \mathrm{j}+1 \Rightarrow 2
$$

Nsde $j-1$ is located in the plenum boundary cell which we identify with the superscript $p$. The general mass/energy finite difference equation for this cell is 


$$
\begin{aligned}
& \frac{\left(\alpha_{k} \rho_{k} \phi_{k}\right)_{1}^{n+1}}{\Delta t} \\
& +\frac{1}{\left(V_{c}^{t}\right)_{1}}\left\{\left(A_{c z}^{t} \hat{\alpha}_{k} \hat{\rho}_{k} \hat{\phi}_{k} C_{k}\right)_{1 / 2}^{n}+\left(A_{c z}^{t} \hat{\alpha}_{k} \hat{\rho}_{k} \hat{\phi}_{k} C_{k}\right)_{3 / 2}^{n}\right\}\left(\delta P^{t}\right)_{1}^{m+1} \\
& \quad-\frac{1}{\left(V_{c}^{t}\right)_{1}}\left\{\left(A_{c z}^{t} \hat{\alpha}_{k} \hat{\rho}_{k} \hat{\phi}_{k} C_{k}\right)_{3 / 2}^{n}\right\}\left(\delta P^{t}\right)_{2}^{m+1} \\
& =\frac{1}{\left(V_{c}^{t}\right)_{1}}\left\{\left(A_{c z}^{t} \hat{\alpha}_{k} \hat{\rho}_{k} \hat{\phi}_{k}\right)_{1 / 2}^{n}\left[\left(B_{k}\right)_{1 / 2}^{n}+\left(C_{k}\right)_{1 / 2}^{n}\left(P^{p}-P_{1}^{t}\right)^{m}\right]\right. \\
& \left.-\left(A_{c z}^{t} \hat{\alpha}_{k} \hat{\rho}_{k} \hat{\phi}_{k}\right)_{3 / 2}^{n}\left[\left(B_{k}\right)_{3 / 2}^{n}+\left(C_{k}\right)_{3 / 2}^{n}\left(P_{1}^{t}-P_{2}^{t}\right)^{m}\right]\right\} \\
& +\frac{\left(\alpha_{k} \rho_{k} \phi_{k}\right)_{1}^{n}}{\Delta t}+\sum_{i}\left\langle S_{i}\right)_{1}^{n+1}
\end{aligned}
$$

In Eq. (3.4.3-32), there is no contribution from the term

$$
\left\{\left(A_{c z}^{t} \hat{\alpha}_{k} \hat{\rho}_{k} \hat{\phi}_{k} C_{k}\right)_{1 / 2}^{n}\right\}\left(\delta P^{p}\right)^{m+1}
$$

This occurs because in cases where the plenum pressure is specified $\delta \mathrm{P}^{\mathrm{p}}$ is identically zero. For cases where the flow rate of component $\mathbf{k}$ is specified as a boundary condition the coefficients $\left(C_{k}\right)_{1 / 2}^{n}$ in the velocity relationship will be identically zero. Therefore, in either case, this term vanishes from the mass/energy difference equation. As before, the coefficients $\left(B_{k}\right)_{1 / 2}^{n}$ and $\left(C_{k}\right)_{1 / 2}^{n}$ are determined from the solution of the momentum equations at this boundary.

The set of difference equations for the first mass/energy cell in the top section are readily obtained from Eqs. (3.4.3-10) - (3.4.3-14) shown above. These equations can be combined into the matrix equation

$$
\mathrm{cm}^{\mathrm{m}}\left[\begin{array}{l}
\left(\delta \mathrm{P}^{\mathrm{t}}\right)_{1}^{\mathrm{m}+1} \\
\left(\delta \alpha^{\mathrm{l}}\right)_{1}^{\mathrm{m}+1} \\
\left(\delta \mathrm{T}_{\mathrm{g}}^{\mathrm{t}}\right)_{1}^{\mathrm{m}+1} \\
\left(\delta \mathrm{T}_{\mathrm{f}}^{\mathrm{t}}\right)_{1}^{\mathrm{m}+1} \\
\left(\delta \mathrm{X}_{\mathrm{a}}^{\mathrm{t}}\right)_{1}^{\mathrm{m}+1}
\end{array}\right]+\underline{\mathrm{cp}}^{\mathrm{n}}\left[\left(\delta \mathrm{P}^{\mathrm{t}}\right)_{2}^{\mathrm{m}+1}\right]=\underline{\mathrm{f}}^{\mathrm{m}}
$$

where $\mathrm{cm}^{\mathrm{m}}$ is a $5 \times 5$ matrix of coefficient values, $\underline{c p}^{\mathrm{n}}$ is now a 5 element vector of pressure coefficients coupling the first fluid cell to the second fluid cell, and $\underline{f}^{m}$ is the 5 element vector of right hand side terms. As with the other fluid cells in the top section, formulation 


\begin{tabular}{|l|ll|l|}
\hline $2 / 15 / 93$ & WSRC-TR-92-532 Rev. 0 & FLOWTRAN-TF Software Design & Page 407 \\
\hline
\end{tabular}

of the mass and energy finite difference equations described above and the inversion of the $\mathrm{cm}^{\mathrm{m}}$ matrix are programmed in subroutine JACOBT.

Last Mass/Energy Cell in Bottom Section: Figure 3.4.3-10 shows a schematic representation of the last mass/energy cell in the bottom section (cell $T_{1}$ in Fig. 3.4.3-1) and the tank bottom boundary cell.

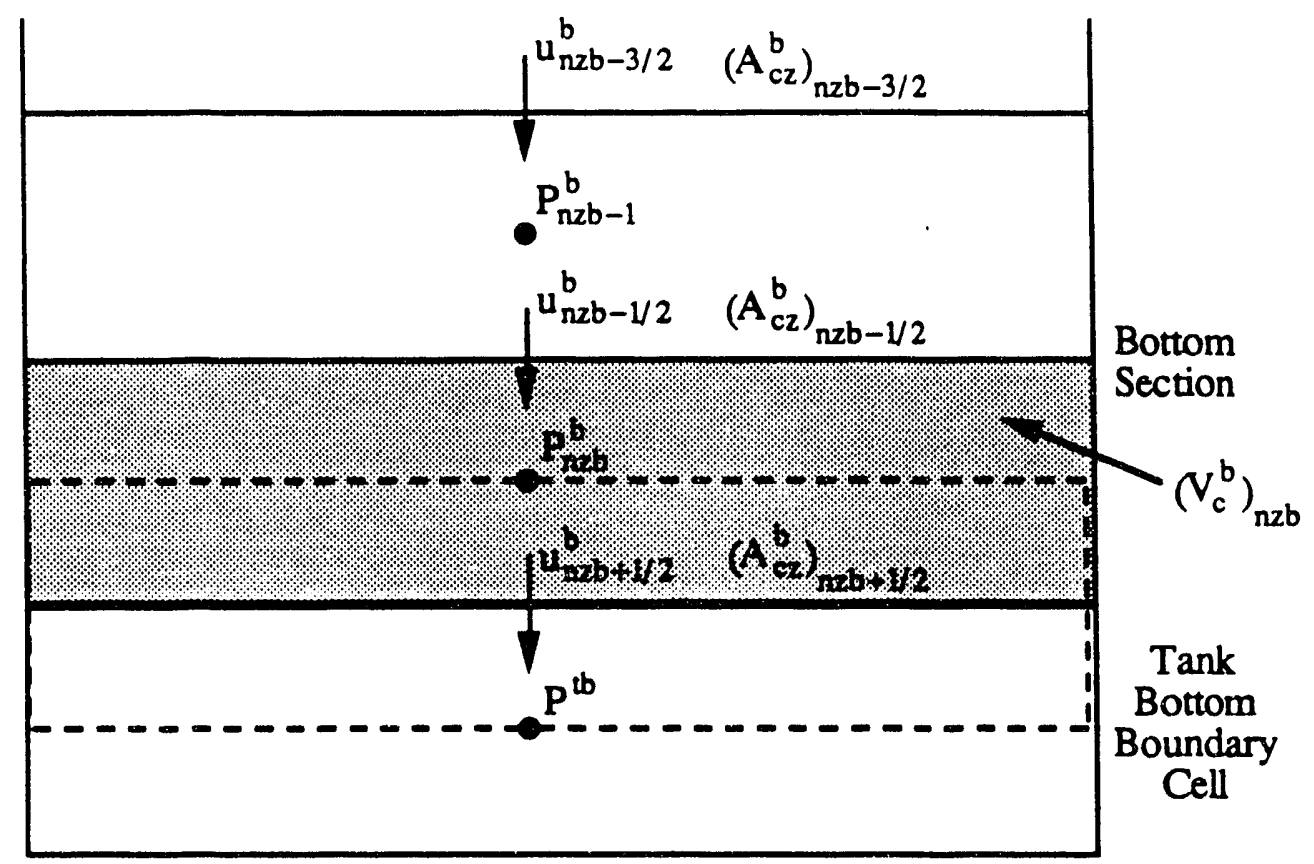

Figure 3.4.3-10 Last mass/energy cell in bottom section.

Considering the last fluid cell in the bottom section, the general differenced form of the mass/energy conservation equation shown in Eq. (3.4.3-9) can be applied with

$$
j \Rightarrow n z b, j-1 / 2 \Rightarrow n z b-1 / 2, j-1 \Rightarrow n z b-1, j+1 / 2 \Rightarrow n z b+1 / 2, j+1 \Rightarrow \text { tb }
$$

Node $j+1$ is located in the tank bottom boundary cell which we identify using the superscript tb. The general mass/energy finite difference equation for this cell becomes 


$$
\begin{aligned}
& \frac{\left(\alpha_{k} \rho_{k} \phi_{k}\right)_{n z b}^{n+1}}{\Delta t} \\
& +\frac{1}{\left(V_{c}^{b}\right)_{n z b}}\left\{\left(A_{c z}^{b} \hat{\alpha}_{k} \hat{\rho}_{k} \hat{\phi}_{k} C_{k}\right)_{n z b-1 / 2}^{n}+\left(A_{c z}^{b} \hat{\alpha}_{k} \hat{\rho}_{k} \hat{\phi}_{k} C_{k}\right)_{n z b+1 / 2}^{n}\right\}\left(\delta P^{b}\right)_{n z b}^{m+1} \\
& \quad-\frac{1}{\left(V_{c}^{b}\right)_{n z b}}\left\{\left(A_{c z}^{b} \hat{\alpha}_{k} \hat{\rho}_{k} \hat{\phi}_{k} C_{k}\right)_{n z b-1 / 2}^{n}\right\}\left(\delta P^{b}\right)_{n z b-1}^{m+1} \\
& =\frac{1}{\left(V_{c}^{b}\right)_{n z b}}\left\{\left(A_{c z}^{b} \hat{\alpha}_{k} \hat{\rho}_{k} \hat{\phi}_{k}\right)_{n z b-1 / 2}^{n}\left[\left(B_{k}\right)_{n z b-1 / 2}^{n}+\left(C_{k}\right)_{n z b-1 / 2}^{n}\left(P_{n z b-1}^{b}-P_{n z b}^{b}\right)^{m}\right]\right. \\
& \left.-\left(A_{c z}^{b} \hat{\alpha}_{k} \hat{\rho}_{k} \hat{\phi}_{k}\right)_{n z b+1 / 2}^{n}\left[\left(B_{k}\right)_{n z b+1 / 2}^{n}+\left(C_{k}\right)_{n z b+1 / 2}^{n}\left(P_{n z b}^{b}-P^{t b}\right)^{m}\right]\right\} \\
& +\frac{\left(\alpha_{k} \rho_{k} \phi_{k}\right)_{n z b}^{n}+\sum_{i}\left\langle S_{i}\right\rangle_{n z b}^{n+1}}{\Delta t}
\end{aligned}
$$

As with the mas/energy cell adjacent to the plenum, Eq. (3.4.3-34) does not contain the term

$$
\left\{\left(A_{c z}^{b} \hat{\alpha}_{k} \hat{\rho}_{k} \hat{\phi}_{k} C_{k}\right)_{n z b+1 / 2}^{n}\right\}\left(\delta P^{t b}\right)^{m+1}
$$

This again occurs because in cases where the tank bottom pressure is specified $\delta \mathrm{P}^{\mathrm{tb}}$ is identically zero while for cases where the flow rate of component $k$ is specified the coefficients $\left(C_{k}\right)_{n z b+1 / 2}^{n}$ in the velocity relationship are identically zero.

Difference equations for the last mass/energy cell in the bottom section can be combined into the matrix representation

$$
\mathrm{cm}^{m}\left[\begin{array}{l}
\left(\delta P^{b}\right)_{n z b}^{m+1} \\
\left(\delta \alpha^{b}\right)_{n z b}^{m+1}
\end{array}\right]
$$

where $\mathrm{cm}^{\mathrm{m}}$ is a $5 \times 5$ matrix of coefficient values, $\mathrm{cp}^{\mathrm{n}}$ is a 5 element vector of pressure coupling coefficients, and $\mathrm{f}^{\mathrm{m}}$ is the 5 element vector of right hand side terms. Formulation of the mass and energy finite difference equations and inversion of the $\mathrm{cm}^{\mathrm{m}}$ matrix are programmed in subroutines JACOBB. 


\subsection{Constitutive Relations}

Computational implementations of several constitutive relations have already been discussed in section 2 for convenience. Some remaining issues applicable to all constitutive models are discussed in this section.

State property and velocity evaluations: Because the FLOWTRAN-TF mesh is staggered, velocities are directly available only at cell faces while state properties are defined at the cell centers. In general, constitutive models require property and velocity input for their evaluation. The wall heat transfer rate is defined directly at the fluid cell centers by using cell center properties and averaging the velocities at the cell faces. Wall drag is defined at cell faces by making use of face velocities directly but averaged properties. Similarly, interfacial drag, heat transfer and mass transfer coefficients are defined on a cell face basis. Interfacial subchannel mixing rates are defined on a cell center basis.

Numerically well-posed evaluations: All of the constitutive term models are required to be computationally well-posed for all conceivable parameter values. For example the physical laminar friction factor is $64 / \mathrm{Re}$. In order to avoid a divide by zero and ill-conditioning, the computational laminar friction factor is defined to be

$$
f_{L}=\frac{64}{\max (\operatorname{Re}, 50)}
$$

as given by (2.5.2.1-13). The modifications needed to make the physical models computationally well-posed are typically obvious. Rather than list the numerous small modifications needed, the reader is referred to the FLOWTRAN-TF source code for more detail. 


\subsection{Boundary Conditions}

Nearly all of the fluid finite difference equations in FLOWTRAN-TF have been presented in section 3.4. This development report discusses the remaining finite difference equations. These involve boundary condition options and their computational implementation. Specifically, fluid momentum finite difference expressions are needed at the cell faces between the Plenum and Top Section and between the Bottom Section and Tank Bottom for various boundary condition options. The various boundary condition options are discussed in section 2.6

\section{Option \#1, prescribed pressure and void fraction}

Figure 3.6-1 shows the momentum computational cell for which finite differenced momentum equations are needed. The gas and liquid finite differenced momentum balances given in section 3.4 are generally applicable to the inlet momentum cell shown in Figure 3.6-1. Special expressions for quantities at $j=-1 / 2$ are needed however. The development of such expressions is presented for the gas momentum balance with the understanding that the liquid momentum balance is handled analogously.

The mean phasic area associated with the pressure term in the gas momentum balance when $\mathrm{u}_{\mathrm{g}, 1 / 2}>0$ is given in section 3.4 as

$$
\langle\alpha \mathrm{A}\rangle_{1 / 2}=\frac{2\left(\hat{\alpha} \mathrm{A}_{\mathrm{cz}}\right)_{-1 / 2}\left(\hat{\alpha} \mathrm{A}_{\mathrm{cz}}\right)_{1 / 2}}{\left[\left(\hat{\alpha} \mathrm{A}_{\mathrm{cz}}\right)_{-1 / 2}+\left(\hat{\alpha} \mathrm{A}_{\mathrm{cz}}\right)_{1 / 2}\right]}
$$

Because a mass/energy cell beyond the Plenum cell is not available, the above term is undefined by section 3.4 logic. For lack of a better choice, define

$$
\left(\hat{\alpha} A_{c z}\right)_{-1 / 2} \equiv\left(\hat{\alpha} A_{c z}\right)_{1 / 2}=\alpha_{p l} A_{c z, 1 / 2}
$$

Then

$$
\langle\alpha \mathrm{A}\rangle_{1 / 2}=\alpha_{\mathrm{pl}} \mathrm{A}_{\mathrm{cz}, 1 / 2}
$$

When $u_{g, 1 / 2}<0$ the quantities needed are defined by section 3.4 .

To evaluate the gas momentum advection term the cell 0 (Plenum) gas velocity must always be computed regardless of velocity sign according to

$$
\left(\tilde{u}_{\mathrm{g}}\right)_{0}=\frac{\left(\mathrm{A}_{\mathrm{cz}} \hat{\alpha} \hat{\rho}_{\mathrm{g}} \mathrm{u}_{\mathrm{g}}\right)_{-1 / 2}\left(\mathrm{~A}_{\mathrm{cz}}\right)_{-1 / 2}+\left(\mathrm{A}_{\mathrm{cz}} \hat{\alpha} \hat{\rho}_{\mathrm{g}} \mathrm{u}_{\mathrm{g}}\right)_{1 / 2}\left(\mathrm{~A}_{\mathrm{cz}}\right)_{1 / 2}}{\left[\left(\mathrm{~A}_{\mathrm{cz}} \hat{\alpha} \hat{\rho}_{\mathrm{g}}\right)_{-1 / 2}+\left(\mathrm{A}_{\mathrm{cz}} \hat{\alpha} \hat{\rho}_{\mathrm{g}}\right)_{1 / 2}\right]\left(\mathrm{A}_{\mathrm{zz}}\right)_{0}}
$$

as discussed in section 3.4. Again for lack of a better alternative, define 


$$
\begin{gathered}
\cdot\left(A_{c z} \hat{\alpha} \hat{\rho}_{g} u_{g}\right)_{-1 / 2} \equiv\left(A_{c z} \hat{\alpha} \hat{\rho}_{g} u_{g}\right)_{1 / 2} \\
\left(A_{c z} \hat{\alpha} \hat{\rho}_{g}\right)_{-1 / 2} \equiv\left(A_{c z} \hat{\alpha} \hat{\rho}_{g}\right)_{1 / 2} \\
\left(A_{c z}\right)_{-1 / 2} \equiv\left(A_{c z}\right)_{1 / 2}
\end{gathered}
$$

Then

$$
\left(\tilde{u}_{\mathrm{g}}\right)_{0}=\frac{\left(\mathrm{A}_{\mathrm{cz}} \mathrm{u}_{\mathrm{g}}\right)_{1 / 2}}{\left(\mathrm{~A}_{\mathrm{zz}}\right)_{0}}
$$

If $\left(\tilde{u}_{g}\right)_{0}<-v_{\min }(\omega=0)$ then the quantities needed to compute the advection term have already been defined in section 3.4. For $\left(\tilde{u}_{g}\right)_{0}>-v_{\min }(\omega>0)$ quantities at face $j=-1 / 2$

$$
\begin{gathered}
\left(A_{c z} \hat{\alpha} \hat{\rho}_{g} u_{g}\right)_{-1 / 2} \equiv\left(A_{c z} \hat{\alpha} \hat{\rho}_{g} u_{g}\right)_{1 / 2} \\
\left(A_{c z} \hat{\alpha} \hat{\rho}_{g} u_{g} u_{g}\right)_{-1 / 2} \equiv\left(A_{c z} \hat{\alpha} \hat{\rho}_{g} u_{g} u_{g}\right)_{1 / 2}
\end{gathered}
$$

Then

$$
\begin{gathered}
\left\langle A_{z z} \alpha \rho_{g} u_{g}\right\rangle_{0}=\left(A_{c z} \hat{\alpha} \hat{\rho}_{g} u_{g}\right)_{1 / 2} \\
\left\langle A_{z z} \alpha \rho_{g} u_{g} u_{g}\right\rangle_{0}=\left(A_{c z} \hat{\alpha} \hat{\rho}_{g} u_{g} u_{g}\right)_{1 / 2}
\end{gathered}
$$

and the advection term is computed as discussed in section 3.4. For the usual case of positive velocities the above assumptions are equivalent to neglecting the advection term. Except for the above modifications, the gas momentum balance at $j=-1 / 2$ is computed as described in section 3.4 and the overall momentum balance takes the form

$$
a_{11}^{z} u_{g}+a_{12}^{2} u_{f}=b_{1}^{z}+c_{1}^{2}\left(P_{p l}-P_{t 1}\right)
$$

where $P_{p l}$ is the Plenum pressure and $P_{t l}$ is the pressure in the first Top Section cell. The liquid momentum balance is handled in an analogous manner to the gas balance. 


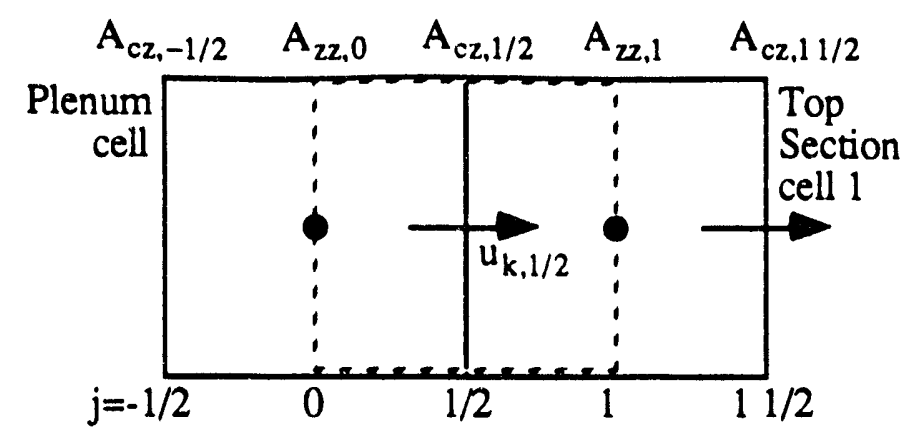

Figure 3.6-1 Momentum computational cell at inlet plenum.

\section{Options \#2-4, prescribed liquid and/or gas volumetric nowrate}

The gas flowrate at $j=1 / 2$ is computed as

$$
Q_{g, 1 / 2}=\left(A_{c z} \hat{\alpha} u_{g}\right)_{1 / 2}
$$

where

$$
\hat{\alpha}_{1 / 2}=\omega\left(u_{g, 1 / 2}\right) \alpha_{p l}+\left(1-\omega\left(u_{g, 1 / 2}\right)\right) \alpha_{t 1}
$$

and

$$
\omega(v)=\left\{\begin{array}{cc}
1 & v \geq v_{\min } \\
\frac{1}{2}\left(1+\frac{v}{v_{\min }}\right) & v_{\min }>v>-v_{\min } \\
0 & v \leq-v_{\min }
\end{array}\right.
$$

In equation (3.6-15), $\alpha_{\mathrm{pl}}$ is the Plenum void fraction and $\alpha_{11}$ is the void fraction in the first Top Section cell. Let $\mathrm{Q}_{\mathrm{g}, \mathrm{bc}}>0$ denote the desired inlet gas flowrate and define

$$
Q_{\text {g.pl }} \equiv A_{c z, 1 / 2} \alpha_{p l} v_{\min }
$$

If $Q_{g, b c} \geq Q_{g, p l}$ then the required gas velocity is defined by

$$
\left(A_{c z, 1 / 2} \alpha_{p l}\right) \cdot u_{g, 1 / 2}=Q_{g, b c}
$$

Otherwise $Q_{g, b c}<Q_{g, p l}$ and the desired gas velocity is determined from

$$
\mathrm{Q}_{\mathrm{g}, \mathrm{bc}}=\frac{\left(\mathrm{A}_{\mathrm{cz}} \mathrm{u}_{\mathrm{g}}\right)_{1 / 2}}{2}\left[\left(1+\frac{\mathrm{u}_{\mathrm{g}, 1 / 2}}{\mathrm{v}_{\min }}\right) \alpha_{\mathrm{pl}}+\left(1-\frac{\mathrm{u}_{\mathrm{g}, 1 / 2}}{\mathrm{v}_{\min }}\right) \alpha_{\mathrm{tl}}\right]
$$


Equations (3.6-18) and (3.6-19) still yield velocity expressions of the general form given by equation (3.6-13). The coefficients are defined as follows

$$
\begin{aligned}
& a_{11}^{z}=\left\{\begin{array}{cc}
A_{c z, 1 / 2} \alpha_{p l} & 0<Q_{g, b c} \geq Q_{g, p l} \\
A_{c z, 1 / 2}\left(\frac{\alpha_{p l}+\alpha_{t 1}}{2}\right) & 0<Q_{g, b c}<Q_{g, p l} \text { and } \alpha_{p l}=\alpha_{t 1} \\
\frac{A_{c z, 1 / 2}}{v_{\min }}\left(\alpha_{p l}-\alpha_{t 1}\right) & 0<Q_{g, b c}<Q_{g, p l} \text { and } \alpha_{p l} \neq \alpha_{t 1}
\end{array}\right. \\
& a_{12}^{2}=0 \\
& b_{1}^{z}=\left\{\begin{array}{cc} 
& 0<Q_{g, b c} \geq Q_{8, p l}, \text { or } \\
Q_{g, b c} & 0<Q_{g, b c} \geq Q_{g, p l} \text { and } \\
-A_{c z, 1 / 2}\left(\frac{\alpha_{p l}+\alpha_{t 1}}{2}\right) & \alpha_{p l}=\alpha_{t l} \\
+\left[\left(A_{c z, 1 / 2} \frac{\alpha_{p l}+\alpha_{t 1}}{2}\right)^{2}+\frac{2 A_{c z, 1 / 2}\left(\alpha_{p l}-\alpha_{t 1}\right)}{v_{\min }} Q_{g, b c}\right]^{1 / 2} & \text { otherwise }
\end{array}\right. \\
& c_{1}^{2}=0
\end{aligned}
$$

A prescribed liquid flowrate boundary condition is imposed in an analogous manner with the liquid phase coefficients being defined by

$$
\begin{gathered}
a_{21}^{z}=0 \\
a_{22}^{2}=\left\{\begin{array}{cc}
A_{c z, 1 / 2}\left(1-\alpha_{p l}\right) & 0<Q_{f, b c} \geq Q_{f, p l} \\
A_{c z, 1 / 2}\left[\frac{\left(1-\alpha_{p l}\right)+\left(1-\alpha_{t 1}\right)}{2}\right] & 0<Q_{f, b c}<Q_{f, p l} \text { and } \alpha_{p l}=\alpha_{t 1} \\
\frac{A_{c z, 1 / 2}}{v_{\min }}\left[\left(1-\alpha_{p l}\right)-\left(1-\alpha_{t 1}\right)\right] & 0<Q_{f, b c}<Q_{f, p l} \text { and } \alpha_{p l} \neq \alpha_{t 1}
\end{array}\right.
\end{gathered}
$$




$$
\begin{aligned}
& \begin{cases} & 0<Q_{\mathrm{f}, b c} \geq \mathrm{Q}_{\mathrm{f}, \mathrm{pl}} \text {, or } \\
\mathrm{Q}_{\mathrm{f}, \mathrm{bc}} & 0<\mathrm{Q}_{\mathrm{f}, \mathrm{bc}} \geq \mathrm{Q}_{\mathrm{f}, \mathrm{pl}} \text { and }\end{cases} \\
& b_{2}^{2}=\left\{\begin{array}{l}
-A_{c z, 1 / 2}\left[\frac{\left(1-\alpha_{p l}\right)+\left(1-\alpha_{t 1}\right)}{2}\right] \\
{\left[\left(A_{c z, 1 / 2} \frac{\left(1-\alpha_{p l}\right)+\left(1-\alpha_{t 1}\right)}{2}\right)^{2}\right]^{1 / 2} \quad \text { otherwise }}
\end{array}\right. \\
& \alpha_{\mathrm{pl}}=\alpha_{\mathrm{t} 1} \\
& +\left[\begin{array}{c}
2 \\
+2 A_{c z, 1 / 2} \frac{\left(1-\alpha_{p l}\right)-\left(1-\alpha_{11}\right)}{v_{\min }} Q_{f, b c}
\end{array}\right] \\
& c_{2}^{2}=0
\end{aligned}
$$

where

$$
Q_{\mathrm{f}, \mathrm{pl}} \equiv A_{\mathrm{cz}, 1 / 2}\left(1-\alpha_{\mathrm{pl}}\right) \mathbf{v}_{\min }
$$

The prescribed liquid flowrate $Q_{f, b c}$ is required to be greater than zero. Table 3.6-I summarizes the definitions of the various gas and liquid coefficients as a function of user boundary condition option.

Table 3.6-I Summary of linear velocity expression coefficients as a function of boundary condition option.

\begin{tabular}{|c|c|c|}
\hline $\begin{array}{c}\text { Boundary condition } \\
\text { at Plenum cell }\end{array}$ & $\begin{array}{c}\text { Gas coefficients } \\
\mathrm{a}_{11}^{2}, \mathrm{a}_{12}^{2}, \mathrm{~b}_{1}^{2}, \mathrm{c}_{1}^{2}\end{array}$ & $\begin{array}{c}\text { Liquid coefficients } \\
\mathrm{a}_{21}^{2}, \mathrm{a}_{22}^{2}, \mathrm{~b}_{2}^{2}, \mathrm{c}_{2}^{2}\end{array}$ \\
\hline $\begin{array}{c}\text { Option 1 } \\
\text { "Prescribed pressure and void" }\end{array}$ & $\begin{array}{c}\text { Gas momentum balance: } \\
\text { see section 3.4 }\end{array}$ & $\begin{array}{c}\text { Liquid momentum balance: } \\
\text { see section 3.4 }\end{array}$ \\
\hline $\begin{array}{c}\text { Option 2 } \\
\text { "Prescribed liquid flowrate" }\end{array}$ & $\begin{array}{c}\text { Gas momentum balance: } \\
\text { see section 3.4 }\end{array}$ & $\begin{array}{c}\text { Equations (3.6-24) through } \\
(3.6-27)\end{array}$ \\
\hline $\begin{array}{c}\text { Option 3 } \\
\text { "Prescribed gas flowrate" }\end{array}$ & $\begin{array}{c}\text { Equations (3.6-20) through } \\
(3.6-23)\end{array}$ & $\begin{array}{c}\text { Liquid momentum balance: } \\
\text { see section 3.4 }\end{array}$ \\
\hline $\begin{array}{c}\text { Option 4 } \\
\text { "Prescribed liquid and gas } \\
\text { flowrate" }\end{array}$ & $\begin{array}{c}\text { Equations (3.6-20) through } \\
(3.6-23)\end{array}$ & $\begin{array}{c}\text { Equations (3.6-24) through } \\
(3.6-27)\end{array}$ \\
\hline
\end{tabular}




\section{Horizontal, stratified flow}

Figure 3.6-2 illustrates the pressure profile in a horizontal stratified two-phase flow. The flow is assumed to be one-dimensional and the properties constant. The pressures shown in Figure 3.6-2 are defined as

$$
\begin{aligned}
& \mathrm{P}_{\mathrm{i}} \equiv \text { interface pressure } \\
& \overline{\mathrm{P}}_{\mathrm{g}} \equiv \text { average pressure in gas phase } \\
& \overline{\mathrm{P}}_{\mathrm{f}} \equiv \text { average pressure in liquid phase }
\end{aligned}
$$

Assuming that the transverse pressure gradients in the gas and liquid are effectively due to hydrostatic head yields

$$
\begin{aligned}
& \bar{P}_{g}=P_{i}-\frac{1}{2} \rho_{g} g \alpha_{g} H \\
& \bar{P}_{f}=P_{i}+\frac{1}{2} \rho_{f} g \alpha_{f} H
\end{aligned}
$$

where

$$
\begin{aligned}
& \alpha_{\mathrm{g}} \equiv \text { gas volume fraction (void fraction, } \alpha \text { ) } \\
& \alpha_{\mathrm{f}} \equiv \text { liquid volume fraction }(1-\alpha) \\
& \mathrm{H} \equiv \text { channel vertical height }
\end{aligned}
$$

The pressure force on the gas phase per unit volume for the control volume shown in Figure $3.6-2$ is

$$
\frac{F_{g}}{V}=\frac{\left(\bar{P}_{g 1} \alpha_{g 1}-\bar{P}_{g 2} \alpha_{g 2}\right) A}{V}-\frac{\left(P_{i 1}+P_{i 2}\right)}{2} \frac{\left(\alpha_{g 1}-\alpha_{g 2}\right) A}{V}
$$

In the limit at $\Delta z$ goes to zero

$$
\lim _{\Delta z \rightarrow 0} \frac{F_{g}}{V}=\frac{\partial \bar{P}_{g} \alpha_{g}}{\partial z}-P_{i} \frac{\partial \alpha_{g}}{\partial z}
$$

Similarly for the liquid phase

$$
\lim _{\Delta z \rightarrow 0} \frac{F_{f}}{V}=\frac{\partial \bar{P}_{f} \alpha_{f}}{\partial z}-P_{i} \frac{\partial \alpha_{f}}{\partial z}
$$

The pressure force per unit volume on the mixture is the sum of equations $(3.6-32)$ and (3.6-33): 


$$
\lim _{\Delta z \rightarrow 0} \frac{F}{V}=\frac{\partial}{\partial z}\left(\bar{P}_{g} \alpha_{g}+\bar{P}_{f} \alpha_{f}\right)
$$

Equation (3.6-34) motivates defining a mixture average pressure as

$$
\overline{\mathrm{P}} \equiv \alpha_{\mathrm{g}} \overline{\mathrm{P}}_{\mathrm{g}}+\alpha_{\mathrm{f}} \overline{\mathrm{P}}_{\mathrm{f}}
$$

In terms of the mixture average pressure, the interface, average gas, and average liquid pressures are expressed as

$$
\begin{gathered}
P_{i}=\bar{P}+\frac{1}{2} \rho_{g} g \alpha_{g}^{2} H-\frac{1}{2} \rho_{f} g \alpha_{f}^{2} H \\
\bar{P}_{g}=\bar{P}-\frac{1}{2} \rho_{g} g \alpha_{g} \alpha_{f} H-\frac{1}{2} \rho_{f} g \alpha_{f}^{2} H \\
\bar{P}_{f}=\bar{P}+\frac{1}{2} \rho_{g} g \alpha_{g}^{2} H+\frac{1}{2} \rho_{f} g \alpha_{g} \alpha_{f} H
\end{gathered}
$$

Substituting equations (3.6-36)-(3.6-38) into equations (3.6-32) and (3.6-33) yields

$$
\begin{aligned}
& \lim _{\Delta z \rightarrow 0} \frac{F_{g}}{V}=\alpha_{g} \frac{\partial \bar{P}}{\partial z}+\Delta p g H \alpha_{g} \alpha_{f} \frac{\partial \alpha_{g}}{\partial z} \\
& \lim _{\Delta z \rightarrow 0} \frac{F_{f}}{V}=\alpha_{f} \frac{\partial \bar{P}}{\partial z}-\Delta p g H \alpha_{g} \alpha_{f} \frac{\partial \alpha_{g}}{\partial z}
\end{aligned}
$$

By comparison, the same quantities for a nonstratified two-phase flow are

$$
\begin{aligned}
& \lim _{\Delta z \rightarrow 0} \frac{F_{g}}{V}=\alpha_{g} \frac{\partial \bar{P}}{\partial z} \\
& \lim _{\Delta z \rightarrow 0} \frac{F_{f}}{V}=\alpha_{f} \frac{\partial \bar{P}}{\partial z}
\end{aligned}
$$

Therefore, the phasic momentum balances are extended to horizontal two-phase flow through the addition of a void gradient term. Specifically, moving terms (3.6-39) and (3.6-40) to the r.h.s. of the respective z-direction momentum balance (i.e. change the sign), replacing $\alpha_{g}$ with $\alpha$ and $\alpha_{f}$ with $(1-\alpha)$ yields the following momentum balances (see section 2.4.1)

$$
\alpha \rho_{g} \frac{\partial u_{g}}{\partial t}+\alpha p_{g} v_{g} \cdot \nabla u_{g}=-\alpha \frac{\partial \bar{P}}{\partial z}-\Delta \rho g H \alpha(1-\alpha) \frac{\partial \alpha}{\partial z}-F_{w_{g}}^{z}-F_{i}^{z}
$$




$$
(1-\alpha) \rho_{\mathrm{f}} \frac{\partial u_{\mathrm{f}}}{\partial \mathrm{t}}+(1-\alpha) \rho_{\mathrm{f}} \mathbf{v}_{\mathrm{f}} \cdot \nabla \mathrm{u}_{\mathrm{f}}=-(1-\alpha) \frac{\partial \overline{\mathrm{p}}}{\partial \mathrm{z}}+\Delta \rho g H \alpha(1-\alpha) \frac{\partial \alpha}{\partial z}-F_{w f}^{z}+F_{i}^{z}
$$

Note that the inter-subchannel mixing terms $\mathbf{M}_{\mathrm{tg}}$ and $\mathbf{M}_{\mathrm{tf}}$ do not appear in the onedimensional momentum balances. See sections 2.4.1 and 2.5.8 for more information. Also the $\mathbf{z}$-component of the gravitational vector $\mathbf{g}$ is zero for a horizontal momentum face so the gravitational body force terms do not appear either.

The liquid momentum balance given by $(3.6-44)$ reduces to a weir flow relationship under the following conditions

- Negligible inertial terms in the momentum balance (steady-state; 1.h.s. =0)

- Negligible interfacial shear $\left(F_{i}^{2}=0\right)$

- Constant properties

- Negligible gas density compared to liquid density

- Form losses only

- Positive velocities

The resulting weir flow relationship is

$$
Q_{\mathrm{f}}=\sqrt{\frac{2 \mathrm{~g}}{\mathrm{~K}^{2}}} \mathrm{LH}_{\mathrm{f}}^{3 / 2}
$$

where

$$
\begin{aligned}
Q_{\mathrm{f}} & \equiv \text { liquid volumetric flowrate } \\
\mathrm{K}^{\mathrm{z}} & \equiv \text { form loss coefficient } \\
\mathrm{L} & \equiv \text { channel width (area A divided by height } \mathrm{H} \text { ) } \\
\mathrm{H}_{\mathrm{f}} & \equiv \text { upstream (Plenum) liquid level }\left(\alpha_{\mathrm{f}} \mathrm{H}\right)
\end{aligned}
$$

In order that the finite differenced momentum equations also yield the weir flow relationship given by (3.6-45), the void gradient terms must be computationally evaluated as

$$
\begin{aligned}
& -\Delta \rho_{p l} g H \alpha_{p l}\left(1-\alpha_{p l}\right) \frac{\left(1-\alpha_{p l}\right) A_{c z, 1 / 2}}{V_{z, 1 / 2}} \\
& +\Delta p_{p l} g H \alpha_{p l}\left(1-\alpha_{p l}\right) \frac{\left(1-\alpha_{p l}\right) A_{c z, 1 / 2}}{V_{z, 1 / 2}}
\end{aligned}
$$

Note that only the upstream Plenum void fraction appears and that for the purpose of evaluating $\partial \alpha \partial z$, the downstream void fraction is assumed to be one. The quantity 


$$
\frac{A_{c z, 1 / 2}}{V_{z, 1 / 2}}
$$

was chosen to achieve compatibility with the finite differenced pressure gradient term (see section 3.4). Unfortunately, the differencing scheme given by equations (3.6-46) may yield non-physical results because

$$
\frac{\partial \alpha}{\partial z} \rightarrow \frac{\left(1-\alpha_{p l}\right) A_{c z, 1 / 2}}{V_{z, 1 / 2}}
$$

is not always a good approximation. To remedy this problem equations (3.6-46) are modified as

$$
\begin{aligned}
& -\Delta \rho_{p l} g H \alpha_{p l}\left(1-\alpha_{p l}\right) \frac{\left(\alpha_{t 1}-\alpha_{p l}\right) A_{c z, 1 / 2}}{V_{z, 1 / 2}} \\
& +\Delta p_{p l} g H \alpha_{p l}\left(1-\alpha_{p l}\right) \frac{\left(\alpha_{t 1}-\alpha_{p l}\right) A_{c z, 1 / 2}}{V_{z, 1 / 2}}
\end{aligned}
$$

Note that equations (3.6-46) and (3.6-47) have different signs when $\alpha_{\mathrm{tl}}<\alpha_{\mathrm{pl}}$. For this situation, equations (3.6-46) are clearly aphysical. Equations (3.6-47) no longer rigorously satisfy the weir flow relationship but are close whenever the top section cell \#1 void is near 1.

Two schemes for treating the void gradient terms are available to the user. The first approach is to use equations (3.6-47) under all conditions. The second is to use equations (3.6-46) whenever $\alpha_{11}>\alpha_{\mathrm{pl}}$ and equations (3.6-47) otherwise. This latter choice appears to be numerically well-behaved as opposed to straight use of equations (3.6-46). The first choice is the more physically accurate and well-behaved one. The second yields the exact weir flow relationship which is desirable for typical FLOWTRAN-TF applications.

In addition to including terms (3.6-46) in the momentum balances, additional modifications are needed to accurately model horizontal, stratified flow. The default flow regime logic developed for vertical flow (section 2.6.3) is overridden for horizontal flow by

$$
\begin{gathered}
\alpha<\alpha_{\text {strat }} \Rightarrow \text { dispersed flow (bubbly regime) } \\
\alpha \geq \alpha_{\text {strat }} \Rightarrow \text { separated flow (stratified) }
\end{gathered}
$$

where $\alpha_{\text {strat }}$ is a user specified ransition void fraction. A value of 0.05 is suggested. In the dispersed regime the default interfacial and wall drag models are used. For the separated regime, the vertical separated regime interfacial drag logic (section 2.6.5.2) is used except that the interfacial drag coefficient is replaced with a user specified value $C_{i, s t r a t}^{z}$. A value near zero is suggested so that the weir flow relationship given by $(3.6-45)$ is recovered. Specifically, the interfacial drag model for horizontal, separated flow is given completely by 


$$
F_{i}^{z}=C_{i, s t r a t}^{z}\left|u_{g}-u_{f}\right|\left(u_{g}-u_{f}\right)
$$

That is, wall drag repartitioning is not part of the separated flow model. The default wall form drag coefficient (section 2.5.1) is retained for horizontal, dispersed flow. For horizontal, stratified flow, the coefficient is replaced with a user specified coefficient $K_{\text {strat }}^{z}$ which should be set by the user to whatever value is required to reproduce a particular weir flow model. Having separate form loss coefficients for dispersed and separated flow permits accurate predictions over the entire range of flows.

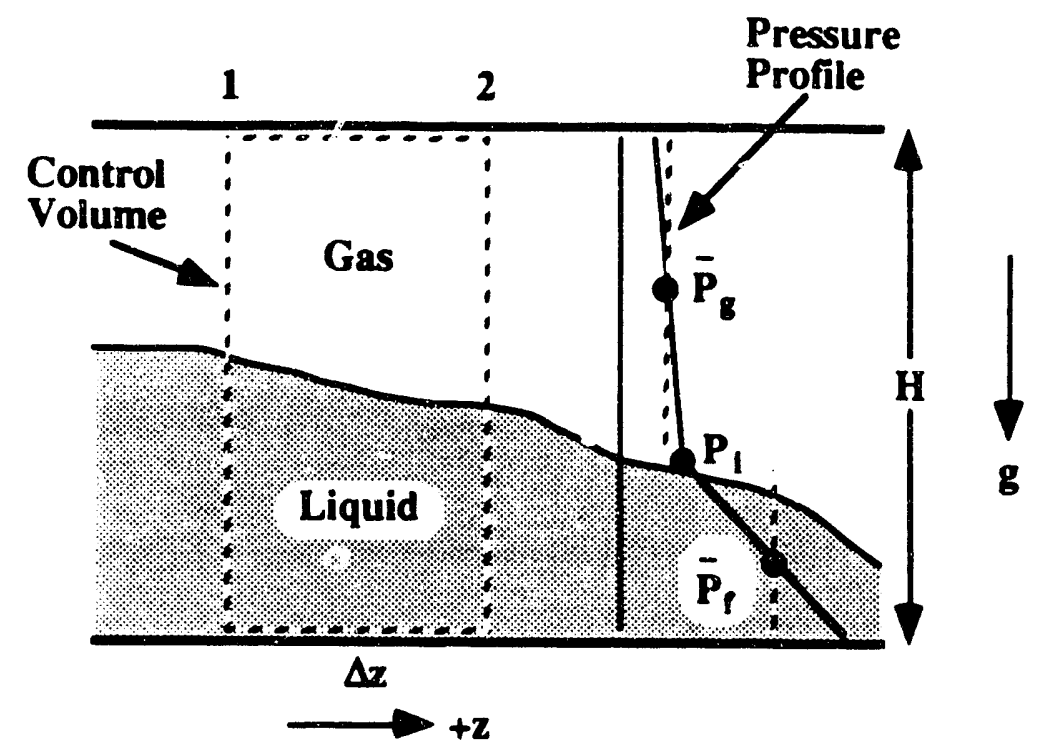

Figure 3.6-2 Control volume and nomenclature for horizontal, stratified flow.

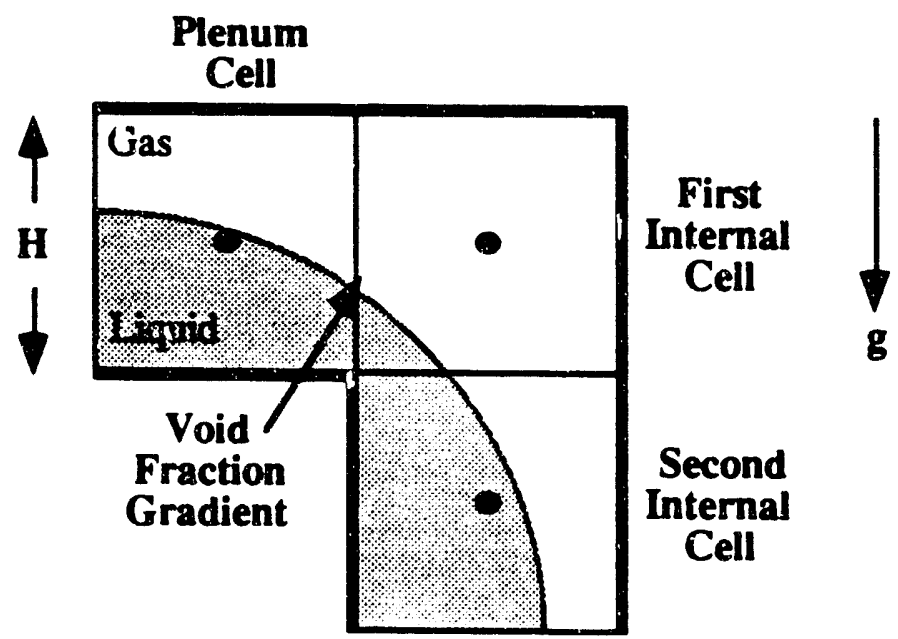

Figure 3.6-3 Horizontal, stratified flow at the cell face between the Plenum and Top Section. 


\section{Outlet boundary conditions}

The finite differenced phasic momentum balances at the face between the last Bottom Section mass/energy cell and the Tank Bottom cell are handled in an analogous manner to the inlet face equations.

\section{Approximate Plenum pressure correction options}

With most FLOWTRAN-TF applications it is very desirable to prescribe the liquid flowrate. An obvious method for accomplishing this is to choose boundary condition option \#2 "Prescribed liquid flowrate". As is, this choice is not generally recommended for two-phase flows because the liquid momentum balance at the inlet face is discarded. The gas and liquid flowrates are generally inconsistent because they do not correspond to a common inlet pressure. The following options for modifying b.c. option \#2 are now added to largely remove this inconsistency between flowrate and pressure.

The idea is to approximately back-out the correct Plenum pressure from the liquid momentum balance using old time values. The liquid momentum balance takes the form

$$
a_{21}^{z} u_{g}+a_{22}^{z} u_{f}=b_{2}^{z}+c_{2}^{z}\left(P_{p l}-P_{t 1}\right)
$$

The user has the option to override the user prescribed Plenum pressure at $n+1$ by

$$
P_{p l}^{n+1}=P_{11}^{n}+\left(a_{21}^{z} u_{g}+a_{22}^{z} u_{f}-b_{2}^{z}\right)^{n} /\left(c_{2}^{z}\right)^{n}
$$

for prescribed liquid flowrate cases. There are three choices dictated by a 'Plenum pressure update' flag i ppu in the input deck. For $i$ ppu $=0$ the Plenum pressure is defined by the user. For ippu $=1$, the user specified Plenum pressure is always replaced by equation (3.6-50). For ippu $=2$, the Plenum pressure is updated by equation (3.6-50) only when the inlet flow is dispersed $\left(\alpha_{\mathrm{pl}}<\alpha_{\text {stral }}\right)$. With these modifications the liquid momentum balance is satisfied approximately. The same treatment could be applied to b.c. option \#3, but, is not because current applications do not motivate it.

\section{Suggested approach for prescribing liquid flowrate}

With b.c. option $\# 2$ and ippu $>0$, the user is capable of prescribing liquid flowrate and still have gas and liquid flowrates which are nearly consistent. This is one recommended approach.

Another recommended approach is to construct the computational mesh such that the inlet momentum cell is horizontal (see Figure 3.6-3) and select boundary condition option \#1. Recall that the momentum balances nearly satisfy the weir flow relationship given by (3.6-45) for $\alpha \leq \alpha_{\text {strat. }}$. Therefore, the liquid flowrate is nearly directly related to the liquid level in the Plenum cell. Liquid flowrate can be effectively prescribed by solving (3.6-45) for the corresponding liquid height, or equivalently void fraction, and then specifying this void fraction for the Plenum cell. The plenum void fraction may be fine tuned to yield the precise flowrate desired. The advantage of this latter approach is that both momentum balances are solved at the inlet face, that is, more physics is retained. 
The liquid flowrate depends weakly on the void fraction in top section cell \#1. For some applications this void fraction will vary through the course of a transient calculation causing the liquid flowrate to vary mildly. If a constant liquid flowrate is desired, first the process described above using boundary condition option \#1 should be used to determine the plenum void fraction which is consistent with the desired flowrate and nominal top section cell \#1 void fraction. Then boundary condition option \#2 may be selected in conjunction with this plenum void fraction to set the liquid flowrate constant. Although the liquid momentum balance is not solved with b.c. option \#2, it will be nearly satisfied nonetheless because the specified inlet void fraction is nominally consistent with the specified liquid flowrate. 


\subsection{Phase Transition Logic}

Two-phase air-water flow is governed by the following set of 10 equations $(\$ 2.4 .1$ and $\S 2.5 .4 .2)$.

Gas momentum

$$
\alpha \rho_{g} \frac{\partial v_{g}}{\partial t}+\alpha \rho_{g} v_{g} \cdot \nabla v_{g}=-\alpha \nabla P+\alpha \rho_{g} g-F_{w_{g}}-F_{i}-M_{t g}
$$

Liquid momentum

$$
(1-\alpha) \rho_{\mathrm{f}} \frac{\partial \mathbf{v}_{\mathrm{f}}}{\partial \mathrm{t}}+(1-\alpha) \rho_{\mathrm{f}} \mathbf{v}_{\mathrm{f}} \cdot \nabla \mathbf{v}_{\mathrm{f}}=-(1-\alpha) \nabla \mathrm{P}+(1-\alpha) \rho_{\mathrm{f}} \mathbf{g}-\mathbf{F}_{\mathrm{wf}}+\mathbf{F}_{\mathrm{i}}-\mathbf{M}_{\mathrm{tf}}
$$

Gas mass

$$
\frac{\partial \alpha \rho_{g}}{\partial t}+\frac{1}{V} \nabla \cdot\left[V \alpha \rho_{g} v_{g}\right]=\Gamma-W_{t g}
$$

Liquid mass

$$
\frac{\partial(1-\alpha) \rho_{\mathrm{f}}}{\partial \mathrm{t}}+\frac{1}{\mathrm{~V}} \nabla \cdot\left[\mathrm{V}(1-\alpha) \rho_{\mathrm{f}} \mathbf{v}_{\mathrm{f}}\right]=-\Gamma-\mathrm{W}_{\mathrm{tf}}
$$

Air mass

$$
\frac{\partial \alpha \rho_{g} X_{a}}{\partial t}+\frac{1}{V} \nabla \cdot\left[V \alpha \rho_{g} X_{a} v_{g}\right]=-X_{a} W_{t g}
$$

Gas energy

$$
\frac{\partial \alpha \rho_{g} h_{g}}{\partial t}+\frac{1}{V} \nabla \cdot\left[V \alpha \rho_{g} h_{g} v_{g}\right]=\alpha \frac{\partial P}{\partial t}+E_{i}+Q_{w g}-Q_{t g}
$$

Liquid energy

$$
\frac{\partial(1-\alpha) \rho_{f} h_{f}}{\partial t}+\frac{1}{V} \nabla \cdot\left[V(1-\alpha) \rho_{f} h_{f} v_{f}\right]=(1-\alpha) \frac{\partial P}{\partial t}-E_{i}+Q_{w f}-Q_{t f}
$$

Interface vapor-liquid equilibrium

$$
0=T_{i}-T_{s a t}\left(y_{s i} P\right)
$$


Interface mass ransfer

$$
0=\Gamma\left(1-y_{s i}+\phi \Delta y_{s}\right)-K_{g} \Delta y_{s}
$$

Interface energy transfer

$$
0=\Gamma\left[\lambda_{i}+\phi\left(c_{p w} \Delta T_{f}-c_{p s} \Delta T_{g}\right)\right]+\left(H_{f i} \Delta T_{f}+H_{g i} \Delta T_{g}\right)
$$

The two momentum balances are vector equations in general yielding 4 scalar momentum equations. The 10 unknowns associated with the above equations are $v_{g}=\left\{u_{g}, w_{g}\right\}^{\mathrm{T}}$, $v_{f}=\left\{u_{f}, w_{f}\right\}^{T}, P, \alpha, T_{g}, T_{f}, X_{a}, T_{i}, y_{s i}$ and $\Gamma$. For single-phase liquid flow the equation/variable set is reduced to

$$
\begin{array}{ll}
\text { Liquid momentum } & \mathbf{v}_{\mathrm{f}}=\left\{\mathrm{u}_{\mathrm{f}}, \mathrm{w}_{\mathrm{f}}\right\}^{\mathrm{T}} \\
\text { Liquid mass } & \mathbf{P} \\
\text { Liquid energy } & \mathrm{T}_{\mathrm{f}}
\end{array}
$$

\begin{tabular}{|c|c|}
\hline $\begin{array}{l}\text { Gas momentum } \\
\text { Gas mass } \\
\text { Air mass } \\
\text { Gas energy }\end{array}$ & $\begin{array}{l}\mathbf{v}_{g}=\left\{u_{g}, w_{g}\right\}^{T} \\
P^{T} \\
X_{g} \\
T_{g}\end{array}$ \\
\hline
\end{tabular}

and for single-phase gas flow with air and water components the equation/variable set is

Note that when the $\alpha=0$ in the general two-phase flow equation set, the gas mass, gas momentum, gas energy and air mass balances each reduce to the trivial statement $0=0$. Also, the interface mass and energy transfer balances both imply $\Gamma=0$ because $\mathrm{K}_{\mathrm{g}}, \mathrm{H}_{\mathrm{gi}}$ and $\mathrm{H}_{\mathrm{fi}}=0$ when there is no interfacial area present. Therefore the number of independent, non-trivial equations has gone from 10 to 4 as should be the case. For $\alpha=1$ the number of non-trivial, independent equations is 5 .

The capability of modeling both single-phase and two-phase flows is expected of FLOWTRAN-TF. The full two-phase flow equation becomes singular at the singie-phase limits $\alpha=0,1$. For nearly single-phase flow the equation is non-singular, but, illconditioned. Numerically, the finite difference equations are singular for conditions sufficiently close to single-phase due to the finite precision of the calculations. Therefore additional logic is needed to numerically model single-phase and near single-phase flows beyond that associated with equations $(3.7-1-10)$.

One option would be to solve reduced sets of equations and unknowns identified above when the flow is single-phase. This option would require extensive additional coding to implement the reduced equations sets. Furthermore, it is not clear how to construct a numerically well-posed problem for nearly single-phase flows.

.The option chosen for FLOWTRAN-TF is essentially to develop substitute equations which replace singular ones under single-phase conditions. The full set of two-phase flow variables, $v_{g}=\left\{u_{g}, w_{g}\right\}^{T}, v_{f}=\left\{u_{f}, w_{f}\right\}^{T}, P, \alpha, T_{g}, T_{f}, X_{a}, T_{i}, y_{s i}$ and $\Gamma$, are solved for under single-phase conditions. The substitute equations effectively assign arbitrary prechosen values to those two-phase variables which lose meaning under single-phase conditions. For single-phase liquid flow the choices 


$$
\begin{gathered}
\mathrm{u}_{\mathrm{r}}=0 \\
\mathrm{w}_{\mathrm{r}}=0 \\
\mathrm{~T}_{\mathrm{B}}=\mathrm{T}_{\mathrm{i}} \\
\mathrm{X}_{\mathrm{a}}=0 \\
\mathrm{y}_{\mathrm{si}}=1 \\
\alpha=0(\Gamma=0)
\end{gathered}
$$

are made and for single-phase gas flow the selections are

$$
\begin{gathered}
u_{r}=0 \\
w_{r}=0 \\
T_{f}=T_{i} \\
y_{s i}=y_{s} \\
\alpha=1(\Gamma=0)
\end{gathered}
$$

The transition from two-phase to single-phase flow and vice versa is smoothly accomplished by blending the physical and substitute equations as single-phase flow is approached. By always solving 10 equations, the coding is simplified and a smooth transition between single-phase and two-phase flow is easy to define.

The following subsections summarize the modifications to the two-phase flow equation set to handle single-phase flow, discuss phase transitions in more detail, address verification issues, and give updated derivatives needed for Newton iterations. Detailed information on the development of the modifications is confined to FLOWTRAN-TF development report TF-36.

Axial momentum modifications: At both single-phase limits we choose to make the relative velocity given by

$$
u_{g}=\left(\frac{1-C_{0} \alpha}{1-\alpha}\right)^{n} u_{g}-\left(C_{0}\right)^{n} u_{f} \equiv C_{g}^{n} u_{g}-C_{f}^{n} u_{f}
$$

equal to zero. The choice is arbitrary but convenient. A well-posed gas momentum balance at the single-phase limit $\langle\alpha\rangle_{j+1 / 2}=0$ requires

$$
\frac{\partial F_{i}^{d z}}{\partial u_{r}} \neq 0
$$

and advection and mixing terms which are zero. The first requirement is implemented by changing the bubbly regime interfacial drag coefficient to 


$$
C_{i b}^{d z}=\frac{\max \left(\varepsilon_{\alpha 1}, \alpha_{b}\right)(1-\alpha)^{3} \Delta \rho g}{u_{g j, b}^{2}}
$$

where $\varepsilon_{\alpha 1}=0.001$ and augmenting one of the relative velocities in $\mathrm{F}_{i}^{\mathrm{dz}}$ as

$$
F_{i}^{d z}=C_{i}^{d z}\left[\left|u_{r}\right|+\varepsilon_{u}\right] u_{r}
$$

The advection and mixing terms both involve fluid cells other than the $\mathrm{j}$ and $\mathrm{j}+1$ cells. Therefore these terms can be non-zero even if $\langle\alpha\rangle_{j+1 / 2}=0$. The second requirement for a well-posed gas momentum equation is implemented by multiplying the advection and mixing terms with the factor

$$
\begin{aligned}
\varphi\left(\langle\alpha\rangle_{j+1 / 2}\right) & =\min \left[1, \max \left(0, \frac{\langle\alpha\rangle_{j+1 / 2}-\varepsilon_{\alpha 1}}{\varepsilon_{\alpha 2}-\varepsilon_{\alpha 1}}\right)\right] \\
& =\left\{\begin{array}{cc}
0 & \langle\alpha\rangle_{j+1 / 2} \leq \varepsilon_{\alpha 1} \\
\frac{\langle\alpha\rangle_{j+1 / 2}-\varepsilon_{\alpha 1}-\varepsilon_{\alpha 1}}{\varepsilon_{\alpha 1}} & <\langle\alpha\rangle_{j+1 / 2}<\varepsilon_{\alpha 2} \\
1 & \langle\alpha\rangle_{j+1 / 2} \geq \varepsilon_{\alpha 2}
\end{array}\right.
\end{aligned}
$$

where $\varepsilon_{\alpha 1}=0.001$ and $\varepsilon_{\alpha 2}=0.01$.

Analogous modifications are required for a well-posed liquid momentum balance at $\langle 1-\alpha\rangle_{j+1 / 2}=0$. The annular regime interfacial drag coefficient is changed to

$$
C_{i \mathrm{a}}^{\mathrm{dz}}=\mathrm{a}_{\mathrm{ia}}^{0} \mathrm{f}_{\mathrm{i}} \frac{1}{2} \rho_{\mathrm{g}}
$$

where $\mathrm{a}_{\mathrm{ia}}^{0}$ is a non-zero annular interfacial area concentration defined by

$$
a_{i \Omega}^{0}=\frac{P_{w}-8 \delta}{A_{c}}
$$

and equation (3.7-13) is again used. The advection and mixing terms are multiplied with the factor

$$
\begin{aligned}
\varphi\left(\langle 1-\alpha\rangle_{j+1 / 2}\right) & =\min \left[1, \max \left(0, \frac{\langle 1-\alpha\rangle_{j+1 / 2}-\varepsilon_{\alpha 1}}{\varepsilon_{\alpha 2}-\varepsilon_{\alpha 1}}\right)\right] \\
& =\left\{\begin{array}{cc}
\frac{\langle 1-\alpha\rangle_{j+1 / 2}-\varepsilon_{\alpha 1}}{\varepsilon_{\alpha 2}-\varepsilon_{\alpha 1}} & \varepsilon_{\alpha 1}<\langle 1-\alpha\rangle_{j+1 / 2}<\varepsilon_{\alpha 1} \\
1 & \langle 1-\alpha\rangle_{j+1 / 2} \geq \varepsilon_{\alpha 2}
\end{array}\right.
\end{aligned}
$$


The need for the above modifications is demonstrated in FLOWTRAN-TF development report TF-36.

Azimuthal momentum modifications: The azimuthal momentum balances are modified in an analogous manner to the axial momentum balances. The interfacial drag coefficient $C_{i}^{x}$ is already non-zero at each single-phase limit so no changes are needed. The interfacial drag term is changed to

$$
F_{i}^{d x}=C_{i}^{d x}\left[\left|w_{r}\right|+\varepsilon_{u}\right] w_{r}
$$

and both the advection and mixing terms in the gas and liquid momentum balances are multiplied by

$$
\varphi\left(\langle\alpha\rangle_{i+1 / 2}\right)=\min \left[1, \max \left(0, \frac{\langle\alpha\rangle_{i+1 / 2}-\varepsilon_{\alpha 1}}{\varepsilon_{\alpha 2}-\varepsilon_{\alpha 1}}\right)\right]
$$

and

$$
\varphi\left(\langle 1-\alpha\rangle_{i+1 / 2}\right)=\min \left[1, \max \left(0, \frac{\langle 1-\alpha\rangle_{i+1 / 2}-\varepsilon_{\alpha 1}}{\varepsilon_{\alpha 2}-\varepsilon_{\alpha 1}}\right)\right]
$$

respectively.

Mass/energy modifications: The following composite function is useful for smoothly introducing modifications to the mass/energy equations as the flow transitions from twophase to single-phase conditions

$$
\varphi\left(x, x_{1}, x_{2}\right) \equiv \varphi_{x}=\left\{\begin{array}{cc}
3\left(\frac{x-x_{1}}{x_{2}-x_{1}}\right)^{2}-2\left(\frac{x-x_{1}}{x_{2}-x_{1}}\right)^{3} & x \leq x_{1} \\
1 & x<<x_{2} \\
& x \geq x_{2}
\end{array}\right.
$$

The function and first derivative are continuous. Continuity of the first derivative is required for Newton iterations.

For single-phase liquid flow we choose to set the air mass fraction $X_{\mathrm{a}}$ equal to zero. This is accomplished by introducing a new air sink term to the right hand side of equation $(3.7-5)$ defined by

$$
\Gamma_{\mathrm{a}}=\left(1-\varphi_{\alpha}\right) \varepsilon_{\Gamma_{\mathrm{a}}}\left(0-\mathrm{X}_{\mathrm{a}}\right)=-\left(1-\varphi_{\alpha}\right) \varepsilon_{\Gamma_{\mathrm{a}}} \mathrm{X}_{\mathrm{a}}
$$

where $\varphi_{\alpha} \equiv \varphi\left(\alpha, 0, \varepsilon_{\alpha}\right)$ and $\varepsilon_{\Gamma}=1.0$. Note that the term is zero for $\alpha>\varepsilon_{\alpha}$. For physical consistency, term $(3.7-22)$ is added to the gas mass balance and $\mathrm{Ea} \equiv \Gamma_{\mathrm{a}} \mathrm{h}_{\mathrm{a}}$ is added to the gas energy balance.

For single-phase liquid flow, we choose $y_{s i}=1$ by making $K_{g}$ non-zero at $\alpha=0$. We choose $T_{g}=T_{i}$ by making $H_{g i}$ non-zero at $\alpha=0$. For single-phase gas flow, we choose 
$\mathrm{y}_{\mathrm{si}}=\mathrm{y}_{\mathrm{s}}$ by making $\mathrm{K}_{\mathrm{g}}$ non-zero at $\alpha=1$. We choose $T_{\mathrm{f}}=\mathrm{T}_{\mathrm{i}}$ by making $\mathrm{H}_{\mathrm{fi}}$ non-zero at $\alpha=1$. Additional changes are needed to enable superheated single-phase liquid to flash and subcooled single-phase gas to condense. The modifications are to make $\mathrm{H}_{\mathrm{fi}}$ non-zero when $T_{f}>T_{i}$ and $H_{g i}$ non-zero when $T_{g}<T_{i}$. Specifically, the interfacial heat and mass transfer coefficients are redefined by

$$
\begin{aligned}
& \mathrm{K}_{\mathrm{g}}=\varphi_{\alpha} \varphi_{1-\alpha} \mathrm{K}_{\mathrm{g}}^{*}+\left(1-\varphi_{\alpha}\right) \mathrm{K}_{\mathrm{g}}^{0}+\left(1-\varphi_{1-\alpha}\right) \mathrm{K}_{\mathrm{g}}^{1} \\
& \mathrm{H}_{\mathrm{gi}}=\varphi_{\alpha}\left[1-\varphi_{\mathrm{T}_{\mathrm{i}}-\mathrm{T}_{\mathrm{g}}}\left(1-\varphi_{1-\alpha}\right)\right] \mathrm{H}_{\mathrm{gi}}^{*}+\varphi_{\mathrm{T}_{\mathrm{i}}-\mathrm{T}_{\mathrm{g}}}\left(1-\varphi_{1-\alpha}\right) \mathrm{H}_{\mathrm{gi}}^{1}+\left(1-\varphi_{\alpha}\right) \mathrm{H}_{\mathrm{gi}}^{0} \\
& \mathrm{H}_{\mathrm{fi}}=\varphi_{1-\alpha}\left[1-\varphi_{\mathrm{T}_{\mathrm{f}}-\mathrm{T}_{\mathrm{i}}}\left(1-\varphi_{\alpha}\right)\right] \mathrm{H}_{\mathrm{fi}}^{*}+\varphi_{\mathrm{T}_{\mathrm{f}}-\mathrm{T}_{\mathrm{i}}}\left(1-\varphi_{\alpha}\right) \mathrm{H}_{\mathrm{fi}}^{0}+\left(1-\varphi_{1-\alpha}\right) \mathrm{H}_{\mathrm{fi}}^{1}
\end{aligned}
$$

where

$$
\begin{aligned}
& \mathrm{K}_{\mathrm{g}}^{*} \equiv \alpha(1-\alpha) \hat{\mathrm{K}}_{\mathrm{g}}^{\mathrm{n}}, \text { previous } \mathrm{K}_{\mathrm{g}} \\
& \mathrm{K}_{\mathrm{g}}^{0} \equiv \varepsilon_{\alpha}\left(1-\varepsilon_{\alpha}\right) \hat{\mathrm{K}}_{\mathrm{g}}^{\mathrm{n}} \\
& \mathrm{K}_{\mathrm{g}}^{1} \equiv \varepsilon_{\alpha}\left(1-\varepsilon_{\alpha}\right) \hat{\mathrm{K}}_{\mathrm{g}}^{\mathrm{n}} \\
& \mathrm{H}_{\mathrm{gi}}^{*} \equiv \alpha(1-\alpha) \hat{\mathrm{H}}_{\mathrm{gi}}^{\mathrm{n}}, \text { previous } \mathrm{H}_{\mathrm{gi}} \\
& \mathrm{H}_{\mathrm{gi}}^{0} \equiv \varepsilon_{\alpha}\left(1-\varepsilon_{\alpha}\right) \hat{\mathrm{H}}_{\mathrm{gi}}^{\mathrm{n}} \\
& \mathrm{H}_{\mathrm{gi}}^{1} \equiv \varepsilon_{\alpha}\left(1-\varepsilon_{\alpha}\right) \hat{\mathrm{H}}_{\mathrm{gi}}^{\mathrm{n}} \\
& \mathrm{H}_{\mathrm{fi}}^{*} \equiv \alpha(1-\alpha) \hat{H}_{\mathrm{f}}^{\mathrm{n}}, \text { previous } \mathrm{H}_{\mathrm{fi}} \\
& \mathrm{H}_{\mathrm{fi}}^{0} \equiv \varepsilon_{\alpha}\left(1-\varepsilon_{\alpha}\right) \hat{\mathrm{H}}_{\mathrm{fi}}^{\mathrm{n}} \\
& \mathrm{H}_{\mathrm{fi}}^{1} \equiv \varepsilon_{\alpha}\left(1-\varepsilon_{\alpha}\right) \hat{\mathrm{H}}_{\mathrm{fi}}^{\mathrm{n}} \\
& \varphi_{\alpha} \equiv \varphi\left(\alpha, 0, \varepsilon_{\alpha}\right) \\
& \varphi_{1-\alpha} \equiv \varphi\left(1-\alpha, 0, \varepsilon_{\alpha}\right) \\
& \varphi_{\mathrm{T}_{\mathrm{i}}-\mathrm{T}_{\mathrm{g}}} \equiv \varphi\left(\mathrm{T}_{\mathrm{i}}-\mathrm{T}_{\mathrm{g}}, 0, \varepsilon_{\mathrm{T}}\right) \\
& \varphi_{\mathrm{T}_{\mathrm{f}}-\mathrm{T}_{\mathrm{i}}} \equiv \varphi\left(\mathrm{T}_{\mathrm{f}}-\mathrm{T}_{\mathrm{i}}, 0, \varepsilon_{\mathrm{T}}\right) \\
& \varepsilon_{\alpha} \equiv 0.001 \\
& \varepsilon_{\mathrm{T}} \equiv 0.001{ }^{\circ} \mathrm{C}
\end{aligned}
$$

The motivation for these modifications is given in FLOWTRAN-TF development report TF-36.

Modifications to donored properties: Cell face properties are defined by a donor cell scheme based on the previous time step. This logic is adequate for nearly all circumstances. An exception occurs when a two-phase cell is upstream of a single-phase 
cell at time $n$. In this case, the both phases are defined to be present at the common face between the cells for the $n+1$ time step using the default logic. If the velocity of the phase not present in the single-phase cell does not change direction between the $n$ and $n+1$ time steps, then no problem occurs. If the velocity does change sign, a converged solution cannot be obtained. The reason is that mass of the phase not present in the single-phase cell is being withdrawn at the face in question. Any finite time step size, no matter how small, results in a void fraction outside the range $[0,1]$. To correct this problem, FLOWTRAN-TF searches for velocity reversals whenever an non-physical void fraction is computed. If the velocity of the non-existent phase reverses, the donored face properties of non-existent phase are set to zero to be consistent.

Phase transitions: The original two-phase flow equations given by $(3.7-1-10)$ have been modified such that the equation set is well-posed for single-phase as well as twophase conditions. The modifications have been smoothly introduced. Transitions from superheated single-phase liquid and subcooled single-phase gas to two-phase conditions have been made possible. Therefore no fundamental problems are expected during a transition from single-phase to two-phase flow and vice versa. The transition zone at the single-phase limits has been made large $\left(\varepsilon_{\alpha}=0.001\right)$ so that the transitions are as gradual as practical, however, small time steps may be needed to traverse some transitions.

Numerical testing has shown $\mathrm{X}_{\mathrm{a}}$ to be a 'problem' variable during phase transitions under some circumstances. Under single-phase liquid conditions, $X_{\mathrm{a}}$ is zero. Under typical airwater conditions at room temperature, $X_{a}$ is nearly one. In going between these states, $X_{a}$ must make an extreme change. Frequently, $X_{a}$ would overshoot the physical bounds of $[0,1]$ during a Newton iteration even for very small time steps. To correct this problem, FLOWTRAN-TF constrains $X_{a}$ to the interval $[0,1]$ during Newton iterations without calling for a time step reduction. This simple scheme has been effective in yielding a converged, physically-correct solution with additional iterations. Analogously, the gas and liquid temperatures are constrained to lie within the ranges $[270,705 \mathrm{~K}]$ and $[270,455 \mathrm{~K}]$, respectively.

Even with the above modifications, convergence problems with both the inner and outer Newton iterations were encountered. Starting guesses were frequently too far from the converged solution for the standard Newton iteration method to handle. To remedy this problem, tne maximum changes in $\mathrm{y}_{\mathrm{si}}, \mathrm{x}_{\mathrm{a}}, \mathrm{T}_{\mathrm{g}}$ and $\mathrm{T}_{\mathrm{f}}$ during iterations are limited to 0.04 , $0.04,5^{\circ} \mathrm{C}$ and $5^{\circ} \mathrm{C}$, respectively. This simple scheme proved effective in ensuring convergence during phase transitions.

Numerical testing also showed that two-phase flow into a single-phase liquid cell is aided by eliminating the wall induced interfacial drag component $\mathrm{F}_{\mathrm{i}}^{\mathrm{Wz}}$ at cell faces adjoining a single-phase liquid cell. This is done by multiplying $F_{1}^{\omega \prime}$ by the factor

$$
\varphi\left(\alpha_{\min }\right)=\min \left[1, \max \left(0, \frac{\alpha_{\min }-\varepsilon_{\alpha 1}}{\varepsilon_{\alpha 2}-\varepsilon_{\alpha 1}}\right)\right]
$$

where $\alpha_{\min }$ is the minimum of the two void fractions of the axial cells adjoining the face in question. The other parameters in equation (3.7-26) are the same as defined above.

Verification: Partial verification is given in FLOWTRAN-TF development report TF-36 that the modifications introduced yield a well-posed equation set and that transitions to or from single-phase flow can be handled. The demonstrations typically assumed $\alpha=0$ or 1. For a rigorous verification, all 10 equations should be solved iteratively for all 10 
unknowns (including $\alpha$ ) without prior assumptions. The equation set is too large and complex for a hand-calculation. Therefore numerical experiments will be used during $V \& V$ to confirm that modifications described here are adequate.

Modified conservation equations: Below is a summary of the newly modified conservation equations

\section{GAS MOMENTUM}

$$
\begin{aligned}
& {\left[\frac{R^{2}\left\langle\alpha \rho_{g}\right\rangle^{n}}{\Delta t}+\left(\frac{\partial F_{w g}^{2}}{\partial u_{g}}\right)^{n}+\left(\frac{\partial F_{I}^{2}}{\partial u_{g}}\right)^{n}\right]_{i, j+1 / 2}\left(u_{g}\right)_{i, j+1 / 2}^{n+1}} \\
& +\left[\left(\frac{\partial F_{w g}^{z}}{\partial u_{f}}\right)^{n}+\left(\frac{\partial F_{l}^{z}}{\partial u_{f}}\right)^{n}\right]_{i, j+1 / 2}\left(u_{f}\right)_{i, j+1 / 2}^{n+1} \\
& =\frac{\langle\alpha A\rangle_{i, j+1 / 2}^{n}}{\left(V_{z}\right)_{i, j+1 / 2}}\left(P_{i, j}^{n+1}-P_{i, j+1}^{n+1}\right) \\
& +\frac{1}{\left(V_{z}\right)_{i, j+1 / 2}}\left\{\left\langle A_{z z} \alpha \rho_{g} u_{g} u_{g}\right\rangle_{i, j}^{n}-\left\langle A_{z z} \alpha \rho_{g} u_{g}\right\rangle_{i, j}^{n}\left\langle u_{g}\right\rangle_{i, j+1 / 2}^{n}\right. \\
& -\left\langle A_{z z} \alpha \rho_{g} u_{g} u_{g}\right\rangle_{i, j+1}^{n}+\left\langle A_{z z} \alpha \rho_{g} u_{g}\right\rangle_{i, j+1}^{n}\left\langle u_{g}\right\rangle_{i, j+1 / 2}^{n} \\
& +\left\langle A_{2 x} \alpha \rho_{g} w_{g}\right\rangle_{i-1 / 2, j+1 / 2}^{n}\left[\left\langle u_{g}\right\rangle_{i-1 / 2, j+1 / 2}^{n}-\left\langle u_{g}\right\rangle_{i, j+1 / 2}^{n}\right] \\
& \left.-\left\langle A_{z x} \alpha \rho_{g} w_{g}\right\rangle_{i+1 / 2, j+1 / 2}^{n}\left[\left\langle u_{g}\right\rangle_{i+1 / 2, j+1 / 2}^{n}-\left\langle u_{g}\right\rangle_{i, j+1 / 2}^{n}\right]\right\} \phi\left(\langle\alpha\rangle_{j+1 / 2}\right) \\
& +\left\langle\alpha \rho_{g}\right\rangle_{i, j+1 / 2}^{n} \frac{\left\langle u_{g}\right\rangle_{i, j+1 / 2}^{n}}{\Delta t}+\left\langle\rho_{g}\right\rangle_{i, j+1 / 2}^{n} g_{i, j+1 / 2}^{z} \frac{\langle\alpha A\rangle_{i, j+1 / 2}^{n}\left(\Delta z_{m}\right)_{i, j+1 / 2}}{\left(V_{z}\right)_{i, j+1 / 2}} \\
& -\left[\left(F_{w_{g}}^{z}\right)^{n}-\left(\frac{\partial F_{w_{g}}^{z}}{\partial u_{g}}\right)^{n} u_{g}^{n}-\left(\frac{\partial F_{w_{g}}^{z}}{\partial u_{f}}\right)^{n} u_{f}^{n}\right]_{i, j+1 / 2} \\
& -\left[\left(F_{I}^{z}\right)^{n}-\left(\frac{\partial F_{I}^{z}}{\partial u_{g}}\right)^{n} u_{g}^{n}-\left(\frac{\partial F_{l}^{z}}{\partial u_{f}}\right)^{n} u_{f}^{n}\right]_{i, j+1 / 2}-\left(M_{t g}^{z}\right)_{i, j+1 / 2}^{n} \phi\left(\langle\alpha\rangle_{j+1 / 2}\right)
\end{aligned}
$$




\section{GAS MOMENTUM (cont'd)}

$$
\begin{aligned}
& \left\langle u_{g}\right\rangle_{i, j+1 / 2}^{n}=\frac{\left(A_{c z}\right)_{i, j+1 / 2}}{\left\langle A_{c z}\right\rangle_{i, j+1 / 2}}\left(u_{g}\right)_{i, j+1 / 2}^{n} \\
& =R_{i, j+1 / 2}^{2}\left(u_{g}\right)_{i, j+1 / 2}^{n} \\
& \left\langle A_{c z}\right\rangle_{i, j+1 / 2} \equiv \frac{\left(V_{z}\right)_{i, j+1 / 2}}{\left(\Delta z_{m}\right)_{i, j+1 / 2}} \\
& (\hat{\alpha})_{i, j-1 / 2}^{n}=\omega \alpha_{i, j-1}^{n}+(1-\omega) \alpha_{i, j}^{n} \\
& \left(\hat{\rho}_{g}\right)_{i, j-1 / 2}^{n}=\omega\left(\rho_{g}\right)_{i, j-1}^{n}+(1-\omega)\left(\rho_{g}\right)_{i, j}^{n} \\
& \left(\tilde{u}_{g}\right)_{i, j} \equiv \frac{\left(A_{c z} \hat{\alpha} \hat{\rho}_{g} u_{g}\right)_{i, j-1 / 2}^{n}\left(A_{c z}\right)_{i, j-1 / 2}+\left(A_{c z} \hat{\alpha} \hat{\rho}_{g} u_{g}\right)_{i, j+1 / 2}^{n}\left(A_{c z}\right)_{i, j+1 / 2}}{\left[\left(A_{c z} \hat{\alpha} \hat{\rho}_{g}\right)_{i, j-1 / 2}^{n}+\left(A_{c z} \hat{\alpha} \hat{\rho}_{g}\right)_{i, j+1 / 2}^{n}\right]\left(A_{z z}\right)_{i, j}} \\
& \left\langle A_{z z} \alpha \rho_{g} u_{g}\right\rangle_{i, j}^{n}=\omega\left(A_{c z} \hat{\alpha} \hat{\rho}_{g} u_{g}\right)_{i, j-1 / 2}^{n}+(1-\omega)\left(A_{c z} \hat{\alpha} \hat{\rho}_{g} u_{g}\right)_{i, j+1 / 2}^{n} \\
& \left\langle A_{z z} \alpha \rho_{g} u_{g} u_{g}\right\rangle_{i, j}^{n}=\omega\left(A_{c z} \hat{\alpha} \hat{\rho}_{g} u_{g} u_{g}\right)_{i, j-1 / 2}^{n}+(1-\omega)\left(A_{c z} \hat{\alpha} \hat{\rho}_{g} u_{g} u_{g}\right)_{i, j+1 / 2}^{n} \\
& \left\langle A_{2 x} \alpha \rho_{g} w_{g}\right\rangle_{i-1 / 2, j+1 / 2}^{n}\left[\left\langle u_{g}\right\rangle_{i-1 / 2, j+1 / 2}^{n}-\left\langle u_{g}\right\rangle_{i, j+1 / 2}^{n}\right]= \\
& \frac{1}{2}\left(A_{c x} \hat{\alpha} \hat{\rho}_{g} w_{g}\right)_{i-1 / 2, j}^{n}\left[\left\{\left\langle u_{g}\right\rangle_{i-1 / \ldots, j+1 / 2}^{n}\right\}_{U_{p p e r}}-\left\langle u_{g}\right\rangle_{i, j+1 / 2}^{n}\right] \\
& +\frac{1}{2}\left(A_{c x} \hat{\alpha} \hat{\rho}_{g} w_{g}\right)_{i-1 / 2, j+1}^{n}\left[\left\{\left\langle u_{g}\right\rangle_{i-1 / 2, j+1 / 2}^{n}\right\}_{\text {Lower }}-\left\langle u_{g}\right\rangle_{i, j+1 / 2}^{n}\right] \\
& \langle\alpha A\rangle_{i, j+1 / 2}=\frac{2\left(\hat{\alpha} A_{c z}\right)_{i, j-1 / 2}\left(\hat{\alpha} A_{c z}\right)_{i, j+1 / 2}}{\left[\left(\hat{\alpha} A_{c z}\right)_{i, j-1 / 2}+\left(\hat{\alpha} A_{c z}\right)_{i, j+1 / 2}\right]} \text { (positive velocity) } \\
& \langle\alpha A\rangle_{i, j+1 / 2}=\frac{2\left(\hat{\alpha} A_{c z}\right)_{i, j+1 / 2}\left(\hat{\alpha} A_{c z}\right)_{i, j+3 / 2}}{\left[\left(\hat{\alpha} A_{c z}\right)_{i, j+1 / 2}+\left(\hat{\alpha} A_{c z}\right)_{i, j+3 / 2}\right]} \text { (negative velocity) }
\end{aligned}
$$


\begin{tabular}{|l|l|}
\hline $2 / 15 / 93$ & WSRC.TR-92-532 Rev. 0 \\
\hline
\end{tabular}

FLOWTRAN-TF Software Design

Page 431

LOUD MOMENTUM

$$
\begin{aligned}
& {\left[\left(\frac{\partial F_{w f}^{z}}{\partial u_{g}}\right)^{n}-\left(\frac{\partial F_{1}^{2}}{\partial u_{g}}\right)^{n}\right]_{i, j+1 / 2}\left(u_{g}\right)_{i, j+1 / 2}^{n+1}} \\
& +\left[\frac{R^{2}\left\langle(1-\alpha) \rho_{f}\right\rangle^{n}}{\Delta t}+\left(\frac{\partial F_{w f}^{2}}{\partial u_{f}}\right)^{n}-\left(\frac{\partial F_{1}^{2}}{\partial u_{f}}\right)^{n}\right]_{i, j+1 / 2}\left(u_{f}\right)_{i, j+1 / 2}^{n+1} \\
& =\frac{\langle(1-\alpha) A\rangle_{i, j+1 / 2}^{n}}{\left(V_{z}\right)_{i, j+1 / 2}}\left(P_{i, j}^{n+1}-P_{i, j+1}^{n+1}\right) \\
& +\frac{1}{\left(V_{z}\right)_{i, j+1 / 2}}\left\{\left\langle A_{z z}(1-\alpha) \rho_{f} u_{f} u_{f}\right\rangle_{i, j}^{n}-\left\langle A_{z z}(1-\alpha) \rho_{f} u_{f}\right\rangle_{i, j}^{n}\left\langle u_{f}\right\rangle_{i, j+1 / 2}^{n}\right. \\
& -\left\langle A_{z z}(1-\alpha) \rho_{f} u_{f} u_{f}\right\rangle_{i, j+1}^{n}+\left\langle A_{z z}(1-\alpha) \rho_{f} u_{f}\right\rangle_{i, j+1}^{n}\left\langle u_{f}\right\rangle_{i, j+1 / 2}^{n} \\
& +\left\langle A_{z x}(1-\alpha) \rho_{f} w_{f}\right\rangle_{i-1 / 2, j+1 / 2}^{n}\left[\left\langle u_{f}\right\rangle_{i-1 / 2, j+1 / 2}^{n}-\left\langle u_{f}\right\rangle_{i, j+1 / 2}^{n}\right] \\
& \left.-\left\langle A_{z x}(1-\alpha) \rho_{f} w_{f}\right\rangle_{i+1 / 2, j+1 / 2}^{n}\left[\left\langle u_{f}\right\rangle_{i+1 / 2, j+1 / 2}^{n}-\left\langle u_{f}\right\rangle_{i, j+1 / 2}^{n}\right]\right\} \phi\left(1-\langle\alpha\rangle_{j+1 / 2}\right) \\
& +\left\langle(1-\alpha) \rho_{\mathrm{f}}\right\rangle_{\mathrm{i}, \mathrm{j}+1 / 2}^{\mathrm{n}} \frac{\left\langle\mathrm{u}_{\mathrm{f}}\right\rangle_{\mathrm{i}, \mathrm{j}+1 / 2}^{\mathrm{n}}}{\Delta \mathrm{t}} \\
& +\left\langle\rho_{\mathrm{f}}\right\rangle_{\mathrm{i}, \mathrm{j}+1 / 2}^{\mathrm{n}} \mathrm{g}_{\mathrm{i}, \mathrm{i}+1 / 2}^{\mathrm{i}} \frac{\langle(1-\alpha) A\rangle_{\mathrm{i}, \mathrm{j}+1 / 2}^{\mathrm{n}}\left(\Delta \mathrm{z}_{\mathrm{m}}\right)_{\mathrm{i}, \mathrm{j}+1 / 2}}{\left(\mathrm{~V}_{\mathrm{z}}\right)_{\mathrm{i}, \mathrm{j}+1 / 2}} \\
& -\left[\left(F_{w f}^{z}\right)^{n}-\left(\frac{\partial F_{w f}^{z}}{\partial u_{g}}\right)^{n} u_{g}^{n}-\left(\frac{\partial F_{w f}^{z}}{\partial u_{f}}\right)^{n} u_{f}^{n}\right]_{i, j+1 / 2} \\
& +\left[\left(F_{1}^{2}\right)^{n}-\left(\frac{\partial F_{l}^{2}}{\partial u_{g}}\right)^{n} u_{g}^{n}-\left(\frac{\partial F_{1}^{z}}{\partial u_{f}}\right)^{n} u_{f}^{n}\right]_{i, j+1 / 2}-\left(M_{t f}^{z}\right)_{i, j+1 / 2}^{n} \phi\left(1-\langle\alpha\rangle_{j+1 / 2}\right)
\end{aligned}
$$




\section{GAS MASS (Nonlinear)}

$$
\begin{aligned}
& \frac{\left(\alpha \rho_{g}\right)_{i, j}^{n+1}-\left(\alpha \rho_{g}\right)_{i, j}^{n}}{\Delta t}+ \\
& \frac{1}{\left(V_{c}\right)_{i, j}}\left\{-\left(A_{c z} \hat{\alpha} \hat{\rho}_{g}\right)_{i, j-1 / 2}^{n}\left[\left(B_{g}\right)_{i, j-1 / 2}^{n}+\left(C_{g}\right)_{i, j-1 / 2}^{n}\left(P_{i, j-1}^{n+1}-P_{i, j}^{n+1}\right)\right]\right. \\
& +\left(A_{c z} \hat{\alpha} \hat{\rho}_{g}\right)_{i, j+1 / 2}^{n}\left[\left(B_{g}\right)_{i, j+1 / 2}^{n}+\left(C_{g}\right)_{i, j+1 / 2}^{n}\left(P_{i, j}^{n+1}-P_{i, j+1}^{n+1}\right)\right] \\
& -\left(A_{c x} \hat{\alpha} \hat{\rho}_{g}\right)_{i-1 / 2, j}^{n}\left[\left(D_{g}\right)_{i-1 / 2, j}^{n}+\left(E_{g}\right)_{i-1 / 2, j}^{n}\left(P_{i-1, j}^{n+1}-P_{i, j}^{n+1}\right)\right] \\
& \left.+\left(A_{c x} \hat{\alpha} \hat{\rho}_{g}\right)_{i+1 / 2, j}^{n}\left[\left(D_{g}\right)_{i+1 / 2, j}^{n}+\left(E_{g}\right)_{i+1 / 2, j}^{n}\left(P_{i, j}^{n+1}-P_{i+1, j}^{n+1}\right)\right]\right\} \\
& =\left(\Gamma_{I}\right)_{i, j}^{n+1}+\left(\Gamma_{w}\right)_{i, j}^{n}+\left(\Gamma_{a}\right)_{i, j}^{n+1}-\left(W_{t g}\right)_{i, j}^{n}
\end{aligned}
$$




\section{GASMASS OLinear)}

$$
\begin{aligned}
& \left\{\frac{\alpha_{i, j}^{m}}{\Delta t}\left(\frac{\partial \rho_{g}}{\partial P}\right)_{i, j}^{m}-\left(\frac{\partial \Gamma_{I}}{\partial P}\right)_{i, j}^{m}\right. \\
& +\frac{1}{\left(V_{c}\right)_{i, j}}\left[\left(A_{c z} \hat{\alpha} \hat{\rho}_{g} C_{g}\right)_{i, j-1 / 2}^{n}+\left(A_{c z} \hat{\alpha} \hat{\rho}_{g} C_{g}\right)_{i, j+1 / 2}^{n}\right. \\
& \left.\left.+\left(A_{c x} \hat{\alpha} \hat{\rho}_{g} E_{g}\right)_{i-1 / 2 . j}^{n}+\left(A_{c x} \hat{\alpha} \hat{\rho}_{g} E_{g}\right)_{i+1 / 2, j}^{n}\right]\right\}(\delta P)_{i, j}^{m+1} \\
& +\left\{\frac{\left(\rho_{g}\right)_{i, j}^{m}}{\Delta t}-\left(\frac{\partial \Gamma_{I}}{\partial \alpha}\right)_{i, j}^{m}-\left(\frac{\partial \Gamma_{z}}{\partial \alpha}\right)_{i, j}^{m}\right\}(\delta \alpha)_{i, j}^{m+1} \\
& +\left\{\frac{\alpha_{i, j}^{m}}{\Delta t}\left(\frac{\partial \rho_{g}}{\partial T_{g}}\right)_{i, j}^{m}-\left(\frac{\partial \Gamma_{I}}{\partial T_{g}}\right)_{i, j}^{m}\right\}\left(\delta T_{g}\right)_{i, j}^{m+1} \\
& +\left\{-\left(\frac{\partial \Gamma_{I}}{\partial T_{f}}\right)_{i, j}^{m}\right\}\left(\delta T_{f}\right)_{i, j}^{m+1} \\
& +\left\{\frac{\alpha_{i, j}^{m}}{\Delta t}\left(\frac{\partial \rho_{g}}{\partial X_{a}}\right)_{i, j}^{m}-\left(\frac{\partial \Gamma_{I}}{\partial X_{a}}\right)_{i, j}^{m}-\left(\frac{\partial \Gamma_{a}}{\partial X_{a}}\right)_{i, j}^{m}\right\}\left(\delta X_{a}\right)_{i, j}^{m+1} \\
& -\frac{1}{\left(V_{c}\right)_{i, j}}\left\{\left(A_{c z} \hat{\alpha} \hat{\rho}_{g} C_{g}\right)_{i, j-1 / 2}^{n}(\delta P)_{i, j-1}^{m+1}+\left(A_{c z} \hat{\alpha} \hat{\rho}_{g} C_{g}\right)_{i, j+1 / 2}^{n}(\delta P)_{i, j+1}^{m+1}\right. \\
& \left.+\left(A_{c x} \hat{\alpha} \hat{\rho}_{g} E_{g}\right)_{i-1 / 2, j}^{n}(\delta P)_{i-1, j}^{m+1}+\left(A_{c x} \hat{\alpha} \hat{\rho}_{g} E_{g}\right)_{i+1 / 2, j}^{n}(\delta P)_{i+1, j}^{m+1}\right\} \\
& =\frac{1}{\Delta t}\left[\left(\alpha \rho_{g}\right)_{i, j}^{n}-\left(\alpha \rho_{g}\right)_{i, j}^{m}\right]+\left(\Gamma_{I}\right)_{i, j}^{m}+\left(\Gamma_{w}\right)_{i, j}^{n}+\left(\Gamma_{a}\right)_{i, j}^{m}-\left(w_{i g}\right)_{i, j}^{n} \\
& +\frac{1}{\left(V_{c}\right)_{i, j}}\left\{\left(A_{c z} \hat{\alpha} \hat{\rho}_{g}\right)_{i, j-1 / 2}^{n}\left[\left(B_{g}\right)_{i, j-1 / 2}^{n}+\left(C_{g}\right)_{i, j-1 / 2}^{n}\left(P_{i, j-1}^{m}-P_{i, j}^{m}\right)\right]\right. \\
& -\left(A_{c z} \hat{\alpha} \hat{\rho}_{g}\right)_{i, j+1 / 2}^{n}\left[\left(B_{g}\right)_{i, j+1 / 2}^{n}+\left(C_{g}\right)_{i, j+1 / 2}^{n}\left(P_{i, j}^{m}-P_{i, j+1}^{m}\right)\right] \\
& +\left(A_{c x} \hat{\alpha} \hat{\rho}_{g}\right)_{i-1 / 2, j}^{n}\left[\left(D_{g}\right)_{i-1 / 2, j}^{n}+\left(E_{g}\right)_{i-1 / 2, j}^{n}\left(P_{i-1, j}^{m}-P_{i, j}^{m}\right)\right] \\
& \left.-\left(A_{c x} \hat{\alpha} \hat{\rho}_{g}\right)_{i+1 / 2, j}^{n}\left[\left(D_{g}\right)_{i+1 / 2, j}^{n}+\left(E_{g}\right)_{i+1 / 2, j}^{n}\left(P_{i, j}^{m}-P_{i+1, j}^{m}\right)\right]\right\}
\end{aligned}
$$




\section{LIQUID MASS (Nonlinear)}

$$
\begin{aligned}
& \frac{\left[(1-\alpha) \rho_{f}\right]_{i, j}^{n+1}-\left[(1-\alpha) \rho_{f}\right]_{i, j}^{n}}{\Delta t}+ \\
& \frac{1}{\left(V_{c}\right)_{i, j}}\left\{-\left[A_{c z}(1-\hat{\alpha}) \hat{\rho}_{f}\right]_{i, j-1 / 2}^{n}\left[\left(B_{f}\right)_{i, j-1 / 2}^{n}+\left(C_{f}\right)_{i, j-1 / 2}^{n}\left(P_{i, j-1}^{n+1}-P_{i, j}^{n+1}\right)\right]\right. \\
& +\left[A_{c z}(1-\hat{\alpha}) \hat{\rho}_{f}\right]_{i, j+1 / 2}^{n}\left[\left(B_{f}\right)_{i, j+1 / 2}^{n}+\left(C_{f}\right)_{i, j+1 / 2}^{n}\left(P_{i, j}^{n+1}-P_{i, j+1}^{n+1}\right)\right] \\
& -\left[A_{c z}(1-\hat{\alpha}) \hat{\rho}_{f}\right]_{i-1 / 2, j}^{n}\left[\left(D_{f}\right)_{i-1 / 2, j}^{n}+\left(E_{f}\right)_{i-1 / 2, j}^{n}\left(P_{i-1, j}^{n+1}-P_{i, j}^{n+1}\right)\right] \\
& \left.+\left[A_{c z}(1-\ddot{\alpha}) \hat{\rho}_{f}\right]_{i+1 / 2, j}^{n}\left[\left(D_{f}\right)_{i+1 / 2, j}^{n}+\left(E_{f}\right)_{i+1 / 2, j}^{n}\left(P_{i, j}^{n+1}-P_{i+1, j}^{n+1}\right)\right]\right\} \\
& =-\left(\Gamma_{I}\right)_{i, j}^{n+1}-\left(\Gamma_{w}\right)_{i, j}^{n}-\left(W_{t f}\right)_{i, j}^{n}
\end{aligned}
$$




\section{LUUDD MASS ainear)}

$$
\begin{aligned}
& \left\{\frac{1-\alpha_{i, j}^{m}}{\Delta t}\left(\frac{\partial \rho_{f}}{\partial P}\right)_{i, j}^{m}+\left(\frac{\partial \Gamma_{I}}{\partial P}\right)_{i, j}^{m}\right. \\
& +\frac{1}{\left(V_{c}\right)_{i, j}}\left[\left(A_{c z}(1-\hat{\alpha}) \hat{p}_{f} C_{f}\right)_{i, j-1 / 2}^{n}+\left(A_{c z}(1-\hat{\alpha}) \hat{\rho}_{f} C_{f}\right)_{i, j+1 / 2}^{n}\right. \\
& \left.\left.+\left(A_{c x}(1-\hat{\alpha}) \hat{\rho}_{f} E_{f}\right)_{i-1 / 2, j}^{n}+\left(A_{c x}(1-\hat{\alpha}) \hat{\rho}_{f} E_{f}\right)_{i+1 / 2, j}^{n}\right]\right\}(\delta P)_{i, j}^{m+1} \\
& +\left\{-\frac{\left(\rho_{f}\right)_{i, j}^{m}}{\Delta t}+\left(\frac{\partial \Gamma_{I}}{\partial \alpha}\right)_{i, j}^{m}\right\}(\delta \alpha)_{i, j}^{m+1} \\
& +\left\{\left(\frac{\partial \Gamma_{I}}{\partial T_{g}}\right)_{i, j}^{m}\right\}\left(\delta T_{g}\right)_{i, j}^{m+1} \\
& +\left\{\frac{1-\alpha_{i, j}^{m}}{\Delta t}\left(\frac{\partial \rho_{f}}{\partial T_{f}}\right)_{i, j}^{m}+\left(\frac{\partial \Gamma_{I}}{\partial T_{f}}\right)_{i, j}^{m}\right\}\left(\delta T_{f}\right)_{i, j}^{m+1} \\
& +\left\{\left(\frac{\partial \Gamma_{1}}{\partial X_{a}}\right)_{i, j}^{m}\right\}\left(\delta X_{a}\right)_{i, j}^{m+1} \\
& -\frac{1}{\left(V_{c}\right)_{i, j}}\left\{\left(A_{c z}(1-\hat{\alpha}) \hat{\rho}_{f} C_{f}\right)_{i, j-1 / 2}^{n}(\delta P)_{i, j-1}^{m+1}+\left(A_{c z}(1-\hat{\alpha}) \hat{\rho}_{f} C_{f}\right)_{i, j+1 / 2}^{n}(\delta P)_{i, j+1}^{m+1}\right. \\
& \left.+\left(A_{c x}(1-\hat{\alpha}) \hat{\rho}_{f} E_{f}\right)_{i-1 / 2, j}^{n}(\delta P)_{i-1, j}^{m+1}+\left(A_{c x}(1-\hat{\alpha}) \hat{\rho}_{g} E_{f}\right)_{i+1 / 2, j}^{n}(\delta P)_{i+1, j}^{m+1}\right\} \\
& =\frac{1}{\Delta t}\left[\left((1-\alpha) \rho_{f}\right)_{i, j}^{n}-\left((1-\alpha) \rho_{f}\right)_{i, j}^{m}\right]-\left(\Gamma_{l}\right)_{i, j}^{m}-\left(\Gamma_{w}\right)_{i, j}^{n}-\left(W_{t f}\right)_{i, j}^{n} \\
& +\frac{1}{\left(V_{c}\right)_{i, j}}\left\{\left(A_{c z}(1-\hat{\alpha}) \hat{p}_{f}\right)_{i, j-1 / 2}^{n}\left[\left(B_{f}\right)_{i, j-1 / 2}^{n}+\left(C_{f}\right)_{i, j-1 / 2}^{n}\left(P_{i, j-1}^{m}-P_{i, j}^{m}\right)\right]\right. \\
& -\left(A_{c z}(1-\hat{\alpha}) \hat{\rho}_{f}\right)_{i, j+1 / 2}^{n}\left[\left(B_{f}\right)_{i, j+1 / 2}^{n}+\left(C_{f}\right)_{i, j+1 / 2}^{n}\left(P_{i, j}^{m}-P_{i, j+1}^{m}\right)\right] \\
& +\left(A_{c x}(1-\hat{\alpha}) \hat{\rho}_{f}\right)_{i-1 / 2, j}^{n}\left[\left(D_{f}\right)_{i-1 / 2, j}^{n}+\left(E_{f}\right)_{i-1 / 2, j}^{n}\left(P_{i-1, j}^{m}-P_{i, j}^{m}\right)\right] \\
& \left.-\left(A_{c x}(1-\hat{\alpha}) \hat{\rho}_{f}\right)_{i+1 / 2, j}^{n}\left[\left(D_{f}\right)_{i+1 / 2, j}^{n}+\left(E_{f}\right)_{i+1 / 2, j}^{n}\left(P_{i, j}^{m}-P_{i+1, j}^{m}\right)\right]\right\}
\end{aligned}
$$




\section{AIR MASS (Nonlinear)}

$$
\begin{aligned}
& \frac{\left(\alpha \rho_{g} X_{a}\right)_{i, j}^{n+1}-\left(\alpha \rho_{g} X_{a}\right)_{i, j}^{n}}{\Delta i}+ \\
& \frac{1}{\left(V_{c}\right)_{i, j}}\left\{-\left(A_{c z} \hat{\alpha} \hat{\rho}_{g} \hat{X}_{a}\right)_{i, j-1 / 2}^{n}\left[\left(B_{g}\right)_{i, j-1 / 2}^{n}+\left(C_{g}\right)_{i, j-1 / 2}^{n}\left(P_{i, j-1}^{n+1}-P_{i, j}^{n+1}\right)\right]\right. \\
& +\left(A_{c z} \hat{\alpha} \hat{\rho}_{g} \hat{X}_{a}\right)_{i, j+1 / 2}^{n}\left[\left(B_{g}\right)_{i, j+1 / 2}^{n}+\left(C_{g}\right)_{i, j+1 / 2}^{n}\left(P_{i, j}^{n+1}-P_{i, j+1}^{n+1}\right)\right] \\
& -\left(A_{c x} \hat{\alpha} \hat{\rho}_{g} \hat{x}_{a}\right)_{i-1 / 2, j}^{n}\left[\left(D_{g}\right)_{i-1 / 2, j}^{n}+\left(E_{g}\right)_{i-1 / 2, j}^{n}\left(P_{i-1, j}^{n+1}-P_{i, j}^{n+1}\right)\right] \\
& \left.+\left(A_{c x} \hat{\alpha} \hat{\rho}_{g} \hat{X}_{a}\right)_{i+1 / 2, j}^{n}\left[\left(D_{g}\right)_{i+1 / 2, j}^{n}+\left(E_{g}\right)_{i+1 / 2, j}^{n}\left(P_{i, j}^{n+1}-P_{i+1, j}^{n+1}\right)\right]\right\} \\
& =\left(\Gamma_{\mathrm{a}}\right)_{\mathrm{i}, \mathrm{j}}^{\mathrm{n}+1}-\left(\mathrm{X}_{\mathrm{a}} \mathrm{W}_{\mathrm{tg}}\right)_{\mathrm{i}, \mathrm{j}}^{\mathrm{n}}
\end{aligned}
$$




\section{AIR MASS (Linear)}

$$
\begin{aligned}
& \left\{\frac{\left(\alpha X_{a}\right)_{i, j}^{m}}{\Delta t}\left(\frac{\partial \rho_{g}}{\partial P}\right)_{i, j}^{m}\right. \\
& +\frac{1}{\left(V_{c}\right)_{i, j}}\left[\left(A_{c z} \hat{\alpha} \hat{\rho}_{g} \hat{x}_{a} C_{g}\right)_{i, j-1 / 2}^{n}+\left(A_{c z} \hat{\alpha} \hat{\rho}_{g} \hat{X}_{a} C_{g}\right)_{i, j+1 / 2}^{n}\right. \\
& \left.\left.+\left(A_{c x} \hat{\alpha} \hat{\rho}_{g} \hat{X}_{a} E_{g}\right)_{i-1 / 2, j}^{n}+\left(A_{c x} \hat{\alpha} \hat{\rho}_{g} \hat{X}_{a} E_{g}\right)_{i+1 / 2, j}^{n}\right]\right\}(\delta P)_{i, j}^{m+1} \\
& +\left\{\frac{\left(\rho_{g} X_{a}\right)_{i, j}^{m}}{\Delta t}-\left(\frac{\partial \Gamma_{a}}{\partial \alpha}\right)_{i, j}^{m}\right\}(\delta \alpha)_{i, j}^{m+1} \\
& +\left\{\frac{\left(\alpha X_{a}\right)_{i, j}^{m}}{\Delta t}\left(\frac{\partial \rho_{g}}{\partial T_{g}}\right)_{i, j}^{m}\right\}\left(\delta T_{g}\right)_{i, j}^{m+1} \\
& +\left\{\frac{\alpha_{i, j}^{m}}{\Delta t}\left(X_{a} \frac{\partial \rho_{g}}{\partial X_{a}}+\rho_{g}\right)_{i, j}^{m}-\left(\frac{\partial \Gamma_{a}}{\partial X_{a}}\right)_{i, j}^{m}\right\}\left(\delta X_{a}\right)_{i, j}^{m+1} \\
& -\frac{1}{\left(V_{c}\right)_{i, j}}\left\{\left(A_{c z} \hat{\alpha} \hat{\rho}_{g} \hat{x}_{a} C_{g}\right)_{i, j-1 / 2}^{n}(\delta P)_{i, j-1}^{m+1}+\left(A_{c z} \hat{\alpha} \hat{\rho}_{g} \hat{x}_{a} C_{g}\right)_{i, j+1 / 2}^{n}(\delta P)_{i, j+1}^{m+1}\right. \\
& \left.+\left(A_{c x} \hat{\alpha} \hat{\rho}_{g} \hat{X}_{a} E_{g}\right)_{i-1 / 2, j}^{n}(\delta P)_{i-1, j}^{m+1}+\left(A_{c x} \hat{\alpha} \hat{\rho}_{g} \hat{X}_{a} E_{g}\right)_{i+1 / 2, j}^{n}(\delta P)_{i+1, j}^{m+1}\right\} \\
& =\frac{1}{\Delta t}\left[\left(\alpha \rho_{g} X_{a}\right)_{i, j}^{n}-\left(\alpha \rho_{g} X_{a}\right)_{i, j}^{m}\right]+\left(\Gamma_{a}\right)_{i, j}^{m}-\left(X_{a} W_{t g}\right)_{i, j}^{n} \\
& +\frac{1}{\left(V_{c}\right)_{i, j}}\left\{\left(A_{c z} \hat{\alpha} \hat{\rho}_{g} \hat{X}_{a}\right)_{i, j-1 / 2}^{n}\left[\left(B_{g}\right)_{i, j-1 / 2}^{n}+\left(C_{g}\right)_{i, j-1 / 2}^{n}\left(P_{i, j-1}^{m}-P_{i, j}^{m}\right)\right]\right. \\
& -\left(A_{c z} \hat{\alpha}_{\hat{\rho}_{g}} \hat{\mathbf{x}}_{a}\right)_{i, j+1 / 2}^{n}\left[\left(B_{g}\right)_{i, j+1 / 2}^{n}+\left(C_{g}\right)_{i, j+1 / 2}^{n}\left(P_{i, j}^{m}-P_{i, j+1}^{m}\right)\right] \\
& +\left(A_{c x} \hat{\alpha} \hat{\rho}_{g} \hat{X}_{a}\right)_{i-1 / 2, j}^{n}\left[\left(D_{g}\right)_{i-1 / 2, j}^{n}+\left(E_{g}\right)_{i-1 / 2, j}^{n}\left(P_{i-1, j}^{m}-P_{i, j}^{m}\right)\right] \\
& \left.-\left(A_{c x} \hat{\alpha} \hat{\rho}_{g} \hat{X}_{a}\right)_{i+1 / 2, j}^{n}\left[\left(D_{g}\right)_{i+1 / 2, j}^{n}+\left(E_{g}\right)_{i+1 / 2, j}^{n}\left(P_{i, j}^{m}-P_{i+1, j}^{m}\right)\right]\right\}
\end{aligned}
$$


$2 / 15 / 93$

WSRC-TR-92-532 Rev. 0

FLOWTRAN-TF Software Design

Page 438

GAS ENERGY (Nonlinear)

$$
\begin{aligned}
& \frac{\left(\alpha \rho_{g} h_{g}\right)_{i, j}^{n+1}-\left(\alpha \rho_{g} h_{g}\right)_{i, j}^{n}+}{\Delta t}+ \\
& \frac{1}{\left(V_{c}\right)_{i, j}}\left\{-\left(A_{c z} \hat{\alpha} \hat{\rho}_{g} \hat{h}_{g}\right)_{i, j-1 / 2}^{n}\left[\left(B_{g}\right)_{i, j-1 / 2}^{n}+\left(C_{g}\right)_{i, j-1 / 2}^{n}\left(P_{i, j-1}^{n+1}-P_{i, j}^{n+1}\right)\right]\right. \\
& +\left(A_{c z} \hat{\alpha} \hat{\rho}_{g} \hat{h}_{g}\right)_{i, j+1 / 2}^{n}\left[\left(B_{g}\right)_{i, j+1 / 2}^{n}+\left(C_{g}\right)_{i, j+1 / 2}^{n}\left(P_{i, j}^{n+1}-P_{i, j+1}^{n+1}\right)\right] \\
& \quad-\left(A_{c x} \hat{\alpha} \hat{\rho}_{g} \hat{h}_{g}\right)_{i-1 / 2, j}^{n}\left[\left(D_{g}\right)_{i-1 / 2, j}^{n}+\left(E_{g}\right)_{i-1 / 2, j}^{n}\left(P_{i-1, j}^{n+1}-P_{i, j}^{n+1}\right)\right] \\
& \left.+\left(A_{c x} \hat{\alpha} \hat{\rho}_{g} \hat{h}_{g}\right)_{i+1 / 2, j}^{n}\left[\left(D_{g}\right)_{i+1 / 2, j}^{n}+\left(E_{g}\right)_{i+1 / 2, j}^{n}\left(P_{i, j}^{n+1}-P_{i+1, j}^{n+1}\right)\right]\right\} \\
& =\alpha_{i, j}^{n} \frac{P_{i, j}^{n+1}-P_{i, j}^{n}}{\Delta t}+\left(E_{I}\right)_{i, j}^{n+1}+\left(E_{w}\right)_{i, j}^{n}+\left(E_{a}\right)_{i, j}^{n+1}+\left(Q_{w g}\right)_{i, j}^{n+1}-\left(Q_{t g}\right)_{i, j}^{n}
\end{aligned}
$$




\section{GAS ENERGY (Linear)}

$$
\begin{aligned}
& \left\{\frac{\left(\alpha h_{g}\right)_{i, j}^{m}}{\Delta t}\left(\frac{\partial \rho_{g}}{\partial P}\right)_{i, j}^{m}+\frac{\left(\alpha \rho_{g}\right)_{i, j}^{m}}{\Delta t}\left(\frac{\partial h_{g}}{\partial P}\right)_{i, j}^{m}-\frac{\alpha_{i, j}^{n}}{\Delta t}-\left(\frac{\partial E_{I}}{\partial P}\right)_{i, j}^{m}-\left(\frac{\partial E_{a}}{\partial P}\right)_{i, j}^{m}-\left(\frac{\partial Q_{w g}}{\partial P}\right)_{i, j}^{m}\right. \\
& +\frac{1}{\left(V_{c}\right)_{i, j}}\left[\left(A_{c z} \hat{\alpha} \hat{\rho}_{g} \hat{h}_{g} C_{g}\right)_{i, j-1 / 2}^{n}+\left(A_{c z} \hat{\alpha} \hat{\rho}_{g} \hat{h}_{g} C_{g}\right)_{i, j+1 / 2}^{n}\right. \\
& \left.\left.+\left(A_{c x} \hat{\alpha} \hat{\rho}_{g} \hat{h}_{g} E_{g}\right)_{i-1 / 2, j}^{n}+\left(A_{c x} \hat{\alpha} \hat{\rho}_{g} \hat{h}_{g} E_{g}\right)_{i+1 / 2, j}^{n}\right]\right\}(\delta P)_{i, j}^{m+1} \\
& +\left\{\frac{\left(\rho_{g} h_{g}\right)_{i, j}^{m}}{\Delta t}-\left(\frac{\partial E_{l}}{\partial \alpha}\right)_{i, j}^{m}-\left(\frac{\partial E_{a}}{\partial \alpha}\right)_{i, j}^{m}-\left(\frac{\partial Q_{w_{g}}}{\partial \alpha}\right)_{i, j}^{m}\right\}(\delta \alpha)_{i, j}^{m+1} \\
& +\left\{\frac{\left(\alpha h_{g}\right)_{i, j}^{m}}{\Delta t}\left(\frac{\partial \rho_{g}}{\partial T_{g}}\right)_{i, j}^{m}+\frac{\left(\alpha \rho_{g}\right)_{i, j}^{m}}{\Delta t}\left(\frac{\partial h_{g}}{\partial T_{g}}\right)_{i, j}^{m}-\left(\frac{\partial E_{I}}{\partial T_{g}}\right)_{i, j}^{m}-\left(\frac{\partial E_{a}}{\partial T_{g}}\right)_{i, j}^{m}-\left(\frac{\partial Q_{w g}}{\partial T_{g}}\right)_{i, j}^{m}\right\}\left(\delta T_{g}\right)_{i, j}^{m+1} \\
& +\left\{-\left(\frac{\partial \mathrm{E}_{\mathrm{I}}}{\partial \mathrm{T}_{\mathrm{f}}}\right)_{i, j}^{\mathrm{m}}-\left(\frac{\partial \mathrm{Q}_{\mathrm{wg}}}{\partial \mathrm{T}_{\mathrm{f}}}\right)_{\mathrm{i}, \mathrm{j}}^{\mathrm{m}}\right\}\left(\delta \mathrm{T}_{\mathrm{f}}\right)_{\mathrm{i}, \mathrm{j}}^{\mathrm{m}+1} \\
& +\left\{\frac{\left(\alpha h_{g}\right)_{i, j}^{m}}{\Delta t}\left(\frac{\partial \rho_{g}}{\partial X_{a}}\right)_{i, j}^{m}+\frac{\left(\alpha \rho_{g}\right)_{i, j}^{m}}{\Delta t}\left(\frac{\partial h_{g}}{\partial X_{a}}\right)_{i, j}^{m}-\left(\frac{\partial E_{I}}{\partial X_{a}}\right)_{i, j}^{m}-\left(\frac{\partial E_{a}}{\partial X_{a}}\right)_{i, j}^{m}-\left(\frac{\partial Q_{w_{g}}}{\partial X_{a}}\right)_{i, j}^{m}\right\}\left(\delta X_{a}\right)_{i, j}^{m+1} \\
& -\frac{1}{\left(V_{c}\right)_{i, j}}\left\{\left(A_{c z} \hat{\alpha} \hat{\rho}_{g} \hat{h}_{g} C_{g}\right)_{i, j-1 / 2}^{n}(\delta P)_{i, j-1}^{m+1}+\left(A_{c z} \hat{\alpha} \hat{\rho}_{g} \hat{h}_{g} C_{g}\right)_{i, j+1 / 2}^{n}(\delta P)_{i, j+1}^{m+1}\right. \\
& \left.+\left(A_{c x} \hat{\alpha} \hat{\rho}_{g} \hat{h}_{g} E_{g}\right)_{i-1 / 2, j}^{n}(\delta P)_{i-1, j}^{m+1}+\left(A_{c x} \hat{\alpha} \hat{\rho}_{g} \hat{h}_{g} E_{g}\right)_{i+1 / 2, j}^{n}(\delta P)_{i+1, j}^{m+1}\right\} \\
& =\frac{1}{\Delta t}\left[\left(\alpha \rho_{g} h_{g}\right)_{i, j}^{n}-\left(\alpha \rho_{g} h_{g}\right)_{i, j}^{m}\right]+\frac{\alpha_{i, j}^{n}}{\Delta t}\left[P_{i, j}^{m}-P_{i, j}^{n}\right]+\left(E_{I}\right)_{i, j}^{m}+\left(E_{w}\right)_{i, j}^{n}+\left(E_{a}\right)_{i, j}^{m}+\left(Q_{w g}\right)_{i, j}^{m}-\left(Q_{t g}\right)_{i, j}^{n} \\
& +\frac{1}{\left(V_{c}\right)_{i, j}}\left\{\left(A_{c z} \hat{\alpha} \hat{\rho}_{g} \hat{h}_{g}\right)_{i, j-1 / 2}^{n}\left[\left(B_{g}\right)_{i, j-1 / 2}^{n}+\left(C_{g}\right)_{i, j-1 / 2}^{n}\left(P_{i, j-1}^{m}-P_{i, j}^{m}\right)\right]\right. \\
& -\left(A_{c z} \hat{\alpha} \hat{\rho}_{g} \hat{h}_{g}\right)_{i, j+1 / 2}^{n}\left[\left(B_{g}\right)_{i, j+1 / 2}^{n}+\left(C_{g}\right)_{i, j+1 / 2}^{n}\left(P_{i, j}^{m}-P_{i, j+1}^{m}\right)\right] \\
& +\left(A_{c x} \hat{\alpha} \hat{\rho}_{g} \hat{h}_{g}\right)_{i-1 / 2, j}^{n}\left[\left(D_{g}\right)_{i-1 / 2, j}^{n}+\left(E_{g}\right)_{i-1 / 2, j}^{n}\left(P_{i-1, j}^{m}-P_{i, j}^{m}\right)\right] \\
& \left.-\left(A_{c x} \hat{\alpha} \hat{\rho}_{g} \hat{h}_{g}\right)_{i+1 / 2, j}^{n}\left\{\left(D_{g}\right)_{i+1 / 2, j}^{n}+\left(E_{g}\right)_{i+1 / 2, j}^{n}\left(P_{i, j}^{m}-P_{i+1, j}^{m}\right)\right]\right\}
\end{aligned}
$$




\section{LIOUID ENERGY (Nonlinear)}

$$
\begin{aligned}
& \frac{\left[(1-\alpha) \rho_{f} h_{f}\right]_{i, j}^{n+1}-\left[(1-\alpha) \rho_{f} h_{f}\right]_{i, j}^{n}}{\Delta t}+ \\
& \frac{1}{\left(V_{c}\right)_{i, j}}\left\{-\left[A_{c z}(1-\hat{\alpha}) \hat{\rho}_{f} \hat{h}_{f}\right]_{i, j-1 / 2}^{n}\left[\left(B_{f}\right)_{i, j-1 / 2}^{n}+\left(C_{f}\right)_{i, j-1 / 2}^{n}\left(P_{i, j-1}^{n+1}-P_{i, j}^{n+1}\right)\right]\right. \\
& +\left[A_{c z}(1-\hat{\alpha}) \hat{\rho}_{f} \hat{h}_{f}\right]_{i, j+1 / 2}^{n}\left[\left(B_{f}\right)_{i, j+1 / 2}^{n}+\left(C_{f}\right)_{i, j+1 / 2}^{n}\left(P_{i, j}^{n+1}-P_{i, j+1}^{n+1}\right)\right] \\
& -\left[A_{c z}(1-\hat{\alpha}) \hat{\rho}_{f} \hat{h}_{f}\right]_{i-1 / 2, j}^{n}\left[\left(D_{f}\right)_{i-1 / 2, j}^{n}+\left(E_{f}\right)_{i-1 / 2, j}^{n}\left(P_{i-1, j}^{n+1}-P_{i, j}^{n+1}\right)\right] \\
& \left.+\left[A_{c z}(1-\hat{\alpha}) \hat{\rho}_{f} \hat{h}_{f}\right]_{i+1 / 2, j}^{n}\left[\left(D_{f}\right)_{i+1 / 2, j}^{n}+\left(E_{f}\right)_{i+1 / 2, j}^{n}\left(P_{i, j}^{n+1}-P_{i+1, j}^{n+1}\right)\right]\right\} \\
& =\left(1-\alpha_{i, j}^{n}\right) \frac{P_{i, j}^{n+1}-P_{i, j}^{n}}{\Delta t}-\left(E_{l}\right)_{i, j}^{n+1}-\left(E_{w}\right)_{i, j}^{n}+\left(Q_{w f}\right)_{i, j}^{n+1}-\left(Q_{t f}\right)_{i, j}^{n}
\end{aligned}
$$




\section{LIOUID ENERGY (Linear)}

$$
\begin{aligned}
& \left\{\frac{\left[(1-\alpha) h_{f}\right]_{i, j}^{m}}{\Delta t}\left(\frac{\partial \rho_{f}}{\partial P}\right)_{i, j}^{m}+\frac{\left[(1-\alpha) \rho_{f}\right]_{i, j}^{m}}{\Delta t}\left(\frac{\partial h_{f}}{\partial P}\right)_{i, j}^{m}-\frac{1-\alpha_{i, j}^{n}}{\Delta t}+\left(\frac{\partial E_{I}}{\partial P}\right)_{i, j}^{m}-\left(\frac{\partial Q_{w f}}{\partial P}\right)_{i, j}^{m}\right. \\
& +\frac{1}{\left(V_{c}\right)_{i, j}}\left[\left(A_{c z}(1-\hat{\alpha}) \hat{\rho}_{f} \hat{h}_{f} C_{f}\right)_{i, j-1 / 2}^{n}+\left(A_{c z}(1-\hat{\alpha}) \hat{p}_{f} \hat{h}_{f} C_{f}\right)_{i, j+1 / 2}^{n}\right. \\
& \left.\left.+\left(A_{c x}(1-\hat{\alpha}) \hat{\rho}_{f} \hat{h}_{f} E_{f}\right)_{i-1 / 2, j}^{n}+\left(A_{c x}(1-\hat{\alpha}) \hat{\rho}_{f} \hat{h}_{f} E_{f}\right)_{i+1 / 2, j}^{n}\right]\right\}(\delta P)_{i, j}^{m+1} \\
& +\left\{-\frac{\left(\rho_{f} h_{f}\right)_{i, j}^{m}}{\Delta t}+\left(\frac{\partial E_{I}}{\partial \alpha}\right)_{i, j}^{m}-\left(\frac{\partial Q_{w f}}{\partial \alpha}\right)_{i, j}^{m}\right\}(\delta \alpha)_{i, j}^{m+1} \\
& +\left\{\left(\frac{\partial E_{1}}{\partial T_{g}}\right)_{i, j}^{m}-\left(\frac{\partial Q_{w f}}{\partial T_{g}}\right)_{i, j}^{m}\right\}\left(\delta T_{g}\right)_{i, j}^{m+1} \\
& +\left\{\frac{\left[(1-\alpha) h_{f}\right]_{i, j}^{m}}{\Delta t}\left(\frac{\partial \rho_{f}}{\partial T_{f}}\right)_{i, j}^{m}+\frac{\left[(1-\alpha) \rho_{f}\right]_{i, j}^{m}}{\Delta t}\left(\frac{\partial h_{f}}{\partial T_{f}}\right)_{i, j}^{m}+\left(\frac{\partial E_{l}}{\partial T_{f}}\right)_{i, j}^{m}-\left(\frac{\partial Q_{w f}}{\partial T_{f}}\right)_{i, j}^{m}\right\}\left(\delta T_{f}\right)_{i, j}^{m+1} \\
& +\left\{\left(\frac{\partial E_{I}}{\partial X_{a}}\right)_{i, j}^{m}-\left(\frac{\partial Q_{w f}}{\partial X_{a}}\right)_{i, j}^{m}\right\}\left(\delta X_{a}\right)_{i, j}^{m+1} \\
& -\frac{1}{\left(V_{c}\right)_{i, j}}\left\{\left(A_{c z}(1-\hat{\alpha}) \hat{\rho}_{f} \hat{h}_{f} C_{f}\right)_{i, j-1 / 2}^{n}(\delta P)_{i, j-1}^{m+1}+\left(A_{c z}(1-\hat{\alpha}) \hat{p}_{f} \hat{h}_{f} C_{f}\right)_{i, j+1 / 2}^{n}(\delta P)_{i, j+1}^{m+1}\right. \\
& \left.+\left(A_{c x}(1-\hat{\alpha}) \hat{\rho}_{f} \hat{h}_{f} E_{f}\right)_{i-1 / 2, j}^{n}(\delta P)_{i-1, j}^{m+1}+\left(A_{c x}(1-\hat{\alpha}) \hat{\rho}_{f} \hat{h}_{f} E_{f}\right)_{i+1 / 2, j}^{n}(\delta P)_{i+1, j}^{m+1}\right\} \\
& =\frac{1}{\Delta t}\left[\left((1-\alpha) \rho_{f} h_{f}\right)_{i, j}^{n}-\left((1-\alpha) \rho_{f} h_{f}\right)_{i, j}^{m}\right]+\frac{1-\alpha_{i, j}^{n}}{\Delta t}\left[P_{i, j}^{m}-P_{i, j}^{n}\right]-\left(E_{I}\right)_{i, j}^{m}-\left(E_{w}\right)_{i, j}^{n}+\left(Q_{w f}\right)_{i, j}^{m}-\left(Q_{t f}\right)_{i, j}^{n} \\
& +\frac{1}{\left(V_{c}\right)_{i, j}}\left\{\left(A_{c z}(1-\hat{\alpha}) \hat{\rho}_{f} \hat{h}_{f}\right)_{i, j-1 / 2}^{n}\left[\left(B_{f}\right)_{i, j-1 / 2}^{n}+\left(C_{f}\right)_{i, j-1 / 2}^{n}\left(P_{i, j-1}^{m}-P_{i, j}^{m}\right)\right]\right. \\
& -\left(A_{c z}(1-\hat{\alpha}) \hat{\rho}_{f} \hat{h}_{f}\right)_{i, j+1 / 2}^{n}\left[\left(B_{f}\right)_{i, j+1 / 2}^{n}+\left(C_{f}\right)_{i, j+1 / 2}^{n}\left(P_{i, j}^{m}-P_{i, j+1}^{m}\right)\right] \\
& +\left(A_{c x}(1-\hat{\alpha}) \hat{\rho}_{f} \hat{h}_{f}\right)_{i-1 / 2, j}^{n}\left[\left(D_{f}\right)_{i-1 / 2, j}^{n}+\left(E_{f}\right)_{i-1 / 2, j}^{n}\left(P_{i-1, j}^{m}-P_{i, j}^{m}\right)\right] \\
& \left.-\left(A_{c x}(1-\hat{\alpha}) \hat{\rho}_{f} \hat{h}_{f}\right)_{i+1 / 2, j}^{n}\left[\left(D_{f}\right)_{i+1 / 2, j}^{n}+\left(E_{f}\right)_{i+1 / 2, j}^{n}\left(P_{i, j}^{m}-P_{i+1, j}^{m}\right)\right]\right\}
\end{aligned}
$$


Derivatives: Derivatives consistent with the newly modified mass/energy equations are needed for Newton iterations. These are summarized below

$\Gamma_{\mathrm{a}}, \mathrm{E}_{\mathrm{a}}$ DERIVATIVES

$$
\begin{aligned}
& \Gamma_{\mathrm{a}}=\left(1-\varphi_{\alpha}\right) \varepsilon_{\Gamma_{\mathrm{a}}}\left(0-\mathrm{X}_{\mathrm{a}}\right) \\
& \Gamma_{\mathrm{a}}=-\left(1-\varphi_{\alpha}\right) \varepsilon_{\Gamma_{\mathrm{a}}} \mathrm{X}_{\mathrm{a}} \\
& \left(\frac{\partial \Gamma_{\mathrm{a}}}{\partial \mathrm{X}_{\mathrm{a}}}\right)=-\left(1-\varphi_{\alpha}\right) \varepsilon_{\Gamma_{\mathrm{a}}} \\
& \left(\frac{\partial \Gamma_{\mathrm{a}}}{\partial \alpha}\right)=\varphi_{\alpha}^{\prime} \varepsilon_{\Gamma_{\mathrm{a}}} \mathrm{X}_{\mathrm{a}} \\
& E_{a}=\Gamma_{a}\left(\alpha, X_{a}\right) h_{a}\left(P, T_{g}\right) \\
& \left(\frac{\partial E_{a}}{\partial x}\right)_{x_{j}}=\left[\Gamma_{a}\left(\frac{\partial h_{a}}{\partial P}\right)_{x_{j}}\right]\left(\frac{\partial P}{\partial x}\right)_{x_{j}}+\left[h_{a}\left(\frac{\partial \Gamma_{a}}{\partial \alpha}\right)_{x_{j}}\right]\left(\frac{\partial \alpha}{\partial x}\right)_{x_{j}} \\
& +\left[\Gamma_{a}\left(\frac{\partial h_{a}}{\partial T_{g}}\right)_{x_{j}}\right]\left(\frac{\partial T_{g}}{\partial x}\right)_{x_{j}}+\left[h_{a}\left(\frac{\partial \Gamma_{a}}{\partial X_{a}}\right)_{x_{j}}\right]\left(\frac{\partial x_{a}}{\partial x}\right)_{x_{j}}
\end{aligned}
$$

\section{$\underline{K}_{q} H_{q j} H_{f i}$ DERIVATIVES}

$$
\begin{aligned}
& \mathrm{K}_{\mathrm{g}}=\varphi_{\alpha} \varphi_{1-\alpha} \mathrm{K}_{\mathrm{g}}^{*}+\left(1-\varphi_{\alpha}\right) \mathrm{K}_{\mathrm{g}}^{0}+\left(1-\varphi_{1-\alpha}\right) \mathrm{K}_{\mathrm{g}}^{1} \\
& \mathrm{~K}_{\mathrm{g}}^{*}=\alpha(1-\alpha) \hat{\mathrm{K}}_{\mathrm{g}}^{\mathrm{n}} \\
& \mathrm{K}_{\mathrm{g}}^{0}=\varepsilon_{\alpha}\left(1-\varepsilon_{\alpha}\right) \hat{\mathrm{K}}_{\mathrm{g}}^{\mathrm{n}} \\
& \mathrm{K}_{\mathrm{g}}^{1}=\varepsilon_{\alpha}\left(1-\varepsilon_{\alpha}\right) \hat{\mathrm{K}}_{\mathrm{g}}^{\mathrm{n}} \\
& \left(\frac{\partial \mathrm{K}_{\mathrm{g}}^{*}}{\partial \alpha}\right)=(1-2 \alpha) \hat{\mathrm{K}}_{\mathrm{g}}^{\mathrm{n}} \\
& \frac{\partial \mathrm{K}_{\mathrm{g}}}{\partial \alpha}=\varphi_{\alpha} \varphi_{1-\alpha} \frac{\partial \mathrm{K}_{\mathrm{g}}^{*}}{\partial \alpha}+\left(\varphi_{\alpha}^{\prime} \varphi_{1-\alpha}-\varphi_{\alpha} \varphi_{1-\alpha}^{\prime}\right) \mathrm{K}_{\mathrm{g}}^{*} \\
& \quad-\varphi_{\alpha}^{\prime} \mathrm{K}_{\mathrm{g}}^{0}+\varphi_{1-\alpha}^{\prime} \mathrm{K}_{\mathrm{g}}^{1}
\end{aligned}
$$




$$
\begin{aligned}
& \mathrm{H}_{\mathrm{gi}}=\varphi_{\alpha}\left[1-\varphi_{T_{\mathrm{i}}-\mathrm{T}_{\mathrm{g}}}\left(1-\varphi_{1-\alpha}\right)\right] \mathrm{H}_{\mathrm{gi}}^{*}+\varphi_{T_{\mathrm{i}}-\mathrm{T}_{\mathrm{g}}}\left(1-\varphi_{1-\alpha}\right) \mathrm{H}_{\mathrm{gi}}^{1}+\left(1-\varphi_{\alpha}\right) \mathrm{H}_{\mathrm{gi}}^{0} \\
& \mathrm{H}_{\mathrm{gi}}^{*}=\alpha(1-\alpha) \hat{\mathrm{H}}_{\mathrm{gi}}^{\mathrm{n}} \\
& \mathrm{H}_{\mathrm{gi}}^{0}=\varepsilon_{\alpha}\left(1-\varepsilon_{\alpha}\right) \hat{\mathrm{H}}_{\mathrm{gi}}^{\mathrm{n}} \\
& \mathrm{H}_{\mathrm{gi}}^{1}=\varepsilon_{\alpha}\left(1-\varepsilon_{\alpha}\right) \hat{H}_{\mathrm{gi}}^{\mathrm{n}} \\
& \left(\frac{\partial \mathrm{H}_{\mathrm{gi}}^{*}}{\partial \alpha}\right)=(1-2 \alpha) \hat{\mathrm{H}}_{\mathrm{gi}}^{\mathrm{n}} \\
& \frac{\partial \mathrm{H}_{\mathrm{gi}}}{\partial \mathrm{T}_{\mathrm{g}}}=\varphi_{\mathrm{T}_{\mathrm{i}}-\mathrm{T}_{\mathrm{g}}^{\prime}}^{\prime}\left(1-\varphi_{1-\alpha}\right)\left[\varphi_{\alpha} \mathrm{H}_{\mathrm{gi}}^{*}-\mathrm{H}_{\mathrm{gi}}^{1}\right] \\
& \frac{\partial \mathrm{H}_{\mathrm{gi}}}{\partial \mathrm{T}_{\mathrm{i}}}=-\frac{\partial \mathrm{H}_{\mathrm{gi}}}{\partial \mathrm{T}_{\mathrm{g}}} \\
& \frac{\partial \mathrm{H}_{\mathrm{gi}}}{\partial \alpha}=\left[1-\varphi_{\mathrm{T}_{\mathrm{i}}-\mathrm{T}_{\mathrm{z}}}\left(1-\varphi_{1-\alpha}\right)\right]\left[\varphi_{\alpha} \frac{\partial \mathrm{H}_{\mathrm{gi}}^{*}}{\partial \alpha}+\varphi_{\alpha}^{\prime} \mathrm{H}_{\mathrm{gi}}^{*}\right] \\
& -\varphi_{T_{i}-T_{g}} \varphi_{1-\alpha}^{\prime}\left[\varphi_{\alpha} H_{g i}^{*}-H_{g i}^{1}\right]-\varphi_{\alpha}^{\prime} H_{g i}^{0} \\
& H_{f i}=\varphi_{1-\alpha}\left[1-\varphi_{T_{f}-T_{i}}\left(1-\varphi_{\alpha}\right)\right] H_{f i}^{*}+\varphi_{T_{f}-T_{i}}\left(1-\varphi_{\alpha}\right) H_{f i}^{0}+\left(1-\varphi_{1-\alpha}\right) H_{f i}^{1} \\
& \mathrm{H}_{\mathrm{fi}}^{*}=\alpha(1-\alpha) \hat{H}_{\mathrm{i}}^{\mathrm{n}} \\
& \mathrm{H}_{\mathfrak{\mathrm { f }}}^{0}=\varepsilon_{\alpha}\left(1-\varepsilon_{\alpha}\right) \hat{\mathrm{H}}_{\mathrm{f}}^{\mathrm{n}} \\
& \mathrm{H}_{\mathrm{fi}}^{1}=\varepsilon_{\alpha}\left(1-\varepsilon_{\alpha}\right) \hat{\mathrm{H}}_{\mathrm{f}}^{\mathrm{n}} \\
& \left(\frac{\partial \mathrm{H}_{\mathrm{f}}^{*}}{\partial \alpha}\right)=(1-2 \alpha) \hat{\mathrm{H}}_{\mathrm{f}}^{\mathrm{n}} \\
& \frac{\partial \mathrm{H}_{\mathrm{fi}}}{\partial \mathrm{T}_{\mathrm{f}}}=-\varphi_{\mathrm{T}_{\mathrm{f}}-\mathrm{T}_{\mathrm{i}}}^{\prime}\left(1-\varphi_{\alpha}\right)\left[\varphi_{1-\alpha} \mathrm{H}_{\mathrm{fi}}^{*}-\mathrm{H}_{\mathrm{f}}^{0}\right] \\
& \frac{\partial H_{f i}}{\partial T_{i}}=-\frac{\partial H_{f i}}{\partial T_{f}}
\end{aligned}
$$




$$
\begin{aligned}
\frac{\partial H_{\mathrm{fi}}}{\partial \alpha}= & {\left[1-\varphi_{T_{\mathrm{f}}-\mathrm{T}_{\mathrm{i}}}\left(1-\varphi_{\alpha}\right)\right]\left[\varphi_{1-\alpha} \frac{\partial \mathrm{H}_{\mathrm{fi}}^{*}}{\partial \alpha}-\varphi_{1-\alpha}^{\prime} \mathrm{H}_{\mathrm{fi}}^{*}\right] } \\
& +\varphi_{T_{\mathrm{f}}-T_{\mathrm{i}}} \varphi_{\alpha}^{\prime}\left[\varphi_{1-\alpha} \mathrm{H}_{\mathrm{fi}}^{*}-\mathrm{H}_{\mathrm{fi}}^{0}\right]+\mathrm{H}_{\mathrm{fi}}^{1} \varphi_{1-\alpha}^{\prime}
\end{aligned}
$$

\section{I.H.M.T, DERIVATIVES}

$$
\begin{aligned}
& \left(\frac{\partial \Gamma}{\partial \mathrm{T}_{\mathrm{i}}}\right)_{\overline{\mathrm{x}}}=-\frac{1}{\mathrm{D}}\left\{\begin{array}{l}
\Gamma\left[\left(\frac{\partial \mathrm{h}_{\mathrm{gi}}}{\partial \mathrm{T}_{\mathrm{i}}}\right)_{\overline{\mathrm{x}}}-\left(\frac{\partial \mathrm{h}_{\mathrm{fi}}}{\partial \mathrm{T}_{\mathrm{i}}}\right)_{\overline{\mathrm{x}}}\right] \\
+\left[\mathrm{H}_{\mathrm{fi}}+\left(\frac{\partial \mathrm{H}_{\mathrm{fi}}}{\partial \mathrm{T}_{\mathrm{i}}}\right)_{\overline{\mathrm{x}}} \Delta \mathrm{T}_{\mathrm{f}}+\phi \Gamma \mathrm{c}_{\mathrm{pw}}\right]+\left[\mathrm{H}_{\mathrm{gi}}+\left(\frac{\partial \mathrm{H}_{\mathrm{gi}}}{\partial \mathrm{T}_{\mathrm{i}}}\right)_{\overline{\mathrm{x}}} \Delta \mathrm{T}_{\mathrm{g}}-\phi \Gamma \mathrm{c}_{\mathrm{ps}}\right]
\end{array}\right\} \\
& \left(\frac{\partial T_{i}}{\partial x}\right)_{x_{j}}\left\{\left[K_{B}+(1-\phi) \Gamma\right] \frac{1}{P}\left(\frac{d T_{s a t}}{d p_{s i}}\right)^{-1}-\left(1-y_{s i}+\phi \Delta y_{s}\right)\left(\frac{\partial \Gamma}{\partial T_{i}}\right)_{\bar{x}}\right\}= \\
& +\left\{\left[\mathrm{K}_{\mathrm{g}}+(1-\phi) \Gamma\right] \frac{\mathrm{y}_{s i}}{\mathrm{P}}-\frac{\mathrm{K}_{\mathrm{g}} \Delta \mathrm{y}_{\mathrm{s}}}{\mathrm{D}}\left(\frac{\partial \mathrm{h}_{\mathrm{gi}}}{\partial \mathrm{P}}\right)_{\mathrm{T}_{\mathrm{i}}}\right\}\left(\frac{\partial \mathrm{P}}{\partial \mathrm{x}}\right)_{\mathrm{x}_{j}} \\
& -\left\{\left(\mathrm{K}_{\mathrm{g}}-\phi \Gamma\right)\left(\frac{\mathrm{M}_{\mathrm{s}}}{\mathrm{M}_{\mathrm{a}}}\right)\left(\frac{\mathrm{y}_{\mathrm{s}}}{1-\mathrm{X}_{\mathrm{a}}}\right)^{2}\right\}\left(\frac{\partial \mathrm{X}_{\mathrm{a}}}{\partial \mathrm{x}}\right)_{\mathrm{x}_{\mathrm{j}}} \\
& +\frac{\left(1-y_{s i}+\phi \Delta y_{s}\right)}{D}\left\{\phi \Gamma\left[c_{p w}-\left(\frac{\partial c_{p w}}{\partial T_{f}}\right)_{T_{i}, x_{j}} \Delta T_{f}\right]-\Delta T_{f}\left(\frac{\partial H_{f i}}{\partial T_{f}}\right)_{T_{i}, x_{j}}+H_{f i}\right\}\left(\frac{\partial T_{f}}{\partial x}\right)_{x_{j}} \\
& +\frac{\left(1-y_{s i}+\phi \Delta y_{s}\right)}{D}\left\{\phi \Gamma\left[-c_{p s}+\left(\frac{\partial c_{p s}}{\partial T_{g}}\right)_{T_{i}, x_{j}} \Delta T_{g}\right]-\Delta T_{g}\left(\frac{\partial H_{g i}}{\partial T_{g}}\right)_{T_{i}, x_{j}}+H_{g i}\right\}\left(\frac{\partial T_{g}}{\partial x}\right)_{x_{j}} \\
& \left(\frac{\partial \Gamma}{\partial x}\right)_{T_{i}, x_{j}}=-\frac{\Gamma}{D}\left(\frac{\partial h_{g i}}{\partial P}\right)_{T_{i}}\left(\frac{\partial P}{\partial x}\right)_{x_{j}}-\frac{1}{D}\left[\Delta T_{f}\left(\frac{\partial H_{f i}}{\partial \alpha}\right)_{T_{i}, x_{j}}+\Delta T_{g}\left(\frac{\partial H_{g i}}{\partial \alpha}\right)_{T_{i}, x_{j}}\right]\left(\frac{\partial \alpha}{\partial x}\right)_{x_{j}} \\
& -\frac{1}{D}\left\{\phi \Gamma\left[-c_{p w}+\left(\frac{\partial c_{p w}}{\partial T_{f}}\right)_{T_{i}, x_{j}} \Delta T_{f}\right]+\Delta T_{f}\left(\frac{\partial H_{f i}}{\partial T_{f}}\right)_{T_{i}, x_{j}}-H_{f i}\right\}\left(\frac{\partial T_{f}}{\partial x}\right)_{x_{j}} \\
& -\frac{1}{D}\left\{\phi \Gamma\left[c_{p s}-\left(\frac{\partial c_{p s}}{\partial T_{g}}\right)_{T_{i}, x_{j}} \Delta T_{g}\right]+\Delta T_{g}\left(\frac{\partial H_{g i}}{\partial T_{g}}\right)_{T_{i}, x_{j}}-H_{g i}\right\}\left(\frac{\partial T_{g}}{\partial x}\right)_{x_{j}}
\end{aligned}
$$


\begin{tabular}{|l|l}
\hline $2 / 15 / 93$ & WSRC.TR-92-532 ReV. 0 \\
\hline
\end{tabular}

FLOWTRAN-TF Software Design

Page 445

$$
\begin{aligned}
\left(\frac{\partial \Gamma}{\partial x}\right)_{x_{j}}= & \left(\frac{\partial \Gamma}{\partial T_{i}}\right)_{\bar{x}}\left(\frac{\partial T_{i}}{\partial x}\right)_{x_{j}}+\left(\frac{\partial \Gamma}{\partial x}\right)_{T_{i}, x_{j}} \\
\left(\frac{\partial E_{g i}}{\partial x}\right)_{x_{j}} & =\left(h_{g i}-\phi c_{p s} \Delta T_{g}\right)\left(\frac{\partial \Gamma}{\partial x}\right)_{x_{j}}+\left[\Gamma\left(\frac{\partial h_{g i}}{\partial T_{i}}\right)_{\bar{x}}-\phi \Gamma c_{p s}+H_{g i}+\left(\frac{\partial H_{g i}}{\partial T_{i}}\right)_{\bar{x}} \Delta T_{g}\right]\left(\frac{\partial T_{i}}{\partial x}\right)_{x_{j}} \\
& +\Gamma\left(\frac{\partial h_{g i}}{\partial P}\right)_{T_{i}}\left(\frac{\partial P}{\partial x}\right)_{x_{j}}+\Delta T_{g}\left(\frac{\partial H_{g i}}{\partial \alpha}\right)_{T_{i}, x_{j}}\left(\frac{\partial \alpha}{\partial x}\right)_{x_{j}} \\
& +\left[-\phi \Gamma\left(\frac{\partial c_{p s}}{\partial T_{g}}\right)_{T_{i}, x_{j}} \Delta T_{g}+\phi \Gamma c_{p s}+\Delta T_{g}\left(\frac{\partial H_{g i}}{\partial T_{g}}\right)_{T_{i}, x_{j}}-H_{g i}\right]\left(\frac{\partial T_{g}}{\partial x}\right)_{x_{j}}
\end{aligned}
$$




\subsection{Solution Methods}

In this section, we discuss the numerical solution methods used in the FLOWTRAN-TF code. The solution methods are implemented as the code loops through subroutine TRANS on each time step of the transient calculation. The basic flow diagram for subroutine TRANS is shown in Fig. 3.8-1. At the start of each pass, the code selects an appropriate time step in subroutine TIMSTP based on Courant limits. The power distribution at the mid point of the time step (time $t+\Delta t$ ) is then calculated in subroutines AXIALP and PDIST. Since we allow the axial power shape to vary with time, a new profile is computed at each time step. In subroutine QWALL, the heat flux at the solidfluid interface is computed as well as the derivative of the wall heat flux with respect to the wall temperature. The power distribution in the solid structures and the surface heat flux are used in subroutine SOLID to calculate the temperature distribution throughout the solid materials in the assembly. The derivative of the heat flux with respect to the surface temperature is used to compute an improved estimate of the heat flux that becomes a boundary condition for the fluid energy equations.

Following the calculation of solid temperatures, the next several subroutines within TRANS solve the finite difference approximations to the fluid governing equations. We first evaluate the boundary conditions at the plenum and tank bottom that apply for this time step in subroutine BOUND. Explicit constitutive relations and turbulent exchange quantities are next evaluated in subroutines CONST and TURBX, respectively. With these parameters we can solve the fluid momentum equations using an explicit formulation coded in subroutine EXPLCT. We are now prepared to solve the fluid mass/energy equations using an implicit Newton iteration scheme. To increase computational efficiency, donored cell parameters that are evaluated explicitly are computed one time in subroutines DONORT, DONORM, and DONORB and stored for use within the iterations. Subroutine NEWGES checks for special conditions in the fluid (e.g. creation of a second phase) and makes an initial guess to the solution for the primary state variables. The implicit solution of the fluid mass/energy equation set is programmed in subroutine NEWTON. Subroutine NEWTON can request time step reductions if errors are detected.

Following the fluid solution, the magnitude of the change in the primary state variables in every fluid cell throughout the computational mesh is compared to user input limits in subroutine ACCCHK. If the change in the state variables exceeds the input limits, the code reduces the time step and repeats the computations. When the solution accuracy is acceptable, the code proceeds to check that material properties have been evaluated within the ranges of applicability of their correlations in subroutine PROPCK. If a property evaluation is outside the correlation range a time step reduction is requested. Subroutine UPDATV then uses the velocity relationships derived from the momentum equations to update all fluid velocities to new time values. To prepare for another time step, we store the solution at time step $n+1$ as the time $n$ values in subroutine RESET. This is followed by an evaluation of the power limit criteria in subroutine CRIT. Finally, subroutines PRTSLD, PRTFLD, and PRTPOW print the solid, fluid, and power solution values, respectively, when user input time intervals have elapsed. The code is now ready to increment the time and proceed to another time step. 


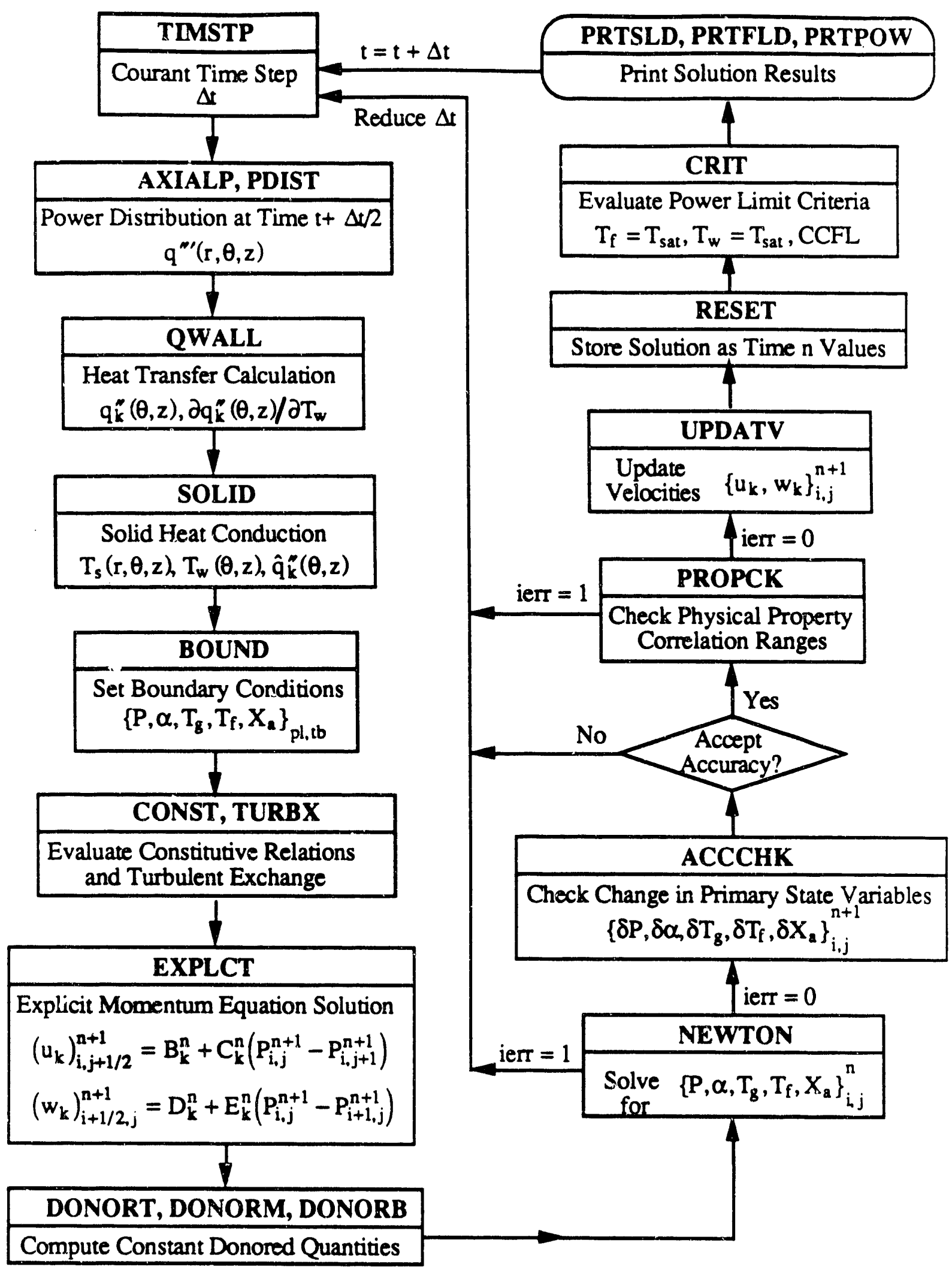

Figure 3.8-1 Flow diagram for subroutine TRANS. 
Much of the numerical solution techniques used in FLOWTRAN-TF have already been presented in other code development reports. De:ailed descriptions of some of the other numerical techniques employed in the solution strategy will be presented in the following sections. We will focus on the coupling between the solid and power equations, the coupling between the solid and fluid equations, and the solution of the fluid finite difference equations.

\subsubsection{Solid-Power}

Power deposited in the metal structures is a source term for the solid energy equation. From Eq. (2.3-4), the basic governing equation that must be solved to obtain the solid temperature distribution is

$$
V_{i, j, k} \frac{\partial E}{\partial t}=\sum_{s=1}^{6}\left[\left(k_{s} \nabla T_{s} \cdot \bar{N}_{s}\right) A_{s}\right]+Q_{i, j, k}^{\prime \prime \prime}
$$

The left hand side of Eq. (3.8.1-1) represents the time rate of change in the energy within a single solid control volume. The first term on the right hand side of the equation is the heat flow into the solid cell across its six faces through conduction from adjacent cells. The last term on the right hand side of Eq. $(3.8 .1-1)$ is the power deposited within the solid cell which varies as a function of time. As noted in \$3.3, when we apply our time differencing scheme to Eq. (3.8.1-1), we evaluate the power distribution at the midpoint of the current time step. We can represent the basic computation of deposited power by the algebraic equation

$$
\begin{aligned}
\left(Q_{i, j, k}^{\prime \prime \prime}\right)^{n+1 / 2} & =\left(V_{i, j, k} R_{f, i} \Theta_{j}\right) Z_{k}^{n+1 / 2} \Phi_{f}^{n+1 / 2} \\
& +\left(V_{i, j, k} R_{w, i} \Theta_{j}\right) Z_{k}^{0} \Phi_{w}^{n+1 / 2} \\
& +\left(V_{i, j, k} R_{d, i} \Theta_{j}\right) Z_{k}^{0} \Phi_{d}^{n+1 / 2}
\end{aligned}
$$

where

$$
\begin{aligned}
V_{i, j, k} & \equiv \text { Solid computational cell volume, } \mathrm{m}^{3} \\
\mathrm{R}_{\mathrm{f}, \mathrm{i}} & \equiv \text { Normalized radial shape function for fission power } \\
\mathrm{R}_{\mathrm{w}, \mathrm{i}} & \equiv \text { Normalized radial shape function for wet decay power } \\
\mathrm{R}_{\mathrm{d}, \mathrm{i}} & \equiv \text { Normalized radial shape function for dry decay power } \\
\Theta_{j} & \equiv \text { Normalized azimuthal power shape function } \\
\mathrm{Z}_{\mathrm{k}}^{\mathrm{n}+1 / 2} & \equiv \text { Normalized axial power shape function } \\
\mathrm{Z}_{\mathrm{k}}^{0} & \equiv \text { Normalized axial power shape function at time } \mathrm{t}=0
\end{aligned}
$$




$$
\begin{aligned}
& \Phi_{\mathrm{f}}^{n+1 / 2} \equiv \text { Fission power transient function } \\
& \Phi_{w}^{n+1 / 2} \equiv \text { Moderated decay power transient function } \\
& \Phi_{\mathrm{d}}^{\mathrm{n}+1 / 2} \equiv \text { Dry decay power transient function }
\end{aligned}
$$

Radial power shapes depend upon the deposited power conditions but are assumed to remain constant in time. The azimuthal power shape is specified as a function of azimuthal position only. Under these assumptions, the products of cell volumes and the normalized radial and azimuthal power distributions shown in Eq. (3.8.1-2) are constant factors that do not vary in time. Therefore, these factors are computed one time in subroutine PNORM prior to entry into subroutine TRANS and are stored for use during the transient calculations. The power transient functions in Eq. (3.8.1-2) are defined to be

$$
\begin{gathered}
\Phi_{f}^{n+1 / 2} \equiv \frac{P_{0}}{\sum_{i, j, k} V_{i, j, k}}\left(1-f_{c}^{n+1 / 2} \delta_{\bmod }\right)\left(\frac{\Delta h^{n+1 / 2}}{\Delta H}\right)_{i, j, k} F_{f}^{n+1 / 2} \\
\Phi_{w}^{n+1 / 2} \equiv \frac{P_{0}}{\sum_{i, j, k} V_{i, j, k}} f_{c}^{n+1 / 2} \delta_{\bmod }\left(\frac{\Delta h^{n+1 / 2}}{\Delta H}\right)_{i, j, k} F_{\bmod }^{n+1 / 2} \\
\Phi_{d}^{n+1 / 2} \equiv \frac{P_{0}}{\sum_{i, j, k} V_{i, j, k}} f_{c}^{n+1 / 2} \delta_{d r y}\left(1-\frac{\Delta h^{n+1 / 2}}{\Delta H}\right)_{i, j, k} F_{d r y}^{n+1 / 2}
\end{gathered}
$$

where:

$$
\begin{aligned}
P_{0} & \equiv \text { Pre-incident assembly power, } W \\
\delta_{\text {mod }} & \equiv \begin{array}{l}
\text { Fraction of initial power attributable to decay products under moderated } \\
\text { tank conditions }
\end{array} \\
\delta_{\mathrm{dry}} & \equiv \begin{array}{l}
\text { Fraction of initial power attributable to decay products under dry tank } \\
\text { conditions }
\end{array} \\
\mathrm{f}_{\mathrm{c}}^{\mathrm{n}+1 / 2} & \equiv \text { Time dependent conservative multiplier }(\geq 1) \\
\Delta \mathrm{h}^{\mathrm{n}+1 / 2} & \equiv \text { Length of solid cell that is moderated, } \mathrm{m} \\
\Delta H & \equiv \text { Total length of solid cell, } \mathrm{m} \\
F_{\mathrm{f}}^{\mathrm{n}+1 / 2} & \equiv \text { Normalized fission power transient } \\
F_{\mathrm{mod}}^{\mathrm{n}+1 / 2} & \equiv \text { Normalized moderated decay power transient } \\
F_{\mathrm{dry}}^{\mathrm{n}+1 / 2} & \equiv \text { Normalized dry decay power transient }
\end{aligned}
$$

Normalizations with respect to the axial distribution and nodal deposited powers are computed within subroutine PDIST. Subroutine AXIALP supplies the axial power distribution at the required time and interpolated to the centers of the axial mesh cells. The 


\begin{tabular}{|l|lll|r|}
\hline $2 / 15 / 93$ & WSRC-TR-92-532 Rev. 0 & FLOWTRAN-TF Software Design & Page 450 \\
\hline
\end{tabular}

axial shape is approximated from the input data using the user specified spline fit (linear or cubic). Linear interpolation in time is applied to the axial shapes. As Eq. (3.8.1-2) indicates, we apply the normalized axial shape at the initial time to the moderated and dry decay heat power distributions. This assumes that the axial shape is burned in at preincident conditions for the decay power components. Complete details of the power computational procedure and normalization techniques are provided in the code development report on power generation.

\subsubsection{Solid-Fluid}

Coupling between the fluid and solid energy equations is obtained through the surface heat flux. The heat flux is calculated explicitly in subroutine QWALL using the heat transfer correlations presented in \$2.5.1. For example, for non-boiling heat transfer, we apply the simple relationship

$$
\left(q_{w}^{\prime \prime}\right)=h\left(T_{w}-T_{f}\right)
$$

where:

$$
\begin{aligned}
\mathrm{q}_{w}^{\prime \prime} & \equiv \text { Surface heat flux, } W / \mathrm{m}^{2} \\
\mathrm{~h} & \equiv \text { Heat transfer coefficient, } W / \mathrm{m}^{2}-K \\
\mathrm{~T}_{w}^{\mathrm{n}} & \equiv \text { Surface temperature, } \mathrm{K} \\
\mathrm{T}_{\mathrm{f}}^{\mathrm{n}} & \equiv \text { Fluid temperature, } \mathrm{K}
\end{aligned}
$$

In subroutine SOLID, the heat conduction equations are solved and solid temperatures at the new time are computed. The heat flux is applied as a radial boundary condition in the computation of the solid temperature distribution (see \$3.3). To obtain an estimate of the boundary condition at time $n+1$, the surface heat flux given by Eq. $(3.8 .2-1)$ is approximated by the single term Taylor series expansion

$$
\left(q_{w}^{\prime \prime}\right)^{n+1}=\left(q_{w}^{\prime \prime}\right)^{n}+\left(\frac{\partial q_{w}^{\prime \prime}}{\partial T_{w}}\right)^{n}\left(T_{w}^{n+1}-T_{w}^{n}\right)
$$

Equation (3.8.2-2) is applied as a solid boundary condition and is used to compute the surface heat flux from updated values of the wall temperature. This same heat flux is used in the solution of the fluid energy equation to conserve energy in the transfer between solid and fluid. From Eq. (3.8.2-1), the derivative of the heat flux with respect to the wall temperature is given by

$$
\left(\frac{\partial q_{w}^{\prime \prime}}{\partial T_{w}}\right)=h+\left(T_{w}-T_{f}\right)\left(\frac{\partial h}{\partial T_{w}}\right)
$$

When the heat transfer coefficient is of the form 


$$
h=c\left(\frac{k_{f}}{D_{H}}\right) \operatorname{Re}^{a} \operatorname{Pr}^{b}
$$

where the Reynolds and Prandtl Numbers are based on bulk fluid properties, the coefficient is solely a function of fluid properties and flow geometry. Therefore, the derivative of the heat flux with respect to the wall temperature is simply the heat transfer coefficient itself and Eq. $(3.8 .2-2)$ reduces to

$$
\left(q_{w}^{\prime \prime}\right)^{n+1}=h^{n}\left(T_{w}^{n+1}-T_{f}^{n}\right)
$$

The adjusted heat flux computed from Eq. (3.8.2-5) is multiplied by the surface area and used as a source term in solving the fluid energy equation. As shown in Fig. 3.8-1, only a single pass is made through the surface heat flux and solid computations at each time step. That is, we do not iterate the fluid and solid solutions to convergence where the derivatives would approach zero but instead use Eq. (3.8.2-5) to provide a single corrected estimate of the fluid energy source term.

The treatment presented above is somewhat simplified (see 82.3). In practice we include an effect from rib heat transfer in the calculation of the surface heat transfer coefficient. When ribs are present within the solid computational cell, Eq. (3.8.2-1) actually uses a modified heat transfer coefficient given by

$$
\hat{h}=\left[1+P_{R i b}\left(\frac{\tanh \left(m R_{L}\right)}{m}-R_{T}\right)\right] h
$$

with

$$
\mathrm{m}=\sqrt{\frac{h}{k_{w} R_{T}}}
$$

where:

$P_{\text {Rib }} \equiv$ Number of half rib surfaces in fluid computational cell divided by the wetted perimeter of the cell wall

$R_{\mathbf{T}} \mathbf{m}$ Rib half thickness, $\mathrm{m}$

$\mathbf{R}_{\mathrm{L}}=\mathrm{Rib}$ length, $\mathrm{m}$

$k_{w} \equiv$ Metal thermal conductivity evaluated at $T_{w}, W / m-K$

Equation (3.8.2-6) introduces a dependence on the metal surface temperature into the heat transfer coefficient. Then Eq. (3.8.2-3) should actually include a contribution from the second term with a coefficient of

$$
\left(\frac{\partial \hat{h}}{\partial T_{w}}\right)=-\frac{h P_{R_{i b}}}{2}\left[\frac{m R_{L} \operatorname{sech}^{2}\left(m R_{L}\right)-\tanh \left(m R_{L}\right)}{m}\right]\left(\frac{1}{k_{w}} \frac{\partial k_{w}}{\partial T_{w}}\right)
$$


In practice, we have neglected this additional correction term arising from rib heat transfer effects and simply apply Eq. (3.8.2-5) to estimate the heat transfer at the new time.

The above discussion applies to the two-phase heat transfer correlations based on SRL data that are implemented in subroutine SRLHTC and the single-phase heat transfer correlations for vapor condensation used in subroutine CDENS. Some of the other heat transfer correlations in the code have more complicated functionality and require additional terms. For example, the Sieder-Tate single phase correlation in subroutine HCONV has the form

$$
h=c\left(\frac{k_{f}}{D_{H}}\right) \operatorname{Re}^{a} \operatorname{Pr}^{b}\left(\frac{\mu_{f}}{\mu_{w}}\right)^{d}
$$

where:

$$
\begin{aligned}
& \mu_{\mathrm{f}} \equiv \text { Fluid viscosity evaluated at the bulk temperature, } \mathrm{kg} / \mathrm{m}-\mathrm{s} \\
& \mu_{\mathrm{w}} \equiv \text { Fluid viscosity evaluated at the wall temperature, } \mathrm{kg} / \mathrm{m}-\mathrm{s}
\end{aligned}
$$

Since Eq. (3.8.2-9) contains a direct dependence on the wall temperature through the fluid viscosity we compute

$$
\left(\frac{\partial h}{\partial T_{w}}\right)=-h d\left(\frac{1}{\mu_{w}} \frac{\partial \mu_{w}}{\partial T_{w}}\right)
$$

and include this factor in Eq. (3.8.2-3) to estimate a corrected surface heat flux.

The boiling heat transfer correlations are also more complicated functions of the wall temperature. The Chen correlation implemented in subroutine CHEN (see \$2.5.1) can be expressed as

$$
\left(q_{w}^{\prime \prime}\right)=h\left(T_{w}-T_{f}\right)+h_{N B}\left(T_{w}-T_{s a t}\right)
$$

where $\mathrm{h}$ is a single-phase heat transfer coefficient that is solely a function of bulk fluid properties and $h_{\mathrm{NB}}$ is a nucleate boiling heat transfer coefficient that is evaluated as

$$
h_{N B}=C\left(T_{w}-T_{s a t}\right)^{x}\left(P_{w}-P\right)^{y}
$$

In Eq. (3.8.2-12), $\mathbf{P}_{\mathbf{w}}$ is the saturation pressure at the wall temperature. To apply Eq. (3.8.2-2) we use Eqs. (3.8.2-11) and (3.8.2-12) to compute

$$
\begin{aligned}
\left(\frac{\partial \mathrm{q}_{w}^{\prime \prime}}{\partial \mathrm{T}_{\mathrm{w}}}\right) & =h+h_{\mathrm{NB}}+\left(\mathrm{T}_{w}-\mathrm{T}_{\text {sal }}\right)\left(\frac{\partial \mathrm{h}_{\mathrm{NB}}}{\partial \mathrm{T}_{\mathrm{w}}}\right) \\
& =h+h_{\mathrm{NB}}\left\{1+x+\left.y \frac{\left(\mathrm{T}_{w}-\mathrm{T}_{\text {sat }}\right)}{\left(\mathrm{P}_{\mathrm{w}}-\mathrm{P}\right)}\left(\frac{\mathrm{dP}_{\mathrm{sat}}}{\mathrm{dT}}\right)\right|_{\mathrm{T}_{w}}\right\}
\end{aligned}
$$


If the code is executing subroutine CHEN but conditions are such that boiling has not started, the nucleate boiling heat transfer coefficient is set equal to zero in the above relationships.

The Mikic-Roshenow boiling correlation in subroutine MIKIC (see \$2.5.1) introduces even more complicated functionality into the derivative of the heat flux. As shown in $\$ 2.5 .1$, we express the surface heat flux as

$$
\left(q_{w}^{\prime \prime}\right)=\sqrt{\left(q_{F C}^{\prime \prime}\right)^{2}+\left(q_{B}^{\prime \prime}-q_{B i}^{\prime \prime}\right)^{2}}
$$

where:

$$
\begin{aligned}
\left(\mathrm{q}_{\mathrm{FC}}^{\prime \prime}\right) & \equiv \mathrm{h}\left(\mathrm{T}_{\mathrm{w}}-\mathrm{T}_{\mathrm{f}}\right)=\text { Forced convection heat flux, } \mathrm{W} / \mathrm{m}^{2} \\
\left(\mathrm{q}_{B}^{\prime \prime}\right) & \equiv \stackrel{\circ}{\mathrm{h}}_{\mathrm{B}}\left(\mathrm{T}_{\mathrm{w}}-\mathrm{T}_{\text {sat }}\right)^{3}=\text { Boiling heat flux, } \mathrm{W} / \mathrm{m}^{2} \\
\left(\mathrm{q}_{B \mathrm{~B}}^{\prime \prime}\right) & \equiv \stackrel{\circ}{\mathrm{h}}_{\mathrm{B}}\left(\Delta \mathrm{T}_{\mathrm{ONB}}\right)^{3}=\text { Incipient boiling heat flux, W/m } \mathrm{m}^{2}
\end{aligned}
$$

Taking the derivative of Eq. (3.8.2-14) we obtain

$$
\left(\frac{\partial q_{w}^{\prime \prime}}{\partial T_{w}}\right)=\frac{1}{\left(q_{w}^{\prime \prime}\right)}\left[\left(q_{F C}^{\prime \prime}\right)\left(\frac{\partial q_{F C}^{\prime \prime}}{\partial T_{w}}\right)+\left(q_{B}^{\prime \prime}-q_{B i}^{\prime \prime}\right)\left(\frac{\partial q_{B}^{\prime \prime}}{\partial T_{w}}-\frac{\partial q_{B i}^{\prime \prime}}{\partial T_{w}}\right)\right]
$$

The heat transfer coefficients are functions of fluid properties only. Therefore, we can immediately write

$$
\begin{gathered}
\left(\frac{\partial q_{F C}^{\prime \prime}}{\partial T_{w}}\right)=h \\
\left(\frac{\partial q_{B}^{\prime \prime}}{\partial T_{w}}\right)=3 h_{B}^{o}\left(T_{w}-T_{s a t}\right)^{2} \\
\left(\frac{\partial q_{B i}^{\prime \prime}}{\partial T_{w}}\right)=\left.3 h_{B}^{\circ}\left(\Delta T_{O N B}\right)^{2}\left(\frac{\partial \Delta T_{O N B}}{\partial T_{w}}\right)\right|_{T_{w}}
\end{gathered}
$$

The expressions used to compute $\Delta \mathrm{T}_{\mathrm{ONB}}$ depend upon the wall temperature since the thermal conductivity is evaluated at the wall temperature. Using the relationships given in $\S 2.5 .1$, we can show that for $h_{F C}<h_{\text {tan }}$

$$
\left(\frac{\partial \Delta \mathrm{T}_{\mathrm{ONB}}}{\partial \mathrm{T}_{\mathrm{w}}}\right)=-\frac{N \mathrm{u}_{\mathrm{cav}}}{1-N u_{\mathrm{cav}}}\left(\Delta \mathrm{T}_{\mathrm{ONB}}+\Delta \mathrm{T}_{\mathrm{sub}}\right)\left(\frac{1}{\mathrm{k}_{\mathrm{w}}} \frac{\partial \mathrm{k}_{\mathrm{w}}}{\partial \mathrm{T}_{\mathrm{w}}}\right)
$$

and for $h_{\mathrm{FC}} \geq h_{\text {Lan }}$ 


$$
\left(\frac{\partial \Delta \mathrm{T}_{\mathrm{ONB}}}{\partial \mathrm{T}_{\mathrm{w}}}\right)=-\left(\Delta \mathrm{T}_{\mathrm{ONB}}+\gamma-\beta\right)\left(\frac{1}{\mathrm{k}_{\mathrm{w}}} \frac{\partial \mathrm{k}_{\mathrm{w}}}{\partial \mathrm{T}_{\mathrm{w}}}\right)
$$

where

$$
\beta=\left\{\begin{array}{ccc}
\left(\Delta \mathrm{T}_{\text {sub }}-\gamma\right) / \sqrt{\tau} & \text { if } & \tau>0 \\
0 & \text { if } & \tau \leq 0
\end{array}\right.
$$

The complete nomenclature used above is explained in $\$ 2.5 .1$.

The film boiling correlations coded in subroutine FILMB and described in $\$ 2.5 .1$ give the heat fluxes into the liquid and gas phases as

$$
\left(\mathrm{q}_{w}^{\prime \prime}\right)_{\mathrm{f}}=(1-\alpha)\left\{\left[(1-\zeta) \mathrm{h}_{\mathrm{BP}}+\zeta \mathrm{h}_{\mathrm{FR}}\right]\left(\mathrm{T}_{\mathrm{w}}-\mathrm{T}_{\text {sat }}\right)+\varepsilon\left[\left(\mathrm{T}_{\mathrm{w}}\right)^{4}-\left(\mathrm{T}_{\text {sat }}\right)^{4}\right]\right\}
$$

and

$$
\left(\mathrm{q}_{w}^{\prime \prime}\right)_{g}=\alpha\left\{\mathrm{h}_{\mathrm{DR}}\left(\mathrm{T}_{\mathrm{w}}-\mathrm{T}_{\mathrm{g}}\right)+\varepsilon\left[\left(\mathrm{T}_{\mathrm{w}}\right)^{4}-\left(\mathrm{T}_{\mathrm{g}}\right)^{4}\right]\right\}
$$

Both the Bromley-Pomeranz ( $h_{\mathrm{BP}}$ ) and Forslund-Rohsenow ( $h_{\mathrm{FR}}$ ) ligujd heat transfer coefficients in Eq. $(3.8 .2-22)$ contain identical factors of $\left(\mathrm{T}_{\mathrm{w}}^{\mathrm{n}}-\mathrm{T}_{\mathrm{sal}}^{\mathrm{n}}\right)^{-0.25}$ and modified heats of vaporization of the form

$$
\hat{\mathrm{h}}_{\mathrm{fg}}=\mathrm{h}_{\mathrm{fg}}+0.5 \mathrm{c}_{\mathrm{pg}}\left(\mathrm{T}_{\mathrm{w}}-\mathrm{T}_{\mathrm{sar}}\right)
$$

It is easy to show that this functionality leads to the following derivative relationship

$$
\left(\frac{\partial q_{w}^{\prime \prime}}{\partial T_{w}}\right)_{f}=(1-\alpha)\left\{0.75 \beta\left[(1-\zeta) h_{B P}+\zeta h_{F R}\right]+4 \varepsilon\left(T_{w}\right)^{3}\right\}
$$

where

$$
\beta=1+\frac{c_{p g}\left(T_{w}-T_{\text {sat }}\right)}{6 \hat{h}_{f g}}
$$

Since the Dougall-Rohsenow heat transfer coefficient in Eq. (3.8.2-23) is only a function of fluid properties, we can immediately evaluate the derivative for the heat flux into the gas phase as

$$
\left(\frac{\partial \mathrm{q}_{w}^{\prime \prime}}{\partial \mathrm{T}_{w}}\right)_{g}=\alpha\left\{\mathrm{h}_{\mathrm{DR}}+4 \varepsilon\left(\mathrm{T}_{w}\right)^{3}\right\}
$$


In the transition boiling regime (coded in subroutine FILMB), we use a simple cubic interpolation scheme to estimate the surface heat fluxes. As shown in $\$ 2.5 .1$, we set

$$
\left(q_{w}^{\prime \prime}\right)_{f}=(1-\delta)\left(q_{f}^{\prime \prime}\right)_{M S F B}+\delta\left(q^{\prime \prime}\right)_{C H F}
$$

and

$$
\left(q_{w}^{\prime \prime}\right)_{g}=(1-\delta)\left(q_{g}^{\prime \prime}\right)_{M S F B}
$$

where $\delta$ is an interpolation parameter that depends upon $\mathrm{T}_{\mathrm{w}}$. Taking the derivatives of Eqs. $(3.8 .2-28)$ and $(3.8 .2-29)$ yields

$$
\left(\frac{\partial \mathrm{q}_{\mathrm{w}}^{\prime \prime}}{\partial \mathrm{T}_{\mathrm{w}}}\right)_{\mathrm{f}}=\left[\left(\mathrm{q}^{\prime \prime}\right)_{\mathrm{CHF}}-\left(\mathrm{q}_{\mathrm{f}}^{\prime \prime}\right)_{\mathrm{MSFB}}\right]\left(\frac{\partial \delta}{\partial \mathrm{T}_{\mathrm{w}}}\right)_{\mathrm{T}_{w}}
$$

and

$$
\left(\frac{\partial q_{w}^{\prime \prime}}{\partial T_{w}}\right)_{g}=-\left(q_{g}^{\prime \prime}\right)_{M S F B}\left(\frac{\partial \delta}{\partial T_{w}}\right)_{T_{w}}
$$

From the definition of the interpolation parameter, we find

$$
\left(\frac{\partial \delta}{\partial \mathrm{T}_{\mathrm{w}}}\right)=\frac{6 \xi(1-\xi)}{\mathrm{T}_{\mathrm{MSFB}}-\mathrm{T}_{\mathrm{CHF}}}
$$

where

$$
\xi=\frac{T_{w}-T_{C H F}}{T_{M S F B}-T_{C H F}}
$$

This completes specification of the derivative terms used to compute improved estimates of the heat flux into the fluid phases. These derivatives are calculated within the heat transfer correlation subroutines and passed into subroutine SOLID where the Taylor series expression given by Eq. (3.8.2-2) is evaluated.

\subsubsection{Fluid}

The techniques used to solve the set of fluid conservation equations are adapted from the THERMIT code (Reed and Stewart, 1978). The finite differencing scheme for the fluid equations is neither fully explicit or fully implicit. The scheme advanced by Reed and Stewart is to treat flow phenomena that occur over short time scales implicitly while phenomena that occur over longer time scales are treated explicitly. Exchange processes such as interfacial mass and energy exchange and the pressure gradient term in the momentum equation, which represent sonic propagation effects, are treated in an implicit manner. However, the convection terms of the mass and energy conservation equations 
are treated in a semi-explicit manner to increase computational efficiency. Fluid property functions in the mass/energy convection terms are evaluated explicitly at time $n$ while the fluid velocities in the mass and energy transport equations are evaluated at the new time $n+1$. These terms represent physical phenomena (i.e. convection) that occur over a longer time scale. Since this differencing is done explicitly, the computational time step is constrained to meet Courant stability limits. However, the Courant limit is not unduly restrictive since it need only account for the longer time scale phenomena. Convective transport terms in the momentum equations are treated in a fully explicit manner.

Donor-cell differencing is used to improve the stability characteristics of the fluid difference equations. The time step is also constrained such that the nonlinear implicit functions can be adequately approximated using a single term in Taylor series expansions about the old time values. This produces function approximations that are linear in the implicit variables and avoids the need to iteratively solve a large system of nonlinear equations.

As shown in $\$ 3.4$, the fluid momentum equations are solved to obtain functions relating velocity components to local pressure differences. These velocity-pressure functions are used to eliminate velocities from the mass/energy equations. Velocity gradient terms in the momentum equations that describe momentum convection are treated explicitly in the difference equations. However, since the interfacial and wall momentum exchange terms are treated implicitly, the liquid and gas velocities are coupled. The linearized axial momentum equations form a $2 \times 2$ system of equations that is inverted to give a set of equations relating the gas and liquid axial velocities to the axial pressure difference at each mesh location. Solving the gas and liquid axial momentum equations simultaneously yields linear velocity relationships of the form

$$
\begin{aligned}
& \left(u_{g}\right)_{i, j+1 / 2}^{n+1}=B_{g}^{n}+C_{g}^{n}\left(P_{i, j}^{n+1}-P_{i, j+1}^{n+1}\right) \\
& \left(u_{f}\right)_{i, j+1 / 2}^{n+1}=B_{f}^{n}+C_{f}^{n}\left(P_{i, j}^{n+1}-P_{i, j+1}^{n+1}\right)
\end{aligned}
$$

where the coefficients $B_{g}^{n}, C_{g}^{n}, B_{f}^{n}$, and $C_{f}^{n}$ are defined in $\$ 3.4 .1$. These coefficients are evaluated explicitly at each grid location (we omit the $i, j+1 / 2$ subscripts for brevity) from parameter values at the old time step. These calculations take place within subroutine EXPLCT (see Fig. 3.8-1).

Similarly, the linearized azimuthal momentum equations form a $2 \times 2$ matrix equation that is inverted to give a set of equations relating the azimuthal fluid and gas velocities to the azimuthal pressure difference across a mesh point. Solving the gas and liquid azimuthal momentum equations simultaneously yields the following velocity relationships

$$
\begin{aligned}
& \left(w_{g}\right)_{i+1 / 2, j}^{n+1}=D_{g}^{n}+E_{g}^{n}\left(P_{i, j}^{n+1}-P_{i+1, j}^{n+1}\right) \\
& \left(w_{f}\right)_{i+1 / 2, j}^{n+1}=D_{f}^{n}+E_{f}^{n}\left(P_{i, j}^{n+1}-P_{i+1, j}^{n+1}\right)
\end{aligned}
$$

The coefficients $D_{g}^{n}, E_{g}^{n}, D_{f}^{n}$, and $E_{f}^{n}$ are defined in $\$ 3.4 .1$. These coefficients are again evaluated explicitly at each grid location from parameter values at the old time step.

Eliminating velocities from the set of five difference equations derived from the fluid mass/energy balances (see \$3.4.2 and \$3.4.3), these equations can be combined into the equivalent matrix representation 


$$
\underline{c m}^{m}\left[\begin{array}{c}
(\delta P)_{i, j}^{m+1} \\
(\delta \alpha)_{i, j}^{m+1} \\
\left(\delta T_{g}\right)_{i, j}^{m+1} \\
\left(\delta T_{f}\right)_{i, j}^{m+1} \\
\left(\delta X_{a}\right)_{i, j}^{m+1}
\end{array}\right]+\underline{c p}^{n}\left[\begin{array}{c}
(\delta P)_{i, j-1}^{m+1} \\
(\delta P)_{i, j+1}^{m+1} \\
(\delta P)_{i-1, j}^{m+1} \\
(\delta P)_{i+1, j}^{m+1}
\end{array}\right]=\underline{f}^{m}
$$

where $\mathrm{cm}^{\mathrm{m}}$ is a $5 \times 5$ matrix of coefficient values, $\mathrm{cp}^{\mathrm{n}}$ is a $5 \times 4$ matrix of pressure coupling coefficients, and $\underline{f}^{m}$ is a 5 element vector of right hand side terms. The superscript $n$ is used to indicate that members of the $\mathrm{cp}^{n}$ matrix are evaluated explicitly at the old time. The superscript $m$ is used to indicate the current step within the Newton iteration scheme. Equation (3.8.3-3) applies within the two-dimensional flow section. As shown in \$3.4.3, several modified versions of this equation apply at special locations in the assembly. All of the modifications simply change the form of the differential pressure vector in the second term. We will use the two-dimensional version shown in Eq. (3.8.3-3) to illustrate the solution methods.

The fundamental fluid conservation equitions are nonlinear since fluid properties at the new time must be evaluated using the equation of state and the exchange terms are evaluated implicitly. To derive Eq. (3.8.3-3), we have linearized the equations by using local Taylor series expansions to approximate the implicit property and exchange terms (see \$3.4.2 and $\$ 3.4 .3$ for details). We then use a Newton iteration strategy to solve the set of equations. Within a single Newton iteration at each time step, we solve the set of linear equations represented by Eq. (3.8.3-3) by inverting the $\mathrm{cm}^{\mathrm{m}}$ matrix to obtain the reduced form

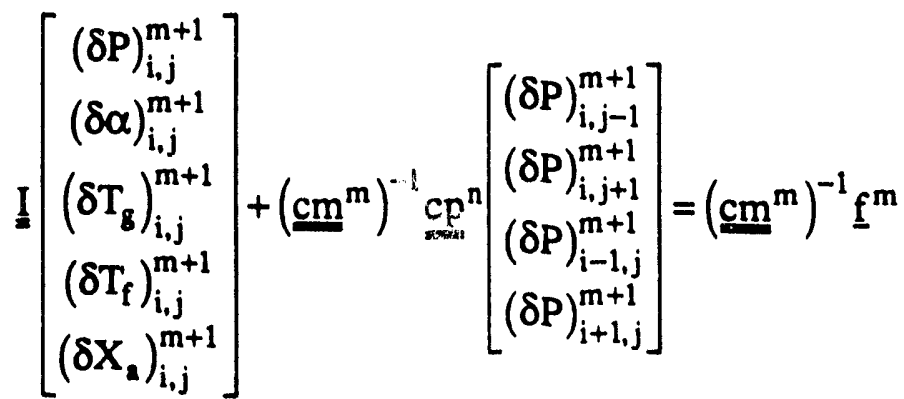

The first equation in this set is an equation involving only the pressure change in the $i, j$ fluid cell and the four neighboring fluid cells. The set of all such equations for the pressure change form a linear algebraic system of equations that can be solved simultaneously for the pressure change in all of the fluid mesh cells. Therefore, the solution of the fluid conservation equations has been reduced to that of solving for the pressure only. The remaining four equations relate the changes in local void fraction, gas temperature, liquid temperature, and air mass fraction to the corresponding pressure changes.

The solution of the mass/energy fluid conservation equations is coded in subroutine NEWTON. A schematic diagram indicating the principal features of this subroutine is shown in Fig. 3.8.3-1. The finite difference analogs to the mass/energy equations are formed separately for the top, middle, and bottom sections of the assembly in subroutines JACOBT, JACOBM, and JACOBB, respectively. Figure 3.8.3-2 shows a schematic diagram of the computations in subroutine JACOBM. All of the JACOB* routines evaluate 
state variables at the current fluid conditions (subroutine STATE) and calculate interfacial heat and mass transport parameters (subroutine GAMMAI). In subroutine JACOBM, which applies in the heated middle section, we must also consider mass and energy generation at the wall. Two user options are available for this calculation as illustrated in Fig. 3.8.3-2. If the input parameter IGAMW is set equal to 1 , the code uses the explicit vapor generation rates calculated in the heat transfer routines (see \$2.5.1) to estimate mass and energy exchange at the wall. If IGAMW is set to any other value, the wall mass and energy exchange terms are set to zero.

Within subroutine NEWTON, we first pass through the three JACOB* subroutines to create the set of finite difference equations for fluid mass/energy conservation. The JACOB* subroutines also perform the matrix inversion shown in Eq. (3.8.3-4). Subroutine INNER is then called to solve the pressure only problem and obtain pressure changes throughout the entire assembly. The solution methods used in subroutine INNER are fully described in \$3.8.4. Following the pressure solution, the primary state variables are updated in subroutine UPDATP using the last four equations in Eq. (3.8.3-4) to compute

$$
(\delta \alpha)_{i, j}^{m+1},\left(\delta T_{B}\right)_{i, j}^{m+1},\left(\delta T_{f}\right)_{i, j}^{m+1}, \text { and }\left(\delta X_{a}\right)_{i, j}^{m+1}
$$




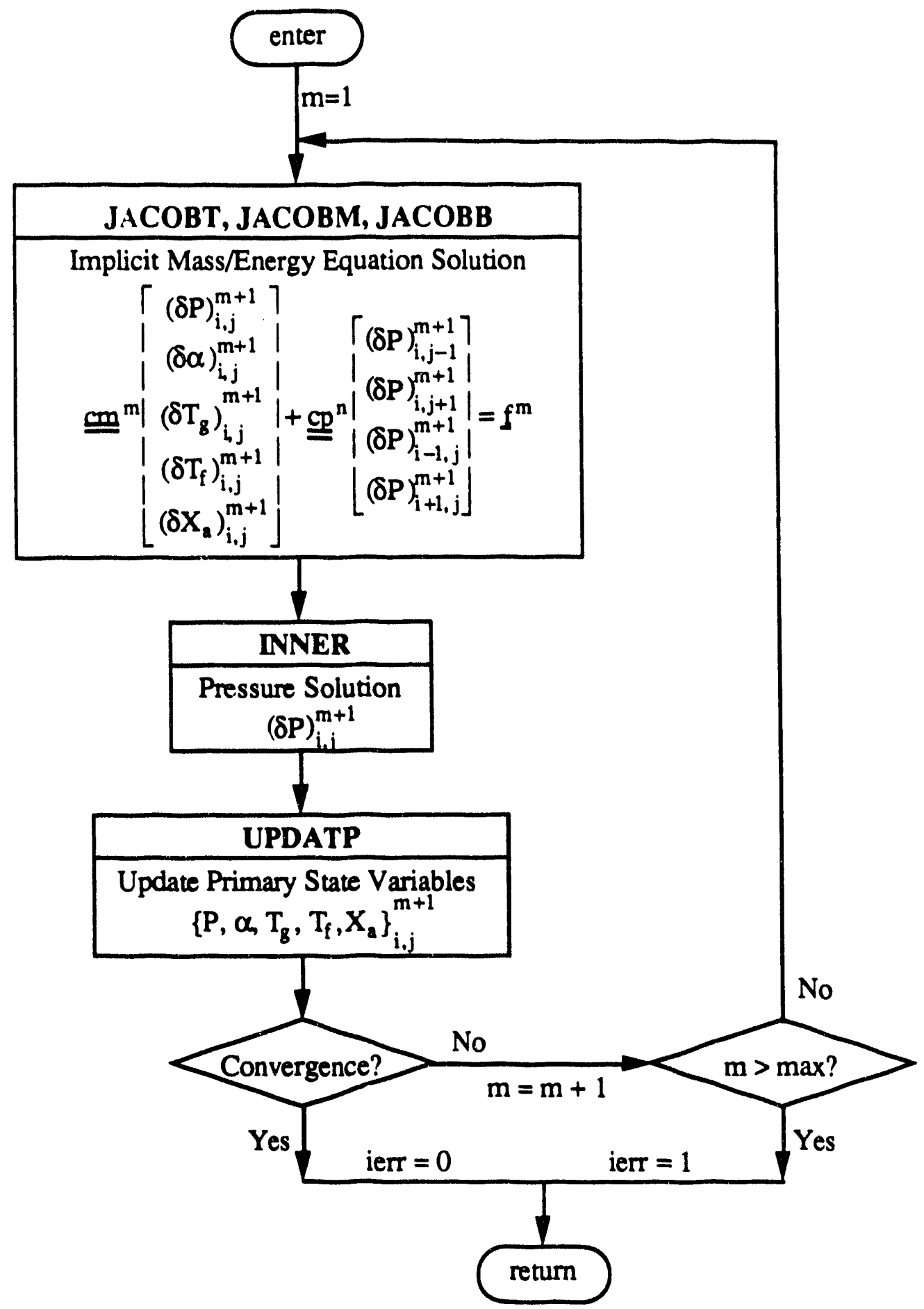

Figure 3.8.3-1 Flow diagram for subroutine NEWTON. 


\section{STATE}

Compute Gas and Liquid Densities and Enthalpies and Derivatives

$$
\begin{aligned}
& \rho_{g}^{m},\left(\frac{\partial \rho_{g}}{\partial P}\right)^{m},\left(\frac{\partial \rho_{g}}{\partial T_{g}}\right)^{m},\left(\frac{\partial \rho_{g}}{\partial X_{g}}\right)^{m} \rho_{f}^{m},\left(\frac{\partial \rho_{f}}{\partial P}\right)^{m},\left(\frac{\partial \rho_{f}}{\partial T_{f}}\right)^{m} \\
& h_{g}^{m},\left(\frac{\partial h_{g}}{\partial P}\right)^{m},\left(\frac{\partial h_{g}}{\partial T_{g}}\right)^{m},\left(\frac{\partial h_{g}}{\partial X_{g}}\right)^{m} h_{f}^{m},\left(\frac{\partial h_{f}}{\partial P}\right)^{m},\left(\frac{\partial h_{f}}{\partial T_{f}}\right)^{m}
\end{aligned}
$$

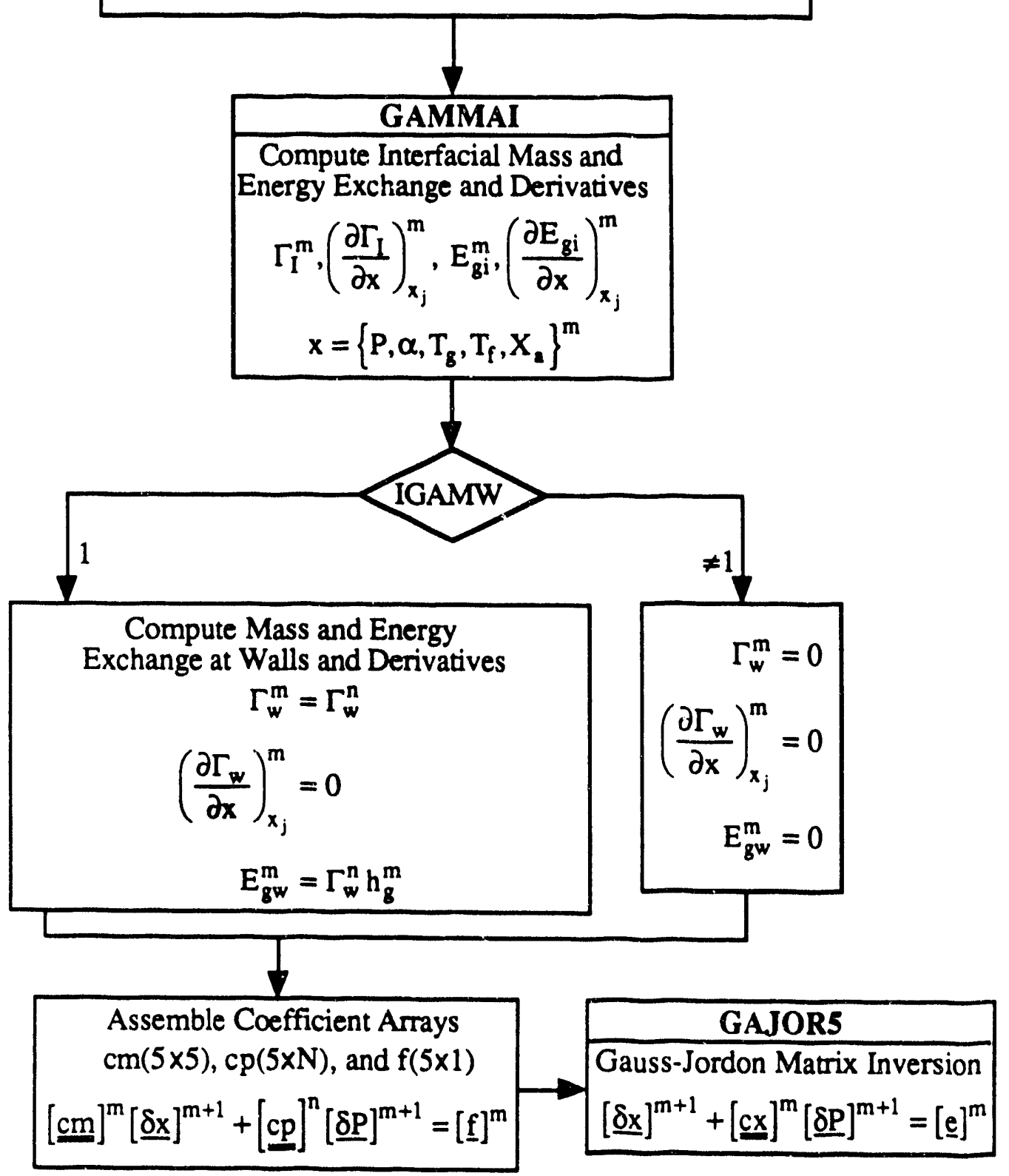

Figure 3.8.3-2 Flow chart of subroutine JACOBM. 
and the relationships

$$
\begin{aligned}
& (P)_{i, j}^{m+1}=(P)_{i, j}^{m}+(\delta P)_{i, j}^{m+1}, \quad(\alpha)_{i, j}^{m+1}=(\alpha)_{i, j}^{m}+(\delta \alpha)_{i, j}^{m+1} \\
& \left(T_{g}\right)_{i, j}^{m+1}=\left(T_{g}\right)_{i, j}^{m}+\left(\delta T_{g}\right)_{i, j}^{m+1}, \quad\left(T_{f}\right)_{i, j}^{m+1}=\left(T_{f}\right)_{i, j}^{m}+\left(\delta T_{f}\right)_{i, j}^{m+1} \\
& \left(X_{a}\right)_{i, j}^{m+1}=\left(X_{a}\right)_{i, j}^{m}+\left(\delta X_{a}\right)_{i, j}^{m+1}
\end{aligned}
$$

Subroutine NEWTON then checks for convergence in the solution by comparing the maximum changes in the state variables to individual tolerances input by the code user and ensuring that

$$
\begin{aligned}
& \max _{i, j}\left|(\delta P)_{i, j}^{m+1}\right| \leq \varepsilon_{P}, \quad \max _{i, j}\left|(\delta \alpha)_{i, j}^{m+1}\right| \leq \varepsilon_{\alpha}, \\
& \max _{i, j}\left|\left(\delta T_{g}\right)_{i, j}^{m+1}\right| \leq \varepsilon_{T_{g}}, \quad \max _{i, j}\left|\left(\delta T_{f}\right)_{i, j}^{m+1}\right| \leq \varepsilon_{T_{f}}, \\
& \max _{i, j}\left|\left(\delta X_{a}\right)_{i, j}^{m+1}\right| \leq \varepsilon_{X_{a}}
\end{aligned}
$$

If all of the convergence criteria are not satisfied, the index $\mathrm{m}$ is incremented by one and the solution is iterated through another pass as shown in Fig. 3.8.3-1. If the maximum number of iterations is exceeded, a flag is set to request a reduction in the time step ( $i$ err $=1$ ) and the computations are terminated. When all of the above convergence criteria are satisfied, the solution is complete and logic proceeds, as shown in Fig. 3.8-1, to subroutine ACCCHK. The accuracy checking procedures are discussed in a separate code development report.

To perform the matrix inversion indicated in Eq. (3.8.3-4), we use a limited Gauss-Jordon procedure specific for a $5 \times 5$ matrix that is coded in subroutine GAJOR5. To accomplish the inversion we first create the augmented matrix obtained by concatenating the $\mathrm{cm}^{\mathrm{m}}$ matrix, the $\mathrm{f}^{\mathrm{m}}$ vector, and the $\mathrm{cp}^{\mathrm{n}}$ matrix. We denote the augmented matrix as the matrix which we represent as

$$
\left[\begin{array}{lllllllll}
a_{11} & a_{12} & a_{13} & a_{14} & a_{15} & f_{1} & p_{11} & \ldots & p_{1 N} \\
a_{21} & a_{22} & a_{23} & a_{24} & a_{25} & f_{2} & p_{21} & \ldots & p_{2 N} \\
a_{31} & a_{32} & a_{33} & a_{34} & a_{35} & f_{3} & p_{31} & \ldots & p_{3 N} \\
a_{41} & a_{42} & a_{43} & a_{44} & a_{45} & f_{4} & p_{41} & \ldots & p_{4 N} \\
a_{51} & a_{52} & a_{53} & a_{54} & a_{55} & f_{5} & p_{51} & \ldots & p_{5 N}
\end{array}\right]
$$

where we have adopted the simplifying notation 


$$
\begin{array}{ll}
c a_{j k} \equiv a_{j k}=c m_{j k}, & j=1 . . .5 ; \quad k=1 . .5 \\
c a_{j 6} \equiv f_{j}=f_{j}, & j=1 . .5 \\
c a_{j k+6} \equiv p_{j k}=c p_{j k} & j=1 . . .5 ; \quad k=1 . . . N
\end{array}
$$

In general, the $c \mathrm{p}^{\mathrm{n}}$ matrix can have a large number of pressure coupling coefficients for assembly cells Tocated at the boundaries between the flow sections (see \$3.4.3). Therefore, we indicate this matrix to be of dimension $5 \times \mathrm{N}$. In the two-dimensional middle section of the assembly, $\mathrm{N}=4$ as each fluid cell is coupled to two neighboring axial cells and two neighboring azimuthal cells. Applying the Gauss-Jordon solution method, we convert the first five columns of the augmented matrix (3.8.3-the $\mathrm{cm}^{\mathrm{m}}$ matrix) into the identity matrix by eliminating off-diagonal elements both above and below the diagonal using the following five step process.

Step 1: To start the solution, the first two rows of the augmented matrix are modified using the relationships

$$
\begin{aligned}
& c a_{1 k}^{\prime}=\frac{c a_{1 k} a_{22}-c a_{2 k} a_{12}}{a_{11} a_{22}-a_{21} a_{12}}, \quad k=3 . . . N+6 \\
& c a_{2 k}^{\prime}=\frac{a_{11} c a_{2 k}-a_{21} c a_{1 k}}{a_{11} a_{22}-a_{21} a_{12}}, \quad k=3 . . . N+6
\end{aligned}
$$

where we use the symbol ca $\mathrm{jk}_{\mathrm{j}}$ to indicate the element of the ca matrix in row $\mathrm{j}$ and column $\mathrm{k}$. This general notation is used to stand for any member of the sugmented matrix (i.e. $a_{j k}$ from $\mathrm{cm}^{\mathrm{m}}, \mathrm{f}_{\mathrm{j}}$ from $\underline{\mathrm{f}}^{\mathrm{m}}$, or $\mathrm{p}_{\mathrm{jk}}$ from $\mathrm{cp}^{\mathrm{n}}$ ). This first elimination procedure creates the modified matrix

$$
\left[\begin{array}{ccccccccc}
1 & 0 & a_{13}^{\prime} & a_{14}^{\prime} & a_{15}^{\prime} & f_{1}^{\prime} & p_{11}^{\prime} & \cdots & p_{1 N}^{\prime} \\
0 & 1 & a_{23}^{\prime} & a_{24}^{\prime} & a_{25}^{\prime} & f_{2}^{\prime} & p_{21}^{\prime} & \cdots & p_{2 N}^{\prime} \\
a_{31} & a_{32} & a_{33} & a_{34} & a_{35} & f_{3} & p_{31} & \cdots & p_{3 N} \\
a_{41} & a_{42} & a_{43} & a_{44} & a_{45} & f_{4} & p_{41} & \cdots & p_{4 N} \\
a_{51} & a_{52} & a_{53} & a_{54} & a_{55} & f_{5} & p_{51} & \cdots & p_{5 N}
\end{array}\right]
$$

where we use the $p$ rime notation to indicate a modified matrix element.

Step 2: We then eliminate the matrix elements in the first two columns of the last three rows by applying

$$
c a_{j k}^{\prime}=c a_{j k}-c a_{j 1} c a_{1 k}^{\prime}-c a_{j 2} c a_{2 k}^{\prime}, \quad j=3,4,5 ; \quad k=3 . . N+6
$$

which creates the modified matrix 


$$
\left[\begin{array}{lllllllll}
1 & 0 & \mathrm{a}_{13}^{\prime} & \mathrm{a}_{14}^{\prime} & \mathrm{a}_{15}^{\prime} & \mathrm{f}_{1}^{\prime} & \mathrm{p}_{11}^{\prime} & \ldots & \mathrm{p}_{1 \mathrm{~N}}^{\prime} \\
0 & 1 & \mathrm{a}_{23}^{\prime} & \mathrm{a}_{24}^{\prime} & \mathrm{a}_{25}^{\prime} & \mathrm{f}_{2}^{\prime} & \mathrm{p}_{21}^{\prime} & \ldots & \mathrm{p}_{2 \mathrm{~N}}^{\prime} \\
0 & 0 & \mathrm{a}_{33}^{\prime} & \mathrm{a}_{34}^{\prime} & \mathrm{a}_{35}^{\prime} & \mathrm{f}_{3}^{\prime} & \mathrm{p}_{31}^{\prime} & \ldots & \mathrm{p}_{3 \mathrm{~N}}^{\prime} \\
0 & 0 & \mathrm{a}_{43}^{\prime} & \mathrm{a}_{44}^{\prime} & \mathrm{a}_{45}^{\prime} & \mathrm{f}_{4}^{\prime} & \mathrm{p}_{41}^{\prime} & \ldots & \mathrm{p}_{4 \mathrm{~N}}^{\prime} \\
0 & 0 & \mathrm{a}_{53}^{\prime} & \mathrm{a}_{54}^{\prime} & \mathrm{a}_{55}^{\prime} & \mathrm{f}_{5}^{\prime} & \mathrm{p}_{51}^{\prime} & \ldots & \mathrm{p}_{5 \mathrm{~N}}^{\prime}
\end{array}\right]
$$

Step 3: We now repeat the elimination procedure used in first step for the third and fourth rows of the matrix by applying the relationships

$$
\begin{aligned}
& c a_{3 k}^{\prime \prime}=\frac{c a_{3 k}^{\prime} a_{44}^{\prime}-c a_{4 k}^{\prime} a_{34}^{\prime}}{a_{33}^{\prime} a_{44}^{\prime}-a_{43}^{\prime} a_{34}^{\prime}}, \quad k=5 \ldots N+6 \\
& c a_{4 k}^{\prime \prime}=\frac{a_{33}^{\prime} c a_{4 k}^{\prime}-a_{43}^{\prime} c a_{3 k}^{\prime}}{a_{33}^{\prime} a_{44}^{\prime}-a_{43}^{\prime} a_{34}^{\prime}}, \quad k=5 \ldots N+6
\end{aligned}
$$

This procedure gives the modified matrix

$$
\left[\begin{array}{ccccccccc}
1 & 0 & \mathrm{a}_{13}^{\prime} & \mathrm{a}_{14}^{\prime} & \mathrm{a}_{15}^{\prime} & \mathrm{f}_{1}^{\prime} & \mathrm{p}_{11}^{\prime} & \ldots & \mathrm{p}_{1 \mathrm{~N}}^{\prime} \\
0 & 1 & \mathrm{a}_{23}^{\prime} & \mathrm{a}_{24}^{\prime} & \mathrm{a}_{25}^{\prime} & \mathrm{f}_{2}^{\prime} & \mathrm{p}_{21}^{\prime} & \ldots & \mathrm{p}_{2 \mathrm{~N}}^{\prime} \\
0 & 0 & 1 & 0 & \mathrm{a}_{35}^{\prime \prime} & \mathrm{f}_{3}^{\prime \prime} & \mathrm{p}_{31}^{\prime \prime} & \ldots & \mathrm{p}_{3 \mathrm{~N}}^{\prime \prime} \\
0 & 0 & 0 & 1 & \mathrm{a}_{45}^{\prime \prime} & \mathrm{f}_{4}^{\prime \prime} & \mathrm{p}_{41}^{\prime \prime} & \ldots & \mathrm{p}_{4 \mathrm{~N}}^{\prime \prime} \\
0 & 0 & \mathrm{a}_{53}^{\prime} & \mathrm{a}_{54}^{\prime} & \mathrm{a}_{55}^{\prime} & \mathrm{f}_{5}^{\prime} & \mathrm{p}_{51}^{\prime} & \ldots & \mathrm{p}_{5 \mathrm{~N}}^{\prime}
\end{array}\right]
$$

where we use the double prime notation to indicate newly modified matrix elements.

Step 4: Repeating the elimination procedure shown in step 2, we eliminate elements in the third and fourth columns of the first, second, and fifth rows of the matrix by using

$$
\mathrm{ca}_{\mathrm{jk}}^{\prime \prime}=\mathrm{ca}_{j \mathrm{k}}^{\prime}-\mathrm{ca}_{j 3}^{\prime} \mathrm{ca}_{3 \mathrm{k}}^{\prime \prime}-\mathrm{ca}_{j 4}^{\prime} \mathrm{ca}_{4 k}^{\prime \prime}, \quad \mathrm{j}=1,2,5 ; \quad \mathrm{k}=5 \ldots \mathrm{N}+6
$$

to obtain a modified matrix of the form

$$
\left[\begin{array}{lllllllll}
1 & 0 & 0 & 0 & \mathrm{a}_{15}^{\prime \prime} & \mathrm{f}_{1}^{\prime \prime} & \mathrm{p}_{11}^{\prime \prime} & \ldots & \mathrm{p}_{1 \mathrm{~N}}^{\prime \prime} \\
0 & 1 & 0 & 0 & \mathrm{a}_{25}^{\prime \prime} & \mathrm{f}_{2}^{\prime \prime} & \mathrm{p}_{21}^{\prime \prime} & \ldots & \mathrm{p}_{2 \mathrm{~N}}^{\prime \prime} \\
0 & 0 & 1 & 0 & \mathrm{a}_{35}^{\prime \prime} & \mathrm{f}_{3}^{\prime \prime} & \mathrm{p}_{31}^{\prime \prime} & \ldots & \mathrm{p}_{3 N}^{\prime \prime} \\
0 & 0 & 0 & 1 & \mathrm{a}_{45}^{\prime \prime} & \mathrm{f}_{4}^{\prime \prime} & \mathrm{p}_{41}^{\prime \prime} & \ldots & \mathrm{p}_{4 \mathrm{~N}}^{\prime \prime} \\
0 & 0 & 0 & 0 & \mathrm{a}_{55}^{\prime \prime} & \mathrm{f}_{5}^{\prime \prime} & \mathrm{p}_{51}^{\prime \prime} & \ldots & \mathrm{p}_{5 \mathrm{~N}}^{\prime \prime}
\end{array}\right]
$$

Step 5: Finally we modify the fifth column of the matrix using

$$
c a_{5 k}^{\prime \prime \prime}=\frac{c a_{5 k}^{\prime \prime}}{a_{55}^{\prime \prime}}, \quad k=6 \ldots N+6
$$




$$
c a_{j k}^{\prime \prime \prime}=c a_{j k}^{\prime \prime}-c a_{j 5}^{\prime \prime} c a_{5 k}^{\prime \prime \prime}, \quad j=1,2,3,4 ; \quad k=6 \ldots N+6
$$

to obtain the final result

$$
\left[\begin{array}{lllllllll}
1 & 0 & 0 & 0 & 0 & \mathrm{f}_{1}^{\prime \prime \prime} & \mathrm{p}_{11}^{\prime \prime \prime} & \ldots & \mathrm{p}_{1 N}^{\prime \prime \prime} \\
0 & 1 & 0 & 0 & 0 & \mathrm{f}_{2}^{\prime \prime \prime} & \mathrm{p}_{21}^{\prime \prime} & \ldots & \mathrm{p}_{2 N}^{\prime \prime \prime} \\
0 & 0 & 1 & 0 & 0 & \mathrm{f}_{3}^{\prime \prime \prime} & \mathrm{p}_{31}^{\prime \prime \prime} & \ldots & \mathrm{p}_{3 N}^{\prime \prime \prime} \\
0 & 0 & 0 & 1 & 0 & \mathrm{f}_{4}^{\prime \prime \prime} & \mathrm{p}_{41}^{\prime \prime \prime} & \ldots & \mathrm{p}_{4 N}^{\prime \prime \prime} \\
0 & 0 & 0 & 0 & 1 & \mathrm{f}_{5}^{\prime \prime \prime} & \mathrm{p}_{51}^{\prime \prime \prime} & \ldots & \mathrm{p}_{5 N}^{\prime \prime \prime}
\end{array}\right]
$$

The matrix inversion procedure is programmed in subroutine GAJOR5 exactly as presented above. For code efficiency, we have made the subroutine specific for a 5 row matrix with an arbitrary number of columns. Note that the identity tensor I that appears in the final result is never really generated by the computational procedure. As the indices on the algorithm equations indicate, we restrict the modification calculations to include only those matrix elements that are actually required by the inversion scheme to avoid unnecessary computations.

Following the pressure solution, updated values for all of the primary state variables are computed in subroutine UPDATP using the relationships

$$
\begin{aligned}
& (\delta \alpha)_{i, j}^{m+1}=f_{2}^{\prime \prime \prime}-\sum_{k=1}^{N} p_{2 k}^{\prime \prime \prime}(\delta P)_{k}^{m+1}, \quad\left(\delta T_{g}\right)_{i, j}^{m+1}=f_{3}^{\prime \prime \prime}-\sum_{k=1}^{N} p_{3 k}^{\prime \prime \prime}(\delta P)_{k}^{m+1}, \\
& \left(\delta T_{f}\right)_{i, j}^{m+1}=f_{4}^{\prime \prime \prime}-\sum_{k=1}^{N} p_{4 k}^{\prime \prime \prime}(\delta P)_{k}^{m+1} \quad\left(\delta X_{a}\right)_{i, j}^{m+1}=f_{5}^{\prime \prime \prime}-\sum_{k=1}^{N} p_{5 k}^{\prime \prime \prime}(\delta P)_{k}^{m+1}
\end{aligned}
$$

In writing Eqs. (3.8.3-12), we use the $\mathbf{k}$ subscript to indicate the generic solution technique. More specifically, for the two-dimensional solution used as the example in Eq. (3.8.3-4), we would compute the void fraction using

$$
\begin{aligned}
(\delta \alpha)_{i, j}^{m+1}:=f_{2}^{\prime \prime \prime}- & p_{21}^{\prime \prime \prime}(\delta P)_{i, j-1}^{m+1}-p_{22}^{\prime \prime \prime}(\delta P)_{i, j+1}^{m+1} \\
& -p_{23}^{\prime \prime \prime}(\delta P)_{i-1, j}^{m+1}-p_{24}^{\prime \prime \prime}(\delta P)_{i+1, j}^{m+1}
\end{aligned}
$$

Similar relations apply for the other state variables. Since the calculations are done for each fluid cell, the matrix coefficients that apply to each cell must be saved in a large array. This storage requirement is not indicated in the above equations.

\section{References:}

Reed, W. H. and Stewart, H. B., 1978, "THERMIT: A Computer Program for ThreeDimensional Thermal-Hydraulic Analysis of Light Water Reactor Cores", M.I.T. Report prepared for EPRI. 


\subsubsection{Inner Pressure Solution}

Subroutine INNER solves for pressure increments throughout the entire assembly. Multichannel middle sections coupled to a single channel top section and single channel bottom section are solved. A generalized grid mesh is illustrated in Fig. 3.8.4-1. In Fig. 3.8.4-1 nzt is the number of axial cells in the top section, $\mathrm{nz}$ is the number of axial cells in the middle section, $\mathrm{nzb}$ is the number of axial cells in the bottom section, and $\mathrm{nx}$ is the number of azimuthal cells in the middle section. In general, more than one middle section or flow channel may be coupled to the top and bottom sections. Each flow channel will have the same number of axial cells ( $\mathrm{nz}$ ) but possibly different numbers of azimuthal cells.

Since a large number of axial and azimuthal positions will be used in most problems of interest, iterative solution strategies are used to avoid the numerical difficulties inherent in direct solution methods of large matrices. However, the overall system matrix is sparse and contains many tridiagonal stripes. These are solved using the Thomas algorithm which is a direct solution technique (Thomas, 1949). Therefore, the solution methods are a combination of direct and iterative solution techniques. This solution strategy was chosen because it was simple, easy to implement, and adequately efficient.

The user is actually able to select one of several solution strategies to use in the pressure calculation. All of the strategies take advantage of a tridiagonal structure along each coordinate direction to develop an efficient solution scheme. The eight solution strategies implemented in subroutine INNER are listed below:

1. Axial tridiagonal solution.

2. Axial tridiagonal solution with a single azimuthally coarse mesh calculation to provide initial estimates of the pressure increments.

3. Axial tridiagonal solution with an azimuthally coarse mesh calculation at the start of each iteration to estimate improved pressure increments.

4. Azimuthal tridiagonal solution.

5. Azimuthal tridiagonal solution with a single axially coarse mesh calculation to provide initial estimates of the pressure increments.

6. Azimuthal tridiagonal solution with an axially coarse mesh calculation at the start of each iteration to estimate improved pressure increments.

7. Alternating tridiagonal sweeps in the axial and azimuthal directions.

8. Alternating direction solution with a single azimuthally coarse mesh calculation to provide initial estimates of the pressure increments.

The numerical methods used to implement these solution strategies are described below. 


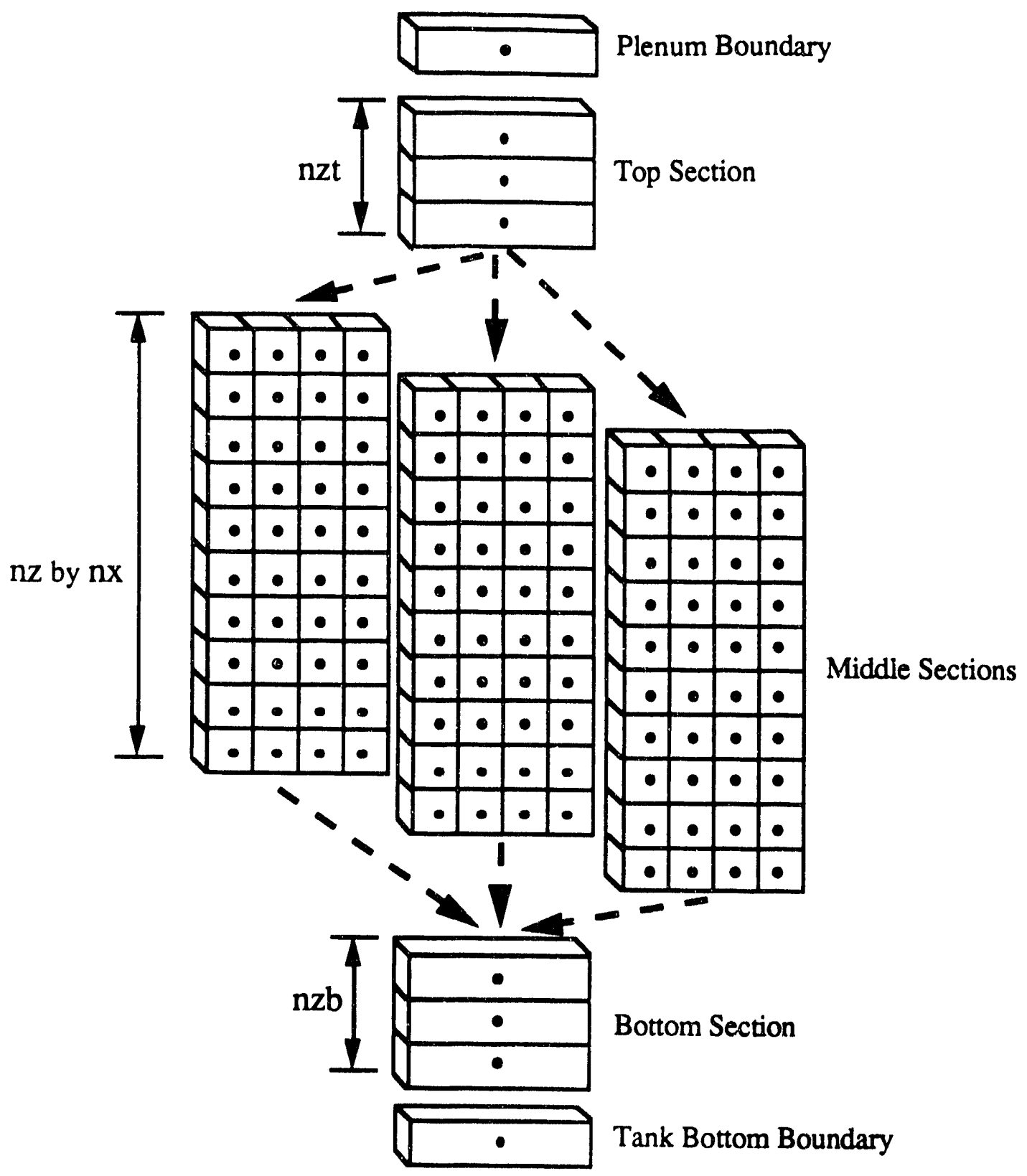

Figure 3.8.4-1 Calculational grid for multiple flow channels showing axial and azimuthal mesh.

Middle Section Solution: First we will consider the pressure solution for a single flow channel in the middle section of the assembly. In general, we are solving a two. dimensional system of finite difference equations for the pressure increments. The five point stencil used to develop the finite difference approximation is illustrated in Fig. 3.8.4-2. 


\begin{tabular}{|l|lll|r|r}
\hline $2 / 15 / 93$ & WSRC-TR-92-532 Rev. 0 & FLOWTRAN-TF Software Design & Page 467 \\
\hline
\end{tabular}

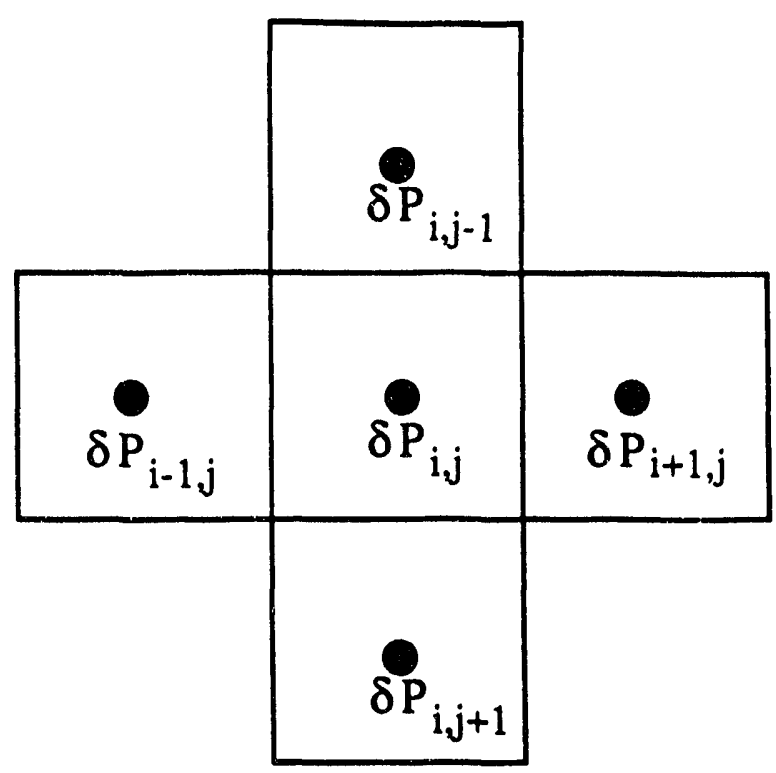

Figure 3.8.4-2 Five point finite difference mesh stencil for middle section.

We then must solve the matrix equation $[A] \delta P=C$ or, using index notation, we are solving a set of linear equations of the form

$$
\begin{aligned}
\phi_{i, j} \delta P_{i, j} & +\alpha_{i, j}^{-} \delta P_{i-1, j}+\alpha_{i, j}^{+} \delta P_{i+1, j} \\
& +\beta_{i, j}^{-} \delta P_{i, j-1}+\beta_{i, j}^{+} \delta P_{i, j+1}=\gamma_{i, j}
\end{aligned}
$$

where $\phi_{i, j}, \alpha_{i, j}^{ \pm}$, and $\beta_{i, j}^{ \pm}$are the components of matrix [A] and $\gamma_{i, j}$ are the components of vector $C$. The $\alpha_{i, j}^{ \pm}$represent pressure coupling coefficients in the azimuthal direction and the $\beta_{i, j}^{ \pm}$are coupling coefficients in the axial direction. The \pm superscripts on the coefficients are used to indicate coupling to either the pressure difference in the positive or negative axial and azimuthal directions. In general, the coefficients also depend on the flow channel; however, this additional index is not included to simplify the expressions. In our cylindrical geometry, the azimuthal mesh wraps around on itself so that, when $\mathrm{i}=1$, we replace $i-1$ with $n x$ and when $i=n x$ we use 1 for $i+1$. At the axial boundaries $(j=1$ and $j=n z)$, the terms involving the axial coefficients $\beta_{i, j}^{-}$and $\beta_{i, j}^{+}$and the pressure increments $\delta P_{i, j-1}$ and $\delta P_{i, j+1}$ are modified to pick up contributions from the cells in the top and bottom sections that bounc the two-dimensional mesh. That is, Eq. (3.8.4-1) strictly applies at internal nodes where for $2 \leq i \leq n z-1$. For $j=1$ we use the equation

$$
\begin{aligned}
\phi_{\mathrm{i}, 1} \delta \mathrm{P}_{\mathrm{i}, 1} & +\alpha_{\mathrm{i}, 1}^{-} \delta \mathrm{P}_{\mathrm{i}-1,1}+\alpha_{\mathrm{i}, 1}^{+} \delta \mathrm{P}_{\mathrm{i}+1,1} \\
& +\beta_{\mathrm{i}, 1}^{-} \delta \mathrm{P}_{\mathrm{nzt}}^{\mathrm{t}}+\beta_{\mathrm{i}, 1}^{+} \delta \mathrm{P}_{\mathrm{i}, 2}=\gamma_{\mathrm{i}, 1}
\end{aligned}
$$

where $\delta P_{n z t}^{t}$ is the pressure difference in the last cell of the top section. For $j=n z$ we write

$$
\begin{aligned}
\phi_{i, n z} \delta P_{i, n z} & +\alpha_{i, n z}^{-} \delta P_{i-1, n z}+\alpha_{i, n z}^{+} \delta P_{i+1, n z} \\
& +\beta_{i, n z}^{-} \delta P_{i, n z-1}+\beta_{i, n z}^{+} \delta P_{1}^{b}=\gamma_{i, n z}
\end{aligned}
$$


where $\delta \mathrm{P}_{1}^{\mathrm{b}}$ is the pressure difference in the first cell of the bottom section.

In practice, the equation set is normalized so that the diagonal coefficients $\phi_{\mathrm{i}, \mathrm{j}}$ are identically equal to one. Equations (3.8.4-1)-(3.8.4-3) are solved in subroutine INNER using a tridiagonal iteration scheme where the user can choose one of the eight different strategies listed in the introduction. These strategies are described below using Eq. (3.8.4-1) for illustration.

Solution Strategy \# 1: Axial Tridiagonal Solution: To solve Eq. (3.8.4-1) along the axial direction, we let pressure increments at adjacent azimuthal locations remain at the most recent values calculated in previous iteration steps and solve for new pressure increments along the axial direction. This strategy is illustrated by Eq. (3.8.4-4) which is written to obtain axial pressure increments at azimuthal position $i$ for iteration step $\mathrm{m}$. The problem is reduced to solving a tridiagonal matrix for updated axial pressure increments at azimuthal position $\mathrm{i}$. The solution is swept over all azimuthal positions and iterated until convergence is obtained. We note that at step $\mathrm{m}$ and azimuthal position $\mathrm{i}$ the pressure increments have already been updated at location $i-1$ so that $m^{\text {th }}$ iterate values for this term appear on the right hand side.

$$
\begin{aligned}
\phi_{i, j} \delta P_{i, j}^{m}+\beta_{i, j}^{-} \delta P_{i, j-1}^{m}+\beta_{i, j}^{+} \delta P_{i, j+1}^{m}= \\
\gamma_{i, j}-\alpha_{i, j}^{-} \delta P_{i-1, j}^{m}-\alpha_{i, j}^{+} \delta P_{i+1, j}^{m-1}
\end{aligned}
$$

Solution Strategy \# 4: Azimuthal Tridiagonal Solution: Alternatively, we could also solve tridiagonal equations along the azimuthal direction keeping adjacent axial pressure increments fixed. This strategy is illustrated by Eq. (3.8.4-5) which is written to obtain azimuthal pressure increments at axial position $\mathrm{j}$ for iteration step $\mathrm{m}$. The solution is swept over all axial positions and iterated until convergence is obtained. Again, at step $\mathrm{m}$ and axial position $j$, pressure increments have already been updated at location $j-1$ so that $m^{\text {th }}$ iterate values for this term appear on the right hand side.

$$
\begin{aligned}
& \phi_{i, j} \delta P_{i, j}^{m}+\alpha_{i, j}^{-} \delta P_{i-1, j}^{m}+\alpha_{i, j}^{+} \delta P_{i+1, j}^{m}= \\
& \gamma_{i, j}-\beta_{i, j}^{-} \delta P_{i, j-1}^{m}-\beta_{i, j}^{+} \delta P_{i, j+1}^{m-1}
\end{aligned}
$$

Solution Strategy \# 7: Altemating Axial and Azimuthal Solution: Another solution strategy that may possibly improve convergence characteristics of the solution is to alternate between axial and azimuthal solutions. That is we alternately make azimuthal sweeps using Eq. (3.8.4-4) and axial sweeps using Eq. (3.8.4-5). We note that this is not a full alternating direction method since we have not split the coefficient matrix into axial and azimuthal components nor have we used an acceleration parameter as is commonly employed in true ADI calculations. At alternate steps in the iteration sequence, we simply switch from solving along the axial direction to solving along the azimuthal direction. This simplified strategy was easily implemented and is included as an option in the subroutine.

Top and Bottom Sections: In both the top and bottom sections of the assembly, the fluid mesh is one-dimensional in the axial direction. The simple three point stencil that applies for this geometry is illustrated in Fig. 3.8.4-3 for the top section. An exactly analogous mesh is used in the bottom section. 


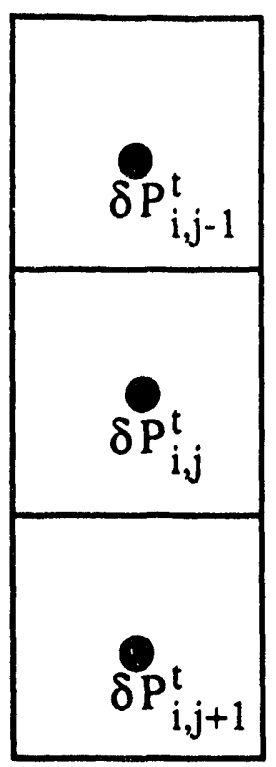

Figure 3.8.4-3 Three point finite difference mesh stencil used for top section.

The pressure problem now naturally reduces to a tridiagonal structure in both sections. Using index notation, the following equations apply for the top section:

for $2 \leq \mathrm{j} \leq \mathrm{nzt}-1$,

$$
\phi_{j}^{t} \delta P_{j}^{t}+\beta_{j}^{t-} \delta P_{j-1}^{t}+\beta_{j}^{t+} \delta P_{j+1}^{t}=\gamma_{j}^{t}
$$

for $\mathrm{j}=1$,

$$
\phi_{1}^{t} \delta P_{1}^{t}+\beta_{1}^{t-} \delta P^{p l}+\beta_{1}^{t+} \delta P_{2}^{t}=\gamma_{1}^{t}
$$

and

$$
\begin{aligned}
& \text { for } j=n z t \text {, } \\
& \qquad \phi_{n z t}^{t} \delta P_{n z t}^{t}+\beta_{n z t}^{t-} \delta P_{n z t-1}^{t}+\sum_{k=1}^{n c} \sum_{i=1}^{n x(k)}\left(\beta_{i, n z t, k}^{t+} \delta P_{i, 1, k}\right)=\gamma_{n z t}^{t}
\end{aligned}
$$

In Eqs. (3.8.4-6)-(3.8.4-8), the $\beta_{j}^{t \pm}$ are the pressure coupling coefficients, $\phi_{j}^{t}$ are the diagonal pressure coefficients, and $\gamma_{j}$ are the right hand side terms for the top section. The \pm superscripts on the coupling coefficients indicate coupling to pressure differences in either the positive or negative axial direction. As in the middle section, the coefficients $\phi_{j}^{t}$ are actually identically equal to one. In writing Eq. (3.8.4-7) for the first cell in the top section, we assume that a plenum boundary pressure increment $\delta \mathrm{P}^{\mathrm{pl}}$ exists and that the $\beta_{1}^{\mathrm{t}}$ coefficient couples it with the pressure in the first cell of the top section. In fact, this term may be identically zero for some of our problems but we include it here for generality. In Eq. (3.8.4-8), the last term on the left hand side couples the last cell in the top section with 
the first cells in all of the middle section flow channels. We have introduced the index $\mathrm{k}$ to indicate flow channels (nc) in Eq. (3.8.4-8). Each flow channel has $n x(k)$ subchannels. All of the equations for the middle section presented previously apply to a single flow channel and the index $\mathrm{k}$ has been eliminated there to simplify the notation.

For the bottom section of the assembly, the following set of equations apply:

for $2 \leq \mathrm{j} \leq \mathrm{nzb}-1$,

$$
\phi_{j}^{b} \delta P_{j}^{b}+\beta_{j}^{b-} \delta P_{j-1}^{b}+\beta_{j}^{b+} \delta P_{j+1}^{b}=\gamma_{j}^{b}
$$

for $\mathrm{j}=1$,

$$
\phi_{1}^{b} \delta P_{1}^{b}+\sum_{k=1}^{n c} \sum_{i=1}^{n x(k)}\left(\beta_{i, 1, k}^{b-} \delta P_{i, n z, k}\right)+\beta_{1}^{b+} \delta P_{2}^{b}=\gamma_{1}^{b}
$$

and

$$
\text { for } j=n z b \text {, }
$$

$$
\phi_{n z b}^{b} \delta P_{n z b}^{b}+\beta_{n z b}^{b-} \delta P_{n z b-1}^{b}+\beta_{n z b}^{b+} \delta P^{t b}=\gamma_{n z b}^{b}
$$

In Eqs. (3.8.4-9)-(3.8.4-11), $\beta_{j}^{b \pm}$ are the pressure coupling coefficients, $\phi_{j}^{b}$ are the diagonal pressure coefficients, and $\gamma_{j}$ are the right hand side terms for the bottom section. The \pm superscripts on the coupling coefficients indicate coupling to pressure differences in either the positive or negative axial direction. As before, all of the coefficients $\phi_{j}^{b}$ are identically equal to one. In Eq. (3.8.4-10), the second term on the left hand side couples the first cell in the bottom section to the last cells in all of the middle section flow channels. Again we note the summation over all flow channels (nc) in Eq. (3.8.4-10). In writing Eq. (3.8.4-11) for the last cell in the bottom section, we assume that a tank bottom boundary pressure increment $\delta \mathrm{P}^{\mathrm{tb}}$ exists and that the $\beta_{\mathrm{nzb}}^{\mathrm{bt}}$ coefficient couples it with the pressure increment in the last cell. This term may also be identically zero for some of our problems but we again include it as a generalizacion of our treatment.

Subroutine inner first solves the top section pressure equations [Eqs. (3.8.4-6)-(3.8.4-8)] using current estimates for the pressure increments in the first cells of the middle section in Eq. (3.8.4-8). Then the middle section pressure problem [Eqs. (3.8.4-1)-(3.8.4-3)] is solved using the selected solution strategy. The solution in the middle section is swept over all flow channels. Finally, the bottom section pressure increments are calculated [Eqs. (3.8.4-9)-(3.8.4-11)] using the current values for pressure increments in the last cells in the middle section in Eq. (3.8.4-10). The solution is checked for convergence and iterative passes through this scheme are made until convergence is achieved or until the number of iterations exceeds the maximum allowed count. A simple flow chart for the subroutine logic is shown in Fig. 3.8.4-4 below.

Coarse Mesh Rebalance Calculations: We seek to enhance the convergence of the pressure solution within our iteration strategy by using a coarse mesh rebalance calculation over the two-dimensional mesh in the middle section. The rebalance technique presented here is actually quite general and the technique can be applied to other types of problems such as solving for temperatures in a two or three dimensional heat conduction mesh. The 
number of coarse mesh rebalance sweeps is controlled by the code user through the input by specifying the parameter ncmr (see Fig. 3.8.4-4).

After each pass through the iterative splution, current estimates for the pressure increments (let these values be denoted by $\delta \mathrm{P}_{i, j}^{\mathrm{m}} \mathrm{-}$ ) will not exactly satisfy Eq. (3.8.4-1). We then assume that an improved estimate for the pressure can be obtained by letting

$$
\delta P_{i, j}^{m}=\delta P_{i, j}^{m-1}+R_{j}^{m}
$$

where $R_{j}^{m}$ is a rebalance correction factor to be applied at axial level $j$. Substituting Eq. (3.8.4-12) into Eqs. (3.8.4-1)-(3.8.4-3), summing the resulting expression over the azimuthal cells (i.e. over index $i$ from $i=1$ to $i=n x$ the number of azimuthal cells), and. rearranging leads to the expressions

for $2 \leq \mathrm{j} \leq \mathrm{nz}-1$,

$$
\begin{aligned}
&\left\{\sum_{i=1}^{n x} \beta_{i, j}^{-}\right\} R_{j-1}^{m}+\left\{\sum_{i=1}^{n x} \phi_{i, j}\right. \\
&\left.+\sum_{i=1}^{n x} \alpha_{i, j}^{-}+\sum_{i=1}^{n x} \alpha_{i, j}^{+}\right\} R_{j}^{m}+\left\{\sum_{i=1}^{n x} \beta_{i, j}^{+}\right\} R_{j+1}^{m} \\
&=\sum_{i=1}^{n x} \gamma_{i, j}-\left[\sum_{i=1}^{n x}\left(\phi_{i, j} \delta P_{i, j}^{m-1}\right)\right.+\sum_{i=1}^{n x}\left(\alpha_{i, j}^{-} \delta P_{i-1, j}^{m-1}\right)+\sum_{i=1}^{n x}\left(\alpha_{i, j}^{+} \delta P_{i+1, j}^{m-1}\right) \\
&\left.+\sum_{i=1}^{n x}\left(\beta_{i, j}^{-} \delta P_{i, j-1}^{m-1}\right)+\sum_{i=1}^{n x}\left(\beta_{i, j}^{+} \delta P_{i, j+1}^{m-1}\right)\right]
\end{aligned}
$$

for $\mathrm{j}=1$,

$$
\begin{aligned}
&\left\{\sum_{i=1}^{n x} \phi_{i, 1}+\sum_{i=1}^{n x} \alpha_{i, 1}^{-}+\sum_{i=1}^{n x} \alpha_{i, 1}^{+}\right\} R_{1}^{m}+\left\{\sum_{i=1}^{n x} \beta_{i, 1}^{+}\right\} R_{2}^{m} \\
&=\sum_{i=1}^{n x} \gamma_{i, 1}-\left[\sum_{i=1}^{n x}\left(\phi_{i, 1} \delta P_{i, 1}^{m-1}\right)\right.+\sum_{i=1}^{n x}\left(\alpha_{i, 1}^{-} \delta P_{i-1,1}^{m-1}\right)+\sum_{i=1}^{n x}\left(\alpha_{i, 1}^{+} \delta, P_{i+1,1}^{m-1}\right) \\
&\left.+\left\{\sum_{i=1}^{n x} \beta_{i, 1}^{-}\right\} \delta P_{n z t}^{i}+\sum_{i=1}^{n x}\left(\beta_{i, 1}^{+} \delta P_{i, 2}^{m-1}\right)\right]
\end{aligned}
$$

and 


$$
\begin{aligned}
& \text { for } j=n z, \\
& \begin{aligned}
&\left\{\sum_{i=1}^{n x} \beta_{i, n z}^{-}\right\} R_{n z-1}^{m}+\left\{\sum_{i=1}^{n x} \phi_{i, n z}\right.\left.+\sum_{i=1}^{n} \alpha_{i, n z}^{-}+\sum_{i=1}^{n x} \alpha_{i, n z}^{+}\right\} R_{n z}^{m} \\
&= \sum_{i=1}^{n x} \gamma_{i, n z}-\left[\sum_{i=1}^{n x}\left(\phi_{i, n z} \delta P_{i, n z}^{m-1}\right)\right. \\
&+\sum_{i=1}^{n x}\left(\alpha_{i, n z}^{-} \delta P_{i-1, n z}^{m-1}\right)+\sum_{i=1}^{n x}\left(\alpha_{i, n z}^{+} \delta P_{i+1, n z}^{m-1}\right) \\
&\left.+\sum_{i=1}^{n x}\left(\beta_{i, n z}^{-} \delta P_{i, n z-1}^{m-1}\right)+\left\{\sum_{i=1}^{n x} \beta_{i, n z}^{+}\right\} \delta P_{1}^{b}\right]
\end{aligned}
\end{aligned}
$$

where we have written Eqs. (3.8.4-14) and (3.8.4-15) to explicitly show the dependence of the solution on the pressure increments in the last cell of the top section $\delta P_{n z t}^{t}$ and in the first cell of the bottom section $\delta \mathrm{P}_{1}^{\mathrm{G}}$. By creating a tridiagonal equation in the axial direction for each channel of the middle section, we can directly couple that channel with the tridiagonal equations for the top and bottom sections. Solving this coupled set, we obtain an approximate solution for the pressure increments in the top and bottom sections and in one flow channel of the middle section in a single calculation.

Inserting Eq. (3.8.4-12), written for flow channel ic, into Eq. (3.8.4-8) that couples pressure increments in the last cell in the top section to pressure increments in the first cells in the middle section, we find the rebalance equation at this location for channel ic to be

$$
\begin{aligned}
\phi_{n z t}^{t} \delta P_{n z t}^{t}+\beta_{n z t}^{t-} \delta P_{n z t-1}^{t} & +\left\{\sum_{i=1}^{n x(i c)} \beta_{i, n z t, i c}^{t+}\right\} R_{1}^{m}=\gamma_{n z t}^{t} \\
& -\sum_{i=1}^{n x(i c)}\left(\beta_{i, n z t, i c}^{t+} \delta P_{i, i, i c}^{m-1}\right)-\sum_{\substack{k=1 \\
k \neq i c}}^{n c} \sum_{i=1}^{n x(k)}\left(\beta_{i, n z t, k}^{t+} \delta P_{i, 1, k}^{m-1 / 2}\right)
\end{aligned}
$$

The $\mathrm{m}-1 / 2$ superscript on $\delta \mathrm{P}$ in the last term of equation (3.8.4-16) denotes that the latest value of $\delta \mathrm{P}$ is used, which may be at either the $\mathrm{m}-1$ or $\mathrm{m}$ level.

Similarly, inserting Eq. (3.8.4-12), written for flow channel ic, into Eq. (3.8.4-10) that couples pressure increments in the first cell in the bottom section to pressure increments in the last cells in the middle section, we obtain the rebalance equation at this location for channel ic as

$$
\begin{aligned}
\phi_{1}^{b} \delta P_{1}^{b}+\{ & \left\{\sum_{i=1}^{n x(i c)} \beta_{i, 1, i c}^{b-}\right\} R_{n z}^{m}+\beta_{1}^{b+} \delta P_{2}^{b}= \\
& \gamma_{1}^{b} \\
& -\sum_{i=1}^{n x(i c)}\left(\beta_{i, 1, i c}^{b-} \delta P_{i, n z, i c}^{m-1}\right)-\sum_{\substack{k=1 \\
k \neq i c}}^{n c} \sum_{i=1}^{n x(k)}\left(\beta_{i, 1, k}^{b-} \delta P_{i, n z, k}^{m-1 / 2}\right)
\end{aligned}
$$




\begin{tabular}{|l|lll|r|}
\hline $2 / 15 / 93$ & WSRC-TR-92-532 Rev. 0 & FLOWTRAN-TF Software Design & Page 473 \\
\hline
\end{tabular}

Solving Eqs. (3.8.4-13)-(3.8.4-17) for the rebalance factors and applying Eq. (3.8.4-12) then leads to an improved estimate of the pressure increments in the middle section. The rebalance factor for a particular axial level is simply added to the existing pressure estimates in each azimuthal cell. Equations (3.8.4-13)-(3.8.4-15) solve the pressure problem over a coarse mesh in the azimuthal direction by lumping all of the azimuthal cells into a single cell. The resulting equation set for the rebalance factors is tridiagonal and can be efficiently solved. A set of rebalance equations is solved separately for each flow channel in the middle section.

With the sets of equations developed above, we are now able to describe the other solution strategies implemented in subroutine INNER.

Solution Strategy \# 2: Axial Tridiagonal Solution with Single Coarse Mesh Rebalance: For this solution strategy, Eqs. (3.8.4-6), (3.8.4-7), and (3.8.4-16) for the top section, Eqs. (3.8.4-13)-(3.8.4-15) for the middle section, and Eqs. (3.8.4-9), (3.8.4-11), and (3.8.4-17) for the bottom section are solved one time at the start of the iteration sequence. The number of coarse mesh sweeps is controlled by the input parameter ncmr. The solution is repeated for each flow channel in the middle section. This solution provides initial estimates for the pressure increments in all sections. After the coarse mesh calculation, the solution follows the axial tridiagonal calculation described for Solution Strategy \#1.

Solution Strategy \# 3: Axial Tridiagonal Solution with Coarse Mesh Rebalance Calculations Every lteration: As in solution strategy \#2, Eqs. (3.8.4-6), (3.8.4-7), and (3.8.4-16) for the top section, Eqs. (3.8.4-13)-(3.8.4-15) for the middle section, and Eqs. (3.8.4-9), (3.8.4-11), and (3.8.4-17) for the bottom section are solved and the solution is repeated for each flow chanrel in the middle section. The number of coarse mesh sweeps is controlled by the input parameter ncmr. However; in contrast to method \#2, with this strategy, the coarse mesh calculation is repeated at the start of each iteration step. This solution provides improved estimates for the pressure increments in all sections at the start of each iteration. After the coarse mesh calculation, the solution again follows the axial tridiagonal calculation described for Solution Strategy \#1.

Solution Strategy \# 8: Altemaring Axial and Azimuthal Tridiagonal Solutions with Single Axial Coarse Mesh Rebalance: This solution strategy is identical to that described for Solution Strategy \#2 except that, after the coarse mesh calculation, the solution alternates between axial and azimuthal tridiagonal calculations as described in Solution Strategy \#7. That is, we perform a single coarse mesh calculation (the number of coarse mesh sweeps is still controlled by the value of ncmr) at the start of the iteration sequence. We did not program a strategy that performs a coarse mesh calculation at every iteration step for the alternating direction method (analogous to Strategy \#3) since this required additional storage arrays to be created.

Similar to the axial coarse mesh rebalance schemes presented above, a rebalance calculation can be performed along the azimuthal direction by defining

$$
\delta P_{i, j}^{m}=\delta P_{i, j}^{m-1}+R_{i}^{m}
$$

and using the same procedure described above. Applying this relationship to Eqs. (3.8.4-1)-(3.8.4-3) and summing over the axial direction, leads to the basic azimuthal rebalance equation 


$$
\begin{gathered}
\left\{\sum_{j=1}^{n z} \alpha_{i, j}^{-}\right\} R_{i-1}^{m}+\left\{\sum_{j=1}^{n z} \phi_{i, j}+\sum_{j=1}^{n z} \beta_{i, j}^{-}+\sum_{j=1}^{n z} \beta_{i, j}^{+}\right\} R_{i}^{m}+\left\{\sum_{j=1}^{n z} \alpha_{i, j}^{+}\right\} R_{i+1}^{m} \\
=\sum_{j=1}^{n z} \gamma_{i, j}-\left[\sum_{j=1}^{n z}\left(\phi_{i, j} \delta P_{i, j}^{m-1}\right)+\sum_{j=2}^{n z}\left(\beta_{i, j}^{-} \delta P_{i, j-1}^{m-1}\right)+\sum_{j=1}^{n z-1}\left(\beta_{i, j}^{+} \delta P_{i, j+1}^{m-1}\right)\right. \\
\left.+\beta_{i, 1}^{-} \delta P_{n z t}^{i}+\beta_{i, n z}^{+} \delta P_{1}^{b}+\sum_{j=1}^{n z}\left(\alpha_{i, j}^{-} \delta P_{i-1, j}^{m-1}\right)+\sum_{j=1}^{n z}\left(\alpha_{i, j}^{+} \delta P_{i+1, j}^{m-1}\right)\right]
\end{gathered}
$$

Equation (3.8.4-19) is solving an axially lumped problem over the azimuthal direction and will yield improved estimates for the pressure increments in each flow channel in the middle section. As before, from the cylindrical geometry, when $\mathrm{i}=1$, we set $\mathrm{i}-1$ to $\mathrm{nx}$ and when $i=n x$, we set $i+1$ to 1 .

Solution Strategy \# 5: Azimuthal Tridiagonal Solution with Single Coarse Mesh Rebalance: In this solution method, Eq. (3.8.4-19) is solved once at the start of the iteration sequence and Eq. (3.8.4-18) is used to provide initial estimates for the pressure increments in the middle section. Thereafter, the solution follows the azimuthal tridiagonal calculation described for Solution Strategy \#4. As before, the number of coarse mesh sweeps is controlled by the input parameter ncmr.

Solution Strategy \# 6: Azimuthal Tridiagonal Solution with Coarse Mesh Rebalance Calculations Every Step: In this solution strategy, Eq. (3.8.4-19) is solved at the start of each iteration sequence and $\mathrm{Eq} .(3.8 .4-18)$ is used to provide improved estimates for the pressure increments in the middle section at the start of each step. After the coarse mesh calculations, the solution follows the method described for Solution Strategy \#4.

Alternative coarse mesh rebalancing strategies exist. For example, rather than performing a rebalance calculation on each flow channel in the middle section separately as described above, we could extend the coarse mesh over all of the channels. This strategy would provide a single pressure estimate, smoothed over all of the flow channels, in one calculation. The strategy developed above allows separate calculations for each flow channel which can be iterated to improve the estimates. That is, some of the work involved in solving the pressure problem can be transferred to the coarse mesh solution. In all of the coarse mesh schemes, a provision is made for the user to specify the number of coarse mesh iterations to be made. This option is useful in coupled multi-channel configurations where a single coarse mesh calculation may not significantly improve the pressure estimate. However, performing multiple passes through the coarse mesh calculations may lead to improved estimates. If only one flow channel exists, the two approaches are identical. 


\begin{tabular}{|l|lll|r|}
\hline $2 / 15 / 93$ & WSRC-TR-92-532 Rev.0 & FLOWTRAN-TF Software Design & Page 475 \\
\hline
\end{tabular}

Flow Chart for Subroutine INNER:

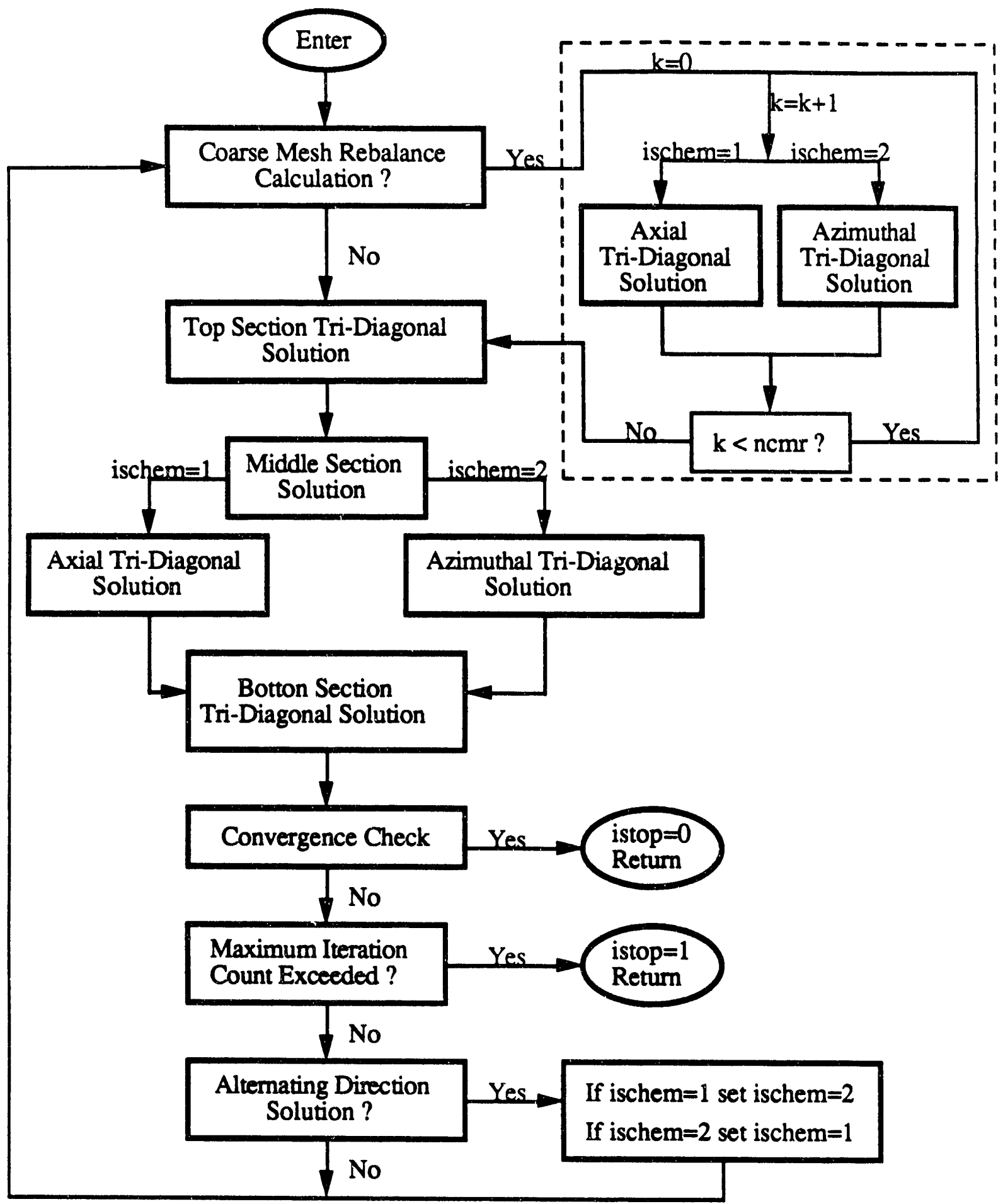

Figure 3.8.4-4 Flow chart for subroutine INNER. 


\begin{tabular}{|l|ll|l|l|}
\hline $2 / 15 / 93$ & WSRC-TR-92-532 Rev. 0 & FLOWTRAN-TF Software Design & Page 476 \\
\hline
\end{tabular}

\section{References}

Thomas, L. H., 1949, "Elliptic Problems in Linear Difference Equations over a Network", Watson Sci. Comput. Lab. Rept., Columbia University, New York. 


\subsubsection{Tridiagonal Matrix Solution}

Subroutine TRIDAG is a generalized tri-diagonal matrix equation solver based on an extension of the Thomas algorithm (Thomas, 1949). The subroutine solves tri-diagonal or periodic tri-diagonal matrix equations $\mathbf{A x}=\mathbf{d}$ where the $\mathrm{n}$ by $\mathrm{n}$ matrix $\mathbf{A}$ can, in general, be of the form

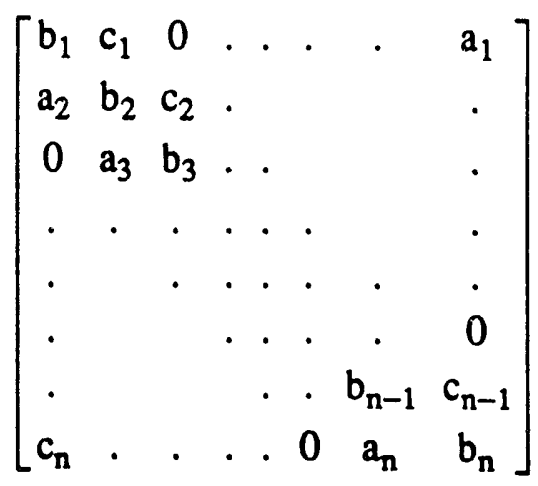

and $\mathbf{d}$ represents the vector

$$
\left[\begin{array}{c}
d_{1} \\
\cdot \\
\cdot \\
d_{n}
\end{array}\right]
$$

The matrix $\mathbf{A}$ is periodic tri-diagonal with terms appearing in the corner positions. This form arises naturally from finite difference schemes in cylindrical coordinates where the starting and ending computational cells in the azimuthal direction are coupled to each other.

Using the usual Gaussian elimination strategy to set diagonal terms in the A matrix to unity and to eliminate all terms below the diagonal, we find that a modified matrix is created having the form

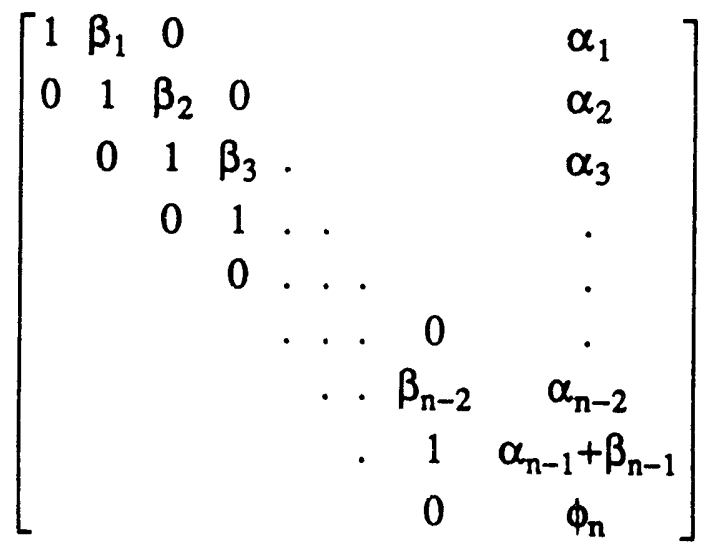


and that the $\mathbf{d}$ vector is modified to become

$$
\left[\begin{array}{c}
\delta_{1} \\
\delta_{2} \\
\cdot \\
\cdot \\
\cdot \\
\delta_{n-1} \\
\rho_{n}
\end{array}\right]
$$

The terms in the modified matrix and vector can be efficiently calculated through the following recursive scheme. First we define initial values for the variables as

$$
\beta_{1}=\frac{c_{1}}{b_{1}}, \alpha_{1}=\frac{a_{1}}{b_{1}} \text {, and } \delta_{1}=\frac{d_{1}}{b_{1}}
$$

Then the following recursive relationships apply for $i=2, \ldots, n-1$

$$
\begin{aligned}
& \beta_{i}=\frac{c_{i}}{\left(b_{i}-a_{i} \beta_{i-1}\right)} \\
& \alpha_{i}=-\frac{a_{i} \alpha_{i-1}}{\left(b_{i}-a_{i} \beta_{i-1}\right)}
\end{aligned}
$$

and

$$
\delta_{i}=\frac{\left(d_{i}-a_{i} \delta_{i-1}\right)}{\left(b_{i}-a_{i} \beta_{i-1}\right)}
$$

For increased efficiency, the reciprocal of the denominator term that is common to Eqs. (3.8.5-5) through (3.8.5-8) is actually calculated only one time and this is multiplied by the appropriate terms to evaluate the relationships.

The terms $\phi_{n}$ and $\rho_{n}$ in the matrix $A$ and the vector $\mathbf{d}$ depend upon $c_{n}$ and must be treated specially. We define three auxiliary variables and initialize their values as

$$
\eta_{1}=c_{n}, \phi_{1}=b_{n} \text {, and } \rho_{1}=d_{n}
$$

The the following recursive relationships apply

$$
\begin{gathered}
\eta_{i}=-\beta_{i-1} \eta_{i-1}, \quad i=2, \ldots, n-2 \\
\phi_{i}=\phi_{1-1}-\alpha_{i-1} \eta_{i-1}, \quad i=2, \ldots, n-1 \\
\rho_{i}=\rho_{i-1}-\delta_{i-1} \eta_{i-1}, \quad i=2, \ldots, n
\end{gathered}
$$


where the special relationships at $\mathrm{n}-1$ and $\mathrm{n}$ given below must also be used

$$
\eta_{n-1}=a_{n}-\beta_{n-2} \eta_{n-2}
$$

and

$$
\phi_{n}=\phi_{n-1}-\left(\alpha_{n-1}+\beta_{n-1}\right) \eta_{n-1}
$$

Finally, we determine the solution to the original set of linear equations through backsubstitution as

$$
x_{n}=\frac{\rho_{n}}{\phi_{n}}
$$

and

$$
x_{i}=\oint_{i}-\beta_{i} x_{i+1}-\alpha_{i} x_{n}, i=n-1, \ldots, 1
$$

The sets of equations in (3.8.5-5) through (3.8.5-16) provide a simple recursive scheme similar to the Thomas algorithm for strictly tri-diagonal problems to solve the original periodic matrix equation. In fact, the scheme shown above will automatically reduce to the tri-diagonal formulation when $a_{1}$ and $c_{n}$ are identically zero. Both strictly tri-diagonal and periodic tri-diagonal matrix equations occur in the FLOWTRAN-TF fluid pressure and solid heat conduction solutions. Subroutine TRIDAG is used to solve all of these equations.

We also note that for iterative solution schemes which typically solve sets of tri-diagonal (or periodic tri-diagonal equations) multiple times until convergence is obtained, the above solution technique can be simplified for subsequent passes. As a solution is iterated, the coefficient matrix remains fixed while the right-hand side vector (d) is updated. Then only the $\delta_{i}$ and $\rho_{i}$ terms need to be recalculated using Eqs. (3.8.5-8) and (3.8.5-12) for another iterative pass through the back-substitution in Eqs. (3.8.5-15) and (3.8.5-16) to solve the same equation set. This can be readily done if the matrix elements $a_{i}$ and the intermediate result $\eta_{i}$ are saved and the denominator terms in Eq. (3.8.5-8) $\left(b_{i}-a_{i} \beta_{i-1}\right)$ are saved after the first pass through the subroutine. Subroutine TRIDAG replaces the diagonal terms in the original matrix with the reciprocal of the denominators.

The symbols in this document correspond to the variable names in subroutine TRIDAG as follows:

$$
\begin{aligned}
& \beta \rightarrow c \text { (input vector } c \text { is subsequently used to store } \beta^{\text {'s }} \text { ) } \\
& \alpha \rightarrow \text { s1 } \quad \delta \rightarrow \text { beta } \quad \eta \rightarrow \mathrm{s} 2 \quad \phi \rightarrow \text { phi } \quad \rho \rightarrow \text { rho }
\end{aligned}
$$

\section{References}

Thomas, L. H., 1949, "Elliptic Problems in Linear Difference Equations over a Network", Watson Sci. Comput. Lab. Rept., Columbia University, New York. 


\subsection{Stability Constraints on Time Step Size}

Before getting into a detailed description, we need to explain the FLOWTRAN-TF fluid differencing scheme and solution strategy briefly. The basic fluid differencing scheme and methodology were adopted from the THERMIT code. That is, our differencing technique is to choose an implicit method for fast phenomena such as local interaction terms between gas and liquid phases and momentum transfer due to pressure change and to treat slower terms like convection and momentum transfer by fluid motion itself in a semi-explicit way. For the remaining terms, which are not sensitive to the change of fluid conditions, a fullyexplicit method was used for an efficient computation time in each step. Thus, a time step constraint for stability was obtained by applying Von Neumann's stability analysis mainly to the time derivative and convection terms of mass and energy equations after dropping the fully-implicit terms in the conservation equations. By this process, a minimum time step size is taken based on conservative assumptions to guarantee the stable running of the code. Throughout this document, unless otherwise defined, the same nomenclature as those of other FLOWTRAN-TF documents is used.

This section describes the main method used to control the computational time step and thus establish numerical stability. Checks are made on each value of the primary state variables at the new time step to ensure that the variations of the new time variables from the old ones are within reasonable limits. Several checks on solution acceptability are done to constrain the time step. These include the maximum change of the primary state variables (pressure, void fraction, air mass fraction, fluid temperature for each phase, and fuel surface temperature) at the new time step and the checks of the material Courant limit. The changes of the new time state variables from the old time values are constrained by input parameters in order to ensure that the linearized equations will sufficiently represent the non-linear equations to provide an acceptable level of accuracy in the computation. In addition, this is done because all fluid and material properties are evaluated in an explicit method. The time step size for stability is checked for the fluid mass and energy conservation equations for each phase before Newton iterations take place in order to solve the fluid conservation equations and, thus, it may reduce the time step. From the mass conservation equation,

$$
\frac{\partial}{\partial t} \alpha_{k} \rho_{k}+\nabla \cdot\left(\alpha_{k} \rho_{k} \bar{v}_{k}\right)=\Gamma_{k}
$$

where $\mathrm{k}=\mathrm{g}$ for gas phase and $\mathrm{k}=\mathrm{f}$ for liquid phase.

After integration of the above differential equation with respect to the control volume of mass $V_{c}$, the forward time difference equation of equation (3.9-1) becomes as follows:

$$
V_{c} \frac{\left(c_{k} \rho_{k}\right)^{n+1}-\left(\alpha_{k} \rho_{k}\right)^{n}}{\Delta t}=\sum_{\substack{\text { control } \\ \text { surface }}}\left(A \hat{\alpha}_{k}^{n} \hat{\rho}_{k}^{n} v_{k}^{n+1}\right)+V_{c} \Gamma_{k}^{n+1}
$$

For the top and bottom sections, equation (3.9-2) becomes

$$
\zeta_{k, j}^{n+1}=\zeta_{k, j}^{n}+\frac{\Delta t}{V_{c}}\left\{\left(A u_{k}^{n+1}\right)_{j-1 / 2} \hat{\zeta}_{k, j-1 / 2}^{n}-\left(A u_{k}^{n+1}\right)_{j+1 / 2} \hat{\zeta}_{k, j+1 / 2}^{n}\right\}+\Delta t \Gamma_{k, j}^{n+1}
$$


where

$$
\zeta_{k, j}^{n}=\left(\alpha_{k} \rho_{k}\right)_{j}^{n}
$$

In equation (3.9-3), $\hat{\zeta}$ denotes the donored quantity at the faces of the mass and energy cells. To assess numerical stability, we evaluate the fluid velocity at the old time step to allow evaluations of the computational time step prior to the calculations. Then, as illustrated in Figure 3.9-1,

$$
\begin{gathered}
\text { for } \begin{array}{c}
u_{k, j-1 / 2}^{n}>0 \text { and } u_{k, j+1 / 2}^{n}>0, \\
\hat{\zeta}_{k, j-1 / 2}^{n}=\zeta_{k, j-1}^{n} \\
\hat{\zeta}_{k, j+1 / 2}^{n}=\zeta_{k, j}^{n} .
\end{array}
\end{gathered}
$$

In this case, equation (3.9-3) can be written as follows:

$$
\zeta_{k, j}^{n+1}=\zeta_{k, j}^{n}+\frac{\Delta t}{V_{c}}\left\{\left(A u_{k}^{n}\right)_{j-1 / 2} \zeta_{k, j-1}^{n}-\left(A u_{k}^{n}\right)_{j+1 / 2} \zeta_{k, j}^{n}\right\}+\Delta t \Gamma_{k, j}^{n+1}
$$

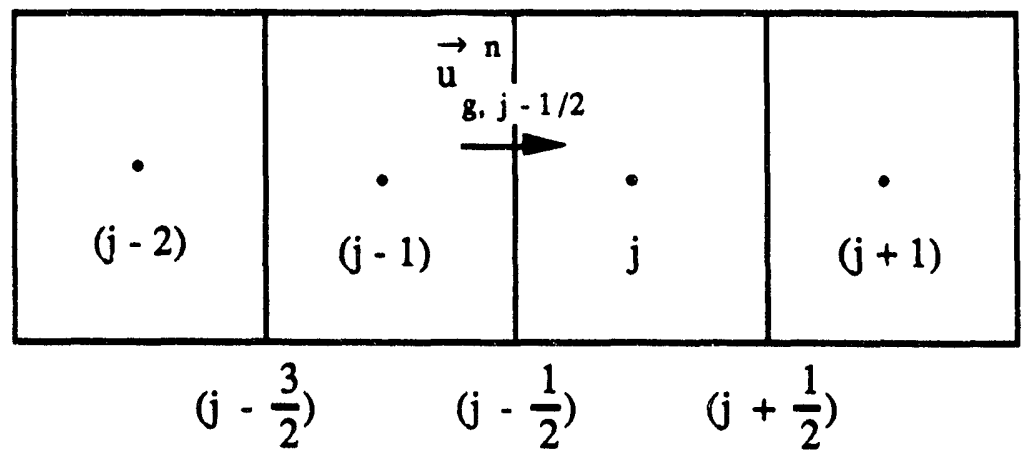

Figure 3.9-1 One-dimensional computational mesh points in the donoring scheme for the mass and energy control volumes.

Convergence is, in general, difficult to examine. Fortunately, stability and consistency guarantee the convergence for the linear partial differential equation. From Lax's equivalence theorem (Roache, 1976), stability is the necessary and sufficient condition for convergency when a linear finite difference approximation of a partial differential equation satisfies the consistency condition. That is, truncation error goes to zero as the time step and spatial mesh sizes become smaller and smaller.

After dropping the fully-implicit term, $\Gamma_{k, j}^{n+1}$, the Von Neumann stability method (Roache, 1976, and Potter, 1972) can be applied to equation (3.9-7). Von Neumann's stability criterion requires that the largest eigenvalue of the amplification matrix of the iteration scheme be less than unity for a consistent finite difference scheme. 
When $\zeta_{k, j}^{n}=g^{n} e^{I k_{z} j \Delta z}$, where $g^{n}$ is the amplitude function at time level $n$ of the paricular componept whose wave number in the flow direction is $k_{z}$ with phase angle of $\beta=k_{z} \Delta z$ and $\mathrm{I}=\sqrt{-1}$, equation (3.9-7), after algebraic rearrangement, becomes

$$
\begin{aligned}
g=1 & +\frac{\Delta t}{V_{c}}\left\{\left(A u_{k}^{n}\right)_{j-1 / 2}(\cos \beta-I \sin \beta)-\left(A u_{k}^{n}\right)_{j+1 / 2}\right\} \\
& \approx 1-\frac{\Delta t}{V_{c}}\left(A u_{k}^{n}\right)_{j+1 / 2}\{(1-\cos \beta)+I \sin \beta\}
\end{aligned}
$$

From Von Neumann's stability criterion, $|\mathrm{g}|<1$ for a stable numerical solution.

When this criterion is applied to equation (3.9-8), the resulting time step constraint for the top and bottom sections becomes

$$
\Delta t<\frac{V_{c}}{\left(A u_{k}^{n}\right)_{j+1 / 2}} \text { for } u_{k, j-1 / 2}^{n_{1}}>0 \text { and } u_{k, j+1 / 2}^{n}>0 \text {. }
$$

For two-dimensional fluid flow in the middle channel section with positive fluid flow axially and azimuthally as illustrated in Figure 3.9-2, the time step criterion can be obtained using the same procedure as that of the top/bottom section (Lee, 1991). For this case we find

$$
\Delta t<\frac{V_{c}}{\left(A u_{k}^{n}\right)_{(i, j+1 / 2)}+\left(A w_{k}^{n}\right)_{(i+1 / 2, j)}}
$$

From the air mass balance equation, the same relations as equations (3.9-9) and (3.9-10) can be obtained using the Von Neumann stability method. Equations (3.9-9) and (3.9-10) correspond to the material Courant limit for stability requirement from the mass conservation of each phase. 


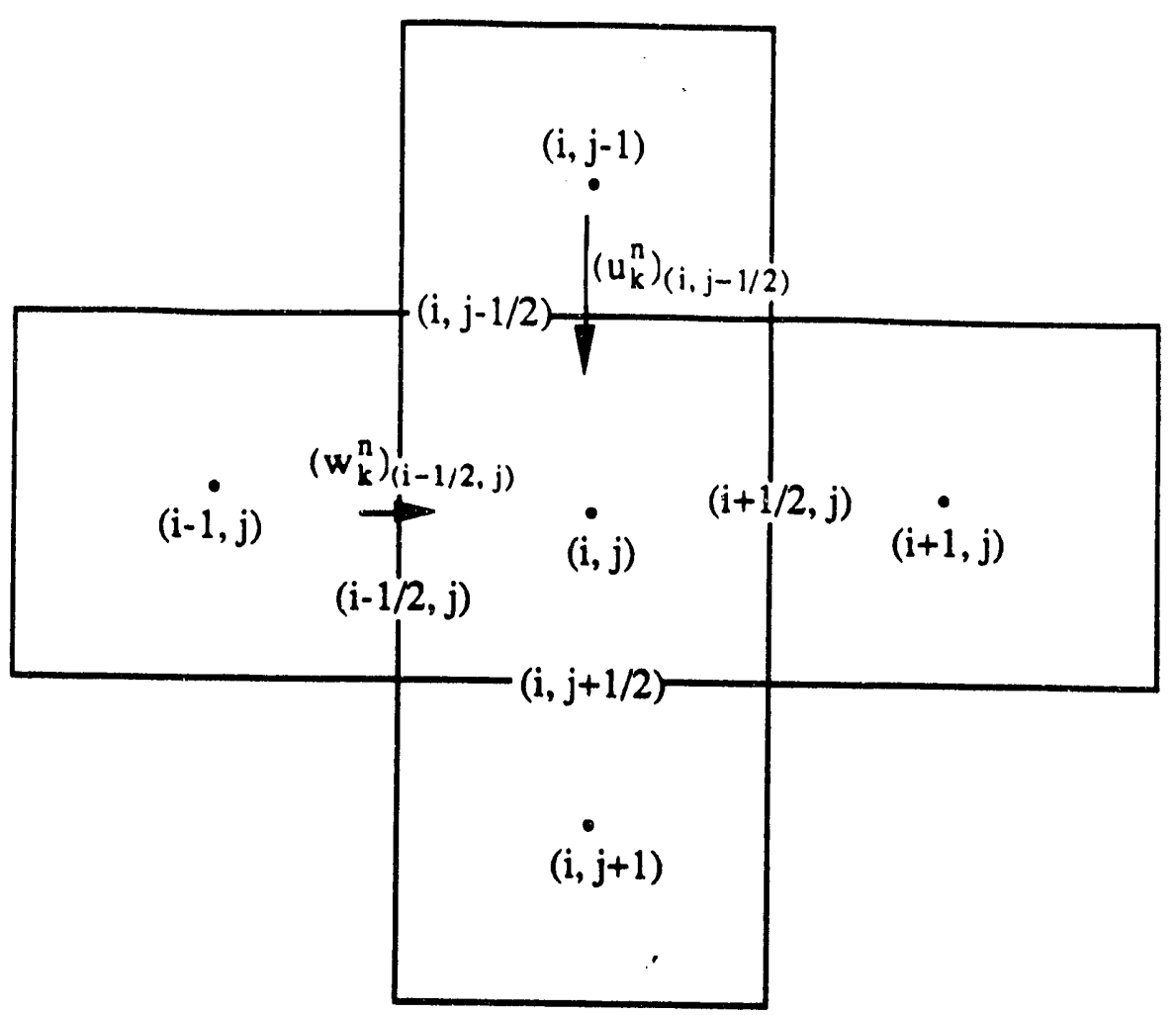

Figure 3.9.2 Two-dimensional computational mesh points in the donoring scheme for the mass and energy control volumes.

In order to check the stable time step for the mass conservation for each phase and air mass continuity before the evaluation of momentum conservation in the code, the following
relation is used:

for top and bottom section,

$$
(\Delta t)_{m t \& b . \min }=\frac{0.9 V_{c}}{\theta_{t \& b}}
$$

where

$$
\boldsymbol{\theta}_{t \& b}=-\left[A\left(\min \left(0, u_{k}^{n}\right)\right\}\right]_{j-1 / 2}+\left[A\left\{\max \left(0, u_{k}^{n}\right)\right\}\right]_{j+1 / 2}
$$

for middle channel section,

$$
(\Delta t)_{\operatorname{mm}, \min }=\frac{0.9 \mathrm{~V}_{\mathrm{c}}}{\theta_{\mathrm{m}}}
$$

where 


$$
\begin{aligned}
\Theta_{m}= & -\left[A\left(\min \left(0, u_{k}^{n}\right)\right\}\right]_{(i, j-1 / 2)}+\left[A\left(\max \left(0, u_{k}^{n}\right)\right\}\right]_{(i, j+1 / 2)} \\
& \left.-\left[A\left\{\min \left(0, w_{k}^{n}\right)\right\}\right]_{(i-1 / 2, j)}+\left[A\left(\max \left(0, w_{k}^{n}\right)\right\}\right]_{(i+1 / 2, j}\right)
\end{aligned}
$$

From the energy conservation equation,

$$
\frac{\partial}{\partial t}\left(\alpha_{k} \rho_{k} h_{k}\right)+\nabla \cdot\left(\alpha_{k} \rho_{k} h_{k} \bar{v}_{k}\right)=\alpha_{k} \frac{\partial P}{\partial t}+Q_{w k}+I_{k} Q_{I}
$$

where $I_{g}=1$ and $I_{f}=-1$.

For the stability analysis, after dropping the fully-implicit terms,

$$
\alpha_{k} \frac{\partial P}{\partial t}+I_{k} Q_{I}
$$

it is assumed that

$$
h_{k}=C_{p_{k}} T_{k}
$$

and

$$
Q_{w k}=\left(\frac{h_{w k} A_{w k}}{V_{c}}\right)\left(T_{w}-T_{k}\right)
$$

Wall heat transfer term is computed by equation (3.9-15) in a semi-implicit way within the code. For the computation of minimum stable time step size, this term is dropped since propagation speed of heat transfer through channel wall surface is expected to be relatively slow compared to convective terms. After applying the above assumptions to equation (3.9-13) and integrating it with respect to the control volume of energy $V_{c}$, the forward time difference equation is obtained after algebraic rearrangement:

$$
\xi_{k,(i, j)}^{n+1}=\xi_{k,(i, j)}^{n}+\left(\frac{\Delta t}{V_{c}}\right) \sum_{\substack{\text { control } \\ \text { surface }}}\left(A v_{k}^{n} \hat{\xi}_{k}^{n}\right)
$$

where

$$
\xi_{k,(i, j)}^{n}=\left(\alpha_{k}^{n} \rho_{k}^{n} C_{p k}^{n} T_{k}^{n}\right)_{(i, j)}
$$

Using the Von Neumann stability method with the same procedure as above, the resulting criterion for the conservative time step with positive fluid flow axially and azimuthally becomes (Lee, 1991)

$$
\Delta t<\frac{V_{c}}{\left(A u_{k}^{n}\right)_{(i, j+1 / 2)}+\left(A w_{k}^{n}\right)_{(i+1 / 2, j)}}
$$


From the energy equation for each phase, the following relation is used to ensure a stable solution in the code:

$$
(\Delta t)_{\mathrm{em}, \min }=\frac{0.9 \mathrm{~V}_{\mathrm{c}}}{\Theta_{\mathrm{m}}}
$$

From equations, (3.9-12) and (3.9-18), minimum stable time step is determined by mass or energy transport for each phase. The time step sizes are computed by equations, $(3.9-11)$ and (3.9-12) or (3.9-18), for each control volume of mass/energy conservation through the entire system and then the minimum time step is chosen before the Newton iteration in order to compute the fluid equations at the new time step. An empirical factor of 0.9 in equations, (3.9-11), (3.9-12), and (3.9-18), was used to imbed additonal conservatism for numerical stability since several assumptions were used in deriving the criterion for a stable time step.

$$
(\Delta t)_{\min }=\min \left\{(\Delta t)_{m t \& b, \operatorname{mir}},(\Delta t)_{m m, \min }(\Delta t)_{e m, \min }\right\}
$$

Based on this minimum time step size determined by equation (3.9-19), the variations of the five primary state variable (pressure, air mass fraction, void fraction, gas temperature, and liquid temperature) and fuel surface temperature after the Newton iteration at each new time step are constrained within a tolerance limit, which is provided by the input deck. This limit is in terms of the ratio of relative difference, or the relative difference between the old time and new time values in order to ensure acceptable accuracy in the linearizations of the nonlinear transport equations. If the maximum variation of main parameters exceed a given limit even after the time reduction by the Courant limit, the time step size is reduced and the computation at this time step is repeated, i.e.,

$$
(\Delta t)=\left(\frac{0.9 \delta}{\gamma_{\max }}\right)(\Delta t)_{\min }
$$

where $\delta$ and $\gamma_{\max }$ are a given tolerance limit and the maximum ratio or absolute amount of relative change respectively.

When the time step size chosen by the above procedure is smaller than the minimum time size given by the input deck, program execution is stopped. Minimum time step size is given as an input parameter (at present $1.0 \times 10^{-5}$ second).

The stability of the solution is further enhanced by using the damping factor between the previous and present time values for the interfacial drag coefficient. The interfacial drag is damped according to

$$
\text { decay }=2^{-\frac{\Delta t}{|c i d a m p|}}
$$

where 'cidamp' is provided by the input deck, 
for cidamp $<0$,

$$
x^{n}=\max \left(\min \left(x^{n}, \frac{x^{(n-1)}}{\text { decay }}\right), x^{(n-1)} * \text { decay }\right)
$$

for cidamp $>0$,

$$
x^{n}=\text { decay } * x^{n-1}+(1-\text { decay }) * x^{n}
$$

where $\mathrm{x}^{\mathrm{n}}$ is the interfacial drag coefficient at the old time step.

Even if the time step size used in the FLOWTRAN-TF is not evaluated on the basis of a rigorous stability analysis without any assumptions, it is concluded that the present analysis is adequate for stable code running since the time step is also controlled by the accuracy checking to reduce unacceptably large time steps before a numerical instability can occur.

\section{References}

Roache, P. J., 1976, Computational Fluid Dynamics, Chapter 3, Hermosa Publishers.

Potter, D., 1972, Computational Physics, Chapter 3, John Wiley \& Sons.

Lee, S. Y., Lab. notebook, WSRC-NB-89-211, p. 63-78. 


\subsection{Onset Criteria Checking and Power Iteration Method}

Input flags exist so that the code user can select which of the criteria discussed in $\$ 2.7$ are applied during the power iteration. The code evaluates each of the selected criteria at every time step during the flow transient. To facilitate the power iteration strategy, the criteria are generally cast in terms of a power ratio which is actual operating power in the assembly divided by the power required to satisfy the particular criterion. An exception is the CCFL criterion ratio. In all cases, the ratio must equal one when the criterion is met. The four criterion ratios are described below.

Fluid Temperature Criterion (\#1): The criterion that the bulk fluid temperature remain below the saturation temperature prevents bulk boiling and significant vapor generation. This criterion also serves as a check on the occurrence of fluid flashing caused by sudden pressure decreases in flow restrictions. Flashing may also generate enough vapor to cause a flow blockage. Noting that

$$
\left[\frac{P_{\text {acraal }}}{P_{\text {criterion }}}\right] \equiv\left[\frac{\dot{m} c_{p}\left(\left(T_{f}\right)_{i, j}-T_{\text {in }}\right)}{\dot{m} c_{p}\left(\left(T_{\text {sat }}\right)_{i, j}-T_{\text {in }}\right)}\right]=\left[\frac{\left(T_{f}\right)_{i, j}-T_{\text {in }}}{\left(T_{\text {sat }}\right)_{i, j}-T_{\text {in }}}\right]
$$

an appropriate fluid temperature criterion ratio is

$$
C_{1}=\phi_{1}\left[\frac{\left(T_{f}\right)_{i, j}-T_{\text {in }}}{\left(T_{\text {sat }}\right)_{i, j}-T_{\text {in }}}\right]
$$

where

$$
\begin{aligned}
C_{1} & \equiv \text { Criterion ratio } \\
\phi_{1} & \equiv \text { Peaking factor } \\
T_{i n} & \equiv \text { Plenum inlet fluid temperature, } K \\
\left(T_{f}\right)_{i, j} & \equiv \text { Local fluid temperature, } K \\
\left(T_{\text {sat }}\right)_{i, j} & \equiv \text { Local fluid saturation temperature, } K
\end{aligned}
$$

Equation (3.10-1) is evaluated for each fluid cell in the computational mesh and the largest value taken as the criterion ratio at the evaluation time.

The power iteration scheme iterates to find operating conditions such that the most limiting criterion is equal to one. The peaking factor is a user input parameter that adjusts the temperature ratio corresponding to the limiting value. With the peaking factor set equal to one, $C_{1}$ equals one when the highest fluid temperature just reaches saturation. When a peaking factor greater than one is input, the criterion reaches one at a maximum fluid temperature below saturation. The value of $\phi_{1}$ required to set a specific temperature limit 
can be easily obtained from Eq. (3.10-1) by substituting the desired limit for $\left(T_{f}\right)_{i, j}$ and solving for $\phi_{1}$. Note that at zero power the fluid temperature will remain at $T_{\text {in }}$ and the criterion ratio will be zero.

Instantaneous Maximum Surface Temperature Criterion (\#2): The surface temperature criterion is chosen to avoid significant vapor generation within the assembly. Some minor vapor generation will occur from evaporation of the heated fluid. However, the metal surface must exceed the local fluid saturation temperature to initiate local boiling and significant vapor generation. In fact, this criterion is quite conservative since initially vapor generated at the wall will condense in the subcooled bulk fluid. Nevertheless, since a surface temperature at saturation is a clear precursor to significant vapor generation this criterion has been adopted as a conservative predictor of operating limits.

The appropriate power ratio is derived by considering single-phase liquid flow through a uniformly heated channel with constant convective heat transfer coefficient and thermal properties. At axial position $\mathrm{z}=\mathrm{L}$

$$
P=A_{h} q^{\prime \prime}=h A_{h}\left[T_{w}(L)-T_{f}(L)\right]
$$

A fluid energy balance from the inlet to position $\mathrm{L}$ yields

$$
T_{f}(L)=\frac{P}{\dot{m} c_{p}}+T_{\text {in }}
$$

Eliminating fluid temperature $T_{\mathrm{f}}$ between these expressions results in

$$
P=\frac{T_{w}(L)-T_{i n}}{\frac{1}{h A_{h}}+\frac{1}{\dot{m} c_{p}}}
$$

The basic $\mathrm{T}_{\mathrm{w}} \leq \mathrm{T}_{\text {sat }}$ criterion power ratio is then derived as

$$
\left[\frac{P_{\text {actual }}}{P_{\text {criterion }}}\right] \equiv\left[\begin{array}{c}
\frac{\frac{T_{w}(L)-T_{\text {in }}}{1}}{h A_{h}}+\frac{1}{\dot{m} c_{p}} \\
\frac{T_{\text {sat }}(L)-T_{\text {in }}}{\frac{1}{h A_{h}}+\frac{1}{\dot{m} c_{p}}}
\end{array}\right]=\left[\frac{T_{w}(L)-T_{\text {in }}}{T_{\text {sar }}(L)-T_{\text {in }}}\right]
$$

The actual criterion ratio in FLOWTRAN-TF can be expressed as

$$
C_{2}=\left[\frac{\left(T_{w}\right)_{\text {is,ia,iz }}-T_{\text {in }}}{\left(T_{\text {sat }}\right)_{i, j}+\Delta T_{\text {sup }}-T_{\text {in }}}\right]
$$

where

$$
\mathrm{C}_{2} \equiv \text { Criterion ratio }
$$


$\mathrm{T}_{\text {in }} \equiv$ Plenum inlet fluid temperature, $\mathrm{K}$

$\left(T_{s a t}\right)_{i, j} \equiv$ Saturation temperature in fluid adjacent to the wall, $K$

$\left(\mathrm{T}_{\mathrm{w}}\right)_{\mathrm{is}, \mathrm{ia}, \mathrm{iz}} \equiv \underset{\mathrm{iz}, \mathrm{K}}{\mathrm{L}}$ wall temperature at surface is, azimuthal location ia, and axial level

$\Delta \mathrm{T}_{\text {sup }} \equiv$ Superheat temperature allowance, $\mathrm{K}$

Equation (3.10-2) is evaluated for each solid surface cell in the middle (heated) section of the mesh and the largest value taken as the criterion ratio at the evaluation time.

Clearly the criterion ratio will be unity when

$$
\left(T_{w}\right)_{\text {is,ia,iz }}=\left(T_{\text {sat }}\right)_{i, j}+\Delta T_{\text {sup }}
$$

The superheating allowance is used as to introduce a conservative factor into the criterion calculation. In practice, to compute operating limits, we specify a $\Delta \mathrm{T}_{\text {sup }}<0$ which is actually some degree of wall subcooling. That is, we let the criterion be satisfied at a wall temperature below saturation as a conservatism to account for model non-idealities. This factor is specified through the input and can be set at the user's discretion. At zero power and thermal equilibrium, the solid temperature will be constant at $T_{\text {in }}$ and the criterion ratio will be zero.

Running Time Average Maximum Surface Temperature Criterion (\#3): Equation (3.10-2) is also computed using a running time average of the wall temperatures at each computational cell in place of instantaneous time values. The running average wall temperature is computed as

$$
\left\langle T_{w}\right\rangle_{i s, i n, i z}=\frac{\sum_{i s=1}^{N_{t}}\left(T_{w}\right)_{i s, i a, i z, i t}}{N_{t}}
$$

where $\mathrm{N}_{\mathrm{t}}$ is the total number of time steps that have been computed at the point in the transient when the evaluation is made. Use of the running average wall temperature averages out sudden changes in the temperature which can occur from rapid changes in the local void fraction, fluid velocity, or fluid temperature. Data obtained from experimental rigs is reported in terms of an average wall temperature meeting the saturation temperature limit. The running average may more closely reflect the true operating conditions and can be selected by the code user to set power limits in place of the instantaneous criterion value.

CCFL Criterion (\#4): Coolant flow to a channel can be blocked if sufficient vapor is generated such that it begins to flow counter-currently (i. e. upward) to the fluid and the flow reaches a counter-current flow limitation (CCFL). The FLOWTRAN-TF interfacial drag model does not permit counter-current flows significantly beyond CCFL. However, instantaneous two-phase conditions may exceed CCFL and even steady operation is allowed at or very slightly beyond CCFL. The CCFL criterion is designed to detect computed two-phase flow conditions at or beyond CCFL. Refer to $\$ 2.5 .5$ for a detailed discussion of the TF interfacial drag model and for more information on CCFL. The CCFL line (shown in Fig. 3.10-1) is modeled as 


$$
\left|j_{g}^{*}\right|^{1 / 2}+m\left|j_{f}^{*}\right|^{1 / 2}=C
$$

where

$$
j_{k}^{*} \equiv j_{k}\left(\frac{\rho_{k}}{\Delta \rho g D_{c}}\right)^{1 / 2}
$$

In Eqs. (3.10-4) and (3.10-5):

$$
\begin{aligned}
\mathrm{m}, \mathrm{C} & \equiv \text { CCFL correlation parameters } \\
\mathrm{j}_{k} & \equiv \text { Superficial velocity of phase } \mathrm{k}(\mathrm{k}=\mathrm{g}, \mathrm{f}), \mathrm{m} / \mathrm{s} \\
\rho_{k} & \equiv \text { Density of phase } \mathrm{k}, \mathrm{kg} / \mathrm{m}^{3} \\
\Delta \rho & \equiv \rho_{\mathrm{f}}-\rho_{\mathrm{g}}, \mathrm{kg} / \mathrm{m}^{3} \\
\mathrm{~g} & \equiv \text { Gravitational acceleration, } \mathrm{m} / \mathrm{s}^{2} \\
D_{c} & \equiv \text { Fluid cell equivalent diameter, } \mathrm{m}
\end{aligned}
$$

Positive fluid flows are taken to be in the downward $(+z)$ direction. At each fluid cell we define

$$
C^{n} \equiv\left\{\begin{array}{ccc}
\left|j_{g}^{* n}\right|^{1 / 2}+m\left|j_{f}^{*} n\right|^{1 / 2} & j_{g}^{* n}<0, j_{f}^{* n}>0 & \text { (counter - current flow) } \\
0 & \text { otherwise } & \text { (co-current flow) }
\end{array}\right.
$$

where $\mathrm{n}$ denotes the actual conditions at time step $\mathrm{n}$.

The chosen CCFL criterion ratio at each fluid cell is

$$
C_{4}=\phi_{4} \frac{C^{n}}{C}
$$

where $\phi_{4}$ is a peaking factor. A conservative pad is added to the criterion ratio by choosing $\phi_{4}>1$ if desired. The maximum valued criterion ratio throughout the fluid computational mesh is identified at each time step. The CCFL criterion ratio is not a power ratio. In practice, we do not attempt to perform a power iteration based on the CCFL criterion so this factor is not important to the power iteration process. 


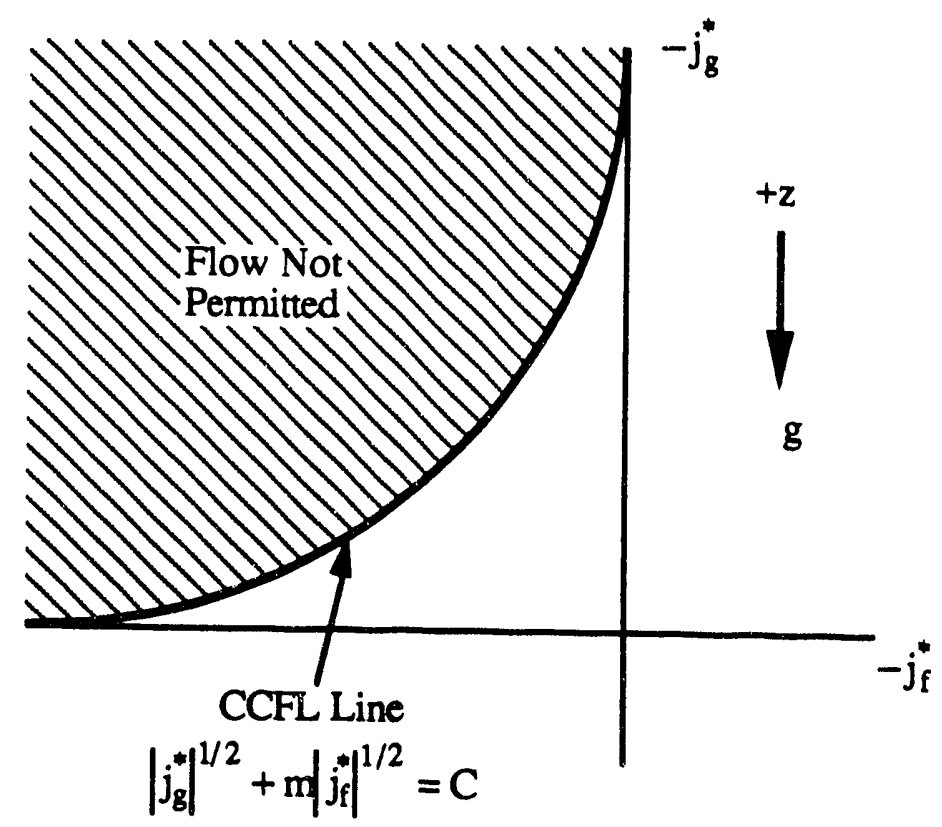

Figure 3.10-1 Schematic representation of downflow CCFL line. 
Power Iteration: As explained above, we write criteria ratios such that they will be identically unity when the criteria are met. Therefore, our power iteration strategy seeks a power level such that the largest (i.e. most limiting) criterion ratio just reaches a value of one during the flow transient. A schematic representation of the power iteration strategy is shown in Fig. 3.10-2. The power iteration strategy implicitly assumes the limiting criterion ratio varies monotonically with power.

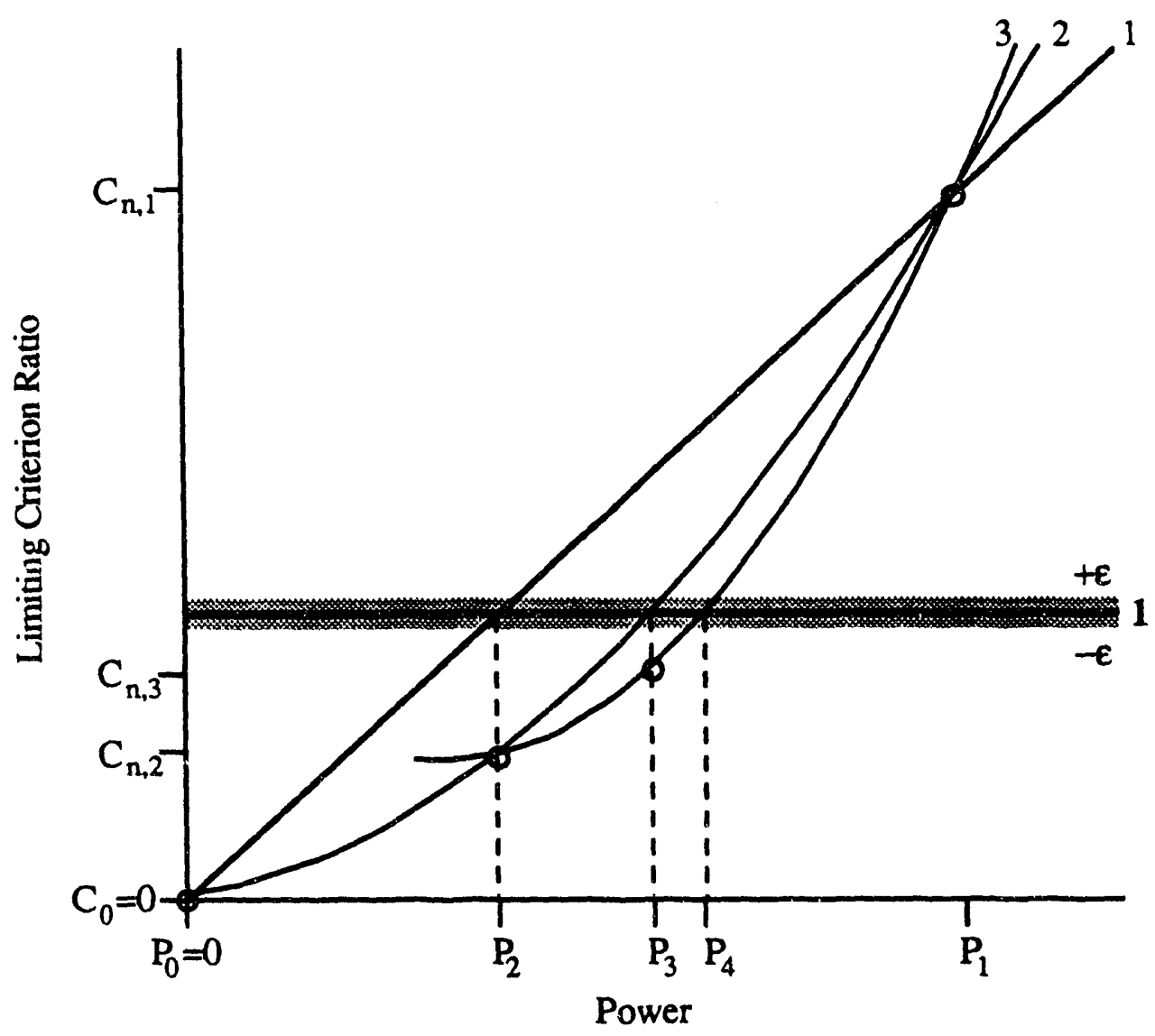

Figure 3.10-2 Schematic representation of power iteration scheme.

The user inputs an initial guess for the pre-incident power level $P_{1}$. The code then evaluates each of the criteria discussed above at every time step through the flow transient. The largest criterion ratio is the limiting value and corresponds to $C_{n, 1}$ on the plot. The subscript $\mathrm{n}$ is used to indicate the criterion number (3.10-1-4) being used. For the first guess at a new power level, we use linear interpo!ation as illustrated by line 1 in Fig. $3.10-2$. That is, we simply take

$$
P_{2}=P_{1} / C_{n, 1}
$$

Note that at zero power $\left(P_{0}=0\right)$ all of the criteria ratios are also zero $\left(C_{0}=0\right)$. This condition determines the origin of our plot and is implicit in Eq. (3.10-8). 
A transient calculation is again performed using the new estimate of assembly power and a new limiting criteria ratio $C_{n, 2}$ is determined. Since we have now located three points in the power - criteria domain, we can use quadratic interpolation to speed up convergence. We fit a quadratic function through the points $\left(P_{0}, C_{0}\right),\left(P_{1}, C_{n, 1}\right)$, and $\left(P_{2}, C_{n, 2}\right)$ and compute the intersection with the line $C=1$. This process is illustrated by curve 2 in Fig. 3.10-2 which determines a new estimate of the limiting power as $P_{3}$. The transient calculation is now performed using pre-incident power $P_{3}$ to obtain criteria ratio $C_{n, 3}$. If required, the power estimation procedure is then repeated using a quadratic fit to the points $\left(P_{1}, C_{n, 1}\right),\left(P_{2}, C_{n, 2}\right)$, and $\left(P_{3}, C_{n, 3}\right)$ to obtain power estimate $P_{4}$ (curve 3 in Fig. $\left.3.10-2\right)$. The power iteration procedure is repeated using quadratic interpolation with the last three data pairs until the limiting criterion is within a user specified tolerance from unity. In Fig. $3.10-2$, we illustrate the accepted tolerance by the shaded band of $\pm \varepsilon$ around the value of $\mathrm{C}=1$. When the limiting criterion ratio falls within the tolerance band the power iteration is terminated. The user also inputs the maximum number of power iterations the code is allowed to make so that excessive run times are avoided if the iteration is not converging to a power limit or if convergence is slow.

The power iteration scheme is coded in subroutine POWER. After the first iteration, where linear interpolation is applied, we fit a quadratic function to the power-criteria relationship for each criterion by letting

$$
a P_{i}^{2}+b P_{i}+c=C_{n, i}
$$

In practice, it is convenient to scale the power values by the latest power estimate to give numbers on the order of unity. Therefore, in place of Eq. (3.10-9), we actually use

$$
a \zeta_{i}^{2}+b \zeta_{i}+c=C_{n, i}
$$

where at iteration $k(k \geq 2)$ the three power values used for the curve fitting are scaled as

$$
\zeta_{i}=P_{i} / P_{k} \quad \text { for } \quad i=k, k-1, k-2
$$

To determine the fitting coefficients $(a, b, c)$, we solve Eq. $(3.10-10)$ using the last three power-criterion data pairs. This problem can be cast into the equivalent matrix form

$$
\left(\begin{array}{lll}
a & b & c
\end{array}\right)\left(\begin{array}{ccc}
\zeta_{k}^{2} & \zeta_{k-1}^{2} & \zeta_{k-2}^{2} \\
\zeta_{k} & \zeta_{k-1} & \zeta_{k-2} \\
1 & 1 & 1
\end{array}\right)=\left(\begin{array}{c}
C_{n, k} \\
C_{n, k-1} \\
C_{n, k-2}
\end{array}\right)
$$

The Vandermonde matrix in Eq. $(3.10-12)$ is easily inverted to obtain the following solution:

$$
\begin{aligned}
& a=f_{1}+f_{2}+f_{3} \\
& b=-f_{1}\left(\zeta_{k-1}+\zeta_{k-2}\right)-f_{2}\left(\zeta_{k-2}+\zeta_{k}\right)-f_{3}\left(\zeta_{k}+\zeta_{k-1}\right) \\
& c=f_{1} \zeta_{k-1} \zeta_{k-2}+f_{2} \zeta_{k-2} \zeta_{k}+f_{3} \zeta_{k} \zeta_{k-1}
\end{aligned}
$$

where 


$$
\begin{aligned}
& \mathrm{f}_{1}=C_{n, k} /\left(\zeta_{k}-\zeta_{k-1}\right)\left(\zeta_{k}-\zeta_{k-2}\right) \\
& f_{2}=C_{n, k-1} /\left(\zeta_{k-1}-\zeta_{k}\right)\left(\zeta_{k-1}-\zeta_{k-2}\right) \\
& f_{3}=C_{n, k-2} /\left(\zeta_{k-2}-\zeta_{k}\right)\left(\zeta_{k-2}-\zeta_{k-1}\right)
\end{aligned}
$$

With the coefficients a,b, and c determined, we solve Eq. $(3.10-10)$ for $\zeta_{k+1}$ by setting $\mathrm{C}_{n, \mathrm{k}+1}=1$ and using the quadratic formula

$$
\zeta_{k+1}=\frac{-b+\sqrt{d}}{2 a}
$$

where $d$ is the discriminant of the quadratic equation

$$
d=b^{2}-4 a(c-1)
$$

We only need to take the positive square root of the discriminant in Eq. (3.10-15) since we expect two real roots and only the positive root will be in the first quadrant in Fig. 3.10-2. Finally we use the normalization factor to calculate an absolute power level as

$$
P_{k+1}=\zeta_{k+1} P_{k}
$$

We would only expect real roots to occur in Eq. (3.10-15) during normal calculations and do not expect to obtain negative discriminants. However, as a precautionary measure, if the discriminant is negative, the code is programmed to bypass Eq. $(3 \cdot 10-15)$ and default to a linear interpolation for the new power estimate using

$$
\zeta_{k+1}=\frac{\zeta_{k}\left(1-C_{n, k-1}\right)-\zeta_{k-1}\left(1-C_{n, k}\right)}{\left(C_{n, k}-C_{n, k-1}\right)}
$$

The procedure outlined in Eqs. (3.10-9) - (3.10-18) above is repeated separately for each criterion selected for use and the minimum power level used as the new estimate. We save the individual criterion ratios from each iteration but only retain the minimum power estimate. In practice the scheme outlined above works quite well. We normally converge to a power limit within a tolerance of \pm 0.005 in the criterion ratio within four iterations. For the few cases we have encountered where power convergence does not occur, we have found that manual adjustment of the pre-incident power estimate and repeated code execution is an adequate procedure. Hence, the need for enhanced power iteration logic has not been identified. 


\subsection{Miscellaneous Topics}

The remaining aspects of FLOWTRAN-TF computational implementation are presented here. These are spline interpolation, property checking, error checking and control, timestep control, and steady-state checking.

\subsubsection{Spline Interpolation}

FLOWTRAN-TF applies first-order or natural cubic splines to interpolation of $\left(t_{i}, y_{i}\right)$ pairs of input forcing functions. The following input forcing functions require spline interpolation:

Table 3.11.1-1 Forcing Functions that Require Spline Interpolation

\begin{tabular}{|c|c|c|}
\hline Forcing Function & SRS Assembly & Experimental Rig \\
\hline Inlet pressure & $\mathrm{x}$ & $\bar{x}$ \\
\hline Inlet void fraction & $\bar{x}$ & $\bar{x}$ \\
\hline Inlet liquid temperature & $x$ & $x$ \\
\hline Inlet gas temperature & $\bar{x}$ & $x$ \\
\hline Inlet air mass fraction & $x$ & $\bar{x}$ \\
\hline Exit pressure & $\bar{x}$ & $\bar{x}$ \\
\hline Exit void fraction & $x$ & $\bar{x}$ \\
\hline Exit liquid temperature & $\bar{x}$ & $\bar{x}$ \\
\hline Exit gas temperature & $x$ & $\bar{x}$ \\
\hline Exit air mass fraction & $\bar{x}$ & $\bar{x}$ \\
\hline Inlet liquid volumetric flow & $x$ & $\bar{x}$ \\
\hline Inlet gas volumetric flow & $\bar{x}$ & $\bar{x}$ \\
\hline $\begin{array}{l}\text { Tank level (set to zero for } \\
\text { experimental rig) }\end{array}$ & $\bar{x}$ & $\bar{x}$ \\
\hline Fission power response & $\mathbf{x}$ & \\
\hline $\begin{array}{l}\text { Moderated tank decay power } \\
\text { response }\end{array}$ & $\bar{x}$ & \\
\hline $\begin{array}{l}\text { Dry tank decay power or } \\
\text { experiment rig power } \\
\text { response }\end{array}$ & $\mathbf{x}$ & $\mathbf{x}$ \\
\hline $\begin{array}{l}\text { Delta multipliers for } \\
\text { conservative decay heat }\end{array}$ & $\mathbf{x}$ & \\
\hline Axial power response & $\mathbf{x}$ & $\mathbf{x}$ \\
\hline
\end{tabular}

The following sections describe the algorithms and subroutines used by FLOWTRAN-TF for spline interpolation. The algorithm discriptions were taken verbatim from reference [1]. They are included in this write-up for completeness. The FORTRAN algorithms were derived from those given by Cheney and Kincaid (1985) and modified to fit the FLOWTRAN-TF code framework. 
First-degree spline: A first-degree spline is a piecewise linear function consisting of linear polynomials joined together to achieve continuity. Given a table of function values, $\left(t_{1}, y_{1}\right), \ldots,\left(t_{n}, y_{n}\right)$, such a function can be constructed as

$$
S(x)= \begin{cases}S_{1}(x) & x \in\left[t_{1}, t_{2}\right] \\ S_{2}(x) & x \in\left[t_{2}, t_{3}\right] \\ \vdots & \\ S_{n-1}(x) & x \in\left[t_{n-1}, t_{n}\right]\end{cases}
$$

where

$$
S_{i}(x)=a_{i} x+b_{i}
$$

It is characterized by the following three properties:

1. The domain of $S$ is an interval $[a, b]$.

2. $S$ is continuous on $[a, b]$

3. There is a partitioning of the interval $a=t_{1}<t_{2}<\cdots<t_{n}=b$ such that $S$ is a linear polynomial on each subinterval $\left[t_{i}, t_{i+1}\right]$.

In FLOWTRAN-TF, $S(x)=S_{n-1}(b)=y_{n}$ when $x>b$. By using the point-slope form of a line, we obtain

$$
S_{i}(x)=y_{i}+m_{i}\left(x-t_{i}\right)
$$

where $m_{i}$ is the slope of the line and is therefore given by

$$
m_{i}=\frac{y_{i+1}-y_{i}}{t_{i+1}-t_{i}}
$$

The form of equation (3.11.1-3) is better than that of equation (3.11.1-2) for the practical evaluation of $S(x)$ because some of the quantities $x-t_{i}$ must be computed in any case simply to determine which subinterval contains $x$. The interval $\left[t_{i}, t_{i+1}\right]$ containing $x$ is characterized by the fact that $x-t_{i}$ is the first of the quantities $x-t_{n-1}, x-t_{n-2}, \ldots, x-t_{1}$ that is non-negative.

Natural cubic spline: The natural cubic spline is available in FLOWTRAN-TF whenever more smoothness is needed in the approximating function. The function $\mathrm{S}$ that we wish to construct consists of $n-1$ cubic polynomial pieces,

$$
S(x)=\left\{\begin{array}{cc}
S_{1}(x) & t_{1} \leq x \leq t_{2} \\
S_{2}(x) & t_{2} \leq x \leq t_{3} \\
\vdots & \\
S_{n-1}(x) & t_{n-1} \leq x \leq t_{n}
\end{array}\right.
$$


In this formula, $S_{i}$ denotes the cubic polynomial that will be used on the subinterval $\left[t_{i}, t_{i+1}\right]$. The interpolation conditions are

$$
\mathrm{S}\left(\mathrm{t}_{\mathrm{i}}\right)=\mathrm{y}_{\mathrm{i}} \quad 1 \leq \mathrm{i} \leq \mathrm{n}
$$

The continuity conditions are imposed only at the interior knots $t_{2}, t_{3}, \ldots, t_{n-1}$. These conditions are written

$$
\lim _{x \rightarrow t_{i}^{-}} S^{(k)}\left(t_{i}\right)=\lim _{x \rightarrow t_{i}^{+}} S^{(k)}\left(t_{i}\right) \quad k=0,1,2
$$

where $S^{(k)}$ implies the kth derivative of $S$.

Two further conditions must be imposed in order to use all the degrees of freedom available. For a natural cubic spline, the choice for these two extra conditions is

$$
S^{\prime \prime}\left(t_{1}\right)=S^{\prime \prime}\left(t_{n}\right)=0
$$

Since $S^{\prime \prime}$ is continuous, the numbers

$$
\mathrm{z}_{\mathrm{i}} \equiv \mathrm{S}^{\prime \prime}\left(\mathrm{t}_{\mathrm{i}}\right) \quad 1 \leq \mathrm{i} \leq \mathrm{n}
$$

are unambiguously defined. We do not yet know the values $z_{2}, z_{3}, \ldots, z_{n-1}$, but, of course, $z_{1}=z_{n}=0$ by equation (3.11.1-5).

If the $z_{i}^{\prime}$ 's were known, we could construct $S$ as now described. On the interval $\left[t_{i,} t_{i+1}\right]$, $S^{\prime \prime}$ is a linear polynomial taking the values $z_{i}$ and $z_{i+1}$ at the endpoints. Thus

$$
S_{i}^{\prime}(x)=\frac{z_{i+1}}{h_{i}}\left(x-t_{i}\right)+\frac{z_{i}}{h_{i}}\left(t_{i+1}-x\right)
$$

with $h_{i}=t_{i+1}-t_{i}$. Clearly, $S_{i}^{\prime \prime}\left(t_{j}\right)=z_{i}, S_{i}^{\prime}\left(t_{i+1}\right)=z_{i+1}$, and $S_{i}^{\prime \prime}$ is linear in $x$. If this is integrared twice, we obtain $S_{i}$ itself:

$$
S_{i}(x)=\frac{z_{i+1}}{6 h_{i}}\left(x-t_{i}\right)^{3}+\frac{z_{i}}{6 h_{i}}\left(t_{i+1}-x\right)^{3}+c x+d
$$

where $c$ and $d$ are constants of integration. By adjusting the integration constants, we obtain a form for $S_{i}$ that is easier to work with,

$$
S_{i}(x)=\frac{z_{i+1}}{6 h_{i}}\left(x-t_{i}\right)^{3}+\frac{z_{i}}{6 h_{i}}\left(t_{i+1}-x\right)^{3}+C\left(x-t_{i}\right)+D\left(t_{i+1}-x\right)
$$

where $\mathrm{C}$ and $\mathrm{D}$ are constants.

The interpolation conditions $S_{i}\left(t_{i}\right)=y_{i}$ and $S_{i}\left(t_{i+1}\right)=y_{i+1}$ can be imposed now and the result is 


$$
\begin{aligned}
S_{i}(x)= & \frac{z_{i+1}}{6 h_{i}}\left(x-t_{i}\right)^{3}+\frac{z_{i}}{6 h_{i}}\left(t_{i+1}-x\right)^{3} \\
& +\left(\frac{y_{i+1}}{h_{i}}-\frac{h_{i}}{6} z_{i+1}\right)\left(x-t_{i}\right)+\left(\frac{y_{i}}{h_{i}}-\frac{h_{i}}{6} z_{i}\right)\left(t_{i+1}-x\right)
\end{aligned}
$$

When the values $z_{1}, z_{2}, \ldots, z_{n}$ have been determined, the spline function $S(x)$ is obtained from equations of this form for $S_{1}(x), S_{2}(x), \ldots, S_{n-1}(\dot{x})$.

We now show how to determine the $z_{i}$ 's. One condition remains to be imposed, the continuity of $S^{\prime}$. At the interior knots $t_{i}(2 \leq i \leq n-1)$, we must have $S_{i-1}^{\prime}\left(t_{i}\right)=S_{i}^{\prime}\left(t_{i}\right)$.

We have from equation (3.11.1-9)

$$
S_{i}^{\prime}(x)=\frac{z_{i+1}}{2 h_{i}}\left(x-t_{i}\right)^{2}-\frac{z_{i}}{2 h_{i}}\left(t_{i+1}-x\right)^{2}+\frac{y_{i+1}}{h_{i}}-\frac{h_{i}}{6} z_{i+1}-\frac{y_{i}}{h_{i}}+\frac{h_{i}}{6} z_{i}
$$

This gives

$$
S_{i}^{\prime}\left(t_{i}\right)=-\frac{h_{i}}{6} z_{i+1}-\frac{h_{i}}{3} z_{i}+b_{i}
$$

where

$$
b_{i}=\frac{1}{h_{i}}\left(y_{i+1}-y_{i}\right)
$$

Analogously, we have

$$
S_{i-1}^{\prime}\left(t_{i}\right)=\frac{h_{i-1}}{6} z_{i-1}+\frac{h_{i-1}}{3} z_{i}+b_{i-1}
$$

When these are set equal to each other, the resulting equation can be rearranged as

$$
h_{i-1} z_{i-1}+2\left(h_{i-1}+h_{i}\right) z_{i}+h_{i} z_{i+1}=6\left(b_{i}-b_{i-1}\right)
$$

for $2 \leq \mathrm{i} \leq \mathrm{n}-1$. By letting

$$
u_{i}=2\left(h_{i-1}+h_{i}\right) \text { and } v_{i}=6\left(b_{i}-b_{i-1}\right)
$$

we obtain a tridiagonal system of equations

$$
\left\{\begin{aligned}
z_{1} & =0 \\
h_{i-1} z_{i-1}+u_{i} z_{i}+h_{i} z_{i+1} & =v_{i} \quad 2 \leq i \leq n-1 \\
z_{n} & =0
\end{aligned}\right.
$$

to be solved for the $z_{i}^{\prime}$ 's. The simplicity of the first and last equations is a result of the natural cubic spline conditions $S^{\prime \prime}\left(t_{1}\right)=S^{\prime \prime}\left(t_{n}\right)=0$. 
Writing (3.11.1-11) in matrix form, we have

$$
\left[\begin{array}{cccccc}
1 & \jmath & & & & \\
h_{1} & u_{2} & h_{2} & & 0 & \\
& h_{2} & u_{3} & h_{3} & & \\
& & \ddots & \ddots & \ddots & \\
& 0 & & h_{n-2} & u_{n-1} & h_{n-1} \\
& & & & 0 & 1
\end{array}\right]\left[\begin{array}{c}
z_{1} \\
z_{2} \\
z_{3} \\
\vdots \\
z_{n-1} \\
z_{n}
\end{array}\right]=\left[\begin{array}{c}
0 \\
v_{2} \\
v_{3} \\
\vdots \\
v_{n-1} \\
0
\end{array}\right]
$$

On eliminating the first and last equation, we have

$$
\left[\begin{array}{ccccc}
u_{2} & h_{2} & & & \\
h_{2} & u_{3} & h_{3} & & 0 \\
& \ddots & \ddots & \ddots & \\
& & h_{n-3} & u_{n-2} & h_{n-2} \\
& 0 & & h_{n-2} & u_{n-1}
\end{array}\right]\left[\begin{array}{c}
z_{2} \\
z_{3} \\
\vdots \\
z_{n-2} \\
z_{n-1}
\end{array}\right]=\left[\begin{array}{c}
v_{2} \\
v_{3} \\
\vdots \\
v_{n-2} \\
v_{n-1}
\end{array}\right]
$$

which is a symmetric tridiagonal system of order $\mathbf{n}-2$. In Gaussian elimination without pivoting, the forwand elimination phase would modify the $u_{i}$ 's and $v_{i}$ 's as follows:

$$
\left\{\begin{array}{c}
u_{3} \leftarrow u_{3}-\left(\frac{h_{2}}{u_{2}}\right) h_{2} \\
v_{3} \leftarrow v_{3}-\left(\frac{h_{2}}{u_{2}}\right) v_{2} \\
\vdots
\end{array}\right.
$$

The general formulas are

$$
\left\{\begin{array}{l}
u_{i} \leftarrow u_{i}-\frac{h_{i-1}^{2}}{u_{i-1}} \\
v_{i} \leftarrow v_{i}-\frac{h_{i-1} v_{i-1}}{u_{i-1}} \quad i=3,4, \ldots, n-1
\end{array}\right.
$$

The back substitution phase yields

$$
\begin{aligned}
& z_{n-1} \leftarrow \frac{v_{n-1}}{u_{n-1}} \\
& z_{n-2} \leftarrow \frac{v_{n-2}-h_{n-2} z_{n-1}}{u_{n-2}} \\
& :
\end{aligned}
$$

The general formulas are 


$$
\left\{\begin{array}{l}
z_{n-1} \leftarrow \frac{v_{n-1}}{u_{n-1}} \\
z_{i} \leftarrow \frac{v_{i}-h_{i} z_{i+1}}{u_{i}} \quad i=n-2, n-3, \ldots, 2
\end{array}\right.
$$

Putting all this together leads to the following algorithm for the tridiagonal system $(3.11 .1-12)$.

Algorithm for solving the natural cubic spline tridiagonal system directly: Given the interpolation points $\left(t_{i}, y_{i}\right)$ for $i=1,2, \ldots, n$ :

1. Compute for $i=1,2, \ldots, n-1$

$$
\begin{aligned}
& h_{i}=t_{i+1}-t_{i} \\
& b_{i}=\frac{1}{h_{i}}\left(y_{i+1}-y_{i}\right)
\end{aligned}
$$

2. Set

$$
\begin{aligned}
& u_{2}=2\left(h_{1}+h_{2}\right) \\
& v_{2}=6\left(b_{2}-b_{1}\right)
\end{aligned}
$$

and compute inductively for $\mathrm{i}=3,4, \ldots, \mathrm{n}-1$

$$
\begin{aligned}
& u_{i}=2\left(h_{i}+h_{i-1}\right)-\frac{h_{i-1}^{2}}{u_{i-1}} \\
& v_{i}=6\left(b_{i}-b_{i-1}\right)-\frac{h_{i-1} v_{i-1}}{u_{i-1}}
\end{aligned}
$$

3. Set

$$
\begin{aligned}
& z_{n}=0 \\
& z_{1}=0
\end{aligned}
$$

and compute inductively for $\mathrm{i}=\mathrm{n}-1, \mathrm{n}-2, \ldots, 2$

$$
z_{i}=\frac{v_{i}-h_{i} z_{i+1}}{u_{i}}
$$

Equation (3.11.1-9) is now rearranged as

$$
S_{i}(x)=A_{i}+B_{i}\left(x-t_{i}\right)+C_{i}\left(x-t_{i}\right)^{2}+D_{i}\left(x-t_{i}\right)^{3}
$$

since nested multiplication can then be utilized.

Equation (3.11.1-13) is the Taylor expansion of $S_{i}$ about the point $t_{i}$. Hence 


$$
A_{i}=S_{i}\left(t_{i}\right), \quad B_{i}=S_{i}^{\prime}\left(r_{i}\right), \quad C_{i}=\frac{1}{2} S_{i}^{\prime \prime}\left(t_{i}\right), \quad D_{i}=\frac{1}{6} S_{i}^{\prime \prime}\left(t_{i}\right)
$$

Therefore, $A_{i}=y_{i}$ and $C_{i}=z_{i} / 2$. The coefficient of $x^{3}$ in equation (3.11.1-13) is $D_{i}$, while the coefficient of $x^{3}$ in equation (3.11.1-9) is $\left(z_{i+1}-z_{i}\right) / 6 h_{i}$. Therefore

$$
D_{i}=\frac{1}{6 h_{i}}\left(z_{i+1}-z_{i}\right)
$$

Finally, equation (3.11.1-10) provides the value of $S_{i}^{\prime}\left(t_{i}\right)$, which is

$$
B_{i}=-\frac{h_{i}}{6} z_{i+1}-\frac{h_{i}}{3} z_{i}+\frac{1}{h_{i}}\left(y_{i+1}-y_{i}\right)
$$

Thus the nest form of $S_{i}(x)$ is

$$
S_{i}(x)=y_{i}+\left(x-t_{i}\right)\left(B_{i}+\left(x-t_{i}\right)\left(\frac{z_{i}}{2}+\frac{1}{6 h_{i}}\left(x-t_{i}\right)\left(z_{i+1}-z_{i}\right)\right)\right)
$$

\section{References:}

Ward Cheney and David Kincaid. Numerical Mathematics and Computing, Second Edition, 1985. 


\subsubsection{Property Checking}

This section describes the applicability checking for the physical property correlations of fluid and solid properties used in the FLOWTRAN-TF code. Checks are made on the value of each correlational parameter to ensure that the fluid and solid properties computed at each time step are within the applicable ranges of property correlations.

\section{Fluid property checking}

The parameters of fluid property checking are pressure $(P)$, liquid temperature $\left(T_{f}\right)$, gas temperature $\left(T_{g}\right)$, and air mass fraction $\left(\mathbf{X}_{\mathrm{a}}\right)$. All the functions of liquid fluid properties were correlated as function of pressure and temperature. The thermodynamic properties of gas mixture, which consists of steam and air, were basically evaluated on the basis of Dalton's law, ideal gas relation and homogeneous ideal gas solution. Transport properties of gas phase were computed in terms of air mass fraction based on the homogeneous mixture of steam and air phases. These fluid property correlations were documented in $\$ 2.4 .2$ and the Appendix. Table 3.11.2-1 summarizes the applicable range of each parameter for fluid thermodynamic and transport property correlations. For all the fluid property computations, local pressure, liquid temperature, and gas temperature are checked at each time step if they are in the following ranges:

For light water,

$$
6.11 \times 10^{2} \leq \mathrm{P} \leq 1.0 \times 10^{6}(\mathrm{~Pa})
$$

For heavy water,

$$
1.03 \times 10^{3} \leq \mathrm{P} \leq 1.0 \times 10^{6}(\mathrm{~Pa})
$$

For light and heavy water

$$
\begin{aligned}
& 283 \leq \mathrm{T}_{\mathrm{f}} \leq 450(\mathrm{~K}) \\
& 275 \leq \mathrm{T}_{\mathrm{g}} \leq 700(\mathrm{~K})
\end{aligned}
$$

In addition, air moss fraction within a gas phase is checked at each time step to guarantee that it is between zero and unity on a physical basis. That is,

$$
0 \leq \mathrm{X}_{\mathrm{g}} \leq 1.0
$$

The above procedure is applied to each spatial mesh through the whole channel section (Top, Middle, and Bottom Sections) at a given time step. If one of these main fluid parameters $\left(P, T_{f}, T_{g}\right.$, and $\left.X_{a}\right)$ is beyond one of the applicable ranges which are given by equations (3.11.2-1) to (3.11.2-5), the code running is stopped. 


\section{Solid property checking}

The checking parameters of solid properties are solid temperature $\left(\mathrm{T}_{\mathrm{s}}\right)$ and, if applicable, weight fraction of metal alloy (W). Correlations of solid material properties were the solid property correlations. In particular, when the applicable range of each parameter in alloy is computed in the FLOWTRAN-TF code, themperature distribution within a metal materials (lithium-aluminium and uranium-aluminium) conductivity correlations of alloy lithium and uranium weight fraction (W a input. The solid property checking is made in the same procedure provided by the user's checking for each spatial mesh through the some procedure as that of fluid property assembly at a given time step. 
Table 3.11.2-1 Applicable ranges of the parameters for fluid property correlations.

\begin{tabular}{|c|c|c|c|}
\hline Two Phases & Fluid & Properties & $\begin{array}{l}\text { Applicable Range } \\
\text { of Parameters }\end{array}$ \\
\hline \multirow{2}{*}{ Liquid } & Light Water & $\begin{array}{l}\text { Thermodynamic } \\
\text { Properties } \\
\text { \& } \\
\text { Transport } \\
\text { Properties }\end{array}$ & $\begin{array}{c}6.11 \times 10^{2} \leq \mathrm{P} \leq 1.0 \times 10^{6}(\mathrm{~Pa}) \\
\& \\
283 \leq \mathrm{T}_{\mathrm{f}} \leq 450(\mathrm{~K})\end{array}$ \\
\hline & Heavy Water & $\begin{array}{l}\text { Thermodynamic } \\
\text { Properties } \\
\quad \& \\
\text { Transport } \\
\text { Properties }\end{array}$ & $\begin{array}{c}1.03 \times 10^{3} \leq \mathrm{P} \leq 10 \times 10^{6}(\mathrm{~Pa}) \\
\& \\
283 \leq \mathrm{T}_{\mathrm{f}} \leq 450(\mathrm{~K})\end{array}$ \\
\hline \multirow{3}{*}{ Gas } & $\begin{array}{l}\text { Light Water } \\
\text { Steam }\end{array}$ & $\begin{array}{l}\text { Thermodynamic } \\
\text { Properties } \\
\quad \& \\
\text { Transport } \\
\text { Properties }\end{array}$ & $\begin{array}{c}275 \leq \mathrm{T}_{\mathrm{B}} \leq 700(\mathrm{~K}) \\
\& \\
6.11 \times 10^{2} \leq \mathrm{P} \leq 1.0 \times 10^{6}(\mathrm{~Pa})\end{array}$ \\
\hline & $\begin{array}{l}\text { Heavy Water } \\
\text { Steam }\end{array}$ & $\begin{array}{l}\text { Thermodynamic } \\
\text { Properties } \\
\quad \& \\
\text { Transport } \\
\text { Properties }\end{array}$ & $\begin{array}{c}275 \leq \mathrm{T}_{\mathrm{g}} \leq 700(\mathrm{~K}) \\
\& \\
1.03 \times 10^{3} \leq \mathrm{P} \leq 10 \times 10^{6}(\mathrm{~Pa})\end{array}$ \\
\hline & Air & $\begin{array}{l}\text { Thermodynamic } \\
\text { Properties } \\
\quad \& \\
\text { Transport } \\
\text { Properties } \\
\text { Air Mass Fraction }\end{array}$ & $\begin{array}{c}275 \leq \mathrm{T}_{\mathrm{g}} \leq 700(\mathrm{~K}) \\
\& \\
0<\mathrm{P} \leq 1.0 \times 10^{6}(\mathrm{~Pa}) \\
0 \leq \mathrm{X}_{\mathrm{g}} \leq 1.0\end{array}$ \\
\hline
\end{tabular}


Table 3.11.2-2 Applicable ranges of temperature and weight fraction of metal alloy for material property correlations in the FLOWTRAN-TF code.

\begin{tabular}{|l|c|c|c|}
\hline \multirow{2}{*}{ Solid Material } & \multicolumn{2}{|c|}{ Solid Material Properties } & \multirow{2}{*}{$\begin{array}{l}\text { Weight Fraction } \\
\text { for Alloy }\end{array}$} \\
\cline { 2 - 4 } & Density & Specific Heat & $\begin{array}{c}\text { Thermal } \\
\text { Conductivity }\end{array}$ \\
\hline Uranium & \multicolumn{2}{|c|}{$273.15<\mathrm{T}_{\mathrm{s}} \leq 923.15(\mathrm{~K})$} & - \\
\hline Aluminium & \multicolumn{2}{|c|}{$273.15<\mathrm{T}_{\mathrm{s}} \leq 923.15(\mathrm{~K})$} & - \\
\hline Stainless Steel & \multicolumn{2}{|c|}{$273.15<\mathrm{T}_{\mathrm{s}} \leq 773.15(\mathrm{~K})$} & - \\
\hline $\begin{array}{l}\text { Lithium- } \\
\text { Aluminium }\end{array}$ & $273.15<\mathrm{T}_{\mathrm{s}} \leq 453.15(\mathrm{~K})$ & $293.15<\mathrm{T}_{\mathrm{s}} \leq 533.15(\mathrm{~K})$ & $0 \leq \mathrm{W}_{\mathrm{Li}} \leq 0.104$ \\
\hline $\begin{array}{l}\text { Uranium- } \\
\text { Aluminium }\end{array}$ & \multicolumn{2}{|c|}{$273.15<\mathrm{T}_{\mathrm{s}} \leq 673.15(\mathrm{~K})$} & $0 \leq \mathrm{W}_{\mathrm{U}} \leq 0.305$ \\
\hline
\end{tabular}


\begin{tabular}{|l|lll|l|}
\hline $2 / 15 / 93$ & WSRC-TR-92-532 Rev.0 & FLOWTRAN-TF Software Design & Page 506 \\
\hline
\end{tabular}

\subsubsection{Error Checking and Control}

This section describes error checking and control in the FLOWTRAN-TF code. When solution errors are detected (e.g. insufficient solution accuracy, convergence failure within a given iteration limit, computed results outside the range of applicability, etc.) in the course of program execution, the FLOWTRAN-TF code sets an error flag (ierr) to indicate the source of the error and to remedy such situations.

The accuracy of the solution is checked within subroutine ACCCHK. When any of the physical variables, pressure $(P)$, void fraction $(\alpha)$, gas and liquid phase temperatures $\left(\mathrm{T}_{\mathrm{g}}\right.$ and $\left.T_{f}\right)$, and air mass fraction $\left(X_{a}\right)$, change by more than a prescribed amount over a single time step an error condition is flagged. The solution is then backed up to the beginning of the time step and the main variables are reset to their old time values. The time step size is reduced and code computations are repeated based on the reduced time step. The prescribed solution tolerance is provided by the user input parameter tol. The tolerance is given in terms of the relative difference or ratio of the difference between the present and old time values to the old time value for the pressure and for the gas and liquid phasic temperatures. For void fraction and air mass fraction, the tolerance is specified in terms of the absolute change in value since these variables are constrained to fall between zero and one. This accuracy check is made on each value of the new time variables to ensure that the variations of the new time variables from the old values fall within reasonable limits. This is done so that the linearized fluid equations will be sufficiently representative of the nonlinear equations to provide an acceptable level of accuracy in the numerical computations. In case of insufficient solution accuracy, the revised time step size is determined by

$$
\Delta t=\frac{0.9 \delta}{\gamma_{\max }}(\Delta t)_{0}
$$

where

$\delta \equiv$ the tolerance provided by the user's input deck

$(\Delta t)_{0} \equiv$ old time step size

In Eq. (3.11.3-1), $\gamma_{\max }$ is defined as the maximum variation of five main variables in the fluid equations with respect to the values from the previous time step, that is, we compute

$$
\gamma_{\max } \equiv \max \left(\gamma_{P}, \gamma_{\alpha}, \gamma_{T_{B}}, \gamma_{T_{f}}, \gamma_{X_{d}}\right)
$$

where

$$
\begin{aligned}
& \gamma_{p} \equiv \frac{\left(P^{n+1}-P^{n}\right)}{P^{n}} \\
& \gamma_{\alpha} \equiv\left(\alpha_{g}^{n+1}-\alpha_{g}^{n}\right)
\end{aligned}
$$




$$
\begin{aligned}
& \gamma_{T_{g}} \equiv \frac{\left(T_{g}^{n+1}-T_{g}^{n}\right)}{T_{g}^{n}} \\
& \gamma_{T_{f}} \equiv \frac{\left(T_{f}^{n+1}-T_{f}^{n}\right)}{T_{f}^{n}} \\
& \gamma_{X_{a}} \equiv\left(X_{a}^{n+1}-X_{a}^{n}\right)
\end{aligned}
$$

In Eqs. (3.11.3-3) through (3.11.3-7), the superscript $\mathrm{n}$ is used to indicate values at the old time and $n+1$ is used to indicate values at the new time. When $\gamma_{\max }>t o l$, the time step is adjusted using Eq. (3.11.3-1) and the computation for time $n+1$ is repeated.

If there is convergence failure within an iteration limit or void fraction falls outside its physically acceptable range (i.e. $\alpha<0$ or $\alpha>1$ ) after fluid computations, or if the values of solid or fluid properties are beyon the applicable range of data correlations, code computations are repeated with the time step size reduced by one half. That is, when an error flag ierr $=1$ is generated in the code, we set

$$
\Delta \mathrm{t}=0.5(\Delta \mathrm{t})_{0}
$$

and repeat the computation for this time step.

When the time step size is less than the minimum time step, dtmin, which is specified through the input, program execution is stopped immediately with the message of "fatal error, time step less than minimum". Table 3.11.3-1 summarizes error checking and control for each of the error indicators used in the FLOWTRAN-TF code. 
Table 3.11.3-1 Summary of error checking and control.

\begin{tabular}{|c|c|c|}
\hline Error Indicator & Error Checking & Control Action \\
\hline ierr $=0$ & $\begin{array}{l}\text { Initial value and value when } \\
\text { no error is indicated. }\end{array}$ & $\begin{array}{l}\text { Program execution is } \\
\text { continued. }\end{array}$ \\
\hline ierr $=1$ & $\begin{array}{l}\text { 1. Convergence failure. }{ }^{1} \\
\text { 2. Diagonal dominance of } \\
\text { pressure solution coefficient } \\
\text { matrix is not satisfied. }{ }^{2} \\
\text { 3. Void fraction is out of } \\
\text { acceptable range. } \\
\text { 4. Solid/fluid physical } \\
\text { properties fall outside of } \\
\text { applicable range. }\end{array}$ & $\begin{array}{l}\text { The time step size is reduced } \\
\text { by half of the old time step } \\
\text { Eq. }(3.11 .3-8) \text {. }\end{array}$ \\
\hline ierr $=2$ & $\begin{array}{l}\text { 1. Time step size is less } \\
\text { than the minimum time step } \\
\text { allowed by the user input } \\
\text { limit. } \\
\text { 2. The determinant is zero } \\
\text { in the calculation of the } \\
\text { explicit momentum terms } \\
\text { before the Newton iteration. }\end{array}$ & $\begin{array}{l}\text { Program execution is } \\
\text { terminated. }\end{array}$ \\
\hline ierr $=3$ & $\begin{array}{l}\text { Insufficient accuracy in the } \\
\text { fluid solution. }\end{array}$ & $\begin{array}{l}\text { Time step is determined by } \\
\text { Eq. }(3.11 .3-1) \text {. }\end{array}$ \\
\hline
\end{tabular}

1 Iteration convergence failures are possible in three separate locations: -ysi; steam mole fraction at the gas-liquid interface in subroutine GAMMAI $-P, \alpha, T_{g}, T_{f}$, and $X_{\mathrm{a}}$; fluid state variables in subroutine NEWTON - $\delta \mathrm{P}$; pressure increment in subroutine INNER

2 Since we impose a Courant limit on the computational time step, the numerical scheme is expected to be stable. Therefore, the pressure solution coefficient matrix is expected to be diagonally dominant and the check for diagonal dominance performed within the code is somewhat redundant. However, this check is included to provide additional assurance that the numerical scheme is stable. 


\subsubsection{Time-Step Control}

The control of time steps in the FLOWTRAN-TF code is accomplished by the solution accuracy check, convergence criteria within a given iteration limit, and applicability check of computed state variables in the course of program execution. Checks are made on the value of each of the new time variables to ensure that the variation of the new time variables from the old ones falls within reasonable limits. For this purpose, the FLOWTRAN-TF code sets an error flag ( $i \in \mathbf{r}$ ) to indicate the source of time step constraints and to execute such situations. The source and its control action for each error flag are discussed in detail in the sections of error checking and control and stability constraints of time step. If the new time variables have nonphysical values (e.g., void fractions less than zero or greater than unity) or if convergence failure in subroutines, GAMMAI, NEWTON or INNER, occurs within a given iteration limit, then the solution is backed up to the beginning of the the time step, the variables are set to their old value, the time step is halved, which is correspnding to the case of ierr $=1$ as illustrated in Figure 3.11.4-1, and the time step is repeated. The time step size is also controlled by the maximum change of the main primary variables (pressure, void fraction, air mass fraction, fluid temperature for each phase, and fuel surface temperature) at the new time step and the checks of the material Courant limit in subroutine ACCCHK. This is done so that the linearized equations will be sufficiently representative of the nonlinear equations to provide an acceptable level of accuracy in the computations. In this case, the new time step size is chosen as the minimum value from the equation

$$
(\Delta t)^{n+1}=\min \left\{(\Delta t)_{c f l},(\Delta t)_{\max },(\Delta t)_{\text {acchk }}\right\}
$$

where

$$
\begin{aligned}
(\Delta t)^{n+1} & \equiv \text { new time step size } \\
(\Delta t)_{\mathrm{cfl}} & \equiv \text { time step size determined by the Courant limit } \\
(\Delta \mathrm{t})_{\max } & \equiv \text { maximum time step size provided by the user's input } \\
(\Delta t)_{\text {acchk }} & \equiv \text { time step size given by solution accuracy check }
\end{aligned}
$$

When the time step size chosen by the above procedure is smaller than the minimum time step, $(\Delta t)_{\min }$ given by the input deck, program execution is stopped. At present, $(\Delta t)_{\min }=$ $1.0 \times 10^{-5}$ seconds.

In addition, when the user sets $(\Delta t)_{\min } \geq(\Delta t)_{\max }$, the code bypasses the computations of the Courant time limit and sets $(\Delta t)^{n+1}=(\Delta t)_{\min }$. Figure 3.11.4-1 shows the summarized block diagram for time step logic in the FLOWTRAN-TF code, which is mainly controlled by the subroutine TIMSTP. 


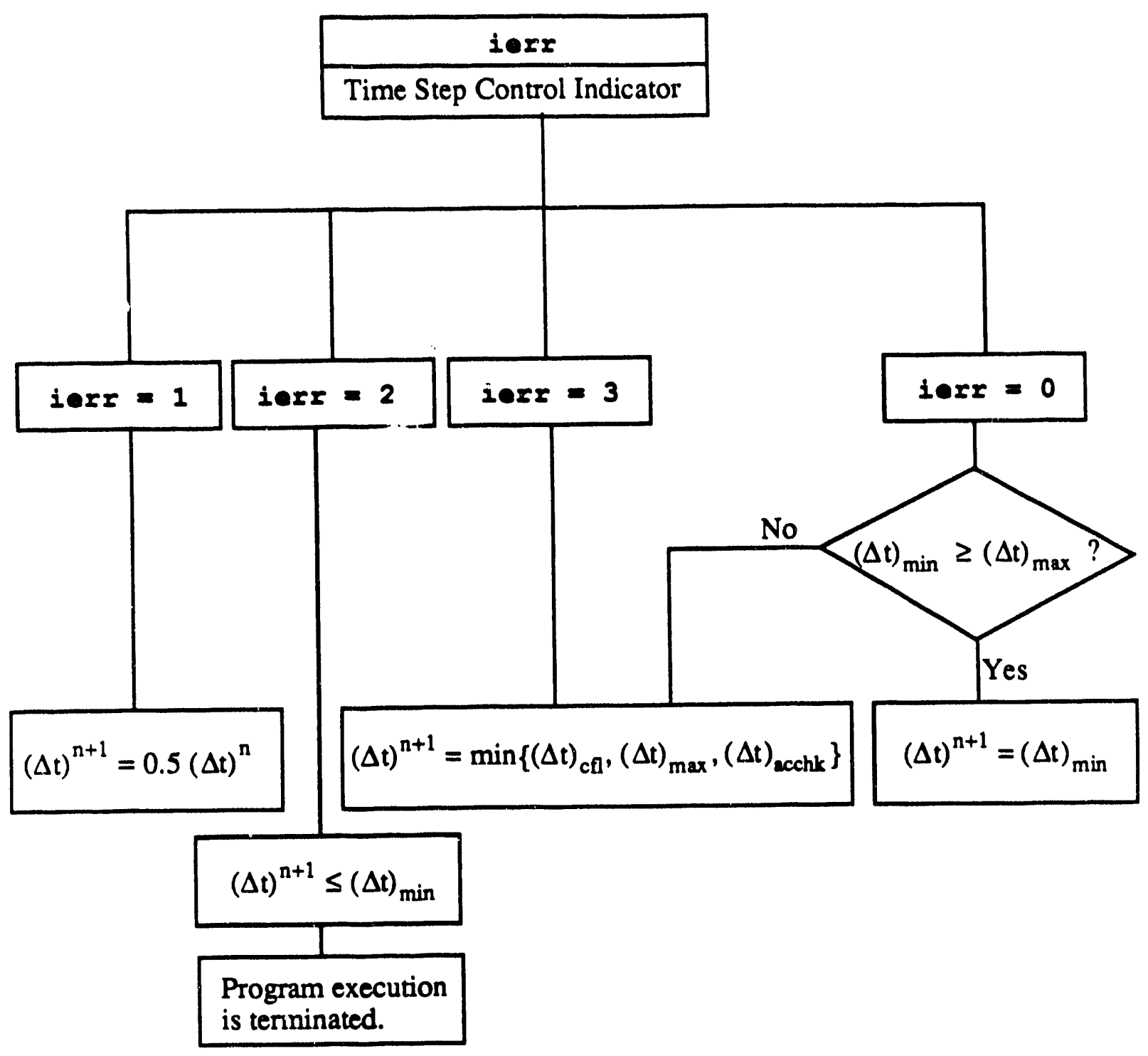

Figure 3.11.4-1 Time step control logic diagram in the FLOWTRAN-TF code. 


\subsubsection{Steady State Checking}

Within subroutine ACCCHK, we perform a test to determine if the solution has reached a steady state where the time rate of change in the fluid variables is zero. The steady state check is based on the overall rate of change in the mixture enthalpy throughout the entire fluid mesh. As the RELAP5/MOD2 code manual (Ransom, et al., 1985) points out fluid enthalpy is a function of the thermodynamic density, internal energy, and pressure as

$$
\hat{\mathrm{H}}=\rho \hat{\mathrm{h}}=\rho \hat{\mathrm{u}}+\mathrm{P}
$$

where

$$
\begin{aligned}
\hat{H} & \equiv \text { Fluid enthalpy density, } J / \mathrm{m}^{3} \\
\hat{\mathrm{h}} & \equiv \text { Fluid specific enthalpy, J/kg } \\
\hat{\mathrm{u}} & \equiv \text { Fluid specific internal energy, J/kg } \\
\mathrm{P} & \equiv \text { Pressure, } \mathrm{Pa} \\
\rho & \equiv \text { Fluid density, } \mathrm{kg} / \mathrm{m}^{3}
\end{aligned}
$$

Therefore, when the fluid enthalpy reaches a steady state value, it is equivalent to the time variation in the fluid density, pressure, and internal energy also reaching steady state. We can then test for a hydrodynamic steady state by requiring that the time rate of change in the fluid enthalpy is zero at every grid location.

Following convergence of the Newton iteration scheme, we test for steady state conditions by evaluating the time rate of change in the mixture enthalpy. We define the fluid mixture enthalpy to be

$$
\hat{h}_{\text {mix }} \equiv \frac{\alpha \rho_{g} \hat{h}_{g}+(1-\alpha) \rho_{f} \hat{h}_{f}}{\alpha \rho_{g}+(1-\alpha) \rho_{f}}
$$

where

$$
\begin{aligned}
\hat{\mathrm{h}}_{\operatorname{mix}} & \equiv \text { Mixture specific enthalpy, } \mathrm{J} / \mathrm{kg} \\
\hat{\mathrm{h}}_{\mathrm{g}} & \equiv \text { Gas specific enthalpy, } \mathrm{J} / \mathrm{kg} \\
\hat{\mathrm{h}}_{\mathrm{f}} & \equiv \text { Liquid specific enthalpy, } \mathrm{J} / \mathrm{kg} \\
\alpha & \equiv \text { Void fraction } \\
\rho_{\mathrm{g}} & \equiv \text { Gas density, } \mathrm{kg} / \mathrm{m}^{3} \\
\rho_{\mathrm{f}} & \equiv \text { Liquid density, } \mathrm{kg} / \mathrm{m}^{3}
\end{aligned}
$$

The change in mixture enthalpy within a fluid cell at time step $\mathrm{n}$ is computed as 


$$
\left(\Delta \hat{\mathrm{h}}_{\operatorname{mix}}\right)_{i}^{\mathrm{n}+1}=\left(\hat{\mathrm{h}}_{\operatorname{mix}}\right)_{i}^{\mathrm{n}+1}-\left(\hat{\mathrm{h}}_{\operatorname{mix}}\right)_{i}^{\mathrm{n}}
$$

where the subscript $i$ is used to indicate any fluid computational cell. The volume averaged change in the mixture enthalpy for the entire system at time step $\mathrm{n}$ can be defined through the relationship

$$
\left\langle\Delta \hat{h}_{\text {mix }}\right\rangle^{n+1} \equiv \frac{\sum_{i=1}^{N} V_{i}\left[\left(\hat{h}_{\text {rnix }}\right)_{i}^{n+1}-\left(\hat{h}_{\text {mix }}\right)_{i}^{n}\right]}{\sum_{i=1}^{N} V_{i}}
$$

where $V_{i}$ is the volume of fluid cell $i$ and the summation is over all (N) fluid cells in the computational mesh. The average time rate of change in the mixture enthalpy for the entire system is then computed using the approximation

$$
\left(\frac{d \hat{h}_{\text {mix }}}{d t}\right)^{n+1} \cong \frac{\left\langle\Delta \hat{h}_{\text {mix }}\right\rangle^{n+1}}{\Delta t}
$$

where $\Delta t$ is the time step used to advance from time $\mathrm{n}$ to time $\mathrm{n}+1$.

Since we wish to use the average time rate of change in mixture enthalpy to test for steady state conditions, applying Eqs. (3.11.5-4) and (3.11.5-5) suffers from the obvious defect that the enthalpy changes within a single fluid cell can be either positive or negative over a particular time step. Terms in the numerator of Eq. (3.11.5-4) may then partially cancel falsely indicating an approach to steady state conditions. This problem is eliminated by replacing the change in enthalpy in Eq. (3.11.5-4) with the root-sum-square value defined to be

$$
\left\langle\Delta \hat{h}_{\text {mix }}\right\rangle^{n+1} \equiv \frac{\sqrt{\sum_{i=1}^{N}\left\{v_{i}\left[\left(\hat{h}_{\text {mix }}\right)_{i}^{n+1}-\left(\hat{h}_{\text {mix }}\right)_{i}^{n}\right]\right\}^{2}}}{\sum_{i=1}^{N} v_{i}}
$$

This gives an approximation to the average time rate of change in the system enthalpy of

$$
\left(\frac{d \hat{h}_{\text {mix }}}{d t}\right)^{n+1} \equiv \frac{\sqrt{\sum_{i=1}^{N}\left\{V_{i}\left[\left(\hat{h}_{\text {mix }}\right)_{i}^{n+1}-\left(\hat{h}_{\text {mix }}\right)_{i}^{n}\right]\right\}^{2}}}{\Delta t \sum_{i=1}^{N} V_{i}}
$$

When the time rate of change in the mixture enthalpy for the system is less than a user input tolerance for several consecutive time steps, the code assumes that steady state conditions 
have been reached. We have fixed the number of test time steps in the code to five. That is, when we find

$$
\frac{\left\langle\Delta \hat{h}_{\text {mix }}\right\rangle^{k+1}}{\Delta t} \leq \tau_{s s}, \quad \text { for } k=n, n-1, \ldots, n-4
$$

where $\tau_{\mathrm{ss}}$ is the input steady state tolerance, we assume that the solution has reached a steady state.

In practice, we find that Eq. (3.11.5-7) can vary significantly in magnitude from time step to time step. These fluctuations usually damp out as the solution approaches a steady state but may persist. For this reason, we base our steady state test on the results of several consecutive time steps. Nevertheless, we have found this formulation to be quite useful in establishing that the flow conditions are reasonably close to a true steady state. When performing an analysis, we often first run the code with initial and boundary conditions that should approach a steady state solution and use the results of this computation as restart conditions for transient calculations. The choice of the $\tau_{\text {ss }}$ tolerance parameter is somewhat arbitrary. For multi-channel assembly calculations, we have found that specifying a tolerance of about $1.0 \mathrm{~J} / \mathrm{kg}-\mathrm{s}$ is sufficiently close to steady state conditions to allow smooth restarts. For simple flow geometries, such as single pipes, the code can achieve absolute steady state where the rate of change in the system enthalpy is identically zero.

\section{References:}

Ransom, V. H., Wagner, R. J., Trapp, J. A., Feinauer, L. R., Johnsen, G. W., Kiser, D. M., and Riemke, R. A., 1985, "RELAP5/MOD2 Code Manual Volume 1: Code Structure, System Models, and Solution Methods", NUREG/CR-4312, EG\&G Idaho, Inc. 


\section{CODE ARCHITECTURE}

Figures 4-1 illustrate the code architecture of FLOWTRAN-TF. Table 4-I is a brief description of each module's function. 


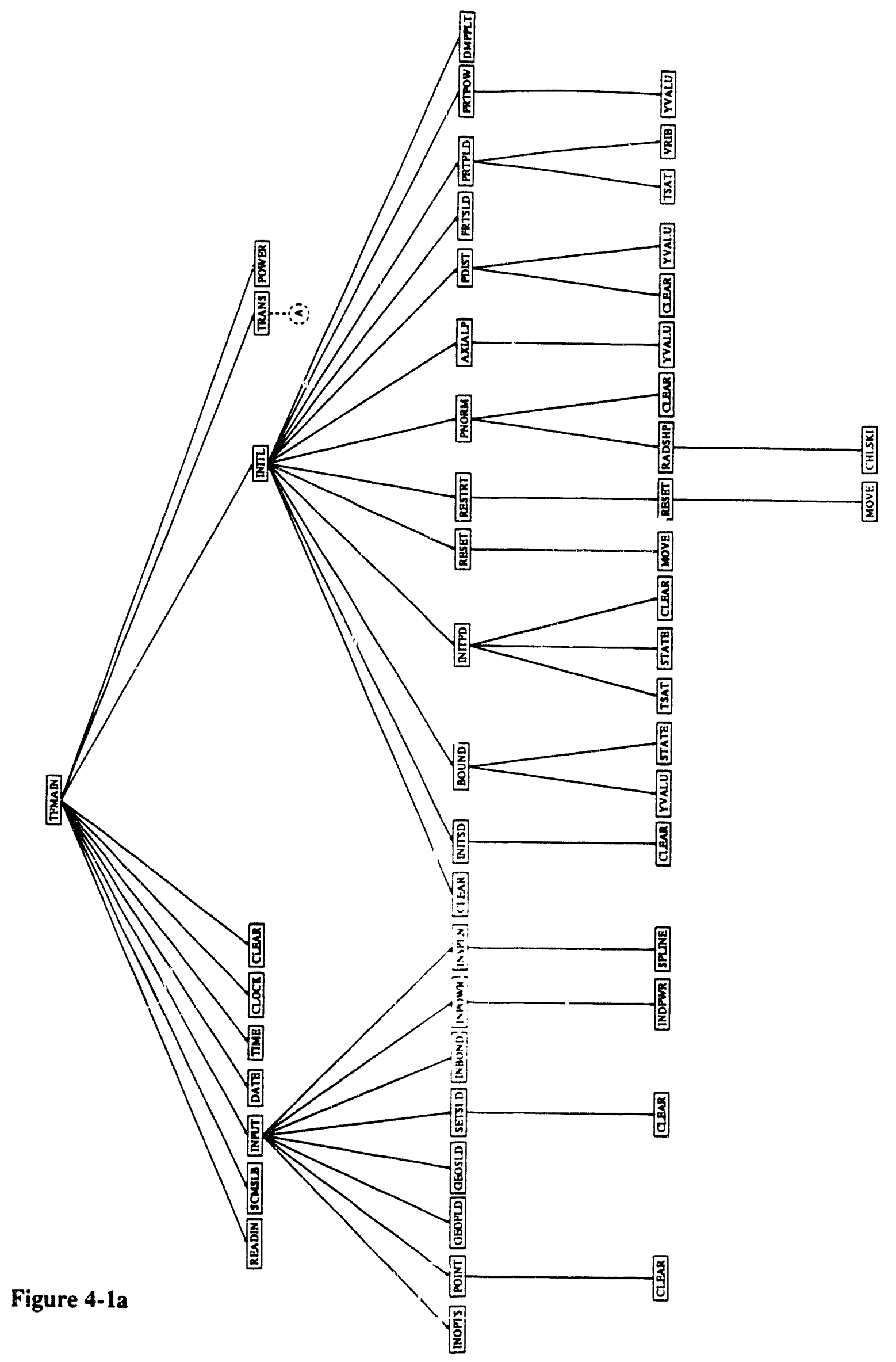




\begin{tabular}{|l|ll|r|}
\hline $2 / 15 / 93$ & WSRC-TR-92-532 Rev. 0 & FLOWTRAN-TF Software Design & Page 516 \\
\hline
\end{tabular}

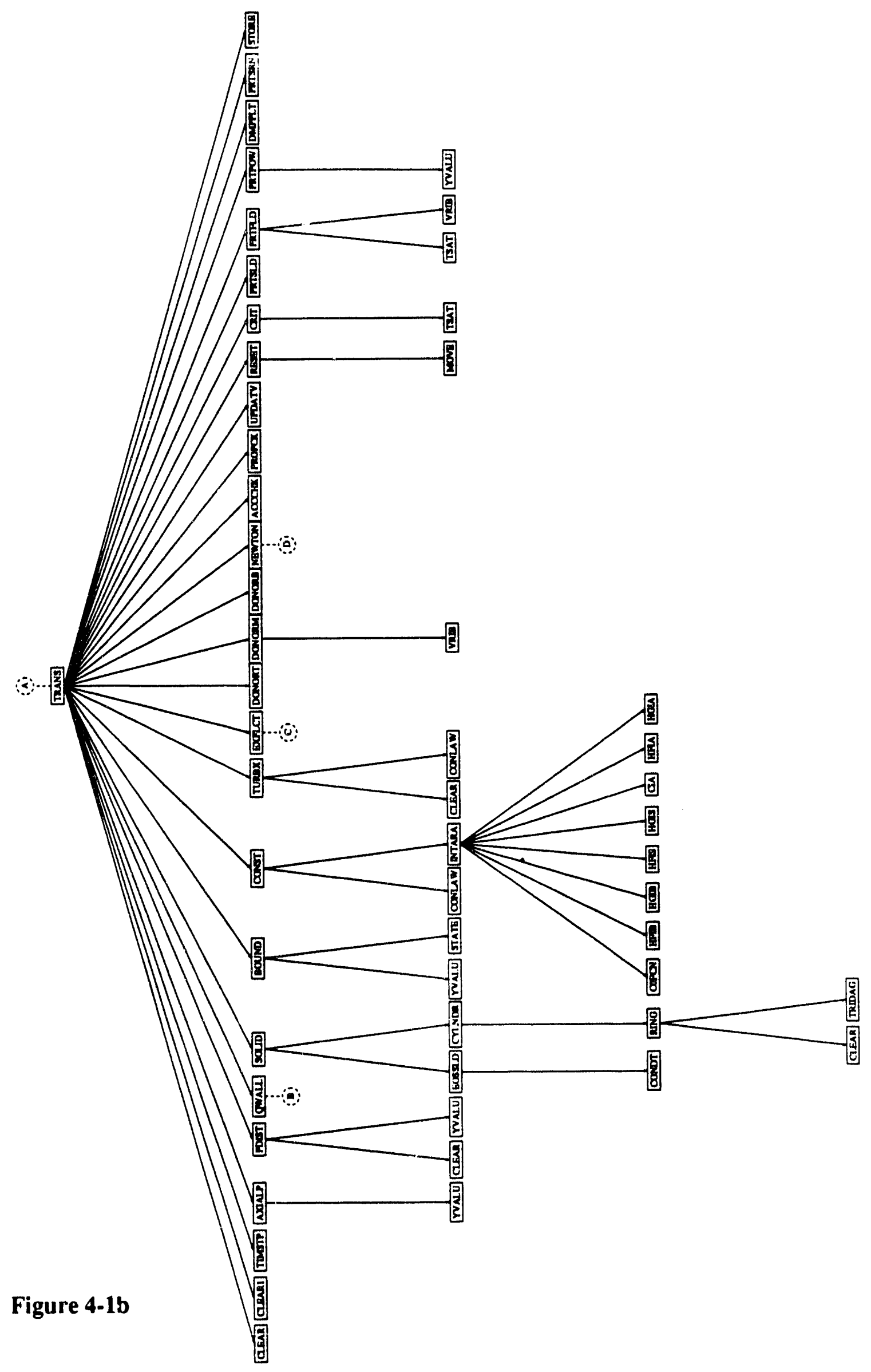




\begin{tabular}{|l|ll|r|}
\hline $2 / 15 / 93$ & WSRC-TR-92-532 Rev.0 & FLOWTRAN-TF Software Design & Page 517 \\
\hline
\end{tabular}

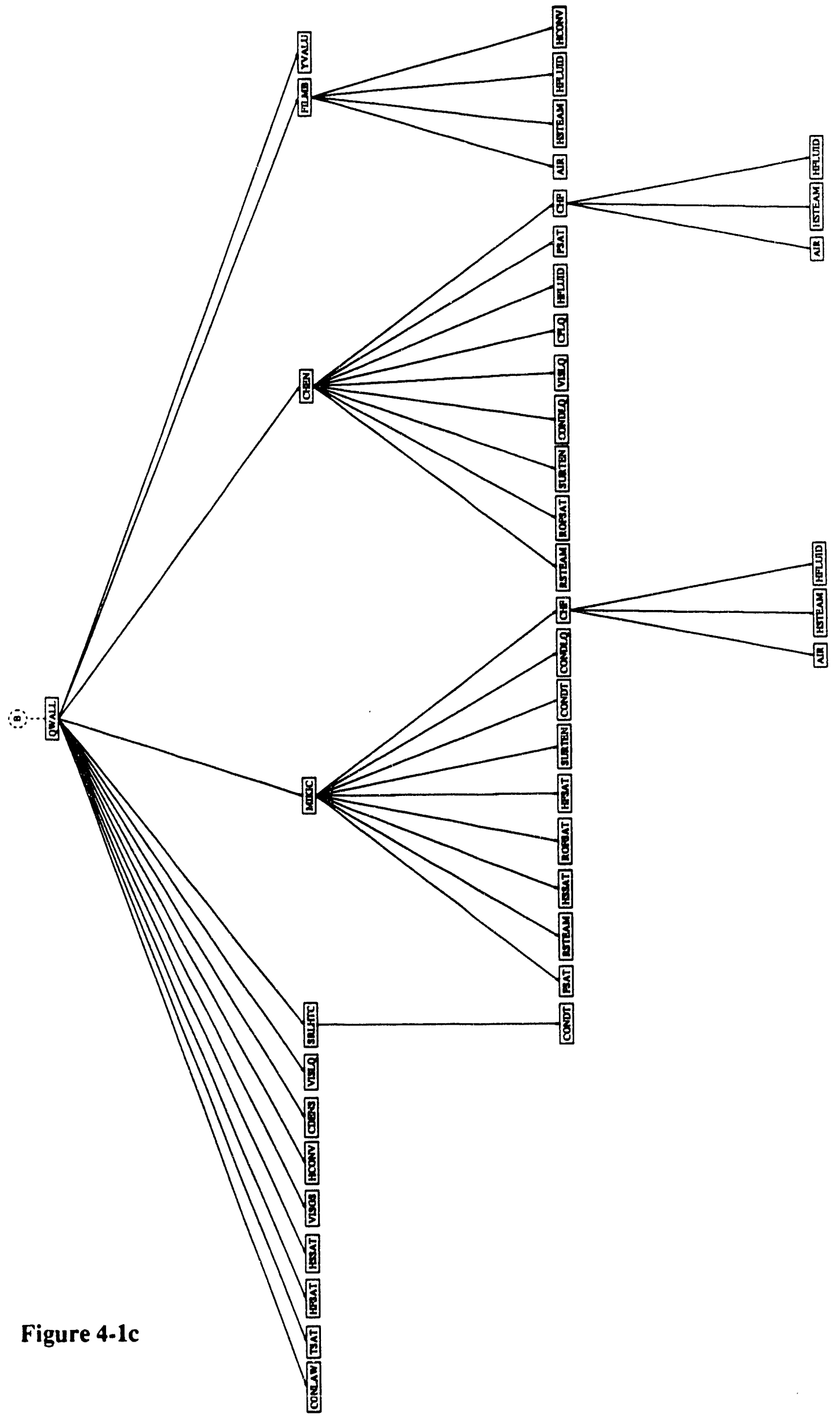




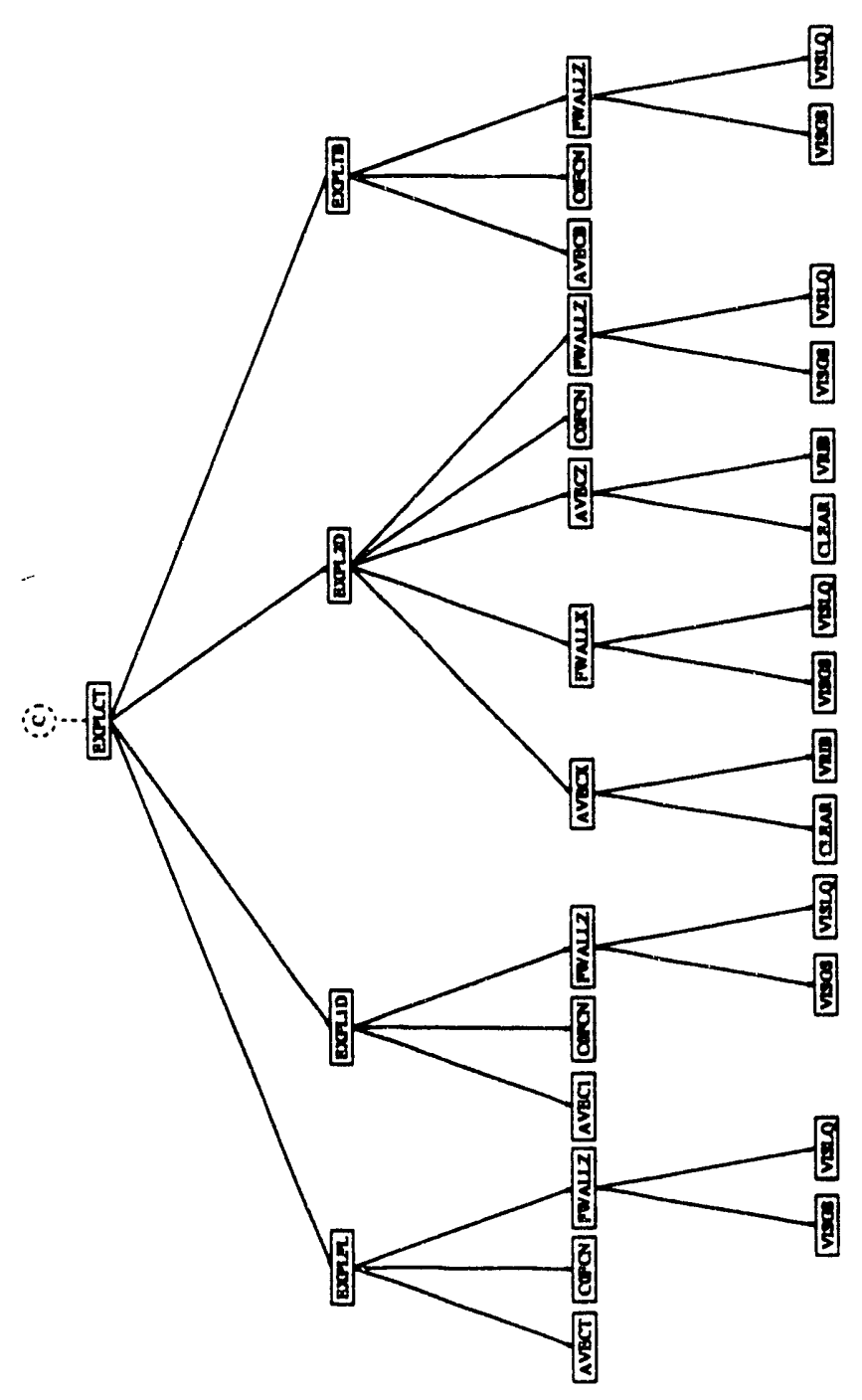

Figure 4-1d 


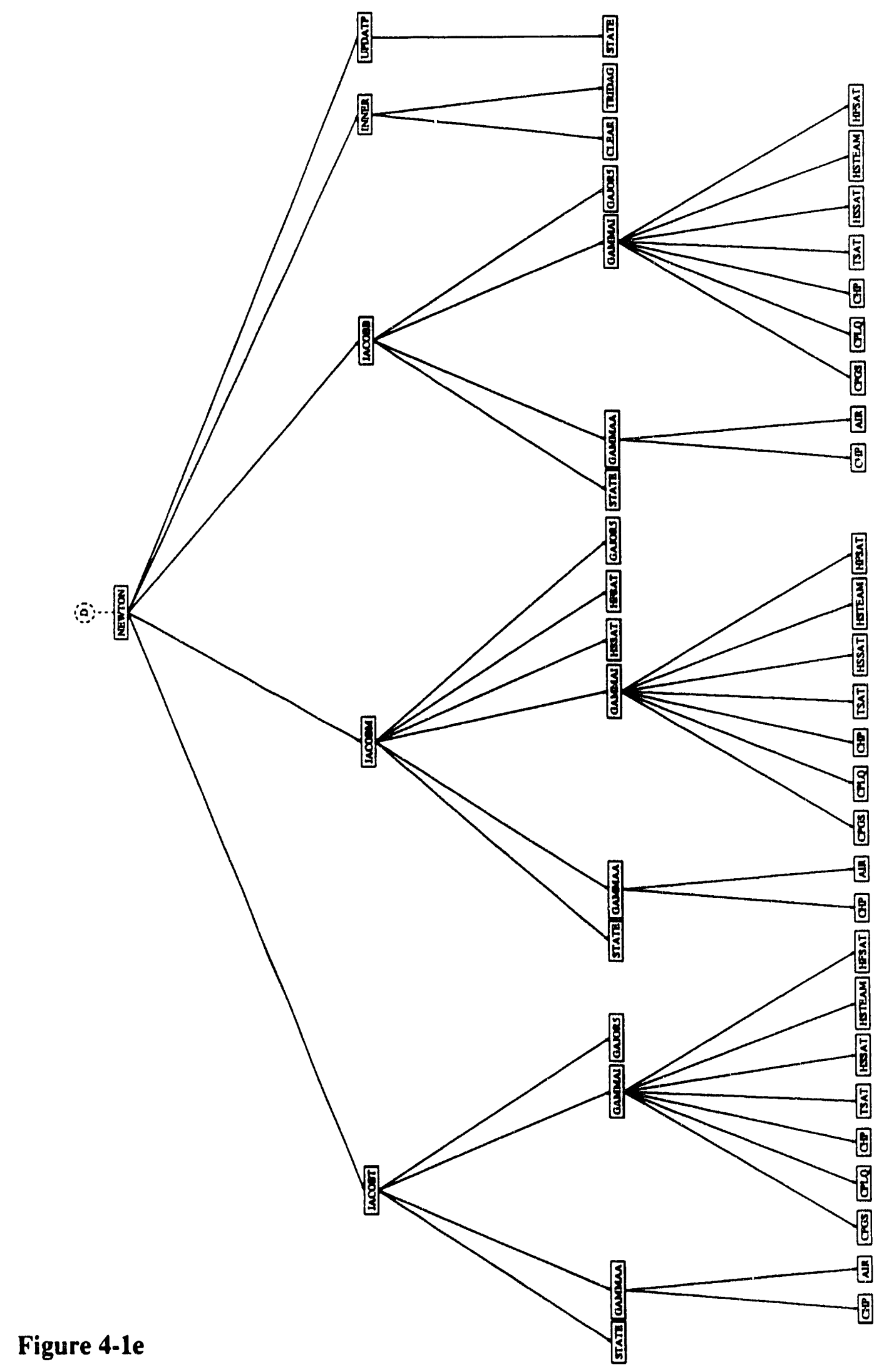


Table 4-I

Module descriptions.

\begin{tabular}{|ll|}
\hline Subroutine & Description \\
ACCCHK & $\begin{array}{l}\text { Checks the solution accuracy. This is made on each value of new time } \\
\text { variables to ensure that the variations of the new time variables from the } \\
\text { old values fall within reasonable limits. }\end{array}$ \\
AIR & $\begin{array}{l}\text { Computes air thermodynamic properties and their derivatives. } \\
\text { AVEC1 }\end{array}$ \\
Calculates the explicit advection terms in the one dimensional axial \\
momentum equations for the top and bottom sections.
\end{tabular}




\begin{tabular}{|c|c|}
\hline CLEARI & Sets all the values in a real array to unity. \\
\hline CONDLQ & $\begin{array}{l}\text { Computes thermal conductivity and its derivative with respect to } \\
\text { temperature of saturated liquid water. }\end{array}$ \\
\hline CONDT & $\begin{array}{l}\text { Computes the thermal conductivity of solid material at the specified } \\
\text { temperature. }\end{array}$ \\
\hline CONLAW & $\begin{array}{l}\text { Computes transport properties of gas and liquid phases at the specified } \\
\text { fluid temperature and air mass fraction. }\end{array}$ \\
\hline CONST & Computes interfacial transport parameters for the whole assembly mesh. \\
\hline CPGS & $\begin{array}{l}\text { Computes the specific heat of air-steam gas mixture at the specified gas } \\
\text { temperature and air mass fraction. }\end{array}$ \\
\hline CPLQ & $\begin{array}{l}\text { Computes the specific heat of saturated liquid water at given fluid } \\
\text { temperature. }\end{array}$ \\
\hline CRIT & Calculates the criterion ratios needed to perform the power iterations. \\
\hline CYLNDR & Solves heat conduction equation for a single cylinder. \\
\hline DMP P LT & $\begin{array}{l}\text { Writes fluid and solid variable arrays to be used for data plotting to a } \\
\text { dump file at the specified unit. }\end{array}$ \\
\hline DONORB & $\begin{array}{l}\text { Computes advective donored quantities at the bottom section of the } \\
\text { assembly that are used to construct the Jacobian matrix within the } \\
\text { Newton iterations. }\end{array}$ \\
\hline DONORM & $\begin{array}{l}\text { Computes advective donored quantities at the middle section the } \\
\text { assembly that are used in the Jacobian matrix within the Newton } \\
\text { iteration. }\end{array}$ \\
\hline DONORT & $\begin{array}{l}\text { Computes dependent advective donored quantities at the top section of } \\
\text { the assembly that are used to construct the Jacobian matrix within the } \\
\text { Newton iterations. }\end{array}$ \\
\hline EOSSLD & $\begin{array}{l}\text { Computes the heat capacity and thermal conductivity of the solid cells as } \\
\text { a function of the material and temperature. }\end{array}$ \\
\hline EXPL L 1D & $\begin{array}{l}\text { Computes the expression for new time step fluid velocities at each cell } \\
\text { face as a linearized function of the new time step pressure difference } \\
\text { across the cell face for the one-dimensional top and bottom sections of } \\
\text { the assembly. }\end{array}$ \\
\hline EXPL L D & $\begin{array}{l}\text { Computes the expression for new time step fluid velocities at each cell } \\
\text { face as a linearized function of the new time step pressure difference } \\
\text { across the cell face for the two-dimensional middle section of the } \\
\text { assembly. }\end{array}$ \\
\hline EXP LCT & Performs explicit fluid calculations for each time step. \\
\hline
\end{tabular}




\begin{tabular}{|c|c|}
\hline EXP LPL & $\begin{array}{l}\text { Computes the expression for new time step fluid velocities at a } \\
\text { boundary cell face as a linearized function of the new time step pressure } \\
\text { difference across the cell face for the top plenum boundary of the } \\
\text { assembly. }\end{array}$ \\
\hline EXP LTB & $\begin{array}{l}\text { Computes the expression for new time step fluid velocities at a } \\
\text { boundary cell face as a linearized function of the new time step pressure } \\
\text { difference across the cell face for the tank bottom boundary of the } \\
\text { assembly. }\end{array}$ \\
\hline E ILMB & $\begin{array}{l}\text { Calculates film boiling and transition boiling heat fluxes using the } \\
\text { literature correlation. }\end{array}$ \\
\hline EWALLX & $\begin{array}{l}\text { Computes the azimuthal liquid and vapor wall friction and local form } \\
\text { loss. }\end{array}$ \\
\hline FWALLZ & Computes the axial liquid and vapor wall friction and local form loss. \\
\hline GAJOR5 & $\begin{array}{l}\text { Performs Gauss-Jordan inversion of } 5 \times 5 \text { matrix to eliminate void } \\
\text { fraction, gas temperature, liquid temperature, and air mass fraction for a } \\
\text { pressure problem only. }\end{array}$ \\
\hline GAMMAA & $\begin{array}{l}\text { Generates artificial air mass sink term required to ensure that the } 5 \times 5 \\
\text { Jacobian matrix is non-singular under single-phase liquid conditions. }\end{array}$ \\
\hline GAMMAI & $\begin{array}{l}\text { Finds the interfacial mass and energy transfer rates to the gas phase per } \\
\text { unit volume. }\end{array}$ \\
\hline GEOELD & Reads fluid geometry input data. \\
\hline GEOSLD & Reads solid geometry input data. \\
\hline HCONV & $\begin{array}{l}\text { Calculates the forced convection single-phase heat fluxes using the } \\
\text { Dittus-Boelter or Sieder-Tate correlations. }\end{array}$ \\
\hline HE IA & $\begin{array}{l}\text { Computes the heat and mass transfer coefficients on the liquid side for } \\
\text { annular flow. }\end{array}$ \\
\hline HE IB & $\begin{array}{l}\text { Computes the heat transfer coefficient on the liquid side for bubbly } \\
\text { flow. }\end{array}$ \\
\hline HEIS & $\begin{array}{l}\text { Computes the heat transfer coefficient on the liquid side for Taylor slug } \\
\text { bubbles. }\end{array}$ \\
\hline HELUID & $\begin{array}{l}\text { Computes liquid enthalpy and its derivatives with respect to temperature } \\
\text { and pressure. }\end{array}$ \\
\hline HFSAT & $\begin{array}{l}\text { Computes saturated liquid enthalpy and its derivative with respect to } \\
\text { temperature. }\end{array}$ \\
\hline HGIA & $\begin{array}{l}\text { Computes the heat and mass transfer coefficients on the gas side for } \\
\text { annular flow. }\end{array}$ \\
\hline
\end{tabular}




\begin{tabular}{|l|ll|l|}
\hline $2 / 15 / 93$ & WSRC-TR-92-532 Rev. 0 & FLOWTRAN-TF Software Design & Page 523 \\
\hline
\end{tabular}

\begin{tabular}{|c|c|}
\hline HGIB & $\begin{array}{l}\text { Computes the heat and mass transfer coefficients on the gas side for } \\
\text { bubbly flow. }\end{array}$ \\
\hline HGIS & $\begin{array}{l}\text { Computes the heat and mass transfer coefficients on the gas side for } \\
\text { slug bubbles. }\end{array}$ \\
\hline HSSAT & $\begin{array}{l}\text { Computes saturated steam enthalpy and its derivative with respect to } \\
\text { pressure. }\end{array}$ \\
\hline HSTEAM & $\begin{array}{l}\text { Computes steam enthalpy and its derivatives with respect to pressure } \\
\text { and temperature. }\end{array}$ \\
\hline INBOND & $\begin{array}{l}\text { Processes thermal-hydraulic boundary conditions input for fluid-phase } \\
\text { calculations. }\end{array}$ \\
\hline INDPWR & $\begin{array}{l}\text { Processes wet or moderated tank decay power radial shape input } \\
\text { assumptions. }\end{array}$ \\
\hline INITED & Initializes fluid variable arrays. \\
\hline INITL & Initializes solid and fluid variables. \\
\hline INITSD & Sets the initial solid temperatures. \\
\hline INNER & Executes iteration scheme to solve the pressure only problem. \\
\hline INOPTS & Reads input options and parameters. \\
\hline INPOWR & Processes power input required for the assembly calculation. \\
\hline INPUT & $\begin{array}{l}\text { Reads input data for a problem and sets storage requirements for } \\
\text { variably dimensioned arrays. }\end{array}$ \\
\hline INSPLN & $\begin{array}{l}\text { Processes transient and spatial input data to generate spline fitting } \\
\text { coefficients. }\end{array}$ \\
\hline INTARA & $\begin{array}{l}\text { Computes the interfacial area concentrations and generalized interfacial } \\
\text { mass, heat, and interfacial drag coefficients for various flow regimes. }\end{array}$ \\
\hline JACOBB & Computes the Jacobian matrix for the bottom section of the assembly. \\
\hline JACOBM & Computes the Jacobian matrix for the middle section of the assembly. \\
\hline JACOBT & Computes the Jacobian matrix for the top section of the assembly. \\
\hline MIKIC & $\begin{array}{l}\text { Computes surface heat flux and the derivative with respect to wall } \\
\text { temperature for laminar and turbulent single-phase forced convection } \\
\text { with subcooled nucleate boiling developed by Mikic and Rohsenow. }\end{array}$ \\
\hline MOVE & Moves $\mathrm{n}$ words from one real array (a) into another real array (b). \\
\hline NEWTON & Performs Newton iterations to solve the fluid equations. \\
\hline
\end{tabular}




\begin{tabular}{|c|c|}
\hline PDIST & Computes the deposited power. \\
\hline PNORM & Normalizes the initial power shapes. \\
\hline POINT & Sets up program storage requirements. \\
\hline POWER & $\begin{array}{l}\text { Performs the interpolation to obtain a new guess for the pre-incident } \\
\text { assembly power and to check the convergence of the power iteration } \\
\text { scheme. }\end{array}$ \\
\hline PROPCK & $\begin{array}{l}\text { Checks the computed fluid and solid material properties for values out } \\
\text { of the applicable ranges of the thermodynamic and transport } \\
\text { correlations. }\end{array}$ \\
\hline PRTELD & Writes fluid variables to output file. \\
\hline PRTPOW & Prints power output at the specified time. \\
\hline PRTSLD & $\begin{array}{l}\text { Prints solid nodal temperatures, surface temperatures and surface heat } \\
\text { fluxes at the specified time step. }\end{array}$ \\
\hline PRTSRN & Prints computation output to screen. \\
\hline PSAT & $\begin{array}{l}\text { Computes liquid saturated pressure and its derivative with respect to } \\
\text { temperature. }\end{array}$ \\
\hline QWALL & Calculates the surface heat fluxes throughout the assembly. \\
\hline RADSHP & Computes unnormalized radial power shapes in each cylinder. \\
\hline READIN & Strips the user's added comments out of the input file. \\
\hline RESET & $\begin{array}{l}\text { Stores new calculated values for the fluid variables in the old time slots } \\
\text { in preparation for the next time step. }\end{array}$ \\
\hline RESTRT & Initializes fluid variable arrays by reading data from the restart file. \\
\hline RING & $\begin{array}{l}\text { Solves the heat conduction equation for a ring of azimuthal and radial } \\
\text { mesh cells at a particular axial location in a single cylinder. }\end{array}$ \\
\hline ROESAT & $\begin{array}{l}\text { Computes liquid saturation density and its derivative with respect to the } \\
\text { temperature. }\end{array}$ \\
\hline RSTEAM & Computes the saturation steam density. \\
\hline SCMSLB & Writes a conñguration control banner to the ASCI output files. \\
\hline SETELD & Sets up fluid geometric data arrays. \\
\hline SETSLD & Initializes the FLOWTRAN-TF solid geometric arrays. \\
\hline SOLID & Drives the heat conduction routines. \\
\hline
\end{tabular}




\begin{tabular}{|c|c|}
\hline SPLINE & Generates the coefficient matrix of the piecewise cubic spline function. \\
\hline SRLHTC & $\begin{array}{l}\text { Computes forced convection single-phase heat fluxes using a modified } \\
\text { Sieder-Tate correlation based on the Heat Transfer Laboratory data of } \\
\text { Savannah River Site. }\end{array}$ \\
\hline STATE & $\begin{array}{l}\text { Computes the equations of state such as density, enthalpy of gas and } \\
\text { liquid and their derivatives with respect to fluid variables. }\end{array}$ \\
\hline STORE & $\begin{array}{l}\text { Saves the solid and fluid variable arrays by storing data for a restart file } \\
\text { at a specified unit. }\end{array}$ \\
\hline SURTEN & Computes the surface tension of liquid water. \\
\hline TEMAIN & Main driver for the FLOWTRAN-TF code. \\
\hline TIMSTP & Determines and controls the time step size for the code computations. \\
\hline TRANS & Controls the transient calculations. \\
\hline TRIDAG & $\begin{array}{l}\text { Solves a generalized tri-diagonal matrix to handle systems of } \\
\text { simultaneous linear equations with tri-diagonal coefficient matrix and } \\
\text { possibly non-zero elements in the comer positions. }\end{array}$ \\
\hline TSAT & $\begin{array}{l}\text { Computes the saturation fluid temperature and its derivative with respect } \\
\text { to pressure. }\end{array}$ \\
\hline TURBX & $\begin{array}{l}\text { Computes the subchannel mixing between adjacent two subchannels in } \\
\text { the middle channel section. }\end{array}$ \\
\hline UPDATP & Updates the outer Newton iteration iterates over the entire grid. \\
\hline UPDATV & Computes the velocity values over the entire grid at the end of time step. \\
\hline VISGS & Computes the dynamic viscosity of air-steam gas mixture. \\
\hline VISLQ & Computes the saturation liquid dynamic viscosity. \\
\hline VRIB & $\begin{array}{l}\text { Computes the void fraction near the rib for a given cell-averaged void } \\
\text { fraction at the middle section of the assembly. }\end{array}$ \\
\hline YVALU & $\begin{array}{l}\text { Computes the piecewise cubic spline function for a given argument } \\
\text { value } x \text {. }\end{array}$ \\
\hline
\end{tabular}




\section{APPENDIX Fluid Thermodynamic and Transport Property Data Correlations}

\section{Table of Appendix Contents}

A Correlations of fluid properties

A.1 The properties of liquid water

A.1.1 Thermodynamic properties of liquid water

A.1.2 Transport properties of liquid water

A.2 The properties of steam

A.2.1 Thermodynamic properties of steam

A.2.2 Transport properties of steam

A.3 The properties of air

A.3.1 Thermodynamic properties of air

A.3.2 Transport properties of air

A.4 The binary gas mass diffusivity of air-steam

B Data of fluid property correlations

B.1 Data of liquid water property correlations

B.2 Data of steam property correlations

B.3 Data of air property correlations

B.4 Data of binary air-steam mass diffusivity

C Plots of fluid property correlations

C.1 Plots of liquid water property correlations

C.2 Plots of steam property correlations

C.3 Plots of air property correlations

C.4 Plots of binary air-steam mass diffusivily 


\section{Nomenclature}

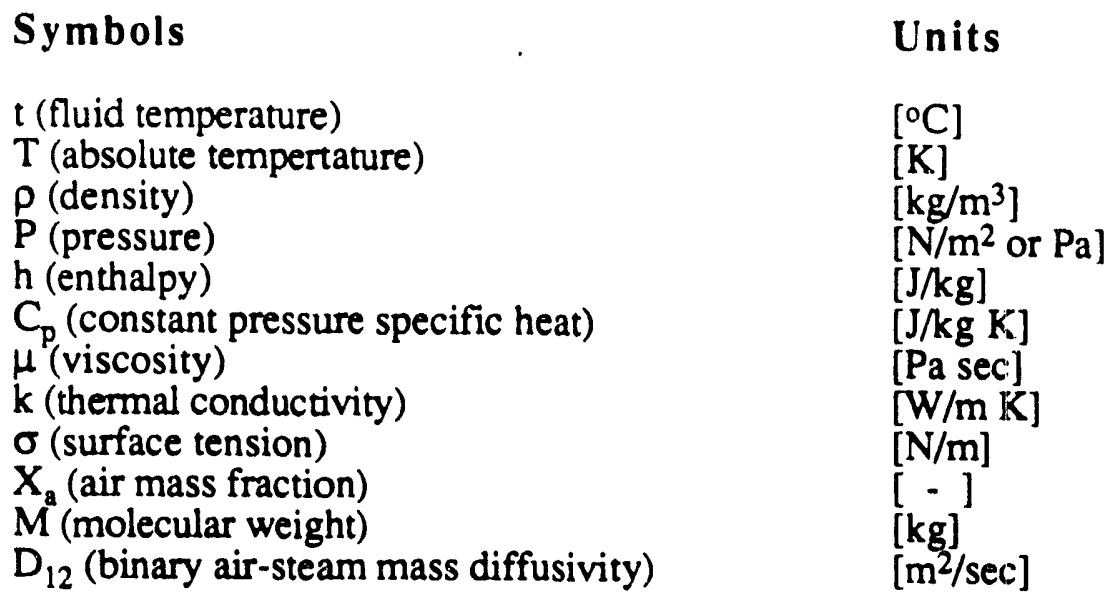

\section{Subscripts}

$\begin{array}{ll}\text { f or } 1 & \text { liquid } \\ \text { s } & \text { steam } \\ \text { a } & \text { air } \\ \text { sat } & \text { saturated }\end{array}$




\section{A Correlations of fluid properties}

\section{A.1 The properties of liquid water}

\section{A.1.1 Thermodynamic properties of liquid water}

Saturation pressure and its derivative with respect to temperature $\left(\mathrm{P}_{\text {sat }}\right.$ and $\left.\left(\mathrm{dP}_{\text {sat }} / \mathrm{dT}_{\mathrm{f}}\right)\right)$ :

$$
\begin{gathered}
P_{s a t}=e^{\left(A+B T_{f}+C T_{f}^{2}+D T_{f}^{3}\right)} \\
\frac{d P_{s a l}}{d T_{f}}=\left(B+2 C T_{f}+3 D T_{f}^{2}\right) e^{\left(A+B T_{f}+C T_{f}^{2}+D T_{f}^{3}\right)}
\end{gathered}
$$

where for light water

$$
\begin{aligned}
& A=-3.8874 \times 10^{1} \\
& B=2.9129 \times 10^{-1} \\
& C=-5.7014 \times 10^{-4} \\
& D=4.0606 \times 10^{-7}
\end{aligned}
$$

and for heavy water

$$
\begin{aligned}
& A=-3.9686 \times 10^{1} \\
& B=2.9393 \times 10^{-1} \\
& C=-5.7036 \times 10^{-4} \\
& D=4.0230 \times 10^{-7}
\end{aligned}
$$

The applicable range of the correlation is $275 \mathrm{~K}<\mathrm{T}_{\mathrm{f}}<450 \mathrm{~K}$. The constants for light water in equation (A.1-1) were determined by curve fitting using the data from Reynolds (1979) and the constants of heavy water were determined by fitting the data of DPSTM-140.

Saturated density and its derivative with respect to temperature $\left(\rho_{\mathrm{f} \text {, sat }}\right.$ and $\left.\left(\mathrm{d} \rho_{\mathrm{f}, \text { sat }} / d T_{\mathrm{f}}\right)\right)$ :

$$
\begin{gathered}
\rho_{\mathrm{f}, \text { sat }}=A+B T_{f}+C T_{f}^{2} \\
\frac{d \rho_{f, \text { sat }}}{d T_{f}}=\left(B+2 C T_{f}\right)
\end{gathered}
$$

where for light water

$$
\begin{aligned}
& A=8.5087 \times 10^{2} \\
& B=1.2788 \\
& C=-2.6526 \times 10^{-3}
\end{aligned}
$$


and for heavy water

$$
\begin{aligned}
& A=9.1986 \times 10^{2} \\
& B=1.5412 \\
& C=-3.0963 \times 10^{-3}
\end{aligned}
$$

The applicable range of the correlation is $275 \mathrm{~K}<\mathrm{T}_{\mathrm{f}}<450 \mathrm{~K}$. The coefficients of light water in equation (A.1-3) were determined by fitting the data from Reynolds (1979) and those of heavy water from DPSTM-140.

Liquid water density derivatives with respect to temperature and pressure ( $\alpha$ and $\beta$ ):

The density slope between saturation and 500 psia was computed for temperatures between $50{ }^{\circ} \mathrm{F}$ and $400^{\circ} \mathrm{F}$ using the compressed $\mathrm{H}_{2} \mathrm{O}$ data given by the literature (Van Wylen and Sonntag, second edition). After converting to SI units, the data for light water was fit with a quadratic equation as shown below. For heavy water case, the density slope between saturation and about 500 psia was computed using the data of AECL-7531 for the temperature range of $50^{\circ} \mathrm{F}$ to $340^{\circ} \mathrm{F}$.

$$
\left.\alpha \equiv \frac{\partial \rho_{f}}{\partial P}\right|_{T_{f}}=A+B T_{f}+C T_{f}^{2}
$$

where for light water

$$
\begin{aligned}
& A=2.3255 \times 10^{-6} \\
& B=-1.1419 \times 10^{-8} \\
& C=1.7192 \times 10^{-11}
\end{aligned}
$$

and for heavy water

$$
\begin{aligned}
& A=2.3373 \times 10^{-6} \\
& B=-1.1316 \times 10^{-8} \\
& C=1.7338 \times 10^{-11}
\end{aligned}
$$

When liquid temperature is given, $\alpha$ can be evaluated, i.e.,

$$
\left.\alpha \equiv \frac{\partial p_{f}}{\partial P}\right|_{T_{f}=373 K}=D
$$

where $D=4.58 \times 10^{-7}$ for light water and $D=5.29 \times 10^{-7}$ for heavy water. In the code implementation, a constant value of $\alpha$, which is evaluated at $T=373 \mathrm{~K}$, is used. Then, the dervative of $\alpha$ with respect to temperature becomes zero.

$$
\begin{aligned}
& \rho_{f}=\rho_{f, \text { sat }}\left(T_{f}\right)+\left.\int_{P_{\text {aat }}\left(T_{f}\right)}^{P} \frac{\partial \rho_{f}}{\partial P}\right|_{T_{f}} d P \\
& =\rho_{\mathrm{f}, \mathrm{sar}}\left(T_{\mathrm{f}}\right)+D\left(P-P_{\text {sat }}\left(T_{\mathrm{f}}\right)\right\}
\end{aligned}
$$




$$
\left.\beta \equiv \frac{\partial \rho_{f}}{\partial T_{f}}\right|_{P}=\frac{d \rho_{f, \text { sat }}}{d T_{f}}-D\left(\frac{d P_{\text {sat }}}{d T_{f}}\right)
$$

$\beta$ can be computed by equation (A.1-8) using equations, (A.1-2) and (A.1-4).

Saturated liquid enthalpy and derivative w.r.t. temperature $\left(\mathrm{h}_{\mathrm{f} \mathrm{sat}}\right.$ and $\left.\left(\mathrm{dh}_{\mathrm{f} \mathrm{sat}} / \mathrm{dT}_{\mathrm{f}}\right)\right)$ :

$$
\begin{gathered}
\mathrm{h}_{\mathrm{f}, \mathrm{sat}}=\mathrm{A}+\mathrm{BT}_{\mathrm{f}}+\mathrm{CT}_{\mathrm{f}}^{2} \\
\frac{\mathrm{dh}_{\mathrm{f}, \mathrm{sat}}}{\mathrm{C} \mathrm{T}_{\mathrm{f}}}=\mathrm{B}+2 \mathrm{CT}_{\mathrm{f}}
\end{gathered}
$$

where for light water

$$
\begin{aligned}
& A=-1.1094 \times 10^{6} \\
& B=3.9602 \times 10^{3} \\
& C=3.6868 \times 10^{-1}
\end{aligned}
$$

and for heavy water

$$
\begin{aligned}
& A=-1.1856 \times 10^{6} \\
& B=4.3402 \times 10^{3} \\
& C=-2.0733 \times 10^{-1}
\end{aligned}
$$

The coefficients of the correiation were determined by the curve fitting of literature data (Reynolds (1979) for $\mathrm{H}_{2} \mathrm{O}$ and AECL-7531 for $\mathrm{D}_{2} \mathrm{O}$ ). The applicable range of the correlation is $275 \mathrm{~K}<\mathrm{T}_{\mathrm{f}}<450 \mathrm{~K}$. For suiucooled liquid enthalpy $\left(\mathrm{h}_{\mathrm{f}}\right)$, saturated liquid enthalpy is used, that is, the pressure dependence of liquid enthalpy is neglected for a given temperature.

$$
\begin{gathered}
\mathrm{h}_{\mathrm{f}}=\mathrm{h}_{\mathrm{f}, \mathrm{sat}}\left(\mathrm{T}_{\mathrm{f}}\right) \\
\left.\frac{\partial \mathrm{h}_{\mathrm{f}}}{\partial \mathrm{T}_{\mathrm{f}}}\right|_{\mathrm{P}}=\frac{\mathrm{dh}_{\mathrm{f}, \mathrm{sat}}}{\mathrm{dT} \mathrm{T}_{\mathrm{f}}}
\end{gathered}
$$

where the r.h.s. of equation (A.1-12) is defined by equation (A.1-10). Also,

$$
\left.\frac{\partial \mathrm{h}_{\mathrm{f}}}{\partial \mathrm{P}}\right|_{\mathrm{T}_{\mathrm{f}}}=0
$$

Constant pressure specific hear of liquid water $\left(\mathrm{C}_{\mathrm{pl}}\right)$ :

Equation (A.1-10) is used to compute the constant pressure specific heat as shown below: 


$$
C_{p l}=\frac{\mathrm{dh}_{\mathrm{f}, \text { sat }}}{d T_{\mathrm{f}}}
$$

\section{A.1.2 Transport properties of water}

Dynamic viscosity of saturated water $\left(\mu_{\mathrm{f}}\right)$ :

$$
\mu_{f}=A e^{\left\{\frac{B}{\left(T_{f}-C\right)}\right\}}
$$

where for light water

$$
\begin{aligned}
& A=2.41277 \times 10^{-5} \\
& B=5.74268 \times 10^{2} \\
& C=1.39160 \times 10^{2}
\end{aligned}
$$

and for heavy water

$$
\begin{aligned}
& A=3.34625 \times 10^{-5} \\
& B=4.90126 \times 10^{2} \\
& C=1.57884 \times 10^{2}
\end{aligned}
$$

The coefficients of viscosity correlation for light water and heavy water were determined by the curve fitting of the data given in DPSTM-140. The applicable range of the correlation is $283.15 \mathrm{~K}<\mathrm{T}_{\mathrm{f}}<453.15 \mathrm{~K}$.

Thermal conductivity of light water $\left(\mathrm{k}_{\mathrm{f}}\right)$ :

$$
k_{f}=\sum_{i=1}^{i=4} C(i) t_{f}^{(i-1)}
$$

where for light water

$$
\begin{aligned}
& C(1)=5.7032432 \times 10^{-1} \\
& C(2)=1.7996615 \times 10^{-3} \\
& C(3)=-7.2881959 \times 10^{-6} \\
& C(4)=3.2412245 \times 10^{-9}
\end{aligned}
$$

and for heavy water

$$
\begin{aligned}
& C(1)=5.6340135 \times 10^{-1} \\
& C(2)=1.4504443 \times 10^{-3} \\
& C(3)=-7.9650470 \times 10^{-6} \\
& C(4)=7.1584948 \times 10^{-9}
\end{aligned}
$$


Coefficients of the thermal conductivity correlation for light water and heavy water were determined by the curve fitting of the data given in DPSTM-140. The applicable range of the correlation is $283.15 \mathrm{~K}<\mathrm{T}_{\mathrm{f}}<573.15 \mathrm{~K}$.

Surface tension of liquid water $(\sigma)$ :

The correlation given by DPSTM-140 is used to compute liquid water surface tension.

$$
\sigma=\sum_{i=1}^{i=4} C(i) t_{f}^{(i-1)}
$$

where

$$
\begin{aligned}
& C(1)=7.5743910 \times 10^{-2} \\
& C(2)=-1.4302481 \times 10^{-4} \\
& C(3)=-2.9108772 \times 10^{-7} \\
& C(4)=2.8387790 \times 10^{-10}
\end{aligned}
$$

The applicable range of the correlation is $283.15 \mathrm{~K}<\mathrm{T}_{\mathrm{f}}<573.15 \mathrm{~K}$. This correlation is used for the computation of light water $\left(\mathrm{H}_{2} \mathrm{O}\right)$ and heavy water $\left(\mathrm{D}_{2} \mathrm{O}\right)$ surface tension.

\section{A.2 The properties of steam}

\section{A.2.1 Thermodynamic properties of steam}

Saturated density $\left(\rho_{s}\right)$ :

From the ideal gas relationship,

$$
\rho_{s}=\frac{P_{s}}{R_{s} T_{g}}
$$

where $R_{s}=461 \mathrm{~J} / \mathrm{kg} \mathrm{K}$ for light water steam (Reynolds and Perkins, 1977) and $R_{s}=415$ $\mathrm{J} / \mathrm{kg} \mathrm{K}$ for heavy water steam (AECL-7531). In equation (A.2-1) $\mathrm{P}_{\mathrm{s}}$ is the partial pressure of steam and $T_{8}$ is the temperature of gas mixture. In sections $B$ and $C$, saturated steam densities computed by the ideal gas relation were compared to the data (Reynolds (1979) data for light water and AECL-7531 (1981) data for heavy water).

Steam density derivatives with respect to pressure and temperature ( $\alpha$ and $\beta$ ):

$\alpha$ and $\beta$ are computed from the derivatives of equation (A.2-1) with respect to pressure or temperature at a given temperature or pressure as shown below. 


$$
\left.\alpha \equiv \frac{\partial \rho_{s}}{\partial P_{s}}\right|_{T_{g}}=\frac{1}{R_{s} T_{g}}
$$

$R_{s}$ is the value used in equation (A.2-1).

$$
\left.\beta \equiv \frac{\partial \rho_{s}}{\partial T_{g}}\right|_{P_{s}}=-\frac{P_{s}}{R_{s} T_{g}^{2}}=-\frac{\rho_{s}}{T_{g}}
$$

$\beta$ is computed by equation (A.2-3) using equation (A.2-1).

Saturated temperanure and its derivative with respect to pressure $\left(\mathrm{T}_{\text {sal }}\right.$ and $\left.\left(\mathrm{dT} \mathrm{T}_{\text {sat }} / \mathrm{dP}\right)\right)$ :

$$
\begin{gathered}
T_{\text {sat }}=A+B(\ln P)+C(\ln P)^{2}+D(\ln P)^{3} \\
\frac{d T_{\text {sat }}}{d P}=\frac{\left\{B+2 C(\ln P)+3 D(\ln P)^{2}\right\}}{P}
\end{gathered}
$$

where for light water

$$
\begin{aligned}
& A=1.4697 \times 10^{2} \\
& B=3.0482 \times 10^{1} \\
& C=-2.6382 \\
& D=1.4715 \times 10^{-1}
\end{aligned}
$$

and for heavy water

$$
\begin{aligned}
& A=1.3795 \times 10^{2} \\
& B=3.3995 \times 10^{1} \\
& C=-2.9758 \\
& D=1.5679 \times 10^{-1}
\end{aligned}
$$

The applicable range of the correlation is $611.3 \mathrm{~Pa}<\mathrm{P}<1.0 \mathrm{MPa}$. The constants for light water in equation (A.2-4) were determined by curve fitting using the data from Reynolds (1979), and the constants of heavy water were determined by fitting the data of DPSTM-140. When local pressure is below the pressure at the triple point $\left(\mathrm{P}_{t}=611.3\right.$ $\mathrm{Pa}), \mathrm{T}_{\text {sat }}$ is computed based on the linear extrapolation in terms of temperature gradient with respect to pressure at the triple point. That is,

$$
T_{\text {sat }}(P)=T_{\text {sat }}\left(P_{t}\right)-\left(\left.\frac{d T_{\text {sat }}}{d P}\right|_{P=P_{t}}\right)\left(P_{t}-P\right)
$$


Saturated steam enthalpy and derivative with repect to pressure $\left(h_{s, s a t}\right.$ and $\left.\left(d_{s, s a t} / d P\right)\right)$ :

The correlation was obtained by the curve fitting of the data found in the literature (Reynolds (1979) for light water and AECL 7531 for heavy water).

$$
\begin{gathered}
h_{s, \text { sat }}=A+B \ln (P)+C(\ln (P)\}^{2} \\
\frac{d h_{s, \text { sat }}}{d P}=\frac{B+2 C \ln (P)}{P}
\end{gathered}
$$

where for light water

$$
\begin{aligned}
& A=2.4057 \times 10^{6} \\
& B=3.9495 \times 10^{3} \\
& C=1.6845 \times 10^{3}
\end{aligned}
$$

and for heavy water

$$
\begin{aligned}
& A=2.2314 \times 10^{6} \\
& B=4.0547 \times 10^{3} \\
& C=1.5260 \times 10^{3}
\end{aligned}
$$

Equation (A.2-7) is applicable to relatively low pressure range $(<1 \mathrm{MPa})$.

Constant pressure specific heat and enthalpy of steam $\left(C_{p s}\right.$ and $\left.h_{s}\right)$ :

$$
\begin{gathered}
C_{p s}\left(T_{g}\right)=\left.\frac{\partial h_{s}}{\partial T_{g}}\right|_{p} \\
=A+B T_{g}+C T_{g}^{2}+D T_{g}^{3} \\
h_{s}=h_{s, ~ s a t}(P)+\int_{T_{s a t}}^{T_{g}}(P) C_{p s}(T) d T \\
=h_{s, \text { sat }}(P)+A\left(T_{g}-T_{s a t}\right)+\frac{B}{2}\left(T_{g}^{2}-T_{\text {sat }}^{2}\right) \\
+\frac{C}{3}\left(T_{g}^{3}-T_{s a t}^{3}\right)+\frac{D}{4}\left(T_{g}^{4}-T_{\text {sat }}^{4}\right)
\end{gathered}
$$

where for light water

$$
\begin{aligned}
& A=1.9014 \times 10^{3} \\
& B=-6.2129 \times 10^{-1} \\
& C=1.9538 \times 10^{-3} \\
& D=-1.0083 \times 10^{-6}
\end{aligned}
$$


and for heavy water

$$
\begin{aligned}
& A=1.6114 \times 10^{3} \\
& B=3.6681 \times 10^{-3} \\
& C=1.3023 \times 10^{-3} \\
& D=-6.6266 \times 10^{-7}
\end{aligned}
$$

The constants for light water in equation (A.2-9) were determined by curve fitting using the data from Reynolds (1979), and the constants of heavy water were determined by fitting the data of AECL-7531. The applicable range of the correlation is $270 \mathrm{~K}<\mathrm{T}_{\mathrm{g}}<700 \mathrm{~K}$.

Derivative of steam enthalpy with respect to pressure at a given temperature is computed using equations, (A.2-8), (A.2-9), and (A.2-10), as shown below.

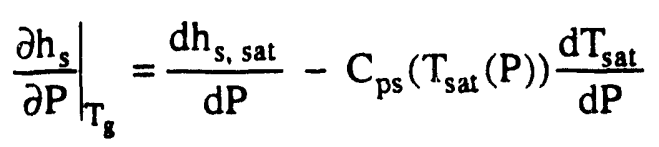

\section{A.2.2 Transport properties of steam}

Viscosity of steam $\left(\mu_{\mathrm{s}}\right)$ :

$$
\mu_{s}=A+B t_{g}-\rho_{s}\left(C-D t_{g}\right)
$$

where for light water

$$
\begin{aligned}
& A=8.040 \times 10^{-6} \\
& B=4.070 \times 10^{-8} \\
& C=1.858 \times 10^{-7} \\
& D=5.900 \times 10^{-10}
\end{aligned}
$$

and for heavy water

$$
\begin{aligned}
& A=8.3224 \times 10^{-6} \\
& B=4.1550 \times 10^{-8} \\
& C=0.0 \\
& D=0.0
\end{aligned}
$$

The constants for light water in equation (A.2-12) were taken from ASME curve fitting function (ASME Steam Tables, 1967), and the constants of heavy water were determined by fitting the data of AECL-7531. The applicable range of light water viscosity correlation is from atmospheric pressure to saturation pressure in the temperature range of $373.15 \mathrm{~K}$ to $573.15 \mathrm{~K}$ and the applicable temperature range of heavy water is from $400 \mathrm{~K}$ to $700 \mathrm{~K}$.

\section{Thermal conductivity of steam $\left(\mathrm{k}_{\mathrm{s}}\right)$ :}

The thermal conductivity of $\mathrm{H}_{2} \mathrm{O}$ steam is computed based on the correlation given by ASME (ASME Steam Tables, 1967). For the curve fitting of $\mathrm{D}_{2} \mathrm{O}$ steam thermal conductivity, data given by AECL-7531 was used. 


$$
\mathrm{k}_{\mathrm{s}}=\mathrm{A}+\mathrm{B} \mathrm{t}_{\mathrm{g}}+\mathrm{Ct}_{\mathrm{g}}^{2}+\mathrm{D} \mathrm{t}_{\mathrm{g}}^{3}
$$

where for light water

$$
\begin{aligned}
& A=1.76 \times 10^{-2} \\
& B=5.87 \times 10^{-5} \\
& C=1.04 \times 10^{-7} \\
& D=-4.51 \times 10^{-11}
\end{aligned}
$$

and for heavy water

$$
\begin{aligned}
& A=1.7880 \times 10^{-2} \\
& B=5.1209 \times 10^{-5} \\
& C=1.1218 \times 10^{-7} \\
& D=0.0
\end{aligned}
$$

The applicable range of $\mathrm{H}_{2} \mathrm{O}$ steam is $373.15 \mathrm{~K}<\mathrm{T}_{\mathrm{g}}<973.15 \mathrm{~K}$, and the applicable range of $\mathrm{D}_{2} \mathrm{O}$ steam is $400 \mathrm{~K}<\mathrm{T}_{\mathrm{g}}<700 \mathrm{~K}$.

\section{A.3 The properties of Air}

\section{A.3.1 Thermodynamic properties of air}

Thermodynamic properties of air are evaluated on the basis of ideal gas assumption.

$$
\begin{gathered}
\rho_{\mathrm{a}}=\frac{\mathrm{P}_{\mathrm{a}}}{\mathrm{R}_{\mathrm{a}} \mathrm{T}_{\mathrm{g}}} \\
\left.\frac{\partial \rho_{\mathrm{a}}}{\partial \mathrm{P}_{\mathrm{a}}}\right|_{\mathrm{T}_{\mathrm{g}}}=\frac{1}{\mathrm{R}_{\mathrm{a}} \mathrm{T}_{\mathrm{g}}} \\
\left.\frac{\partial \rho_{\mathrm{a}}}{\partial \mathrm{T}_{\mathrm{g}}}\right|_{\mathrm{P}_{\mathrm{a}}}=-\frac{\rho_{\mathrm{a}}}{\mathrm{T}_{\mathrm{g}}} \\
\left.\frac{\partial \mathrm{h}_{\mathrm{g}}}{\partial \mathrm{T}_{\mathrm{g}}}\right|_{\mathrm{P}}=\mathrm{A}+\mathrm{BT} \mathrm{T}_{\mathrm{g}}+\mathrm{CT}_{\mathrm{g}}^{2} \\
\left.\frac{\partial \mathrm{h}_{\mathrm{a}}}{\partial \mathrm{P}}\right|_{\mathrm{T}_{\mathrm{g}}}=0
\end{gathered}
$$




$$
\begin{aligned}
h_{a} & =h_{a, \text { ref }}+\int_{T_{\text {ref }}}^{T_{g}} C_{p a}(T) d T \\
& =h_{a, \text { ref }}+A\left(T_{g}-T_{\text {ref }}\right)+\frac{B}{2}\left(T_{g}^{2}-T_{\text {ref }}^{2}\right)+\frac{C}{3}\left(T_{g}^{3}-T_{\text {ref }}^{3}\right)
\end{aligned}
$$

where

$$
\begin{aligned}
R_{\mathrm{a}} & =287.0 \mathrm{~J} / \mathrm{kg} \mathrm{K} \text { (Reynolds, 1979) } \\
\mathrm{T}_{\mathrm{ref}} & =375 \mathrm{~K}(\mathrm{Van} \text { Wylen and Sonntag, second edition) } \\
\mathrm{h}_{\mathrm{a}, \mathrm{ref}} & =3.757 \times 10^{5} \mathrm{~J} / \mathrm{kg} \text { (Van Wylen and Sonntag, second edition) } \\
\mathrm{A} & =1.0059 \times 10^{3} \\
\mathrm{~B} & =-8.6140 \times 10^{-2} \\
\mathrm{C} & =2.6295 \times 10^{-4}
\end{aligned}
$$

Equation (A.3-4) was obtained by fitting the data given by Reynolds (1979). The applicable range of the correlation is $275 \mathrm{~K}<\mathrm{T}_{\mathrm{g}}<700 \mathrm{~K}$.

\section{A.3.2 Transport properties of air}

\section{Viscosity of air $\left(\mu_{\mathrm{a}}\right)$ :}

The correlation of air viscosity is given by the literature (Hilsenrath et al., 1960) as shown below:

$$
\mu_{\mathrm{a}}=\frac{\mathrm{AT}_{\mathrm{g}}^{1.5}}{\mathrm{~B}+\mathrm{T}_{\mathrm{g}}}
$$

where

$$
\begin{aligned}
& A=1.458 \times 10^{-6} \\
& B=1.104 \times 10^{2}
\end{aligned}
$$

The applicable range of the correlation is $273.15 \mathrm{~K}<\mathrm{T}_{\mathrm{g}}<773.15 \mathrm{~K}$.

Thermal conductivity of air $\left(\mathbf{k}_{\mathbf{a}}\right)$ :

$$
\mathrm{k}_{\mathrm{a}}=\mathrm{A}+\mathrm{BT}_{\mathrm{g}}+\mathrm{CT}_{\mathrm{g}}^{2}
$$

where

$$
\begin{aligned}
& A=3.3350 \times 10^{-3} \\
& B=8.0021 \times 10^{-5} \\
& C=-1.8975 \times 10^{-8}
\end{aligned}
$$

The constants of curve fit function in equation (A.3-8) were determined by the data given in Table 94-1 of Raznjevic (1976). The applicable range of the correlation is $273.15 \mathrm{~K}<\mathrm{T}_{\mathrm{g}}$ 


\section{A.4 The binary gas mass diffusivity of air-steam}

The correlation given by Marrero and Mason (1972) is used to compute mass diffusivity under binary gas mixture of air-steam.

$$
\begin{aligned}
& D_{12}=0.187 C_{D}\left(\frac{T^{2.072}}{P}\right) \quad(282 K<T<450 K) \\
& D_{12}=2.750 C_{D}\left(\frac{T^{1.632}}{P}\right) \quad(450 K \leq T<1070 K)
\end{aligned}
$$

where $C_{D}$ (conversion factor) $=1.01325 \times 10^{-4}$. The applicable range of the correlation is $282 \mathrm{~K}<1070 \mathrm{~K}$. The values computed by the correlation are compared to the data available from the literature (Treybal, 3rd ed.) in the appendix B and C. This correlation is used for the computation of the binary gas mixture mass diffusivity under air-light water $\left(\mathrm{H}_{2} \mathrm{O}\right)$ steam or air-heavy water $\left(\mathrm{D}_{2} \mathrm{O}\right)$ steam.

\section{References}

Hilsenrath, Joseph, Hoge, Harold J., Beckett, Charles W., Masi, Joseph F., Benedict, William S., Nuttall, Ralph L., Fano, Lilla, Touloukian, Yeram S., and Woolley, Harold W., Tables of Thermodynamic and Transport Properties of Air, Argon, Carbon Dioxide, Carbon Monoxide, Hydrogen, and Steam, Pergamon Press, 1960.

1967 ASME Steam Tables, ASME, Second Edition.

Raznjevic, K., Handbook of Thermodynamic Tables and Charts, Hemisphere Publishing Corporation, 1976.

Reynolds, W. C., Thermodynamic Properties in SI, 1979.

Aleman, S. E., Gregory, M. V., Hamm, L. L., Koffman, L. D., Pevey, R. E., Reed, W. H., and Smith, F. G., FLOWTRAN: An Alogorithm For Describing The Thermalhydraulic behavior of SRP Assemblies, DPSTM-140.

Hill, P. G., MacMillan, R. D., and Lee, Victor, Tables of Thermodynamic Properties of Heavy Water in SI. Units, AECL-7531, December, 1981.

Reynolds, W. C. and Perkins, H. C., Engineering Thermodynamics, 1977.

Van Wylen, G. J. and Sonntag, R. E., Fundamentals of Classical Thermodynamics, Second Edition, John Wiley and Sons, Inc.

Treybal, R. E., Mass Transfer Operations, 3rd edition, McGraw-Hill, New York.

Marrero, T. R. and Mason, E. A., "Gaseous Diffusion Coefficients", Journal of Physical Chemistry Reference Data, pp 3-118, Vol. 1, No. 1, 1972. 


\section{B Data of fluid property correlations}

\section{B.1 Data of liquid water property correlations}

Saturation pressure of light water:

\begin{tabular}{|llcl|}
\hline $\mathrm{T}(\mathrm{K})$ & $\mathrm{P}_{\text {sat }}-$ Data & $\mathrm{P}_{\text {sat }}-$ Corr. & \% error \\
\hline 273.16 & 611.3 & 619.8 & -1.390 \\
275 & 698 & 705.3 & -1.053 \\
276.92 & 800 & 805.9 & -0.733 \\
280.13 & 1000 & 1003.1 & -0.308 \\
282.81 & 1200 & 1199.9 & 0.010 \\
285.13 & 1400 & 1397.5 & 0.178 \\
287.17 & 1600 & 1594.8 & 0.323 \\
288.99 & 1800 & 1791.5 & 0.472 \\
290.65 & 2000 & 1989.5 & 0.527 \\
294.23 & 2500 & 2484.0 & 0.642 \\
297.23 & 3000 & 2979.3 & 0.690 \\
302.12 & 4000 & 3975.3 & 0.618 \\
306.03 & 5000 & 4971.5 & 0.569 \\
309.31 & 6000 & 5969.6 & 0.507 \\
314.66 & 8000 & 7974.0 & 0.326 \\
318.96 & 10000 & 9985.2 & 0.148 \\
322.57 & 12000 & 11997.9 & 0.017 \\
325.7 & 14000 & 14016.1 & -0.115 \\
328.47 & 16000 & 16038.1 & -0.238 \\
330.96 & 18000 & 18063.1 & -0.351 \\
333.22 & 20000 & 20085.6 & -0.428 \\
338.12 & 25000 & 25138.1 & -0.553 \\
342.26 & 30000 & 30205.7 & -0.686 \\
349.02 & 40000 & 40313.6 & -0.784 \\
354.48 & 50000 & 50406.8 & -0.814 \\
359.09 & 60000 & 60481.7 & -0.803 \\
366.65 & 80000 & 80564.7 & -0.706 \\
372.78 & 100000 & 100598.1 & -0.598 \\
373.14 & 101325 & 101890.5 & -0.558 \\
377.96 & 120000 & 120538.6 & -0.449 \\
382.46 & 140000 & 140369.3 & -0.264 \\
386.47 & 160000 & 160201.9 & -0.126 \\
390.09 & 180000 & 180006.2 & -0.003 \\
393.38 & 200000 & 199688.9 & 0.156 \\
400.59 & 250000 & 248988.6 & 0.405 \\
406.7 & 300000 & 298171.4 & 0.610 \\
416.78 & 400000 & 396678.7 & 0.830 \\
425.01 & 500000 & 496048.6 & 0.790 \\
432 & 600000 & 596399.8 & 0.600 \\
443.59 & 800000 & 802188.6 & -0.274 \\
453.06 & 1000000 & 1015813.8 & -1.581 \\
\hline & & & \\
\hline
\end{tabular}


Saturation pressure of heavy water:

\begin{tabular}{|llll|}
\hline $\mathrm{T}(\mathrm{K})$ & $\mathrm{P}_{\text {sat }}-$ Data & $\mathrm{P}_{\text {sat }}-$ Corr. & \% error \\
\hline 283.15 & 1026.4 & 1037.9 & -1.124 \\
288.15 & 1442 & 1447.8 & -0.403 \\
293.15 & 1999 & 1997.2 & 0.090 \\
298.15 & 2737 & 2725.4 & 0.424 \\
303.15 & 3702 & 3680.1 & 0.591 \\
308.15 & 4950 & 4918.8 & 0.631 \\
313.15 & 6549 & 6509.4 & 0.605 \\
318.15 & 8575 & 8531.9 & 0.502 \\
323.15 & 11121 & 11079.1 & 0.376 \\
328.15 & 14283 & 14257.7 & 0.177 \\
333.15 & 18200 & 18188.9 & 0.061 \\
338.15 & 22987 & 23009.6 & -0.098 \\
343.15 & 28800 & 28872.8 & -0.253 \\
348.15 & 35820 & 35948.2 & -0.358 \\
353.15 & 44230 & 44422.7 & -0.436 \\
358.15 & 54230 & 54500.6 & -0.499 \\
363.15 & 66070 & 66404.8 & -0.507 \\
368.15 & 79980 & 80376.6 & -0.496 \\
373.15 & 96250 & 96676.9 & -0.444 \\
378.15 & 115170 & 115587.3 & -0.362 \\
383.15 & 137060 & 137411.3 & -0.256 \\
388.15 & 162270 & 162476.6 & -0.127 \\
393.15 & 191100 & 191137.9 & -0.020 \\
398.15 & 224100 & 223779.7 & 0.143 \\
403.15 & 261500 & 260821.9 & 0.259 \\
408.15 & 303900 & 302724.7 & 0.387 \\
413.15 & 351700 & 349995.9 & 0.485 \\
418.15 & 405300 & 403199.9 & 0.518 \\
423.15 & 465300 & 462968.5 & 0.501 \\
428.15 & 532300 & 530013.5 & 0.430 \\
433.15 & 606700 & 605142.8 & 0.257 \\
438.15 & 689200 & 689279.6 & -0.012 \\
443.15 & 780300 & 783484.9 & -0.408 \\
448.15 & 880800 & 888985.0 & -0.929 \\
\hline
\end{tabular}




\section{Saturation density of light water:}

\begin{tabular}{|c|c|c|c|}
\hline $\mathrm{T}(\mathrm{K})$ & $\rho_{\text {sat }}(T)$-Data & $\rho_{\text {sat }}(T)-$ Corr. & $\%$ error \\
\hline 275 & 1000.0 & 1001.9 & -0.194 \\
\hline 280 & 1000.0 & 1001.0 & -0.097 \\
\hline 285 & 999.0 & 999.9 & -0.087 \\
\hline 290 & 999.0 & 998.6 & 0.036 \\
\hline 295 & 998.0 & 997.3 & 0.073 \\
\hline 300 & 996.0 & 995.8 & 0.024 \\
\hline 305 & 995.0 & 994.1 & 0.088 \\
\hline 310 & 993.0 & 992.4 & 0.067 \\
\hline 315 & 991.1 & 990.5 & 0.060 \\
\hline 320 & 989.1 & 988.5 & 0.067 \\
\hline 325 & 987.2 & 986.3 & 0.088 \\
\hline 330 & 985.2 & 984.0 & 0.123 \\
\hline 335 & 982.3 & 981.6 & 0.075 \\
\hline 340 & 979.4 & 979.0 & 0.042 \\
\hline 345 & 976.6 & 976.3 & 0.024 \\
\hline 350 & 973.7 & 973.5 & 0.021 \\
\hline 355 & 970.9 & 970.6 & 0.033 \\
\hline 360 & 967.1 & 967.5 & -0.035 \\
\hline 365 & 964.3 & 964.2 & 0.008 \\
\hline 370 & 960.6 & 960.9 & -0.028 \\
\hline 375 & 956.9 & 957.4 & -0.048 \\
\hline 380 & 953.3 & 953.8 & -0.051 \\
\hline 385 & 949.7 & 950.0 & -0.038 \\
\hline 390 & 945.2 & 946.1 & -0.102 \\
\hline 395 & 941.6 & 942.1 & -0.054 \\
\hline 400 & 937.2 & 938.0 & -0.082 \\
\hline 405 & 932.8 & 933.7 & -0.092 \\
\hline 410 & 928.5 & 929.3 & -0.083 \\
\hline 415 & 924.2 & 924.7 & -0.056 \\
\hline 420 & 920.0 & 920.0 & -0.009 \\
\hline 425 & 914.9 & 915.2 & -0.035 \\
\hline 430 & 909.9 & 910.3 & -0.041 \\
\hline 435 & 905.8 & 905.2 & 0.065 \\
\hline 440 & 900.9 & 900.0 & 0.100 \\
\hline 445 & 895.3 & 894.7 & 0.067 \\
\hline 450 & 890.5 & 889.2 & 0.145 \\
\hline
\end{tabular}


Saturation density of heavy water:

\begin{tabular}{|cccc|}
\hline $\mathrm{T}(\mathrm{K})$ & $\rho_{\mathrm{f}, \text { sat }}$-Data & $\rho_{\mathrm{f}, \text { sat }}-$ Corr. & \% error \\
\hline 283.15 & 1106 & 1108.0 & 0.182 \\
293.15 & 1105.3 & 1105.6 & -0.025 \\
303.15 & 1103.3 & 1102.5 & 0.070 \\
313.15 & 1100 & 1098.9 & 0.104 \\
323.15 & 1095.7 & 1094.6 & 0.104 \\
333.15 & 1090.5 & 1089.7 & 0.077 \\
343.15 & 1084.7 & 1084.1 & 0.053 \\
353.15 & 1078.2 & 1078.0 & 0.020 \\
363.15 & 1071 & 1071.2 & 0.020 \\
373.15 & 1063.4 & 1063.8 & -0.040 \\
383.15 & 1055.2 & 1055.8 & 0.059 \\
393.15 & 1046.5 & 1047.2 & 0.067 \\
403.15 & 1037.2 & 1038.0 & 0.073 \\
413.15 & 1027.5 & 1028.1 & 0.057 \\
423.15 & 1017.3 & 1017.6 & 0.030 \\
433.15 & 1006.5 & 1006.5 & 0.001 \\
443.15 & 995.2 & 994.8 & 0.042 \\
453.15 & 983.5 & 982.4 & 0.107 \\
\hline
\end{tabular}

Light water density derivatives with respect to pressure:

\begin{tabular}{|cccl|}
\hline $\mathrm{T}(\mathrm{K})$ & $\alpha^{*}$-Data & $\alpha^{*}-$ Corr. & \% error \\
\hline 283.15 & 0.47142 & 0.47056 & 0.183 \\
310.93 & 0.43007 & 0.43707 & .1 .629 \\
338.71 & 0.43885 & 0.43011 & 1.991 \\
366.48 & 0.44765 & 0.44968 & -0.454 \\
394.26 & 0.49665 & 0.49579 & 0.174 \\
422.04 & 0.56503 & 0.56842 & -0.601 \\
449.82 & 0.66920 & 0.66759 & 0.241 \\
477.59 & 0.79464 & 0.79328 & 0.171 \\
\hline
\end{tabular}

- $\frac{\partial \rho}{\partial P h_{f}} ;\left(10^{-6} \mathrm{~kg} / \mathrm{m}^{3} \mathrm{~Pa}\right)$ 
Heavy water density derivatives with respect to pressure:

\begin{tabular}{|cccl|}
\hline $\mathrm{T}(\mathrm{K})$ & $\alpha^{*}$ - Data & $\alpha^{*}$-Corr. & \% error \\
\hline 283.15 & 0.5300 & 0.5232 & 1.277 \\
303.15 & 0.5010 & 0.5002 & 0.157 \\
323.15 & 0.4720 & 0.4911 & -4.041 \\
343.15 & 0.5040 & 0.4958 & 1.627 \\
363.15 & 0.5100 & 0.5144 & -0.862 \\
383.15 & 0.5520 & 0.5469 & 0.931 \\
403.15 & 0.6050 & 0.5932 & 1.951 \\
423.15 & 0.6470 & 0.6534 & -0.990 \\
443.15 & 0.7250 & 0.7275 & -0.343 \\
\hline$\left.\frac{\partial \rho}{\partial \mathrm{P}}\right|_{\mathrm{T}_{\mathrm{f}}} ;\left(10^{-6} \mathrm{~kg} / \mathrm{m}^{3} \mathrm{~Pa}\right)$ & \\
\hline
\end{tabular}




\begin{tabular}{|l|lrl|r|}
\hline $2 / 15 / 93$ & WSRC-TR-92.532 Rev. 0 & FLOWTRAN-TF Software Design & Page 544 \\
\hline
\end{tabular}

Saturated enthalpy of light water:

\begin{tabular}{|llll|}
\hline $\mathrm{T}(\mathrm{K})$ & $\mathrm{h}_{\mathrm{f}, \text { sat }}$ Data & $\mathrm{h}_{\mathrm{f}, \text { sat }}-$ Corr. & o o error \\
\hline 275 & 7500 & 7536.4 & -0.486 \\
276.92 & 15400 & 15530.7 & -0.849 \\
280.13 & 28600 & 28902.2 & -1.057 \\
282.81 & 39700 & 40071.7 & -0.936 \\
285.13 & 49300 & 49745.2 & -0.903 \\
287.17 & 57800 & 58254.4 & -0.786 \\
288.99 & 65500 & 65848.6 & -0.532 \\
290.65 & 72400 & 72777.3 & -0.521 \\
294.23 & 87500 & 87726.8 & -0.259 \\
297.23 & 100100 & 100261.5 & -0.161 \\
302.12 & 120700 & 120707.4 & -0.006 \\
306.03 & 137200 & 137068.5 & 0.096 \\
309.31 & 151000 & 150802.1 & 0.131 \\
314.66 & 173700 & 173219.9 & 0.276 \\
318.96 & 191800 & 191253.2 & 0.285 \\
322.57 & 207100 & 206403.4 & 0.336 \\
325.7 & 220300 & 219546.9 & 0.342 \\
328.47 & 231900 & 231184.7 & 0.308 \\
330.96 & 242400 & 241651.0 & 0.309 \\
333.22 & 251900 & 251154.4 & 0.296 \\
338.12 & 272600 & 271772.2 & 0.304 \\
342.26 & 289900 & 289205.9 & 0.239 \\
349.02 & 318300 & 317699.7 & 0.189 \\
354.48 & 341300 & 340738.6 & 0.164 \\
359.09 & 360600 & 360207.9 & 0.109 \\
366.65 & 392300 & 392169.8 & 0.033 \\
372.78 & 418000 & 418116.9 & -0.028 \\
373.14 & 419500 & 419641.6 & -0.034 \\
377.96 & 439700 & 440064.5 & -0.083 \\
382.46 & 458600 & 459147.0 & -0.119 \\
386.47 & 475500 & 476164.2 & -0.140 \\
390.09 & 490800 & 491536.5 & -0.150 \\
393.38 & 504700 & 505515.9 & -0.162 \\
400.59 & 535200 & 536179.5 & -0.183 \\
406.7 & 561200 & 562194.8 & -0.177 \\
416.78 & 604300 & 605173.9 & -0.145 \\
425.01 & 639800 & 640320.6 & -0.081 \\
432 & 670100 & 670210.9 & -0.017 \\
443.59 & 720700 & 719851.1 & 0.118 \\
453.06 & 762500 & 760484.7 & 0.264 \\
\hline
\end{tabular}


Saturated enthalpy of heavy water:

\begin{tabular}{|cccl|}
\hline $\mathrm{T}(\mathrm{K})$ & $\mathrm{h}_{\mathrm{f}, \text { sat }}$ Data & $\mathrm{h}_{\mathrm{f}, \text { sat }}$ - Corr. & \% error \\
\hline 283.15 & 26180 & 26705.2 & -2.006 \\
293.15 & 68570 & 68912.3 & -0.499 \\
303.15 & 110990 & 111078.0 & -0.079 \\
313.15 & 153360 & 153202.2 & 0.103 \\
323.15 & 195630 & 195285.0 & 0.176 \\
333.15 & 237770 & 237326.3 & 0.187 \\
343.15 & 279780 & 279326.1 & 0.162 \\
353.15 & 321670 & 321284.5 & 0.120 \\
363.15 & 363440 & 363201.4 & 0.066 \\
373.15 & 405110 & 405076.8 & 0.008 \\
383.15 & 446720 & 446910.8 & -0.043 \\
393.15 & 488290 & 488703.3 & -0.085 \\
403.15 & 529870 & 530454.3 & -0.110 \\
413.15 & 571510 & 572163.9 & -0.114 \\
423.15 & 613240 & 613832.0 & -0.097 \\
433.15 & 655130 & 655458.6 & -0.050 \\
443.15 & 697230 & 697043.8 & 0.027 \\
453.15 & 739580 & 738587.5 & 0.134 \\
\hline
\end{tabular}




\begin{tabular}{|l|lll|r|}
\hline $2 / 15 / 93$ & WSRC-TR-92.532 Rev. 0 & FLOWTRAN-TF Software Design & Page 546 \\
\hline
\end{tabular}

Dynamic viscosity of light water:

\begin{tabular}{|lccl|}
\hline $\mathrm{T}(\mathrm{K})$ & $\mu_{\mathrm{f}}(\mu \mathrm{Pa} \mathrm{sec})$-Data $\mu_{\mathrm{f}}(\mu \mathrm{Pa} \mathrm{sec})-$ Corr. $\%$ error \\
\hline 283.15 & 1304.000 & 1301.939 & 0.158 \\
293.15 & 1002.000 & 1004.872 & -0.287 \\
303.15 & 798.300 & 800.477 & -0.273 \\
313.15 & 653.900 & 654.545 & -0.099 \\
323.15 & 547.800 & 547.056 & 0.136 \\
333.15 & 467.300 & 465.754 & 0.331 \\
343.15 & 404.800 & 402.839 & 0.484 \\
353.15 & 355.400 & 353.181 & 0.624 \\
363.15 & 315.600 & 313.303 & 0.728 \\
373.15 & 283.100 & 280.789 & 0.816 \\
383.15 & 254.800 & 253.919 & 0.346 \\
393.15 & 231.000 & 231.446 & -0.193 \\
403.15 & 210.900 & 212.449 & -0.734 \\
413.15 & 194.100 & 196.234 & -1.099 \\
423.15 & 179.800 & 182.272 & -1.375 \\
433.15 & 167.700 & 170.157 & -1.465 \\
443.15 & 157.400 & 159.567 & -1.377 \\
453.15 & 148.500 & 150.250 & -1.178 \\
463.15 & 140.700 & 142.003 & -0.926 \\
473.15 & 133.900 & 134.663 & -0.570 \\
483.15 & 127.900 & 128.098 & -0.155 \\
493.15 & 122.400 & 122.197 & 0.166 \\
503.15 & 117.500 & 116.870 & 0.536 \\
513.15 & 112.900 & 112.042 & 0.760 \\
523.15 & 108.700 & 107.650 & 0.966 \\
533.15 & 104.800 & 103.641 & 1.106 \\
543.15 & 101.100 & 99.968 & 1.120 \\
553.15 & 97.500 & 96.594 & 0.929 \\
563.15 & 94.100 & 93.485 & 0.654 \\
573.15 & 90.700 & 90.612 & 0.097 \\
\hline
\end{tabular}


Dynamic viscosity of heavy water:

\begin{tabular}{|cccc|}
\hline $\mathrm{T}(\mathrm{K})$ & $\mu_{\mathrm{f}}(\mu \mathrm{Pasec})-$ Data $\mu_{\mathrm{f}}(\mu \mathrm{Pasec})$-Corr. $\%$ error \\
\hline 283.15 & 1679 & 1674.2 & 0.284 \\
293.15 & 1247 & 1253.7 & -0.537 \\
303.15 & 971.8 & 976.9 & -0.528 \\
313.15 & 784.8 & 786.1 & -0.168 \\
323.15 & 651.2 & 649.4 & 0.271 \\
333.15 & 551.8 & 548.3 & 0.627 \\
343.15 & 475.7 & 471.5 & 0.880 \\
353.15 & 415.9 & 411.8 & 0.993 \\
363.15 & 367.9 & 364.4 & 0.958 \\
373.15 & 328.8 & 326.1 & 0.815 \\
383.15 & 296.5 & 294.8 & 0.584 \\
393.15 & 269.4 & 268.7 & 0.248 \\
403.15 & 246.5 & 246.8 & -0.141 \\
413.15 & 226.9 & 228.3 & -0.599 \\
423.15 & 210 & 212.3 & -1.106 \\
433.15 & 195.3 & 198.5 & -1.658 \\
443.15 & 182.4 & 186.5 & -2.261 \\
453.15 & 171.1 & 176.0 & -2.852 \\
\hline
\end{tabular}




\begin{tabular}{|l|lll|r|}
\hline $2 / 15 / 93$ & WSRC-TR-92-532 Rev. 0 & FLOWTRAN-TF Software Design & Page 548 \\
\hline
\end{tabular}

Thermal conductivity of light water:

\begin{tabular}{|lccl|}
\hline $\mathrm{T}(\mathrm{K})$ & $\mathrm{k}_{\mathrm{f}}-$ Data & $\mathrm{k}_{\mathrm{f}}-$ Corr. & \% error \\
\hline 283.15 & 0.587 & 0.58760 & -0.1014 \\
293.15 & 0.603 & 0.60343 & -0.0710 \\
303.15 & 0.618 & 0.61784 & 0.0255 \\
313.15 & 0.631 & 0.63086 & 0.0226 \\
323.15 & 0.643 & 0.64249 & 0.0790 \\
333.15 & 0.653 & 0.65277 & 0.0357 \\
343.15 & 0.662 & 0.66170 & 0.0453 \\
353.15 & 0.67 & 0.66931 & 0.1026 \\
363.15 & 0.676 & 0.67562 & 0.0559 \\
373.15 & 0.681 & 0.68065 & 0.0514 \\
383.15 & 0.684 & 0.68441 & -0.0605 \\
393.15 & 0.687 & 0.68693 & 0.0095 \\
403.15 & 0.688 & 0.68823 & -0.0335 \\
413.15 & 0.688 & 0.68832 & -0.0468 \\
423.15 & 0.687 & 0.68723 & -0.0332 \\
433.15 & 0.684 & 0.68497 & -0.1416 \\
443.15 & 0.681 & 0.68156 & -0.0825 \\
453.15 & 0.677 & 0.67703 & -0.0042 \\
463.15 & 0.671 & 0.67139 & -0.0578 \\
473.15 & 0.664 & 0.66466 & -0.0992 \\
483.15 & 0.657 & 0.65686 & 0.0212 \\
493.15 & 0.648 & 0.64801 & -0.0021 \\
503.15 & 0.639 & 0.63814 & 0.1351 \\
513.15 & 0.628 & 0.62725 & 0.1195 \\
523.15 & 0.616 & 0.61537 & 0.1020 \\
533.15 & 0.603 & 0.60252 & 0.0793 \\
543.15 & 0.589 & 0.58872 & 0.0475 \\
553.15 & 0.574 & 0.57399 & 0.0024 \\
563.15 & 0.558 & 0.55834 & -0.0608 \\
573.15 & 0.541 & 0.54180 & -0.1475 \\
\hline
\end{tabular}




\begin{tabular}{|l|lll|l|}
\hline $2 / 15 / 93$ & WSRC-TR-92.532 Rev. 0 & FLOWTRAN-TF Software Design & Page 549 \\
\hline
\end{tabular}

Thermal conductivity of heavy water:

\begin{tabular}{|clll|}
\hline $\mathrm{T}(\mathrm{K})$ & $\mathrm{k}_{\mathrm{f}}$ - Data & $\mathrm{k}_{\mathrm{f}}$ - Corr. & \% error \\
\hline 283.15 & 0.5750 & 0.5771 & -0.368 \\
293.15 & 0.5890 & 0.5893 & -0.048 \\
303.15 & 0.6000 & 0.5999 & 0.010 \\
313.15 & 0.6100 & 0.6091 & 0.142 \\
323.15 & 0.6180 & 0.6169 & 0.177 \\
333.15 & 0.6250 & 0.6233 & 0.272 \\
343.15 & 0.6290 & 0.6284 & 0.102 \\
353.15 & 0.6330 & 0.6321 & 0.138 \\
363.15 & 0.6350 & 0.6346 & 0.056 \\
373.15 & 0.6360 & 0.6360 & 0.007 \\
383.15 & 0.6360 & 0.6361 & -0.016 \\
393.15 & 0.6350 & 0.6351 & -0.020 \\
403.15 & 0.6320 & 0.6331 & -0.170 \\
413.15 & 0.6290 & 0.6300 & -0.158 \\
423.15 & 0.6250 & 0.6259 & -0.146 \\
433.15 & 0.6200 & 0.6209 & -0.143 \\
443.15 & 0.6140 & 0.6150 & -0.156 \\
453.15 & 0.6070 & 0.6082 & -0.191 \\
\hline
\end{tabular}


Surface tension of light water and heavy water:

\begin{tabular}{|llll|}
\hline $\mathrm{T}(\mathrm{K})$ & $\sigma_{\mathrm{f}}-$ Data & $\sigma_{\mathrm{f}}-$ Corr. & \% error \\
\hline 283.15 & 0.07424 & 0.07428 & -0.060 \\
293.15 & 0.07278 & 0.07277 & 0.015 \\
303.15 & 0.07123 & 0.07120 & 0.044 \\
313.15 & 0.06961 & 0.06958 & 0.050 \\
323.15 & 0.06793 & 0.06790 & 0.044 \\
333.15 & 0.06619 & 0.06618 & 0.021 \\
343.15 & 0.0644 & 0.06440 & -0.005 \\
353.15 & 0.06257 & 0.06258 & -0.023 \\
363.15 & 0.06069 & 0.06072 & -0.051 \\
373.15 & 0.05878 & 0.05881 & -0.059 \\
383.15 & 0.05683 & 0.05687 & -0.065 \\
393.15 & 0.05485 & 0.05488 & -0.054 \\
403.15 & 0.05283 & 0.05285 & -0.047 \\
413.15 & 0.05079 & 0.05079 & -0.008 \\
423.15 & 0.0487 & 0.04870 & 0.002 \\
433.15 & 0.04659 & 0.04657 & 0.041 \\
443.15 & 0.04444 & 0.04441 & 0.063 \\
453.15 & 0.04226 & 0.04222 & 0.086 \\
463.15 & 0.04005 & 0.04001 & 0.105 \\
473.15 & 0.03781 & 0.03777 & 0.115 \\
483.15 & 0.03553 & 0.03550 & 0.082 \\
493.15 & 0.03323 & 0.03321 & 0.053 \\
503.15 & 0.0309 & 0.03090 & -0.012 \\
513.15 & 0.02856 & 0.02858 & -0.055 \\
523.15 & 0.02619 & 0.02623 & -0.154 \\
533.15 & 0.02382 & 0.02387 & -0.207 \\
543.15 & 0.02144 & 0.02149 & -0.254 \\
553.15 & 0.01907 & 0.01911 & -0.196 \\
563.15 & 0.01671 & 0.01671 & 0.002 \\
573.15 & 0.01439 & 0.01430 & 0.603 \\
\hline
\end{tabular}




\section{B.2 Data of steam property correlations}

Steam density of saturated light water:

\begin{tabular}{|lllll|}
\hline$T(K)$ & $P_{\text {sat }}(\mathrm{Pa})$ & $\rho_{\text {s, sat }}$ - Data & $\rho_{\text {s, sat }}^{*}$ & \% error \\
\hline 275 & 698 & 0.00550 & 0.00551 & -0.041 \\
300 & 3536 & 0.02558 & 0.02557 & 0.031 \\
325 & 13530 & 0.09058 & 0.09031 & 0.303 \\
350 & 41660 & 0.26015 & 0.25820 & 0.749 \\
375 & 108200 & 0.63573 & 0.62589 & 1.548 \\
400 & 245600 & 1.36836 & 1.33189 & 2.666 \\
425 & 499900 & 2.66667 & 2.55149 & 4.319 \\
450 & 931500 & 4.80769 & 4.49024 & 6.603 \\
\hline
\end{tabular}

computed by ideal gas assumption

Steam density of saturated heavy water:

\begin{tabular}{|lllll|}
\hline $\mathrm{T}(\mathrm{K})$ & $\mathrm{P}_{\text {sat }}(\mathrm{Pa})$ & $\rho_{\text {s, sat }}$ - Data & $\rho_{\text {s, sat }}$ & \% error \\
\hline 283.15 & 1026.4 & 0.00874 & 0.00873 & 0.035 \\
293.15 & 1999.0 & 0.01645 & 0.01643 & 0.086 \\
303.15 & 3702.0 & 0.02946 & 0.02943 & 0.122 \\
313.15 & 6549.0 & 0.05050 & 0.05039 & 0.210 \\
323.15 & 11121.0 & 0.08319 & 0.08293 & 0.319 \\
333.15 & 18200.0 & 0.13224 & 0.13164 & 0.456 \\
343.15 & 28800.0 & 0.20356 & 0.20224 & 0.651 \\
353.15 & 44230.0 & 0.30440 & 0.30179 & 0.858 \\
363.15 & 66070.0 & 0.44342 & 0.43840 & 1.133 \\
373.15 & 96250.0 & 0.63084 & 0.62154 & 1.473 \\
383.15 & 137060.0 & 0.87846 & 0.86197 & 1.876 \\
393.15 & 191100.0 & 1.19979 & 1.17126 & 2.378 \\
403.15 & 261500.0 & 1.61012 & 1.56299 & 2.927 \\
413.15 & 351700.0 & 2.12666 & 2.05124 & 3.547 \\
423.15 & 465300.0 & 2.76863 & 2.64966 & 4.297 \\
433.15 & 606700.0 & 3.55745 & 3.37511 & 5.126 \\
443.15 & 780300.0 & 4.51732 & 4.24290 & 6.075 \\
453.15 & 991200.0 & 5.67460 & 5.27074 & 7.117 \\
\hline
\end{tabular}

- computed by ideal gas assumption 


\begin{tabular}{|l|ll|r|}
\hline $2 / 15 / 93$ & WSRC.TR-92.532 Rev.0 & FLOWTRAN-TF Software Design & Page 552 \\
\hline
\end{tabular}

Saturation temperature of light water:

\begin{tabular}{|c|c|c|c|}
\hline $\mathrm{P}(\mathrm{Pa})$ & $T_{\text {sat }}(K)-$ Data & $\mathrm{T}_{\text {sat }}(\mathrm{K})-$ Corr. & $\%$ error \\
\hline 611.3 & 273.16 & 272.80 & -0.132 \\
\hline 698 & 275 & 274.77 & -0.085 \\
\hline 800 & 276.92 & 276.80 & -0.044 \\
\hline 1000 & 280.13 & 280.15 & 0.007 \\
\hline 1200 & 282.81 & 282.92 & 0.037 \\
\hline 1400 & 285.13 & 285.28 & 0.053 \\
\hline 1600 & 287.17 & 287.35 & 0.063 \\
\hline 1800 & 288.99 & 289.19 & 0.071 \\
\hline 2000 & 290.65 & 290.86 & 0.072 \\
\hline 2500 & 294.23 & 294.44 & 0.072 \\
\hline 3000 & 297.23 & 297.43 & 0.066 \\
\hline 4000 & 302.12 & 302.26 & 0.047 \\
\hline 5000 & 306.03 & 306.13 & 0.032 \\
\hline 6000 & 309.31 & 309.37 & 0.019 \\
\hline 8000 & 314.66 & 314.65 & -0.004 \\
\hline 10000 & 318.96 & 318.89 & -0.022 \\
\hline 12000 & 322.57 & 322.46 & -0.033 \\
\hline 14000 & 325.7 & 325.56 & -0.042 \\
\hline 16000 & 328.47 & 328.31 & -0.049 \\
\hline 18000 & 330.96 & 330.78 & -0.055 \\
\hline 20000 & 333.22 & 333.03 & -0.058 \\
\hline 25000 & 338.12 & 337.92 & -0.060 \\
\hline 30000 & 342.26 & 342.05 & -0.062 \\
\hline 40000 & 349.02 & 348.83 & -0.055 \\
\hline 50000 & 354.48 & 354.32 & -0.046 \\
\hline 60000 & 359.09 & 358.96 & -0.036 \\
\hline 80000 & 366.65 & 366.59 & -0.017 \\
\hline 100000 & 372.78 & 372.77 & -0.002 \\
\hline 101325 & 373.14 & 373.14 & 0.001 \\
\hline 120000 & 377.96 & 378.00 & 0.012 \\
\hline 140000 & 382.46 & 382.56 & 0.026 \\
\hline 160000 & 386.47 & 386.60 & 0.035 \\
\hline 180000 & 390.09 & 390.25 & 0.041 \\
\hline 200000 & 393.38 & 393.58 & 0.050 \\
\hline 250000 & 400.59 & 400.82 & 0.058 \\
\hline 300000 & 406.7 & 406.95 & 0.062 \\
\hline 400000 & 416.78 & 417.02 & 0.058 \\
\hline 500000 & 425.01 & 425.18 & 0.040 \\
\hline 600000 & 432 & 432.08 & 0.018 \\
\hline 800000 & 443.59 & 443.41 & -0.042 \\
\hline 1000000 & 453.06 & 452.57 & -0.108 \\
\hline
\end{tabular}


Saturation temperature of heavy water:

\begin{tabular}{|c|c|c|c|}
\hline$P(P a)$ & $T_{\text {sat }}(K)-$ Data & $\mathrm{T}_{\text {sat }}(\mathrm{K})-$ Corr. & $\%$ error \\
\hline 1026.4 & 283.15 & 282.86 & -0.101 \\
\hline 1442 & 288.15 & 288.12 & -0.011 \\
\hline 1999 & 293.15 & 293.26 & 0.039 \\
\hline 2737 & 298.15 & 298.33 & 0.061 \\
\hline 3702 & 303.15 & 303.35 & 0.064 \\
\hline 4950 & 308.15 & 308.32 & 0.055 \\
\hline 6549 & 313.15 & 313.27 & 0.040 \\
\hline 8575 & 318.15 & 318.22 & 0.021 \\
\hline 11121 & 323.15 & 323.16 & 0.004 \\
\hline 14283 & 328.15 & 328.10 & -0.015 \\
\hline 18200 & 333.15 & 333.07 & -0.025 \\
\hline 22987 & 338.15 & 338.03 & -0.035 \\
\hline 28800 & 343.15 & 343.01 & -0.042 \\
\hline 35820 & 348.15 & 348.00 & -0.043 \\
\hline 44230 & 353.15 & 353.00 & -0.042 \\
\hline 54230 & 358.15 & 358.01 & -0.038 \\
\hline 66070 & 363.15 & 363.04 & -0.031 \\
\hline 79980 & 368.15 & 368.07 & -0.023 \\
\hline 96250 & 373.15 & 373.10 & -0.013 \\
\hline 115170 & 378.15 & 378.14 & -0.003 \\
\hline 137060 & 383.15 & 383.18 & 0.007 \\
\hline 162270 & 388.15 & 388.22 & 0.017 \\
\hline 191100 & 393.15 & 393.24 & 0.024 \\
\hline 224100 & 398.15 & 398.28 & 0.033 \\
\hline 261500 & 403.15 & 403.30 & 0.036 \\
\hline 303900 & 408.15 & 408.31 & 0.040 \\
\hline 351700 & 413.15 & 413.32 & 0.041 \\
\hline 405300 & 418.15 & 418.30 & 0.036 \\
\hline 465300 & 423.15 & 423.27 & 0.029 \\
\hline 532300 & 428.15 & 428.23 & 0.019 \\
\hline 606700 & 433.15 & 433.17 & 0.004 \\
\hline 689200 & 438.15 & 438.08 & -0.015 \\
\hline 780300 & 443.15 & 442.98 & -0.039 \\
\hline 880800 & 448.15 & 447.85 & -0.067 \\
\hline
\end{tabular}


Saturated steam enthalpy of light water:

\begin{tabular}{|llll|}
\hline $\mathrm{P}(\mathrm{Pa})$ & $h_{\mathrm{s}, \text { sat }}$ Data & $\mathrm{h}_{\mathrm{s}, \text { sat }}-$ Corr. & \% error \\
\hline 611.3 & 2500900 & 2500372.0 & 0.021 \\
698 & 2504300 & 2503792.3 & 0.020 \\
800 & 2507800 & 2507371.3 & 0.017 \\
1000 & 2513700 & 2513361.5 & 0.013 \\
1200 & 2518700 & 2518380.8 & 0.013 \\
1400 & 2522900 & 2522711.8 & 0.007 \\
1600 & 2526700 & 2526528.0 & 0.007 \\
1800 & 2530000 & 2529944.3 & 0.002 \\
2000 & 2533000 & 2533039.5 & -0.002 \\
2500 & 2539600 & 2539719.0 & -0.005 \\
3000 & 2545100 & 2545300.8 & -0.008 \\
4000 & 2553900 & 2554336.3 & -0.017 \\
5000 & 2561000 & 2561536.5 & -0.021 \\
6000 & 2566900 & 2567544.3 & -0.025 \\
8000 & 2576500 & 2577251.5 & -0.029 \\
10000 & 2584200 & 2584973.0 & -0.030 \\
12000 & 2590600 & 2591406.5 & -0.031 \\
14000 & 2596100 & 2596933.3 & -0.032 \\
16000 & 2601000 & 2601785.5 & -0.030 \\
18000 & 2605300 & 2606115.3 & -0.031 \\
20000 & 2609300 & 2610028.0 & -0.028 \\
25000 & 2617700 & 2618438.3 & -0.028 \\
30000 & 2624800 & 2625434.5 & -0.024 \\
40000 & 2636300 & 2636701.8 & -0.015 \\
50000 & 2645400 & 2645633.0 & -0.009 \\
60000 & 2653000 & 2653055.3 & -0.002 \\
80000 & 2665300 & 2664994.0 & 0.011 \\
100000 & 2675000 & 2674446.5 & 0.021 \\
101325 & 2675600 & 2675009.3 & 0.022 \\
120000 & 2683100 & 2682294.3 & 0.030 \\
140000 & 2690000 & 2689016.8 & 0.037 \\
160000 & 2696000 & 2694905.0 & 0.041 \\
180000 & 2701400 & 2700148.5 & 0.046 \\
200000 & 2706200 & 2704878.5 & 0.049 \\
250000 & 2716500 & 2715020.0 & 0.054 \\
300000 & 2724900 & 2723430.5 & 0.054 \\
400000 & 2738100 & 2736929.3 & 0.043 \\
500000 & 2748200 & 2747591.8 & 0.022 \\
600000 & 2756400 & 2756428.0 & -0.001 \\
800000 & 2768700 & 2770598.5 & -0.069 \\
1000000 & 2777600 & 2781782.0 & -0.151 \\
\hline & & & \\
\hline
\end{tabular}




\begin{tabular}{|l|lll|l|}
\hline $2 / 15 / 93$ & WSRC-TR-92-532 & Rev. 0 & FLOWTRAN-TF Software Design & Page 555 \\
\hline
\end{tabular}

Saturated steam enthalpy of heavy water:

\begin{tabular}{|lllll|}
\hline $\mathrm{T}(\mathrm{K})$ & $\mathrm{P}_{\text {sat }}(\mathrm{T})$ & $\mathrm{h}_{\text {s, sat }}-$ Data & $\mathrm{h}_{\mathrm{s} \text { sat }}-$ Corr. & \% error \\
\hline 283.15 & 1026.4 & 2334100 & 2332881 & 0.052 \\
293.15 & 1999 & 2350800 & 2350368 & 0.018 \\
303.15 & 3702 & 2367500 & 2367741 & -0.010 \\
313.15 & 6549 & 2384100 & 2384855 & -0.032 \\
323.15 & 11121 & 2400600 & 2401631 & -0.043 \\
333.15 & 18200 & 2416900 & 2418005 & -0.046 \\
343.15 & 28800 & 2433000 & 2433927 & -0.038 \\
353.15 & 44230 & 2448800 & 2449393 & -0.024 \\
363.15 & 66070 & 2464300 & 2464368 & -0.003 \\
373.15 & 96250 & 2479300 & 2478853 & 0.018 \\
383.15 & 137060 & 2493900 & 2492856 & 0.042 \\
393.15 & 191100 & 2507300 & 2506371 & 0.037 \\
403.15 & 261500 & 2521200 & 2519433 & 0.070 \\
413.15 & 351700 & 2533800 & 2532051 & 0.069 \\
423.15 & 465300 & 2545600 & 2544215 & 0.054 \\
433.15 & 606700 & 2556500 & 2555967 & 0.021 \\
443.15 & 780300 & 2566300 & 2567311 & -0.039 \\
453.15 & 991200 & 2575000 & 2578274 & -0.127 \\
\hline
\end{tabular}


Constant pressure specific hear of light water steam:

\begin{tabular}{|llll|}
\hline $\mathrm{T}(\mathrm{K})$ & $\mathrm{C}_{\mathrm{ps}}-$ Data & $\mathrm{C}_{\mathrm{ps}}-$ Corr. & \% error \\
\hline 270 & 1857.43 & 1856.24 & 0.064 \\
280 & 1859.01 & 1858.48 & 0.028 \\
290 & 1860.99 & 1860.95 & 0.002 \\
300 & 1863.33 & 1863.63 & -0.016 \\
310 & 1866.01 & 1866.52 & -0.027 \\
320 & 1868.99 & 1869.62 & -0.034 \\
330 & 1872.24 & 1872.91 & -0.036 \\
340 & 1875.74 & 1876.39 & -0.035 \\
350 & 1879.47 & 1880.06 & -0.031 \\
360 & 1883.41 & 1883.90 & -0.026 \\
370 & 1887.53 & 1887.92 & -0.021 \\
380 & 1891.84 & 1892.11 & -0.014 \\
390 & 1896.3 & 1896.46 & -0.008 \\
400 & 1900.92 & 1900.96 & -0.002 \\
410 & 1905.68 & 1905.61 & 0.004 \\
420 & 1910.57 & 1910.41 & 0.009 \\
430 & 1915.58 & 1915.34 & 0.013 \\
440 & 1920.7 & 1920.40 & 0.016 \\
450 & 1925.94 & 1925.58 & 0.019 \\
460 & 1931.27 & 1930.89 & 0.020 \\
470 & 1936.69 & 1936.30 & 0.020 \\
480 & 1942.21 & 1941.83 & 0.020 \\
490 & 1947.8 & 1947.45 & 0.018 \\
500 & 1953.48 & 1953.17 & 0.016 \\
510 & 1959.23 & 1958.97 & 0.013 \\
520 & 1965.05 & 1964.86 & 0.010 \\
530 & 1970.94 & 1970.83 & 0.006 \\
540 & 1976.89 & 1976.86 & 0.001 \\
550 & 1982.9 & 1982.96 & -0.003 \\
560 & 1988.97 & 1989.12 & -0.007 \\
570 & 1995.09 & 1995.32 & -0.012 \\
580 & 2001.27 & 2001.58 & -0.015 \\
590 & 2007.5 & 2007.87 & -0.019 \\
600 & 2013.77 & 2014.20 & -0.021 \\
610 & 2020.09 & 2020.56 & -0.023 \\
620 & 2026.45 & 2026.93 & -0.024 \\
630 & 2032.86 & 2033.33 & -0.023 \\
640 & 2039.3 & 2039.73 & -0.021 \\
650 & 2045.78 & 2046.14 & -0.017 \\
660 & 2052.3 & 2052.54 & -0.012 \\
670 & 2058.85 & 2058.94 & -0.004 \\
680 & 2065.44 & 2065.32 & 0.006 \\
690 & 2072.06 & 2071.68 & 0.018 \\
700 & 2078.71 & 2078.01 & 0.034 \\
\hline & & & \\
\hline
\end{tabular}


Constant pressure specific heat of heavy water steam:

\begin{tabular}{|llll|}
\hline $\mathrm{T}(\mathrm{K})$ & $\mathrm{C}_{\mathrm{ps}}-$ Data & $\mathrm{C}_{\mathrm{ps}}-$ Corr. & \% error \\
\hline 283.15 & 1702.4 & 1701.8 & 0.035 \\
293.15 & 1708.2 & 1707.7 & 0.029 \\
303.15 & 1714.1 & 1713.7 & 0.021 \\
313.15 & 1717.1 & 1719.9 & -0.163 \\
323.15 & 1726.5 & 1726.2 & 0.016 \\
333.15 & 1732.8 & 1732.7 & 0.008 \\
343.15 & 1739.4 & 1739.2 & 0.010 \\
353.15 & 1746.0 & 1745.9 & 0.004 \\
363.15 & 1752.8 & 1752.7 & 0.003 \\
373.15 & 1759.7 & 1759.7 & 0.002 \\
383.15 & 1766.7 & 1766.7 & -0.001 \\
393.15 & 1773.9 & 1773.9 & 0.002 \\
403.15 & 1781.1 & 1781.1 & -0.001 \\
413.15 & 1788.5 & 1788.5 & 0.001 \\
423.15 & 1795.9 & 1795.9 & -0.002 \\
433.15 & 1803.5 & 1803.5 & 0.002 \\
443.15 & 1811.1 & 1811.1 & -0.000 \\
453.15 & 1818.8 & 1818.8 & -0.001 \\
463.15 & 1826.6 & 1826.6 & -0.001 \\
473.15 & 1834.5 & 1834.5 & 0.001 \\
483.15 & 1842.4 & 1842.4 & -0.002 \\
493.15 & 1850.4 & 1850.4 & -0.003 \\
503.15 & 1858.5 & 1858.5 & -0.001 \\
513.15 & 1866.6 & 1866.7 & -0.004 \\
523.15 & 1874.8 & 1874.9 & -0.003 \\
533.15 & 1883.1 & 1883.1 & -0.000 \\
543.15 & 1891.4 & 1891.4 & -0.000 \\
553.15 & 1899.7 & 1899.7 & -0.002 \\
563.15 & 1908.1 & 1908.1 & -0.001 \\
573.15 & 1916.5 & 1916.5 & -0.002 \\
583.15 & 1924.9 & 1925.0 & -0.005 \\
593.15 & 1933.4 & 1933.5 & -0.004 \\
603.15 & 1941.0 & 1942.0 & -0.004 \\
613.15 & 1950.4 & 1950.5 & -0.005 \\
623.15 & 1959.0 & 1959.0 & -0.002 \\
633.15 & 1967.5 & 1967.5 & -0.005 \\
643.15 & 1976.1 & 1976.2 & -0.003 \\
653.15 & 1984.6 & 1984.7 & -0.006 \\
663.15 & 1993.2 & 1993.3 & -0.004 \\
673.15 & 2001.8 & 2001.9 & -0.003 \\
683.15 & 2010.4 & 2010.4 & -0.001 \\
693.15 & 2019.0 & 2019.0 & 0.002 \\
703.15 & 2027.5 & 2027.5 & 0.001 \\
\hline & & & \\
\hline
\end{tabular}


Viscosity of light water steam:

\begin{tabular}{|lccc|}
\hline $\mathrm{T}(\mathrm{K})$ & $\mu_{\mathrm{s}}(\mu \mathrm{Pa}$ sec $)-$ Data & $\mu_{\mathrm{s}}(\mu \mathrm{Pa}$ sec $)$-Corr. \% error \\
\hline 373.15 & 12.1100 & 12.0363 & 0.609 \\
423.15 & 14.1500 & 14.0951 & 0.388 \\
473.15 & 16.1800 & 16.1489 & 0.192 \\
523.15 & 18.2200 & 18.1991 & 0.115 \\
573.15 & 20.2500 & 20.2467 & 0.016 \\
623.15 & 22.3000 & 22.2922 & 0.035 \\
648.15 & 23.3000 & 23.3144 & -0.062 \\
673.15 & 24.3000 & 24.3362 & -0.149 \\
698.15 & 25.3000 & 25.3577 & -0.228 \\
723.15 & 26.4000 & 26.3789 & 0.080 \\
\hline
\end{tabular}

Viscosity of heavy water steam:

\begin{tabular}{|cccc|}
\hline $\mathrm{T}(\mathrm{K})$ & $\mu_{\mathrm{s}}(\mu \mathrm{Pa} \mathrm{sec})-$ Data & $\mu_{\mathrm{s}}(\mu \mathrm{Pa} \mathrm{sec})-$ Corr. & \% error \\
\hline 400 & 13.6805 & 13.5930 & 0.639 \\
450 & 15.7009 & 15.6705 & 0.193 \\
500 & 17.6809 & 17.7480 & -0.380 \\
550 & 19.7408 & 19.8255 & -0.429 \\
600 & 21.8430 & 21.9030 & -0.275 \\
625 & 22.9064 & 22.9418 & -0.154 \\
650 & 23.9806 & 23.9805 & 0.000 \\
675 & 25.0578 & 25.0193 & 0.154 \\
700 & 26.1489 & 26.0580 & 0.347 \\
\hline
\end{tabular}

Thermal conductivity of light water steam:

\begin{tabular}{|llll|}
\hline $\mathrm{T}(\mathrm{K})$ & $\mathrm{k}_{\mathrm{s}}$ - Data & $\mathrm{k}_{\mathrm{s}}$-Corr. & \% error \\
\hline 373.15 & 0.0248 & 0.0245 & 1.351 \\
423.15 & 0.1287 & 0.0286 & 0.374 \\
473.15 & 0.0332 & 0.0331 & 0.183 \\
523.15 & 0.0382 & 0.0381 & 0.339 \\
573.15 & 0.0434 & 0.0434 & 0.110 \\
623.15 & 0.049 & 0.0490 & 0.099 \\
648.15 & 0.0519 & 0.0519 & 0.079 \\
673.15 & 0.0549 & 0.0548 & 0.121 \\
698.15 & 0.058 & 0.0579 & 0.223 \\
723.15 & 0.0611 & 0.0610 & 0.221 \\
\hline
\end{tabular}


Thermal conductivity of heavy water steam:

\begin{tabular}{|llll|}
\hline $\mathrm{T}(\mathrm{K})$ & $\mathrm{k}_{\mathrm{s}}$ - Data & $\mathrm{k}_{\mathrm{s}}-$ Corr. & \% error \\
\hline 400 & 0.02623 & 0.02618 & 0.187 \\
450 & 0.03038 & 0.03044 & -0.213 \\
500 & 0.03524 & 0.03527 & -0.084 \\
550 & 0.04067 & 0.04066 & 0.036 \\
600 & 0.04663 & 0.04660 & 0.060 \\
625 & 0.04981 & 0.04979 & 0.049 \\
650 & 0.05312 & 0.05311 & 0.020 \\
675 & 0.05657 & 0.05657 & -0.006 \\
700 & 0.06015 & 0.06018 & -0.046 \\
\hline
\end{tabular}

\section{B.3 Data of air property correlations}

Constant pressure specific heat of air:

\begin{tabular}{|cccc|}
\hline$T(K)$ & $C_{p a}-$ Data & $C_{p a}-$ Corr. & \% error \\
\hline 275 & 1003 & 1002.1 & 0.090 \\
300 & 1004 & 1003.7 & 0.028 \\
325 & 1006 & 1005.7 & 0.032 \\
350 & 1007 & 1008.0 & -0.096 \\
375 & 1010 & 1010.6 & -0.057 \\
400 & 1013 & 1013.5 & -0.051 \\
425 & 1016 & 1016.8 & -0.077 \\
450 & 1020 & 1020.4 & -0.038 \\
475 & 1024 & 1024.3 & -0.030 \\
500 & 1028 & 1028.6 & -0.055 \\
525 & 1033 & 1033.2 & -0.015 \\
550 & 1039 & 1038.1 & 0.090 \\
575 & 1044 & 1043.3 & 0.066 \\
600 & 1050 & 1048.9 & 0.107 \\
625 & 1056 & 1054.8 & 0.116 \\
650 & 1061 & 1061.0 & -0.001 \\
675 & 1067 & 1067.6 & -0.053 \\
700 & 1073 & 1074.4 & -0.135 \\
\hline
\end{tabular}


Dynamic viscosity of air:

\begin{tabular}{|cccc|}
\hline $\mathrm{T}(\mathrm{K})$ & $\mu_{\mathrm{a}}-$ Data & $\mu_{\mathrm{a}}$-Corr. & \% error \\
\hline 273.15 & 0.000017456 & 0.000017161 & 1.691 \\
283.15 & 0.000017848 & 0.000017652 & 1.101 \\
293.15 & 0.000018240 & 0.000018134 & 0.581 \\
303.15 & 0.000018682 & 0.000018609 & 0.392 \\
313.15 & 0.000019123 & 0.000019076 & 0.247 \\
323.15 & 0.000019515 & 0.000019536 & -0.105 \\
333.15 & 0.000019907 & 0.000019988 & -0.408 \\
343.15 & 0.000020398 & 0.000020434 & -0.178 \\
353.15 & 0.000020790 & 0.000020874 & -0.403 \\
363.15 & 0.000021231 & 0.000021307 & -0.358 \\
373.15 & 0.000021673 & 0.000021734 & -0.282 \\
393.15 & 0.000022555 & 0.000022571 & -0.071 \\
413.15 & 0.000023340 & 0.000023386 & -0.198 \\
423.15 & 0.000023732 & 0.000023786 & -0.228 \\
433.15 & 0.000024124 & 0.000024181 & -0.237 \\
453.15 & 0.000024909 & 0.000024957 & -0.192 \\
473.15 & 0.000025693 & 0.000025714 & -0.084 \\
523.15 & 0.000027557 & 0.000027537 & 0.073 \\
573.15 & 0.000029322 & 0.000029268 & 0.185 \\
623.15 & 0.000030989 & 0.000030918 & 0.228 \\
673.15 & 0.000032754 & 0.000032498 & 0.781 \\
773.15 & 0.000035794 & 0.000035475 & 0.891 \\
\hline
\end{tabular}


Thermal conductivity of air:

\begin{tabular}{|clll|}
\hline $\mathrm{T}(\mathrm{K})$ & $\mathrm{k}_{\mathrm{a}}$ - Data & $\mathrm{k}_{\mathrm{a}}$ - Corr. & \% error \\
\hline 273.15 & 0.0237 & 0.0238 & -0.325 \\
283.15 & 0.0744 & 0.0245 & -0.294 \\
293.15 & 0.0251 & 0.0252 & -0.249 \\
303.15 & 0.0258 & 0.0258 & -0.192 \\
313.15 & 0.0265 & 0.0265 & -0.124 \\
323.15 & 0.0272 & 0.0272 & -0.045 \\
333.15 & 0.0279 & 0.0279 & 0.043 \\
343.15 & 0.0286 & 0.0286 & 0.140 \\
353.15 & 0.0293 & 0.0292 & 0.246 \\
363.15 & 0.03 & 0.0299 & 0.359 \\
373.15 & 0.0307 & 0.0306 & 0.480 \\
393.15 & 0.032 & 0.0319 & 0.430 \\
413.15 & 0.0333 & 0.0332 & 0.430 \\
423.15 & 0.0336 & 0.0338 & -0.590 \\
433.15 & 0.0344 & 0.0344 & -0.105 \\
453.15 & 0.0357 & 0.0357 & -0.000 \\
473.15 & 0.037 & 0.0369 & 0.138 \\
523.15 & 0.04 & 0.0400 & -0.012 \\
573.15 & 0.0429 & 0.0430 & -0.153 \\
623.15 & 0.0457 & 0.0458 & -0.288 \\
673.15 & 0.0485 & 0.0486 & -0.212 \\
773.15 & 0.054 & 0.0539 & 0.258 \\
\hline
\end{tabular}




\section{B.4 Data of binary air-steam mass diffusivity}

Binary mass diffusivity of air-steam ${ }^{+}$

\begin{tabular}{|c|c|c|c|}
\hline $\mathrm{T}(\mathrm{K})$ & $\mathrm{D}_{12}^{*} \times 10^{5}-$ Data & $\mathrm{D}_{12}^{*} \times 10^{5}$-Corr. & \% error \\
\hline 273.15 & & 2.090 & \\
283.15 & & 2.251 & \\
293.15 & & 2.419 & \\
299.05 & 2.580 & 2.521 & 2.287 \\
303.15 & & 2.593 & \\
308.15 & & 2.683 & \\
313.15 & 2.880 & 2.774 & 3.681 \\
323.15 & & 3.960 & \\
328.15 & & 3.056 & \\
332.15 & 3.050 & 3.252 & -2.754 \\
338.15 & & 3.352 & \\
343.15 & & 3.454 & \\
348.15 & & 3.558 & \\
353.15 & & 3.663 & \\
358.15 & & 3.770 & \\
363.15 & & 3.878 & \\
368.15 & & 3.988 & \\
373.15 & & 5.175 & \\
423.15 & & 6.382 & \\
473.15 & & 7.518 & \\
523.15 & & 8.726 & \\
573.15 & & 10.002 & \\
623.15 & & & \\
\hline
\end{tabular}

*; $\mathrm{m}^{2} / \mathrm{sec}$

+ ; Mass diffusivity was computed under $\mathrm{P}=1 \mathrm{~atm}$. 


\section{Plots of fluid property correlations}

\section{C.1 Plots of liquid water property correlations}

Saturation pressure of liquid water:

Light water:

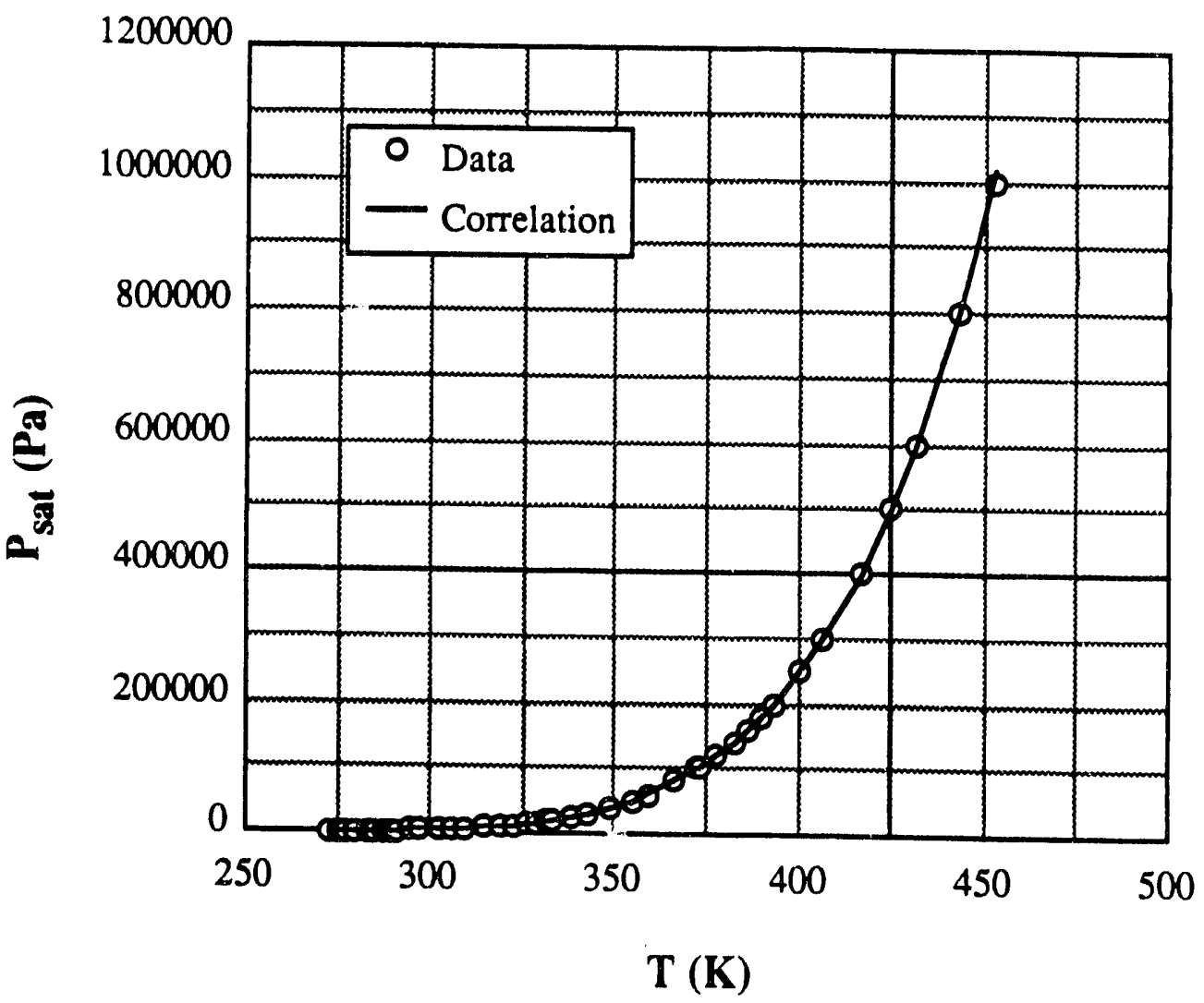


Saturation pressure of liquid water (continued):

Heavy water:

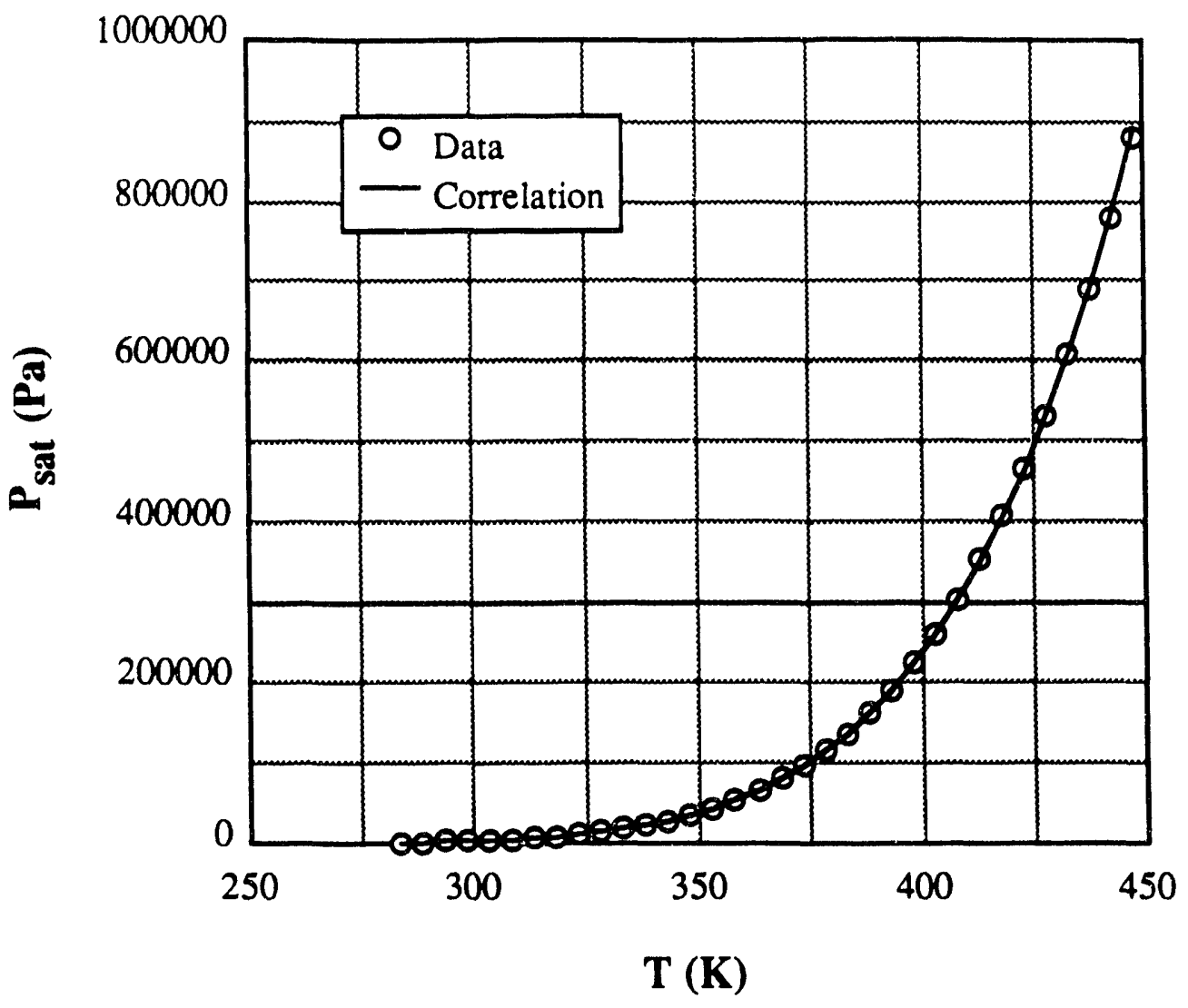


Sarurated density of liquid water:

Light water:

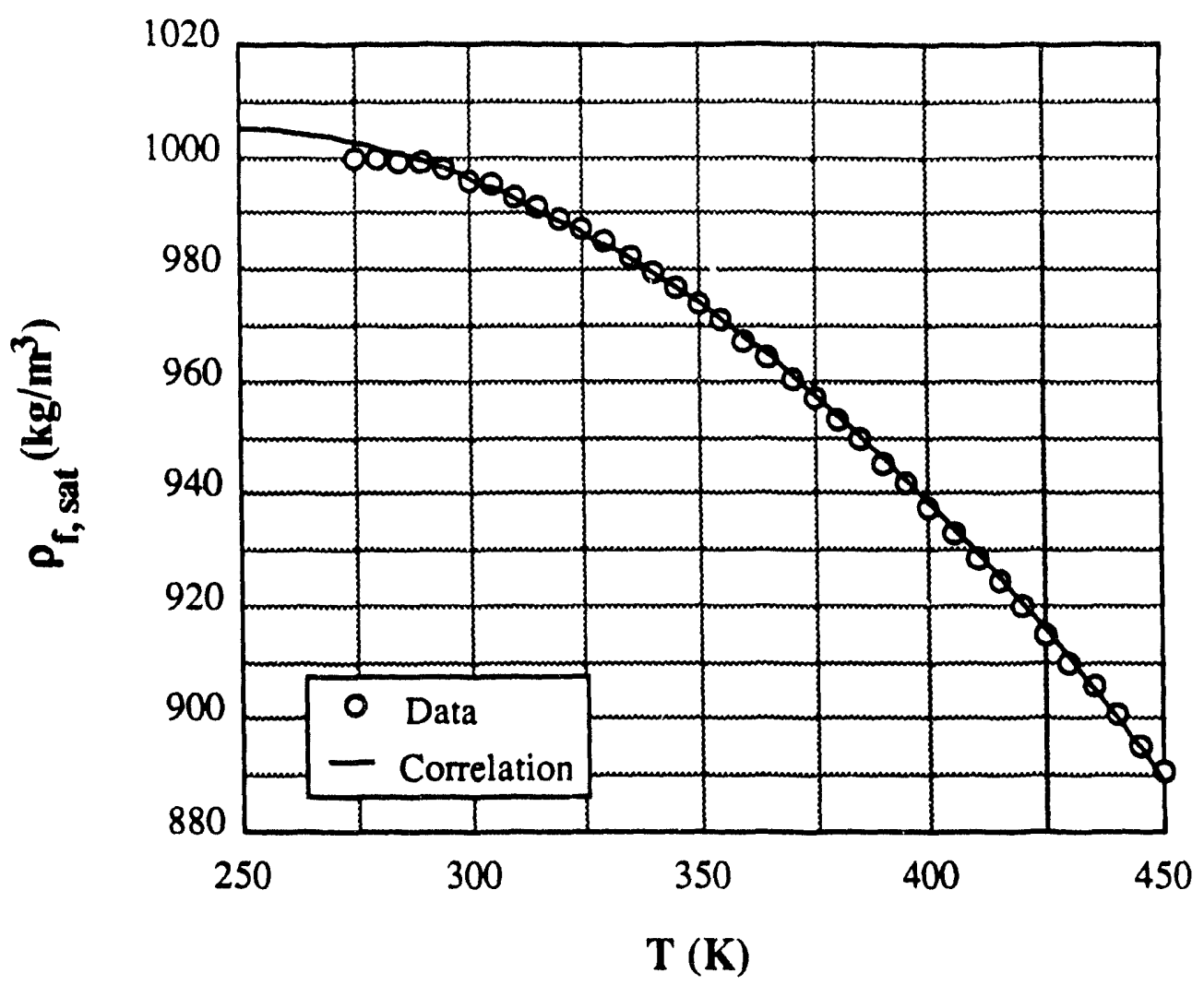




\section{Saturaced density of liquid water (continued):}

Heavy water:

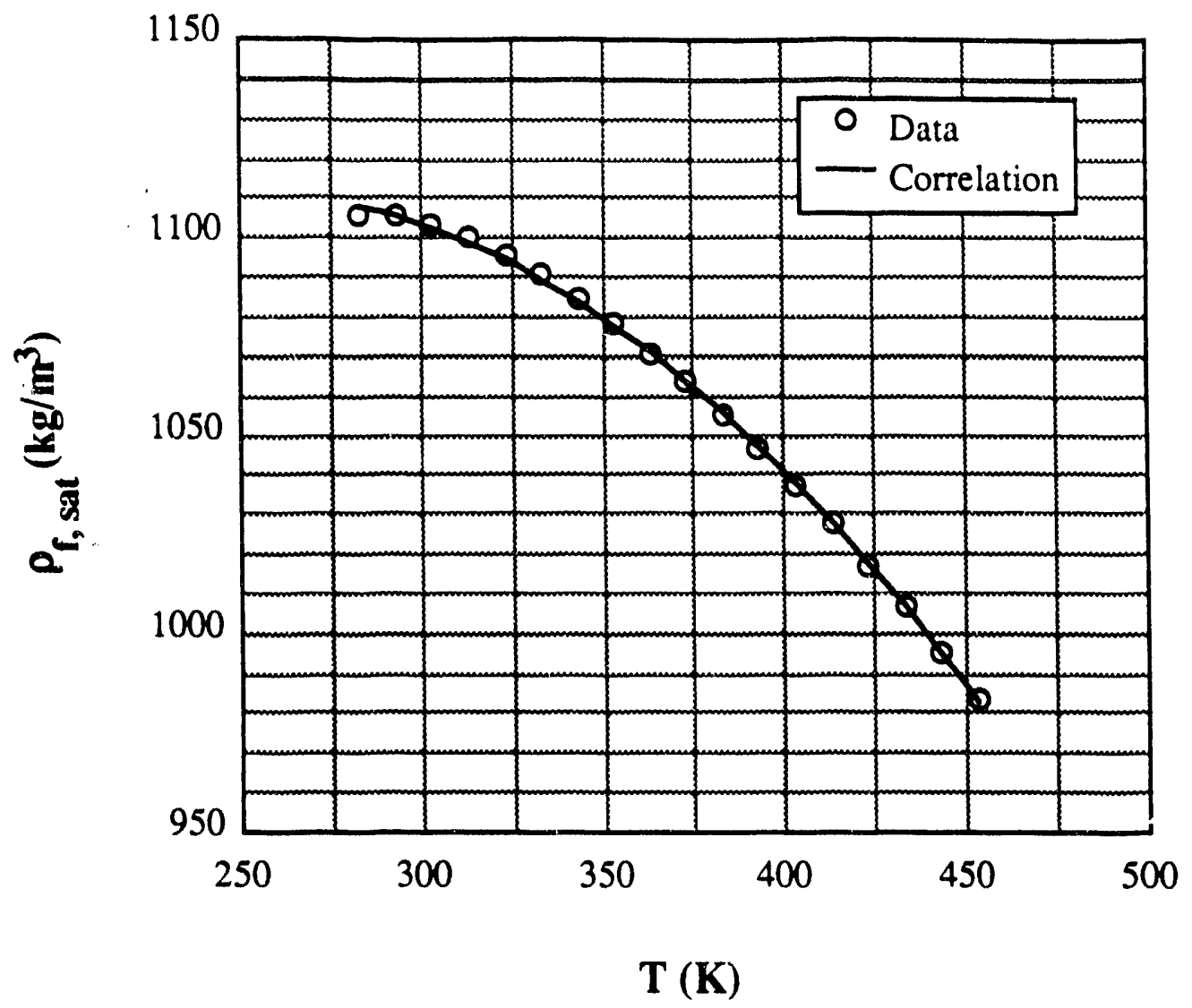


Liquid water density deravative with respect to pressure:

Light water:

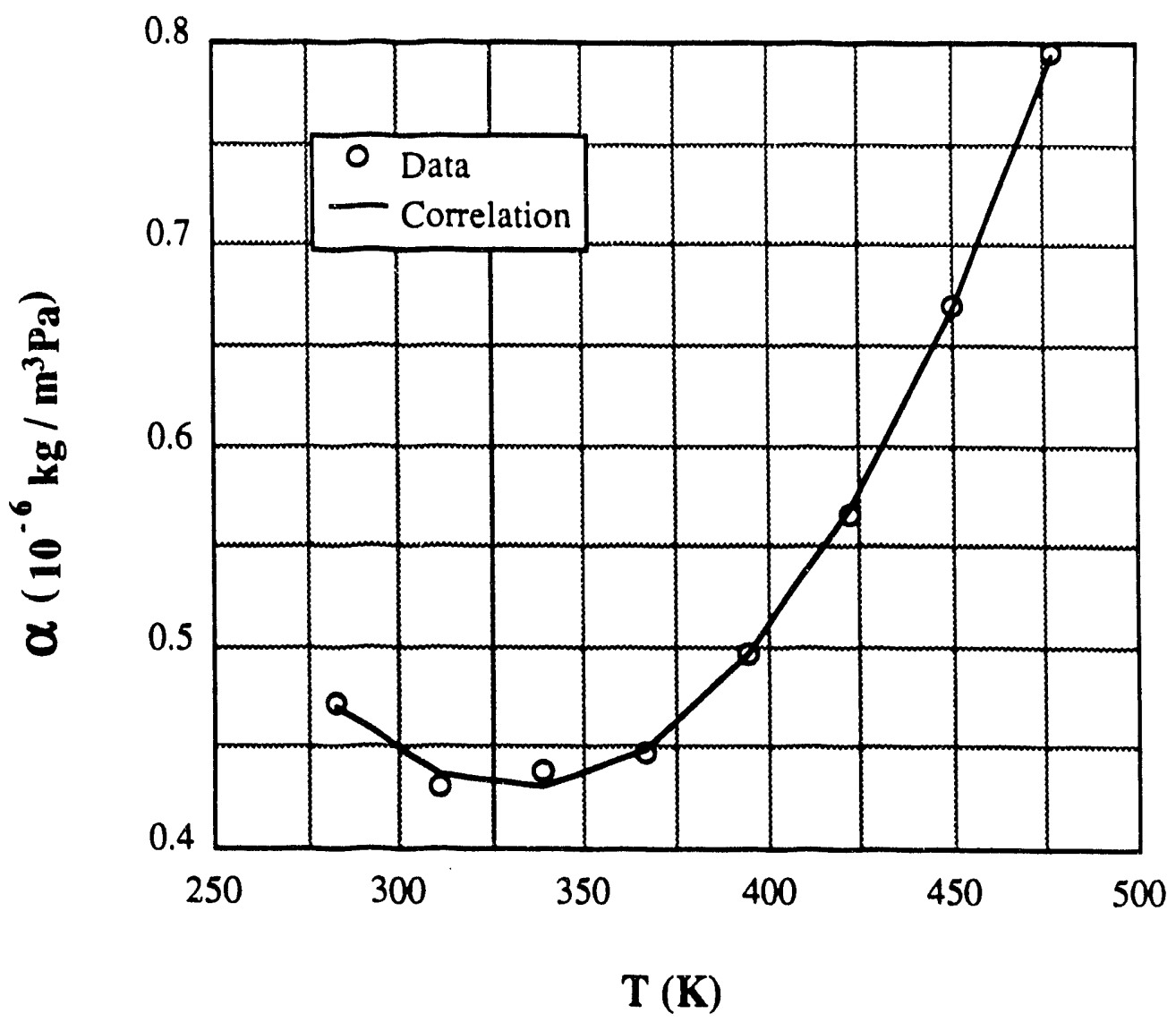


Liquid water density deravative with respect to pressure (continued):

Heavy water:

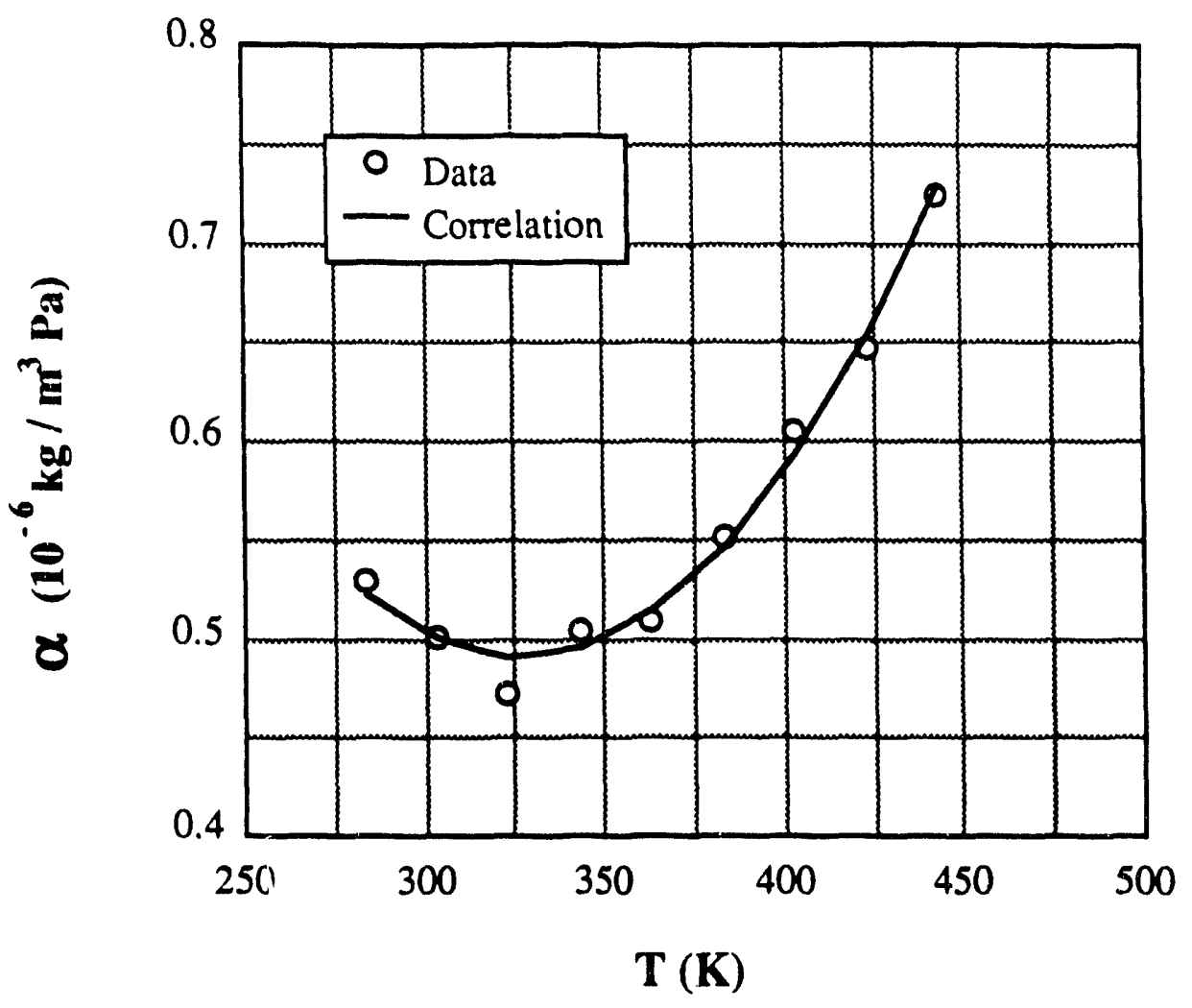




\begin{tabular}{|l|lll|l|}
\hline $2 / 15 / 93$ & WSRC-TR-92-532 Rev.0 & FLOWTRAN-TF Software Design & Page 569 \\
\hline
\end{tabular}

Saturated liquid enthalpy:

Light water:

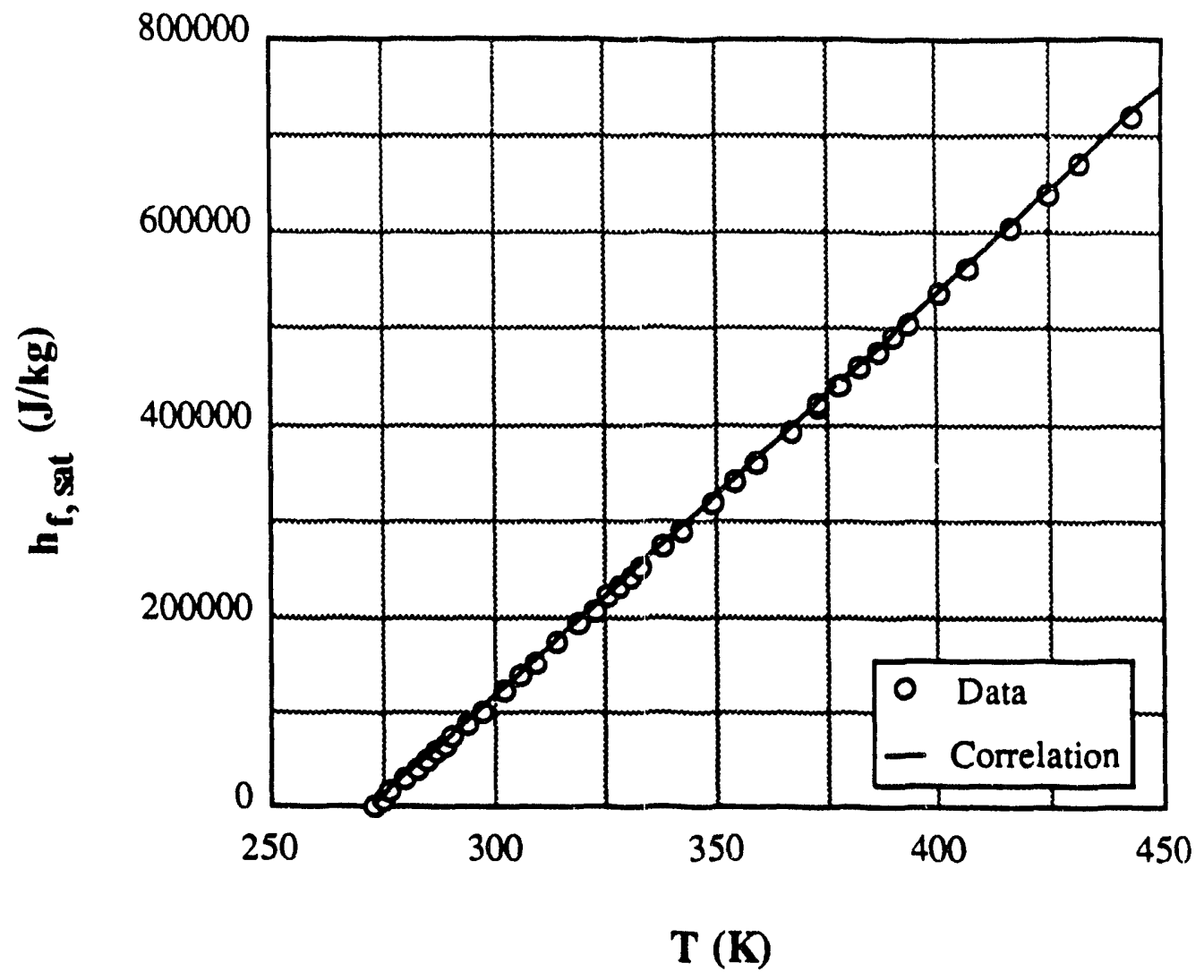


Saturated liquid enthalpy (continued):

Heavy water:

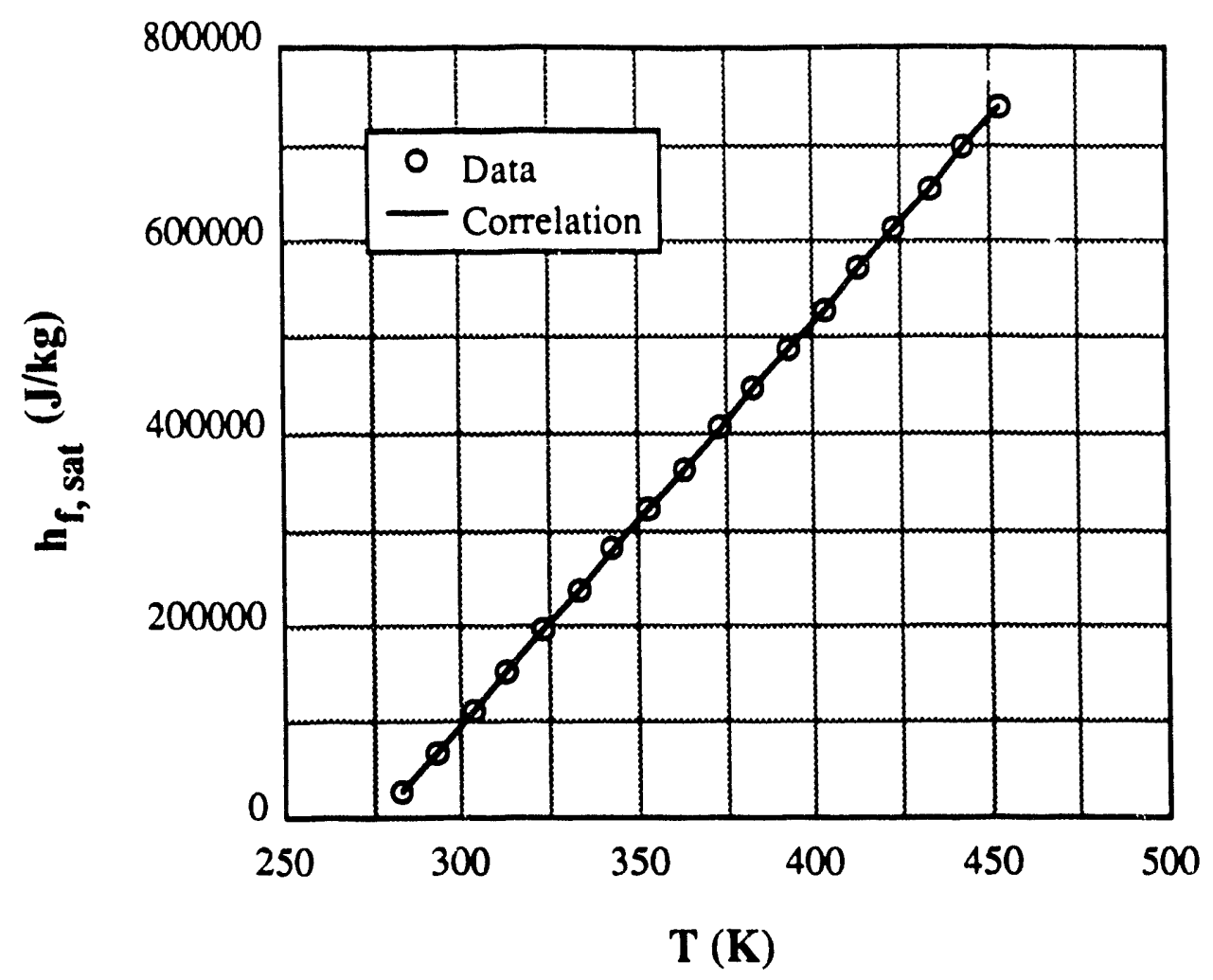


Dynamic viscosity of liquid water:

Light water:

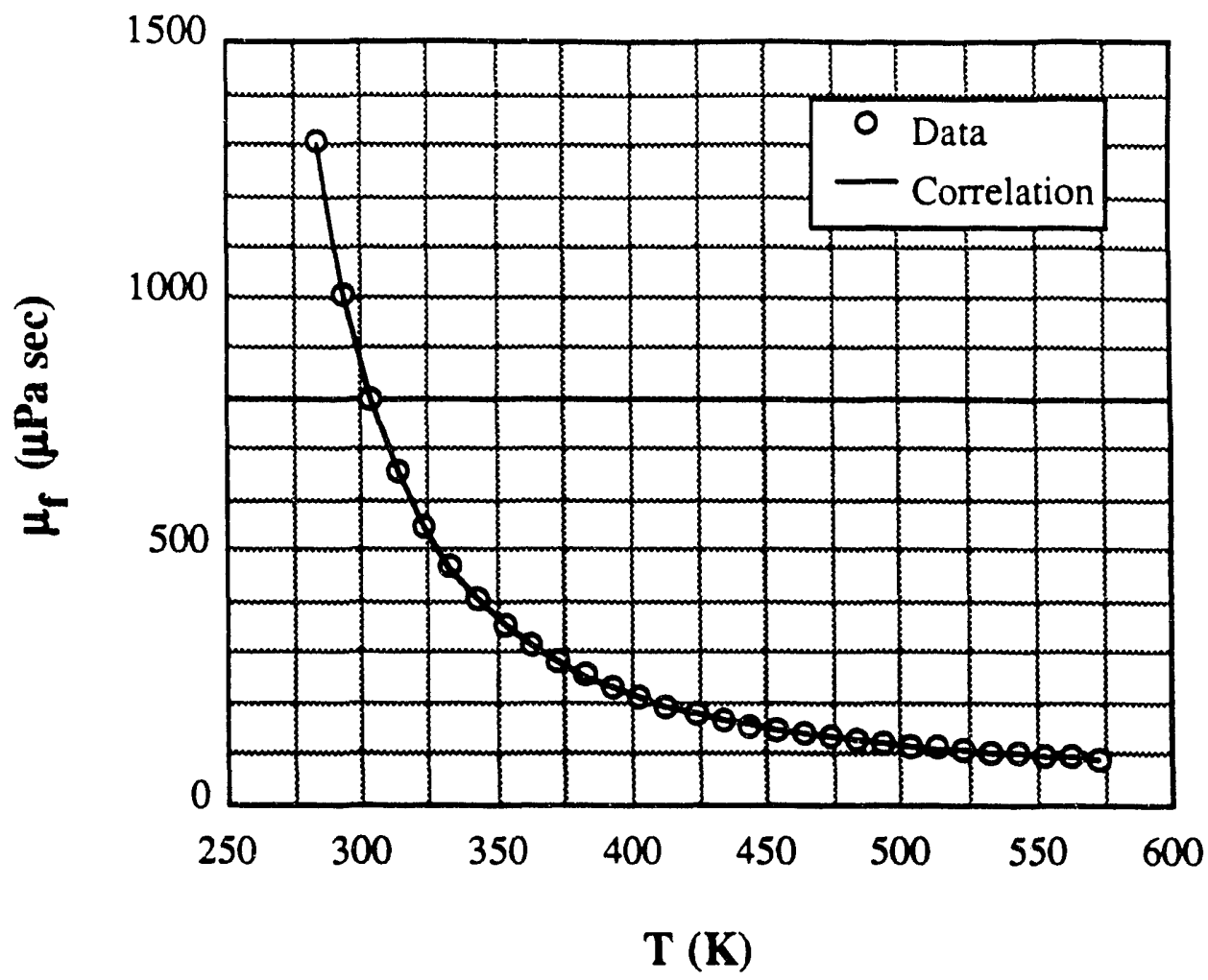


Dynamic viscosity of liquid water (continued):

Heavy water:

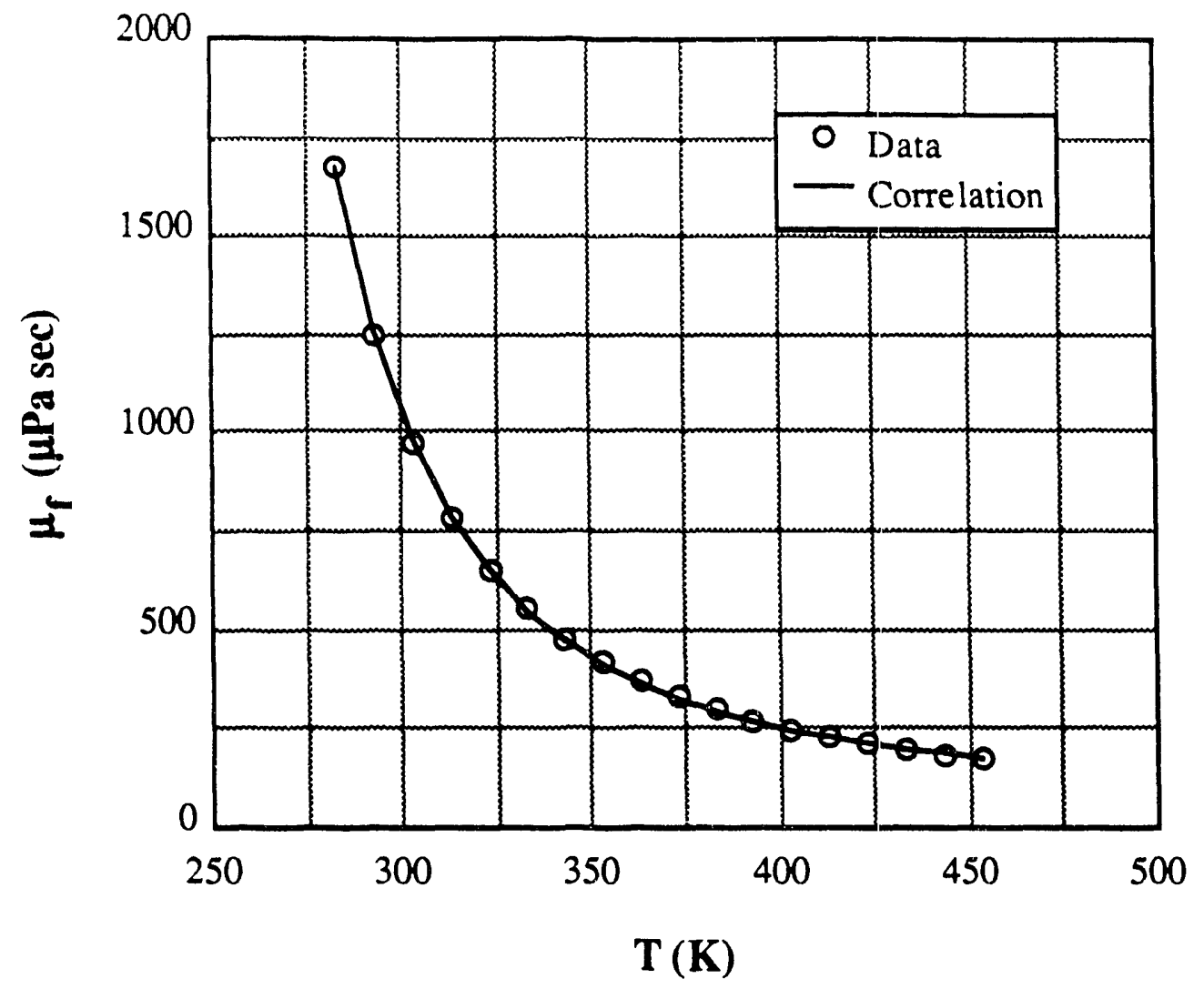


Thermal conductivity of liquid water:

Light water:

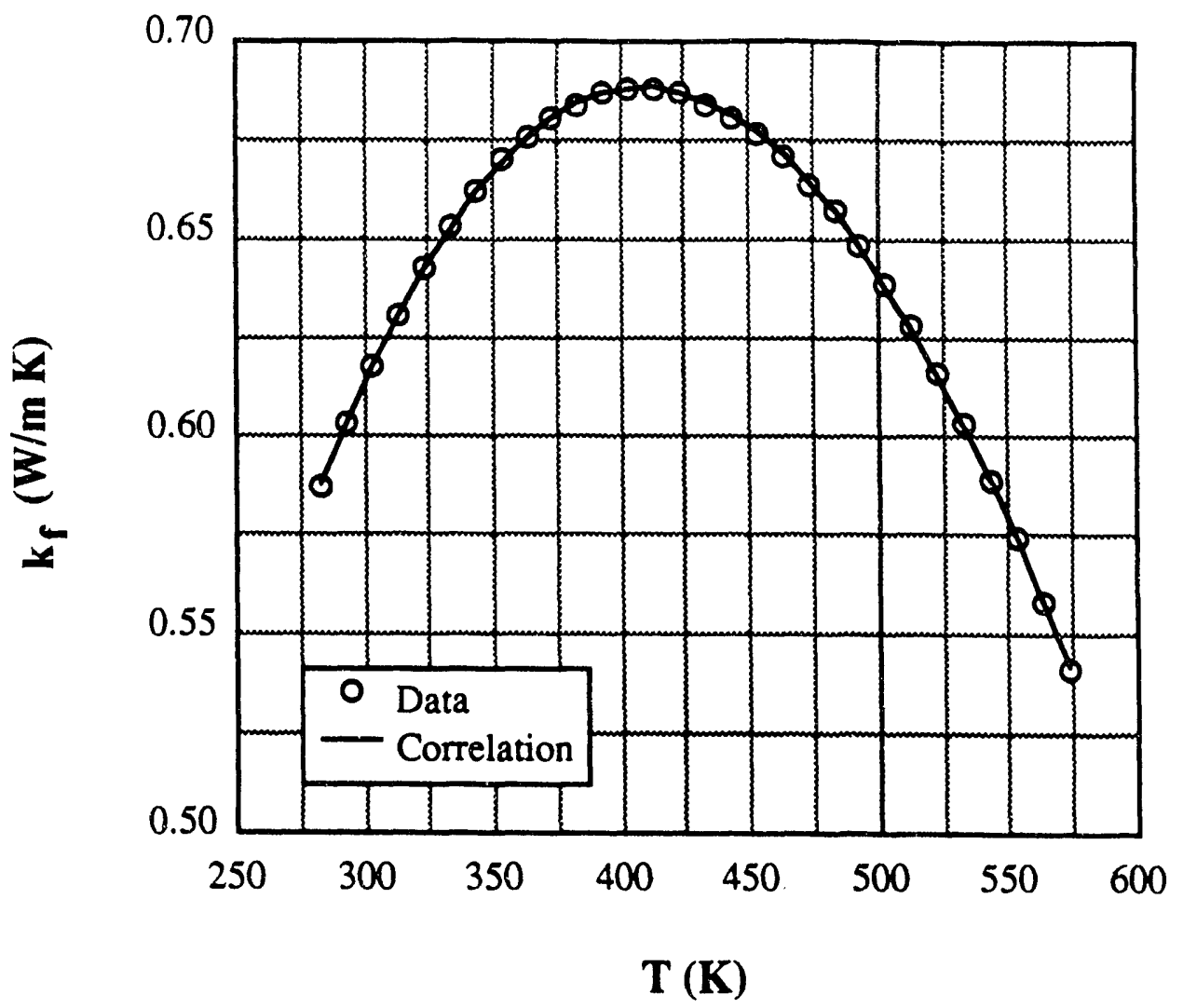


Thermal conductivity of liquid watu (continued):

Heavy water:

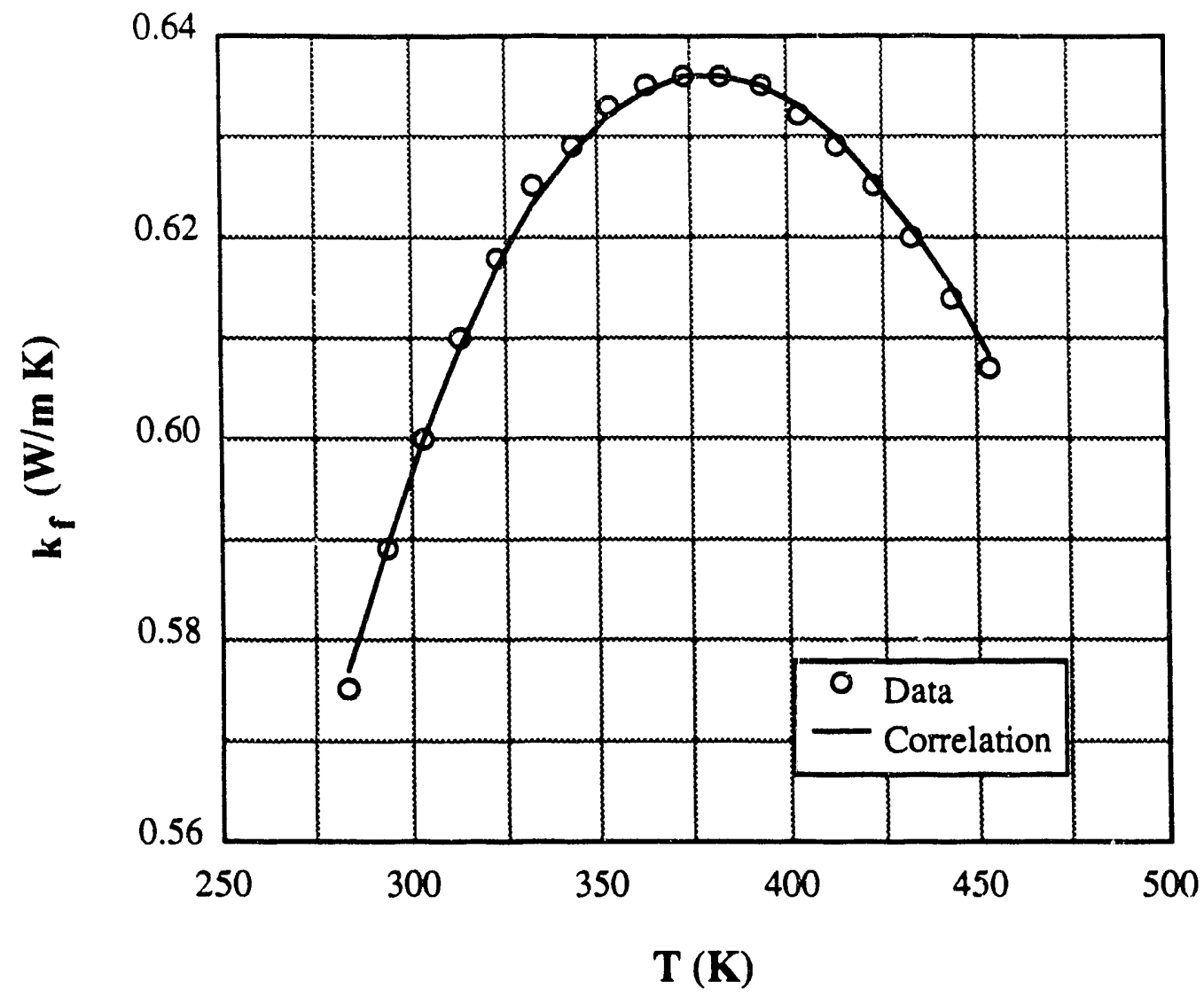


Surface tension of light water and heavy water:

nn 


\section{C.2 Plots of steam property correlations}

\section{Saturated steam density:}

Light water:

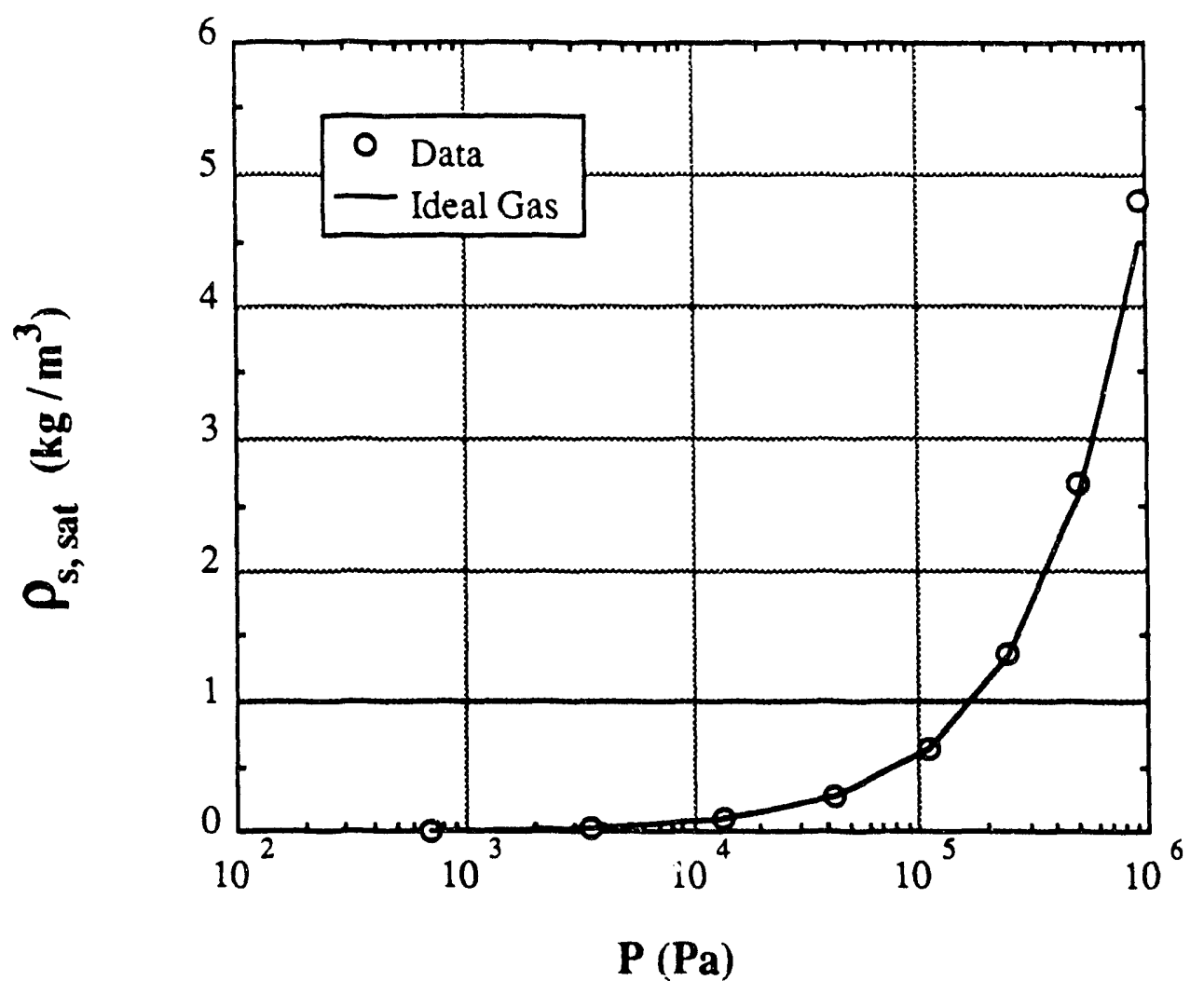


Sarurated steam density (continued):

Heavy water:

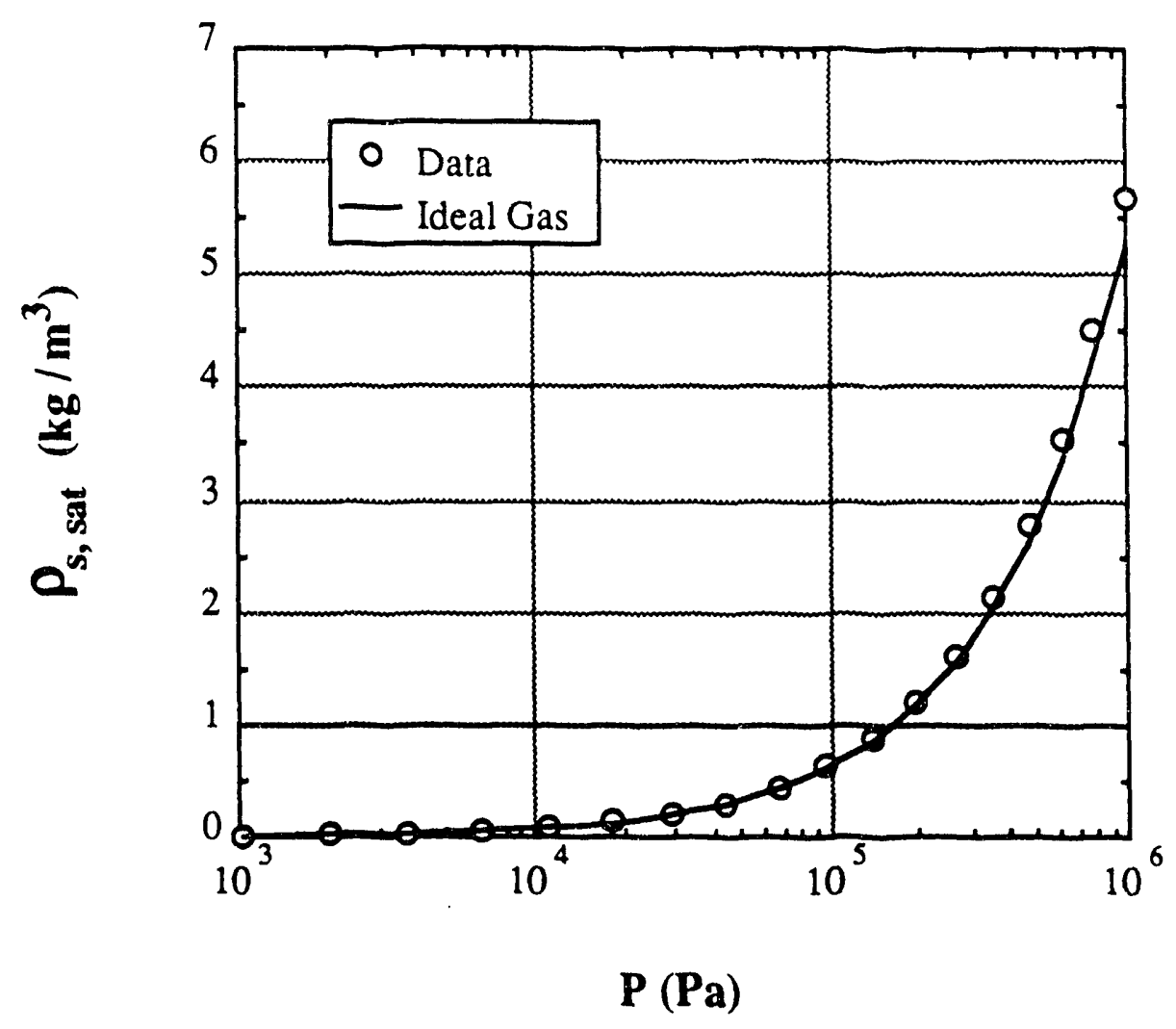


Saturation temperature of liquid water:

Light water:

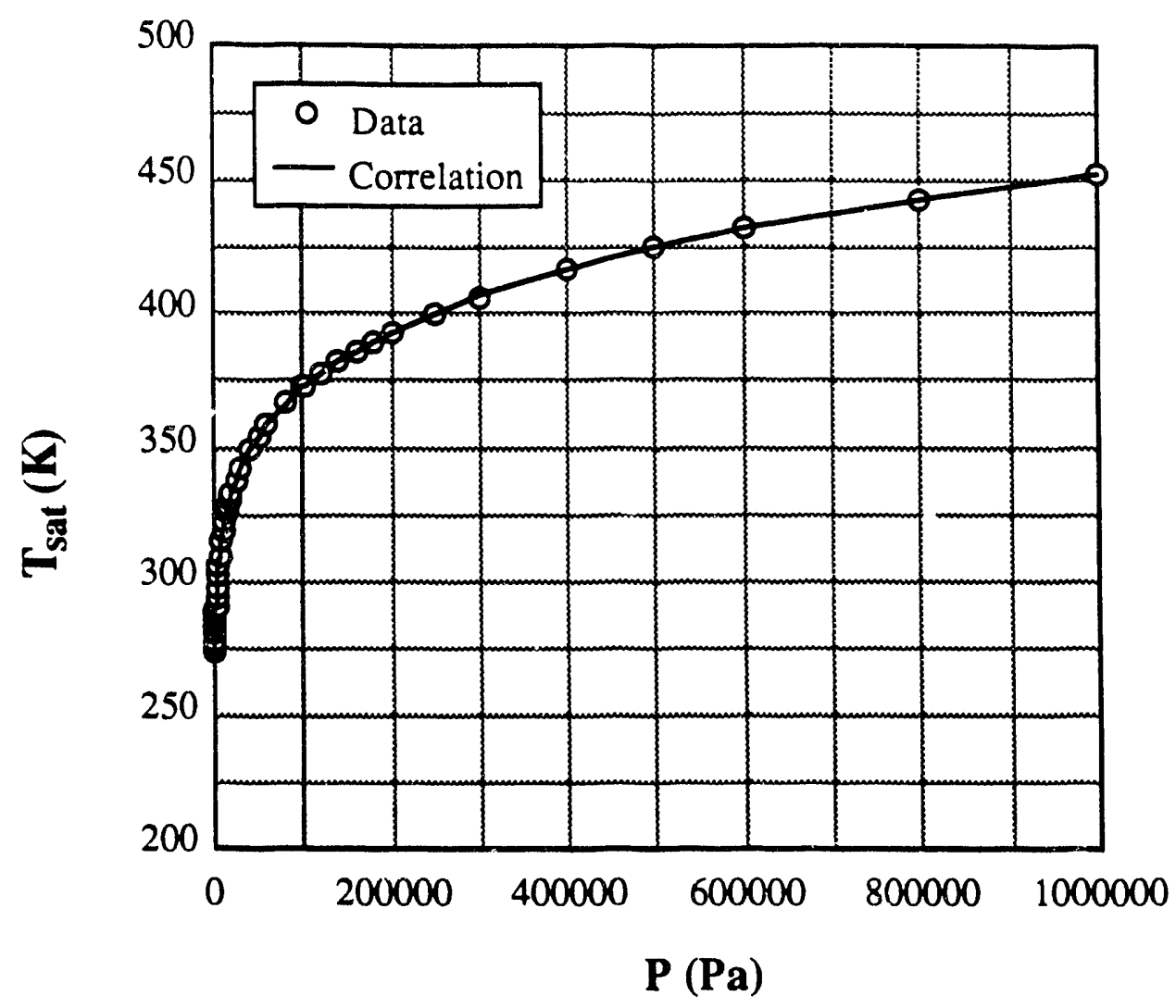


Saturation temperarure of liquid water (continued):

Heavy water:

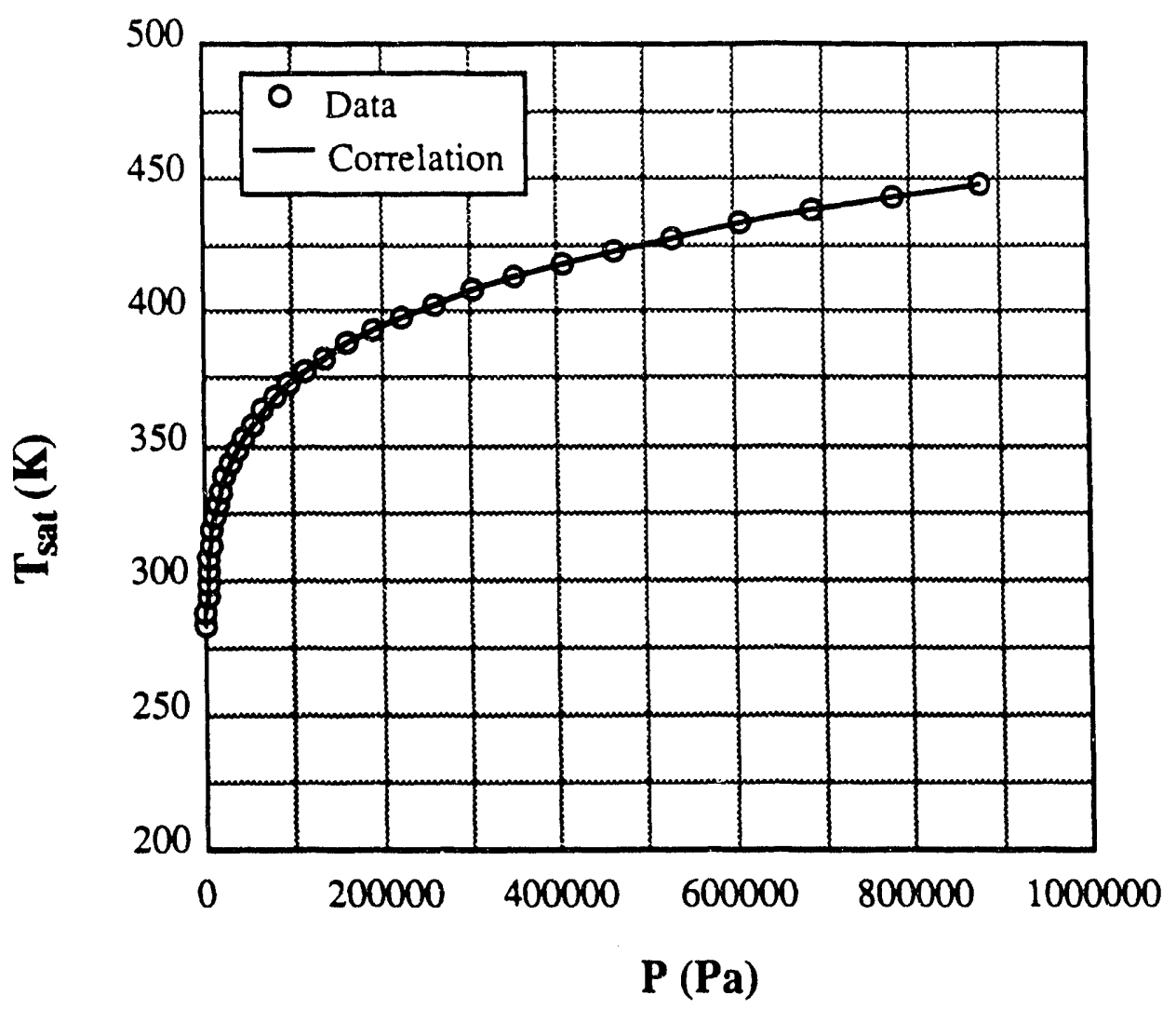


Saturated steam enthalpy:

Light water:

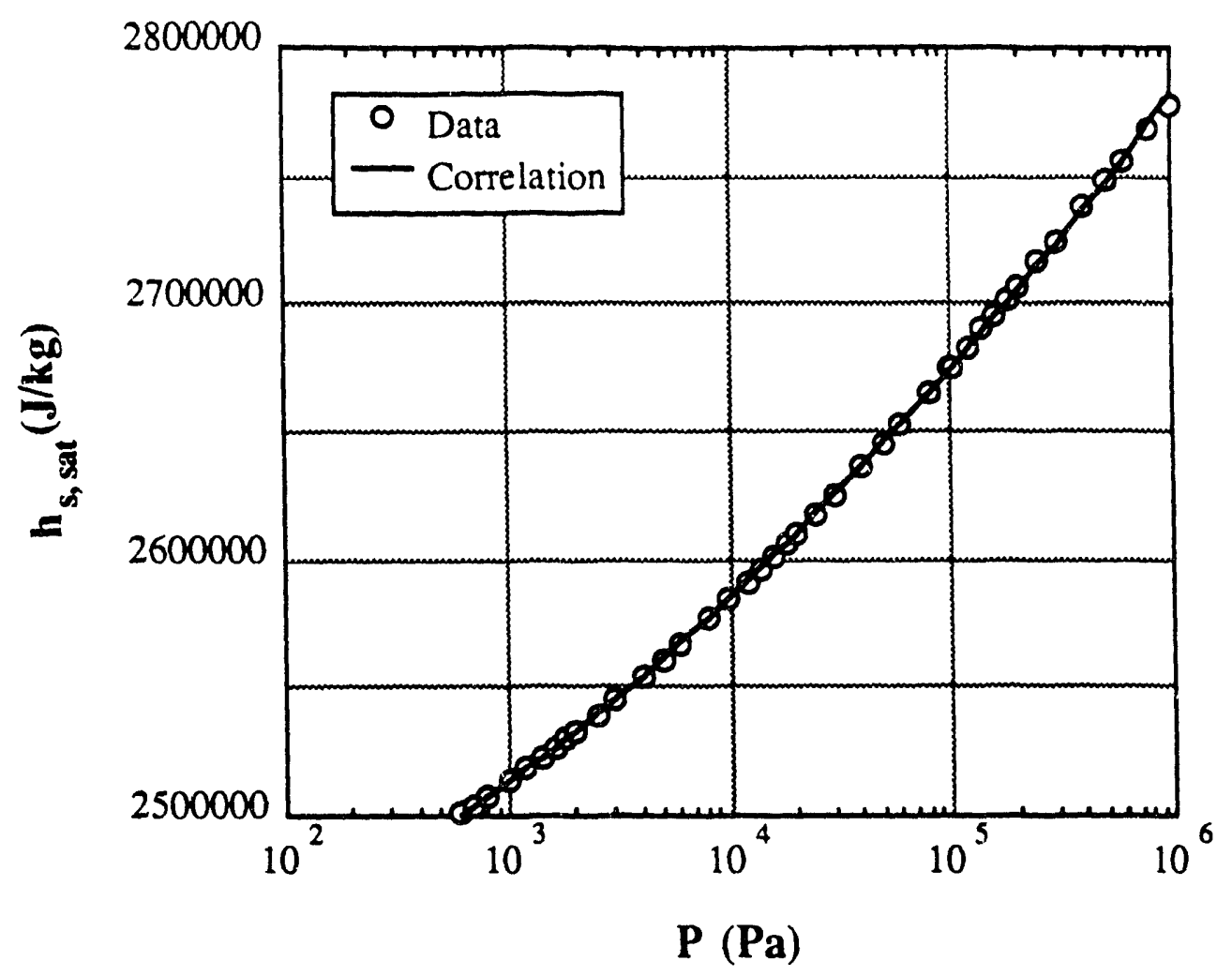




\section{Saturated steam enthalpy (continued):}

Heavy water:

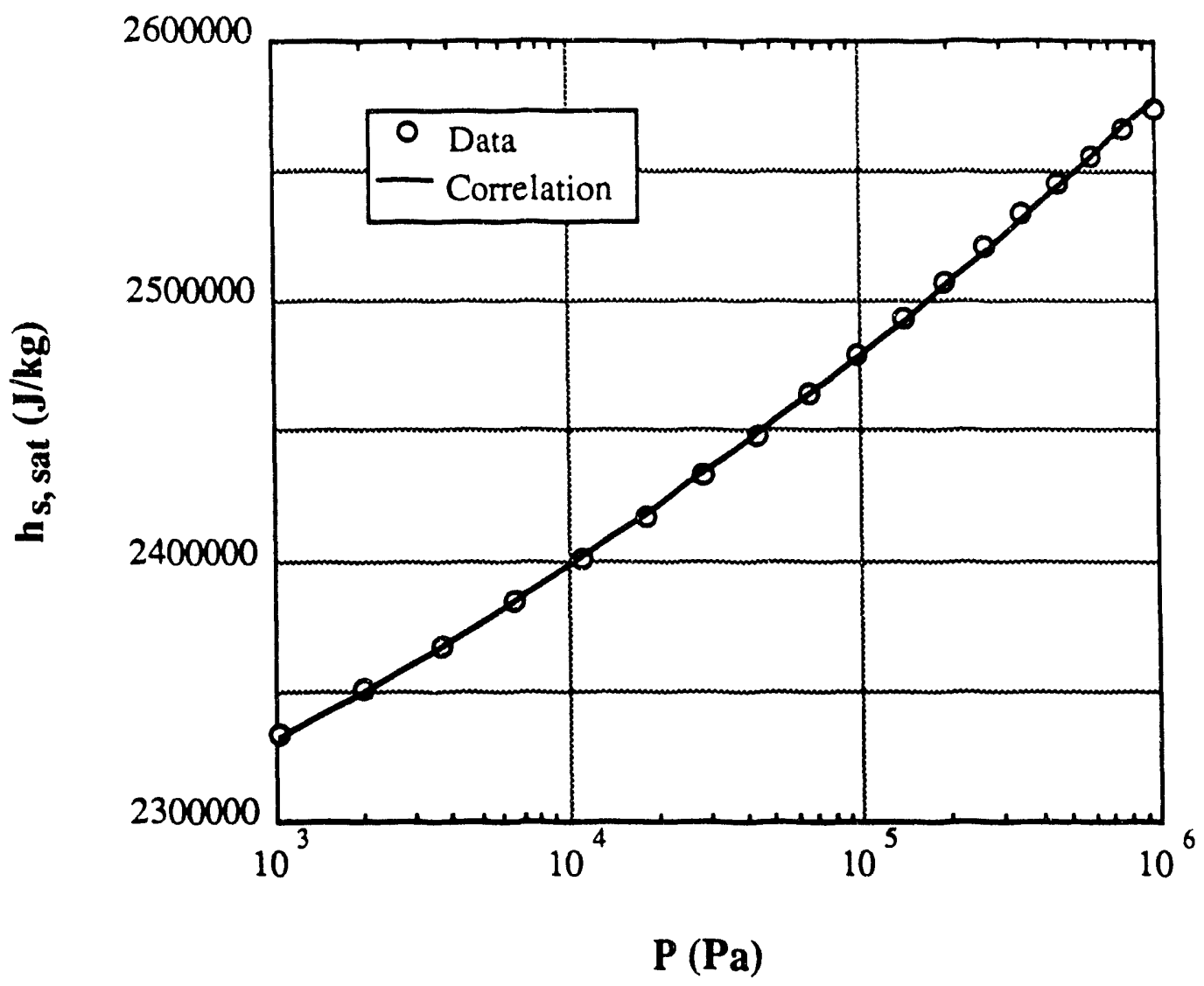




\section{Constant pressure specific heat of steam:}

Light water:

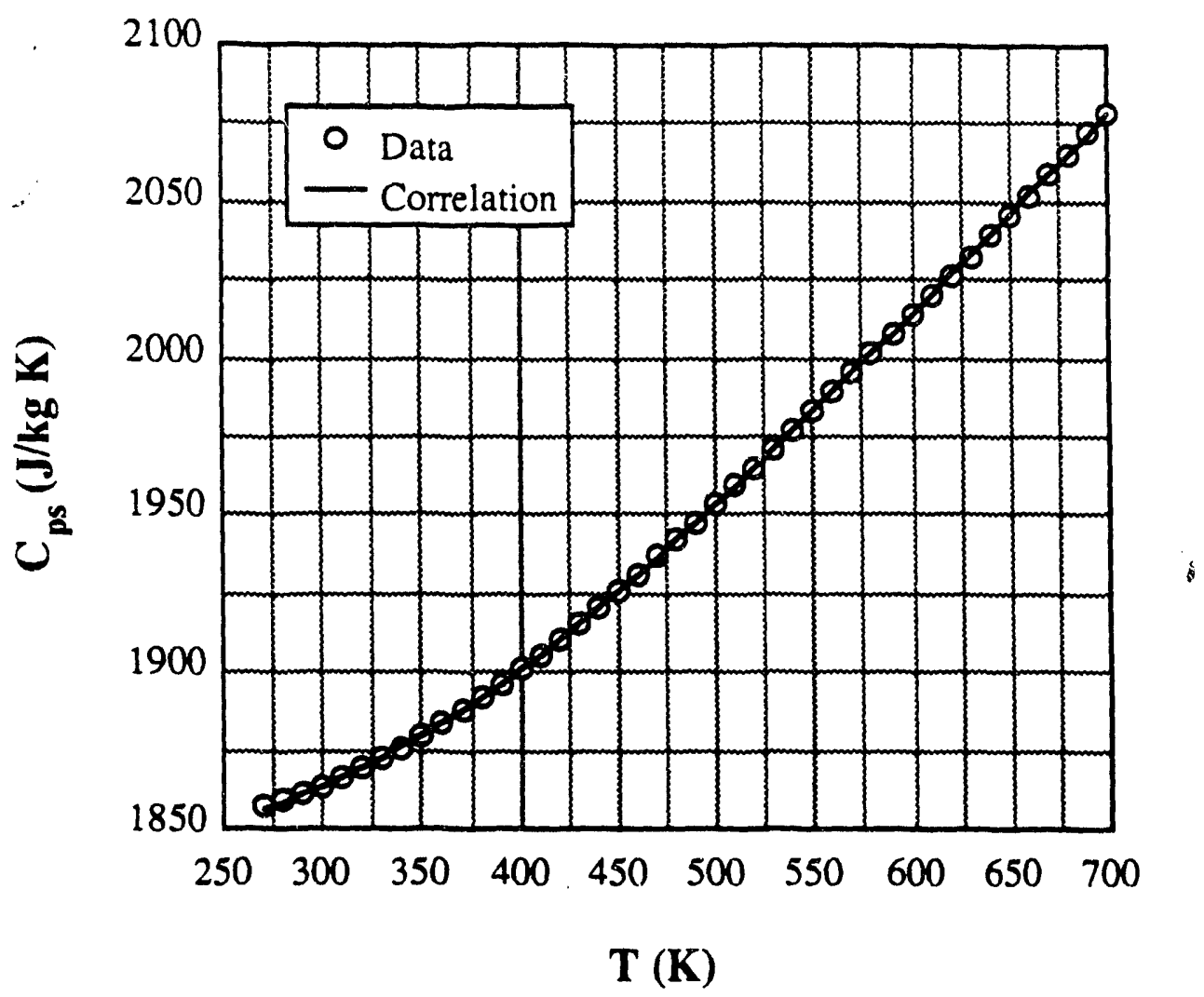


Constant pressure specific heat of steam (continued):

Heavy water:

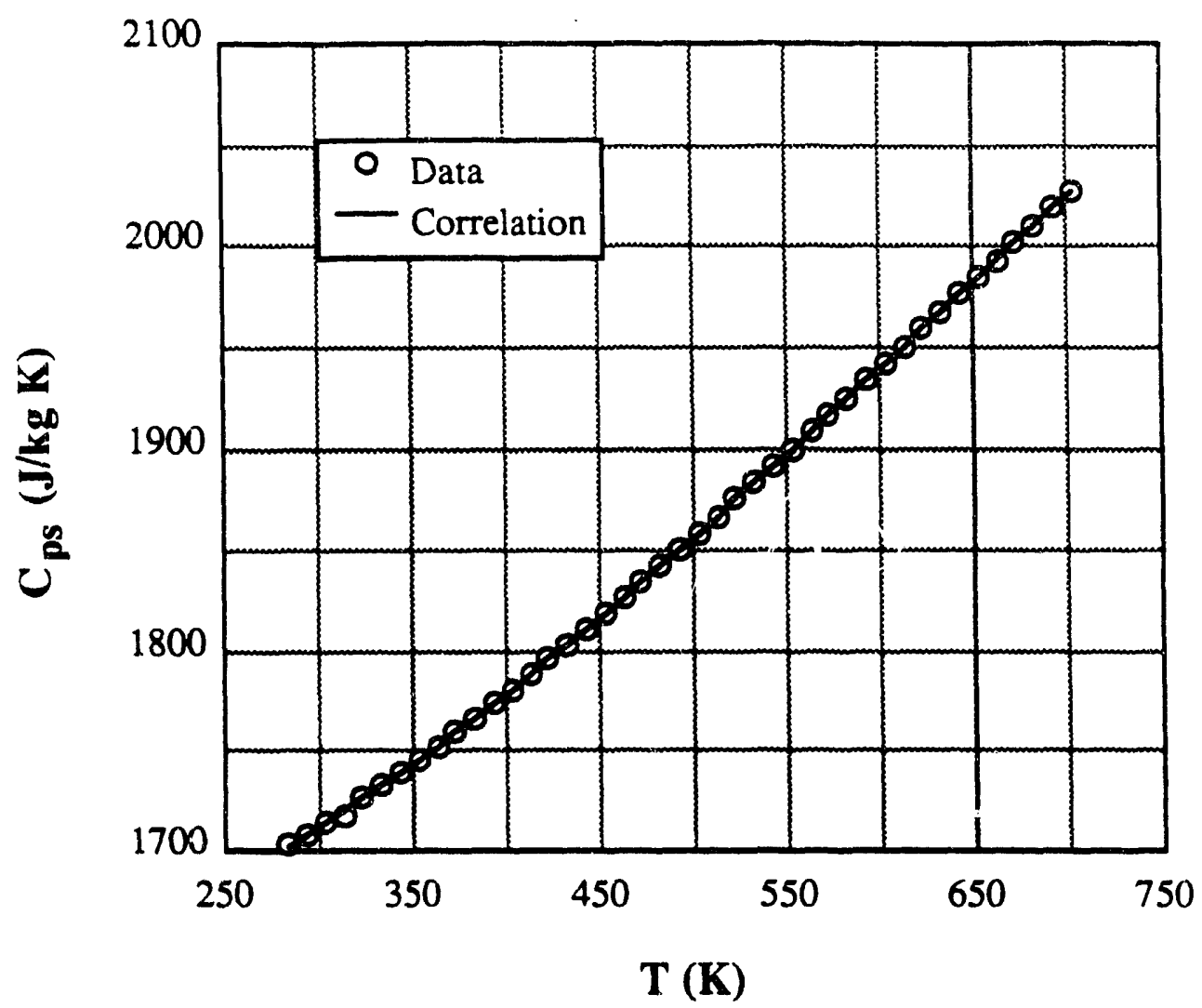




\section{Dynamic viscosity of steam:}

Light water:

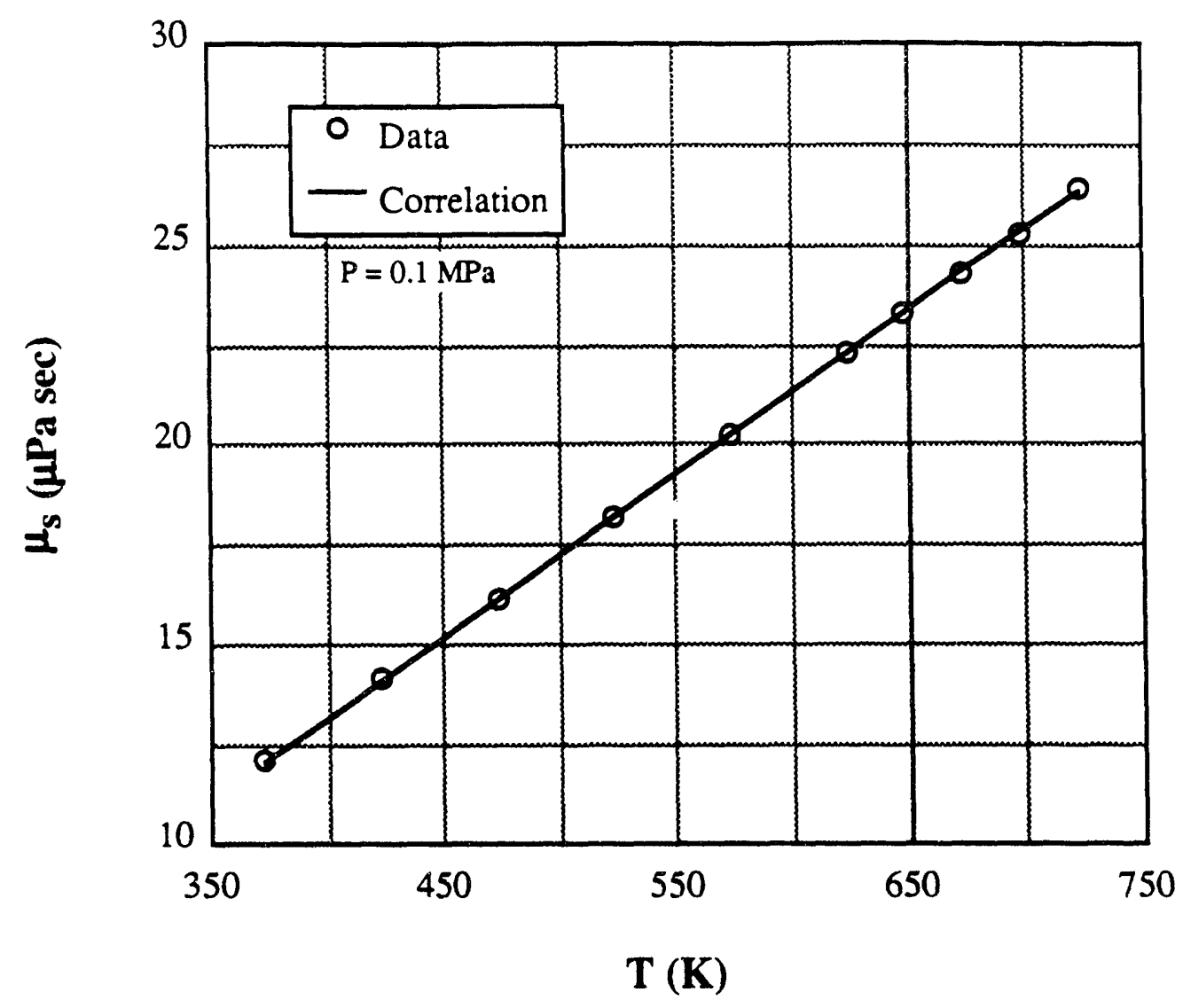


Dynamic viscosity of steam (continued):

Heavy water:

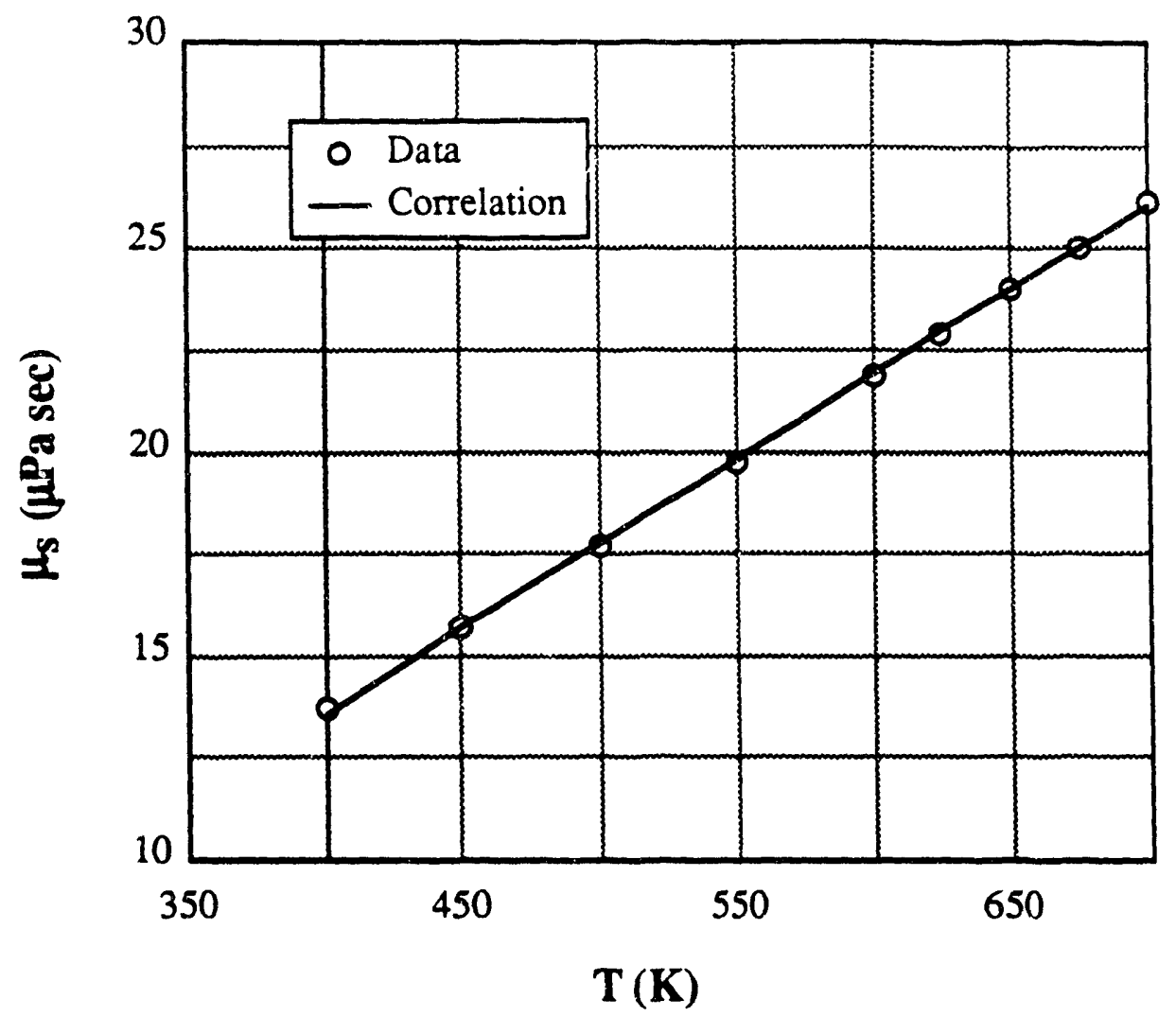


Thermal conductivity of steam;

Light water:

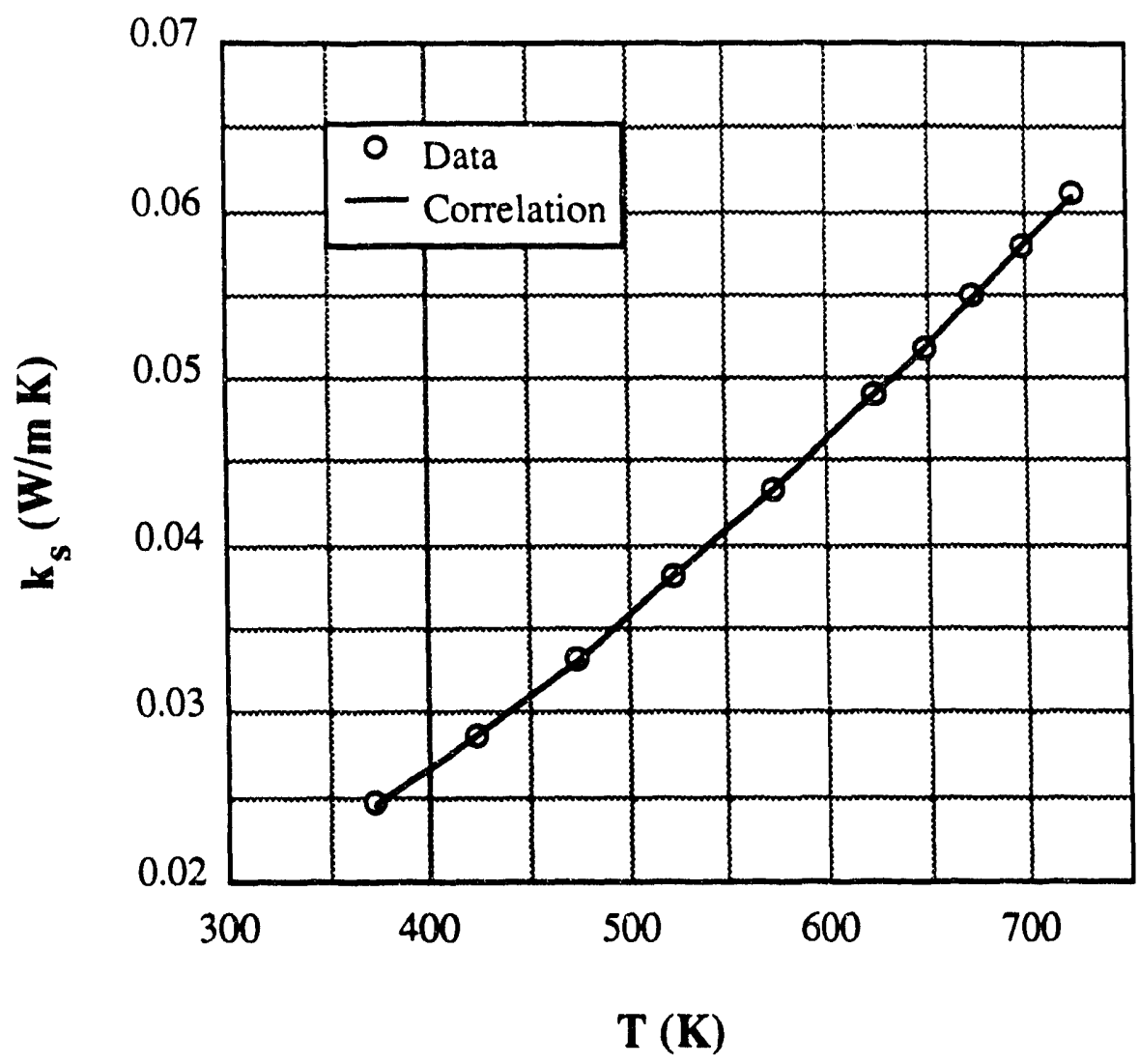


Thermal conductivity of steam (continued):

Heavy water:

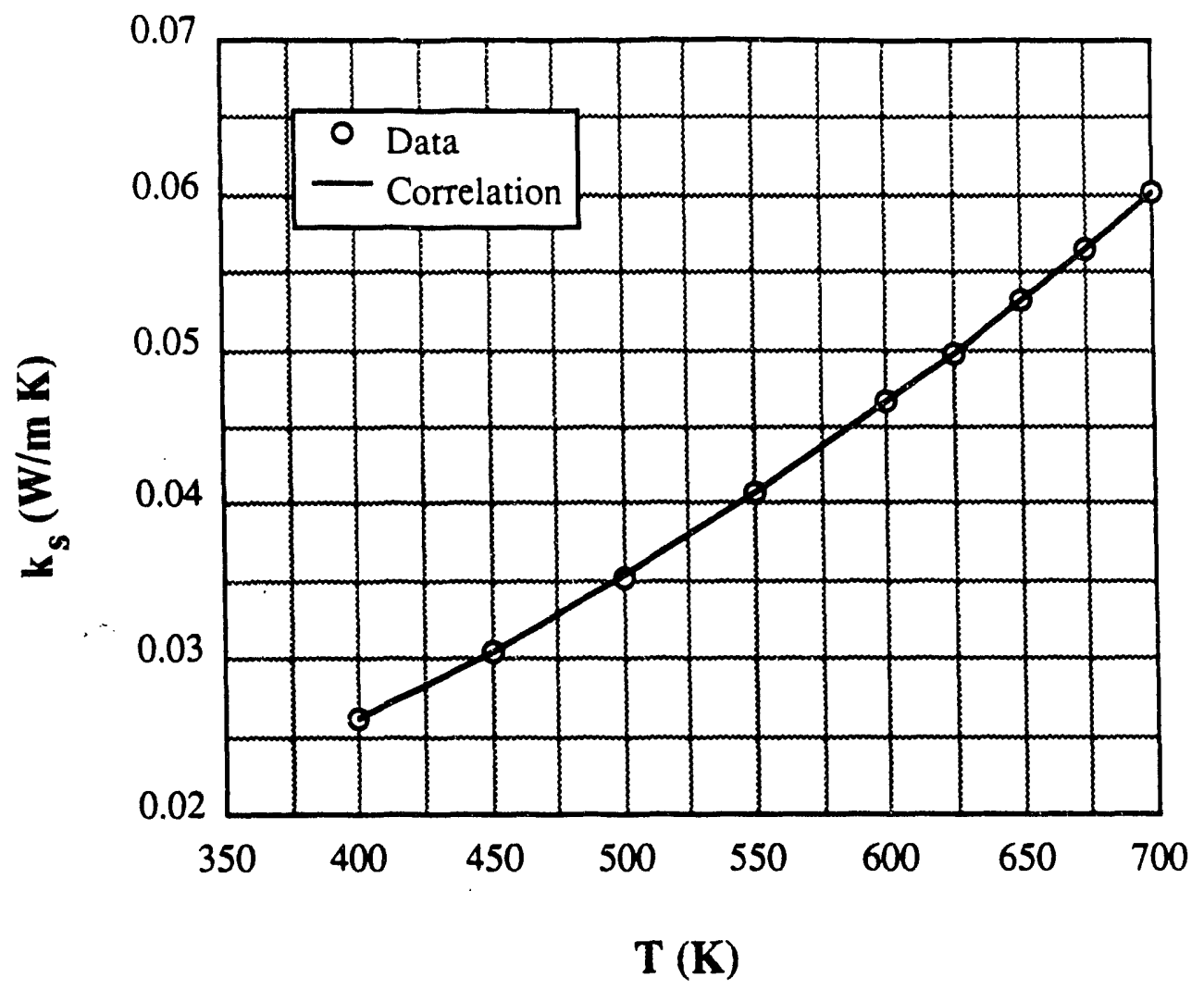




\section{C.3 Plots of air property correlations}

Constant specific heat of air:

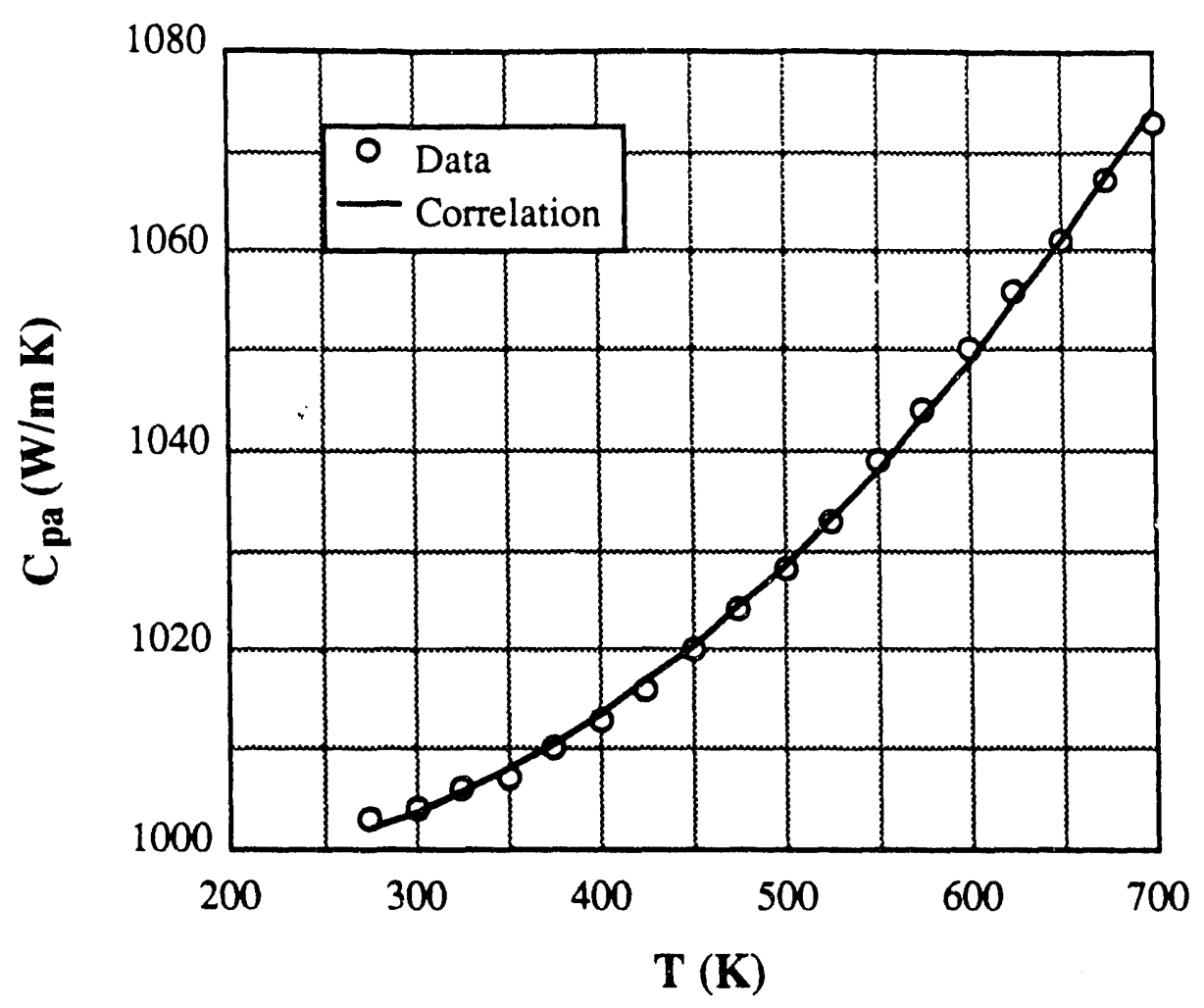


Dynamic viscosity of air:

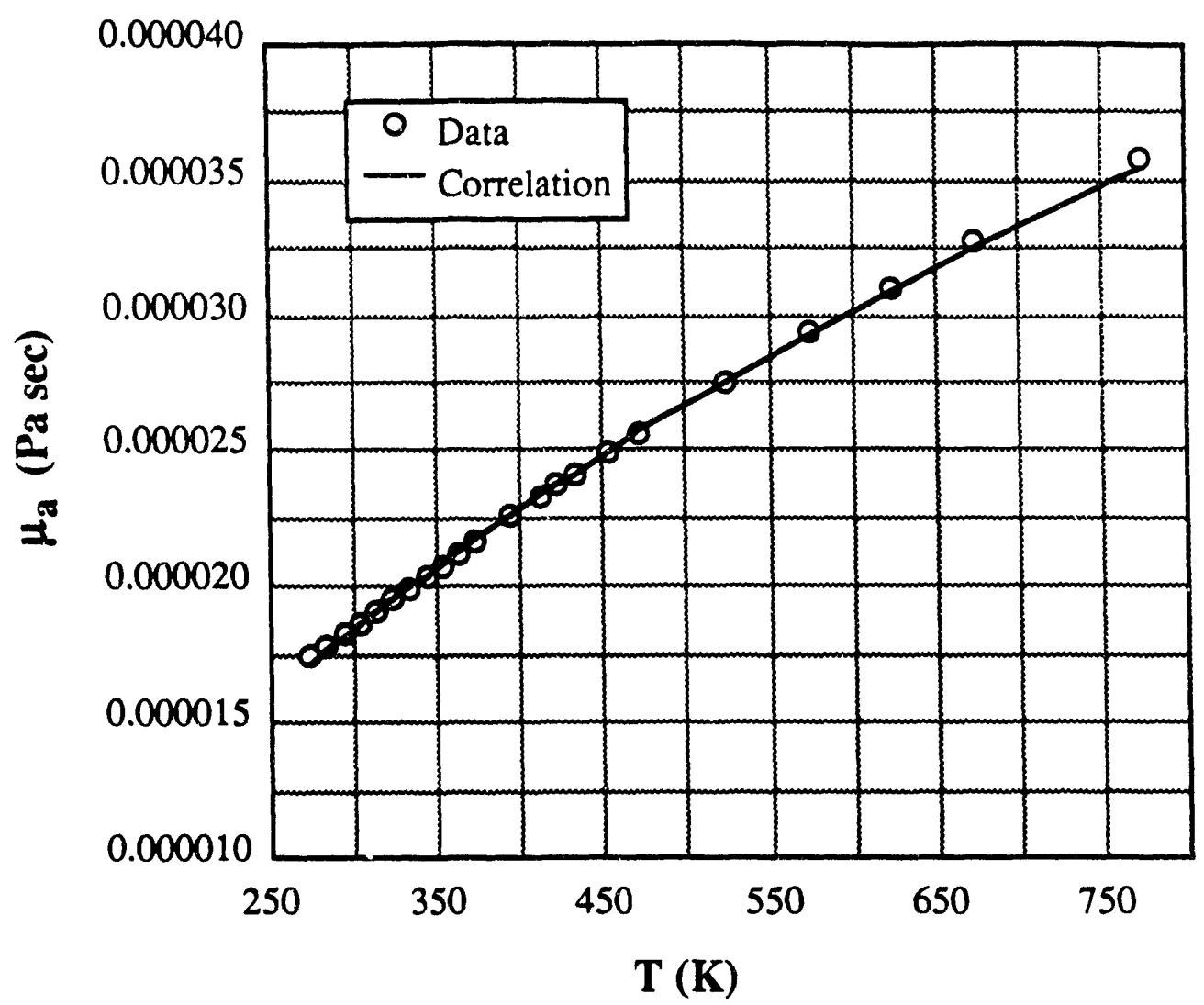


Thermal conductivity of air:

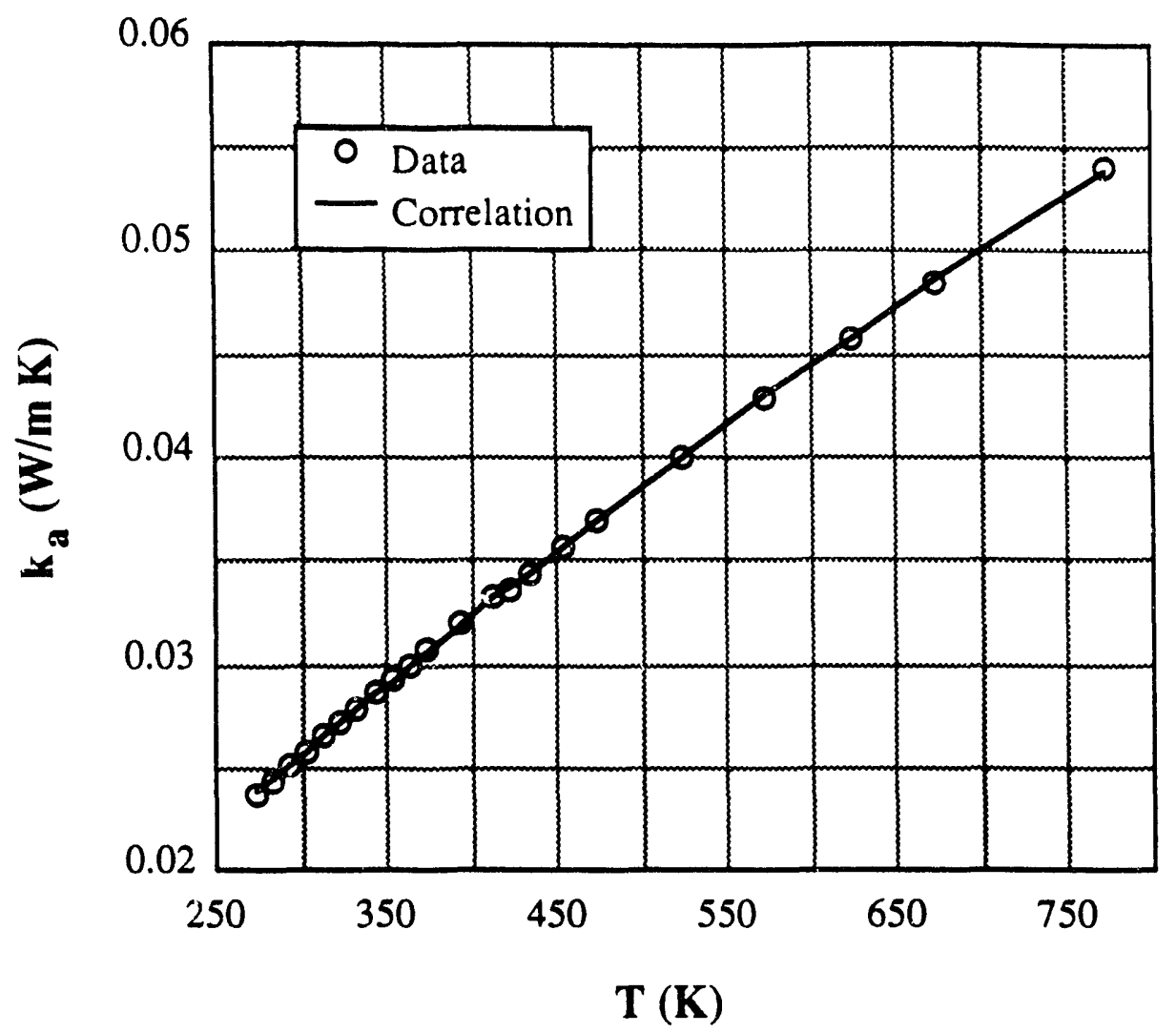




\section{C.4 Plots of binary air-steam mass diffusivity}

Binary gas mass diffusivity of air-steam:

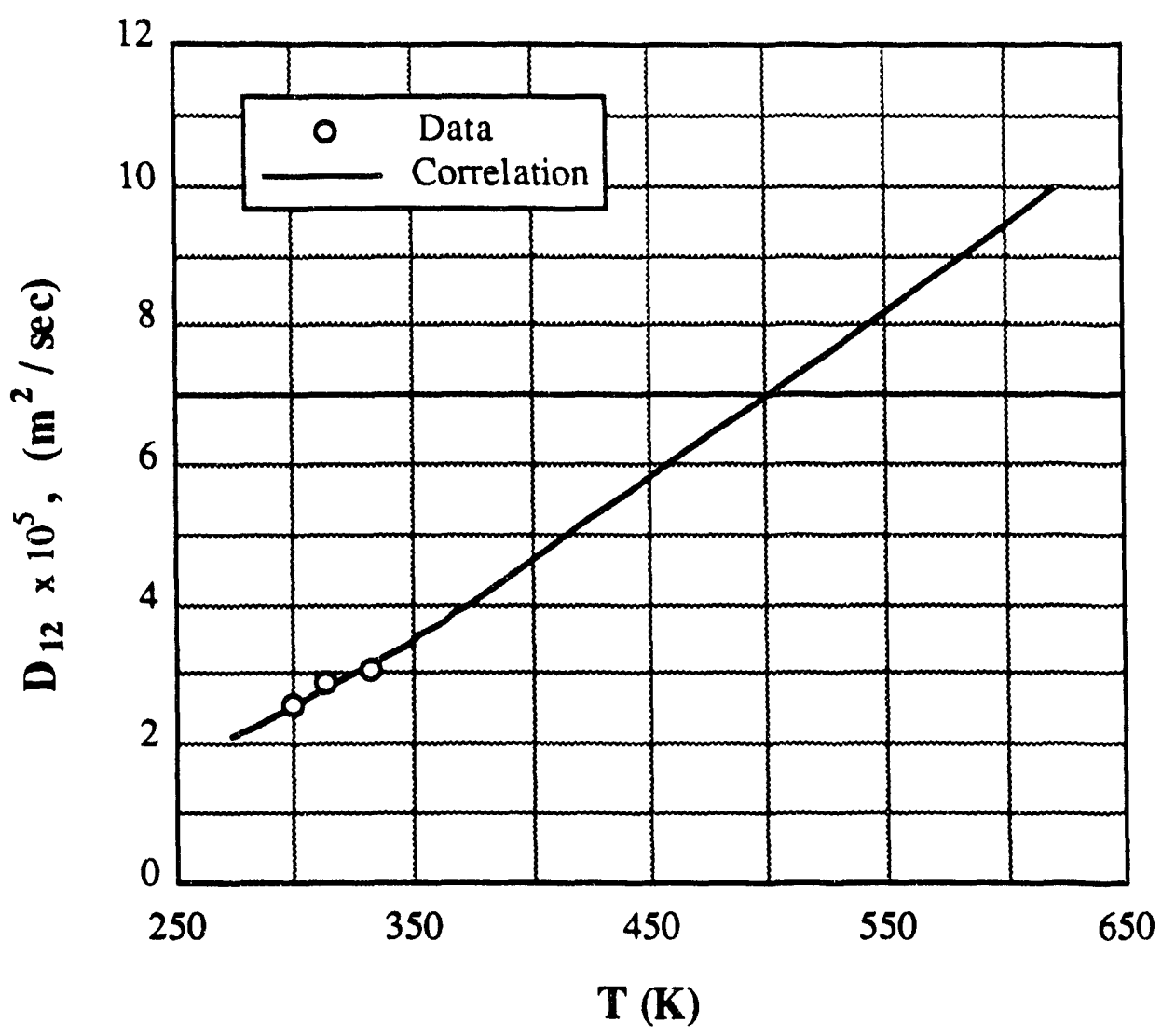



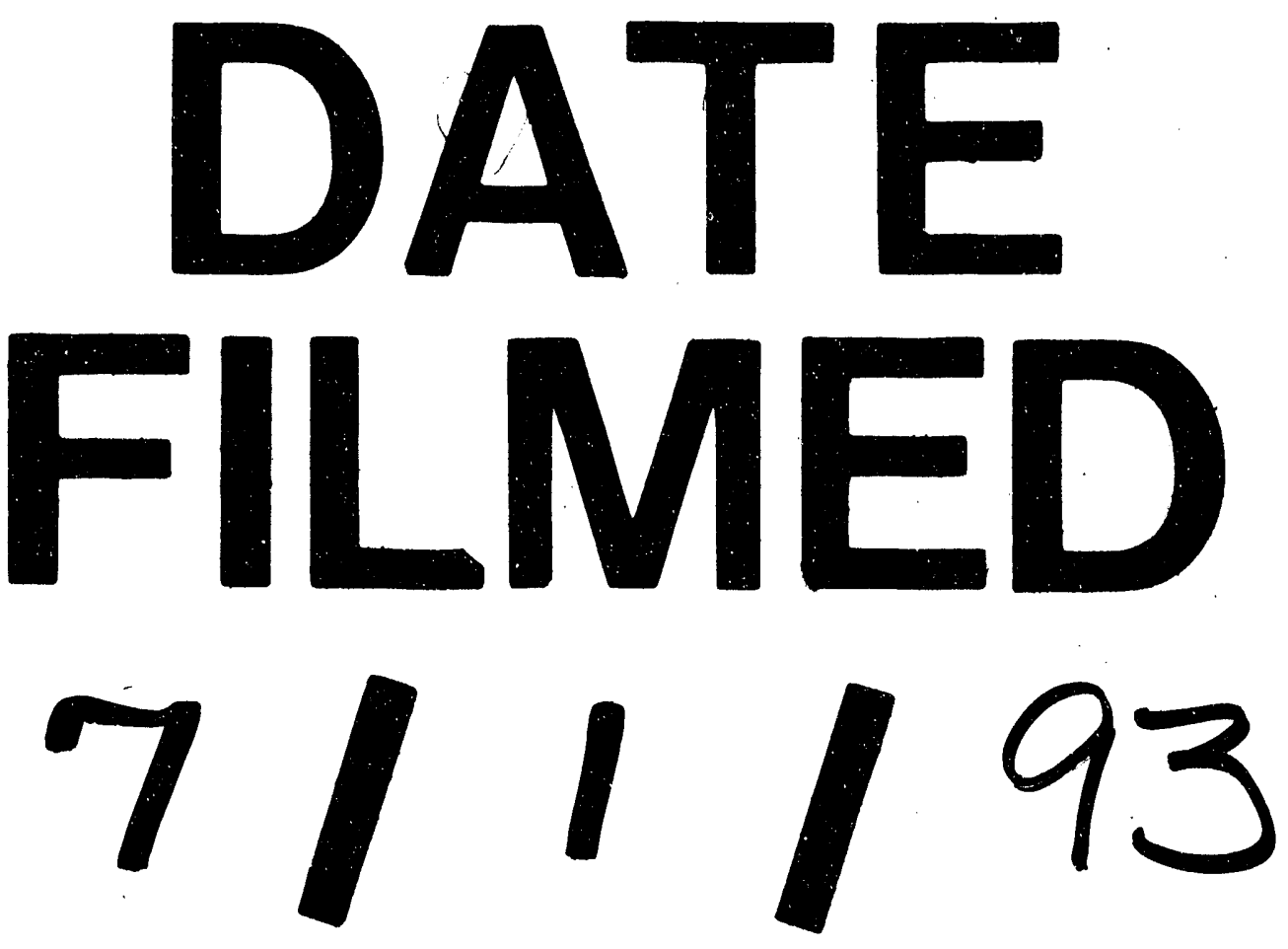
